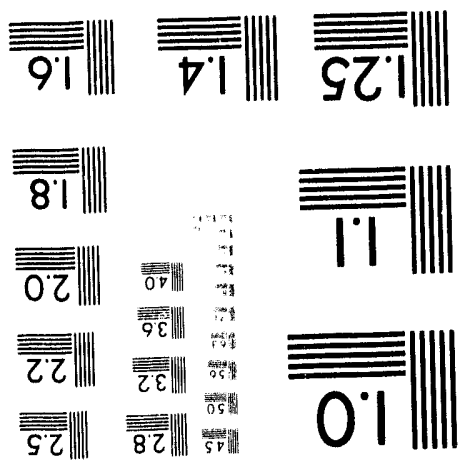



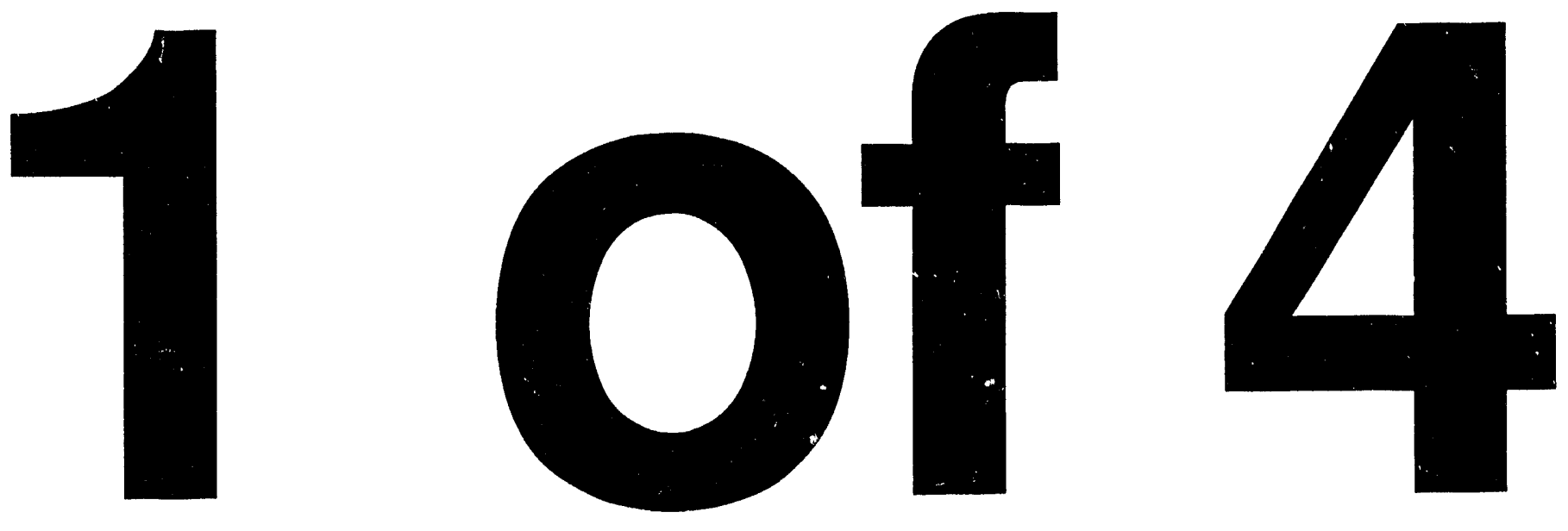


\section{Hanford Site Environmental Report for Calendar Year 1992}

Scientific Editors

R. K. Woodruff

R. W. Hanf

Technical Editor

R. E. Lundgren

June 1993

Prepared for

the U.S. Department of Energy

under Contract DE-AC06-76RLO 1830

Pacific Northwest Laboratory

Richland, Washington 99352 


\section{Preface}

U.S. Department of Energy (DOE) Order 5400). I, "General Environmental Piotection Program," establisher. the requirement for environmental protection programs. These programs ensure that DOE operations comply with applicable federal, state. and local environmental laws and regulations, executive orders, and department policies. The DOE. Richland Operations Office (RL), has established a plan for implementing this order. United States Department of Energy Richland Field Office Envirommental Protection Implementation Plam. Norember 9, 1992. 10 Norember 9. 199.3 (DOE 1992g). This plan is updated annually.

The Hanford Site Environmental Report is prepared annually pursuant to DOE Order 5400.1 to summarize envirommental data that characterize Hanford Site environmental management performance and demonstrate compliance status. The report also highlights significant environmental programs and efforts. More detailed environmental compliance, monitoring, surveillance, and study reports maly be of value: therefore, to the extent practical, these additional reports have been referenced in the text.

Although this report is written to meet DOE reporting requirements and guidelines, it is also intended to be useful to members of the public, public officials, regulators, and Hanford Site contractors. "at The Summary has been written with a minimum of technical terminology. The Helplul Information section lists acronyms, abbreviations, conversion information, and nomenclature useful for understanding the report.

This report is prepared for the RL Quality, Sallety. and Medical Programs Division as an activity of the Public Safely and Resource Protection Program, Pacilic Northwest Laboratory, Office of Health and Environment. Pacific Northwest Laboratory is operatted for DOE by Battelle Memorial Institute. Battelle Memorial Institute is a not-for-profit independent contratet research institute.

Incuiries regarding this report may be directed to the RL Quality, Salfety, and Medical Programs Division, P.O. Box 55(). Richland, Washington 99352, or to Pacific Northwest Laboratory, Office of Health and Environnient, P.O. Box 999. Richland. Washington 99352 .

(a) A brief general summary of this report in pamphlet form is also available by contacting the Pacific Northwest Laboratory at the address given above. 


\section{Summary}

The Hanford Site Environmental Report is prepared annually to summarize envirommental data and informatlion, describe envirommental management performance. and demonstrate the status of compliance with environmental regulations. The report also highlights major envirommental programs and efforts.

The report is written to meet reporting requirements and guidelines of the U.S. Department of Energy (DOE) and to meet the needs of the public. This summary hats been written with a minimum of technical terminology.

The following sections:

- describe the Hanford Site and its mission

- stummarie the status in 1992 of compliance with environmental regulations

- describe the environmental programs at the Hanford Sitc

- discuss public dose estimates from 1992 Hanford activities

- present intormation on eflluent monitoring and environmental surveillance, including ground-water protection and monitoring

- discuss activities to ensure quality.

More detailed information can be found in the hody of the report, the appendixes, and the cited relerences.

\section{The Hanford Site and its Mission}

The Hanford Site in southeentral Washington State is about 1,450 square kilometers $(560$ square miles) of semi-arid shrub-steppe located just north of the confluence of the Snake and Yakima tivers with the Columbia
River. This land, with restricted public aceess, provides a butfer for the smaller areas historically used for the production of nuclear materials, waste storage, and waste disposal. About $6 \%$ of the land area has heen disturbed and is actively used. This $6 \%$ is divided into operational areas:

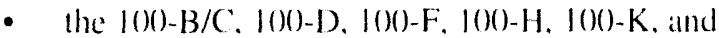
1()()-N Areas, which lie along the Columbia River in the nerthern portion of the Hanford Site

- the 2()()-East and 2()()-West Areats, which lie in the center of the Hanford Site near the basalt outcrops of Gable Mountain and Gable Butc

- the 300 Area. near the southern border of the Hantord Site

- the 400 Area, between the 300) and 200 Areals fhome of the Fast Flux Test Facility (FFTF) |

- the 1100 Area a corridor northwest of the city of Richland used for vehicke maintenance and onther support activitics.

The 6()() Area is the designation for land between the operational areas. Areas used for research and development and administrative functions can be found in Richland, Kennewick, and Pasco, the nearest cities.

The Hanford Site was aceuired by the federal gevernment in 1943 and was dediciled for many years primalrily to the production of plutonium for national defense and the management of the resulting wastes. With the shutdown of the production facilities, missions were diversified to include research and development in the areas of energy, waste management, and environmental restoration.

The DOE has ended the production of nuclear materials all the Hantord Site for weapons. The mission being implemented by the DOE. Richland Operations Office (RL.), includes: 
- wastc management

- environmental restoration

- research and development

- technology development.

Current waste management activities at the Hanford Site include primarily managing wastes with high and low levels of radioactivity (from the nuclear materials production activities) in the 20()-East and 200-West Areas. Key waste management facilities include the waste storage tanks. Plutonium Uranium Extraction (PUREX) Plant, Plutonium Finishing Plant, Central Waste Complex, Low-Level Burial Ground. B Plant, and 242-A Evaporator. In addition. used nuclear fuel is stored in the 10()$-\mathrm{K}$ fuel storage basins.

Envirommental restoration includes adtivities to decontaminate and decommission facilities and to clean up or restore inactive waste sites. The Hanford surplus facilities program conducts surveillance and maintenance of such facilities, and has hegun to clean up and dispose of more than 100 facilities. Current activities include decommissioning of the $\mathrm{CX} 7($ )-tanks (strontium semiworks) and preparing the 18.3-H Solar Evaporation Basins for Resource Conservation and Recovery Act (RCRA) closure.

Rescarch and technology development activities are also conducted on the Hanford Site in the 200, 300, and $4(0)$ Areas and an administrative area south of the Hanford Site boundary. Many of these activities are intended to improve the techniques and reduce the costs of waste management, environmental protection. and Site restoration.

Operation and activities on the Hanford Site are managed by RL through four prime contractors and numerous subcontractors. Each contractor is responsible for the safe, envirommentally sound maintenance and management of its facilities and operations, waste management, and monitoring of operations and effluents for environmental compliance.

The principal contractors include:

- Westinghouse Hanford Company

- Battelle Memorial Institute
- Kaiser Engineers Hanford

- Hanford Environmental Health Foundation.

Non-DOE operations and activities include commercial power production hy the Washington Public Power Supply System:s WNP-2 Reactor (near the 40() Area) and commercial low-level radioalctive waste burial at a site leased and licensed by the state of Washington and operated by U.S. Ecology (near the 200 Areas). Siemens Nuclear Power Corporation operates a commercial nuclear fuel labrication facility, and Allied Technology Group Corporation operates a low-level radioactive waste decontamination, supercompaction, and packaging disposal facility adjacent to the southern boundary of the Hanford Site.

\section{Compliance With Environmental Regulations}

The DOE Order 54(0).1. "General Environmental Protection Program," describes the environmental standards and regulations applicable at DOE facilities. These environmental standards and regulations fall into three categories: 1) DOE directives, 2) federal legislation and executive orders, and 3) state and local statutes, regulations, and requirements. The following subsections summarize the status of Hanford's compliance with these applicable regulations and list envirommental occurrences for 1992.

A key element in Hanford's compliance program is the Hanford Federal Facility Agreement and Consent Order (Tri-Party Agreement). The Tri-Party Agreement is an agreement among the U.S. Environmental Protection Agency (EPA), Washington State Department of Ecology (Ecology), and DOE for achieving the compliance with the remedial action provisions of the Comprehensive Environmental Response, Compensation, and Liability Act (CERCLA) |including Supertund Amendments and Reauthorization Act (SARA) | and with treatment. storage. and disposal unit regulation and correstive action provisions of RCRA.

\section{Compliance Status}

This section summarizes the activities conducted to ensure that the Hanford Site is in compliance with envirommental protection regulations. 


\section{Comprehensive Environmental Response, Compensation, and Liability Act}

The CERCLA established a program to ensure that siles contaminatled by hazatrdous substances atre cleance up by responsible parties or the government. The SARA broadened CERCLA and established provisions lor lederal facilities.

The preliminary assessments conducted for the Hanford Site revealed approximately 1.100 known individual waske sites where hazardous substances may hate been disposed of in a manner that requires furlhere evaluatton (o) determine impact on the enviromment.

The 1$)$ ()t: is actively pursumg the remedial investigattion/ featsibility study (RI/FS) process all some operable units on the Hanford Site. The selection of the operable units currently under investigation is a result of Tri-Party Agreement megotiations. All milestenes related to the RI/fS process established for 1992 were achieved, and the Hanford Site was in compliance with these (CRC I.A/ SARA repuirements. This takes into consideration several milestones delatyed through the change request process.

\section{Emergency Planning and Community Right-To-Know Act}

The Emergency Planning and (ommunity Right-ToKonw Act provides the public with information about hatardous chemicals in the community and establishes emergency plamning and notification procedures lo protect the public from a releatse. Subtille $A$ of the law calls for creation of stale emergency response commissions fo gutele planning for chemicall emergencies. Stalle commissions have also crealled local emergency planming commitles to ensure community participation and planning.

To provide the public with the basis for emergency planning, Subtitle B of the Act contains requiremente for periodic reporting on hataldous chemicals stored and/or used near the community. The 1992 Hemford Tier Tines

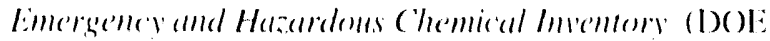
199.3a) was issued to the State Emergency Response ('ommission. local county emergency management committess, and local lire department. The report contained information on hazardous malerials in storage

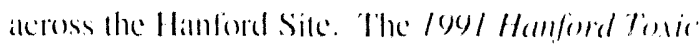

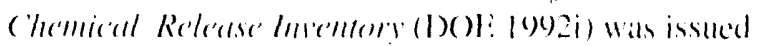
July 1. 1992, wh the I:PA and the stalle. This report confaims intermattion on releases to the enviromment of elemicals that were used in exeress of mandated hresholds. Accordingly, during /092. the Hanford Site wats in compliance with the reporting and notification recuirements comtained in this $A \mathrm{c}$.

\section{Resource Conservation and Recovery Act}

The RCRA establishes regulatery standards for the gencrattion. tramsportation. storage, trealment, and dispesal of havadous waste. Feology has been atuthorized by the I:PA to implement its dangereus waste program in lieu of the l:PA for Washinglon State. exeept for some provisions of the Hazaldous and Solid Waste Antendments of lo84. Deology also implements the stale' regulations. which are ofien more stringent.

Al the Hanford Sile. approximaltely 6.3 treatment, storage. and disposial units have been identified that must be permitted or closed in aceordatree with RC RA amb Washington State regulations. These units are reguired 10 operalle under Ecology s interim-slatus compliance repuirements. Approximately onc-half of the units will be clused.

The Tri-Party Agreement provides the famework for meeting RCRA reguirements. ()f the f(0) milestomes scheduled for lo92. 96 were completed, although some were delayed as approved through the change request process. At the end of 1092, 2.34 Tri-Pitly Agrement milestones hatd heen completed on or ahead of schedule over the previous 3 years.

During 1992. Ecology issmed six moncompliance letters to RI for Hanford contractors for alleged violations on watste management requirements.

A Part B permit applicallon for the Hanlond Site Was issted for public comment in January 1992. Comments were received from licology. Responses to these comments were submilted to DO OE-Headegtarters (HQ) for fimal review. No comments have been received from HQ. Twenty-six ground-water monitoring wells were constructed at seven RCRA treatment, storage, and disposal facilities in 1992. This satisfied Tri-Party Agreement milestone M-24-()0. 
Subtilk I of RC'RA deals with regulation of underground storage lanh systems. These regulations were atded lo RCRA by the Hazalldous and Solid Waste Amendments of 1984. The I:P'A hats developed regulations implementing kechicical standards for tank performance and maln-

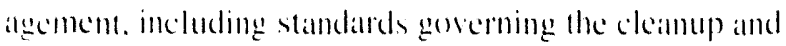
closure of keaking tanks. These regulations do not apply (1) the single-and double-shell muckear waste tanks, which are regulatted as treatment. storigere and disposal filcilities.

\section{Clean Air Act}

The purpose of the (leian Air Act is lo protect public heallh and wellate by salfeguarding air cuality. hringing polluted air into compliance. and prolecting cleall air from degratattion. In Washinglon State. the provisions of the Act ate implemented by l:PA. Washington Stalle Department of Health (D)H), and locial air atuthoritices.

The Hanlerd Site in operated under a Prevention of Sig-

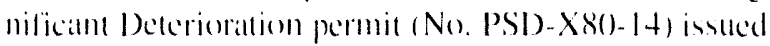
by the LPA in 1980. The permit sets specifie limits for cmissions of nitrogen oxides from the PUREXX and Uranium Trioxide (10), Plants.

The DOH, Division of Radiation Prolection. Air Limissions and Defense Waste Section, hats developed regutatory controls for radionative air emissions under Section 116 of the ('lean Air Act. Washington Slate regulations |Watshington Administration Code (WAC') 2+6-2+7)] require registration of all radioactive air emission point sources with the D()H. All significint Hanferd Site slacks emilting raddiation hate been registered in atcordance with applicable regulations.

Revised Clean Air Act requirements for radienative air emissions were issued December 15. 1989, under Nattional Emission Standards for Hazardous Air Pollutants (NESHAP), 40 Code of liederal Regulations (CFR) 61. Subpart H. Emissions from the Hanford Sile are well within the new EPA orfsite emissions standand of 10 millirem/year leffectise dese equivalent sece Appendix B. "(ilensary")|. However. Hanlourd Site sources de not yed meet the new procedural reguirements for flow measurement. emissions measurement, puality assurance, and simpling documentation. The Patcilic Northwest Latholattory completed thee emission-pointsampler upgrades to comply with sampling and flow rate measurement reguirements of the Cleall Air Act.
The I:PA insted al compliance order and inlombation reguest to RL. on the basis of noncompliance with the NESHAP. The compliance order reguires RI. Io comply with NESHAP Subpant H in the following manner:

1. evaluate all raddionuclide emission points on the Henford Site

2. measure continuous emissions where applicable.

A plan lo describe to the l:PA how RI. will comply is heing written. It will be submilled lo EPA hy April 30. (4) 3.3 .

Pursuant to the NISSHAP program. LPA has developed regulations ypecilically addressing ansestos emissions (4) CFR 61. Subpart M). These regulations alpply at the Ilanferd Site in building demolition/disposal and watse disposil operations. During 1992.998 cuhic meters (1,30.5 cubic yalds) of anbeston were remosed.

The local air atuhority, the Tri-Countien Air Pollution Control Authority. enforesen (ieneral Regulation 80)-7. This regulation pertians to detrimental elfects. fugitive dust. incineration products, odor, opicity. asbestos. and sulfur oxide emissioms. The Authority has also been delegilled responsibility to enfored the EPA anbestom regulations under NISHAP. The Site remains in compliance with the regulations.

Hanford Site contractors prepared Fateility l:ffluent Monitoring Plans (Fl:MPs) in I09) specific to sartous facilitien acress the Site. The FEMPs include sections that outline compliance with 40 ( CFR 61 (ambiem air emissions). A summary of eatch likMP has been incorporated into a Sitewide convironmental monitoring plan covering colluent monitoring and envirommental surveillince. The Westinghouse Hanford Company FlaMPs were revised in 1992.

\section{Clean Water Act}

The ('lean Water Act applies to all dischatrges lo watlers of the Uniled States. At the Hanford Site. the regulations are alpplied hrought a Niltional Pollutant Discharge Elim-

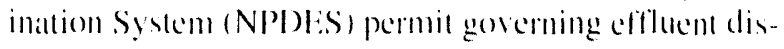
chatrges to the Columbia River. The NPIDLS permit

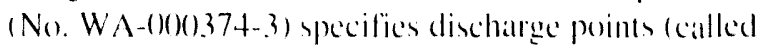
oultalls, of which there alre eight). cflluent limititions. and monitoring reyuirements. 


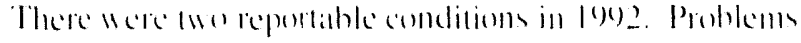

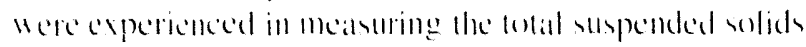

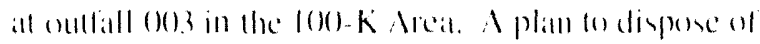
the cllluem to an allermative sile in heing evaluated.

The yuatery limis lon irom was exceded in the N Springes sulfall. The escedance was calused hy a huildup of iron in at genund watter moniloring well. The well had not heell purged since the previous simpling. allowing the buildup.

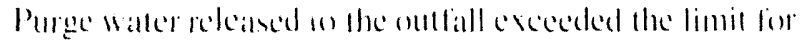
irom. The well was repurged and resimpled. No limits ware exceceded atter repurging.

\section{Safe Drinking Water Act}

The Nallinnal Primally Drinhing Walce Regulations of the Salie d)rinking Walter Act apply lo dhe drinking Walle supplies all the Hantord Site. These regulations are entenced

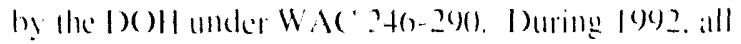

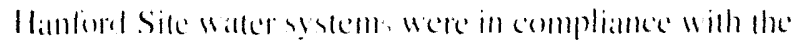
reyuirements of the applicathe regulations.

\section{Toxic Substances Control Act}

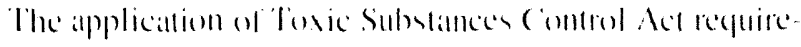

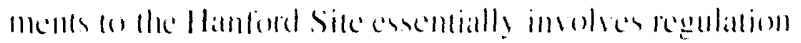

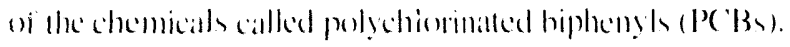
The llantord Site is correnty in compliance with regenta

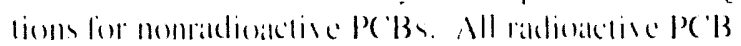
Wastes atre being stored whlh FPA alpproval. pending

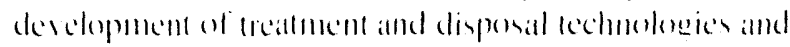
cappobititices.

\section{Federal Insecticide, Fungicide, and Rodenticide Act}

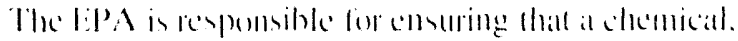

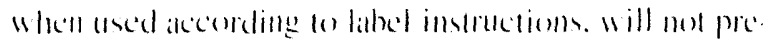

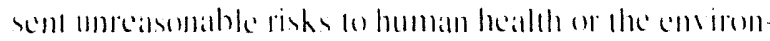
ment. This Act and the Revesed coke of Washingenten IRCW, 17.21. "Warhingen Pesticide Applicallion :Act."

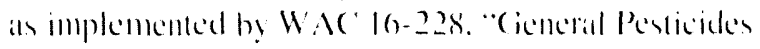
Regulatioms." apply to storagere and ane of pesticides. The Hanford Site is in compliance with the Acl"s regutements

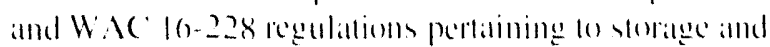
applicintion of persicides.

\section{Endangered Species Act}

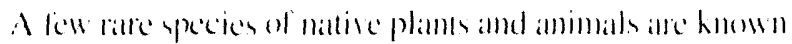

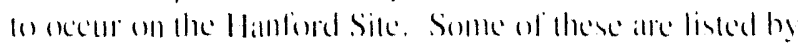

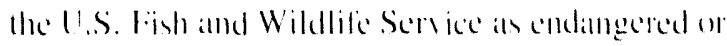

dheallened (lederally liskeds. (Mhers alle lisked by dhe Washinglon Slate o department of Wildlite as endangered.

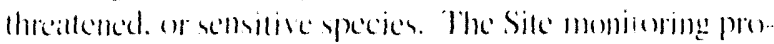
glam is discussed in Section t.2. "Wikllite." Hamford

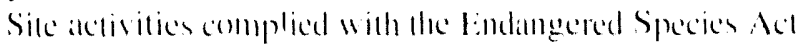
in $19(9)$ ?

\section{National Historic Preservation Act and Archaeological Resources Protection Act}

Cultural resources on the Hanlond site are subject to the provisions of the Nattomal Historic Presemation Act and the Ardateological Resources Protection Act. Compliallece with these Ack is alecemplished throegh a monitor-

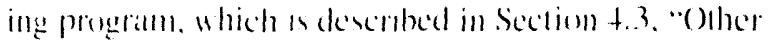
fim irommental Studies and Programs." In I092. Hantord Sile opreations complied will these Acts.

\section{National Environmental Policy Act}

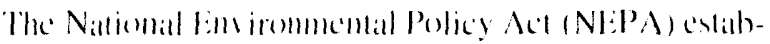
lisher envirommental policy an prevent of climinate dam-

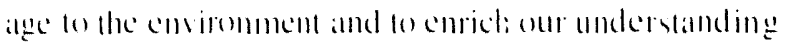

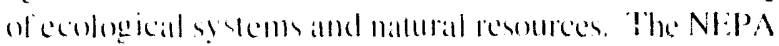
requires that matjor federall projects with signilicant impaces be carchilly revicued and reported to the public

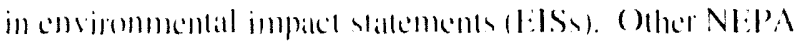

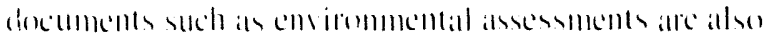
prepared in accordance with NI:PS repuirements

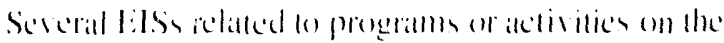

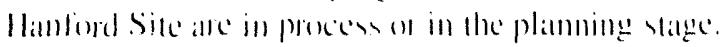
These atre:

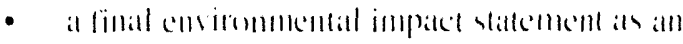
addendum on the decommons boning of eight

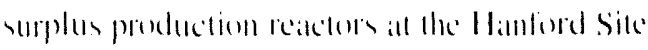

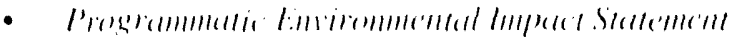

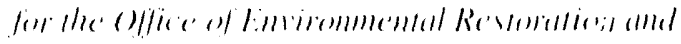

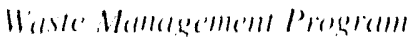

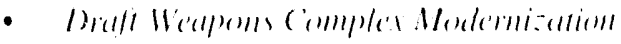
Prostermmantic lis.

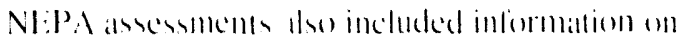

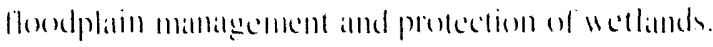

\section{Environmental Occurrences}

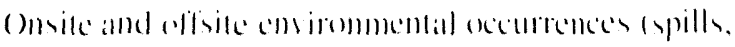

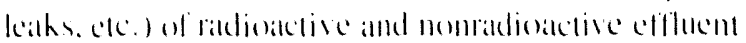

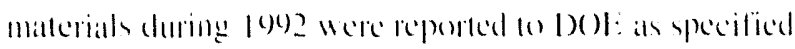




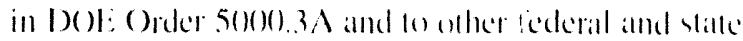
agencies as required by law. All emergency, unusual. and off-normal aceorrence reports. including erent descriptoms and corrective actions. ance al aibable for revien in the Rl. Public Reading Room. Richland. Washinglon. There were no emergency aceurenees reported in 1942. There were 1.5 .31 off-mormal ens iromemental ocementere reports filed al the Hanlord Site during 1902. covering everylhing from leaks from onerbealted motor vehick cooling systems to leaking waste oil drums. Becatuse of the volume of reported off-mormal eceurrences. exent stmmaries atre mot included here.

The 1902 untustal aceorrences with the most potential for envirommental impact and their ocentrence numbers ire:

- Release of (inmaminated Watte to the ciround (RI-KI:H-|(1)) 2-(1)(0)

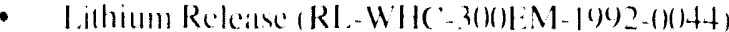

- Waste Tank leak rRI.-WHC:TANKIARM (1) $2 .-(1)(173)$

- Discharge on the (olumbia Riser (RI-Whe"NRI:AC"T()R-10()-(1)()6I)

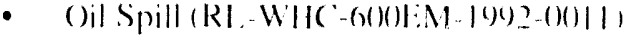

- Radiation läah IRI-WIIC TANKI:ARMI(1)92-(10)74)

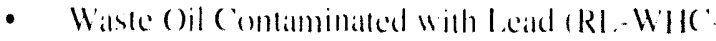
TPI.ANT-1902-(1)181.

\section{Environmental Programs}

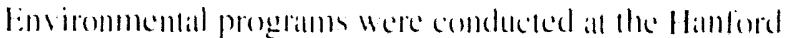

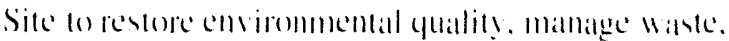
derelop approprialte technologe for ckeanup atclivities. and study the enviromment. These progerams are discorsed helows.

Wildlife inhathiting the Hanford Site is monitored lo delermitce the stattus and comdition of the populations, alld to

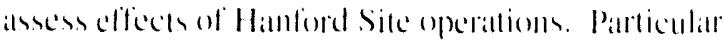

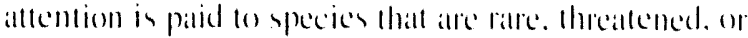
endangered nationally or statew ide and those specters that alle of commerciatl. recreational, or ateshedic importance

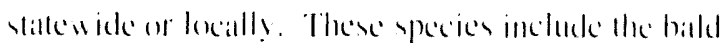

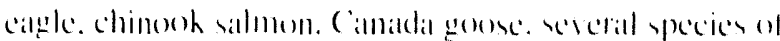
hank. Rock! Mommain clk. muk deer. white pelicall. and onher bird species

fluctuations in wildlife and plann ypecies an the Hanford

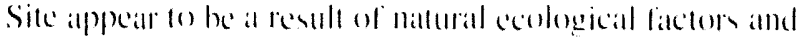
matnagement of the columbial Riser sylem. The estahlishment and mallagement of the Hantorel Site has hate al

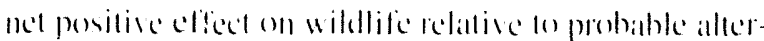
nallice uses of the Sile.

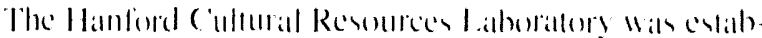
lished by RI, in lo87 as part of the Pateilic Nonthwes

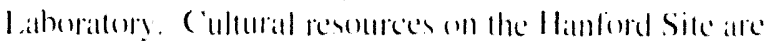

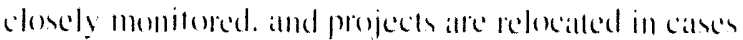
where there is a posibility of allering an! signilicant historicial siles.

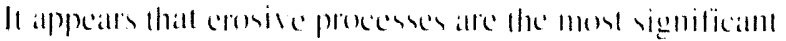

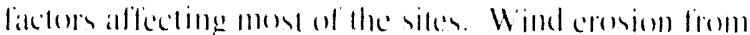

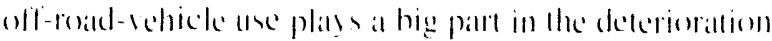
of sites imside and outside of the secorits perimeder.

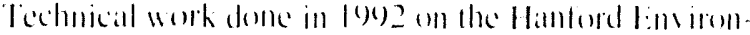

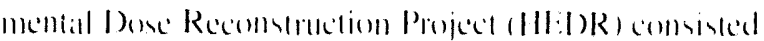

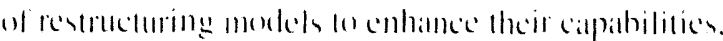

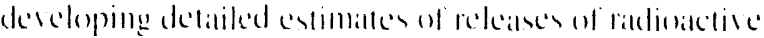
materials. and evaluatting adelitiomal indormation meeded to prodlecérlimitles

The commmonity - -nperated em irommental surveillances

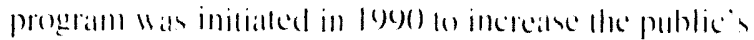

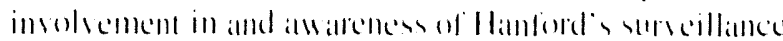

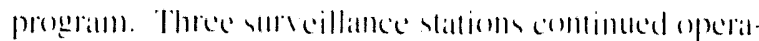
lion in 190?

An edeatlon outreateh program estahlished with the Yahima Indian Nation in l(x)| was continned in ley?

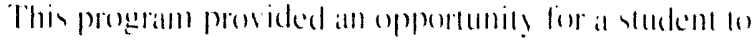
stads Columbia Riser waller yualits and fish heallh and

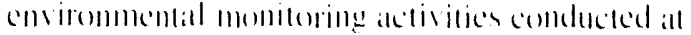
Hanlord.

\section{Environmental Monitoring Information}

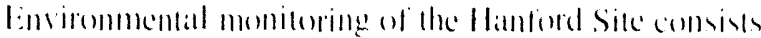
of l effluent montering and 2) emsirommental surveit

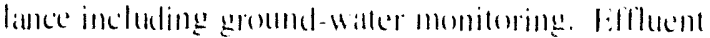


monitoring is pertormed as appropriate by the Sile ficility operatom all the fatcility or at the point of release to the enviromment. Additional monitoring is condected in the emviromment near fateilities that discharge or hate discharged effluents. Envirommental surseillance comsists of sampling and antly $/$ ing convirommental media on and wif the Hanford Site lo detect and quantily ponential contaminants. and to assest their emsirommental alld human healh significances.

The onerall objectives at the moniloring and surseillance programs are 10 demomstrate compliance with lederal. state. and local regulations: confirm adlerence to l)( )l: emvirommental prolection policies: and support emsironmental mambement decisioms.

The following sections discuss the desces calculated form

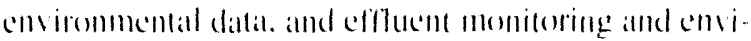
rommental surseillatsec an or trear the Hamford Sile in 14) 2 .

\section{Potential Radiation Doses from 1992 Hanford Operations}

In 1092. ponemtial public doses resulting from exposure (1) Hantord liquid and gasenus effluents were evalualed 10 determinte compliance with pertintent regulations and limis. These doses nere calculated from repented edthe ent rekatses and envirmmental surveillance dalla using

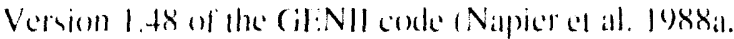
1988b. 19880 and Hanford Site-specilic patrameders. Specilic intormation on sample collection and allalyser

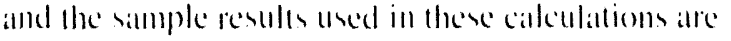
hrielly discussed in the summary sectioms discossing cifluent monitoring and envirommental surveillance.

The potential dese to the hypothetical maximally exposed

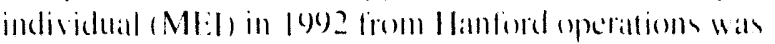

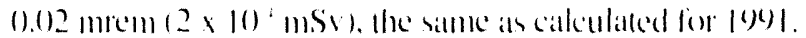

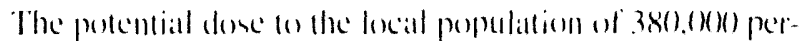

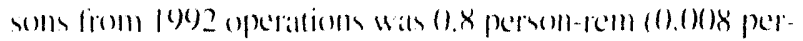

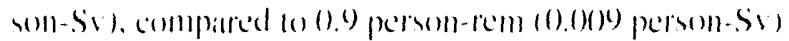

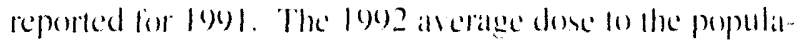

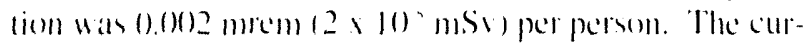
rent 1)( ) : raddiation limil for an individeal member of the

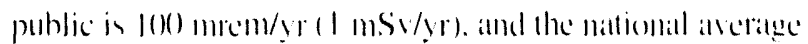

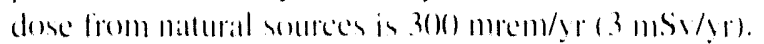

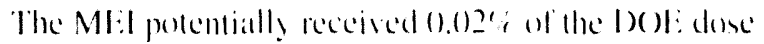
limit and (1).(0)7\% of the national allerage bateheround dose from matural soureses. The arserage individual

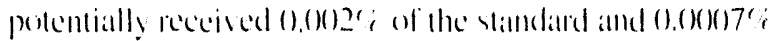

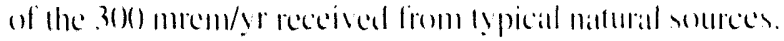

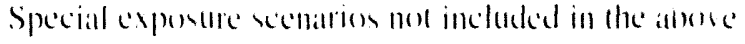

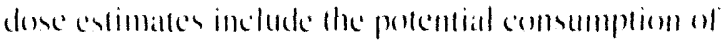
gantere residing on the llantord Site and exposure on radiallon all the puhlicly alcesessible location with the maximum exposture rate. Doses firom these soures would also hare been small compared to the dose limil.

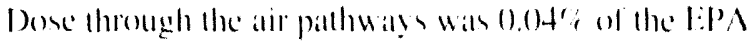
limil (H) (TR Ol).

In addition to the doses estimblled from monitored statch releases, the potential maliation dose to the Mlt lom

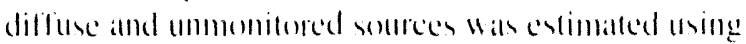

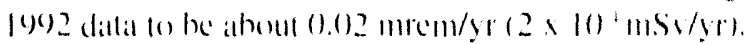

\section{Effluent Monitoring}

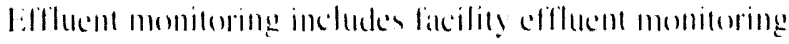

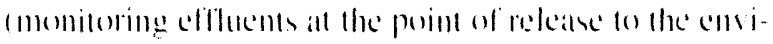
romment) and neall-ficility ens irommental monitoring

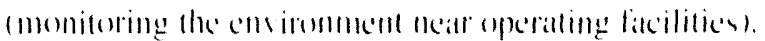

\section{Facility Effluent Monitoring}

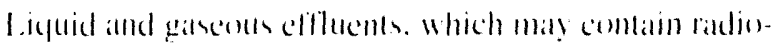
active and hatatrdem comsthents. are continually moni-

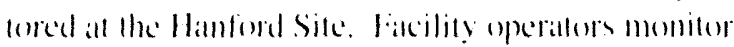

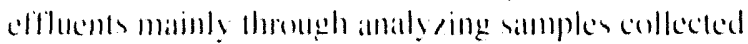

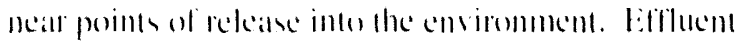
momitoring datta are evaluated to determine heir degres of compliance with alpplicahle lederal. statce and local regulations and permits.

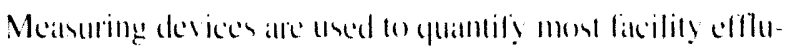
ent flows, with a smaller number of flow s calculated osing

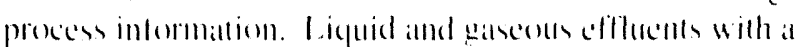

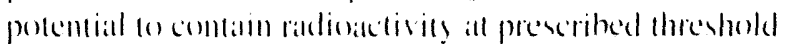

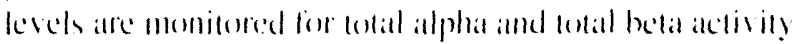

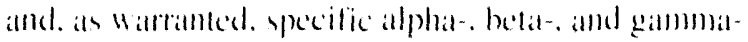

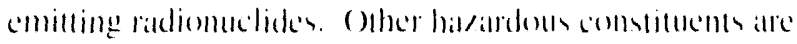
also monilosed. an appliciole.

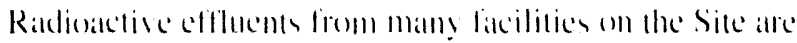
approaching levels practically indistinguishathe from the comtributioms of natural backeround radionctivit!. The new Site mission of envirommental resteration rather than

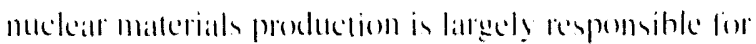

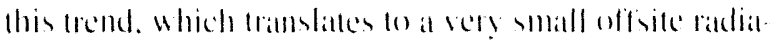

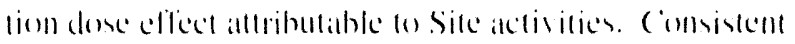

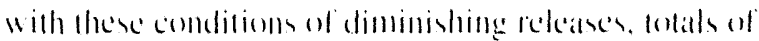

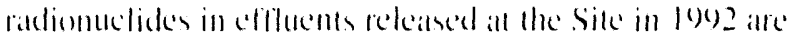

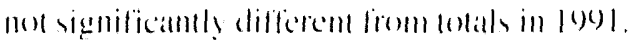




\section{Near-Facility Environmental Monitoring}

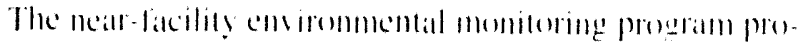

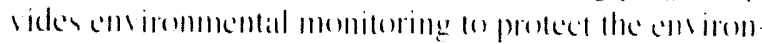
ment alliacent to lacilitice and ensure compliance with

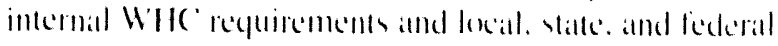
'mo irommental regulations.

Specilically. the near-facilits ems irommental monitoring program monitored new and existing siles. processes.

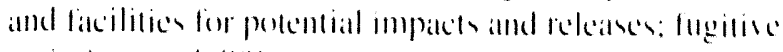
embsions and diffuse sources form contaminatled arreas: and surplus lateilities hefore decomlanminating an decom-

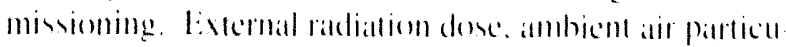

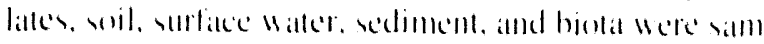

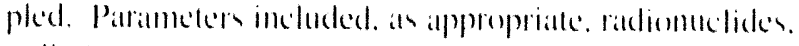

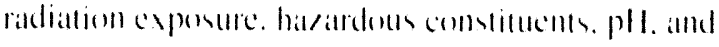
walter kemperillure.

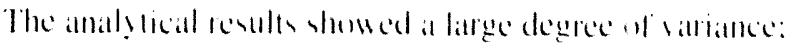

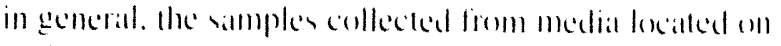

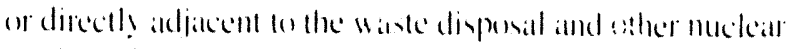

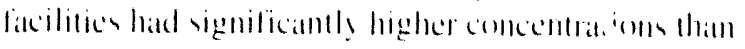

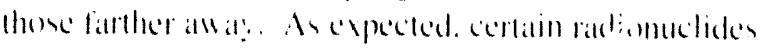

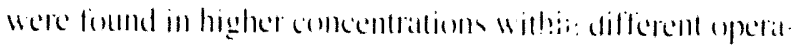

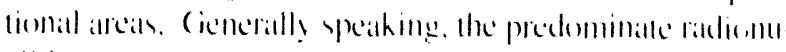

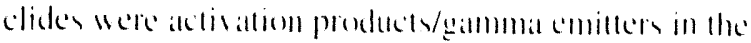

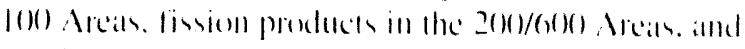
uranium in the $3(1)$. Nerat.

Air Monitoring. Raldualctis its in ail wals sitlupled by a

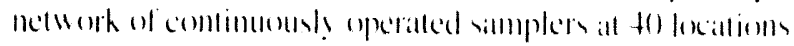

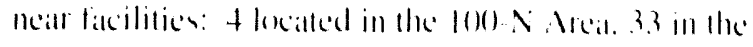

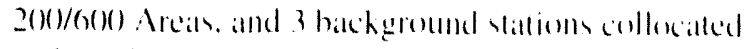

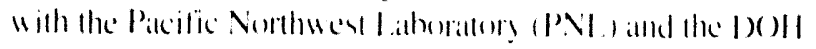
all the Yakima and Wye Barricatere and the old Hantend

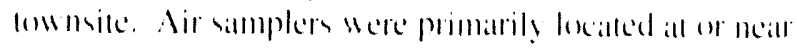

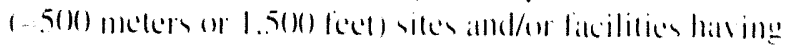
the polential or history for reckatse. With all comphasis an

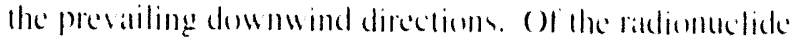

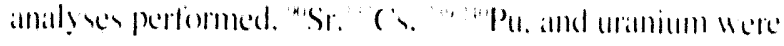
comsistenty delectable in the 200) Areas: "( 11 wats detect-

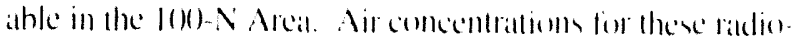

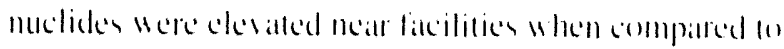
the concentrattoms measured ofleite by PNo.

\section{Monitoring of Surface-Water Disposal Units and}

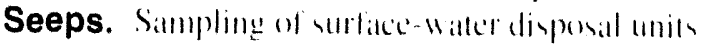
included waller. sediment. and alyuallic kegetattion. Samb.

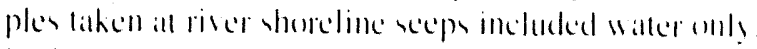
Radiological analy sio of licpuid samples from surliase.

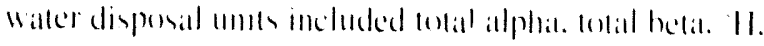

"Pu. and gammateminting radionuclides. Radiolegei

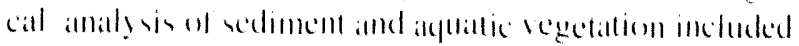

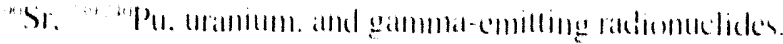

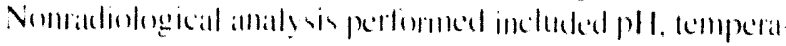
turce, allnd nillatles.

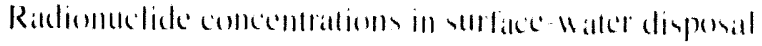
whits were below the applicable Derived comcentratlon

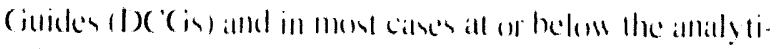

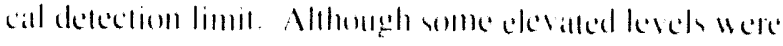

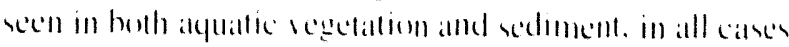

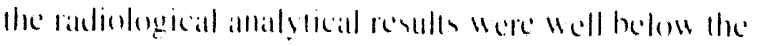

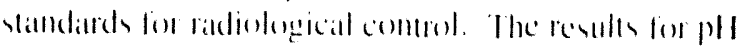
were well withim the pll of 2.1 and 12.5 vallatad for

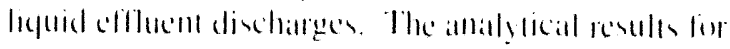

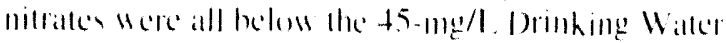
Slamdard (I) IS Si.

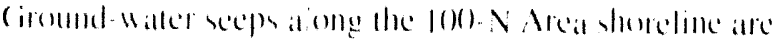

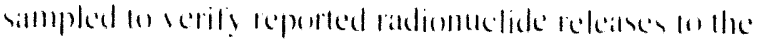

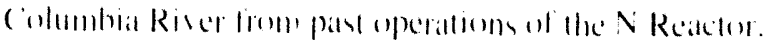

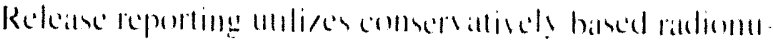

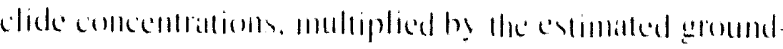

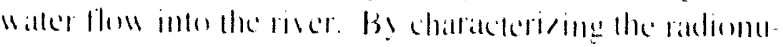

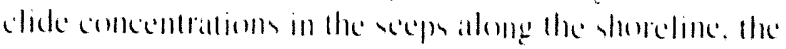

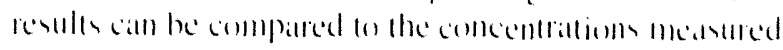

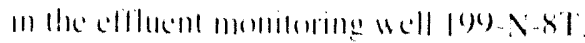

In 1092. the concentrations delecked in the seop samples

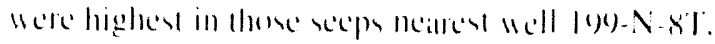

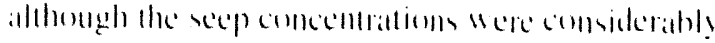

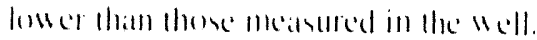

Radiological Surveys. There werc alpmmimalely

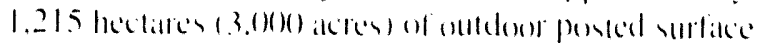

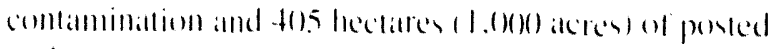

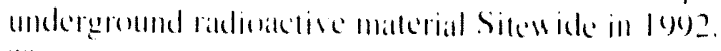

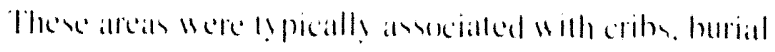

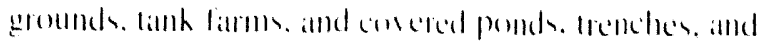
ditches. The number of poused surfiece contamination

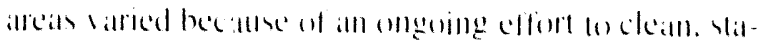
hilise. and remedialle arcas of hmown contamminallon while me" ancal of comtamination were heing identified.

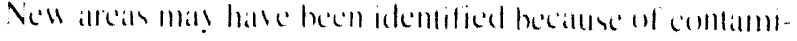

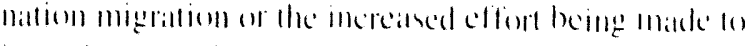

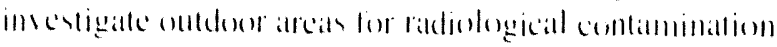

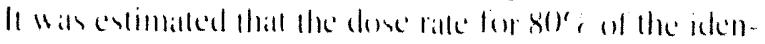

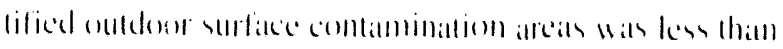
I millirem/hom. allhomgh indalled yechs comld be comsiclerably higher. (ambammation levels of this!ype would

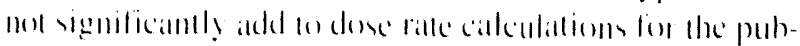
lic ar Silc empluseres. 
Soil and Vegetation Monitoring Suil illid leptlil

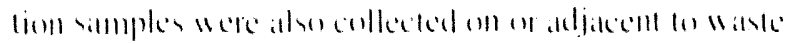

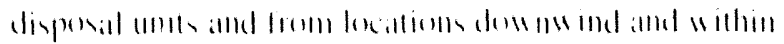

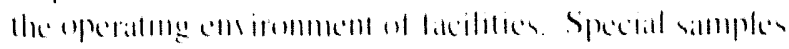

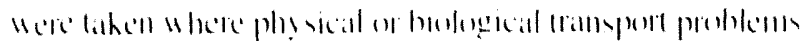

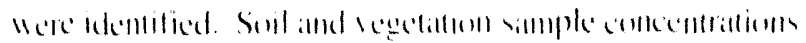

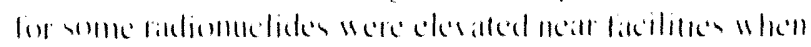

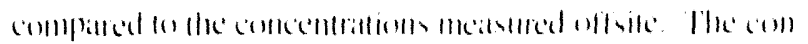

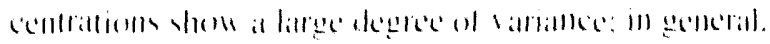

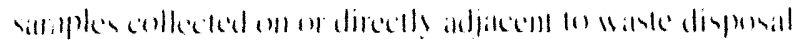

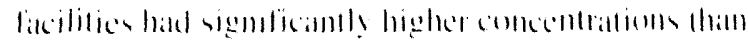
Hure farther all

External Radiation. I: vernal radiation lich were

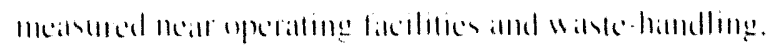

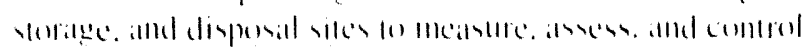

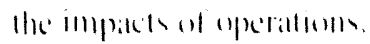

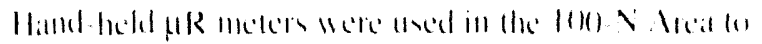

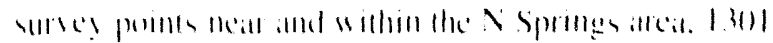

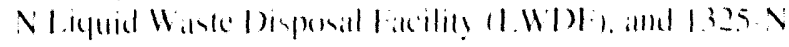

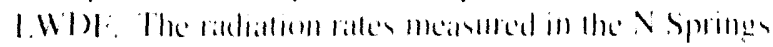

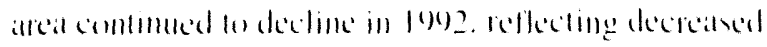

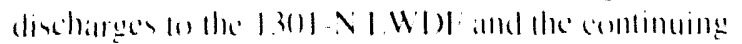

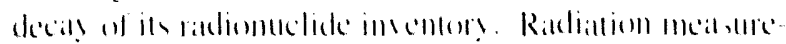

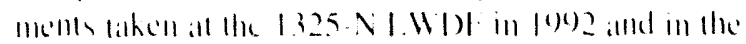

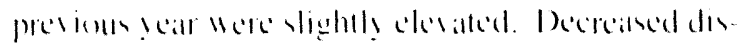

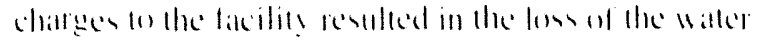

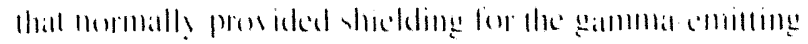

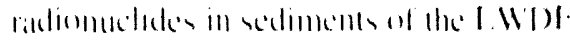

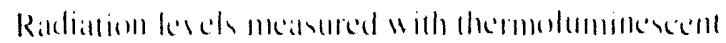

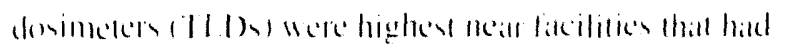

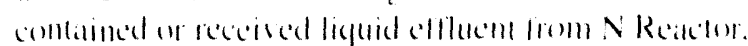

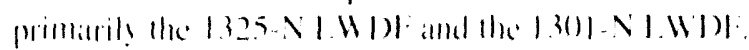

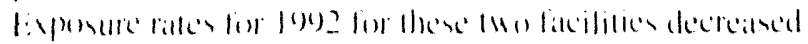

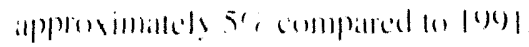

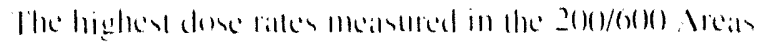

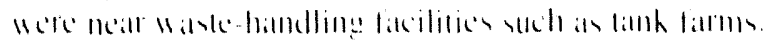

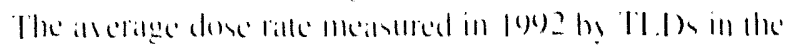

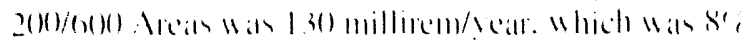

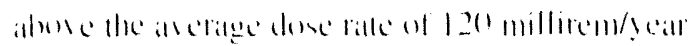
mkiatured in |(x)

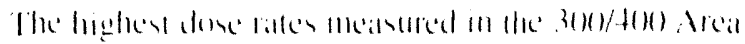

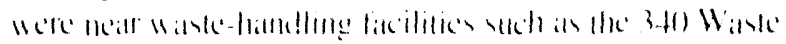

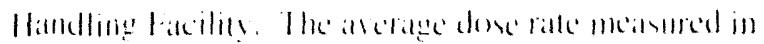

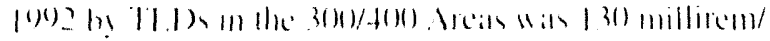

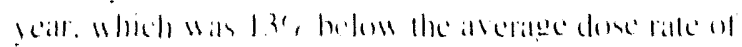

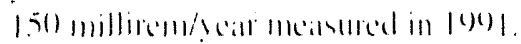

\section{Environmental Surveillance}

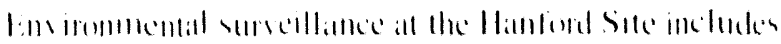

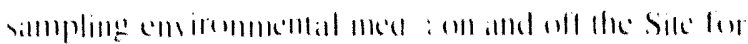

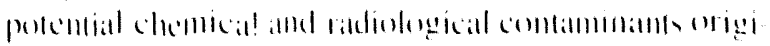

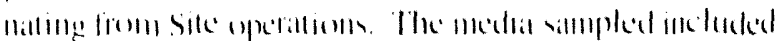

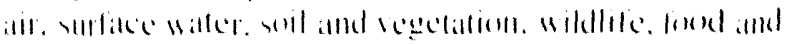

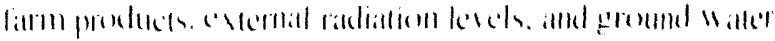

\section{Air Surveillance}

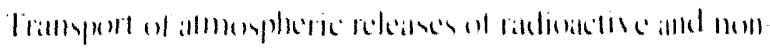

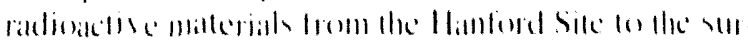

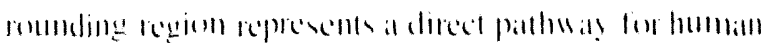

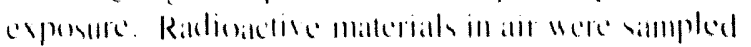

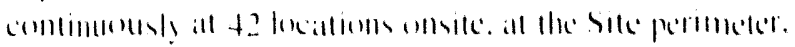

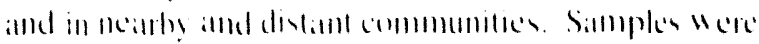

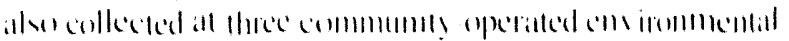

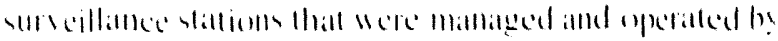

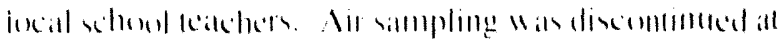

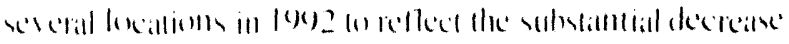

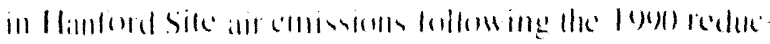
fion in (1)

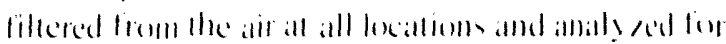

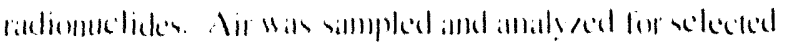

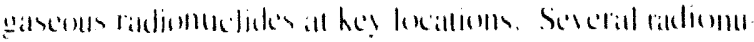

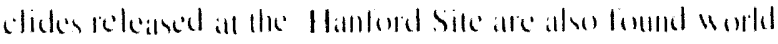

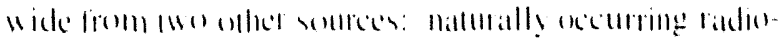

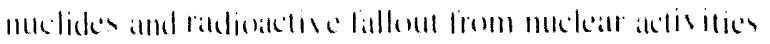

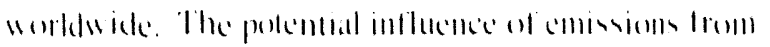

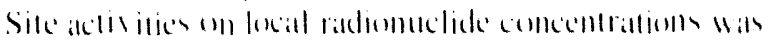

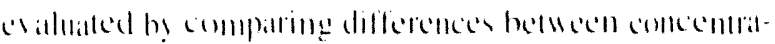

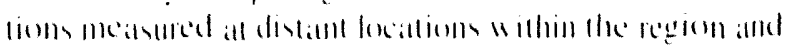

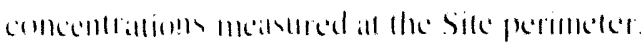

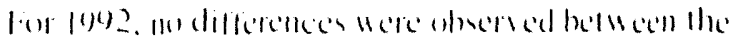

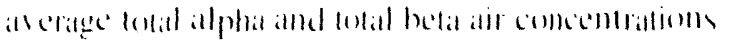

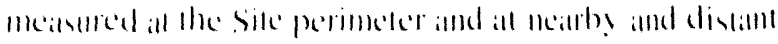

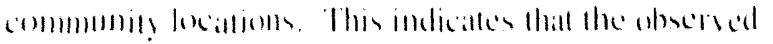

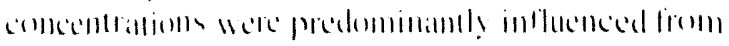

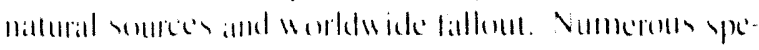

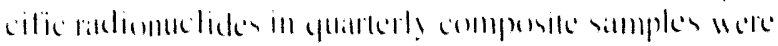

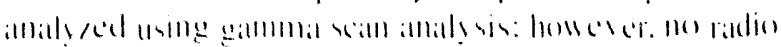

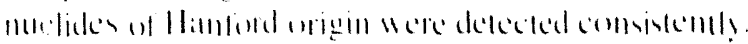

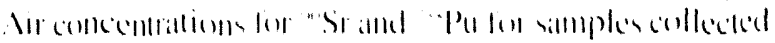

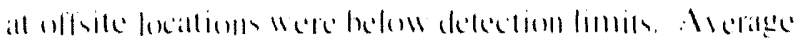

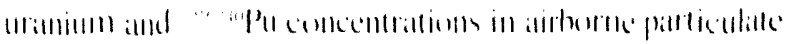

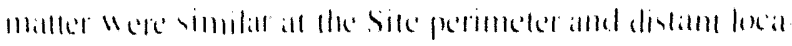

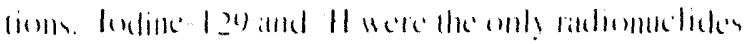

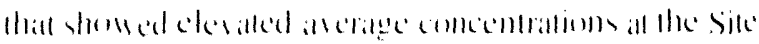


perimeter relative to the distant locitions. Tritium samples collected from January to May 1902 may have been contaminated during the analytical process because most focations including the distant communities reported unusually high concentrations. From June on December 1992 the average sample results for 'H returned 10 normal concentrations with litte difference between the distant locations and the Site perimeter. Average concentrations al the Sile perimeter were higher than the mean concentration reported for the distant locations: however, the average concentration at the Site perimeter

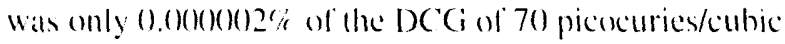
meter. The $1 x$ ( $i$ is the concentration that would result in a radiation dose eyual to the DOE public dose limit (10) millircin/ycar).

Air samples were collected al three Hanford Site locattions for volatile organic compounds and PCBs. All measured air concentrations of these oreanic compounds were well below applicable maximum allowathle concentration standards lor air contaminamts.

\section{Surface-Water Surveillance}

The Columbia River was one of the primaty environmental exposure pathway to the public during 1992 as a result of eperations at the Hanford Site. Radiological and chemical contaminamts entered the river along the Hanlord Reach als dired eflluem discharges and through the seepage of contaminated ground watler. Water samples were collected from the river all sarious lecations throughoul the year to determince compliance with applicathle slandairds.

Although radionuclides associaled with Hanford operations continued to he routinely identified in columbia River water during the year. concentrations remained extremely how at all hocations and were well below applicable standards. The concentrations of 'H. "'t, and uranium were higher at the Richland Pumphouse (downstream from the Site) than att Priest Rapids Dam (upstream from the Site). Differences in concentrations meatsured all the two focattions Here statlistically signilicant $1.5 \%$ signilicance level), indicating a comtribution along the Hanlord Reateh. Chemical water quality comstimeme measured in Columbia Rirer waller during 19) 2 were gencrally similar upstream and downstream and in compliance with applicable standards

1)uring 1092 samples were colleceded fom threc ('olumbia Rirer shoreline yprings. contaminated as a result of pas waste disposal praterices all the Hanford Sile. Comtaminam concentrations in the springs were similar to those found in the ground water. Radionuclide concentrations were generally less than the DOE DCGs. However. "Sr in $N$ Springs water was greater than the $D C G$ als well as the DWS. Tritium, while less than the DCG, was greater than the DWS at the old Hanford townsite springs.

Samples of Columbia River surfiace sediments were col lected from behind MeNary Dam (downstream from the Site) and Priest Rapids Dam and from four shoreline locations along the Hanford Reach of the Columbia River during 1992. As in the past. radionuclide concentrations in sediments hehind McNary Dam were generally slightly higher than those observed in sediments collected from behind Priest Rapids Dam and along the Site.

Three onsite ponds were sampled lo determine radionuclide concentrations. These ponds are aceessible to migratory waterfowl and other animals. As a result. a potential biological pathway exists for the removal and dispersal of contaminants that maty be in the ponds. Concentrations of radionuclides in water collected form these ponds during 1992 were similar lo those observed during past vears. In all cases. madionuclide concentrations in the onsite pond water were helow applicable DOE DCC is.

Offisite water, used for irrigation and/or drinking water. wats sampled to determine radionuclide concentrations in water used hy the nearby public. Likevated total alpha and wat beta concentrations. attributed to naturally oceurring urantum. Were observed al some locations. Average rationuclide concentrations in offsite wated during foes were within applicable I)WSis.

\section{Soil and Vegetation Surveillance}

In log2, three soil simples were collected from the I()()-N Areal and six others were collected oflisite. Most of the offisite sampling was conducted all the Site's downwind perimeter: other samples were collected at relatively upwind and distant lecattons ( Yakimal and Sumbside) fo establish hatekground concemtrations. Radionuclides comsistenty detected were "K. "Sis. "s. "U. and "wn'pu.

The analytical results were used to make two comparisons. The first compatrison, between the onsite and the combined ofleite samples, did not indicalle at difference in

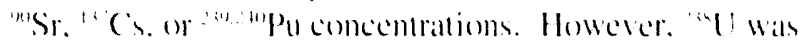
identilied in higher comentrations omsile. The secend comparisen was between the perimeter and distant 
upwind localtons. No significant differences in concentrations were found. indicating no measurable elfects from Hanford operations.

In 1992. one onsite and six offsite vegetation samples were collected. Vegetation was sampled using the same rationale ats soil sampling. Strontium-9) was identitied in five out of seven samples. The sample collected firom the Yakimat atea (upwind of Hanford) Wats identifjed als containing very low levels of ${ }^{-1 \times} \mathrm{U}$. Mlso ${ }^{17} \mathrm{C}$ 's and 231.2. Pu were found in the Sagemoor sample (downwind from Hanford) at very low concentrations. No radionuclick acemulation conld be detected from the vegetation samples liken.

\section{Wildlife Surveillance}

The Hanterd Sile contains latge tratets of undeveloped land that serve as a refuge for many species of wildlife. The Columbia River. which borders the Site. also provides hahitat for wildlife and lish that are of exomomic and recreational importance to the area. Terrestrial wildlife like deer, rabbits, and upland gamebirds have alecess (1) parts of the Site that contain low levels of radionuclides attribulable fo courent and past site operations. Wildlife are monitored for radionuclides as indicitors of possible exposure to the Site surface combamination. Similaty. Columbia River fish are monitored to detect any radiosictivity that maly arise from Site activities as well as to belp estimate the dose to those who may consume these fïsh.

Analysis of wildilie for radionetivity indicalted that some species had accumulated levels of radioalctivity grealer than background levels. Backgeround samples collected for a munber al species over the patst.3 years are summariaed in this year's repont. Strontium-90) was detected in deer and rabhil bone als well ats Columbia River fish carcalsses all levels exceeding concentrations reported in batekground localtons. Ducks collected in August from B Pond. a low-level waste pond focalled neatr the 20 () Lats Area, had signilicantly higher concentrations of " Cs thatl decks collected in November alter migrating decks had arrived. Cesium-1.37 wats also detected al higher concentrations in the muscle of deer collected from a background focallon in Stevens County. north of spokine. than has heen observed in Hanford Site populattions of mule deer. The levels of "Cin the deer from Stevens ('onenty wats altribuked lo past anmospheric lallsul from weaproms kesting. Collectively. the shocrantions of radienctivity in Hanlord fish and wildife indicalte accumulation of small amounts of specific radionuclides originating from the Hanford Site.

The radionuclide concentrations measured in fish and wildlile were used to estimate potential doses to sportsmen who may eonsume Hanford Site game. The resulting doses were much less than applicable guidelines developed to protect the public.

\section{Food and Farm Product Surveillance}

The Hanford Site is situated in a large agricultural allea that produces a wide variety of food products and alfalta. Milk. egges. poultry, bed, vegetables, fruit, wheat, alfallat. and wine were collected from areals generally downwind from the Site and upwind and distant locations. The principal downwind lecations include Wahluke.

Sigemoor, and Riverview. Allallat and larm prodacts

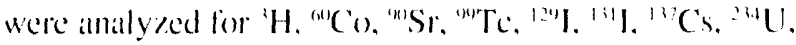

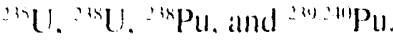

Most of the farm products simpled did not contain meatsurable eoneentrations of radion aclicles. Tritium wals meatsured al levels very close to the detection level, and there was no apparent upwind or downwind effect noted. lodine-129) was found all slightly elevalled levels in downwind milk samples, but the levels were very fow and have been lecreasing over the past 5 years. About $0.2 \%$ of the (1.)2 ntrem MEI annual dose results from ${ }^{1 . "} \mathrm{I}$ in milk.

A special study also investigatted the apparent elevation of "sin in allallit irrigatled with Columbia River waller downstream from the Hanford Site compared to alfallat. irrigaled with other sources of waller. The study showed that levels of " "Sr in the downstream study group exceeded concentrations in the onther group. There wats no monitored difference in "sr in Columbia River water upstream and downstream of the Site. The lindings are inconclusive: however, the levels of " $\mathrm{Sr}$ in the allatla do not constitute a significant dose to farm animals or humans. Overall. the potential offsite dose fo consumers of fim products grown near the Hanford Site in 1992 is a very small fratetion of the public dose guideline of 100 millirem/year lor exposure we envirommental madiclativily.

\section{External Radiation Surveillance}

In 1992. radiological dose lates were meatsured at al number of lociations on and oft the Hantond Site using 
TI.Ds. Comtributors to the ratdiological doses measured include natural (uranium and its progeny in soil and other primordial radionuclides) and artilicial sources. Onsitc dose rates, an a whole. appear lo be decreansing. while oflisite dose rales appear to have increased slightly.

The arerage batekground radiological dosc rate. calculated from TL.Ds at Yakimat and Sunnyside (distant and upu ind locattions relative to the Hantord Sile), Wals 93 millirem/yeart6\% as compared fo the arerage of 102 millirem/yeat $\pm 6 \%$ measured all the downwind perimeter of the Site. These alle increatses of $6 \%$ and $2 \%$. respentively. over last year"s meanured dose rates. Dose rattes at the (olumbia River shoreline around the 10()-N Arat were approximately two times higher than typical shoreline dose rates. This areal of higher dose rates may be attributable to styshine from the $10(0-\mathrm{N}$ Area liquid waste disposal facilities (Brown and Perkims |90) Onsite dose rates measured near operatiomal atreas were higher than the alverage background dose rate.

Vartous road and railroad comtamination surveys were performed during le92. No contamination on roads or railroads was found.

\section{Ground-Water Protection and Monitoring}

Radiological and chemical comstituents in ground watter were monitored during 1992 throughout the Hanlond Site in support of the orerall ohjectives dexerihed in Section 5.0. Monitoring atetivities were conducted lo identify and puantily existing. emenging. or potential ground-water quality problems: assess the potential for contaminamts to migrate off the Hantord Site: and prepalre an integrated assessment of the condition of geround water on the Sitc. To comply with RC RA. atditional monitoring wats conducted lo anses the impatct thatl

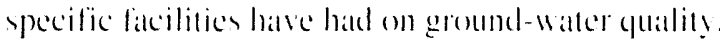
During fov2, 72 () Hantiond Site wells were sampled to saltisly ground-waller monitoring needs. As discussed in Sece-tion 5.3. four additional wells lecaled aterom the Columbia River and east of the Site were sampled to determine whether Hantord operations hatd allected waller cuality oflisite.

Analytical resulus for samples were compared with EPA'S DWS (Tables (.2 and ('.3. Appendix (') and DOF:'s DC'(i Table ('.6. Appendix C). Ciround waller beneath the Hantord Sile is losed lor drinking an live focations. Only the drinking water in the fol) Areal at the FFTF Visitors center is arailable for public comsumptions: this source is discussed in Section 5.8 . In addition.
Water supply wells for the cily of Richland are lecilted adjacent to he soubern boundary of the Hanford Sile.

Radiological monitoring results indicalled that total alphat.

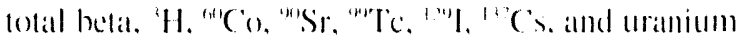
concentrations in wells in or neatr operating areals were al levels greater than the DWS. (oncentrations of uranium in the 2()()-West Areal were greater than the De(i. Comcentrations of 'th in the $20(1)$ Areas and " $\mathrm{Sr}$ in the $10(0)-\mathrm{N}$

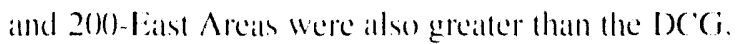
Tritium comtinued a move slowly with the general ground-waller finew and discharge on the Columbia River.

Certain chemicals regulated by the EPA and the State of Washingten were also present in Hanford Site ground water near operational areas. Nitrate concentrations exceeded the DWS at isolatted lecations in the 100. 20(0. and $30(0)$ Areals and in several 600$)$ Areal localtions. (hromium concentrations were greater than the DWS an the 1()()-1), 10()-H, and I()()-K Areas. and in the surrounding areas. (hromium concentrattoms greatler than the DWS Here also found in the 20()-latst and 20(0-West Areals. (yanide was present in grenend water nomth of the 20() lats Area. High concentrations of carthon tetrachloride ind chlorolion were found in wells in the 20)(-West Areat. Trichlorocthylene was foumd all levels exceeding the I)WS al wells in and near the 100)-1: 10(0)-K. 20(0-West, and 3(3)

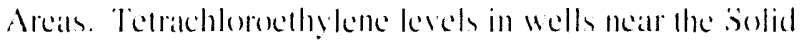
Waste Landtill remain just greater that the DWS. Samples from monitoring wells near Richland water supply wells shomed ihat concentrations of regulatted groundwater comstiflemts in this alleal were len than the I)WS

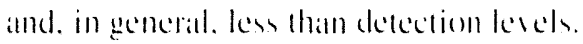

A comprehensice review of all ground-walter monitoring work on the Sice in publisled ammally. Before loge, these reports comtaned complete listing on all radiorlegical and chemical dattal colleceded during the reponting periods. Corrently, complete listings for grented-waller emsirommental surveillance data can he found in a comm. panion wolume to this report fo complement data listinge published by wher programs.

\section{Quality Assurance}

(omprehensive (yuality assurance (2) A) progerams. Which include varions quality comtrol practices and methods to verily dillat. alre matintained to emsure datat quality. The () A programs atre implemented through () A plams designed to meet reguirements in the American National 
Standiards Institute/A merican Society of Mechanical Engineers NQA-I QA program document and DOE

Orders. Quality assurance plans are maintained for all activities, and conformance is verified through anditors. Quality control methods include but are not limited to replicale sampling and analysis, analysis of blanks and reference standards, participation in interlaboratory crosscheck studies, and splitting samples with other laboritories. Sample collection and laboratory analyses are conducted using documented and approved procedures. When sample results atre received. they are screened for anomalous values by comparing them to recent results and historical data. Analytical laboratory performance on the EPA Laboratory Intercomparison Studies Program and the national DOE Quality Assessment Program indicated that laboratory performance was adequate overall, wals excellent in some areas, and needed improvement in others. 


\section{Report Contributors}

The production of the Hanford Site Environmental Report requires the knowledge, skills, experience, and cooperation of many people and several organizations. The contributions and cooperation, often under demanding time constraints, of the following individuals are gratefully acknowledged. The lead authors are listed for the main sections. (The authors are from the Pacific Northwest Laboratory unless otherwise indicated.)
Section

1.0) Introduction

1.1 Site Mission

1.2 Introduction to the Hanford Site

1..3 Major Operations and Activities

2.0) Environmental Compliance Summary

2.1 Environmental Compliance and Cleanup

2.2 Compliance Status

2.3 Current Issues and Actions

2.4 Environmental Occurrences

2.5 Compliance Status Update

3.0 Effluent Monitoring Information

3.1 Facility Effluent Monitoring

3.2 Near-Facility Environmental Monitoring

3.3 Solid Waste Management and Chemical Inventories

4.0 Environmental Program Information

4.1 Climale and Meteorology

4.2 Wildlile

4.3 Other Envirommental Sudies and Programs

5.0) Environmental Surveillance Information

5.1 Environmental Surveillance al Hanford

5.2 Air Surveillance

5.3 Surface-Water Surveillance

5.4 Food and Farm Product Surveillance

5.5 Wildlife Surveillance

5.6 Soil and Vegetation Surveillance

5.7 External Radiation Surveillance

5.8 Ground-Water Protection and Monitoring Program

6.0) Potential Radiation Doses from 1992 Hanford Operations

7.0 Quality Assurance
Authors

R. K. Woodruff

R. K. Woodruff:

J. M. Nickels, WHC

C. F.. Cushing

J. M. Nickels, WHC

J. M. Nickels, WHC

J. M. Nickels, WHC

J. M. Nickels, WHC

J. M. Nickels. WHC

R. W. Hanl; J. M. Nickels, WHC

J. M. Nickels, WHC

D. I. Rokkan, SAIC; L. P. Diediker, WHC

D. J. Rokkan, SAIC; L. P. Diediker, WHC

J. W. Schmidt, WHC; A. R. Johnson. WHC;

S. M. McKinney, WHC; C. J. Perkins, WHC

D. J. Rokkan, SAIC; L. P. Diediker, WHC

R. K. Woodruff

D. J. Hoitink

L. L. Cadwell

W. T. Farris; R. W. Hanf;

M. K. Wright; R. K. Woodruff

R. K. Woodruff

R. K. Woodrulf

G. W. Patton

R. L. Dirkes

T. M. Posion

T. M. Poston

E. J. Antonio

E. J. Antonio

P. E. Dresel; R. W. Bryce; J. C. Evans

J. K. Soldat

T. M. Poston; L. P. Diediker, WHC;

J. W. Schmidt, WHC 


\section{Acknowledgments}

The Palcific Northwest Lahoratory (PNL) programs described in this report were managed by the office of Health and Environment under the direction of R. E. Jacpuish. Environmental samples were collected for PNL by Radiation Protection Technologists M. E. Alnuarode. G. L. Indersen, L. L. Bell. L. W. Hankel, J. D. ilarrison, J. A. Jahnke, T. R. Lakey, J. J. Lope\% D. L. Macklict. D. I. Merrill. D. A. Mueller, and J.J. Reck. The environmental monitoring supervisor is E. W. Lusty. Technical assistance for sample collection was provided by A. T. Cooper, B. L. Tiller, and T. L. VanArsdale.

The atuthors appreciate the reviews by M. W. Tiernan. R. D. Hildehrand. and E. B. Dalgin of the U.S. Department of Energy, Richland Operations (Office: J. J. Dorian of Westinghouse Hanford Company: and E. B. Moore. D. J. B. :e. J. L. Devary, P. C. Hays, J. N. Holloway. and M. J. Sula of the PNI.

Community-operated envirommental surveillance stations were mallaged by local leachers who were responsible for collecting the samples and matintaining the stations. The managers and allernate mantgers for each station included:

\section{I deslie Groves Park, Richland}

C. A. Wagner. Manager

T. E. Gilmore. Alternate Manager

\section{Basin City Elementary School, Basin City}

C. L. Stevenson. Manager

\section{Fdwin Markham Elementary School,} North Franklin County

M. P. Madison. Manager

K. A. Darrington. Alternate Manager
The Westinghouse Hanford Company (WHC) programs described in this repert were managed by Regulatory Support Department under the direction of (i. W. Jackson.

Near-ficility environmental samples were collected by the Site Surveillance Health Physics group: H. A. Besel. D. R. Berup. R. L. Bumgarner, L. Corgatelli,

R. M. Frederick. D. S. Gumnink, B. M. Markes, R. G. Mikulecky, R. Olveda. K. S. Steffen, and R. I. Walts.

Croups and organizations participating in the WHC monitoring programs include Health Physics technicians and supervisors, facility operators, congnizant lacility envionmental engineers. Process Analytical Lahoratories. Ventilation Balance. and Field Maintenance. Individuals in these organizations collected and analyzed samples, maintained monitoring and sampling equipment, meatsured stack thow rales. ensured facility operations adhered to environmental process controls. identified needed monitoring uperades, aided in the interpretation and implementation of environmental regulations, and ensured effluent data reported are accurate.

This report was produced on Macintosh using Aldus Pagemaker. Valuable lext processing suppont was provided by R. M. Watt. N. L. Johnson. L. M. Andor, H. C. Morgan, A. Jewell, D. J. Kennedy, K. K. Chase. J. E. Ciority, and R. M. Urbina. Publication assistance was provided by M. K. DeSmet. C. L. Salvard. and S. K. Schult\%. Graphices for the report were designed by J. P. Noland, K. A. Corcoran, K. K. Kalchele, and L. G. Wallenhurger (Boeing Computer Services, Richland) and W. R. Gorst using Aldus Frechand and DeltaPoint Inc. Delta Graph. Cover and dividers were designed by H. M. Bullock (Bocing Computer Services. Richliand). 


\section{Contents}

Preface

Summary

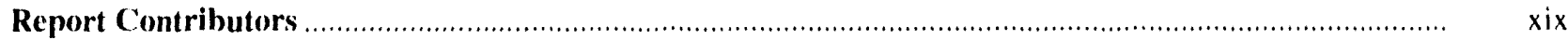

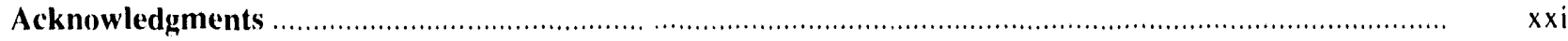

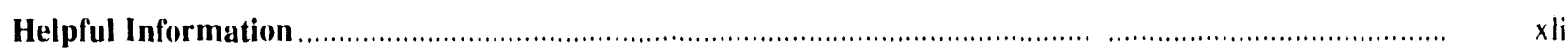

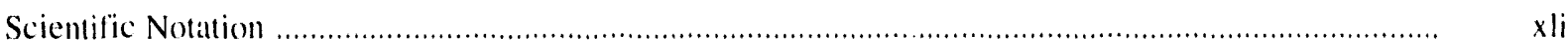

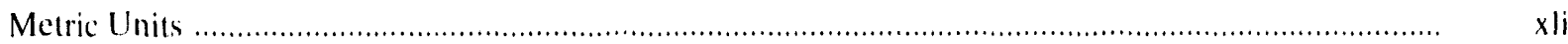

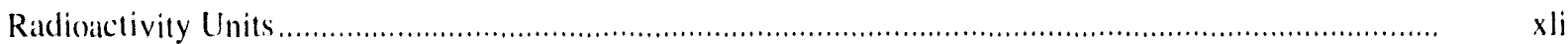

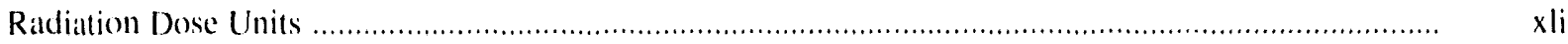

Understanding the Dalta Tables ................................................................................................... xliii

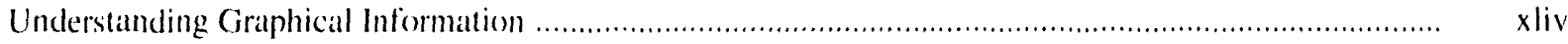

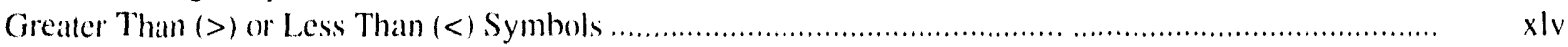

Elcmental and Chemical Constituent Nomenclature .......................................................................... xlvi

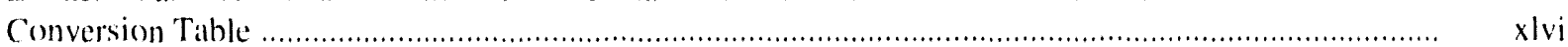

Acronyms and Abbreviations........................................................................................................ $\quad$ lvii

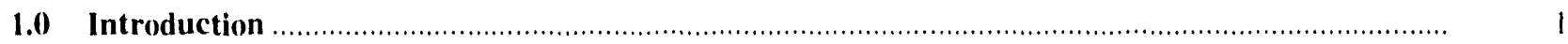

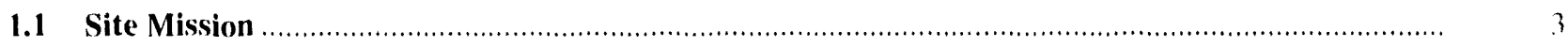

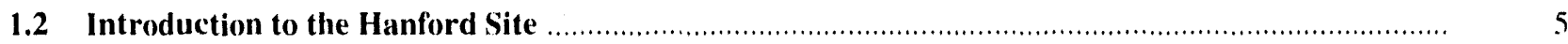

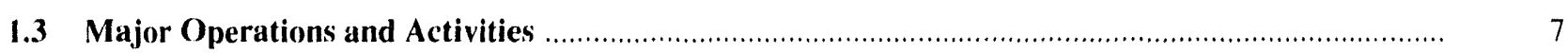

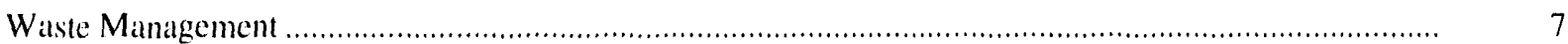

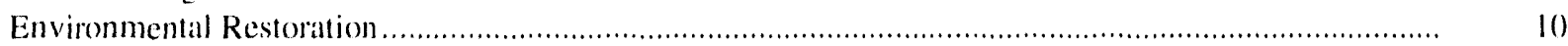

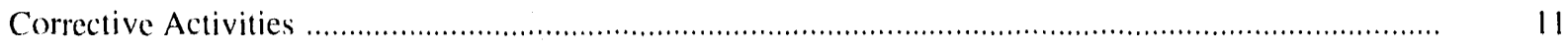

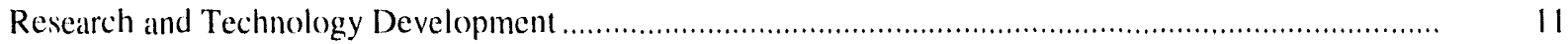

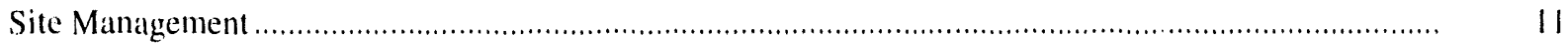

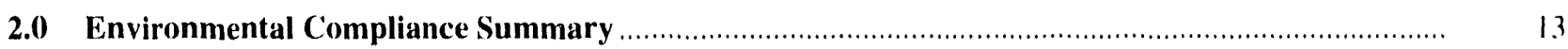

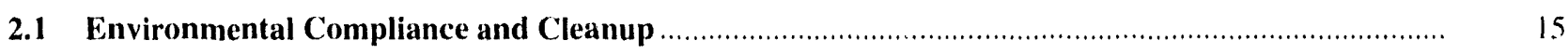

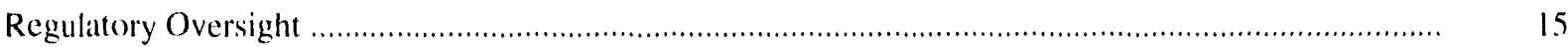

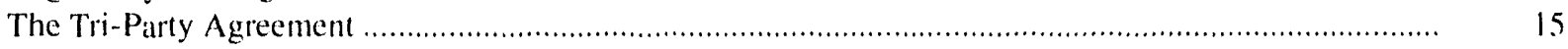

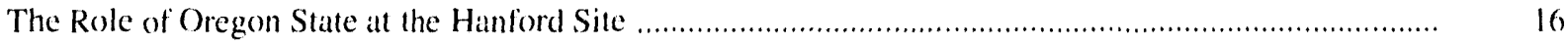

The Role of Indian Nations at the Hanford Site ............................................................................... 16

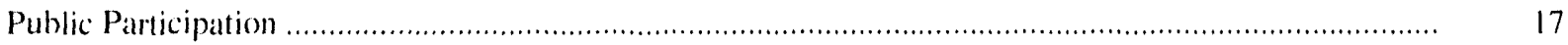


Comprehensive Environmental Response, Compensallon, and l iability Act

Resource Comservation and Recovery Act

Cleill Air Act

Cleall Waller Act

Sille Drinking Walter $\Lambda \mathrm{cl}$.....

Toxic Substances (ombrol $\Lambda \mathrm{c}$.

fecleral lnsecticide, Fungicide, and Rodenticide Acl.

Endangered Species Act.

National Historic Preservattion Acl. Archaeological Resonuces

Prolection Act, and American Indian Religions Frecedom Act

National Envirommental Policy Acl

\subsection{Current Issues and Actions}

Handord Federal Fateility Agreement and (insent ()rder

Hanford future Site Use/Cleanup Stralegy

The columbia River's Ilanlerd Reach

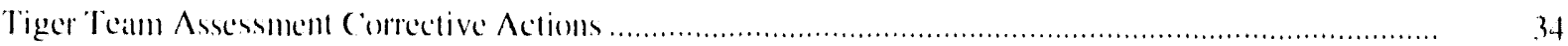

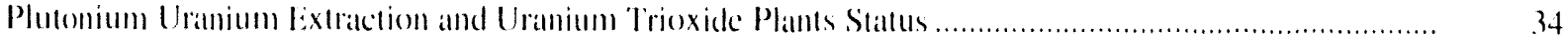

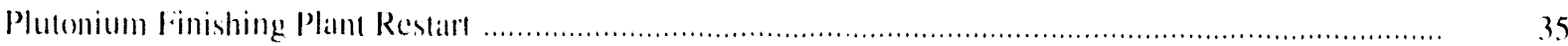

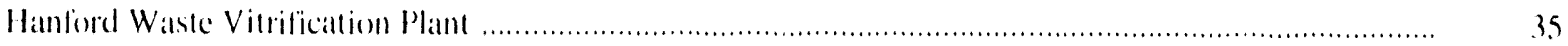

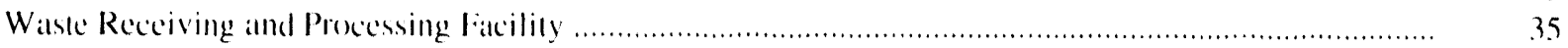

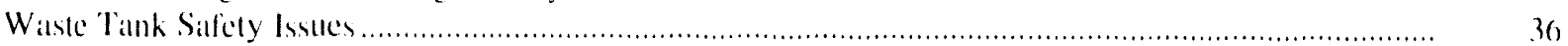

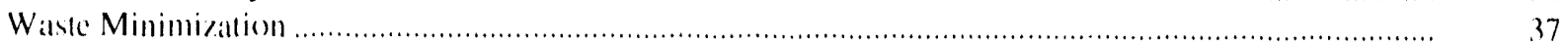

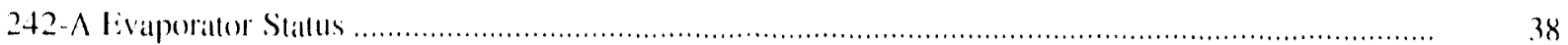

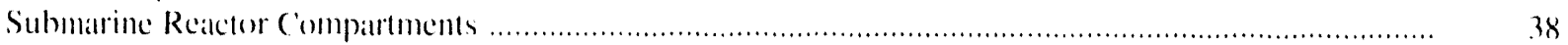

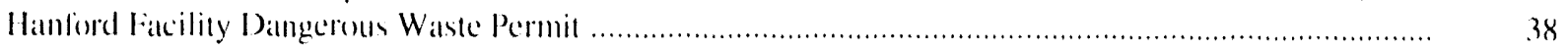

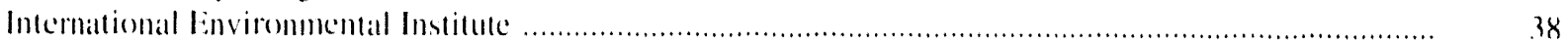

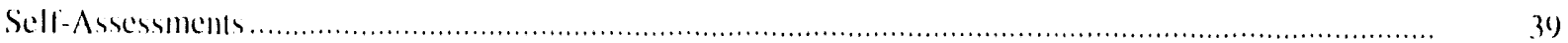

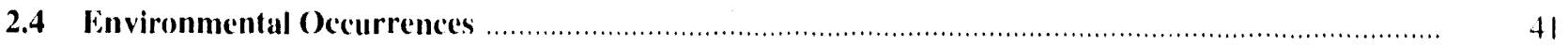

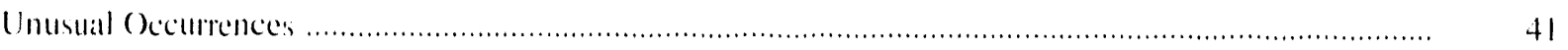

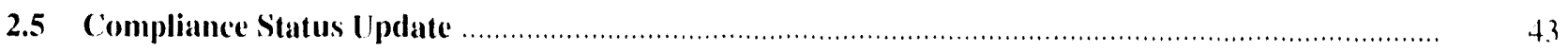

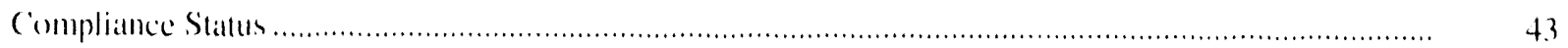

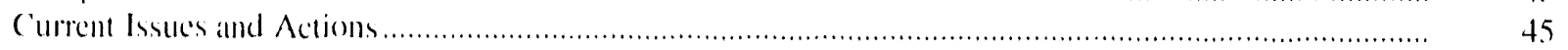

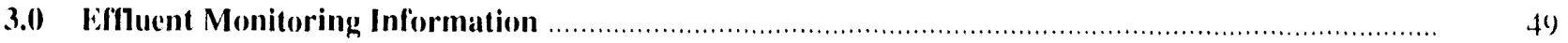

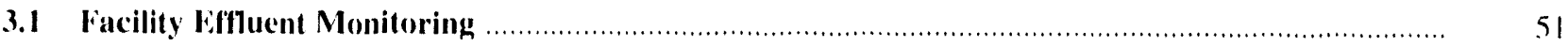

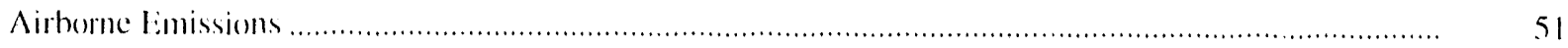

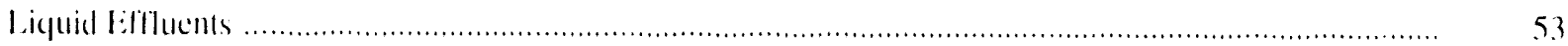

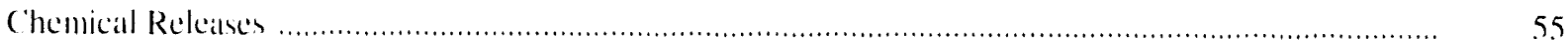




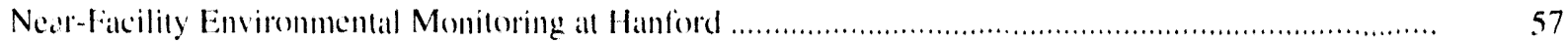

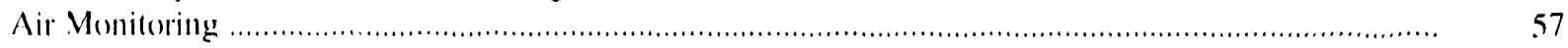

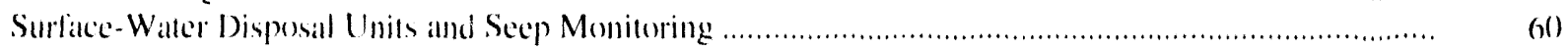

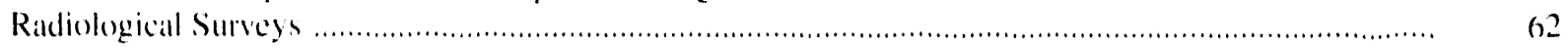

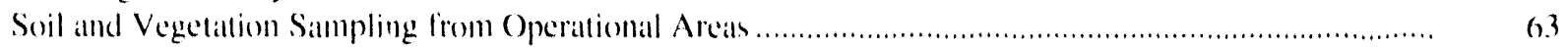

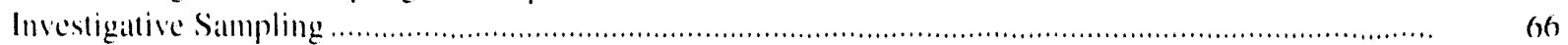

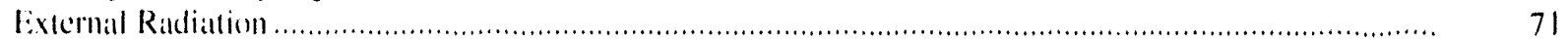

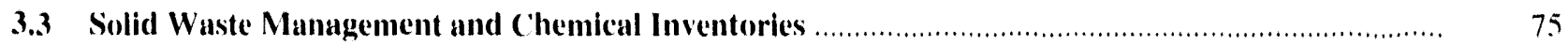

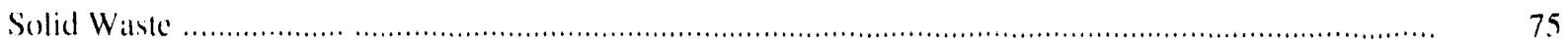

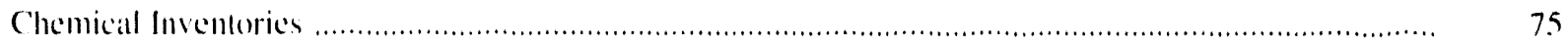

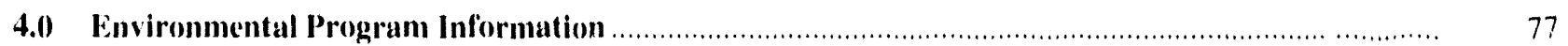

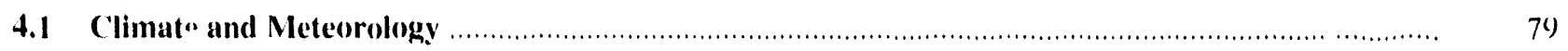

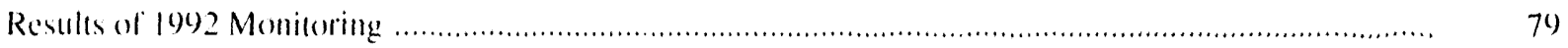

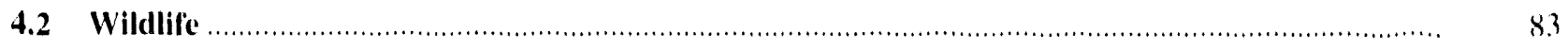

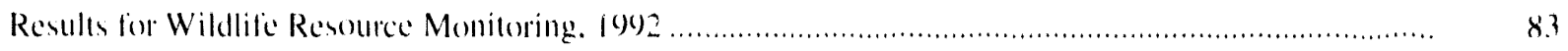

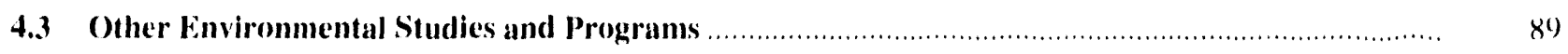

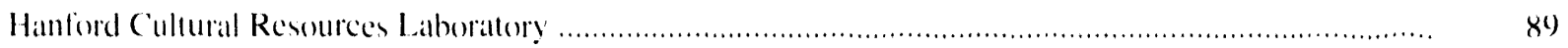

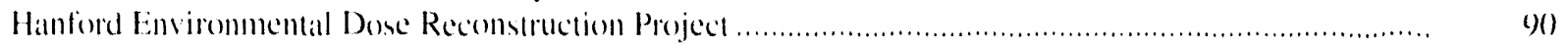

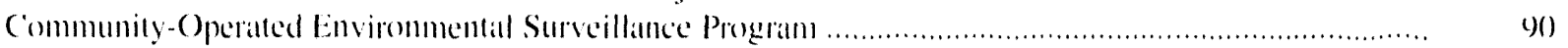

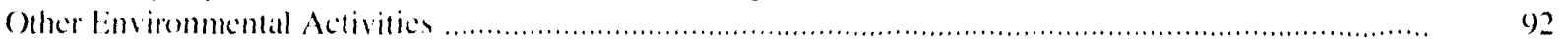

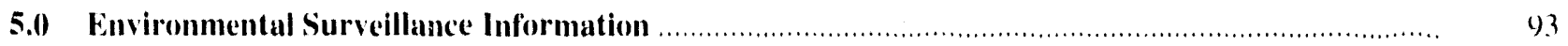

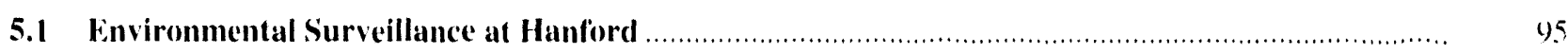

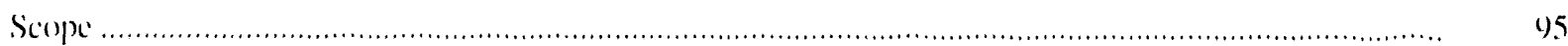

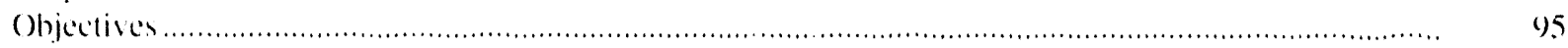

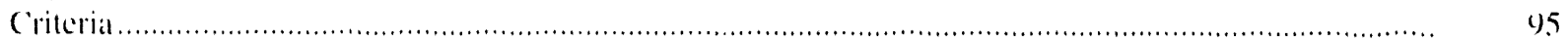

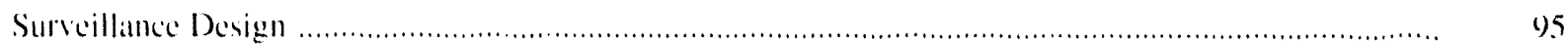

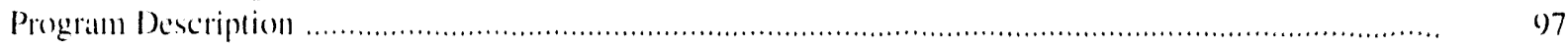

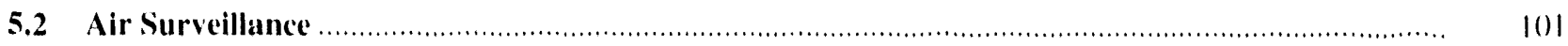

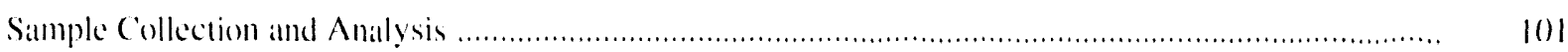

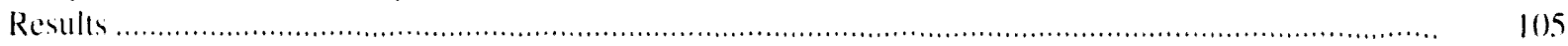

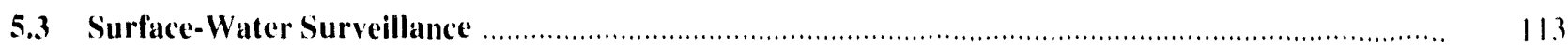

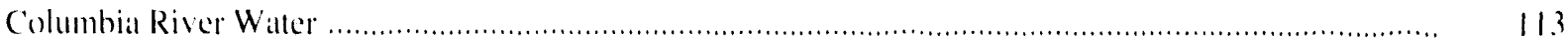

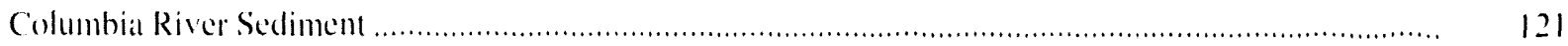




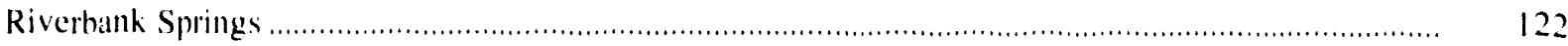

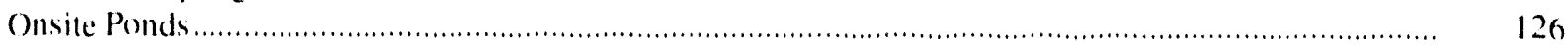

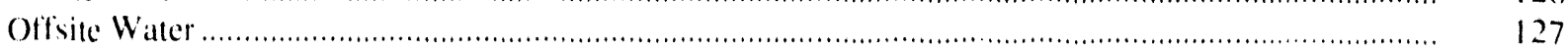

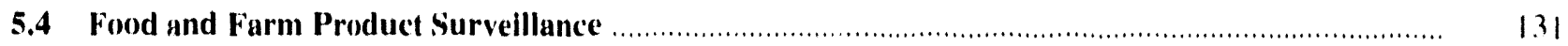

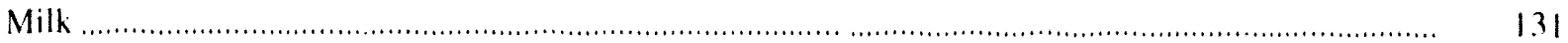

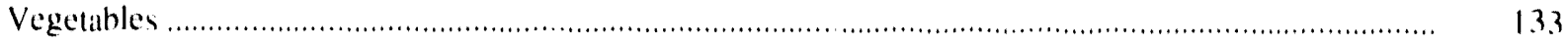

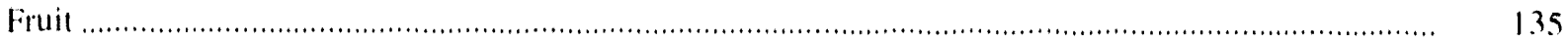

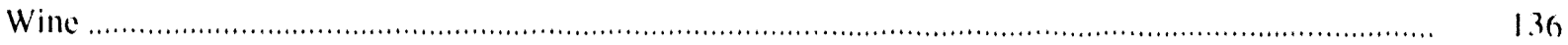

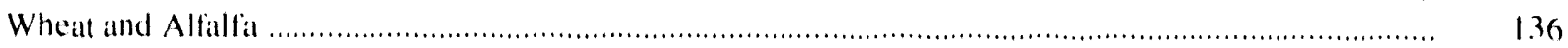

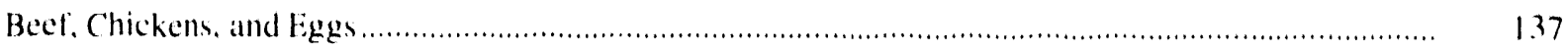

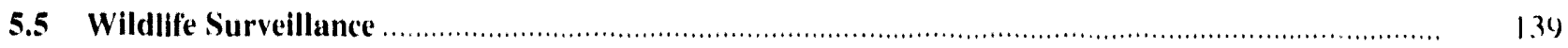

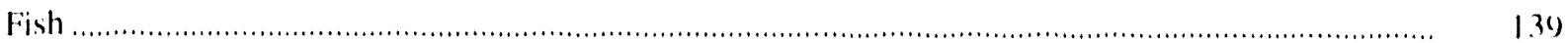

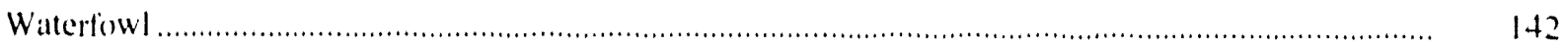

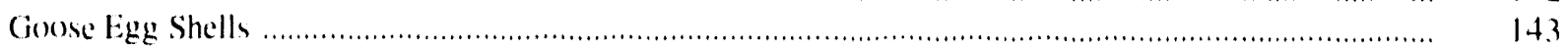

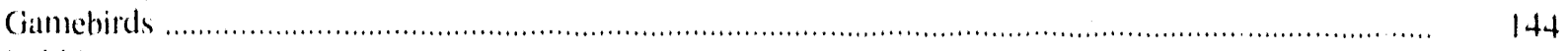

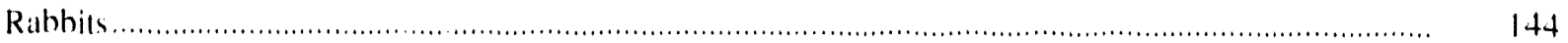

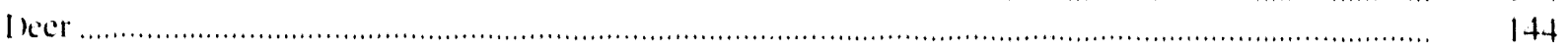

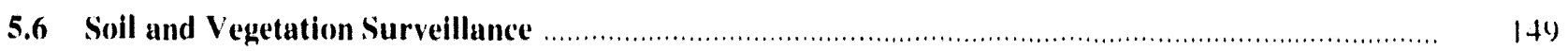

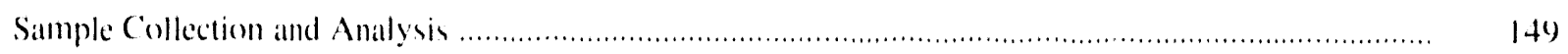

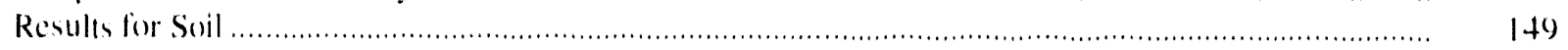

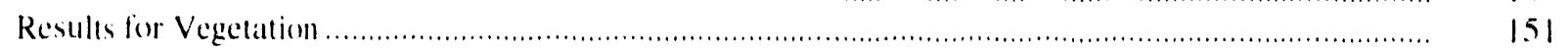

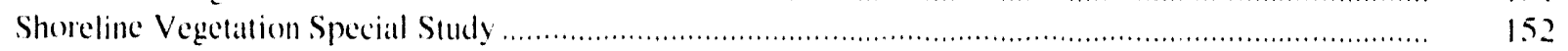

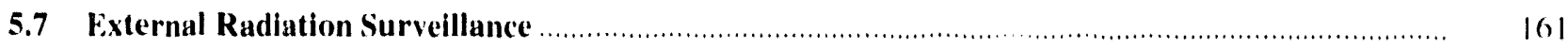

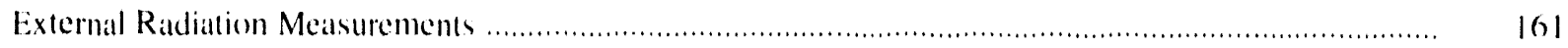

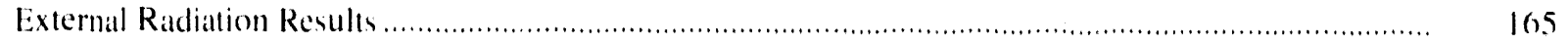

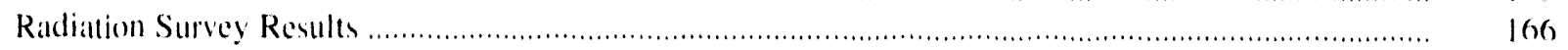

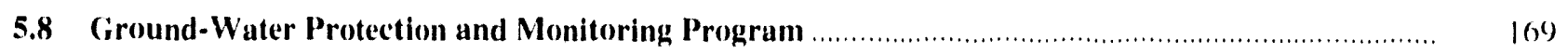

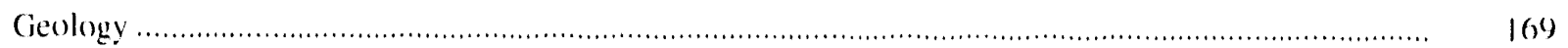

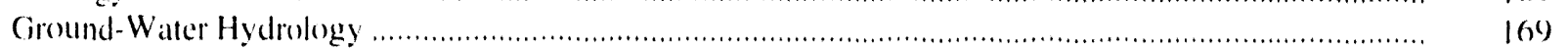

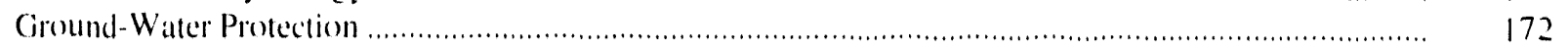

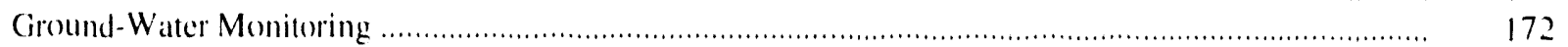

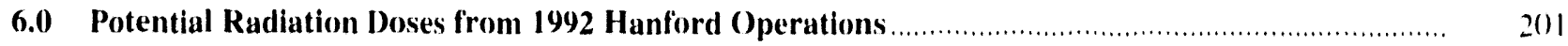

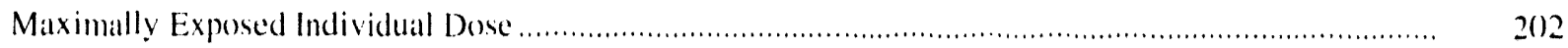

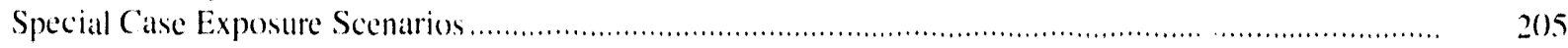

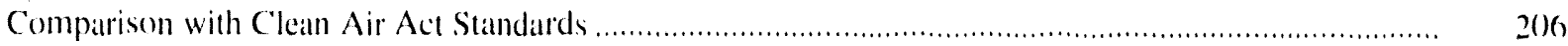

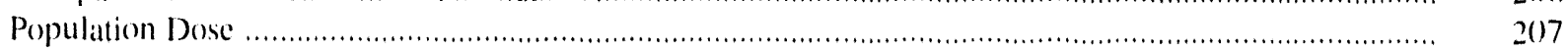

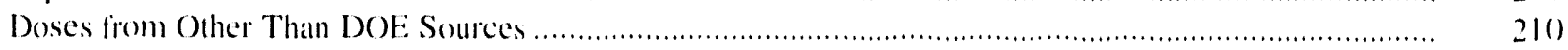

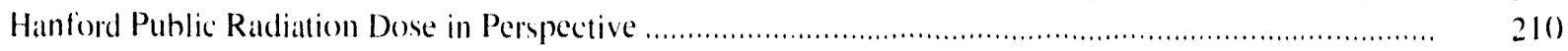

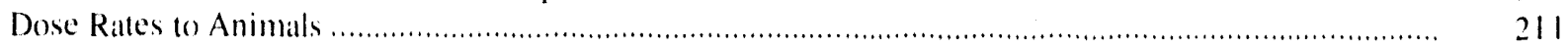




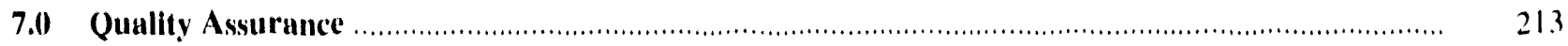

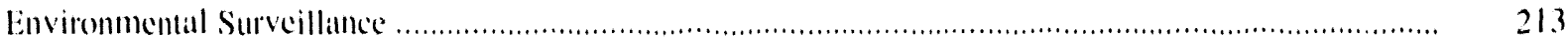

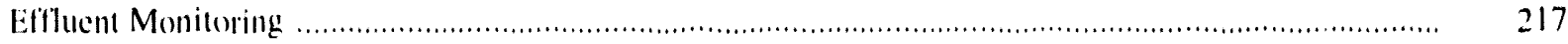

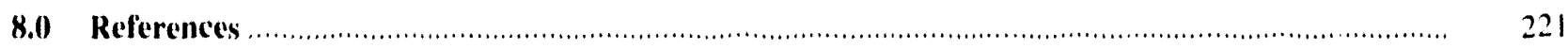

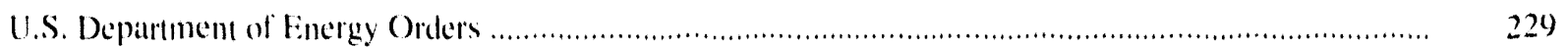

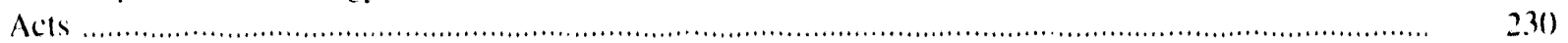

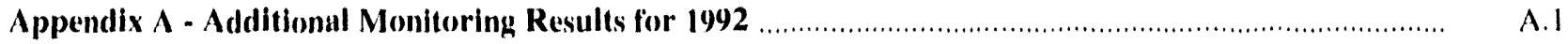

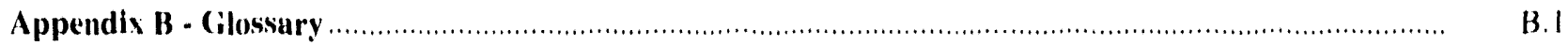

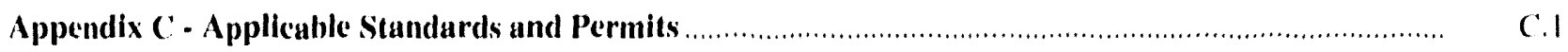

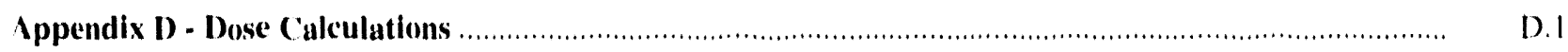

Appendix E - RCRA and CERCLA Monitoring Documents ........................................................... E..

Appendix F - Radionuclides Detected by Ciamma Spectroscopy ........................................................

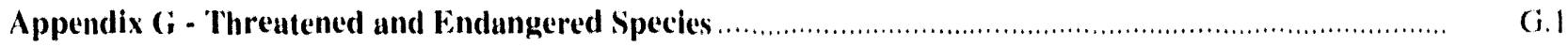




\section{Figures}

H.I Datta Plotled Using a Linear Sciale ............................................................................................. xliv

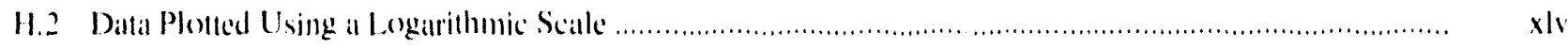

H.3 Datla With Error Bars Plotted Using a Linear Stale ........................................................................ xiv

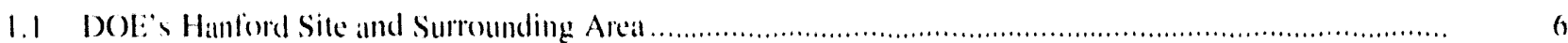

2.1 Location of Aggregate Areals of the National Prioritien List for the Hantord Site ....................................

2.2 Six (jeographir Study Areas for the Hanford Future Site Usen Working Group) .................................... 32

3.1 Airhorne Releases of Selected Riddionuclides to the Almosphere. 1987 Through 1992 .......................... 52

3.2 Radionuclide Concentrations in Near-Facility Air Samples Compared to

Background 1.ocations. 1987 Through 1992

3.3 Radionuclide Concentrations in Near-Facility Soil Samples Compared to

Background Concentrattions. 1987 Through 1902

3.4 Radionuclide Concentrations in Near-Facility Vegetation Compared to

Background Concentrations. 1987 Through 1992

3.5 Radiation Survey Meamurements Along the $10(0-\mathrm{N}$ Area Shoreline.

1987 Through 1092

4.1 Hantord Meteorological Monitoring Network Wind Roses. 1902

4.2 Bald Eagles Observed Along the Hantond Reach. Fall and Winter Months. 1902 Through 1992

4.3 ('hinowk Salmon Spawning Rededs in the Hanford Reach. 1948 Through 1942

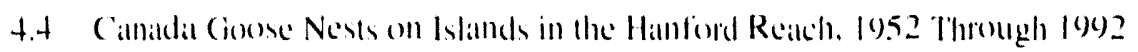

4.5 Red-Tatiled. Swainson's, and Ferruginous Hawks on the Hanford Site.

1975 Through 1992 .....

t.6 Elk on the Hantord Sile Counted by Acrial Surveillance During the Post-Calving

Period: August Through September: and the Post-Hunting Period: December

Through January, 1975 Through 1992.

4.7 Area Comsidered in Estimaning Doses from Pans Hanford Operations in the

Hanford Envirommental Dose Recomstruction Project 


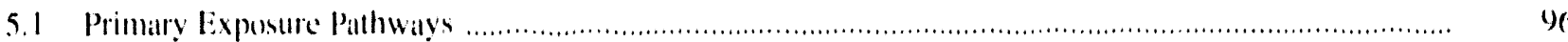

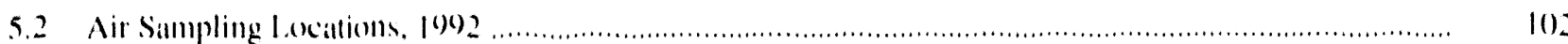

5.3 Monthly Arerage Tolal Beta Radioatcisity in Airhornc Patticulate Simples. 1986 Through 1692

5.4 Anmual Concentrations of hodine-120 in Air neatr the Hanford Site. 1987 Through 1992

5.5 Anmual Average (oncentrations of Tritium in Air near the Hantord Site. 1087 Through 190?

5.6 Ammal Average Concentrations of Plutonimm-2.39.240 in Air al the Hantord Envirolls, 1987 Through 1092

5.7 Ammal Average Concentrations of (tranium in Air at the Hantord Environs. 1987 Through 1902

5.8 Walter and Sediment Simpling Localtioms, 1962

5.9 Anmual Average Tolal $A$ phat Concentrations in Columbia River Waller. 1087 Through 1002

5. I0 Ammual Arerage Tolal Beta Coneontrations in Columbia River Walter. 1987 Through 1992

5.11 Annual Average Tritum concentrations in Columbia River Waller. 1987 Through 1092

5.12 Ammual Average Strontium-9) (oncentrations in (olumbia River Waller. 1987 Through 1062

5.1.3 Annual Average Granium Concentrations in Columbia River Watcr. 1987 Through 1902

5.14 Anmual Average lodine-124) (oncentrations in (olumbia River Waller. 1987 Through 190)

5.15 Columbia Rirer Waller Quality Measurements, 1987 Through 196)2

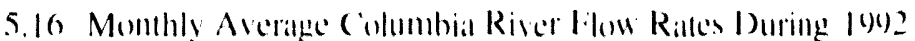

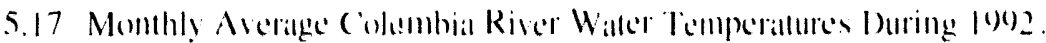

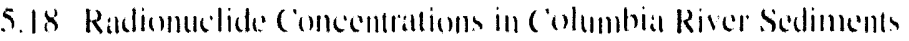

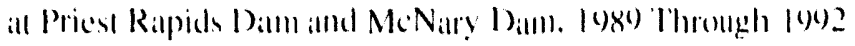

5.14 Radionuclide ('oncentrations in N Springs, 1988 Through 1042

5.20 Radionuclide ('mecontrations in Riverhank Springs mear the () ld Handiond Timmsile, lose Through l(x)2 
5.22 Annual Average Radionuclide Concentrations in B Pond. 1987 Through 1902

5.23 Average Total Beta and Tritium Concentrations in FFTF Pond. 1087 Through 1992

5.24 Annual Average Radionuclide Comeentrations in West Lake, 1987 Through 199?

5.25 Fond and Farm Product Sampling loncitions. 1992

5.26 Average lodine-129) Concentrations in Milk. 1987 Through 1992

5.27 Strontium-90 Concentrations in Milk, 1987 Through 1992

5.28 Annual Average Stromtium-9)( Concentrations in Leary Vegetables. 1987 Through 1902

5.29) Strontium-9) (oncentrations in Alfalfa Routinely Collected all Riverview and Richland and All ()her Sampling locations. 1987 Through 1092

5.30 Fish and Wildlifi Simpling L.onations. 1902

5.31 Concentrations of Cesium-1.37 in Duck Muscle Samples from

B Pond. 1987 Through 1992

5.32 Soil and Vegetaltion Sampling Locations, 1902

5.3.3 Selected Radionuclide Maximum. Median. and Minimum Concentrations in Soil, 1987 Through 1902

5.34 Selected Radionuclide Maximum. Median, and Minimum Concentrations in Soil at Perimeter and Distant l.ocittons, 1987 Through 1092....

5.35 Selected Radionuclide Maximum. Median, and Minimum concentrations in Vegetation, 1087 Through 1092

5.36 Tritiun (innementatoms in Columbia River Shoreline Vegetation for Individual Simples colleceled from lege Through ley2

5.37 Coball-6) (oncentrations in Columbia River Shoreline Vegetation for Individual Samples colleced from lego Through log)

5.38 Stromtium-1)( ) oncentrations in Columbia River Shoreline Vegetaltion

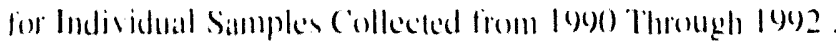

5..39) Cesitum-1.37 Concentrations in Columbia River Shoreline Vegetation for Individual Simples collected from loge) Thromgh 1092 
5.40 Uramum Concentrations in Columbia River Shoreline Vegetation for Individual Samples collected from 1990) Through 1992

5.41 Plutonium Concentrations in Columbia River Shoreline Vegetation for Individual Samples collected from 1990) Through 1992.

5.42 Thermoluminescent Donimeter Locattoms and Station Numbers on the Hantord Site. 1902

5.4.3 Thermoluminescent Dosimeter Measurement Locations and Statton Numbers for Perimeder and Community Sites. 1992

5.ty Thermoluminescent Dosimeler I ocattons and Station Numbers on the Hanford Reach of the Columbia River. 1992

5.45 Annual Average Done Ralles. 1987 Through 1992.

5.46 Roald and Railroald Survey Routes. 1992

5.47 Ciendogic Cross Section of the Hanford Site

5.48 Water-Tahle Elevalioms for the Unconfined Aquifer at Hanford. June 1992

5.49) Hanford Sile Lnconfined Ayuifer Monitoring Well Locations. 1992

5.50 Hanford Site Confined Aquifer Monitoring Well Locations, 1992

5.51 Monitoring Well locations in the 2()()-1 tats Areat. 1992

5.52 Monituring Well lociations in the 200)-West Area. 1992

5.53 I.ocations of RCRA Ground-Waler Monitoring Projects and landmaths on the Hanford Sitc

5.54 Tritium (oncentrations in the Lenconfined Ayuiler. 1992

5.65 Trifum (oncentration in Well 6,90-24-33. 1962 Through 1992

5.56 Tritium (incentrations in Well 699-4(1)-1. 1962 Through 1992

5.57 Tritium Cimcentrations in Well 699-S19-1:13. 1975 Through 1992

5.58 Tritium (incentration in Well 209-W22-9. 1976 Through 1092

5.59) Distrihution of Solected Radinnudieles (ireater Than the Drinking Watce Standard near the 200 Areas. 1992

5.(3) Distribution of Selecked (ombaninams (irealled Than the Drinhing

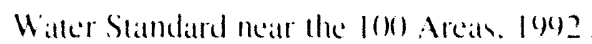


5.62 Distribution of Iodine-129 in the Unconfined Ayuiler, 1992

5.6 .3 Uranium Concentrations in Well 299-W19-18, 1987 Through 1992

5.64 Uranium Concentrations in Well 399-1-17A. 1987 Through 1992.

5.6.5 Distribution of Nitrate in the Unconfined Ayuiler. 1992

5.66 Nitrate Concentrattons in Well 299-W19-18, 1986 Through 1992

5.67 Distrihution of Selected Hazardous Chemicals Greater Than the Drinking Watter Standard near the 200) Areas, 1992

5.68 Distribution of Carbon Tetrachloride in the 20()-West Area, 1992

5.69) Distribution of Chloroform in the Unconlined Acpuiler near the 20()-West Areal, 1092

6.1 Iocations Important to Dose Calculations

6.2 Calculated Eiflective Dose Equivalem to the Hypothetical Maximally Exposed Individual. lose Through 1092.

0.3 Calculated Elledive Dose Equivalent to the Population Within $80 \mathrm{~km}$ of the Hanford Sile. 1988 Through 1992

o.t National Ammual Average Radiation Doses from Various Sources

7.1 (omparisom of Thermoluminescent I)osimeter Results with Known Exposures, 1902 


\section{Tables}

H.I Names and Symbols for Units of Measure ....................................................................................

H.2 Names and Symbols for Units of Radioactivity ........................................................................... xlii

H.3 Names and Symbols for Units of Radiation Dose ...................................................................... xlii

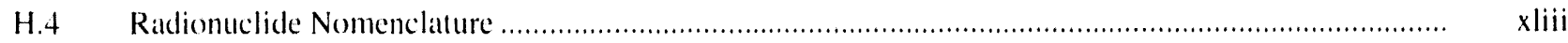

2.1 Seventeen Priority Chemicals of the Emergency Planning and

Community Right-To-Know Act Pollution Prevention Program ........................................................ 21

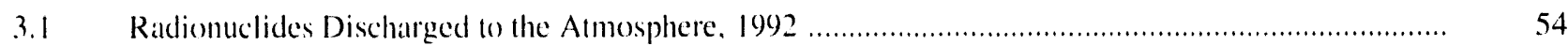

3.2 Nonradioactive Constituents Discharged to the Atmosphere, 1992 _................................................... 55

3.3 Radionuclides in Liquid Effluents Discharged to Ground Disposal

Facilitics. 1992

3.4 Radionuclides in Liquid Etfluents Discharged to the Columbia River

from the 100 Areas, 1992

3.5 Nonradioactive Liquids Eflluents Discharged to Ground Disposal

Facilities, 1992

Disposal Units, 20(0/60() Areals, 1992

3.8 Radtiological Results for Aquatic Vegetation Samples from

Surface-Water Disposal Units. 200/(6)() Areas. 1992.

3.9 Radiological Results for Sediment Samples from Surfice-Water

Disposial Units, 200/60)(0) Areias. 1992

3.10) Concentrations of Radionuclides in 100(-N Area Shoreline Seeps, 1992

3.11 Nonradiological Results for Liquid Samples from Surface-Water

Disposal Units. 1992

3.12 Outdoor Contaminition Status. 1992

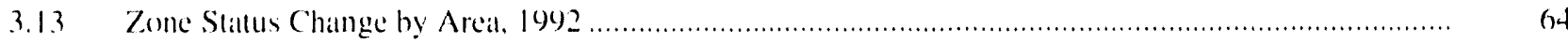

3.14 Special Samples Collected from the Operations Areas, 1992 ........................................................ 
3.15 10()-N Liquid Waste Disposall Facilities Direct Radiation

Measurements, |94) and 1092

3.16 Thermolumineseent Dosimeler Results for Waste-Handling

Pacilities in the Operatting Areats, 1091 and 109?

3.17 Radioatctive Solid Waste Disposal. 1902

3.18 Hanford Site Tier-Two Emergency and Hazardous Chemical Inventory

Average Balance of Ten Chemicals Stored in Greatest Quantity. 1902

4.1 Monthly (Cimatelogical Data from the Hanford Meteorology Stattion. 10)2

5.1 Environmental Surveillance Sample Types and Measurement Locattoms. 1902

5.2 Air Sampling Locallons, Sample Composite Ciroups, and Analyses, log2

5.3 Airbone Radionuclide Concentrations in the Hanford Environs. 1992 Compared to Values from the Previous 5 Years

5.4 Average Concentrations of Selected Volatile Organic Compounds in Air on the Hanford Site, 1992

$5.5 \quad$ Surfice-Walcer Surveillance, 1092

5.6 Sedimem Surveillance, 1002...

5.7 Numbers of l.ocalloms, Sampling Frequency. and Analyses Porformed

for Routinely Sampled food and Farm Products, 190?

5.8 Raddionuclide Concentrations in Milk. 1092 compared to Values

from the Previous 5 Years

5.9 Tritium (oncentrations in Winc. 1092 Companced 10 Values

from the Previous 5 Years

5.10 Strontium-40) in Alfaltia Samples. 1992

5,11 Locations. Species. and Raddonuclides Simpled for lishls and

Wilditic. 1002

5.12 Summary of (esium-1.37 in Bass Muscle and Strontium-4) in Bam (ancias.

1992 Compared lo Valtes from the Previous 5 Yeals

5.1.3 Summary of (esium-1.37 in Duck Muscle. 1992 (ompared lo Villues

from the Previous 5 Years

5.14 Concentrations of Strontium-9) in (ionse ligg sholls, 1987. 1988. 1901. and 1942

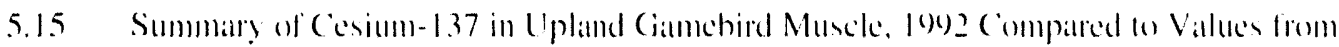
the Previous 5 Yeats 
5.16 Summary of Cesium-1.37 in Rabbil Muscle. 1902 Companed to Values

from the Previous 5 Years

5.17 Summary of Sirontium-90) in Rabhit Bone, 1992 Compared lo Values from the Previous 5 Years

5.18 Soil and Vegetalton Samples, 1902

5.19 Radenuedide Concentrations in Soil Samples on and olit the

Hanford Site 1992 companed to Values from the Previous 5 Years, 1992

5.20) Concentrations of Three Radionuclides (onsistemly found in

Vegetation. 1992

5.21 Raddionuclide Concentrations in Vegetattion Samples on and off

the Hanford Site. 1992 companed to Values from the Previous 5 Years

5.22 Dose Ratles Meisured hy Thermoluminescent Dosimeters all Perimeler and

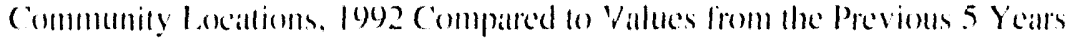

5.2.3 Dose Ratles Measured Along lo Hanford Reach of he Columbia River.

1902 Compared to Values from the Previous 5 Youts

5.2t Dose Ralles for Thermoluminescent Dosimeter Locitions on ho

Hanford Site. 1002 compared to Valeses from the Previous 5 Years

5.25 Radionuclides and Chemicals Analyzed for in Ciround Waller

5.26 Major (hemical and Radiological Ciround-Wanter Contaminames

and Their link a Site (Operations

6.1 Denes to the Hypothedicial Maximally Lxponed Individual from

Hanford ()perations, foge

6.2 Population Dones fiom Hanford (Operations, f902

6.3 Summary of Doses (o the Public in the Vicinity of Hantord

from Varrious Sumbes, 100)

6.4 Evimaled Risk from Various Activitionand lixporates

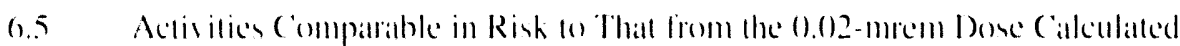

for the lou? Maximally lixponed londividual

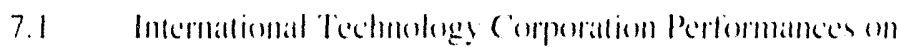

I)( )l: (Quality Assessment Program Simples, |092

7.2 Intemational Techmology (inponalion Pertormances on

ISA Intereompartion Program Samples. 1902

7.3 International Technolegy Componation Performances on PN.

Quality commol Simples. I()) 
7.4 Comparison of Quality Assurance Task Force 1092 Intercomparison Simples ................................... 216

7.5 222-S Analytical Laboratory Performance on DOE Quality

Assessment Program Samples, 1992.

7.6 222-S Analytical Laboratory Pertormance on EPA Intercomparison

Program Samples, 1992

A.1 Radionuclide Concentrations Measured in Columbia River Water al

Priest Rapids Datm. 1992 Compared to Values from the Previous 5 Years

A.2 Radionuclide Concentrations Measured in Columbia River Water all the 300 Areat. 1992 Compared to Values from the Previous 5 Years

A.3 Radionuclide Concentrations Measured in Columbia River Water alt the Richland Pumphouse, 1992 Compared to Values from the Previous

5 Years

A.4 Radionuclide Concentrations Measured in Columbia River Water Along

Cross Sections Established all the Vernita Bridge and Richland

Pumphouse, 1092

A.5 Columbia River Waller Quality Dalla, 1992

A.6 Radionuclide Concentrations in Columbia River Sediment.

1992 Compared to Values from the Previous 4 Years

A.7 Summary of Cesium-1.37 in Milk. 1992 Compared to Values from

the Previous 5 Yeits

A.8 Strontium-90) in Lealy Vegelables. 1992 compared to Values from

the Previous 5 Years

A.9) (ohall-(6). Strontium-9), and (e'sium-1.37 in Riverview (anrots. 1992

Compared o Values from the Previous 5 Years

A.10 Ammual Average Concentratton of Strontium-90) in Altallia, 1982 (6 1092

A.11 Summary of Strontium-e) in (aup (ancass and ('esium-1.37 in (anp)

Muscle, 1992 Compared lo Values from |09) and 1901

A.12 Concentrations of Strontium-4) in Whitefish (ancass and Cesium-1.37 in

Whitefish Muscle, 1902 Compared to Values from the Previous 5 Years

A.1.3 Summary of Plutonium-2.38 and Plutonium-2.39,240 in Rabhit liver.

1092 compared an Values from the Previous 5 Years

A.14 Summary of Strontium-9) in Deer Bone and Cesium-1.37 in Deer Muscle.

1992 Compared to Values from the Previous 5 Years

A.15 Strontium-9) Concentrations in Soil, 1987 Through 1992 


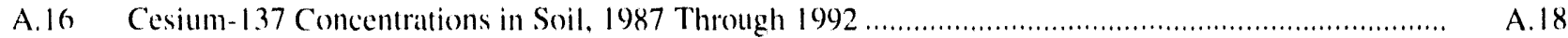

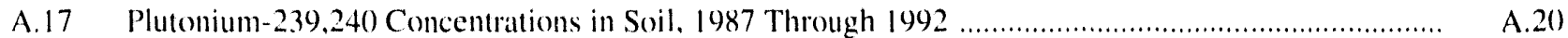

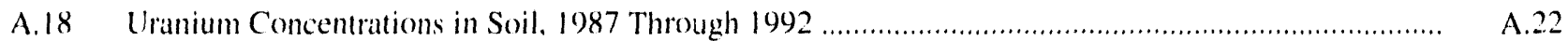

C.1 Washington State Water Quality Standards for the Hanford Reach of the Columbia River.

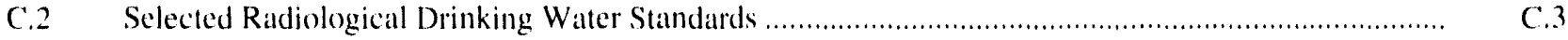

C.3 Selected Chemical Drinking Water Standards ..........................................................................

C.4 Benton-Franklin-Walla Walla Counties Air Pollution Control Authority Ambient Air Quality Standards

C.5 Radiation Standards for Protection of the Public from

All Routine DOE Activities

C.6 Selected Derived Concentration Guides

C.7 Envirommental Permits

D.1 Food Pathway Parameters Used in Dose Calculations, 1992

D.2 Dietary Parameters Used in Dose Calculations, 1992

D.3 Residency Parameters Used in Dose Calculations, 1902

D.4 Recreational Parameters Used in Dose Calculations, 1992

D.5 Documentation of 10()-N Area Airborne Release Dosc Calculations, 1992

D.6 Documentation of 1()()-N Area Liquid Release Dose Calculations. 1992

D.7

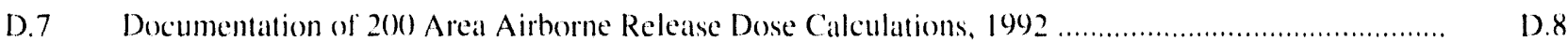

D.8 Documentation of 300 Area Airborne Release Dose Calculations, 1992 ........................................... D.9

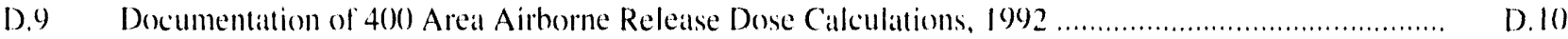

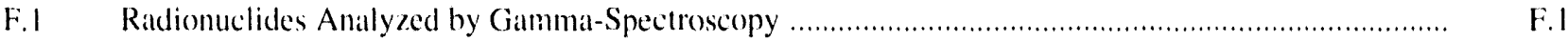

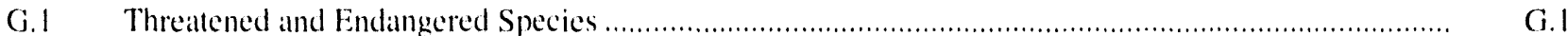

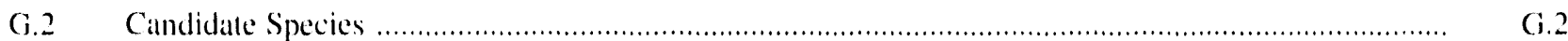

G.3 Washington State Plant Species of Concern Oecurring on the Hanford Sitc ............................................ 
Helpful
Information

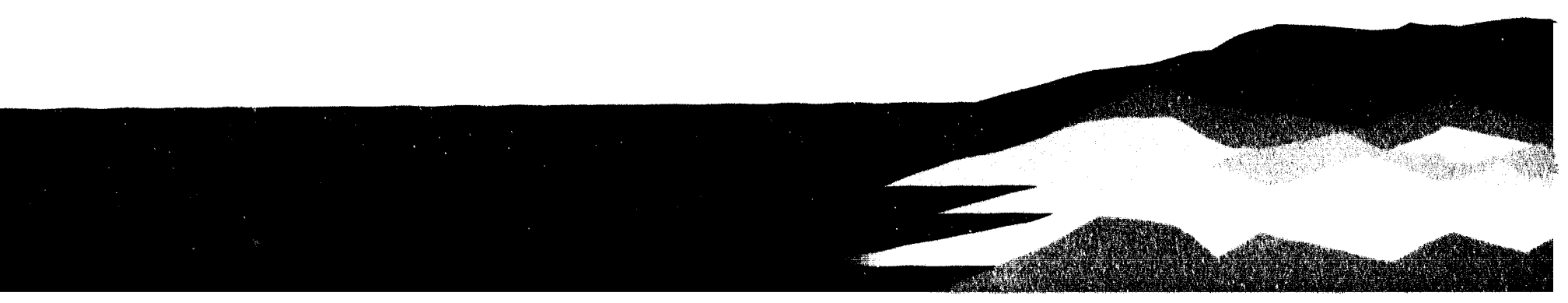




\section{Helpful Information}

The following intormalion is provided an ansist the reader in understanding the repont. Definitions of lechnical lerms cian he found in Appendix B. "(jhonsally." A public intermation stmmong pamphlet is as ailable by following the directions in he "Prefices."

\section{Scientific Notation}

Scientitic notallon is used in this repont lo expess reng large of very small mumbers. For example. Ihe number

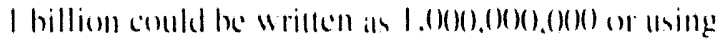

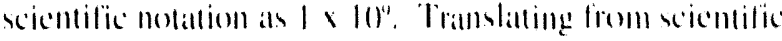
molittion to at more traditional mumber reguires mon ing the decimal point either left or right from the number. II the value given is 2.1) $\times 10$. whe decimal poime stomld be

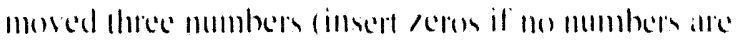

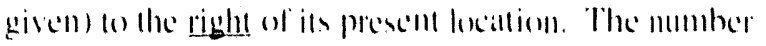

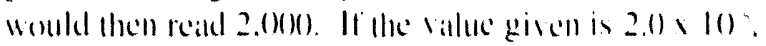
the decimal poimt shoukl be moved live mumbers lo the left of is present lecallion. The resull would heorme $0.0(1)(1) 2$

\section{Metric Units}

The primary units used in this repont alte metric.

Tahle 11.1 semmmarises and delines the kerms and corres

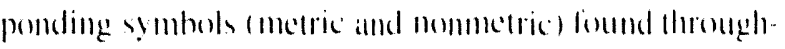
ottl this report.

\section{Radioactivity Units}

Much of this report deals with levels of radiondetivily in

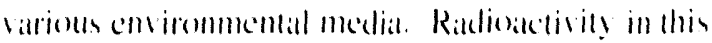
report is usually discussed in units of curtice (ci) (Table 11.2). The surie is the basic mit used to describe the anomont of radioatctivity present. and concentrations

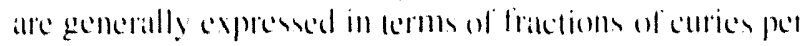

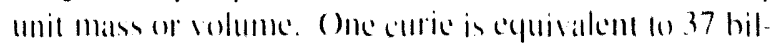
lion disintegratioms per second or is a yuantiey al all! riddionuclide that decalys all the ralle of 37 hillion disintegrallions per secomd. Disintegrattoms gentherally

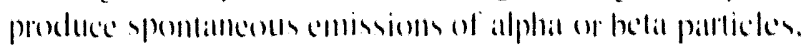

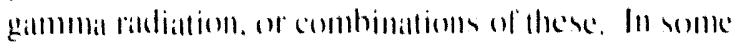

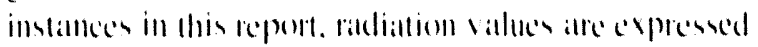

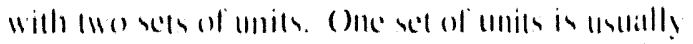

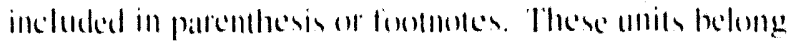

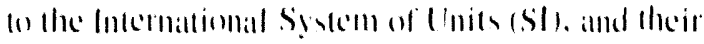
inclusion in this repont is mandated hy 1$)(3)$ :. Sl units atre lhe "nsw" incernationally alcépled units and will

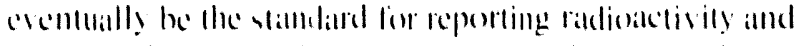
radiation dese in the Inited Stalles. The basic lan for

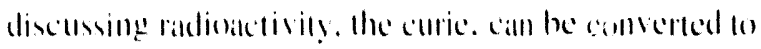

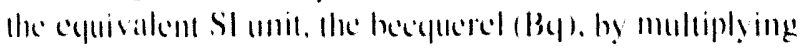

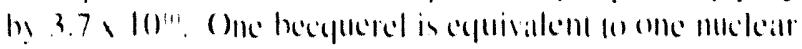
disinlegration per secomd.

\section{Radiation Dose Units}

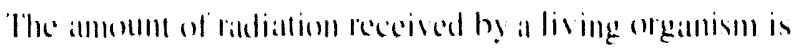
expressed in terms af radiallion dose. Ratdiatlonn dose in this repott is astally writlen in ferms of effectise dose equivaleme and reperted mumerically in unils of rem or in

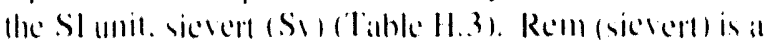
lerm llat relates ionising radiandon and hiological ellect or risk. A dose of I millirem has a biolegical effect simi-

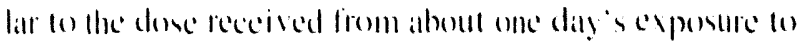

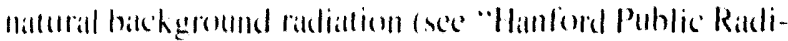

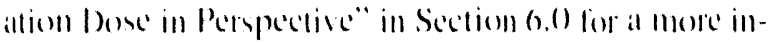

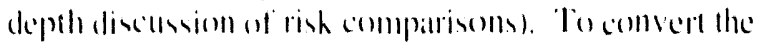
mose commonomy used dose kerm in this repont. the millirem. on the sl equisalemt. He millisievent. multiply mililim by (1).1)1.

Additumal informatton on radianion and done lerminol-

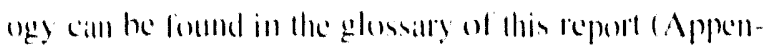
(lix B). A list of the radiomuclides discussed in this

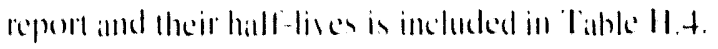

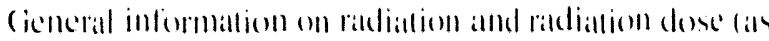

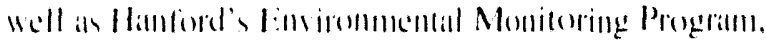

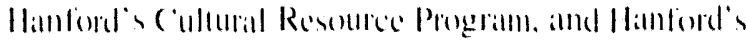
wildiles has leen sompriled in inlormattional palmphlets 
Table H.1. Names and Symbols for Units of Measure

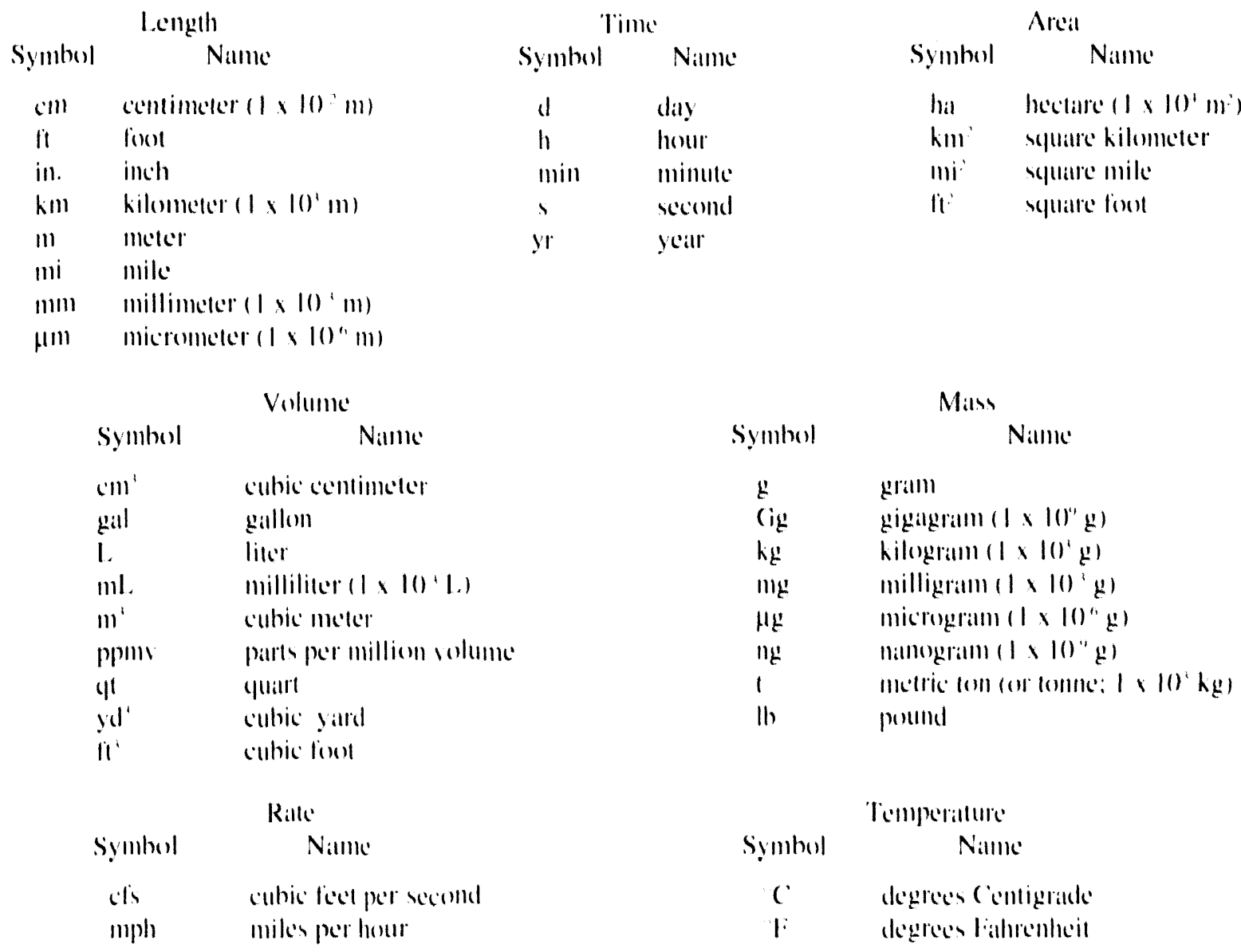

Table H.2. Names and Symbols for Units of Radioactivity

\begin{tabular}{|c|c|}
\hline \multicolumn{2}{|c|}{ Ratlionativity } \\
\hline Sỵmhol & Nillicic \\
\hline Ci & curic \\
\hline $\mathrm{mCi}$ & millicuric (l $\times 10^{\circ}$ ('i) \\
\hline$\mu \mathrm{Ci}$ & miconourie (l $810^{\prime \prime}(\mathrm{i})$ \\
\hline$n C^{\prime} i$ & manocurie (I $\times 10$ "(i) \\
\hline$p C i$ & picocuric $11 \times 10 \%$ (i) \\
\hline $\mathrm{iCi}$ & fentosurice $1 \times 1)^{\prime \prime}(\mathrm{i})$ \\
\hline aC'i & altocuric (1 $\times 10^{10}$ (i) \\
\hline $\mathrm{By}$ & becefuerel \\
\hline
\end{tabular}

Table H.3. Names and Symbols for Units of Radiation Dose

\begin{tabular}{|c|c|}
\hline \multicolumn{2}{|c|}{ Raldiallion 1)ans } \\
\hline Symhol & Nitme \\
\hline mrad & millimal 1 × 10 ' radl) \\
\hline mrem & millirem (1 $\times 10$ 'rem) \\
\hline Siv & sicvert \\
\hline MSis & millisievert (1 $\times 10$ Siv) \\
\hline MSi & microsicuent $(1 \times 10$ "Sw) \\
\hline R & menllgen \\
\hline$m R$ & millirountgen (I $\times(0)^{\prime} R$ ) \\
\hline$\mu R$ & micorocontgen $1 \times 10$ " R/ \\
\hline (iv) & grail \\
\hline
\end{tabular}


Table H.4. Radionuclide Nomenclature

\begin{tabular}{|c|c|c|c|c|c|}
\hline Raldionnclide & Sỵmbul & Hill-lile & Rondionuclide & Symbol & Halli-life \\
\hline mitium & 'H & $12.3 \mathrm{yr}$ & crsillm-1.37 & $1 ;$ & $30.2 y$ \\
\hline heryllimm-7 & $\mathrm{Hc}$ & $5.3 .2 \times 1$ & corium-14t & "lc & $2 x+1$ \\
\hline carthon-14 & $\mathrm{C}$ & $5730 \mathrm{yr}$ & promethimm-147 & "PIII & $2.02 \mathrm{yr}$ \\
\hline sodium-22 & $\mathrm{Ni}$ & $2.6 \mathrm{yr}$ & curopiuml-1.52 & Wu & 1231 \\
\hline polatsiumlutu & "k & $1.26 \times 1010$ & cur(p)ium-1.54 & "In & $16 y$ \\
\hline $\arg (1) 1-+1$ & $" \Delta \mathrm{r}$ & $1.8 \mathrm{~h}$ & curopium-1.5.5 & 1:u & $1.8 \pm 1$ \\
\hline chromimim-.51 & $\therefore \mathrm{Cr}$ & $27.7 \mathrm{~d}$ & Ihallium-20x & "Nl & $3.1 \mathrm{~min}$ \\
\hline matngantuse -54 & $" \mathrm{M} \|$ & 3124 & bismull-212 & $\because \mathrm{Bi}$ & $611.6 \mathrm{~min}$ \\
\hline coball -57 & ( Ci) & $271.8 \mathrm{~d}$ & lead.212 & "Ph & $10.6 \mathrm{~h}$ \\
\hline colball-(n) & "(i) & $5.3 y$ & polonium-212 & $P_{0}$ & $0.3 \times 101$. \\
\hline nickel-6.3 & $" \mathrm{Ni}$ & $92 y$ & polonium-216 & $\therefore H_{1}$ & $11.15 \mathrm{~s}$ \\
\hline sink -0.5 & $\cdots / .11$ & $24.3 .8 \mathrm{~d}$ & riadon-220) & $\therefore \mathrm{R} n$ & $55.6 \mathrm{~s}$ \\
\hline kryplon-8.5 & $\mathrm{Kr}$ & $10.7 \mathrm{yr}$ & rialium-226 & $\therefore K i l$ & $16(x) y r$ \\
\hline strmtium-89) & ${ }^{\circ \prime} \mathrm{Si}$ & 521 & radium-228 & $\therefore \mathrm{Ka}$ & $5.75 y$ \\
\hline stromlimm-s) & $" \mathrm{Sr}$ & $28.8 \mathrm{yr}$ & Homium-2.32 & Th & $1.4 \times 10^{111} \mathrm{yr}$ \\
\hline miohium-95 & "Nh & 36.1 & Itranium lotal & U or mranium & $\ldots$ \\
\hline irc(n)ium-9. & $" \% /$ & $(1+.01)$ & mannium-2.34 & $\therefore$ & $2+110 \%$ \\
\hline molyhdenum-90 & ${ }^{\prime 2,} \mathrm{Mo}$ & $60.0 \mathrm{~h}$ & uranium-2.35 & $\because U$ & $7 \times 111 y$ \\
\hline technetium-s) & $\cdots$ & $2.12 \times 10 \% \mathrm{yr}$ & uranium-2.36 & "1! & $2.3 \times 111 \mathrm{yr}$ \\
\hline rublenium-10.3 & $\cdots R \|$ & $39 .+1$ & uranium-2.38 & $\cdots 1$ & $4.5 \times 100^{11} ! 1$ \\
\hline rulhenimm 160 & $\|" \cdot R\|$ & $.367 \mathrm{~d}$ & plutminum-2.38 & "xpll & $87.7 \mathrm{yr}$ \\
\hline 1 inl-11.3 & "Sin & 1151 & meplunimm-234 & $" \mathrm{~Np}$ & $2 .+1$ \\
\hline anllimuny-125 & $\because \mathrm{Sh}$ & $2.7 \mathrm{y}$ & plutonimm-230 & $\because P \|$ & $2+\times 10^{4}: 1$ \\
\hline indinc- 129 & | & $1.0 \times 1011$ & plulomium - $2+10$ & "ll & $0.537 \mathrm{gr}$ \\
\hline indime-1.31 & 11 & $8.1) \mathrm{d}$ & plutonimm-241 & $P_{11}$ & $14.4 ! 1$ \\
\hline harium-1.33 & 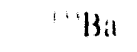 & $10.53 y$ & ancricium-241 & "NIII & $4.33 \mathrm{y}$ \\
\hline cesium-13. & 'cis & 2.111 & & & \\
\hline
\end{tabular}

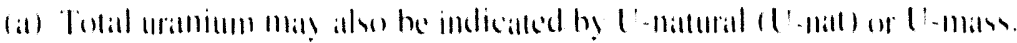

What call he obtaimed. free hy writing lo

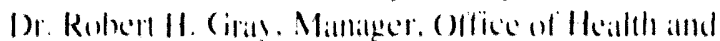

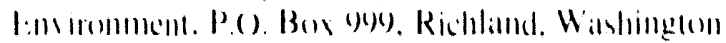

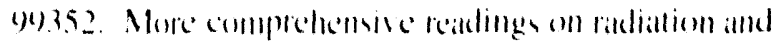

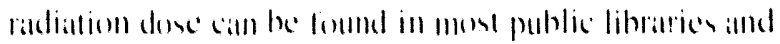
in matn! lexal beroh steres.

\section{Understanding the Data Tables}

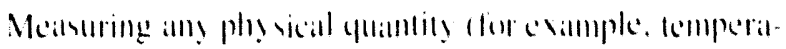

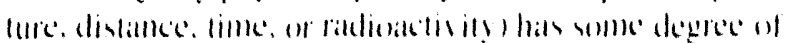


inherent uncertants. This uncertionly resules from the

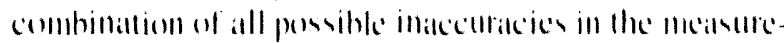
ment process. including such liactors as the reading of the resul. the callibrattom of the measurement devices and numerical roumding errors. In this report, indis idual

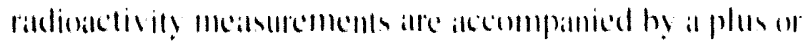

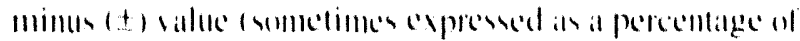

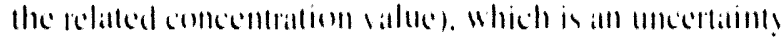

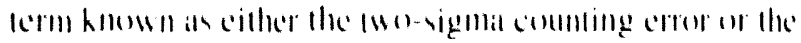

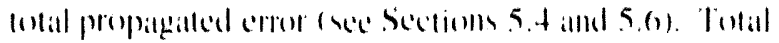

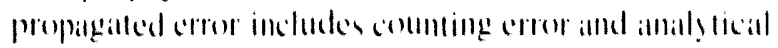

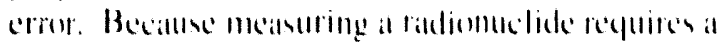

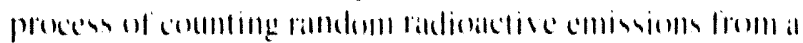

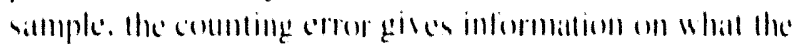
meintrement might he if the sillme simple were commled

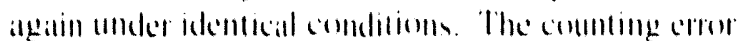

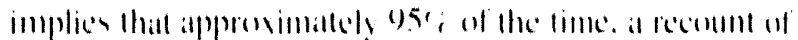

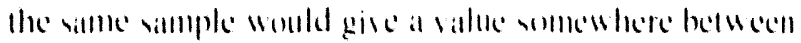

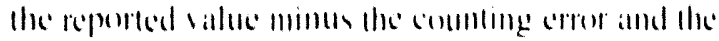

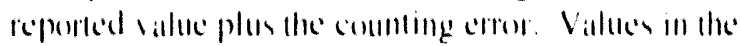

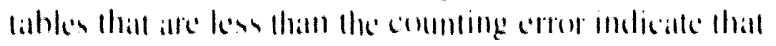
the repented result might hatse comle from a silmple will

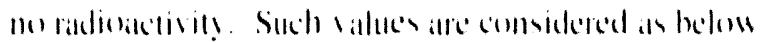

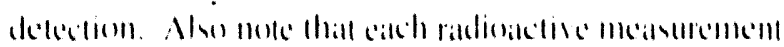

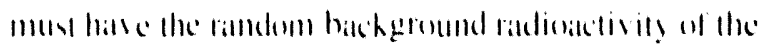

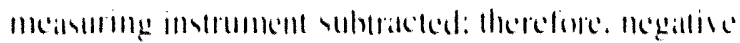

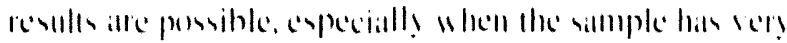
little radinationils.

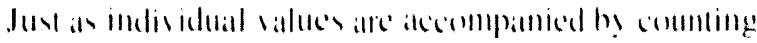

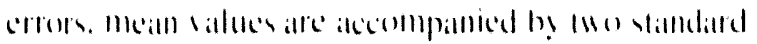

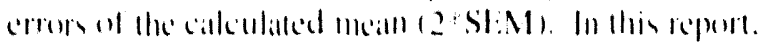

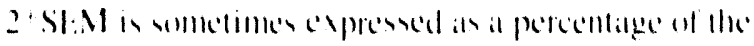

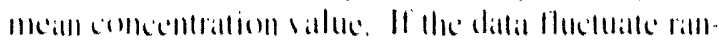

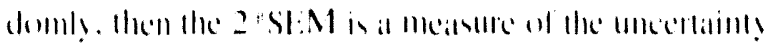

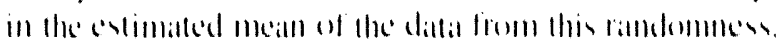

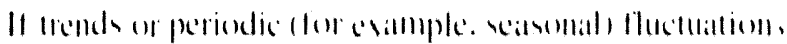

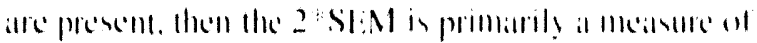

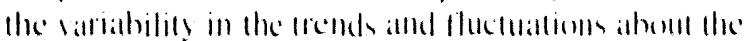
meill of the dilt.1.

\section{Understanding Graphical Information}

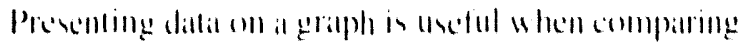

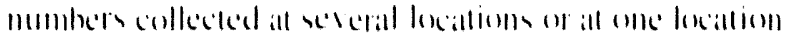

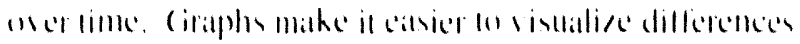

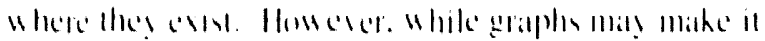

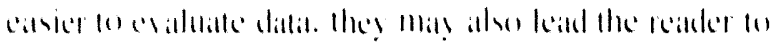

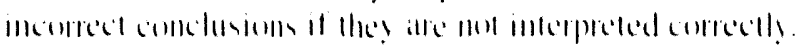

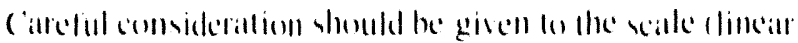

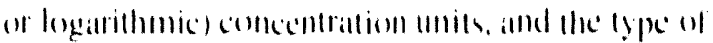
uncortaint! uscol.

Some of the datla graplece in this report alle plolled using

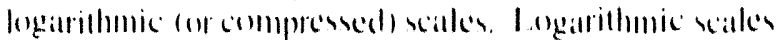

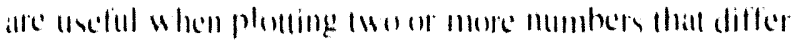
greally in sice. forr exallmple. a sillmple will a collecelllat fioll of $5 \mathrm{~g} / \mathrm{l}$. Would get low all the boltom of the glaph if plotled on a limeatr scalce 11 ith a simple has ing a concen-

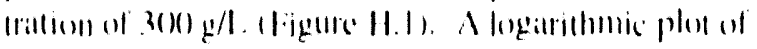

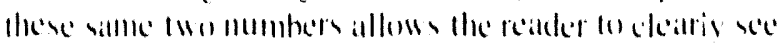
buth dillat points arigute H.21.

The meitl salues glaphed in this report hatse ertical

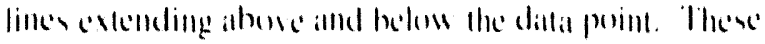

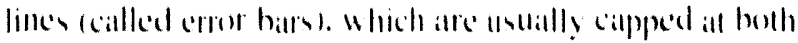

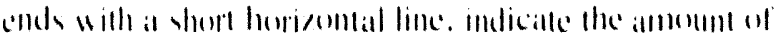

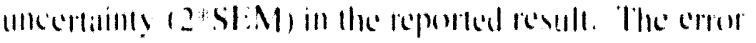

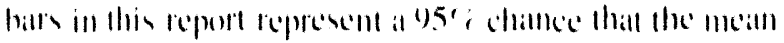

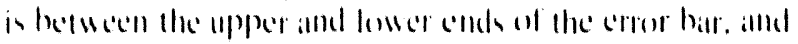

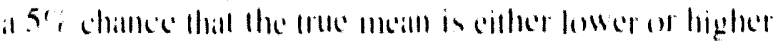

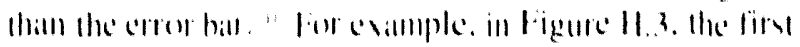

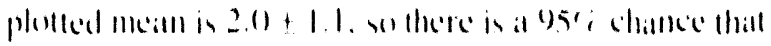

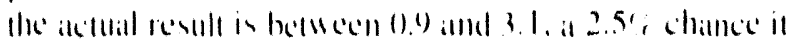

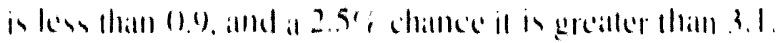

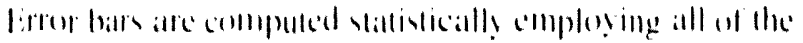

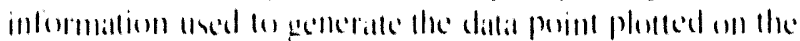

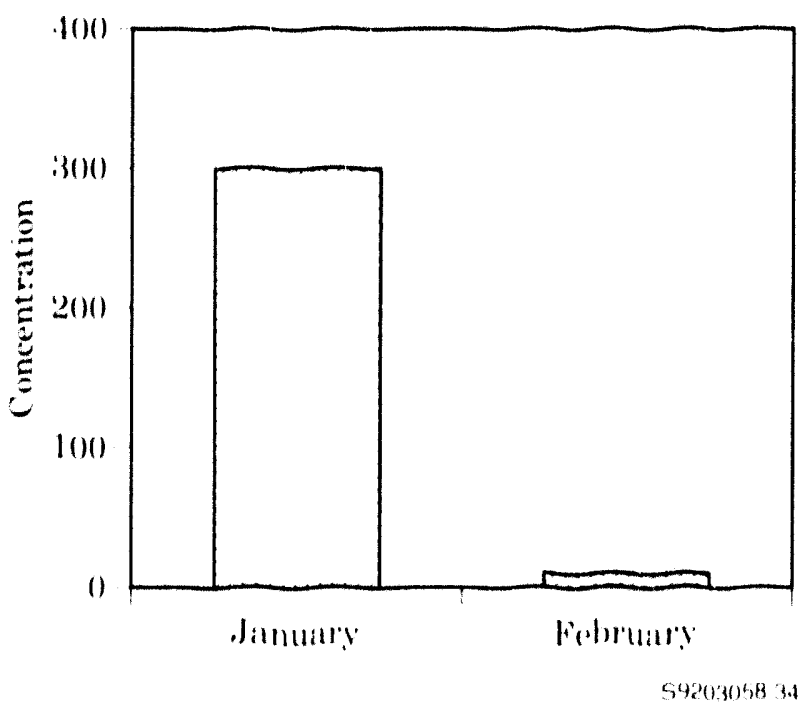

Figure H.1. Data Plotted Using a Linear Scale

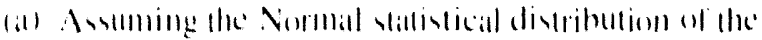
litlit. 


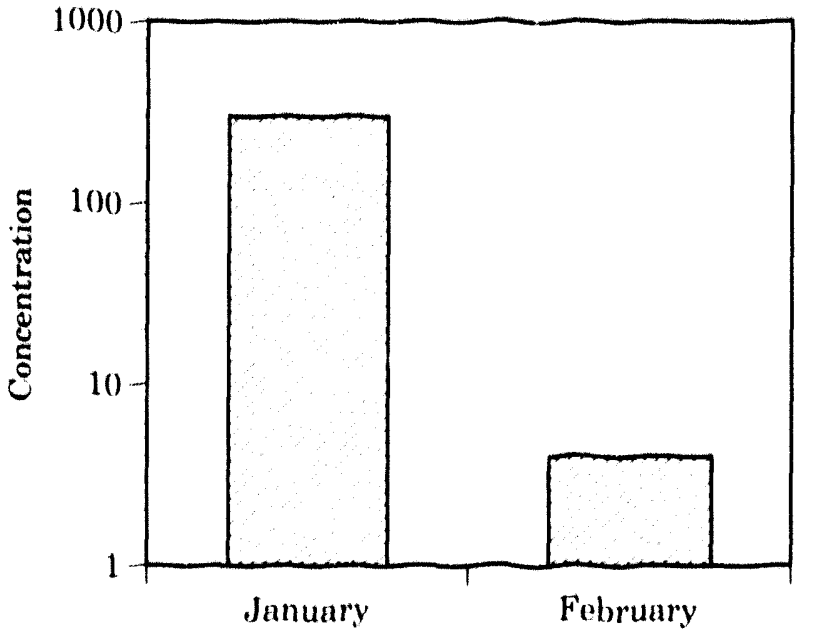

$\$ 9203058.33$

Figure ti.2. Data Plotted Using a Logarithmic Scale

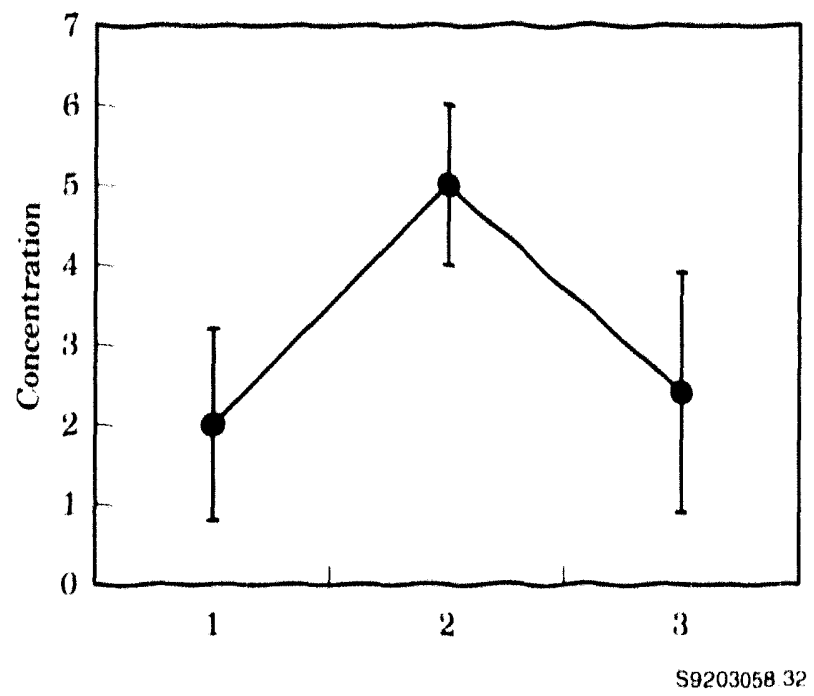

Figure H.3. Data With Error Bars Plotted Using a Linear Scale graph. These bars provide a quick visual indication that one mean may be statistically similar to or different from another mean. If the error bars (or range of values) of two or more means overlap. as is the case with means 1 and 3 and the ans 2 and 3. the means may be similar. statistically. If the errer bars do not overlap (means I and 2), the means may be statistically different. Meians that appear to be very different visually (meanns 2 and 3 ) may actually be quite similar when compared stati-lically.

Uncertainties (error bars) are not plonted in Section 5.6. "Soil and Vegetation Surveillance." Insteud, sample median. maximum, and minimum values are illustralled. Uncertainties are not used becaluse of the small number of soil and vegetition samples collected and analyzed during the years.

\section{Greater Than $(>)$ or Less Than (<) Symbols}

Grealler than ( $>$ ) or less than (<) symbols are used to indicate that the actual value may either be latger than the number given or smaller than the number given. For example. $>0.09$ woteld indicalle that the actual value is greatler than (1.)(0). An incequality symbol pointed in the opposite direction $(<0 .(19)$ would indicate that the number is less than the vallec presented. If an inequality symbol is used in associattion with an underscone $(\leq$ or 2). this indicilles that the actual vallue is lews-than-orequal-to or greater-than-er-equal-10 the number given, respectively. 


\section{Elemental and Chemical Constituent Nomenclature}

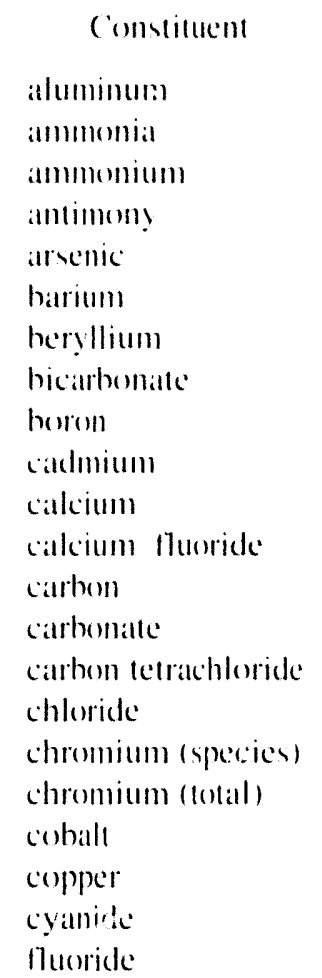

\section{Conversion Table}

\begin{tabular}{|c|c|c|}
\hline Multiply & By & To ohtatin \\
\hline in. & 2.54 & $\mathrm{~cm}$ \\
\hline 11 & 0.30 .5 & III \\
\hline $\mathrm{mi}$ & 1.01 & $\mathrm{~km}$ \\
\hline Ih & 0.454 & $k !$ \\
\hline licy yl & 0.946 & 1 \\
\hline 11 & 0.119 .3 & III \\
\hline acres & 0.4115 & hal \\
\hline$m i$ & 2.54 & hm \\
\hline ii & 0.018 & $\mathrm{II}^{\prime}$ \\
\hline dpוn & $(1.4 .51)$ & $\mathrm{pCi}$ \\
\hline 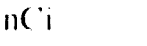 & $(1 .(1)) 1$ & $\mathrm{pci}$ \\
\hline $\mathrm{p}(\ddot{i} / \mathrm{l}$ & 10 & $\mu(\dddot{i} / 11,1$. \\
\hline$p(i / m$ & 111 & $(\mathrm{i} / \mathrm{m})$ \\
\hline$p(i / m$ & 111 & $m(i / c m)$ \\
\hline $\mathrm{m}(\mathrm{i} / \mathrm{hm}$ & 1.11 & $\mathrm{n}(\mathrm{i} / \mathrm{m}$ \\
\hline heculerel & $2.7 \times 111$ & curic \\
\hline gril! & $1(1)$ & $\mathrm{rad}$ \\
\hline vier & $1(1)$ & $r(m)$ \\
\hline pph & $(1) .(1)(1)$ & ווחיות \\
\hline $\mathrm{F}$ & $(1 \div-32) \div 9 / 5$ & $c^{\circ}$ \\
\hline
\end{tabular}

Symbol

Al

NHI

NH,

Sh

As

BaI

$\mathrm{Bc}$

H(C)

B

(id

Cal

Call

C

( ()$^{\prime}$

$\mathrm{CCl}$

(i)

(ir)

$\mathrm{Ci}$

(i)

(iI

( N

I.
Comstituent

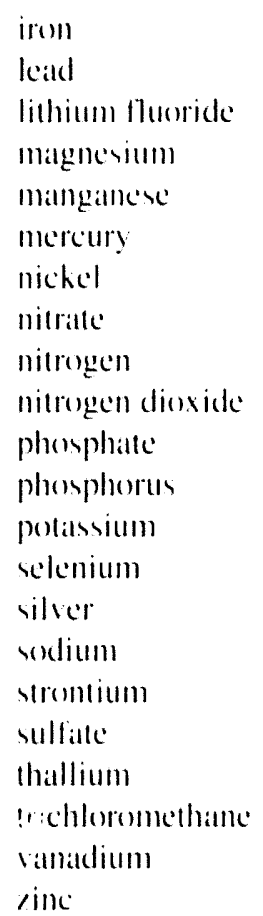

Symbol

$1 \mathrm{c}$

$\mathrm{Ph}$

I.it:

Mg

Mn

$\mathrm{Hg}$

$\mathrm{Ni}$

No ;

$N$

N()

$P()_{i}$

$\mathrm{P}$

k

Se

Ag

$\mathrm{Nal}$

Si

SO:

TI

chol. $\mathrm{V}$

$\% .11$
Muliply

\begin{tabular}{|c|}
\hline (') \\
\hline$m$ \\
\hline hIII \\
\hline$k !$ \\
\hline 1. \\
\hline$m$ \\
\hline hat \\
\hline km \\
\hline 111 \\
\hline$p(i$ \\
\hline ('i \\
\hline$\mu(\mathrm{C} / \mathrm{ml}$. \\
\hline ( $\mathrm{C} / \mathrm{m}$ \\
\hline ncillo' \\
\hline $\begin{array}{l}\text { cillic } \\
\text { lial }\end{array}$ \\
\hline rim \\
\hline p' \\
\hline
\end{tabular}

$$
\text { B! }
$$$$
\text { (1. } 304
$$$$
3.28
$$$$
\text { (1). } 621
$$$$
2.2015
$$$$
1.057
$$$$
\text { 11).76 }
$$$$
2.47
$$$$
0.386
$$$$
3.7
$$$$
2.22
$$$$
\text { I. (1)(1) }
$$$$
11)^{\prime \prime}
$$$$
\text { (1) }
$$$$
10
$$$$
1.11
$$$$
3.7 \times 10^{10}
$$$$
\text { (1).(1) }
$$$$
\text { (). (1) }
$$$$
\text { I. (1)() }
$$

$(\times 99 / 5)+.32$
Tu (ohtain

in.

ii

mi

Ih

licy yl

11

acres

$111 i^{\circ}$

ii

upm

1)

$p(i / \mathrm{l}$.

p(i/mi

pe i/m m( i/km" hecquerel graly sicuer pph 


\section{Acronyms and Abbreviations}

AALC; ambient air level goals

Al.ARA as low as reasonably achievable

Al.E Arid Lands Ecology (Reserve)

ANSI American National Standards Institute

ASME American Society of Mechanical Engineers

ASTM American Socicty for Testing and Materials

CERCIA Comprehensive Environmental Response. Compensation, and Liability Act

CFR Code of Federal Regulations

COES community-operated envirommental surveillance

DCE dichloriethylene

ICC; Derived Concentration Guide

DIT

DOE

DOH Washington State Department of Health

DOI U.S. Department of the Interior

IDW Drinking Waller Standard

EA envirommental assessment

Ecology Washington Statc Department of Ecology

EIDE effective dose equivalent

EIS envirommental impact statement

EM-50 Office of Technology Development

EMI. environmental measurement laboratory
EMSL environmental monitoring and surveillance laboratory

EPA U.S. Environmental Protection Agency

ERA Expedited Response Actions

ERMC Environmental Restoration/Remediation Management Contractor

ERRA Environmental Restoration Remedial Action (Program)

ES\&H environment, salesty, and health

ES\&QA Environmental Safety and Quality Assurance

FEMP Facility Effluent Monitoring Plan

FFCA Federal Facility Compliance Agreement

FFTF Fast Flux Test Facility

FR Federal Register

FY liscal year

HCRI Hanlord Cultural Resources Laboratory

HEDR Hanford Environmenti' Dose Reconstruction (Project)

HQ U.S. Department of Energy-Headquarters

HCPP Hanford generating plant

HRA Hanford remedial action

HMS Hanford Meteorological Station

HWVP Hanford waste vitrification plant

IC.RP International Commission on Radiological Protection

I'T International Technology Corporation 


\begin{tabular}{|c|c|c|c|}
\hline KEH & Kaiser Engineers Hanford & PEIS & $\begin{array}{l}\text { programmatic envirommental impact } \\
\text { statement }\end{array}$ \\
\hline I.EPS & low-energy photon & PFP & Plutonium lanishing Plant \\
\hline L.L.BC; & low-level burial ground & PNI. & Pilcific Northwest I aboratory \\
\hline I,WDF & licpuid waste disposal facility & $\mathbf{P Q I}$ & Process Quality Department \\
\hline MCL. & maximum contammant level & PSD & prevention of significant deterionation \\
\hline MCIC; & maximum contaminant level goals & PUREX & Plutonium Uranium Extraction (Plant) \\
\hline MI)A & minimum detectable activity & $\mathbf{Q A}$ & quality assurance \\
\hline MI)C: & minimum delectable concentration & QATF & Quality Assurance Task lonce \\
\hline MEI & maximally exposed individual & $Q C$ & yuality control \\
\hline NASQAN & $\begin{array}{l}\text { Natural Stream Quality Accomnting } \\
\text { Network }\end{array}$ & $\mathbf{R C A}$ & radi angically controlled area \\
\hline NC:RP & $\begin{array}{l}\text { National Council on Radiation Protection } \\
\text { and Measurements }\end{array}$ & RCHN & Richland North \\
\hline NEPA & National Envirommental Policy Act & $\begin{array}{l}\text { RCRA } \\
\text { RCW }\end{array}$ & $\begin{array}{l}\text { Resource Conservation and Recovery Act } \\
\text { Revised Code of Washington }\end{array}$ \\
\hline NESHAP & $\begin{array}{l}\text { National Emission Standards for } \\
\text { Hazardous Air Pollutants }\end{array}$ & REDOXX & Reduction Oxidation (Plann) \\
\hline NOV & notice of violation & RI/FS & remedial investigation/feasibility study \\
\hline NPDES & $\begin{array}{l}\text { National Pollutant Discharge Elimination } \\
\text { System }\end{array}$ & RI. & $\begin{array}{l}\text { U.S. Department of Energy Richland } \\
\text { operations office }\end{array}$ \\
\hline NPR & new production reater & SAIC & $\begin{array}{l}\text { Science Application International } \\
\text { Corporation }\end{array}$ \\
\hline NPS & National Park Service & & \\
\hline NRC' & U.S. Nuclear Regulatory Commission & SARA & $\begin{array}{l}\text { Superfund Amendments and } \\
\text { Reatulhorizaltion Ace }\end{array}$ \\
\hline NS & no standard or no sample & SC'A & surface comlamination anca \\
\hline NTU & nephelometric turbidity unit & SE & standard error \\
\hline NWR & national wildlife refuge & SEM & standard error of the me:an \\
\hline OEMP & $\begin{array}{l}\text { Operational Environmental Monitoring } \\
\text { Program }\end{array}$ & SEN & Secretary af Encrgy Notice \\
\hline PC'B & polychlorinated biphenyl & SI & International System of Inits \\
\hline & & TCE & trichlorocthylene \\
\hline
\end{tabular}


thermoluminescent dosimeter

USGS

U.S. Cieologicial Survey

TRIDEC Tri-Cities Industrial Develupment Council

USRADS Ultratsonic Ranging and Data System

TRU

transuranic

UST

Underground Storage Tank

TSD

treatment, storage. and disposial

V()A

volatile organic analyses

TSS

total suspended solids

VOC

volatile organic compound

TWRS Tank Waste Remediattion Systems

WAC

Washington Administrative Code

UNSCEAR United Nations Science Committee on the

WIPP

Waste Isolation Pilot Plant

Effects of Atomic Radiation

WHC Westinghouse Hanford C'ompany

UO, Plant Uranium Trioxide Plant

URM underground radiondive malterials

WRA Wildlife Recreation Area 


\section{Introduction}

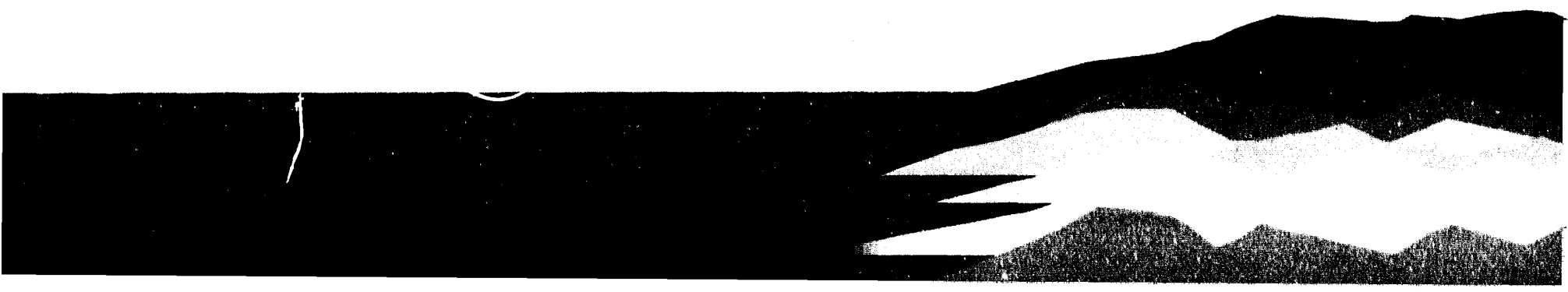




\subsection{Introduction}

The purpose of this report is 10 stommarise information and datat that chatracterise Hanford Sile environmental management pertentmance and demonstrate the status of compliance wilh applicable federal. statle, and local envirommental laws and regulations. The repent also highlights signilicant emvirommental programs and ciforts.

The report describes the Site mission and atctivities, gencral emsirommental features, radiological and chemicall redeases from operations, stallus of compliatlece will

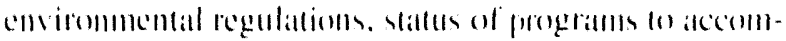
plish compliance. and envirummental monitoring activilies and results.
Those interested in more detail than the stummary information presented in this report are referred to the technicial reports cited in the text. Report sources include lecal community libraries and the National Technical Information cenler. Springlichl. Virginial 22161. Descriptions of amalytical and sampling medhods. formerly part of this repert. alle contatined in the Manford

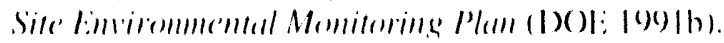

Readers less familiall with the concepts. Eerminology. and units used in this report ma!y find the preceding Helpful Informallion section useful. 


\subsection{Site Mission}

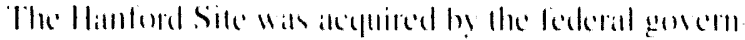

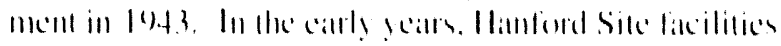

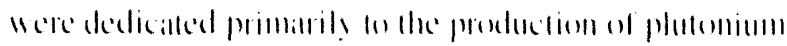

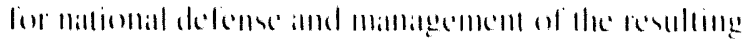

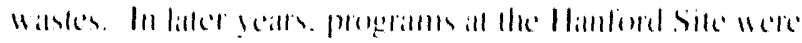

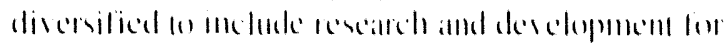

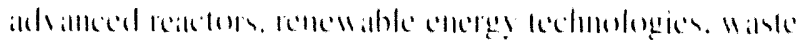

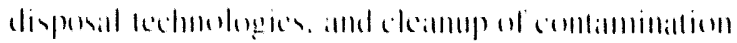
fiom part praticen.

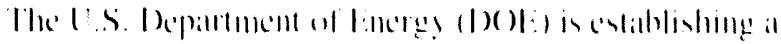

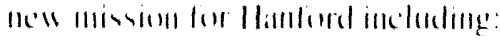

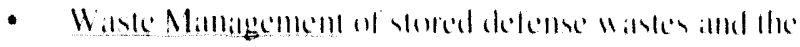

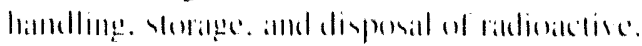

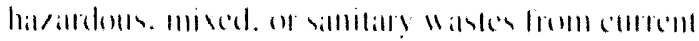
illititices

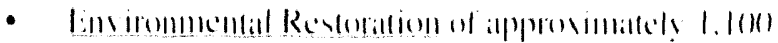

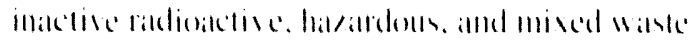
sites and aboul 10() sutplus litcilitices

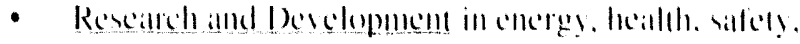

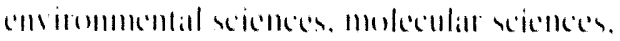

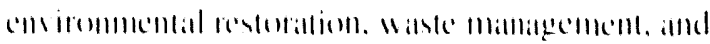
mationall securil!

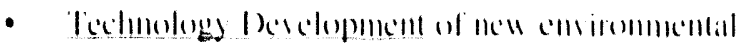

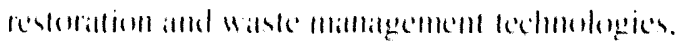

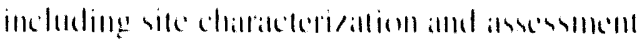

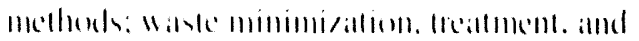

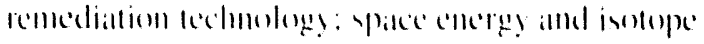

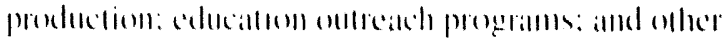
yecial intialtisas

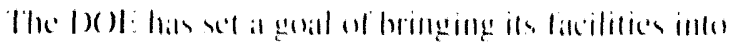

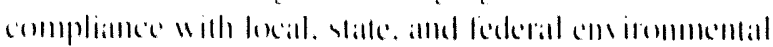
lims ('allup h 2018 . 


\subsection{Introduction to the Hanford Site}

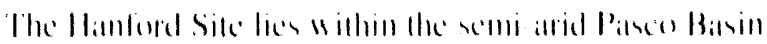

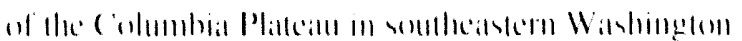

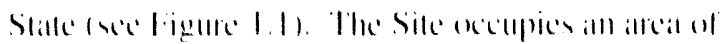

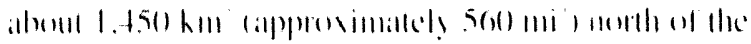

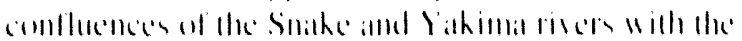

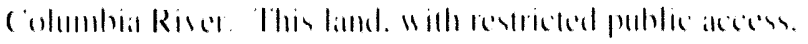

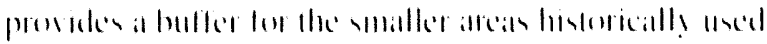

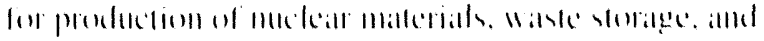

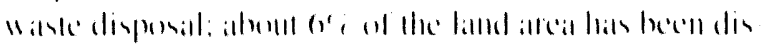

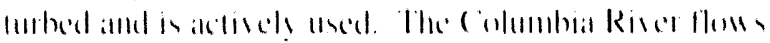

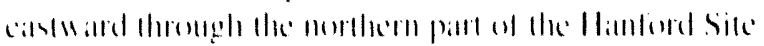

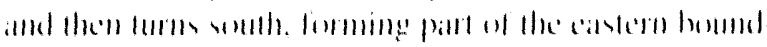

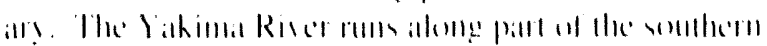

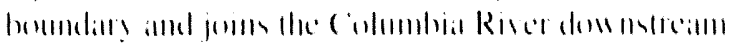

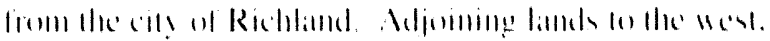

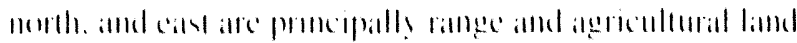

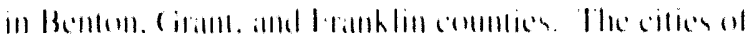

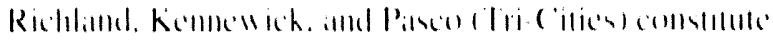

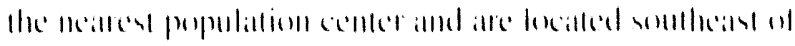
he Hanlund Sile.

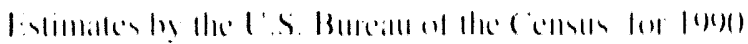

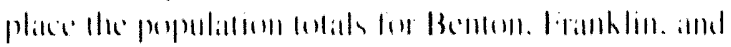

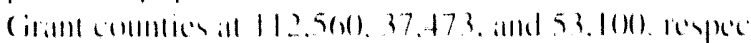

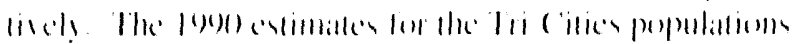

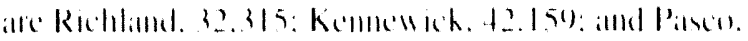

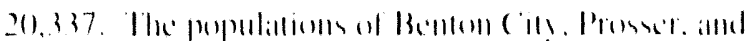

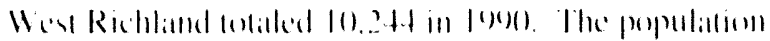

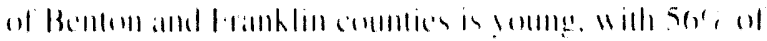

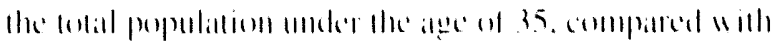

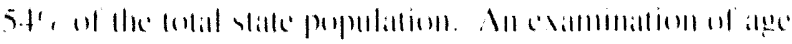

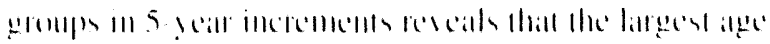

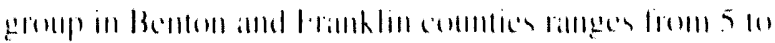

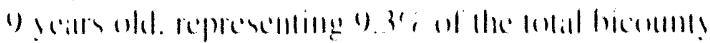

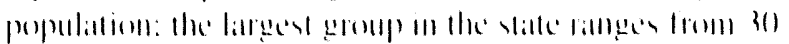
10.3 .4 ? popmination.

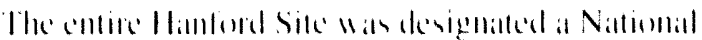

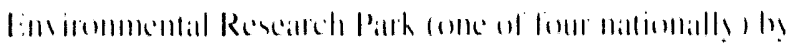

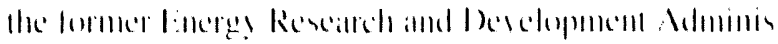

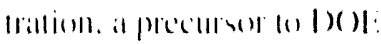

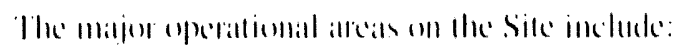

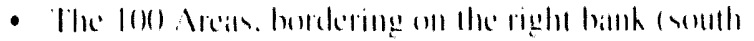

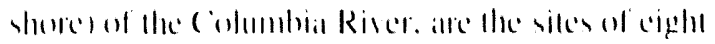

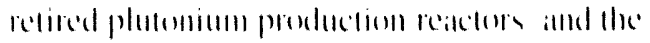

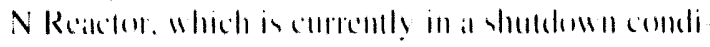

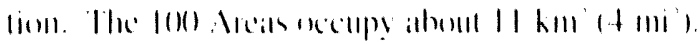

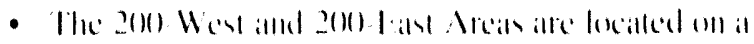
plilleall and allo ahoul 8 andd 11 hmo 15 and 7 mil).

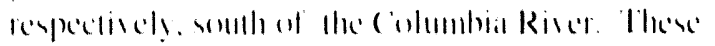

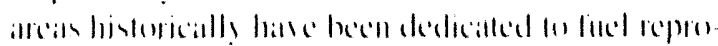

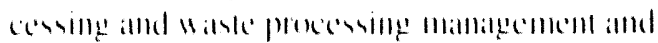

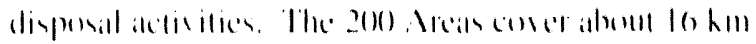
(1) IIIi)

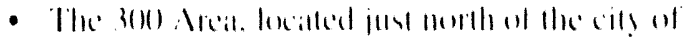

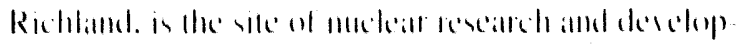
ment. The alleal (1)

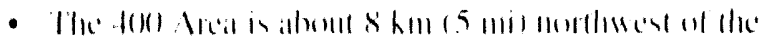

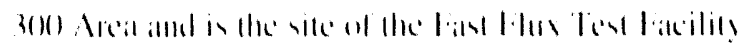

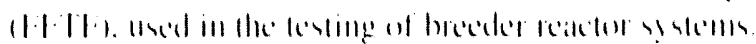

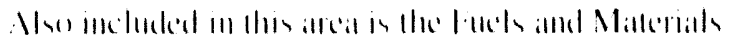
I: vamminallonl landils

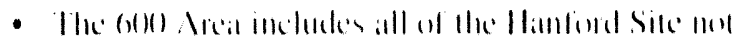

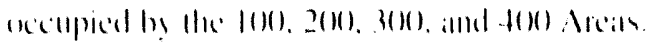

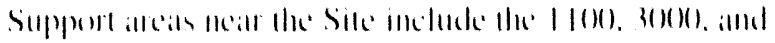

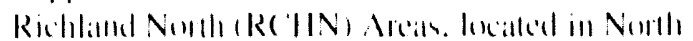

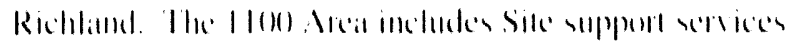

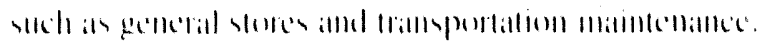

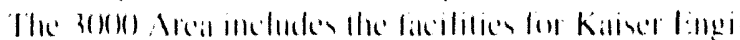

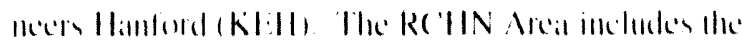

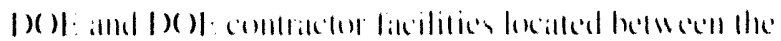

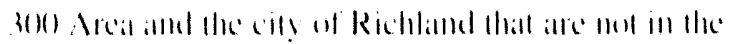
11011) ambl 3(1)10) Areats

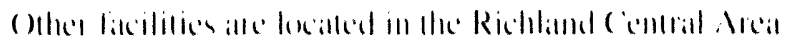

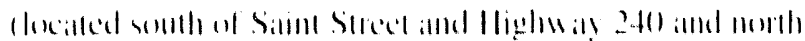




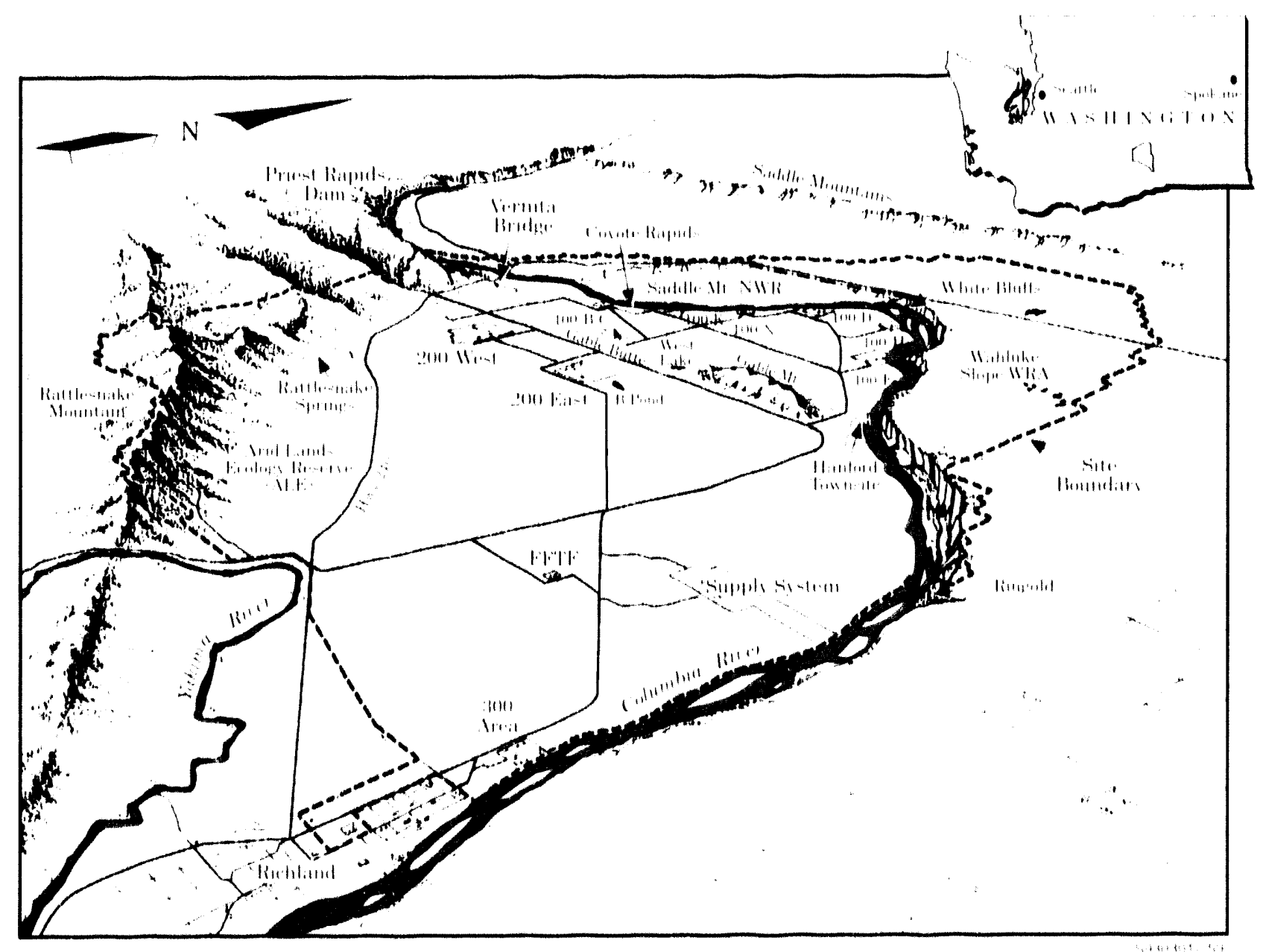

Figure 1.1. DOE's Hanford Site and Surrounding Area

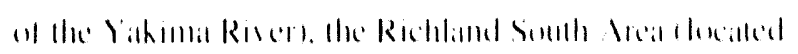

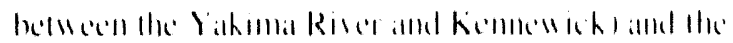

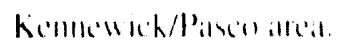

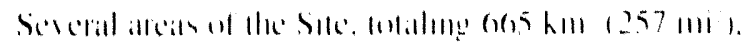

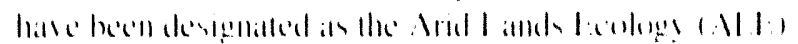

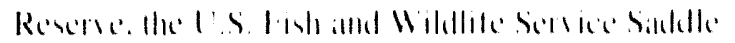

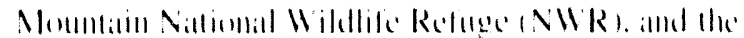

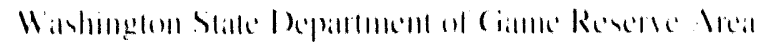

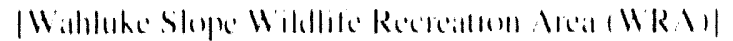

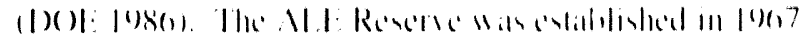

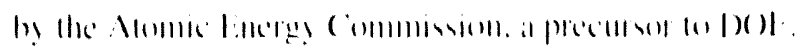

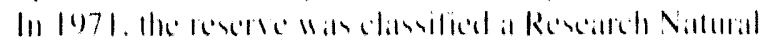

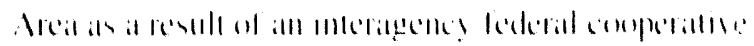
itgle'sllm.

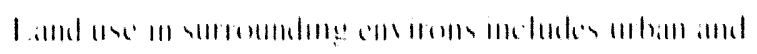

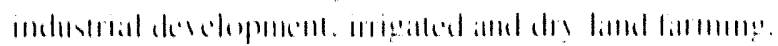

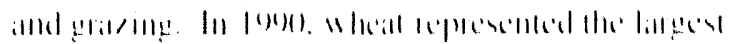

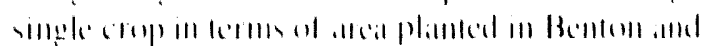

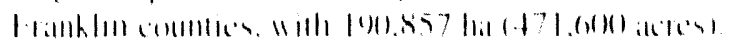

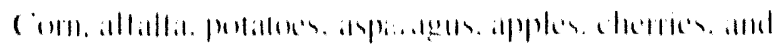

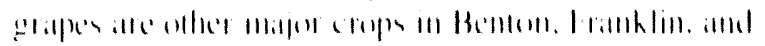

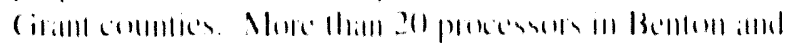

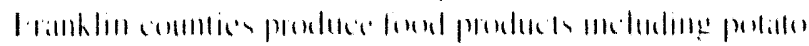

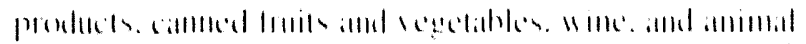
lind

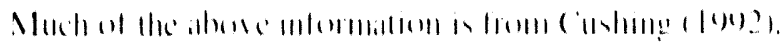

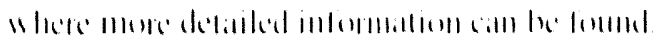




\subsection{Major Operations and Activities}

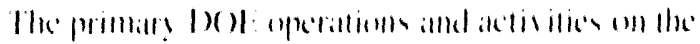

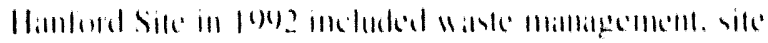

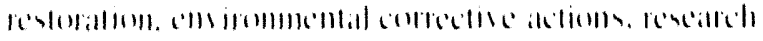

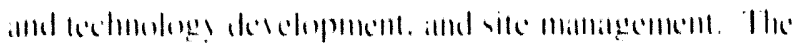

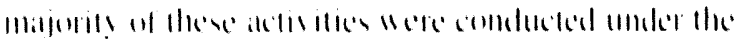

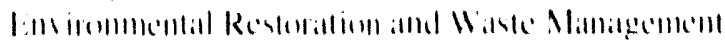

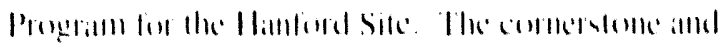

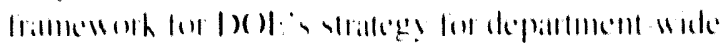

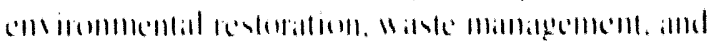

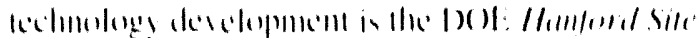

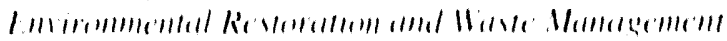

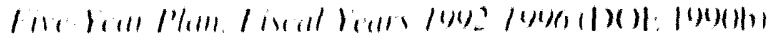

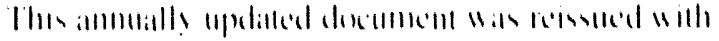

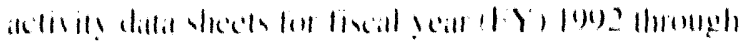
10)

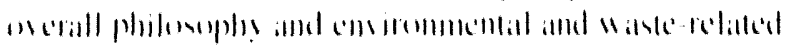

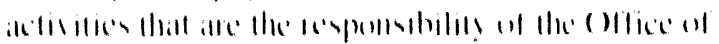

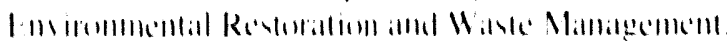

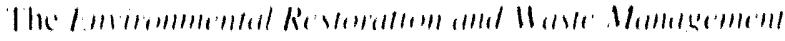

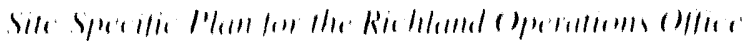

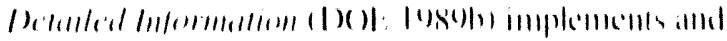
s"ppons

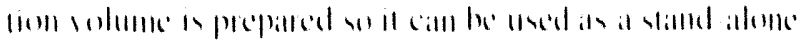

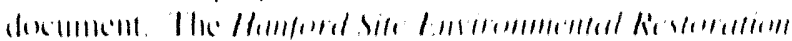

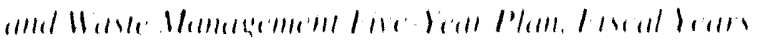

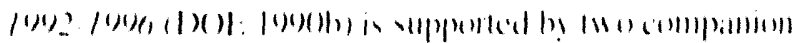

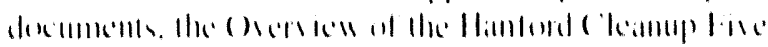

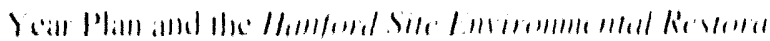

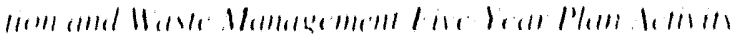

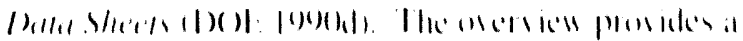

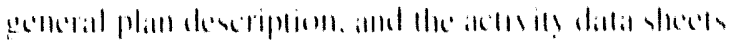

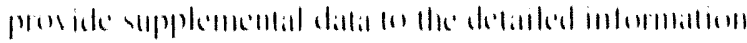
dactumtill.

\section{Waste Management}

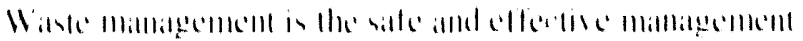

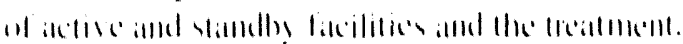

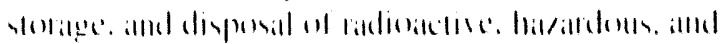

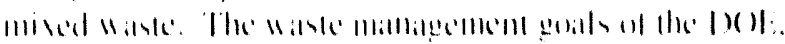

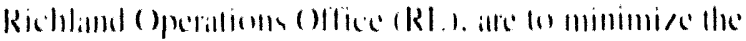

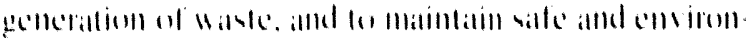

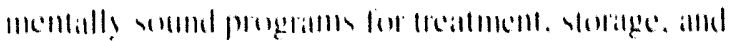

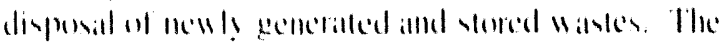

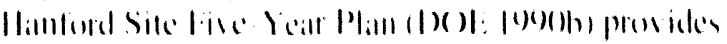

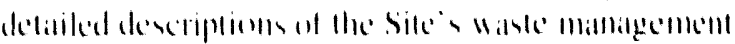

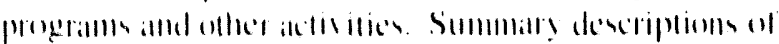

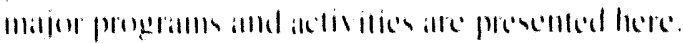

\section{Waste Minimization}

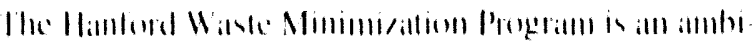

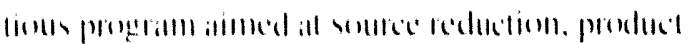

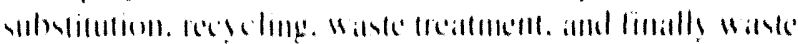

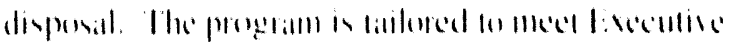

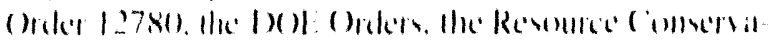

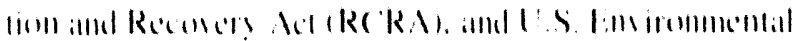

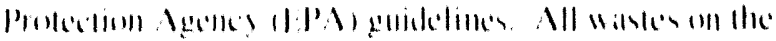

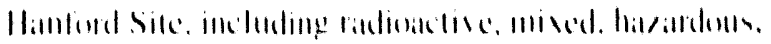

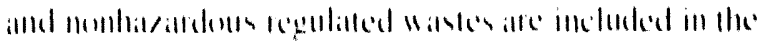

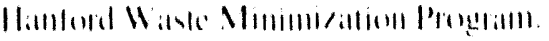

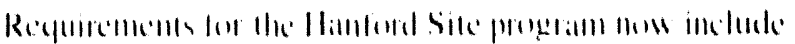
He lollowing:

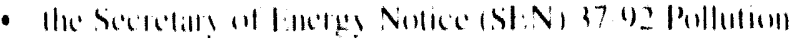

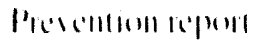

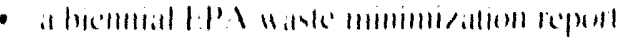

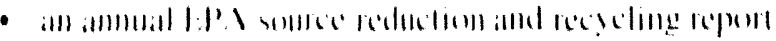

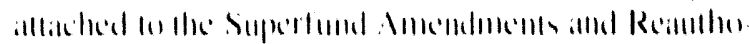

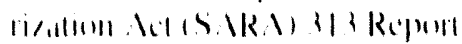

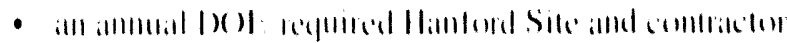
ycerlis

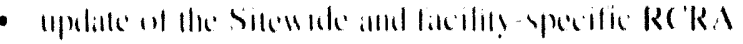
P'illt B perminis

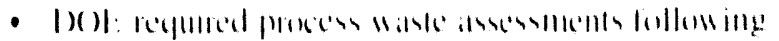
stille gurdelimes 


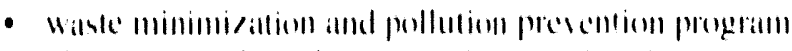

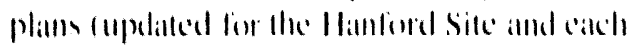
(ontritulur).

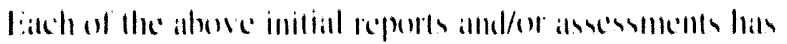
hecell complyleted.

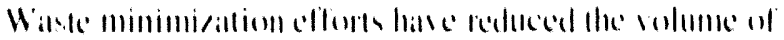

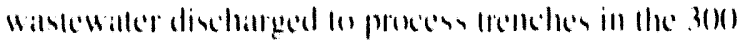

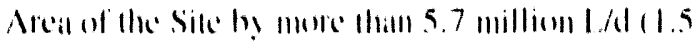

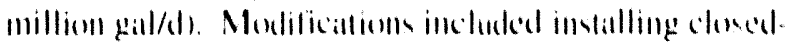

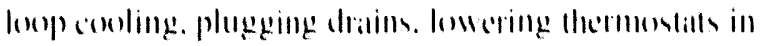

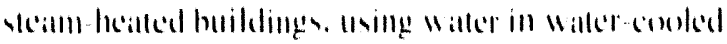
eyuipmentol anly when repuired. and pulling andministra live combors in place where pusible.

In addition, at multifumedion lamh Spate Mambegement

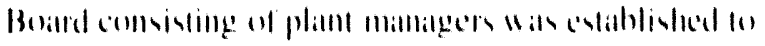

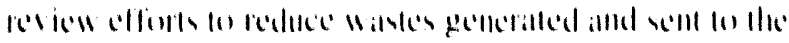

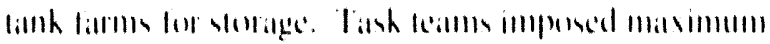

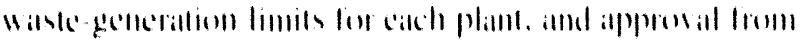

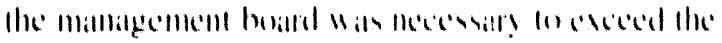

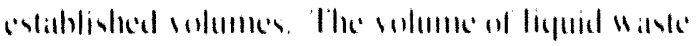

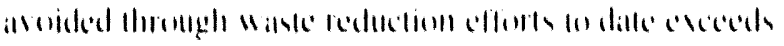
22 million I. (5.5 million gall).

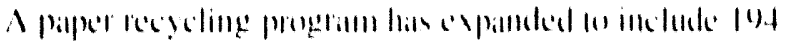

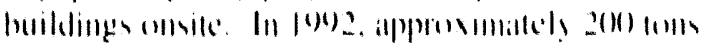

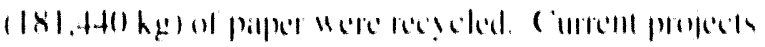

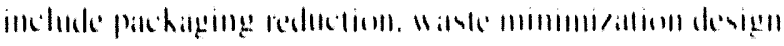
ahechlist. and lecholmoleg! mambles

\section{Soll Column Discharges}

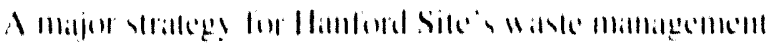

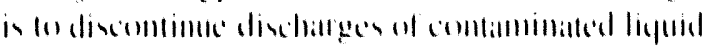

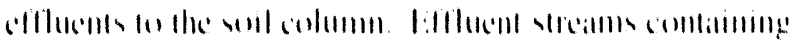

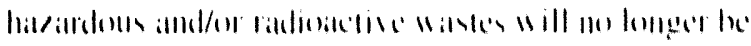

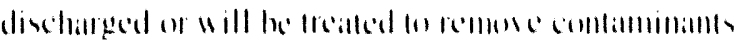

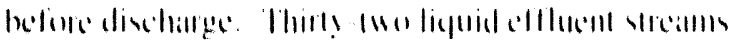

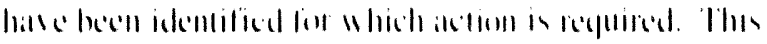

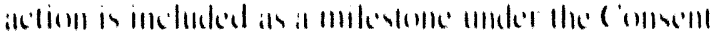

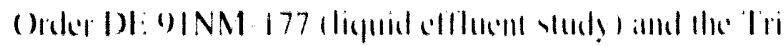

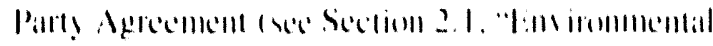

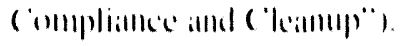

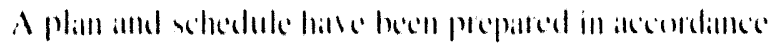

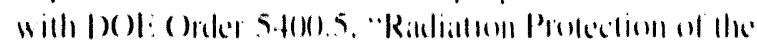

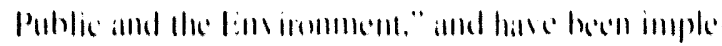

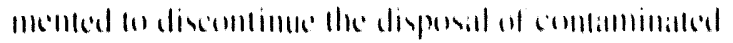

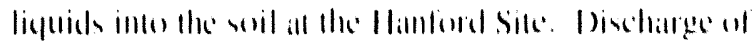

comlaminamls in the major Waske streamms will be discon

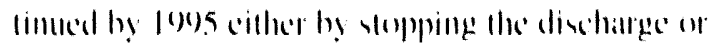

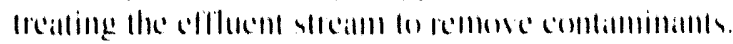

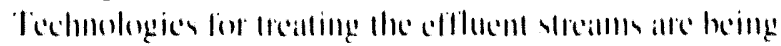
evaluated fo dekemime which would hes med regulattor! revuirements.

\section{Stored Wastes}

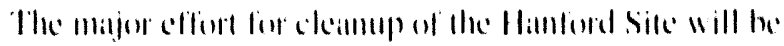

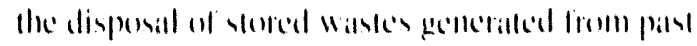

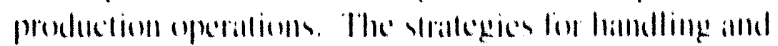

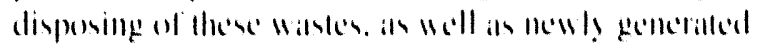

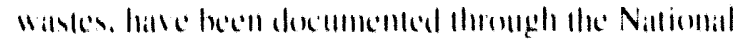

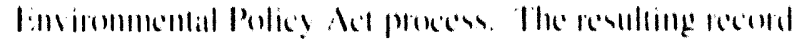

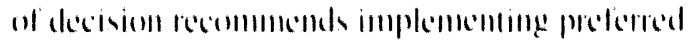

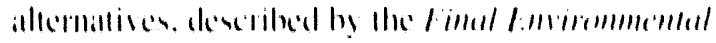

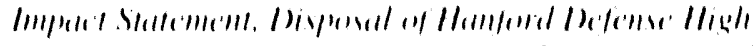

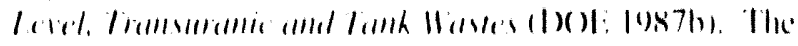

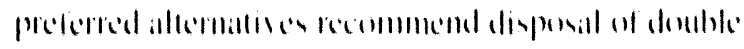

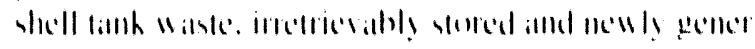

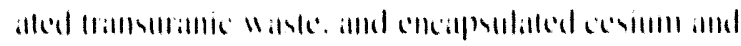
slmmliam Manke as follum.

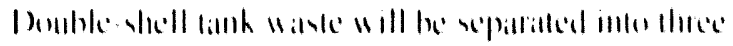

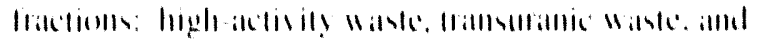

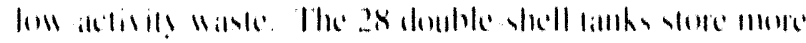

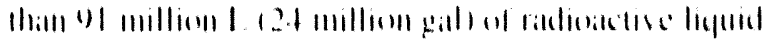

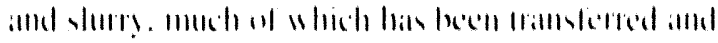

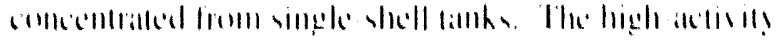

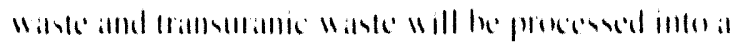

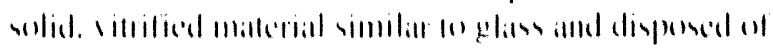

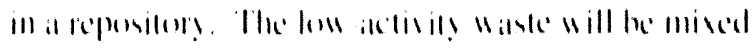

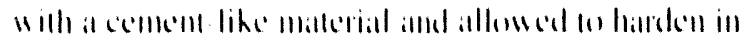

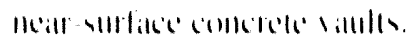

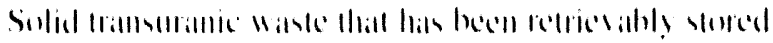

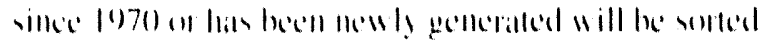

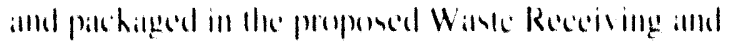

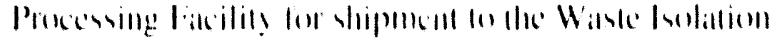

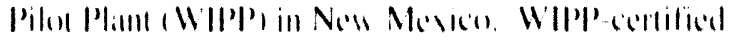

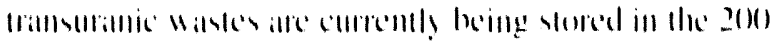

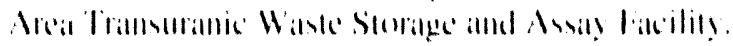

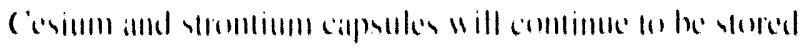

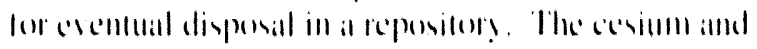

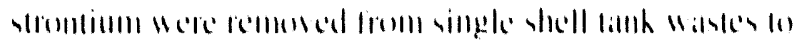

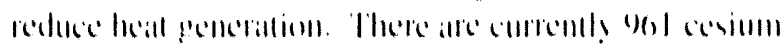

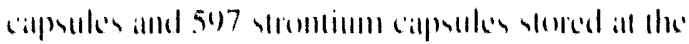

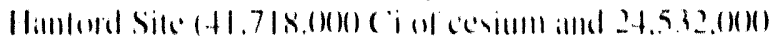
('i) vinllitum) 


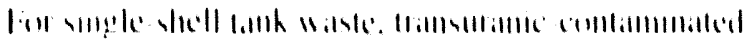

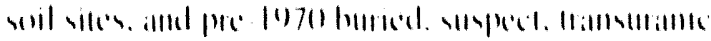

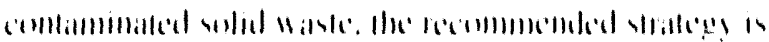

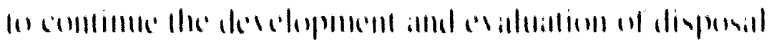

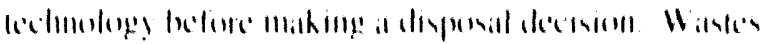

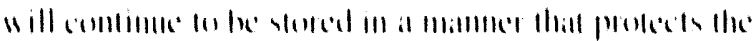

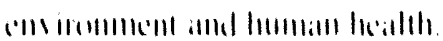

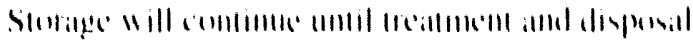

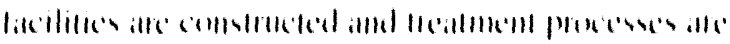

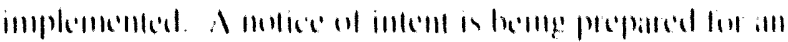

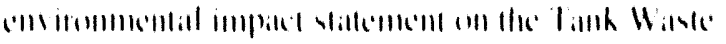

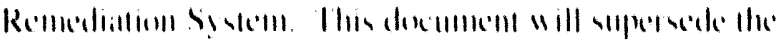

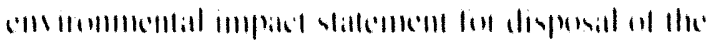
single sholl tallh wistis

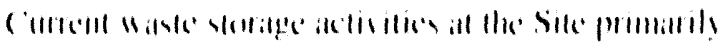

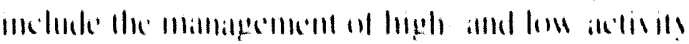

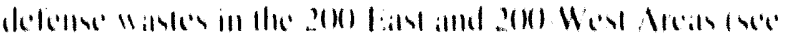

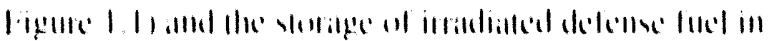

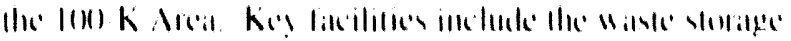

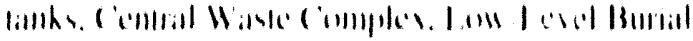

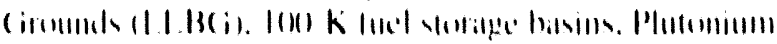

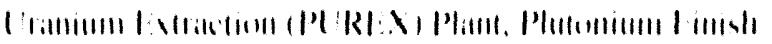

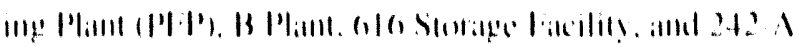
1:1 in|mintuा

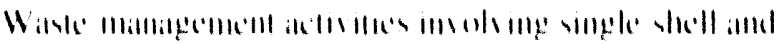

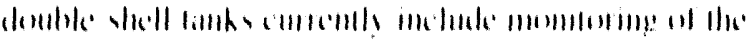

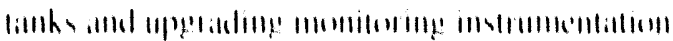

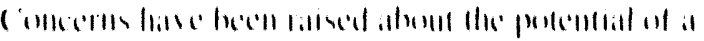

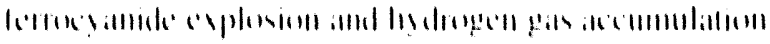

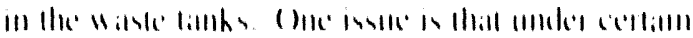

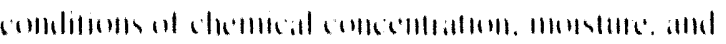

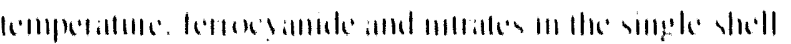

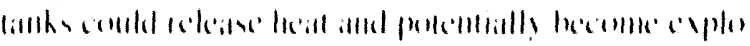

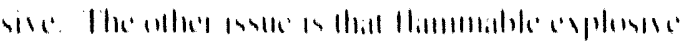

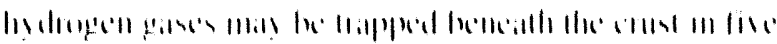

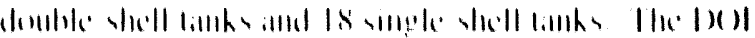

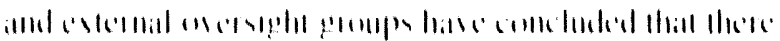

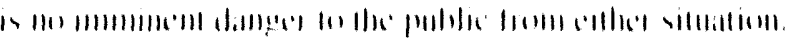

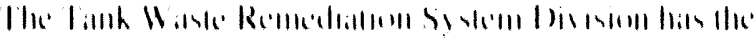

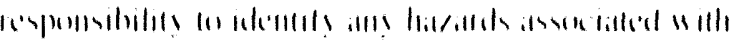

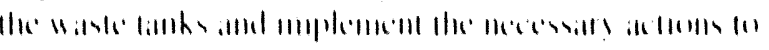

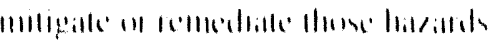

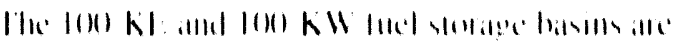

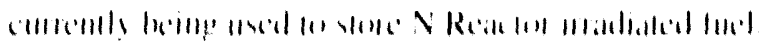

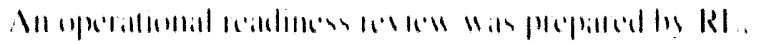

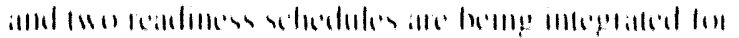

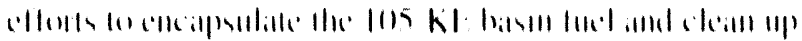

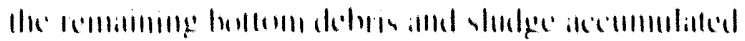

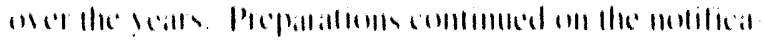

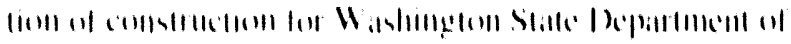

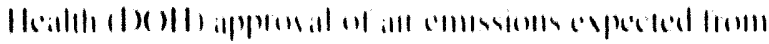

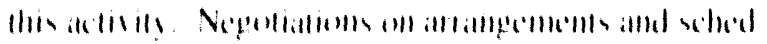
ules ills conllumumy

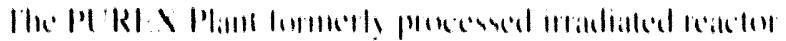

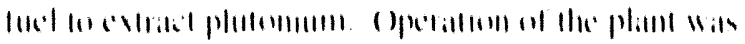

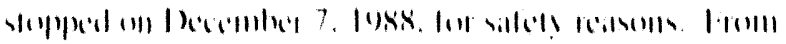

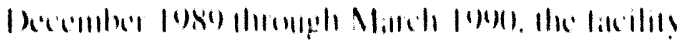

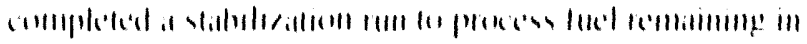

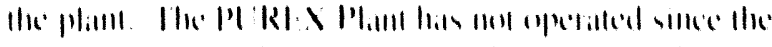

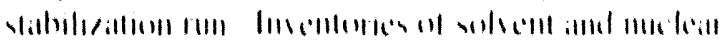

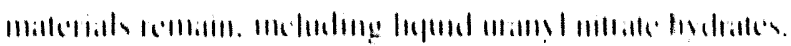

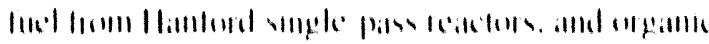

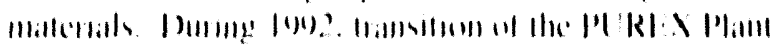

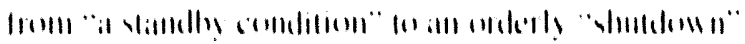

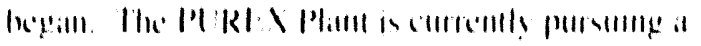

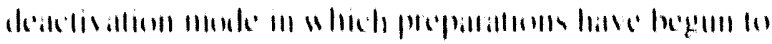

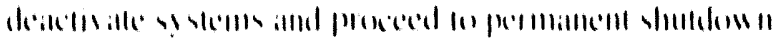

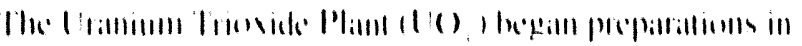

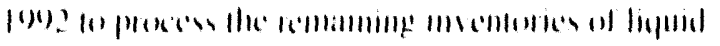

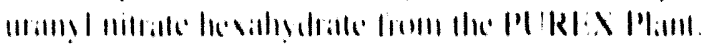

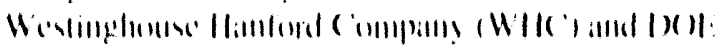

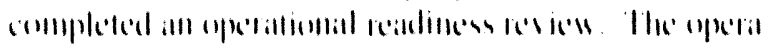

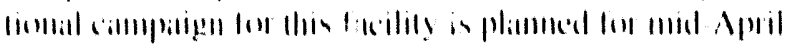

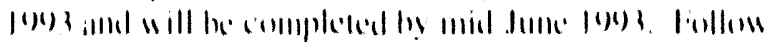

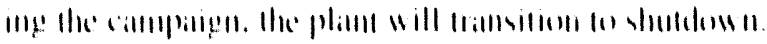

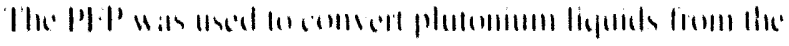

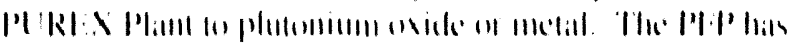

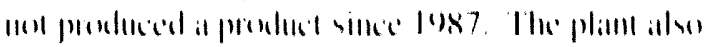

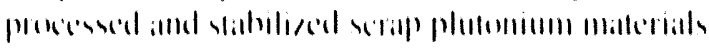

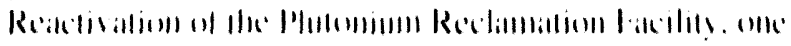
al Ile (1)

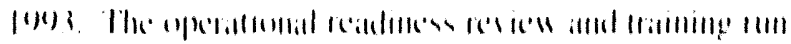

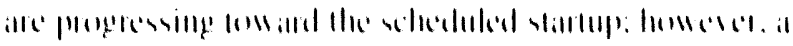

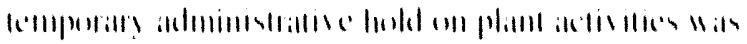

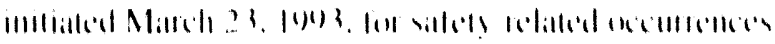

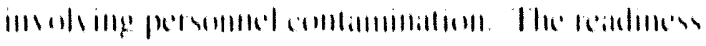

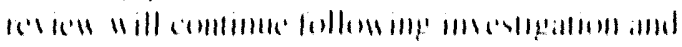

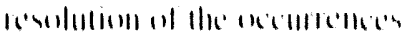

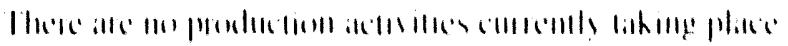

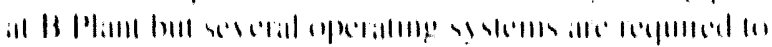

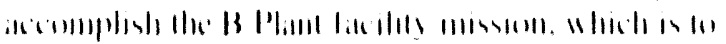

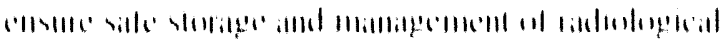
IIIrinllillin's 


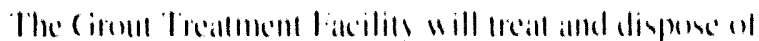

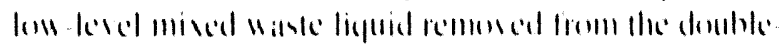

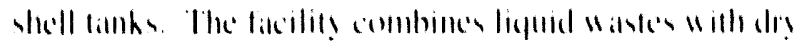

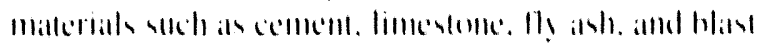

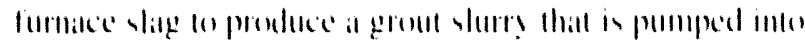

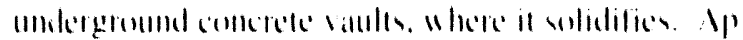

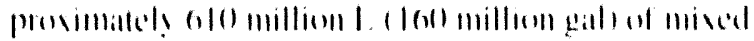

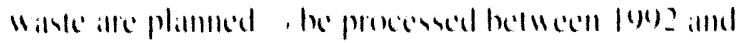

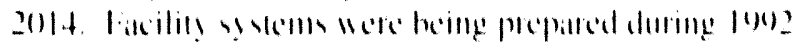

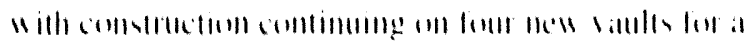

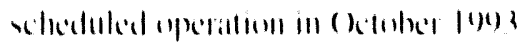

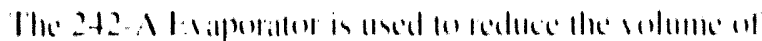

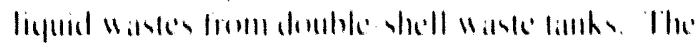

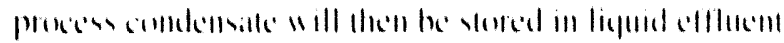

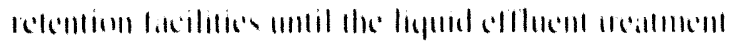

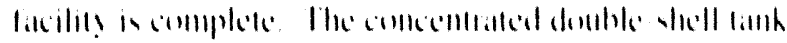

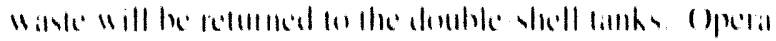

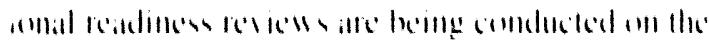

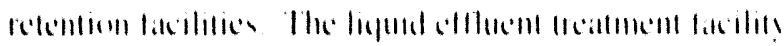

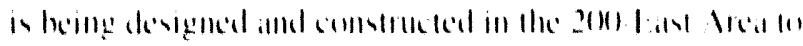

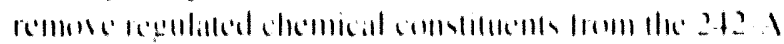

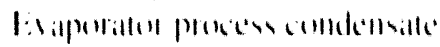

\section{Environmental Restoration}

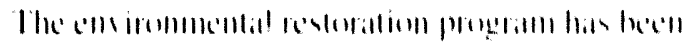

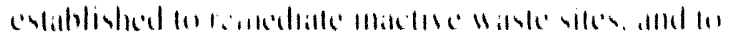

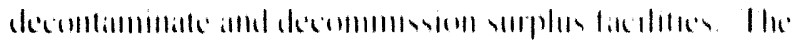

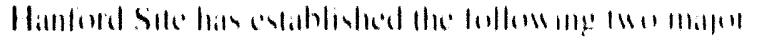

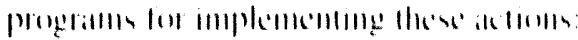

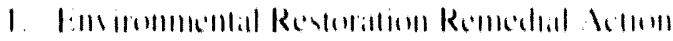
IIRR W PULIIIII

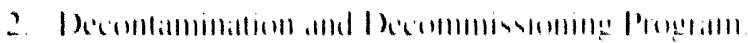

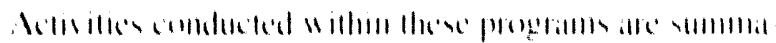

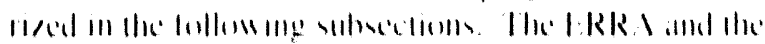

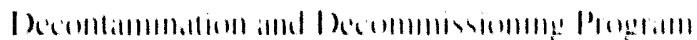

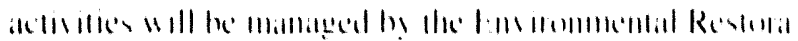

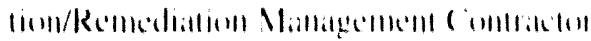

\section{Environmental Restoration Remedial Action Program}

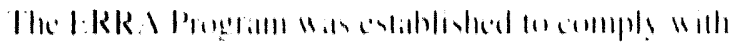

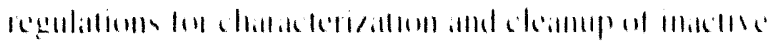

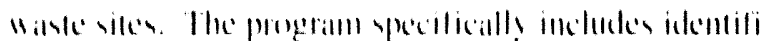

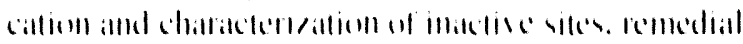

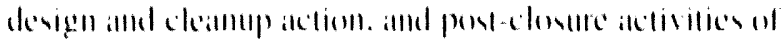

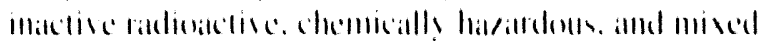
"Iisle vill's

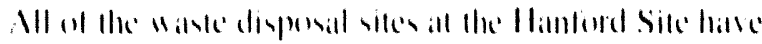

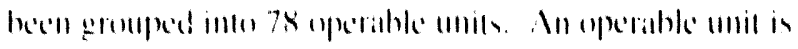

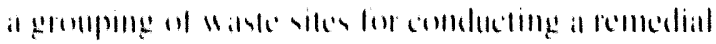

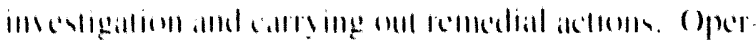

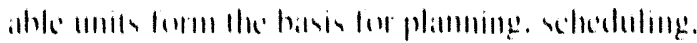

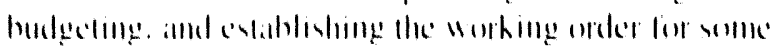

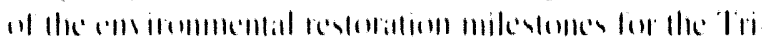

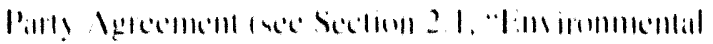

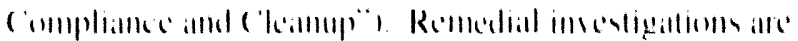

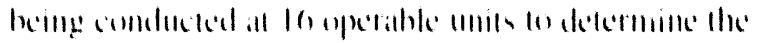

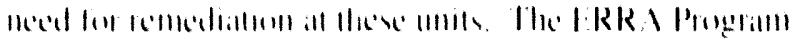

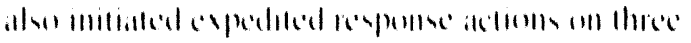

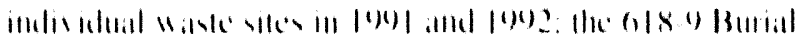

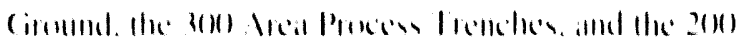

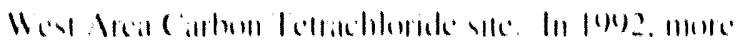

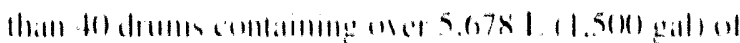

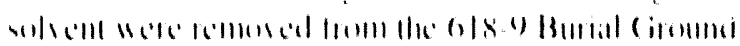

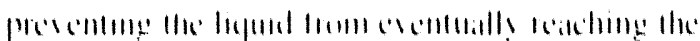

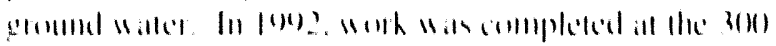

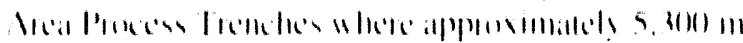

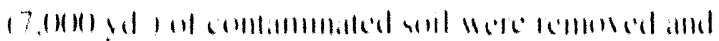

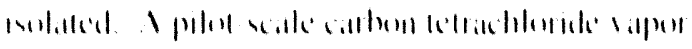

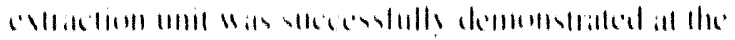

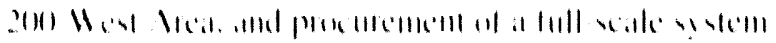
III IIIIllatid

\section{Decontamination and Decommissioning Program}

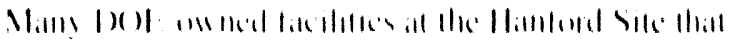

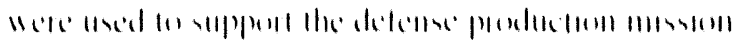

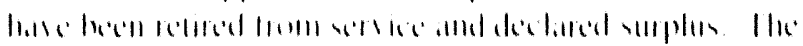

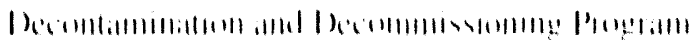

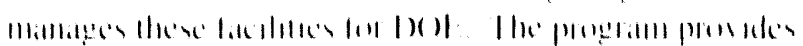

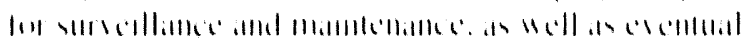 \\ da'colllatmm
}

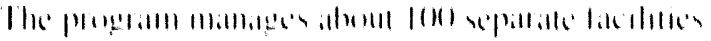

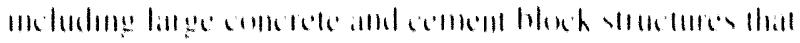

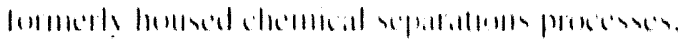

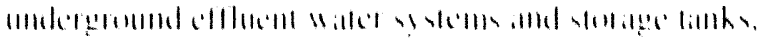

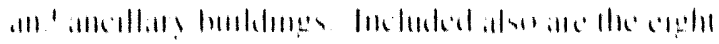

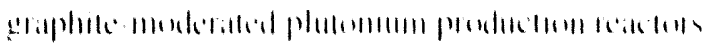




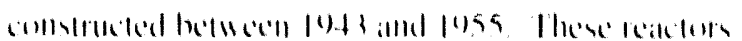

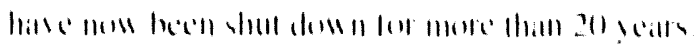

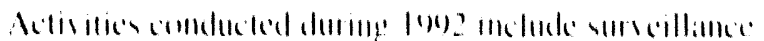

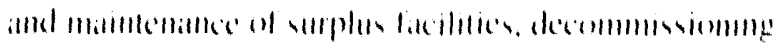

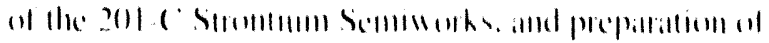

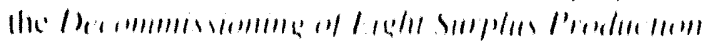

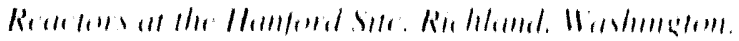

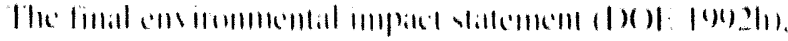

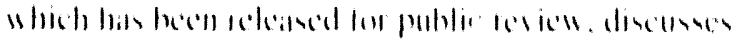

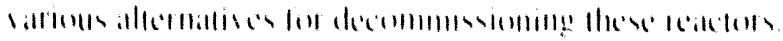

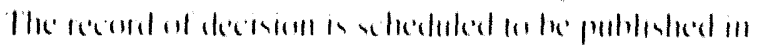
191003

\section{Corrective Activities}

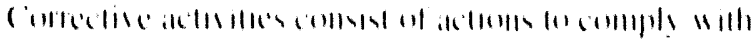

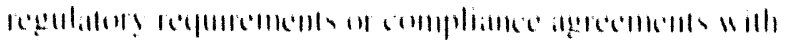

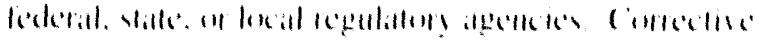

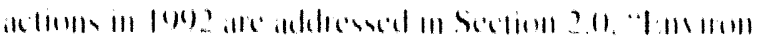

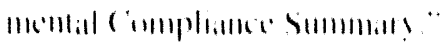

\section{Research and Technology Development}

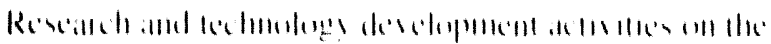

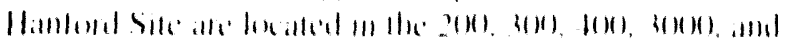

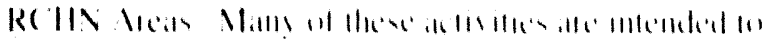
IIIp)

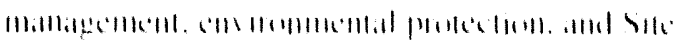

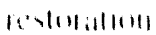

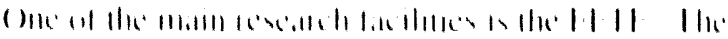

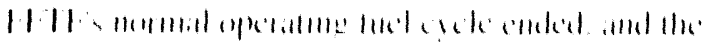

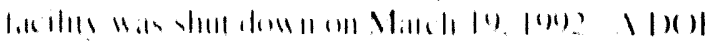

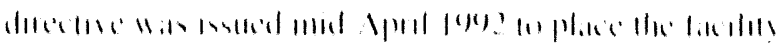

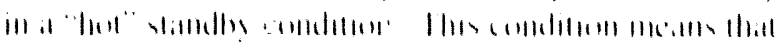
licelin! y

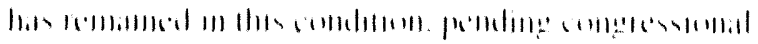

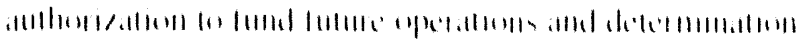
11 11 16.

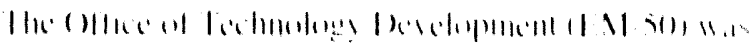

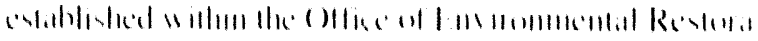

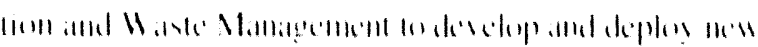

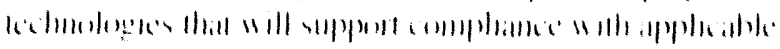

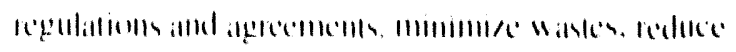

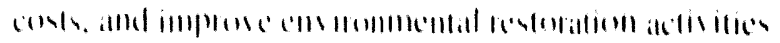

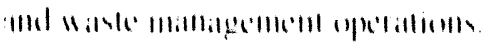

IS 611 ,

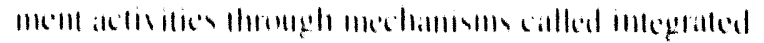

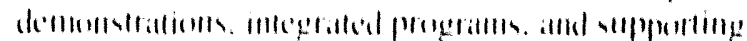

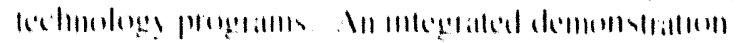

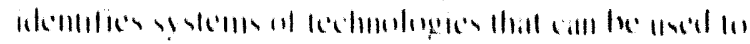

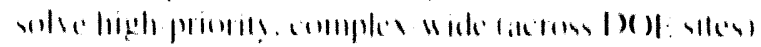

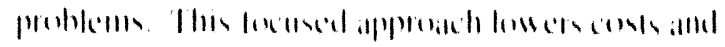

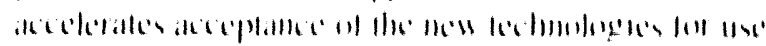

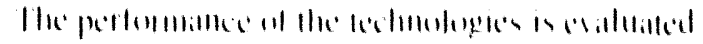

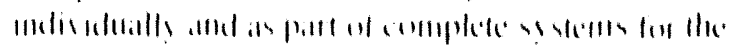

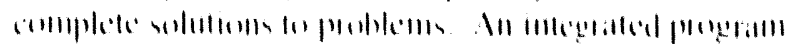

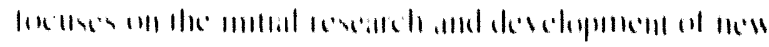

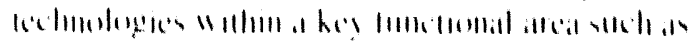

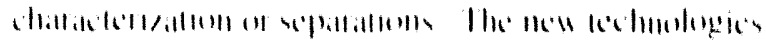

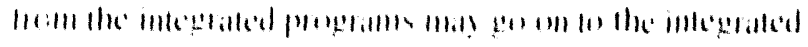

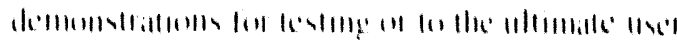

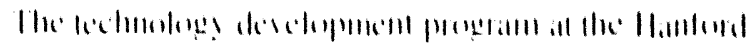

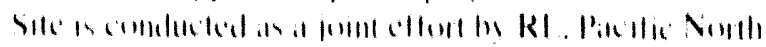

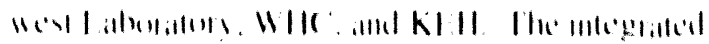

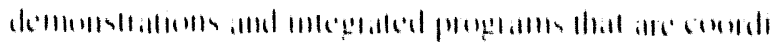

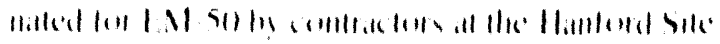

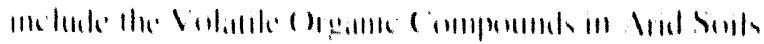

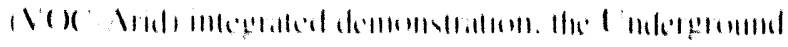

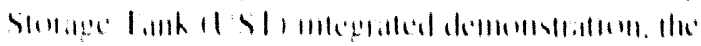

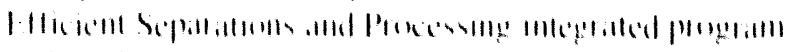

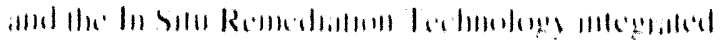

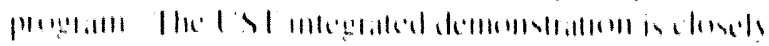

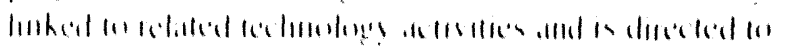

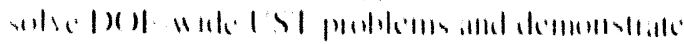

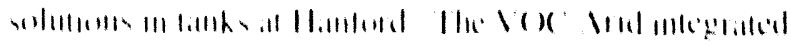

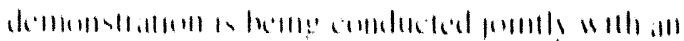

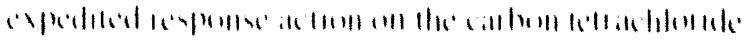

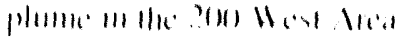

\section{Site Management}

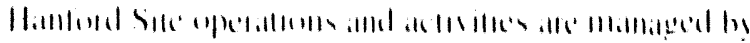

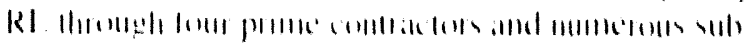

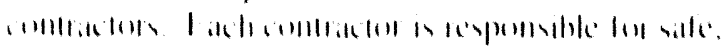

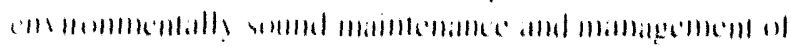

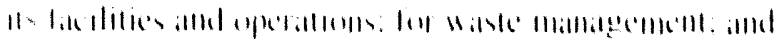

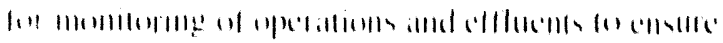

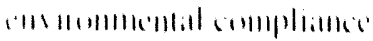


The principal contractors and their respectie reyponsihilitice include:

- Westinghouse Hanford company, the operating and engincering contratefor. conducts emsirommental

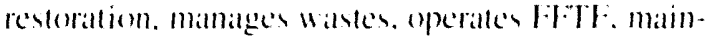
taims $N$ Reactor and its fuel fahrication litcilities, and provides support services stech ats life protection. stores. and electrical power distribution.

- Baltelle Memorial Institule. the resciach and derelopment contractor. operates the Patcitic Northwest Laboratory for DOE. conducting reseatreh and development in envirommental restoration and waste management. environmental science. molecular science. energy, health and salety. and national security.

- Kaiser Engineers Hantord. the engineering and construction services contratelor. provides archilestural. construction. and engincering services.
- Hanford Environmental Heallh foundation is the oceupational and emsirommental health services contractors.

Non-D)F operattons and activities on the Hanford Site include commercial power production by the WashingIon Puhlic Power Supply System WNP-2 Realctor and commercial low-level radionctive waste burial al a site laased and licensed by the State of Washington and operated by U.S. Ecology. Immediately adjacent to the southern boundary of the Site, Siemens Nuclear Power Corporation operates a commercial nuclear fucl fabrication lacility, and Allied Technology Group Corporation aperalles a low-level radiondive waste decontamination. super compaction. and packaging disposal facility. 


\section{Environmental Compliance Summary}

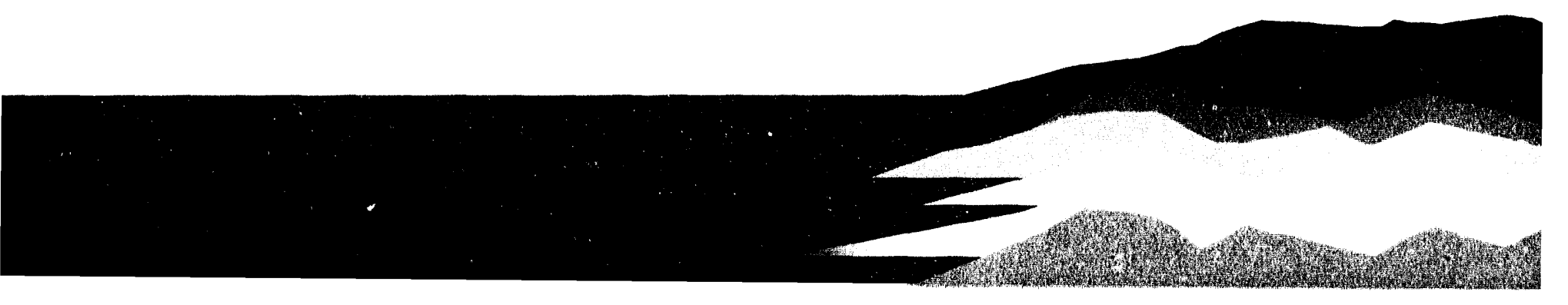




\subsection{Environmental Compliance Summary}

This section briefly describes how enviromental compliance is heing atehieved for the Hanford Site. Included are subsections describing I) the regulations and oversight of compliance at the Site. 2) the current stitus of the Sile's compliance with the principal regulations, 3) the issues and actions arising from these compliance efforts. and th the envirommentally significant environmental accurrences. The linal subsection provides all updiate of these issues for Jannuary I through April 1. 1(29)3. per 1)(OE: Orders. 


\subsection{Environmental Compliance and Cleanup}

Many entities have a role in the I)( ) la's new mission of envirommental restoration and waste mamagememe. These include lederal. stalce, and local regulatory agencies: ensirommental groups: regional communities: Indian nations: and individual citizens. The following subededions describe the roles of the principal agencies, organisattions. and public in envirommental compliance and cleanup of the Hanford Sile.

\section{Regulatory Oversight}

Several federal. statce and local government agencies ance responsible for enforeing and oversecing envirommental regulations all the Hanlond Site. These agencies include the F:PS. he Washinglon State Depantment of Lecologes (ficology), the De) and the Tri-Countices (Bentonlanklin-Walla Walla Countices) Air Pollution Combol Authority. These agencices issue permits. review compliance reports. participate in joint monitoring

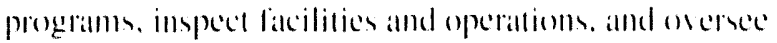
compliance will applicable regulattions. The I)( )I: through compliance andits and its directives to operattoms offices, initiates and ansesses atedions for comforming to ensirmmental recpuirements.

The l:PA is the principal federal envirommental regulator in Washinglon State. The LIPA derelops. implements. and enforeses envirommental profection regulations and fechomology-batsed standards as direcled hy statules passed by compress. In some instancess low lips has dedegated eme irommental regulatory antherily fo the stalle ar atullorised the state program to operante in lien of the feckeral progeram when the stille's program meets of exceded the $1: P A$ s requirements. For instance. He l:PA

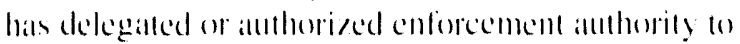
leoblogy for air pollution control and many areab of

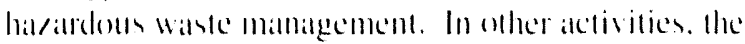
salk program is enforced direcolly upen federal agencies

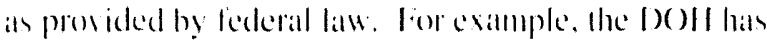
atulhorily to implement the state program for radionuclick air emissoms on the allmosphere all the Hanloned Site in accordanse with the federal lakeilities section of the ('leam Air Act. Whore regulatory atulhority is not delegatced on allohorised lo the stalle liPA Region Io is repousible for revien ing and enforcing compliance wills I:PA regulatiom an they pertain to the Ilantord Site.

\section{The Tri-Party Agreement}

The Hamlond federal lateility Comsent and Agreement ()eder (also known an the Tri-Panly Agreement) is an

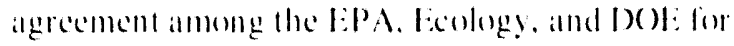
achieving compliance with the comprehensive lanvirommental Reyponse. (ompensillion, and liability $\mathrm{Act}$ (CISRCLA) (including S $A R A)$ remedial atem provisions and wilh RC RA trallment, storage, and disponal unil regulanton and corrective action provisions. The Tri-Panly Agrecment I) defines and ranks RC RA and Cl:RC'A cleanup commitments. 2) establishes respomsihilitices. 3) provides a basis for budgeting, and th rellects a concorled goal of achiesing full regulattory compliance and remediation. with entorcente milestones, in an aggeressise bul achievahle manmer. The Tri-Parly Agreement wals estathlished with impul from the public. (oppies of the agreement and quallerly progeses reports of activilies alre publicly alvailable all the RL. Public Readding Romm in Richland. Washington, and all inlormatton repositories in Seattle and Spokanc. Washington, and Portand. ()regon. To ged on the mailing list to obtain Tri-Parly Agreement updates, contact lla $I: P \Lambda$ or RI. directly, or call Jecology all 1-80()-321-20)8. Requests hy mail cill be seml to:

Manlond Mariling I,ist

P.(). Bux 1970 13.3-3.5

Richland. WA 99.352

(1)

Hantiond leplate

Departiment of licology

P.(). $130 \mathrm{OS}+70(0)$

() Iympial. WA $98.5(0+4-76() 0$ 


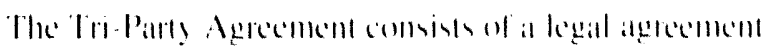

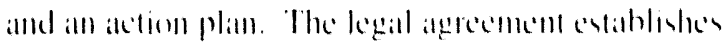

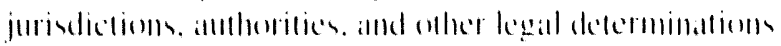

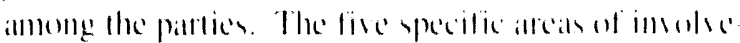

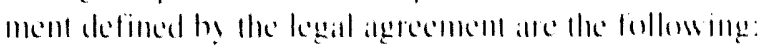

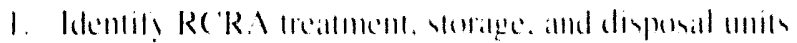

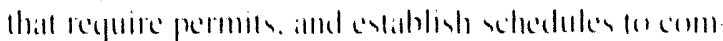
ply wilh interim and limal status reguiremems.

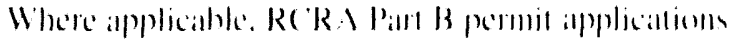

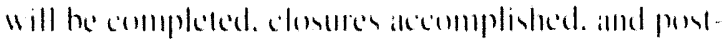
closure carte implementerl.

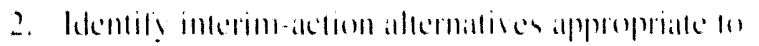

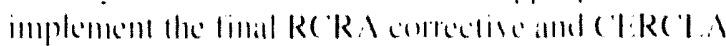
remediall aldioms.

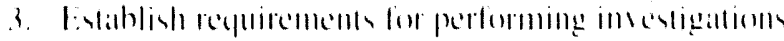

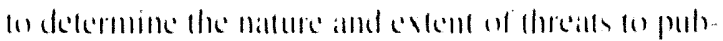

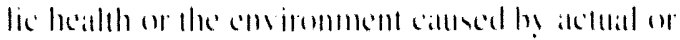

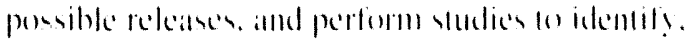

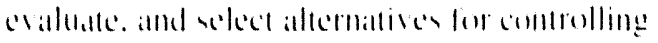
pusible relames.

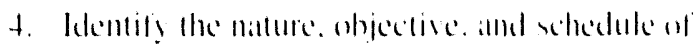
reyponse aletions for cleamup on hatardous mallerial yills.

5. Implemem the selected interim and linal RCRA

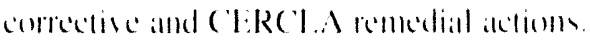

The action plan implements the lexal agrement by 11 delining how the pattien will woth together. 21 describ.

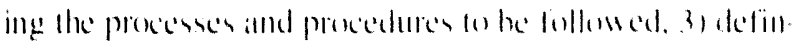

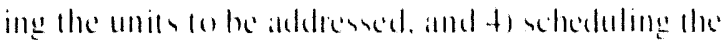

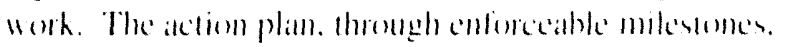
establishes a plant and achedule for bringenge the llanfond Site into compliance with applicahle reguirements on

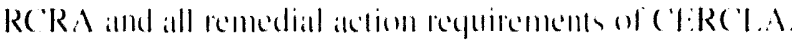

\section{The Role of Oregon State at the Hanford Site}

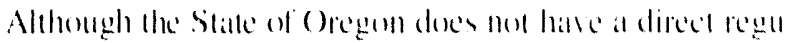

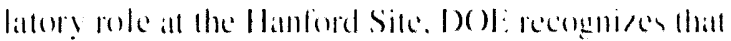

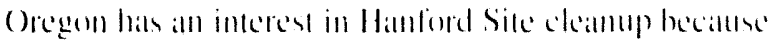

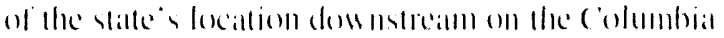

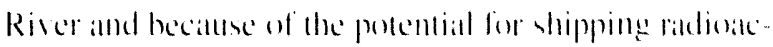

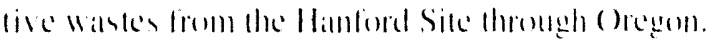

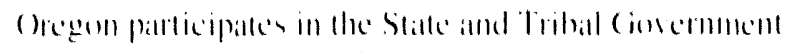

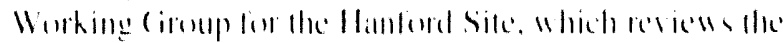

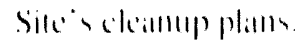

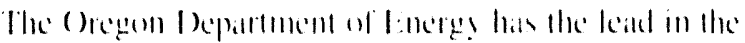

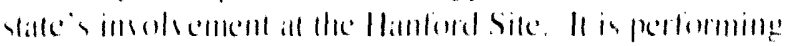

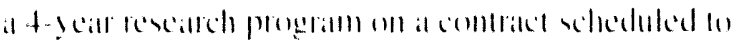

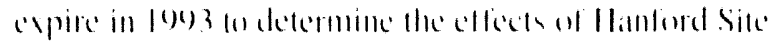

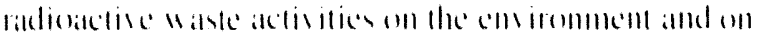

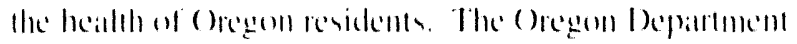

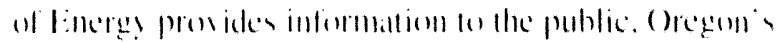

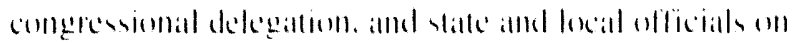

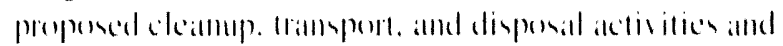

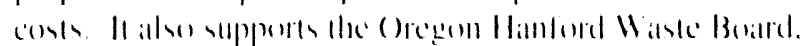

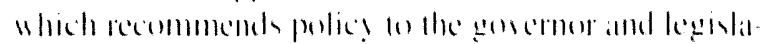

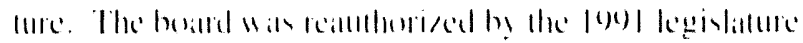

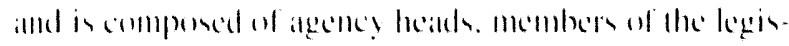
latture, and citisms.

\section{The Role of Indian Nations at the Hanford Site}

The Ilanforel site is lecalled an land ceded in treatlies in I855 with the Yathimal Indian Nattom and the Cimled-

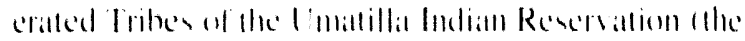

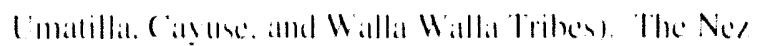

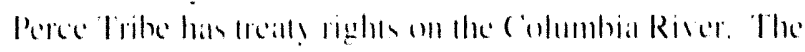

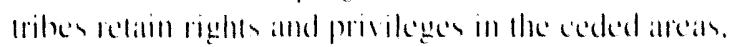

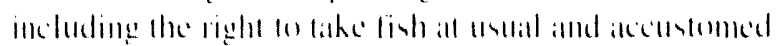
plices.

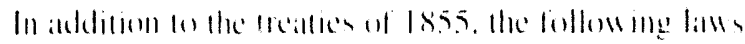

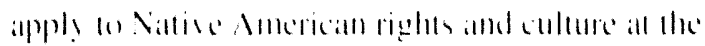

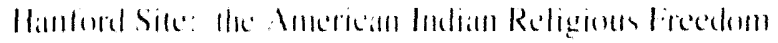

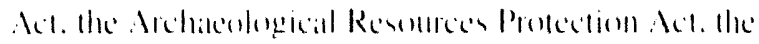

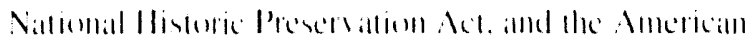

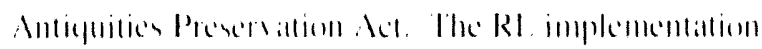

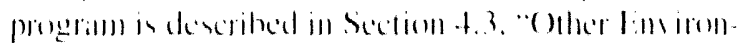

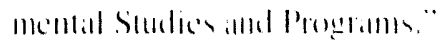

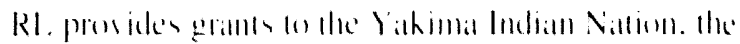

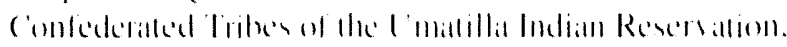

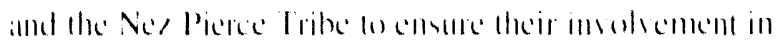

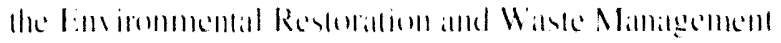

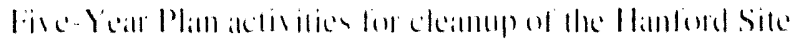

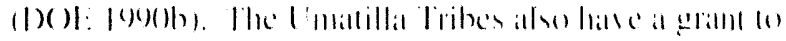

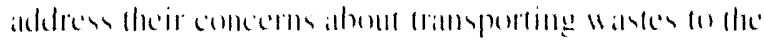

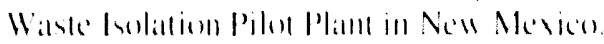




\section{Public Participation}

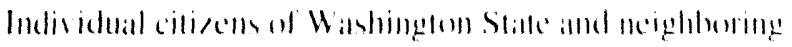

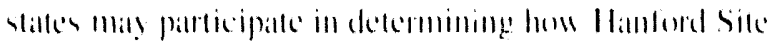

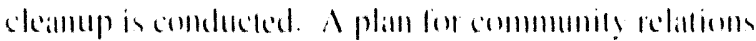

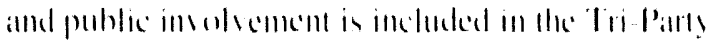

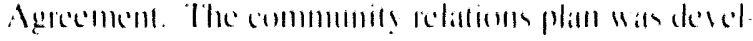

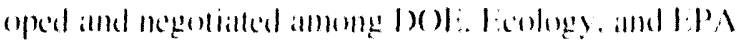
Region 10 with public commentm and war jombly appromed in |(0)().

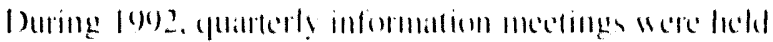

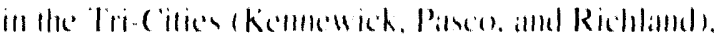

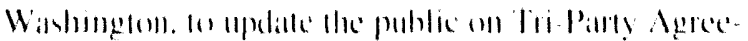

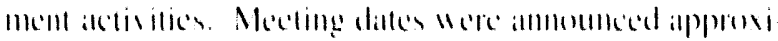

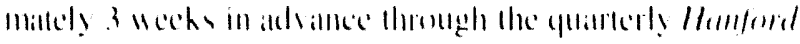

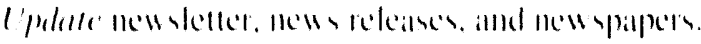

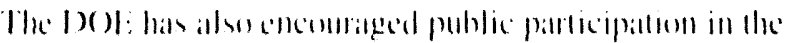

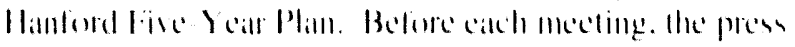

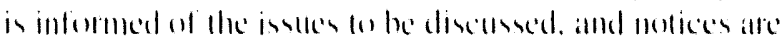
seml to elected allicials. commonmils leaders. and interest 니만

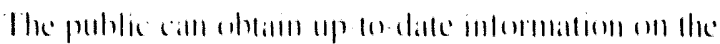

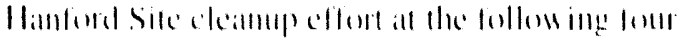
memllaters:

1. Hoe RI. Puhlic Re'iding Romm. Richland. Washinglun

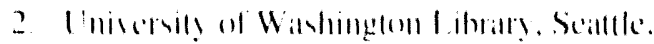
Wishington

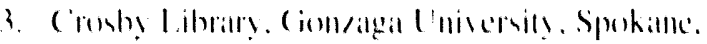
Washinglun

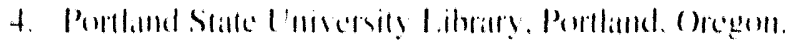

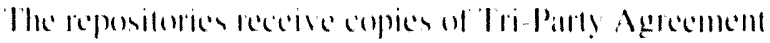

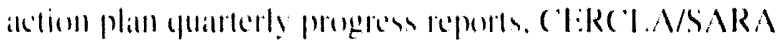

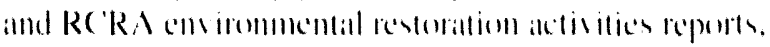

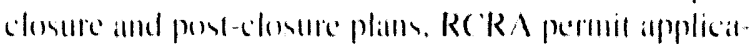

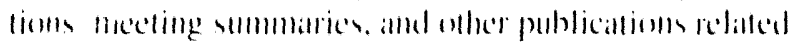

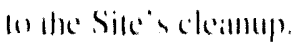




\subsection{Compliance Status}

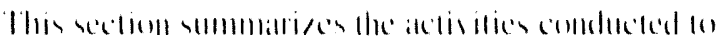

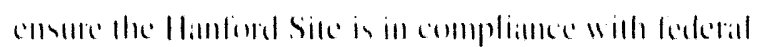

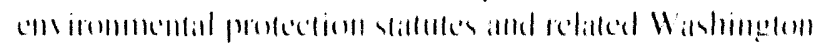

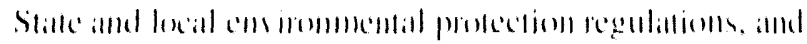

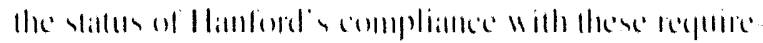

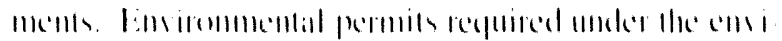

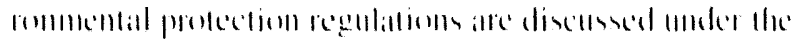

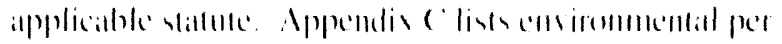

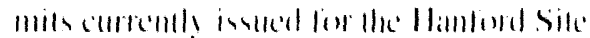

\section{Comprehensive Environmental Response, Compensation, and Liability Act}

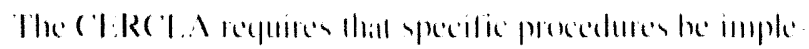

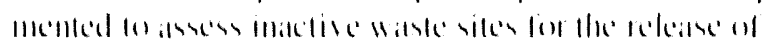

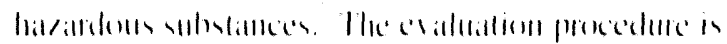

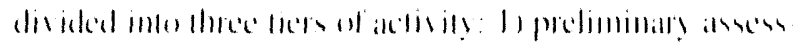

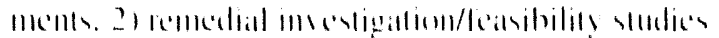

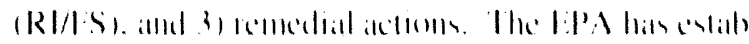

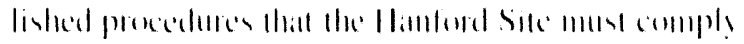

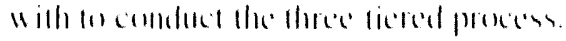

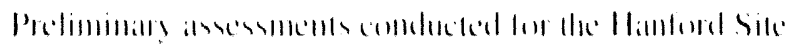

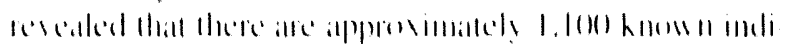

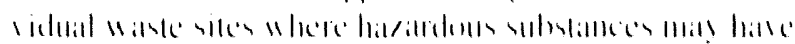

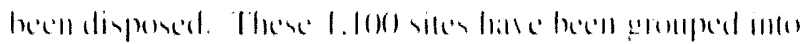

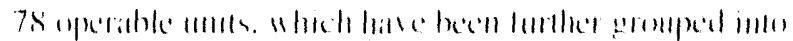

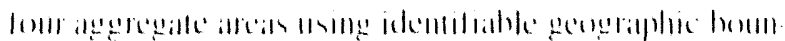

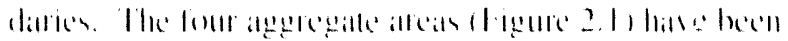

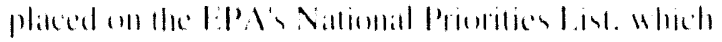

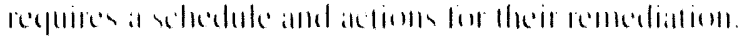

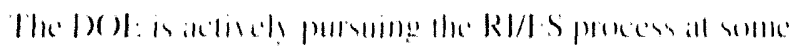

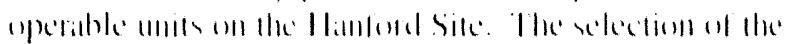

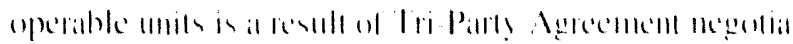

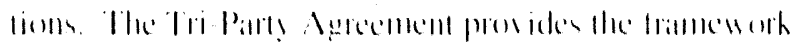

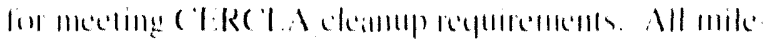

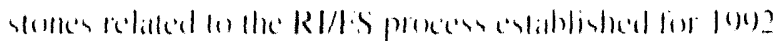

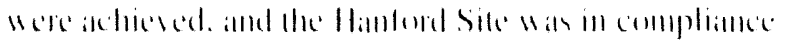

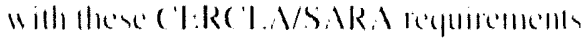

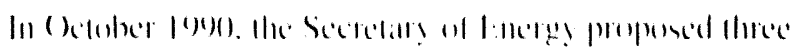

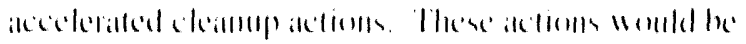

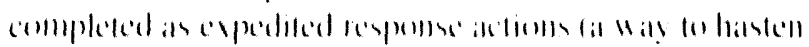

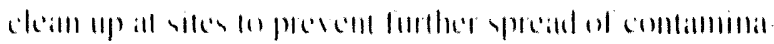

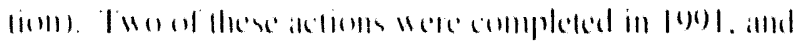

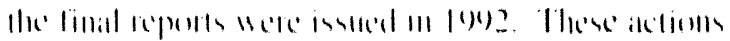

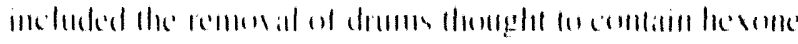

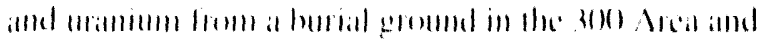

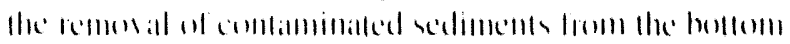

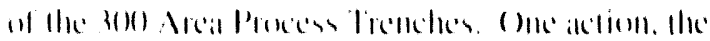

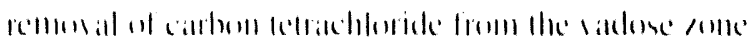

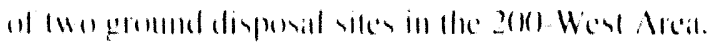

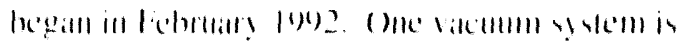

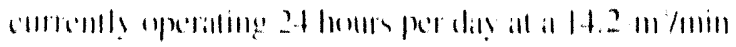

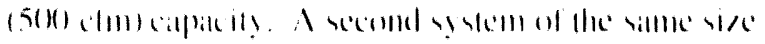
will be (1)

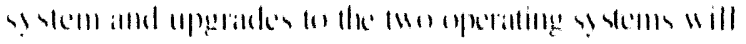

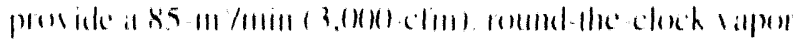

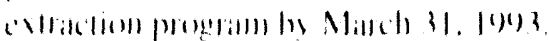

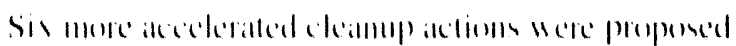

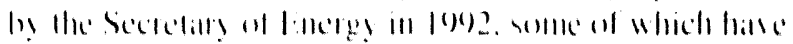

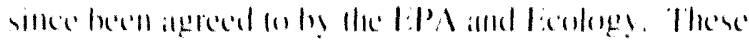

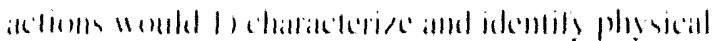

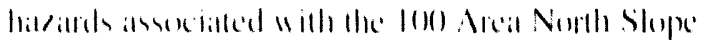

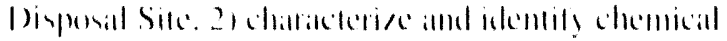

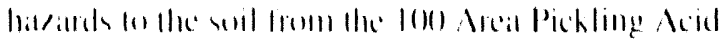

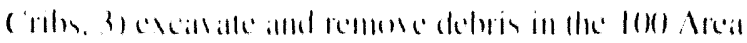

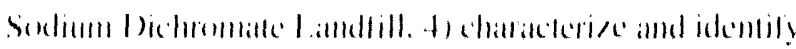

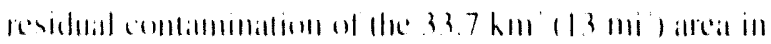

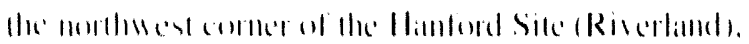

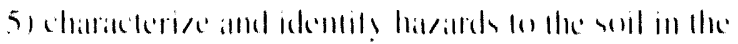

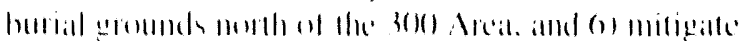

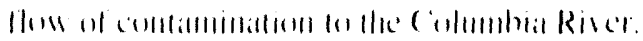

\section{Lawsuit Filed}

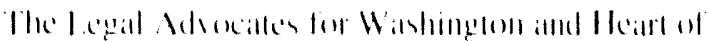

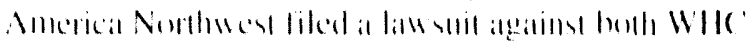

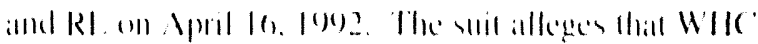

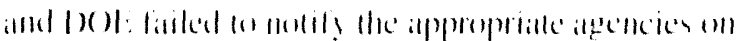

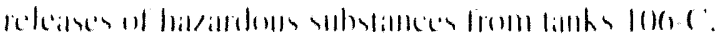

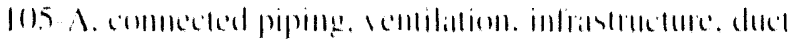

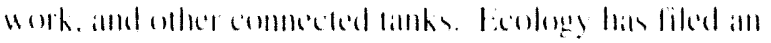

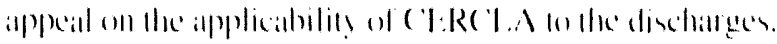




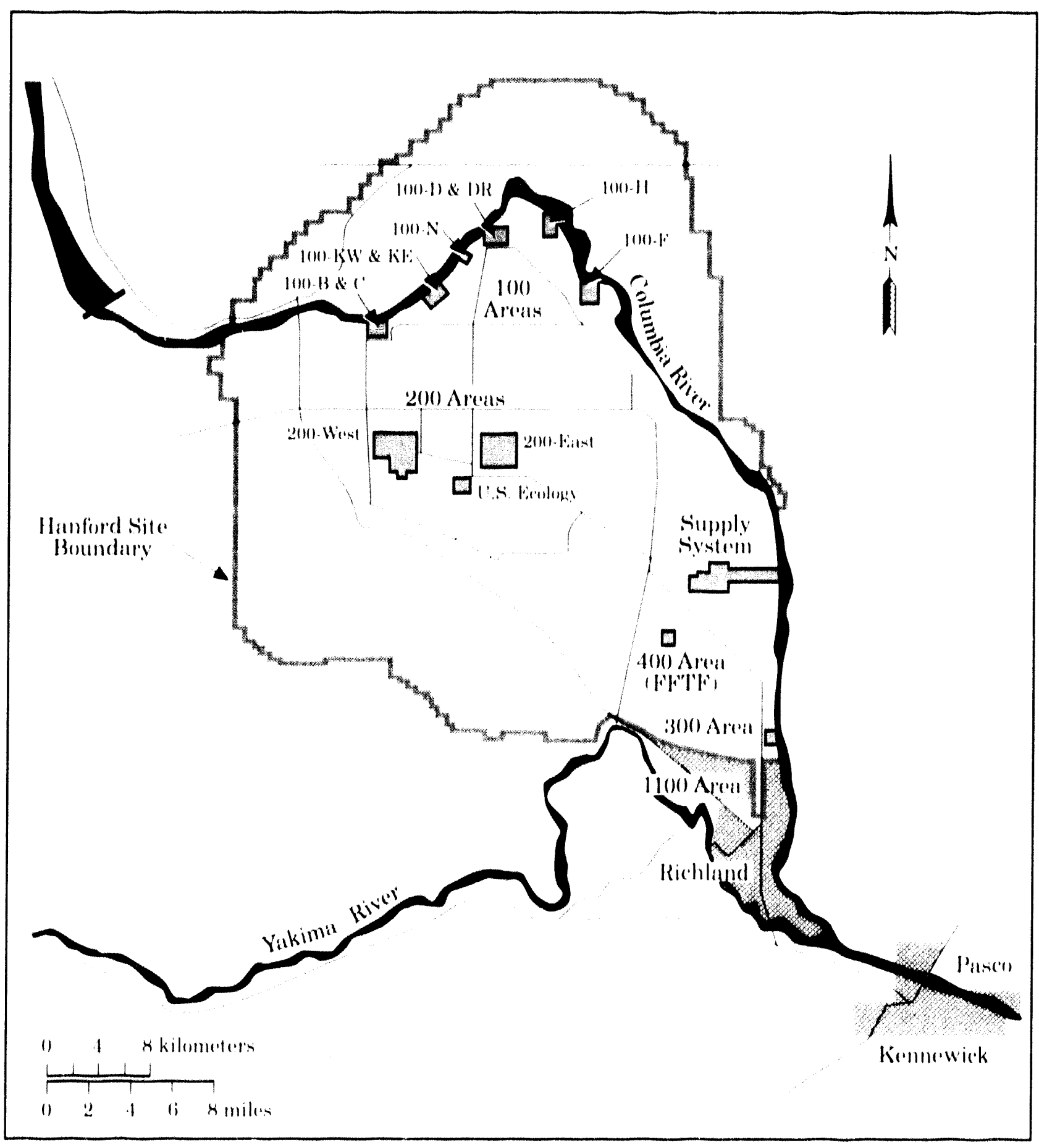

$590^{2} 305815$

Figure 2.1. Location of Aggregate Areas of the National Priorities List for the Hanford Site (100 Areas, 200 Areas, 300 Area, and 1100 Area) 


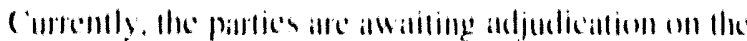

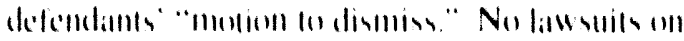

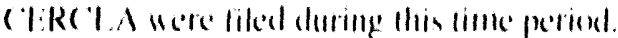

\section{Emergency Planning and Community Right-To-Know Act}

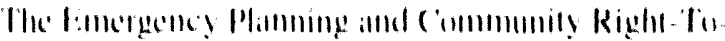

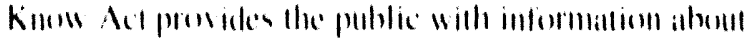

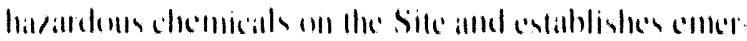

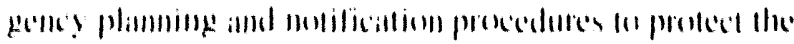

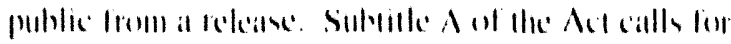

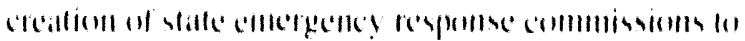

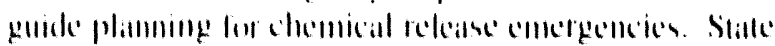

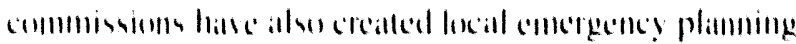

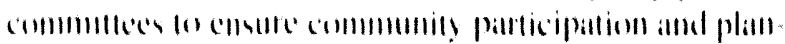

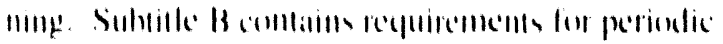

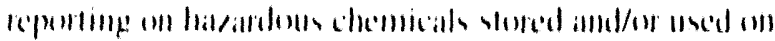

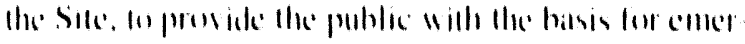
fents plimming

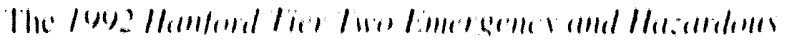

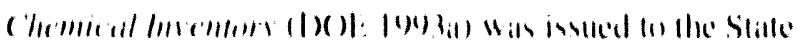

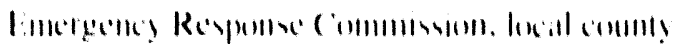

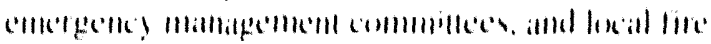

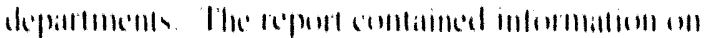

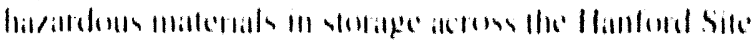

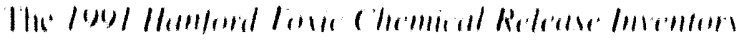

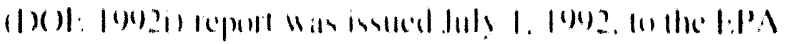

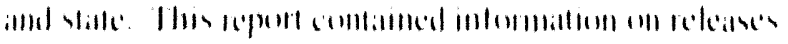

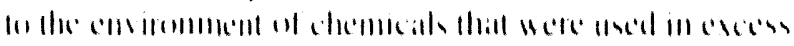

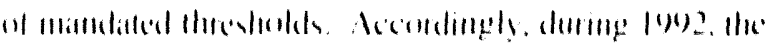

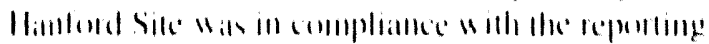

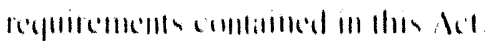

\section{Pollution Prevention Program}

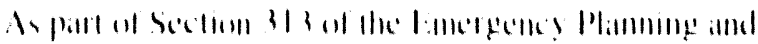

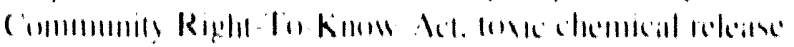

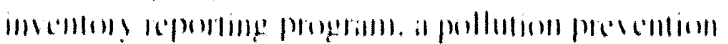

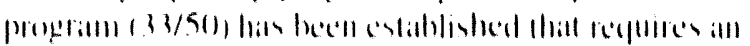

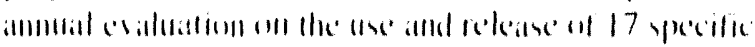

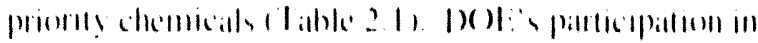

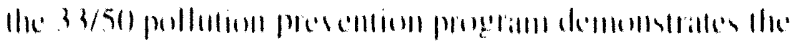

Table 2.1. Seventeen Priority Chemicals of the Emergency Planning and Conımunity Right-ToKnow Act Pollution Prevention Program

B०IINIIL

('admium and compunmols

('arhon ledrachloride

(hlorolorm

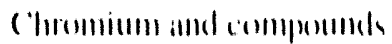

cyathider

Dichloromlenhanc

l.cill and commponmols

Mercury and commpunnds

Meillyl siliyl ketome

Mellyyl isobutyl ketome

Nichel and comprommols

Tetrathlomedigyene

Tillache

1.1.1 Hedhoresthans

Trichloroculisglente

xylenews

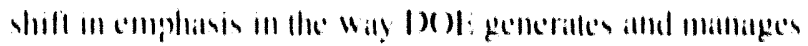

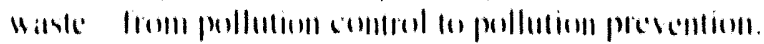

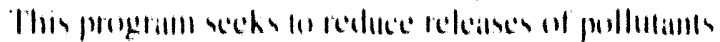

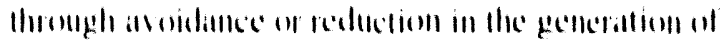

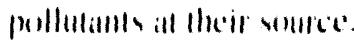

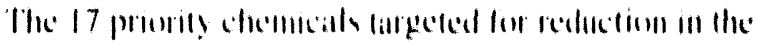

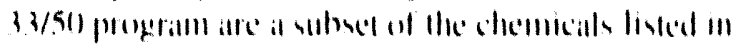

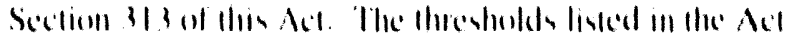

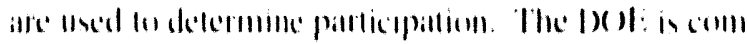

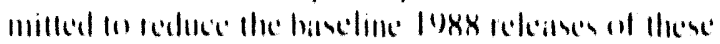

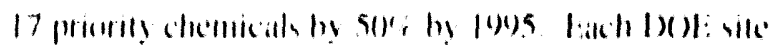

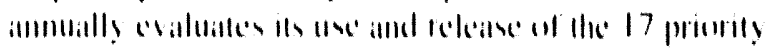

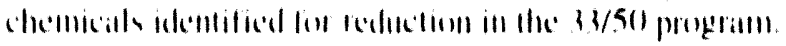

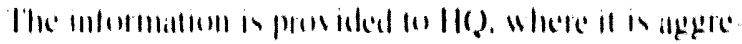

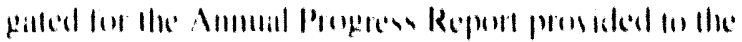
III)

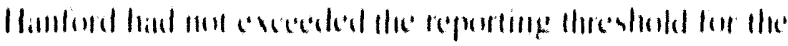

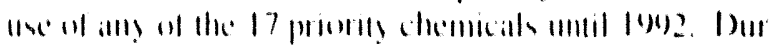

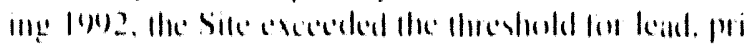

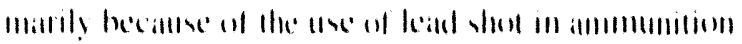

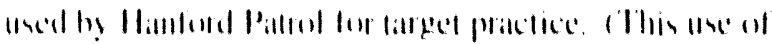

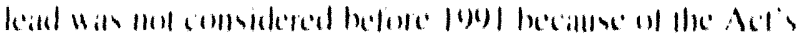

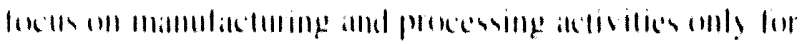

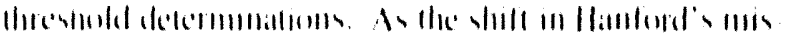

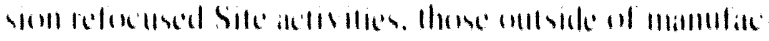

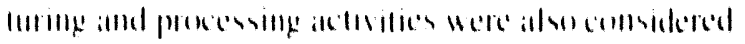

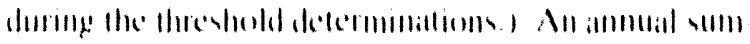

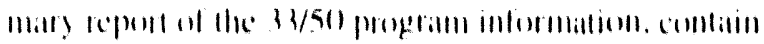

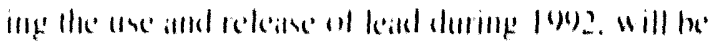
completed for the Hamford Sile hy Augus lous. 


\section{Resource Conservation and Recovery Act}

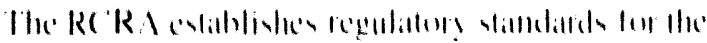

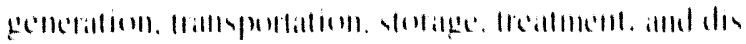

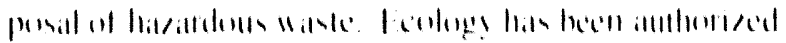

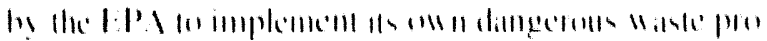

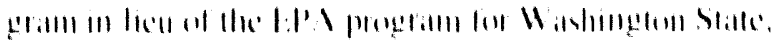

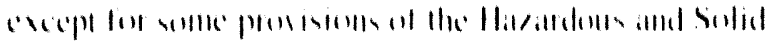

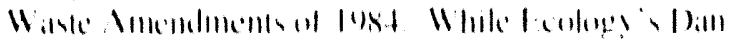

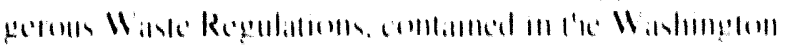

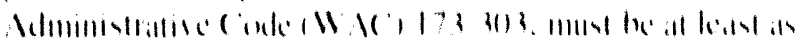

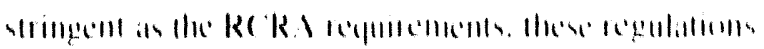
ille wll'th more vingents

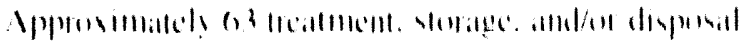

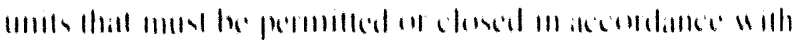

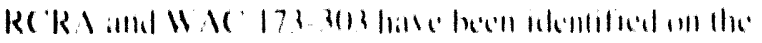

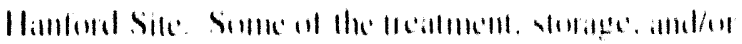

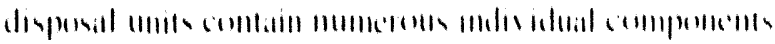

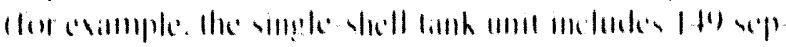

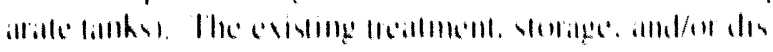

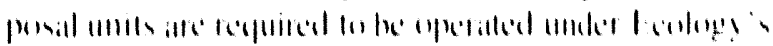

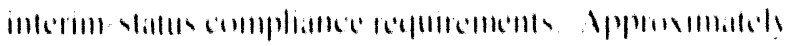

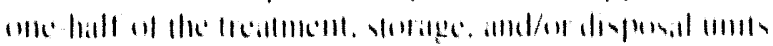

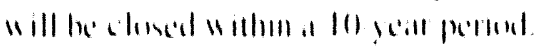

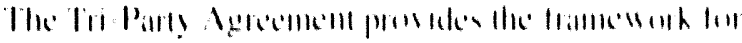

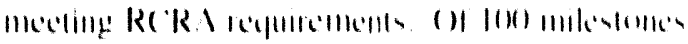

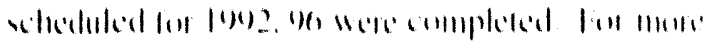

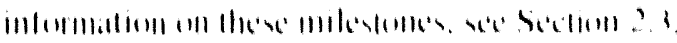

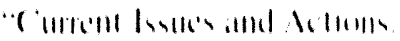

\section{Enforcement Action}

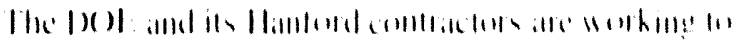

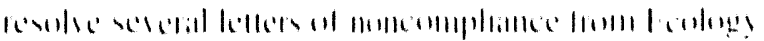

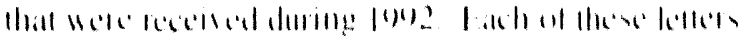

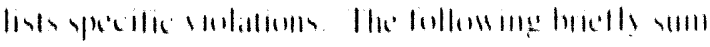

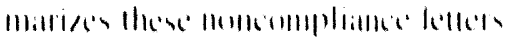

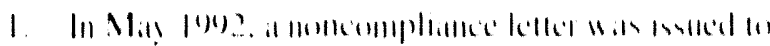

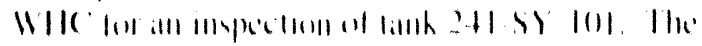

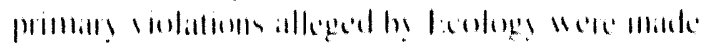

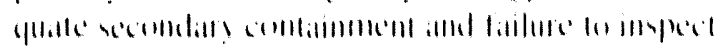

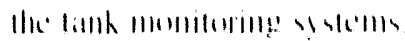

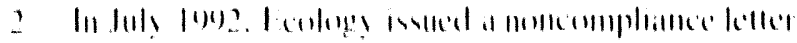

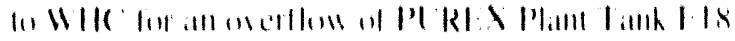

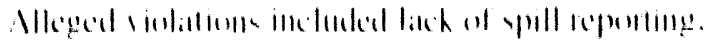

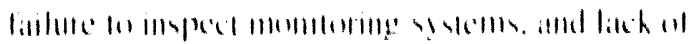

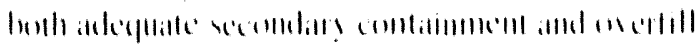

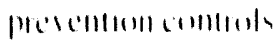

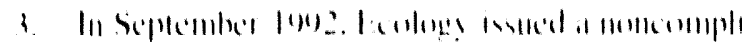

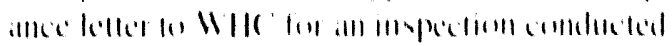

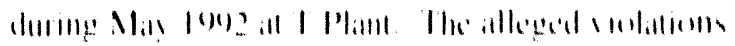

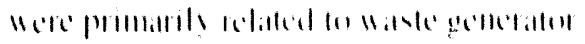

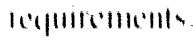

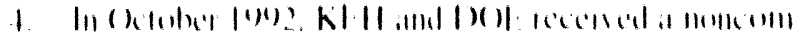

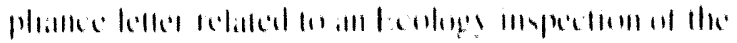

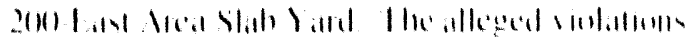

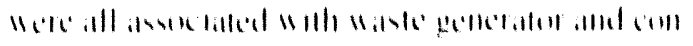

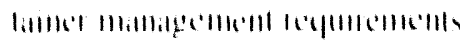

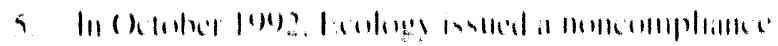

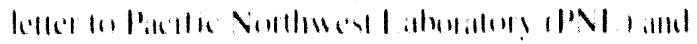

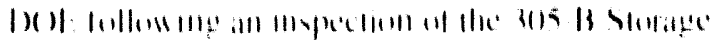

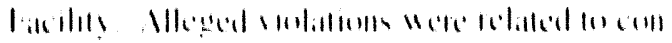

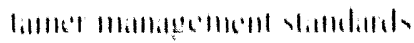

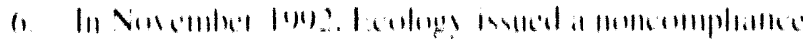

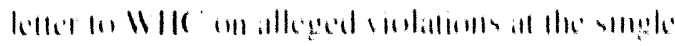

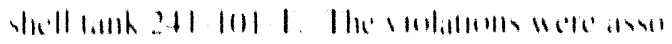

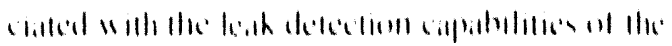

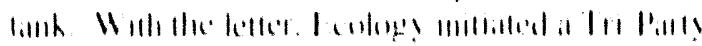

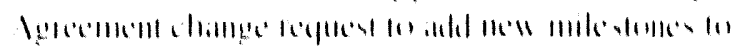

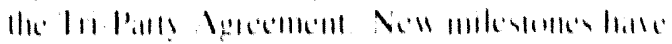

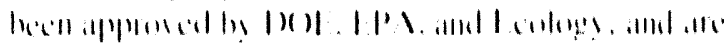

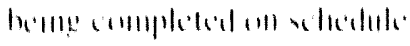

\section{Hanford RCRA Part B Permit}

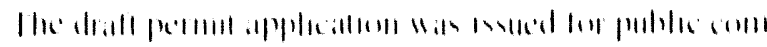

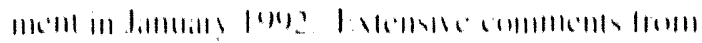

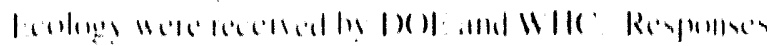

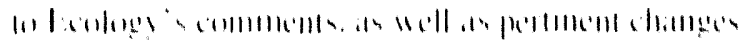

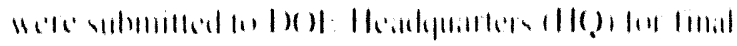

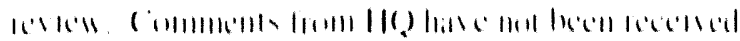

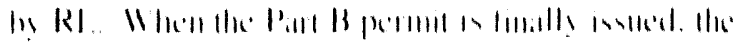

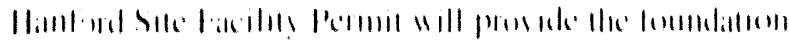

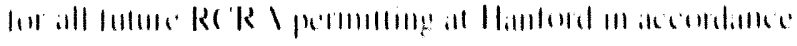

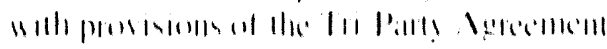




\section{Resource Conservation and Recovery Act Ground-Water Monitoring}

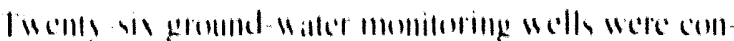

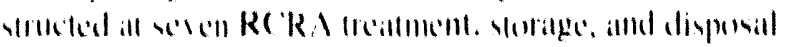

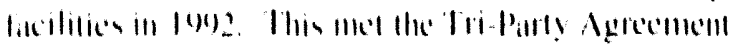
Nilevtume $112+101$

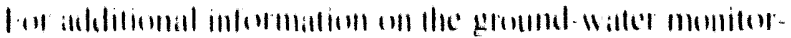

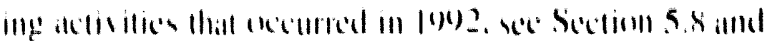
spendist

\section{Resource Conservation and Recovery Act Undnrground Storage Tanks}

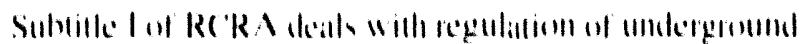

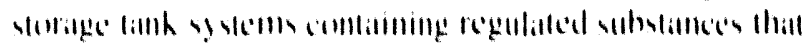

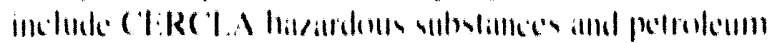

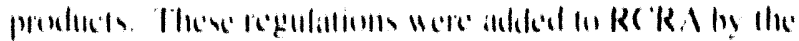

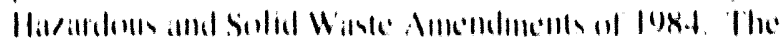

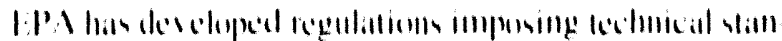

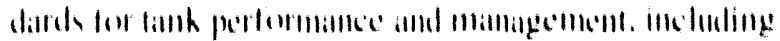

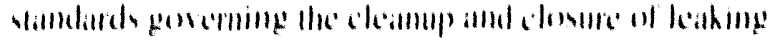

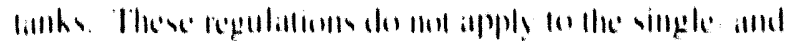

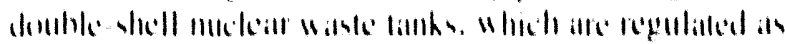

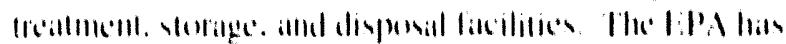

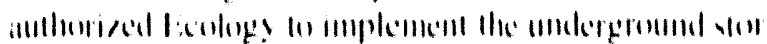

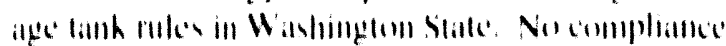

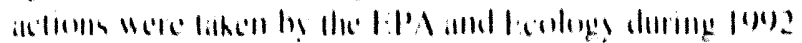

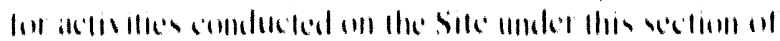
RI'RA

\section{Clean Air Act}

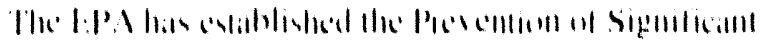

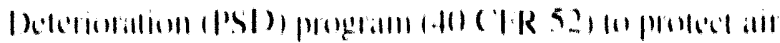

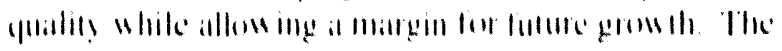

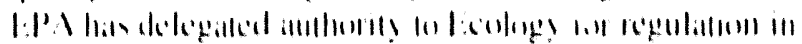

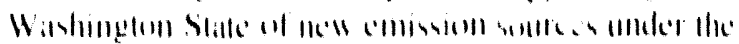
('Si) pringutill

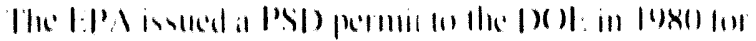

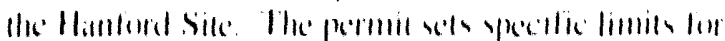
nitrogen oside Platils

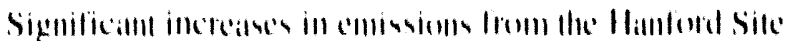

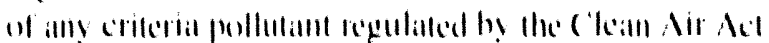

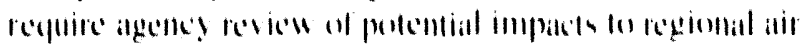
yuallily. Additionall limils ma!n he necensilly in accordante will he lest) permit.

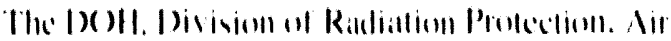

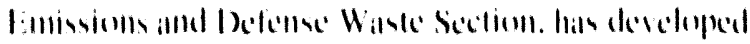

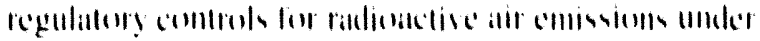

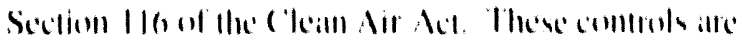

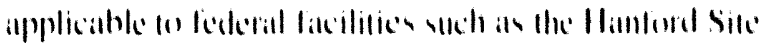

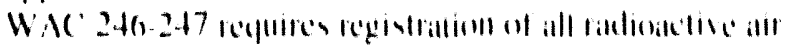

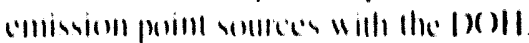

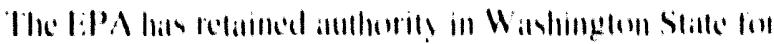

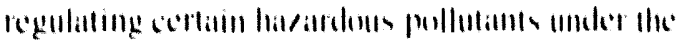

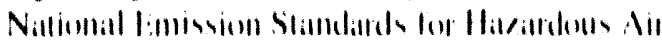

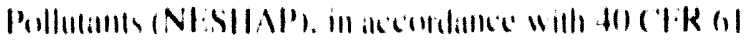

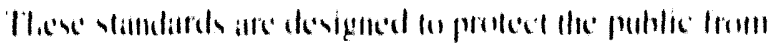

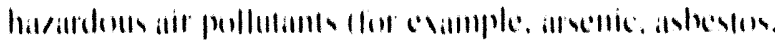

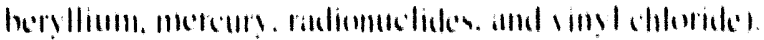

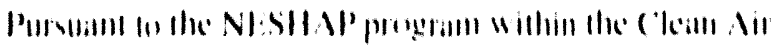

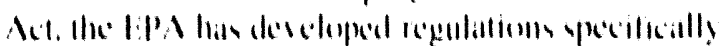

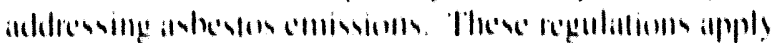

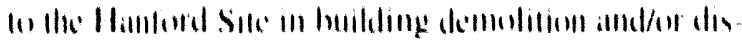

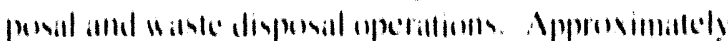

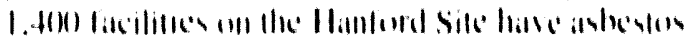

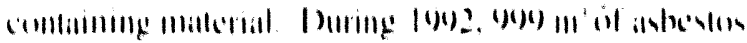

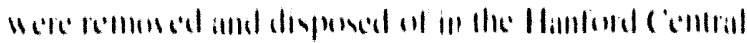

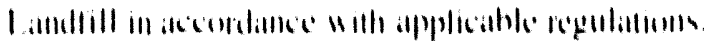

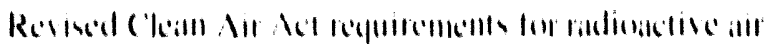
(IIIIS)

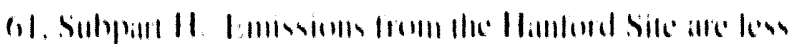

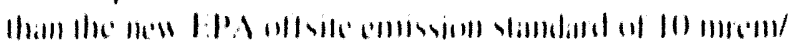
$\begin{array}{llll}11 & 0\end{array}$

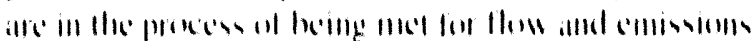

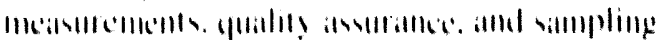

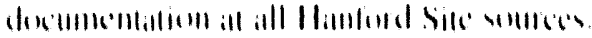

Thes -

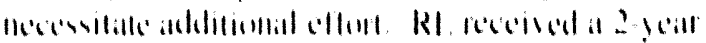

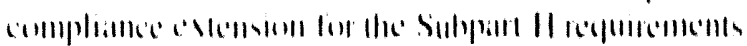

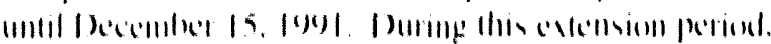

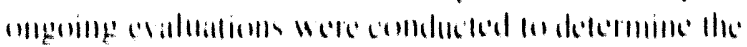

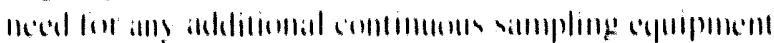

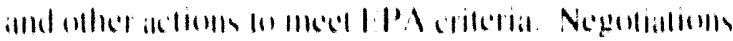

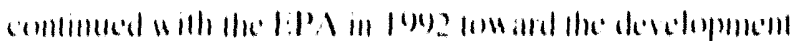

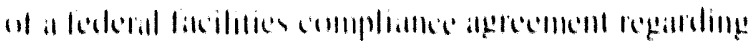

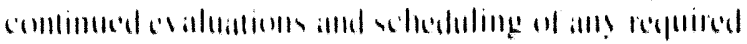

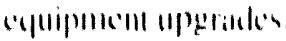




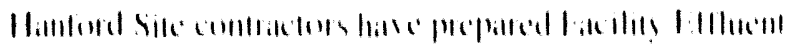

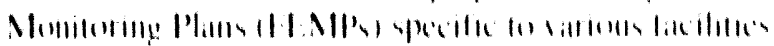

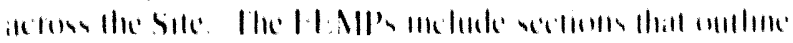

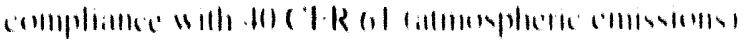

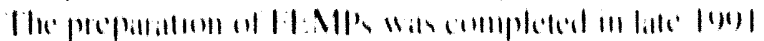

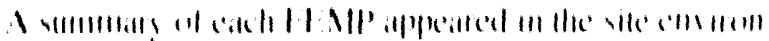

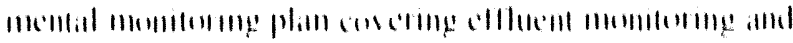

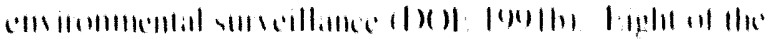

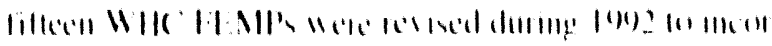

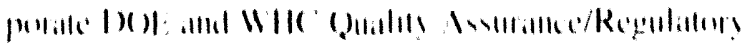

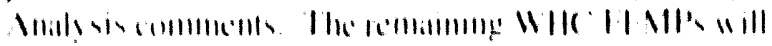

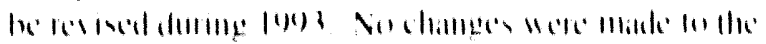
I'NI IIIII III IM!)

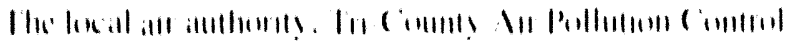

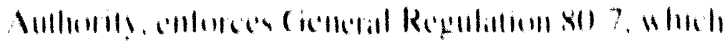

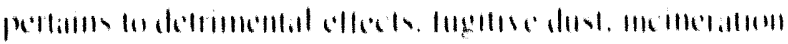

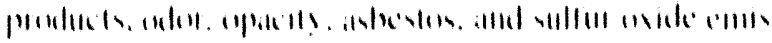

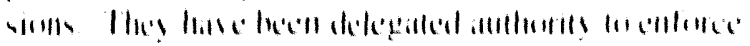

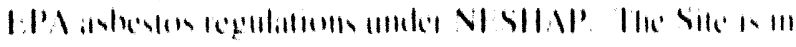

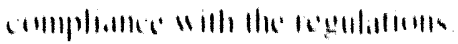

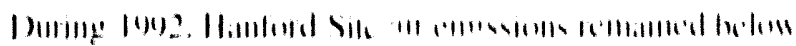

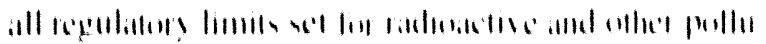

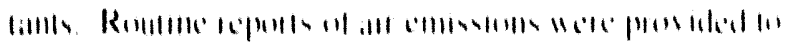

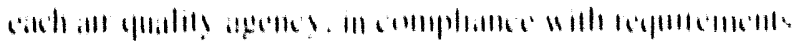

\section{Clean Water Act}

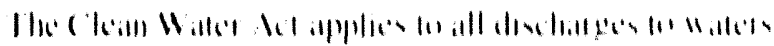

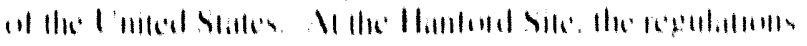

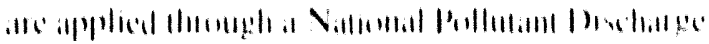
| IIIIII)

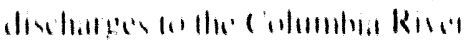

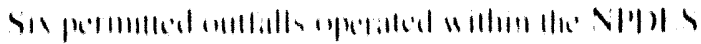
pelomel |

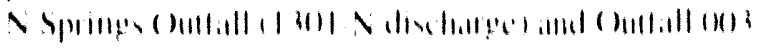

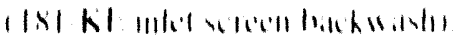

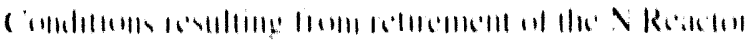

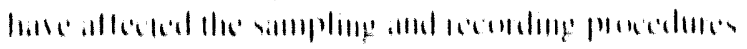

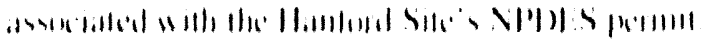

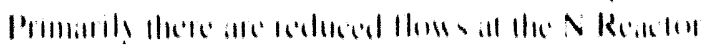

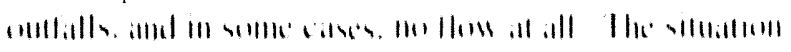

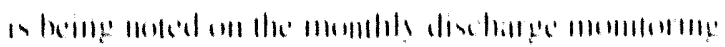
लإ)

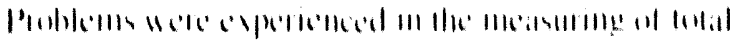

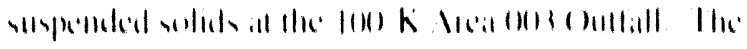

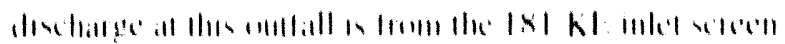

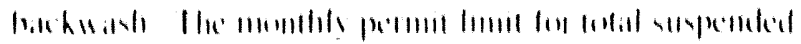

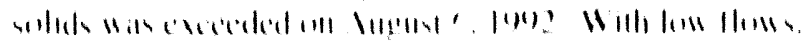

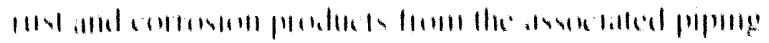

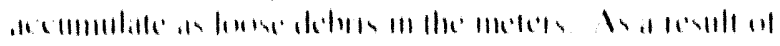

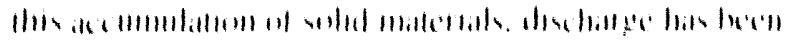

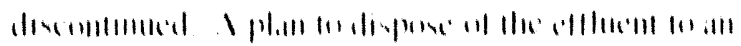
allemente vlle

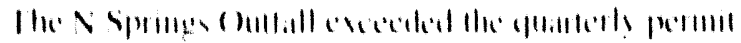

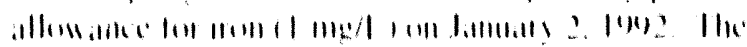

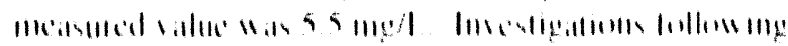

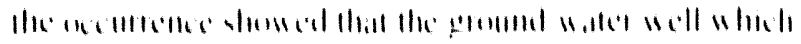

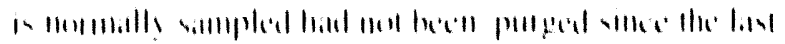

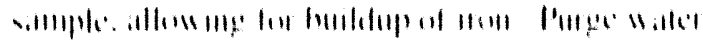

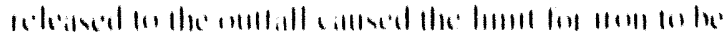

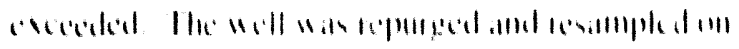

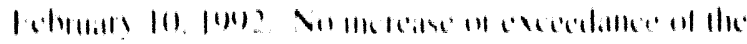

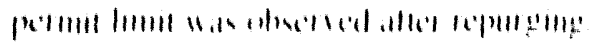

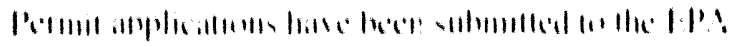

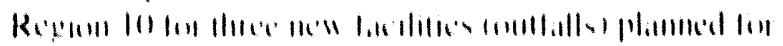

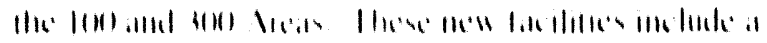

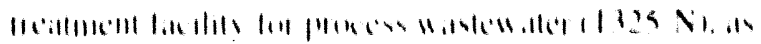

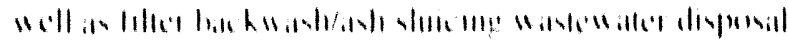

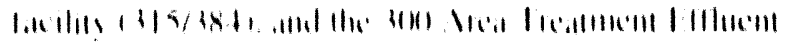

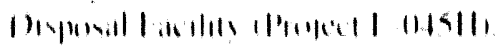

\section{Lawsult Flled}

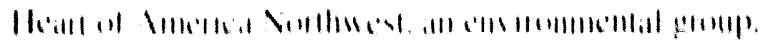

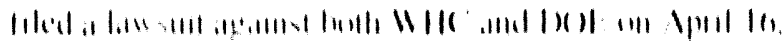

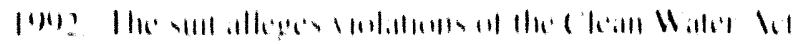

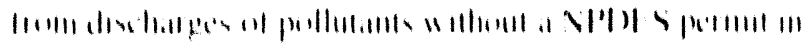

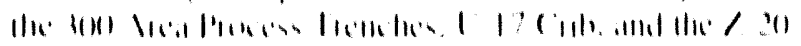

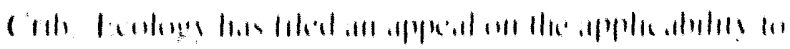

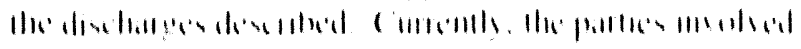

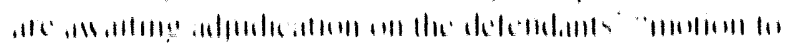
ل11,11110

\section{Safe Drinking Water Act}

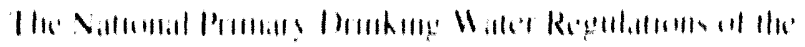

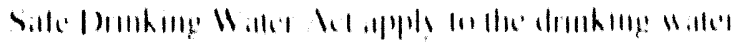
-

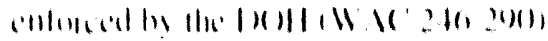

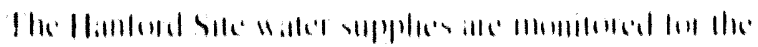

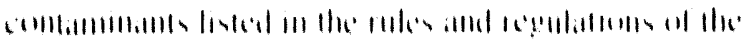

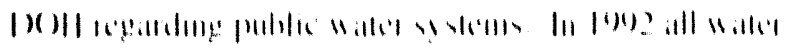




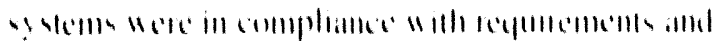
itgrentillils

\section{Toxic Substances Control Act}

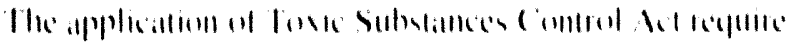

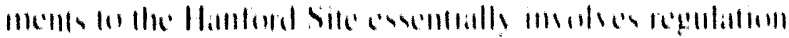

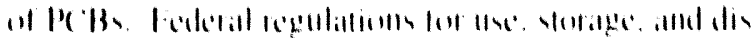

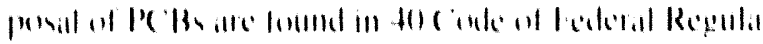

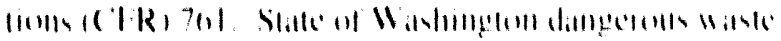

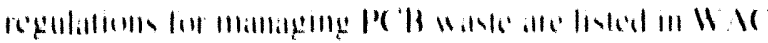
173.313

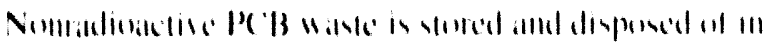

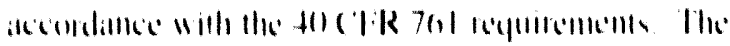

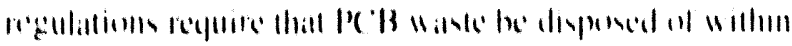

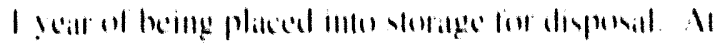

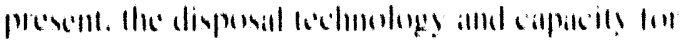

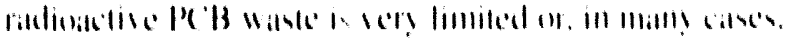

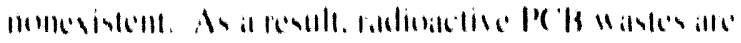

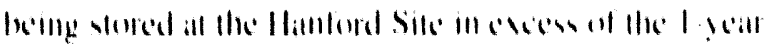

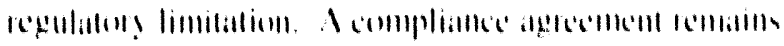

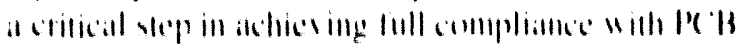
repulations.

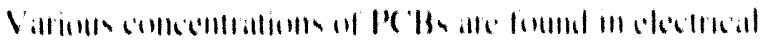

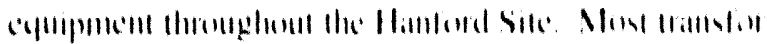

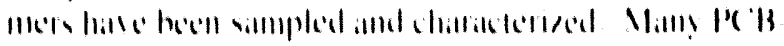

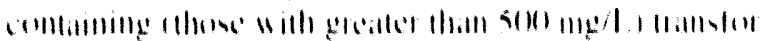

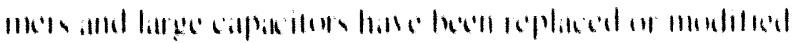

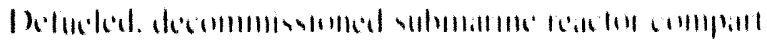

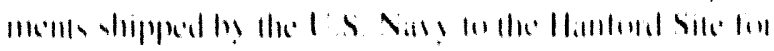

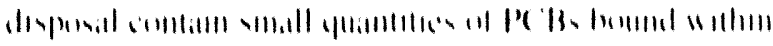

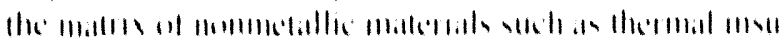

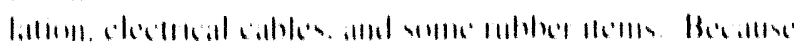

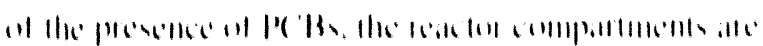

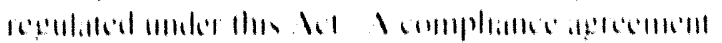

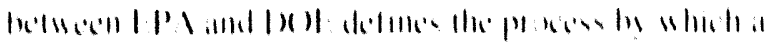

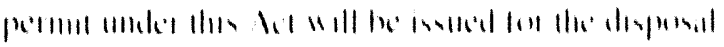

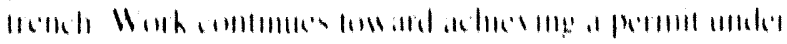
Ihi, 1,1

\section{The United States Environmental Protection Agency}

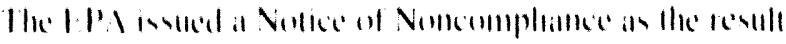

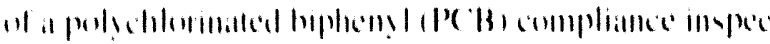

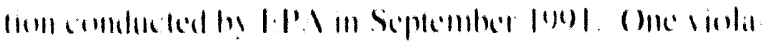

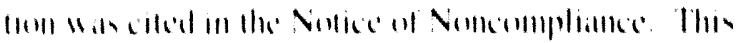

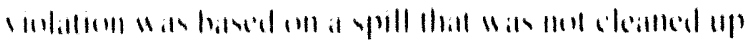

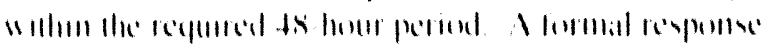

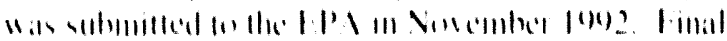

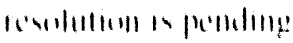

\section{Federal Insecticide, Fungicide, and Rodenticide Act}

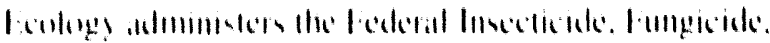

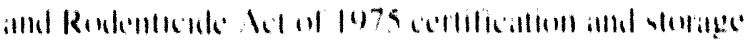

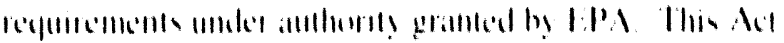

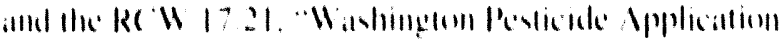

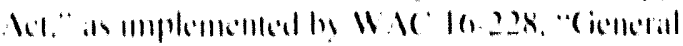

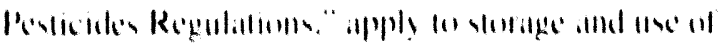

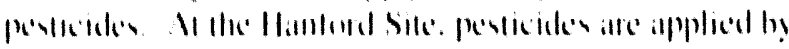

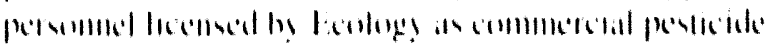

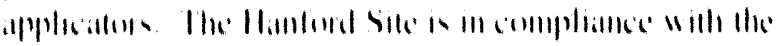

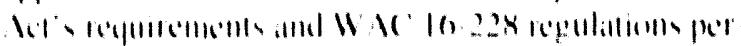

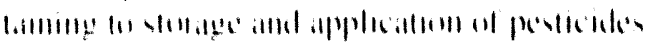

\section{Endangered Species Act}

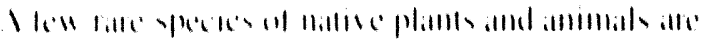

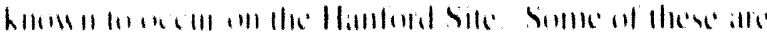

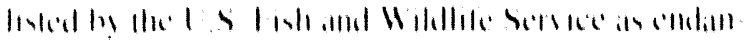

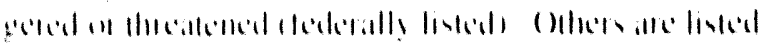

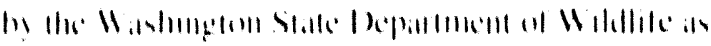

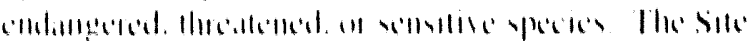

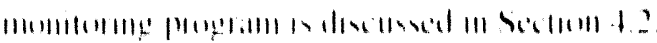

"Willlil:" 


\section{National Historic Preservation Act, Archaeological Resources Protection Act, and American Indian Religious Freedom Act}

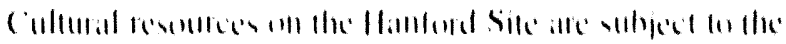

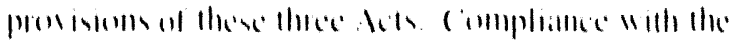

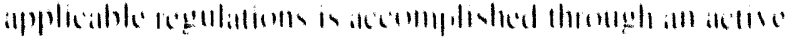

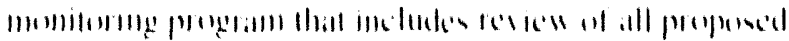

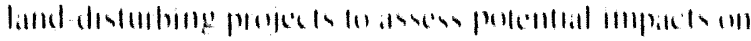

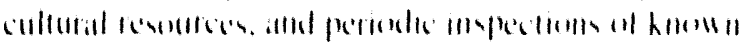

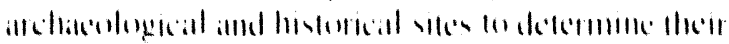

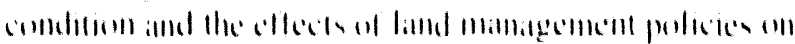

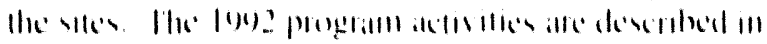

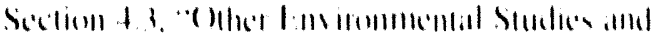

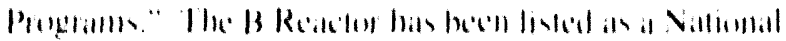
Ilivintic Sill

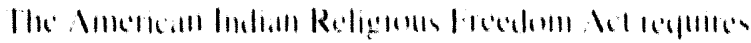

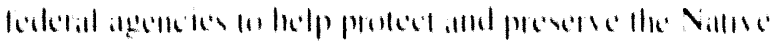

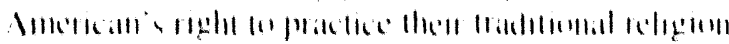

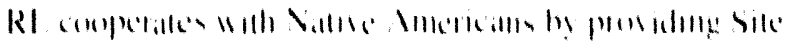
acsolat

\section{National Environmental Policy Act}

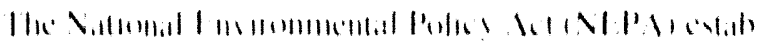

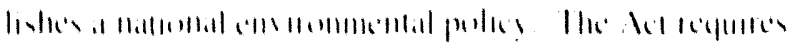

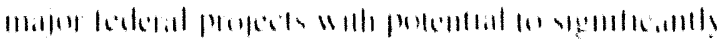

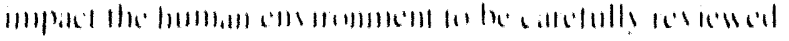

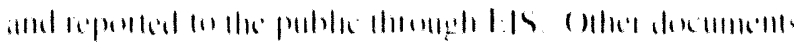
sich a 1 .

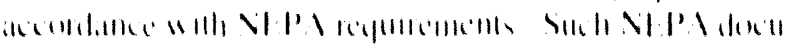

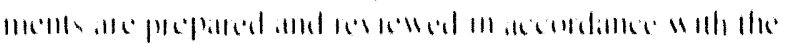

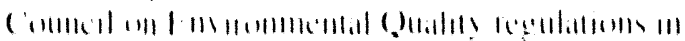

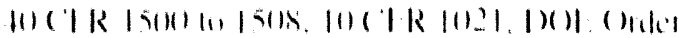

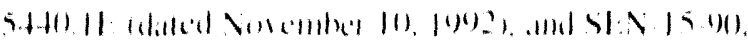

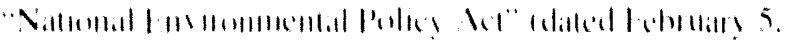
|अ(1)

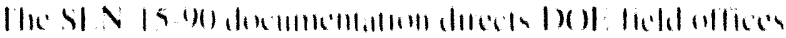

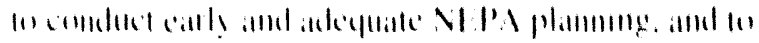

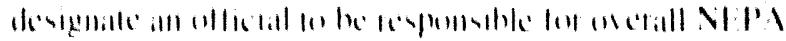

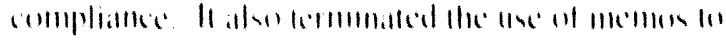

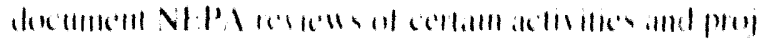

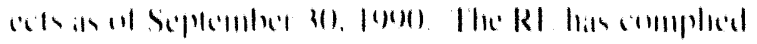

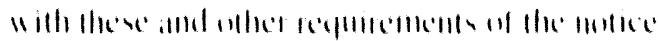

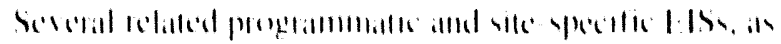

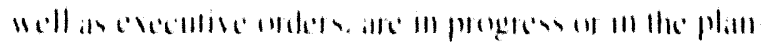

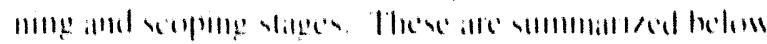

\section{Environmental Impact Statement, Decommissioning of Eight Surplus Production Reactors at the Hanford Site, Richland, Washington}

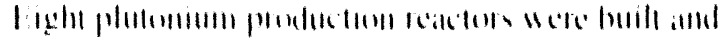

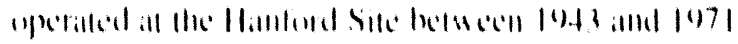

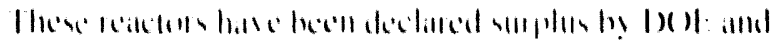

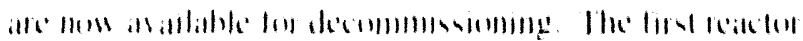

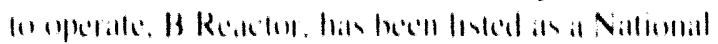
Ilinterts Sile

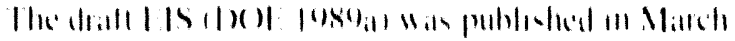

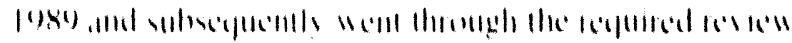

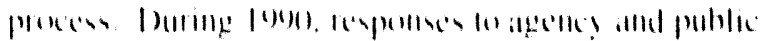

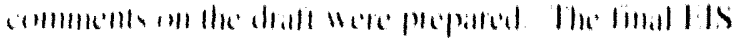
(19)

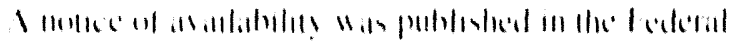

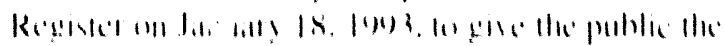

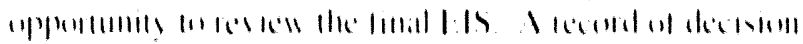

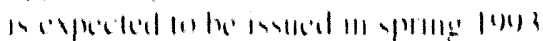

\section{Programmatic Environmental Impact Statement for the Environmental Restoration and Waste Management Program}

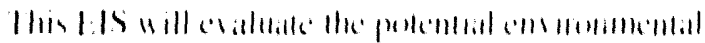

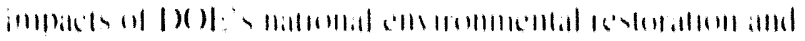

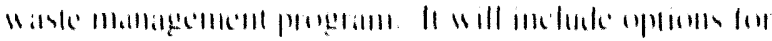

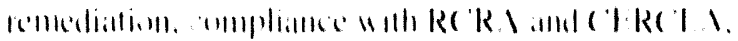

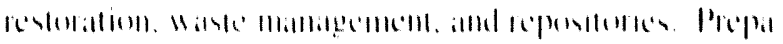

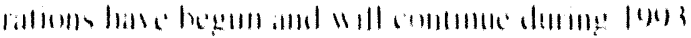




\section{Weapons Complex Modernization Programmatic Environmental Impact Statement}

The RI. centractors ansisted Argonne Natlomall I aborat-

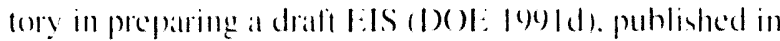
April | $99 \mid$ f. for siting. construction. and operattion of a new production reactor o NPR) lo prodace tritiom. The

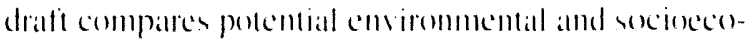
nomic impacts from the siting of an NPR all the Hanford Site. Laho Nottonal Ingincering I ahoratory. and the Salvamah River Plant. The kechonologien proposed for trition production are the light-Maller reateder. modulat high-temperature gab-costed reacter. and heatsy-water reacter. The siter were enaluated regarding eateh of the

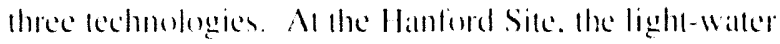
reater would he WNP-1. which is 6.34; complete. The fucl/hritum target fahricateon and tritum processing would he housed in the existing fuck and Matcrials Fiamination Facility in the f(o) Areat.

In Nowember 1901. 1)(0): announced that it will incorporate the com ironmental impact analysis for the DO): NPR ciplecily proposal into the Weapons (omplex Moderni/ation Programmatic Ens irommental Impatel Stallement and include NPR siting and lechnology decisions in the Weipoms (omplex Modernizallian Record of Decision. The D()I: inviled the public on comment on incerporating the tritium calpacity analy sis on November 29. |(20) 1.56 Federal Register (FR) 6(1)85. The implementation plant takes the resulting comments into accoumt. During 1992. public comments were received. No further actions ocecurred.

\section{Executive Order 11988 - Floodplain Management}

To minimis potential ham to or within the l() () year flockplatin as a relates to the columbia River. the

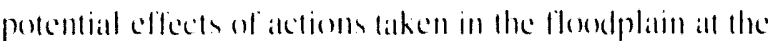
Hantored Site are evaluated and altermattives are con-

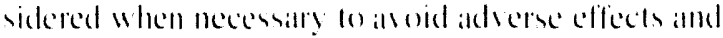

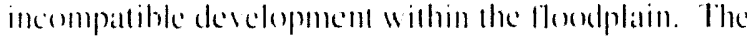

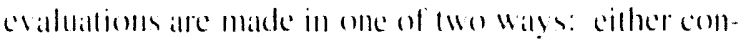
currently with the Nl:PA process, or sepalrattely. Will the required public notice and stallement of lindings pub lished in the liR. If the action reguires an envirommental

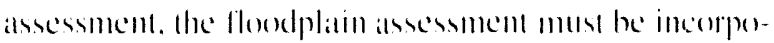
rated. The satement of findings maty be incorporated in

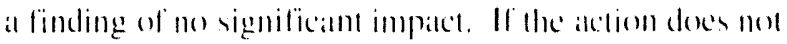
equire an envirommental assessment, the floodplain assessment accompanices an information hulletion. 'Three

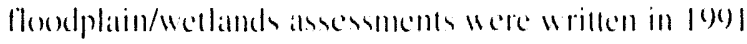
and 1992. and indeded in NI:PA desementation.

\section{Executive Order 11990 - Protection of Wetlands}

Protection is comsidered lor any action proposed in a wetand al the Hanlond Site on minimise the destruction. loss. or degradation of those wedlands. An evalluallon lakes into atcentunt envirommental concerms and is contducted cither concurrenty with the NISPA process or separately. with the reguired public motice published in the fiR. If the action requires an envirommental ansessmemt. the wedlands assessment must be incorpontaded. The statement of findings may be incorponated in the finding of no signilicant impated. If the atction does not reguire an emvironmental atsesesmemt. Whe wedands assessment accompanies an informattion bulletin.

D()): issted regulations a support compliance with these

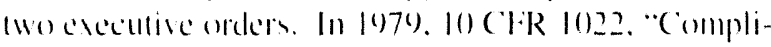

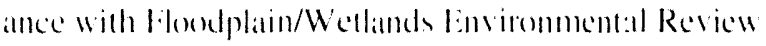
Reyuirements." wats published to address the profection

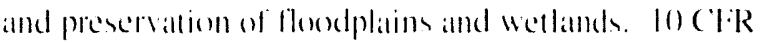
1022 reguires that the dectmentation for lloselplation and lletands assessment be prepalled concumenty with and incolponatled into the NIPA documentation. 


\subsection{Current Issues and Actions}

Progeres has heen made doward alchicving full regulatory compliance all the Hanford Site. ()ngengeself-alsse'ssments of the eompliance status. implementation of the Tri-Panty Agrecoment. and public medinges continte lo identify convironmentat compliance issters. These isstes are discossed openly with the regulattory agencies and with the public to consure thall all emvirommental compliance issues alle addressed.

\section{Hanford Federal Facility Agreement and Consent Order (Tri-Party Agreement)}

()riginally signed on May 15. 1989. the Tri-Paty Agreement is all agrecment among EPA, Ecology. and D)OF 10 achieve compliance with CERC I.A rentedial action provisions and with RC RA treatment. storage, and disposal unit regulation and corrective atetion provisions. The TriParty Agreement 1 ) detines and rankis RC RA and (TRCI A cleanup commitments. 2 ) estahlishes responsihilities. 3) provides a basis for hudgeting. and 4 ) reflects a concerted goal of achieving full regulatory compliance and remediation, with enforceable milestones, in all alggressive but achievable manner. Milestemes scheduled for 1992 were completed. Included in these completed milestones were the following activities:

- Two RC'RA Part B permit applications and four chosure plans for Hanford treatment. storages and disposal facilitics were submilted lo ficology.

- Ten Aggregate Area Mantagement Study Reports lon the 20) Areas of the Hanford Sile were submitted to licology.

- Actions to meed 38 Tri-Party Agrecment milestomes dealing with mantegement of licpuid effluents at the Hanford Site nere completed.

- Twenty-six RCRA ground-watcr monitoring wells were installed.
At the end of 1992. a total of 2.34 enforceable Tri-l'arty Agrecment milestomes for include liscial yealls (liY) lese

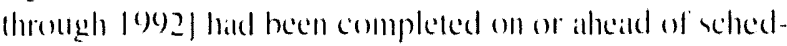

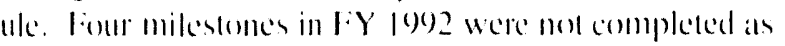
planned. 1)( )t: was assessed penaltien for hailure to follow change procedures in the Tri-Parly Agreement.

Two of the Tri-Party Agreement milestomen (M-1).5-(0.3 and $M-(15-(1) 4$ require the stabilization of 1.3 single-shell limks. In Augun 109). Headyuarters (II) halted progress on stabilising the lanks to resolve the isste of polenlially adding licuaid on "watch lisl" (Public law 10)1-510. Section 31.37 Wyden Amendmemt lanks. "Watch list" lanks include those thall contain ferrocyanides. hydrogengats-generaling waste, high-heal-generatting waste. and Waste with high concentrations of organic chemicals. Salety issties still exind with single-sloll tanks. and pumping continues to be alliected. Because of these

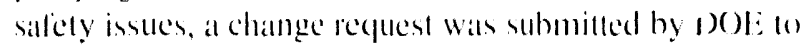
delaty the milestone for the "Interim Stahilization of an additional nime single-shell lanks" (M-0.5-(1)4), dac in September 1902. This milestone will be completed when the sillety issues can be adecuately resolved.

Another milestone (M-14-()) requires the comstruction and operation of a low-level mixed-waste laboratory. Dol: proposed redefining the milestone to require Dor: (o) provide the necessilly lathorillory ciapacity lo handle Tri-Parly Agrecment analytical requirements through privaltiation. An agerement was reatehed lo use commercial bahomatories. will penallies assessed for failure fo comply with the Tri-Party Agreement. if mandated sample furmatround times are not mel with commercial laborateries, In addition. He agreement repuires the procurement of locial laboratory facilities.

An extemsion was requested for a milestone requiring the evaluation of adrlitional double-shell tanh requirements (M-31-(1)2), so Hatl necessaty information on fulure lank needs maly be incorporated from the ongeing lank waste remediation system planning and integlation eflont. This panning and integration effort became necessary in part becatuse of the determination that B Plant could not meet RCRA repuirements allowing lank waste prelrealment and hecause of the need lo address lank waste saldely 


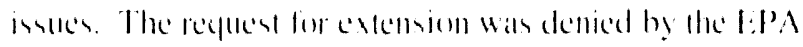
and licologe and dispalce resolution. an provided hy he

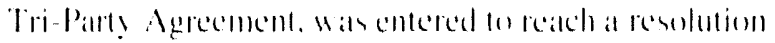
(on) this inste.

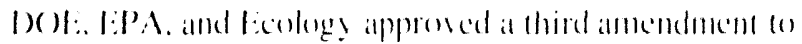

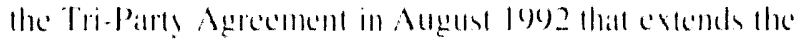
resiew and revision lime periods low Re RA Pant B permit applicattions and chesure plans. This annendment became necessaly becalmse of the complesily af permit

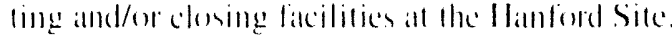

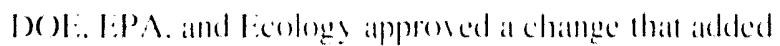

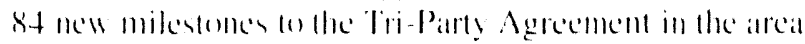

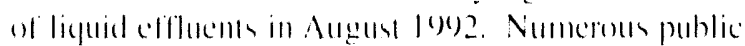

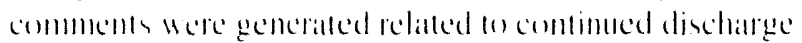
of liguid effluents at the Hanford Site and the changer.

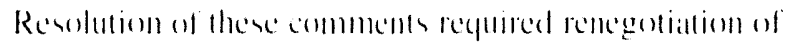
some of the $8+$ milestences and the adeption of restriclioms on the wolume to be discharged from some of these seams. This change patehige also deleted the proposed milestences for the decontamination latundey lacility becallse 1)( )I: will purste a prisale. olf site vendor for Horeservices.

The Tri-Panly Agecement requires the preparation of indis idual work plame for conducting remedial imestigalion and fearihilit! study worh on the approximalely 80 devignated operable mits. The work in heing actively condecterl at selected operable units on the site in accordance with the schedules sipulated in the Tri-Pant? Agreconent action plan.

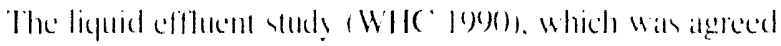

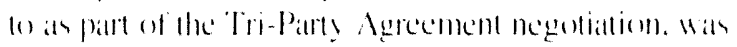

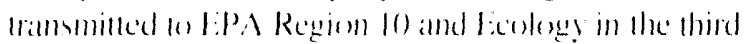

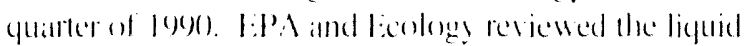

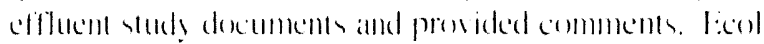

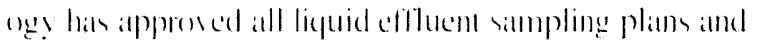

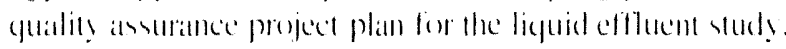

\section{Hanford Future Site Use/ Cleanup Strategy}

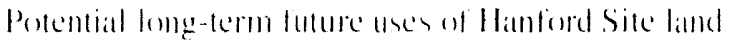

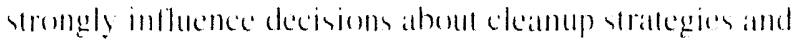
cleamup standards. Indersanding puhlic and onher

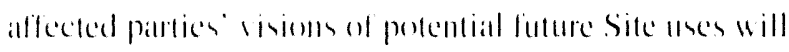
help |)( ) : make cleamup decisiome thall will be publicly silpported and that will stand the test of times.

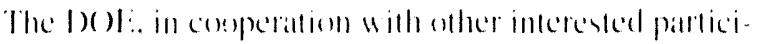

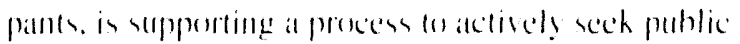
impul to the development of cleammp stattegice, laking

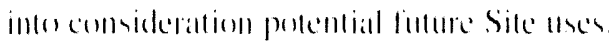

\section{Hanford Future Site Uses Working Group}

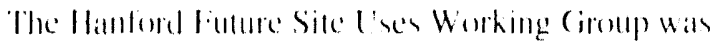

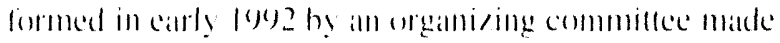

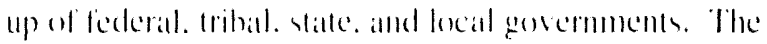
Working (iroup wal chatged with dereloping a range of fullue use optioms for the Sile and asesesing the implications of these use an cleanup. The Working (iroup)

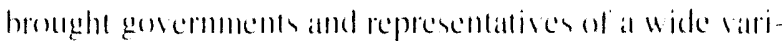

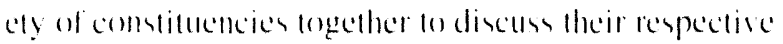
future sisome for the Hanford Sile. The Working (iroup) did nol sech comsemsts on one single vision. hut endeasored to proside decision makers with a full range of visions for the future. Cleanup decisioms necessally to make the ene fulure use options pessible will be allatlyed in depth in an upcoming enviremmental impatel slitcriment.

The members of the Working (iroup included representa-

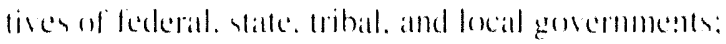

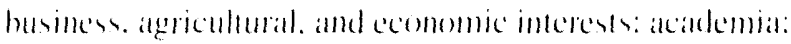
emsirommental gromps: and grompe with a special interest in the Hamford Site

The Wurking (imup med monthly from April 1902 a becomber lyo2. The Working (iroup engaged in at joint edecallion prexess lo bring all of its members to a common base of underelanding about the sike.

The! accomplished this by hringing in experts lo address the Working (iroup on lopice that included the history of the Sike from the perspectives of Native imericans. sedters. and the federal gesernment: the habitall that the Sice prosides for plame and amimals: the ecomennic impace of the Site on the Tri-citice: and the combaminat-

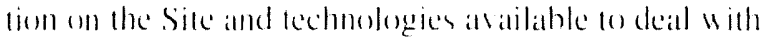
that contamination. 


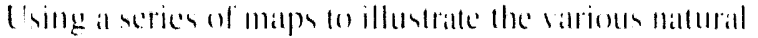
and artificial features af aix geopgaphic arean of the Silc

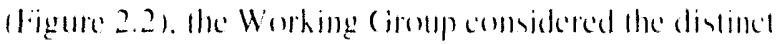

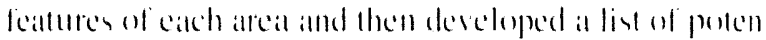

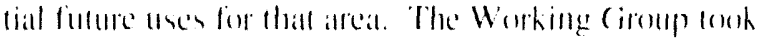

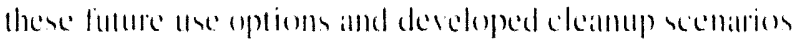

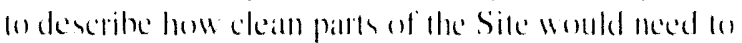
be to atecommondate the future uses. Future unes were described in generic lerms, stch in agriculame or wild-

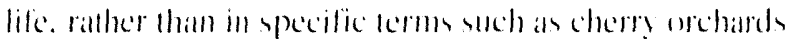
or cilk herds.

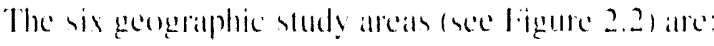

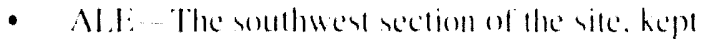
undisturbed for security and salfers reabssms, is

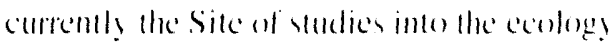
and hathitall of shrub-steppe lands.

- Nonth af the Riser Wabluke Slopes ....The area morth of the Collumbial River. hept undereloped as a securits buffer. in corrently derignated in part ats a willite refuge managed hy the L S. firb and Wibllife Service. The remainder is at rildlite recreation area mamaged by the Washingen Department of W'ildlife.

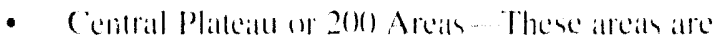
where mextear mallerials were processed. moch of the waster atre stored. and much of the known comtaminamls atre concentralled.

- Columbia River The stredeh of the risce that rum through the Sile. known as the llantorel Reach. is home to spaw ning grounds for more than a third of the river s lall chinowe salmon.

- Reaterson the Risce. The arcal along the river contains nine retired plutomium prodection reatedors and the land in hedween. Light of the reactom were shut down by 1971: the nimh (N Reatedon wat shut down in less.

- All ()ther Areas-.-This areal comsise of all af the Site that is not included in the other geographice areas. This includes the $30(1)$ and 1100 () Areas just north of Richland. the Washington Public Pouer Supply Sysem. Whe Pacific Northuest J ahomallory. as well as latrege tracts of relatively undisturbed land herween those ficilities.
The Working Girump develuped al sel of nine majof recommentantoms for the Sile as a whole. These recom mendations. as well as the luture une optioms and cleanup

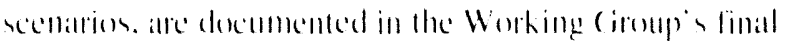

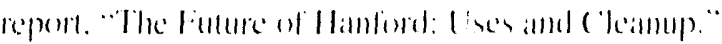

The Working Ciroup secommendations were:

- profed the columbia River. Al some locatiomson the Site. contimninamb ane known lo be entering the riser. Stopping atctual and potential contaminatton of the collombiat is at high priorit!

- deall realiatically and forcefully wilh gromme-water

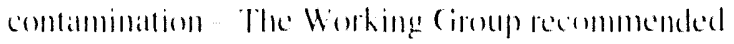
that groumd water should mon be uned if it would jeopardise public heallh or safely or adsersely

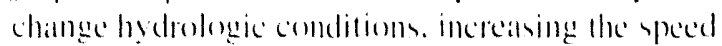

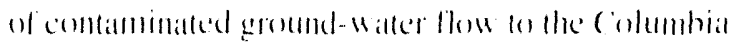
Rirer. The Worting (iroup axpected that ground

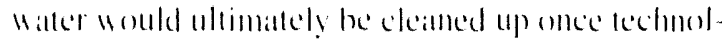

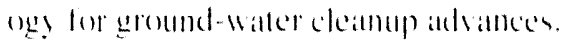

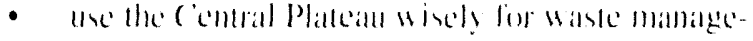

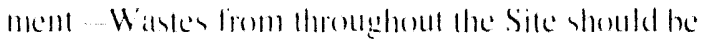
concentrated in the central Plateatu rincleseleng and surrounding the 200) Areas). Wilhin the Central Matcatu, the Working (irtup stgegented that waste

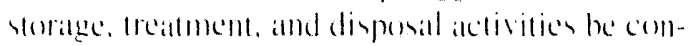
centratled lo minimise the amment of land devoled

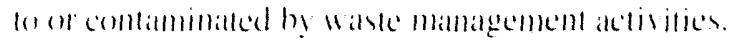

- do mo harm doring cleamen ar with new denclop. ment the primsary goal of cleanup is a protect humantheallh and puhlic siltels. In addition, comimommentat salues of the Site are lo be proteceded and restoned. Decisions dealing with ckanmp and

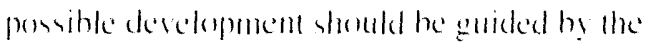
primciple. "(h) nu hamm."

- cleamup of arcias of high future une vallac is imporetant The Working (iroup beliened that allean of high fulure use value should be camdidates for

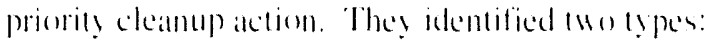

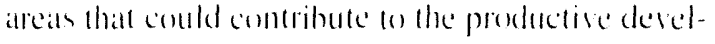
opment or use of the site for ontere purposes and areas that contd he cleaned up yuichly for a very small pereentate of the cleamup hudget, making large trace of land an ailable for wher uses. 
1992 Environmental Report

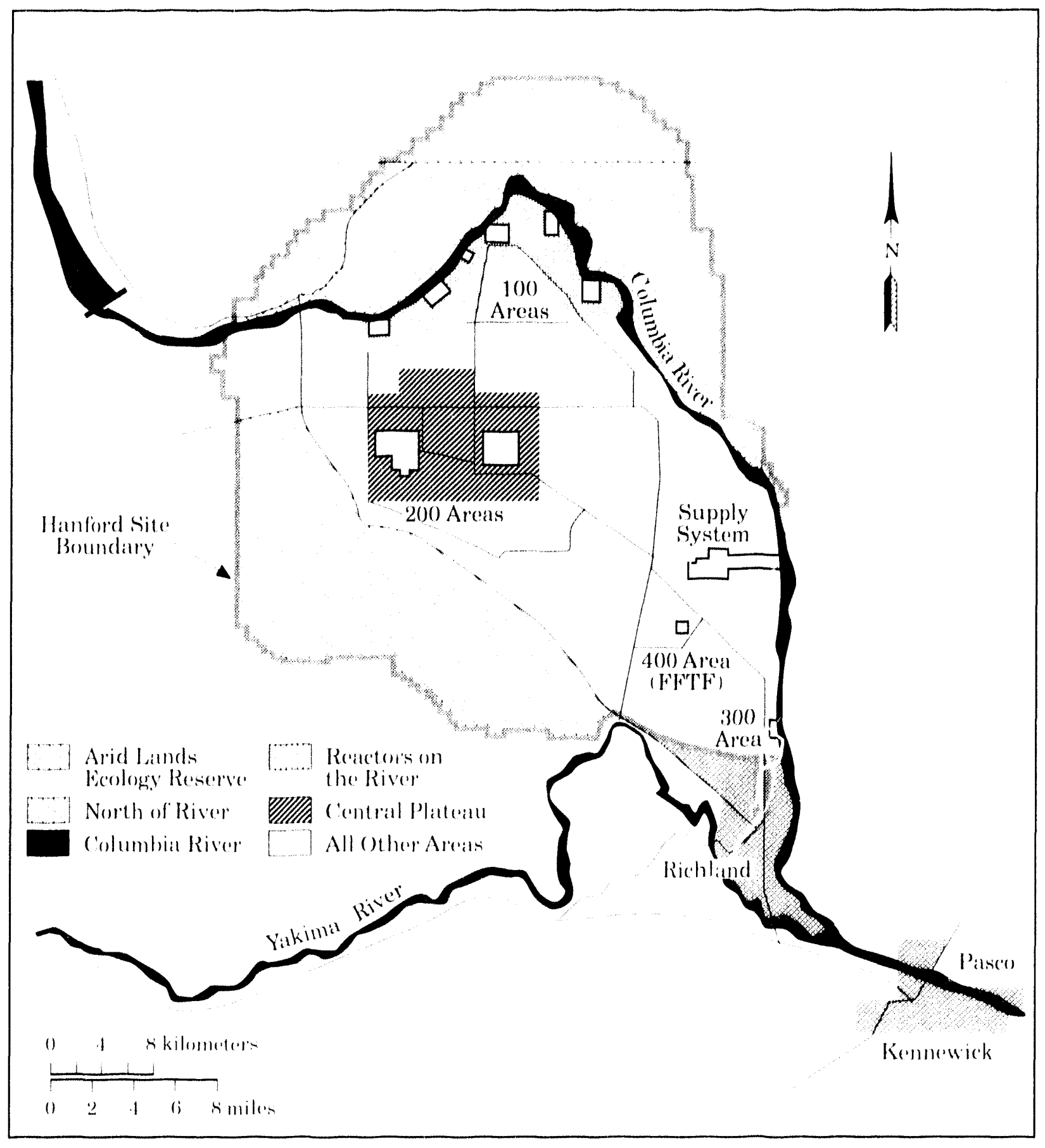

$\$ 930301244$

Figure 2.2. Six Geographic Study Areas for the Hanford Future Site Uses Working Group

32 


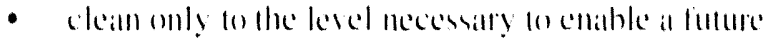
use option lo recur The Working (iroup believed

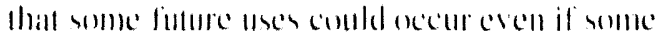

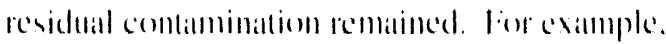

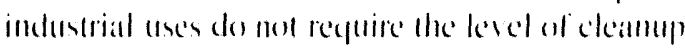

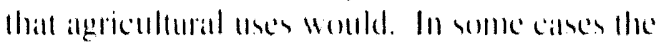

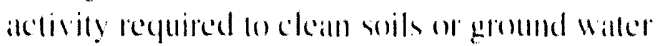
might be detrimentall to all alleal designated for wildlilic.

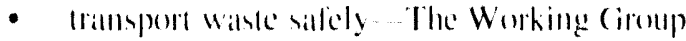
recognised that decisioms related an Hantord cleamup will reguire the manyportation of radionalctive and halatledous malerials within the Site.

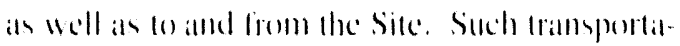

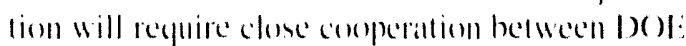
and tribill. slitke, and local gerermments. The Working (iroup endersed llke Hatzardent Matterials Mantagement fimergency Response Training ('enter.

- captere ecomomic devedopment apportunitices locally 10() : has announced its intemtioms to

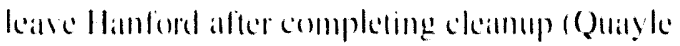

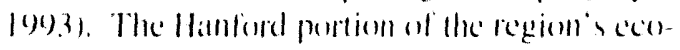
memic batse must ultimitely be replated hy non-1)( )l: activities within the state. The Working (iroup) urged D) ()t: and its contriators lo liactor locial development and ecomemic diversificattion into cleanup decisions, Research and develepment

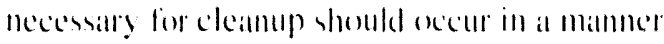
that crealles addelitional privalle sector economic dereremenem opportunitios.

- imvolve the public in lubure decisions about Hanfiond Puhlic involvement and comsultation must be incorperated inne fulue decision-making at Ilanford. The Working (iroup process bhould be a model for invole ing the public. The public should be consulted on decisions insolving trallsportation and entergency preparedness, ecomomic

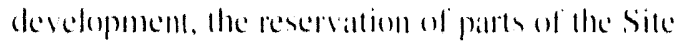
by 1)(ot: for other missioms. use of ground waller. and exposure rist resulting from land use decisions.

The Working (iroup completed its linal report in december 1002.

\section{The Columbia River's Hanford Reach}

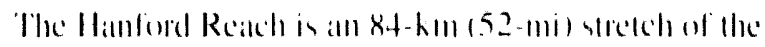
(olumbia River from Priest Raphede Dam lo lle head of latke Wallula near Richland. Comgeres parsed at law

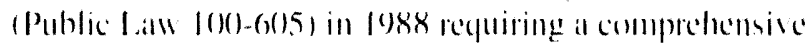
stedy of the Hanford Reated. The Secrelary of Interion, in

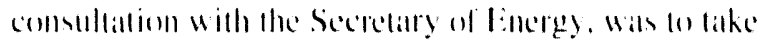

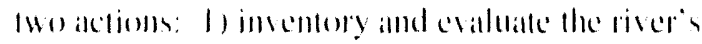
resurees, and 2) develop and analy a a series of prolec fion altermatives. Including designation of the Reakeh in lle Natlomal Wild and Scenic Riters system. The

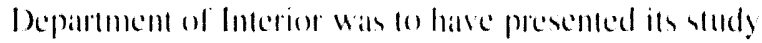
and a finat las to Congeres hy November logl: how erer. his has mot yed occoured.

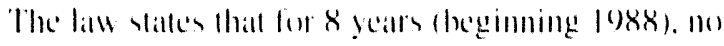

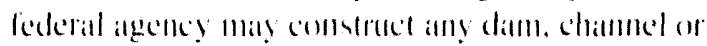

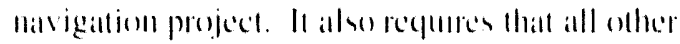
aclivities, lo the extemt practicable. he planned and

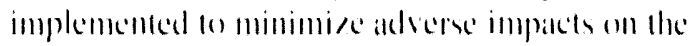

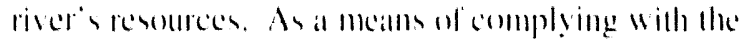
law. RI. Inotities the Natlomal Park Service of all proposed atctivities subject on the Nattional Pallk Services semsultallion and condediattion process agreed upen hy the two insolved agencies.

Analysis of the allematives hegan in Maly 100). Options range from entablishing a resource protection alreal to lathing! no andion. Which state of lederal agencies would manage the alleit. Whether development would he limiled. and how far from the shere the protection would extend are among aller issues lo be addressed in lle bis. forr example. il He Hanford Reach llere declatred al

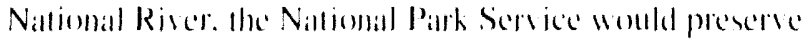

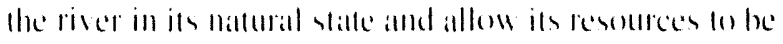
used but mol chatuged. allered. ar depleted.

The Nattomal Path Service is the katel angency for the Department of lonerion and announced is preterred allemallive in a drall liss in 1902. A public comment period followed. and public hearings were held. The

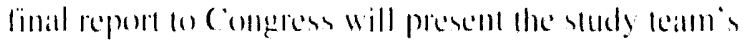
final recommendiations. 


\section{Tiger Team Assessment Corrective Actions}

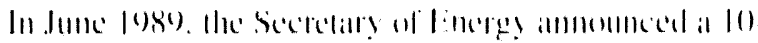

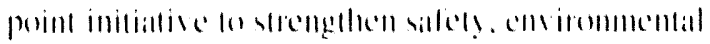

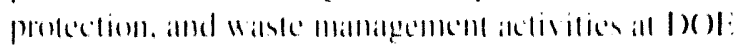

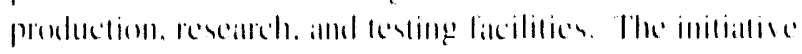

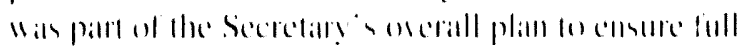

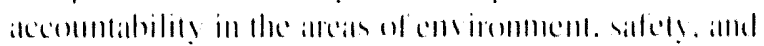

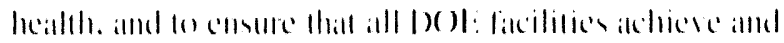

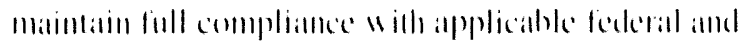

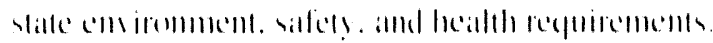

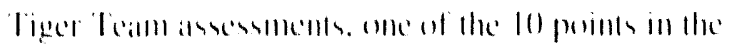

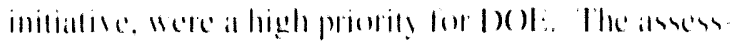

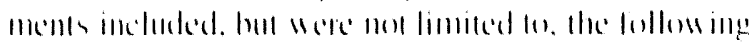

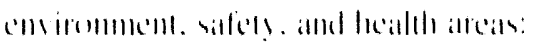

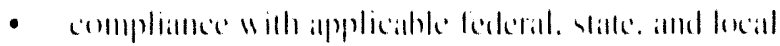

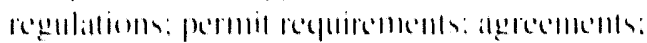

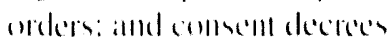

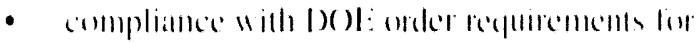

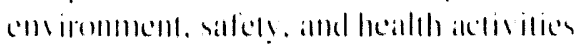

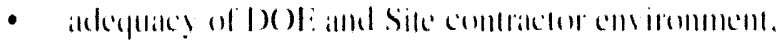
sately. and health mallatgement programs. inclading

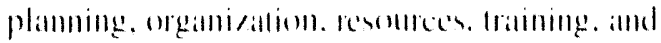
relationship with regulattery agencices

- combormanter will applicinhle best and ancepted industr! mikdices

- identificallion af rom ciallses.

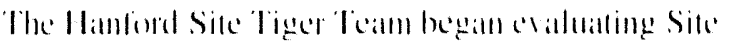

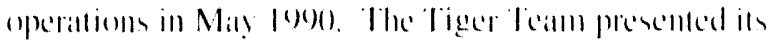

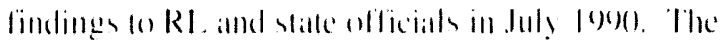

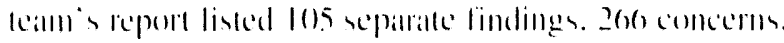

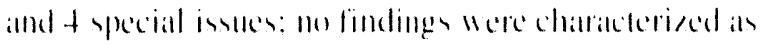

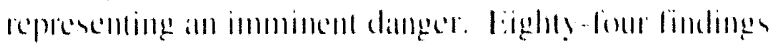

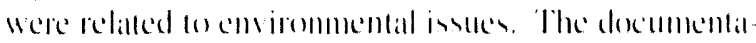
lion of the resules of the arsessment is puhlinfled in

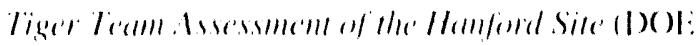

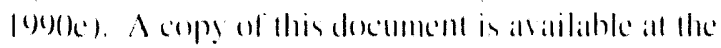
RI. Puhlic Residing Romm in Richlind. Wanhinglon.

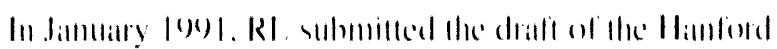

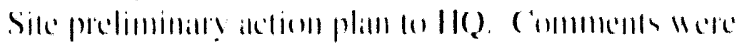

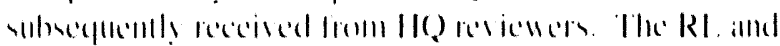

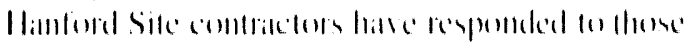

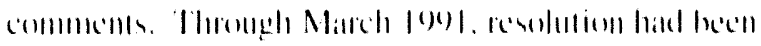

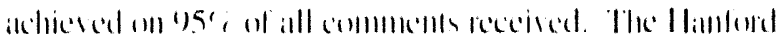

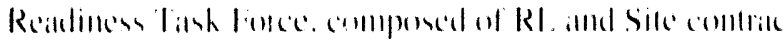

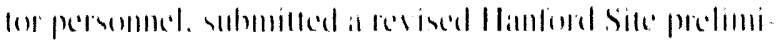

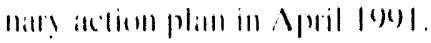

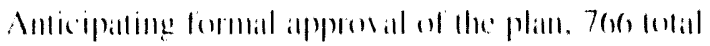

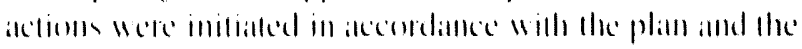

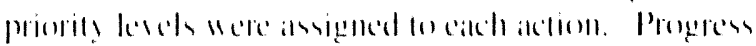

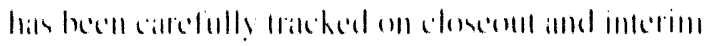

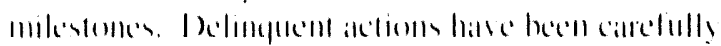
illally

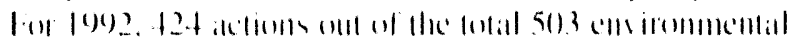

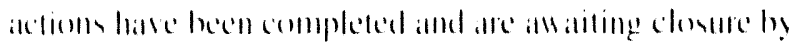

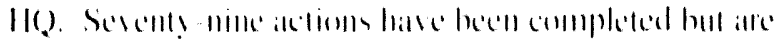

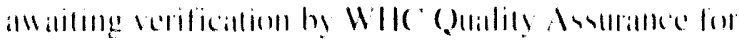

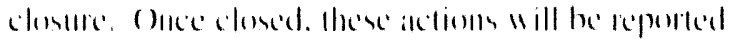
(1) H()

\section{Plutonium Uranium Extraction and Uranium Trioxide Plants Status}

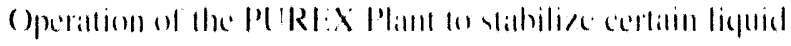

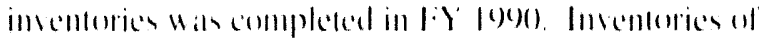

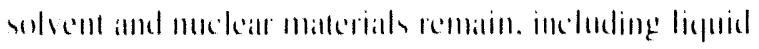

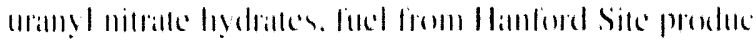
tion reakdors, and organte materials. Tramsition of the

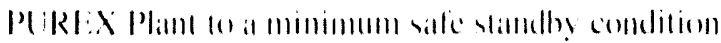

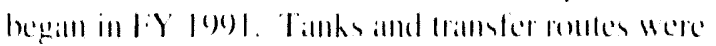

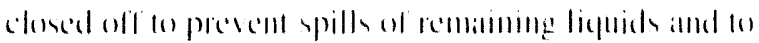

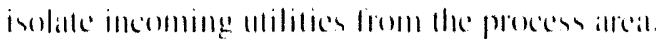

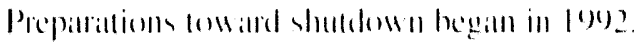

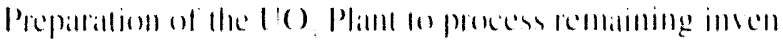

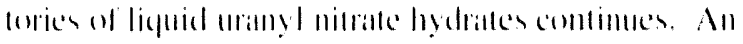

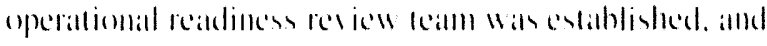

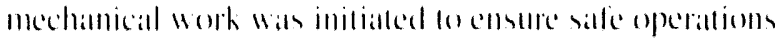
The operational campatign was plammed for mid-spril 109.3 . 


\section{Plutonium Finishing Plant Restart}

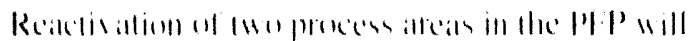

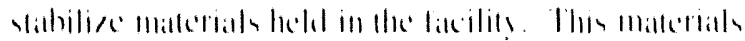

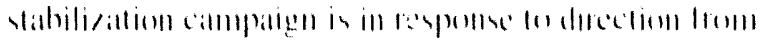

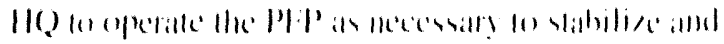

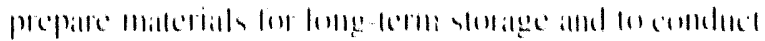

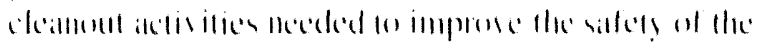
liscilin!

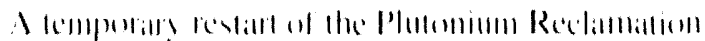

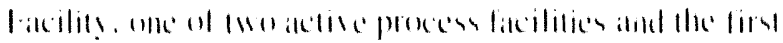

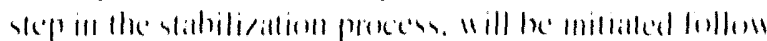

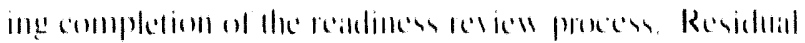

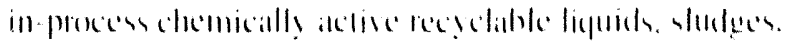

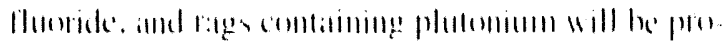

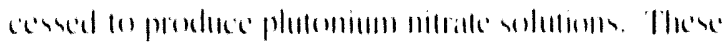

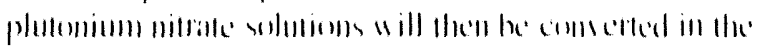

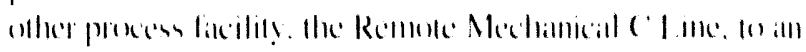

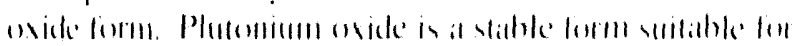

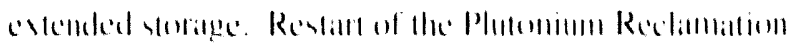

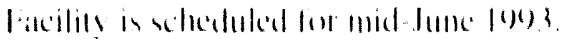

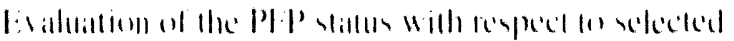

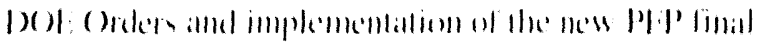

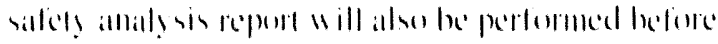

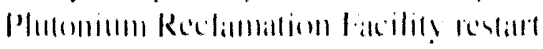

\section{Hanford Waste Vitrification Plant}

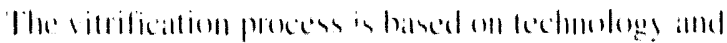

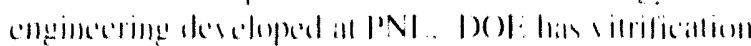

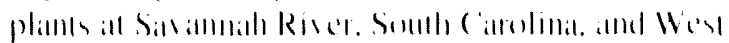

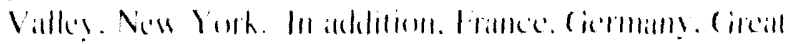

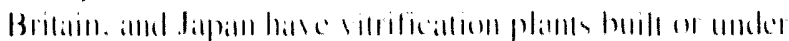

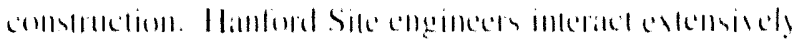

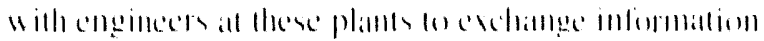

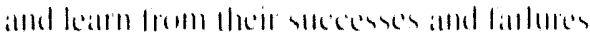

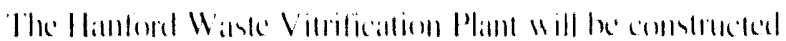

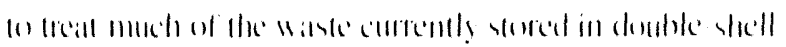

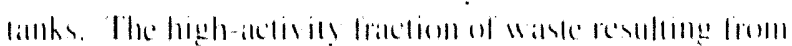

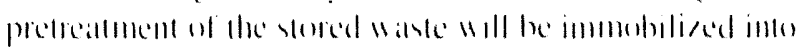

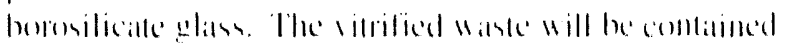

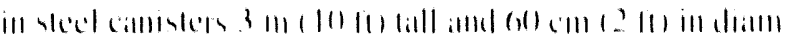

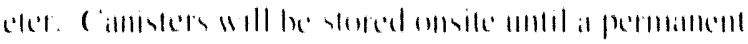

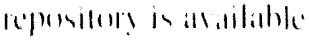

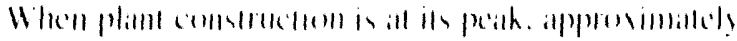

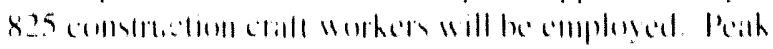

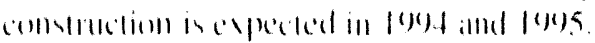

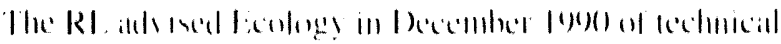

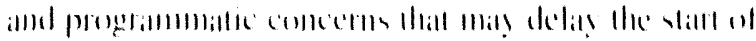

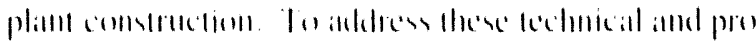

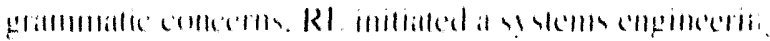

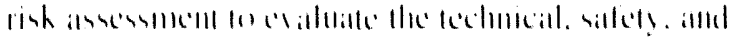

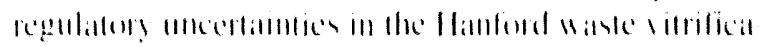
tim prillatrill

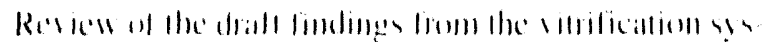

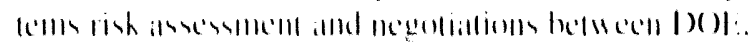

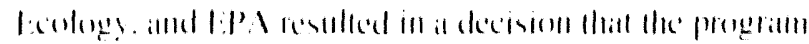

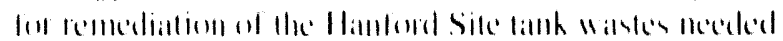
lab berdelines.

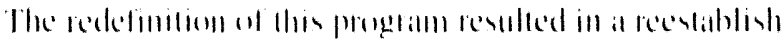

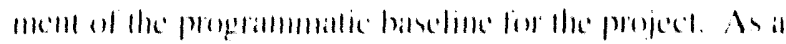

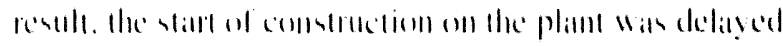

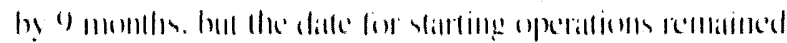

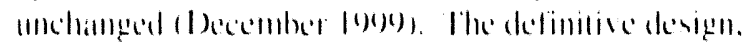

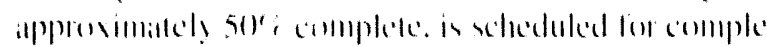

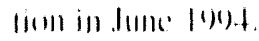

\section{Waste Receiving and Processing Facility}

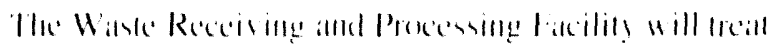

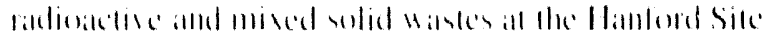

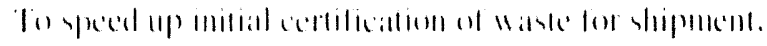

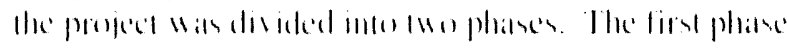

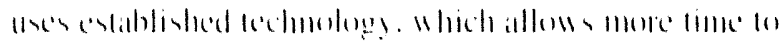

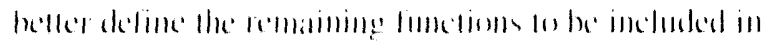

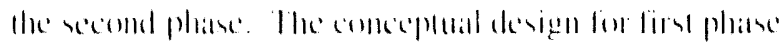

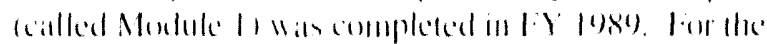

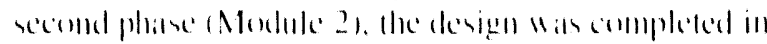
1) (1)!.

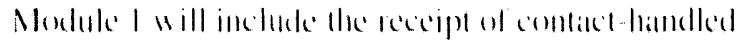

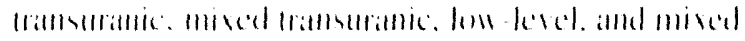

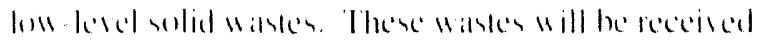




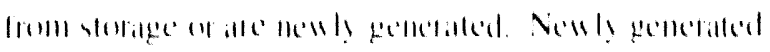

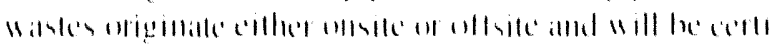

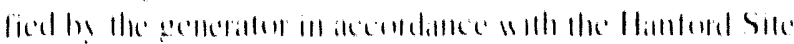

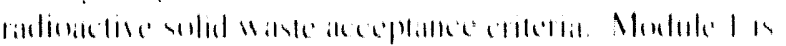

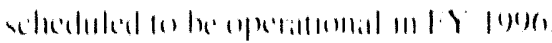

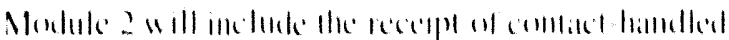

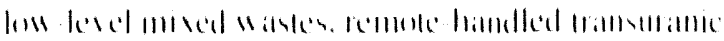

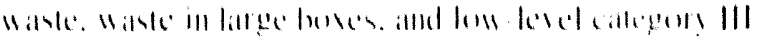

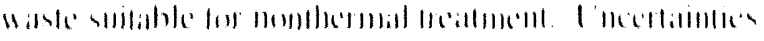

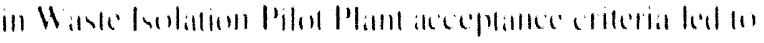

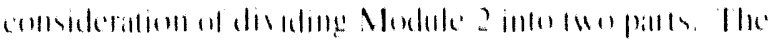

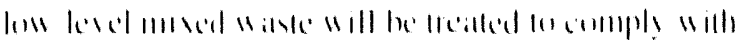

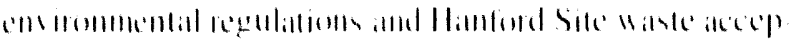

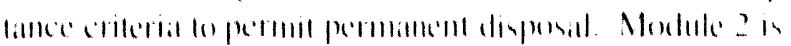

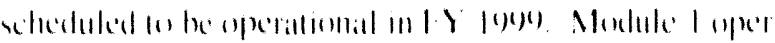

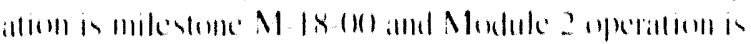

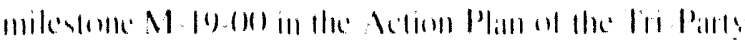
Aptrome'll!

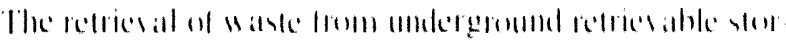

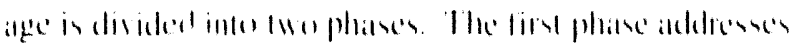

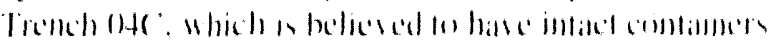

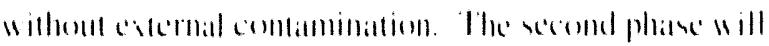

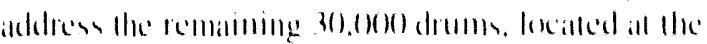

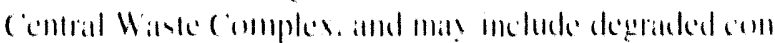

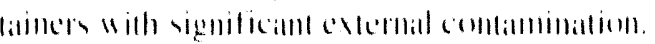

\section{Waste Tank Safety Issues}

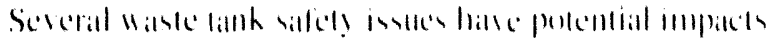

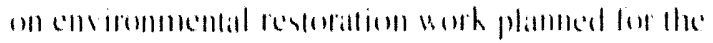

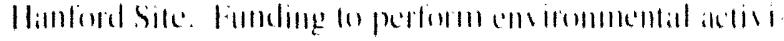

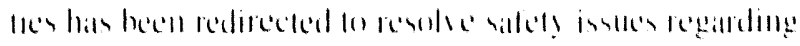

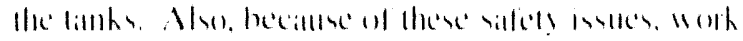

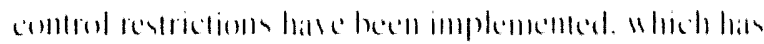

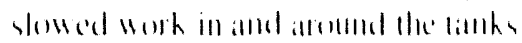

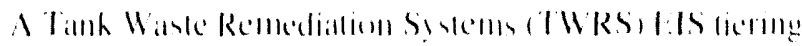

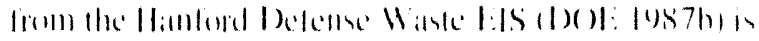

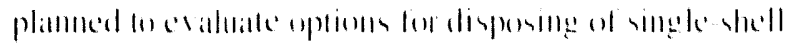

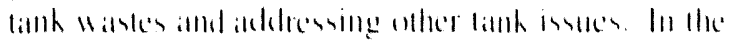

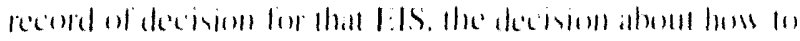

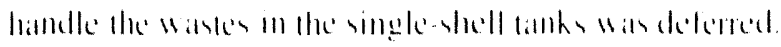

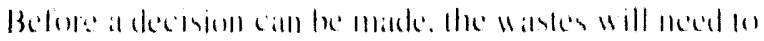

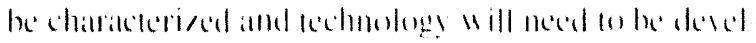

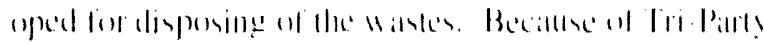
Agreoment milentomen, the IWRSI:Is, scheduke is propereal for acceleration.

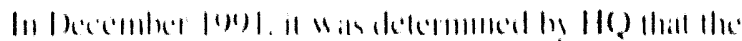

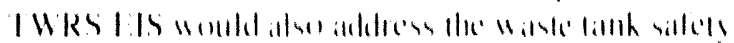

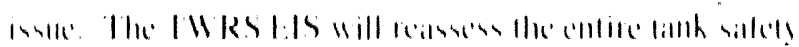

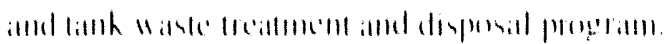

\section{Background}

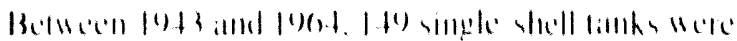

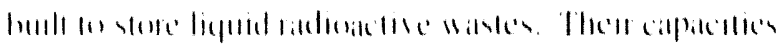

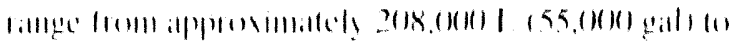

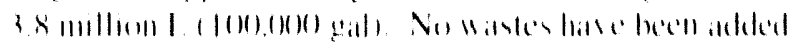

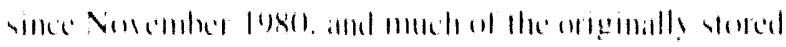

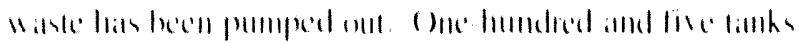

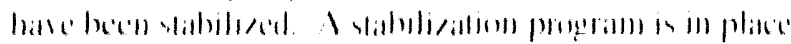

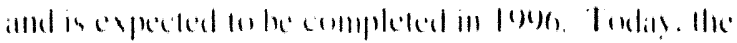

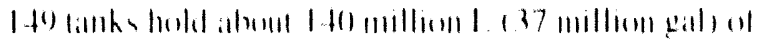

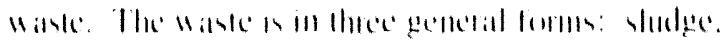

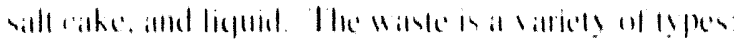

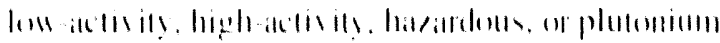

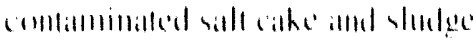

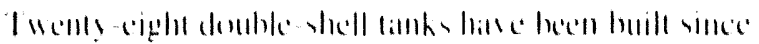

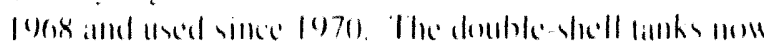

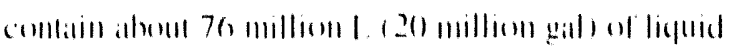

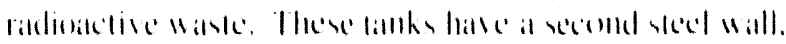

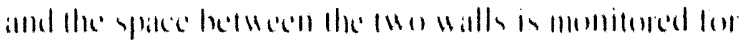
liaks.

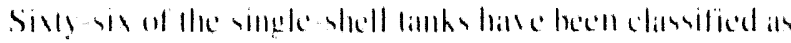

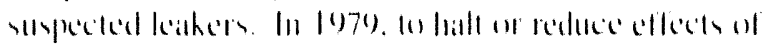

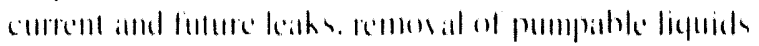

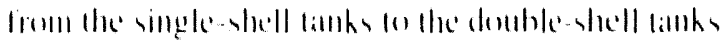

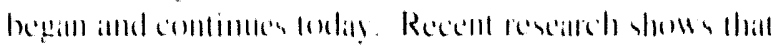

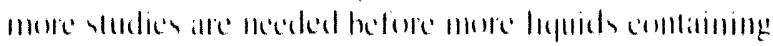

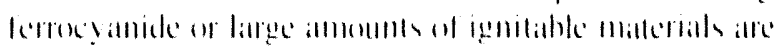

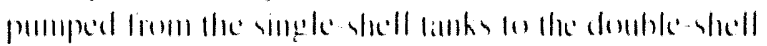

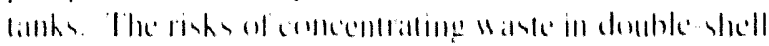
tanke munt allow be arallualed.

\section{Issues}

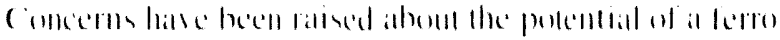

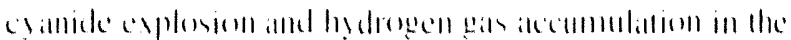

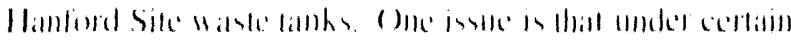

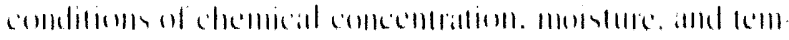

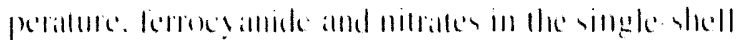

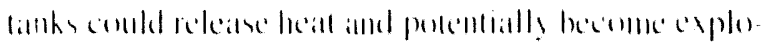

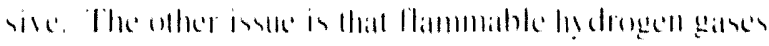

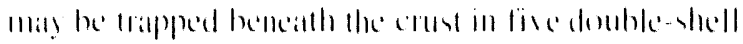
lamks and is simgle shell limhs. OHe lituh in particulats. 


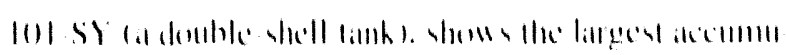

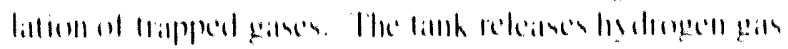

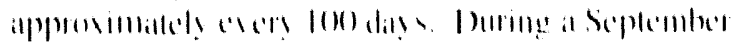

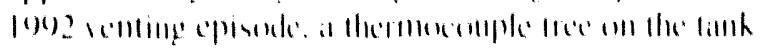

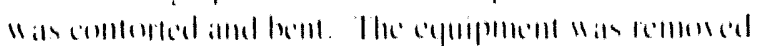

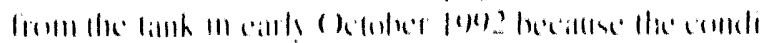

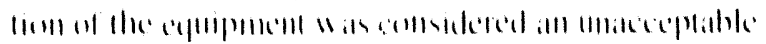

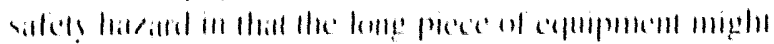

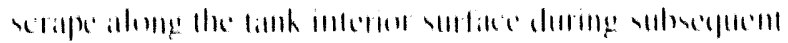

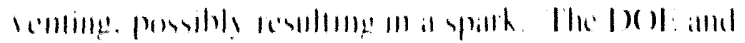

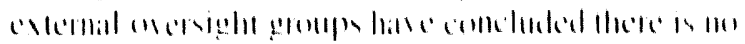

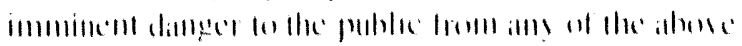
villillimill

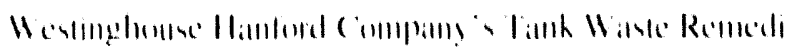

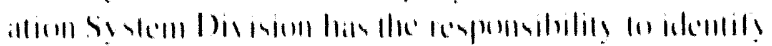

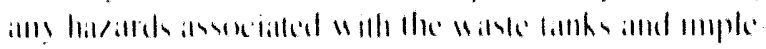

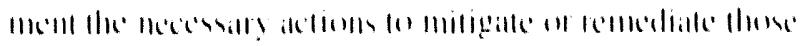

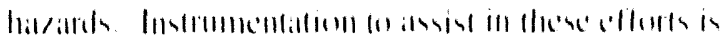

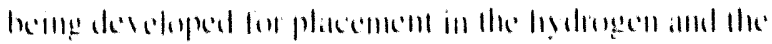

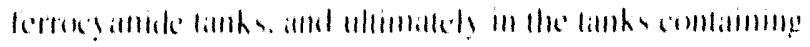

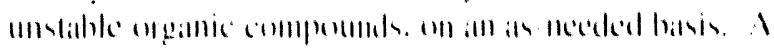

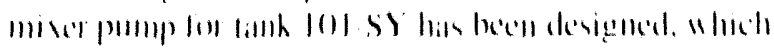

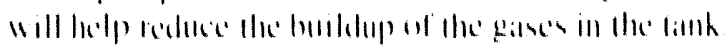

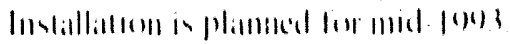

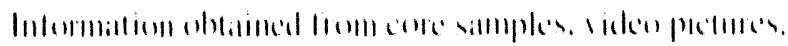

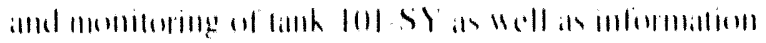

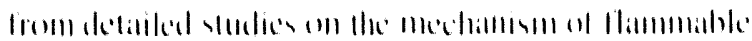

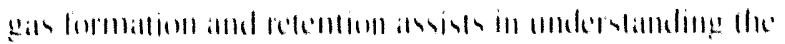

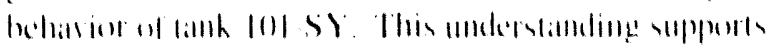

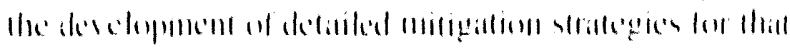

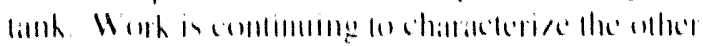

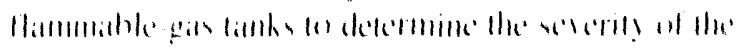
liatillil.

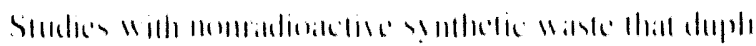

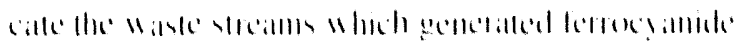

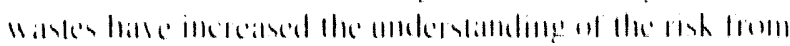

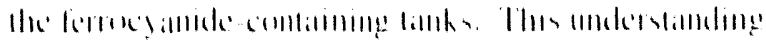

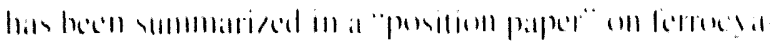

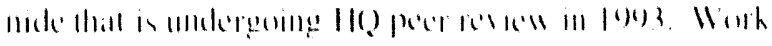

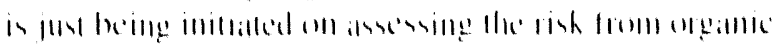
colltaminge lanhs.

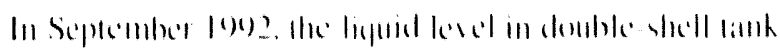

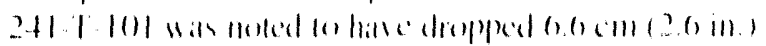

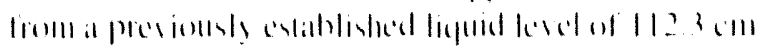

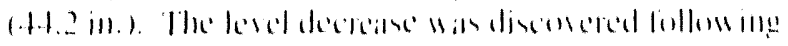

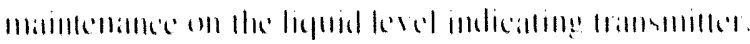

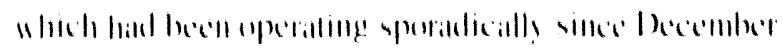

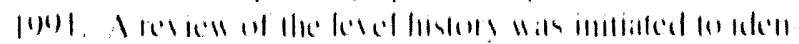

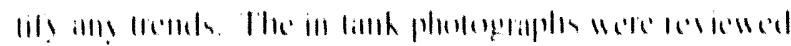

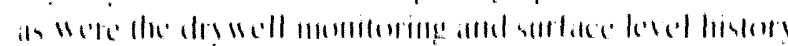

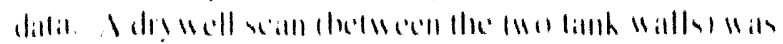

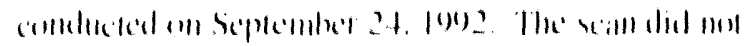

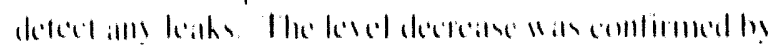

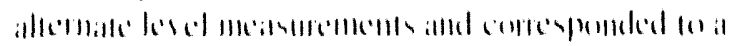

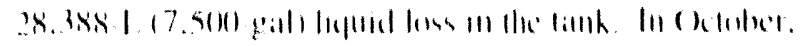

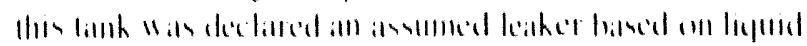

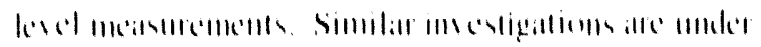

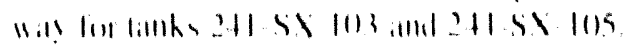

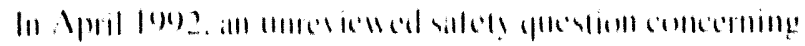

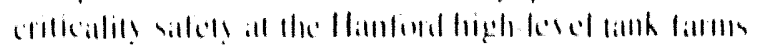

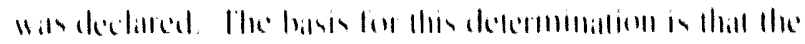

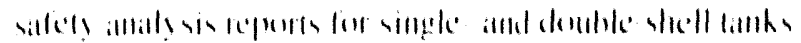

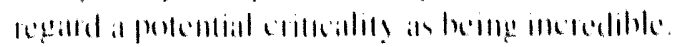

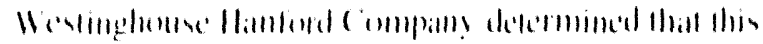

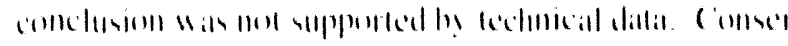

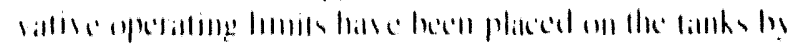

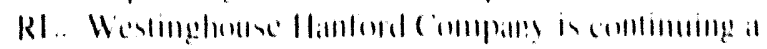

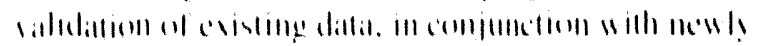

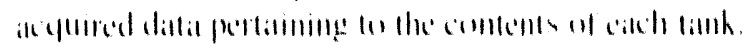

\section{Waste Minimization}

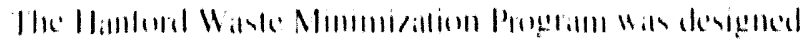

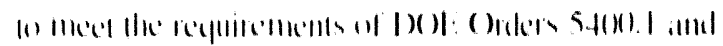

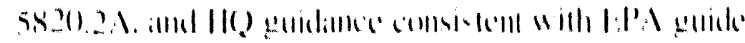

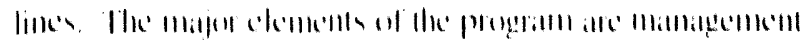
งा1)

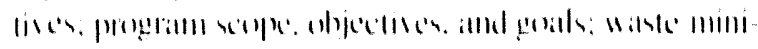

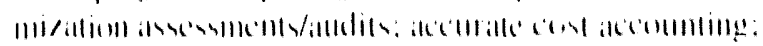

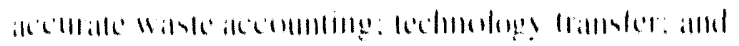

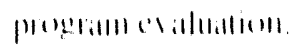

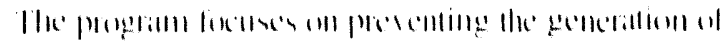

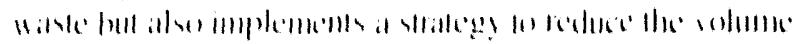

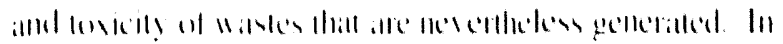

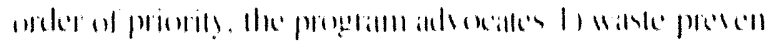

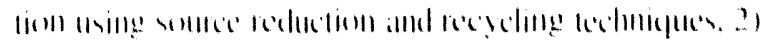

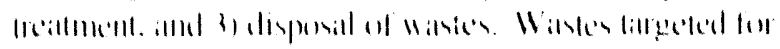

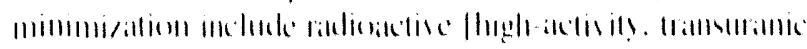

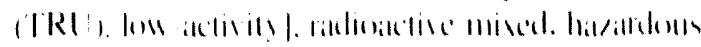

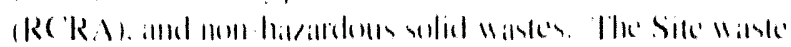

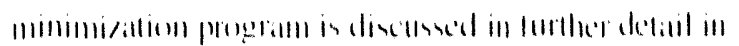

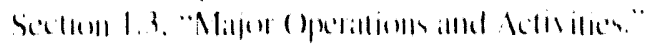




\section{2-A Evaporator Status}

Ihe 212 \1 1

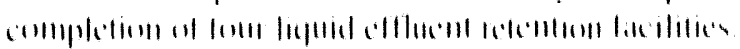

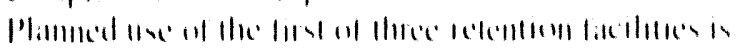

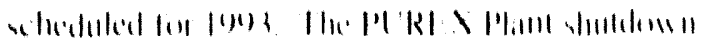

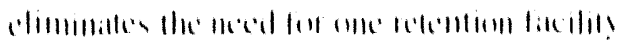

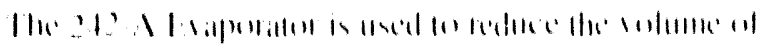

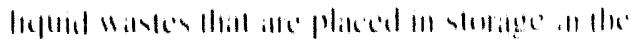

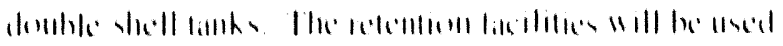

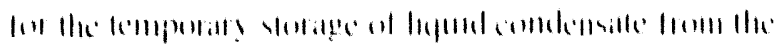

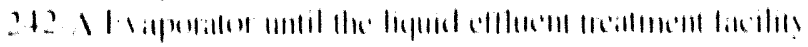

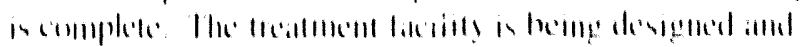

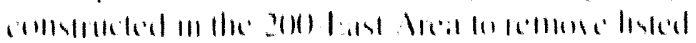

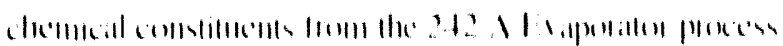

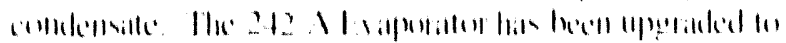

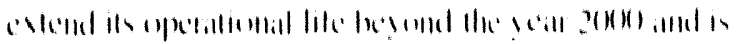

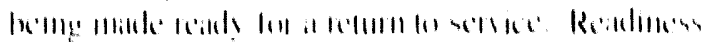

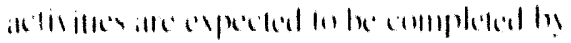

M.11 7.11113

\section{Submarine Reactor Compartments}

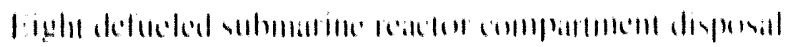

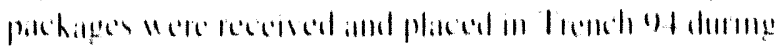
(') (1) ?

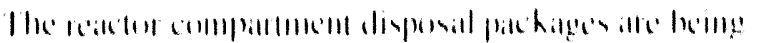

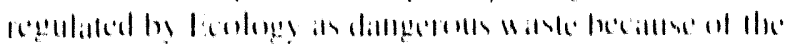

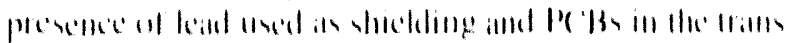

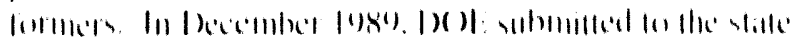

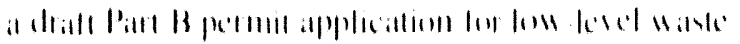

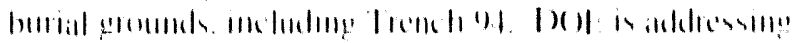

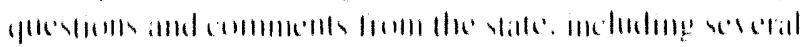

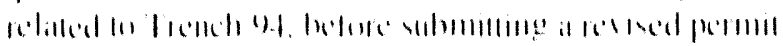
al)

\section{Hanford Facility Danger- ous Waste Permit}

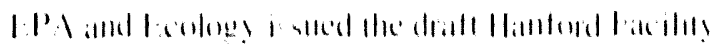

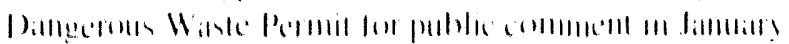

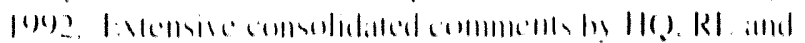

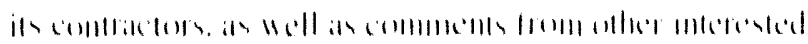

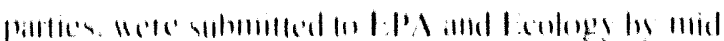

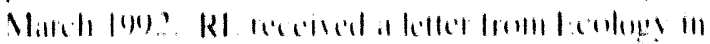

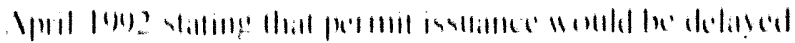

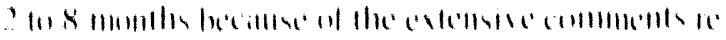

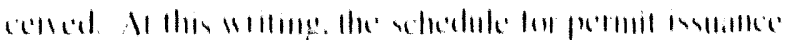

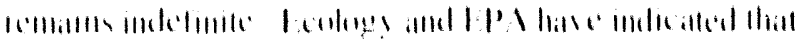

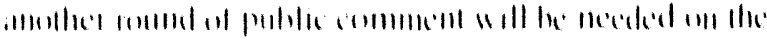

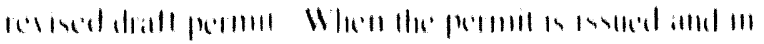

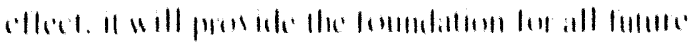

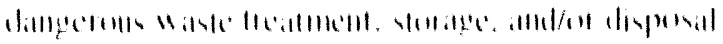

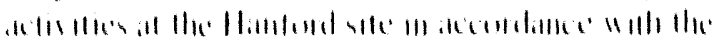

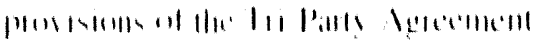

\section{International Environmental Institute}

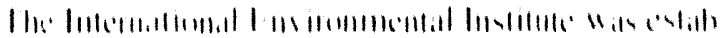

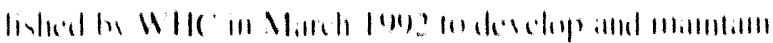

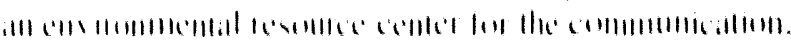

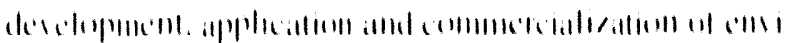

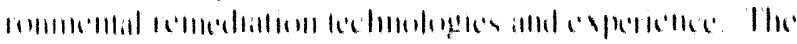

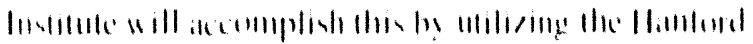

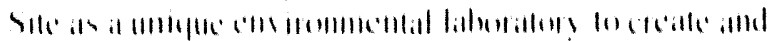

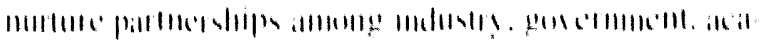

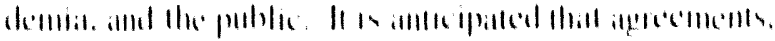

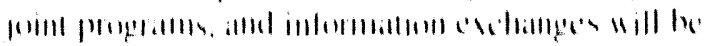

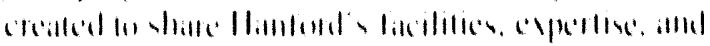

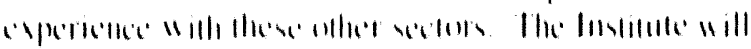
act a

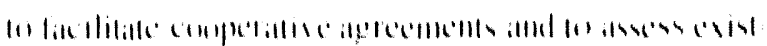

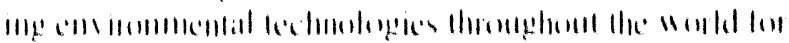

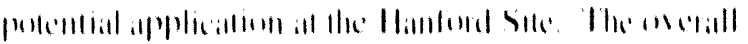

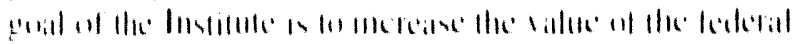

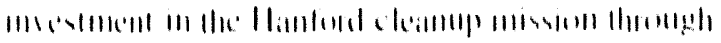

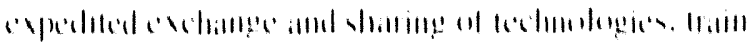

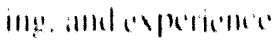

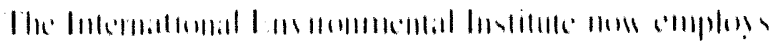

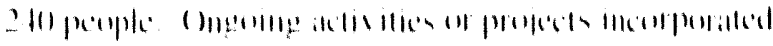

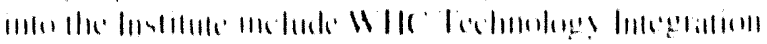

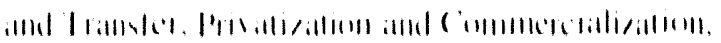

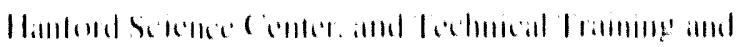

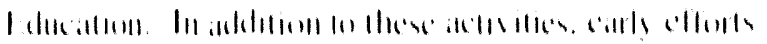

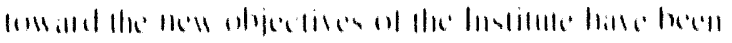

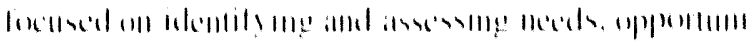

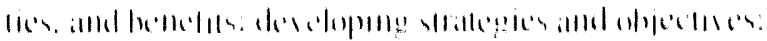

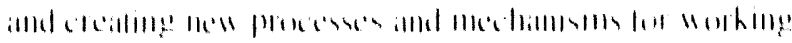

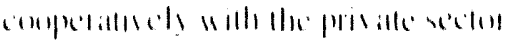




\section{Self-Assessments}

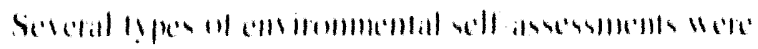

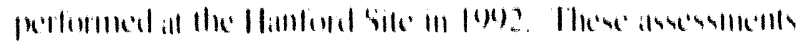

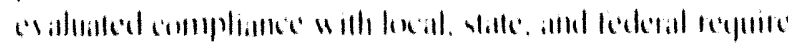

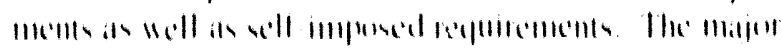
forden

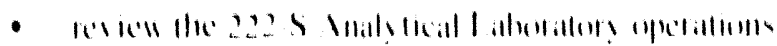

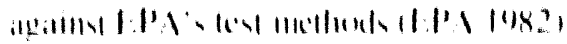

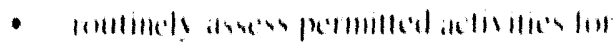

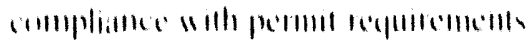

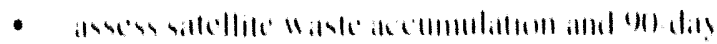

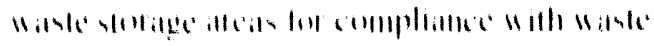

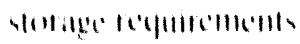

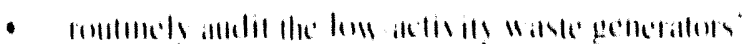

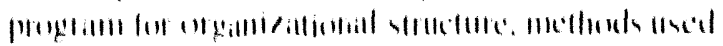

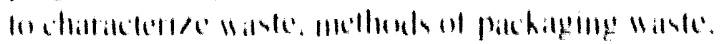

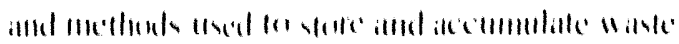

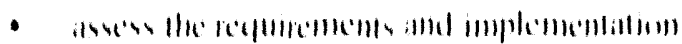
(1) I) 1 (t) Nitlitgenthell"

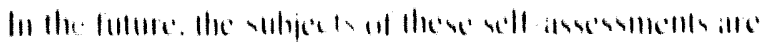

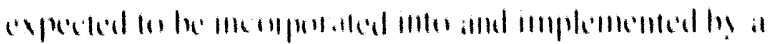

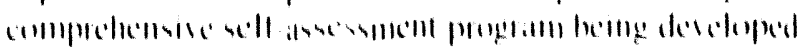

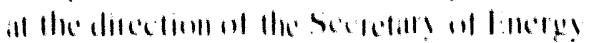




\subsection{Environmental Occurrences}

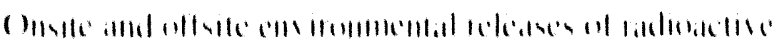

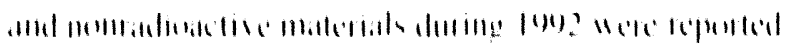

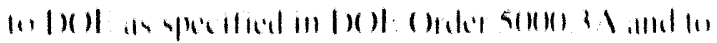

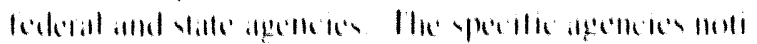

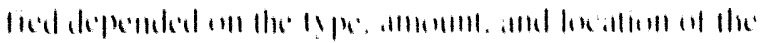

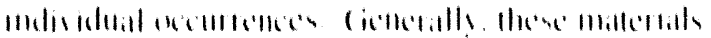

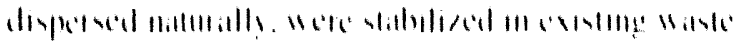

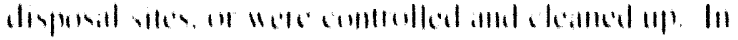

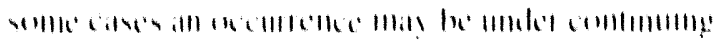
ohem.

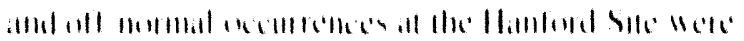

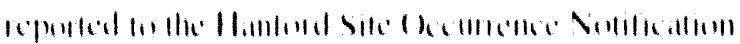

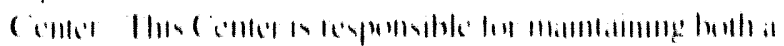

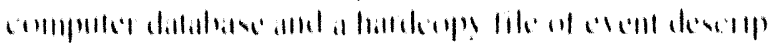

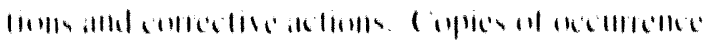

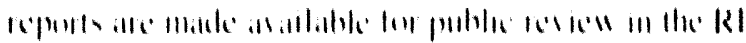

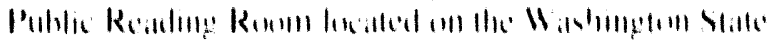

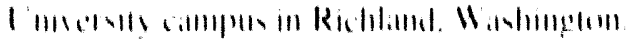

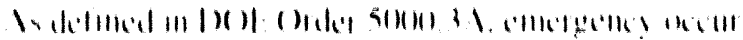

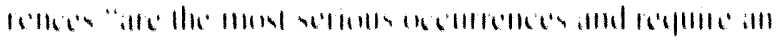

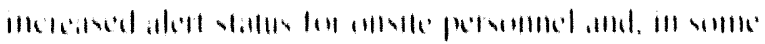

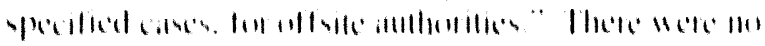

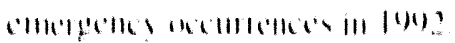

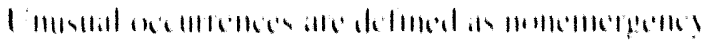

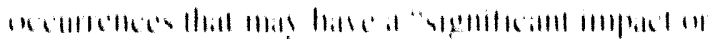

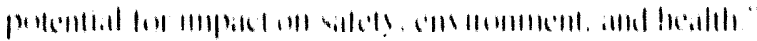

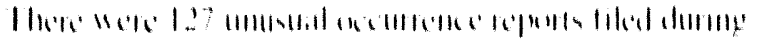

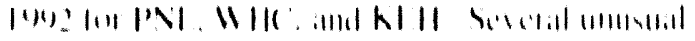

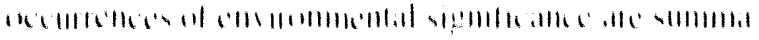
n/Mllim

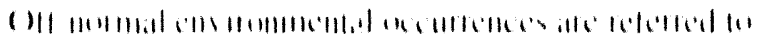

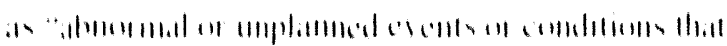

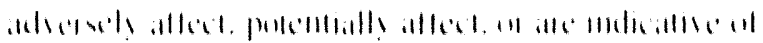

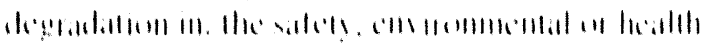

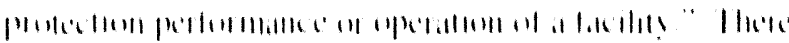

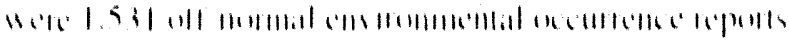

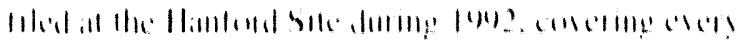

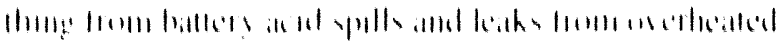

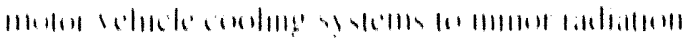

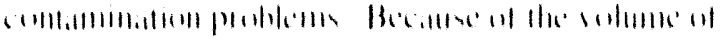

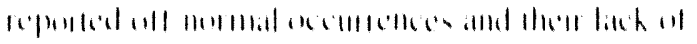

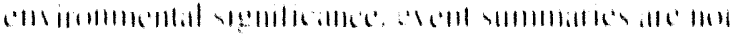

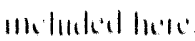

\section{Unusual Occurrences}

\section{Release of Contaminated Water to the Ground (RL-KEH-1992-0061)}

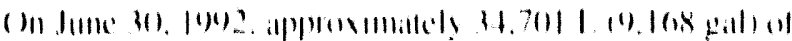

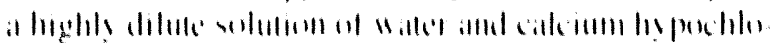

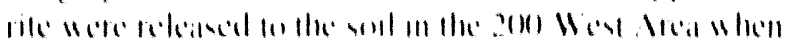

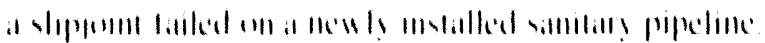

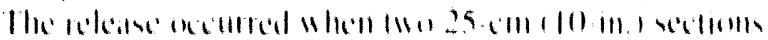

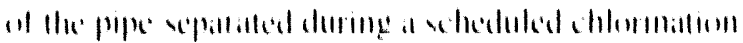

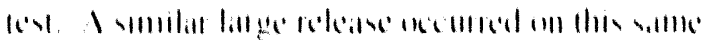

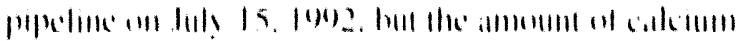

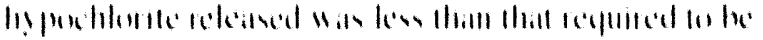

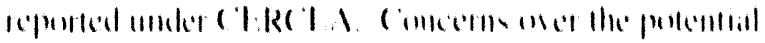

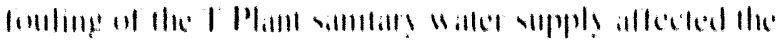

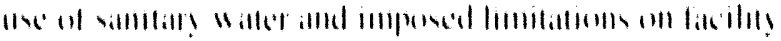

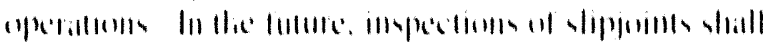

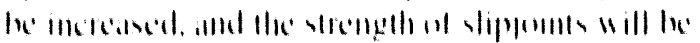

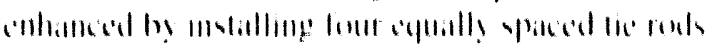
ill He fuills.
\end{abstract}

\section{Lithium Release (RL.WHC.300EM-1992- 0044)}

1)

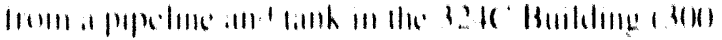

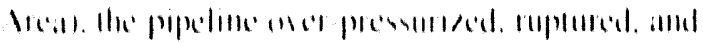

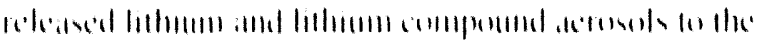

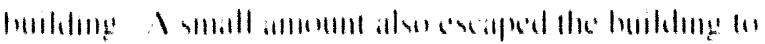

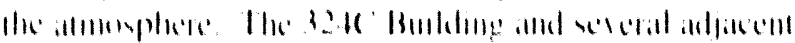

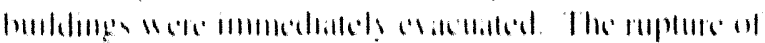
He ply

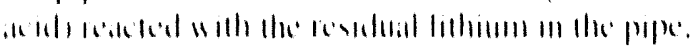

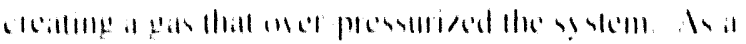

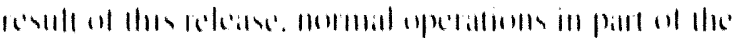

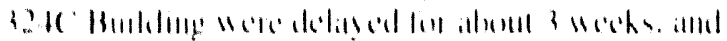
decommmon-

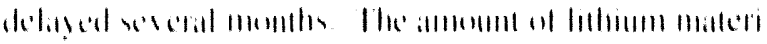

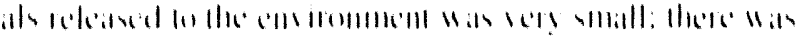
mureputialste vill 


\section{Waste Tank Leak (RL-WHC- TANKFARM-1992-0073)}

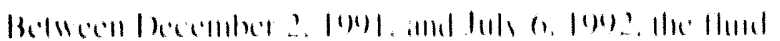

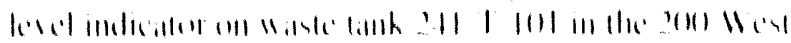

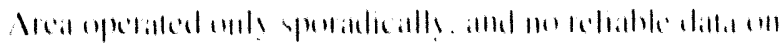

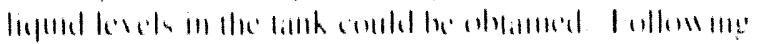

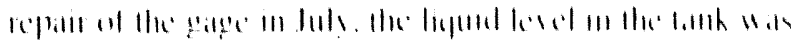

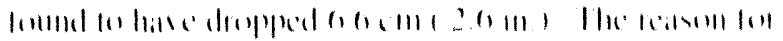

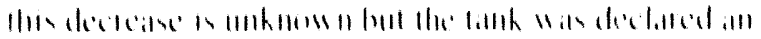

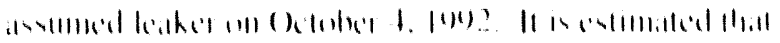

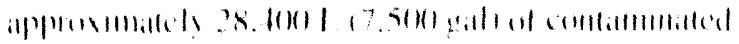

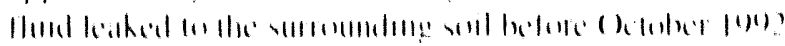

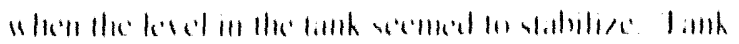

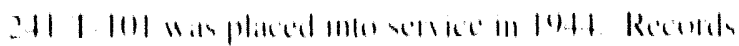

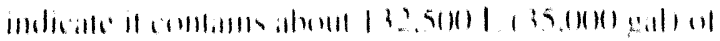

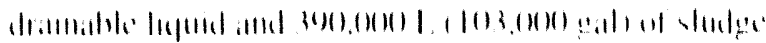

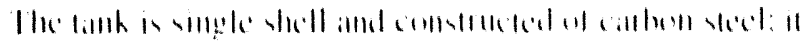

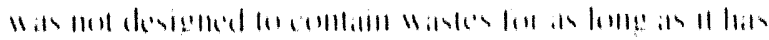

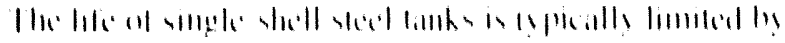

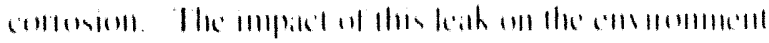

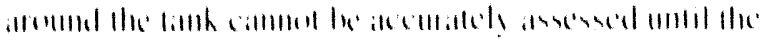

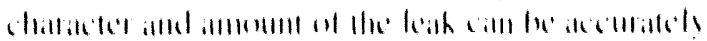

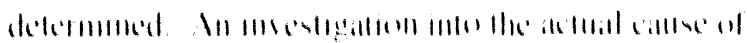

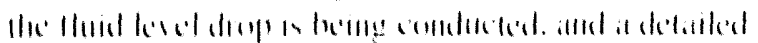

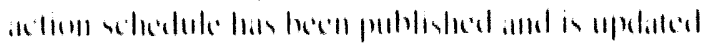

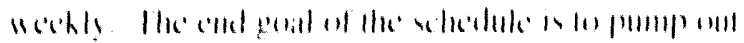

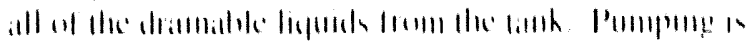

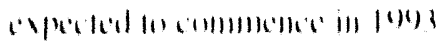

\section{Discharge to the Columbia River (RL-WHC-NREACTOR -1992-0061)}

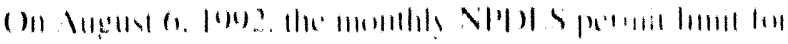

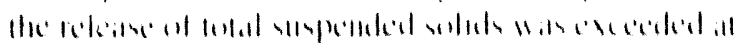

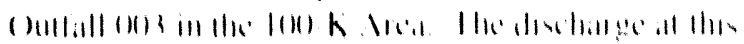

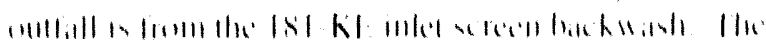

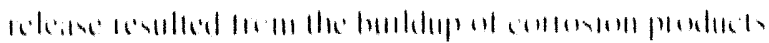

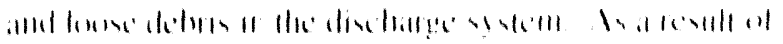

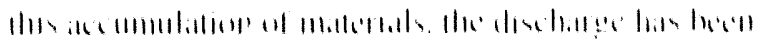

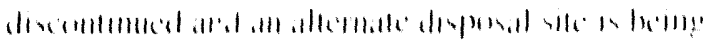
(r). llaininl

\section{Oil Spill (RL-WHC-600EM-1992-0011)}

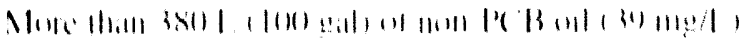

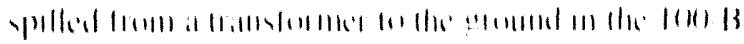

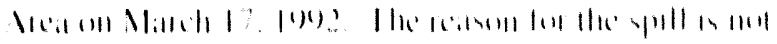

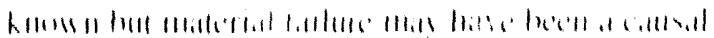

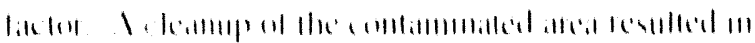

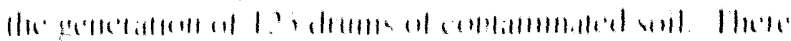

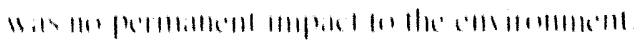

\section{Radiation Leak (RL-WHC-TANKFARM- 1992.0074)}

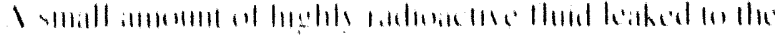

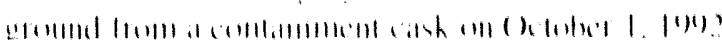

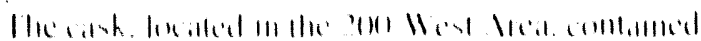

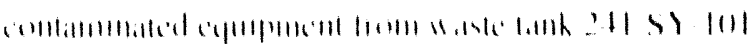

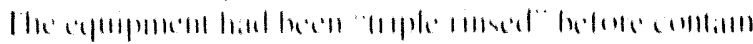

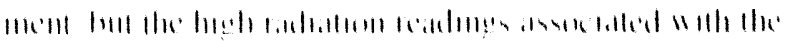

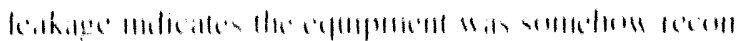

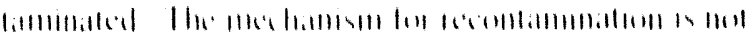

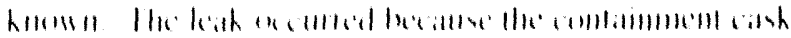

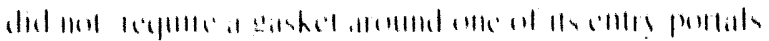

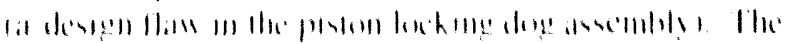

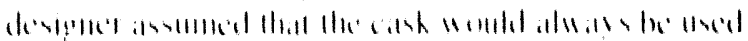

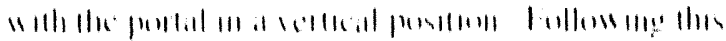

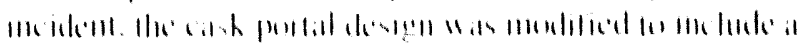

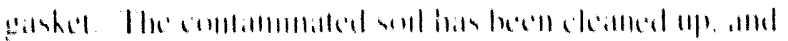

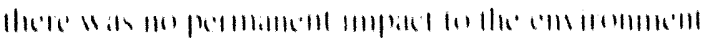

\section{Waste Oll Contaminated with Lead (RL-WHC-TPlant-1992-0018)}

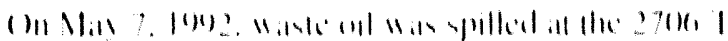

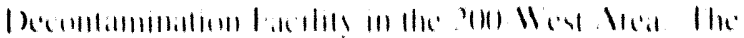

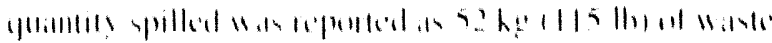

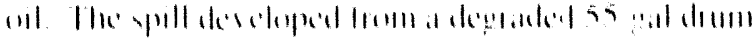

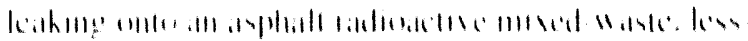

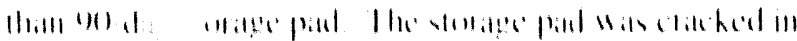

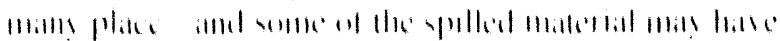

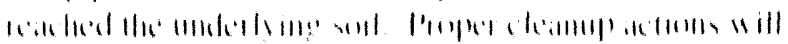

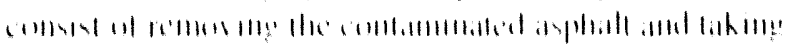

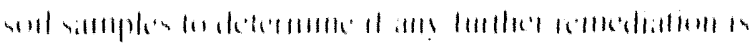

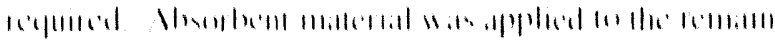

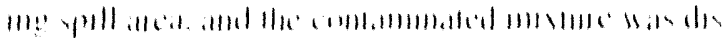

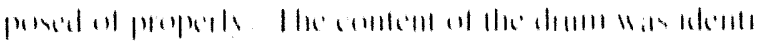

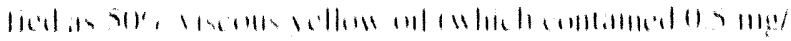

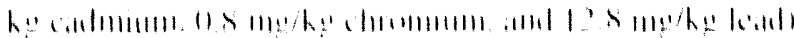

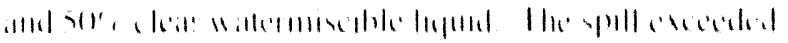

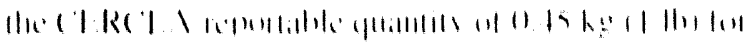

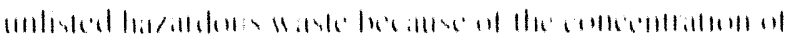

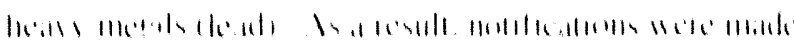

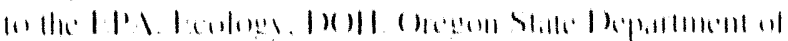

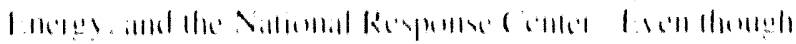

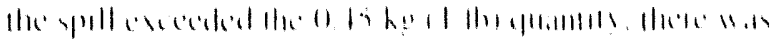

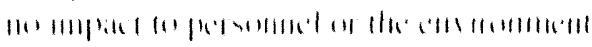




\subsection{Compliance Status Update (January 1 to April 1, 1993)}

The following summany supplemems Section 2.? 2. 10

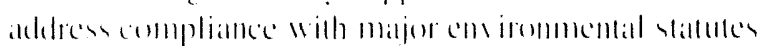

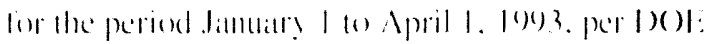
reyuirements

\section{Compliance Status}

\section{Comprehensive Environmental Response, Compensation, and Liability Act}

\section{Expedited Response Actions}

Fxpedited reyponse actioms lor the follow ing ypecilic

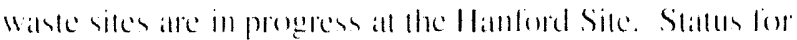

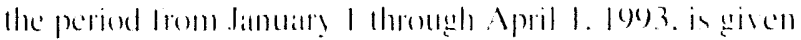
helow:

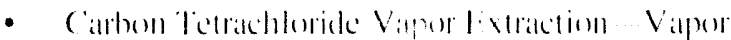
extrateden from the vadere sone heneath the

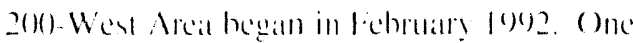
vilcuum st stem is currenty operalling 24 hours per

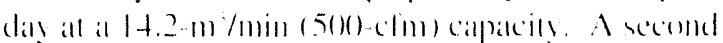

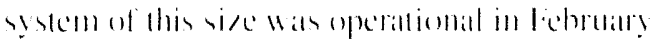

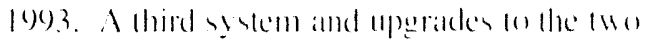

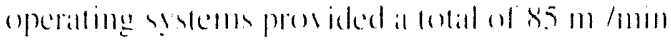

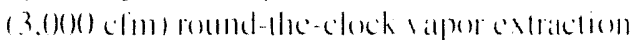

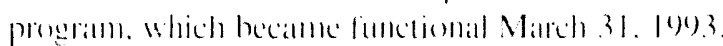

- North Slope doisponal Sile The North Slope

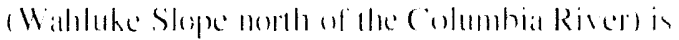
fecaled on the mortheren and castern bordere ol the

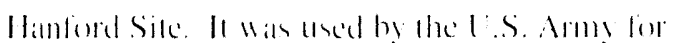

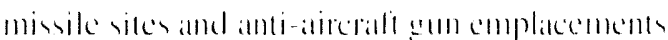

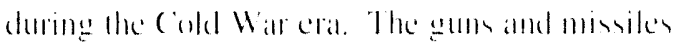

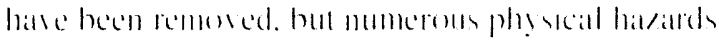

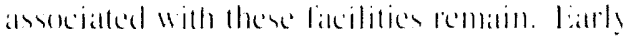

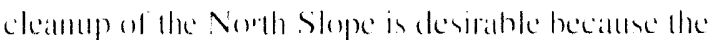

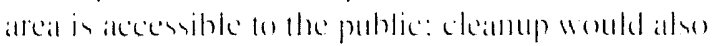

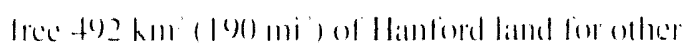

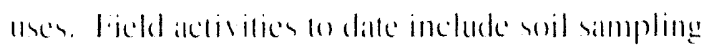
and getophysical smorys

- Pickling Acid c ribs Nitric and hydrollumerce acide

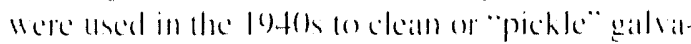
nised pipe before the pipe being used in the conn. strection of the los Areal reactors. The Pickling Acid cribs. lecalted south of the White Blutits

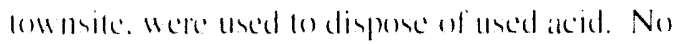

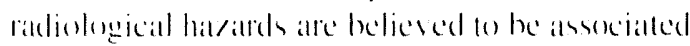

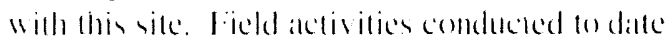

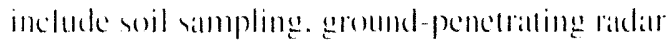
surseillance. and lest pil excalvallioms.

- Sodium Dichromale Iandlill During Hanford's carly prodection lears, sodium dichromate was

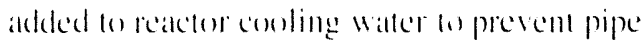

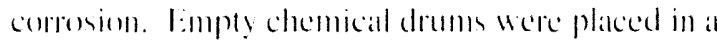
ravine and concered with soil. Comstruction debris maly also hate been disposed of all this site. In Jimuary 1993, I:PA and lacolong recommended excatration and remomal of the dehris to accomplish at record of decision. (hatraterization andivities include wit simpling and geophysicial surveys.

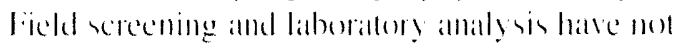
revaled anl comtamnination. ('leanne and excatratlion actisitien were completed on April 1.3. 1993.

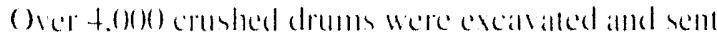

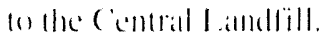

- Riverland Riverland in lecilled ini lle northuest corner of the Hantord Sile. Hest ol Highwaly $2+10$.

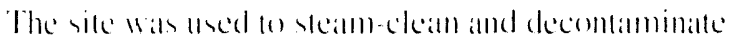

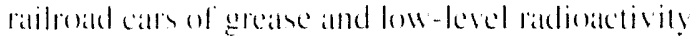
lom 19+4.3 10 1957. The area inclueles approxi malks it hom (1.3 mi of properly and contained

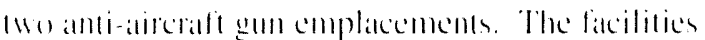
were decommissomed in 190,3. Recemt site chatracerisallon has been alceomplashed lo delermine if all! residual combamination exists that would contlice with current release criteriat. 
- 6lX-11 Burial Cirounds The $018-11$ (WYI) Burial (iround is locilled $12 \mathrm{~km}(7.5$ mi) north of the 300$)$ Areal. adjacem on Washington Public Power Supply System WNP-2. I.ow-ked. intermediate. high-level activity. and transuranic Wastes from 300 Areal research fareilities were disposed ol into trenches. caissons. and pipe storage units from 1962101967. fäcld screconing and charackerization icsults continte forompare with release criteria.

- N Springs. An conninering study is being condected and will le compleded April 30. 1993.

\section{CERCLA-Reportable Releases}

There were thee releases under the CERCi.A-reportathle quantity regutements helween dimuary I and April 1. 1093. The first spill involsed a contimuous release of carbon ketrabloticle during the solvent exaration presess at the Plutonium Reclamation Facility. The second spill involved a small amount of antifreese (ethylene glycol)

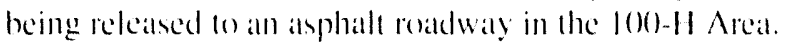
The third spill insolved a small amoum of highly concentratled PCB-contaminated oil heing released in the 1()()-I) Area. The P(B level for this spill wats below the ClRC A.A-reportable gamity. hut wats required to be reported per 40 Cl.R 761.125(c)(1)(i). Absorbent material was applied to the first and secend spills, and the contaminated mixtures were disposed of according an the proper regulations. Approprialle notilications were made to the Natlonal Response (enter, Ecology. and EPA in alcerdance with ClERC LA. There was no impatel to personnel or the enviromment.

\section{Emergency Planning and Com- munity Right-To-Know Act}

There were no new compliance isstes identified regarding the Emergency Pamming and Community Right-ToKnow Act during the period from damualy I through April I. 199.3.

\section{Resource Conservation and Recovery Act}

\section{Washington State Department of Ecology}

()n January 1.5. 1093. 1)( )1: and WHe received a compliance letter from Eeolngy. identifying violations and other
Walste storige issters all lank 241-SY-161. The violations noted include execeding the waske acemmulation limil of 120 days and compliance problems associalled with

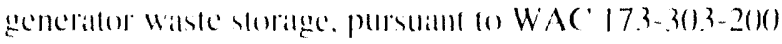
and WAC 17.3-301,3-6,30. D)( ) l has issued a formal respomse. No additional atedioms were necessalty.

\section{Hanford Part B Permit}

The dratt permit application was issted for public comment on damuany 15. 1002. (ommlonts by licology were ieceited. Responses lo leology scomments. als well ats pertinent changes. Were submitted on He for linal review. (omments bom Ho have non been received. When the Part B permil is finally issued. He Hamford Sitc lacility Permit will provide the foundation for all future RC RA permitting all I Ianlord in akeordance with provisions of the llanlend lederal Facility Agrement and Comsent (order rliri-Party Agrecment).

\section{Ground-Water Monitoring}

four wells sere comstructed for projects alsoscialded with the 20) Area lifluent Trealmemt Disposal Facility. Pursuant lo WAC 17.3-160). a regulatory valtiance on well 290-W/8-32 in the low-kevel burial grounds was issmed by ecology for noncompliance with the construction standiards.

\section{Clean Air Act}

\section{Compliance with the Revised 40 CFR 61 Emission Measurement Requirements}

() February 3. 1903. 1he Dot: and the Hantord Sile

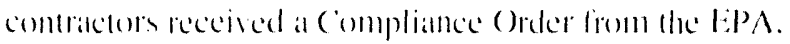
purstant lo Sections 11.3 and 114 of the ('lean Air Act.

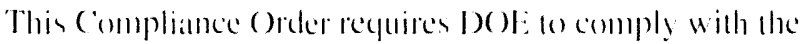
radionuclide NISSHAP hy evaluating radiontuclide emis som points at the Hanford Site and perloming continuous emission meanurememts. as specilied by fO) (TR 61.9.3. In andition. I:PA hats required hat D)( )l: prepare a writen ecompliance plan by Apsil 3(1. 1943. 10 address the requirements of the Compliance order. The compliance plan must proside schedules for implementation and mombly reporting of work completed and work planned for the following momth. BPA expects on use this schedwle as the basis lor a lederal lateility (ompliance Agree-

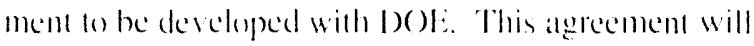
formalize the schedule of emissions atsessment and any measurement compliance actions determined necessary. 


\section{Washington State Department of Health}

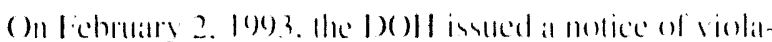

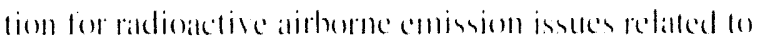

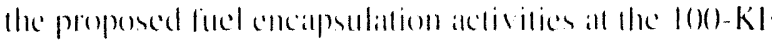

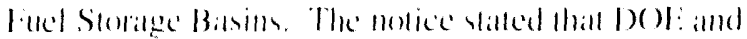

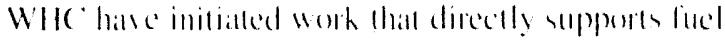

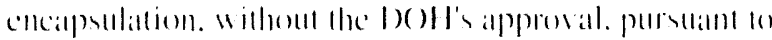

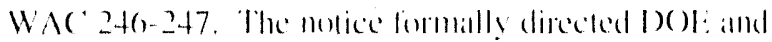

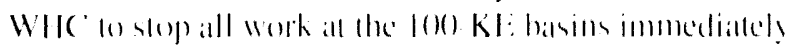

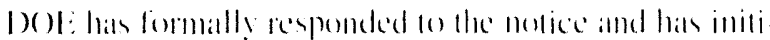

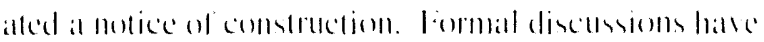

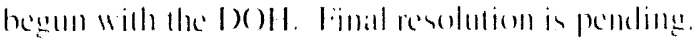

\section{Prevention of Significant Deterioration}

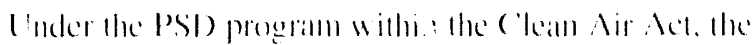

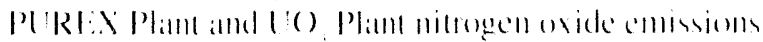

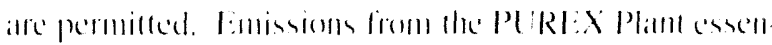

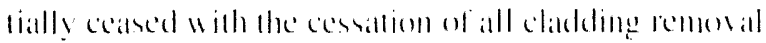

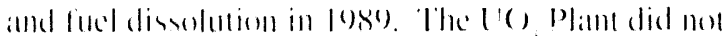

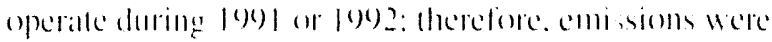

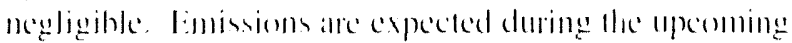

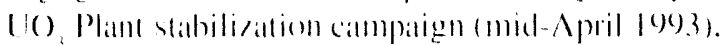
which will comsist of a precontemlattion phase and a calcination phase. The campaign is expected lo lates approximalcely I momb on complete

\section{Clean Water Act}

There were no compliance issues idembilied wilh NPI) :S permitling requirements during the perion liom Janually I through spril I. |40)3.

\section{Safe Drinking Water Act}

There Here no new compliance issters identified regarding this Aco from dombary I through April I. 1993.

\section{Toxic Substances Control Act}

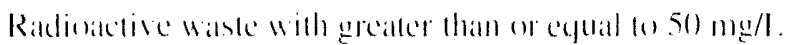
$P($ Bs is being stored all the llanford central Waste complex. Merting with the LPP hate commenced and

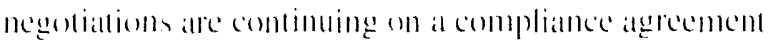

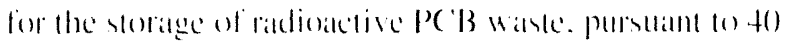

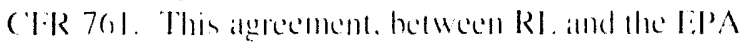

Region I0, is intended an allow the aleceptance and

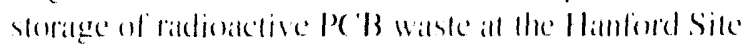

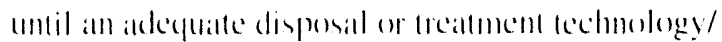

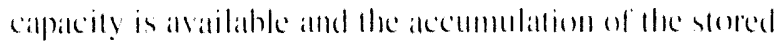
waske is eliminalled.

\section{Federal Insecticide, Fungicide, and Rodenticide Act}

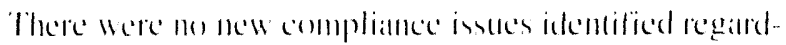

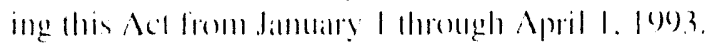

\section{Endangered Species Act}

There were no new eompliance isstes idembified regard-

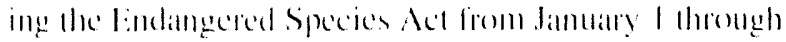
April 1. 1093.

\section{National Historic Preservation Act}

There were mo new enmpliance inster identified reganding Une Nattomal Historic Preservatton Act from Jamuary I hromgh April 1. |093.

\section{Current Issues and Actions}

\section{Liquid Effluent Consent Order}

(imsent ()reler D) I: 1 NM-177 wass signed December 23. I09). hy RL. and fochogy regulating Hanford Site liguid effluent discharges. This comsent order contations compliance milestomes for Hanford licpuid eflluent streams designated as Phase l. Phase Il. and miscellaneons streams. In alceordante with kerims of the consemt oreler. simpling and andalysis plans have been submitted to

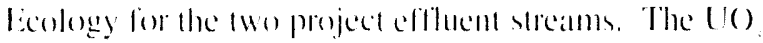
Plant process comblemsile to the 216-17-17 Crib. the (10) Plant wastewater to the 216-1-1+1)itch, the PlP wasteWalker. and $4(x)$ Areal secondars conling walle simpling and analysis plams hate heell approwed hy lacology. In

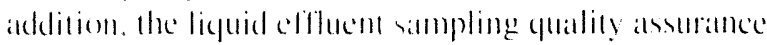
projecl plan was alp proved by lacology. 


\section{Hanford Federal Facility Agree- ment and Consent Order}

The follow ing is a summatry of ale more signilic:ant

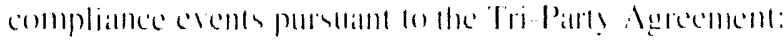

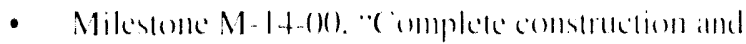

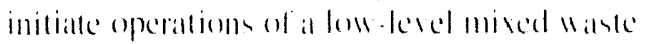

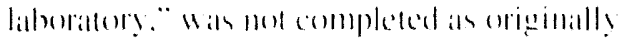

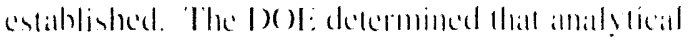
needs all the Hanford site would be heller sallistied

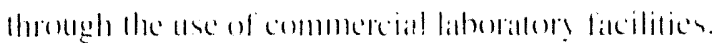
Dispute resolution was entered as prow ided by the Tri-lants Agrecoment. A linal resolutum uals reached on dimnany 11. 1993. which inclucked an agerement on ase lecally prosided commercial

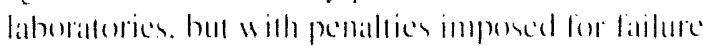
lo comply with the Tri-l'arly Agrecment.

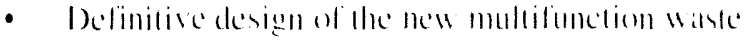
lank latcility wats initialled.

- Improved in-tank monitoring. a revised contingens? plan lor leaks alld all physical preparations lion ensergency pumping of licyuids were implemesned lor single-shell lank T- $\mid(1)$.

- Comstraction of the Hanford Waste Vitrilicallom Pland (aniscer Slorige Building/Multipurpore Stomege Building was initialed.

- A drall RCRA closure plan for the l(0) 1) pond was completed and submitled on the J:PA and licologey.

- () April 2. 1993. RI. invoked dispule resolution lior the solid thaste designation compliallece isste originated by foolegy s Nolice of Penally

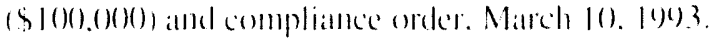
Approximaltely 2.0000 contatiners of solid waste were all alleged redoltion of WAC 17.3-3013-17001 1)allated the implementition of WA $17.3 .3013-1) 70$. ().

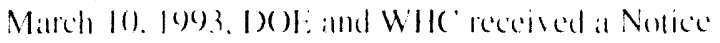

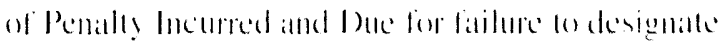
these solid waskes at dangeroms of extremely

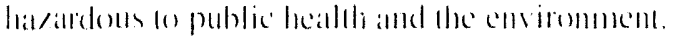

\section{Federal Facility Compliance Agreement}

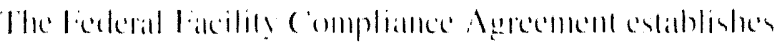

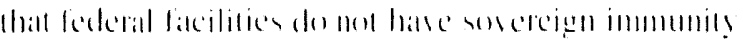

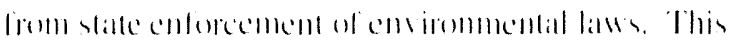

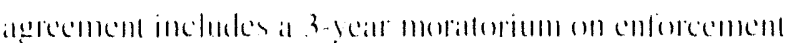

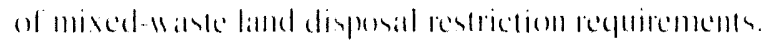

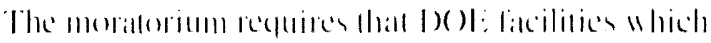

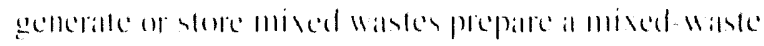

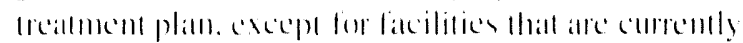

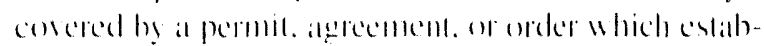

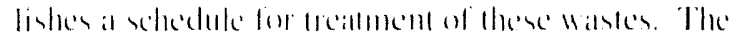

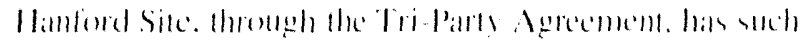

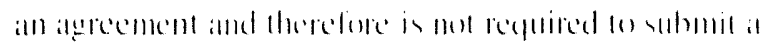

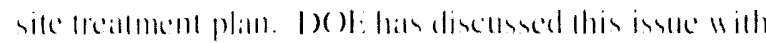

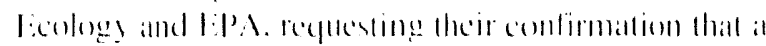

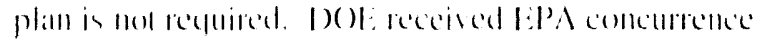

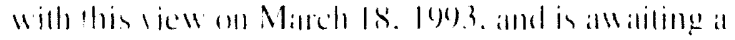
respense form ficology.

\section{Waste Minimization}

The ISA Bicmnial Waste Minimization Repont 4 an completed in March 10)2. RI. issted guidante uncrealle semiannual pregerese reports in stpporet of implementing

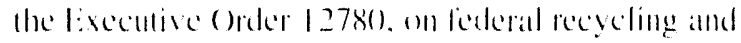
adfirmative procurement programs. The first progesess report was completed on December 15. 1092.

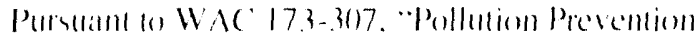
Panning." a repont on hatratrdous waste generaltion and

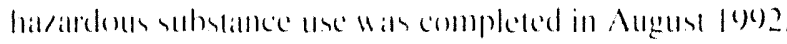

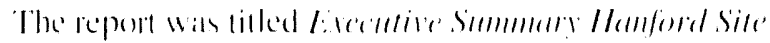

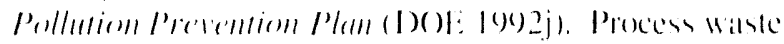
assessmemts ance continumg in 1093.

\section{Tiger Team Update}

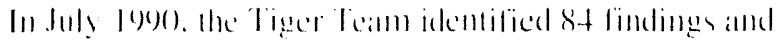

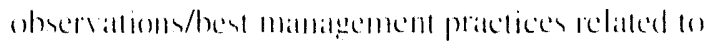

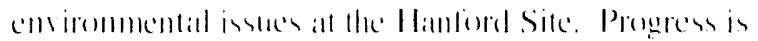
beime mate an ale completion of corrective actioms

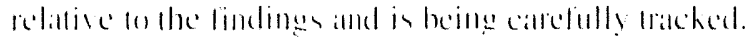

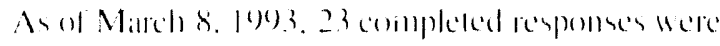

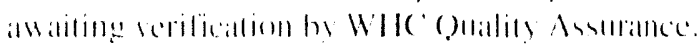




\section{Hanford Waste Vitrification Plant}

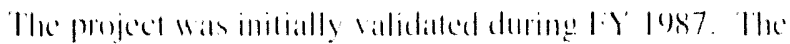

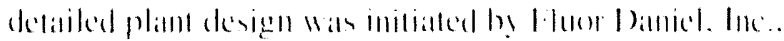

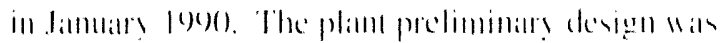

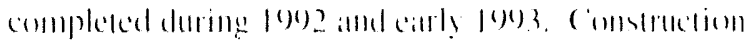

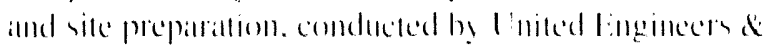

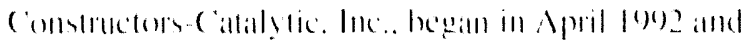

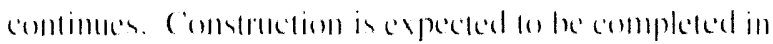

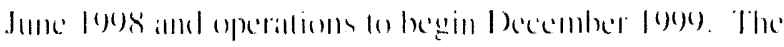

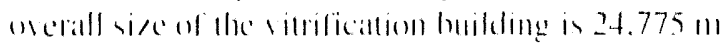
$120(1)+40(1) 11$ 1.

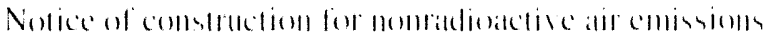

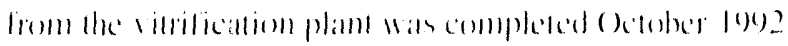

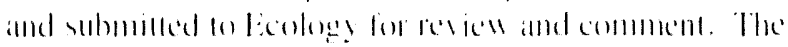

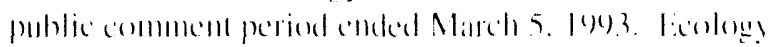

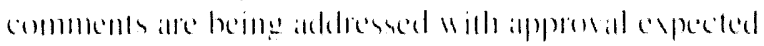
hy milleart.

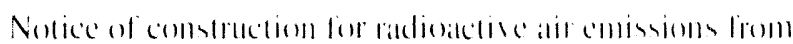

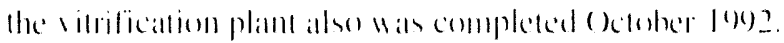

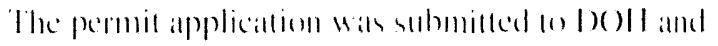
appromal is expected catrly foc)?.

\section{Hanford Site Waste Safety Issues}

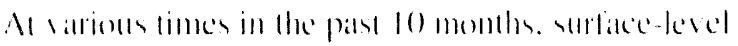

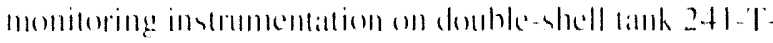
10) has shown unexpected flectuation in waste surlate

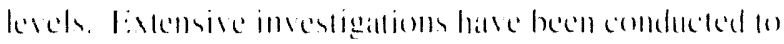

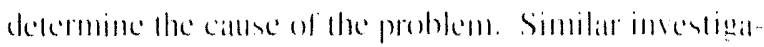

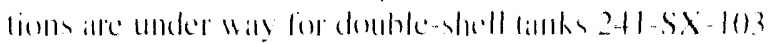
and $2+1-5 x-105$

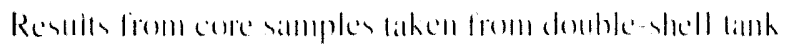

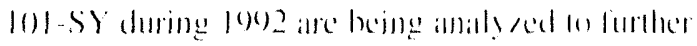

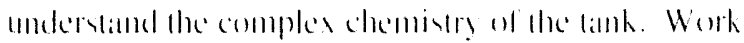
Was iniliated fo develop a mixer pump. Which will he installed mid le93. The miser pump will support eflorts

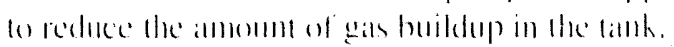

\section{International Environmental Institute}

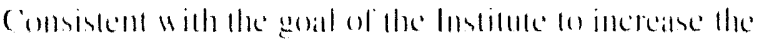

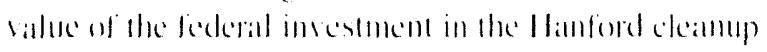
mision through expedited exchange and shatring on

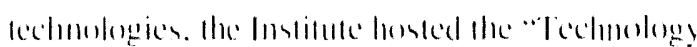

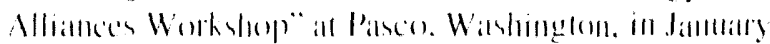

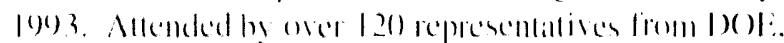

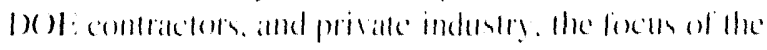

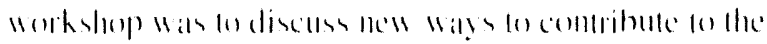

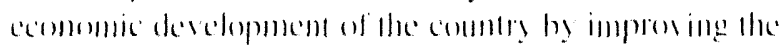

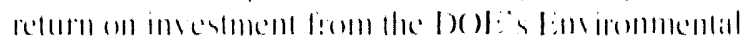

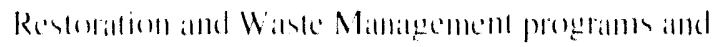

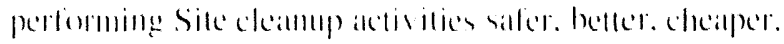
allal firster.

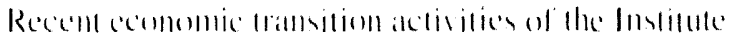

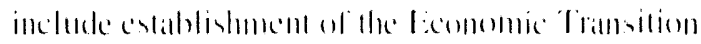

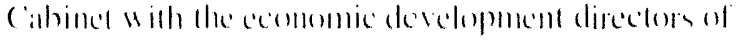

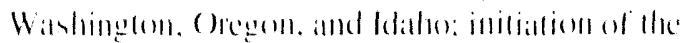

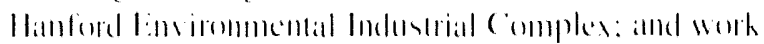

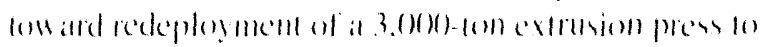

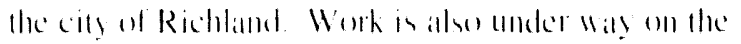

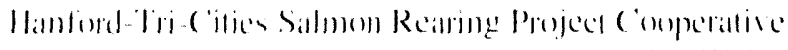

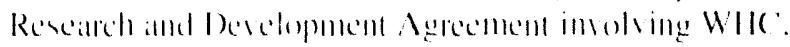

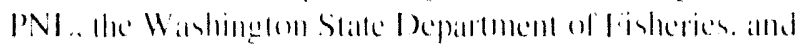

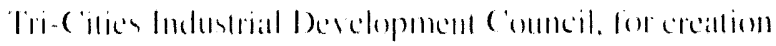

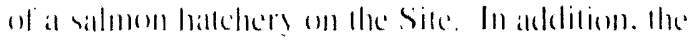

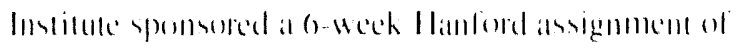

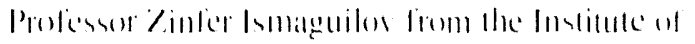

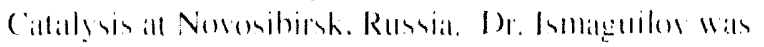

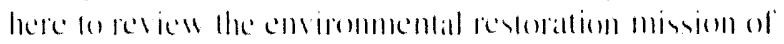

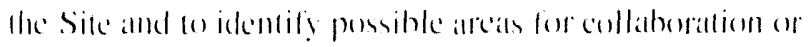

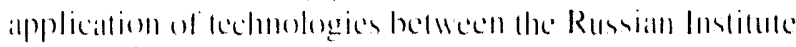
ind Hanfurd.

\section{Russian - U.S. Environmental Restoration Workshop}

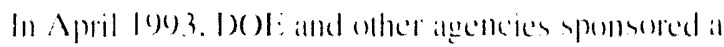

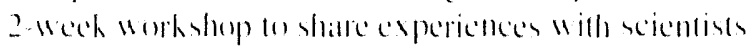
and decisiommaters fom instifules asserialed will

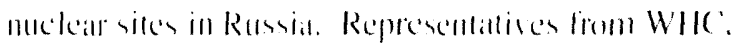

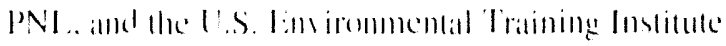

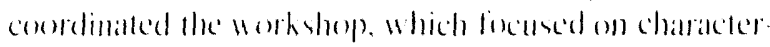

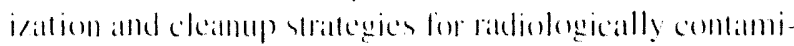
nated siles, A delegation of high-kevel olliciats from Russial parlicipalled. The firs weet was held in Wasls-

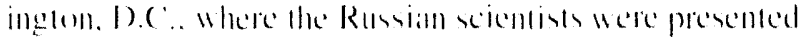

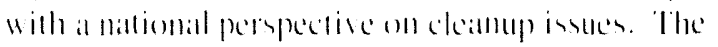

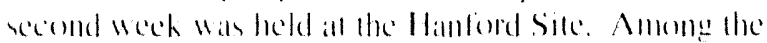

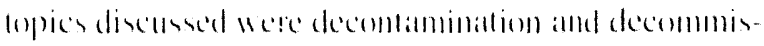

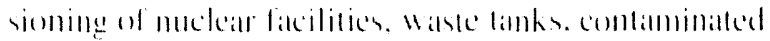

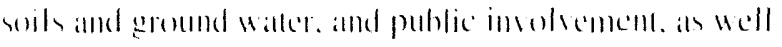
as associated envirommental lectunologies. 


\section{Effluent Monitoring Information}

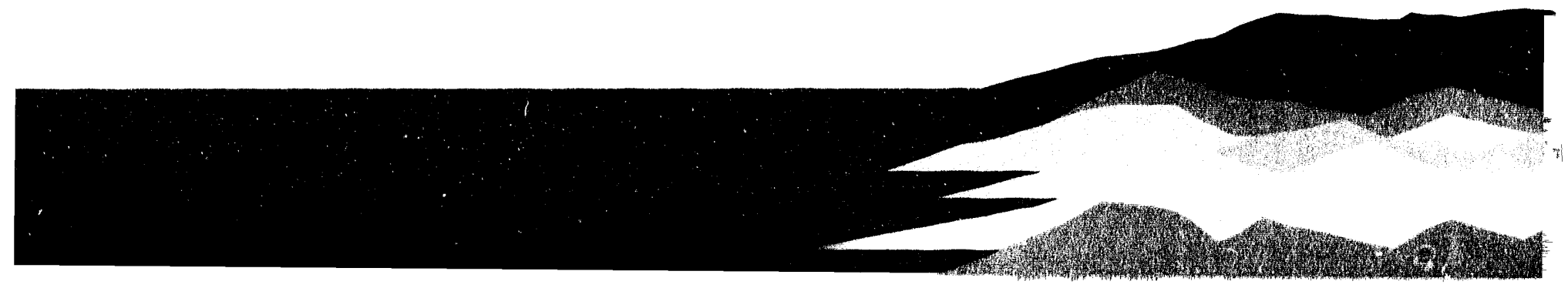




\subsection{Effluent Monitoring Information}

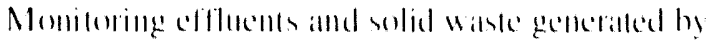

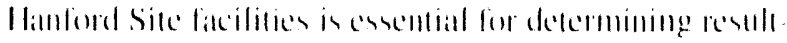

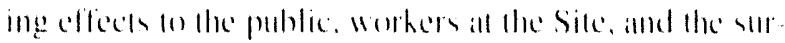

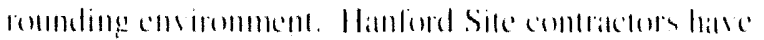

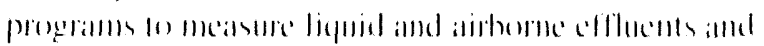

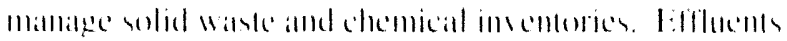

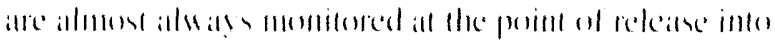

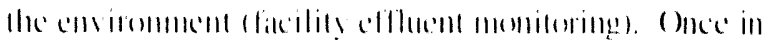

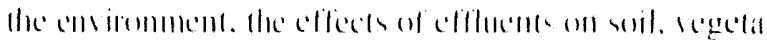

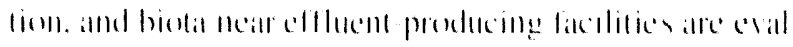

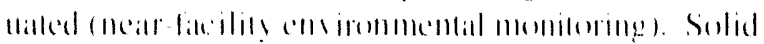

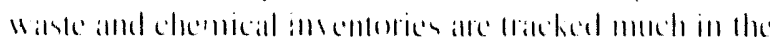

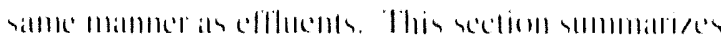

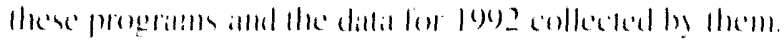




\subsection{Facility Effluent Monitoring}

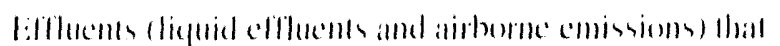

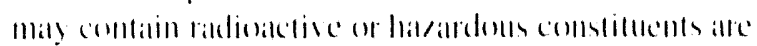
combinmally momilemed when releared all lle Hantord Site.

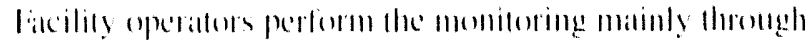
allaly /ing samples collected neall points of release into

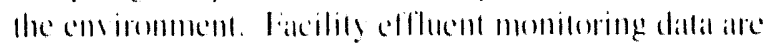

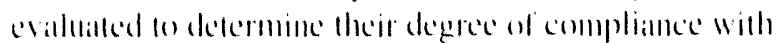
alpplicable federal, statce and local regulations and per

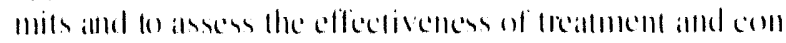

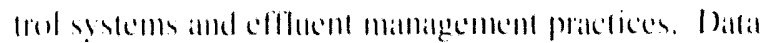

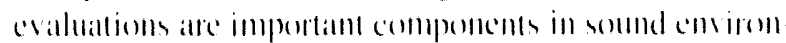

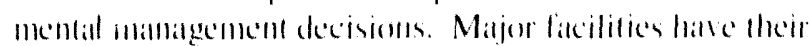

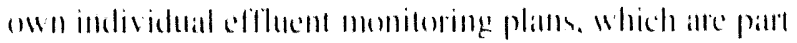
of the comprelensive Site envirommental monitoring

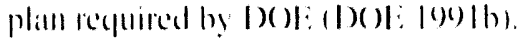

Measuring devices yuantify most fiabily effleme flows. with a smaller number of flows callewlined asing process inlormation. I iquid and airhorne elfluents with a polen

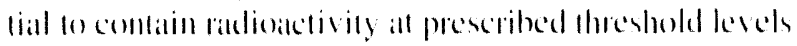
are monitored for total alphat and tolal belat activily and.

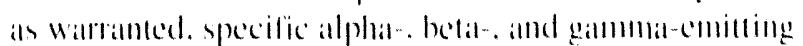

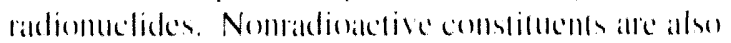
monitored. an inplicible.

The majority of radionedive elfluents fom facilities at the Site atre alpproathing levels pratedially indistinguishat-

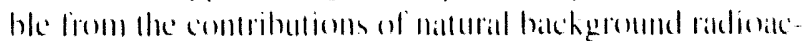

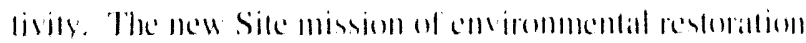

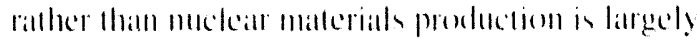

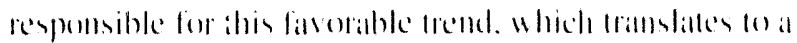
vers small offsite radiation dose clfect illributable w

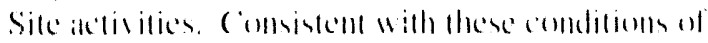

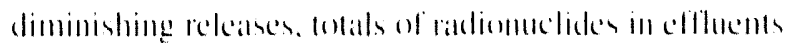
released all the Site in 1992) are not significintly diflerent

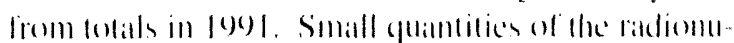

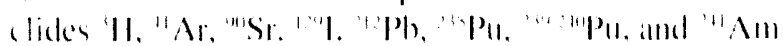
were present in airburne and liguid dischatrges during

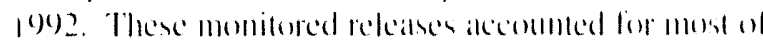

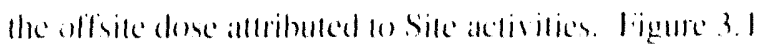

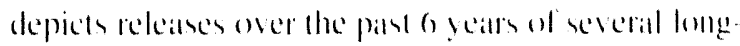

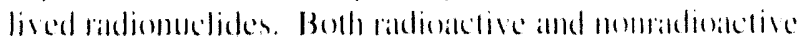

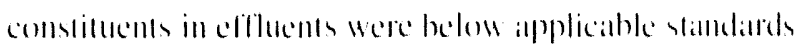
in $1(1)$ ?

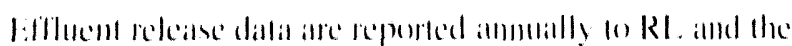

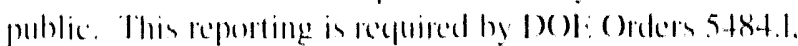

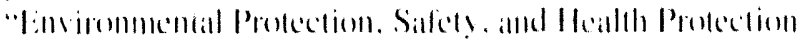

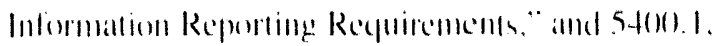

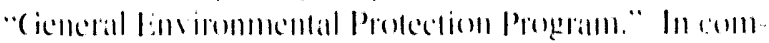

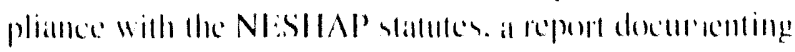

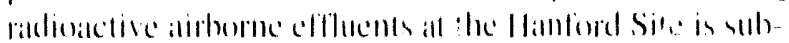

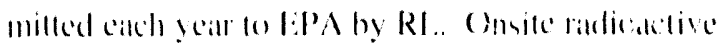

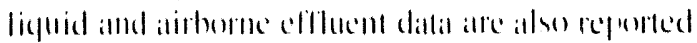

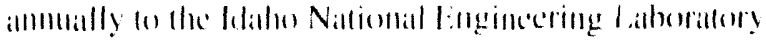

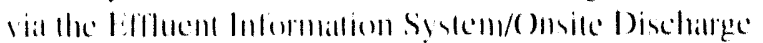

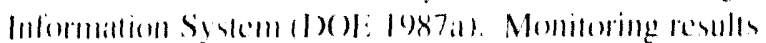

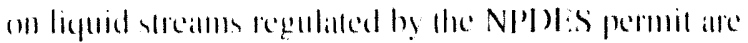

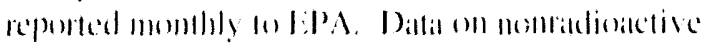

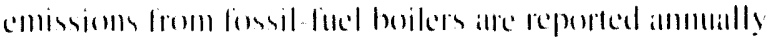
to the Bonton-1 tanklin-Wallal Wallal comntion sil Pollution comtrol Aullorily.

\section{Airborne Emissions}

\section{Radioactive Airborne Emissions}

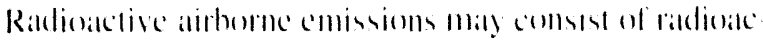

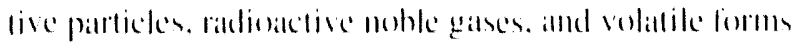

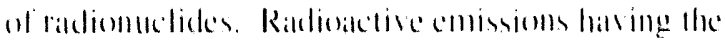

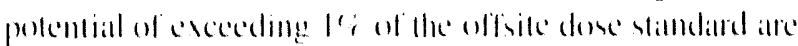
contintums) munitored.

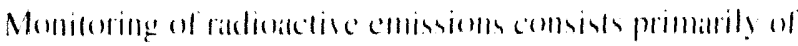
allaly sing samples comtimmons collected before the

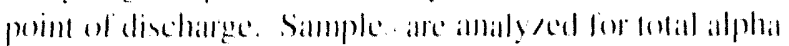

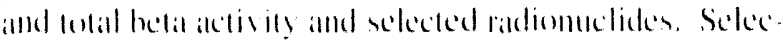
fioll of the yeceilic ratliomeclides that will be simpledel. allaly sed. and reported is based on 1 ) all evallattion ol

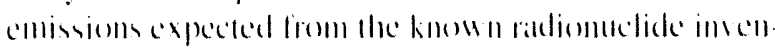

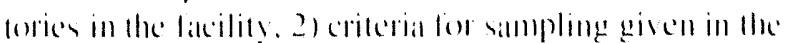

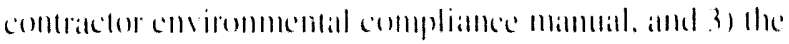
potential contribution to dle offite dese recened by

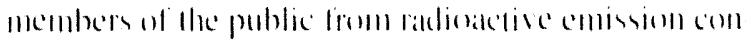
stllemts. Continturus radiatlon monitoring systems alle used all certain discharge poimts lo the ems iromment when 

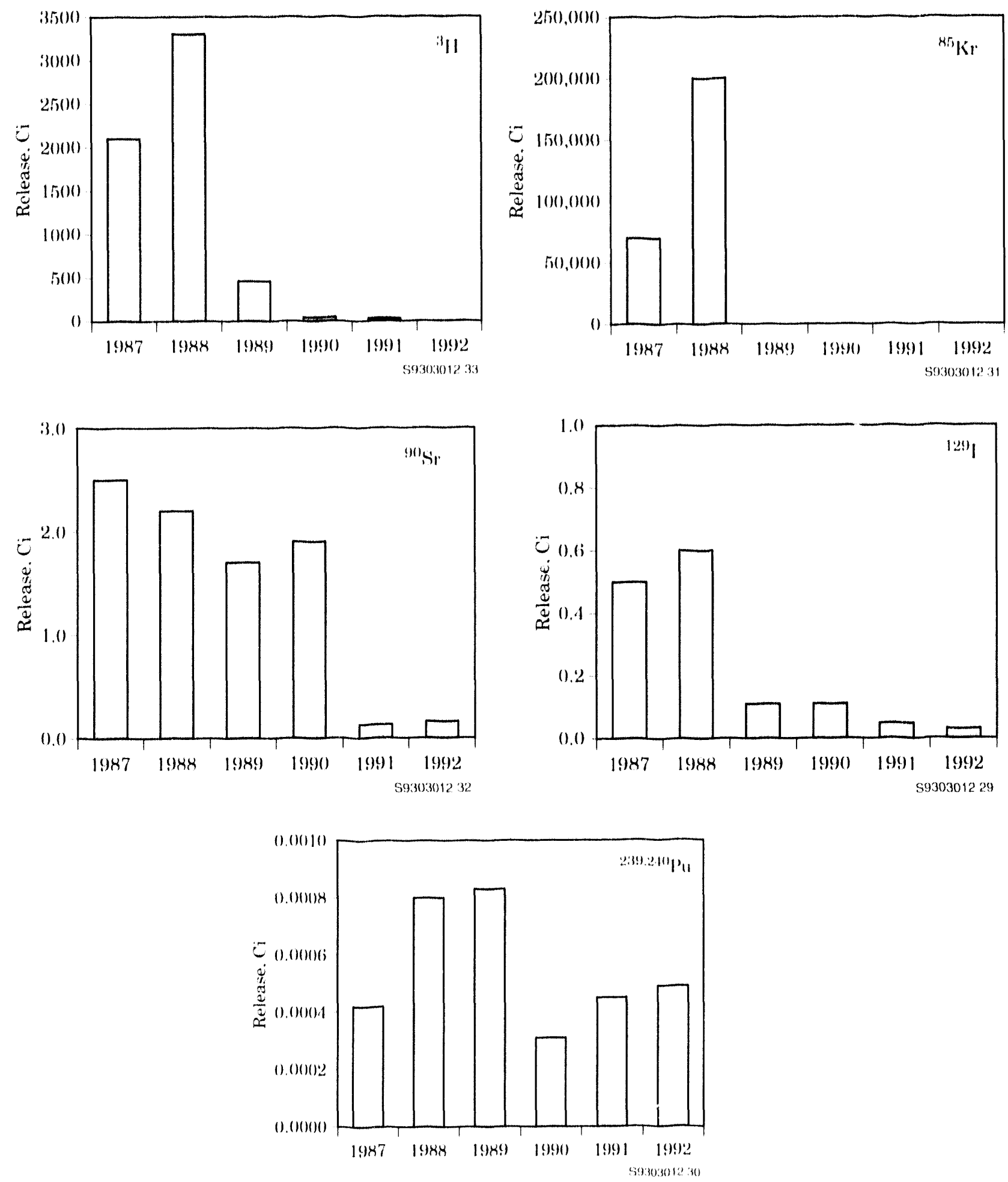

Figure 3.1. Airborne Releases of Selected Radionuclides to the Atmosphere, 1987 Through 1992. Concentrations of some radionuclides have been very low over the last few years and appear to be zero (no bar) on the graphs. 


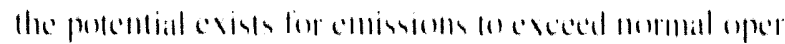

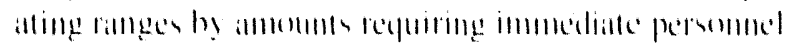
illert.

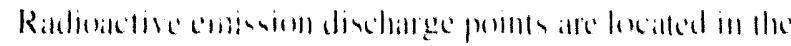

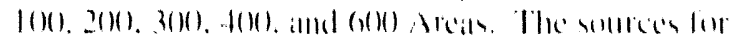

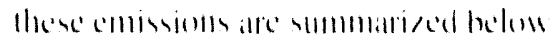

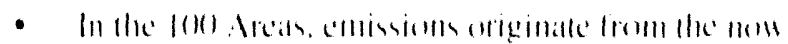

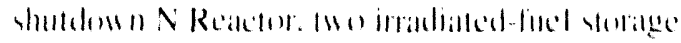

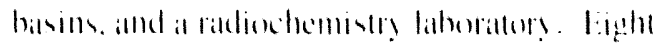

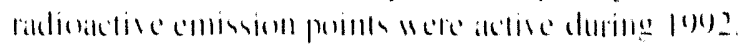

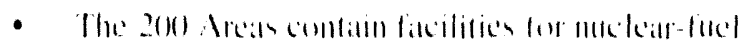

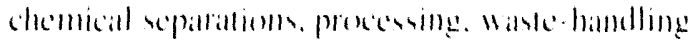

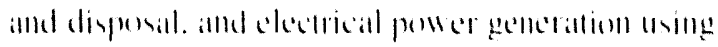

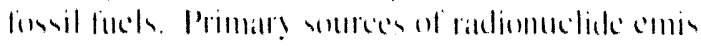

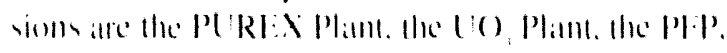

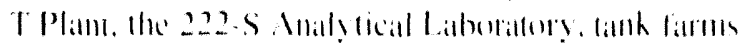

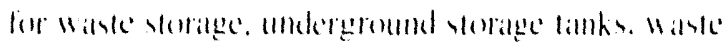

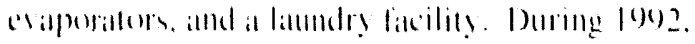

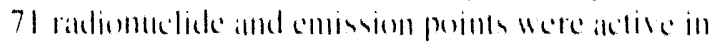
the 2010 Arean.

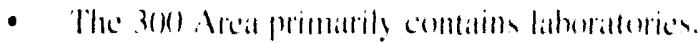

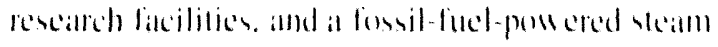

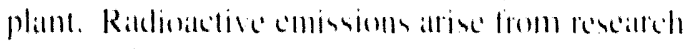
and development and wask-handling activitices

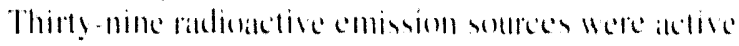

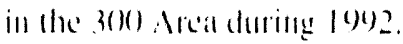

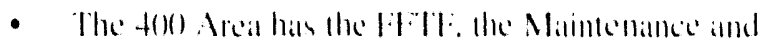

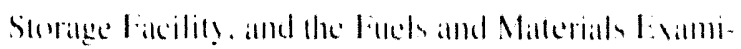

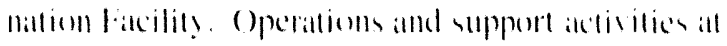

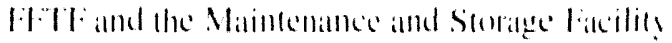

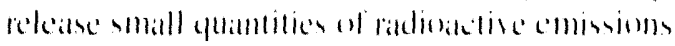

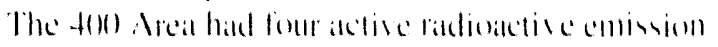
mules during lous.

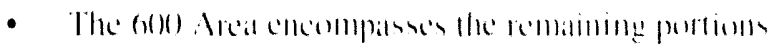

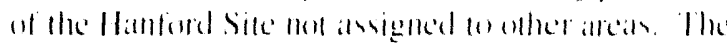

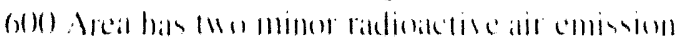
prints.

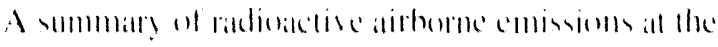
Hanlord sile in 1092 in given in tible 3.1.

\section{Nonradioactive Airborne Emissions}

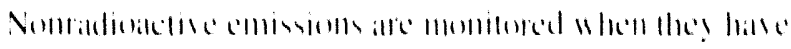

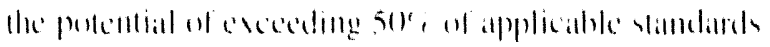

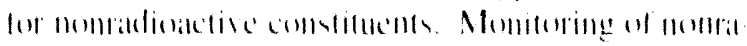

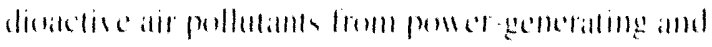

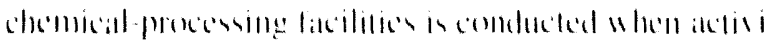

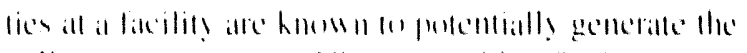

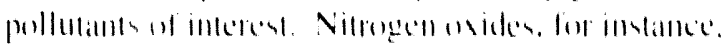

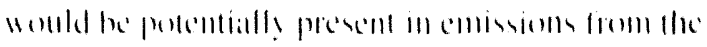

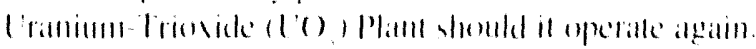

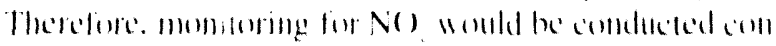

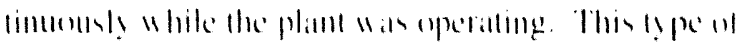

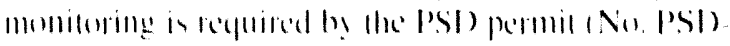

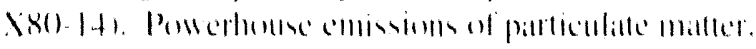

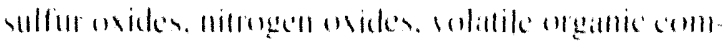

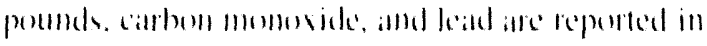

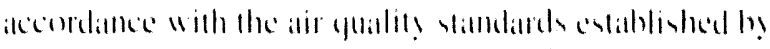

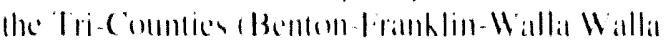

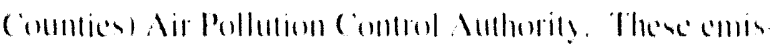

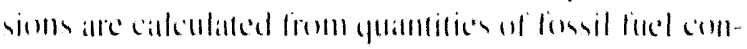

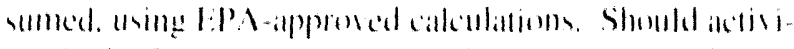

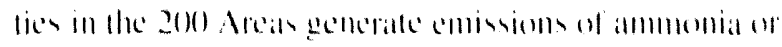

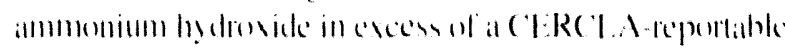

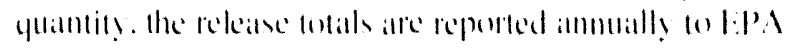

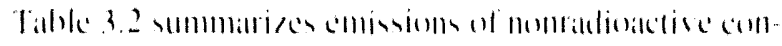

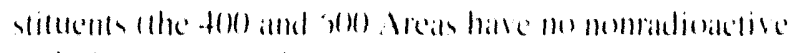

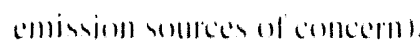

\section{Liquid Effluents}

\section{Radioactive Liquid Effluents}

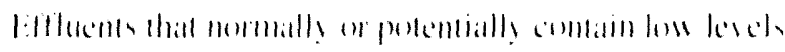

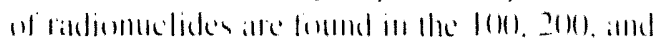

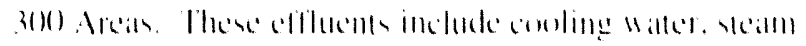

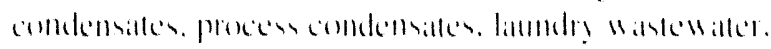

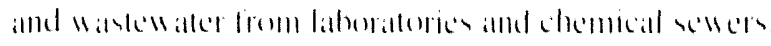

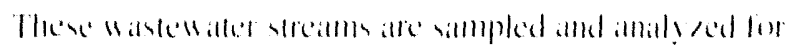

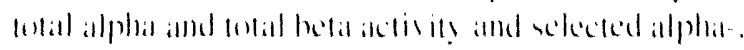

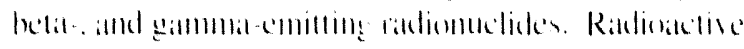

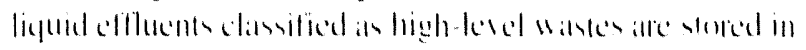

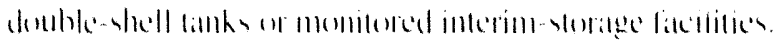


Table 3.1. Radionuclides Discharged to the Atmosphere, 1992

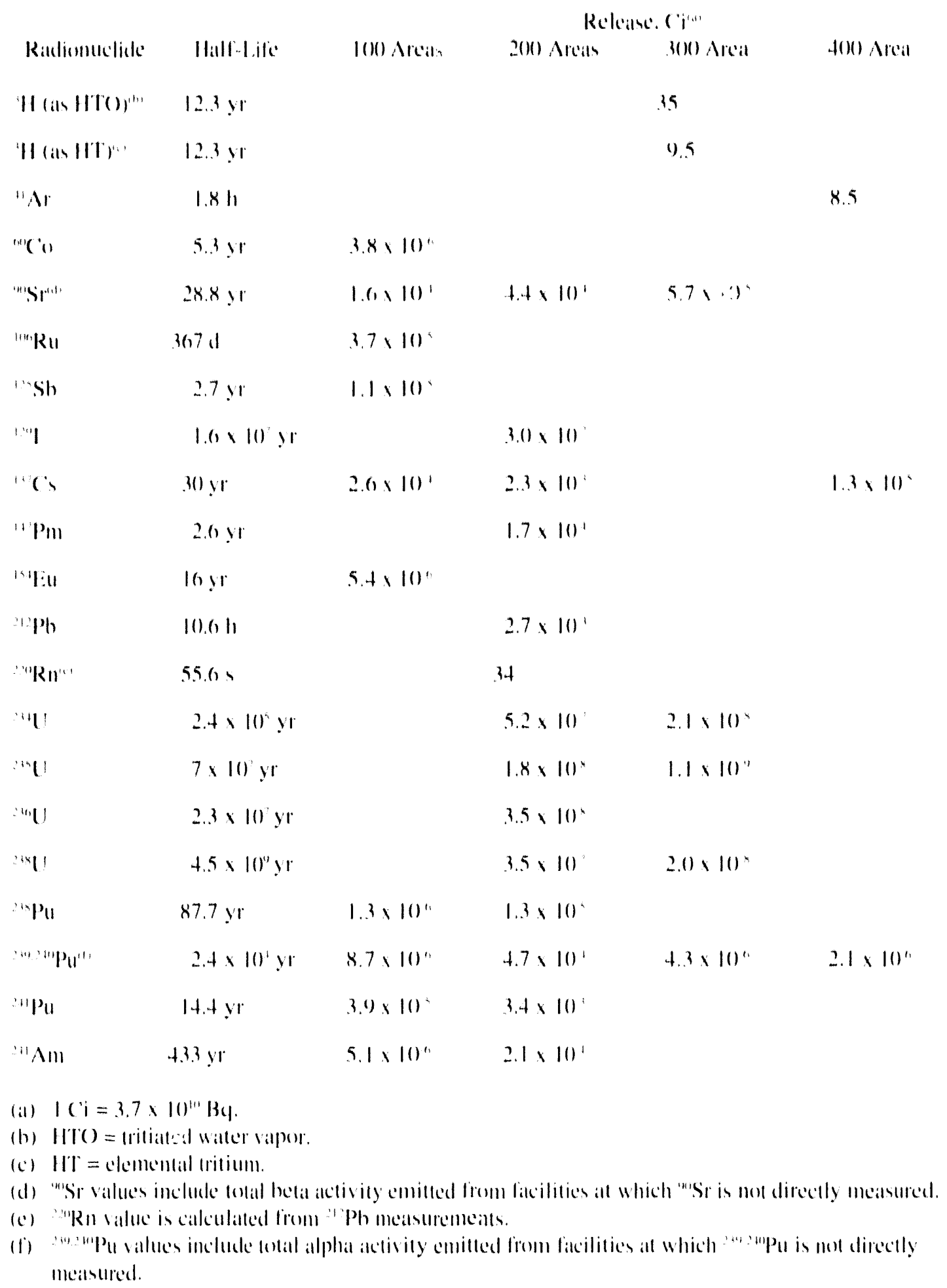


Table 3.2. Nonradioactive Constituents Discharged to the Atmosphere. 1992

\begin{tabular}{|c|c|c|}
\hline \multirow[b]{2}{*}{ ('ombluturdn } & \multicolumn{2}{|c|}{ Rol'tiat: $k !$} \\
\hline & 2011 Nrias & 3110) Ariat \\
\hline P'intriculitle milller & $7.11,111$ & 37,10 \\
\hline Nillogellowiles & $12 \times 110$ & $5.1 \times 11^{i}$ \\
\hline Sullur miden & 4.2111 & 25110 \\
\hline C:Hbun memenside & $111 \times 111$ & 10.101 \\
\hline 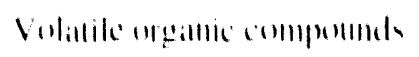 & $1.5,111$ & 20,10 \\
\hline Ammmenial & $7.6,111$ & 11.11 \\
\hline lentel & $2.7 \times 111$ & 3.19 \\
\hline
\end{tabular}

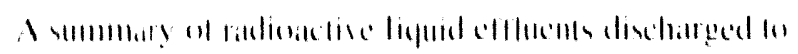

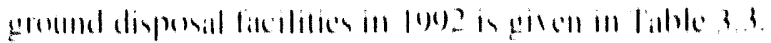

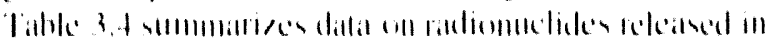

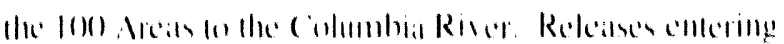

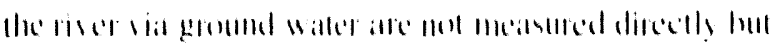

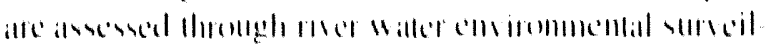

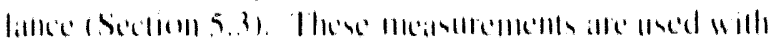

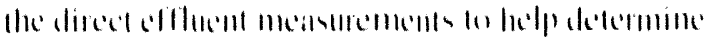
pelcollial public dones.

\section{Nonradioactive Hazardous Constituents in Liquid Effluents}

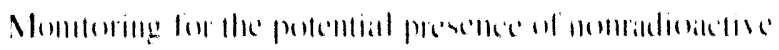

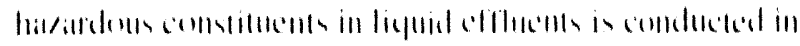

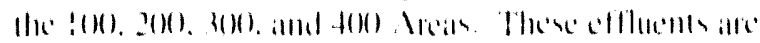

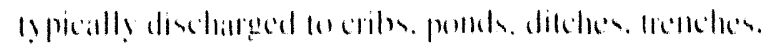

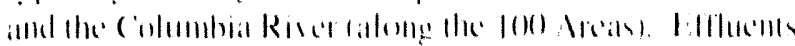

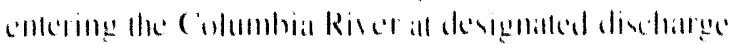

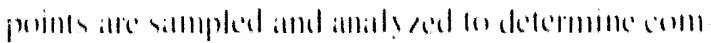

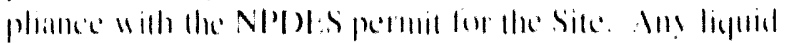

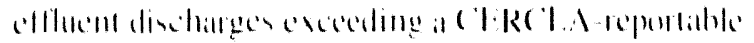

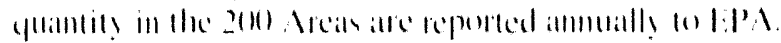

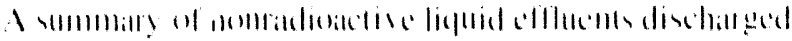

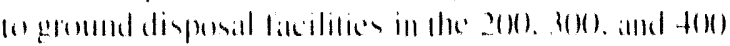
Arein in lege is given in lithle 3.5

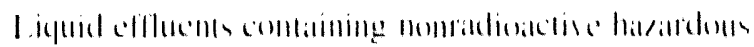

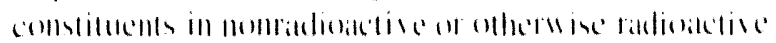

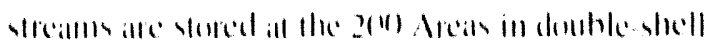

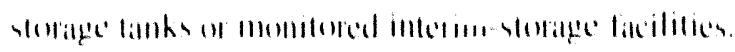
Activilies in the (1)

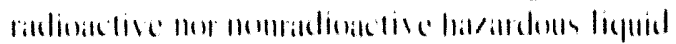
clllicills

\section{Chemical Releases}

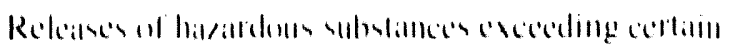

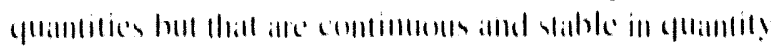

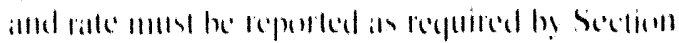

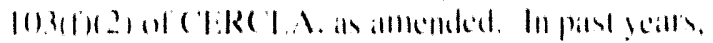

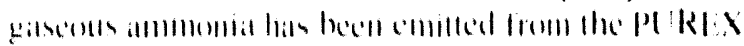

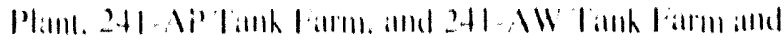

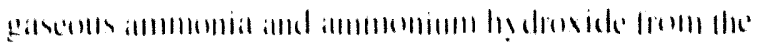

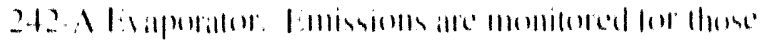

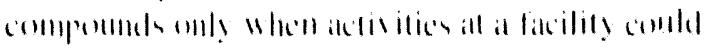

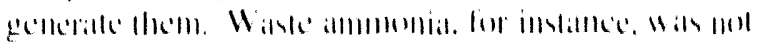

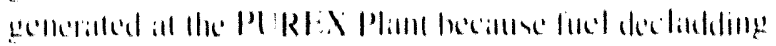

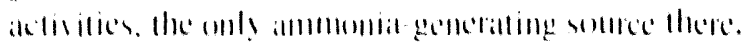

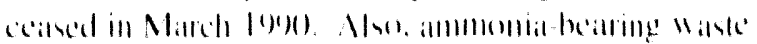

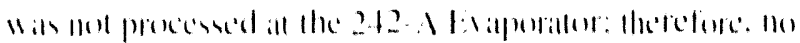

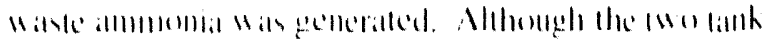

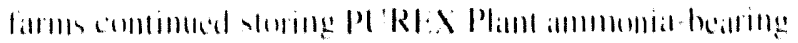

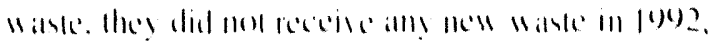

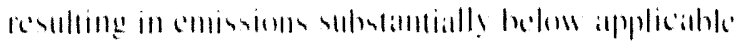
repurtahle gutmotiter. 
Table 3.3. Radionuclides in Liquid Effluents Discharged to Ground Disposal Facilities, 1992

Releiser, $\mathrm{C}^{\prime}$

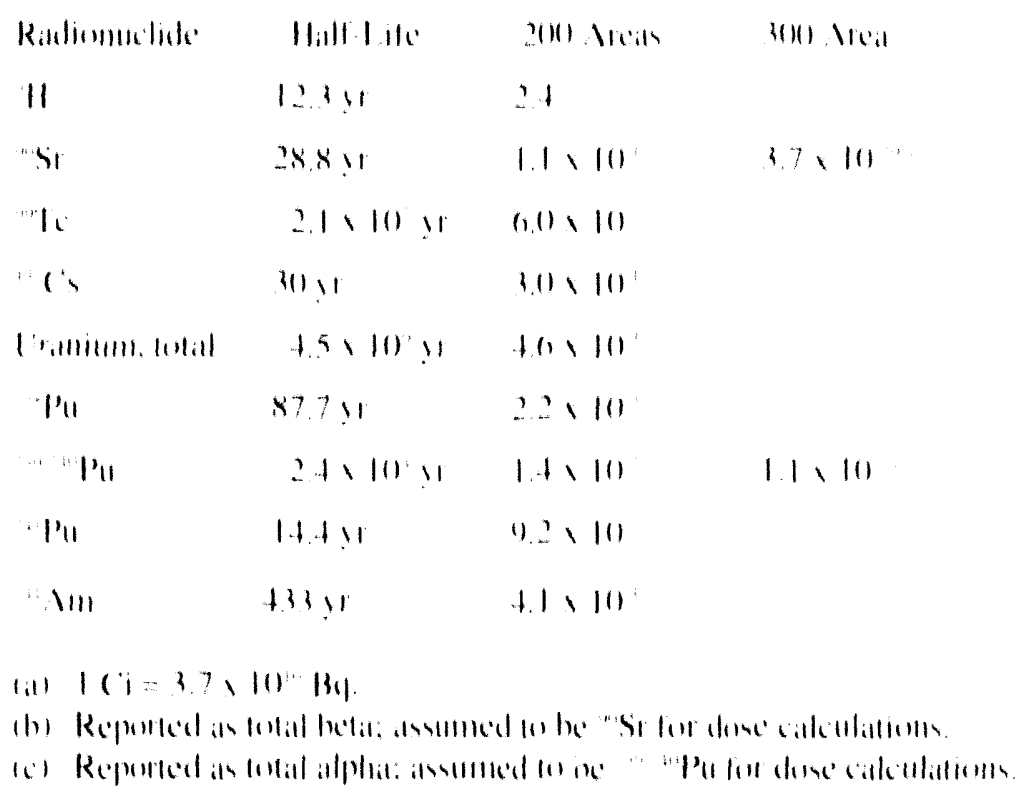

Table 3.4. Radionuclides in Liquid Effluents Discharged to the Columbia River from the 100 Areas, 1992

Rilliomuclisk

\begin{tabular}{|c|c|c|}
\hline 11 & 12.111 & 1 \\
\hline (i) & 5.3 .11 & 46111 \\
\hline "Sir & $28.5 \div$ & 10110 \\
\hline "k॥ & 30711 & $8.3>111$ \\
\hline $\mathrm{Sh}$ & $2.7 ! 1$ & $5+111$ \\
\hline 15 & $31 ! 3$ & $4.5,111$ \\
\hline$l^{\prime \prime \prime}$ & 87.711 & $29 \times 111$ \\
\hline N" & $24 \times 111 \div$ & 1.5111 \\
\hline
\end{tabular}

(a) $103=3.7 \times 10 \mathrm{By}$.
Table 3.5. Nonradioactive Liquid Effluents

Discharged to Ground Disposal Facilities. 1992

\begin{tabular}{|c|c|c|c|c|}
\hline \multirow{3}{*}{ 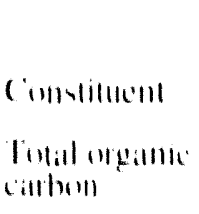 } & \multicolumn{4}{|c|}{ Roleiser ( } \\
\hline & 2010 Nroas & अ11 \ra: & $41111:$ & Aria \\
\hline & 50,110 & & & \\
\hline Nillilles & 11,111 & & 5.1 & $110^{\circ}$ \\
\hline Nitriles & & $3,3,111$ & $\therefore 2$ & $\sqrt{111}$ \\
\hline Sultalles & & $11 \times 111$ & 2.1 & 110 \\
\hline 1.luntile & & $1.8 \times 111$ & 1.1 & 111 \\
\hline ('inpler & & 12 & 5.5 & $110:$ \\
\hline 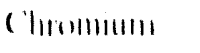 & & 13 & $x+1$ & $\sqrt{10}$ \\
\hline leat & & 35 & $\therefore 6$ & $1111^{:}$ \\
\hline Cithlminmm & & 12 & 1,1 & $\sqrt{111}$ \\
\hline Silser & & 11.8 & & \\
\hline chlinme' & & $1.1,10^{\circ}$ & 031 & 1111 \\
\hline
\end{tabular}




\subsection{Near-Facility Environmental Monitoring}

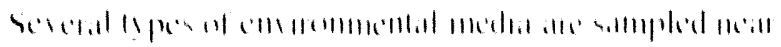

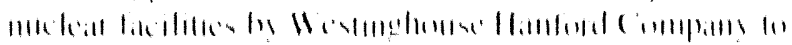

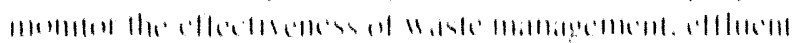

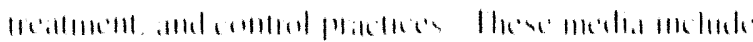

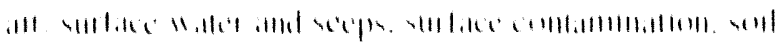

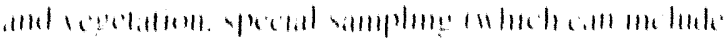

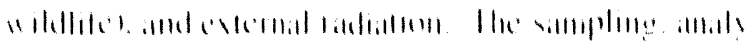

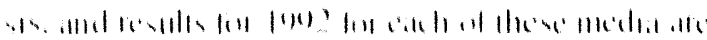

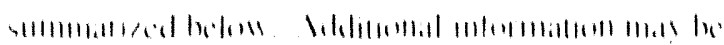

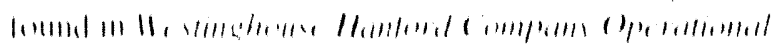

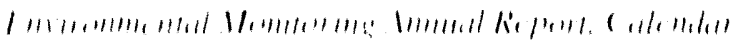

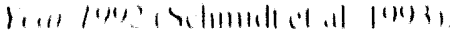

\section{Near-Facility Environmental Monitoring at Hanford}

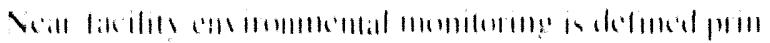

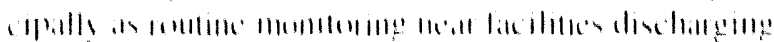

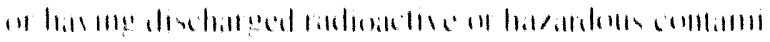

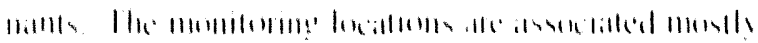

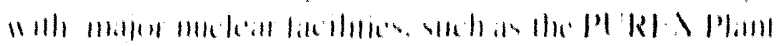

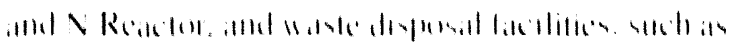

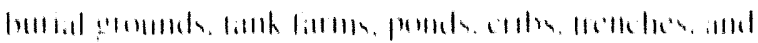
Mlline

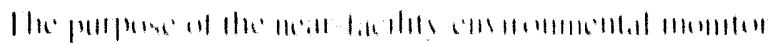

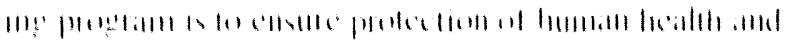

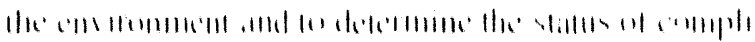

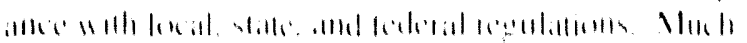

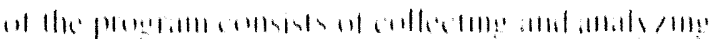

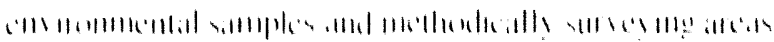

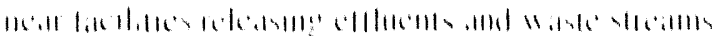

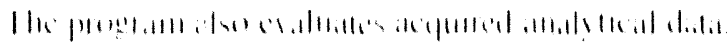

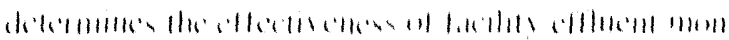

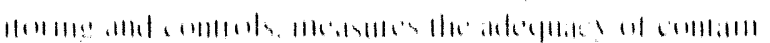

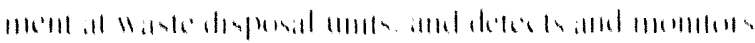

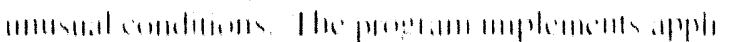

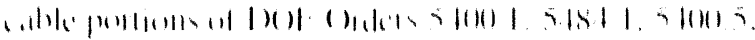
ind is 211 . I

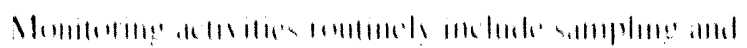
Hom

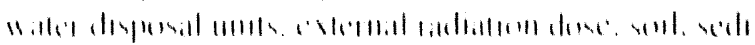

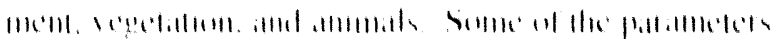

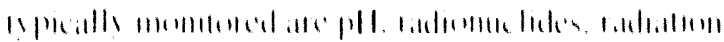

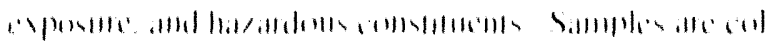

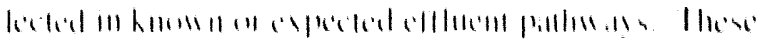

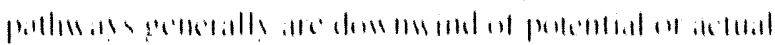

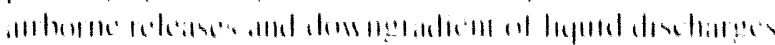

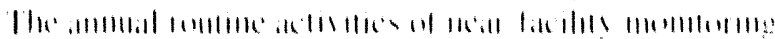

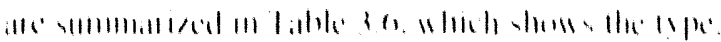

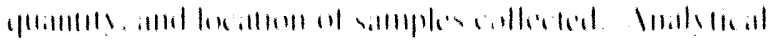
16

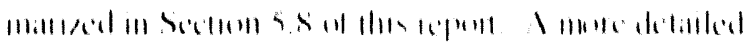

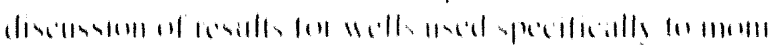

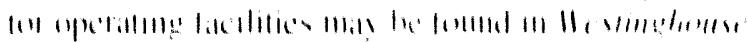

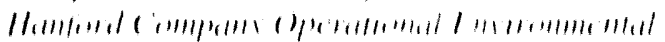

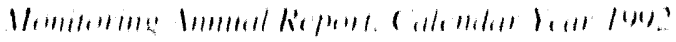

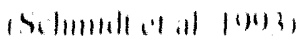

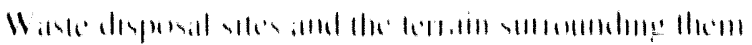

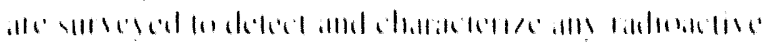

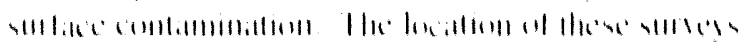

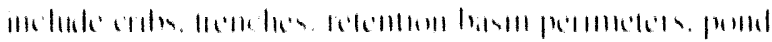

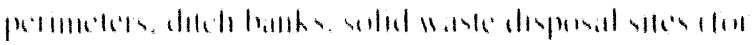

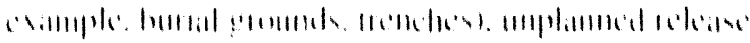

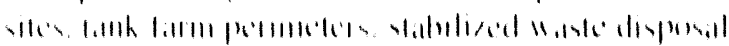

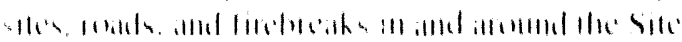

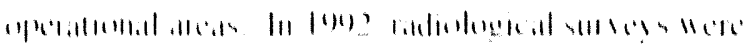

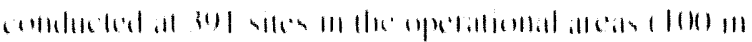

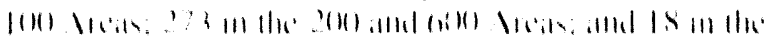

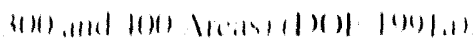

\section{Air Monitoring}

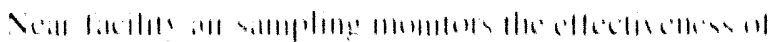

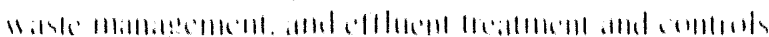

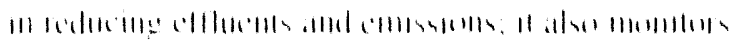

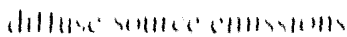




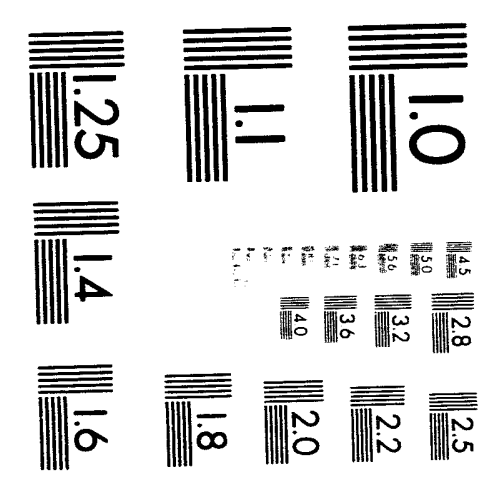



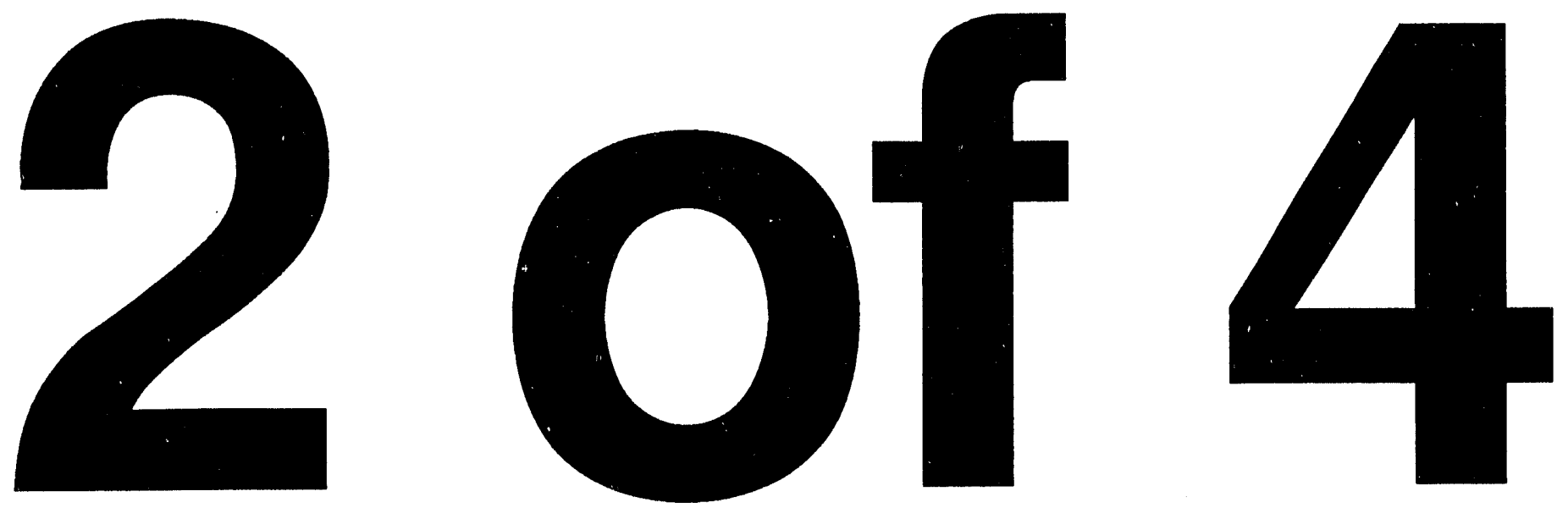
Table 3.6. Near-Facility Routine Environmental Samples and Locations

\begin{tabular}{|c|c|c|c|c|}
\hline Samples & Total & 100 Areals & $2(0) / 60(0)$ Areats & $3(0) / 400$ Areas \\
\hline Air & 40 & 4 & 36 & \\
\hline Surface waller & .32 & 22 & 10 & \\
\hline External radiation & 289 & $213^{1+1}$ & 61 & 15 \\
\hline Soil & 1.57 & 32 & 110 & 15 \\
\hline Vegetation & 95 & 40) & 4) & 15 \\
\hline
\end{tabular}

(a) 41 TLDs and 172 survey points.

\section{Sample Collection and Analysis}

Radioactivity in air was sampled by a network of eonlimuously operated samplers al 40 locations near nuclear facilities: + localled in the $10(0)-\mathrm{N}$ Area, $3.3 \mathrm{in}$ the $200 / 6(0)$ Areas, and 3 background stations collocated with samplers operalled by PNL and the Washington State Department of Health at the Yakima and Wye Barricades and the old Hanford townsite. To avoid duplication of sampling, the near-lacility envirommental monitoring program used existing PNL air samplers in the $3(0)$ and 40() Areals (results reported in Section 5.2. "Air Surveillance"). Air samplers were primarily located all or neall $(250) \mathrm{mor} 1.500$ (t) sites and/or facilities having the potential for. or history of, release, with an emphasis on the prevailing downwind directions.

Simples were collected according (1) al schedule established before the monitoring year. Airborne particles were sampled all ealch of these stations by drawing air through a glass tiber filter. The filters were collected weekly, field-surveyed for gross radiondetivity lo dedect any unusual trends or off-normal oceurences. held for all least 7 days, and then analyzed for total alphat and heta activity. The 7-daly holding period wats necessary to allow for the decaly of maturally oceurring radionuclides that would otherwise ohseure detection of longer-lived radionuclides associated with emissions liom nuclear facilities. The otal radionctivity measurements were used to indicate changes in trends in the neall-lacility enviromment.

For most radionuclides, the amount of radioatetive material collected on a filler during a l-week sampling period was foo small to be measured accurately. The alceuracy of the simple analysis was increased by compositing the samples into a biammal compessite for catch location. Each biamnal composite was then sent to lonternational
Technology Corporation to be analyod for strontium, plutonium. uranium, and gamma-emitting radionuclides.

\section{Radiological Results}

()f the radionuclide analyses pertormed, "Sr. 1 "Cs. 231.2."Pu, and uranium were consistently detectable in the 20() Areals: "Co wals also detedable in the 10()-N Area. Air concentrations for these radionuclides were elevalted near facilities when compared to the concentrations measured offsite. Figure 3.2 shows average values for 1992 and the preceding 5 years compared to the Derived Concentration Guides (DCO) and the background air comedntration as meitsured by PNI. As the data indicate. the concentrations show a large degree of variance. In general, the samples collected from air samplers localed on or directly adjaxent to waste disposal and other nuclear facilities had significantly higher concentrations than those larther awaly. The data also show. as expected, that erertain radiontelides had higher concentrations within different operational areas. Generally speaking, the predominate radionuclides alle activattion products/gammat emitters in the 100 Areas and fission products in the $20(0) / 6(0)$ Areas. A more detailed datta summary maly be found in the Westinghonses Hanford compram ()perational Enviranmental Momitoring Ammual Report. Calendar Yo'or 1992 (Schmidt et al. 199.3).

\section{0-N Area}

Analytical results from air samples taken in the 10()-N Area were on a downward trend for most radionuclides as a resule of facility shutdowns. better elflueme controls, and improved waste malnagement practices. These levels were well helow the I)C $i$; however, they were above those meisured offsite. 

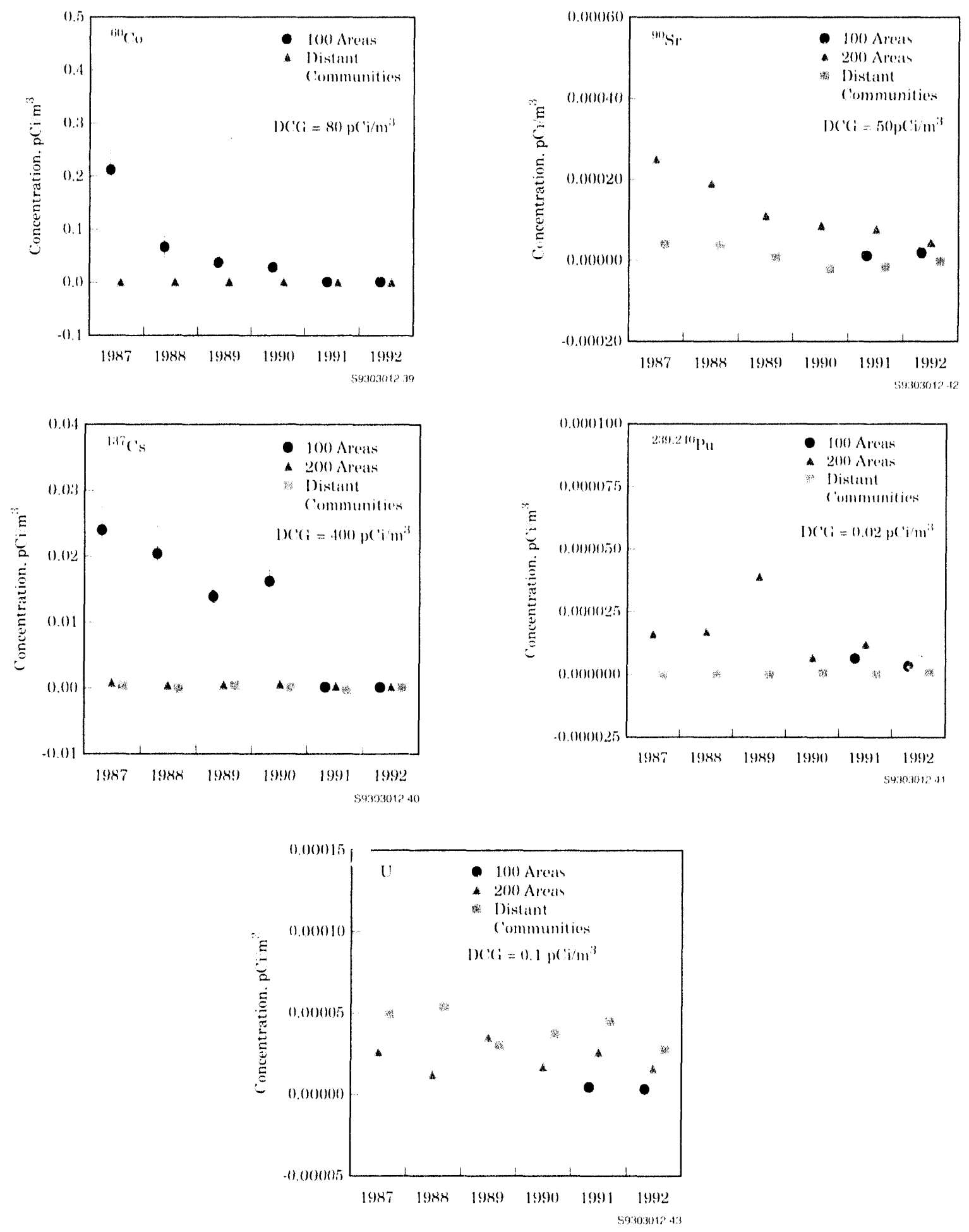

Figure 3.2. Radionuclide Concentrations ( \pm 2 SEM) in Near-Facility Air Samples Compared to Background Locations, 1987 Through 1992. As a result of figure scale, some uncertainties (error bars) are concealed by point symbol. 


\section{Areas}

Analytical resules from air s:mples laken in the $20(0) / 60()$ Areas were on al dowmwand trend for most radionnelides

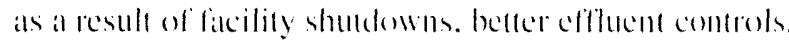
and improsed waste management practices. These kevels. allhough well helow the 1 (') Were above hose meatsured offsite and were higher lor " tranium when sompared lo levels in the (0)()-N Area.

\section{Surface-Water Disposal Units and Seep Monitoring}

Surlate-waller disposal units lased by the operating facilitices and their seceps atre monitored lo arsess the eflectiveness of edflent and contamination controls.

\section{Sample Collection and Analysis}

Samples firom surface-waller disposal units and river shoreline secps rere collected from various locilloms in the operational areass. A more detailed description of

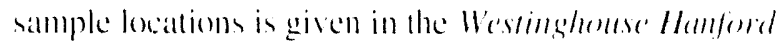

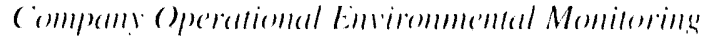

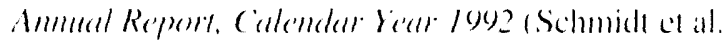
1993). Sampling of surfice-water disposal unis included water sediment, and alpuattic regetation. Samples laken at river shoreline seeps included watler only. The simpleling methods are discussed in detail in loe Whe mantual

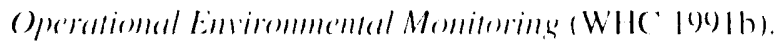
To atroid duplication of sampling. the nealr-fictiling environmental meniloring program used PNI, sulates-

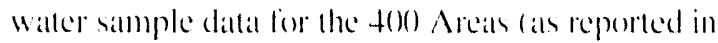
Section 5.3. "Surface-Waller Surveillance").

Raddological analysis of waller samples firm surface

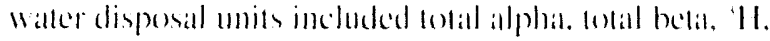

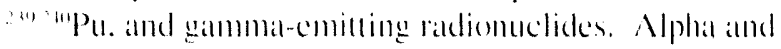

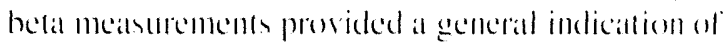
radioatctive contamination. Ratdiologeical analysion

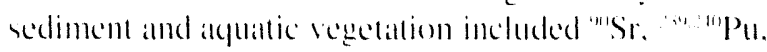
tramium. and galmmma-cmitume radionuclides. Nonradiological analysis performed included pll. lemperat-

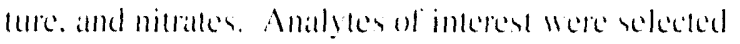
batsed on their presence in effleme dischateges. and the if importance in verifying edlleme comerol and determining complianse with applicables standards. Surfice-waller

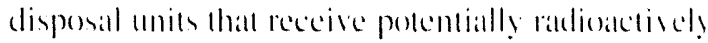

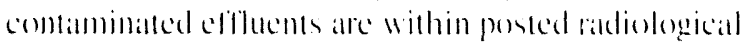
comtrol alreas.

\section{Radiological Results}

\section{Surface-Water Disposal Units}

Radiological analytical results for individual surfatecwaller disposall mits alle summanted in Table 3.7. In all

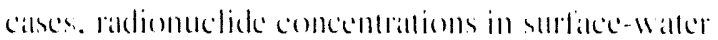

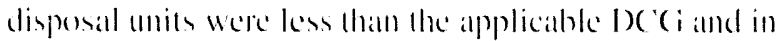

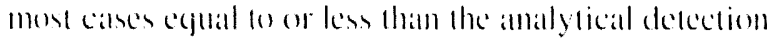
limil. However. the maximum total belat concentration

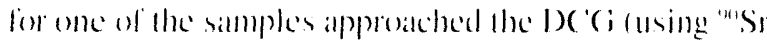

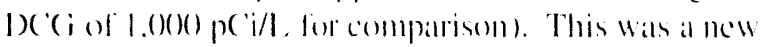

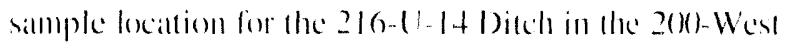
Area. The simpling locition batd been changed an a result of the stabilization the pacement of lill matterial over combiminated soilst of the original sampling location al the lower portion of the $216-1-1+$ Dilch in early 1992. This sutike-watler disposatl unil had recerived

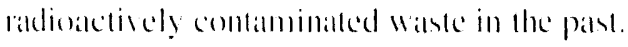

Raddiologicall allalylical results for alyuatlic vegetallonn and sedimeml simples laken from surfice-Watler disposal units focilted in the $20(0 / 6)(0)$ Areats atre stummatrized in Tahles 3.8 and 3.9. respectisely. Nlihomgh some ele. rated levels catl be seen in both aquatlic regetation and sedimeme, in all calser the radiologicall amalytical results were well below the standatrds for radiological comtrol.

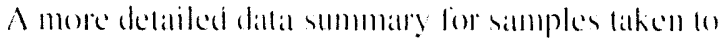
monitor strliace-waller disposal unils maly be found in

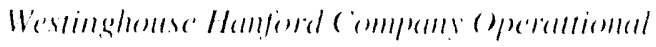

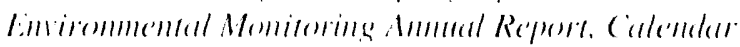
'roter 1992) (Schmidt (la al. 1993).

\section{Seeps}

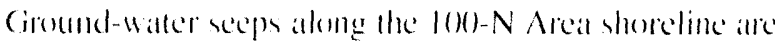
simpled ammally a verify reported radiomelelide relearses to the Columbia River from the palst operation of the

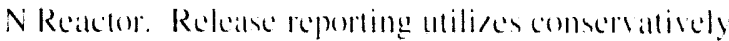
batsed radiontaclide concentrations, muldiplied by the

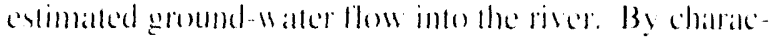
cerizing the radiontedide concentrations in the seceps

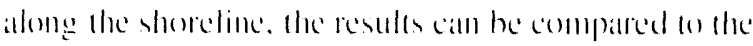
comecontrations maasmed in he ellhem monitoring well ()()-N-ST.

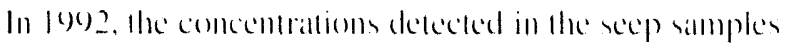

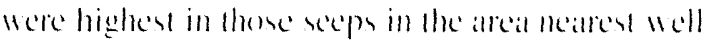

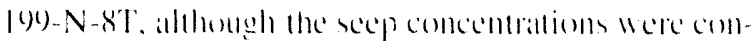
siderably lower than those measured in the well. The 
Table 3.7. Radiological Results for Liquid Samples from Surface-Water Disposal Units, 200/600 Areas (pCi/L), 1992

\begin{tabular}{|c|c|c|c|c|c|c|c|}
\hline 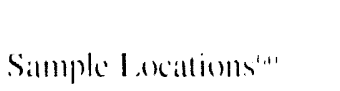 & $\begin{array}{l}\text { No. ol } \\
\text { Siamples }\end{array}$ & & Total Alphal & Tolal Belar & 'II & "Sr & $11 \mathrm{Cs}$ \\
\hline \multirow[t]{2}{*}{ 2(x)-West Area Ditches } & 21 & M('ill) & 5.5 & 32 & +50 & 9.6 & 4.3 \\
\hline & & Maximumn & 24 & 920 & 4.5() & 28 & 7.5 \\
\hline \multirow[t]{2}{*}{ 2(1) West Area Ponds } & If & Mc:all & $(1.20$ & 1.5 & $0(1)$ & 11 & +1 \\
\hline & & Niaximum & $1) .88$ & 0.2 & $1 .(0)(0)$ & 78 & te \\
\hline \multirow[t]{2}{*}{ 2(1)- East Areal Ditches } & 12 & Mčall & 0.31 & 5.5 & 1.20() & 7.1 & 42 \\
\hline & & Maximum & 0.8 .3 & 40 & $2.1(10)$ & 10 & f() \\
\hline \multirow[t]{3}{*}{ 2()()-Last Area Ponds } & $(1)$ & M('ill & 0.71 & 3.1 & 770 & 10 & 42 \\
\hline & & Maximum & 12 & 21 & $2.1(1)$ & 04 & (1) \\
\hline & & I)( & 3()$^{\mathrm{in}}$ & $1.0(0)()^{(n)}$ & $2.00(0) .0(0)$ & 1.0000 & $3 .(1)(0)$ \\
\hline
\end{tabular}

(a) 200-West Areal Ditches: 216-1'-4. 216-11-14.

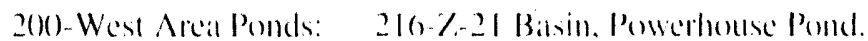

20)- lant Area Ditches: $210-13-3-3$.

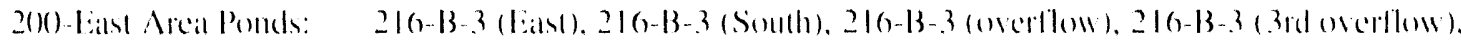
Pencihouse Pend.

(b) Using : "Pu d)(ci lor compartisom.

(c) Using "Si Ix (i line comparisum.

Table 3.8. Radiological Results for Aquatic Vegetation Samples from Surface-Water Disposal Units 200/600 Areas (pCi/g), 1992

\begin{tabular}{|c|c|c|c|c|c|c|}
\hline Sample L.ocallioms & $\begin{array}{l}\text { No. of } \\
\text { Silmples }\end{array}$ & & "Sir & $" C$ & :"1"'Pu & 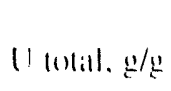 \\
\hline \multirow[t]{2}{*}{ 20(0)-West Area Dikde: } & 2 & ML:an & 0.97 & $<2.9$ & 0.7 & $5.4 \times 10$ \\
\hline & & Maximmm & 1.2 & $<+.9$ & 1.3 & $2.4 \times 10$ \\
\hline \multirow[t]{2}{*}{ 20)(1)-West Areal Ponds } & 2 & Me:ill & $<0.58$ & $<0.8 .3$ & $<0.48$ & $5 .+\times 10$ \\
\hline & & Maximum & $<(1.04)$ & $<0.80$ & $<(1.48$ & $8.6 \times 11 \%$ \\
\hline \multirow[t]{2}{*}{ 2(0)-Latst Areal Ditches } & 1 & Mein & 1.4 & 7.8 & $<0.38$ & $3,6 \times 10$ \\
\hline & & Maximum & 1.4 & 7.8 & $<(1) .38$ & $3.6 \times 10^{5}$ \\
\hline \multirow[t]{2}{*}{ 20()-latst Acail Ponds } & 5 & Mc:an & $<1.8$ & $<2.8$ & $<(1 .+2$ & $4.5 \times 11^{n}$ \\
\hline & & Miximum & 5.0 & $<5.3$ & $<1.40$ & $7.7 \times 10 \times$ \\
\hline
\end{tabular}

(a) 2(1)-West Areal bitches: 216-1-4. 210-(1-14.

20()-West Area Ponds: 216\%.-21 Basin. Powerhouse Pond.

20)-Jasl Area Dilches: 210-13-3-3.

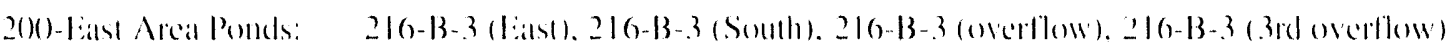

Powcrhouse Pond. 
Table 3.9. Radiological Results for Sediment Samples from Surface-Water Disposal Units, 200/600 Areas (pCi/g), 1992

\begin{tabular}{|c|c|c|c|c|c|c|}
\hline Sample Locationstin & $\begin{array}{l}\text { No. of } \\
\text { Simpless }\end{array}$ & & ${ }^{2} \mathrm{Si}$ & "C's & $\therefore 414: 24 \mathrm{Pu}$ & U totitl. g/g \\
\hline \multirow[t]{2}{*}{ 20(0)-West Areal Ditches } & 2 & Meill & 0.4 .3 & 19) & 0.74 & $0.1 \times 10^{10}$ \\
\hline & & Maximum & 0.55 & 34 & 1.2 & $1.2 \times 10^{5}$ \\
\hline \multirow[t]{2}{*}{ 20(0)-West Area Ponds } & 2 & Me:inl & $<0.35$ & $<(1.14$ & $<0.4 .3$ & $4.7 \times 10$ \\
\hline & & Maximum & $<0.58$ & $<0.1 .5$ & $<0.50$ & $6.3 \times 10=$ \\
\hline \multirow[t]{2}{*}{ 20(0)-East Areal Ditches } & 1 & Me:ill & 1.1 & 110 & 14 & $7.9 \times 10$ \\
\hline & & Maximum & 1.1 & 110 & 14 & $7.9 \times 10$ \\
\hline \multirow[t]{2}{*}{ 20)()-East Area Ponds } & 5 & Mcan & 0.89 & 8.1 & 2.3 & $8.0 \times 10 ?$ \\
\hline & & Maximum & 2.3 & 16 & 7.5 & $1.7 \times 10^{\circ}$ \\
\hline
\end{tabular}

(a) 20()-West Area Ditches: 216-T-4. 210-(1-14.

2(0)-West Areal Ponds: 216-\%-21 Basin. Powerhouse Pond.

2()()-Fast Area Ditches: 216-B-3-3.

20)-1ast Area Ponds: 216-B-3 (East). 216-B-31 South). 216-B-3(overtlow). 216-B-3(3rdovertlow)

Powerhousc Pond.

datia from seep silmpling are summitrized in Tible 3.10.

A more detailed dalta summintry mar be found in

Westinghouse' Hanford (omprom! (Operational

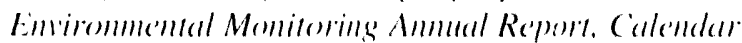

Yectl 1992 : Echmide el all. 199.3).

\section{Nonradiologica! Results for Surface-Water Disposal Units}

Nonradiological antalytical cesults for water samples laken from surfitede-water disposil units localted in the 200 and 60)( Areas are summatrized in Table 3.11. The results lor pH were well within the $\mathrm{pH}$ of 2.0 and 12.5 standard for liquid efllenen discharges based on the discharlge limits listed in RCRA. The analytical results for

Table 3.10. Concentrations of Radionuclides in 100-N Area Shoreline Seeps, 1992 (results in pCi/L)

\begin{tabular}{|c|c|c|c|c|}
\hline Radiommclisk & $\begin{array}{l}\text { W(ell |c0)-N-Sl } \\
\text { (Nkill) }\end{array}$ & Misimum & $\begin{array}{l}\text { Sexps } \\
\text { Mean }\end{array}$ & $1)\left(i^{\prime}+1\right.$ \\
\hline$H$ & $+3.0(0) 0$ & 7711 & 3010 & $2.00(0) .010101$ \\
\hline "ili) & 7.5 & 5.2 & 1.2 & $5.0100)$ \\
\hline$" \mathrm{Si}$ & $0,5(1)$ & 1.50 & $f(1)$ & 1.000 \\
\hline
\end{tabular}

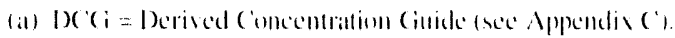

nitrates were all less than the delection limit axeept fior those simples from the $216-T-4$ Ditch. The average nitrate result for that locattion wats $2.0 \mathrm{mg} / \mathrm{l}$. Which was slightly greater than the antalytical delection limit of $1.4 \mathrm{mg} / \mathrm{l}$. and is kess than the $4.5-\mathrm{mg} / \mathrm{l}$, drinking watter standard for public watcer supplies.

\section{Radiological Surveys}

Another aspect of the near-ficility envirommental menitoring program is radiologicial surveying. Which monitors and helps direct the reduction of the radiologically controlled atreas on the Hanferd Site. There are two lype of radiologicially controlled areas. which ane posted: underground radiestetive materials (URM⿻) and surfiace contamination arreiss (SC'As).

URM alleits are posted atreas with contamination contained below the soil surfice. These areats are lypically "stabilized" cribs, burial grounds, and corered ponds. trenches, and ditches. Batriers over the contimination sources are used on inhibit radionuclide transport to the surface environs. Waste sites atre routinely surveyed (all least annually) fo document the corrent raddistogical stillus. 
Table 3.11. Nonradiological Results for Liquid Samples from Surface-Water Disposal Units, 1992

\begin{tabular}{|c|c|c|c|c|c|c|c|}
\hline \multirow[b]{2}{*}{ Simple Locallioms } & \multicolumn{4}{|c|}{$\mathrm{pH}$} & \multicolumn{3}{|c|}{ Nitrate (NO), mg/l. } \\
\hline & $\begin{array}{l}\text { No, of } \\
\text { Simmples }\end{array}$ & Meill & Maximum & Minimum & $\begin{array}{l}\text { No. ol } \\
\text { Sillimples }\end{array}$ & Mciall & Maximumm \\
\hline 2()()-West Area Ditches & 80 & 7.3 & 9.5 & 6.5 & 8 & 1.7 & 3.9 \\
\hline 200-West Area Ponds & 100 & 8.9 & $10 . .3$ & 7.1 & 8 & $<1.4$ & $<1.4$ \\
\hline 20()-last Area Ditches & 50 & 8.1 & 8.7 & 7.4 & 4 & $<1.4$ & $<1.4$ \\
\hline 20()-lias Area Ponds & 25.5 & 8.8 & 9.7 & 7.8 & 20 & $<1.4$ & $<1.4$ \\
\hline 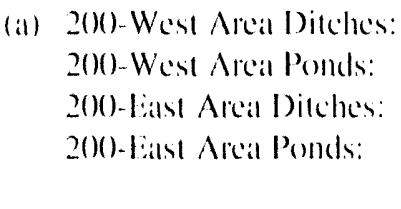 & $\begin{array}{l}216-\mathrm{T}- \\
210-\%- \\
216-\mathrm{B}- \\
216-\mathrm{B}- \\
\text { Powerl }\end{array}$ & 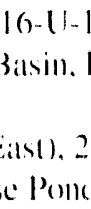 & 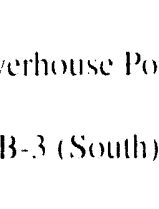 & $10-13-310$ & $11.216-13$ & $3 n d o$ & low) \\
\hline
\end{tabular}

SCAs may or may mot have been associalled with an underground radioaledive malterial structure. A breech in the barrier of URM maly have resulted in the growth of combaninated vegetation. Insects or animals might have hurrowed into an (IRM and brought contaminatfion of the surfike. Vent pipes or risers from an undergereund structure could have been a source of speck contamination. Fallout froms stachs. of unplanned

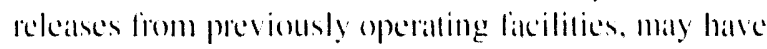
catused all area of surfice contaminalion thall wals not

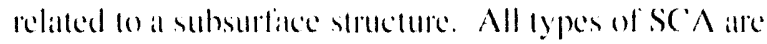
susceptible to comtaminatlon mighalion.

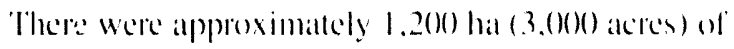

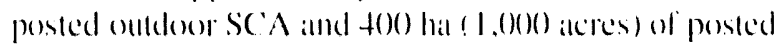
(IRM. not including atedive liacilities, at Hantond. The mumber of SC $A$ aceres was bree times larger than the URM aceres primarily becamse of the BC Comtrolled

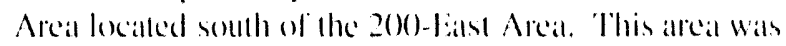
posted ats a Radiologically (ontrolled Area in 1950) hecaltse of widespread speck comtaminatiom, and encomparsed approximately $1,0(0)$ hat $(2,50()$ acters). Inhle 3,12 contaims the alcreage for SC A and IRM areats

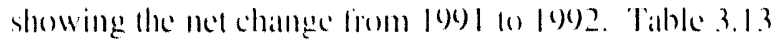
stmmanias the number of contaminaled akes that changed stallus in 1092. Twenly aleres were reclatssilied from SC'A lo llRM, and 16 alcres were sampled and unconditionally released.

The area of posed surlice contamination viried becalsse of all ongoing elfort fo cleam, slabilize, and remediatle alleas of known contanimatton while new arean of conlamination were being ickentilied. Table 3.1 .3 indicalles the changes resulting from stabilization alctivilies during 1992. Newly identified areas maly be from contamination migration or the result of an increased effort to

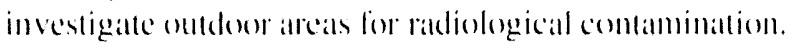
Vehicles erguipled with radiation deledion devices and the Ullarasonic Ranging and D )ala System (l!SRADS) have identilied ancals of contamination that were previnusly undelected.

It wats entimated that $80 \%$ of the identified antedor surlace contamination would result in an external dose rate of less than I merm/h, although the dese from isolalled spectis could be comsiderably higher. Contamimatton levels of this magnifude would not signilicantly add to dose rate calculations lon the public or employedes.

\section{Soil and Vegetation Sampling from Operational Areas}

Soil and regetalton simples were colleceded on or adjitcent lo walske disposal units and from lociltions downwind and neall or wilhin the boundanters of the operalling facilities. Simples are collected lo detect potential migralion and aleposition of facility elflemes. Migration call oceur as the resull of resuspension from radionalively combanimaled surlate arcias, absorption of radio-

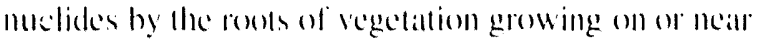
undergeround and surface-water disposill units, or by waste site intrusion by animals. Special samples were 
Table 3.12. Outdoor Contamination Status, 1992 (approximate surface area in acres)

\begin{tabular}{|c|c|c|c|c|}
\hline Hanford Site Area & Surlike Conlaminationn" & $\begin{array}{l}\mathrm{Net} \\
\text { (hamge'th) }\end{array}$ & $\begin{array}{l}\text { Underground } \\
\text { Radioactive Malteriall" }\end{array}$ & $\begin{array}{l}\mathrm{Nel} \\
\text { Chillng }\end{array}$ \\
\hline 100) Areas & 160 & .6 & 460 & 5 \\
\hline 200 Areils & .329 & -.32 & 366 & 17 \\
\hline $6(0)$ Areia & 120 & () & 230 & () \\
\hline BC Control Areal & 2.5()()$(R C A)^{t+1}$ & () & 30 & () \\
\hline $3(1)$ Area & 4.5 & () & .30 & () \\
\hline Totals & 3.154 & -38 & 1.116 & 22 \\
\hline
\end{tabular}

(a) Includes arteas posted as "Surfike Combanination Areas" or as "Radiological" Controlled Areas" and areas that had both underground and surface contamination.

(b) $=$ decreances.

(c) Includes areas with only underground contamination. Does not include areats that had surface an well as underground radioatetive material.

(d) Radliologically Controlled Arcia.

Table 3.13. Zore Status Change by Area, 1992
Location

100 Areis

2(x)-Eass Areal

I()) Arcias

2()()-Easl Areal

20)-West Area

Zome Change
SCA IO Releilsed
SCA IO Releised
SCA IO URM
SCA IO URM
SCA to URM

Acreilge

1

15

5

5

12 also laken where physical or biologicall tramsport prohlems were identified. The results of simpling effore are discussed below.

\section{Sample Collection and Analysis}

The simpling methods and locittions used atre discussed in detail in the WHC manual Operational Eintrommentul

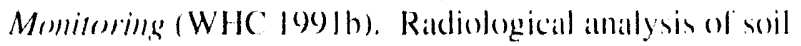
and vegetittion samples included "Sr. ${ }^{24}$ ?"Pu, uranium, and gammit-cmilling radionuclides.

\section{Radiological Results}

\section{Soil}

(O) the radionuclide andlyses pertormed, "'Co, "Sr. " ('s, 2.4. Put and uranium were consistently detectable. Sovil concentrattions for these raddionuclides were elevated neatr and within lixility boundaries when compared to the centcentrations measured offsite. fïgure 3.3 shows alverage values for 1092 and the preceding 5 vears. As the data indicite. the concentrations show a large degeree of vatriance. In general, the samples collected on or directly addiacent to the waste disposal faceilities had significantly higher concentrations than those firther alwaly. The datta also show, an expected. that certain radiontedides will have higher concentrations within diflerent operational atreis. (ienerally opeaking. the predeminatle radionuclides arte activation products/gammon emilters in the 100) Areas. lission products in the $200 / 60(0)$ Arcias. and tratuiunn in the $3(x)$ Areas. A more detailed dillia summiry may

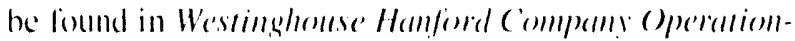
al Einvironmental Monitoring Anmual Re'port. Callender. rear 1992 (Schmidl el al. 190.3).

100 Areas. Analyticall results from soil simples collected in the 100 Areats were on a downwital trend. showing effects of the shudewn of the preduction reacers and the effectiveness of effluent controls dhat have bee 1 implemented in recent years. However. these fevels were greater than those meisused offsite. and the concentration of "w'Co wats greater than thatl measured in the $20(0) /(0)(0)$ and $3(0) / 40(0)$ Areals. The " in the l()( Areals was the resule of past discharges 10 waste dispossal structures, primarily the 1.301-N Liquid Waste Disposall Facility. Measures hare been laken in recent years on identify and minimise the migration of contamination from these structures. 

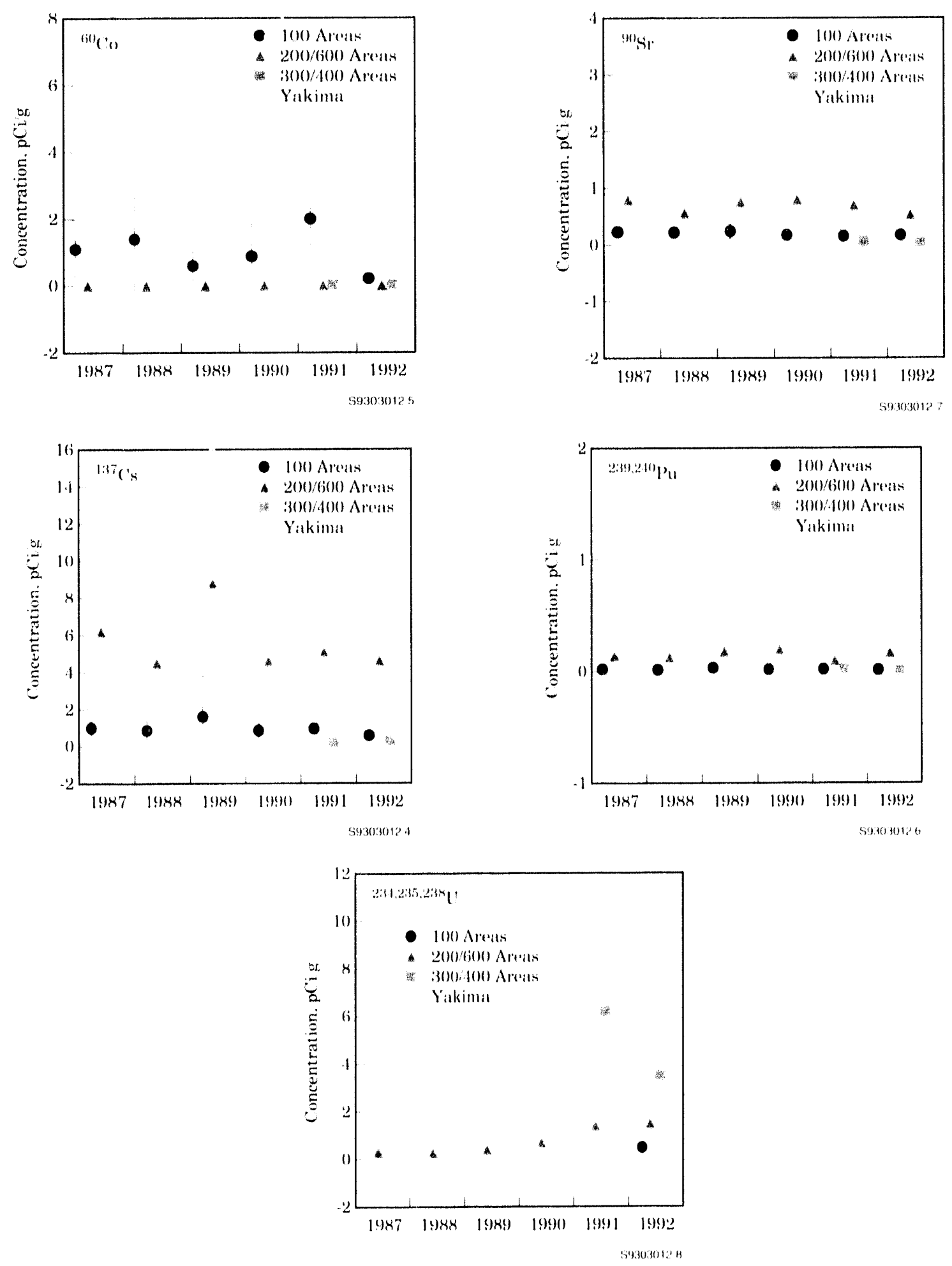

Figure 3.3. Radionuclide Concentrations ( \pm 2 SEM) in Near-Facility Soil Samples Compared to Background Concentrations, 1987 Through 1992. As a result of figure scale, some uncertainties (error bars) are concealed by point symbol. 
200/600 Areas. Anilyglicill resulls flom soil simples lathen in the $20(0) / 6(0)$ Areas nere on a downward lrend

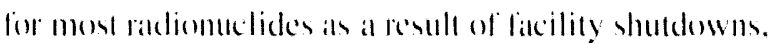

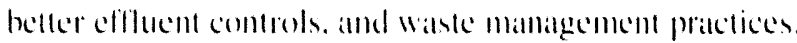
However. these levels were grealle than those measured offisite, and were shown lo be higher for "Si. " ('s, and

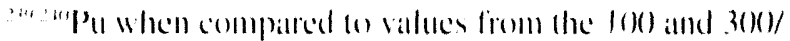
f(x) Areas. Granium kevels were slighly increased over those meisured in log).

300/400 Areas. This was the second sampling yeall for the $3(x) / 40)$ Areas neatr-like ilty envirommental monitoring program. The datta for these areas were

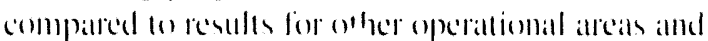

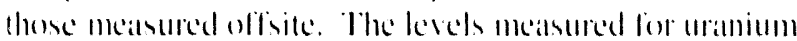

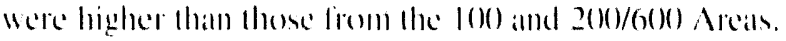
This diflerence is expected hecatuse the mantum is the

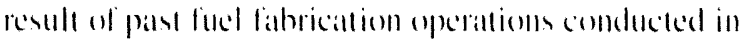
(lic $3(0)$ Areial.

\section{Vegetation}

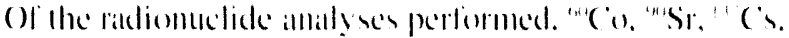

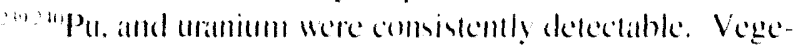

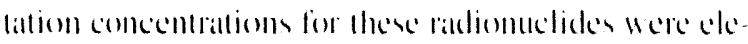
valced neall and within lateility houndarties companted to

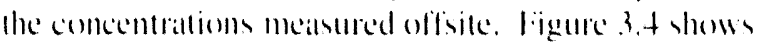

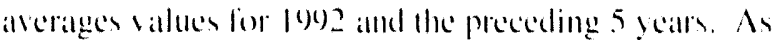
the dala indicalle. the comecontratioms show al latge degres of vartiance. In germerall the simples collected on or

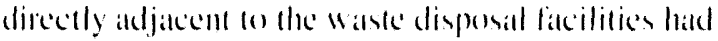
significantly higher concentrations than these farther allaty. As with the soil samples, the dalla shom that cerltain radionuclides will be found in vegetaltion in higher concentrations within different operational atreas. Except for "sir (at fission prodect) delected in segetation from the $N$ Springs, gencrally speaking the prodominalle radiomuclides alle activallon products/gamman emillers in the 1010 Areas. lission products in the $20(0 / 60)(1$ Arcils, and uranium in the $3(x)$ Areats. A more delitiled dallat stmmmary

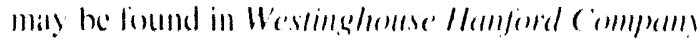

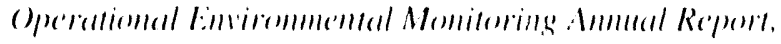

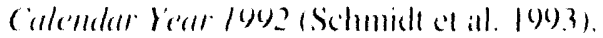

100 Areas. Analylicial resulls from regetallion sillmple collected in the 10) Areas were all on a downward trend

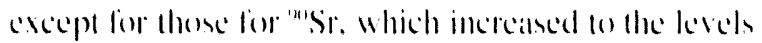
se'll in 1989. The maximum values were from simples collected from the $\mathrm{N}$ Springes area lecated in the $10(1)-\mathrm{N}$

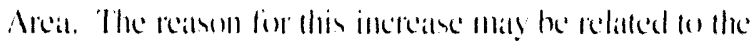
time of year for simpling. and here were no N Springs

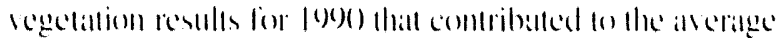

concentratlom on "nst in the 100 Areal vegetallom.

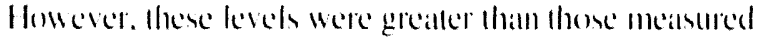

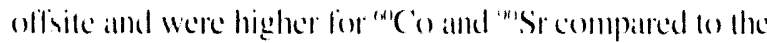
$2(1) /(0)(0)$ and $3(0) / 40)$ Areas.

200/600 Areas. Analytical results limm vegetaltion salmples lithen in the $20(8 / 6)(0)$ Arean were on a downward

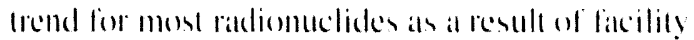
sholdowns. beller eflluent controls. and improved waste

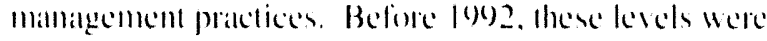
greater than those measured ofteite and were higher for

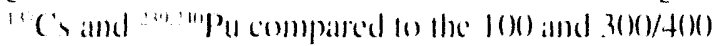

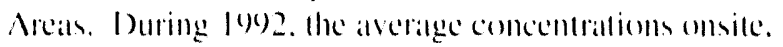
offsite. and within the varions operational ancas were similar for these two radionnelides.

300/400 Areas. This wath the second sampling yeall for the $3(0)(8) /(0)$ Arear near-fateility envirommental monitoring program. (iencrally, most of the levels in the 300 Areat were greater thath these meastred offsite and were higher for aramiun compared to the for) and 20(0)/60)( Areas. This diflerence is expected becillse

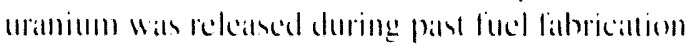

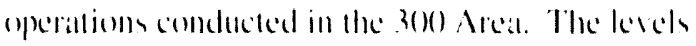
meabured in the for) Areal were all ar mear these meamured allitic.

\section{Investigative Sampling}

The purpose of imsestigatlice sampling was fo determine whelher eflluent combrols and Walste containment were adequatce. An impentant pant of the mear-lacility program, investigative momitoring wate comducled and ypecial samples were taken in the operations areats lo contion the

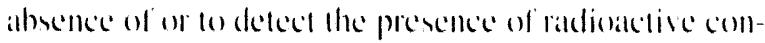
tamminams. This imsentigatlise sampling louk plate neall lateilities such as slorage and disposal sites for an least me of the following reasoms:

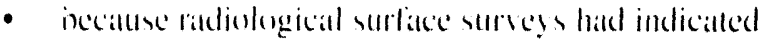

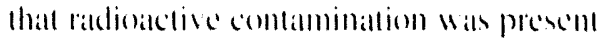

- Io yuantily the radiological condition of a sile belore facility comstruction or operation

- Io yuantily the radiological condition of a site belone remediatlion

- 10 deferminc if hiolic intrusion (c.e... animal burroms

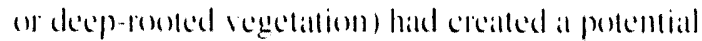
for the spreatd of contaminams 

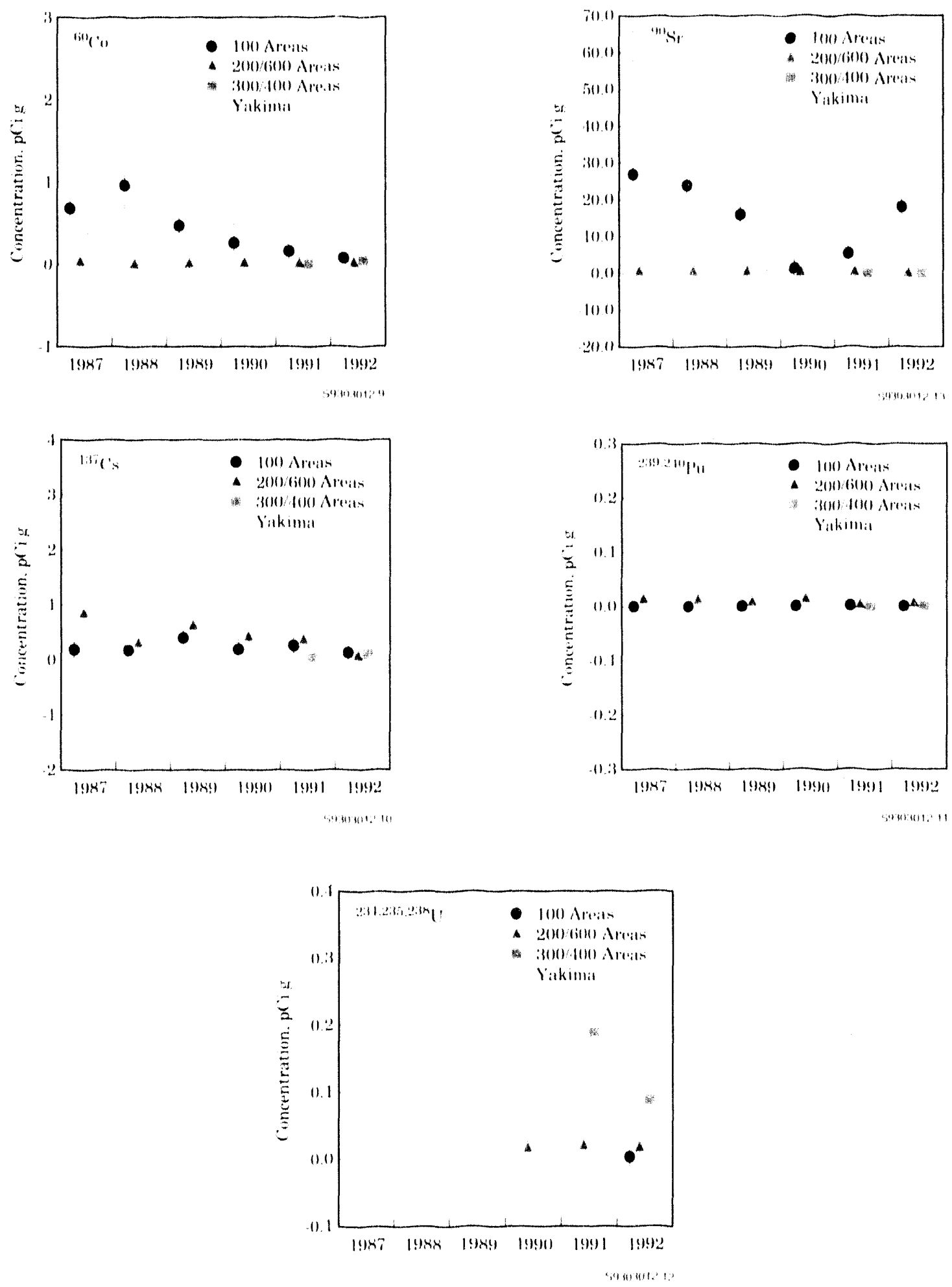

Figure 3.4. Radionuclide Concentrations ( \pm 2 SEM) in Near-Facility Vegetation Compared to Background Concentrations, 1987 Through 1992. As a result of figure scale, some uncertainties (error bars) are concealed by point symbol. 
- to determime the integrity of waste comtaimment I!scills.

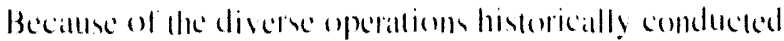
al each of the operating atreas (10). 20(1). and $30(0)$ Areas). radiomelide comecontratioms in silmples from the different arear were gencrally prediclable.

\section{Sample Collection and Analysis}

Typersol insertigattive samples arer the years hate included atis: Maller: stom: sediments: soil: vegetation

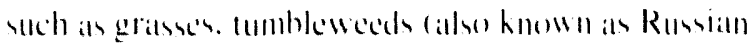

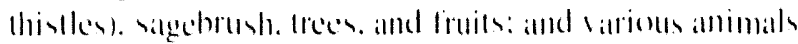

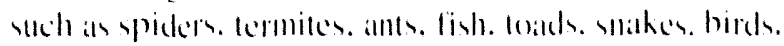
mices rabhits. conotes, and boheals.

Special simples in 1902 indeluded soil: lumbleweeds:

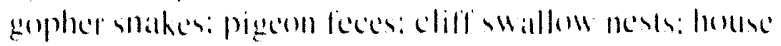

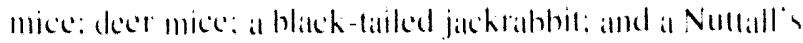
collontail rabhit.

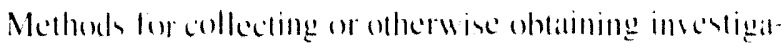

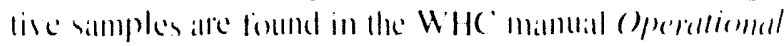

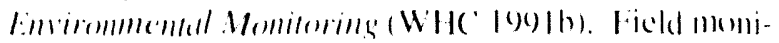

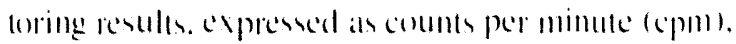

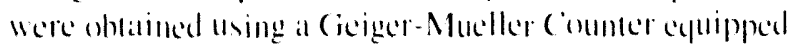

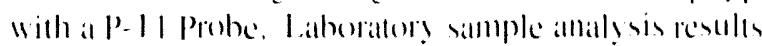
are expressed in pe $\mathrm{g} / \mathrm{g}$. Maximum concentrations of radionuclides rather than ancrages atre presented in this subscition.

\section{Results}

Special simples were collected where known or sts pected radinatetive comlamination was present. A direcel relattomship an the general status of the different media within the epreatting atreats lo contamination led. reported for special simples cammon be dratwn. A more

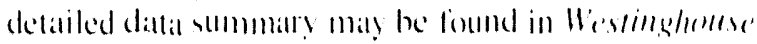

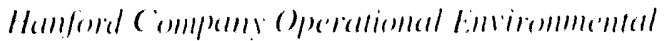

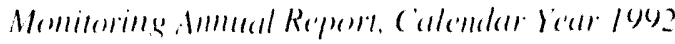
(Sichmidl cel al. 1093).

\section{Soil}

In 1992. 30 imsertigalior soil silmples were taken. The

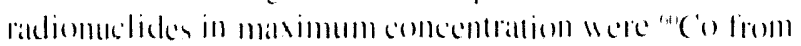

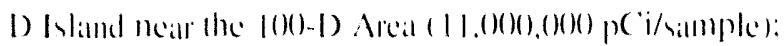

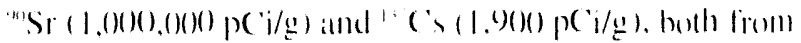

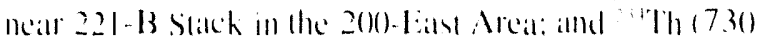

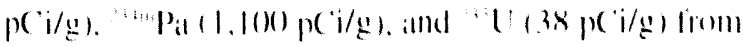

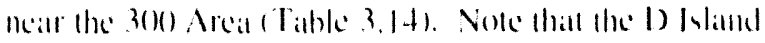
sample analysers result was for a single speck af

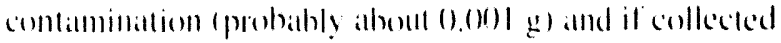

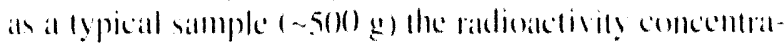

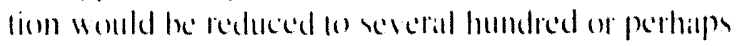
thousand picocurice pere gram. Further discussion af the shoreline radiological sursey mat be found in Section 5.7. The number of contamination incidents.

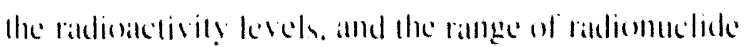

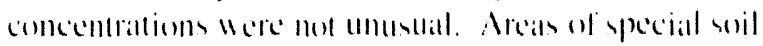
simpling that were outside radiological combend ancias and that had levels grealter thath Whlle radiologgical control

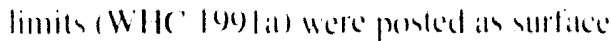
comtamination atreas.

\section{Vegetation}

There were 1.3 imstances of entaminalled regetation

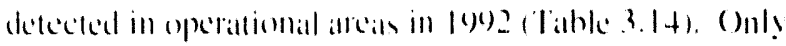

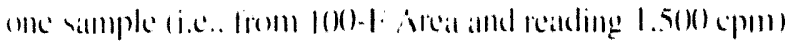

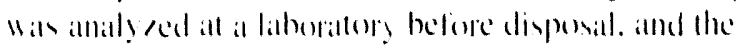

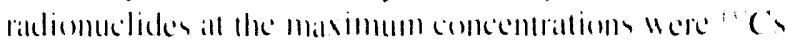

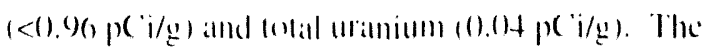
rematining 12 instatleses of contaminalled lumbleweds

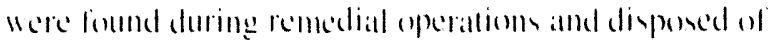

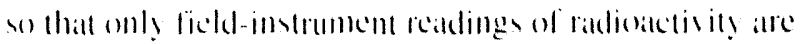
inailable. fickd-imstrument realings ranged fom 350 ( ) reported for the past 3 years. The mumber of plants

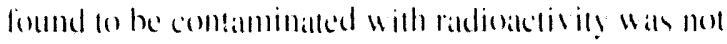
umusual. The greatey mumber of combaminated regelation (+2) submilted for analyses was in 1978. In the 200) Areas hefore 1980, " hen legetation combol was limiled ar monexistemt. comlanminated regetation was counted in aceser rather han individual specimems. Vegetation control in |902. an in |99). Wais motictably more effectice than in 109() and 1989 and hopefully represents a return lo all improving trend. An improsing

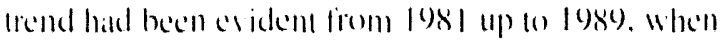
resistance to the herbicide in use wats lirst moled. Improsed vegetation control was probahly the result al improsed equipment and use of more elfective herbicides.

\section{Wildlife}

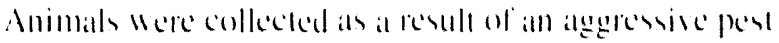
comtrol program designed to limit the exponome and

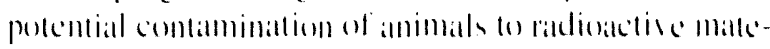
rial. Animals were collected direcelly from ar near facilitien lo identily problems in preventatise meansures derigned to inhibit animbal inlrusion. Survens were 
Table 3.14. Special Samples Collected from the Operations Areas, 1992

\begin{tabular}{|c|c|c|c|}
\hline $\begin{array}{l}\text { Simple Type } \\
\text { (Number) }\end{array}$ & $\begin{array}{c}\text { Arcal } \\
\text { (Number) }\end{array}$ & $\begin{array}{l}\text { Elevaled } \\
\text { Radionuclides }\end{array}$ & $\begin{array}{c}\text { Maximum } \\
\text { Concentration, } \mathrm{pCi} / \mathrm{g}\end{array}$ \\
\hline $\begin{array}{l}\text { Soil } \\
(3(1))\end{array}$ & $\begin{array}{l}10() \\
(2)\end{array}$ & (1) $\mathrm{Co}$ & $11,0(0), 0(0)(1)$ \\
\hline & $\begin{array}{l}200 \\
(26)\end{array}$ & $\begin{array}{l}{ }^{137} \mathrm{Cs} \\
{ }^{19} \mathrm{Sr}\end{array}$ & $\begin{array}{r}1,900 \\
1.000 .00(0)\end{array}$ \\
\hline & $\begin{array}{r}3(00) \\
(2)\end{array}$ & $\begin{array}{l}{ }^{23+4} \mathrm{Th} \\
23+m \mathrm{~Pa} \\
2{ }^{24} \mathrm{U}\end{array}$ & $\begin{array}{r}730 \\
1.100 \\
.38\end{array}$ \\
\hline $\begin{array}{l}\text { Tumbleweed } \\
\text { (1.3) }\end{array}$ & $\begin{array}{l}100 \\
(3)\end{array}$ & $\begin{array}{l}{ }^{17} \mathrm{Cs} \\
U \text { Total }\end{array}$ & $\begin{array}{r}<0.96 \\
0.31\end{array}$ \\
\hline & $\begin{array}{l}2(1) \\
(10)\end{array}$ & $N A^{\prime \prime \prime}$ & NA \\
\hline $\begin{array}{l}\text { Gopher snake } \\
\text { (2) }\end{array}$ & $\begin{array}{l}2()(1) \\
(2)\end{array}$ & $\begin{array}{l}{ }^{12 \mathrm{CS}} \\
{ }^{\prime \prime S r}\end{array}$ & $\begin{array}{r}7.9()(0 \\
3()(1)\end{array}$ \\
\hline $\begin{array}{l}\text { Pigeon lieces } \\
\text { (1) }\end{array}$ & $\begin{array}{l}200 \\
(1)\end{array}$ & $17 \mathrm{CS}$ & 280 \\
\hline $\begin{array}{l}\text { Clifi swallow } \\
\text { nest } \\
(2)\end{array}$ & $\begin{array}{l}200 \\
121\end{array}$ & $\begin{array}{l}" \mathrm{Cu} \\
" \mathrm{Cs} \\
" \mathrm{Sr} \\
" \mathrm{Mn} \\
{ }^{14} \mathrm{Eu} \\
{ }^{156} \mathrm{Eu} \\
\therefore \mathrm{Pu} \\
\therefore \mathrm{Pu}\end{array}$ & $\begin{array}{r}74.00(0) \\
2.30(0) \\
340 \\
160 \\
470 \\
180 \\
8 \\
47\end{array}$ \\
\hline $\begin{array}{l}\text { House mouse } \\
\text { (9) }\end{array}$ & $\begin{array}{l}2(1) \\
(9)\end{array}$ & 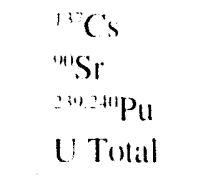 & $\begin{array}{r}1.5(x) \\
3.10(0) \\
<5.0 \\
1.3\end{array}$ \\
\hline $\begin{array}{l}\text { Deer mouse } \\
\text { (10) }\end{array}$ & $\begin{array}{l}100 \\
(2)\end{array}$ & $\begin{array}{l}\text { "Cs } \\
\therefore \cdots+P u\end{array}$ & $\begin{array}{l}100 \\
<1\end{array}$ \\
\hline & $\begin{array}{l}2(1)(0) \\
(6)\end{array}$ & $\begin{array}{l}\because \mathrm{CS} \\
\therefore \because \mathrm{P}_{\mathrm{H}}\end{array}$ & $\begin{array}{r}<2.5 \\
<.5\end{array}$ \\
\hline & $\begin{array}{l}3(1)(1) \\
(2)\end{array}$ & $\begin{array}{l}\text { "Con } \\
1 ; C)\end{array}$ & $\begin{array}{r}97 \\
5.5(1)\end{array}$ \\
\hline
\end{tabular}


Table 3.14. Special Samples Collected from the Operations Areas, 1992 (contd)

Sample Type (Number)

Deer mouse (contd)

Black-tailed jackrabbit

(1)

Nulall's collontail (1)

(a) $\mathrm{pCi} / \mathrm{sample}$.

(b) NA - not analyzod.
Area

(Number)

Elevated

Radionuclides
6.3

4.2()()

$1,6(0)$

$38,(0)(0)$

6.50

1.70()

18

56

1,100

15

19

5

$<5$ performed after collection to determine if an animal is radioactively contaminated. If an animal was found free of contamination, it would be taken to a suitable habitat area and released. If an animal was contaminated, a decision was made fo collect a sample or dispose of the animal. This decision was based on the level of eontaminatlon, sampling facility, and frequency of eccurrence.

There were 26 special animal or nest samples analyed in 1992. The radionuclides found at the maximum concentrations were " $\mathrm{Co}(74.00() \mathrm{pCi} / \mathrm{g})$, " $\mathrm{Sr}(3,10() \mathrm{pCi} / \mathrm{g})$. $\left.{ }^{134} \mathrm{Ru}(4.20() \mathrm{pCi} / \mathrm{g}),{ }^{17} \mathrm{Cs}(7.9)(0) \mathrm{pCi} / \mathrm{g}\right)$, and ${ }^{154.155} \mathrm{Eu}$ $(1,7)(1) \mathrm{pCi} / \mathrm{g}$ ) (see Table 3.1.3). The lotal number of animals found 10 be contaminated with radioactivity, the radioactivity levels, and the range of radiontelides concentrations were not untsual: however, the number of incidents decreased slightly compared to $32 \mathrm{in} 1991$. The greallest number of contaminated anmimals was found in 1982 144. mostly pigeons): however, before 1981 fewer representative samples were submitted lor radionuclide analyses.

\section{Special Characterization Projects}

Special chatracterization projects were conducted to verify the status of contaminated soil at 216-1/-14 Ditch in the 20)()-West Area before stabilizing the lower end, anround B Stack in the 2()()-East Areat. and on D Island in the Columbia River, which was the site of the old liquid effluent outfall from D Reactor. Radionuclides found al the maximum concentrattons at these siles were, respeclively, ${ }^{17} \mathrm{Cs}\left(1,6(0) p(\mathrm{Ci} / \mathrm{g}):{ }^{17} \mathrm{Sr}(1,0(0),()(0) \mathrm{pCi} / \mathrm{g})\right.$ and

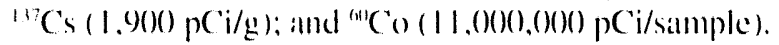
Note that the D Island sample analyses result was for a single speck of contamination (probahly about ().(0)| g) and if collected as a lypical simple $(-50)($ g) the radionetivity concentration would be reduced lo several hunded or perhaps thousand picocuries per gram. 


\section{External Radiation}

Exlermal radiation fields were measured near operallions lakeilities and waste handling. storage. and disposal sites (o) meisures. alssess, and control the impacts of operattions.

\section{Sample Collection and Analysis}

Two methods of measurement are employed. one heing hand-held $\mu$ R meders used to survey multiple survey points and the onter being thermoluminescent dosimeters (TLD). The meistmement metheds used for external radiation measurements and sample locallions are discussed in detail in the WHC manual ()peranional Emvirommental Monimains (WHC |00)|b).

\section{Results}

\section{Radiation Measurements}

Hand-held $\mu$ R meters were used to survey points neat and within there waste disposal localtions in the 10()-N Area: the N Springs alreal. 1.3()I-N liquid Waste Disposal Ficility (L.WDI:), and 1.325-N I.WDF: These radiation measturements were laken all a height of approximallely I m (3.28 fit) to alssess the effects of changes in operations of these filcilities and ane not necessarily a true measurement of exposure rate. The hand-held $\mu R$ meters are known to over-respond to lowencrgy gamma radiation. The radiation rate measured along the $100-\mathrm{N}$ Area shoreline wats still declining in 1992 and is companed to rates during the past 5 years in Figure 3.5. The shith in the dose rate levels show the eflects of lle decreased discharges of the 1.3()1-N I.WDE: and the contimuing deciag of "'Co. the principal residual ratdenuclide. The radiation measurements latedn all the 1.301-N I.WI)1: in |992. als in previous years, continue (1) show dhe decisy of " (o) (Table 3.15). The radiation measurements taken all the 1.325-N I.WI)l in 1992 and in the previous year were elevated. compared lo the carlicr years. This increase indiealles the effece of decreased dischatrges of liguid waste to that lateility ('Table 3.15). The decreased discharges resuled in the loss of the walle that normally provided shickding from the gimmol-emitting radiontedides comtatined in the sediments of the L.WDF, principally " (c) and "s. A more detailed data stmmmary maly be foumd in
Westinghomse Hemplond commpany. Operational

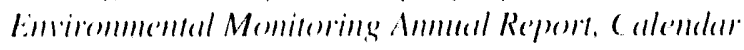
Ye(11/ 1992 (Sclminidt all. 1993).

\section{TLDs}

100 Areas. All TI.Ds in the 100 Areats were locilled in the 10()-N Area: results are presented in Tahle 3.10. The 1992 TLD results indicate that direct radiation levels were highest neat lateilities that had contained or received licpuid effluent from $\mathrm{N}$ Reacerer. These fakilites primarily include the 1.325-N I.WDI: and the 1.301-N I.WDF: While the results were noticeably higher than those for other Io()-N Area TI.D locillions, the overall results for these (wo fatcilites decreased in exposure rate by approximately 5\% when compatred to |0)

In 1992, thee TL.D locillions were moved from previously oceupied buildings lo onther lecaltions. Measurements were initialed all two locations, between the 1.30) -N L.WDF and the Columbia River shoreline (N Springs). A more detailed data summary may he

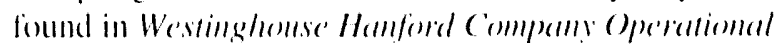

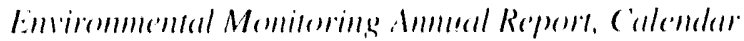
Yo(11/ /992 (Schmide al. 1993).

200/600 Areas. TI.1) results for 1902 are compancel (1) those of 10)! for the $20(0) / 6(0)$ Areas in Table 3.16. The highest dose ralles were measured neall waste-handling facilities such ats tamk liams. The highest dose rate wats measured at the $241-A$ Tank farm complex localled in the 200-bast Area. The arerage dose rate measured in 1992 by Th. Ds in the $200 /(0)(0)$ Areals was $1.30 \mathrm{~mm} \times \mathrm{m} / \mathrm{yt}$. which was an increatse of $8 \%$ over the anerage dose rate of $120 \mathrm{mrem} / \mathrm{yr}$ meatsured in |40). A more detatiled dattil summary maly be loumd in Westinghumse Hemford

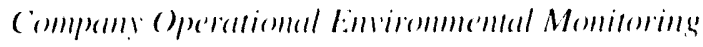

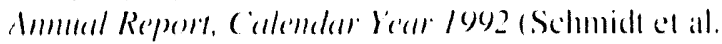
10) 31 .

300/400 Areas. A comparison of $1(0) 2$ Th. D results fo those of 199) for the $30(0) / 40(0)$ Areats alle presented in Table 3.16. The highest dose rates in the 30) Area were measured near waske-handling facilities such als the 340 Waste Handling lacility. The alveratge dose ralle meistured in 1992 by Th.1) in the 300 () Areats wats 100 mremlyr, which was a decreatse of 10 th of the alserage dose ralle of 180 morem/yr measured in 1001 . 

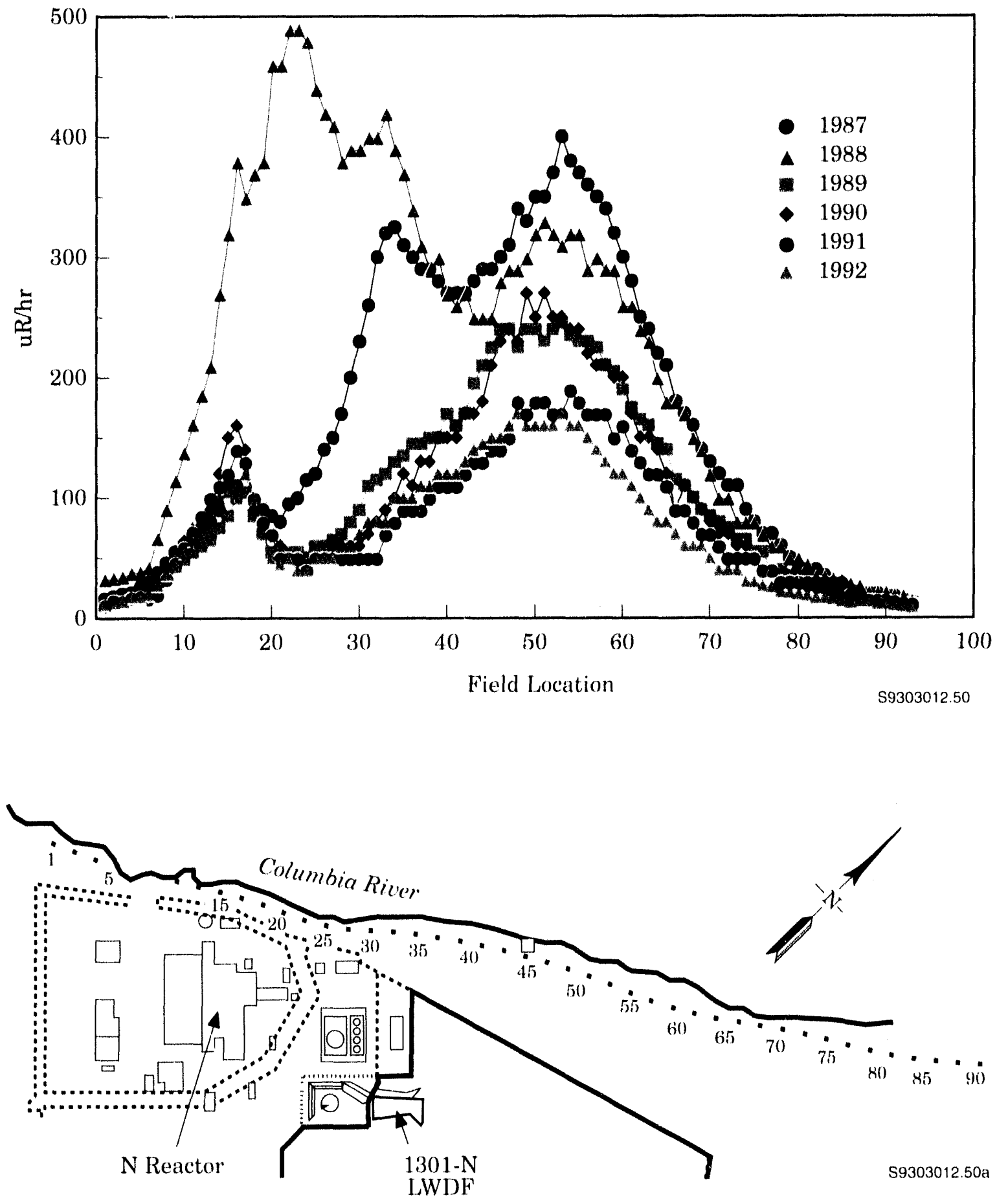

Figure 3.5. Radiation Survey Measurements Along the 100-N Area Shoreline, 1987 Through 1992 
Table 3.15. 100-N Liquid Waste Disposal Facilities (LWDF) Direct Radiation Measurements, 1991 and $1992(\mu \mathrm{R} / \mathrm{h})$

LWDF

$$
1991 \text { average }
$$

1992 Average

1.3() $1-\mathrm{N}$

2,100

2,000

$1.325-\mathrm{N}$

950

940)
The highest dose rates, although not significantly elevated above background, measured in the 400 Area was near the 4718 Building. The average dose rate measured in 1992 by TLDs in the 400) Area was 90) mrem/yr, which was a decrease of $20 \%$ of the average dose rate of $110 \mathrm{mrem} / \mathrm{yr}$ measured in 1991 . A more detailed data summary may be found in Westinghouse Hanford Company Operational Environmental Monitoring Annual Report, Calendar Year 1992 (Schmidt et al. 1993).

Table 3.16. Thermoluminescent Dosimeter (TLD) Results for Waste-Handling Facilities in the Operating Areas, 1991 and 1992 (mrem/yr, based on $24 \mathrm{~h} / \mathrm{d}$ )

\begin{tabular}{|c|c|c|c|c|c|c|}
\hline \multirow[b]{2}{*}{ Area } & \multirow[b]{2}{*}{ No, of Siunples } & \multicolumn{2}{|c|}{$\mid 991$} & \multicolumn{2}{|c|}{1992} & \multirow[b]{2}{*}{$\%$ Change $^{\text {tal }}$} \\
\hline & & Maximum & Mean & Maximum & Mean & \\
\hline $10(0)-\mathrm{N}$ & 41 & 14,260 & 1,210 & 13,280 & $1.200)$ & -1 \\
\hline $200(60)(0)$ & 62 & 840 & 120 & 700 & 1.30 & 8 \\
\hline 300$)$ & 8 & 72() & 180 & 610 & 160 & -10 \\
\hline 400 & 7 & 190 & 110 & 110 & 90 & -20 \\
\hline
\end{tabular}

(a) Numbers indicate a decrease (-) or increase from 1991. 


\subsection{Solid Waste Management and Chemical Inventories}

\section{Solid Waste}

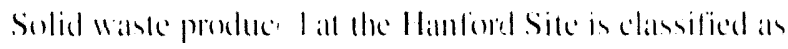

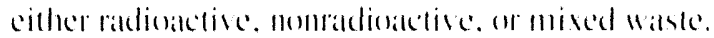

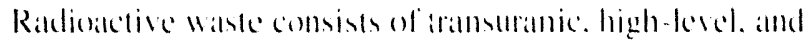
low-ked wastes. Radientetive mived waste has boll

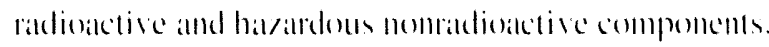

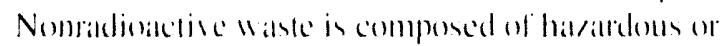

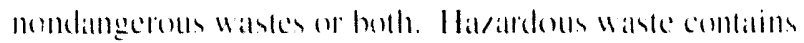

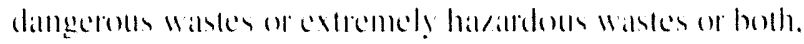

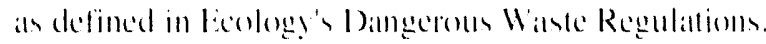

Radlioative and mised waste is comremty handled in seremal ways. High-level wasle is stomed in double-shell lamks. I.om-kedel wask also is stomed in double-shell

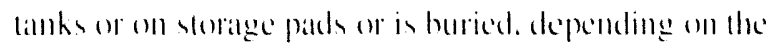

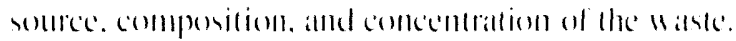

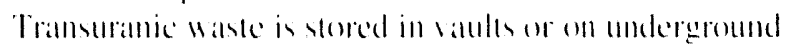
storage pads liom which it can be redrieved.

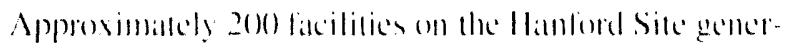

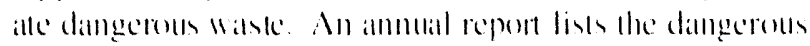
waskes alld extremely hatardeme wasker gencratled.

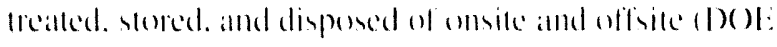

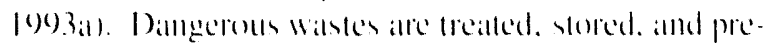
pared for dispesal all serenal Hanturd Sile faccilities.

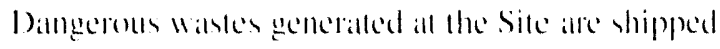
allite for disposall. destruction. or recycling.

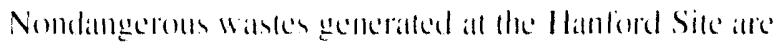
burted in the Solid Waste lamdlill. hated in the 20) Areats. These wastes orighate all hoth process and

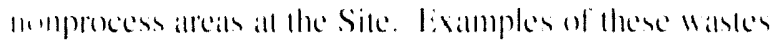

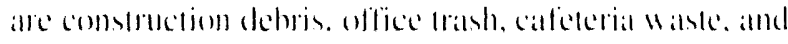
packiging matterials. ()here materials and items chassified as waste are solidified filter bach wash and sledge from the treatment al tise watler. latiled and broken

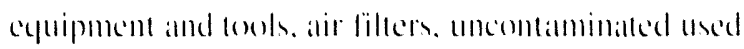

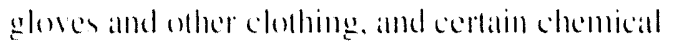
precipitates such als oxalales. Nombaliondetive triable

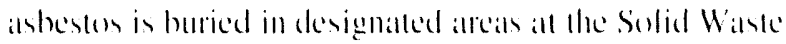

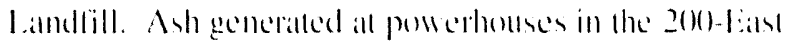

and 20(1)-West Arean is huried in devignated sites meall those powerhouses. Demolition waste fom lom Areas decommissioning projocts is huried in situ or in designatled sites in the lo() Areals.

A smmmatry of solid waste disposed of at the Hantiond Silc in 1902 is shomn in Table 3.17. Solid waste program atctivition ane regulated by the Toxic Substances (omtrol Act, discussed in Section 2.(1) "Iimsirommental compliance Summany."

\section{Chemical Inventories}

\section{Emergency Planning and Community Right-To-Know-Act}

Title III of the Suprefund Amendments and Reaumbori-

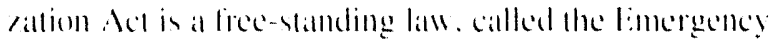
Pamming and community Right- To-Knom Acl. This Act reguires that the public be given informattion about hatandous chemicals in their commmumities. It also estab-

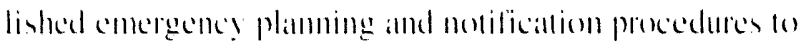
proted the puhlic in the erent of a hatardous chemical releine.

Subtille B contaims reguirements for reponting informat-

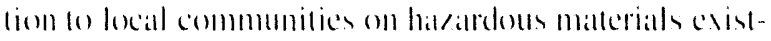
ing in or releated from a lacility near those locales. 'The Hantond Sile was in compliance w ith the reporting and

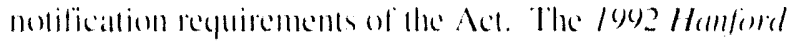

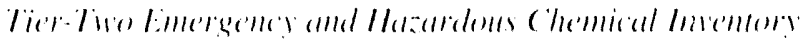

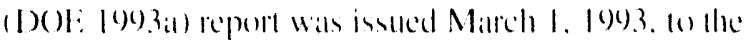
Stalle limergency Reyponse (ommissiom, locial connly

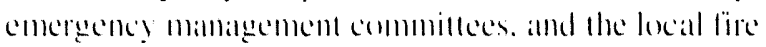
departments. This repont combans intormation on

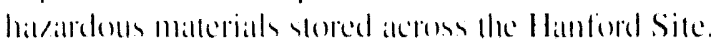

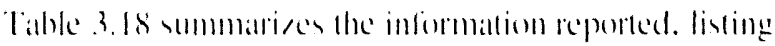
the 10 chemicals sored in greallest guamtity on the Hamlinel Sile. 
Table 3.17. Radioactive Solid Waste Disposal, 1992

\begin{tabular}{|c|c|c|c|c|c|c|c|}
\hline Constituent & Units & Low-Level & $\begin{array}{l}\text { Low-Level } \\
\text { Mixed }\end{array}$ & $\begin{array}{l}\text { Low-Level } \\
\text { Plus's" }\end{array}$ & $\begin{array}{l}\text { Low-Level } \\
\text { Mixed Plus's' }\end{array}$ & Transuramic & $\begin{array}{l}\text { Trimsuranic } \\
\text { Mixed }\end{array}$ \\
\hline Uranium & $g$ & $9.9 \times 11^{10}$ & $2.9 \times 10 \%$ & 0.0 & (0.) & $2.6 \times 10^{4}$ & $2.6 \times 100^{n}$ \\
\hline Plutonium & $g$ & 3.2 & $4.1 \times 10^{1}$ & $1.6 \times 10^{4}$ & $2.5 \times 10$ & $5.5 \times 10^{\prime}$ & $3.6 \times 10^{2}$ \\
\hline Americium & $g$ & $7.3 \times 10^{1}$ & $1.9 \times 10^{1}$ & $1.0 \times 10$ & $2.2 \times 10^{111}$ & $1.0 \times 10^{1}$ & 8.8 \\
\hline Thorium & $g$ & $8.6 \times 10^{2}$ & $2.0 \times 10^{0}$ & $0.0)$ & 0.0 & $1.0 \times 10^{1}$ & 0.0 \\
\hline Strontium & $\mathrm{Ci}$ & $3.5 \times 10^{3}$ & $2.0 \times 10^{1}$ & 0.0 & $5.0 \times 10^{10}$ & (i.) & $0.4 \times 10^{1}$ \\
\hline Europium & $\mathrm{Ci}$ & $3.7 \times 10^{2}$ & $2.0 \times 10^{2}$ & 0.0 & (1) 1 & $7.2 \times 10^{4}$ & 0.0 \\
\hline Cosium & $\mathrm{Ci}$ & $3.7 \times 10^{1}$ & $3.6 \times 10^{\prime}$ & 0.0 & $1.4 \times 10^{\circ}$ & $7.2 \times 10 \%$ & 2.8 \\
\hline $\begin{array}{l}\text { Giher lission } \\
\text { and activation } \\
\text { products }\end{array}$ & $\mathrm{Ci}$ & $3.6 \times 1()^{1}$ & 3.4 & 0.0 & $1.8 \times 100^{\prime \prime}$ & $3.9 \times 10^{4}$ & $9.6 \times 10^{6}$ \\
\hline
\end{tabular}

(a) Low-level with polychlorinated biphenyls.

(b) Low-level mixed with polychlorinated hiphenyls.

Table 3.18. Hanford Site Tier-Two Emergency and Hazardous Chemical Inventory Average Balance of Ten Chemicals Stored in Greatest Quantity, 1992

\section{Hazardous Malerial}

Coal

Mineral oil

Sodium

Uranium nitrate hexahydrate

Diencl fuel

Fuel oil. No. 6

Nitrogen

Nitric acid

Ethylene glycol

Sodium chloride
Averiage Daily

Balince, $k g$

$7.0 \times 10)^{\circ}$

$4.1 \times 10^{\prime \prime}$

$2.7 \times 10^{\prime \prime}$

$1.6 \times 10^{16}$

$1.0 \times 10^{n}$

$1.0 \times 10^{\prime \prime}$

$7.0 \times 10^{4}$

$6.7 \times 10^{4}$

$0.0 \times 10^{4}$

$5.2 \times 10^{7}$
The annual toxic chemical release inventory (D)E 1992i) report is pre vided to EPA as information only, as directed by HQ. EPA no longer reguires this report since the primary missiem of the Hanford Site has shifted from production operations to envirommental restoration. Available to the public, the report has Site intormation on foxic chemicall releastes and transiers, an well als waste management practices. 


\section{Environmental Program Information}




\subsection{Environmental Program Information}

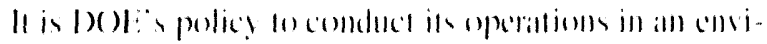
rommentally responsible manmer and comply willo appli

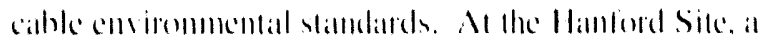
variely of envirommental atctivitics alle pertentmed focom-

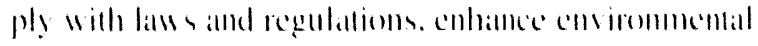

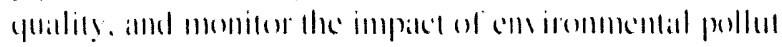
ants lowm Sitcoperattoms.

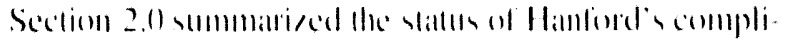

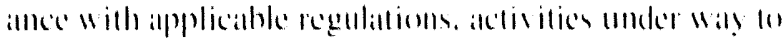

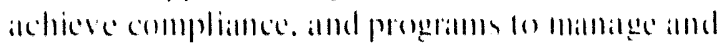
improse convirommential puility.

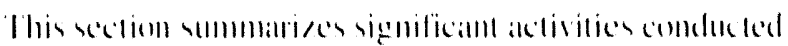

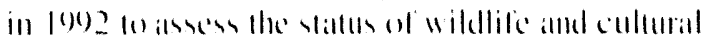

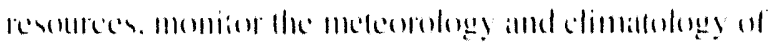

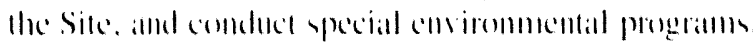




\subsection{Climate and Meteorology}

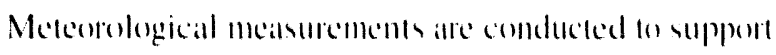

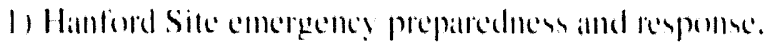
21 atmospleric dispersion calculations. and 3) Hanlond

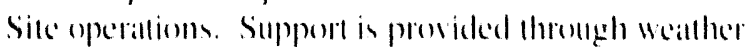
forecisting and the mathlenance and distribution al

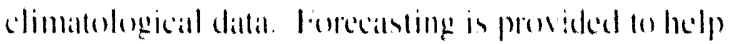

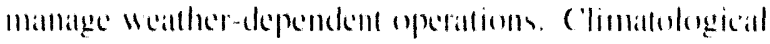
dalla are prosided to help plan weallere-dependent

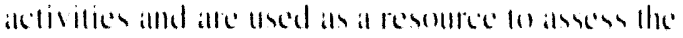

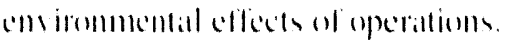

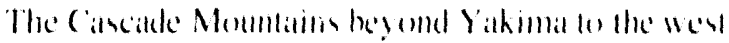
greatly miluence the climate of the Ilanforel Site. This

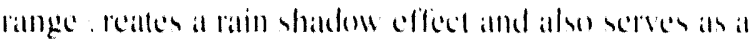
source of cold air drainage. Which has a comsiderable chlect on the wind eqgime

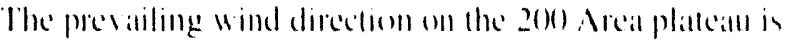

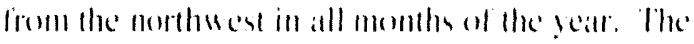
secondatr! 1 ind direction is from the southwest. Sum marien of wind direction indicale that winds frem the morthmest guadrant acelle mont ollen during the winter

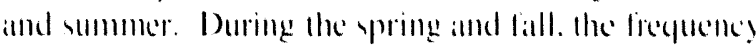

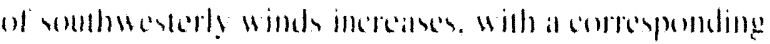

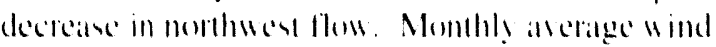

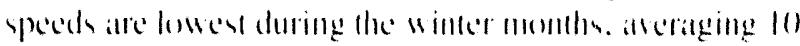

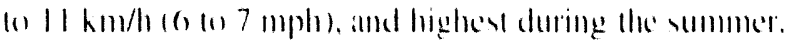
areraging $141010 \mathrm{hm} / \mathrm{h} 191010 \mathrm{mph}$. Wind yeces

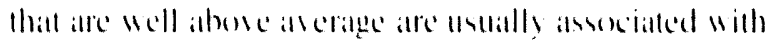

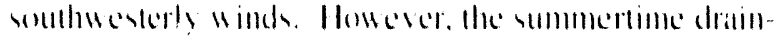
age wind a are gencrally nonthwesterly and frepuent!

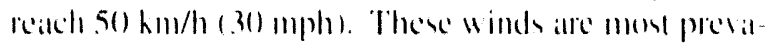
lemt oner the northern pertion of the site.

Dimmal and mombly alseriges and extremes of kempera-

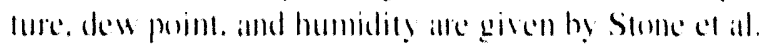
1983). The record maximum temperature is to (" $(11.5 \mathrm{f})$ and the record mimimum temperallure is $-32.8 \mathrm{C}^{\circ}$

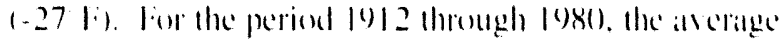
monthly kemperatures ranged from a low an - 1.5 c

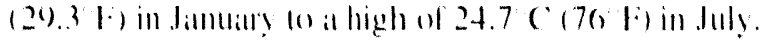
Dorimg the winter. the highest mombly atserage temperallure all the llantiond Medeorological Station athMS

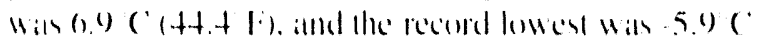

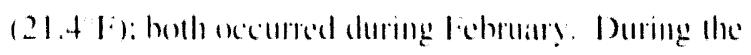
stommer. the recerd maximum momblhy alserage lemperat

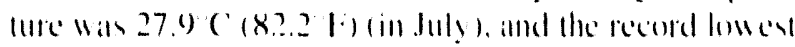
was 17.2 ( (6.3 fi) (in Jume). The anmual an crige relat

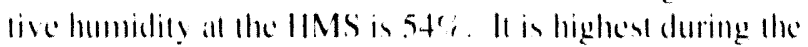
winter months, anelaging about 75'i, and lomest doring

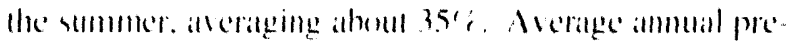

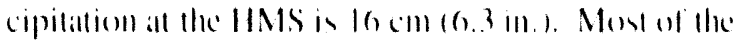
precipitation wecus during the winler. with meatly hall of

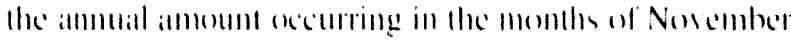
through lechruilly.

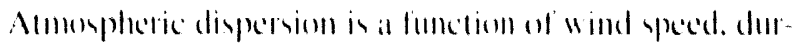

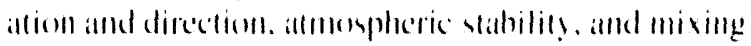
depth. Dispersion comditions ance gemerally gend if wimk

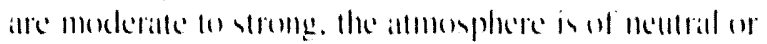

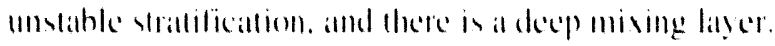

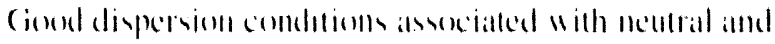

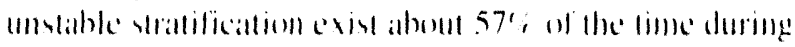

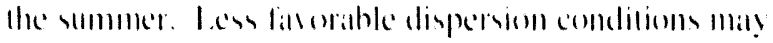
ocedr when dhe wind yeed is light and the mising laster

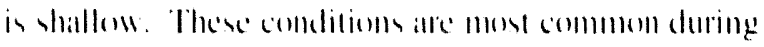
the winter. when moderately la extremely stable statl-

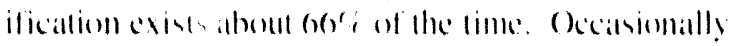
llete are excemeded perionds. primatrils during winter

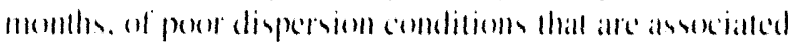

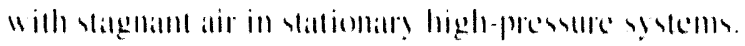

\section{Results of 1992 Monitoring}

The weather in 1902 was much warmer and wetler than

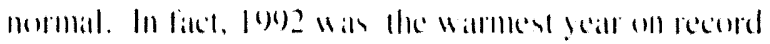

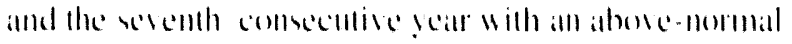
anmual ancrage temperanture. The arerage temperallure

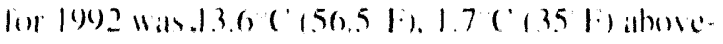

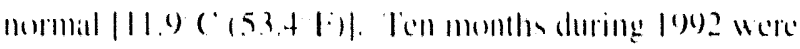

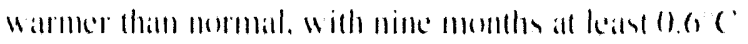

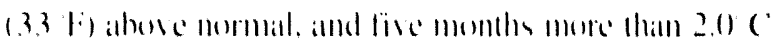

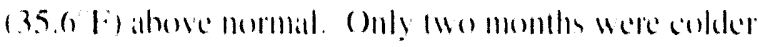

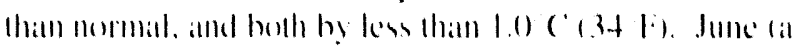
recond warm mombh had the langest positive departure.

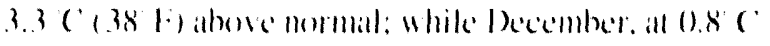

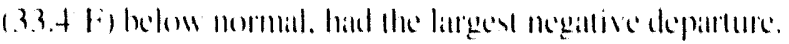




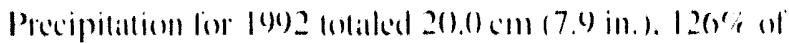

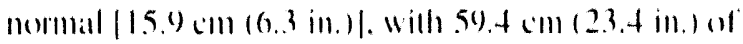

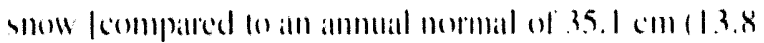
in.)1. Becallse 1902 was Wallmed ham normal. will above normal precipitation and no signilicant cold

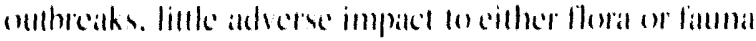
nould be inticipalcel.

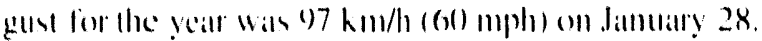

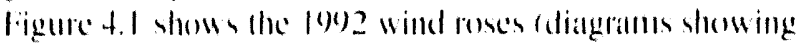

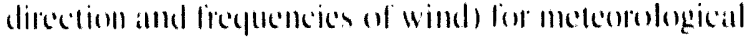
monituring valioms on and alrome the Hantord Site.

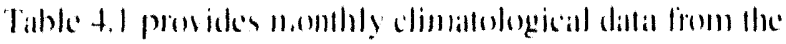

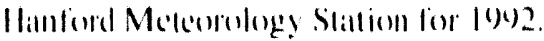

The average wind speed lor 1902 was 11.1$) \mathrm{km} / \mathrm{h}(6.8$ mph). $1 .+\mathrm{km} / \mathrm{h}(1) .9$ mploh helow mormal. and the peath

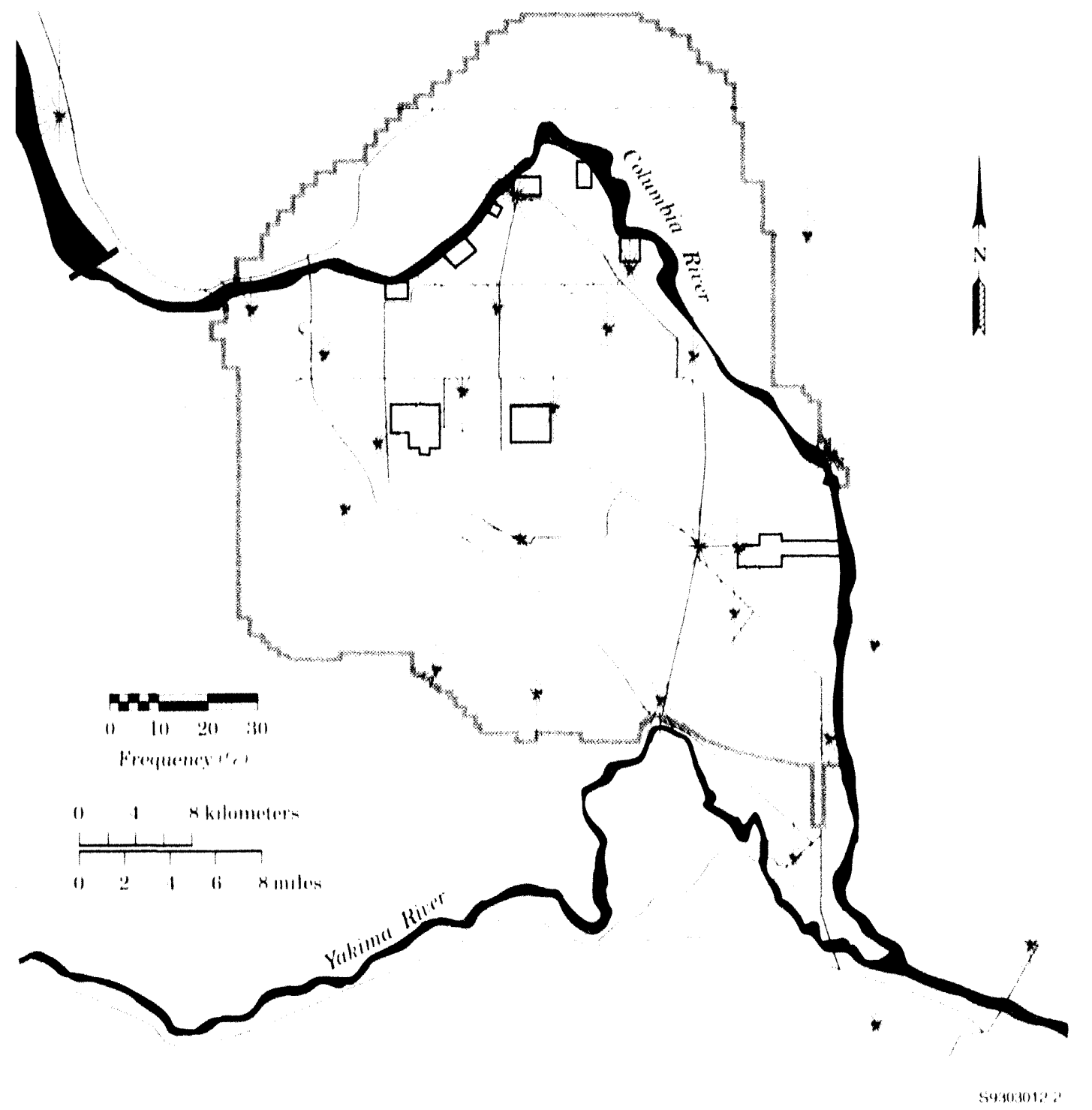

Figure 4.1. Hanford Meteorological Monitoring Network Wind Roses, 1992. Individual lines indicate direction from which wind blows. Length of line is proportional to frequency of occurrence from a particular direction. 
Table 4.1. Monthly Climatological Data from the Hanford Meteorology Station. 1992

Hanford Meteorology Station. 25 Mile X.W. of Richland. WA

Latitude to $34 \mathrm{X}$. Longitude 119 :511. Elevation $733 \mathrm{~F}$

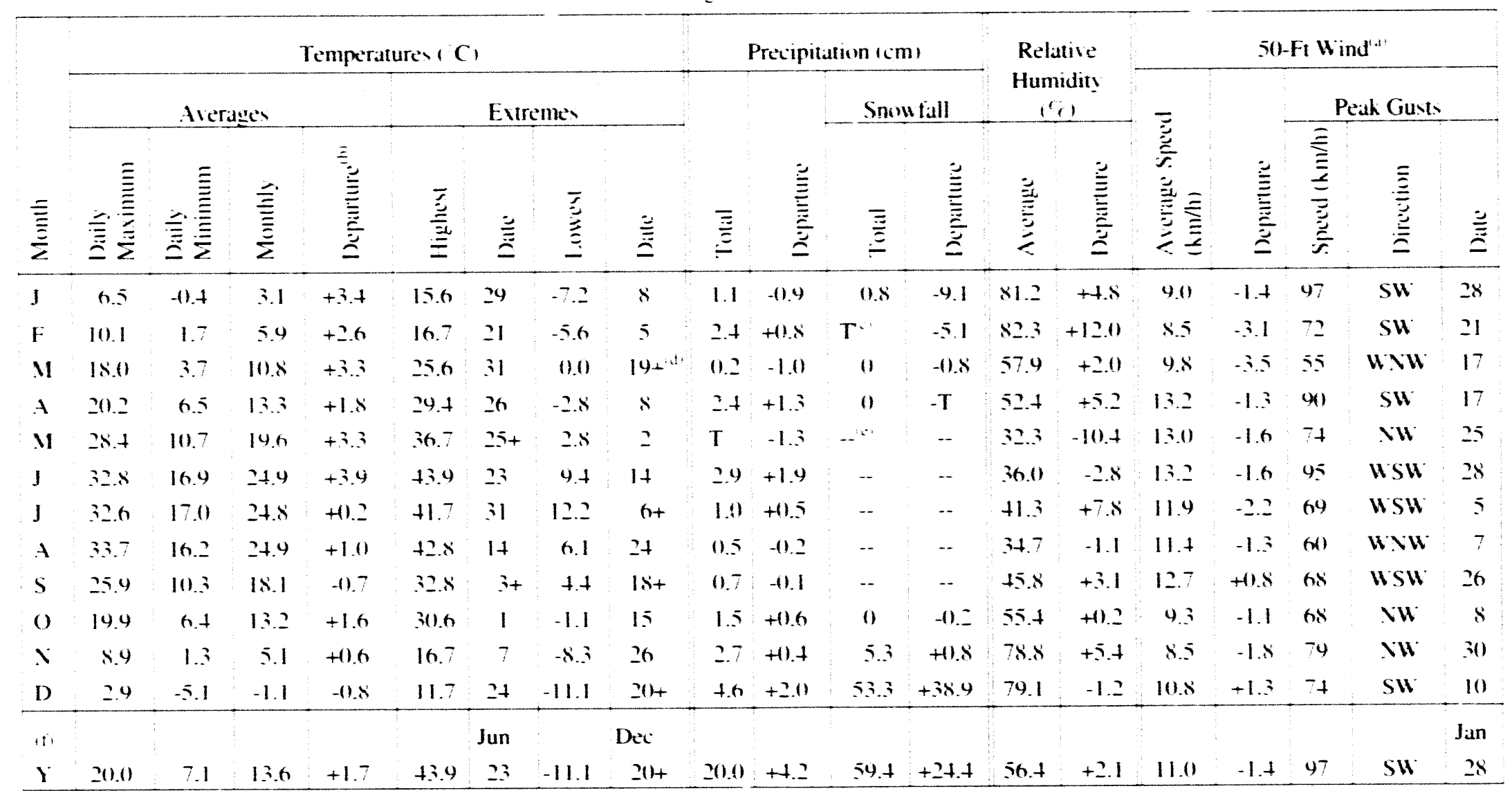

(a) Measured on a touer 50 fit above the ground

(b) Departure columns indicate povitive or negative departure of meteorohogical parameters from 30-year $11961-19901$ climatolingical normals.

(ic) Trace.

td + after date indicates latest of everal occurrences.

(e) -- means no record of an! sou fall during these months.

(f) Yearl alerages. extremes. and totals. 


\subsection{Wildlife}

The Hamford Sile is a relations lange. undisturbed areal

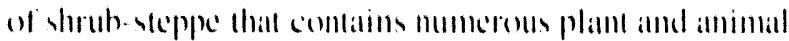

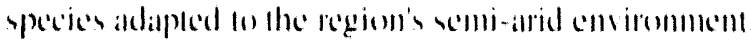

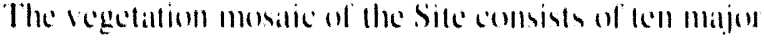

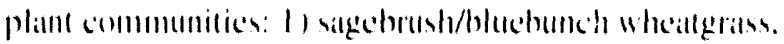

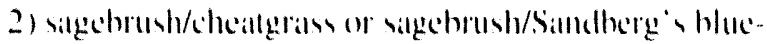
gratss. 3) siggehrush-billerbrush/chealtgrass, th grease

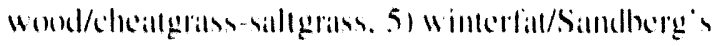

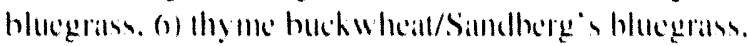
7) chealgram-1umble musland. 8 ) willom (1) ri, antian. 9) sping hopsiger. and lol silld dunes (cushing 1992).

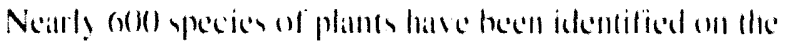

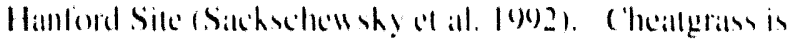

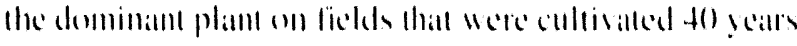
algu.

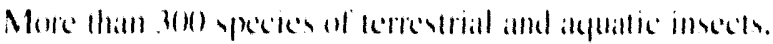

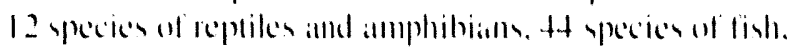

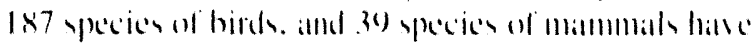

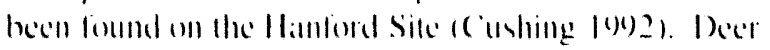

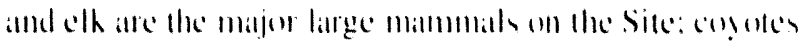

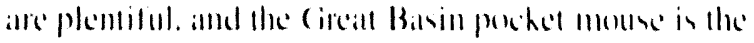
most abumdiml mammall. Watterfom latre mumeroms an the

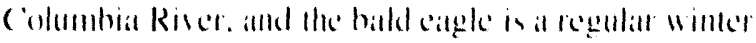
sistor alomg the riser. Sallmom and seelleval alle the lish yecices off most inlerest.

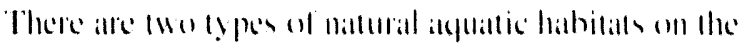

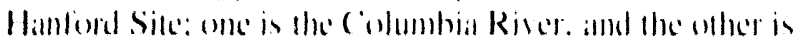

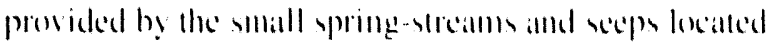
mainly on the Ml.1: Reserve in the Ratllesllake Hills.

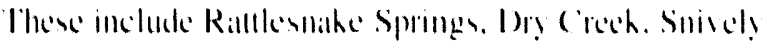

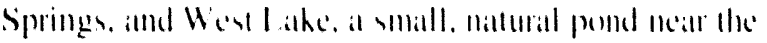

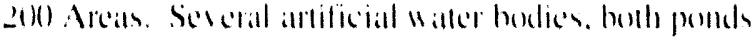

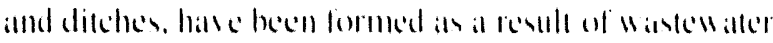

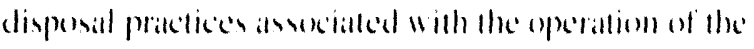

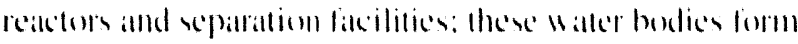

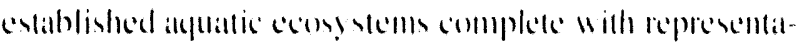

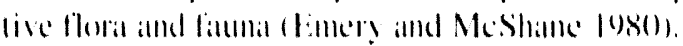

Noplams on mammals om the loderal lis al lindamgered and Theallemed Wildlite and Plans 1.50 ( IR 17.11. 17.121 are hnown an reside fulleme on the Hamlord Sile.

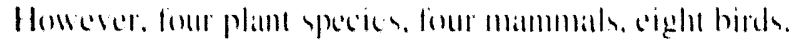

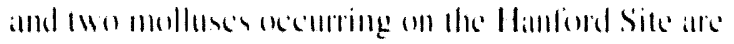

comrently candidales lon formal linding by the federal gorermment and/or Wishinglon State. The lederal gorermment lists the peregring labem an endangered and

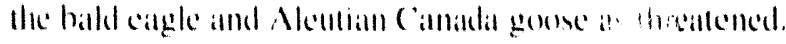
The peregrime falcon and Alemian Camada gonse are migrants lorough the Hanford site. and the bald eagle in at commonn winter resident. Appendix (i lises speciall.

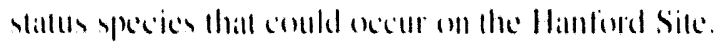

\section{Results for Wildlife Resource Monitoring, 1992}

Wildlile populattom inhathiling the Hanfond Site ane

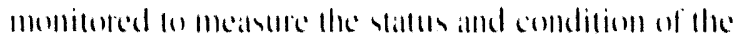

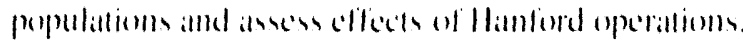

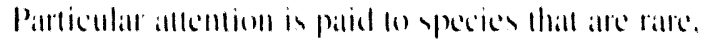
threatlemed. or endamgered mattionally ar statewide and

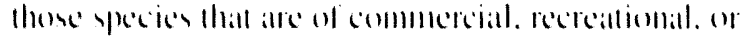

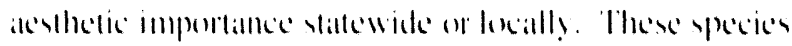

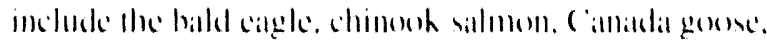

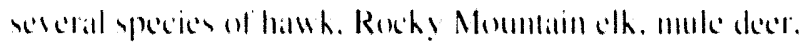
white pelicant. and wher bird yecies.

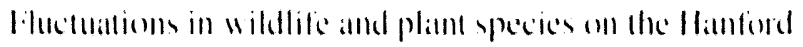
Site aly manigement of the columbia Rier sysem. The cestahlishmeme and mantagement of the llanford Site has had al

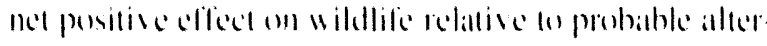
mative mes of the Silce.

\section{Bald Eagle}

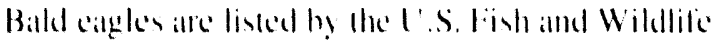

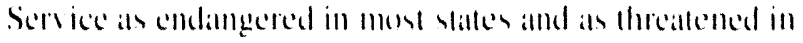
the stalle of Washimglom. Hesterically, hald eagles hatve winlered along the Hanlond Reash of the columbial River. Homerer. when monitoring begall in the carly 196(k. mumbers were very low (ligure 4.2). Following

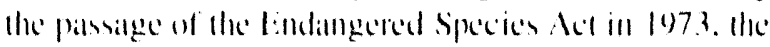
mumber af winlering bald eatyles increased. Passible reasoms for the observed increare are the added 


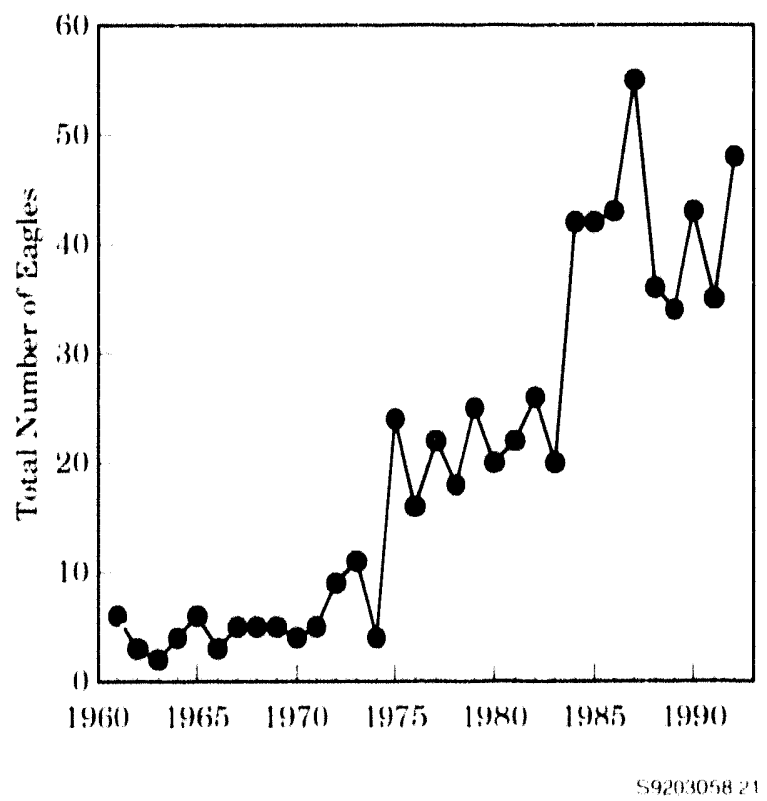

Figure 4.2. Bald Eagles Observed Along the Hanford Reach. Fall and Winter Months, 1962 Through 1992

protection of bald eatgles at menting locallioms off the Hantord Site and the nattomw ide climinatton of dichloro

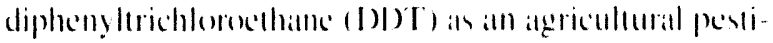

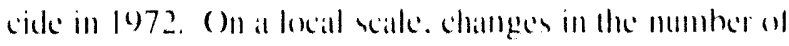

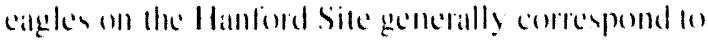

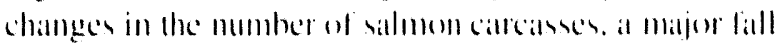
and winter food source for eagles. Mous of the cigles

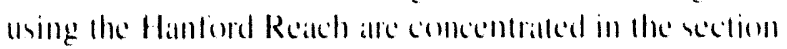

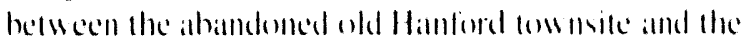
(1)(1)-K Nrcil.

The Hanford Reitch in expected wo continte prosiding wintering hathitit. an long is the criticall resourcen stech ats

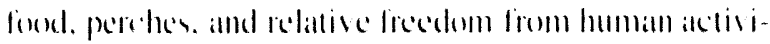
tien are maintained. I. imited nest building by hild eagles

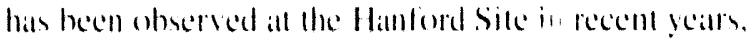

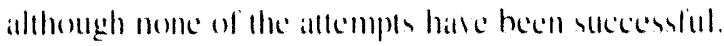

\section{Chinook Salmon}

Chinosh salment ans an important resource to the citisens

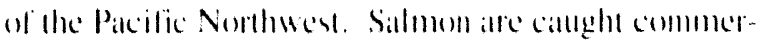
cially and for recreation. The commercial and recreat

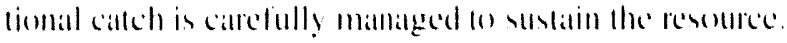

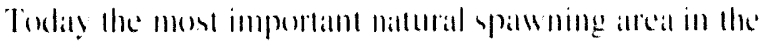
mainstrean Columbia Riser for the Lall chinosoh race in found in the free-flowing Hanlord Reash. In the carly gears of the Hantord Site. Here were few spalwning nests (redds) in the Hanterd Reich (ligune t.3). In the years between 194.3 and 1971. a number of dians were contstructed on the columbia River. The reservoim crealled behind the damb eliminated most mainstem ypawning areas and increased salmont spawning in the Hantord Realch. Fisheries malligement strategies aimed al mainlaining spatu ning populations in the mainsem columbia

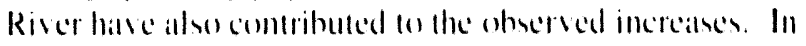
recent ycars. numbers of liall chimosh silmon ypawning

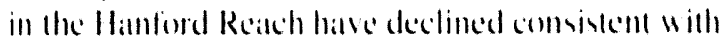
reduced run sisen refurning wo the (olumbia River. The

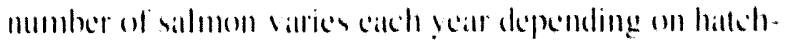

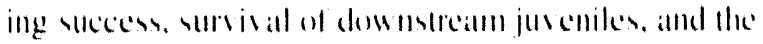
se whe commercial and recreational callehes. The

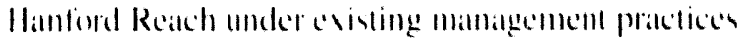

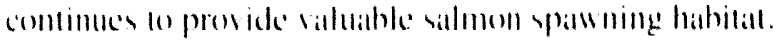

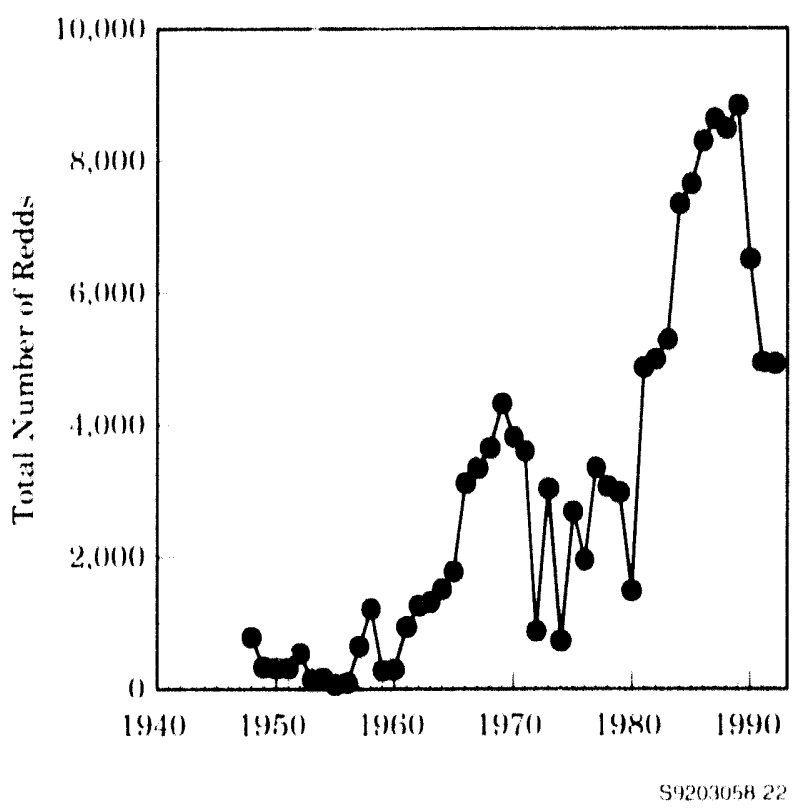

Figure 4.3. Chinook Salmon Spawning Redds in the Hanford Reach, 1948 Through 1992

\section{Canada Goose}

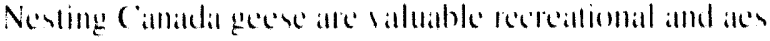

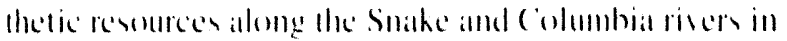
ristern Woshinglon. (

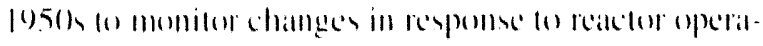
tions lfigare t.t). The gradual decline oberved in the

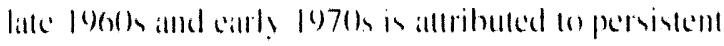




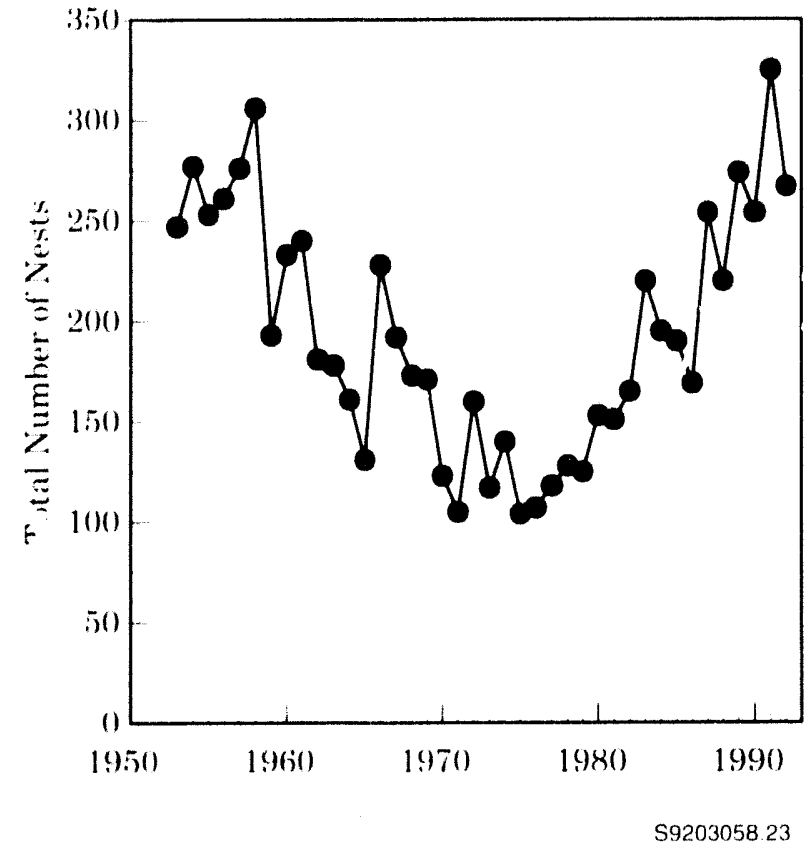

Figure 4.4. Canada Goose Nests on Islands in the Hanford Reach. 1952 Through 1992

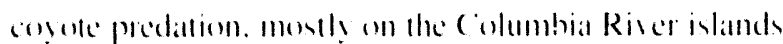
uputeam from the ald Hanford low mile. Since the

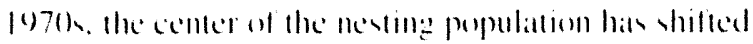

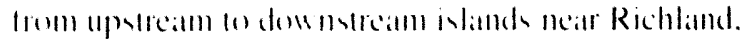

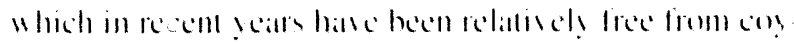

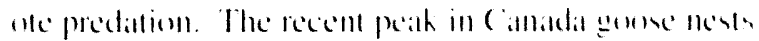

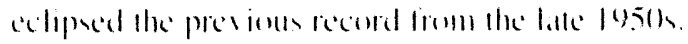

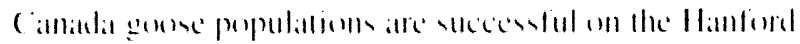

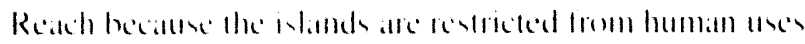

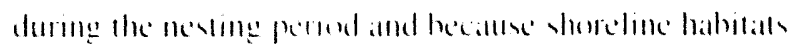

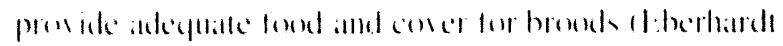

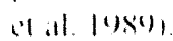

\section{Hawks}

The undereloped lamel of the semi-arid areas at the

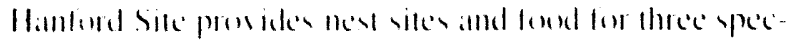

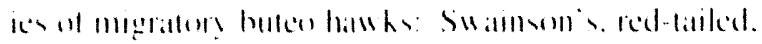

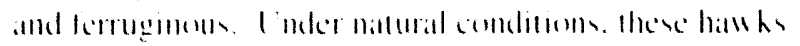

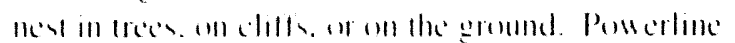

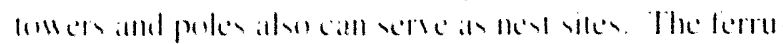

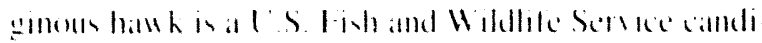

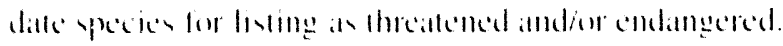

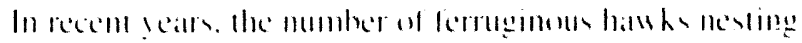

(1) the Hanford Site hat increased frigure +.5 ). Hawks raised on the Hanford Site die during offinite migration and while wintering on ranges far from the Hanford Site. The Site contimues to pros ide hawh mesting habitats administratively profected from human intrusioms, as well as pros idling suitahle foraging areas. The bhatp declines in red-tailed and Swainsonts hawh nests in the latce 1980 atre probably not a resull of Hanford Site activitio because the number of nests lon the very sensilive fermginous hawk did nol decline (lighure 4.5 ). Decreases in nesting red-lailed and Swainson's hawks "lere prohathly related to impales that oceurred during lheir migratton and/or while they were on their wintering grounds.

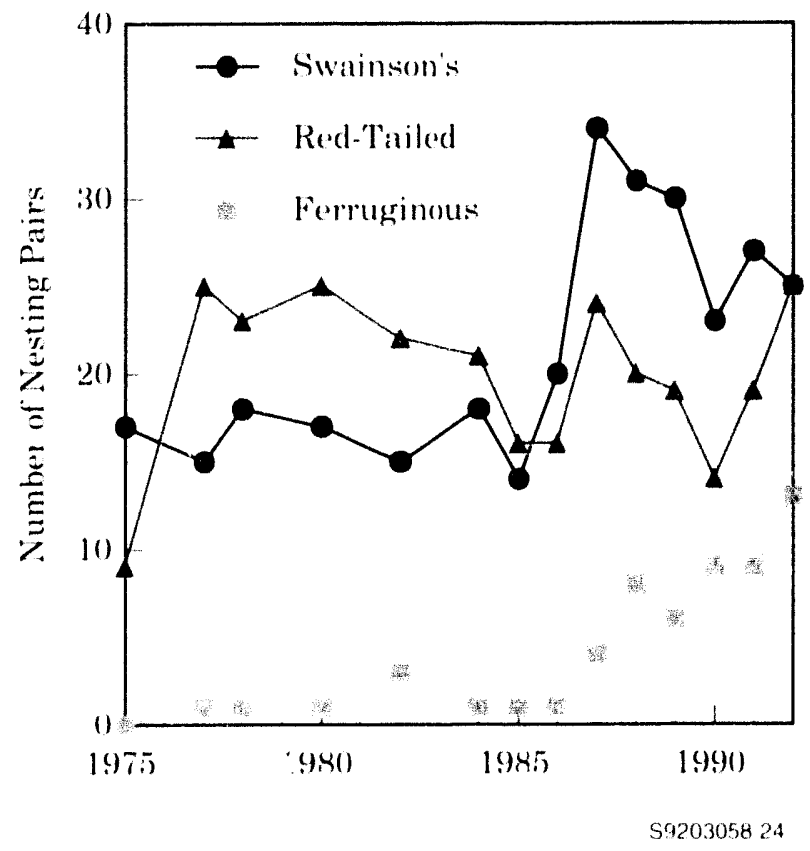

Figure 4.5. Red-Tailed. Swainson's, and Ferruginous Hawks on the Hanford Site. 1975 Through 1992

\section{Rocky Mountain Elk}

Roch! Moumtain elh did not inhabit the Hanford Site

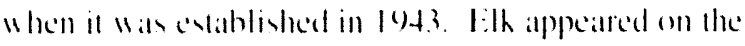
N1.1: Resere in the winter of |972. A lew amimals tated and repredaced. The grealley number of elh

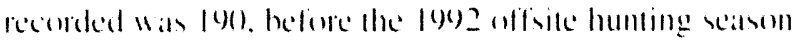

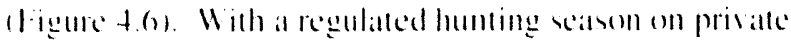




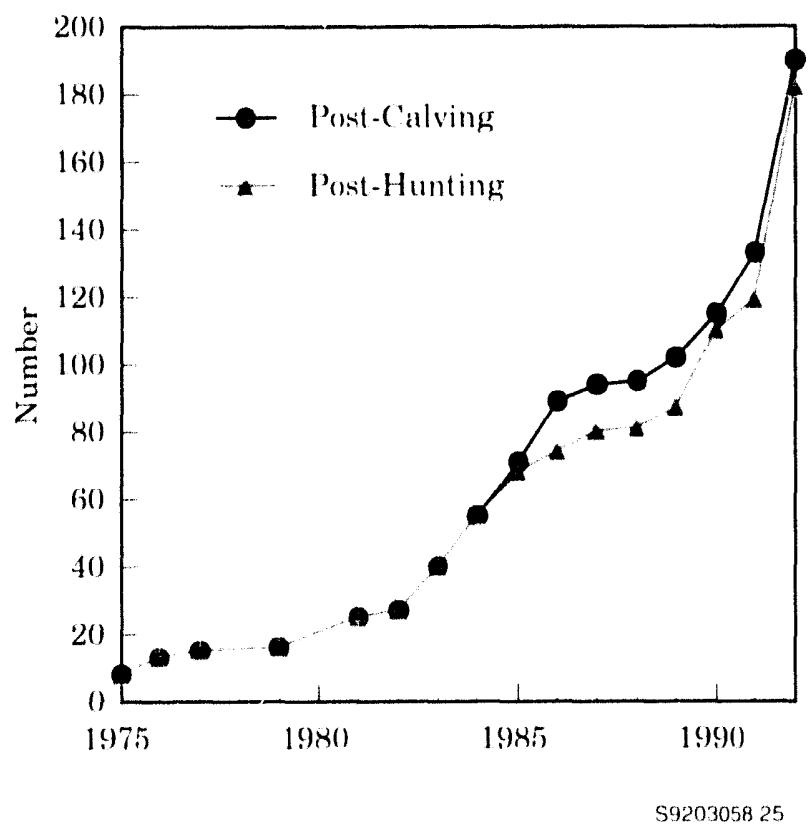

Figure 4.6. Elk on the Hanford Site Counted by Aerial Surveillance During the Post-Calving Period: August Through September; and the Post-Hunting Period: December Through January, 1975 Through 1992

lands atjoining the NI.E: Rescrese the cth population

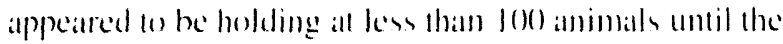
spring of 1900 . Howerer. compatratively lew elk were killed during the past three oflisice hunting seatsoms. and the herd has expanded to ils corrent population of 182 animils.

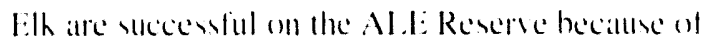
I1 at ailahle forage withoul competition firom domestic liscolect: 2) unrentricted alecess lo drinhing waller all springe located on the AI.E Reserve: 3 l relatively mild winters: f) ability (1) accommodate cxtreme summer temperatlures. exen in the absence of shade: and 5 ) absertec of humbing on the sitc.

\section{Mule Deer}

Mule deer are a commonen resident of the Hantord Site and are important hecatuse of the recreational fort site humting) and alesthetic values they prowide. Becaluse muke deer hase been prolected form humbing on the Hanlord Site lor approximales 50 seams the herd has developed a number of unique pespulation chatracteristics that are in comtrast to most ofher herds in the semianid region of the Northuest. These chatracteristices include a large proportion of old-iage animals and lange-intlered males. This herd provides a unique opportunily for comparison to other more heavily harrested herds in this region.

Becallse of the unigue nature of the herd and high degere of public interest, a study was initialled in 199() (1) 1) ohtain estimatles of the number of deer on the Hanford Site. 2) determine the extent and frepuency of uffeste movements by Hanford Site deer, and 3) evalualle the

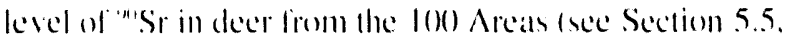
"Wildife Surveillance").

Thity-six mule dee were catprured and litted with eall tages and/or radio collats in 190) herween the old Hanford lownsile and the $N$ Realctor. Frepuent ofliste movements were made by these deer during the year. and one animall wats killed by a hunker during one of these mosements. In general. the mule deer pepulation on the Hamtord Site appears a be healthy. The mumbers of deer an the Hemford Sile do not appeatl to hatre changed dramatlically oner the hast lew deciales, and mally very old $1>5$ gears) animals are present.

\section{White Pelican}

Histerically. the white pelican has risited the Hanford Reach of the (olumbia Rirer in small numbers catch winter. This larger lish-eatling bird has donly recenty heen listed by the Washingten Statce Department of

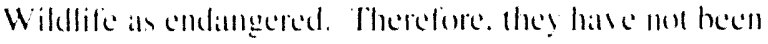
momitored an extemsicely an hald eatgles. Mans of the feillures of the Hamlond Reath that mathe it antratedied wintering hald eagles also mathe it attratelise to white pelicills.

\section{Shrub-Steppe Birds of Special Concern}

The Warhington I epartment of Wildlite has listed ser cral shrub-seppe birk als species of special concern becaluse their pepulations hate heen diminished by massice lomes of nallive shrub-steppe habitall an a resull of expanding agriculare and urbanisation. The Hanford Site contaims large contignums atreals of relattiscly undisturbed shrub-sceple hathilat thall provides nesling hahilat for these hirds. Tu dedermine the spatial distribution and

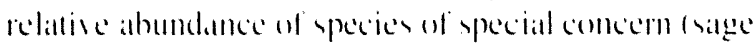


sparr.sws, sage lhrashers, loggerhead shrikes, and longinlled curlews). (wo transects have been monitored on the Hanlord Site over the past 5 years using U.S. lïsh and Wildlife Service procedures. These mansects cross a variety of habitats including relatively undisturbed shrub-steppe, recently hurmed sirub-steppe, and algricultural lields that were abandoned in the early 1940s and alre now dominated by cheatgrass.

The only shrub-steppe species of special concern that nested in the abandoned fields was the long-billed curlew. The sage thrasher was seldom seen along either transect route, which is in agreenent with onlher studies of shrub-steppe birds thatl indicate thatl sage thrashers atre mot abunclant in low-clevation shruh-steppe habitats. Sage sparrows were most common in places that supported stands of sagebrush which hate escaped burning by recent wildtires. Loggerhead shrikes were less plentifiul than sage sparrows and accurred in plates that supported ciller sagehrush or hillerbrush shrubs.

The lower elevations of the Hanford Site provide habitall suitable for viable populations of long-hilled curlews, sage sparrows, and loggerhead shrikes but mot sige thrashers. The long-billed curlew nests on the ground and is not dependent on desert shrubs for mest placement. However, sage sparrows and loggerhead shrikes place their nests in the branches of desert shrubs; thus. the loss of sagebrush and hilterbrush shrubs lhrough burning is detrimental (o) these species.

\section{Special Plants and Invertebrate Animals}

The Washinglon Nattural Heritage Program ( $190(0)$ hats

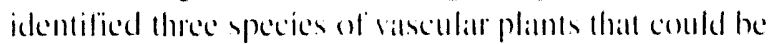
jeopardised by comstruction and/or cleammp atctivitics pertarmed on the Hanford Sile. These species alle
Columbia yelloweress. Columbia milk vetch, and Hower's desert parsley. Columbial yelloweress is listed as an endangered taxon in Washington Statce. Il occurs along the shoreline of the Columbia River on the Hanford Site, Columbia milk veteh is listed as a thealtened laxom and ocedors on dry land of the Hantord Site upstream from the Vermita Bridge. Hoover's desert parsley. also lisked as a lloreatened taxom, oceurs on talus slopes of the Hanfond Site in the same general areat as columbia milk vetch.

The U.S. Fish and Wildlite Service lists the Columbia pebhlestlail and shortiace limper as candidate species for protection as threatened or endangered species. Both inhathit the Hanford Reach of the Columbia River and appear to have been widespread historically in the mainstem Columbia River Basin hetore the installation of dams. Boh species are now apparently redaced within the Columbia Basin to isolated populations that alle separated by large alleas of unsuitable habital.

Only two sizathle populations of ( onlumbia pehblesmail remain: those in the Medhow and Okanogan rivers of north eentral Washington. Neither of these latger populations are protected. Smaller populations survive in the Hanford Reach and elsewhere. Becillse of the lack 1) habitat protection and the substantial redection in the species' hisenrical range, the ('olumbia pebblesmail will probahly he listed federally as endangered

Currently. large populatioms of shortlace limpets persist in four streams: He Dexchules River. ()egents; the Hanlend Reach of the (olumbia River, Washingent Ilells ('anyon, ldaho and ()regent: and the ()kamogan River. Washingtom. Smaller populattoms exist elsewhere. While substantial range reduction has accurred in this species. and the large populations are not protected, the shorllake limper will probably be listed lederally as lineillened. 


\subsection{Other Environmental Studies and Programs}

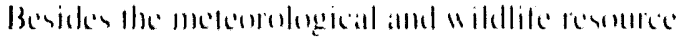
monituring on the Hanford Site. wher vodien and

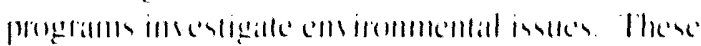

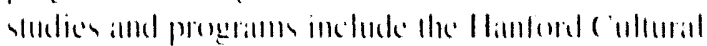

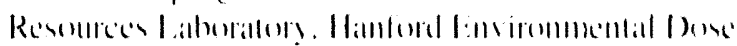

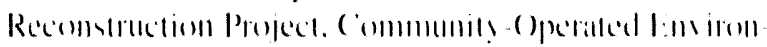

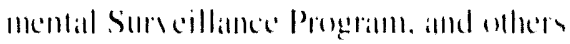

\section{Hanford Cultural Resources Laboratory}

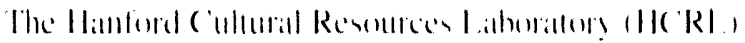

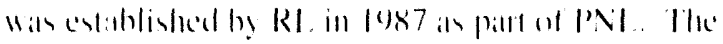

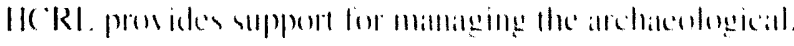

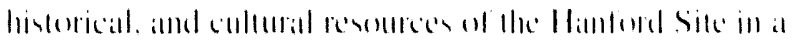

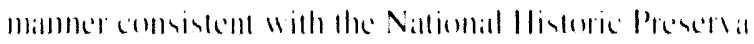

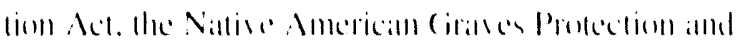

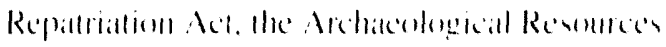

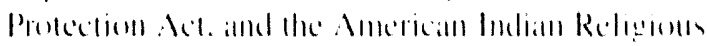
frection Ail.

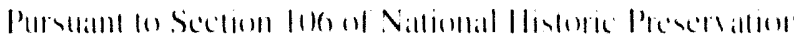

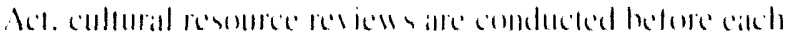

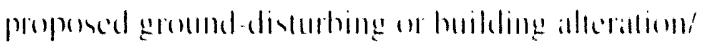

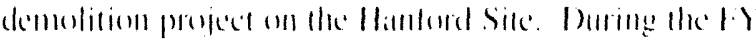

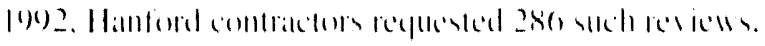

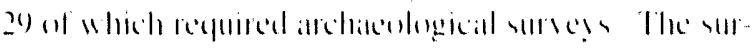

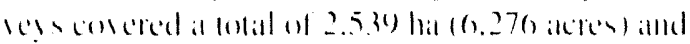

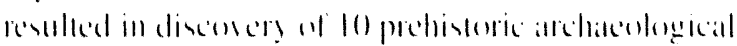

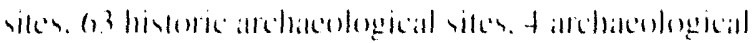

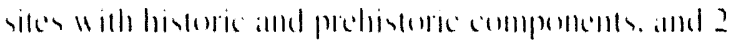

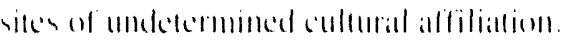

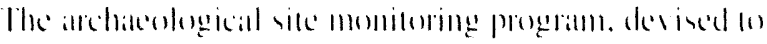

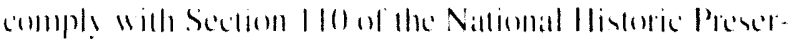

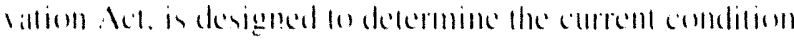

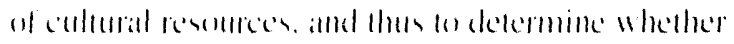

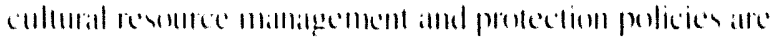

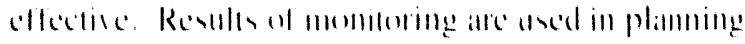

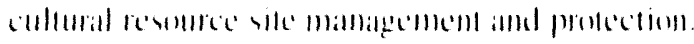

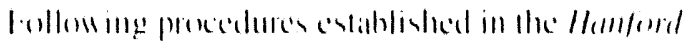

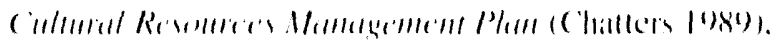

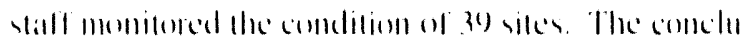

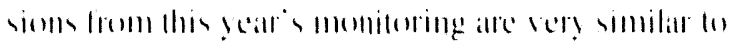

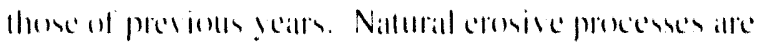

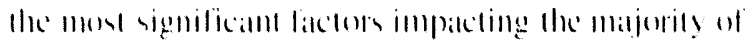

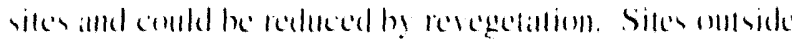

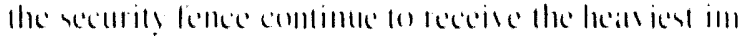

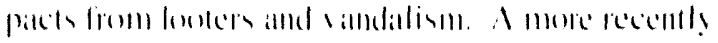

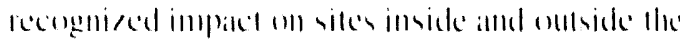

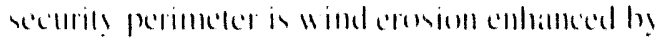

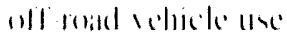

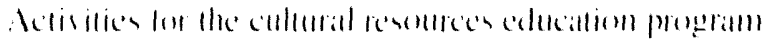

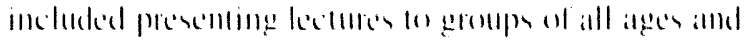

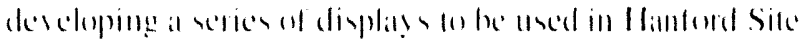

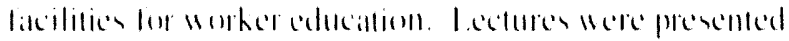

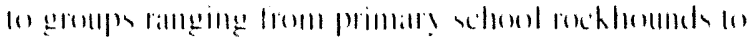

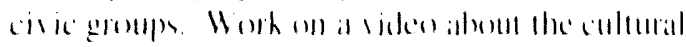

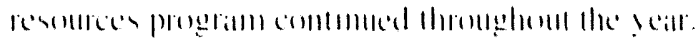

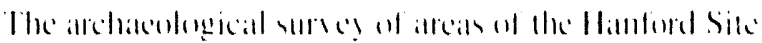

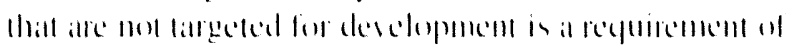

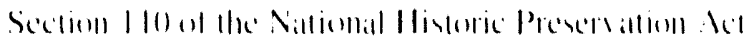
and of los

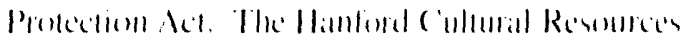

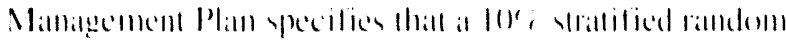

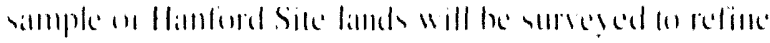

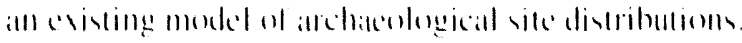

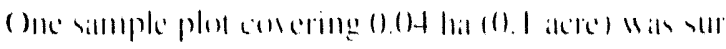

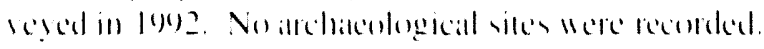

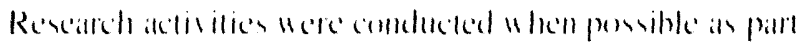

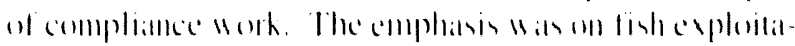

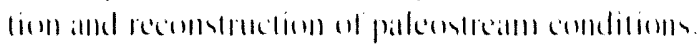




\section{Hanford Environmental Dose Reconstruction Project}

In 1987. alfer receiving a recommondation by the

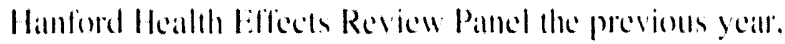

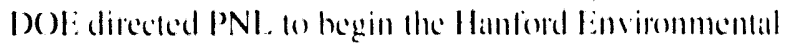
Dose Reconstruction (HI:D)R ) Project. The Hantord Heallh laflects Review Pancl hald been formed lo comsider the pencential heallh implicalloms of historic Hambord Sile releases of radionetive materials.) The objectise of the HI:DR Project is to develop estimatles of the radiation deses that people may hase recened frem parst llanford operattions. An independent Technicall Slecring Pandel was selected by the Viee Presidents lon Rescarch all miljor moiversities of Washingten and oregon to direct the werth of the project. The 18 member pance comsists of experts in valrous technical

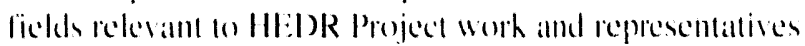
from the states of Washinglon, Oregent. and ldaho: Nallice American tribes: and the publice In $|90|$. responsibility for mantaging the HISDR Project tramsferred to the U.s. Department of Heallh and Human

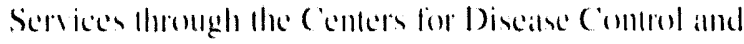

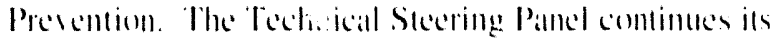
role ats the rechnical direstor at the work.

In 199(). scientios compleded the libst phase of the III: )R Project, which was to delermine whelher enough

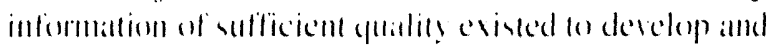

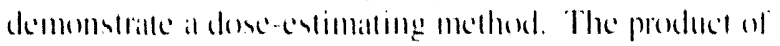
this phase was a set of mote Hatll 20 decumems that dencribe:

- He preliminars information foumd or reconvilleded

- meliminary dose-estimating models amd computcir couks

- preliminary entimatles of done and their ancer-

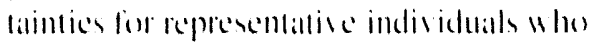
maly hate lived neatr the Hanford Silc during cally years of operallioms.

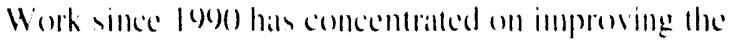

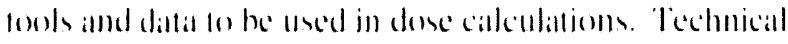
work lor loc) 2 comsisted of restructuring modelo lo

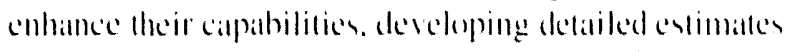

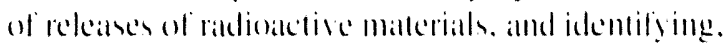

acequiring, and evaluating additional inlomantion needed 10 produce estimalles. This information is heing devel.

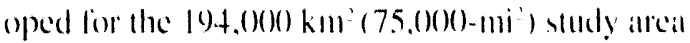
highlighted in Figure 4.7. lor major exposture pathwalys. and for he full history of the Hanliond Sile 194t through 1901.

In addition lo work heing performed al PNI. ceghts northwest Native American tribes are condeding rescatrch fos support dose estimales lor their tribal members.

The key deliserables of the project are the fouls lor the estimation of doses lo real individuals comrently being interviewed by stalf from the fred Hutchimson (innere

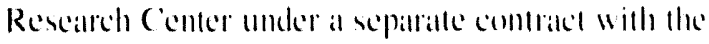

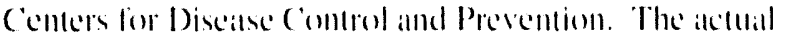
life histories of these individuals will he ased to prodece accuratle dose estimalles.

\section{Community-Operated Environmental Surveillance Program}

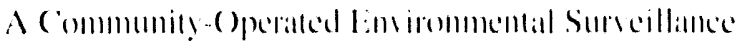

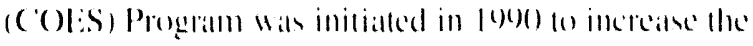

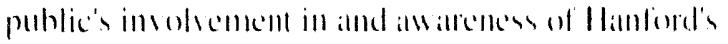

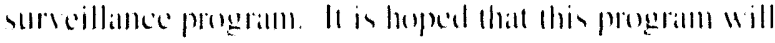
increane public understandeng of surveillance resulls,

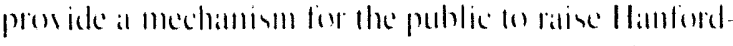

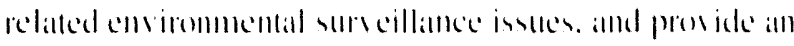

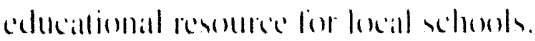

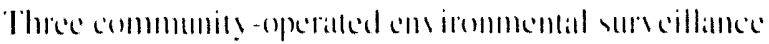

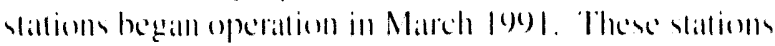

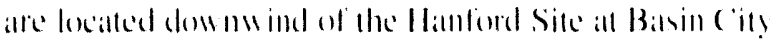

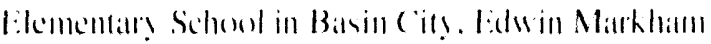

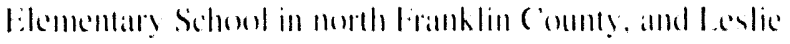

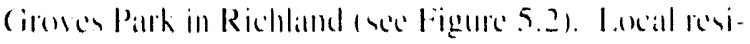

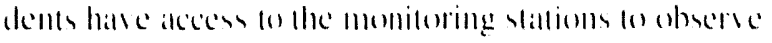
the imstuments alld resules.

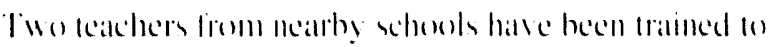

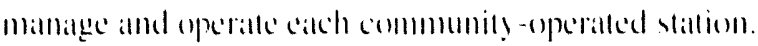

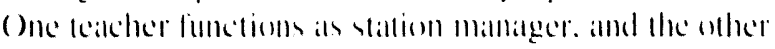

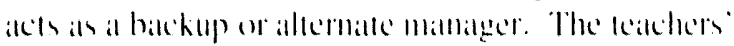

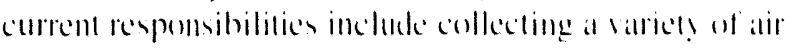
silmples. preparring simples and conllections records lon 


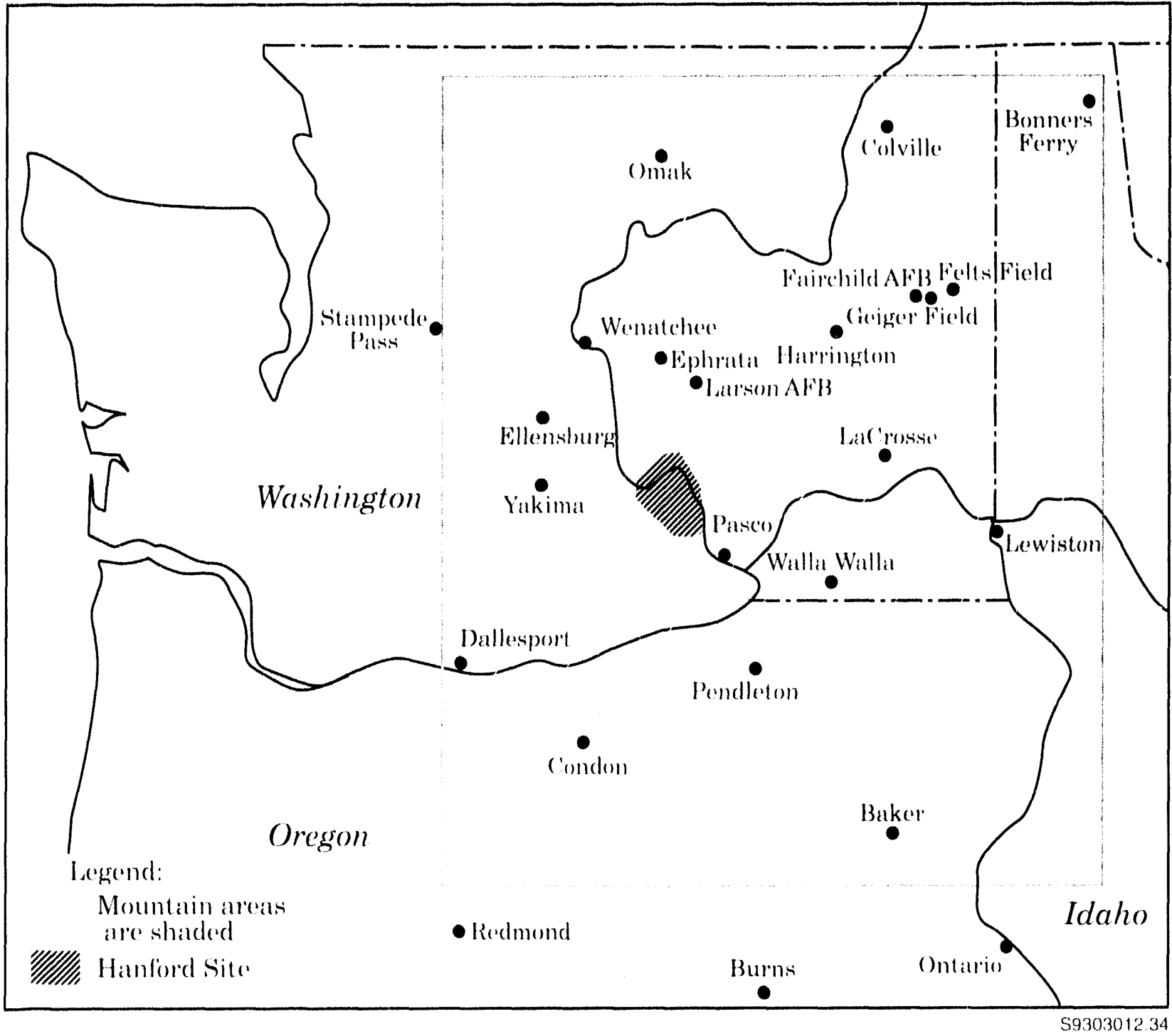

Figure 4.7. Area Considered in Estimating Doses from Past Hanford Operations in the Hanford Environmental Dose Reconstruction Project

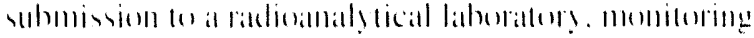

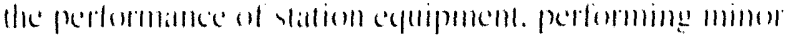

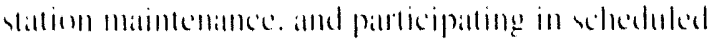

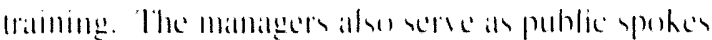

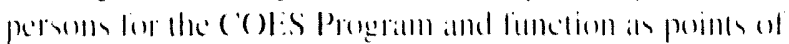

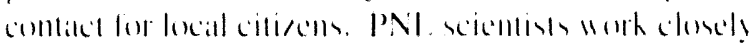

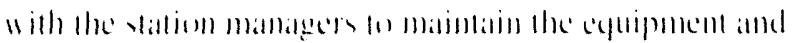

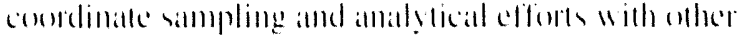

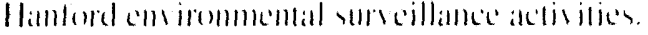

Resulls from llese statloms alle discunsed in Sections 5.2.

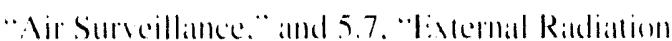
Survillante." 


\section{Other Environmental Activities}

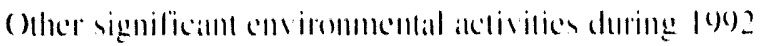
included comprilation of a Hanlond Sile land use planning intialtive. the contimuation of a Nattemall Park Semice

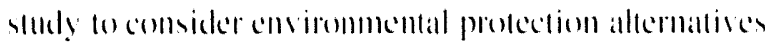

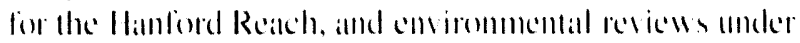

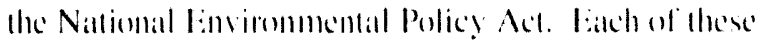
activitices is stummarised in Section 2.1). "Ins irommemtal compliance Summary."

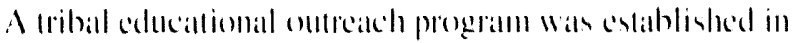
f(9)| lo monitor water guality and lish heallh in the

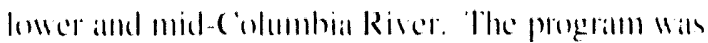

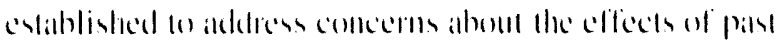
and present (1)perallioms all the Hanforel Sile on waller yuality and the heallh of silmon and fiste comsumers.

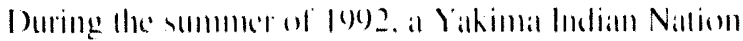

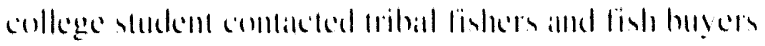
alomg the Columbia River helween MeNally and

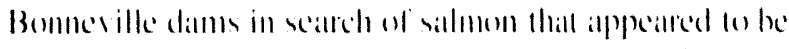

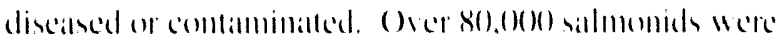
callyght dering lle study, and + lish were reported ats

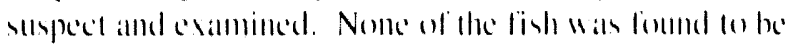

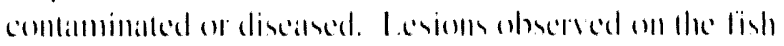

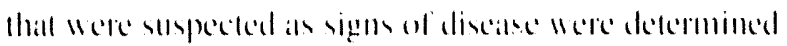

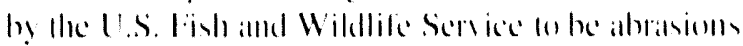
calluced by gill mels. 


\section{Environmental Surveillance Information}

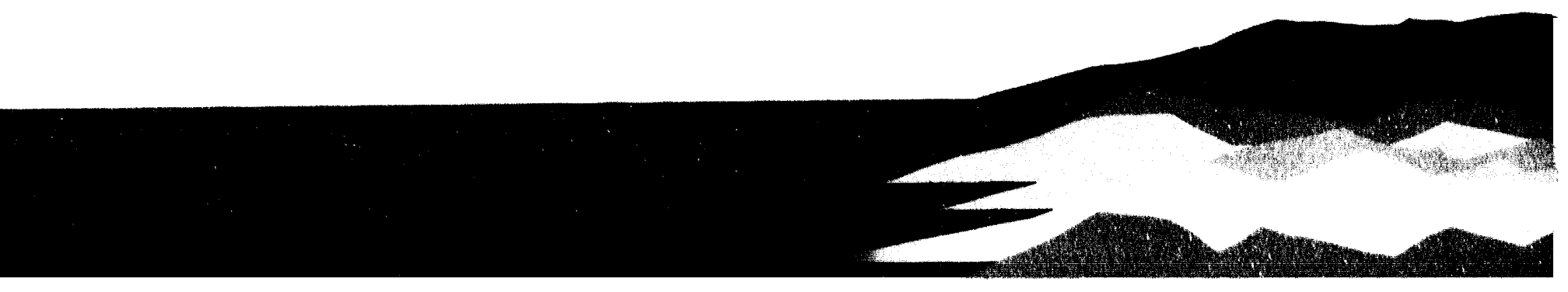




\subsection{Environmental Surveillance Information}

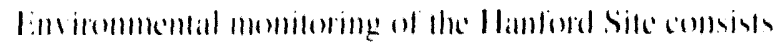

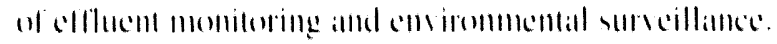

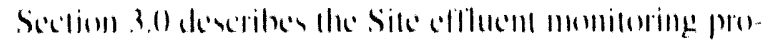

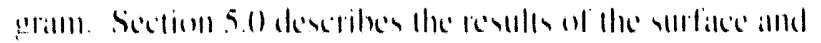

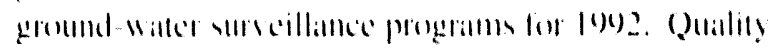
aburamce and control for moniloring proghams are

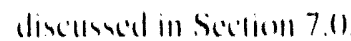

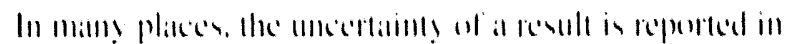

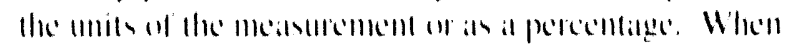

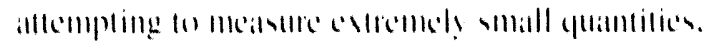

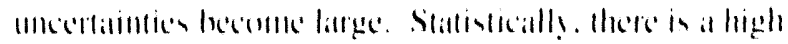

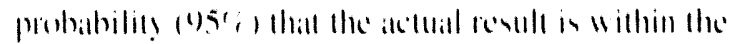

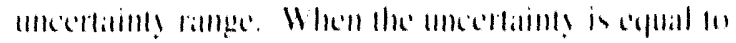

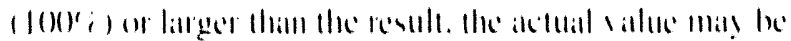

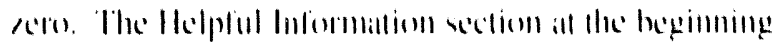

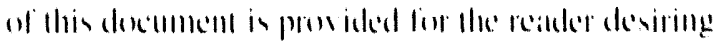

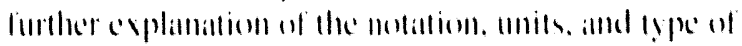
intirmantion heing reperted.

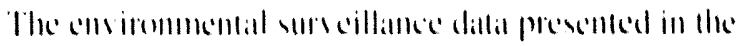

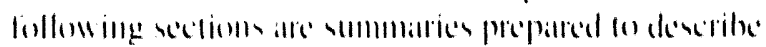

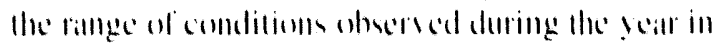

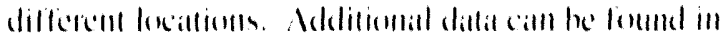

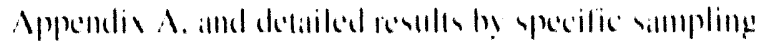

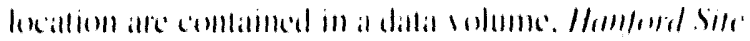

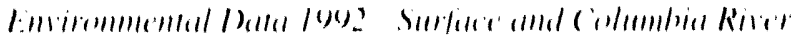
(Bisping and Wimdrull l(2)3). 


\subsection{Environmental Surveillance at Hanford}

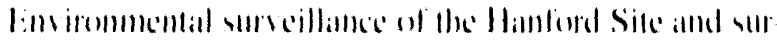

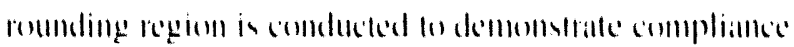

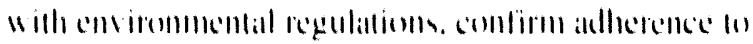

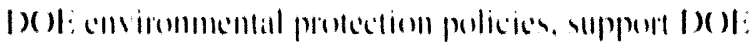

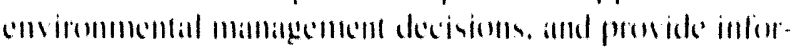
mation for the public. Sum cillante is comducted an all

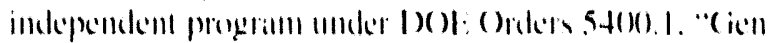

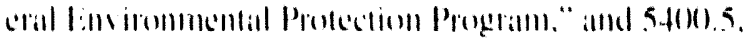

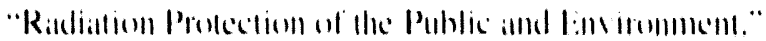

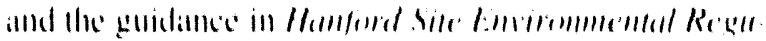

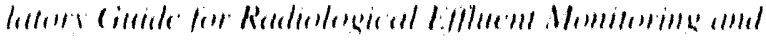

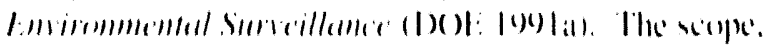

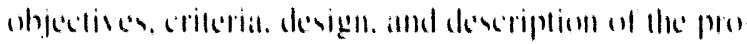
glamm atre stommarised belum and pros ided in detail in the

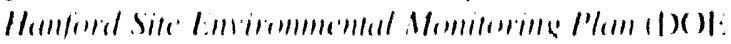
(1) $) 1111$.

\section{Scope}

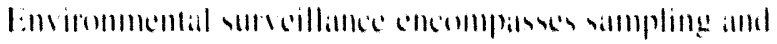

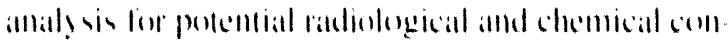

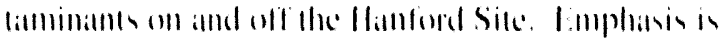

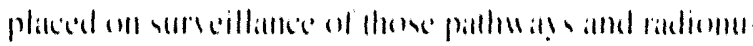

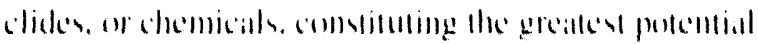

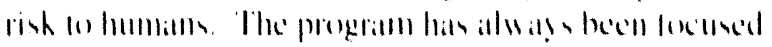

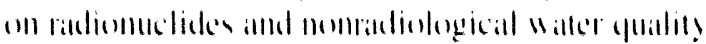

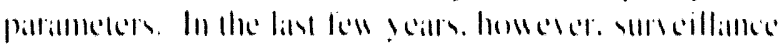

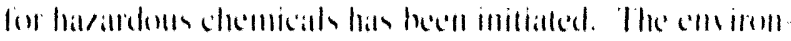

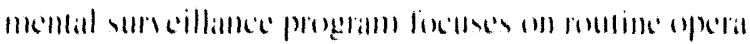

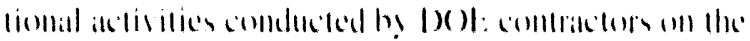

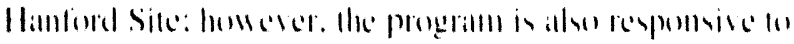

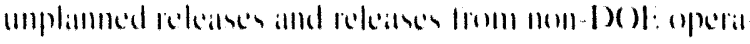

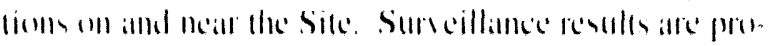
vided almmally through this repunt series. In additions.

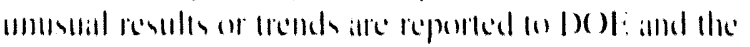

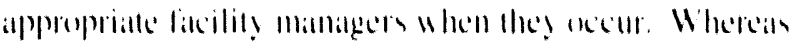

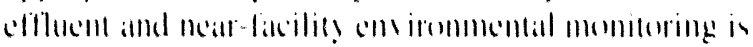

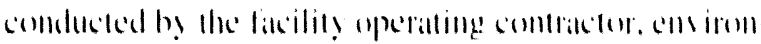

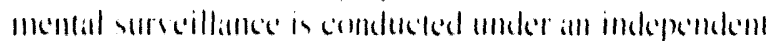

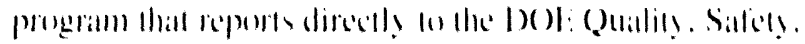
indel Heallh Proglatmo Divinion.

\section{Objectives}

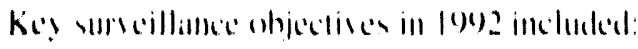

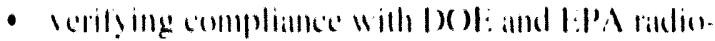

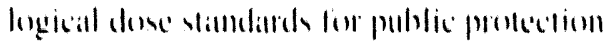

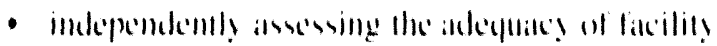
pollutions conllow

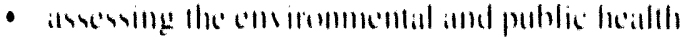

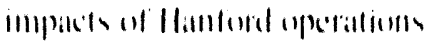

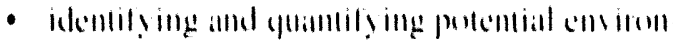
monlitl yualim! poublems

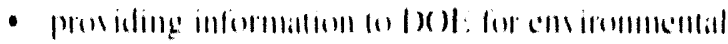

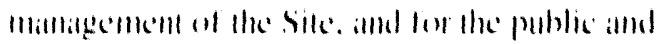

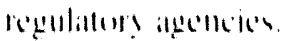

\section{Criteria}

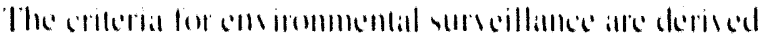

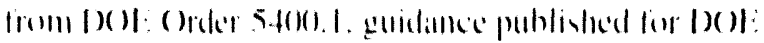

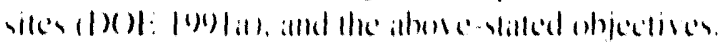

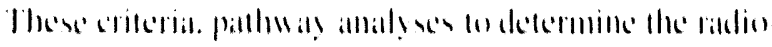

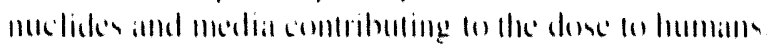

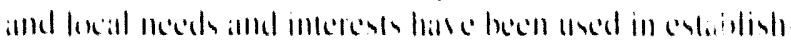

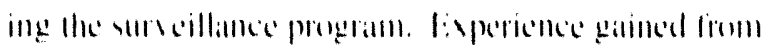

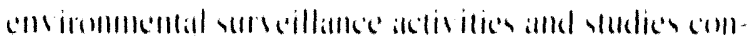

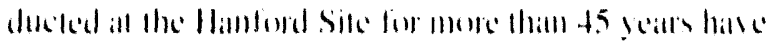

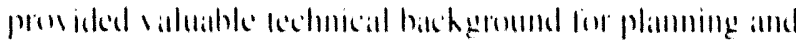
datla inlerpretitions.

\section{Surveillance Design}

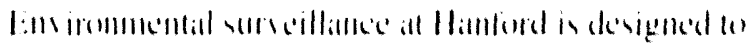

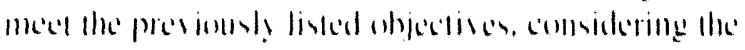

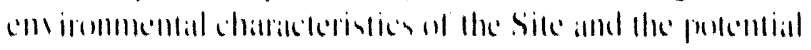


and actual releases from Sile andivities. The main focus is on defermining envirommental impacts and compliantes will puhlic heallh standards, an well as envirommental

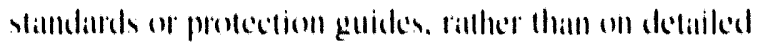
radiological and chemiscal chatracterisationl.

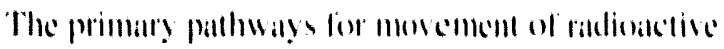
materials and chemicals bom the Sile fo the publis are

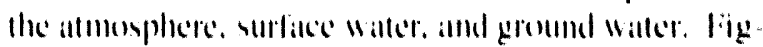

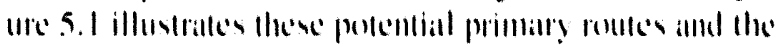

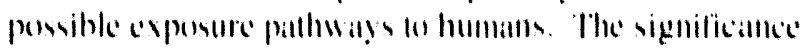

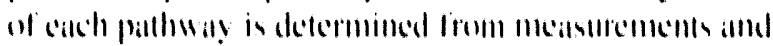

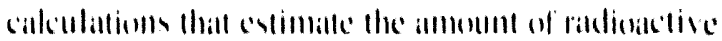

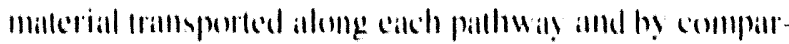

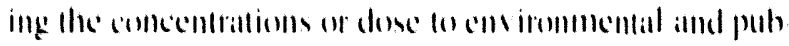

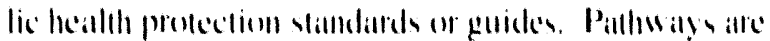

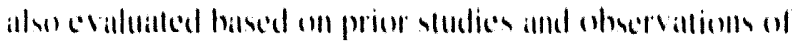

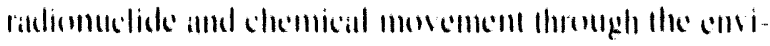

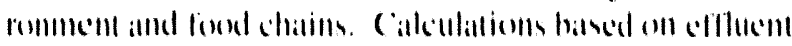
data show the expected concentrations aff the Hamberd

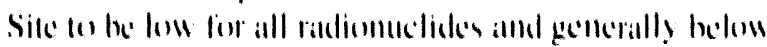

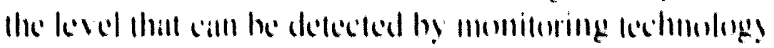

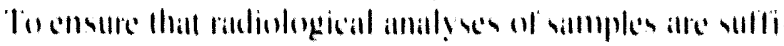

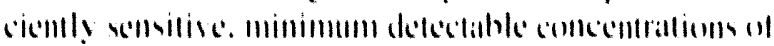

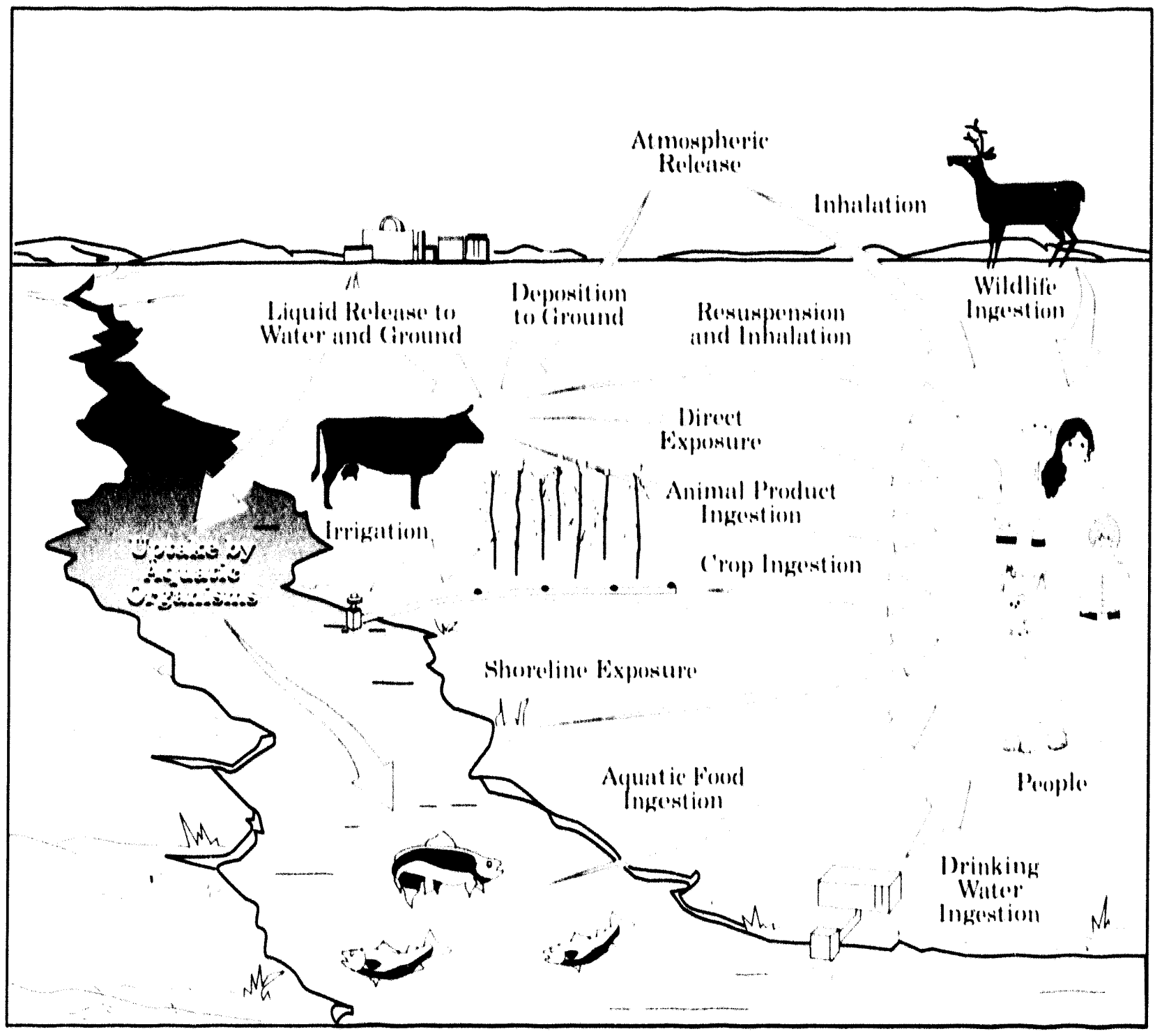

S920:3058 1346

Figure 5.1. Primary Exposure Pathways 


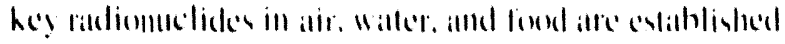

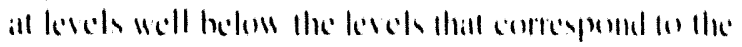
standiats.

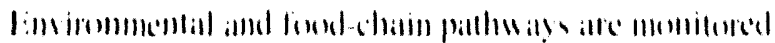

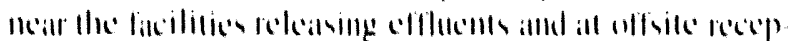

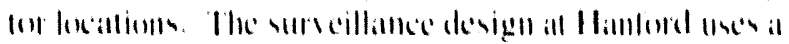

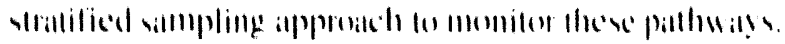

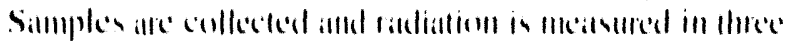

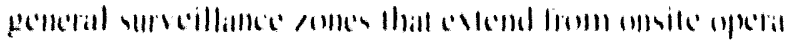

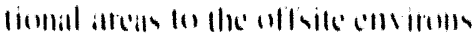

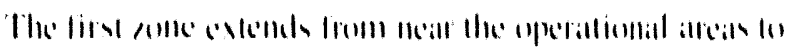

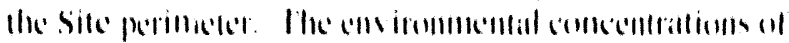

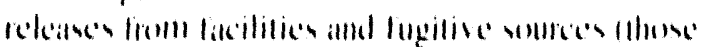

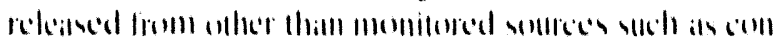

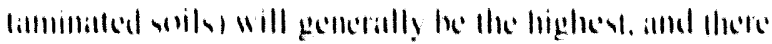

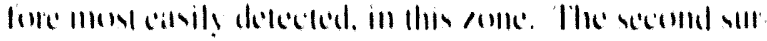

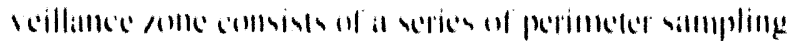

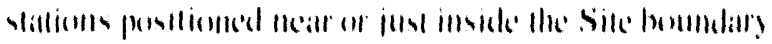

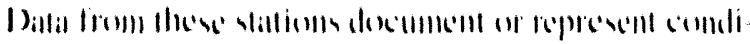

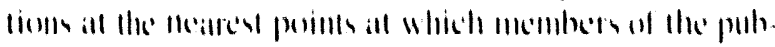

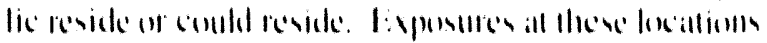
alle lypicially the maisimum thal an! member of the pub.

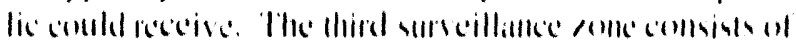

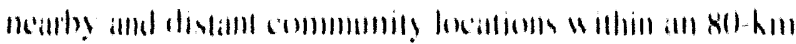

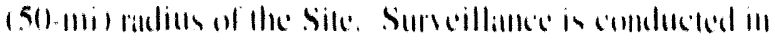

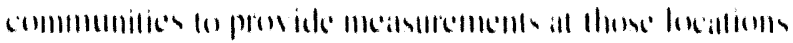

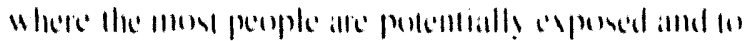

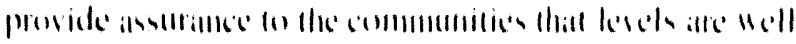

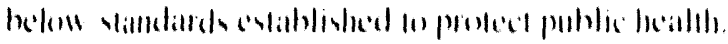

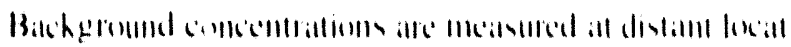

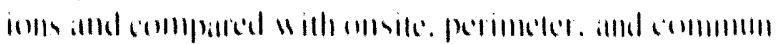

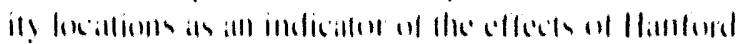

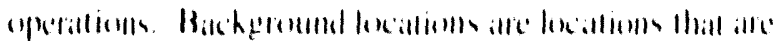

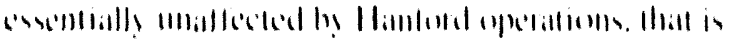

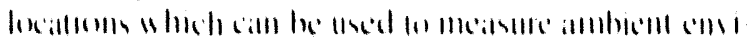

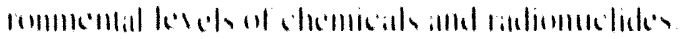

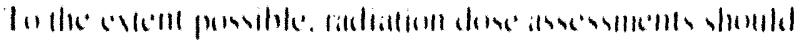

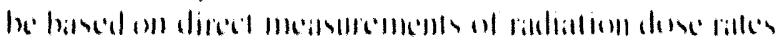

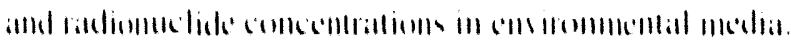

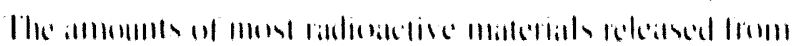

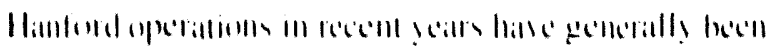

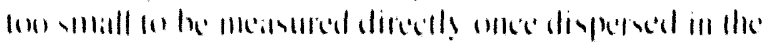

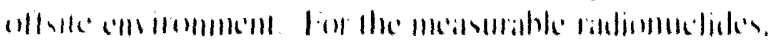

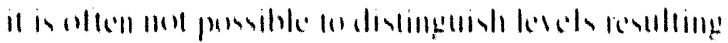
from

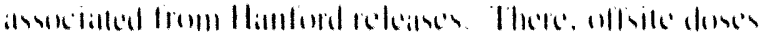

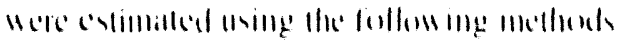

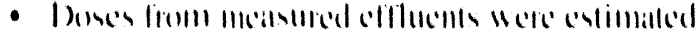

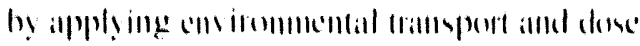

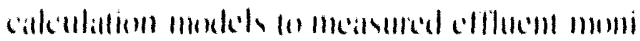

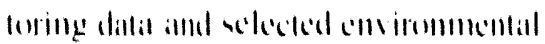
mleisuremlents.

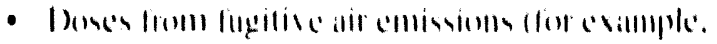

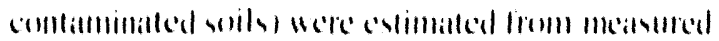

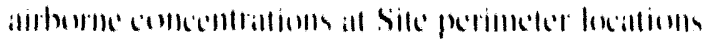

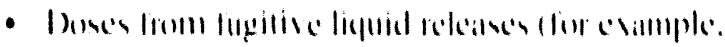

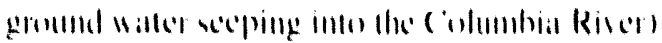

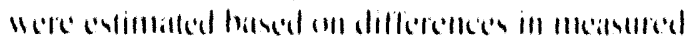

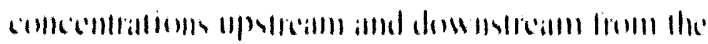
Ilanlonil Sile.

\section{Program Description}

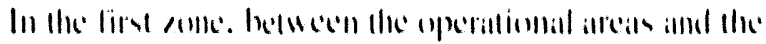

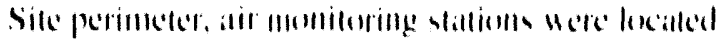

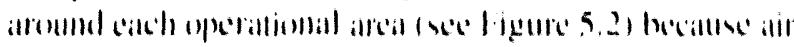

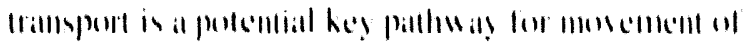

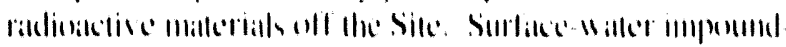

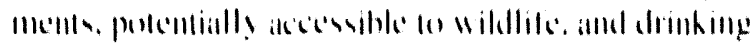

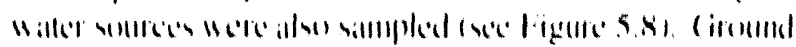

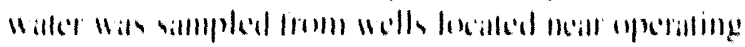

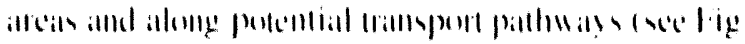

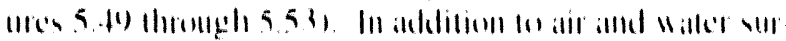

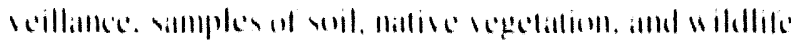

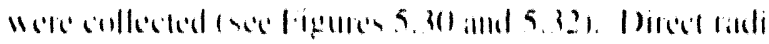

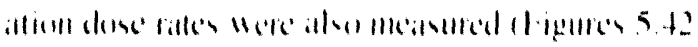

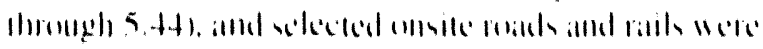

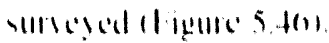

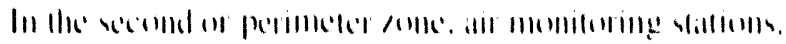

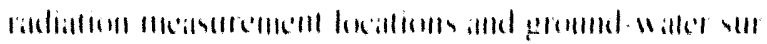

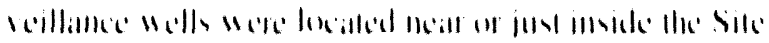

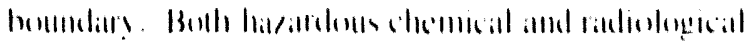

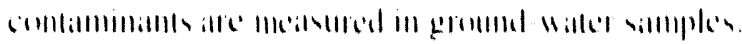

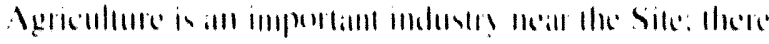

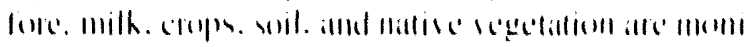

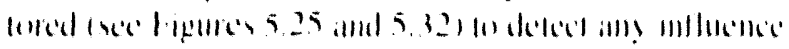

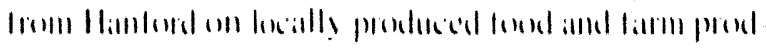

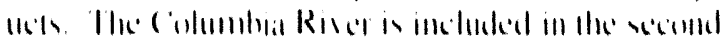

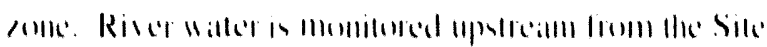

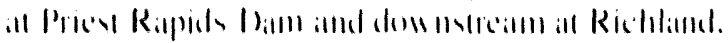

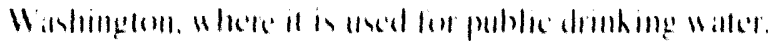

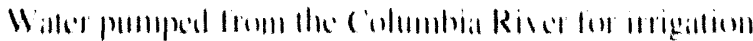

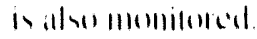




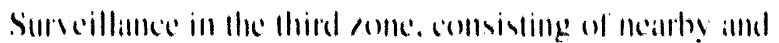

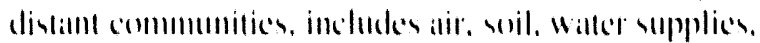

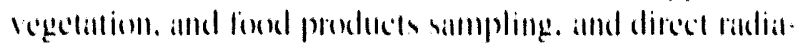

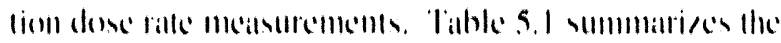
geographic distrihution of measurement lecittoms.

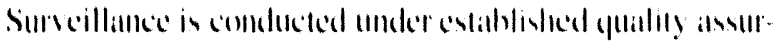

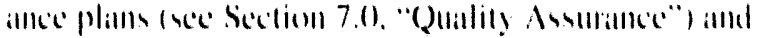

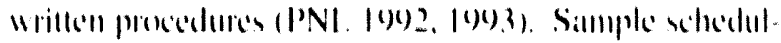

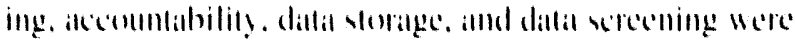

mamiged and comtrolled by compunteriad systems. I abo

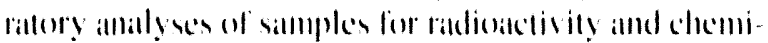

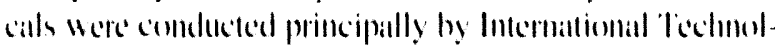

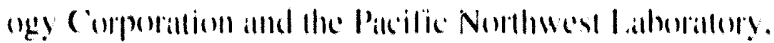
besh in Richlamd. Washinglent. Selected river waller gual-

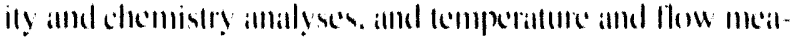
strements were pertemmed by the U.S. (icological Survey. benver. cindorido. 
Table 5.1. Environmental Surveillance Sample Types and Measurement Locations. 1992

\begin{tabular}{|c|c|c|c|c|c|c|c|c|}
\hline \multirow[b]{3}{*}{$\begin{array}{l}\text { Totial } \\
\text { Number }\end{array}$} & \multicolumn{7}{|c|}{ Sample Locations } & \multirow[b]{3}{*}{ Downstream } \\
\hline & & & & & & \multicolumn{2}{|c|}{ Columbia River } & \\
\hline & Onsite & $\begin{array}{l}\text { Site } \\
\text { Perimeter }^{\text {h }}\end{array}$ & $\begin{array}{l}\text { Nearby } \\
\text { Communities. }\end{array}$ & $\begin{array}{l}\text { Distant } \\
\text { Communities }\end{array}$ & $\begin{array}{l}\text { COES } \\
\text { Stations }\end{array}$ & Upstream & $\begin{array}{l}\text { Hanford } \\
\text { Reach }^{\text {hi }}\end{array}$ & \\
\hline 45 & 23 & 13 & 4 & 2 & 3 & & & \\
\hline+36 & 436 & & & & & & & \\
\hline 3 & & & & & & & & 3 \\
\hline 4 & & & & & & 2 & 1 & 1 \\
\hline 1 & & 1 & & & & & & \\
\hline 13 & 8 & $5:$ & & & & & & \\
\hline 6 & & & & & & 1 & 3 & 2 \\
\hline 3 & 3 & & & & & & & \\
\hline 11 & & 5 & 5 & $\mathbf{i}$ & & & & \\
\hline 9 & 9 & & & & & & & \\
\hline 16 & 4 & $s$ & & 4 & & & & \\
\hline 74 & 28 & $37^{t}$ & 4 & 2 & 3 & & & \\
\hline 15 & 14 & $!$ & & & & & & \\
\hline 16 & & 16 & & & & & & \\
\hline
\end{tabular}

(a) Surveillance Zone 1 .

(b) Surveillance Zone 2 .

(c) Surveillance Zone 3.

(d) COES = community -operated environmental surveillance.

(e) Approximately 729 wells were sampled for all ground-w ater monitoring programs onsit:

(f) Includes four off site water supplies.

(g) TLD: = thermoluminescent dovimeter

(hi Includes locations in and along the Columbia River. 


\subsection{Air Surveillance}

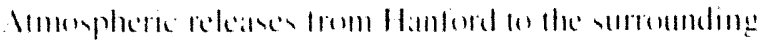

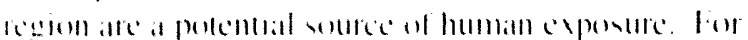

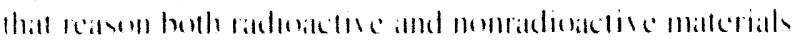

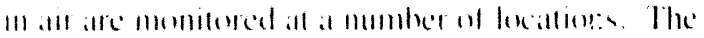

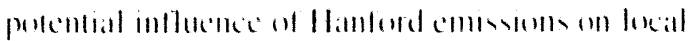

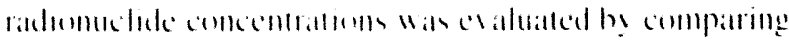

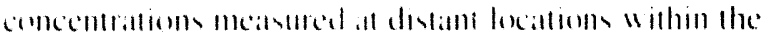

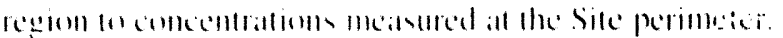

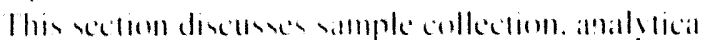

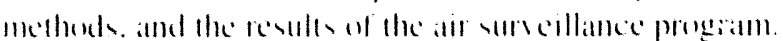

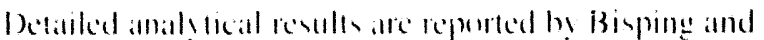
Hindrulf (1)031.

\section{Sample Collection and Analysis}

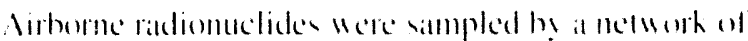

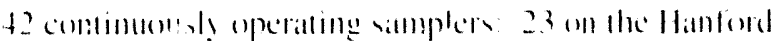
Site. 13 near the Silc perimeder. 4 in meath commmmitices.

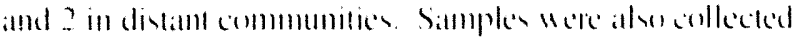

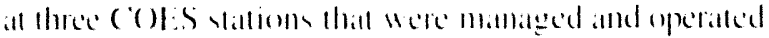

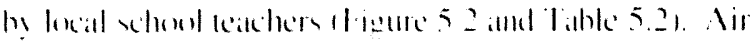

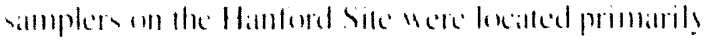

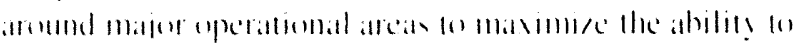

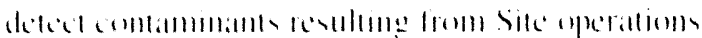

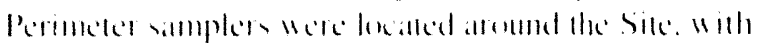

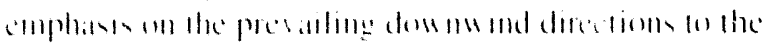

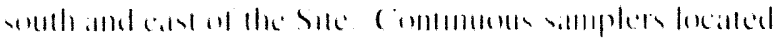

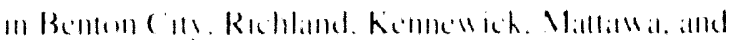

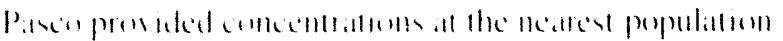

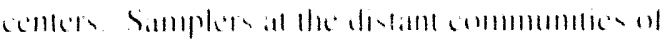

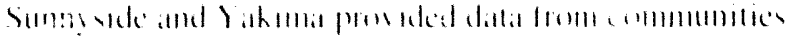

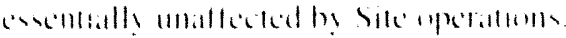

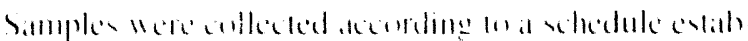

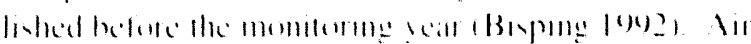

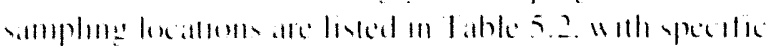

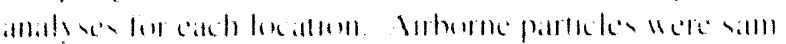

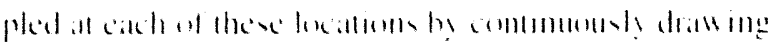

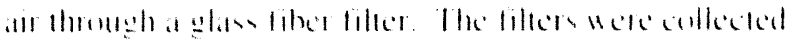

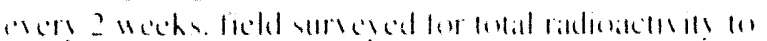

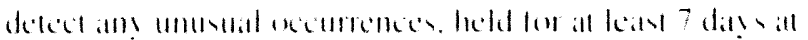

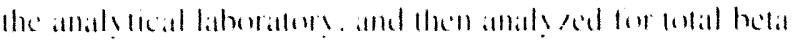

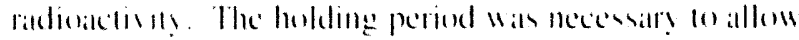

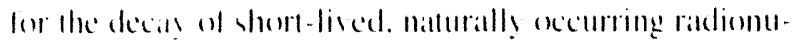

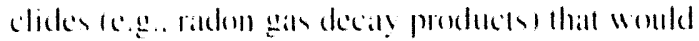

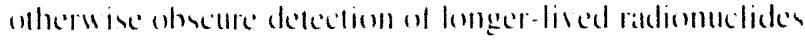

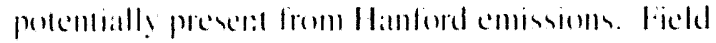

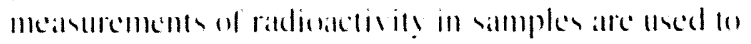
monitor chatnger in em irommental comblitions that condel Warrant allention hetore the more detatled and sensities

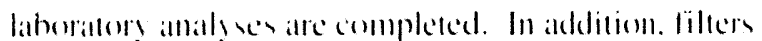

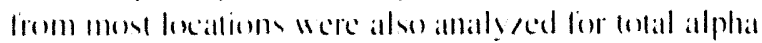
radiontction!s.

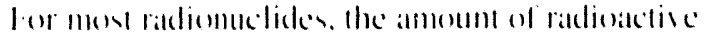
material collected on the filler during the 2-week period was low small to be readily measured. The semsitisily allal accurac! of sample antaly sis was increased by combining himechls samples lor nearhy localloms for in

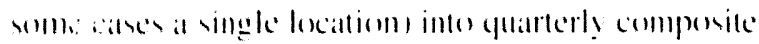
simples. The yuatterly compunite samples were then

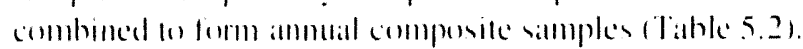

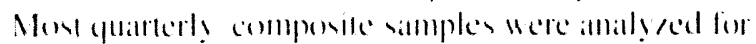
mumerour yecilic g:ammat emoming radionuclider

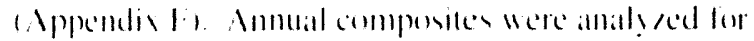

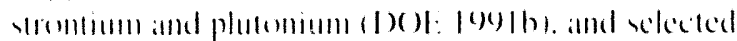

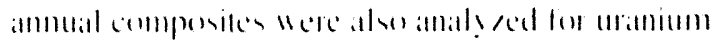
istupes

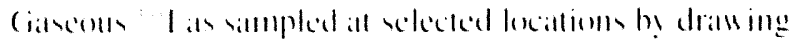

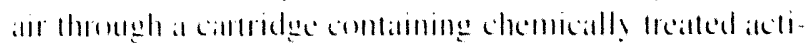

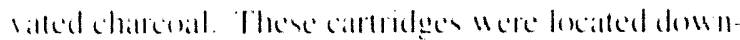

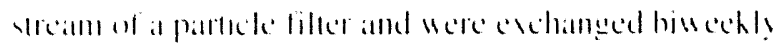

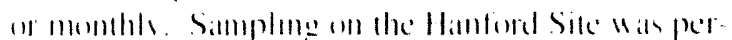

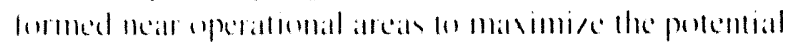

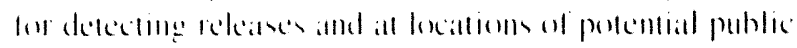

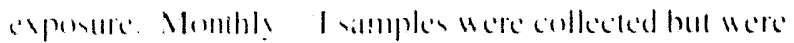

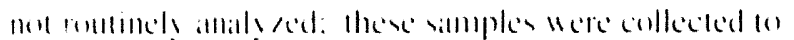

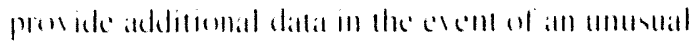

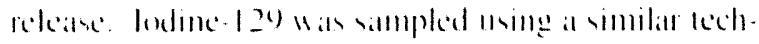

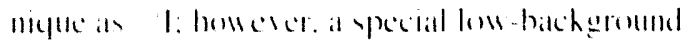

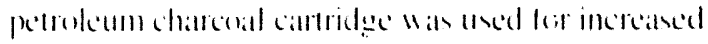

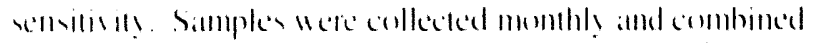

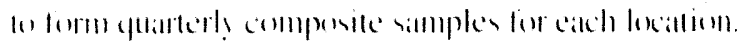

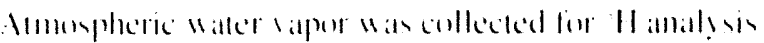

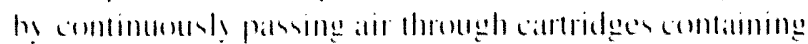




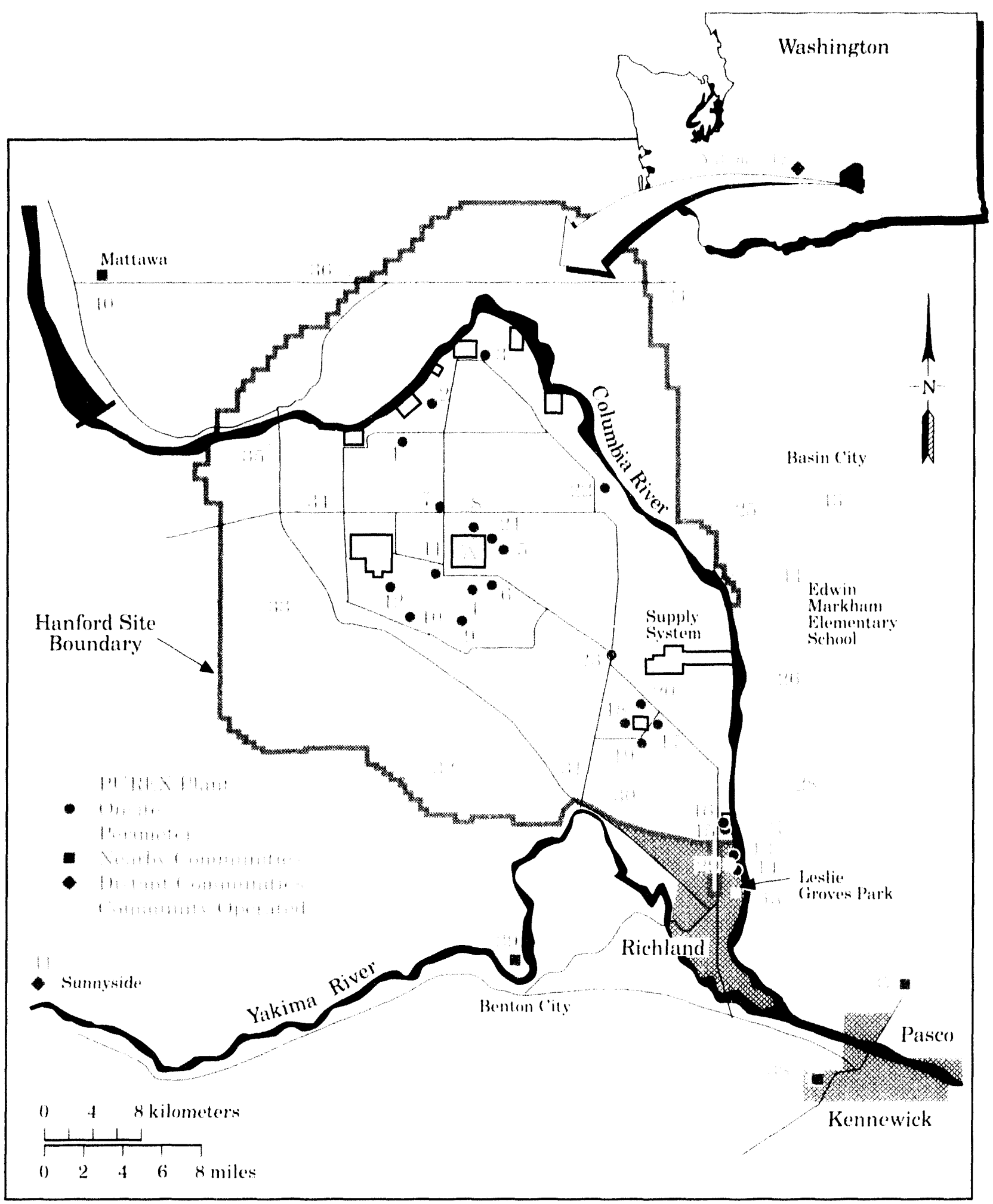

5930301257

Figure 5.2. Air Sampling Locations, 1992 (see Table 5.2 for location key) 
Table 5.2. Air Sampling Locations, Sample Composite Groups, and Analyses, 1992

\begin{tabular}{|c|c|c|c|c|}
\hline $\begin{array}{l}\text { Matp' } \\
\text { l.ncilliull }\end{array}$ & Sampling laciation. & Allily & ('impunite (imul) & Analy sex \\
\hline \multicolumn{5}{|l|}{ Onsite } \\
\hline 1 & $1011 \cdot k$ & Butia. alphia. H & \multirow{3}{*}{ 1010 Areits } & \multirow{3}{*}{ (iammllia. Sis. P'u } \\
\hline 2 & IOH-N & Belit. alphat & & \\
\hline 3 & $(1(x)-1)$ & Betat. illphiat & & \\
\hline$t$ & Suf 2010 tans & Bctia, allpha & \multirow{3}{*}{$2(101.1: a 141$} & \multirow{3}{*}{ (iammma. Sr. P'u. I } \\
\hline 5 & $1:$ a $20(1)$ Tals & Bclat, alpha & & \\
\hline 1 & 2(1) fiast sl: & Butlis. alphat. II. "I & & \\
\hline 7 & R1. IIA. Mi." & $N R N^{\prime:}$ & & \multirow{5}{*}{ (iillmmil. Sr. P'u. I } \\
\hline$s$ & $N$ or $2(0)-$ lans & NRA & & \\
\hline ) & SW of B/C ribly & Hetil. illphit & \multirow{3}{*}{ 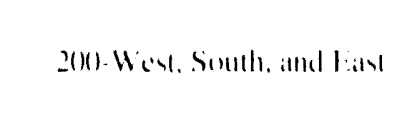 } & \\
\hline 111 & Arm lomp ( Camp & Belat. alphas & & \\
\hline 11 & (ill Building & Butit. illphat. II & & \\
\hline 12 & 2(10) Hen Sl: & Belial. alphial. Vox: M & 2111. West & Gilmmina. Sir. P'u. 1 \\
\hline 13 & $300)$ Witler intithe & Biclia & \multirow[t]{2}{*}{$3(11)$ Areil } & \multirow[t]{2}{*}{ (iallmmit. Sir. Pu. 1} \\
\hline 11 & 3(10) Simulh ( ialle & Belit, ilphis. H & & \\
\hline 15 & $3(10) \mathrm{NI}$ & 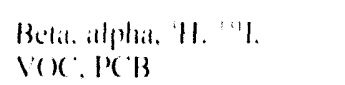 & \multirow[t]{2}{*}{$3111 \mathrm{NI}:$} & \multirow[t]{2}{*}{ Gilmmal. St. P'u. I' } \\
\hline 16 & 3n Trench & Belit. alphat. II & & \\
\hline 17 & 4101 tialst & Belat. alphat. |1." & \multirow{4}{*}{$f(101$ Arcia } & \multirow{4}{*}{ (itmmlat. Si, Pu } \\
\hline Is & H(N) Hers & Belat. alphal I NRA, & & \\
\hline 19 & Hol Sinuh & Betat alphat I NRA, & & \\
\hline 20 & f(I) Nonth & Betil alphial I NRA & & \\
\hline 21 & B Pond & Betal ilphial & \multirow[t]{3}{*}{$B I^{\prime}(m) \mathrm{I}$} & \multirow[t]{3}{*}{ (iammint. Sr. Pu.l: } \\
\hline$\therefore$ & Himford Timsnilc & NRI & & \\
\hline 23 & M!c Bartricinde & I hertat allud alphas NRA, & & \\
\hline \multicolumn{5}{|l|}{ Perinuter } \\
\hline 21 & Burge Ramch & NRA & & \\
\hline 25 & Rimguld Nal Tintrer & 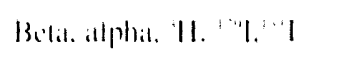 & Ringuld Met Tumer & (iammmat. Sir. P'u \\
\hline 26 & W I nnd al I ir Ronal & Betit. alphat & II land Wlin Roind & Giammmil, Sir. l: \\
\hline 27 & Bụor l.anding & 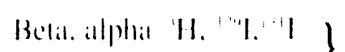 & 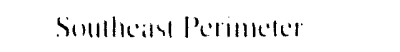 & (iallumlit. Sr. Pu, I \\
\hline $2 x$ & 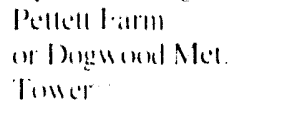 & Belit, alphat. II & & \\
\hline 24 & Balledle (immples & NRA & & \\
\hline 30 & 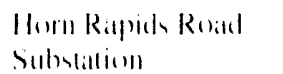 & Belit alphai & & \\
\hline 31 & Promere Bartricake & Belit. alphat. H. "1 & Premsel Barriciak & (jimmmlas. Si. Plu. I \\
\hline$\because$ & Al.1:|c|l|l.al & NR: & & \\
\hline
\end{tabular}


Table 5.2. Air Sampling Locations, Sample Composite Groups, and Analyses, 1992 (contd)

\begin{tabular}{|c|c|c|c|c|c|}
\hline \multicolumn{2}{|c|}{ Mil } & 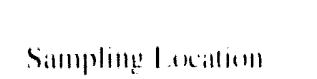 & Anallyen" & (imingusle (imotep) & Aliblỵu \\
\hline \multicolumn{6}{|c|}{ Perineter } \\
\hline \multicolumn{2}{|c|}{$\begin{array}{l}31 \\
34\end{array}$} & Ratllesllithe Sprimgy & 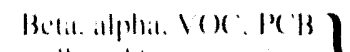 & \multirow[b]{2}{*}{ Ment Perimeder } & \multirow[b]{2}{*}{ (iammmat. Sr. l'u } \\
\hline 34 & & Yahima Barricanke & 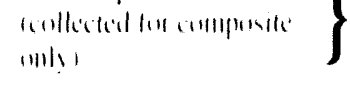 & & \\
\hline \multicolumn{2}{|l|}{35} & Vomila Bridger & Betil & \multirow{2}{*}{ 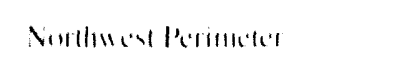 } & \multirow{2}{*}{ Gimmmit. Sir. I'u } \\
\hline 31 & & Willuhe slope & Heltat. ilphit. II & & \\
\hline \multicolumn{6}{|c|}{ Neurby ('ommumunities } \\
\hline 37 & & l'incin & Belit & \multirow{2}{*}{ Incilices } & \multirow{2}{*}{ (iillumalı. Si. I'u } \\
\hline $3 x$ & & kimlewich & Betat alphlat & & \\
\hline \multicolumn{2}{|l|}{$31)$} & 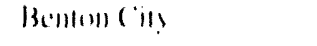 & \multicolumn{3}{|l|}{ NRI } \\
\hline \multicolumn{2}{|l|}{+11} & Mallatwa & \multicolumn{3}{|l|}{ NR I } \\
\hline \multicolumn{6}{|c|}{ Dislant ( ommmonnitics } \\
\hline \multicolumn{2}{|c|}{+1} & Summ! side & Bitit. inlphis. H. I & Sullm! vikle & (iammma, St. I'ul! \\
\hline \multicolumn{2}{|l|}{12} & Yathimal & Butat alphat. H. I, I & lishinlas & (iammollat Si. Pu, I' \\
\hline \multicolumn{6}{|c|}{ (ommunity -(Operated Stulloms } \\
\hline \multicolumn{2}{|c|}{+3} & Bilsill ( ily & Bulla. alphial. II. I & 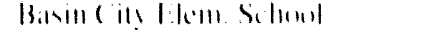 & (i.tmmma. St. I'to. I \\
\hline \multicolumn{2}{|l|}{4.4} & Nonth Itranklin ( 'umm! & Helit. alphat. H. I & 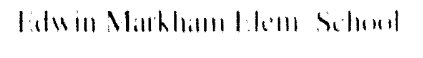 & (iammma. Si. I'u. I \\
\hline \multicolumn{2}{|l|}{15} & Richland & Hallit. ilphat. II. 1 & lenlic cimeses liuk & (iallmmil. Si, In, I: \\
\hline \multicolumn{6}{|c|}{ (i) Siceligure 52} \\
\hline \multicolumn{6}{|c|}{ 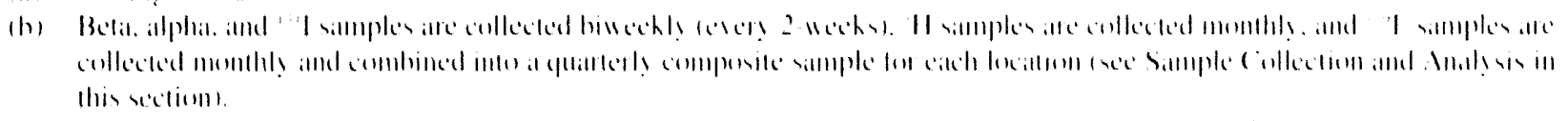 } \\
\hline (c) & \multirow{2}{*}{\multicolumn{5}{|c|}{ 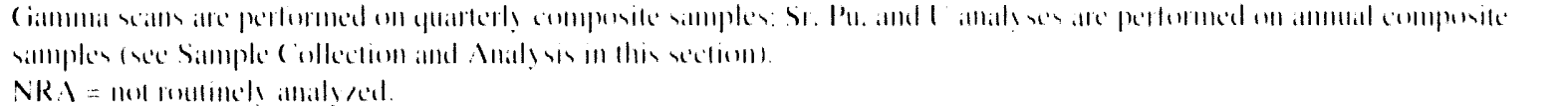 }} \\
\hline \multirow{2}{*}{$\begin{array}{l}\text { (d) } \\
\text { (c) }\end{array}$} & & & & & \\
\hline & $\begin{array}{l}\text { The I } \\
\text { (1) is }\end{array}$ & 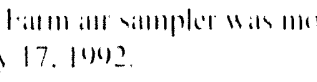 & 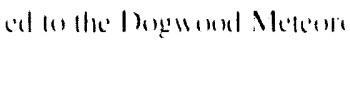 & 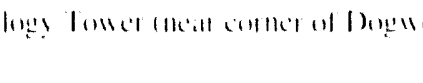 & $2,1.1111$ \\
\hline
\end{tabular}

silical gel. which were exchanged enery t wewh. The trapped water wale remomed from the silica gel and analysa.

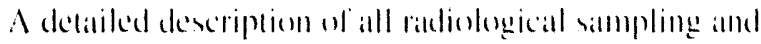

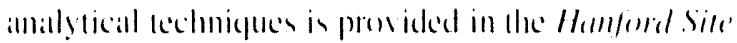

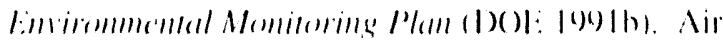
momitoring was discombinted all sereral locallioms in

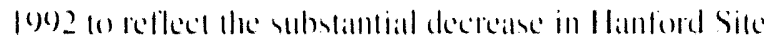

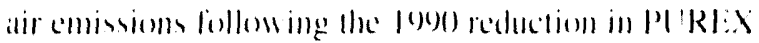
Pant operations. Air silmpling was discomtimled all

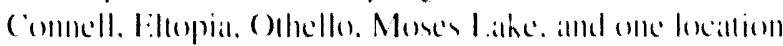

in Richland. In addition. ail samples were collected hul

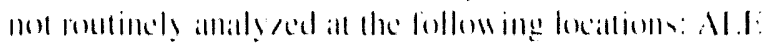

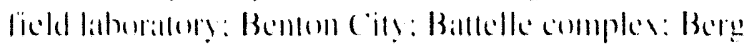

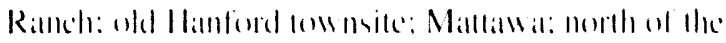

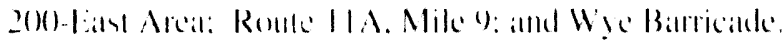

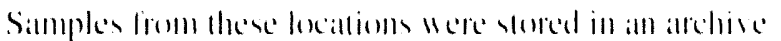

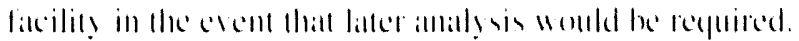

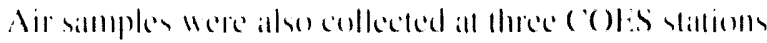

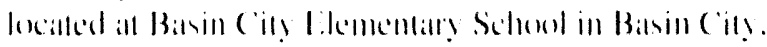

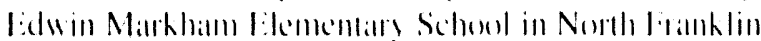




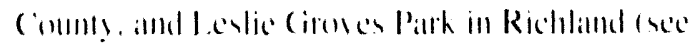

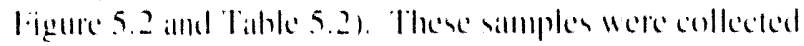

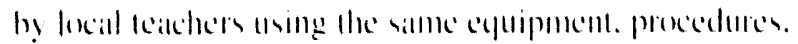

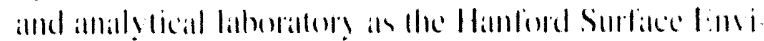
fommental Surveillance Program. This moth was pall w

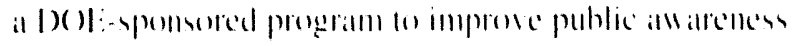
al Itanford ens irommental monitoring program and the eflects af Sile nerations.

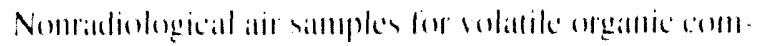

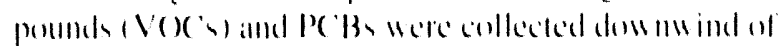

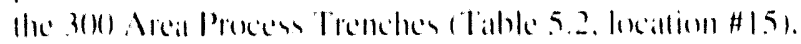

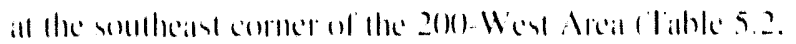

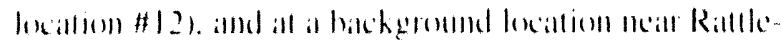

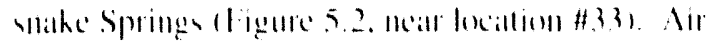

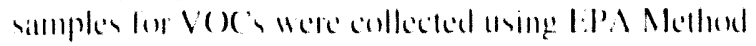

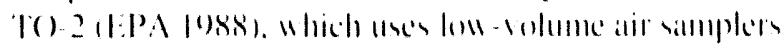

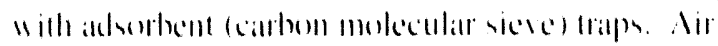

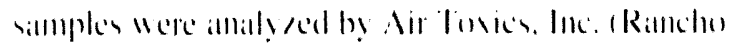

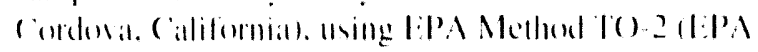

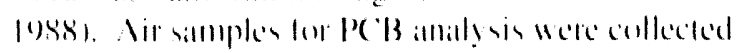

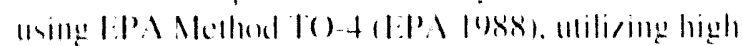

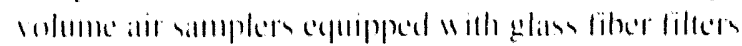

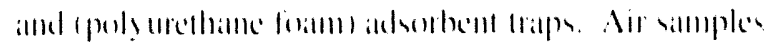

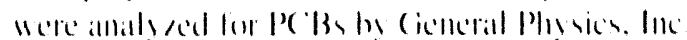

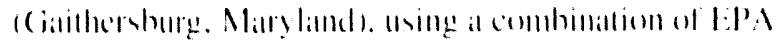

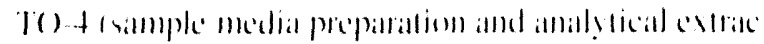

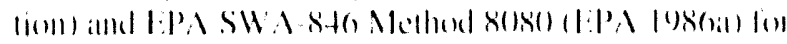

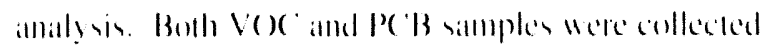

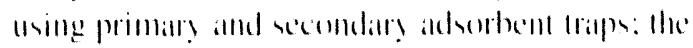

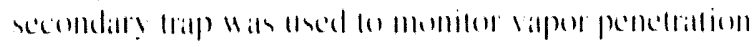

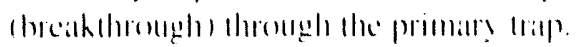

\section{Results}

\section{Radiological Results}

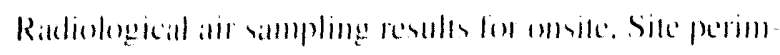

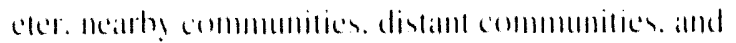

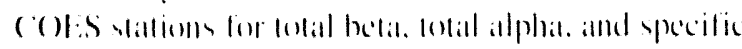

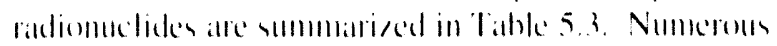

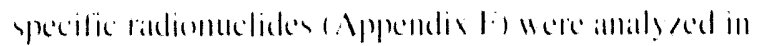

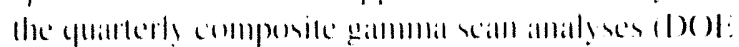

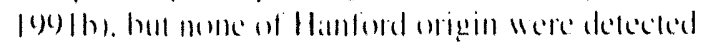
(n)

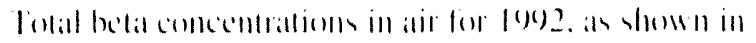

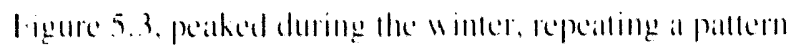

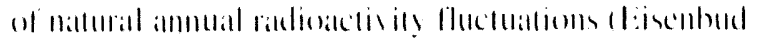

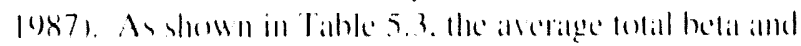

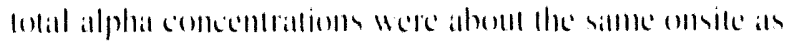
at the Sile perimeter and in nearly and distanl commmmon-

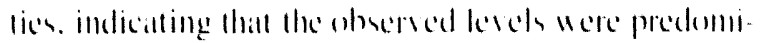

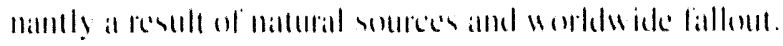

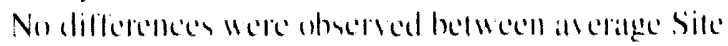

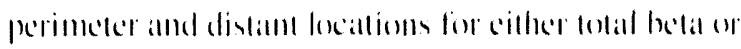
lotill illphat concentrallions.

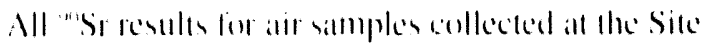
perimeder. commmunily enperated surverllance stations.

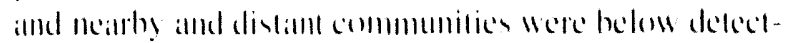

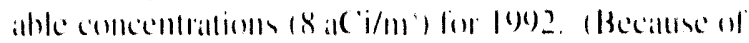

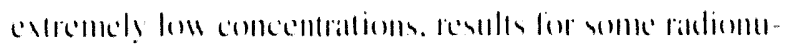

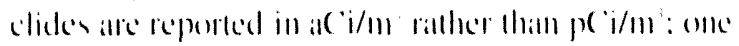

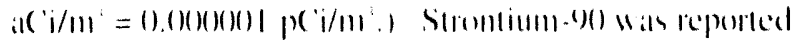

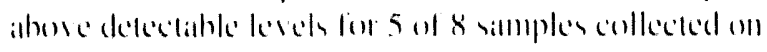

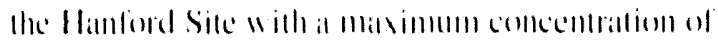

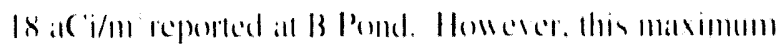

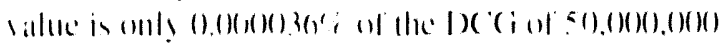

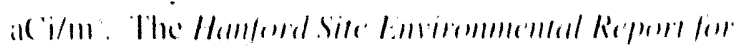

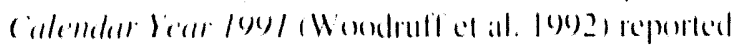

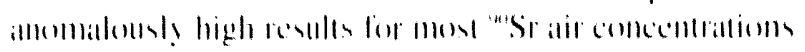
for the fomtle quatrler of loy an well an for all ammual

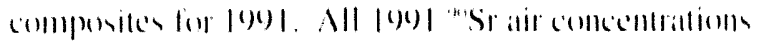

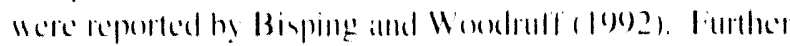

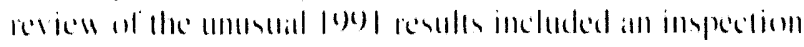

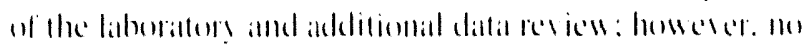

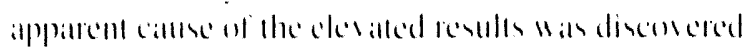

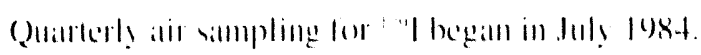

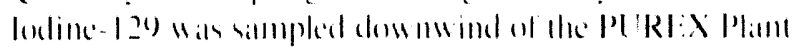

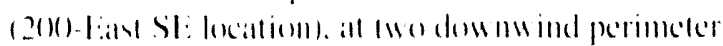

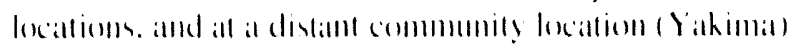

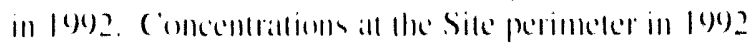

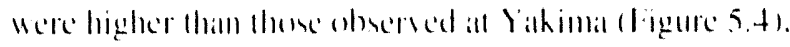
and the dillerence was stattistically signilicant (ome - lailed

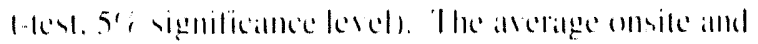

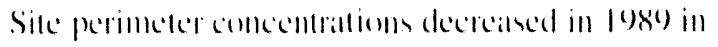

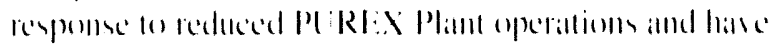

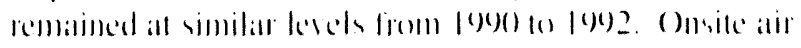

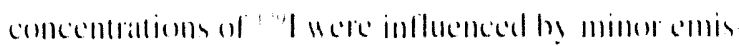

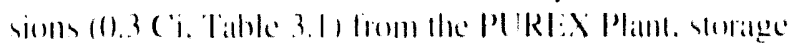

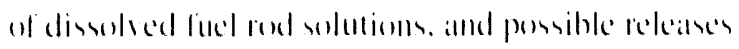

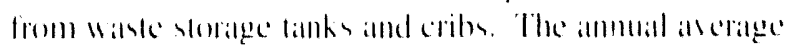

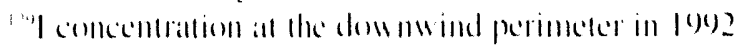

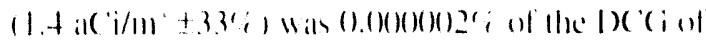

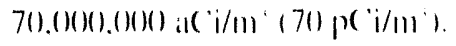

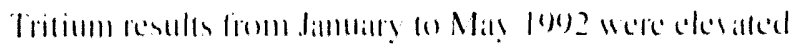

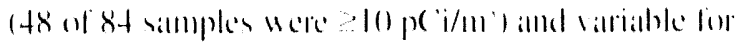

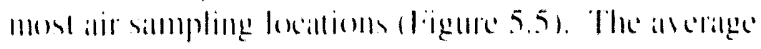

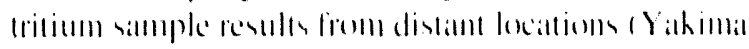


Table 5.3. Airborne Radionuclide Concentrations in the Hanford Environs, 1992 Compared to Values from the Previous 5 Years

1942

\begin{tabular}{|c|c|c|c|}
\hline Radionuclide & Componite Group & $\begin{array}{c}\text { Sir. of } \\
\text { Simples }\end{array}$ & $\begin{array}{l}\text { Maximum" } \\
\mathrm{pCi} / \mathrm{m}\end{array}$ \\
\hline $\mathrm{H}$ & Onvite & 35 & $770 \pm 6.0$ \\
\hline \multirow[t]{3}{*}{ Jan-Max 1442 } & Perimeter & 24 & $16(x) \pm 9.4$ \\
\hline & Distant Communities & 10 & $350 \pm 3.7$ \\
\hline & COES - Stations & 15 & $12(x) \pm 7.7$ \\
\hline $\mathrm{H}$ & Onsite & (n) & $2 \mathrm{x} \pm 1.1$ \\
\hline \multirow[t]{3}{*}{ June-Dec 1942 } & Perimeter & 4) & $330 \pm 50$ \\
\hline & Diviant Communities & 16 & $3801 \pm 5.4$ \\
\hline & COES Station & 23 & $14 \pm 2.1$ \\
\hline \multirow[t]{6}{*}{ Toral Beta } & Onvite & 495 & $0.11 \pm 0.000035$ \\
\hline & Perimeler & 234 & $0.15 \pm 0.0(k) 39$ \\
\hline & Nearh! Communities & 54 & $0.10 \pm 0.00033$ \\
\hline & Distant Communitie & 54 & $0.12 \pm 0.000038$ \\
\hline & COES Station, & 80 & $0.057 \pm 0.0 k 0) 34$ \\
\hline & & & $\mathrm{aCi} / \mathrm{m}^{\circ}$ \\
\hline \multirow[t]{5}{*}{${ }^{*} \mathrm{Sr}$} & Onvite & $x$ & $18 \pm 7.9$ \\
\hline & Perimeter & 6 & $30 \pm 53$ \\
\hline & Nearhy Communities & 1 & $2.8=5.2$ \\
\hline & Distant Communities & 2 & $1.9 \pm 4.9$ \\
\hline & COES Station. & 3 & $0.13 \pm 5.7$ \\
\hline \multirow[t]{5}{*}{$" R u$} & Onvite & 31 & $1.9(x)=1.5(x)$ \\
\hline & Perimeter & 23 & $1.9(x)=1.8(x)$ \\
\hline & Nearhy Communitier & 3 & $-450 \pm 2.6(x)$ \\
\hline & Divtani Communiter & $s$ & $0.30=2.200$ \\
\hline & COES Station- & 12 & $1.7(x)=2.6(x)$ \\
\hline \multirow[t]{3}{*}{$\therefore 1$} & Onsite & + & $74 \pm 7.2$ \\
\hline & Perimeter & $s$ & $2.2 \pm 10.15$ \\
\hline & Diviant Communitiev & 4 & $0.13 \pm 0.013$ \\
\hline \multirow[t]{4}{*}{1} & Onvile & 74 & $f .4(x) \pm 5.1(x)$ \\
\hline & Perimeter & 78 & $x .1(x) \pm 5.5(x)$ \\
\hline & Distant Communitie & 53 & $2(x \times) \pm 1.9(x)$ \\
\hline & COES Station & 80 & $3.6(x) \pm 3.1(x)$ \\
\hline
\end{tabular}

$$
1987-1991
$$

$\begin{array}{ll}\underset{\text { Average }}{\text { pCi } / \mathrm{m}} & \text { No. of } \\ & \end{array}$

$$
\underset{\mathrm{NCL} / \mathrm{m}}{\text { Maximum }}
$$

$130 \pm 69 \%$

$190 \pm 160 \%$

$71 \pm 81 \%$

$2(x) \pm 150 \%$

$2 . + \pm 11.46 \%$

$1.5 \pm 1.1 \%$

$1.4 \pm 1.1 \%$

$1.7 \pm 1.2 \%$

$0.020 \pm 5.80$,

0.0 $20104.0 \%$

$0.024 \pm 226$

0.1 $121=25 \%$

$0.014 \pm 11 \%$

$\mathrm{aCl} / \mathrm{m}$

$8.1 \pm 50 \%$

$0.98 \pm 140 \%$

$2.8 \pm 190 \%$

$-2.7 \pm 56 \%$

$-0.087 \pm 28 \%$

$-470=114 \%$

$-62 \pm 720 \%$

$-1+(x)=10 x ?$

$-410 \pm 160 \%$

$150 \pm 370 \%$

$$
\begin{aligned}
& 50 \pm 35 \% \\
& 1.4 \pm 33 \%
\end{aligned}
$$

$0.142 \pm 320$

$-5- \pm 6.8(4)$
$-390 \pm 1.30 \%$
$-20 \pm 1.20 \% \%$

$-(x) \pm 170 \%$

355
464
123
24
355
464
123
24
2598
1747
1125
701
62

$7 \pm 2.3$

$12 \pm 1.3$

$9.0 \pm 0.45$

$10 \pm 1.3$

$71 \pm 2.3$

$9.0 \pm 0.45$

$10 \pm 1.3$

$0.13 \pm 0.00136$

$0.11=0.0(1)+10$

$0.088 \pm 0.0(1041$

$0.077 \pm 0.0(0) 30$

$0.043 \pm 0 .(0) 18$

$\mathrm{aCi} / \mathrm{m}$

$+2(x) \pm 1+11$

$2.3(x) \pm+3$

$6.3(x) \pm 110$

$150 \pm 79$

$6+ \pm 36$

$1+.0(x) \pm 9.4(x)$

$1+(0)(x) \pm x(x)$

$12.000 \pm 7.500$

$20 .(x)=16.0(x)$

$2 . t(x) \pm 3 . t(x)$

$710=54$

$1 x=2.7$

$0.47 \pm 0.22$

$-.9(x) \pm 7.4(x)$

$13.000) \pm 11 .(x)$

$f .3(x) \pm f .3(x)$

$28 .(000) \pm 19.14 x)$
1942

Concentration

Guide

$\mathrm{pCi} / \mathrm{m}$

100.0000

$2.2 \pm 2.9 \%$
$1.4 \pm 4.3 \%$
$1.2 \pm 18 \%$
$2.4 \pm 5 \%$

$2.2 \pm 2.9 \%$

$1.2 \pm \pm 18 \%$

$2.4 \pm 50 \%$

$0.021 \pm 2.4 \%$ $0.020 \pm 30 \%$ ， $0.020 \pm 3.7 \%$ $0.019 \pm 4.3 \%$ $0.016=14 \%$

\section{$\mathrm{dCi} / \mathrm{m}$}

$\mathrm{aCi} / \mathrm{m}$

$80 \pm 89 \%$

$110 \pm 68 \%$

$130 \pm 12 \%$

$18+44 \%$

$2.2 \pm 1 .(6) \%$

$3.7 \pm 130.10 \times 6 \%$

$52 \pm 1.200 \%$

$-460 \pm 170 \%$

$180 \pm+40 \%$

$-6 h \pm 1.5(6)$ \%

$1811 \pm+6 \%$

$+.9 \pm 27 \%$

$0.31=35 \%$

$51=340 \%$

$-130 \pm 190 \%$

$-130=250 \%$

$1.1(x)=110 x$
50.0000 .0000

$30.0(0) .0000$

$70.000 .0(10)$

Hog. (x) (x). (x) 
Table 5.3. Airborne Radionuclide Concentrations in the Hanford Environs. 1992 Compared to Values from the Previous 5 Years (contd)

\begin{tabular}{|c|c|c|c|c|c|c|c|c|}
\hline & & & 194 & & & $198:-194$ & & 1402 \\
\hline Radionuclide & Componite Group & $\begin{array}{l}\text { Sir. of } \\
\text { Samples }\end{array}$ & $\begin{array}{c}\text { Maximum } \\
\text { aCi/m }\end{array}$ & $\begin{array}{l}\text { Alerage } \\
\mathrm{aCl} / \mathrm{m}\end{array}$ & $\begin{array}{l}\text { Vis. if } \\
\text { Samples }\end{array}$ & $\underset{\mathrm{aCi} / \mathrm{m}}{\text { Maximum }}$ & $\begin{array}{l}\text { Alerage } \\
\mathrm{aCi} / \mathrm{m}\end{array}$ & $\begin{array}{l}\text { Concentration } \\
\text { Guide } \\
\mathrm{aCi/m}\end{array}$ \\
\hline C. & Onvite & 31 & $350 \pm 250$ & $75 \pm 71 \%$ & 301 & $1.2(x) \pm 870$ & $52=84 \%$ & $f(x) .(x)(x) .(x))$ \\
\hline & Perimeter & 23 & $2(x)=180$ & $H=5 N^{\prime}$ & $29 x$ & $1 .(6 x) \pm 1.4(x)$ & $27 \pm 2+\%$ & \\
\hline & Nearb! Communities & 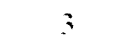 & $1601 \pm 230$ & $36 \pm f(x)$ & 245 & $2(x) \pm 4501$ & $7 s=l 0 \mathrm{kr} r$ & \\
\hline & Distant Communitie & $x$ & $240 \pm 220$ & $f(x)=x y^{\prime} ;$ & $2(x)$ & $2.2(x)=1 . f(x)$ & $44=44^{\prime}$ & \\
\hline & COES Station & 12 & $390) \pm 270$ & $7+ \pm 11 \%$ & 4 & $130 \pm 240$ & $26 \pm 230$ & \\
\hline Iriotall & Onvite & 6 & $61 \pm 34$ & $37 \pm 28 \%$ & 115 & $6.2(x) \pm 73$ & $230 \pm 554$ & {$[(x) \cdot(x))$} \\
\hline & Perimeler & $\therefore$ & $5 x=411$ & $H=34 \%$ & i4 & $f(x)=31$ & $s x \pm 30 \%$ & \\
\hline & Distant Communitie & $=$ & $(x) \pm 3.5$ & $4=73 \%$ & 41 & $250 \pm 20$ & $5 n \pm 23$ & \\
\hline & COES Station & 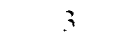 & $h_{2}=50$ & $H \pm H^{\prime}$ & 4 & $57 \pm 16$ & $5 x \pm 14^{\prime}$ & \\
\hline$\therefore \mathrm{Pu}$ & Onvic: & $s$ & $0 . \mathrm{Nh}=0.53$ & $0.21 \pm 1(x)^{\prime}$ & 153 & $2.7=0.020$ & $0.26=42 \%$ & Bnosuno \\
\hline & Pernmeter & 5 & $0.29 \pm 0.36$ & $0.11 \pm 11 \times x$ & lik & $3.0 \pm 0.0125$ & $0.0 \times 7=150 \%$ & \\
\hline & Nearby Communtier & 1 & $0.30 \pm 0.42$ & $10.3010160 \%$ & 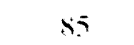 & $1.2 \pm 0.0115$ & $-0.077 \pm 120 \%$ & \\
\hline & Distant Communtite & 2 & $0.27=0.45$ & $0.17=12 \%$ & 7 & $53 \pm 0.1130$ & $0.25 \pm 110 \%$ & \\
\hline & COFS Station & $\vdots$ & $0.17 \pm 0.33$ & $10.082 \pm 110 \%$ & y & $1.8 \pm 0.016$ & $0.46 \pm 8 t^{\prime}$ & \\
\hline$\therefore \mathrm{Pu}$ & Onsite & $s$ & $7.7 \pm 1.6$ & $\therefore 3 \pm+I^{\prime}$, & 153 & $x 6 \pm 11$ & $1 . x \pm 65$ & $20 .(x)$ \\
\hline & Perimeler & 5 & $2.3=01.89$ & $12=45$ & 116 & $32 \pm 23$ & $0 .+3=35 r$ & \\
\hline & Nearby Cimmunitie & 1 & $10 \pm 0.71$ & $10=71 \%$ & 85 & $22 \pm 1.5$ & b.sh $=42$ & \\
\hline & Distani Conimunities & 2 & $3 y=1.2$ & $2+ \pm 12 \%$ & 7 & $2 x=24$ & $0.2+=7 x$ & \\
\hline & COES Station & $\vdots$ & $1.7 \pm 0.65$ & $15=15 \%$ & $y$ & $\therefore .3=1.5$ & $1.1=74$ & \\
\hline Total Alphat & Onvitc & +64 & $x .7(x)=1.2(x)$ & $6211=11 \%$ & 2.196 & $56 .(x)=2.6(x)$ & $1.3(x)=11 \%$ & \\
\hline & Perimeter & $21 x$ & $h . X(n)=1.2(k)$ & $h(x)=1 y^{\prime \prime}$ & $1.24 k$ & $\therefore 2(x)=2.3(x)$ & $1.2(x)=11 \%$ & \\
\hline & Nearts Communitio & 27 & $1.60101 \pm 510$ & $h(x)=1 y^{\prime \prime}$ & 225 & $\ln (x)=1.5(x)$ & $1.2(x)=2(\%$ & \\
\hline & Distani Communitie- & 54 & $x .3(x)=1.1(x)$ & $550 \pm 4 x^{\prime}$ & $2 \mathrm{si}$ & $22(n)=1.6(x)$ & $1.2(x)=2 x$ & \\
\hline & COES Statuon & si & $f x(x)=7(x)$ & $h(x)=23$ & 62 & $B . x(n)=1.1(n)$ & $5(x)=65 \%$ & \\
\hline (a) Onsite. S & perimeter. nearhy com & ither. and & tivtunt sumpling 1 & are identufied ir & igure $5.1 \mathrm{a}$ & ad Tahle 5.2 & & \\
\hline (b) Maximu & ingle smple results \pm ? & ma countin & E errors. The une & ative concentrati & valuen & plained in the $x$ & Helptul Information." & \\
\hline a tierage & of all smples t2 lime t & ercent van & dard error of the & ted mean iSEMi. & & & & \\
\hline (d) From Dx & Derived Concentration & deree Ap & pendix $\mathrm{Cl}$ & & & & & \\
\hline (e) Alerage & annual mean. & 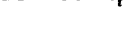 & & & & & & \\
\hline (i) The Jan & fas 1 wo 2 result are vus & and are lit & di at the revult, & aton contaminat & & & & \\
\hline$\because \quad C O E S=$ & mmunity-eperated enu & nental sur & ellance retations & mo are identilied & Figure 5 & nd Table 52 & & \\
\hline (h) Tuos: & at the Promer Barricad & re exdus & drom the averat: & and $330 \mathrm{pCl} / \mathrm{m}$ ) & & & & \\
\hline (1) One 1 & Sunn vide uatercle & om the & erage $1.350 \mathrm{pCi}$ & & & & & \\
\hline
\end{tabular}




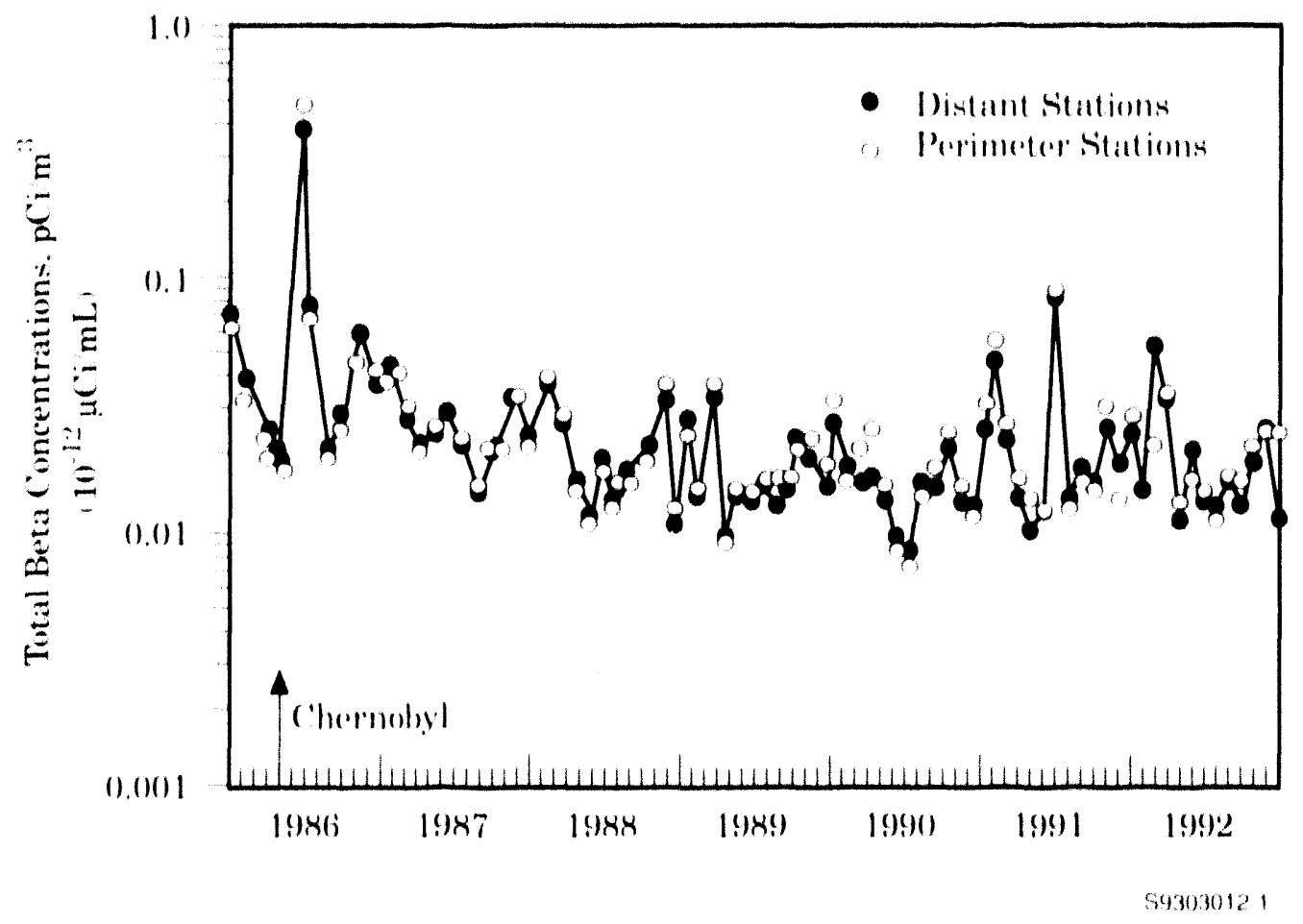

Figure 5.3. Monthly Average Total Beta Radioactivity in Airborne Particulate Samples. 1986 Through 1992

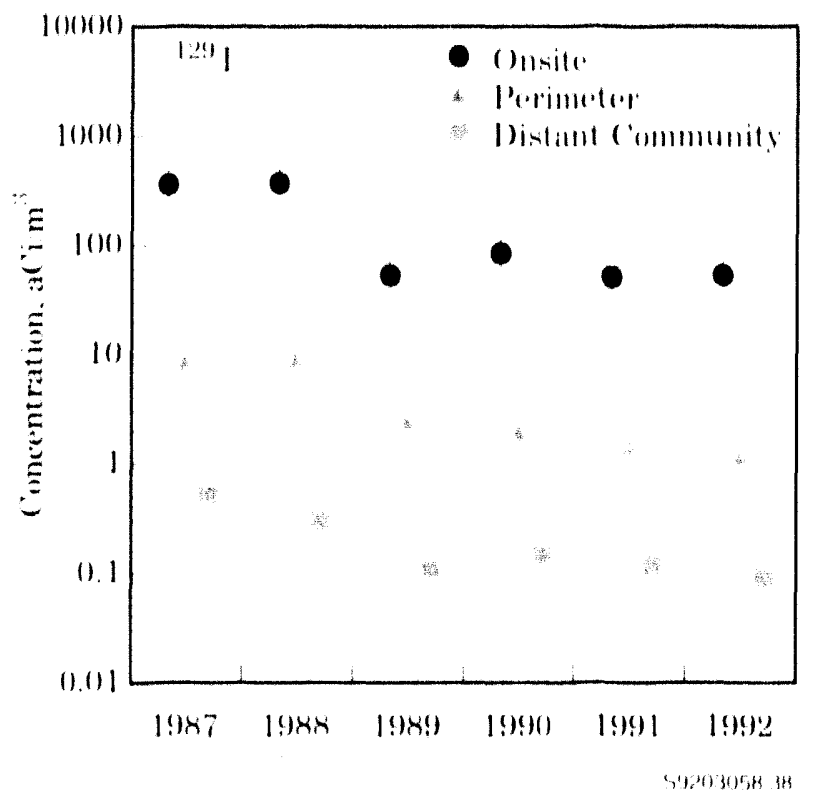

Figure 5.4. Annual Concentrations ( $t 2$ SEM) of lodine-129 (1) in Air near the Hantord Site, 1987 Through 1992. As a result of figure scale, some uncertainties (error bars) are concealed by point symbol.

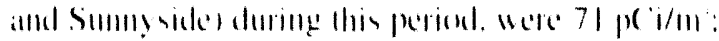

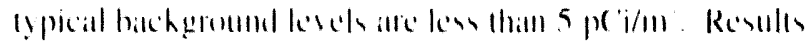

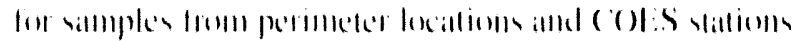

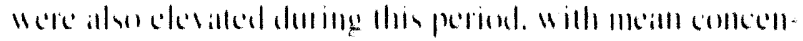

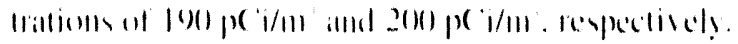

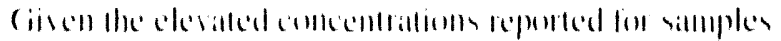

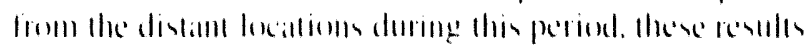

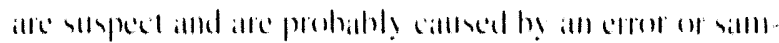

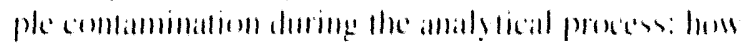

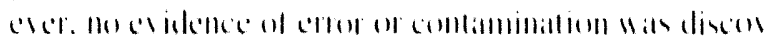

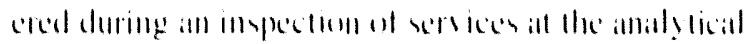

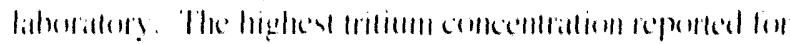

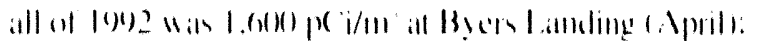

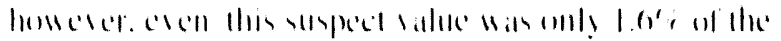

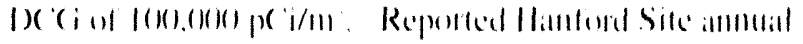

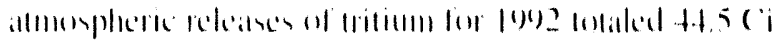

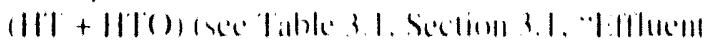

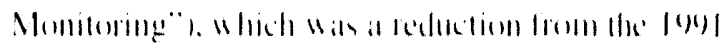

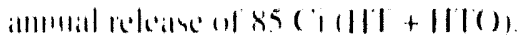

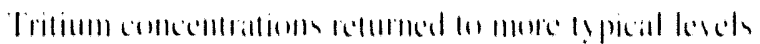

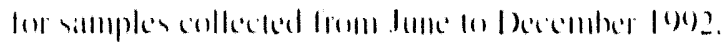

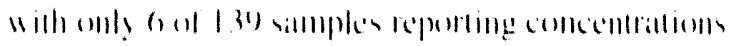




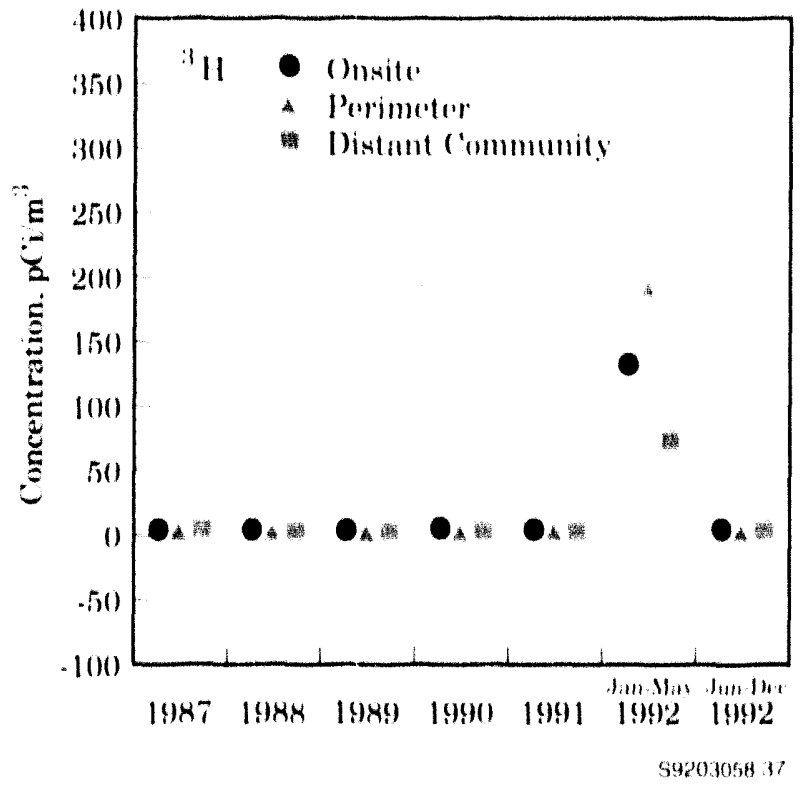

Figure 5.5. Annual Average Concentrations $\left( \pm 2\right.$ SEM) of Tritium ( $\left.{ }^{3} \mathrm{H}\right)$ in Air near the Hanford Site. 1987 Through 1992. As a result of figure scale. some uncertainties (error bars) are concealed by point symbol. January through May 1992 results are suspect and may be the result of laboratory contamination. The June through December averages were calculated excluding one value $\left(380 \mathrm{pCl} / \mathrm{m}^{\prime}\right.$ at Sunnyside) for the distant locations and two values $\left(280\right.$ and $330 \mathrm{pCi} / \mathrm{m}^{3}$ at the Prosser Barricade) for the perimeter locations.

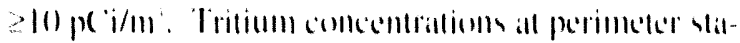

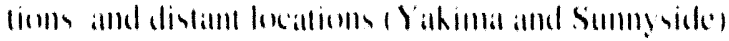
were similat during this period, wilh andrage salues of

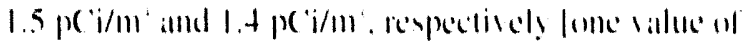

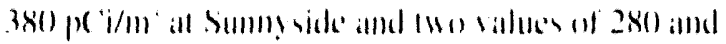

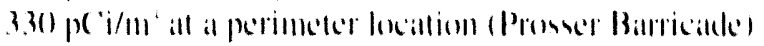

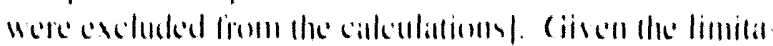

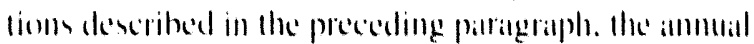

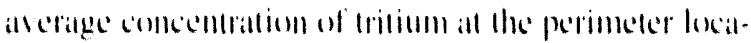

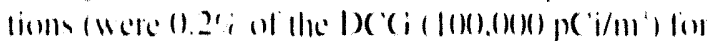

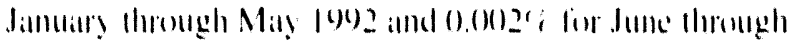
becember 10)?.

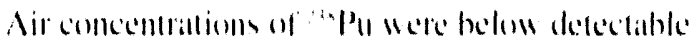

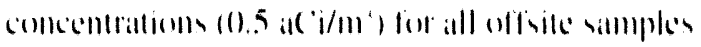
collected for lov) (Table 5.3), The masimum Hanford

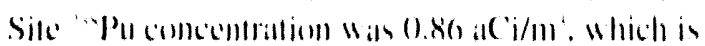

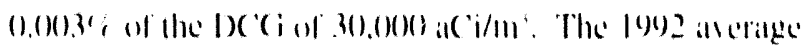

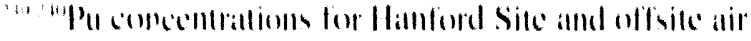

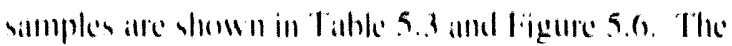
1002 Site perimeler anmual "." :"Pu comecentrallion was

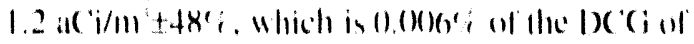

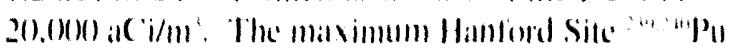

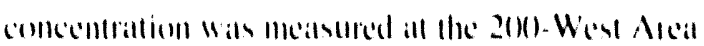

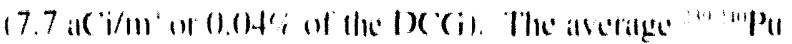

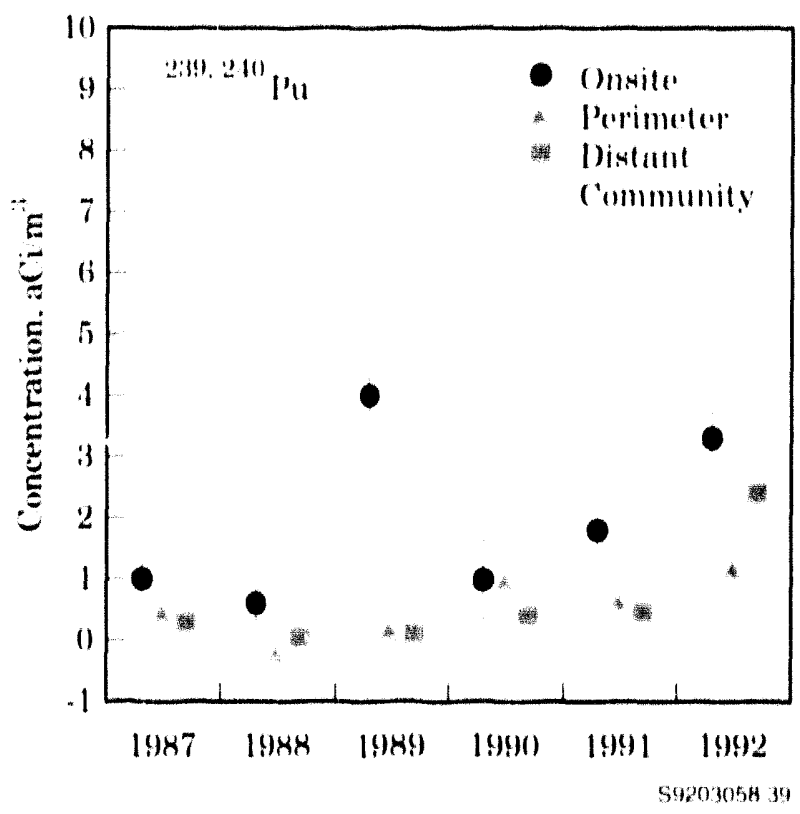

Flgure 5.6. Annual Average Concentrations ( \pm 2 SEM) of Plutonium-239,240 (24: Pu) in Air at the Hanford Environs, 1987 Through 1992

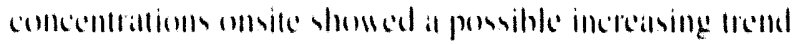

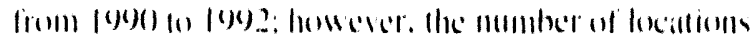

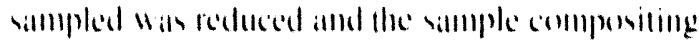

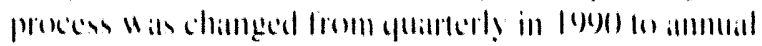

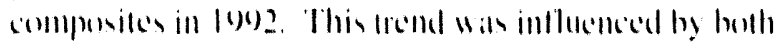

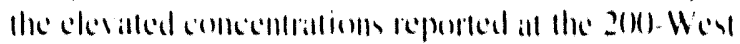

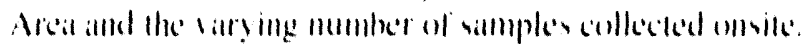

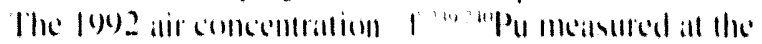

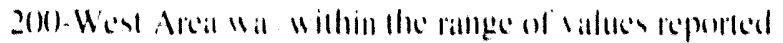

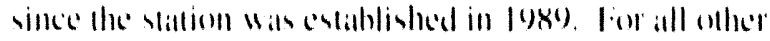

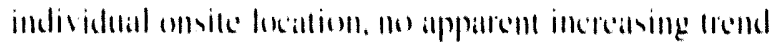

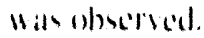

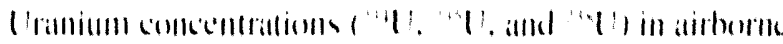
particulate maller in |90) 2 uere smilat an the sile peri-

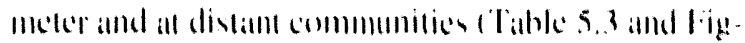

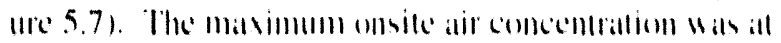

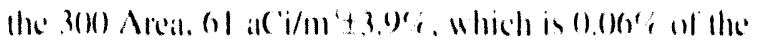


Table 5.4. Average Concentrations of Selected Volatile Organic Compounds in Air on the Hantord Site. 1992 (ng/L \pm 2 standard deviation)

\begin{tabular}{|c|c|c|c|c|c|}
\hline (imp) & 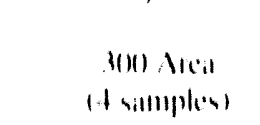 & 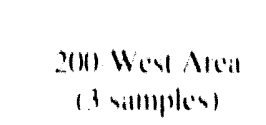 & 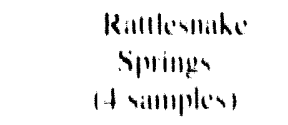 & MACM & $M N G$ \\
\hline 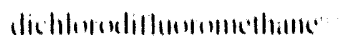 & 16,90 & 4.510 .0 & $21+111$ & 4050 & $\mathrm{NA}$ \\
\hline mishlorollmonomethame & $11.52+11.4 x$ & 11.361 .111 & $0.2 \times 1010$ & $5(1)(x)$ & 0.1 \\
\hline dichlomonedhalle & $10.115 x+0.1116 .8$ & $011111+11.11018$ & $0116077+010111.1 \times$ & 1.81111 & $(1.1132$ \\
\hline muhhuromedhams: & $01020+100118$ & $11.1118 \times, 11.11311$ & $.01111175+11.11159$ & 9.78 & Ni \\
\hline 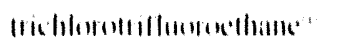 & $0.18+11311$ & $0111+011 \%$ & $11.11+11.21$ & Ni & Ni \\
\hline 1.1.1. Hidhlumenhatme & $11.70+11.94$ & $0.37+10.50$ & $111210.2 x$ & 1.4111 & $10.810)$ \\
\hline 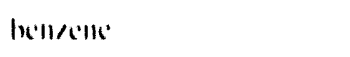 & $11.41+11.31$ & $0.11+11.18$ & $11.15+10.22$ & 5 & $(1.1106$ \\
\hline 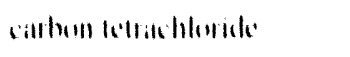 & $11111+1130$ & $11.2 x+012$ & $10.17+10.311$ & 120 & 10.1151 \\
\hline 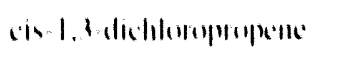 & $01113+101140$ & $.01011++0.10122$ & $.0111799^{\circ}, 11011152$ & 5 & $\mathrm{Ni}$ \\
\hline 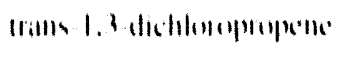 & $111115,10.10158$ & $(11.111+10.10122$ & $41111175+11.10192$ & 5 & $\mathrm{~N} I$ \\
\hline foluente & $10.7 .4+1.1$ & 11.2410 .311 & $11.22+1120$ & 17.9 & $1+1101$ \\
\hline$m+11, y=m$ & $012+10.50$ & $010190,+1101160$ & $11.1111+1101117$ & 115 & 97 \\
\hline "Mkit & $11.11+11.10$ & $011113+110142$ & $11011++110115$ & 135 & 2011 \\
\hline 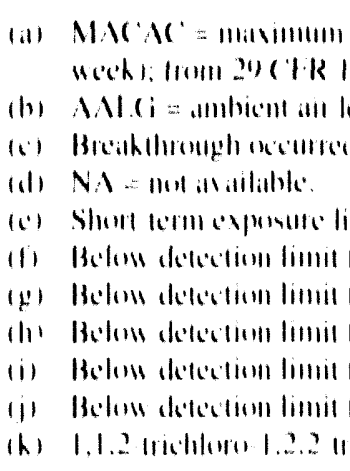 & 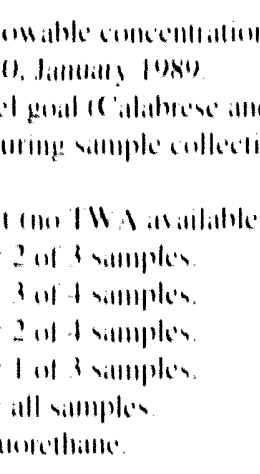 & 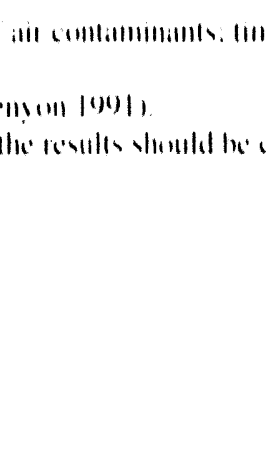 & 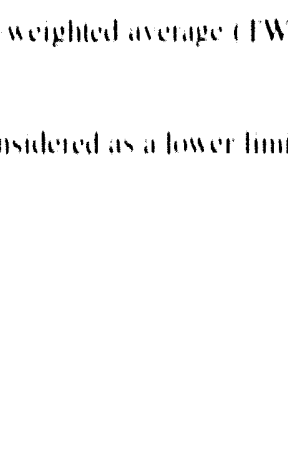 & $\begin{array}{l}\text { Ix. In dily. }+ \\
\text { onl! }\end{array}$ & \\
\hline
\end{tabular}

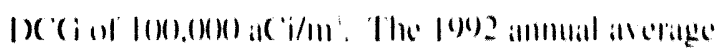

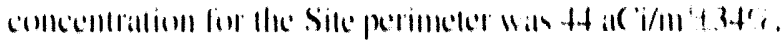
which was ().(1)t'i ol the i) ( $(i$.

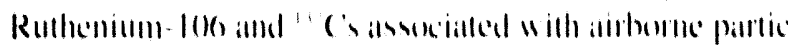

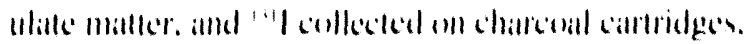

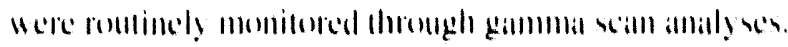

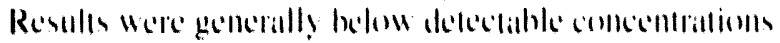

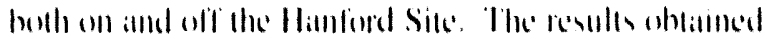

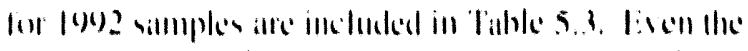

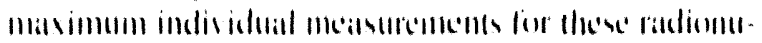

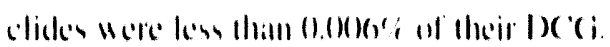

\section{Nonradiological Results}

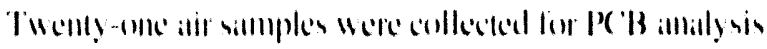

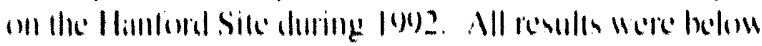




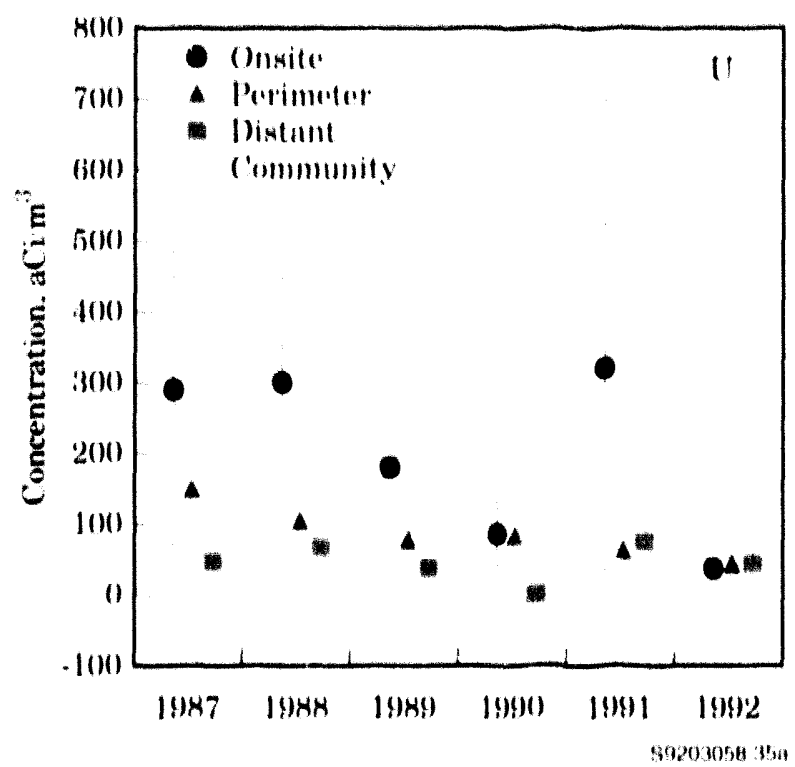

Figure 5.7. Annual Average Concentrations ( +2 SEM) of Uranlum (" $U, \cdots, U$, Hantord Environs, 1987 Through 1992. As a result of ligure scale, some uncertainties (error bars) are concealed by point symbols.

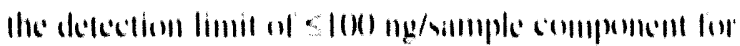

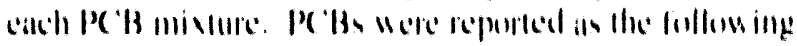

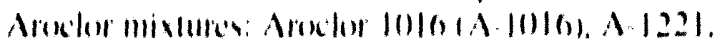
A.1232. A.1242. A.12.4x. A.1254. ind A.1260. Air

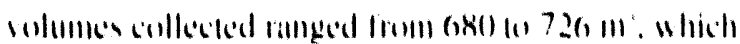

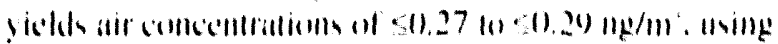

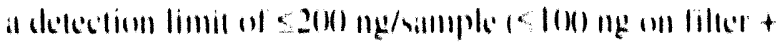

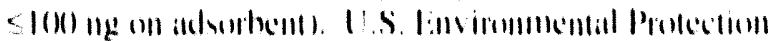

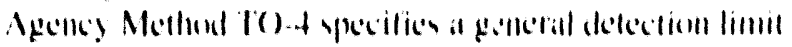

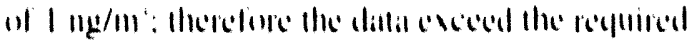

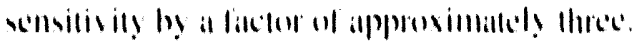

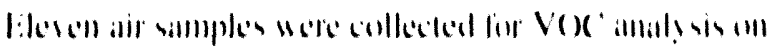

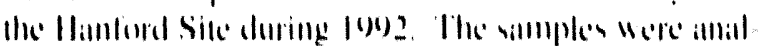

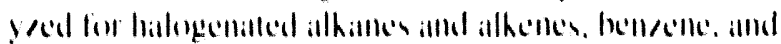

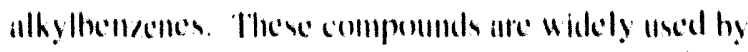

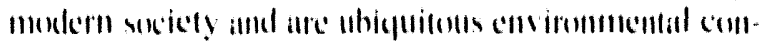

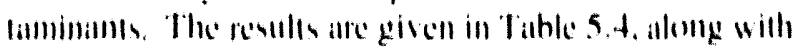

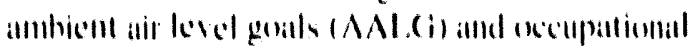

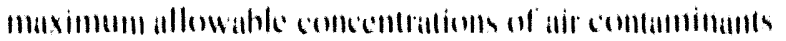

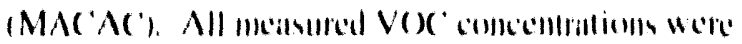
well below MAC AC values. The AN regulitury, monhinding limits thit were develened hy

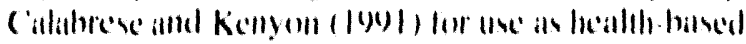

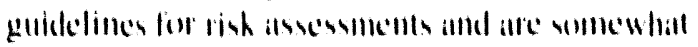

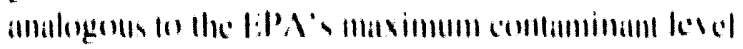

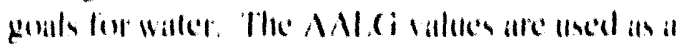

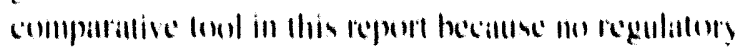

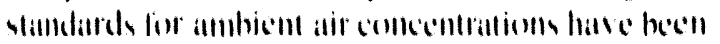

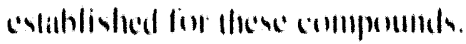

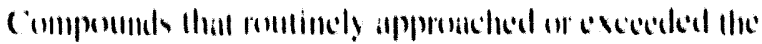

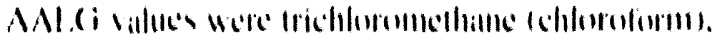

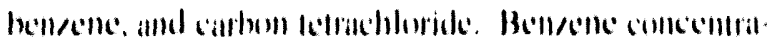

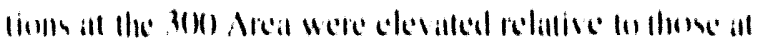

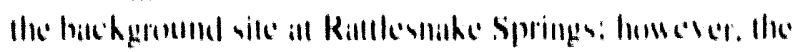

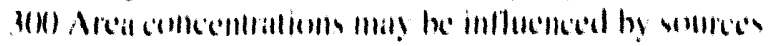

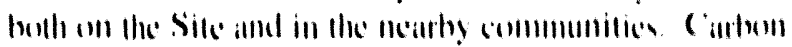

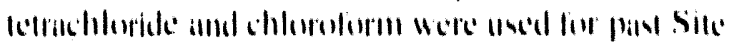

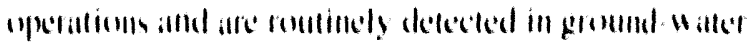
menitoring "

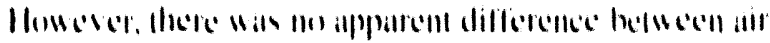

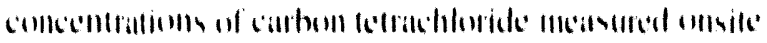

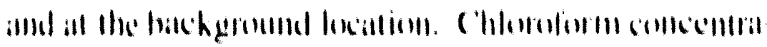

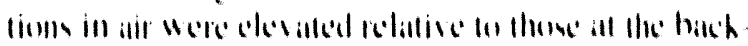

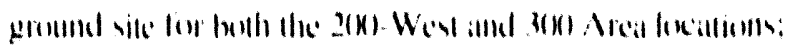

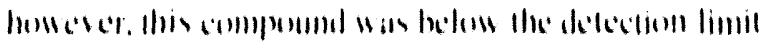

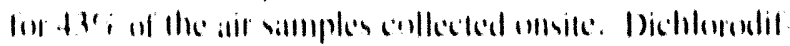

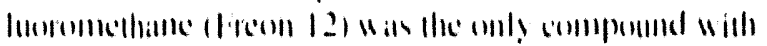

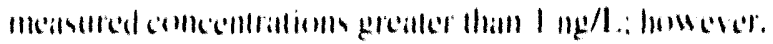

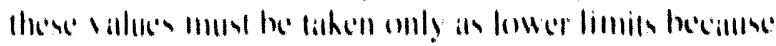

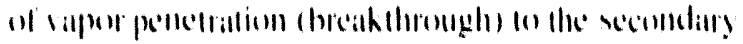

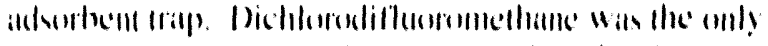

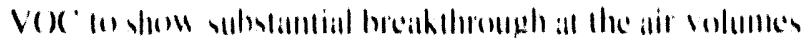
sillinkled. 


\subsection{Surface-Water Surveillance}

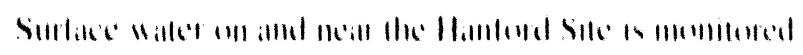

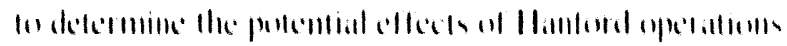

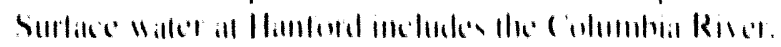

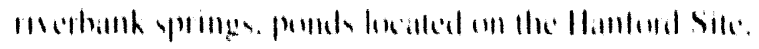

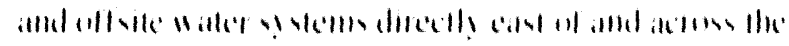

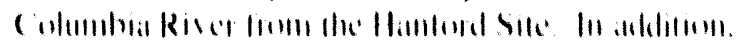

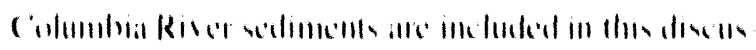

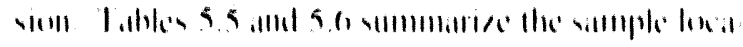

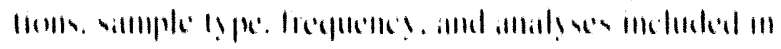

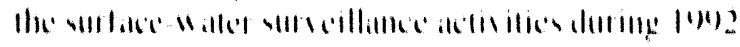

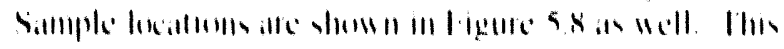

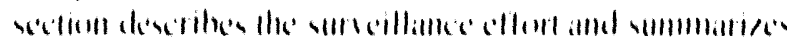

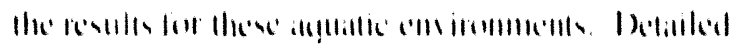

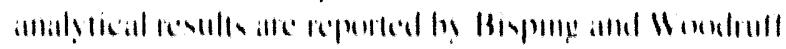
110031.

\section{Columbia River Water}

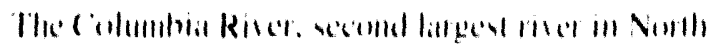

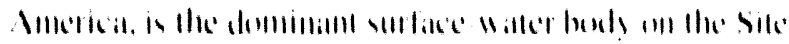

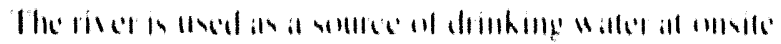

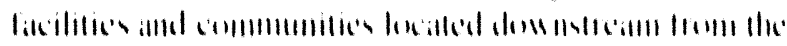

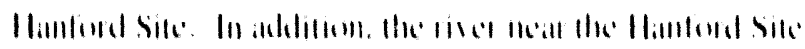

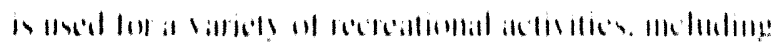

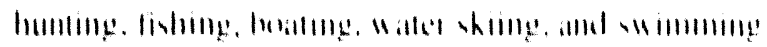

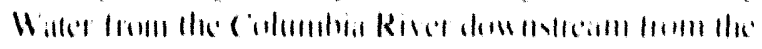
Silte is and

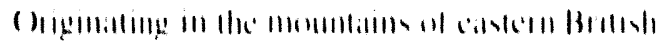

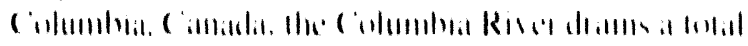

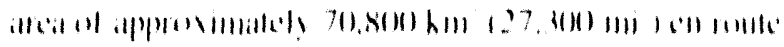

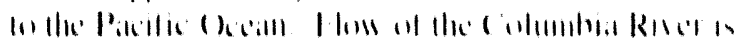

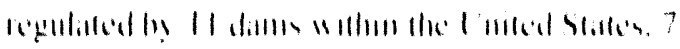
IIp)

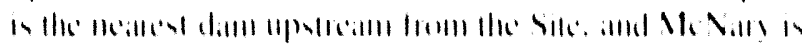

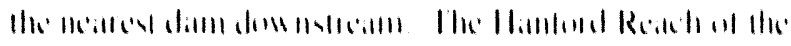

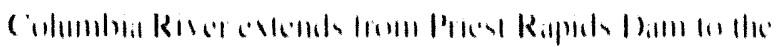

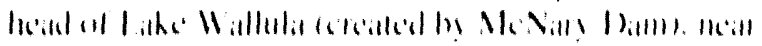

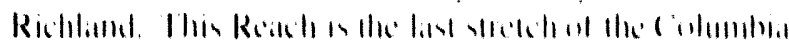

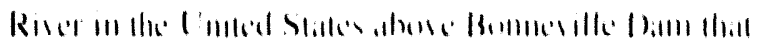

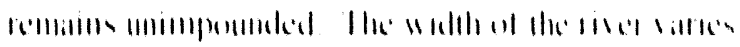

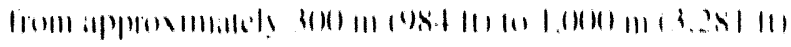

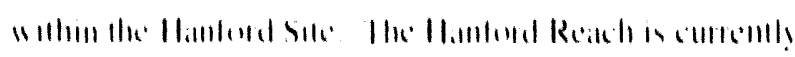

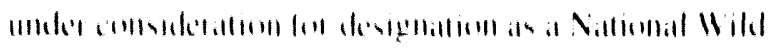

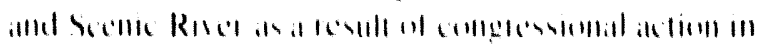

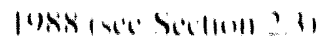

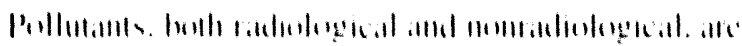

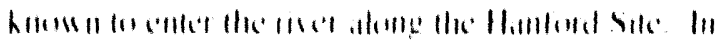

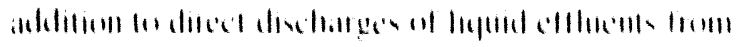

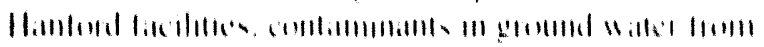

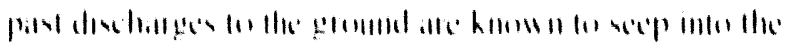

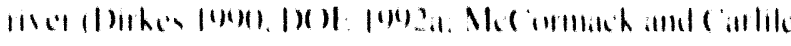

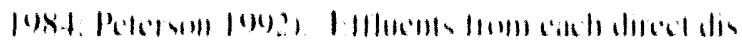

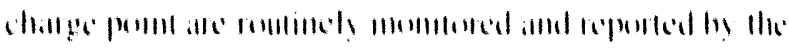

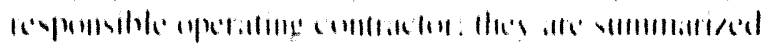

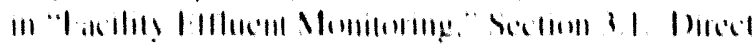

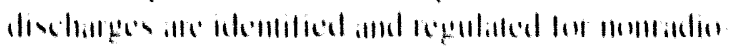

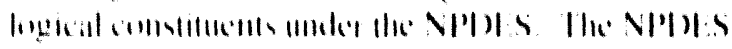

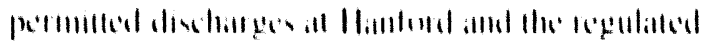

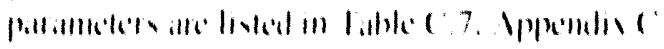

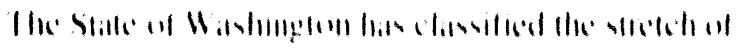

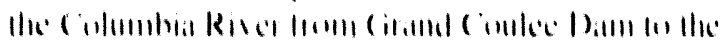

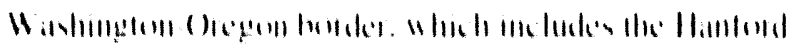

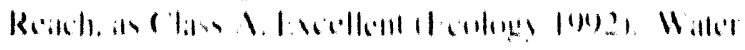

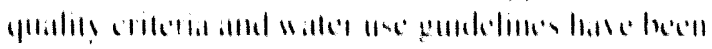

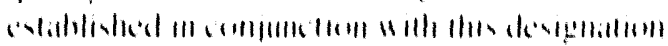

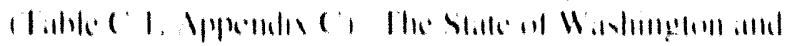

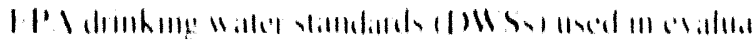

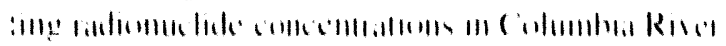

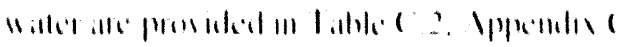

\section{Sample Collection and Analysis}

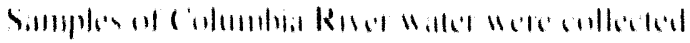

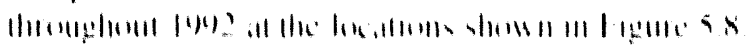

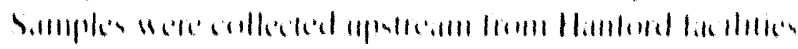

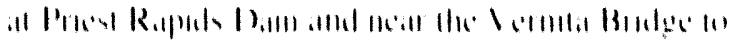

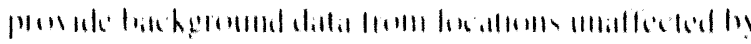

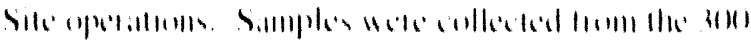

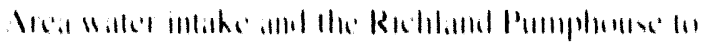

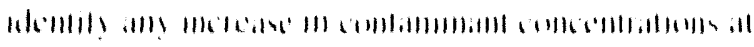

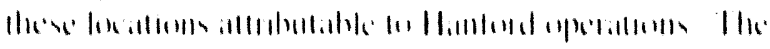

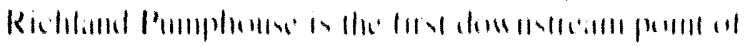


Table 5.5. Surface-Water Surveillance, 1992

lacillinil

Simple Ty

Irepuens:

Analysurs

\section{(olumblu River · Rudiologicul}

Prices Ralpids Dillu and Richland

('IIImulatline

$M(\cdots, n)^{n}$

(collested incehls)

Particulanc alfilters

\section{$M$}

() ( (IIII)

3(10) Areil Walled lillake

Prien Rapids Dilm. Richlamil. antl

Suluble (rovin)

M

() (i)III)

(irah ilramedels)

()

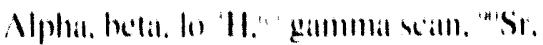
"lic. 1 !"

(iillmIma! scilll

P'I'

3110) Areal Willer lillike

Virmila Bridge alnd Richland

Colimbla River - Nonradiolongleul

Vermila and Rishlambl

(itill)

()

W(2) NAS(Q) N. Lemperallure, dimolled

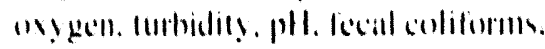

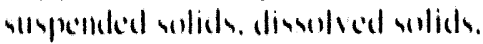

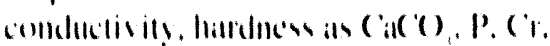

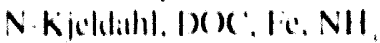

Vernitat and Richlint

(itablenamsects) (?)

Vermila and Richland

Thermongaph

(imlintilile

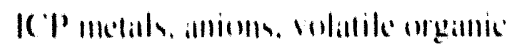

\section{()nsilfe Ponds}

Wenl lahe

$\begin{array}{ll}\text { (iriah } & (\text { ) } \\ \text { (iralh } & \mathrm{N} \\ \text { (ival } & (\text { ) }\end{array}$

()

M

|3 Pu|l|

1.1. Pund

\section{(ontivite Winter}

Ringuld Hilldery.

(irill)

Manlew Cormer.

While Blult, Mhallum.

White Blalls de(e). and

Nesinder lanm

Riscritull Gimal

(iril)

1

Mphat. helat. H. "Si. 1: Hillmmill seill

\section{Rlverbunk Siprings}

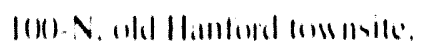

(itill)

1

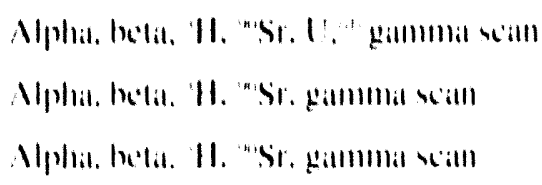

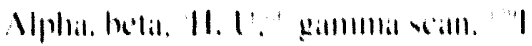
atml 3010 ) Nreat

Mpha. helat. H. "Si. "The gimmmat will, 1

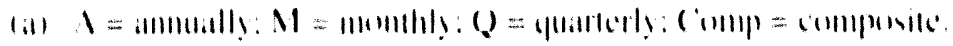

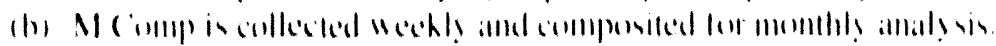

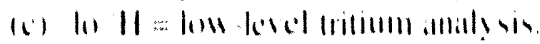

(d) Intuplic mratmium

(c) Somplic plumminum.

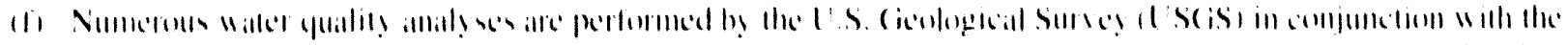

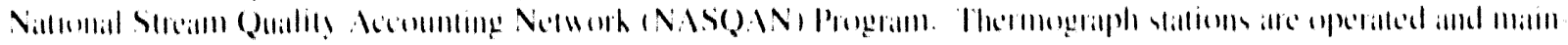

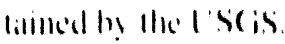

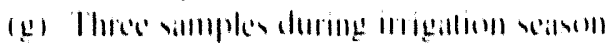


Table 5.8. Sediment Surveillance, 1992

l.mittin)

\section{MeNury Inam}

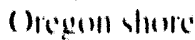

$1 /$ from ()regun shore

$2 / 3$ /rwा () /

Walinglont vinte

Priest Ruplels I)

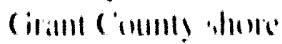

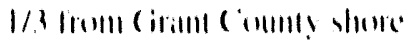

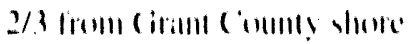

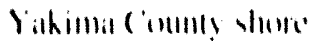

While Blults Slowph

1010-1: Slough

Hunford Slough

Richlund
Irifulisticy

A

$A$

$A$

$\wedge$

$\lambda$

$\Lambda$

$A$

$\lambda$

$\wedge$

A

$\lambda$

$\lambda$

Anilymen

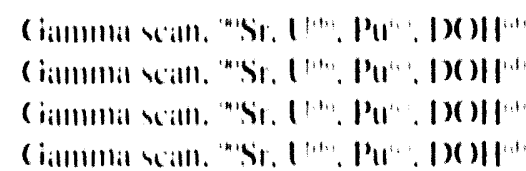

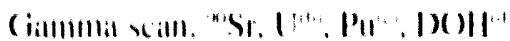

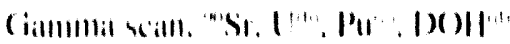

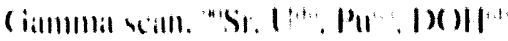

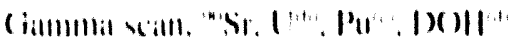

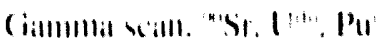

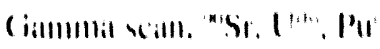

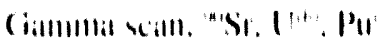

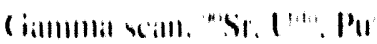

(i) S(ce ligutere $5 . x$.

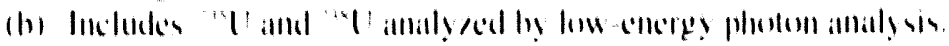

(i) Bollopic plutuminum.

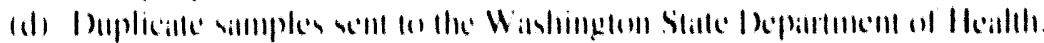

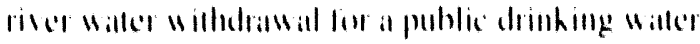

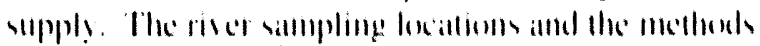

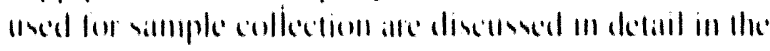

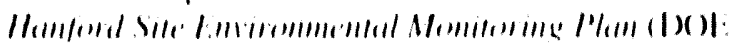

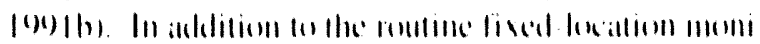

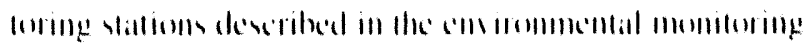

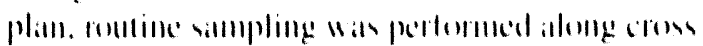

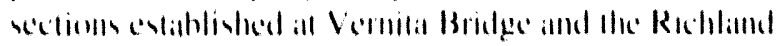

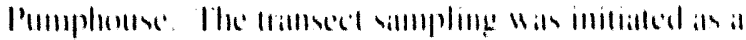

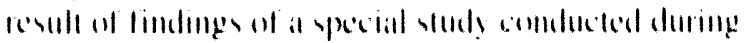

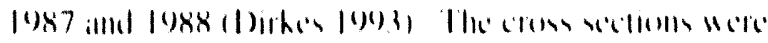
tampled yuaterly durme (W).

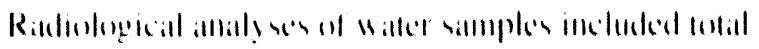

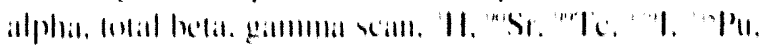

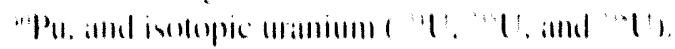

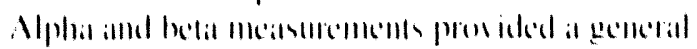

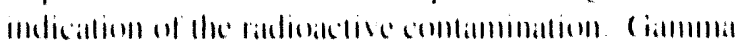

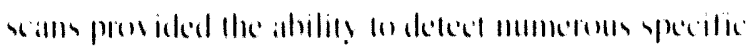

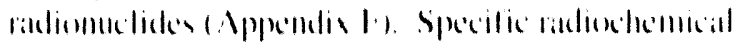

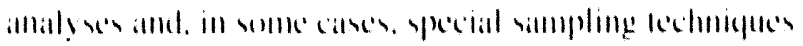

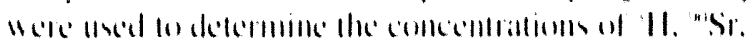

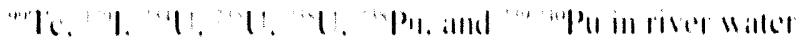

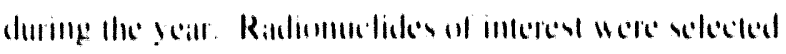

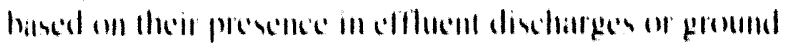

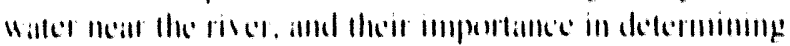

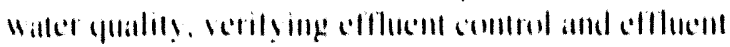

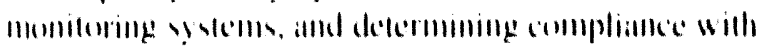

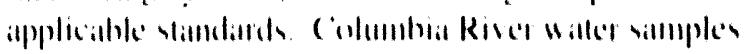

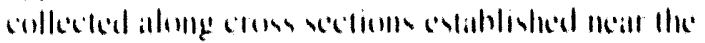

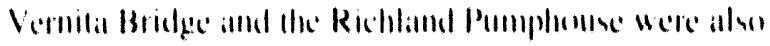

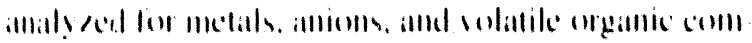

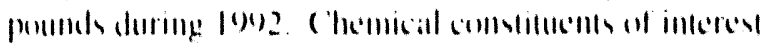

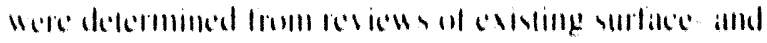

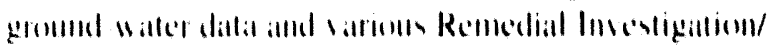

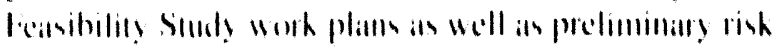

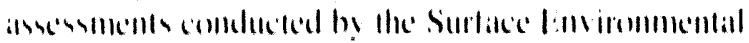

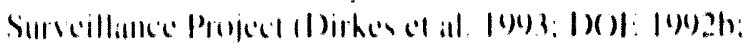
l:

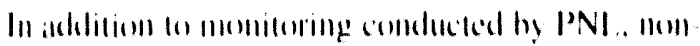

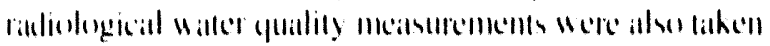

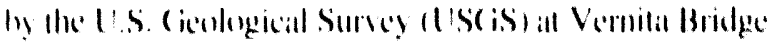

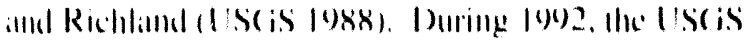
simplen were collected allonge croms sectioms every 2momlls all Vermita Bridge and yuarterly al Richlamol. 


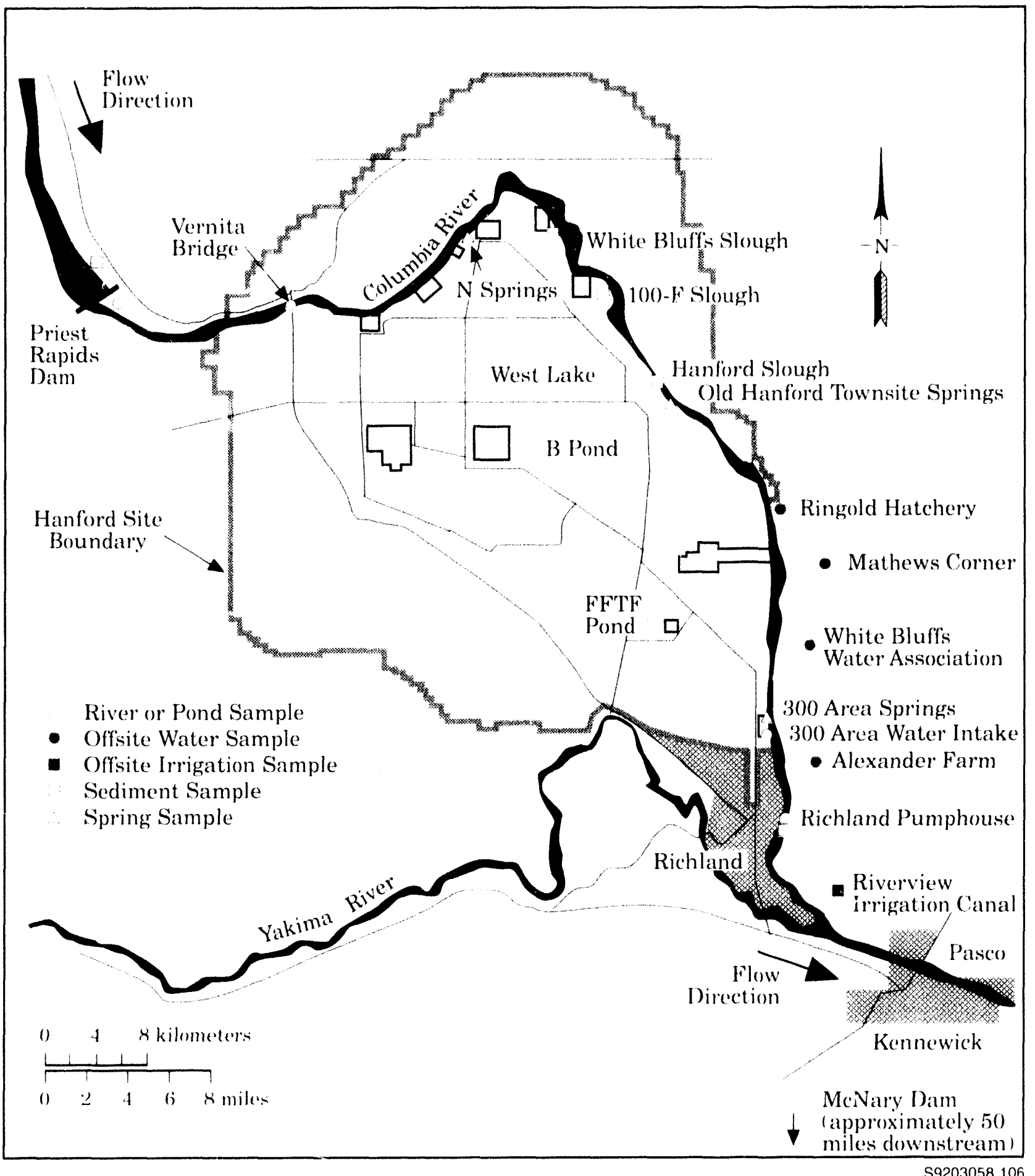

Figure 5.8. Water and Sediment Sampling Locations, 1992 


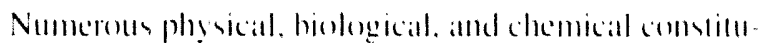

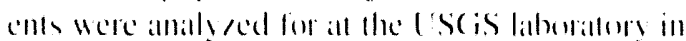

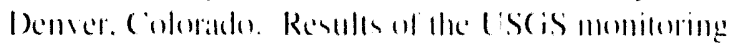
activities atre dexumented in Biyping and Wondrull (1093). In addition on ampling. Whe l se is provided

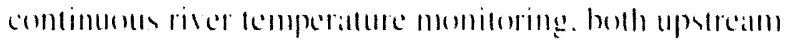
from the Site near Prient Rappieh Daln and all Richland.

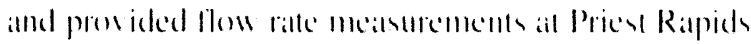
1):III.

\section{Radiological Results for River Water}

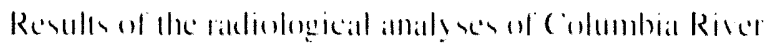

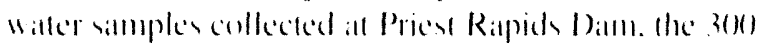

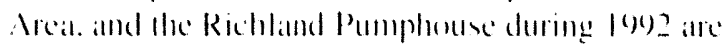

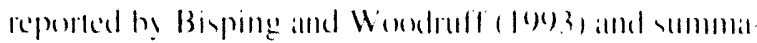

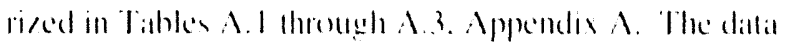
summatries also include the masimum indievidual revell

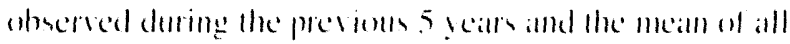

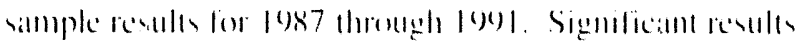

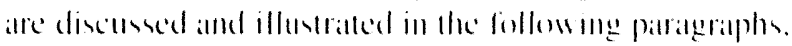

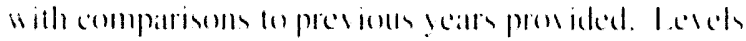

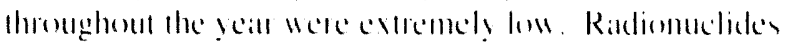

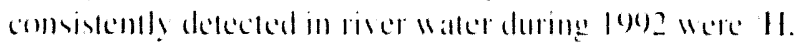

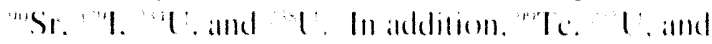

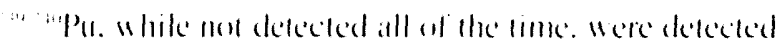

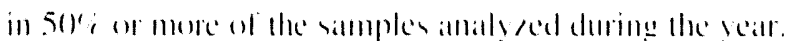

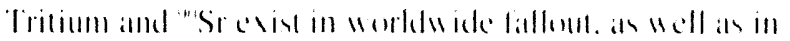

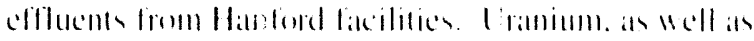
H. excurs nallurall! in the eme iromment in aldelitum to being present in Hantord effllems

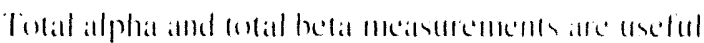

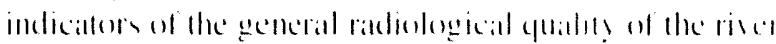

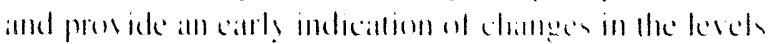

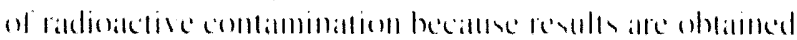

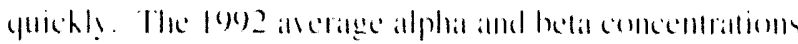

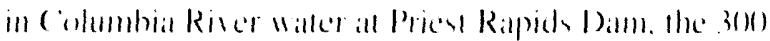

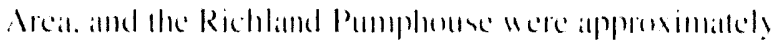

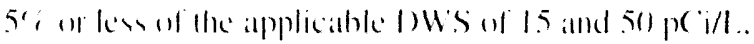

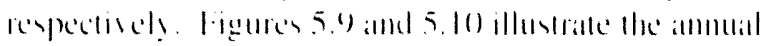

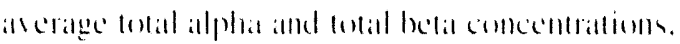

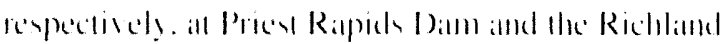

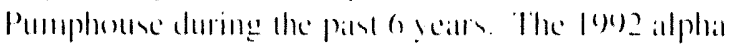

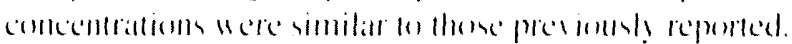

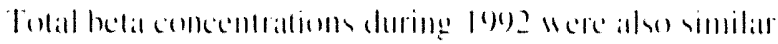

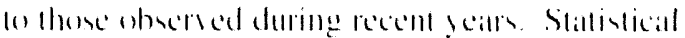

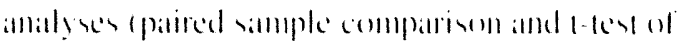

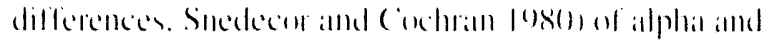

hetal concentrations at Priest Rappide Datm and the Richland Pumphouse indicalled the dilferences were not significanl 15's significance lesell.

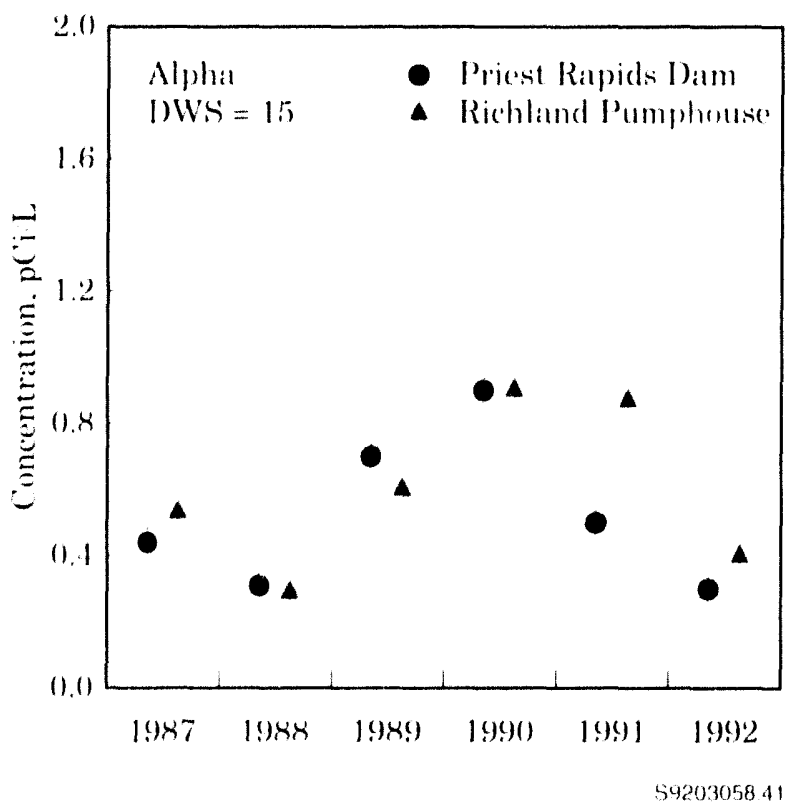

Figure 5.9. Annual Average Tol.1 Alpha Concentrations $( \pm 2$ SEM) in Columbia River Water, 1987 Through 1992

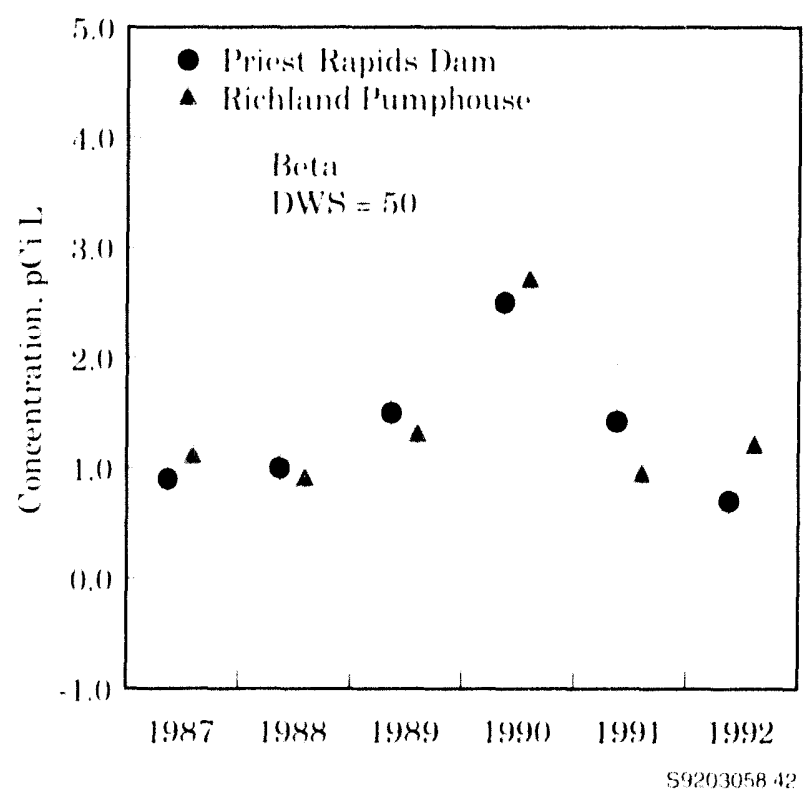

Figure 5.10. Annual Average Total Beta Concentrations ( +2 SEM) in Columbia River Water, 1987 Through 1992 


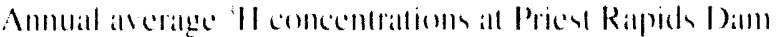

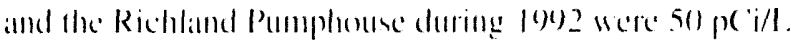

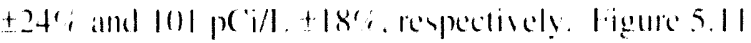

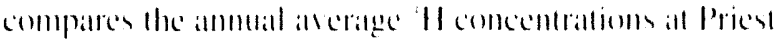
Raprid Dam and the Richand Pamphouse lanm 1987

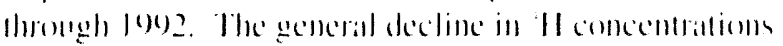

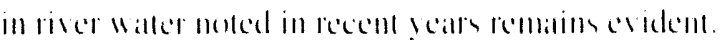

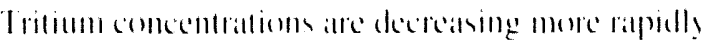

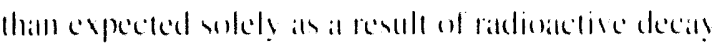

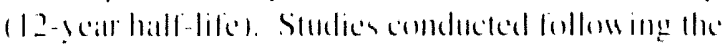

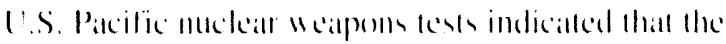
effectise residence time of 11 deposiled on the

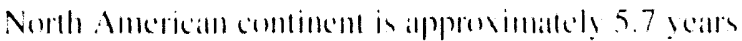

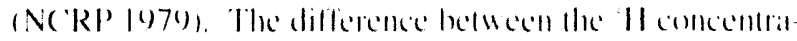

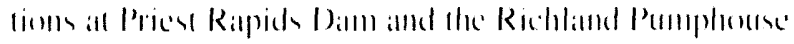

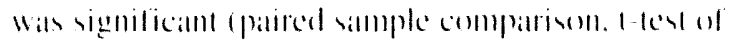

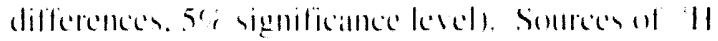

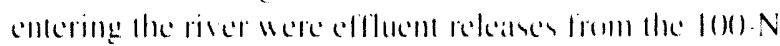

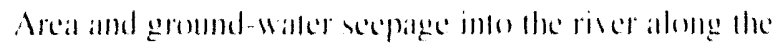

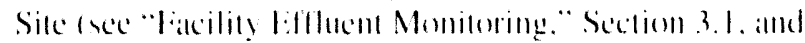

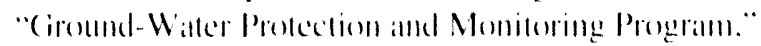
Section 5.8). Tritium concentralloms meabented all the Richland Pumphouse, while representative of the walled comsumed by asem of the cily of Richland drinking

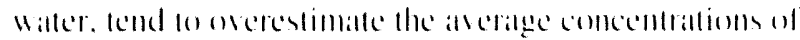

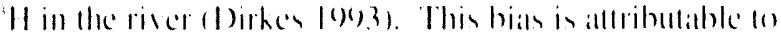

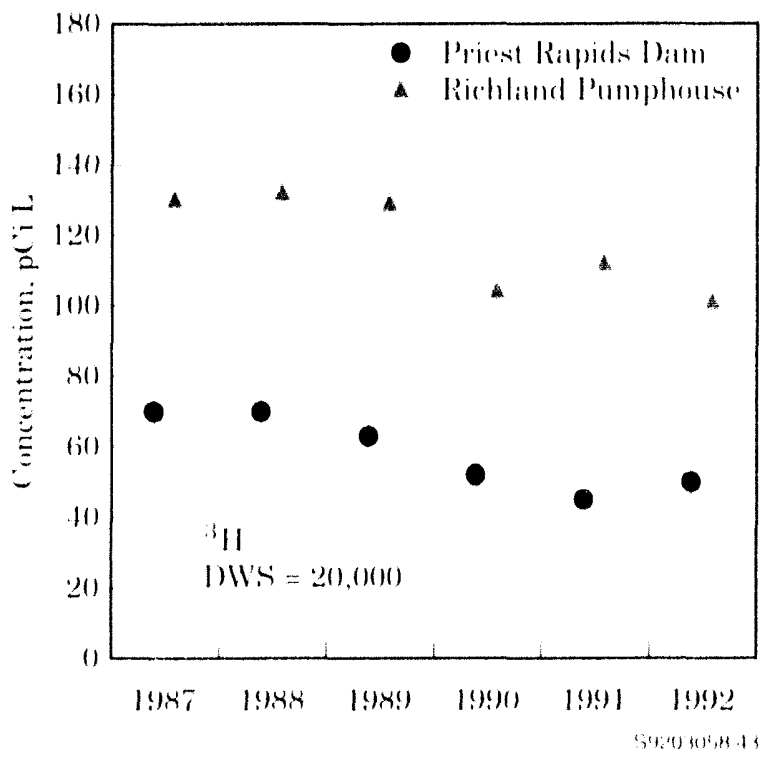

Figure 5.11. Annual Average Tritium ('H) Concentrations ( +2 SEM) in Columbia River Water, 1987 Through 1992. As a result of figure scale, some uncertainties (error bars) are concealed by point symbol.

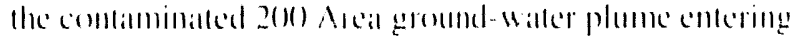

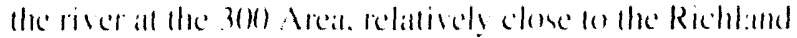
simple intake: his plome is mol completely mised willin the riser at the Richland Plumphouse. Sampling along a

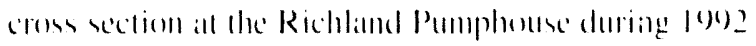
contiomed this concentration gradient in the river ander

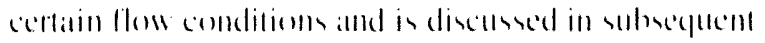

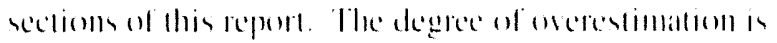

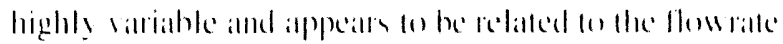

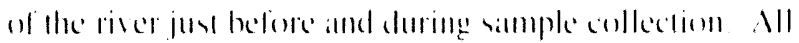

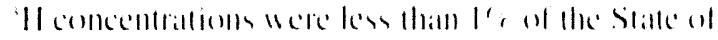

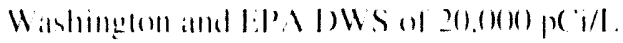

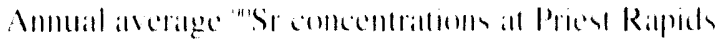

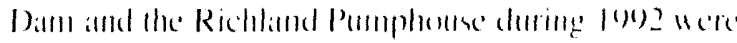

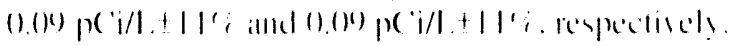

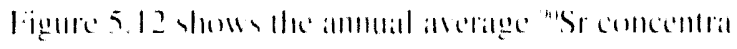

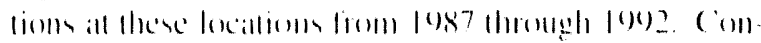

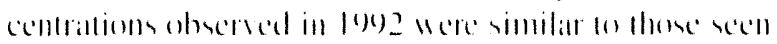

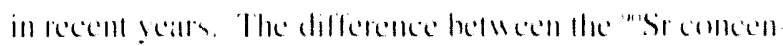

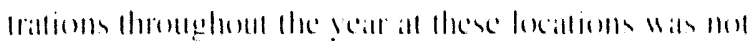

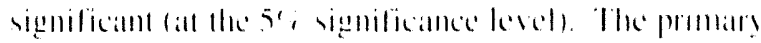

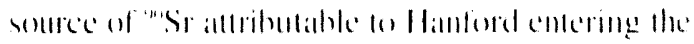

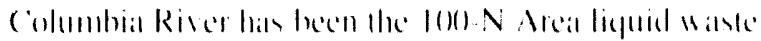

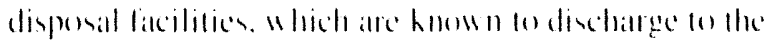

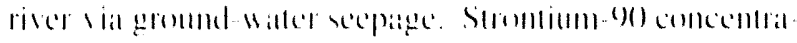

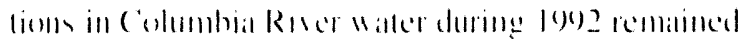

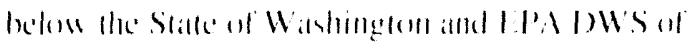

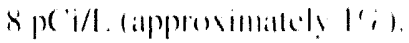

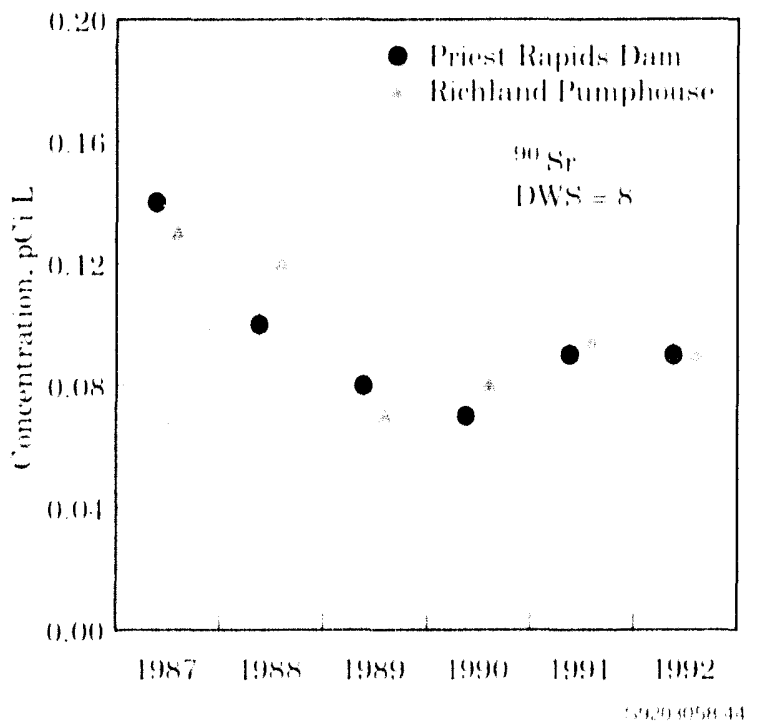

Figure 5.12. Annual Average Strontium-90 ("Sr) Concentrations 12 SEM) in Columbia River Water. 1987 Through 1992 


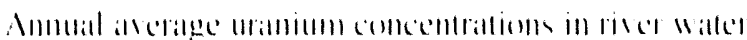

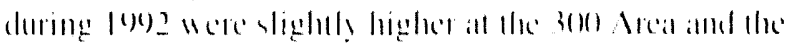

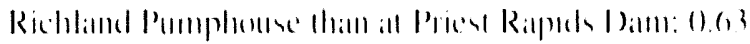

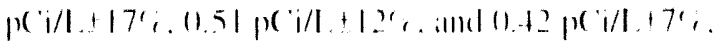

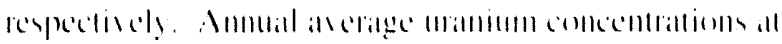

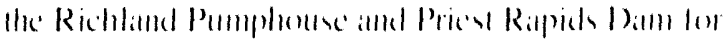

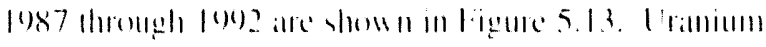

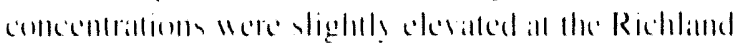

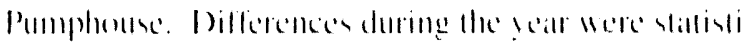

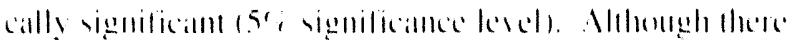

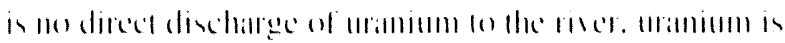

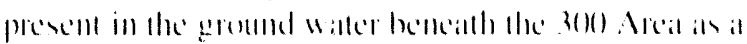

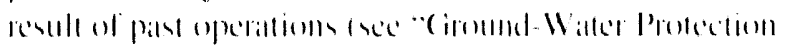

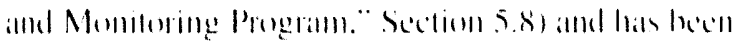

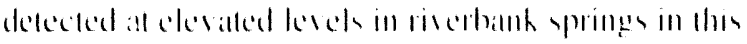

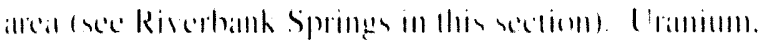

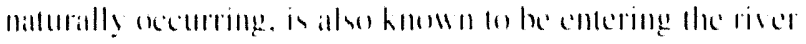

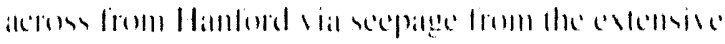

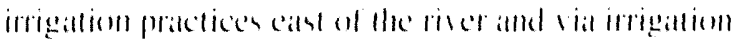

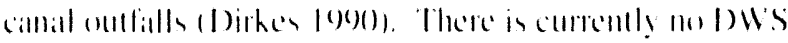

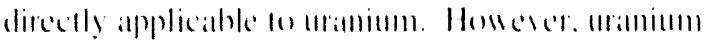

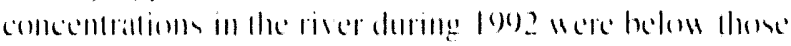

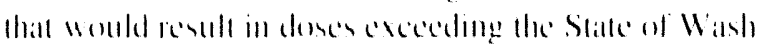

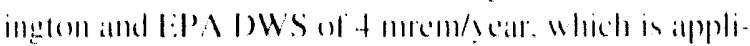
cable wambropogenic radionuclides.

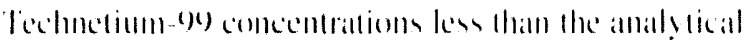

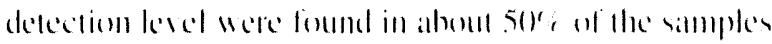

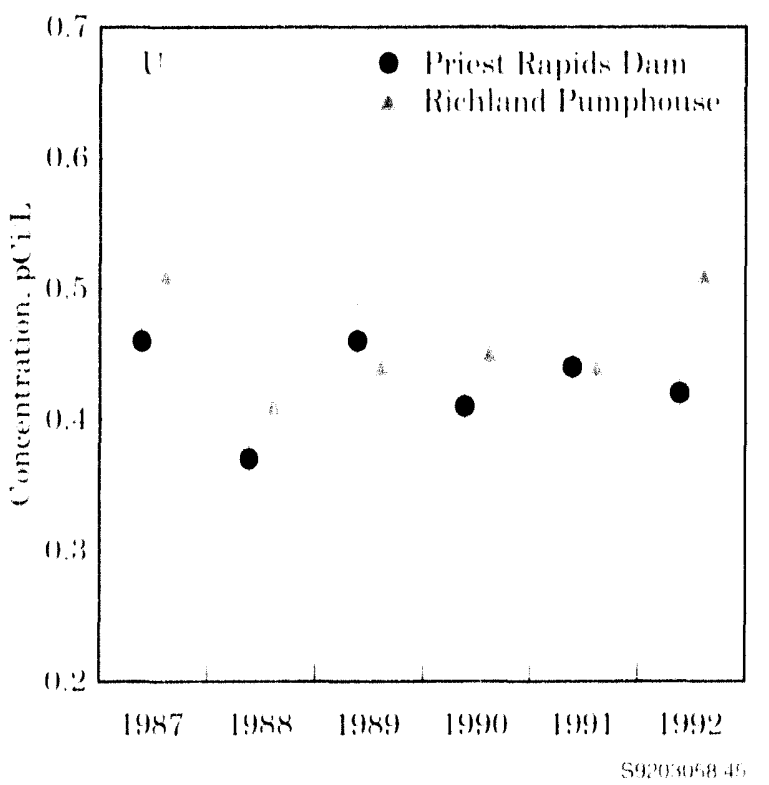

Figure 5.13. Annual Average Uranium Concentrations (+2 SEM) in Columbia River Water, 1987 Through 1992

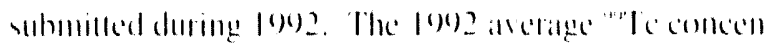

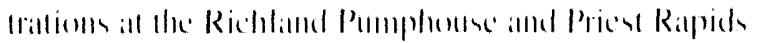

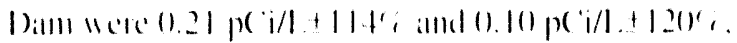

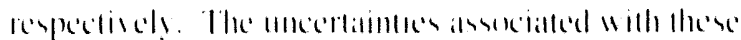

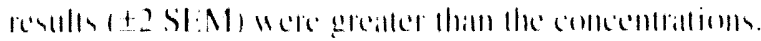

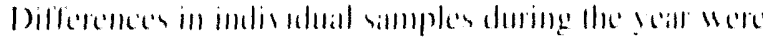

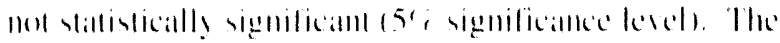

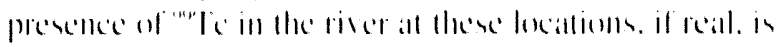

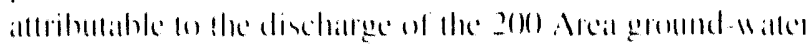

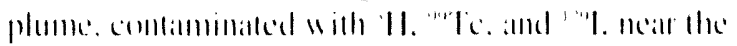

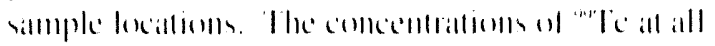

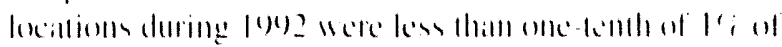
the I)WS (1)

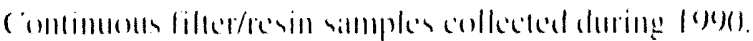

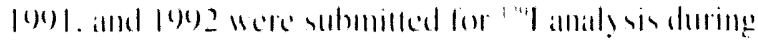

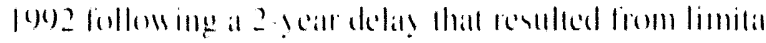

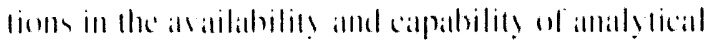

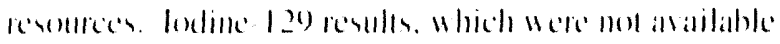

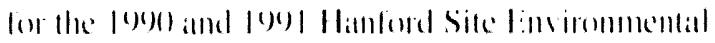
Reports atre provided in this report. The comedentrations of

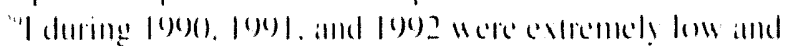

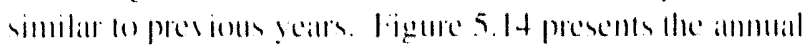

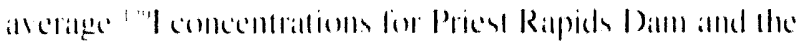

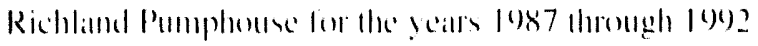

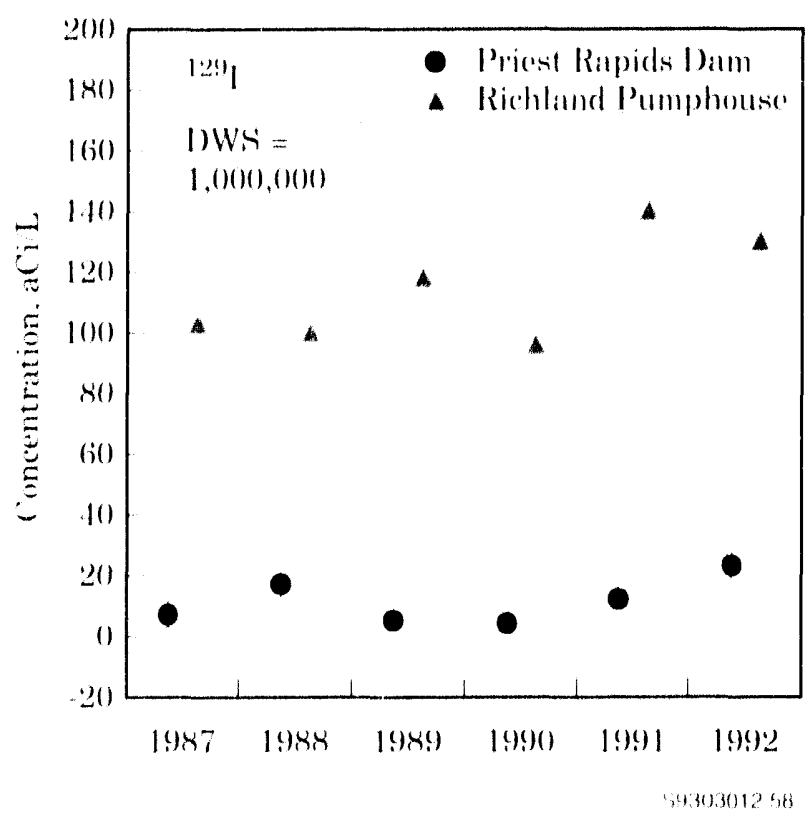

Figure 5.14. Annual Average lodine-129 (", Concentrations $1+2$ SEM) in Columbia River Water, 1987 Through 1992. As a result of figure scale, some uncertainties (error bars) are concealed by point symbol. 


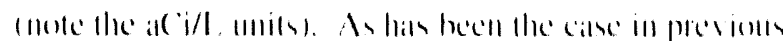

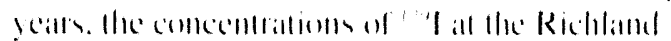

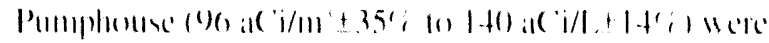

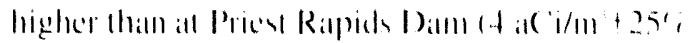

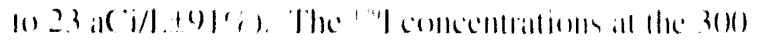

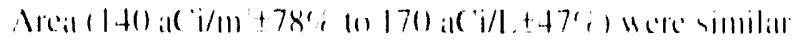

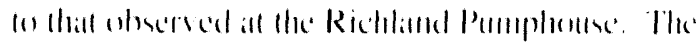

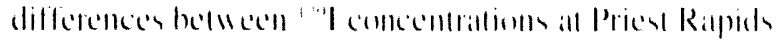

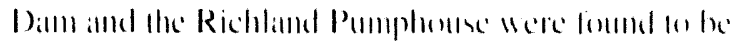

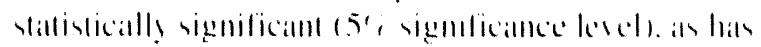

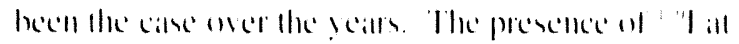

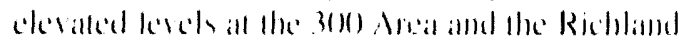
Pumphomse is allributahle to the How of combaminalled

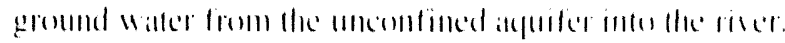

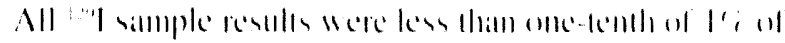
He I)WS

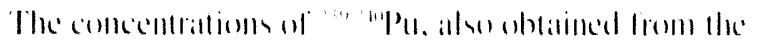

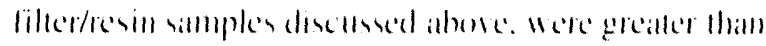
the deketion level apporimaltely 50 ,

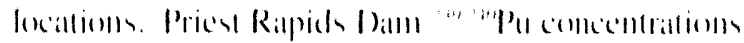

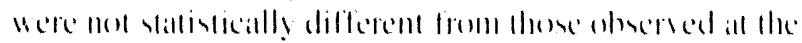

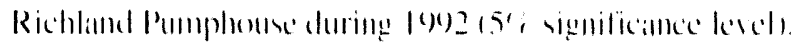

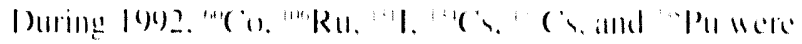
mol comsistently found in meanurable gutmmitices in

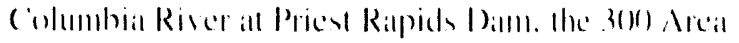

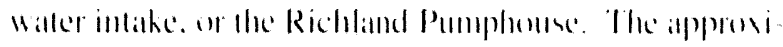

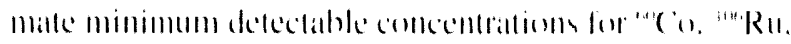

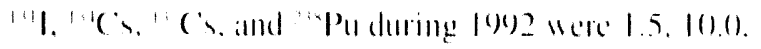

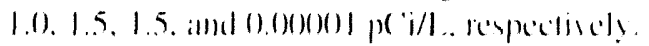

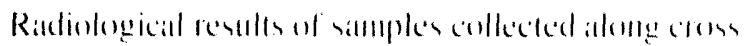
sections established an Vermita Bridge and the Richland Pumplouse during ley 2 are presented in lable $\mathrm{A} .4$.

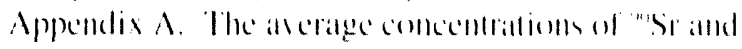

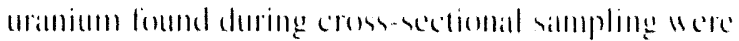

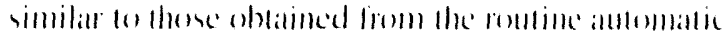

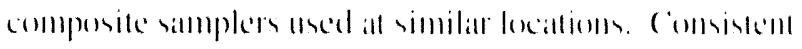

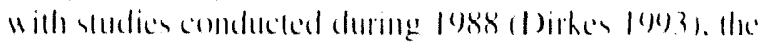

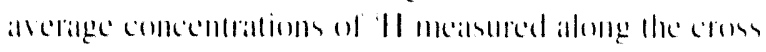

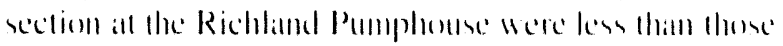

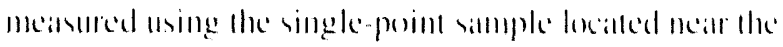

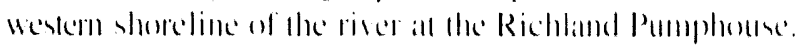

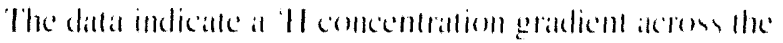

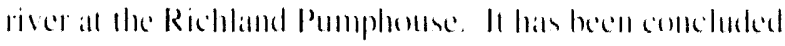

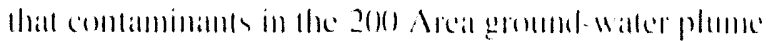

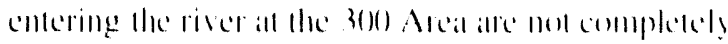
mised all the Richland Pemphemser. comsistem with pas

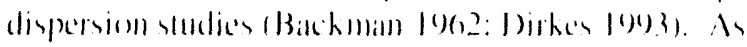

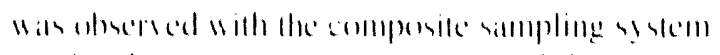

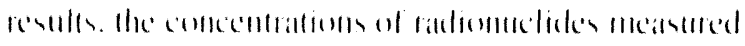

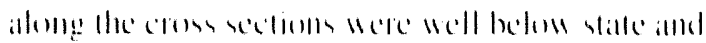

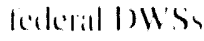

\section{Nonradiological Results for River Water}

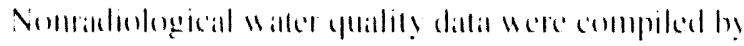

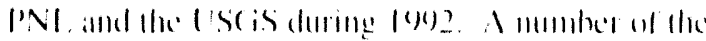

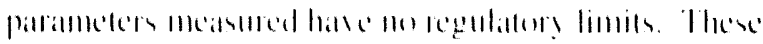

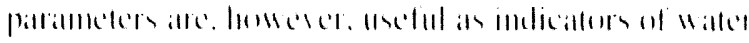

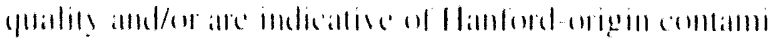

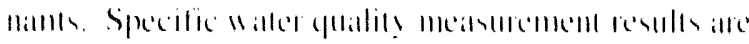

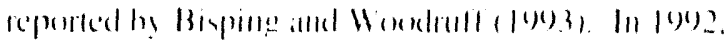

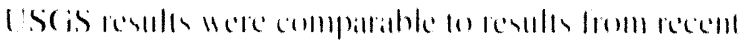

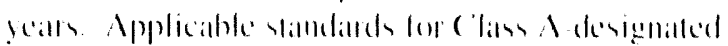

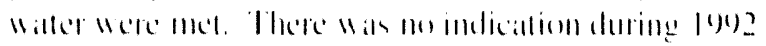

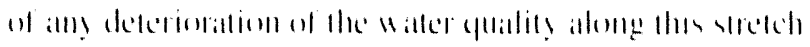

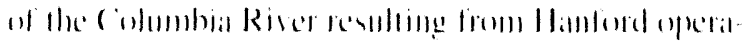

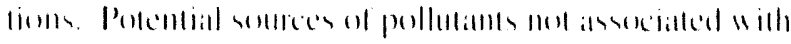

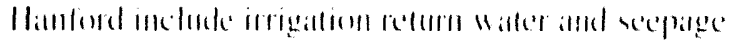

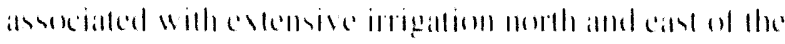
(i)lumblat Rirer.

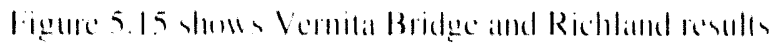

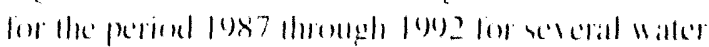

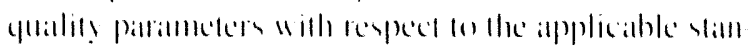

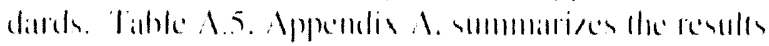

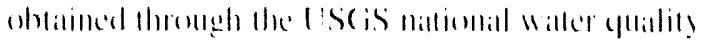

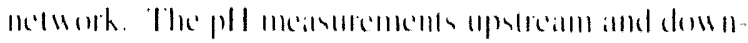

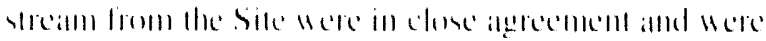

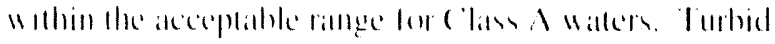

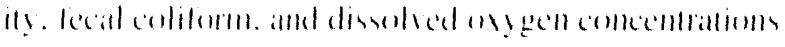

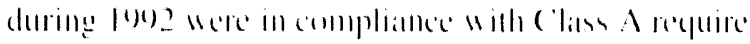

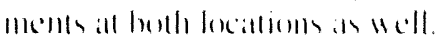

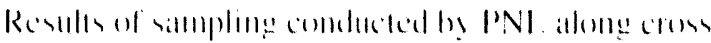

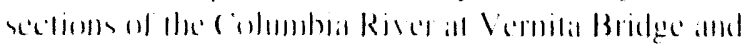

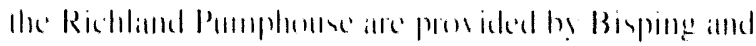

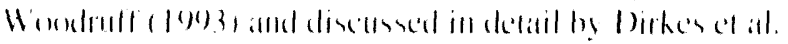

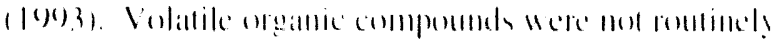

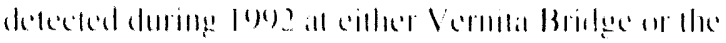

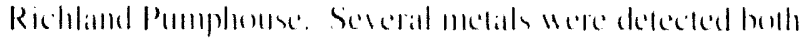

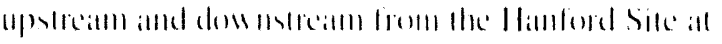

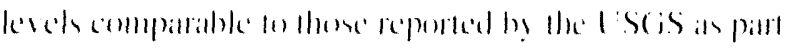

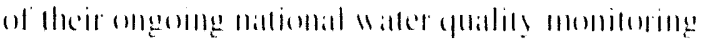

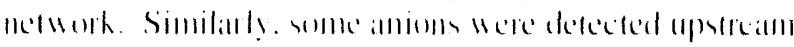

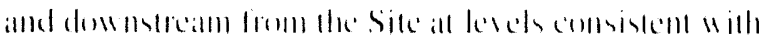



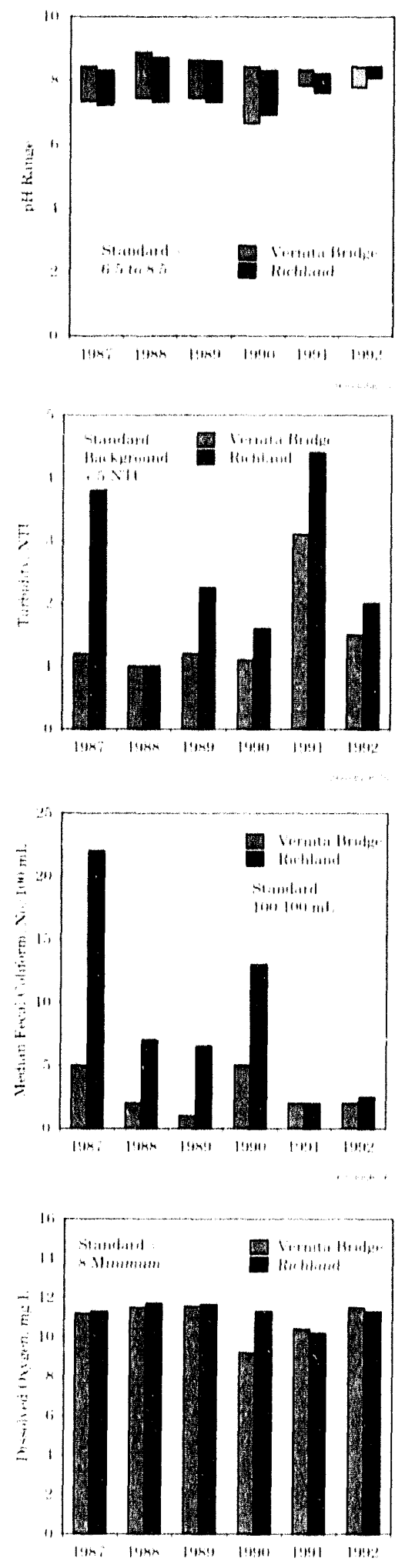

Figure 5.15. Columbia River Water Quality Measurements, 1987 Through 1992
Howe reported by the lise is. There was no meinterible difference between the confecentrations of metals or anions delected in lle river watter along the cross sections upstream from the Site all Vermila Bridge and down-

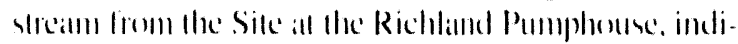

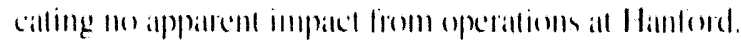

The anmual ancrage flow rate of the Colmonhia River was

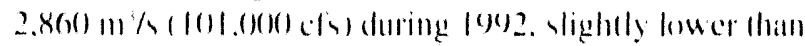

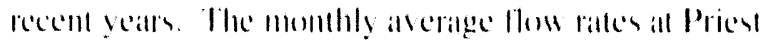
Rapide 1)am alle slome in lighure 5.16. The peak

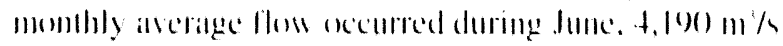

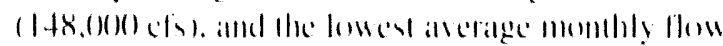

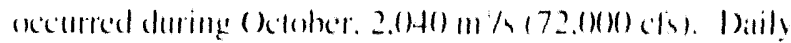

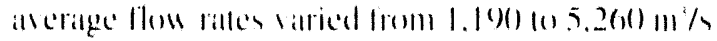

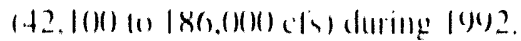

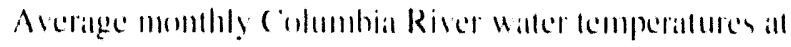

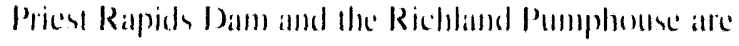

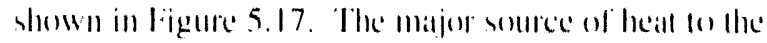

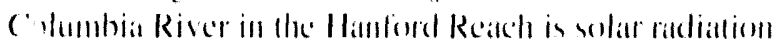
(1)auble (4) al. 1987). Riser kemperalures and the

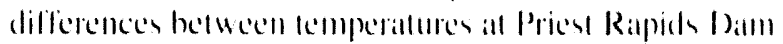

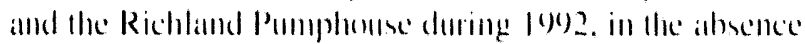
of reacerer aperations. Here similat an these in the past

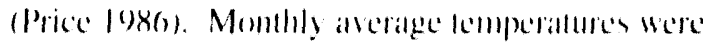
slighlly higher all the Richland Pumphenese ohall all Priest Rapide Dilm from fobruary through August alld

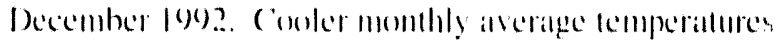
Here observed all the Richland Pumplomse during damuary and Nowember. Monthly ancrige kemperallures

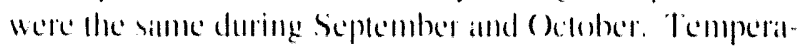
fures allong the Hatmlond Reade were in compliance with

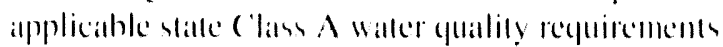
doring lle year.

\section{Columbia River Sediment}

\section{Sample Collection and Analysis}

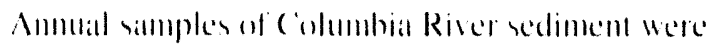

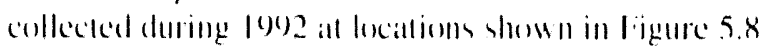

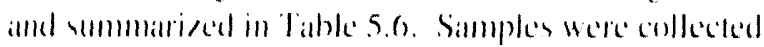
upstreann from the Hanford Sile hehind Rries Ratpids

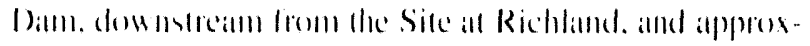

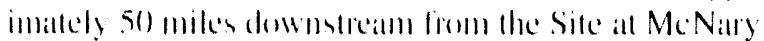
Dam. Samples were also collected along the Ilantord

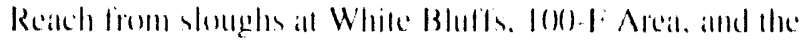

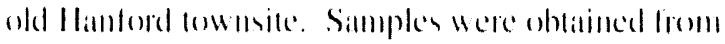
approximately $1.5 \mathrm{~cm}$ (6 im. 1 al the lop sedimem matlerial 


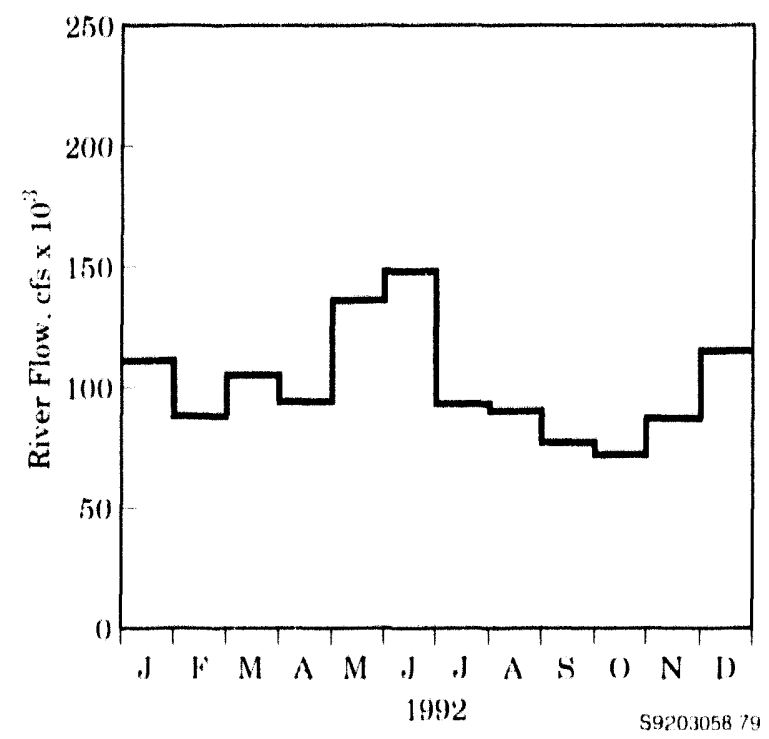

Flgure 5.16. Monthly Average Columbia River Flow Rates During 1992 (measured at Priest Rapids Dam)

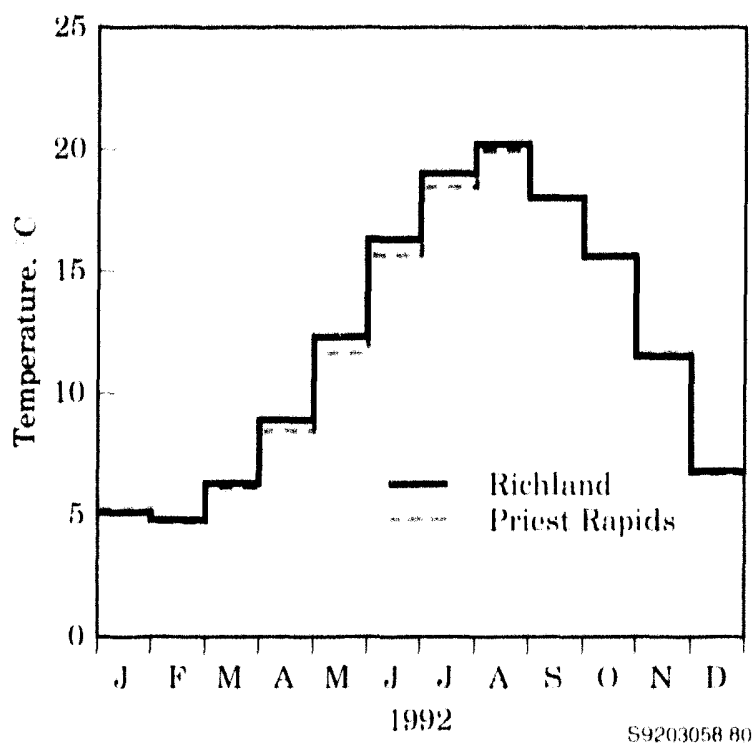

Figure 5.17. Monthly Average Columbia River Water Temperatures During 1992

using a dredge simpler. Antalysers of the sedimem

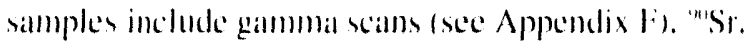

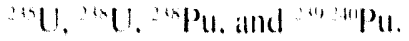

\section{Radiological Results for Sediments}

Surface sediments in the columbia Riser ane known lo

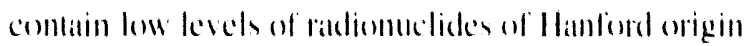
and from nuclear weipoms lesting fiallow r Beistey et all.

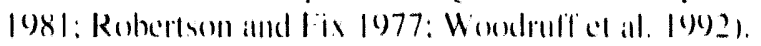
Analytical results for surfice sediment simples collected during 1992 alle summarised in Tahle A.6. Appendix A. and presented in detail by Bisping and Woudrulf (1993). Tithle A.6. Appendix A, illso incledes summonary data for

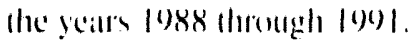

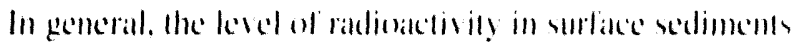
hehind M.Nary Ditnl was slightly higher than that

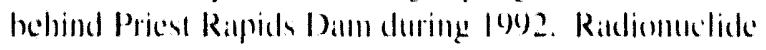
concentrattions in sediments collected from the whogh

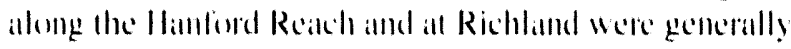

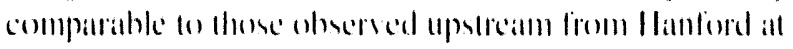

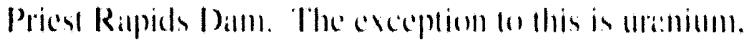
which was present in sediments collected all Richland all levele compartable to these al MeNally Dam.

Figure 5.18 shows the concentrations of selected radionucliden in Collumbia River sedimente all Priest Ralpids

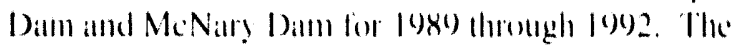
comcentrations of hadionuclides measured during 1992

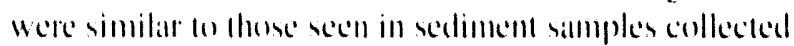
during the previous ty years. The concentrations of "we during f(9)2, which were les than the detection kevel (1).015 p ( 'i/g) in sediments belinal Priest Rapids Dam. were highes in sediments collected from MeNary pool.

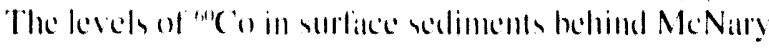

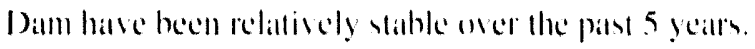

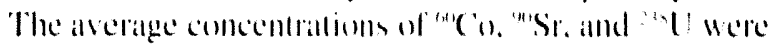
higher in sedimemb collected an Me Narly Dallm thill in these collected from Rries Rapids Dami during 1992.

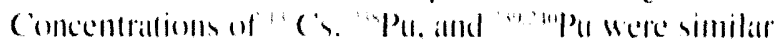
at the twoth lecillioms.

\section{Riverbank Springs}

The seeprage of geround watler into the Collumbia River

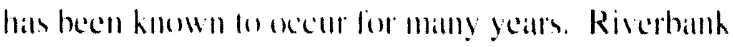

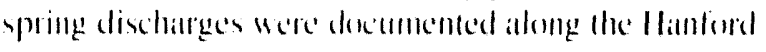



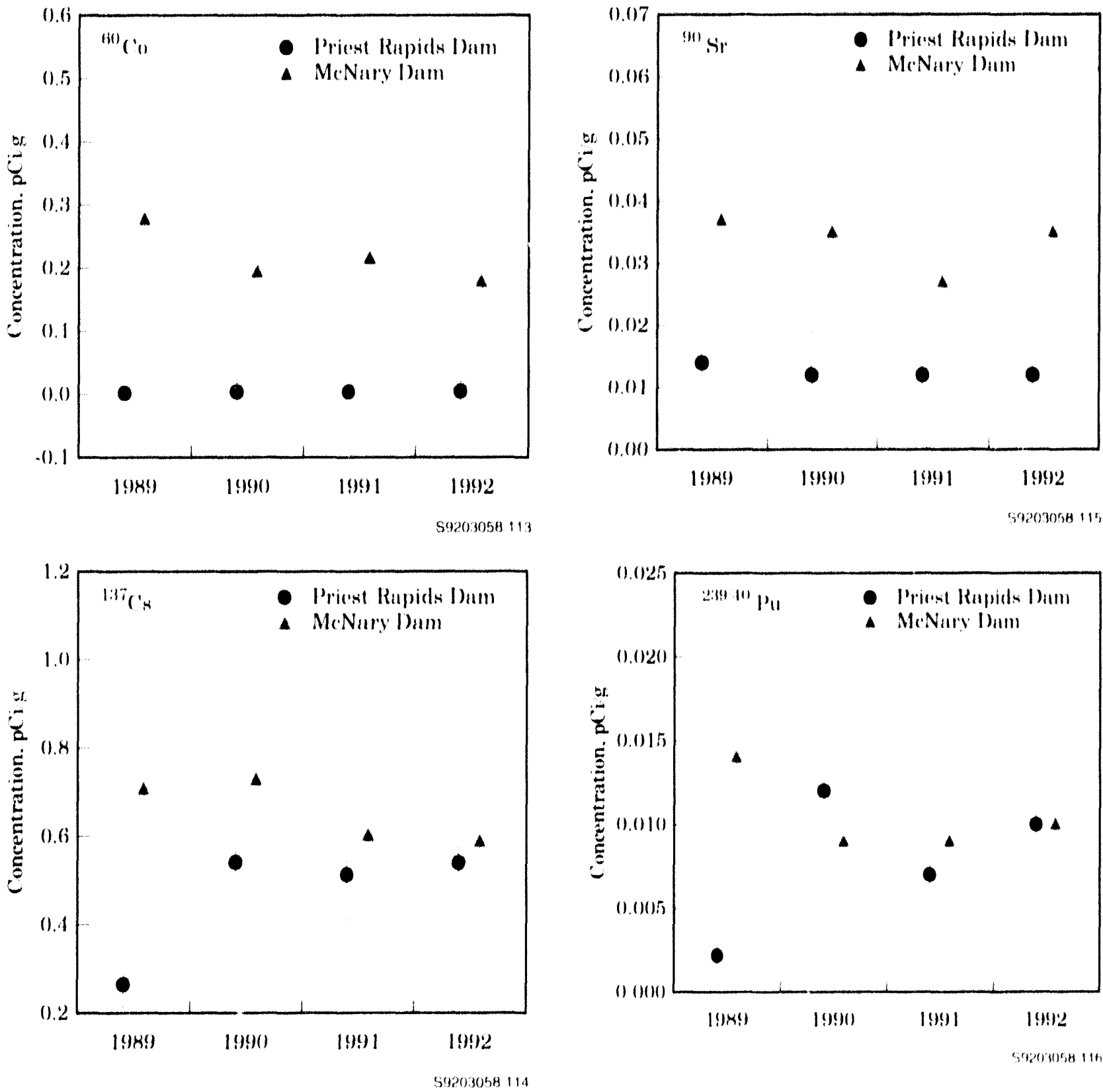

Figure 5.18. Radionuclide Concentrations ( \pm 2 SEM) in Columbia River Sediments at Priest Rapids Dam and McNary Dam, 1989 Through 1992. As a result of figure scale, some uncertainties (error bars) are con. cealed by point symbol.

Reach long before the startup of Hanteral operations denkins 19221. These relitively smbill springe llow intermiltenly. alpparently influenced primalrily by changes in river kevel. Hanford-origin combaminams

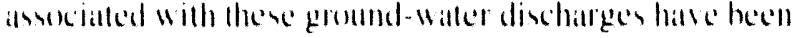
decumented los enter the river allong the Hanterd Reich

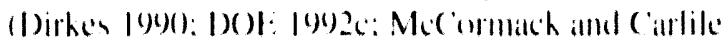

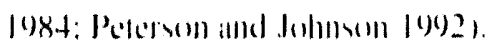




\section{Sample Collection and Analysis}

Silmplas

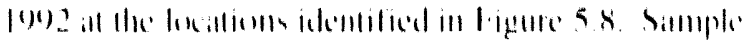

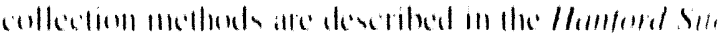

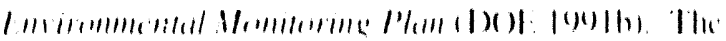

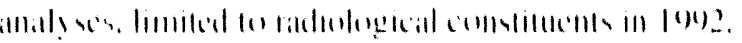

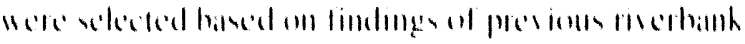

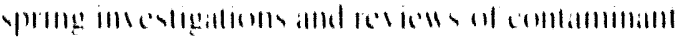

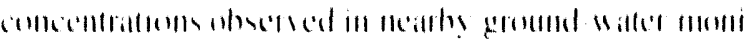

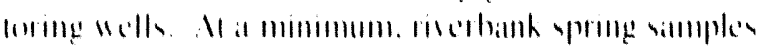

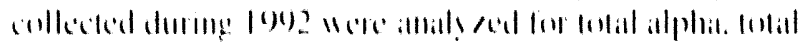

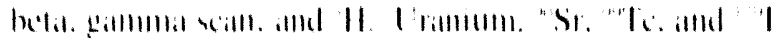

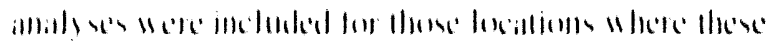

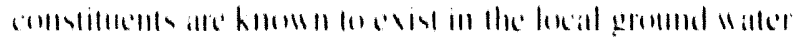

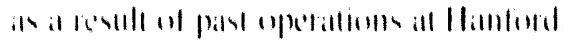

\section{Results}

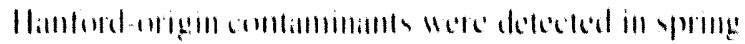

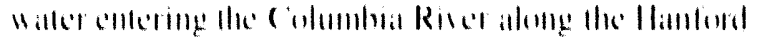

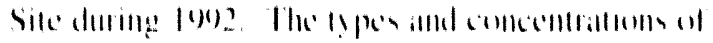

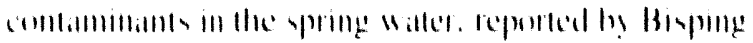

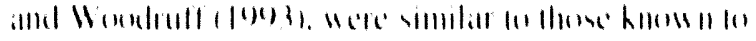

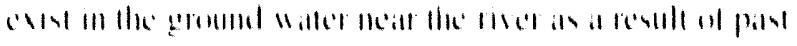
Drerallom

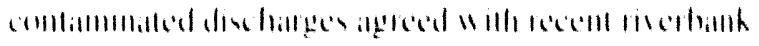

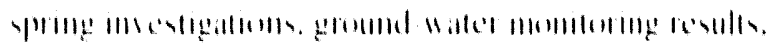

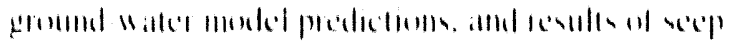

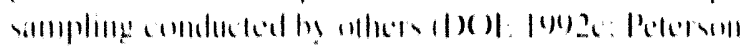

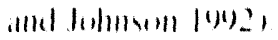

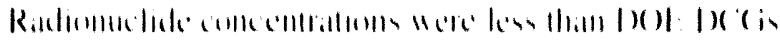

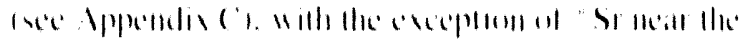

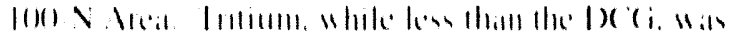

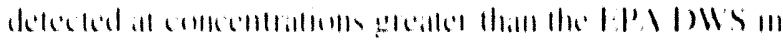

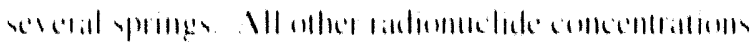

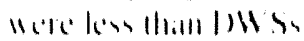

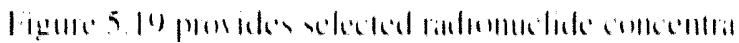

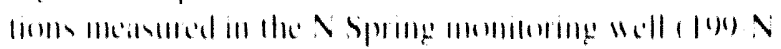

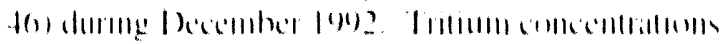

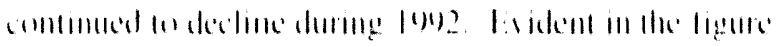

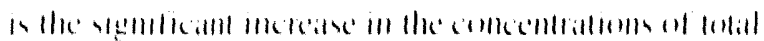

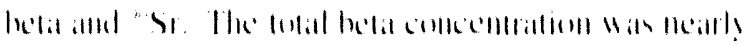

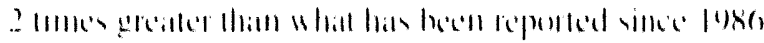

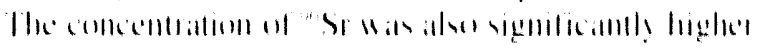

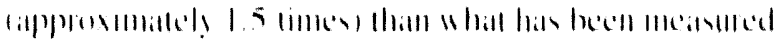

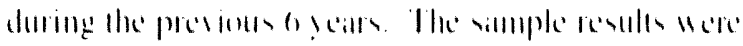

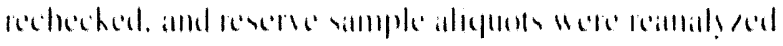

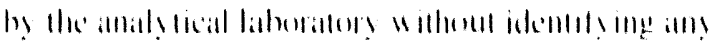
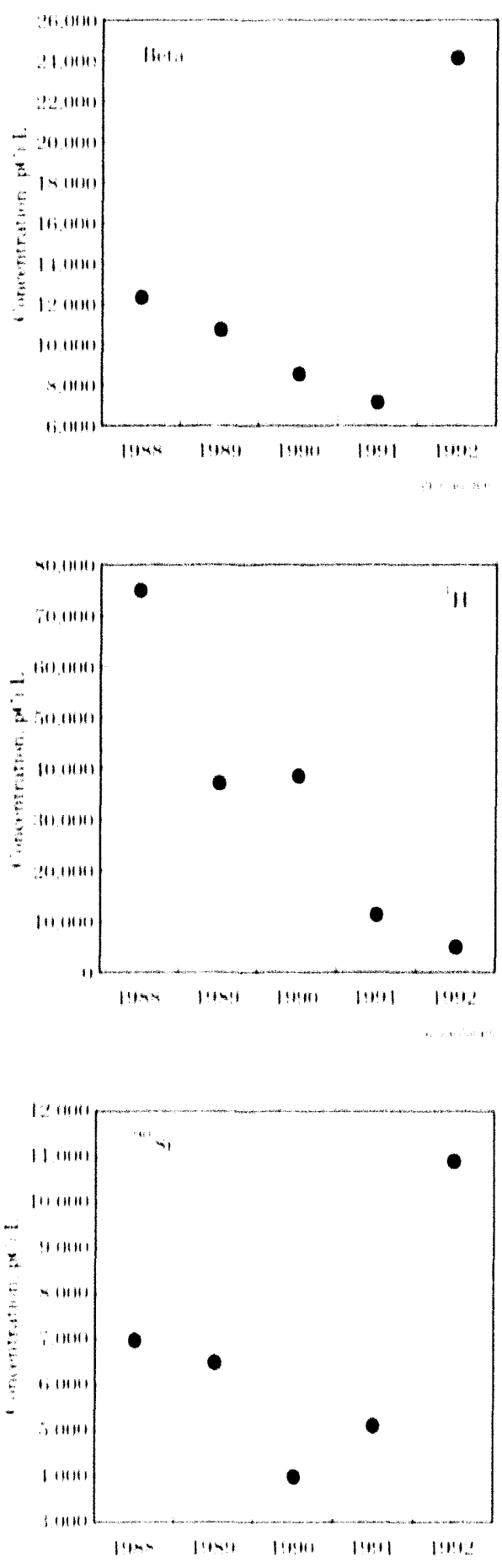

Figure 5.19. Radionuclide Concentrations 1+2 SEM) in N Springs, 1988 Through 1992. As a result of figure scale. some uncertainties (error bars) are concealed by point symbol. 


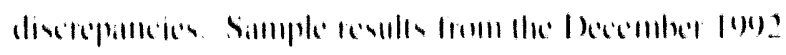

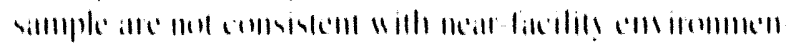

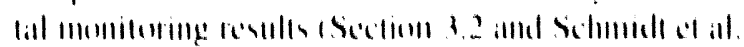

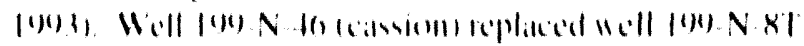

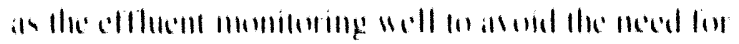

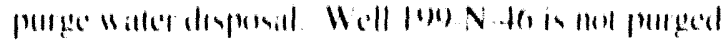

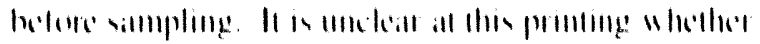

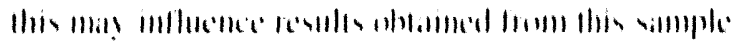
lixillinil.

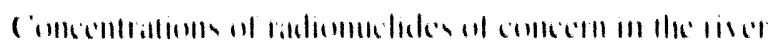

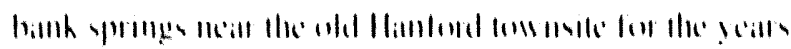

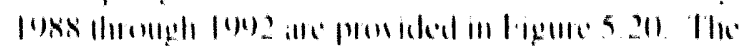

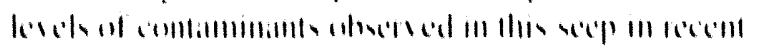

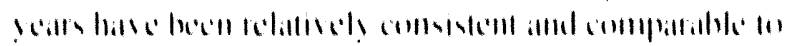

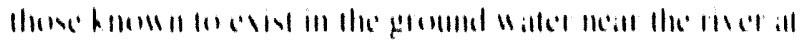

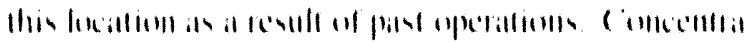

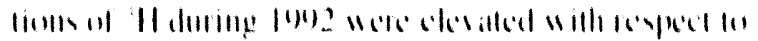

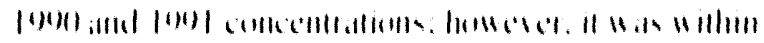

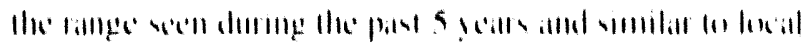

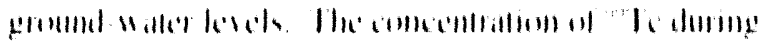

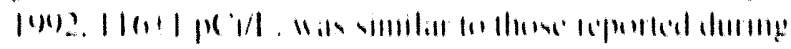

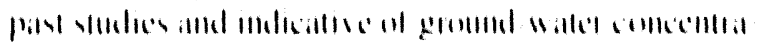

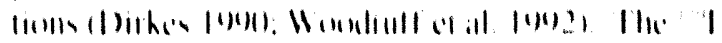

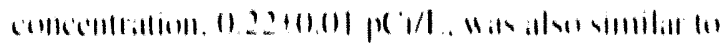

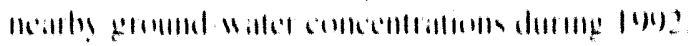

1.

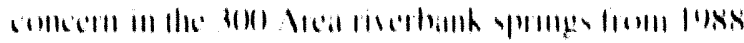

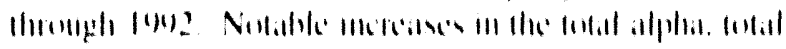

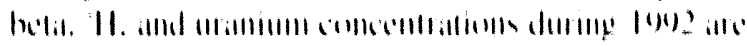

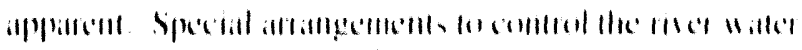

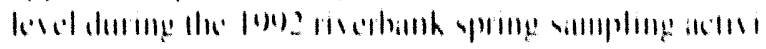

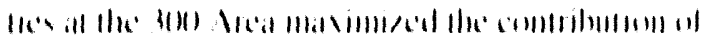

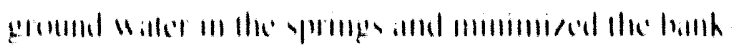

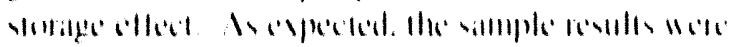

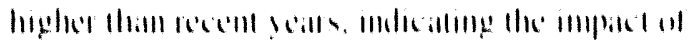

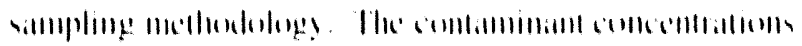

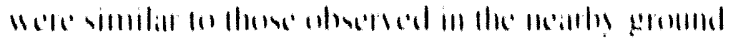

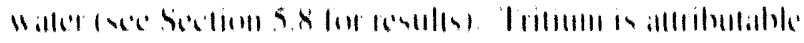

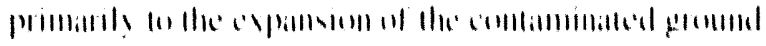

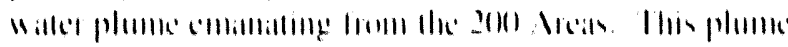

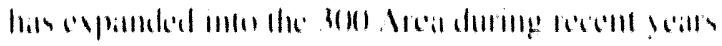

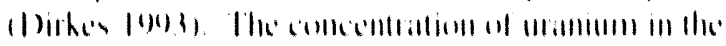

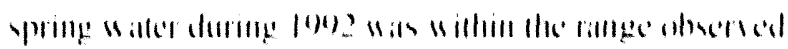

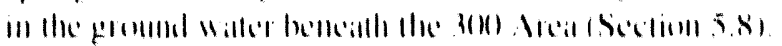

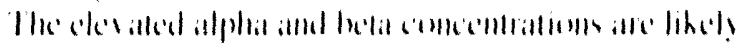

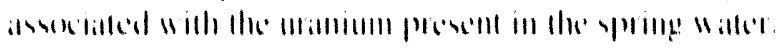
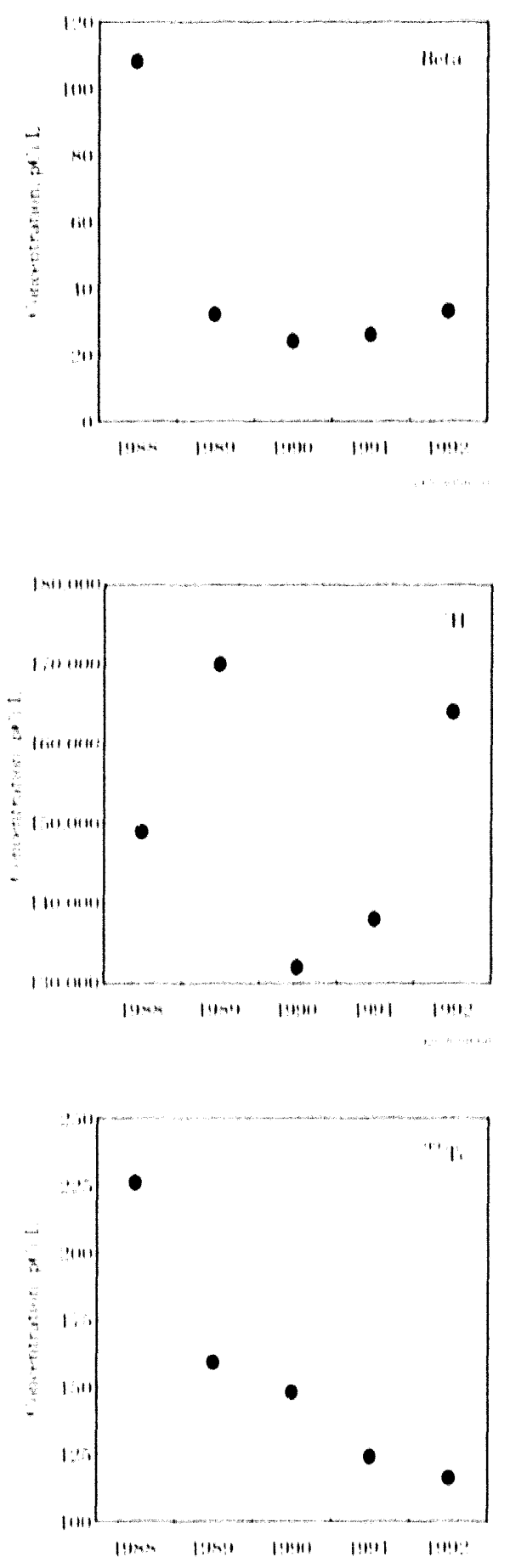

Figure 5.20. Radionuclide Concentrations 1,2 SEM) in Riverbank Springs near the Old Hanlord Townsite, 1988 Through 1992. As a result of figure scale, some uncertainties (error bars) are concealed by point symbol. 

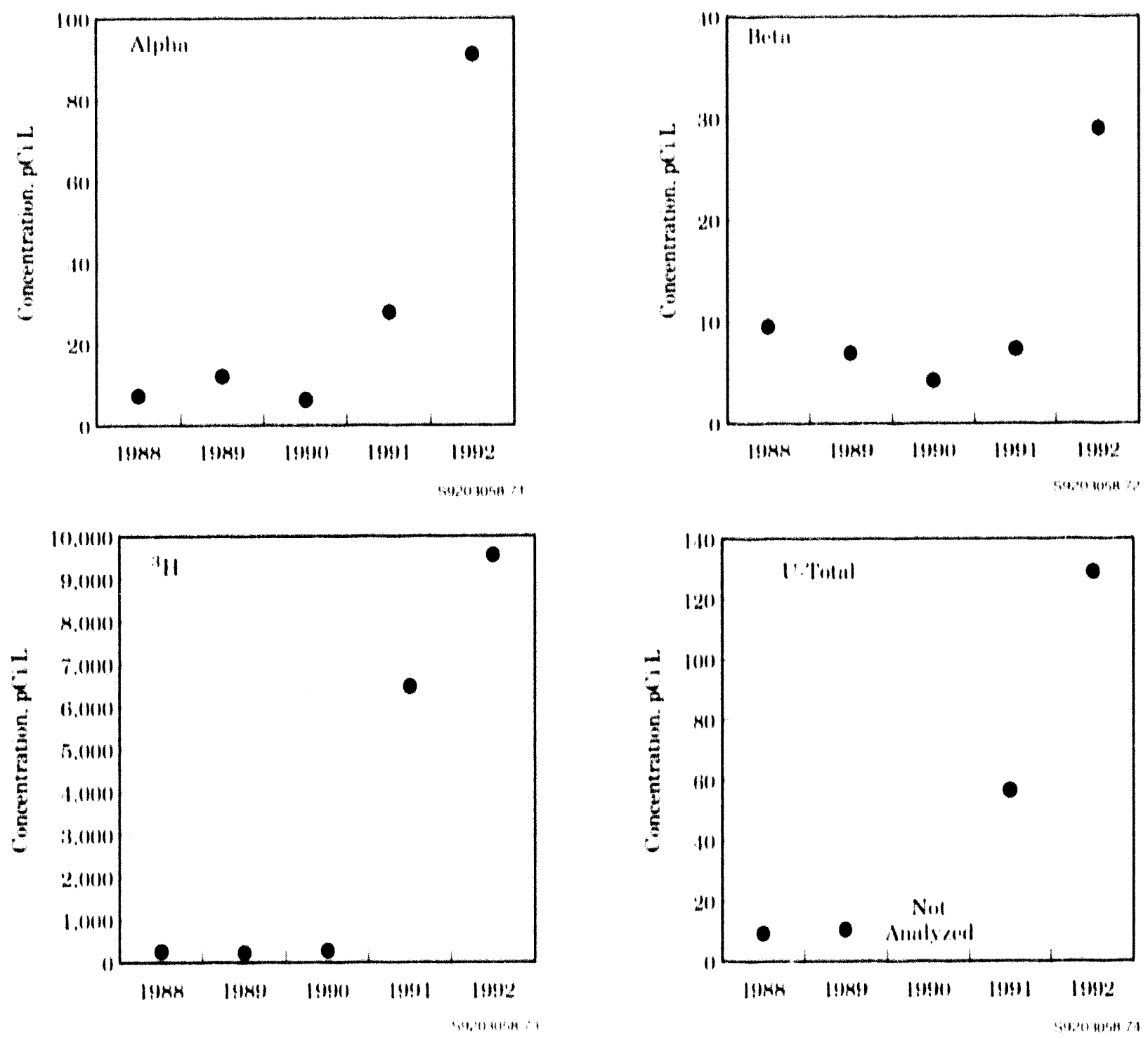

Figure 5.21. Constituents of Concern in 300 Area Riverbank Springs. 1988 to 1992. Concentrations are 12 SEM. As a result of figure scale, some uncertainties (error bars) are concealed by point symbol.

\section{Onsite Ponds}

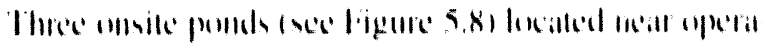

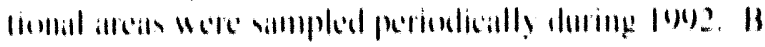

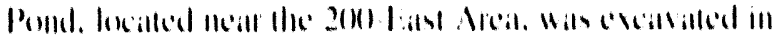

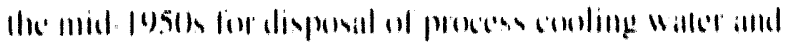

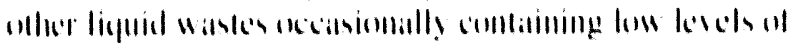

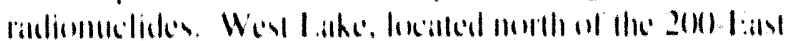

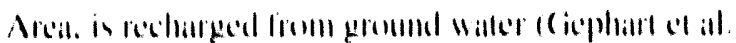

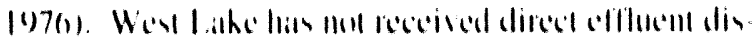

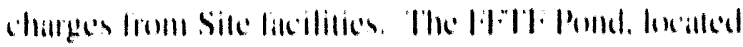

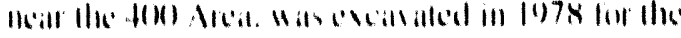

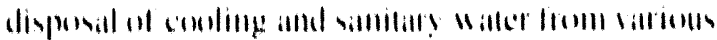

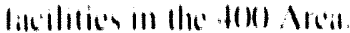

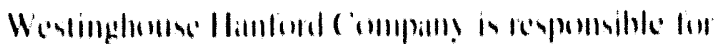

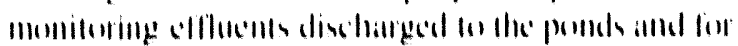

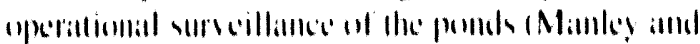

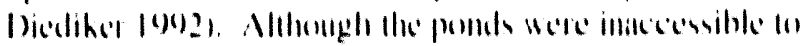

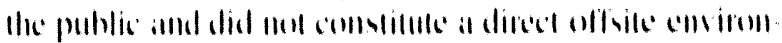

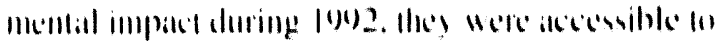

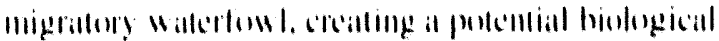




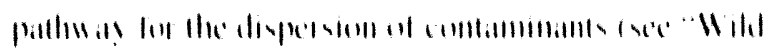

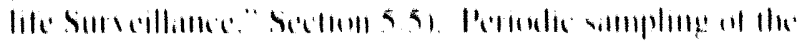

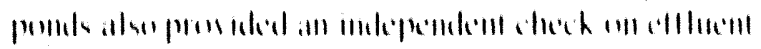

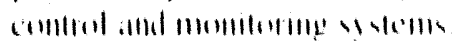

\section{Sample Collection and Analysis}

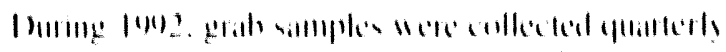

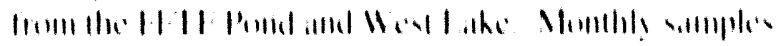

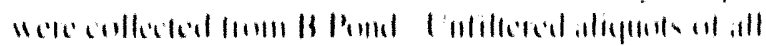

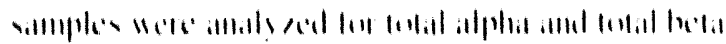

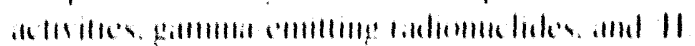

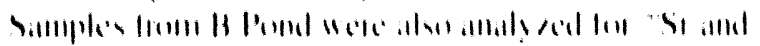

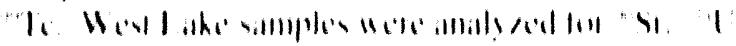

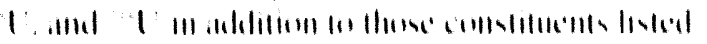
. $11011 \mathrm{~s}$

\section{Results}

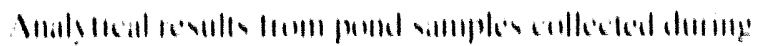

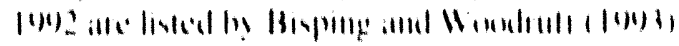

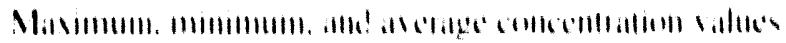

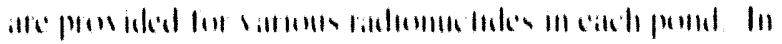

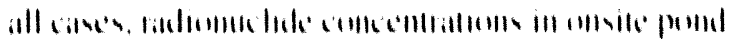

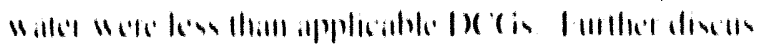

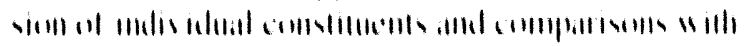

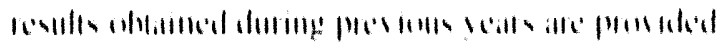
In'lin

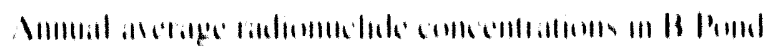

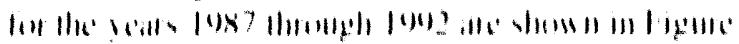

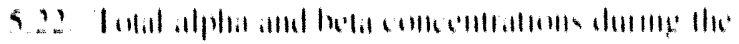

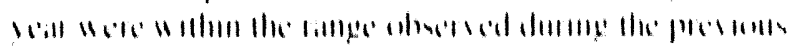

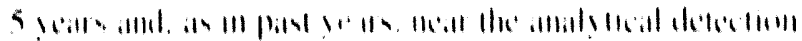

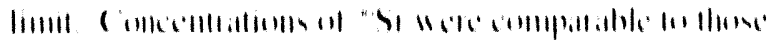

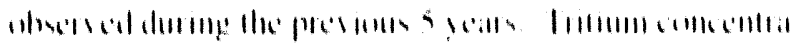

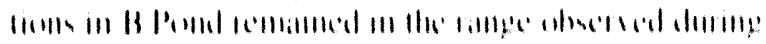

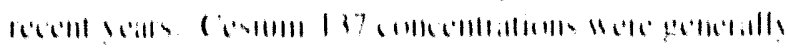

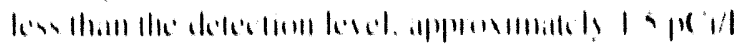

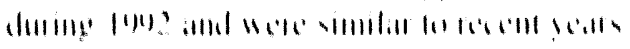

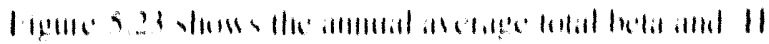
colluc

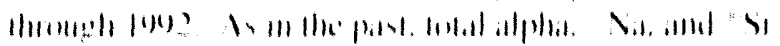

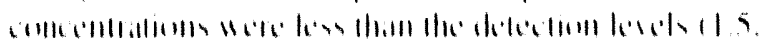

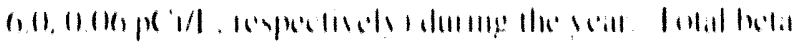

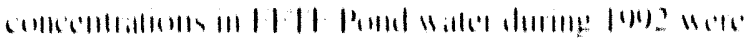

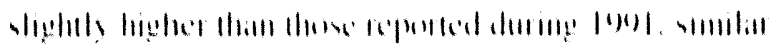

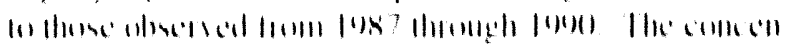

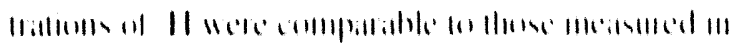

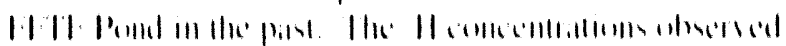

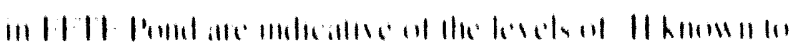

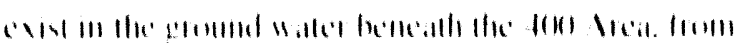

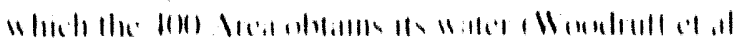
(4)!

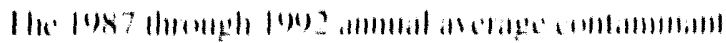

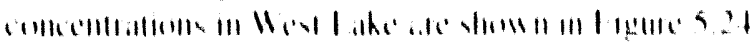

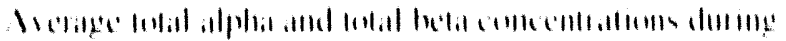

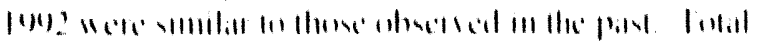

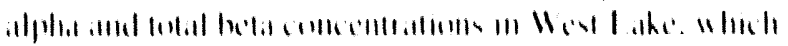

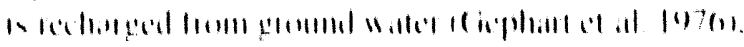

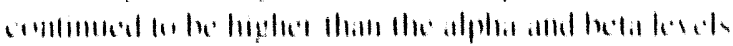

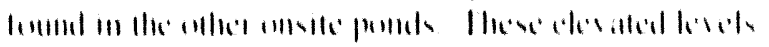

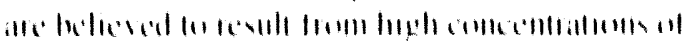

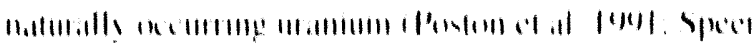

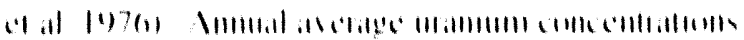

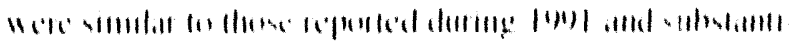

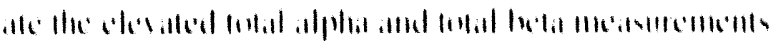

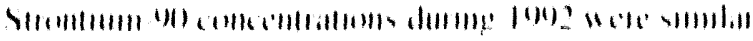

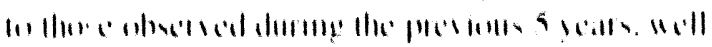

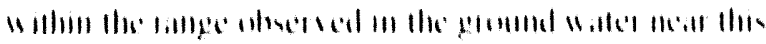

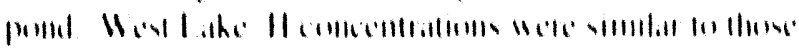

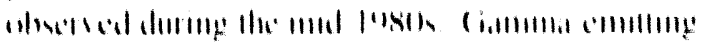

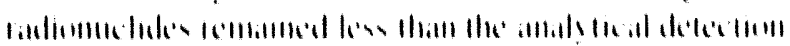

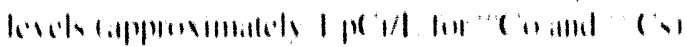

\section{Offsite Water}

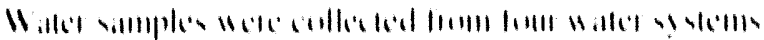

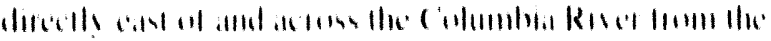

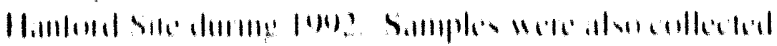

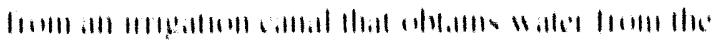

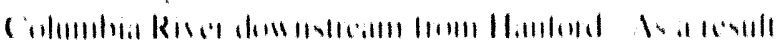

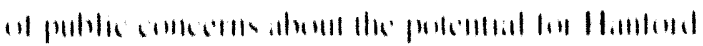

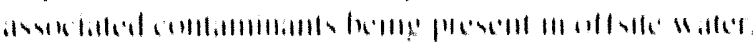

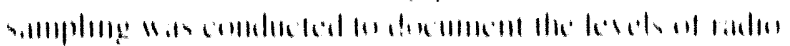

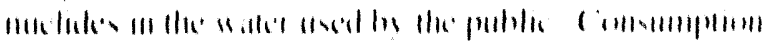

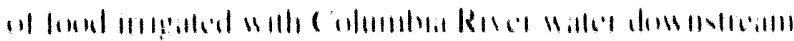

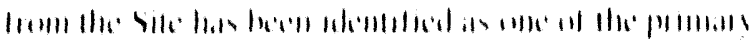

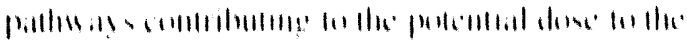

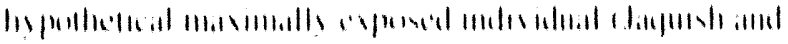
Nllikell lestio.

\section{Sample Collection, Analysis, and Results}

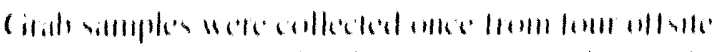

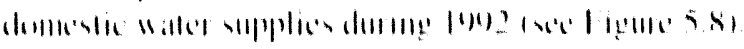

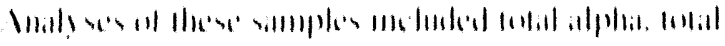



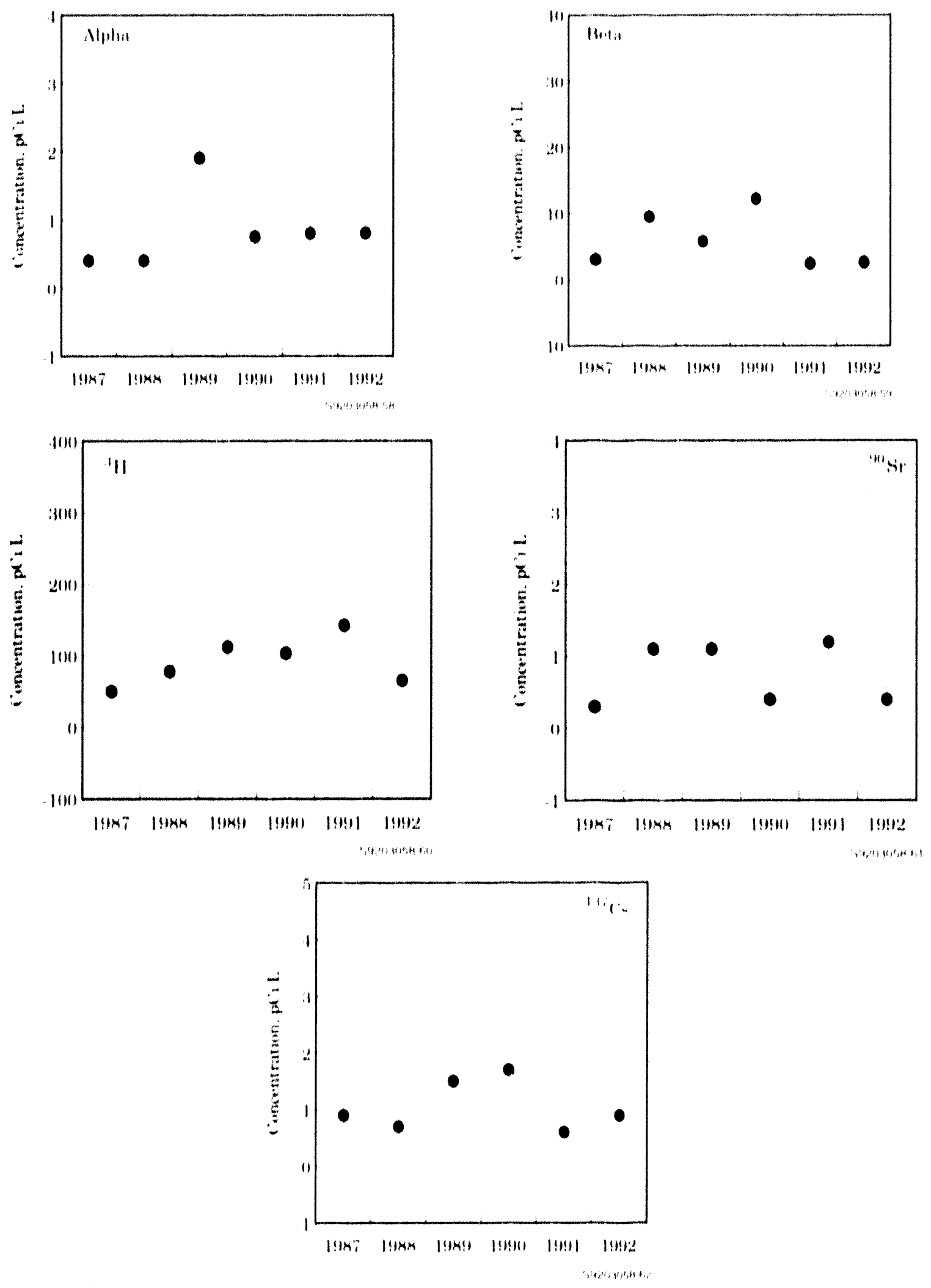

Figure 5.22. Annual Average Radionuclide Concentrations (+2 SEM) in B Pond. 1987 Through 1992 

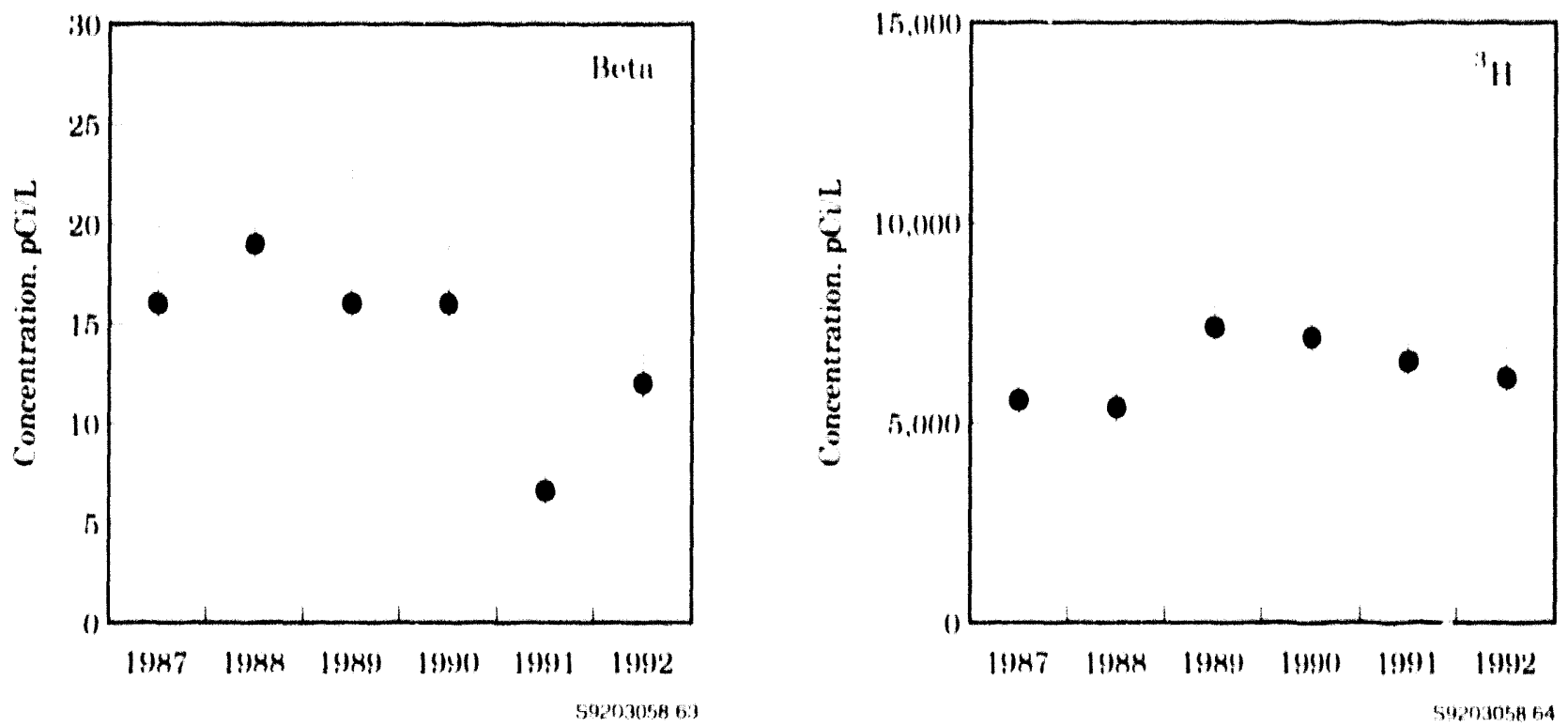

Figure 5.23. Average Total Beta and Tritium ('H) Concentrations ( +2 SEM) in FFTF Pond, 1987 Through 1992.

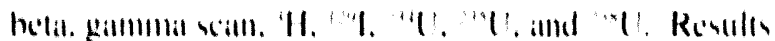

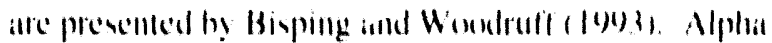

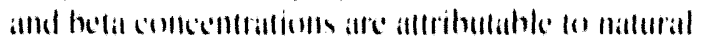

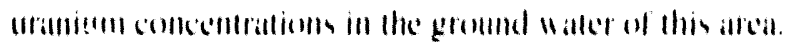

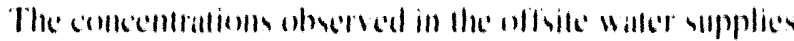
were comparable lo llowe reponted by the Slate at

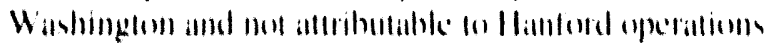

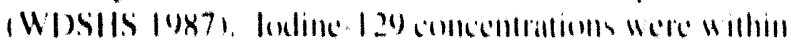

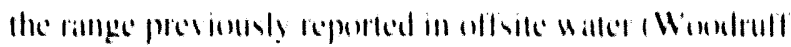

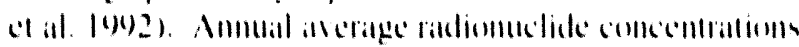

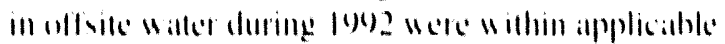
I)WS

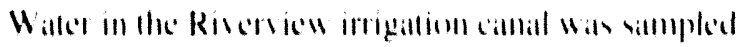

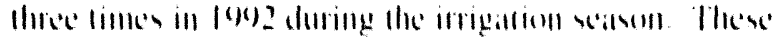

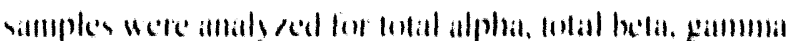

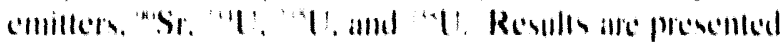

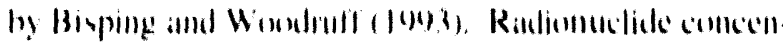

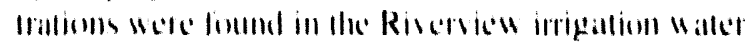

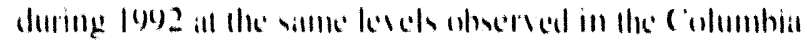

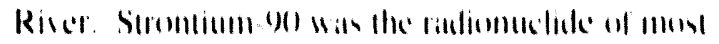

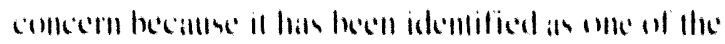

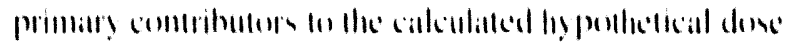

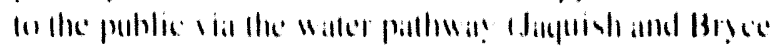

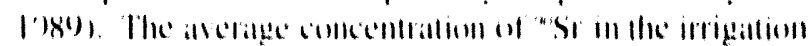

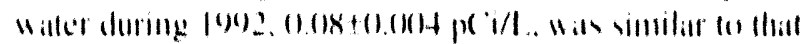

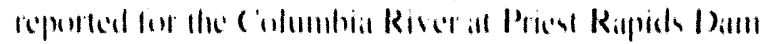

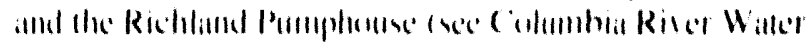
viliscetionil) 

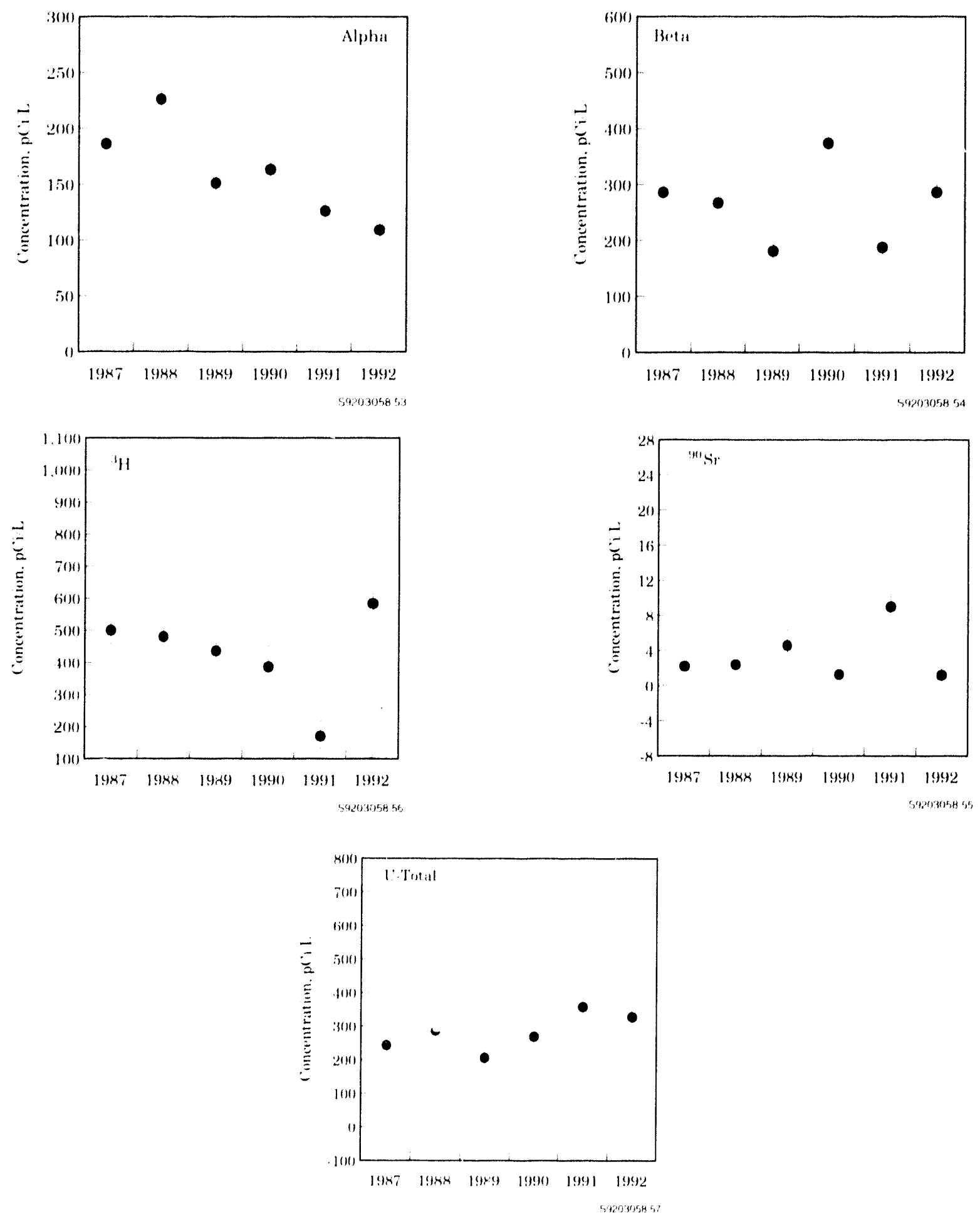

Figure 5.24. Annual Average Radionuclide Concentrations ( \pm 2 SEM) in West Lake, 1987 Through 1992. As a result of figure scale, some uncertainties (error bars) are concealed by point symbol. 


\subsection{Food and Farm Product Surveillance}

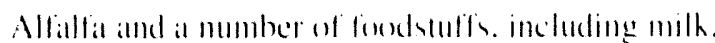
regetables, fruits. wince. Wheall, heel, chichems. and egges. llere collected all sereral locattom surtounding

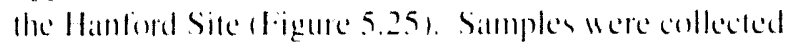
primatrily form locattom in the perationg downm ind

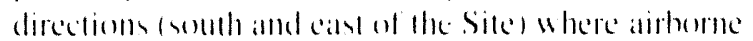

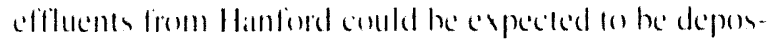
ited. Samples were also colleceded in gentratly upwind directions somewhall distant from the Site te proside information on background radioativil!. This secton

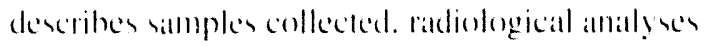

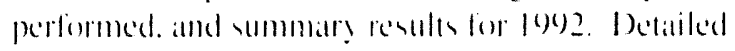
analylical resule are an ailathe in Bisping and Wordent (1993). some of which has heen summatred in Appendix $A$. The potential dose lo members al the public from the comstomption of locial food and latrm products is addresed in Section 6.0). "Potential Radiattom Dones from lo92 Hanford Operations." Results for licyuids atre reported in pe "i/l. of liquid prodect or distillante from

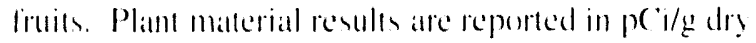
weight and animal products in pe'i/g wet weight. Many simples had concentrations that were less than delectahle.

By comparing several downwind locatioms to generally upu ind ar distant locitions (liggure 5.25). the simmpling approach addreseses the potential influence of Hanford Site releatses. Specific detath of the sampling derign including sampling locations and radionuclides analyod

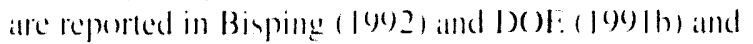
have heen stmmmaried in Tahle 5.7. (iammal seams rea

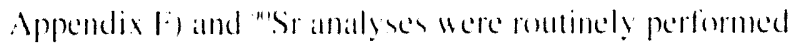
for nearly all products. Selected larm products were specificall! analyed for additional radiontelides including H. "Te. "I. Uramium. and plutonium.

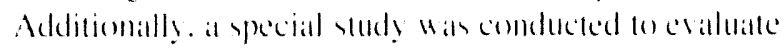
"Sireselu in allalla

\section{Milk}

\section{Sample Collection and Analysis}

Simples of raw. Whole milk were collected from taas Wahlute and Sagemonor areal dairy fimms near the Site perimeter in the prevalingly dow mwind direction to

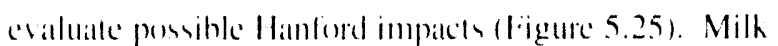
samples were aluo collected from at Sumngide dairy lo indicatce the genteral background concentrations af radion-

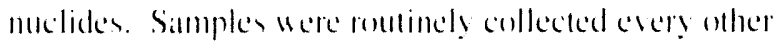
week throughout the year from the Sagemoner areat and momlly from the athere areas

Tritium amalyses were conducted on one sample either monthly or yuarterly. "Sr analyeses were conducted quarterls and tamaly ses were conducted on two semit. ammual compmeite samples. (jatmmat scams of milk were performed on samples from each localtion all frequencies varying from biweckly lo quarterly.

\section{Results}

Iodine-129 comtributed ahoul 30\% of the dose to the maximally exposed individual (Mlil) through the comsumption of fored products lece Section 6.01). lodine-129 was identilied by high-resolution mass spectroscopy in all six milk simples tested. In recent years. the levels of "."I in milk collected from Sagemon and Ealst Wahluke (downwind locallioms) have persisted all levels $20+4$ times grealler than levels measured in Sumnyside (lighte 5.26): however, concentrations were fow (Tahle 5.8 ).

About 11\% of the 35 milk samples colleceded and

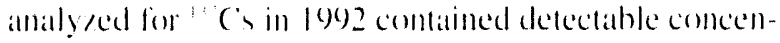

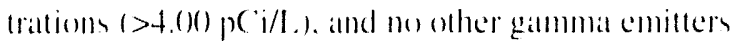
Were consistently delectahle (Appendix $A$. Tahle $\mathrm{A.7}$ ). However. $88^{\prime}$, of all milk samples atmalyed for " $\mathrm{Sr}$ in 199 2omtained measumahle levels with no apparent differences between upwind and downwind locations (Table 5.8). Both "Sr and "('s are expected lo some degree in milk samples heciatese of the presence of these radionuclides in worldwide fillout and mosemem through the air-pasture-oow-milk forkl chain. Figure 5.27 shows the o-year record for " ${ }^{k} \mathrm{Sr}$ in milk samples from all sillmpling areas. Concentrations of "Sir hate rematined relattively constann over the past 6 years. Tritium was meatsured in athout 1t'i of the 22 samples analyed. with maximum concentrations near a detection limit of 28() p (i/l.. There was no apparent diflerence belween resulle upwind and downwind of the Site (Table 5.8). 


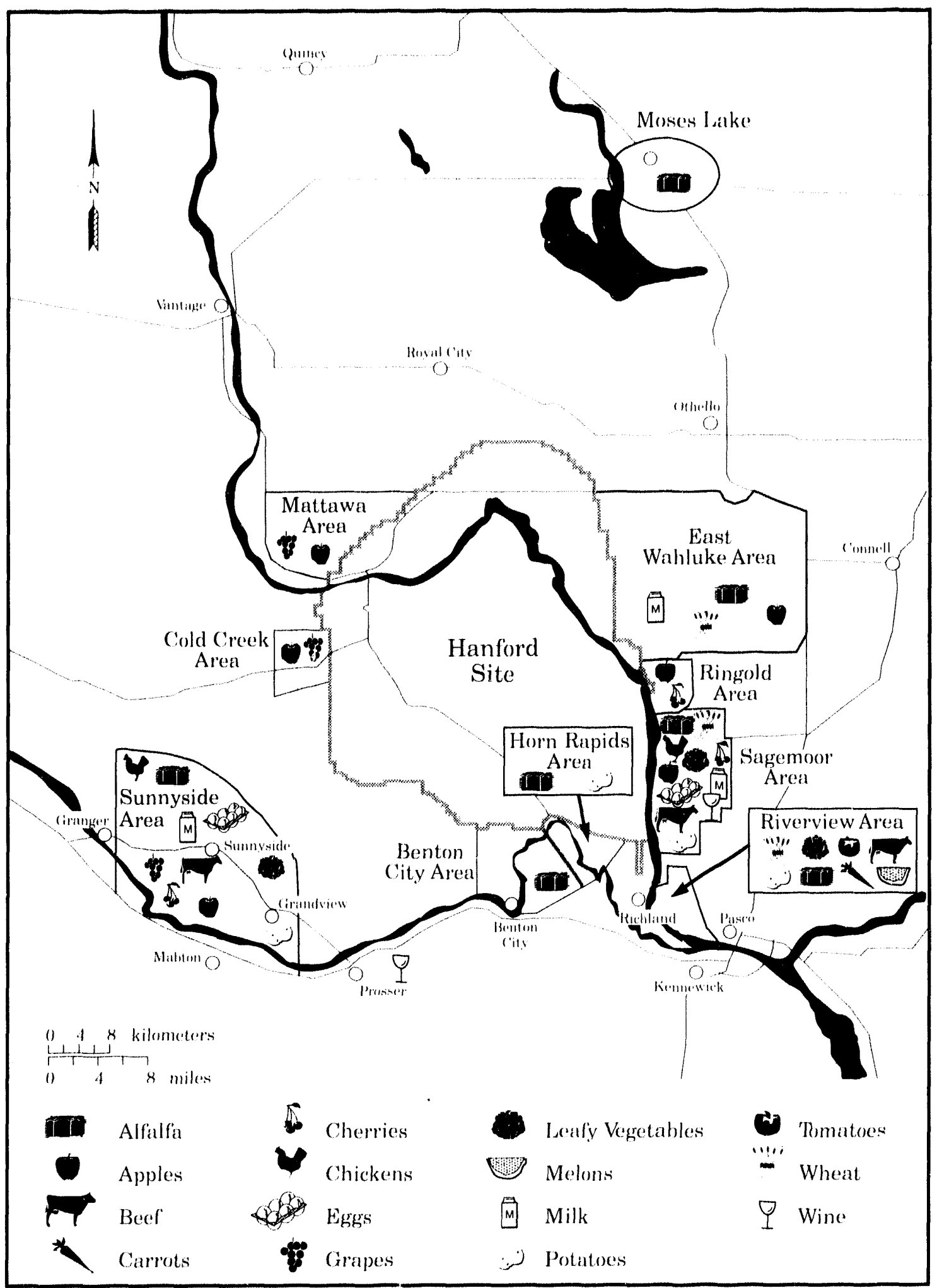

S9203058.12b

Figure 5.25. Food and Farm Product Sampling Locations, 1992 
Table 5.7. Numbers of Locations, Sampling Frequency, and Analyses Performed for Routinely Sampled Food and Farm Products, 1992

\begin{tabular}{|c|c|c|c|c|c|c|c|c|c|c|}
\hline \multirow[b]{2}{*}{ Media } & \multicolumn{2}{|c|}{ Number oll locitions } & \multirow{2}{*}{$\begin{array}{l}\text { Simpling } \\
\text { Prefulency }\end{array}$} & \multicolumn{7}{|c|}{ Number of I.ocations Analyzed } \\
\hline & Upwind & Downuind & & H & Gatmma & "'Sr & 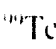 & $1 \cdots 12 j^{\prime}$ & U & $\mathrm{Pu}$ \\
\hline Milk & 1 & 2 & B.M.Q. WSA & 3 & 3 & 3 & () & 3 & () & () \\
\hline $\begin{array}{l}\text { leggn. meial } \\
\text { ind poulters }\end{array}$ & 1 & 2 & $S A$ or A & () & 3 & 3 & () & (1) & () & () \\
\hline Vegetiales & 1 & 3 & A & () & 5 & 5 & + & 2 & 2 & 2 \\
\hline Irruit & 3 & + & A & + & $t$ & 4 & () & 2 & () & 3 \\
\hline $\begin{array}{c}\text { Wheilt and } \\
\text { alfialfia }\end{array}$ & 2 & 5 & $A$ & () & 3 & 3 & () & () & 0 & 2 \\
\hline Wine & 2 & 2 & $A$ & + & 4 & (1) & () & () & 0 & () \\
\hline
\end{tabular}

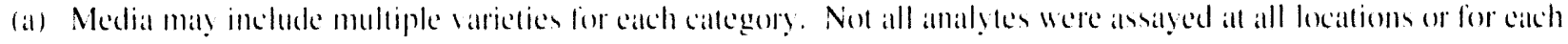
variely of media.

(h) $\mathrm{M}=$ monthly: $\mathrm{Q}=$ qualterly: $\mathrm{SA}=\mathrm{x}$ (m)iammually: $\mathrm{A}=$ ammually: $\mathrm{B}=$ biweckly.

(c) 'I was only measured in milk.

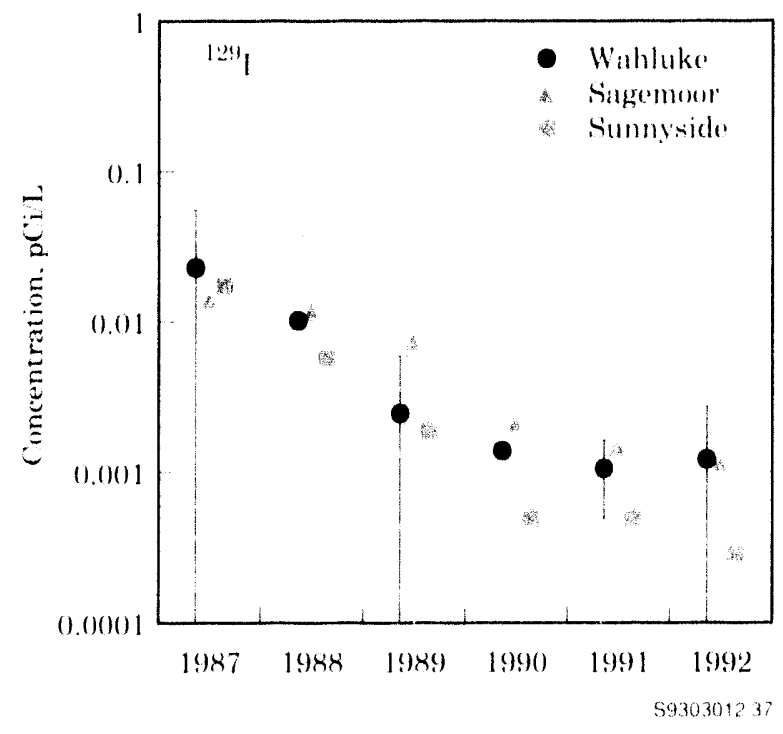

Figure 5.26. Average lodine-129 (129) Concentrations ( \pm 2 SEM) in Milk, 1987 Through 1992. As a result of figure scale. some uncertainties (error bars) are concealed by point symbol. Uncertainties for some annual averages for Sunnyside samples were less than zero and cannot be shown on a logscaled figure.
Thirty-four milk samples were collected and antyal for I during |092. No allmospleric releases of "I from Hanford were reported for legen see Table 3.11). and "I was mot delected in any milk simple.

\section{Vegetables}

\section{Sample Collection and Analysis}

Samples of lealy vegetathles lcabhatge. brocioli lealves. heet tops. or turnip) greens). tomatoes. ciarrots. and potitees were obtitined during the summer from gardens and farms located within the simpling arean ree figute 5.25 ). Lealy regetahles are simpled becallse of the potential deposition of airborne contaminants. At some locattions they may also receise deposition from overheat irrigation. Three replicate simplen of eatch vegetable were collected at calch sampling locittion. If antalysis of one of the replicalles showed detectable levels of radionuclides. the remaining two replicaltes were also

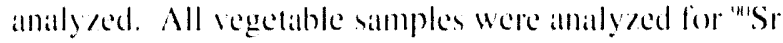
and gatnmina-emitting radionuclides: in addition. potatoes

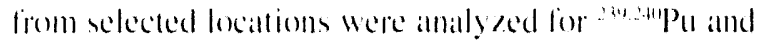
uranium isotopes. Samples were collected from the 
Table 5.8. Radionuclide Concentrations in Milk, 1992 Compared to Values from the Previous 5 Years $(\mathrm{pCi} / \mathrm{L})$

1042

I.rciltion

Maximum!'t!

Mcill!"

No. I.c's Thian

Delectiont

'H

Downwind

Ipwind

"Sir

Downwind

Upwind

12"I

Downwind

Upwind
Wahluke Area

Siggemoor

Sunnyside

Willuke Areai

Silgemoor

Sunnyside

Willuluke Areil

Sigcomoor

$$
\begin{aligned}
& 210 \pm 10(0 \% \\
& 300 \pm 8(1 \% \\
& 170 \pm 120 \%
\end{aligned}
$$

$0.62 \pm 6(1) \%$

$0.5 .3 \pm 70 \%$

$0.62 \pm 80 \%$

$$
\begin{array}{r}
0 .(0) 119 \pm 0 \% \\
0 .(0) 15 \pm 0 \% \\
0 .(0)(0) .5 \pm \pm 0 \%
\end{array}
$$

I.ciltion
Maximum!'m

$3(x) \pm 7(0 \%$

$330 \pm 70 \%$

$310 \pm 70 \%$

Sunnyside

\section{"ST}

Downwind

Williluke Areil

Silgemoor

Upwind

Sumnyside

12"I

Downwind

Willuke Areil

Silgemoor

Upwind

Sumnyside

$$
\begin{aligned}
& 1.8 \pm(60 \% \\
& 1.3 \pm 50)^{r} \\
& 3.2 \pm 6(10 \%
\end{aligned}
$$

$$
\begin{aligned}
& 0.0 .38+10 \% \\
& 0 .(1) 7 \pm 10 \% \\
& 0.031+10 \%
\end{aligned}
$$

(9) $\pm 12(1) \%$

$200 \pm 2(1 \%$

(x) $\pm 10(1) \%$

3014

70114

4014

$$
\begin{aligned}
& (1.52 \pm 20 \% \\
& 0.39 \pm 5.5 \% \\
& (1.54 \pm 20 \%
\end{aligned}
$$

2014

$101+$

1 of $t$

$$
\begin{aligned}
& 0 .(0) 12 \pm 120 \% \% \\
& 0.0012 \pm 50 \% \\
& 0 .(00(1) 28 \pm 50 \%
\end{aligned}
$$

$|987.190|^{\prime \cdots 1}$

Meill':

No. I.es Thanl

Delectiont

$5(1)$ of 55

57 ol 6.3

5.3 of 5.5

1()$) \pm 2(1 \%$

(0) $\pm .50 \%$

$0.80 \pm 20 \%$
$0.80 \pm 20 \%$
$0.73 \pm+(1 \%$

2 of 2()

() 1 () 20

$3(1) 2(1)$

() (1)

() of (c)

(1)

(a) Results have shown a decreasing trend over the period of 1987 10 1962).

(b) Maximum \pm 2 sigma analytical propagialted error. expressed as a percentige.

(c) Meanl \pm 2 standard error of the calleulated meinl. expressed an a percentige.

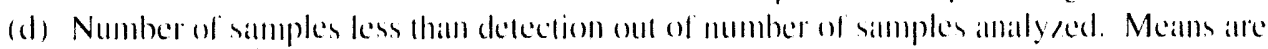
baised on ill samples collected. 


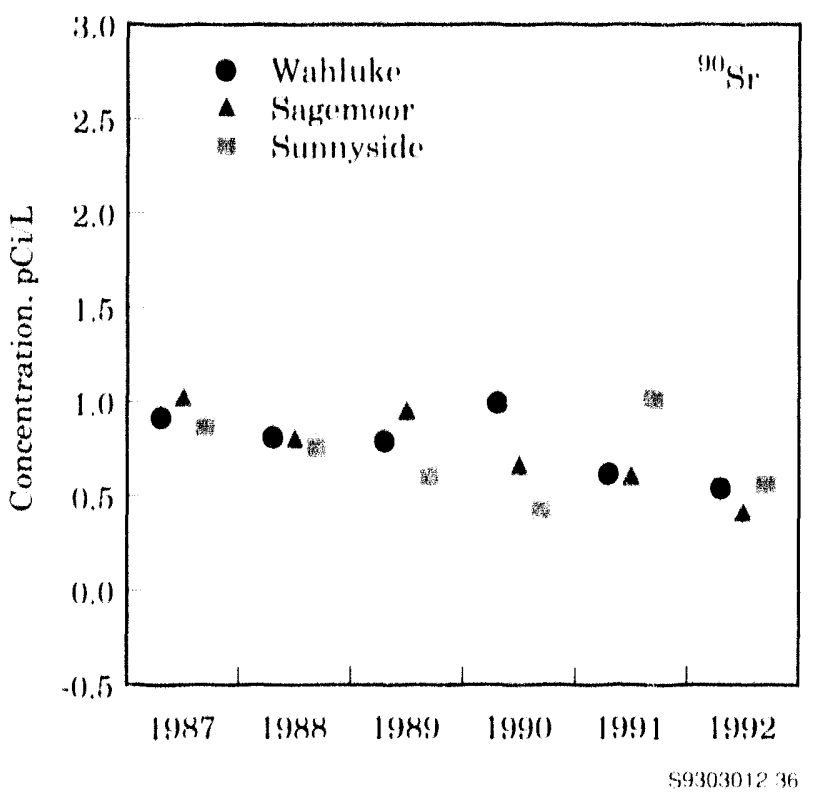

Figure 5.27. Strontium-90 (") Sr) Concentrations ( \pm 2 SEM) in Milk, 1987 Through 1992. As a result of figure scale, some uncertainties (error bars) are concealed by point symbol.

Riverview and Hom Rappids atreas lo alssess potential comtamination from the irrigation of erops at these locitlions. Irrigation watler for Horn Rapids and Riverview is withdrawn from the Columbia River downstream fiom Hantiord.

\section{Results}

Strontium-9) wats identified in mos leafy vegetiahle

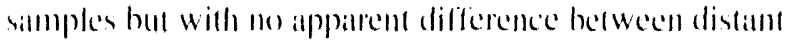
and nearby locitions. indicitting that Hantord wats mot al significinn source $(\Lambda$ ppendix $\Lambda$. Table $A .8)$. The comcentrations were varbahle and near dedection $1-0.000 .5$

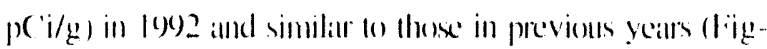
une 5.28). ('esium-1.37 wats lound in leally vegelahles

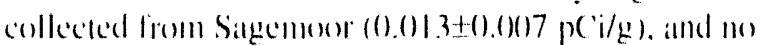
onler gianmintemilters were consistently detectible.

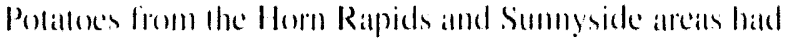
(wo) delectable raddiation measurements in 1992:

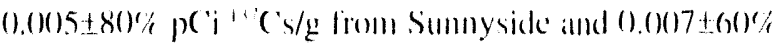

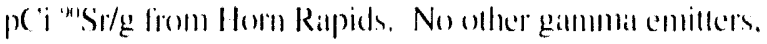

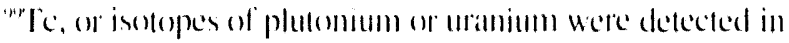

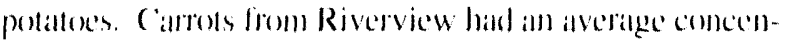

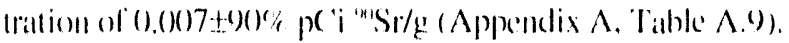

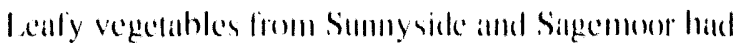

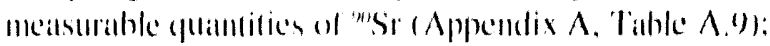
however. there were nor apprarent dilferences between upwind and downwind lekilloms (ligurere 5.28).

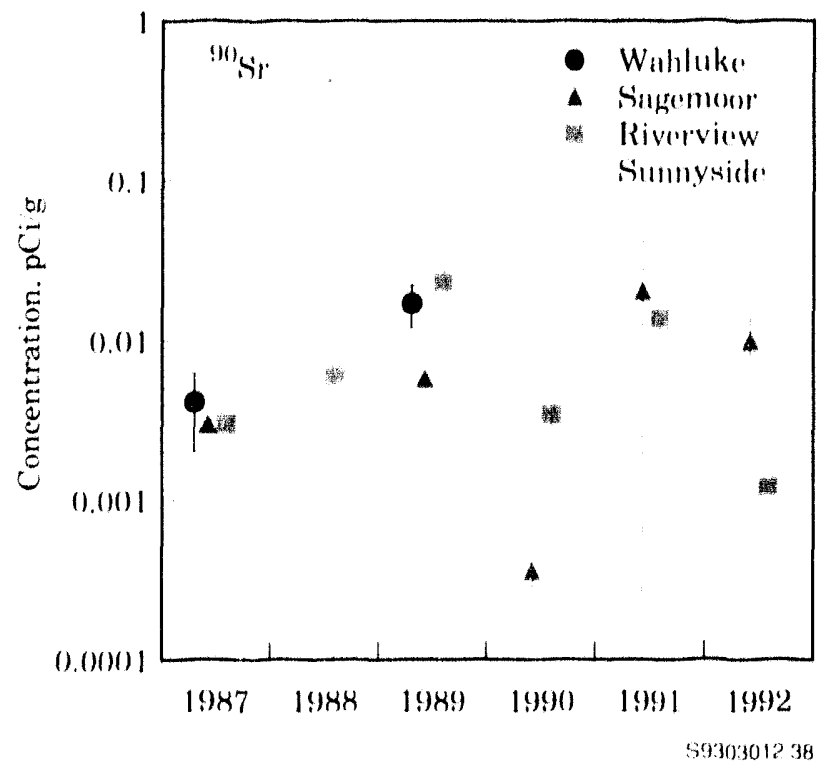

Figure 5.28. Annual Average Strontium-90 ( $\left.{ }^{90} \mathrm{Sr}\right)$ Coricentrations ( +2 SEM) in Leafy Vegetables, 1987 Through 1992. As a result of figure scale, some uncertainties (error bars) are concealed by point symbol.

\section{Fruit}

\section{Sample Collection and Analysis}

Simples of apples. cherries, grapes. and melons were collected in triplicalle during hatreses from the areals

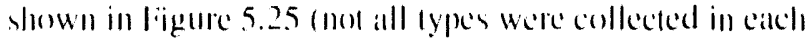
areit). The edible portions were antilyed for "II. "'Sr. garmma comillers and, for selected samples. "'l and

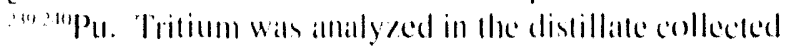
limoln linut samples.

\section{Results}

Meisurable levels of ratlonativily were not delected in alpples, cherrices, concord grapes, or melons collected in 1992 fiom cither upwind or downwind locillions. Minimum levels of deection were 300 p ('i/l. plant 


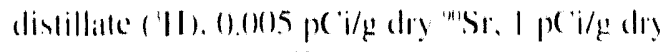

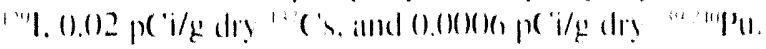

\section{Wine}

\section{Sample Collection and Analysis}

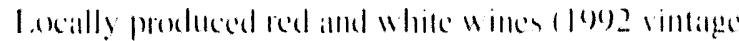

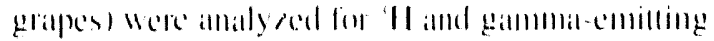

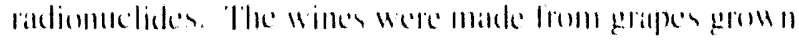

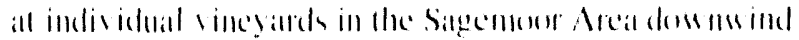
of the Sile alld in the Yathima Valley near Prosere

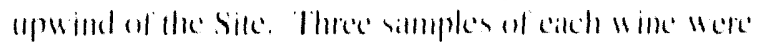
whailled from carch atreal

\section{Results}

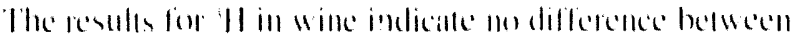

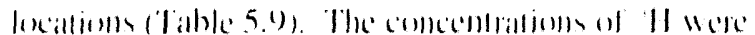

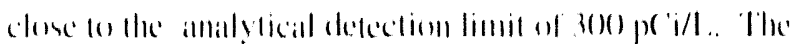

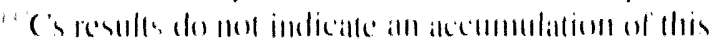

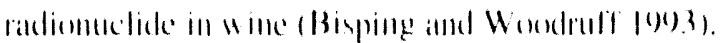

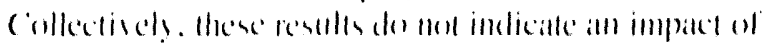

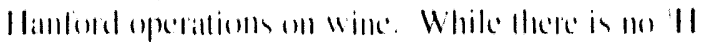
standard for wime: He sambland for drinking walle is $20 .(1)(3) \mathrm{p}(\mathrm{C} i / \mathrm{l}$.

\section{Wheat and Alfalfa \\ Sample Collection and Analysis}

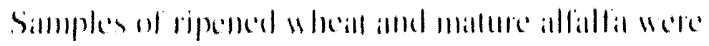

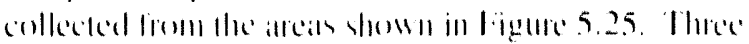

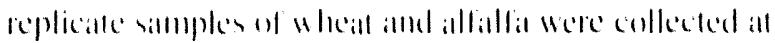

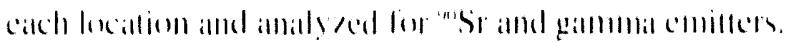

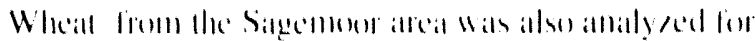

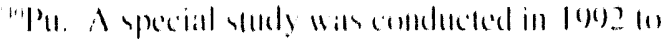
exallmime "Si ill allalla.

Table 5.9. Tritium ( $\left.{ }^{3} \mathrm{H}\right)$ Concentrations in Wine, 1992 Compared to Values from the Previous 5 Years (pCi/L)

\begin{tabular}{|c|c|c|c|c|}
\hline \multirow[b]{2}{*}{ Type of Wime } & \multirow[b]{2}{*}{ I.ncillion } & \multicolumn{2}{|r|}{ (x)! } & \\
\hline & & Mitximulu!"." & Meilll: & 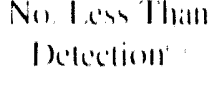 \\
\hline While Will & $\begin{array}{l}\text { Columbial Basill } \\
\text { Yathimat Villey }\end{array}$ & $\begin{array}{l}570)+4(1) \% \\
550+4(1)\end{array}$ & $\begin{array}{l}38(1)+3()^{\prime} \\
3.5(1)+3()^{r}\end{array}$ & $\begin{array}{l}\ln 110 \\
01110\end{array}$ \\
\hline Red Winc & $\begin{array}{l}\text { Columbia Balsill } \\
\text { Yathimal Valley }\end{array}$ & $\begin{array}{l}70(1)+3(1) ; \\
0.50)+4(1)^{2} ;\end{array}$ & $\begin{array}{l}4(0)+4()^{\prime} ; \\
4(0)+5()^{\prime} ;\end{array}$ & $\begin{array}{l}0,110 \\
\operatorname{lol} 10\end{array}$ \\
\hline
\end{tabular}

1987.|19)

Incillin)

White Winc

(inlumbia Barsin

MinximmII!

Maill'

$930+30 \%$ Yakima Valle'y

$821)+4(1) ;$

$370)+1()^{\prime}$,

$2(1)+5()^{\prime}$

$(10)+.5()^{2}$

Red Winc

(illumhial Barin

Yakima Valley

$5(1)+4() \%$

$320)+301$
No. I.in Thall

|chciliul

$+11112$

() (1) 11

20111

$f(1)$

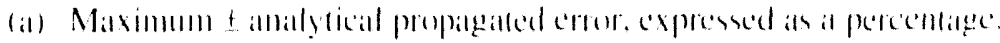

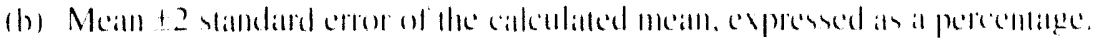

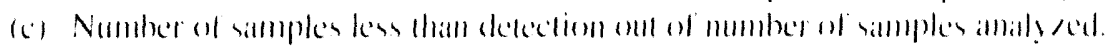




\section{Results}

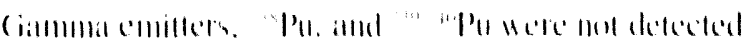

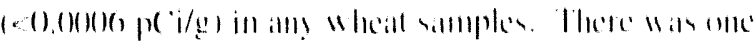

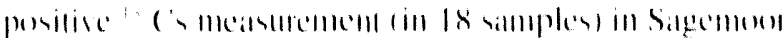

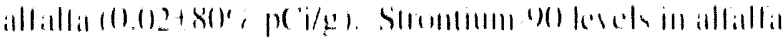

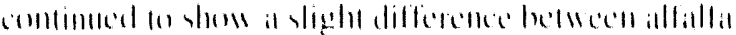

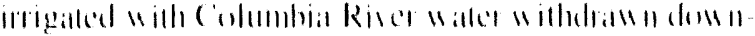

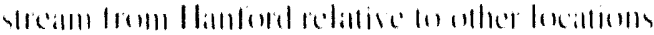

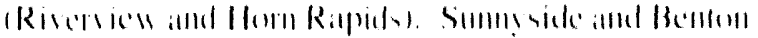

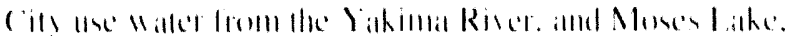

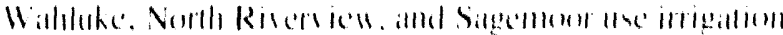

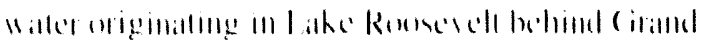

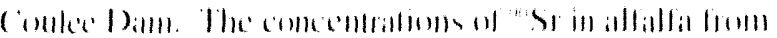

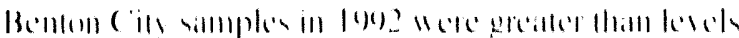

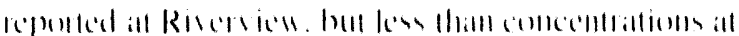

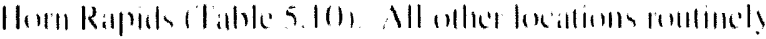

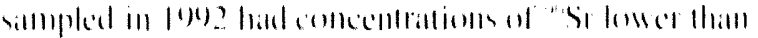

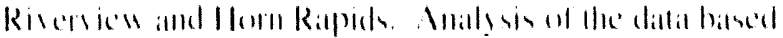

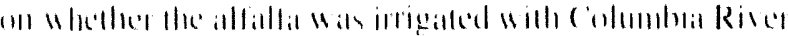

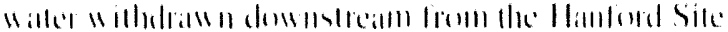

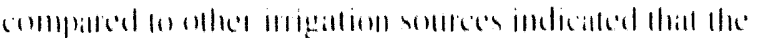

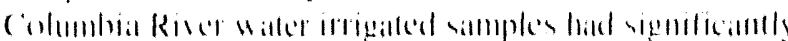

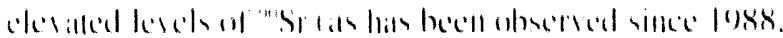

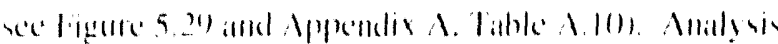

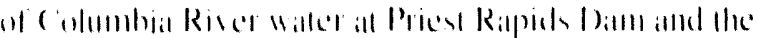

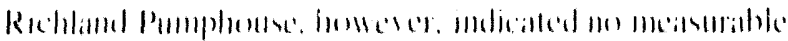

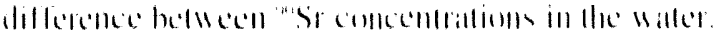

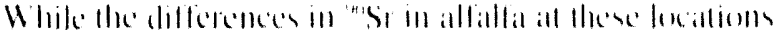

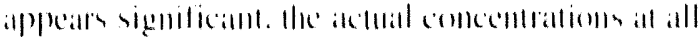

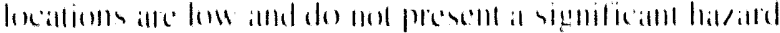

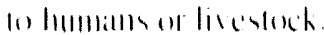

Table 5.10. Strontium-90 ("Sr) in Alfalfa Samples. 1992.

\begin{tabular}{|c|c|c|}
\hline I matllon & (intecontrition & $\begin{array}{l}\text { Nin, ul } \\
\text { Sillnplinen }\end{array}$ \\
\hline
\end{tabular}

\begin{tabular}{|c|c|c|c|}
\hline 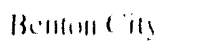 & $111.2+11.117$ & 3 & rakimal Rour \\
\hline Hann Rilpils & $(1.201011100$ & 8 & ( whlumbia Risu \\
\hline Mlus' l.aks & $0115,110110 ?$ & 1 & Romenclel I ithe \\
\hline 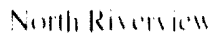 & $0103,10.113$ & 3 & 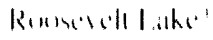 \\
\hline Risenter & $0,11,11,12$ & 4 & (whmmbla Rider \\
\hline Singermm, & 0110,101013 & (1) & Romencell late" \\
\hline SHIIII >ルk & $01017+11.061$ & 3 & Jathmail Risel \\
\hline II alinhe & $0005+0.014$ & 3 & Remand ll I athe \\
\hline
\end{tabular}

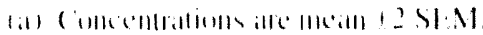

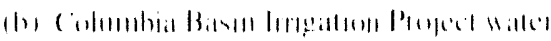

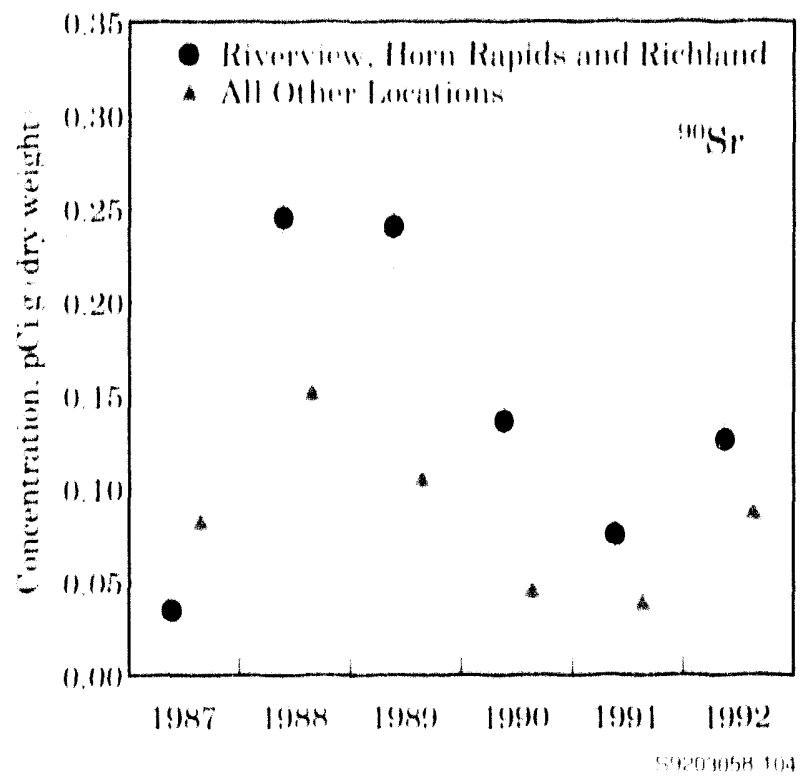

Figure 5.29. Strontium 90 ("Sr) Concentrations in ( +2 SEM) Alfalfa Routinely Collected at Riverview and Richland (irrigated with Columbia River water) and All Other Sampling Locations, 1987 Through 1992. As a result of figure scale, some uncertain ties (errur bars) are concealed by point symbol.

\section{Beef, Chickens, and Eggs Sample Collection and Analysis}

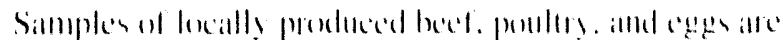

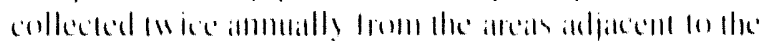

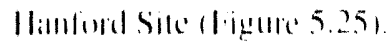

\section{Results}

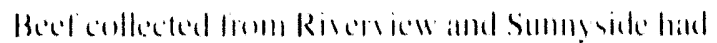

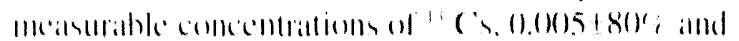

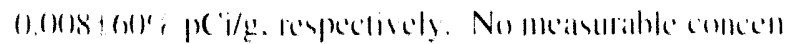

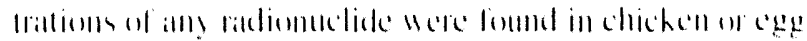

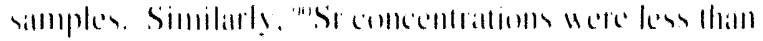

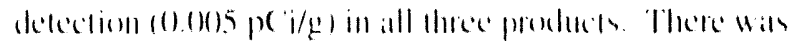

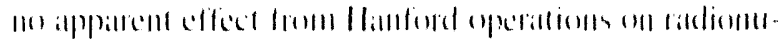

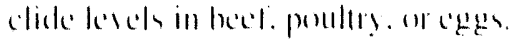




\subsection{Wildlife Surveillance}

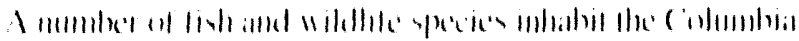

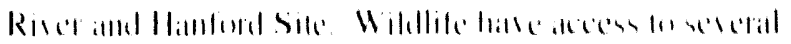

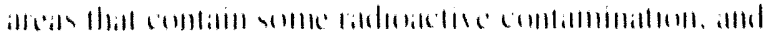

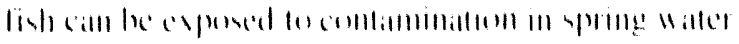

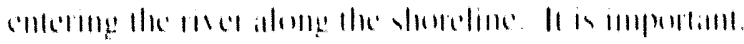

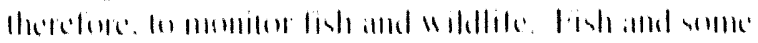

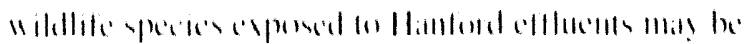

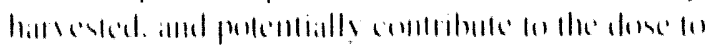

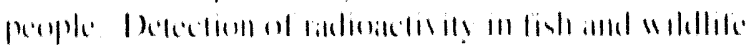

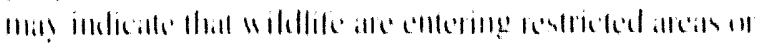

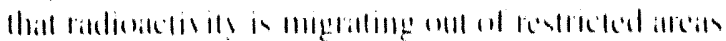

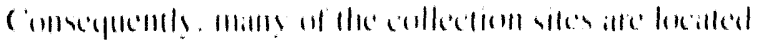

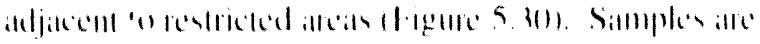

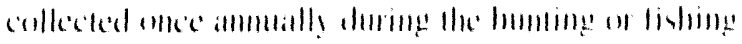

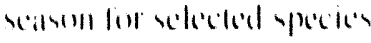

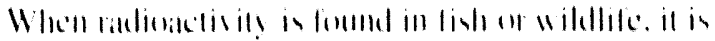

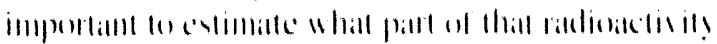

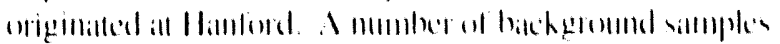

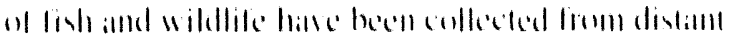

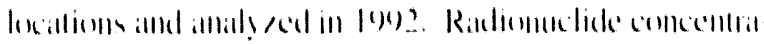

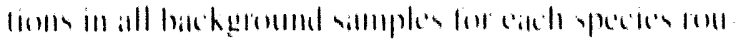

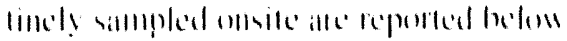

Sirombium-9)

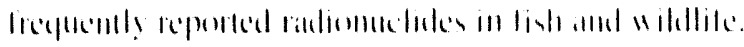

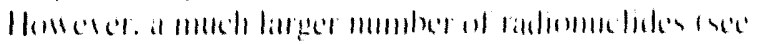

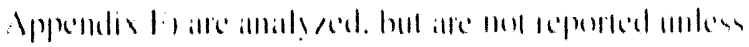

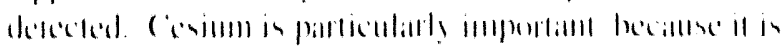

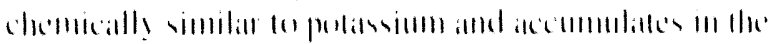

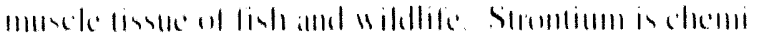

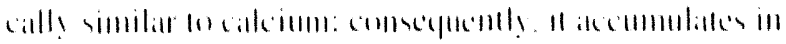

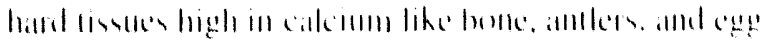

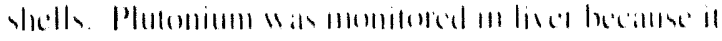

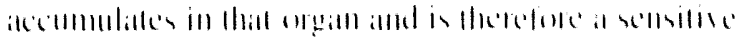

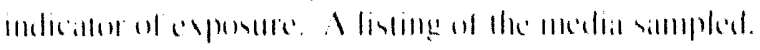

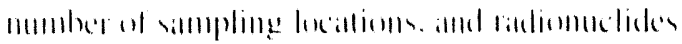

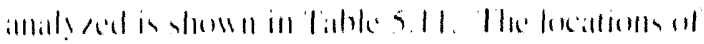

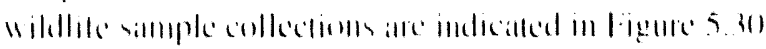

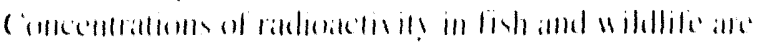

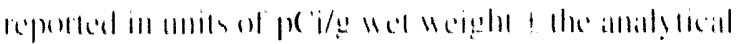

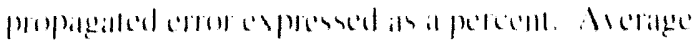

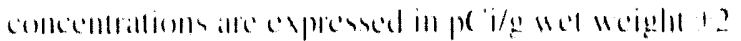

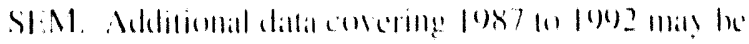

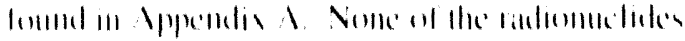

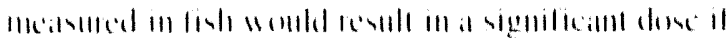

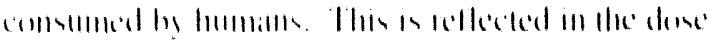

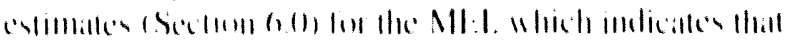
coms

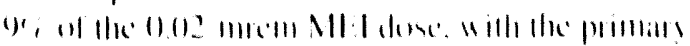

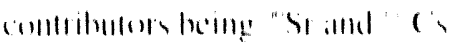

\section{Fish}

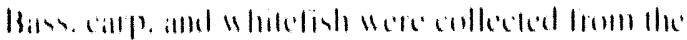

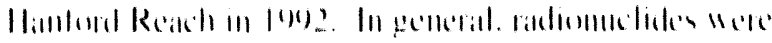

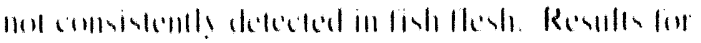

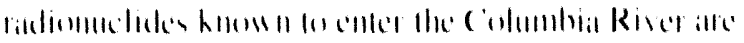

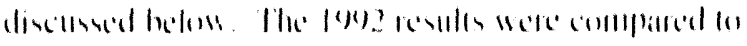

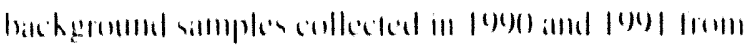

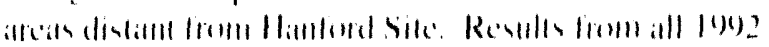

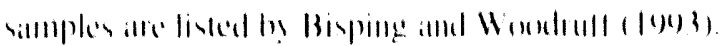

\section{Bass}

\section{Sample Collection and Analysis}

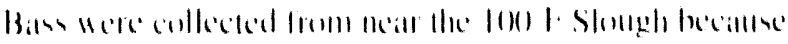

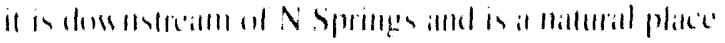

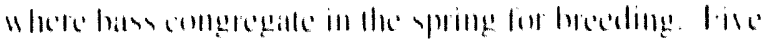

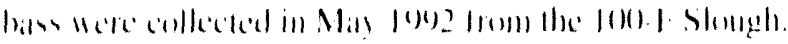

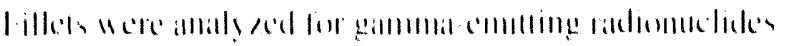

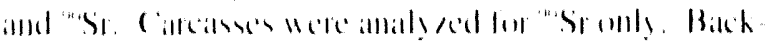

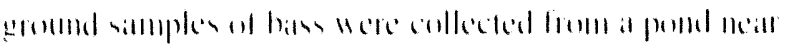

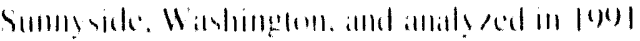

\section{Results}

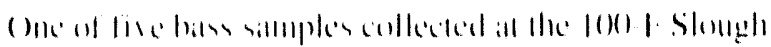

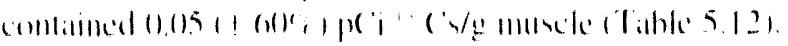

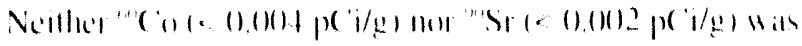

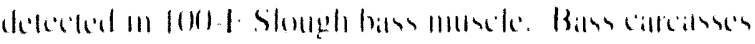

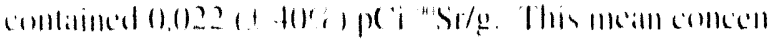

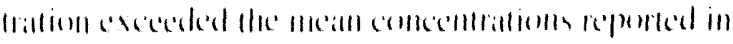

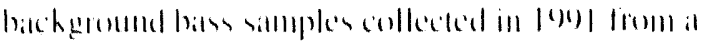

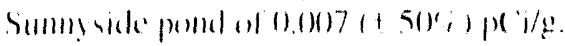




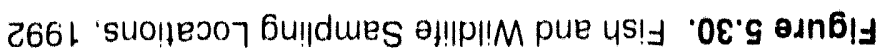

ce e.

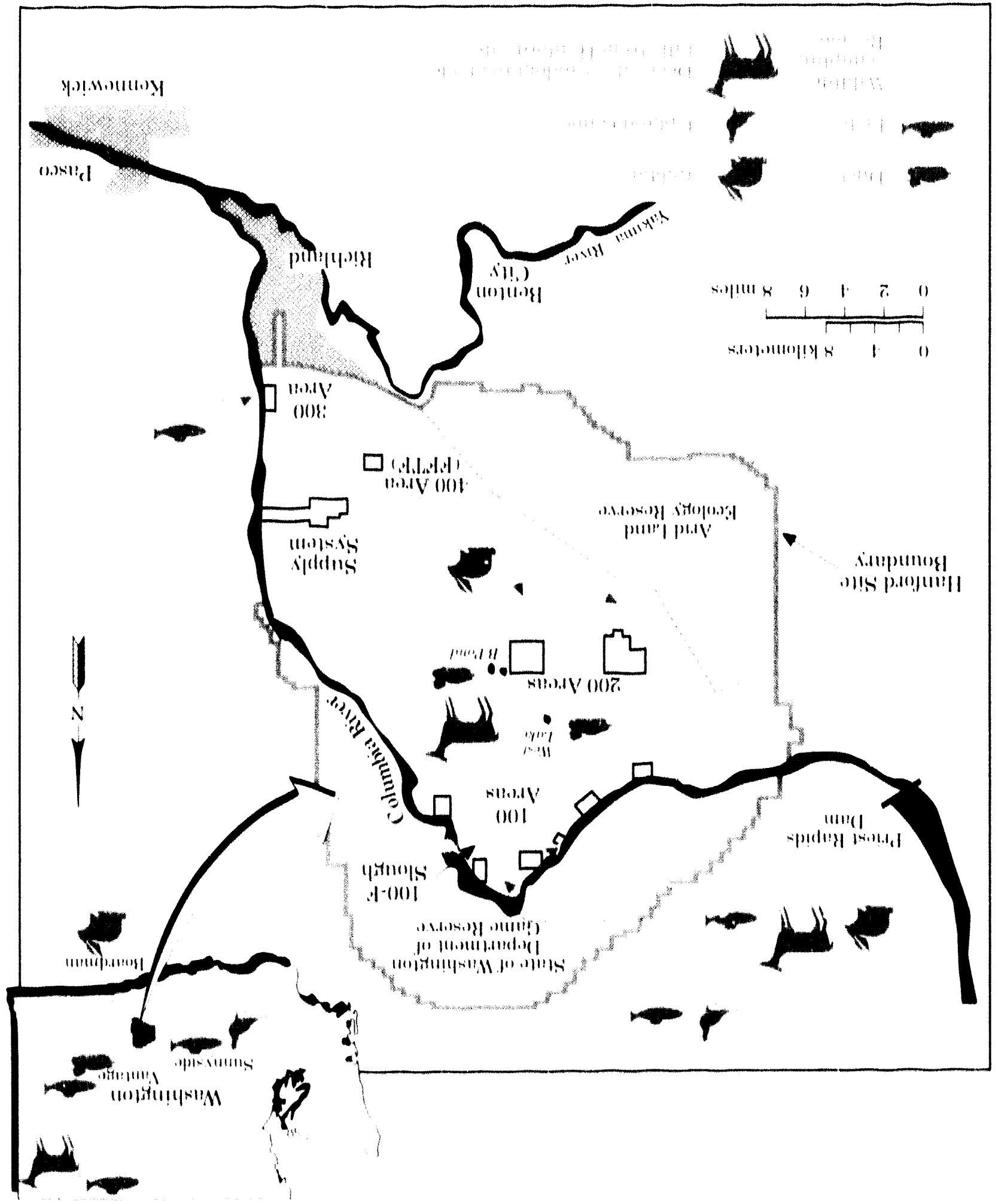


Table 5.11. Locations. Species, and Radionuclides Sampled for Fish and Wildile, 1992

\begin{tabular}{|c|c|c|c|c|c|c|c|}
\hline \multirow{2}{*}{ Mediai } & \multirow{2}{*}{$\begin{array}{l}\text { Number of } \\
\text { Spuction }\end{array}$} & \multirow{2}{*}{$\begin{array}{l}\text { Othile } \\
\text { I. oritionss }\end{array}$} & \multirow[b]{2}{*}{ (iammina } & \multicolumn{3}{|c|}{ 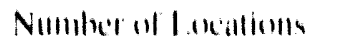 } & \multirow[b]{2}{*}{$\mathrm{Pu}$} \\
\hline & & & & "Sr" & "lic & 11 & \\
\hline 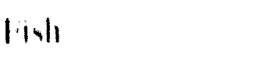 & 1 & 1101 & 3 & 3 & 1 & 1 & 11 \\
\hline 1)miks & 1 & 1 & 2 & 3 & 1 & 1) & 1) \\
\hline Inland giannchirsh & $1^{3}$ & 1 & 1 & 1) & 11 & 11 & 11 \\
\hline Mule deer & 1 & 3 & 1 & 2 & 11 & 11 & 1 \\
\hline 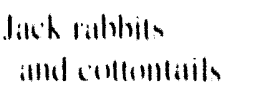 & 2 & 1 & 3 & 3 & 11 & 11 & 3 \\
\hline
\end{tabular}

Table 5.12. Summary of Cesium-137 ("'C Cs) in Bass Muscle and Strontium-90 ("' $\mathrm{Sr}$ ) in Bass Carcass (pCi/g wet), 1992 Compared to Values from the Previous 5 Years

(以) 2

Nir.

I.ncalton

Maเiminu!i!"

Meilli"

I.'m Hhill

Detcoliunt
$|(1) \times 7|(1) \mid$

$\mathrm{Nor}$ lem Than

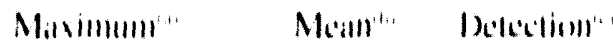

"17's In Mluscles

I(1)1.1. Slough

$01.115+6.0 \%$

$(1.1) 2+111)^{\prime}$

4115

$(1)(114)+501)^{\prime}$

11.11.4 +30\%

501211

Stam!n vide.t.

$0.118+9(1)$,

$(1.1) 1+16(1) \%$

(1) 11211

"sir in C arciuss

(1)1) f. Stongh

$(1.19 .310+31 \%$

$0.1022+404$

01115

$0.0600+50 \%$

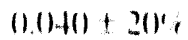

(1) 111211

Summysick

$(1,(1) 32+31)^{r}$

$01 .(1017+501$

2 (1) 211

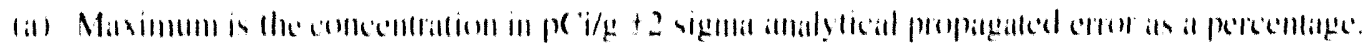

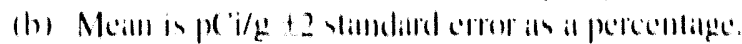

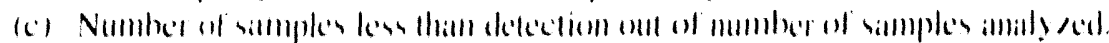

(d) Cillecied in |w) 


\section{Carp}

\section{Sample Collection and Analysis}

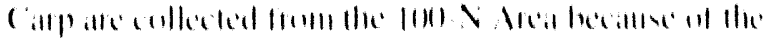

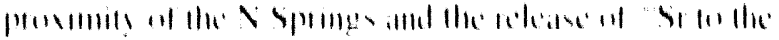

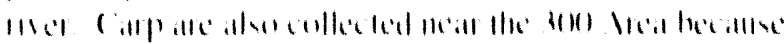

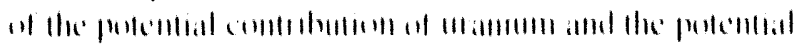

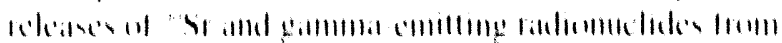

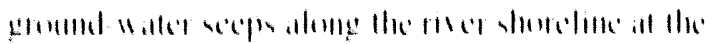

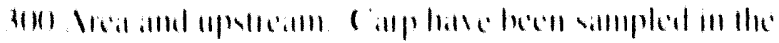

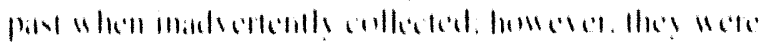

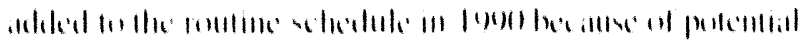
conkw

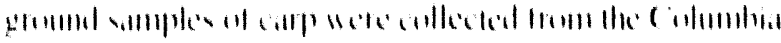

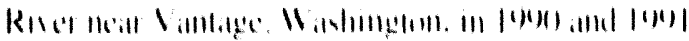

\section{Results}

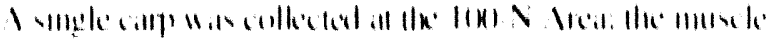

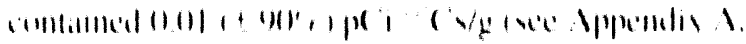

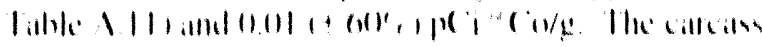

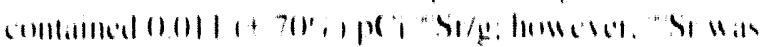

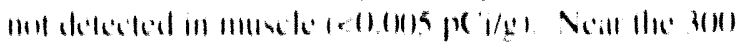

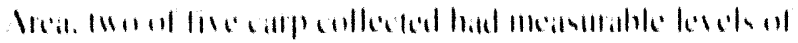

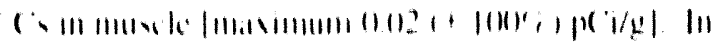

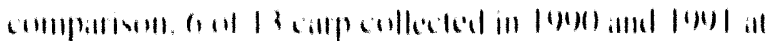

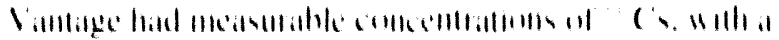

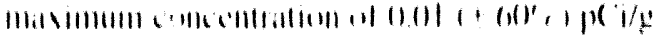

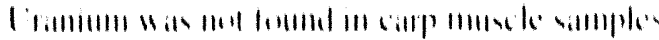

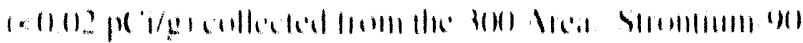

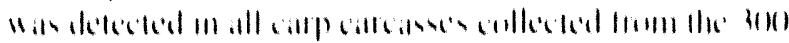

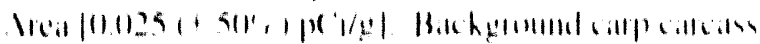

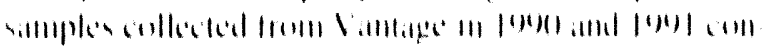

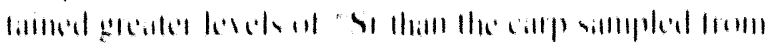

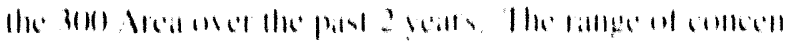

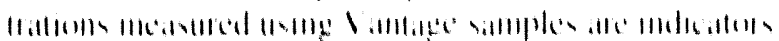

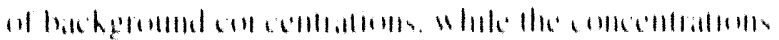

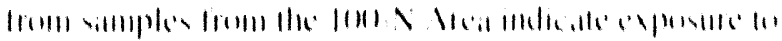
chasted st m the mater

\section{Whitefish}

\section{Sample Collection and Analysis}

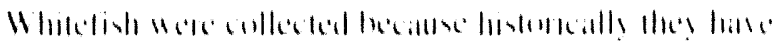

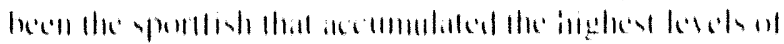

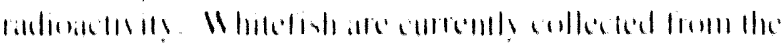

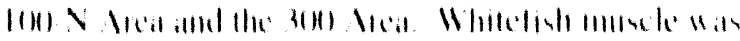

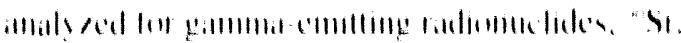

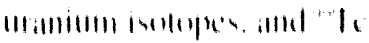

\section{Results}

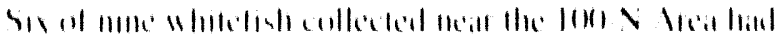

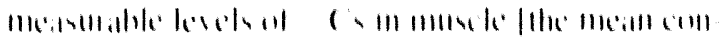

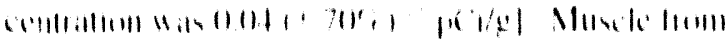

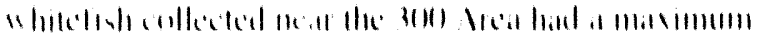

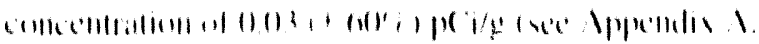

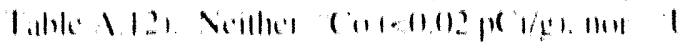

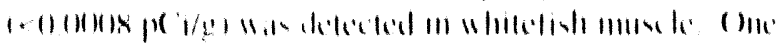

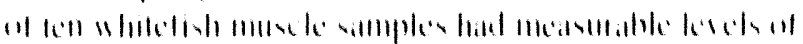
1. $1111+111+1101 / 21$

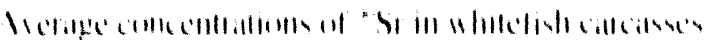

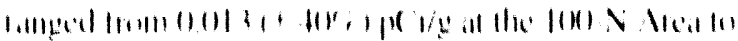

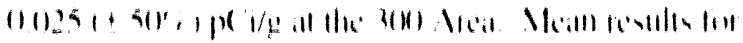

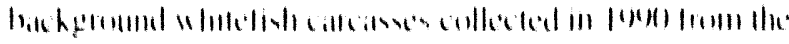

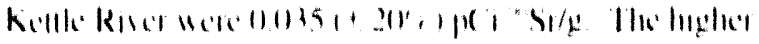

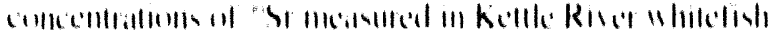

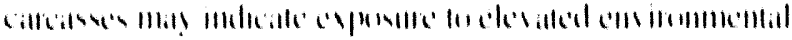

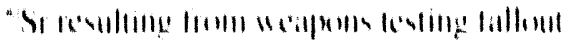

\section{Waterfowi}

\section{Sample Collection and Analysis}

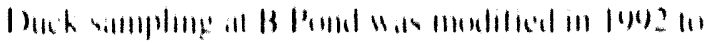

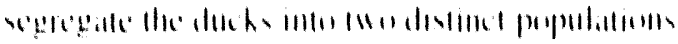

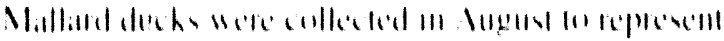

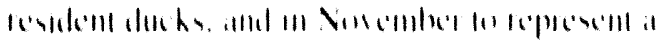

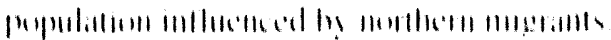

\section{Results}

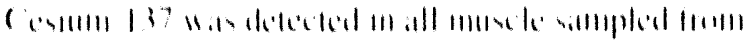

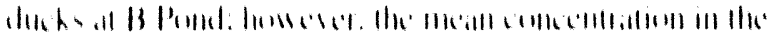

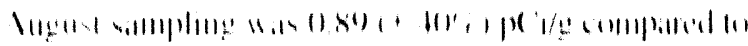

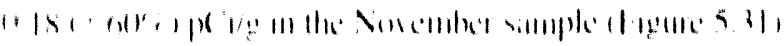

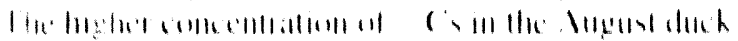

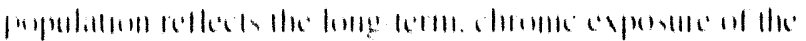

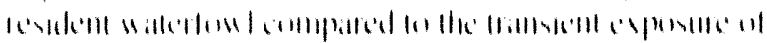

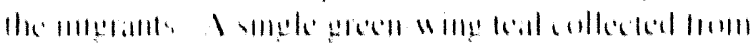

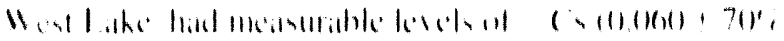

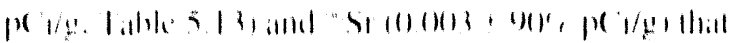

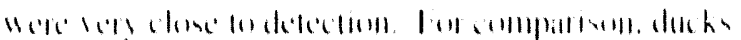




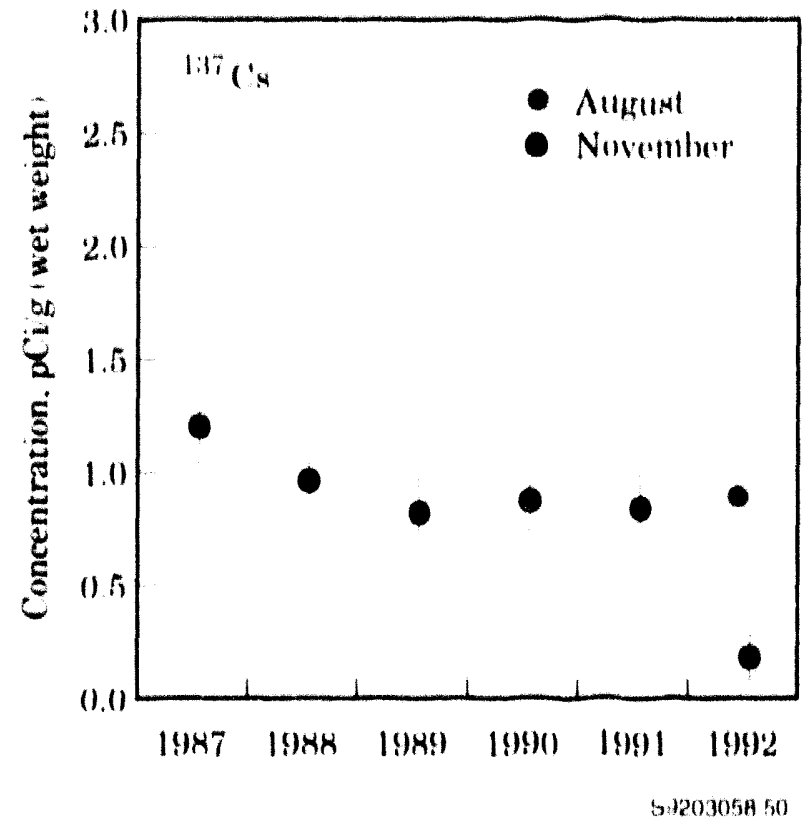

Figure 5.31. Concentrations ( 12 SEM) of Cesium137 (" "Cs) in Duck Muscle Samples from B Pond, 1987 Through 1992

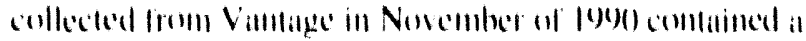

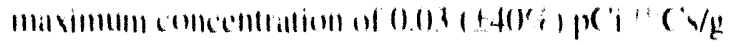

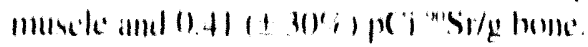

\section{Goose Egg Shells} Sample Collection and Analysis

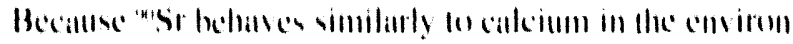

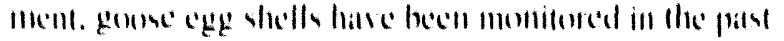

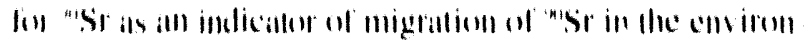

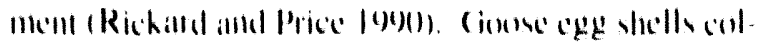

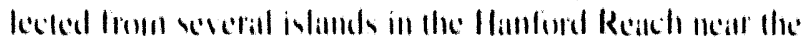

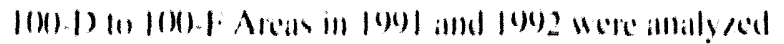

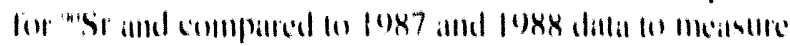

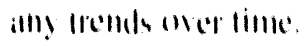

\section{Results}

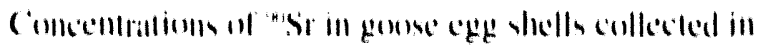

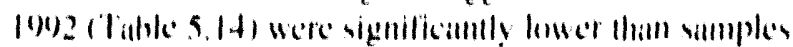

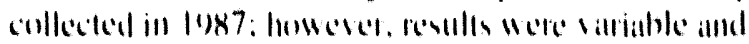

Table 5.13. Sumınary of Cesium-137 ('"Cs) in Duck Muscle, 1992 Compared to Values from the Previous 5 Years

149) I.we llanl

I.tuillin! Mlinimum!" Mrill':

$0.5 t+511 \%$

$1.32+10 \%$

III) N SRit

Wist lake

$11.111,71)^{4}$

Vallitipe

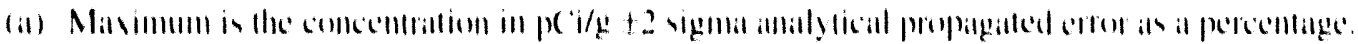

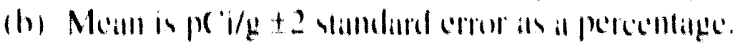

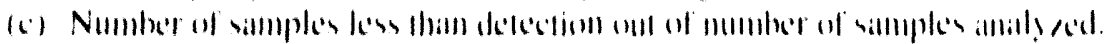

(d) Nome colleciled.

(c) (i)llecterl in lout).
No.

$|11 \times 7 \cdot|(1) \mid$

No.

I.wo Thinn

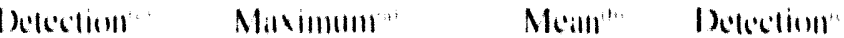

(1) 1110

$1.1+111^{\prime}$

$11.80+301 \%$

110101

$11.113+8(1)$

$(1,0(1)+1204$

110112

$\| 111$

$1+1+10 \%$

$11.31+10111 \%$

$4(1)$

(1). 1$)+f(1)$

$0.6112+1+10(1)$

$7(111$ 
Table 5.14. Concentrations of Strontium-90 (" $\mathrm{Sr}$ ) in Goose Egg Shells, 1987, 1988, 1991, and 1992

N(1, (1)

Yeall

(interintritiont)

Sillinples

1987

II)

|(1)

(1)! 2

$$
\begin{aligned}
& 1.26+0.22 \% \\
& 11.84+0.15 \% \\
& 1.01+0.34 \%
\end{aligned}
$$

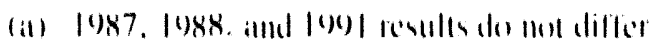

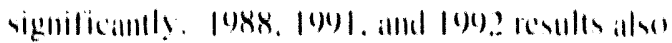
(10) mot difler signilicinnly.

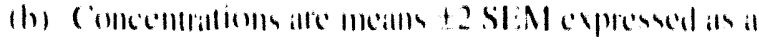
percentige.

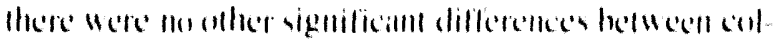

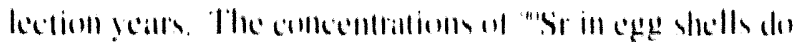

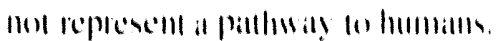

\section{Gamebirds}

\section{Sample Collection and Analysis}

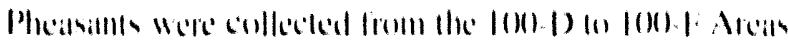

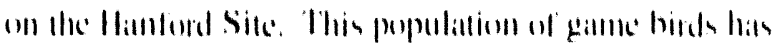

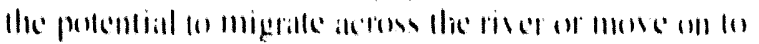

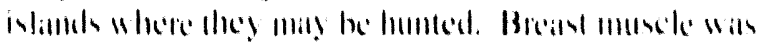

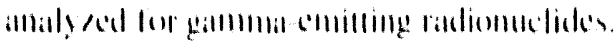

\section{Results}

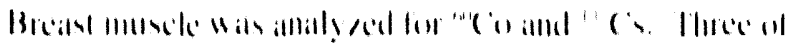

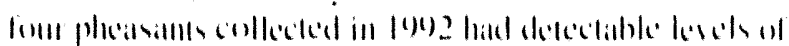

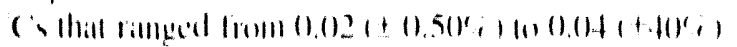

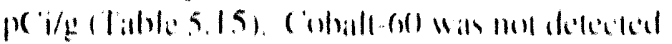
(a) (1)2 p) (i/g) in muscle.

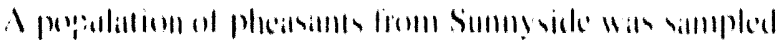

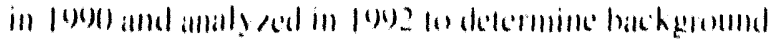

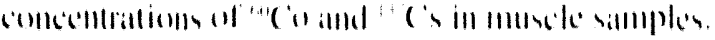

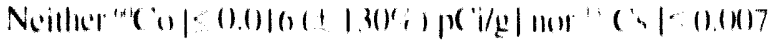
1t $\mid 8(1) ;$ )

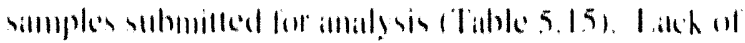

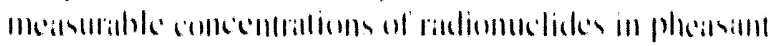

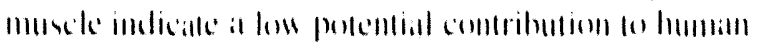

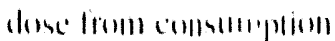

\section{Rabbits}

\section{Sample Collection and Analysis}

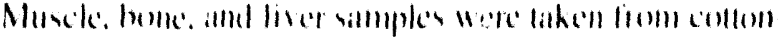

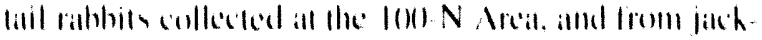

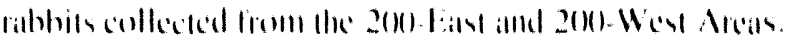

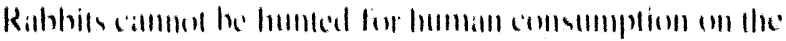

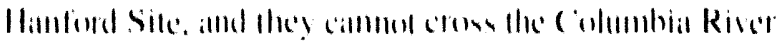

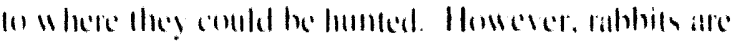

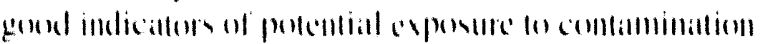

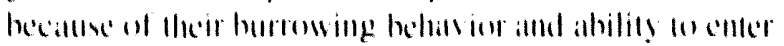

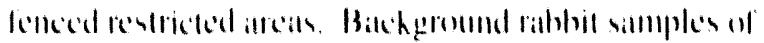

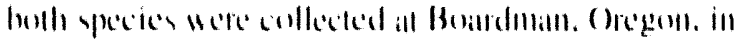

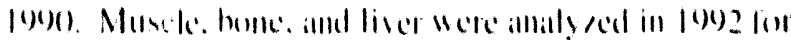

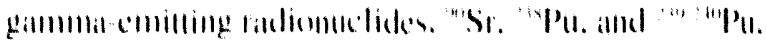
respestivils.
\end{abstract}

\section{Results}

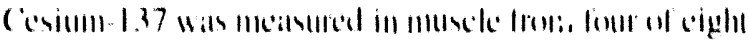

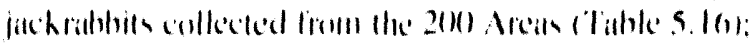

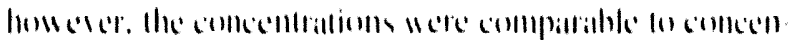

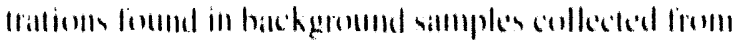

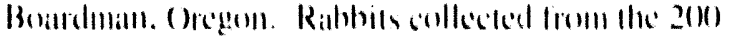

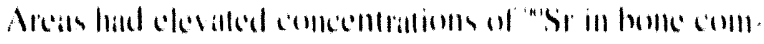

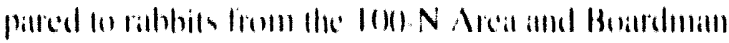

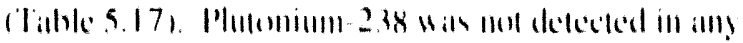

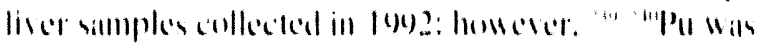

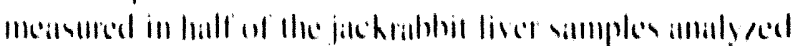

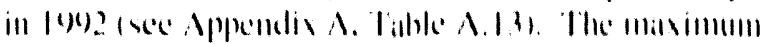

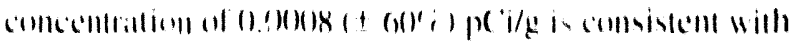

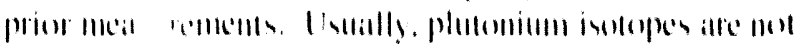

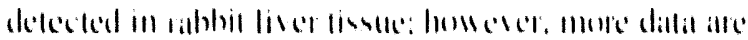

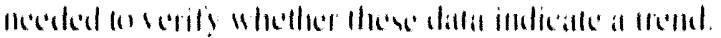

\section{Deer}

\section{Sample Collection and Analysis}

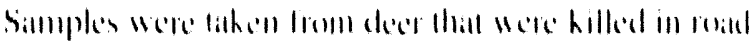

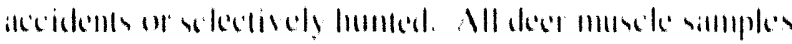

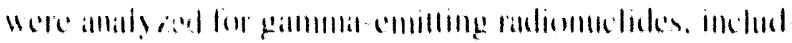

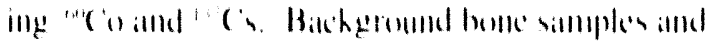

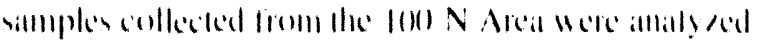

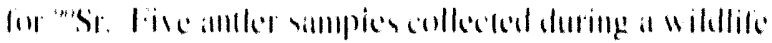

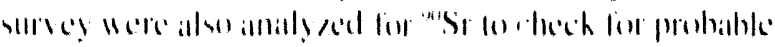

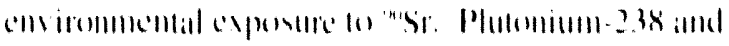


Table 5.15. Summary of Cesium-137 ( $\left.{ }^{137} \mathrm{Cs}\right)$ in Upland Gamebird Muscle (pCi/g wet), 1992 Compared to Values from the Previous 5 Years

1992

No.

$|987-199|$

Less Than

Lociation

Maxinumm'."

Meanin

Detection

1()()$-D) 1010(0-\mathrm{F}$

Areas

$0.04+40 \%$

$0.02 \pm(60 \%$

1014

1(1)-N Arcai

Yakima County

$--^{(4)}$

$-n^{\prime \prime \prime) ~}$ $-\cdot$ $-\cdot$
Maximumbiti

Less Than

Delection"

20 of 31

1 of 1

$0.007 \pm 180 \% \quad 0.001 \pm 680 \% \quad 10$ of 10

(a) Maximum is the concentration in $\mathrm{pCi} / \mathrm{g} \pm 2$ sigma total error as a pereentage.

(b) Mean is pCi/g \pm z standard error as a percentage of all samples analyzed including less-than-detection values.

(c) Number of samples less that detection out of number of simples analyzed.

(d) None collected.

(c) Collected in 1990)

Table 5.16. Summary of Cesium-137 ( $\left.{ }^{137} \mathrm{Cs}\right)$ in Rabbit Muscle (pCi/g wet), 1992 Compared to Values from the Previous 5 Years

1992

N()

Less Than

Location/Species

Maxim!:ın!

Mean't'

Detection"

20)-Eansl Areal

jackrabbit

$0.022 \pm 5() \%$

$0.008 \pm 1.30 \%$

3014

1014

$0.011 \pm 40 \%$

200-West Areal

$0.014 \pm 70 \%$

jackrabbit

10()-N Area/

cottontail

$0.032 \pm 1.30 \% \quad-(0.001 \pm 2.000 \% \quad 4014$

Boardman'dy/

jackrabbit

coltontail
$1987-199 \mid$

No.

Less Than

Detection"

Mean'th'

$0.25 \pm 20 \% \quad(0.04 \pm 90 \%$

7 of 13

$0.15 \pm 20 \%$

$0.0 .3 \pm 110 \%$

7 of 9

$0.15 \pm 30 \% \quad 0.0 .3 \pm 100 \%$

10 of 15

$\begin{array}{llr}0.03 \pm 70 \% & 0.005 \pm 200 \% & 9 \text { of } 10 \\ 0.03+130 \% & 0.006 \pm 150 \% & 10 \text { of } 10\end{array}$

(a) Maximum is the concentration in $\mathrm{pCi} / \mathrm{g} \pm 2$ sigma analytical propagated error ats a percentage.

(b) Mean is $\mathrm{pCi} / \mathrm{g} \pm 2$ standard error as a percentage.

(c) Number of samples less than detection out of number of samples analyzed.

(d) Collected in 1990. 
Table 5.17. Summary of Strontium-90 ( $\left.{ }^{90} \mathrm{Sr}\right)$ in Rabbit Bone (pCi/g wet), 1992 Compared to Values from the Previous 5 Years

1992

\begin{tabular}{|c|c|c|c|c|c|c|}
\hline & & 19 & & & $|987-190|$ & \\
\hline Location/Species & Miximum!n!' & Meann'" & $\begin{array}{l}\text { No. } \\
\text { Less Than } \\
\text { Delection" }\end{array}$ & Maximum! & Mean'th' & $\begin{array}{l}\text { No. } \\
\text { Less Than } \\
\text { Detection" }\end{array}$ \\
\hline $\begin{array}{l}\text { 20(1)-Lasul Areal } \\
\text { jackrabhil }\end{array}$ & $18.0 \pm 1 \%$ & $7.9 \pm 90 \%$ & () of 4 & $49 \pm 20 \%$ & $11 \pm 70 \%$ & 0 of 1.3 \\
\hline $\begin{array}{l}\text { 2(0)-West Areal } \\
\text { jacckrabbil }\end{array}$ & $14.8 \pm 20 \%$ & $4.1 \pm 170 \%$ & () of at & $140 \pm 20 \%$ & $21 \pm 1.50 \% /$ & 0 of 9 \\
\hline $\begin{array}{c}\text { 10(0)-N Areal } \\
\text { collontail }\end{array}$ & $(0.41 \pm 2() \%$ & $0.24 \pm 40 \%$ & () of 4 & $4(60 \pm 20 \%$ & $98 \pm 70 \%$ & () of 15 \\
\hline $\begin{array}{l}\text { Boardmantity } \\
\text { jackrabbit } \\
\text { cottontail }\end{array}$ & & & & $\begin{array}{l}0.91 \pm 10 \% \\
0.36 \pm 20 \%\end{array}$ & $\begin{array}{l}0.47 \pm 2(1) \% \\
(0.27 \pm 10 \%\end{array}$ & $\begin{array}{l}\text { 0) of } 10 \\
0 \text { of } 10\end{array}$ \\
\hline
\end{tabular}

(a) Maximum is the concentration in $\mathrm{pCi} / \mathrm{g} \pm 2$ sigma analytical propagated error als a percentage.

(h) Mean is $\mathrm{pCi} / \mathrm{g} \pm 2$ stindiard error as al percentage.

(c) Number of simples less than detection out of number of samples analyzed.

(d) Collected in 1990.

2mesu were analyzed in liver samples from all deer sampled in 1992. While deer hunting is not allowed onsite. deer can leave the Site, and a small number of deer potentially from Hanford are harvested annually from Columbia River islands and Grant. Benton, and Franklin counties. Background deer samples were donited by a hunter who collected them in Stevens County, locitted about $210 \mathrm{~km}(1.31 \mathrm{mi})$ northeast of the Hanford Site.

\section{Results}

Generally, "Co and other gatmmit-emitting radionuclides were not detectable in deer muscle; the maximum concentration was (1).()I $( \pm 9) \%) \mathrm{pCi} / \mathrm{g}$. However. three of nine deer sampled al Hanford had positive mealsurements of " $" \mathrm{Cs}$ (Bisping and Woodruff 1993). These measurements ranged from $0.006( \pm 50 \%)$ p $\left(\mathrm{i}{ }^{1.17} \mathrm{Cs} / \mathrm{g}\right.$ to 0.017

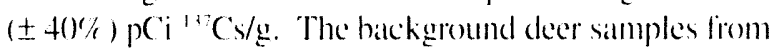
Stevens County contained $0.33( \pm 10 \%)$ and 0.52 $( \pm 10 \%) \mathrm{pCi}{ }^{17} \mathrm{Cs} / \mathrm{g}$. These background deer samples contain about ken times higher concentrations of " $" \mathrm{C} S \mathrm{~s}$ because the Stevens County area historically received more precipitation and thus received more deposition of fallout from attmospheric weapons testing than the arid Hanford Site.

A positive relationship between elevated levels of ${ }^{17} \mathrm{C}$ in Hanford workers has been established with the consumption of wild game taken from other areas that received relatively higher levels of falloun (Makleellan et al. 1993). In this study, Hanford workers who had elevated whole-body measurements consumed large anounts of deer or elk meat from animals that had accumulated elevated levels of fallout ${ }^{19} \mathrm{C}(\mathrm{s}$ in their natural habitat. The annual effective dose to the hunters was about 2.5 mrem.

In 1992. whe three deer simpled from the 10(1)-N Areat hat measurable amounts of "se $\mathrm{Sr}$ in bone (range 1.4 to 20.8 $\mathrm{pCi} / \mathrm{g}$ ) that exceeded the levels measured in background deer (maximum value of $(0.81 \mathrm{p}(\mathrm{j} / \mathrm{g})$. Concentrations of "'Sr in antlers from Hanford deer ranged from 0.3. $( \pm .30 \%) 100.5+( \pm 10 \%) \mathrm{pCi} / \mathrm{g}$ antler. The se concentrations of "St are comparable to levels in deer bone from background samples from stevens County 
(see Appendix A. Table A.14) and rabbil bone samples collected from Boardman, ()regon (Tahle 5.17). Fiven though one deer antler was found close to the 100 - $\mathrm{N}$ Area, the relatively low "Sr concentrations maly indicate that the deer hatd not been exposed to elevatted levels $\left.{ }^{0}\right)^{\circ}$ "Sr in their labital. Analysis of antlets is a nondestructive method of monitoring deer populations.

In 1992, no plutonium was detected in any deer liver sample $(<0.0)(0) 5 p(\mathrm{i} / \mathrm{g})$. 


\subsection{Soil and Vegetation Surveillance}

Surfike soil samples were collected from nime lecaltions

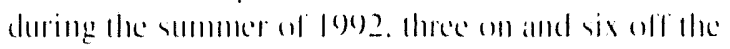
Hanford Sile. The oflsile samples were collected at lone

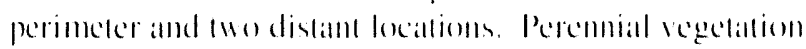

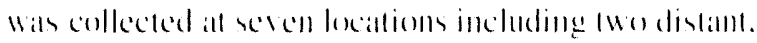
ane onsite and four perimeter leciations. The purpense of

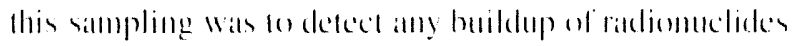
firm deposition of airhorme efflemens releared from

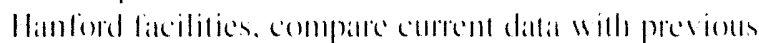

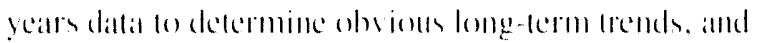

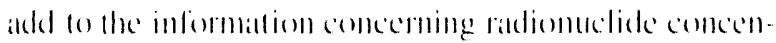
frations fore soil and regedaltion hold and and oll the llanlind Sile.

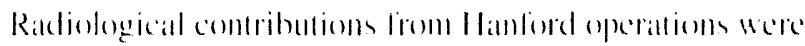
ansessed by compratring resulls from samples laken:

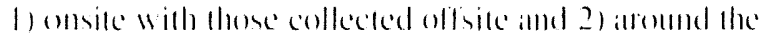

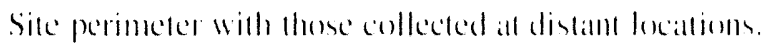

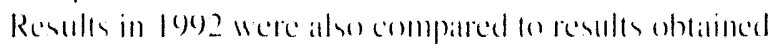
in provious yeits.

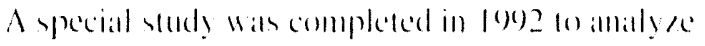
shereline regetation simpled from andeas where elevalted

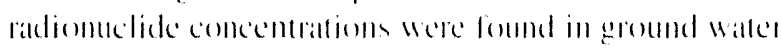
secping into the (olumbial River.

\section{Sample Collection and Analysis}

Soil and regetattion samples mere collected at the locat lioms shown in ligute 5.3.2 and stummatried in Table 5.18 . ()msile simpling wats concentralled around

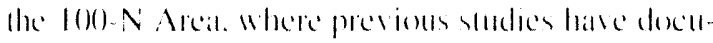

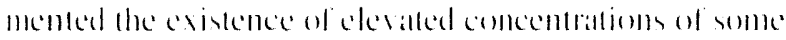

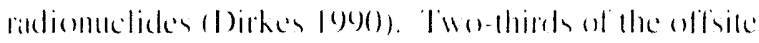
simples Here collected all dom

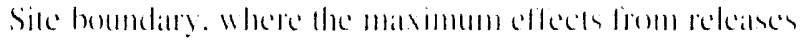

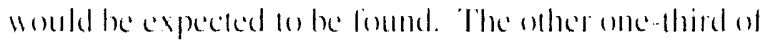
the oflisite simples were collected all relatisely distant and upwind locattoms, where buildup of Itantord's

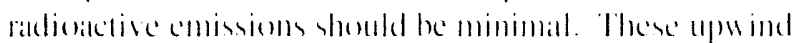

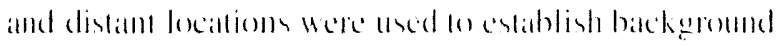

randiunuclide concentration levels for 1092. The areas

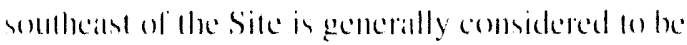
downwind and the areal month to monthwest of Hanford is comsidered upwind.

Soil simples were composiles af live soil cores $2.54 \mathrm{~cm}$

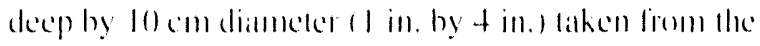

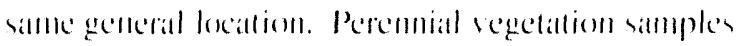

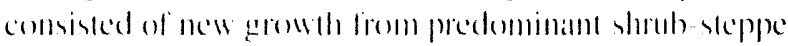

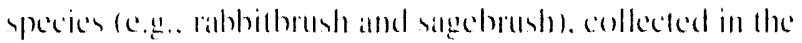
same vicinity an the soil simples. Sample alligutsots were

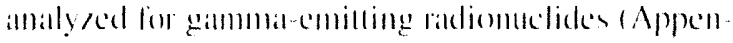

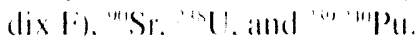

A shoreline aededation study was statted in log) by simpling shoreline vegetation in areas reported to have radioleggical contaminattion in grommd-water seeps. Areals of particular interest included the $1(1)-\mathrm{N}$ Areit. the old

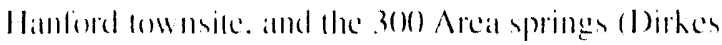

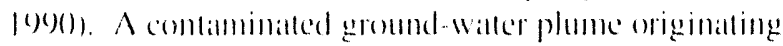

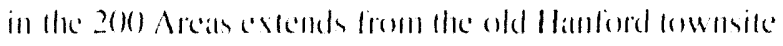

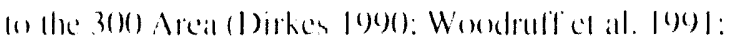
Woudrulf (el al. 1902). In 1092, additional simplen were collected at the same locations and all a backeround focitfon uputreimn from the Vernita Bridge.

\section{Results for Soil}

() the soil andyses performed. the only comsistenty

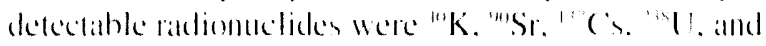

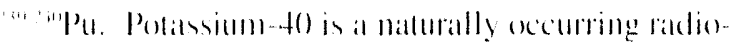
muclick with a half-life longer thatl ane hillion years. and

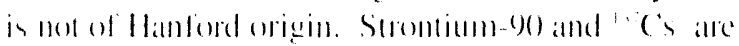
bolh fission produck illd halde hall-lises ol 27.7 and

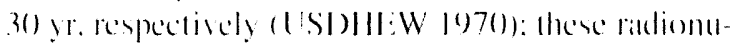
clides maly he of Hamlord origin or liom global allmos-

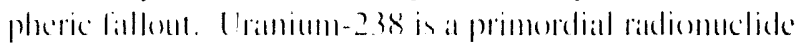
havimg a hall lile of 4.51 hillion gears and is mallurally found in soils ofl the Hantord Sile. Howeres. It as

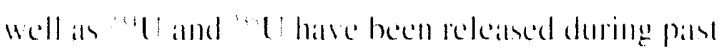

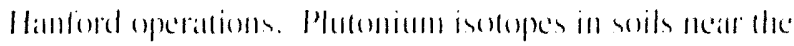

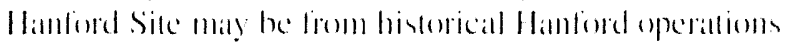
or may be the resule of globat atmompleric lialloul. 


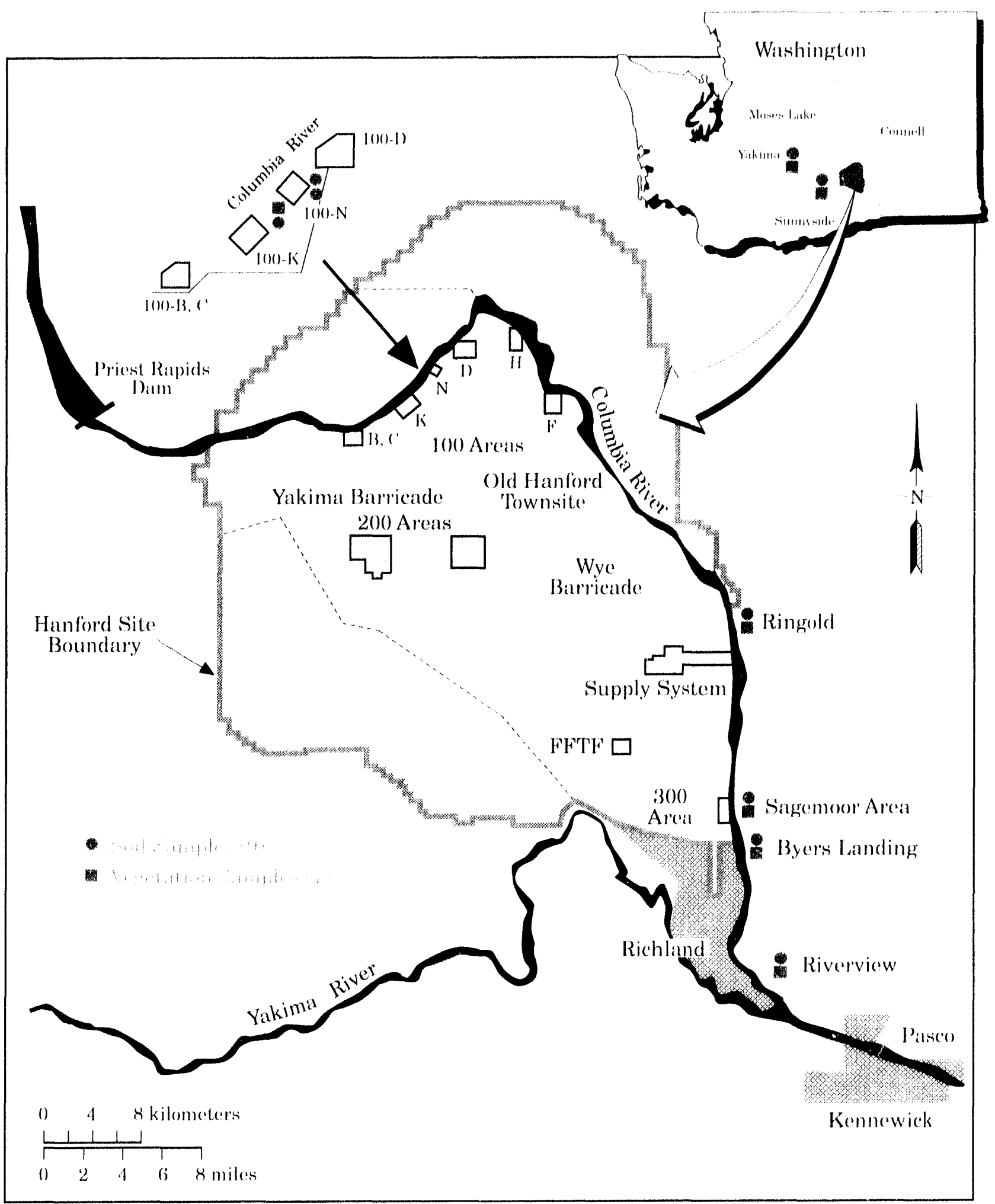

$\$ 9303012.14$

Figure 5.32. Soil and Vegetation Sampling Locations, 1992 
Table 5.18. Soil and Vegetation Samples, 1992

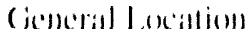

Sivil

()isile

(1)() $\mathrm{N}$ Area

() Militedit

N('ill

I TistinII

Vegetation

()mike

1(1) $\mathrm{N}$ Arua

()fisilent:

Ne:ill

1)islanl
No. in

Silliples liecfuciley

$\wedge$

Al1 3 y

$\wedge$ or $5 \mathrm{yl}$

$\Lambda$

$A 10.3 \mathrm{yr}$

A 115 yr
Aliilyı's

(i) $\mathrm{A}=$ intmlially.

$3 y=0$ mecoeve 3 years

$5 \mathrm{yl}=$ mecerery 5 years.

(b) Neill = lecittims all the Sile perimeler.

1)istame = Yak imat and Summy side (sece liggune 5.32) .

Roldennelide concentrations in soil are reported in Table .5.19 alld Appendix A. Tiables $A .15$ through $A .18$. and ance shown in ligumes 5.3 .3 and 5.34. Nompanamedric stallistical antalyses of the analytical resulds indicatted no significant differenees betweedn lle distant upwind and

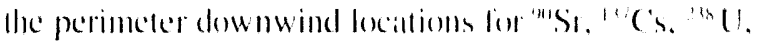

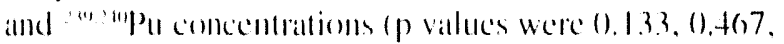
0.73.3, and 0.2(0), respectively). A p vallec represents the

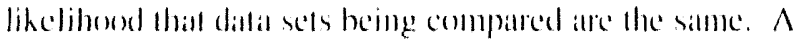

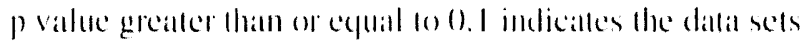
are similatr. and a p value less than 0. I indicates they atre not similar. Likewise. there were me statistical difleseneses between the onsite and the combined off site radionuclide

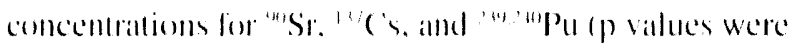
0.952 .0 .05 .5 , alld 0.714. respectively). A stallistical difference was found when companting onsite and com-

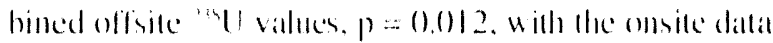
having a higher range of values. Thios means thall the all

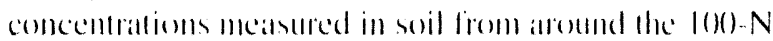
Area were signilicanlly different (and highen) hand con cembratioms measured in offsite soil samples.

\section{Results for Vegetation}

Activity concentrations of the three most comsistently detected radiontuclides found in vegetation during 1992. Be. "K, alldel" Sr, alle presented in Tiable 5.20. Beryllimm-7 and "K are naturally oceurring radionuclides and alle not of Handord origin. Strontium-(9) is a fission producl and maly be of llantord origin or from global fiallout. Historically, other tadionuclides of interest hatve been uranium. " clickes were only sporadically delected (Tahle 5.21). Uranium-2.38 wats only seen in one simple collected all

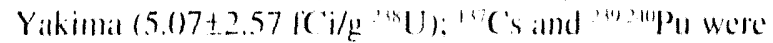
anly defected in a sillople conlleceded at Sigemonor, al down-

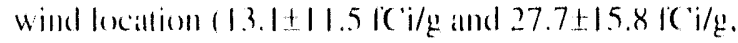

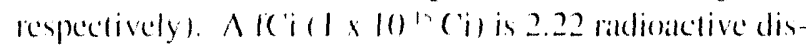
integrations every $1 .(0)()$ minntes. The rematining samples haderers alssocialled with the analyses sted that the $9.5 \%$ confidence interval encompassed 2 ero (i.e.. not $95 \%$ comfjedent that the trate radiontelide concentration wats above

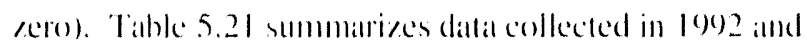

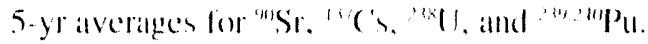


Table 5.19. Radionuclide Concentrations in Soil Sarnples Collected on and off the Hanford Site, 1992 Compared to Values from the Previous 5 Years (units are $\mathrm{pCi} / \mathrm{g}$ dry weight)

$109)$

\begin{tabular}{|c|c|c|c|c|c|c|c|}
\hline \multirow[b]{2}{*}{ Lacillim } & \multirow[b]{2}{*}{$\begin{array}{l}\text { Raldiu- } \\
\text { muclide }\end{array}$} & \multirow[b]{2}{*}{$\begin{array}{l}\text { No, ol } \\
\text { Sillmples }\end{array}$} & & & \\
\hline & & & 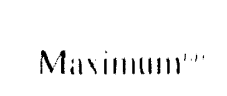 & Mc'inl'." & $\begin{array}{l}\text { Nol of } \\
\text { Sillmple's }\end{array}$ & Manimlum!". & Me:an't" \\
\hline \multirow[t]{4}{*}{ ()msite } & "Si & 3 & $0.24+5 \%$ & $0.12+10.3 \%$ & $6(1)$ & $2.7+34$ & $0.24 \pm 3.54$ \\
\hline & 113 & 3 & $1.0 \pm+1 / 6$ & $0.73+5.4 \%$ & $0(1)$ & $20+14$ & $1.9 \pm 0(1) \%$ \\
\hline & $\therefore 11$ & 3 & $1.3 \pm .30 \%$ & $1.2+14 \%$ & .13 & $3.3+1.4 \%$ & $0.77 \pm 18^{1 / 1}$ \\
\hline & " $" P_{11}$ & 3 & $(1 .(1) 2() \pm 0 \%$ & $0.011 .3 \pm 7.3 \%$ & $0,1)$ & $0.71 \pm 1 \%$ & $(0.06,3+0,3 \%$ \\
\hline \multirow[t]{5}{*}{ Perimeter } & "Sir & 4 & $0.15+0 \%$ & $0.11+37 \%$ & 48 & $0 .+1) \pm 0 \%$ & $0.13 \pm 18 \%$ \\
\hline & 118 & 4 & $0.95+8 \%$ & $0.00+50 \%$ & +8 & $1.8+34$ & $0.58+221 /$ \\
\hline & $" \|_{1+m}$ & 3 & $11.91 \pm 25 \%$ & $0.72+26 \%$ & .37 & $1.8+226$ & $0.76+1.3 \%$ \\
\hline & 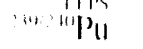 & 4 & $0.0121+8^{\prime} / 1$ & $0.013 \pm 68 \%$ & 3.3 & $11 .(120) \pm 11 \%$ & $0.0(1) 72+34 \%$ \\
\hline & & & & & & $(1.1) 33+7 \%$ & $(1.0) 11 \pm 21 \%$ \\
\hline \multirow[t]{4}{*}{ Distant } & "Ni & 2 & $0 .(14.5+111 / 4$ & $(1.0142+176$ & 3.3 & $0.3 .5+t^{\prime \prime} .6$ & $(1.10+28 \%$ \\
\hline & $11 C$ & 2 & $0.4 .5 \pm 6 \%$ & $0.43+61 \%$ & 33 & $1.2 \pm 5 \%$ & $0.30 \pm 20 \% / 1$ \\
\hline & $\because x I_{111}$ & 2 & $0.8+1 \pm 3+1 / 1$ & $0.76+226$ & 24 & $1.7 \pm 5 \%$ & $0.75 \pm 21 \%$ \\
\hline & : we : inlu & 2 & $0.0107 x+1+4$ & $0.001077 \pm 1 / 4$ & 3.3 & $(1,1120) \pm 11 \%$ & $(1,0)\left(172+34^{\prime} / 6\right.$ \\
\hline
\end{tabular}

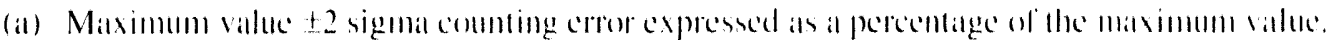

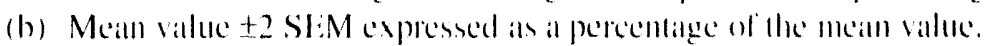

(c) Maximum value in previous 5 years 22 SEM expressed as a perecentage of the maximum value.

(d) five year meall valle \pm 2 SIEM expressed als a pereenlage of the meall value

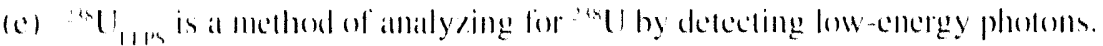

Basced on these resulls (ligure 5.35 ) and the associated

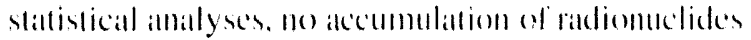

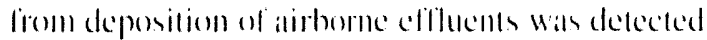

\section{Shoreline Vegetation Special Study}

Collumbiat River shomelinte regetillom silmples were

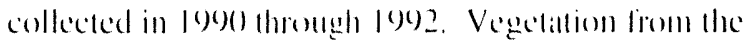
10)-N Areat. He old Hanlond lownsite, and the 300 Areat

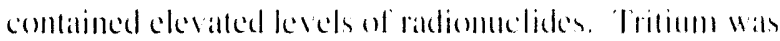
clevated above background in all alreas (ligene 5.36):

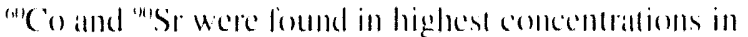

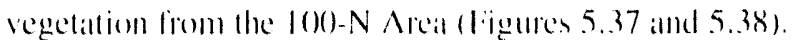

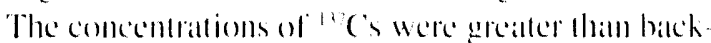

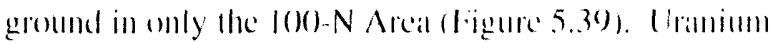

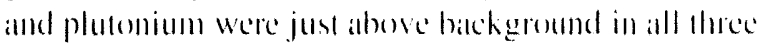
arean (Figures 5.40 and 5.41 ). The maximum

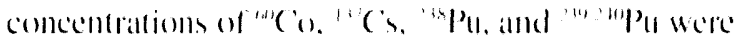

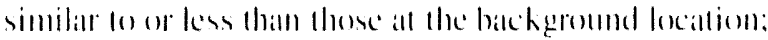
however. here vere imstances of plutomium in shoreline regetation that appeared fo be slightly alevalled abore the bateground locattom. The commilled alledise dose equivalent was estimated based on comsumplion of a kilogram of plants containing the highest concentrilloms foumd in the edihle portions of regetartion. The highest

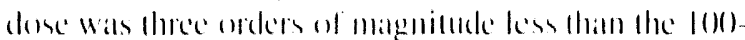

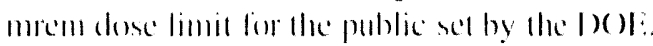

As part of the shoreline vegetallon study, silmples of

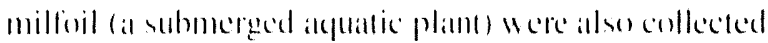

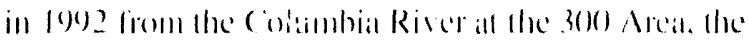
I(1)-N Areat. and upstream from the Vernila Bridge (upstream from the Site). Results showed higher

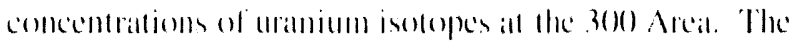

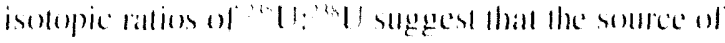
Iramium around lle 30 () Areat is enriched will 

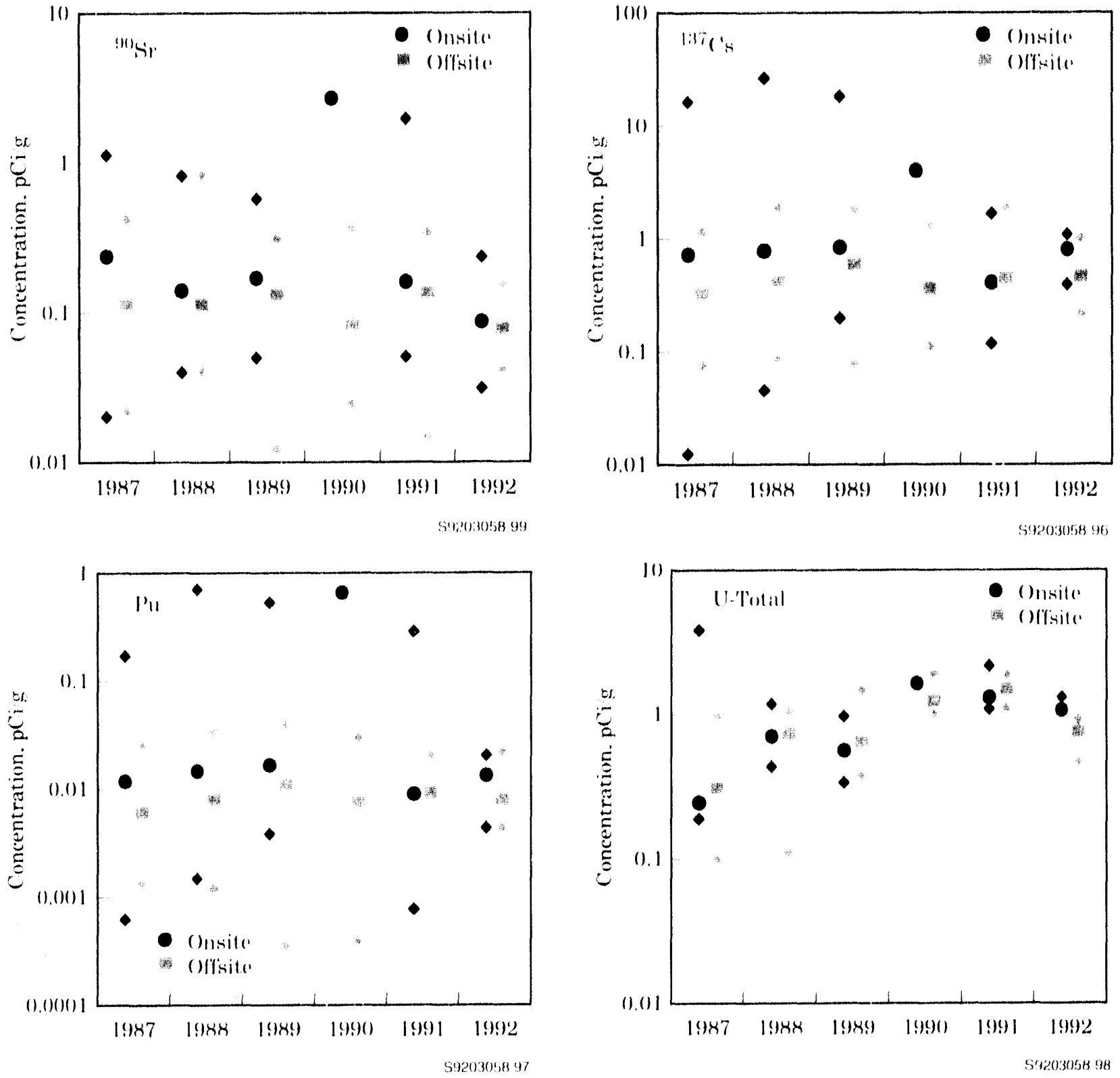

Figure 5.33. Selected Radionuclide Maximum, Median, and Minimum Concentrations in Soil, 1987 Through 1992. Units are $\mathrm{pCi} / \mathrm{g}$ (dry weight). For the U-total graph, samples trom 1987 were analyzed by $U_{\text {NAA }} ; 1988$, 1989 , and 1992 by $U_{11, \ldots,}$; onsite 1990 by $U_{w: 1,}$ and offsite by $U_{\text {HAI }} ;$ and 1391 by $U_{\ldots,}$. 

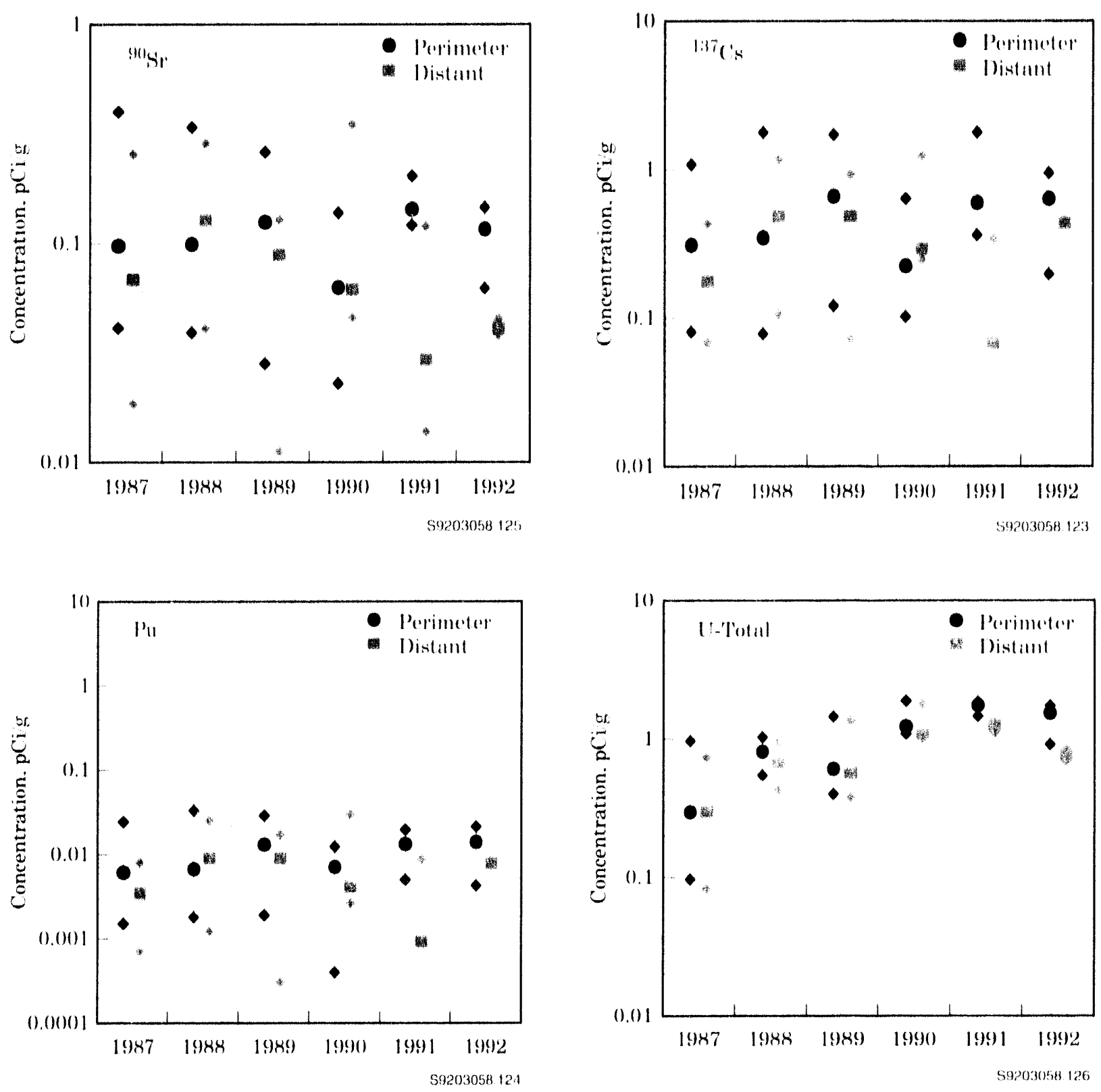

Figure 5.34. Selected Radionuclide Maximum, Median, and Minimum Conceritrations in Soil at Perimeter and Distant Locations, 1987 Through 1992. Units are pCi/g (dry weight). As a result of figure scale, some values overlap. 
Table 5.20. Concentrations of Three Radionuclides Consistently Found in Vegetation, 1992 (units are $\mathrm{pCl} / \mathrm{g}$ dry weight)

Sitmpling I.ncittion

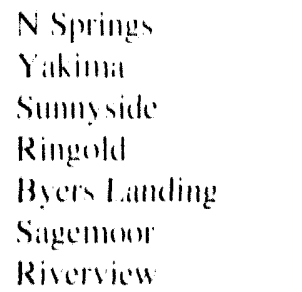

$130^{\circ}$

$$
\begin{aligned}
& 7.11+(1.30 \\
& 1.7 \pm 0.32 \\
& 2.110 .20 \\
& 2.7 \pm 0.27 \\
& 3.8+0.41 \\
& 4.6 \pm 0.27 \\
& 4.4 \pm 0.31
\end{aligned}
$$

"K
$16+01.88$
$17+01.90$
$1.7+11.83$
$17+0.78$
$19+12$
$1.5+11.67$
$17+11.68$

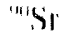

\begin{tabular}{|c|c|c|}
\hline Maximumm"s & Mc:alli": & $\begin{array}{l}\text { No. ol } \\
\text { Sillmple }\end{array}$ \\
\hline $0.0115+20 \%$ & 0.011 .5 & 57 \\
\hline $0 .(10.50 \pm .388 \%$ & $0 .(11) .50$ & 57 \\
\hline-1$)(1)(1) .4$ & $0.11(1.4+4$ & 56 \\
\hline $0.000000+ \pm 1.50 \%$ & $0 .(1)(1)(1)(1.4$ & .57 \\
\hline $0.1118 \pm 20 \%$ & $(1.1)(10) 5 \pm 87 \%$ & 53 \\
\hline (1).(1)1.3 +88\% & $-(1.00006 \pm 17.30 \%$ & 5.3 \\
\hline$(1) .(1)(1)+1)$ & $(1) .(1)(10) \pm 221 \%$ & 53 \\
\hline$(1.1)(1)(128+57 \%$ & $(0.0)(1) 1.3 \pm 78 \%$ & 5.3 \\
\hline$(1) .(1)+28 \%$ & $0.0(18.5 \pm 1.30 \%$ & 26 \\
\hline $0.00(0.51+30+1$ & $-(1.0)(1) .3+60621 / 6$ & 26 \\
\hline$-(1.01017$ & 0.010117 & If \\
\hline $0.01077+47 \%$ & $0 .(1)(177$ & 2 \\
\hline $0.010011010+111 \%$ & $0.0(1)(1) 10.3 \pm .31 \%$ & 26 \\
\hline
\end{tabular}

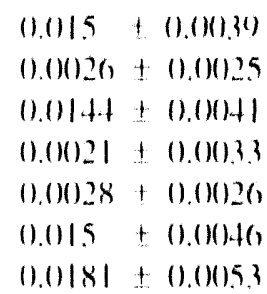

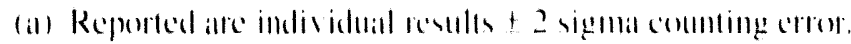

Table 5.21. Radionuclide Concentrations in Vegetation Samples on and off the Hanford Site, 1992 Compared to Values from the Previous 5 Years (units are $\mathrm{pCi} / \mathrm{g}$ dry weight)

1012

\begin{tabular}{|c|c|}
\hline $0.74+3 \%$ & $(1.01)+8.5 \%$ \\
\hline$(1.1) 79+204$ & $0.017 \pm 40 \%$ \\
\hline 0.47 & $0.015 \pm 811$ \\
\hline $0.15 \pm 8 \%$ & $(1.079$ \\
\hline (1)(1) $1.3+2$ & $(1.0()(1) \geq 0$ \\
\hline
\end{tabular}

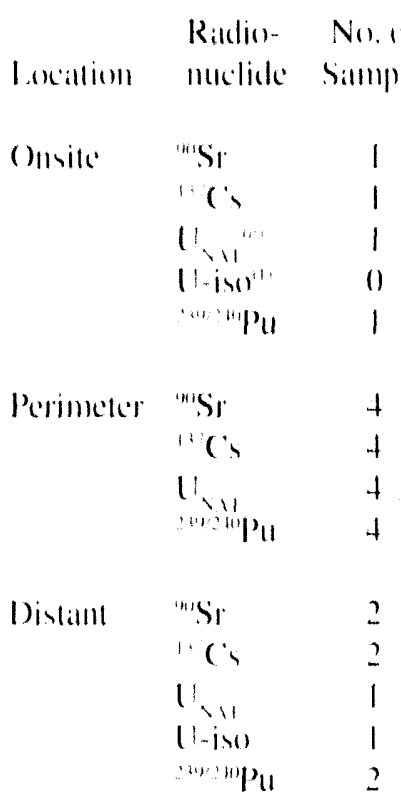

$|987-109|$

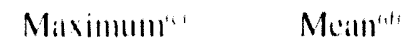

$$
\begin{aligned}
& 0 .+1+5 \% \quad(1.089 \pm 24 \% \\
& \text { (1).32+8\% } \quad 0.0 .53+.35 \% \\
& \text { (1).1) } 36 \quad 0.1112 \pm 18 \% \\
& \text { (1).(0)(0) } 2+24^{\prime} \% \quad(0.0)(1) 2 \\
& 0.041 \pm 6 \% \quad(0.0) 1010+0.5 \%
\end{aligned}
$$

$$
\begin{array}{cr}
0.36 \pm 4 \% & 0.0150 \pm 28 \% \\
0.11 \pm 24 \% & 0.0118 \pm .2 \% \\
0.0 \% & 0.0118 \pm 19 \% \\
0.0(10) 7.5 \pm+2 \% & 0.0(0010 \pm 22 \%
\end{array}
$$

(a) Maximum value t2 sigma coumling error expressed as a percentige of the maximum value.

(b) Meall value $22 \mathrm{Sl}: \mathrm{M}$ expressed an a percentige of the meall valle.

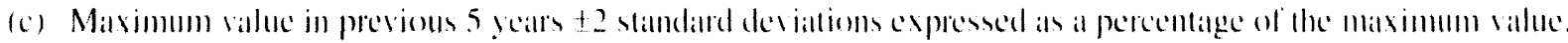

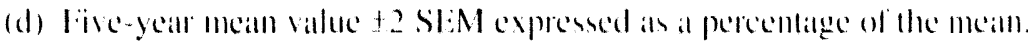

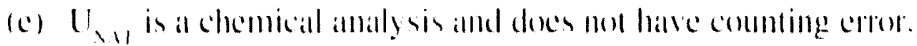

(i) Il-iso is al sum of isoltopic results. 

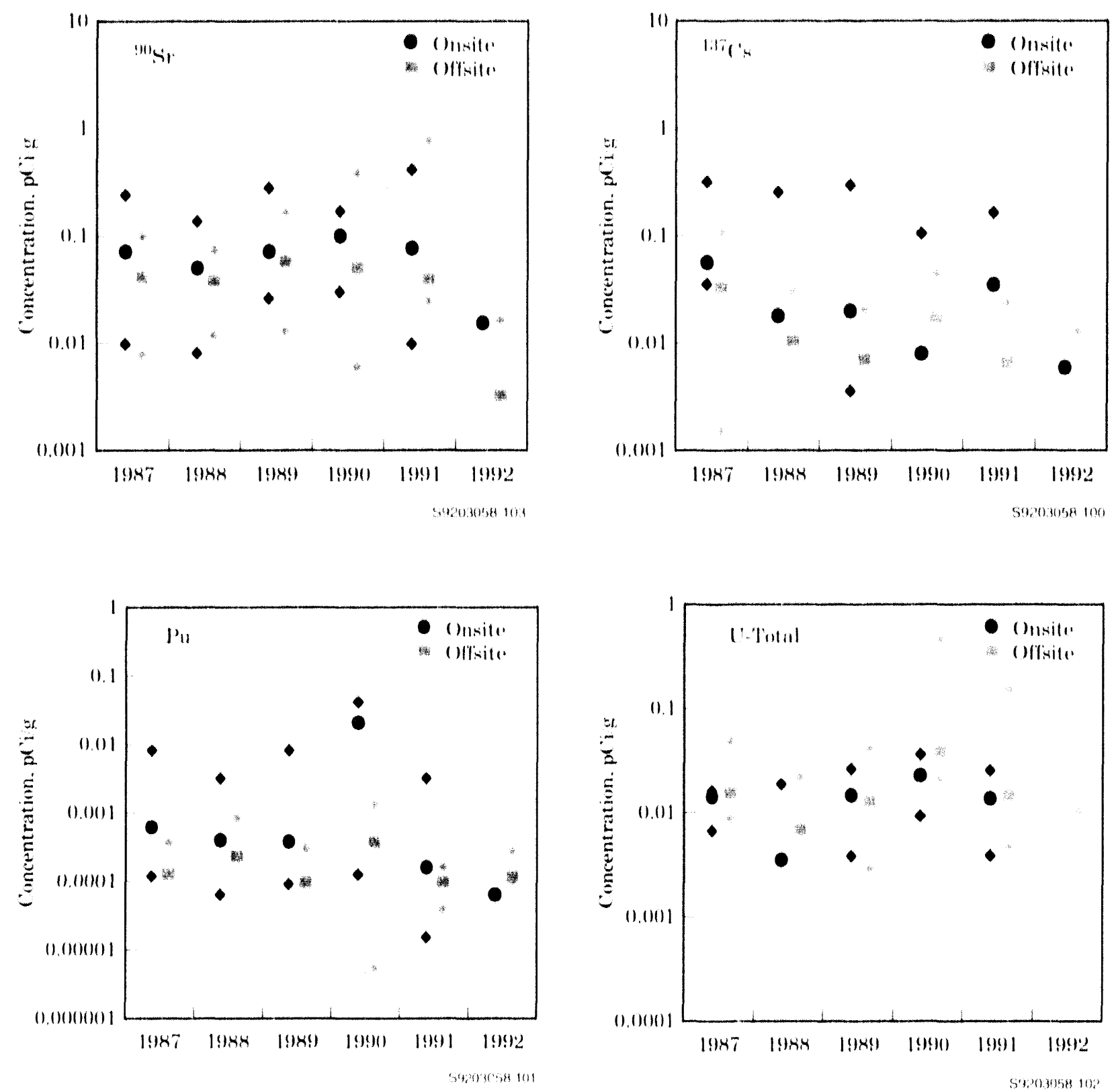

Figure 5.35. Selected Radionuclide Maximum. Median, and Minimum Concentrations in Vegetation, 1987 Through 1992. Units are $\mathrm{pCi} / \mathrm{g}$ (dry weight). As a result of figure scale, some values overlap. 


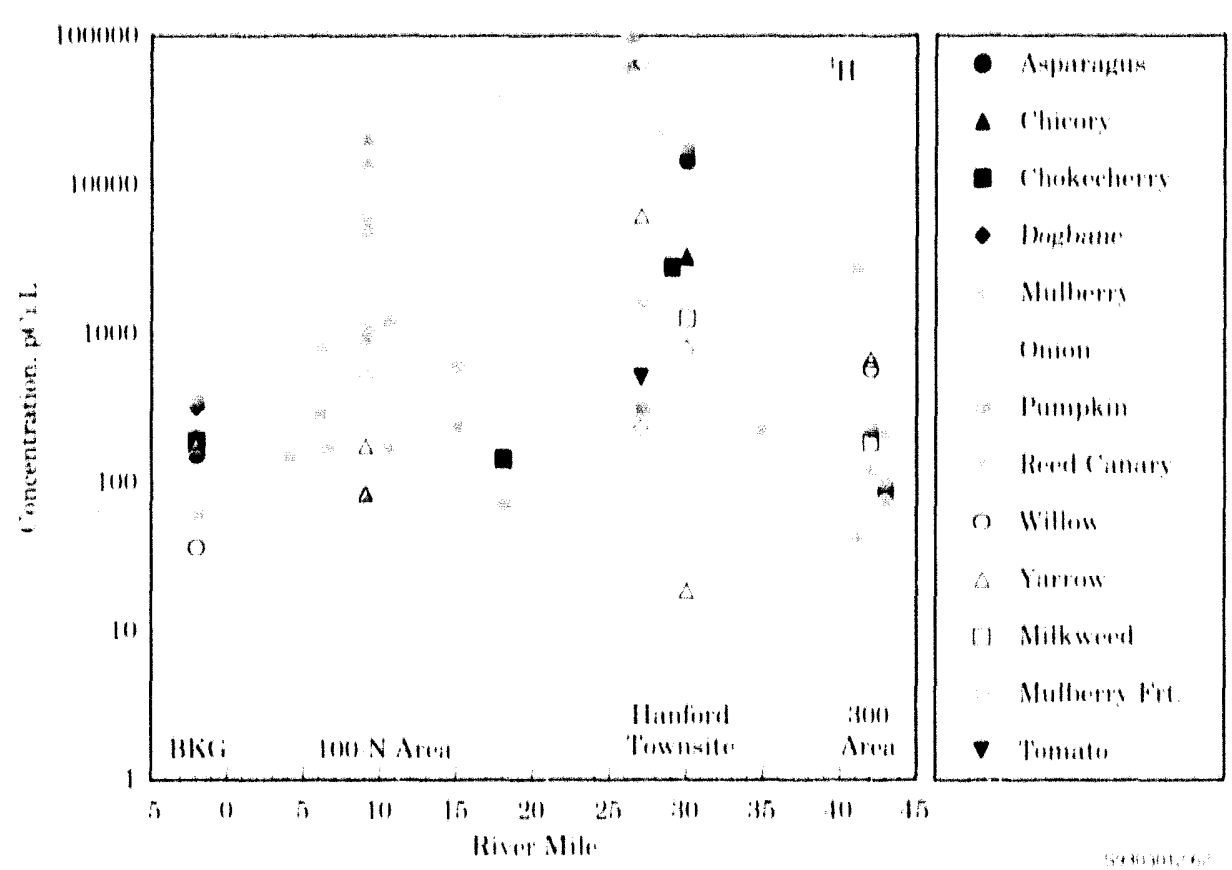

Figure 5.36. Tritium ('H) Concentrations in Columbia River Shoreline Vegetation for Individual Samples Collected from 1990 Through 1992. Pumpkin and tomatoes had been planted along the shoreline at the old Hanford townsite and are not normal shoreline vegetation.

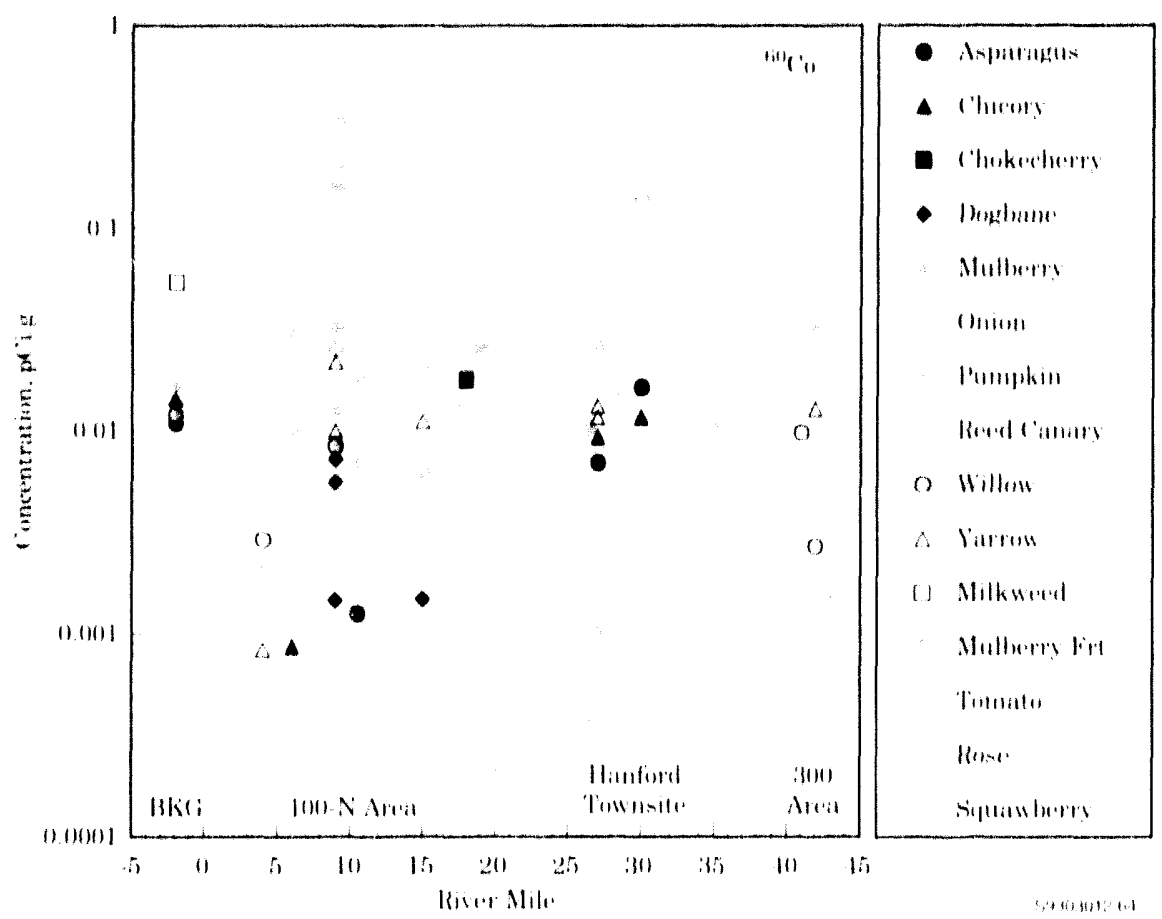

Figure 5.37. Cobalt-60 ("i) $\mathrm{Co}$ ) Concentrations in Columbia River Shoreline Vegetation for Individual Samples Collected from 1990 Through 1992. Pumpkin and tomatoes had been planted along the shoreline at the old Hanford townsite and are not normal shoreline vegetation. 


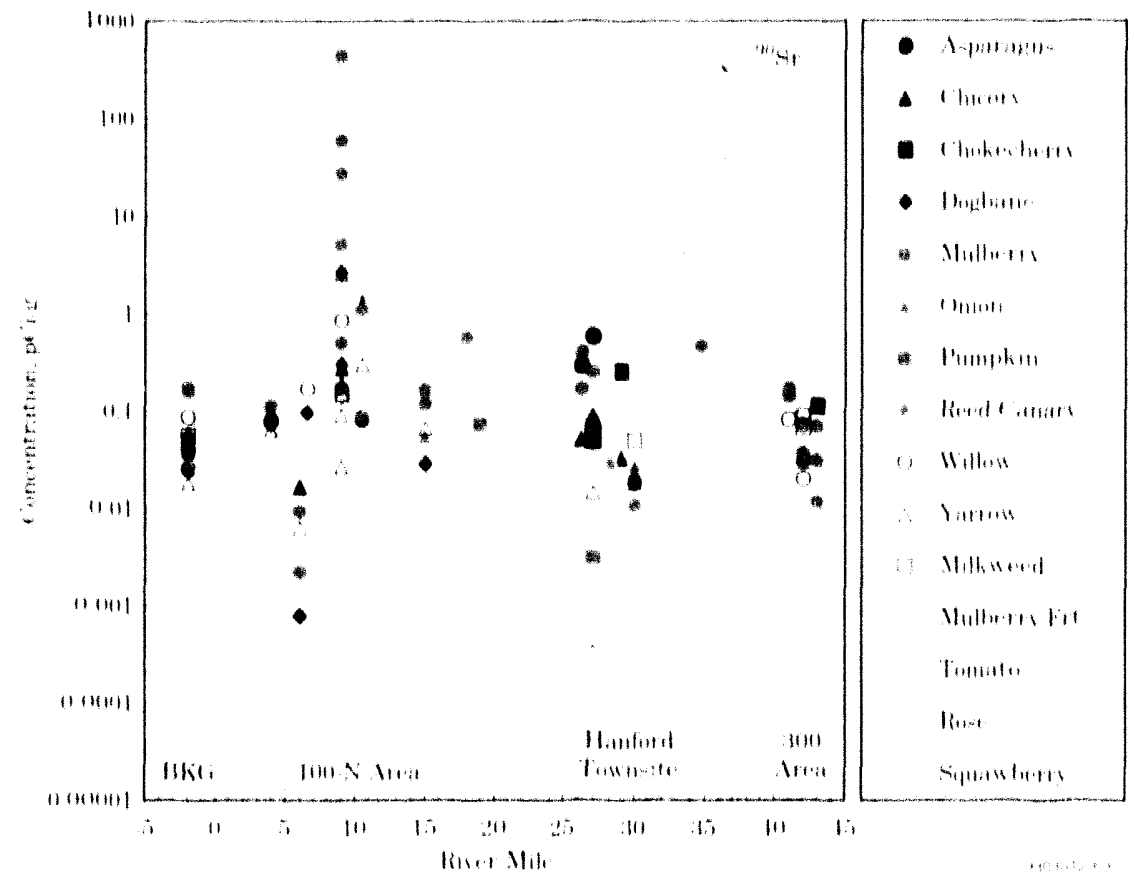

Figure 5.38. Strontium-90 (" ${ }^{*} \mathrm{Sr}$ ) Concentrations in Columbia River Shoreline Vegetation for Individual Samples Collected from 1990 Through 1992. Pumpkin and tomatoes had been plan 11 'ing the shoreline at the old Hanford townsite and are not normal shoreline vegetation.

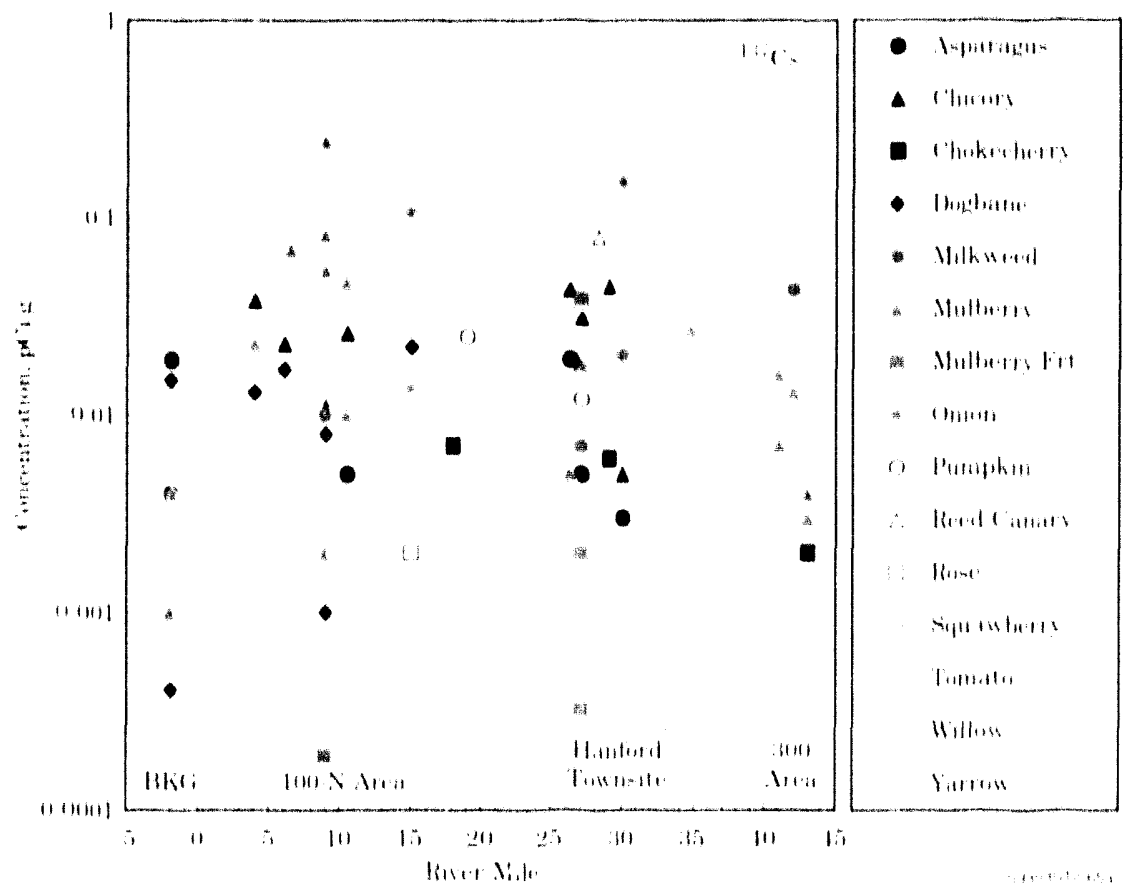

Figure 5.39. Cesium-137 ("Cs) Concentrations in Columbia River Shoreline Vegetation for Individual Samples Collected from 1990 Through 1992. Pumpkin and tomatoes had been planted along the shoreline at the old Hanford townsite and are not normal shoreline vegetation. 


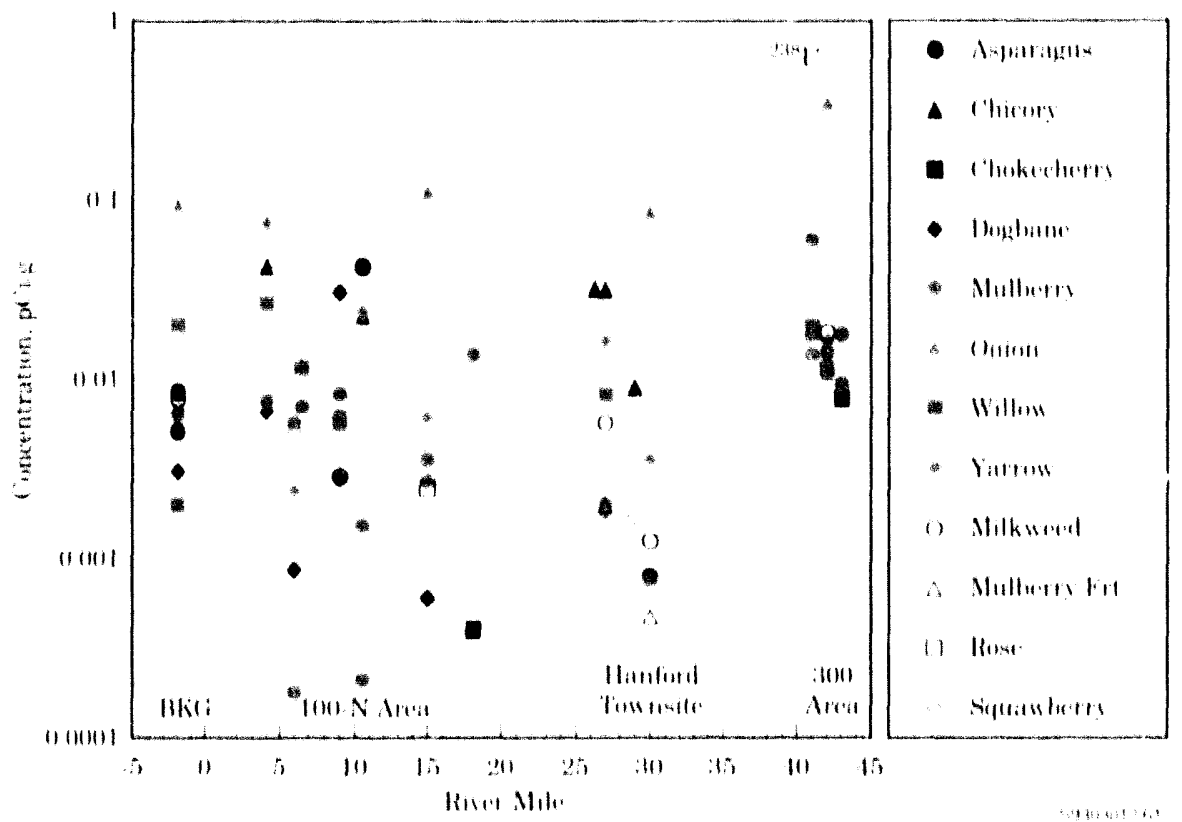

Figure 5.40. Uranium Concentrations in Columbia River Shoreline Vegetation for Individual Samples Collected from 1990 Through 1992

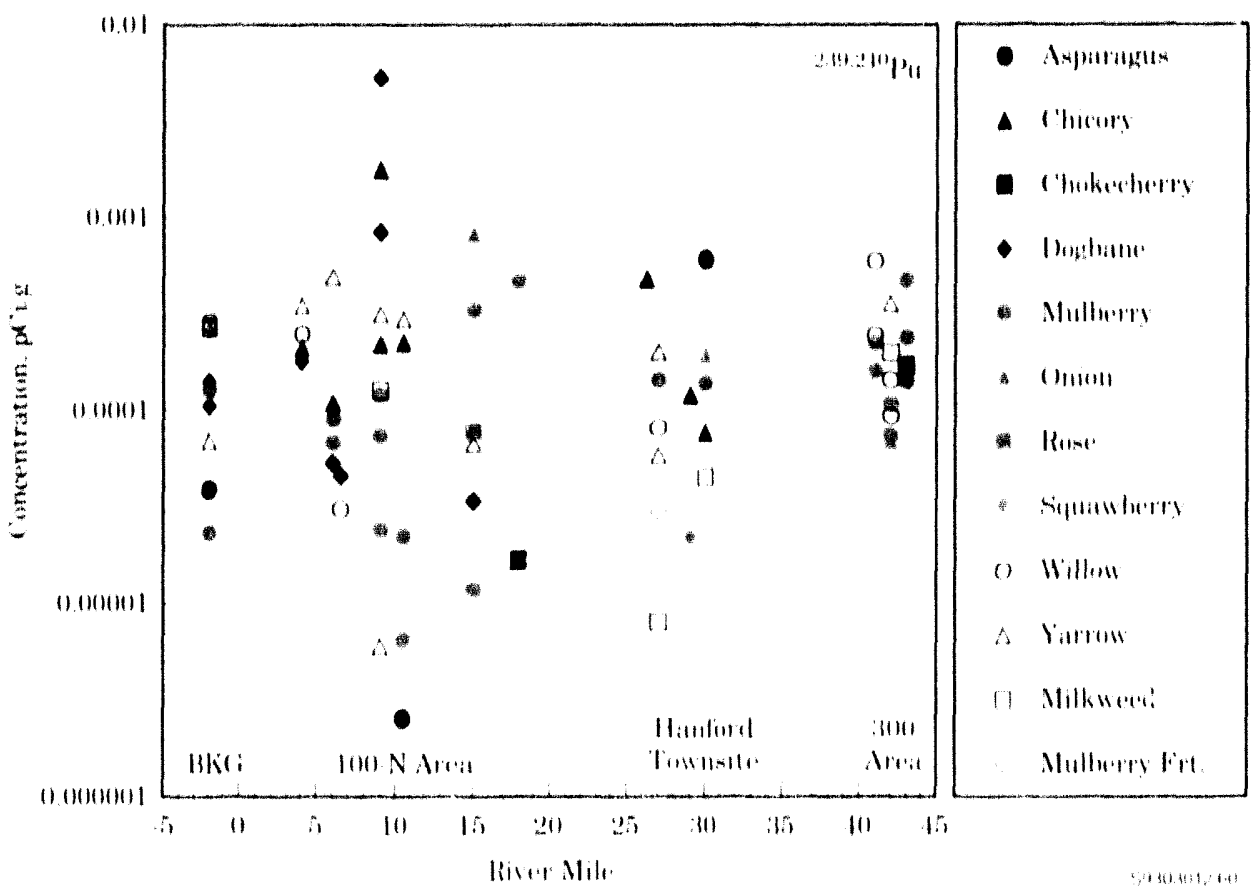

Figure 5.41. Plutonium ( Samples Collected from 1990 Through 1992 


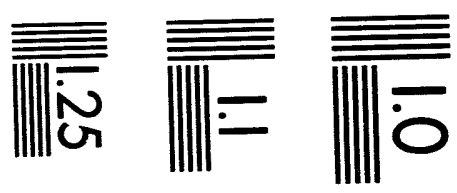

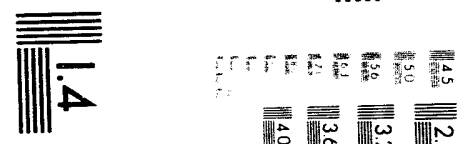

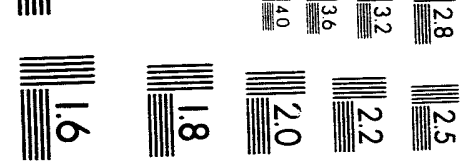



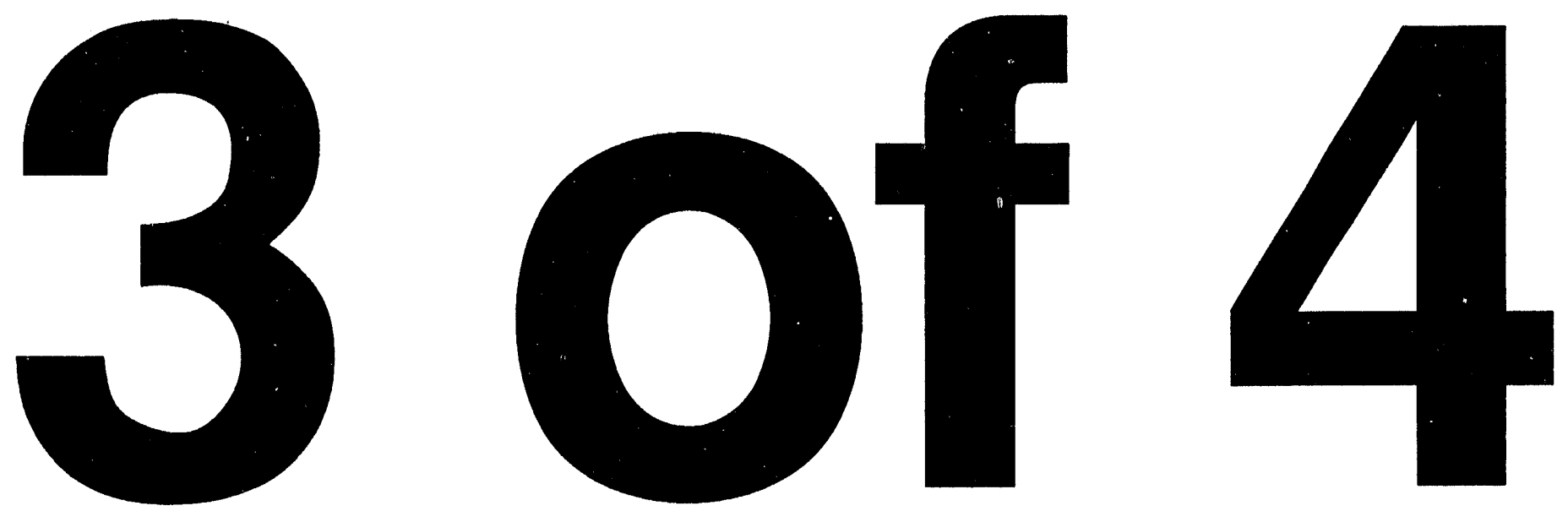


\subsection{External Radiation Surveillance}

External radiation is definedal as radiation originating from a sourec outside the hody (Appendix B). Lixtermal raddiation fields consist ol a natural componeme and an antilicial of human-made componem. The natural component can be divided into: 1) cosmic ladiantons. 2) primordial radionuclides in the carthe scrust primarily "K. "Th. and "(l), and 3) an airbornc component. primarily radon. The human-made component may be divided into medical $x$ rays. mocelear power. nucleatr rescarch, nuclear waste management, allol consumer products. Envirommental radiattion lickds may be influenced by the presence of artiticially produced

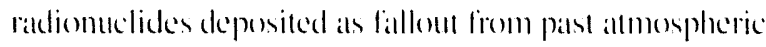
testing of nucleall weapons or hose produced and releatsed to the environment during the making or use of nuckear luel. The interation of radiattion with matter results in energy being deposiled in that material. The concept of energy deposited in a mass of material is called radiattion absorbed dose. A special unit called lle rad was introduced for this concept in the early 1950s. and more recently. an Internallionall System (SI) unit called the garay (ciy) has heen delined.

Extemal radiation exposure rates were measured all locations on and off the Hamlond Silce using Tl.Ds. lixtemal radiation and contamination surveys were also performed all locations on and aromel the Hamford Site. This section describes how external ladiallom was measured. how the surveys were performed. and ile results of these meatsmements.

\section{External Radiation Measurements}

Thermoluminescence. Th. or light output whihited hy

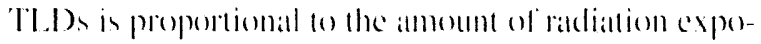
sure, X. which is measured in moits of reentgen (R). The

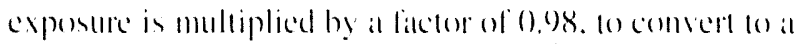

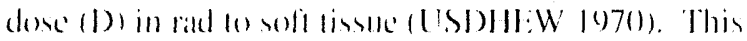
comversion facker relating $R$ lo rad is. homerer. alssumed fo be unity throughoul his report for consistency with past reports. This dose is lurther modified by a cuallits

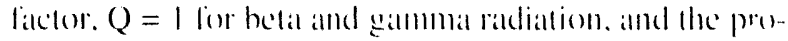
doct of all other modifying fictors (N). N is atssumed to be I lo obtain dose equivalence (H), mealsured in rem

$$
\begin{aligned}
& \text { 1) }(\text { rad })=X(R) * 1.0 \\
& H(\text { rem })=1) * N *()
\end{aligned}
$$

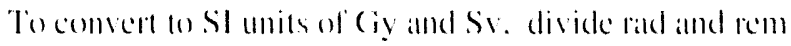
by 10() . respectively.

An envirommental Th. De (omprises thee plastic cards that

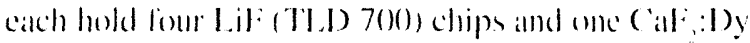
(TI.D) $2(1)$ ) chip. The Th.1) is positioned I $\mathrm{m}(3,3,3$ (t) ahove the ground all various focattions boll on and all the Hanlord Sile. The Tl.1) atre collected and read puarcerly. except lor llose at the lo()-N Areal shoreline, which are processed monthly. The 12 'T1.1) $7(0)$ chips al cach lecallion are used fo delermine the alserage envirommental done all that locillom and that puantity is divided by the length of time the Tl.1) wats in the tield to determine the

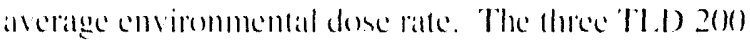
chips are included to permil dome determination in the evem of a radiological emergency.

The Th.1 ss are positioned all mumerous lecalloms onsite (figgure 5.+2), atround the Site perimeter. in neathy and

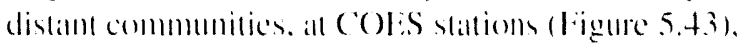
and alomg the Hanford Reach of he columbia River (lïgurc 5.4t). All community and most of the omsite alnd perimeter locations are collocited with air monitor-

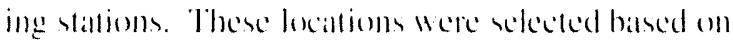
historical delemomatioms of the highes polemtials for

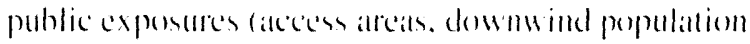
centers) from parst and current Hanfore' operations.

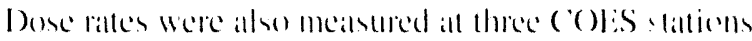
lecalled al lidwin Markham likencontary School. Basin

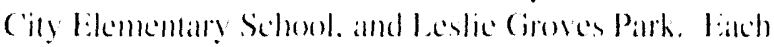

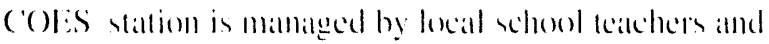

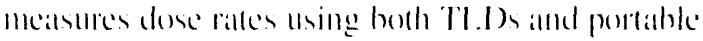
starey instruments.

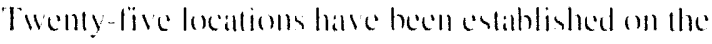
(olumbia Riser shorelime lom upstream fiom the 10()-13 


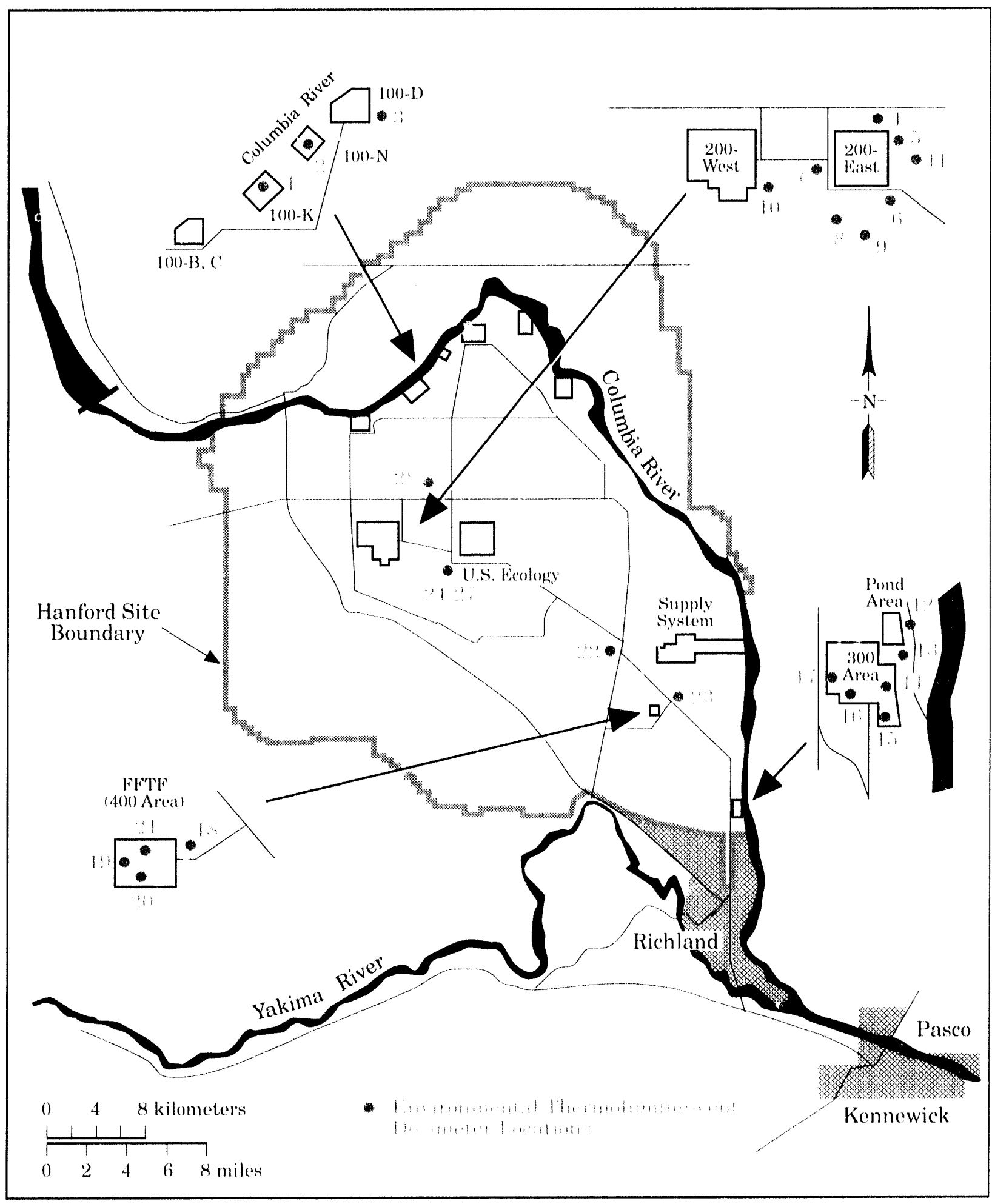

S9203058.7

Figure 5.42. Thermoluminescent Dosimeter (TLD) Locations and Station Numbers on the Hanford Site, 1992 


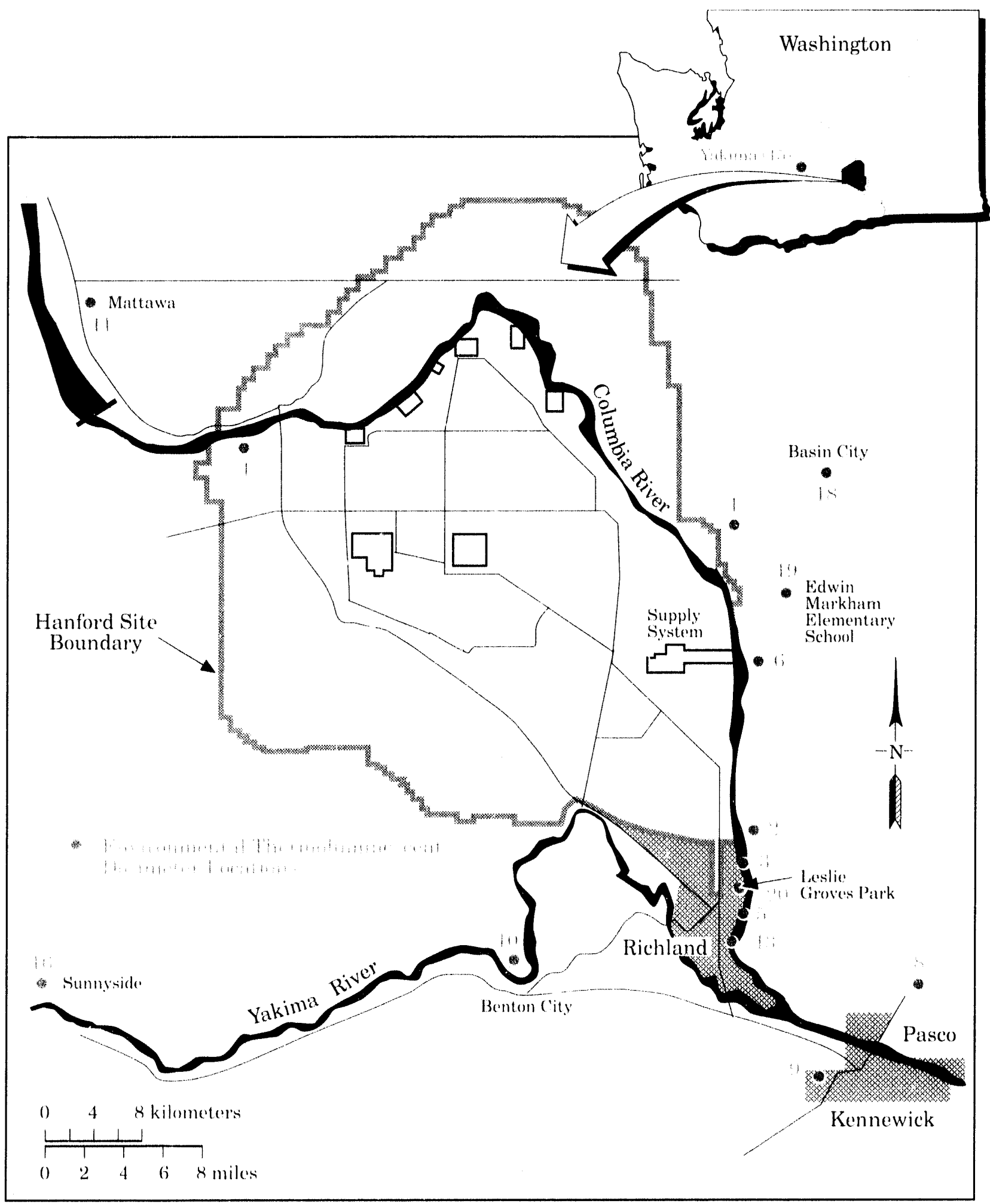

$\$ 9203058.5$

Figure 5.43. Thermoluminescent Dosimeter (TLD) Measurement Locations and Station Numbers for Perimeter and Community Sites, 1992 


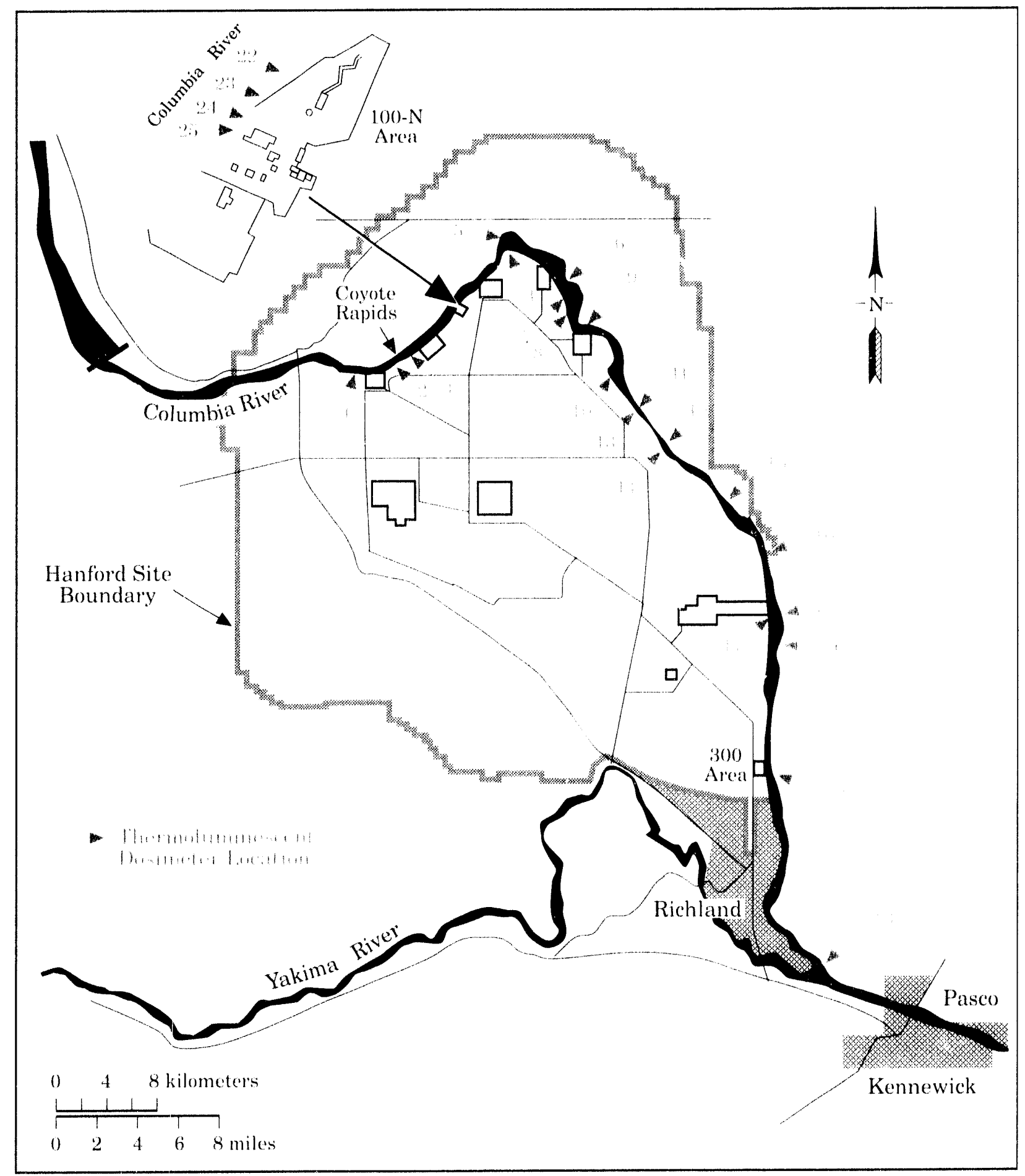

$\$ 9203058.6$

Figure 5.44. Thermoluminescent Dosimeter (TLD) Locations and Station Numbers on the Hanford Reach of the Columbia River, 1992 
Area to just upstratm from the 30) Areat. The general public has aceess 10 some of these locations. Hisfortcally, dose ralles measured along the shoreline have been higher than typical background rates. Sula (1980)

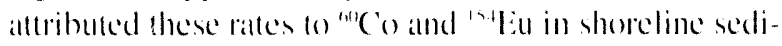
ments ats a result of liquid releases during past reacent operations in the 100) Areas. Two locations. Coyote Rapids and Richland Punphousc, had 'Th I)s submerged in the Columbia River. These awo locations were discon. linued aller the second guarter of le92, hecatuse suhmerged TLD realdings were less than lerrestrial background readings and radiontelick concentrations in the Columbia River have decreased lo levels such that no difference wats seen herween the upstreatm (Coyote Rapids) and downstream (Richland Pumphouse) Tl.D) raidings.

\section{External Radiation Results}

Perimeter and offsite localtoms, primatrily downwind of lhe Site and neall population centers. Here monitored with Tl.1)s. Th.1) expostmes hatre hee'll comberted lo dose cyuisalent rates by the process described above. Table 5.22 shows arerage dose ralles for perimeder and ofleste lexafions. Quarterly exposure ralles were alseraged by malp focation. ranked within the location classificallom. and then converted to dose equivalents per years.

Perimeter dose rates for 1002 nere similat to these observed in 109), but on alreratge all ofliste dose rattes increased slighlly. Vartalloms in nallural hackground radiation caln occur ais a result of changes in annual cosmic radiation (up) fo $10 \%$ ) and terrestrial radiation (1.510 25\%, NC RP 1087). () Her factors possibly atfecting annual dose lates reponted here may include variations in the sensitivity of individual Th. Ds aero dose readings, fadling. random errors in the readout expupment or procedures (Rathbun 1989), and changes in Tl.1) station locillions.

The hakegeround exemal radiation dese rate was 9.3 morem/ yr $\pm 6 \%$ ats compared to the perimeter alserage of $102 \mathrm{mrem} / \mathrm{yr}+6 \%$. This slight difference in alverage dose rates maly be dece to natural ge(ographlice vatriations in terrestrial radiatlion / many of the perimeder location: ale rich in "K and horium isolopes (Rathbun l989) and variations resulting from human alelivily. The 1092 ancrage "'K concentration in soil all perimeler localloms (19) pe $\mathrm{i} / \mathrm{g}$ ) is higher than the anerage " $K$ enencentrattion in soil all distant locattions (1.3pe (i/g). Allhough this diflerence in "kencentrattions does not accouml lor a

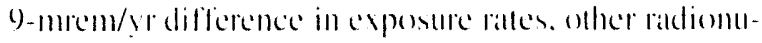
clides were found in higher soil concentrattioms all the Site perimeder than all distant commonity focalloms lsee Section 5.6). Humaln akdivitices alfecting the anerage dose later maly include fandscalpe changes sted as huildings and other construction. Which maty sheld a portion of the lerrestrial component. Figure 5.45 graphically displaty al comparison between. and trends of perimeter and distant TI.J) locatloms during 1987 through 1092. Year-lo-ycar

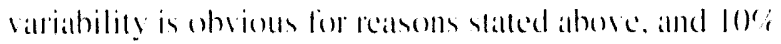
variability is mon unlikely (NCRP 1987).

Table 5.22. Dose Rates Measured by Thermoluminescent Dosimeters (TLDs) at Perimeter and Community Locations, 1992 Compared to Values from the Previous 5 Years

\begin{tabular}{|c|c|c|c|c|c|c|c|}
\hline & & & & Jose Ralle. & mrem/yr & & \\
\hline & & & 1002 & & & $1987-1901$ & \\
\hline I.xaltion & $\begin{array}{c}\text { Mily } \\
\text { Locilliont }\end{array}$ & $\begin{array}{l}\text { No. of } \\
\text { Silmples }\end{array}$ & Maximum & Mčill & $\begin{array}{l}\text { No.of } \\
\text { Simmples }\end{array}$ & Maximum & Mc:ill \\
\hline Perimeler & $1-6$ & 6 & $111 \pm 5 \%$ & $102+6 \%$ & 1.53 & $168+18 \%$ & $89 \pm 3 \%$ \\
\hline Neatby commmunity & 7.10 & 4 & $90+101^{6}$ & $9 \pm 4 \%$ & 170 & $112+15 \%$ & $79 \pm 3 \%$ \\
\hline Distamll commmunity & $11 \cdot 12$ & 2 & $96+74$ & $93 \pm 0 \%$ & 13 & $107+20 \%$ & $77 \pm t^{\prime \prime}$ \\
\hline (C)ISS stattions & 13.15 & 3 & $10.3 \pm 0.01 / 6$ & $93+12 \%$ & 10 & $80 \pm 0 \%$ & $70 \pm 7 \%$ \\
\hline
\end{tabular}

(a) So rigure 5.43. 


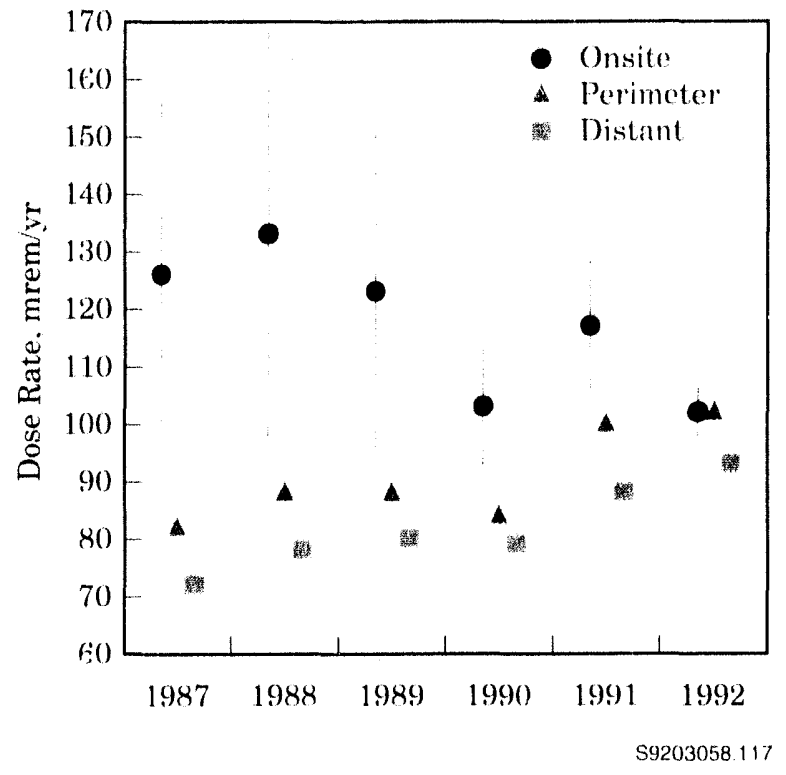

Figure 5.45. Annual Average Dose Rates, 1987 Through 1992

Figure 5.44 shows lociations of TLDs positioned along the Columbia River shoreline, and Table 5.23 shows the arerage measured dose ralles for shoreline locations. Dose rates were highest near the 1(0)-N Area shoreline. Iwo to three times higher than typical shoreline dose rates. The higher rates measured in the 10()-N Area are attributed to past waste management practices in thall areat. The public does not have open alecess to the 10()$-\mathrm{N}$ Area shoreline, but dee"; have access to the addiacent water body. The dose implications at this locittion are discussed in Section 6.0.

Figure 5.42 displays the 28 onsite locitions where TL.Ds were placed in 1992. Tahle 5.24 sumbmatrizes the results of 1942 measurements, which are divided by operational areal. All areas had higher average desce rates thatn backgreund, The highest alverage done rate was seen in the 60) Area and is due to the waste-handling aldivities at U.S. Ecology. a mon-D()E licility.

\section{Radiation Survey Results}

Onsite roads and railways, the Columbia River shoreline, and perimeter locations were routinely surveyed using portable radiation detectors in 1992. The fiecuency of surveys on specific roads and railroids wass determined by their use and the potential for contamination. These routes and frecuencies were reported by Bisping (1992).

Railroads and roads were surveyed using mobile scintillatton detectors. The detector consisted of leur sodium iodide detectors mounted on the rear humper of a fourwhec-drive truck. The detectors were mounted $0.46 \mathrm{~m}$ (1.5 fi) ahove the ground. Output from the defectors and the associalled electronics wats recorded on strip charts for review and documentation after surveys were complete. In 1992. matd and railroild surveys revealed no areas of defectable contamination. Routers atre slown in Figure 5.46.

Hand-held survey instruments were used to perform radiation surveys at many of the Columbia River shoreline Tl.D lecations. These surveys provide a coarse screening for elevaled raddiation fiedds. The shoreline surveys showed that radiation levels all some locattions were comparatale fo levels measured at those locattions in previous years. The highes levels were recorded on the $10(0-\mathrm{N}$ Areal shoreline and ranged from I0) 10100$) \mathrm{mrem} /$ hr using a Bicron Micro Rem meter. Results for ratdiation surveys alle nol tithulated by Bisping and Woodrull (1993), but are in the Surface Envirommental Surveillance Project files all PNI. 
Table 5.23. Dose Rates Measured Along the Hanford Reach of the Columbia River, 1992 Compared to Values from the Previous 5 Years

$19(92$

Milp

Locattonl't.

Typical shoreline arcal

1()()-N shoreline"

All shoreline

Immorsed in Columbial River"ts

lomallion"

Maximum

$$
\mid-21
$$

$1+1 \pm 8 \%$

$22-25$

$32+ \pm 8 \%$

$32+ \pm 8 \%$

(6) $\pm 2 \%$
Dose Ralle," mrem/yr

$|987-109|$

No, of

Meian't)

Simples

Maximum Mcan

$108 \pm 5 \%$

2()

$1.32 \pm .59 \% \quad 100 \pm 7 \%$

$239 \pm 20 \%$

4

$3.33 \pm 8 \%$

$250 \pm 3() \%$

$130+16 \%$

24

$33.3 \pm 8 \%$

$16.5 \pm 9 \%$

(6) $\pm 29 \%$

2

$5.3 \pm 00 \%$

$51 \pm 16 \%$

(a) Quarterly integraled readings in $\mathrm{m} R / \mathrm{d}$ were comserted to annual dose equivalent ralles (mmem/yr).

(h) All locallions alle shown in Figure 5.44.

(c) Locations are identilied in Figure 5.44.

(d) Means \pm 2 SL:M computed using station alverages.

(c) Monthly integrated exposume readings in $m \mathrm{R} / \mathrm{d}$ converted to anmual dose cequivalent rates in mrem/yr.

(i) Immersion points all Richland Pumphouse and ('oyole Rapids. Measurement discontinued after second puanter 1992.

Table 5.24. Dose Rates for Thermoluminescent Dosimeter (TLDs) Locations on the Hanford Site, 1992 Compared to Values from the Previous 5 Years

1092

Dowe Rate."1' mrem/yr

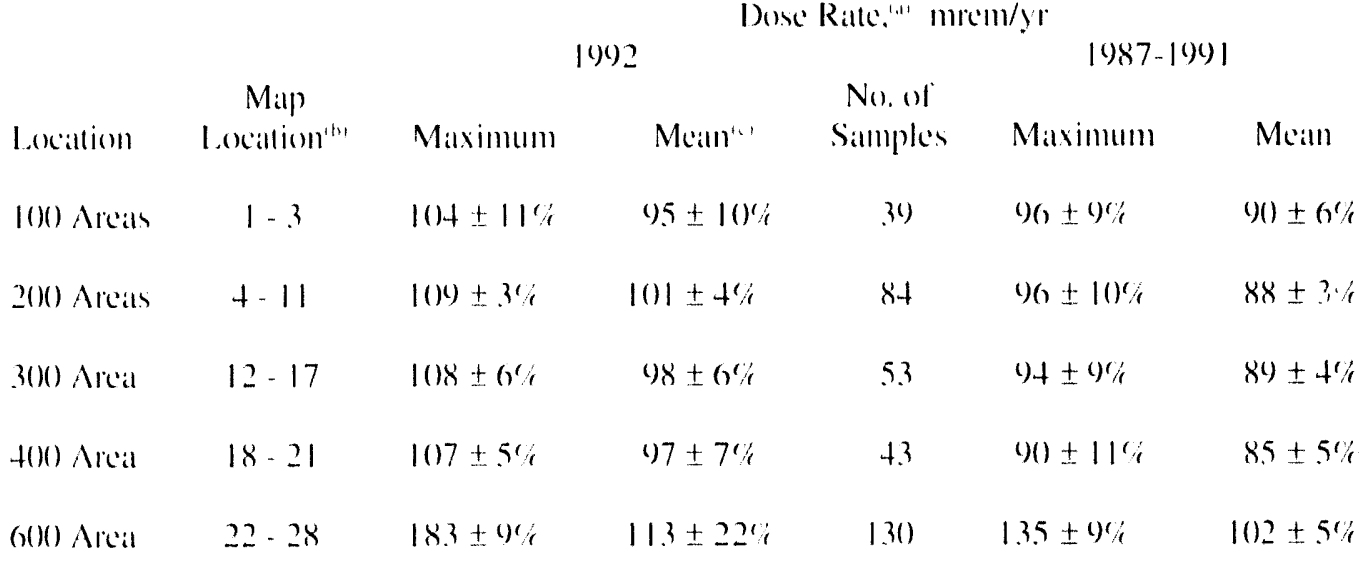

(a) Quarterly integrated readings in mem were comverted fo anmual dose equivalent ralles.

(b) Lexaltions are idemlilied in ligure 5.42.

(c) Means \pm 2 SEM computed usimg stallon arragess. 


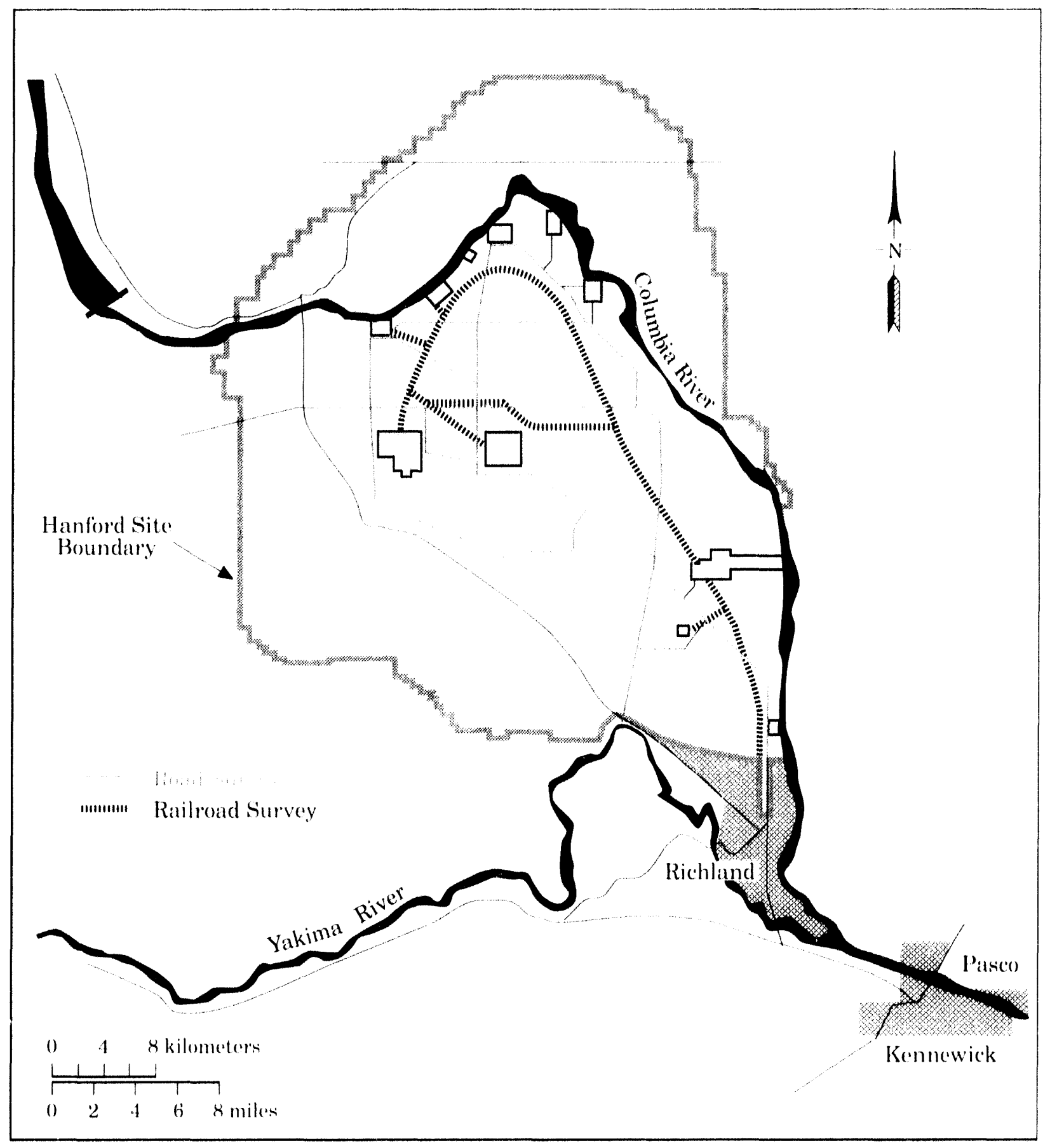

$\$ 92030584$

Figure 5.46. Road and Railroad Survey Routes, 1992 


\subsection{Ground-Water Protection and Monitoring Program}

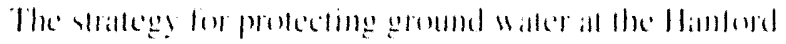

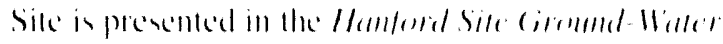

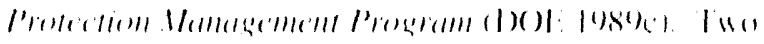

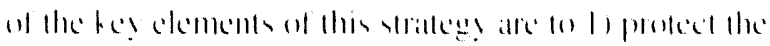

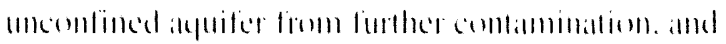

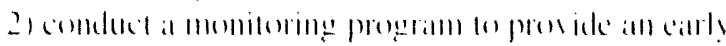

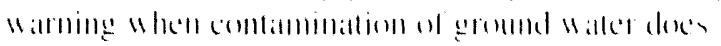

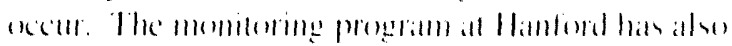

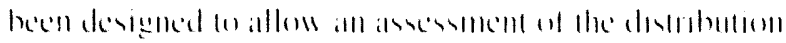

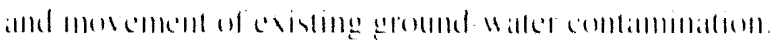

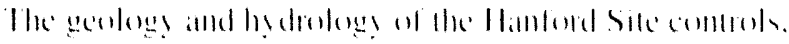

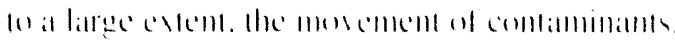

\section{Geology}

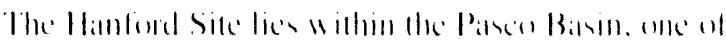

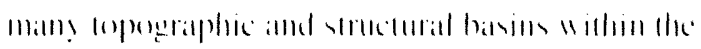

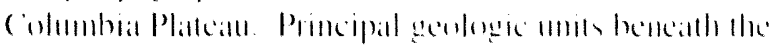

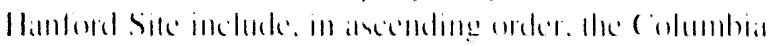

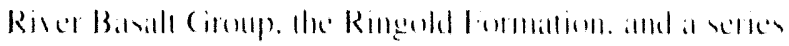

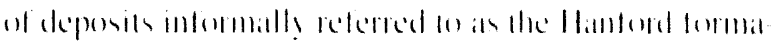

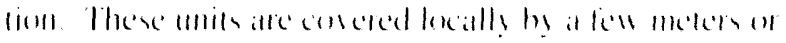

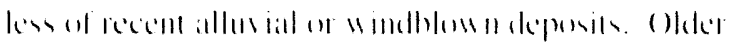

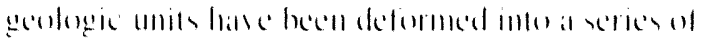

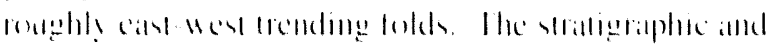

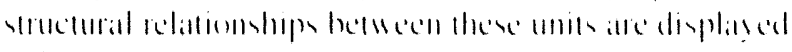
ii) 1.igure 5.47

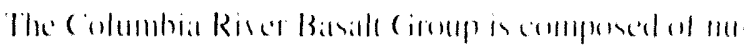

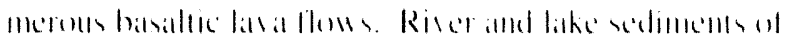

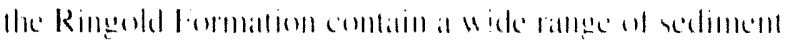

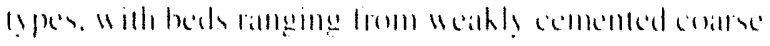

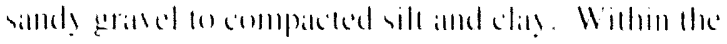

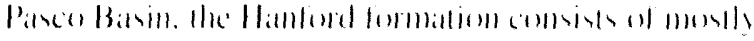

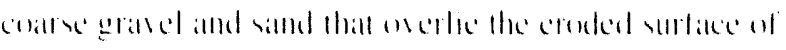

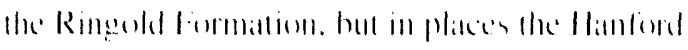

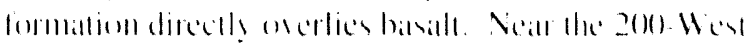

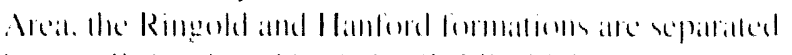

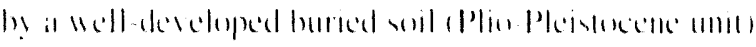

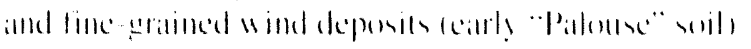

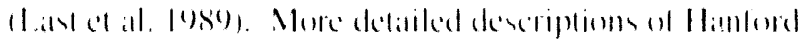

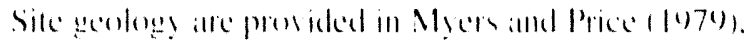
1)(1) (l)

\section{Ground-Water Hydrology}

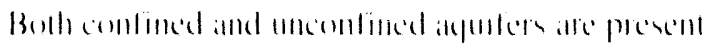

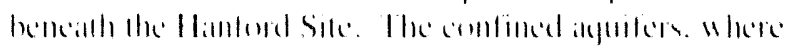

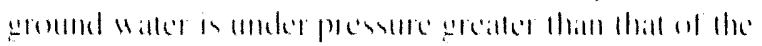

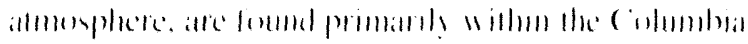

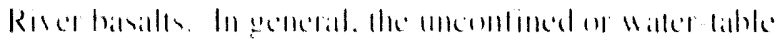

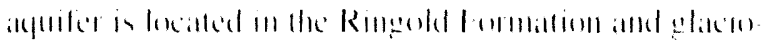

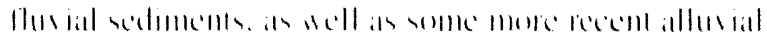

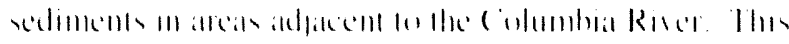

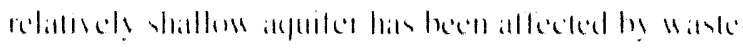

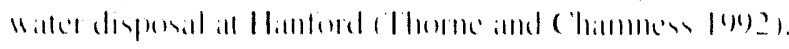

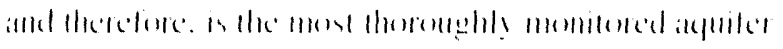
hencillh ile Sile:

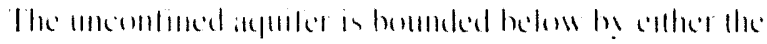

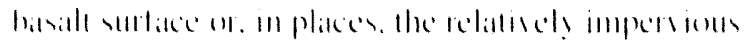

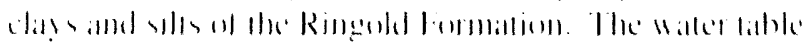

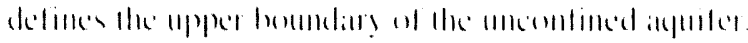

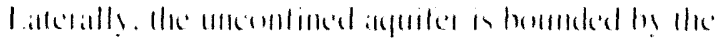

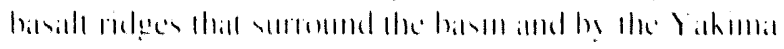

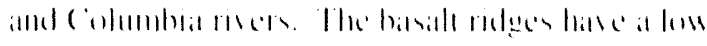

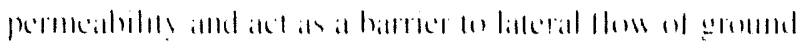

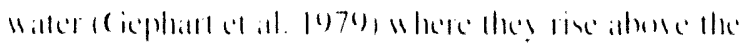

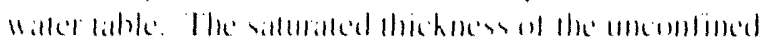

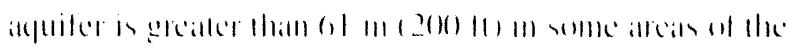

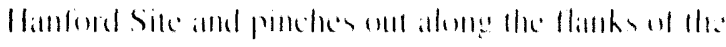

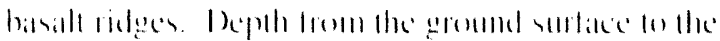

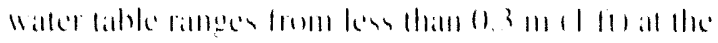

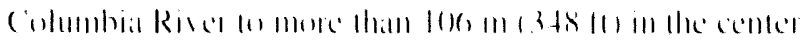

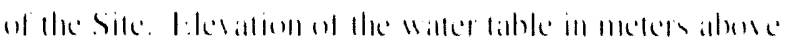

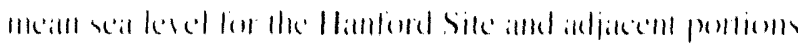

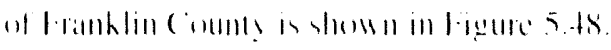

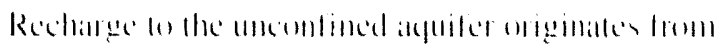

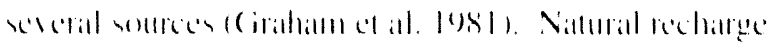




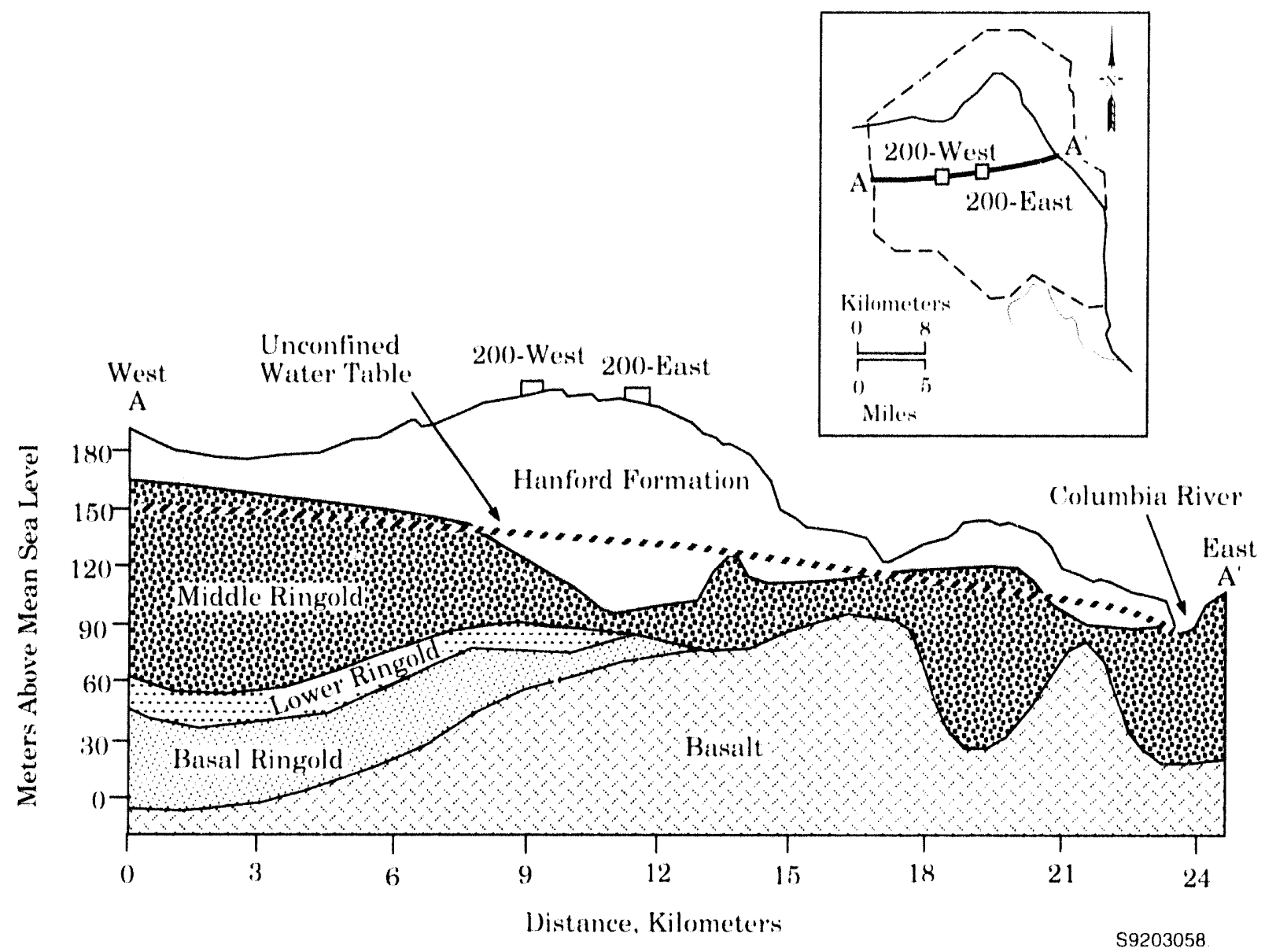

Figure 5.47. Geologic Cross Section of the Hanford Site (modified from Tallman et al. 1979)

oceurs from precipitation at higher elevaltoms and rumoli from intermitent streams, steh as Cold Creck and Dry Creck on the western mangin of the Site. The uncontined aquiter is recharged by the Yakima River as it llows along the somblowest boundary of the Hanford Site. The Columbia River rechatres the unconfined ayuiter during high stages when river waler is transterred to the alyuiler along the river bank. The uncomfined ayuiler receises litte, if any, recharge from precipitation directly on regelated atreas of the Hantord Site becilluse of a high rate of evapontramspiration from native soll and regetalton.

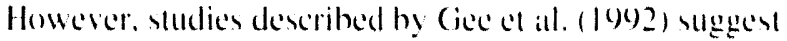
that precipitatton maly contribule rechatrge to the ground water in alreas where solk are course textured and hare of regetaltion.

Large-scale artificial recharge oceur liom oflisite agricultural irrigation and liguid-Waste disposal in the opelating areas. Recharge from imigation in the Cold Creek Valles enters the Hannford Site at gromond-water flow across the western boundary. Recharge lo groumd Walter aleross lle (olumbia Rive from the Hanterd Sile is primarily from irrigatlon and imrigation canall leakage.

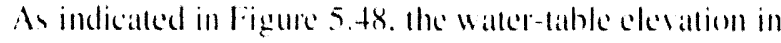

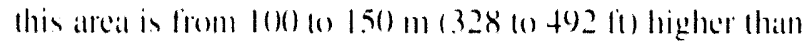
the water-table clevaltion on the Hantord Site.

The operational dischatge of water has crealled gromendwaller mounds near seiteh of the major wastewalled disposal facilitien in the 200 Areas. The se momols hatse

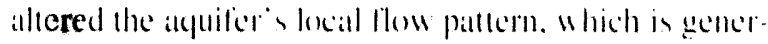
ally from the recharge areas in the west fo the discharge alleas (primatrily the columbia River) in the cast. Walter levels in the unconfincel alyuifer hate changed combinually during Sile operallioms becillese of varialtoms in the volume of waste water dischatrged. Comseguently, the 


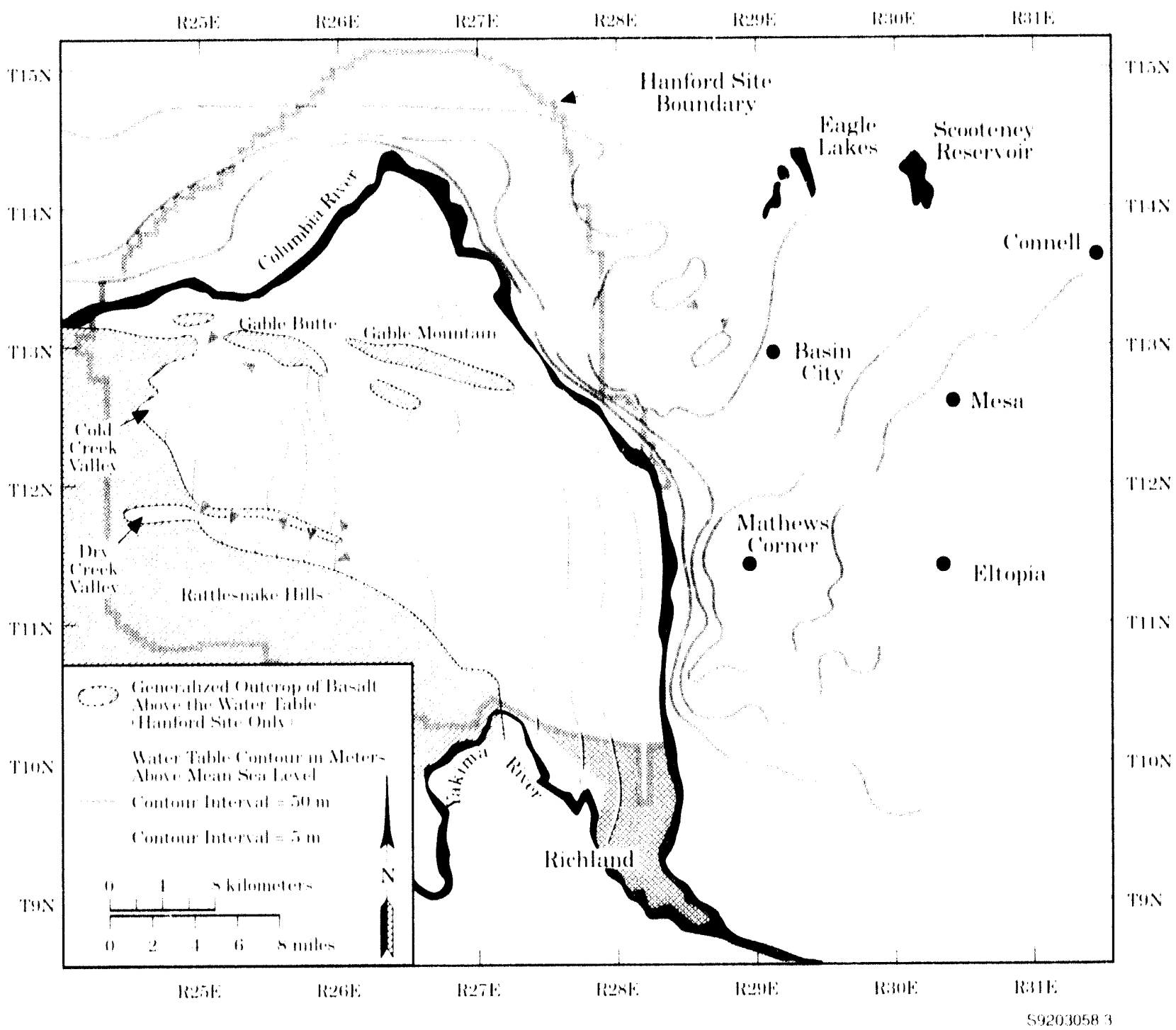

Figure 5.48. Water-Table Elevations for the Unconfined Aquifer at Hanford, June 1992

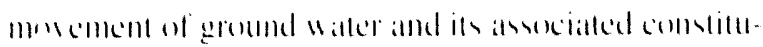
colls has also changed with timle.

(iround- Haller monnding also (x)

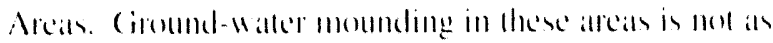

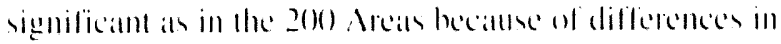

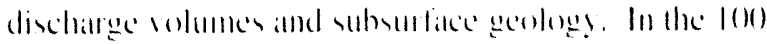

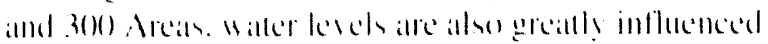
b) riscr stiges.

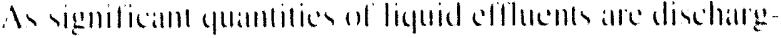

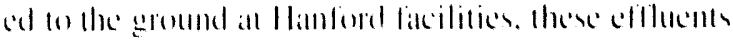

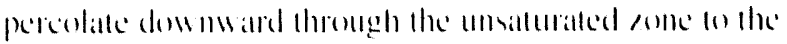

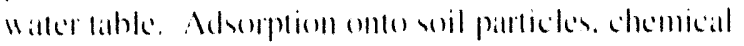

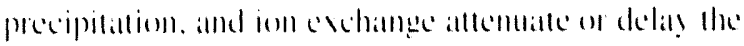

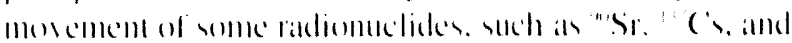

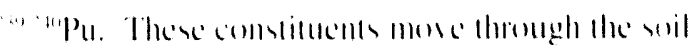

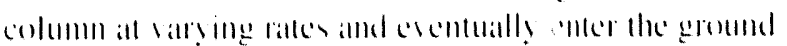
waller. ()!her ioms. stch as mitrate. and radiontedides. 


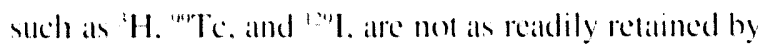
the soil and move downgradient in the same direction ats. ant all at rate nearly equal to. the llow of ground water. When the leyuid eflluents reach the ground water. their concentrations ane reduced by dilution. As these constituents mone with the ground water. radiontelide and chemical concentrations are further reduced by spreading (dispereion). and radionuclide concentrations are redaced by radionative decily.

\section{Ground-Water Protection}

The ceflort to protect ground-water quality is heing implemented through programs 10 minimise wastes being discharged to the stil column and through sike remediation activitios heing carried out in accordance with an agreement hetween the Washington Slate Deparmem of licology (Foology). D)(o). and EPA. This agreement. called the Tri-Party Agreement. provides a framewort for remediation of the Hanford Site oner a 30-ycall period. A summaty of accomplishments in Mask minimizallon and site remediation is presented in

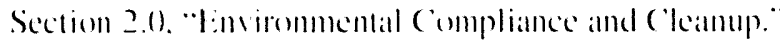

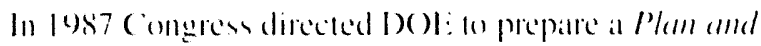

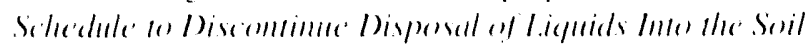

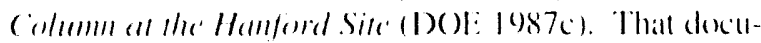
ment presents an implementation plan for providing allernative treatment and disposill of contaminalled ellient discharged to the soil on the Hanford Site. The 33 major wask streams that have been identilied will be addressed in lwo phases. Phase / projects are comsidered

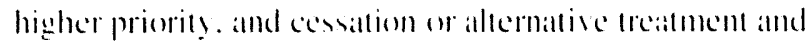
disposal ysems will he implemented by 1945 for these streams. Phase II weams will be dealt with alfer commpletion of Phatse I projects.

\section{Ground-Water Monitoring}

Giround-waller mombloring al the Handerd Site is ant

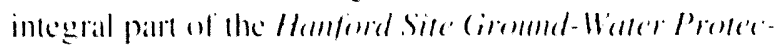

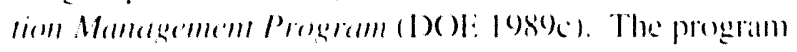

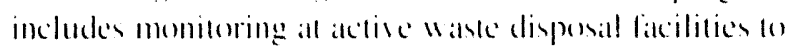

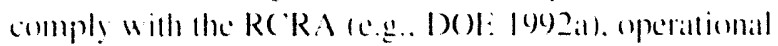
momiloring in and aldjacent for reatere and chemical precesing facilitices. and em imemmental surecillance.

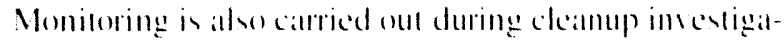

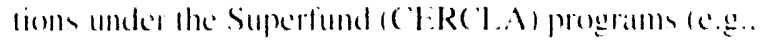

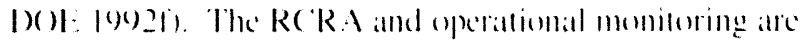

managed by the Site operating contractor. CLRC'LA chatacterizations are mallaged by the operating contracfor and the U.S. Army Corps of Engineers. Additional details on RCRA compliant monitoring are presented in Section 2.0. "Envirommental Compliance and Cleanup." The Envirommental Surveillance Program ansesses the impact of Hanford operations on ground water. hoth onsite and offisite. indepeindently of the operating contraciors programs.

\section{Sample Collection and Analysis}

Ciround-water samples were collected ats part of the Hanford Ground-Water Envirommental Surveillance Program and other monitoring programs. The GroundWatce Emvirommental Surveillance Program utilizes the dalla from onher programs to provide als complete an inlepretation ats possible. Wells monitored by the various programs in 10)2 alre shown in figures . 5. 40 through 5.52. (iround-waller monitoring was conducted all the lacilities shown in figure 5.53 (1) comply with RC'RA.

(iround-water simples were collected firmalppoximaltely 720 wells for the monitoring programs during 1992. The monitoring fiegueney for the wells was selected hased on regulatory reyuirements. proximity to waske sources. and the chataleteristics of the groundwater low sysem at the simpled location. (One hunded and rincty-ninc of the wells were simpled ance. 16.3 were simpled wice. 20 2 were sampled approximallely yuarterly. and 156 wells were simpled more licyucn!ly during lhe ycar.

Allhough the programs are mallaged by dillerent aganifalloms. a commonom dallabase is ased to stome geroundWaller dallat so that ciach monitoring program has alceses to dalla collected by oflee programs. Samples for the emitummembal smecillance. RC RA. and operational programs alle collected hy simpling teams fo!lowing a single sed of procedures. Giround-llatler simples were analyed by a combination of subcomtateter and in-house lahoratorices

Mont ground-Matter momituring wells on the Site ance 10 (1) 20) com in diameler and are comstructed af steel or stainless-slect cissing. Moniloring wells for the unconlined alepuiter are comblrucked will well sereens or pertoralled

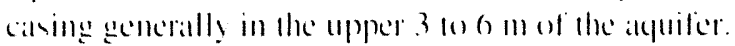
This comstrection allows selmple collection neatr the top of the alyuiter. Where mas immon comcentratsoms for

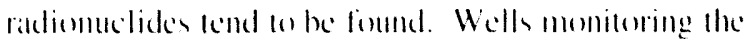




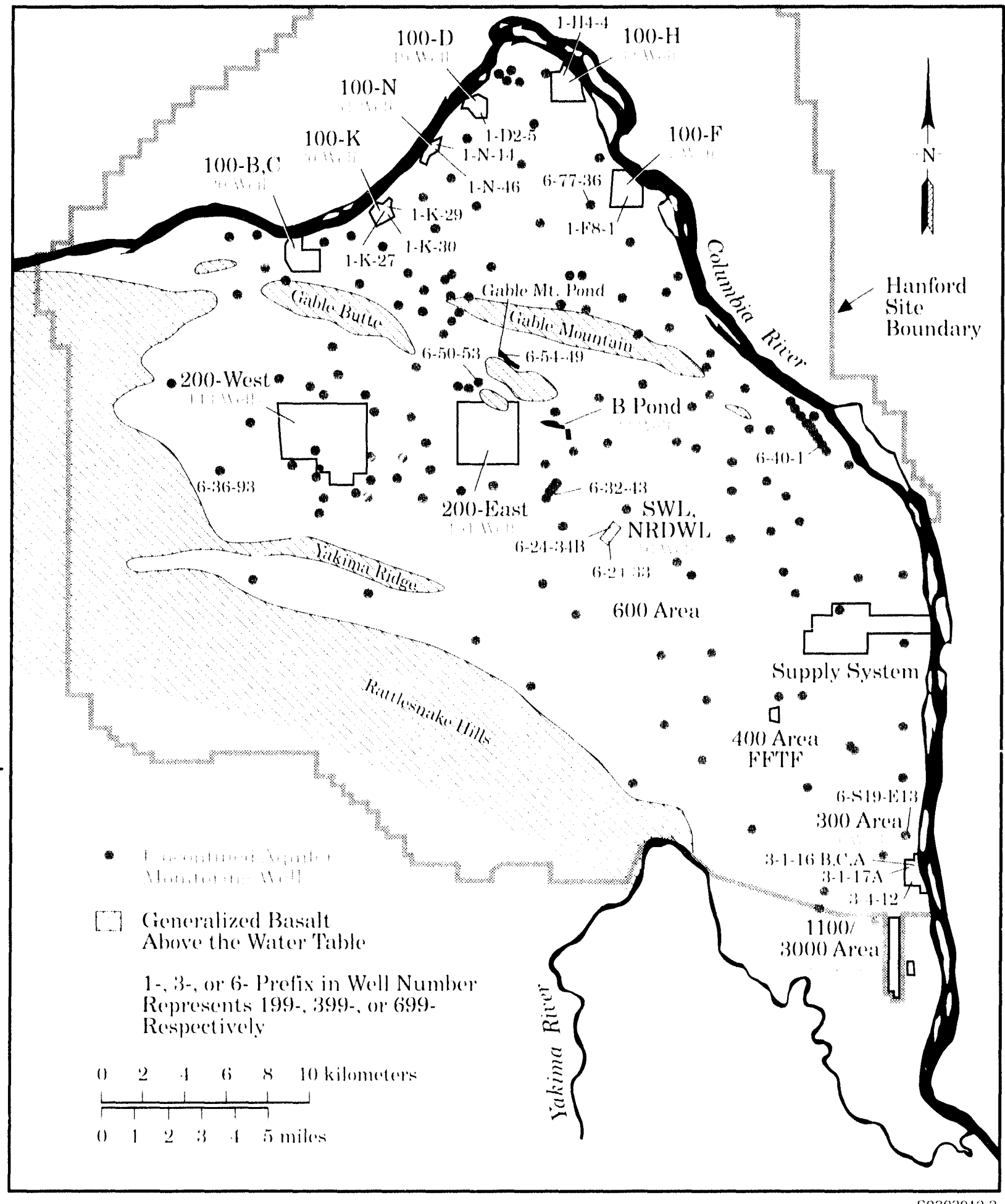

$\$ 93030123$

Figure 5.49. Hanford Site Unconfined Aquifer Monitoring Well Locations, 1992. Numbered well locations are discussed in the text. 


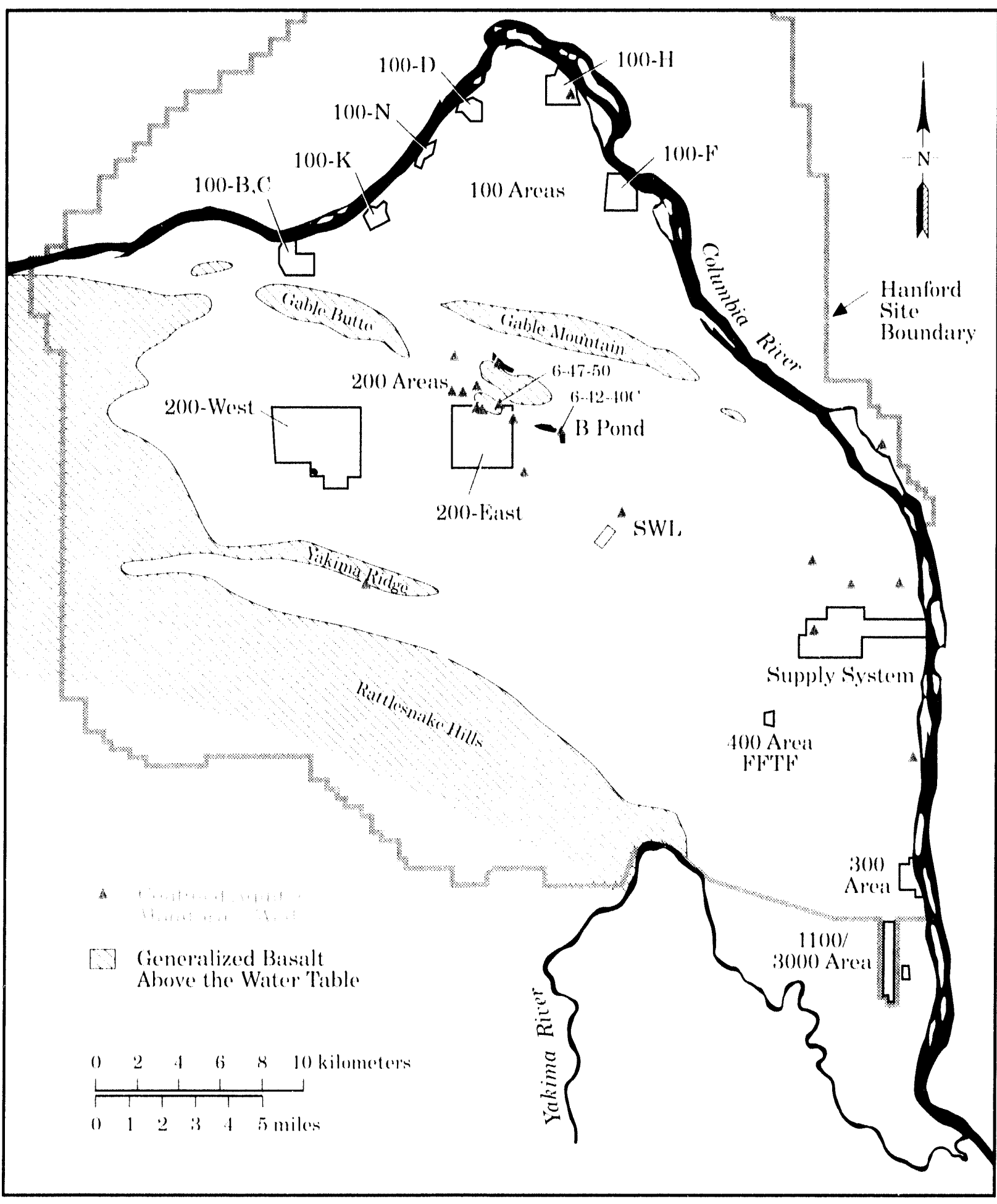

5930301215

Figure 5.50. Hanford Site Confined Aquifer Monitoring Well Locations, 1992. Numbered well locations are discussed in the text. 


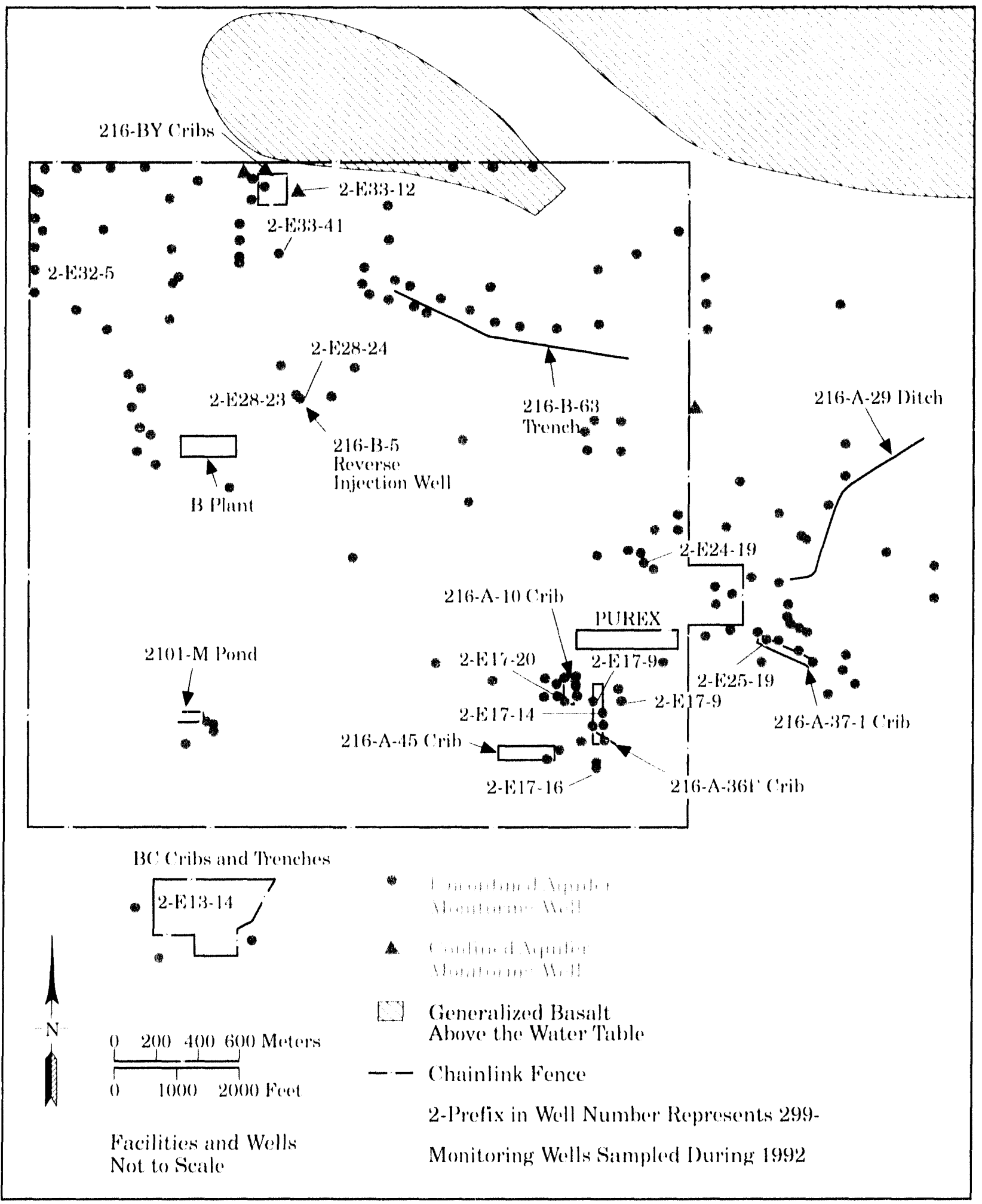

59303012.18

Figure 5.51. Monitoring Well Locations in the 200 East Area, 1992. Numbered well locations are discussed in the text. 


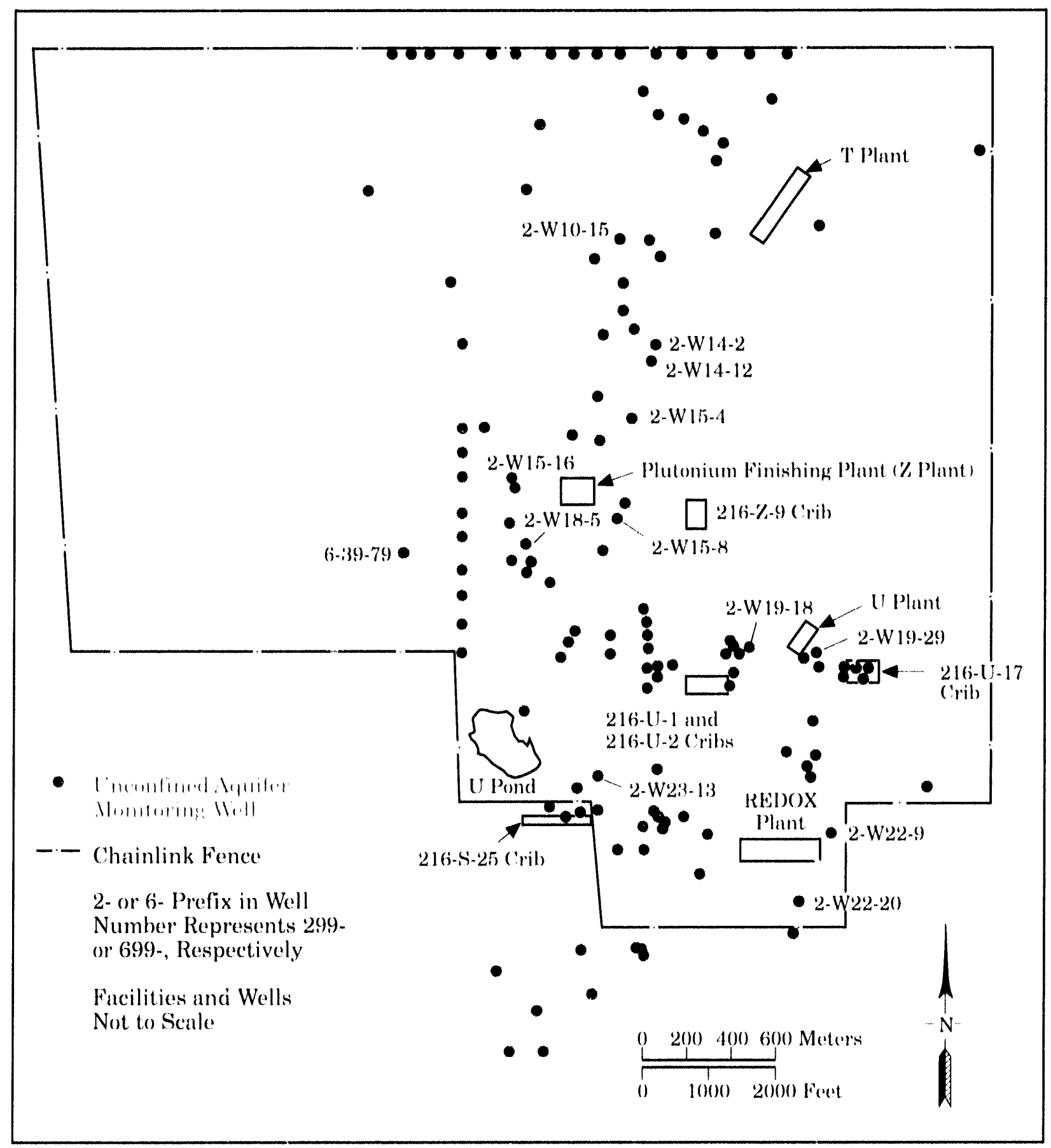

$\$ 9303012.17$

Figure 5.52. Monitoring Well Locations in the 200-West Area, 1992. Numbered well locations are discussed in the text. 


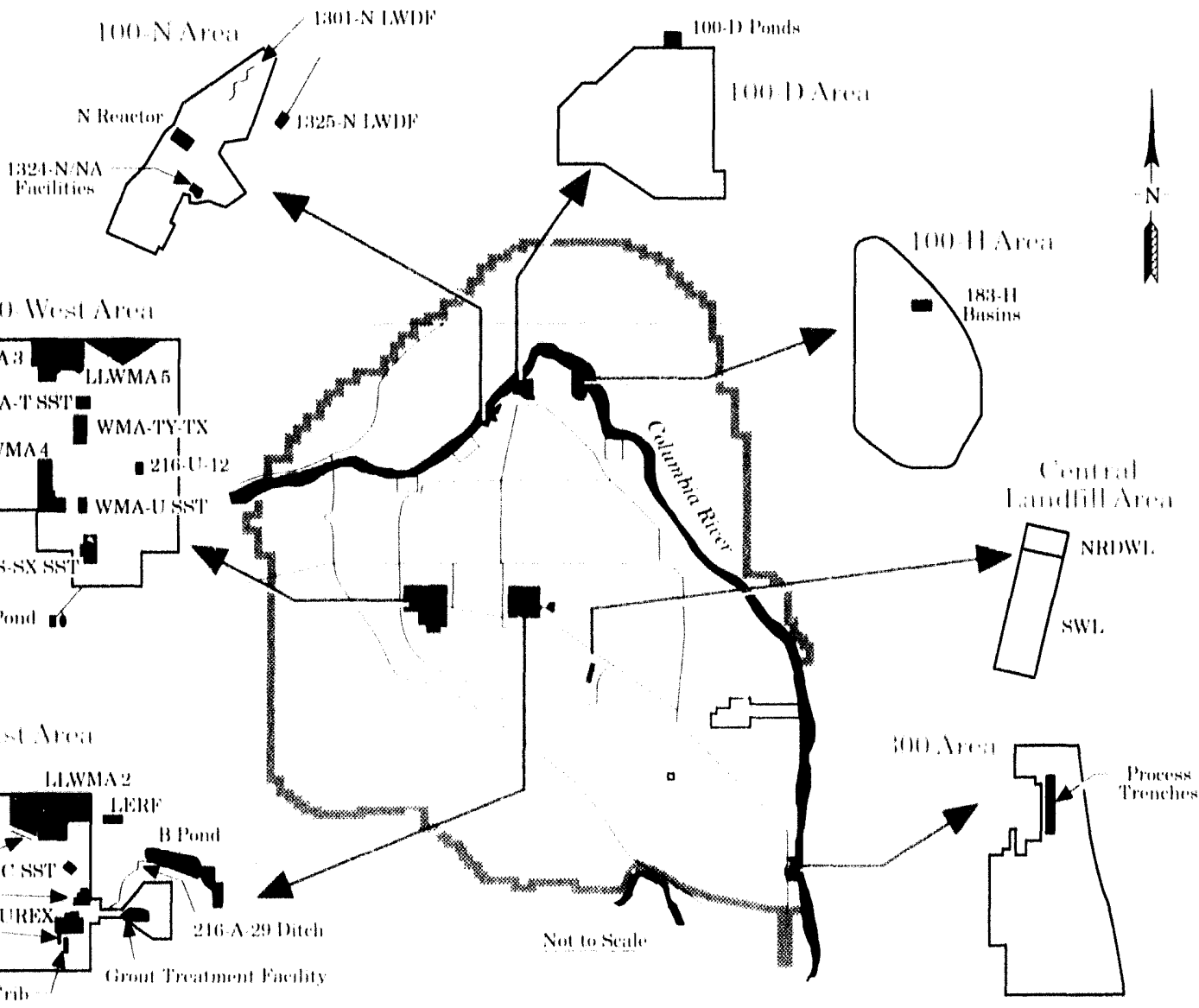

LERF Liguid Ellluent Retention Fateility l.LWMA Low-lavel Waste Management Area LWIOF ligund Wasto Dispowal Facility

NkI)WL, Nomearlionative l)angerems Winste landlill
PlREx Plutonium-Lmanim Extration (Plant)

Sist Singlo.sholl Tank

SWl. Sulid Wasto landlill

WMA Waste Management Arrat

s9o030rid 16

Figure 5.53. Locations of RCRA Ground-Water Monitoring Projects and Landmarks on the Hanford Site

confined aypifer have screens, pertorated casing or an open hole within the monitored ilyuiler. Wells drilled before 198.5 were generally comstructed with carthon steel calsing. Wells recently comstructed for RC RA monitoring projects and CIERCI.A chatracterizattions have hee'l constructed with stitinless-sted culsing.

Samples were collected for all programs following documented sampling procedures (PNI. 1993: WHC

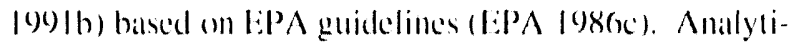
call lechnicues used atre described in the Hemfored Site

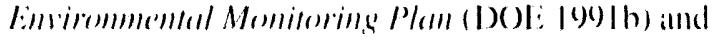
CIERCI.A work plans. The species antalyad for are listed in Tithle 5.25.

Latch antilysis of a ground-waller sample provides informattion on the composition of ground witter all one time all one locition in the atpuifer. Hencertainty in the antalysis results from a number of sources. Some of the sources of uncertainty are discussed below. Several tedmiches are used in this discussion to interpres the salmple results given these uncertatinties and are also discolssed. 
Table 5.25. Radionuclides and Chemicals Analyzed for in Ground Water

Radiological
Parramelers

'H

(") $\mathrm{C}(1)$

"'Sr

"w'Tc

III'Ru

"m!'Ru

$1: \mathrm{Sh}$

(.)I

"1"1

"Cs

"HAm

Total Alphai

Total Beta

Plutonium Isolopes

Uranium lsotopes

Urimium (total)
Chemical Patrameters

$\mathrm{pH}$ (ficld and laboratory)

(onductance (field)

Alkalinity

Total carthon

Total organicciaton

Total organic halogens

B. Be. Nil. Mg. AI. K. Co. Si

Ca. V. (r. Mn. lic. Ni

(u. Zn. Sr, Ag. Cil. Sh. Ba

$\mathrm{F}, \mathrm{Cl}, \mathrm{NO}, \mathrm{PO}, \mathrm{SO}, \mathrm{NO}, \mathrm{Br}$

$\mathrm{CN}$

$\mathrm{NH}$

Volatile organic :onstituents

Semivolatile organic comstituents

PC'Bs

Pesticides/herhicides

Biological oxygen demand/chemical oxygen demand

Dissulved oxygen
Simpling lechnicfues are designed to provide a sample that is reatsonably representative of the atuifer concentrattion when the silmple is taken. However, there atre limitattons in our ability to collect representattive sillmples or even to deline precisely what volume of aquifer is represented in the sample. Proper well construction. well purging, sample preservation, and, in some instances. liltering are used to help ensure consistent and represenlative samples. Carreful sample labeling protecols, chainof-custody documentation, and bottle preparation anoid many gross errors in silmple results. Duplicalle simples and field blanks help alssess the simnpling proceduie.

Uncertainties are inherent in laboratory antalysis of sallnples. Ciross errors can be introduced in the laboratory or at other stages in the simpling. Ciross errors include transcription errors. calculattion errors, mislabeling results, or other errors that result from not lollowing established procedures. Often. llese gross crrors call be recognized hecilluse unreatsomably high or unreatsonathly low vialues result. Ditta review procedures are used (1) invertigate and correct geross errors. Leven if the source of a possible gross error cannol be identitied, a llag is entered into the dattibase indiciting the review hat oceured and the dettum maty be suspect.

Random errors are unavoidably introduced in the analyticill procedures. Usually there ane insulficient replicate antilysen 10 assess the overall random crerer. Instraments for analysis of radionative constituents count the number of radionatetive decily products all a dedector. and hatekground counts atre subtrateded sut. The nature of radioatetive decaly and the instrument design result in at random counting crorer, which is reported with the antilyticial result. Gencrally, silnple results less than the counting error indicalle the constituent was not detected. The backeround sublatetion maly resule in the reporting of results that alle less than ero. Although this is physically imponsible. lhe negallive villues maly be of use for some statistical antalyses. 
Systematlic errors may result from instrument callibration. standard or simple preparations. chemical interferences in allalylicall echmigues, as well an sampling mellondology and rimple hamelling. Sample and lahoraltory protecols have beell designed to minimis ystematic errots. The laborateries used by the ground waller surveillance and ollere programs participalle in incerlaboratery comparisoms in which many laborallorics antaly a hlind samples prepared by the laps. The liborateries used have companced lavorably with oller laboratories. indicalling the levelat systematic error from mally soluters is alcecetable.

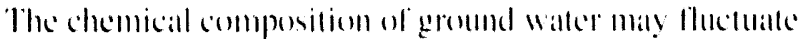
from differences in the contaminame source. rechanges ar lle low-lield. The range of this concentration llastualion cian be estimbtled hy taking mally simmples. but there is a limit on the mumber that can be pratetically laken.

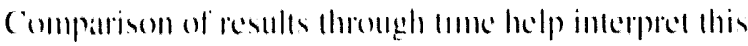
mattural variahility.

When more than one simple wats collected from a well dering 1992, the variability of the datal can be indicalled

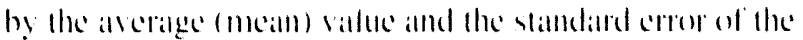

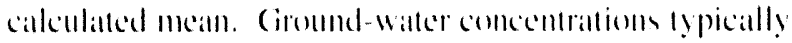

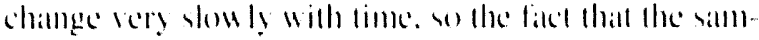
ples are mol alwaty sollected all the same time is not a matjor problem.

() Nerall sample uncertainty may be latedored into data calluatem hy comsidering the comecoltration trend in at

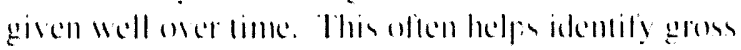
errors, and orerall lomp-lem trends can be distinguished

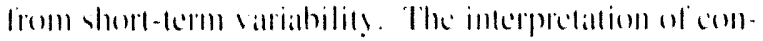

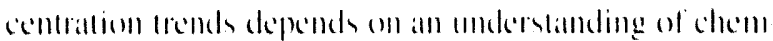

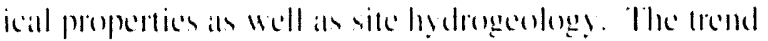

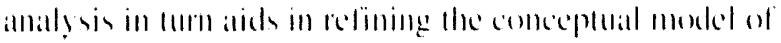
the chemical trimspurt.

Plume malp presented in this section ance diatgrammantic representalloms of the interprelation of Site grommel-wated

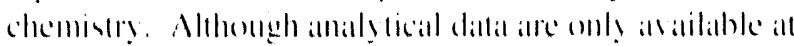
specilie points where wells were simpled. conterms alle

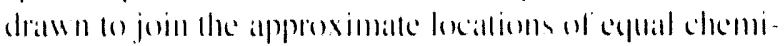

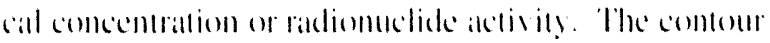

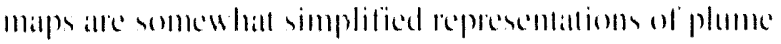

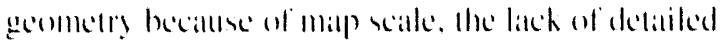

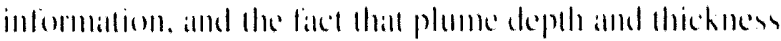

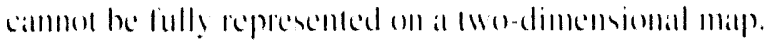

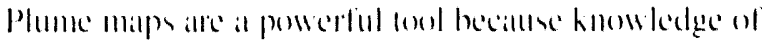
comecontrattioms in surrounding wells, know kedge al

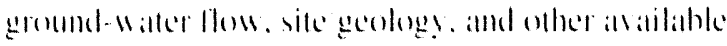

infermation mas be factored into the preparation. This minimise the impace of uncertainty or error in any parliculatr sample.

\section{Radiological Analysis}

Most ground-Maller samples collected onsite in 1992 were allalyed for 'H. Selected simples were subjected to more extensive radiologgical antalysis by alphat-. hetat-.

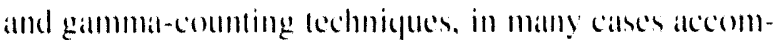
panied by selective radiochemical separtations. These lechmigues allow the antalysis ol individual radionaclicles. Uranimm analyes were performed by a laser lluorescence melhod on by alpha connting. Results from the former method are reported in $\mu g / l . ;$ results for the latter

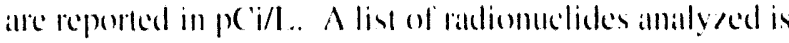
presented in Table 5.25. The radiological monitoring network for most allean on the Hanford Site is shown in ligures 5.49 and 5.50 . liggures 5.51 and 5.52 show monitoring wells in the 2(0)-lanst and 20)-Wost Areats.

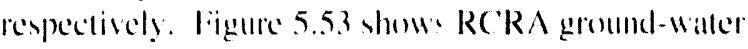
monitoring projects horenghoul he Hantiond Sile.

\section{Chemical Analysis}

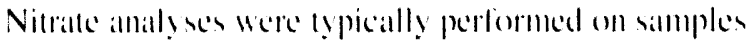

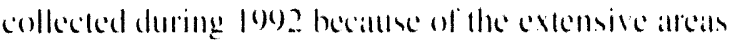
with elevalled nitrate concentrations originating firmom onand olfsite sources. Selected monitoring wells were used for adelitional chemicial surveillance. ('hemicall salmpling wells were chosen by comsidering the results of previous chemical analyses and the proximity o homom active and

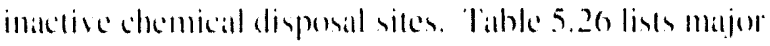

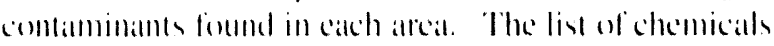

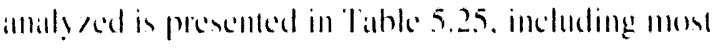

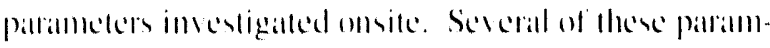

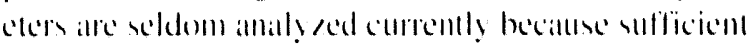
challateriatlion has heell ohtained hy past antaly ues

\section{Results}

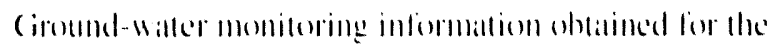

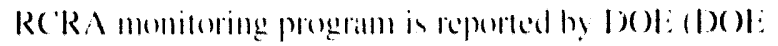
(9) 3b) and for drimking waller stpplices on the llantord

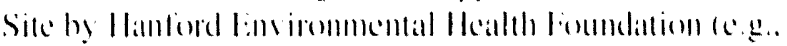
Thumam 1992). (Insite drinking watler supply wells all

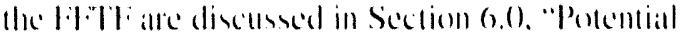

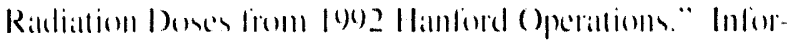

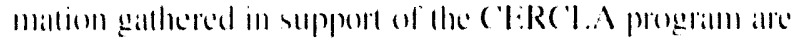
repented in Remedial Imsentigation reperts (c.g. I)( )):

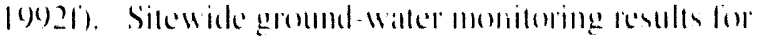




\section{Table 5.26. Major Chemical and Radiological Ground-Water Contaminants and Their Link} to Site Operations

\begin{tabular}{|c|c|c|}
\hline facilitics ly & Areal & (imstillents \\
\hline Reactor (Opcrations & $1(1)$ & Iritium. " (c). "Sir. ( $\mathrm{r}+\mathrm{s} . \mathrm{S}()_{1}$ \\
\hline Irradiated linel Processing & $2(1)$ & 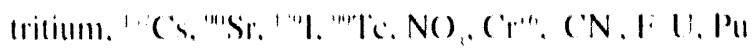 \\
\hline Plutonium Puriliciation & $2(11)$ & 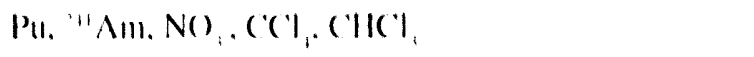 \\
\hline Fucl labricallion & 3()$(1)$ & 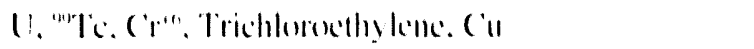 \\
\hline
\end{tabular}

the year ane in the (iround-Walce Lnvirmmental Surveil-

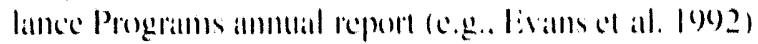
and lisicd in a complementary volume to lhis report. Highlights of those resules alle discossed below.

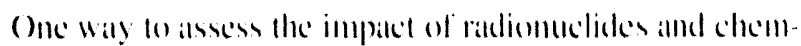
jeals in ground-waller is lo commpare the comerentratloms (n)

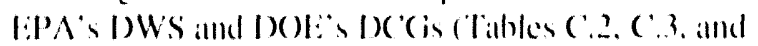
('o. Appendix ("). Specilic drinking watter standands hare only heen proposed lor a lew radiological comstituents all the lime this menert was preparted. Drinking waller standards have heen calculated for onler radiontedieles by comsidering the hall-lite of the isclopese the entergy and

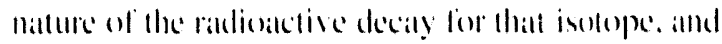
physiolegeical fiketors stach as the huildup of the isotope in particulatr organs. Drinking waller stamblards alle mone

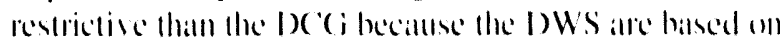

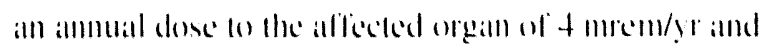

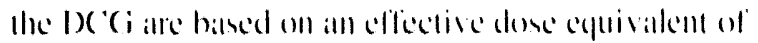

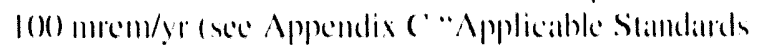
and Permis and lanvirommental (ompliance bocumental-

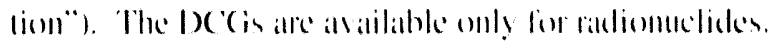

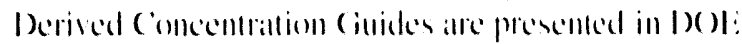
()rder $5+40(1) .5$.

\section{Radlological Monitoring Results for the Unconfined Aquifer}

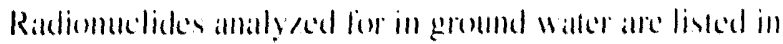

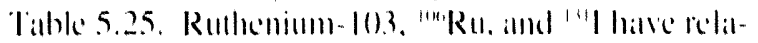

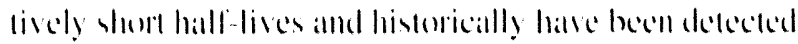

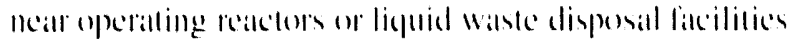

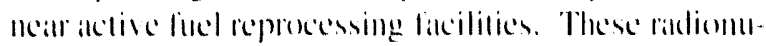

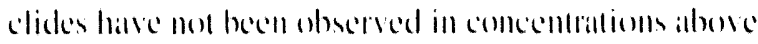
lhe 1)WS. and in general. hase not heen detected since

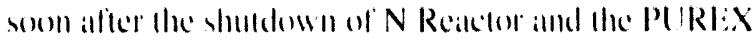

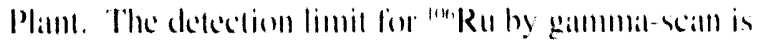
higher than he I)WS hut the hall-life ol only I yearl

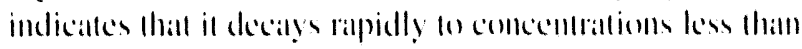
the DWS. (iruss llotill) alphat and beta ane used as indicallors of radionuclide distrihution and alle mot discussed

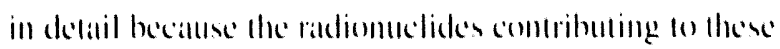

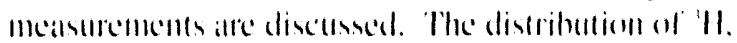

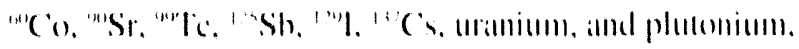
will be disconsed in the lollowing sections. The Iype al

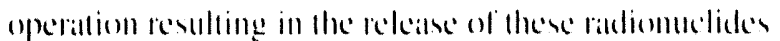

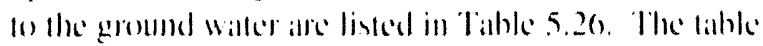
also lists the locattoms where these aperatloms were perfinmed.

Tritium. Tritium is preseme in mamy Warke streams dischallged fo the soil columm and is the most mobile radio

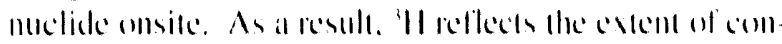
laminattion in the ground walle from Sitc operations and

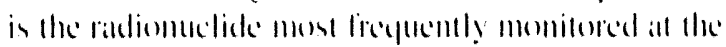

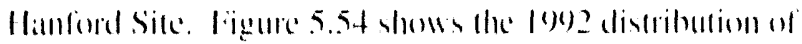

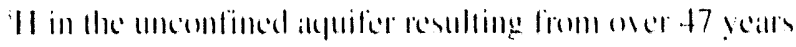

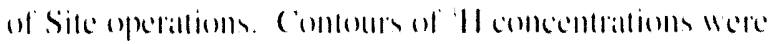

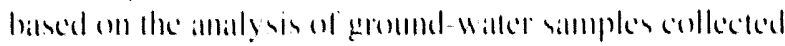
form moniloring wells.

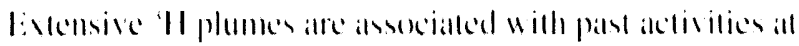

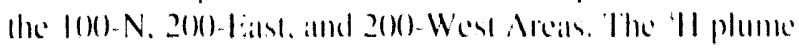

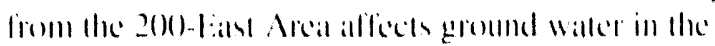

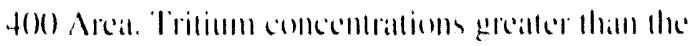

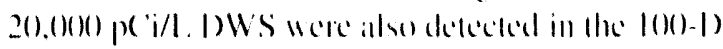
and $1(1) \cdot k$ srials.

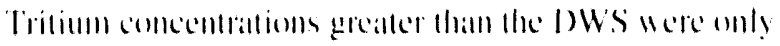

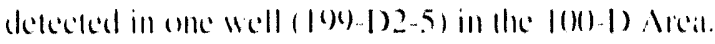




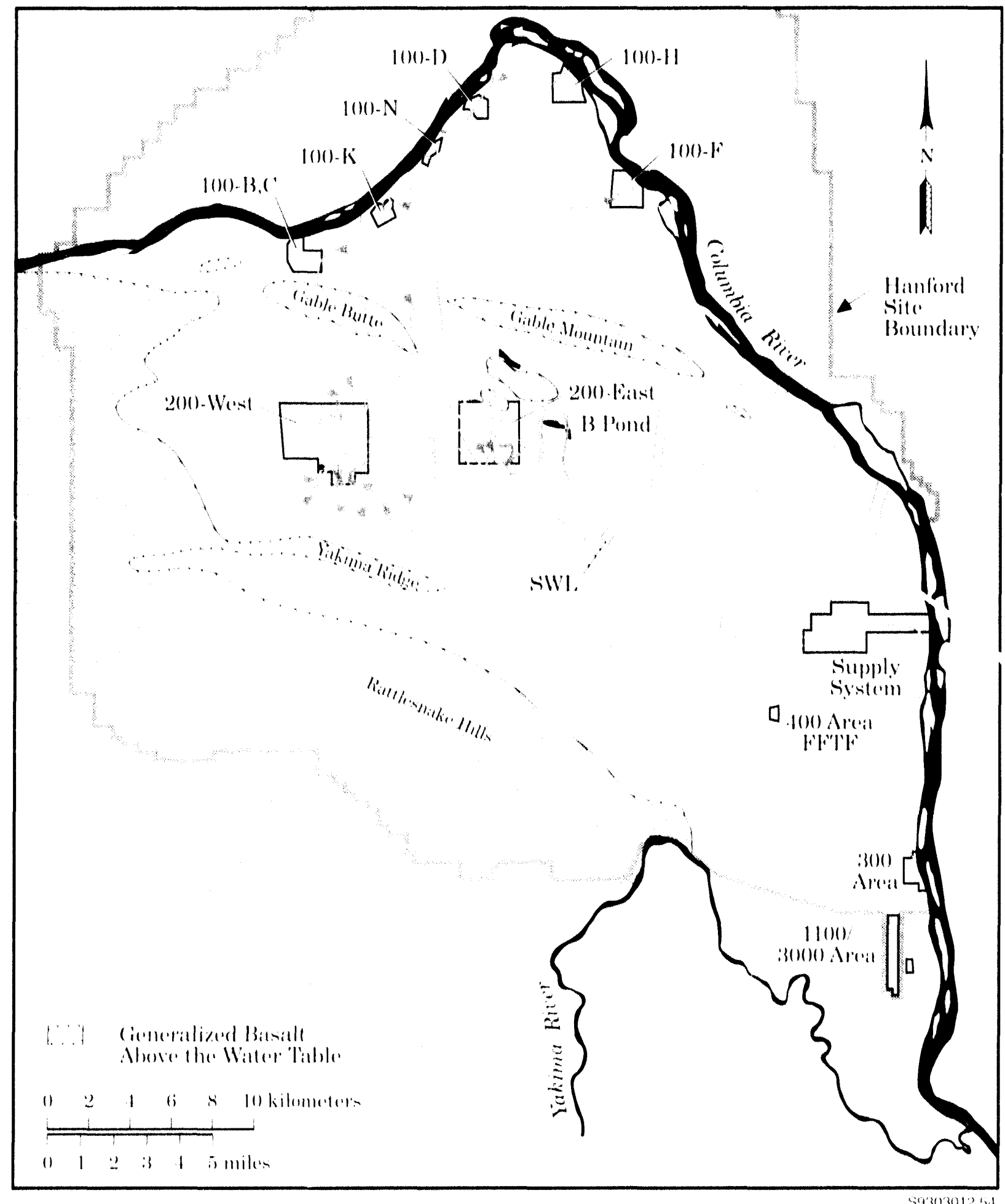

Figure 5.54. Tritium ('H) Concentrations in the Unconfined Aquifer, 1992 


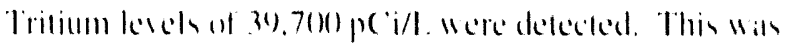

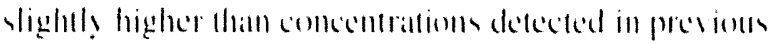
cials.

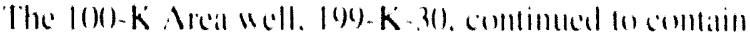

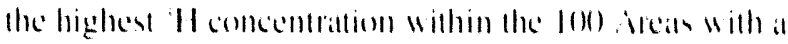

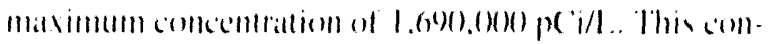
centration was comsiderathly higher than that dedected in

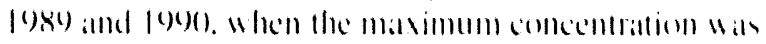

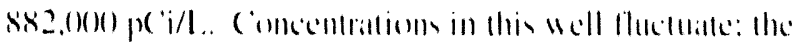
presious high wis in late les7. Thus, the long-kerm

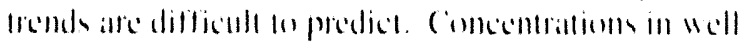

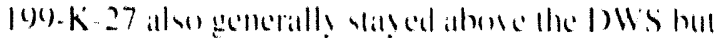

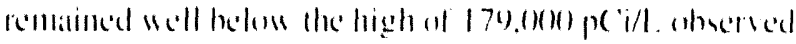
in (1)

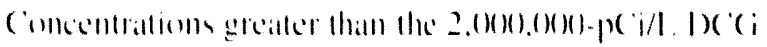

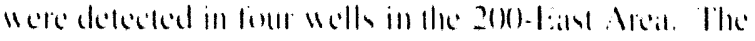

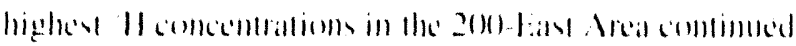

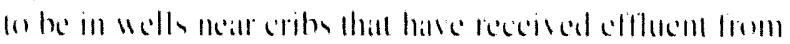

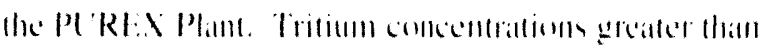
the I) ( $($ i w

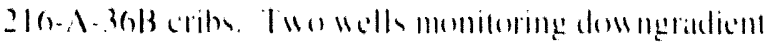

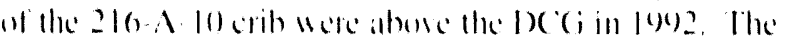

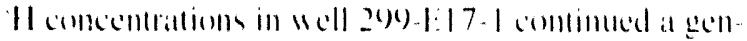

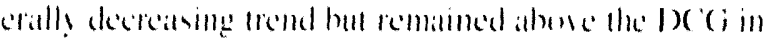

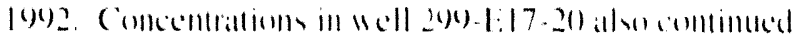

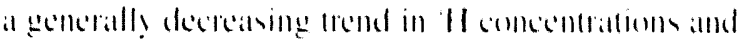

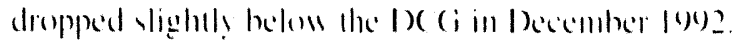

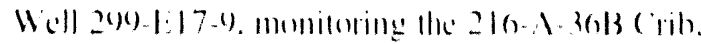

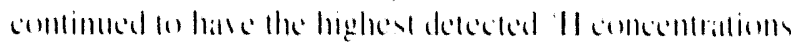

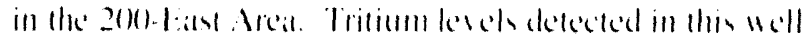

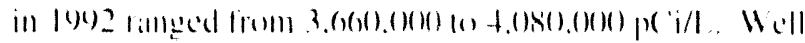

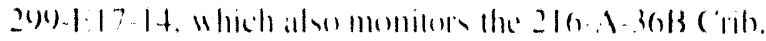

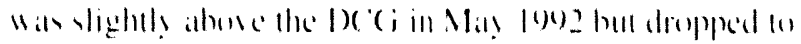

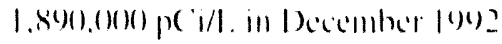

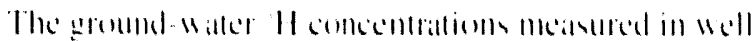

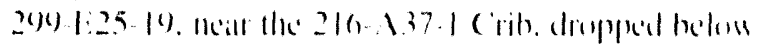

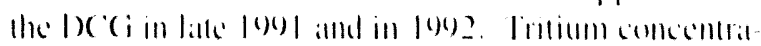

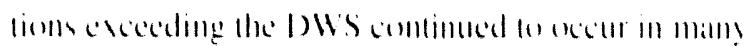

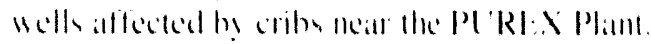

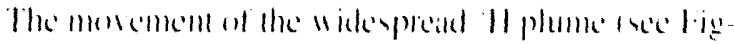

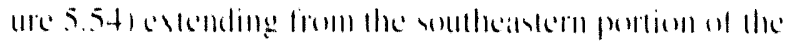

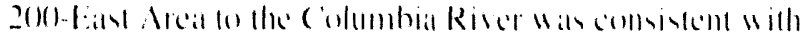

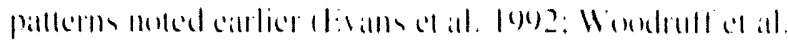

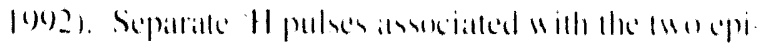

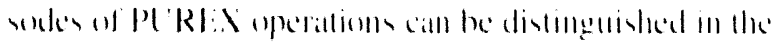

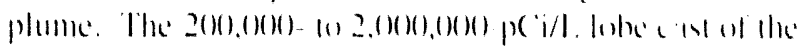

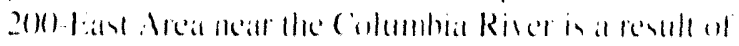

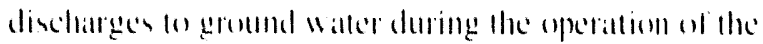

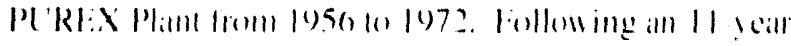

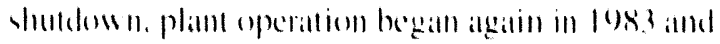

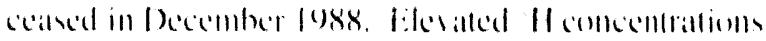

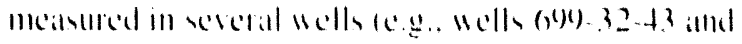

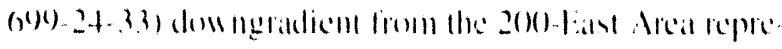

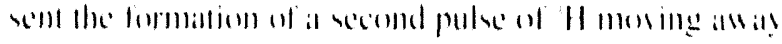

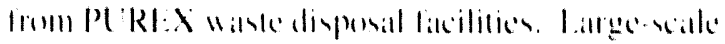

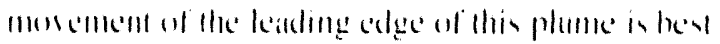

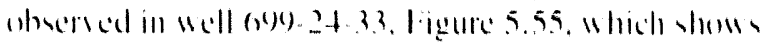

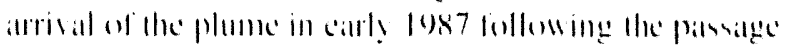

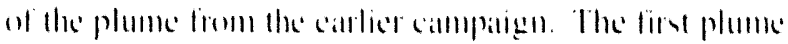

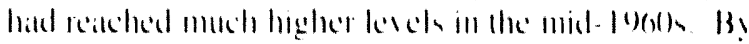

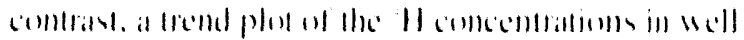

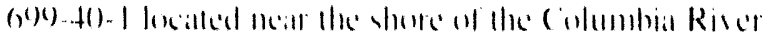

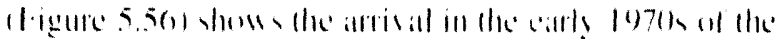

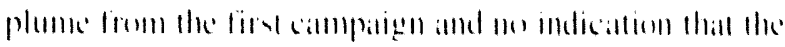
xcound plume has !co arriscid.

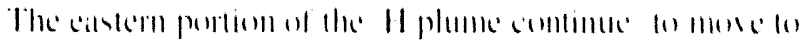

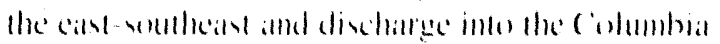

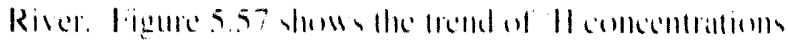

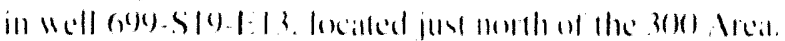

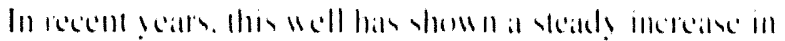

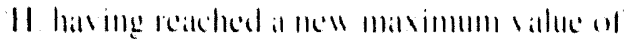

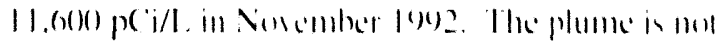

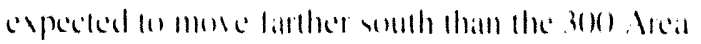

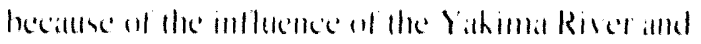

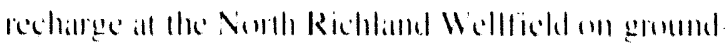

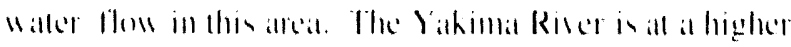

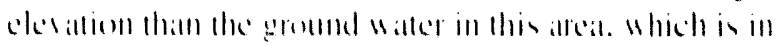

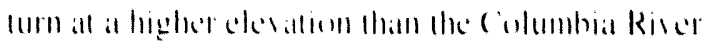

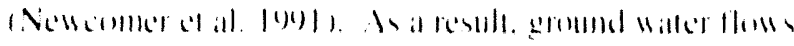
from 1 w

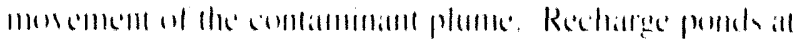

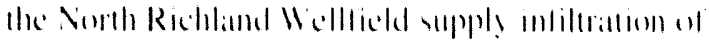

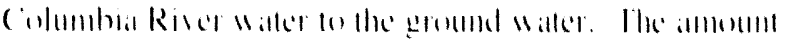

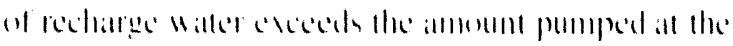

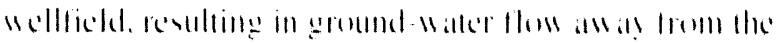

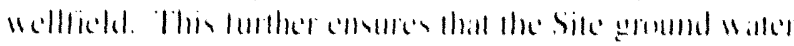

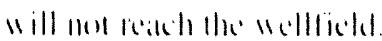

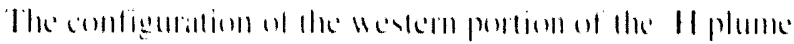

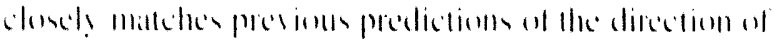

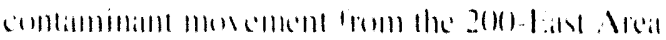

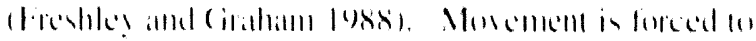

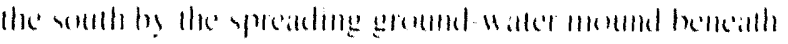

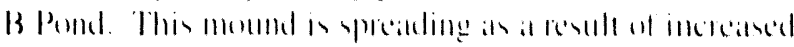

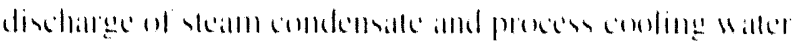

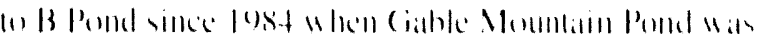

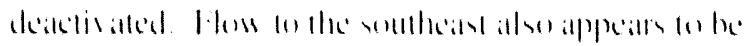




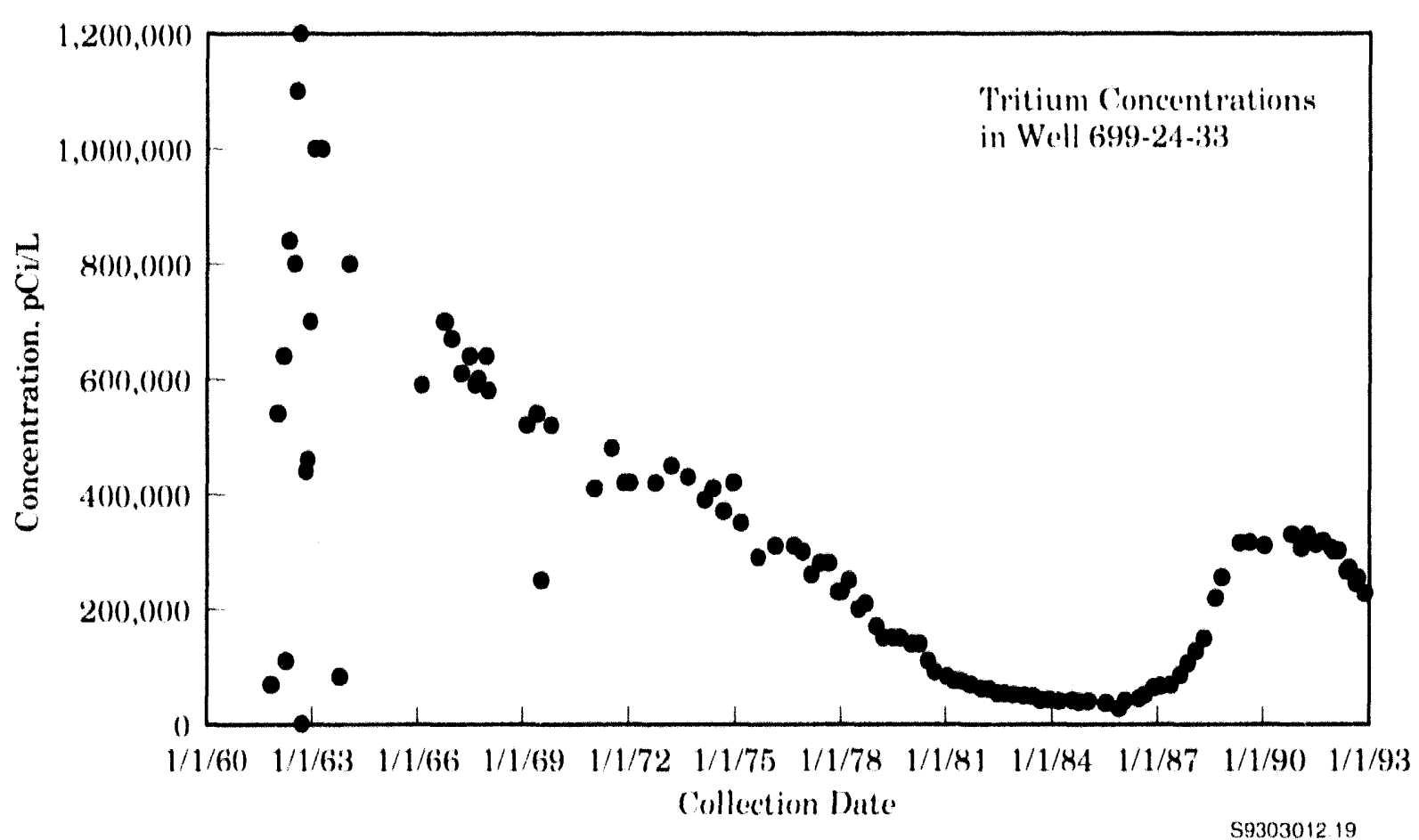

Figure 5.55. Tritium ( $\mathrm{H} H$ ) Concentrations in Well 699-24-33, 1962 Through 1992

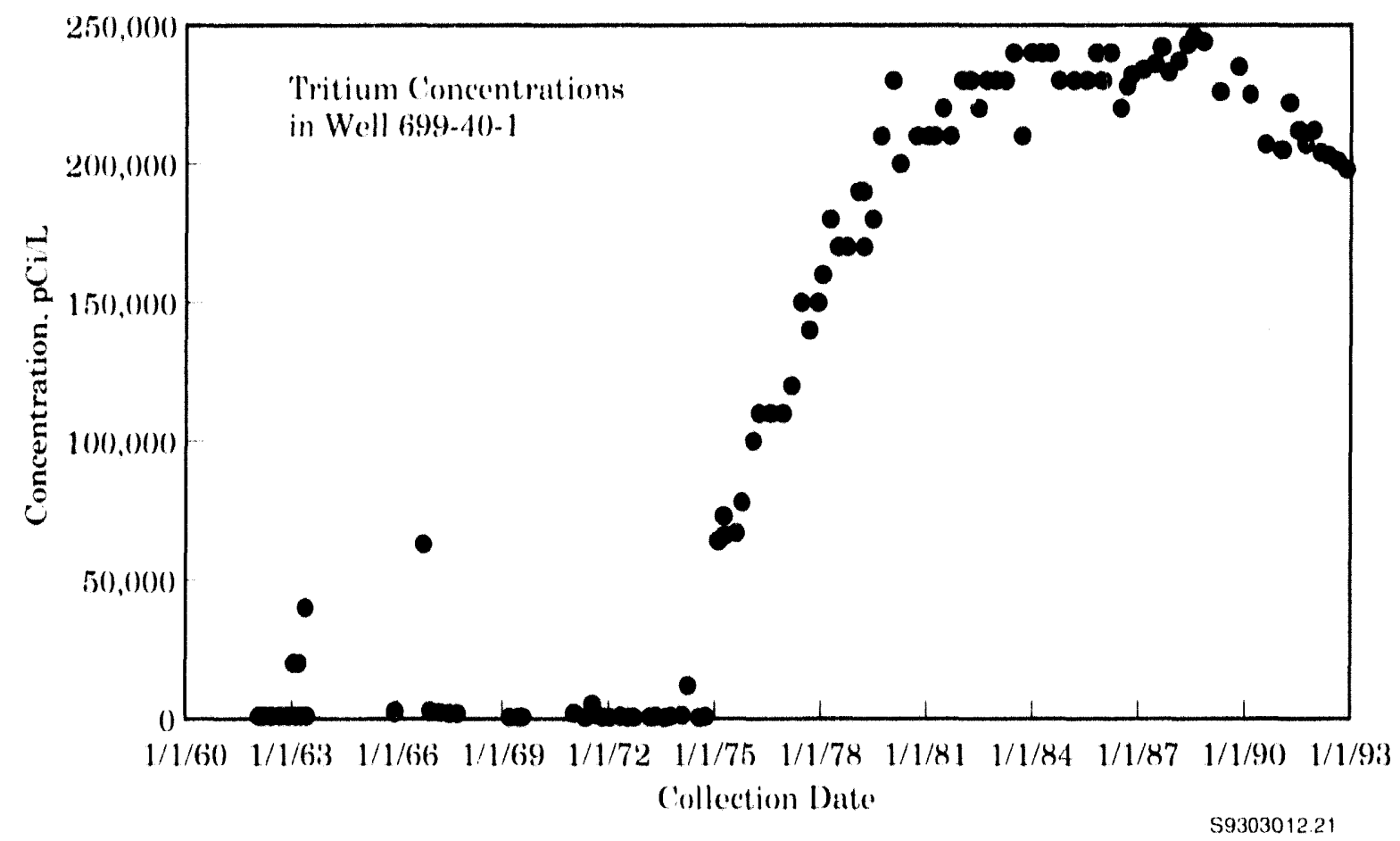

Figure 5.56. Tritium ( ${ }^{3} \mathrm{H}$ ) Concentrations in Well 699-40-1, 1962 Through 1992 


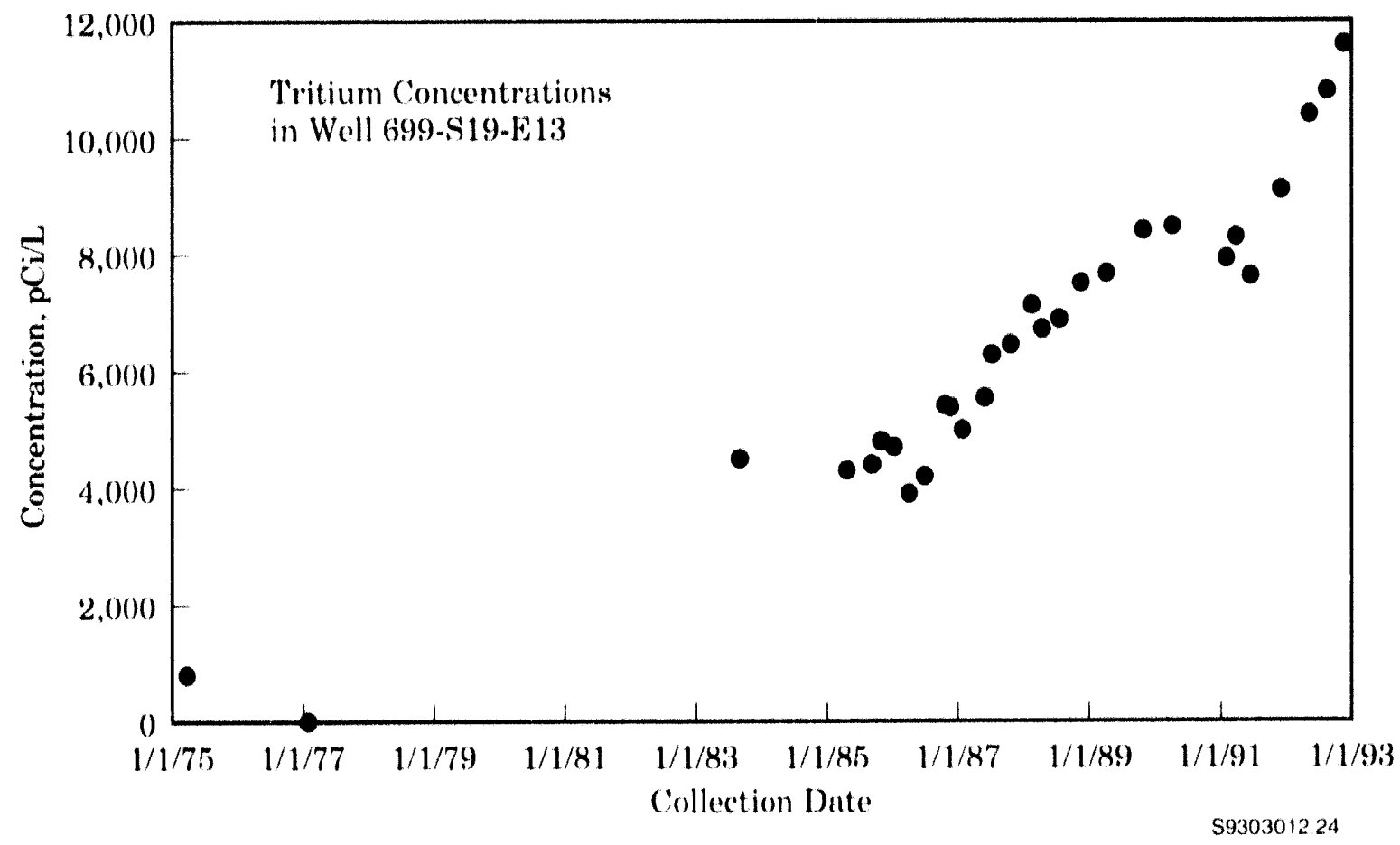

Figure 5.57. Tritium $\left({ }^{3} \mathrm{H}\right)$ Concentrations in Well 699-S19-E13, 1975 Through 1992

promoled by a ame of high permeability sediments

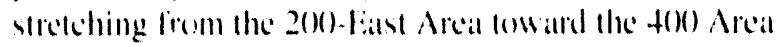
(dacobsull and Freshley 190()).

The monement of th plumes in the 20)(0-West Areal was also comsistent with previous observations. The plume extending from neall the Redection ( )xidation (REDOOX) Pant in the southern part of the 20)-West Areal contin-

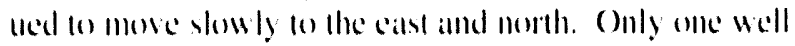

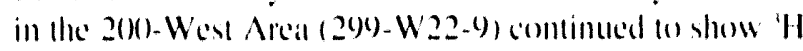

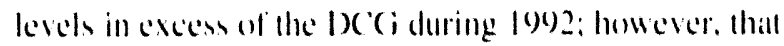

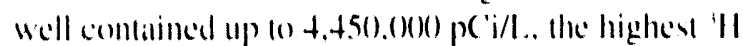
levels of any ground-water monitoring wells on the Site. The 'H soncentrations detected in well 209. W22.9 hate' decreasced stcidily since 1977 (fightre 5.58).

Movement of the Rl:I)( )X Plant 'H plume is expected lo be slow because of the low permeahility of the sediments in this alfea and the declining flow from the gromend-water mound bencall the neathy it Pond since the pond's deacetivattion. Tritium concentratioms in individual wells alle

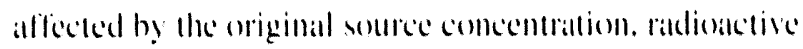
decity dering the tranel time to the well. and dispersion ar dilution of the plume.
Cobalt-60). All "'Co concentrations were consistenty near or below the delection limit 20 p(i/l.) for wells monitored in log) e exeept for two wells. The firs well. 20).1:17-16, is in the allea south of the PUIRIXX Plant in

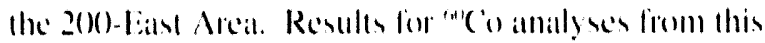

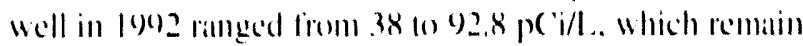

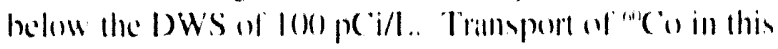
allea is rery slom. als indicaled by the limited extent of "Co detected in this highly monitored allea. The secomel well with defectable "(o) is well 60)-5(1)-5.3. which is localed in a region morth of the 200 Areas allected by Waske disponal in the BY (ribs (liggure 5.59). The concentrattons of "(") in this well in 1992 were up l(1 $3.32 \mathrm{p}$ ( $\mathrm{i} / \mathrm{L}$. Which is lower than the $/ 90)$ maximum of

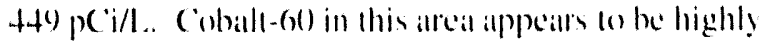
mobile, probably hecaluse of the presence of a soluble coball-cyanide (or ferrogyande) complex associalled with the plume originating in the BY Cribs.

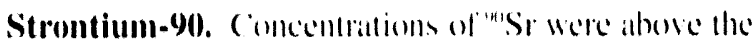
8 p(i/l. I)WS in wells in the 10()-B. 1(0)-1). 1(1)-1:

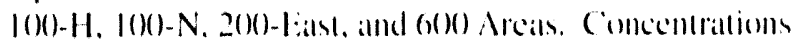

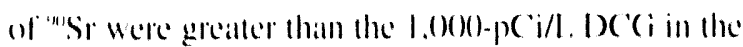




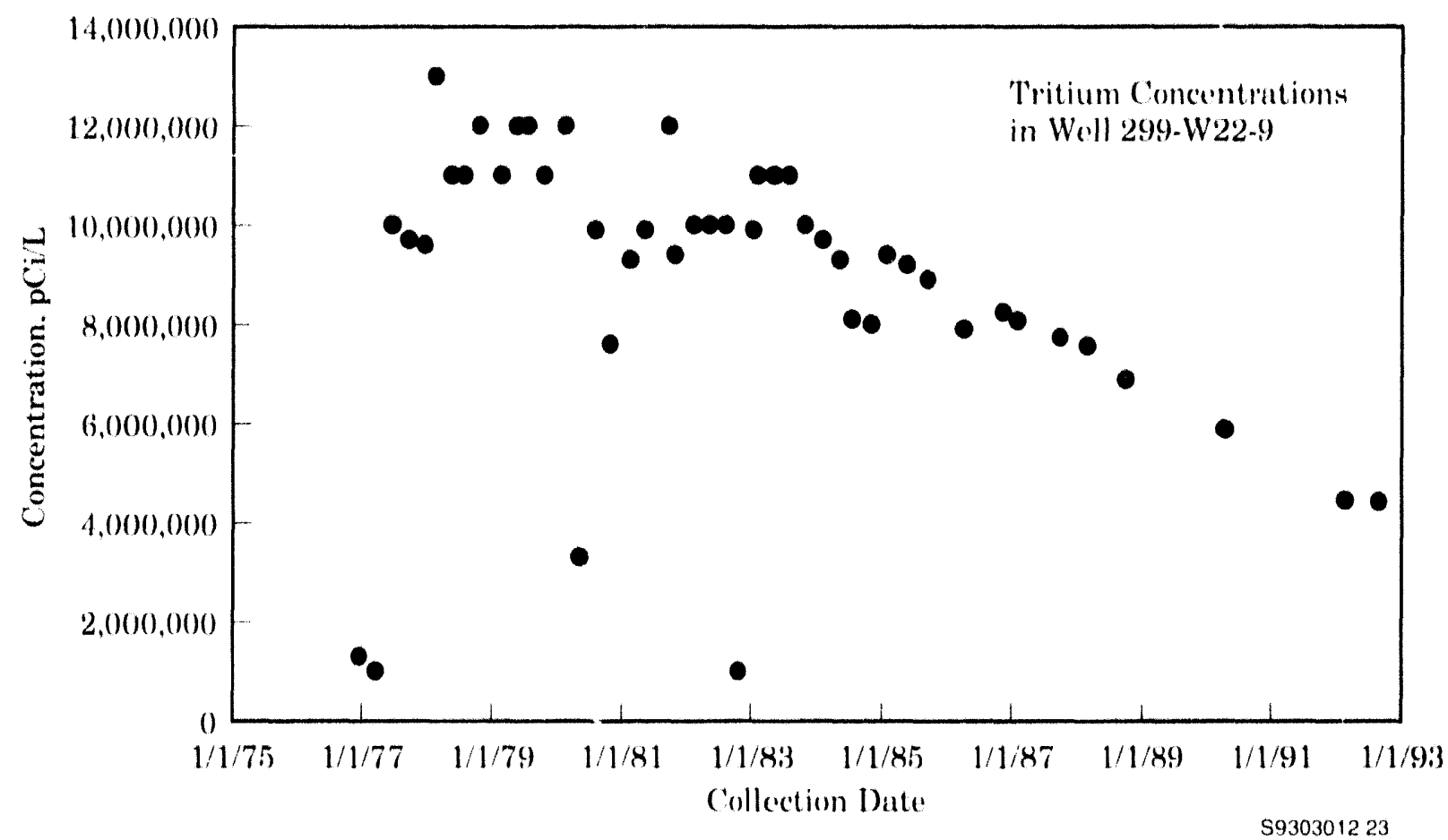

Figure 5.58. Tritium ('H) Concentrations in Well 299-W22-9. 1976 Through 1992

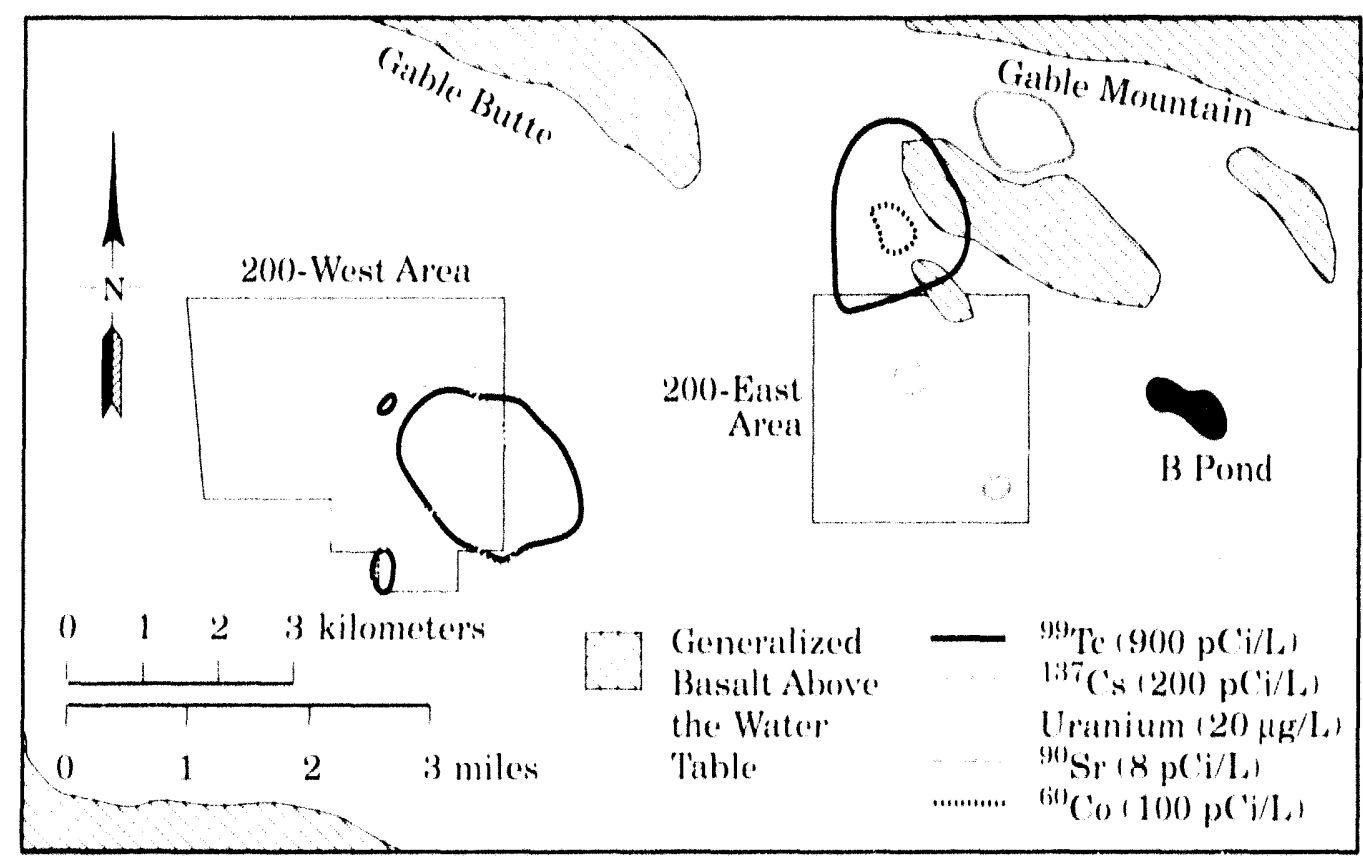

5930301248

Figure 5.59. Distribution of Selected Radionuclides Greater Than the Drinking Water Standard near the 200 Areas, 1992. Drinking water standard for each constituent is shown in parantheses. 


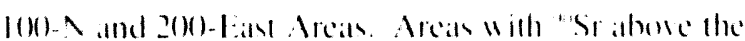
oWs ane vhow $n$ in figumes 5.54 and 5.00

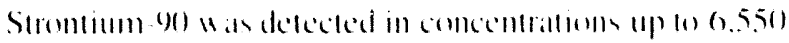

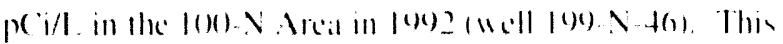
well is lexited helle

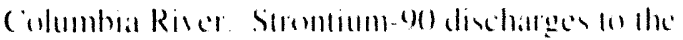

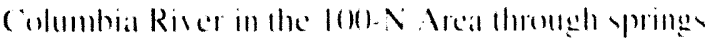

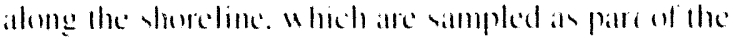

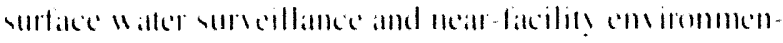

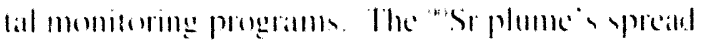

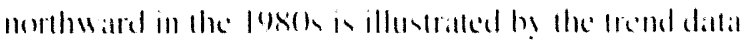

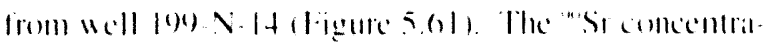

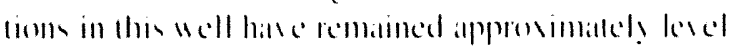

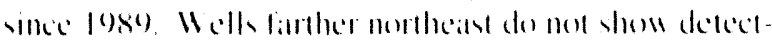
athe "St The steids lesels indicalle the plume is not yreading all an! discermible ralle all this lime.

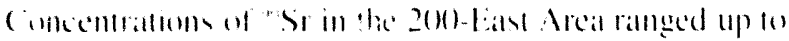

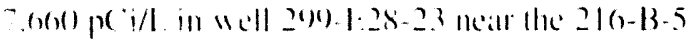

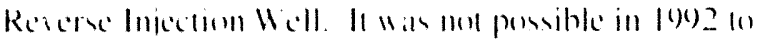

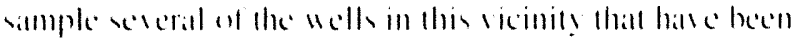

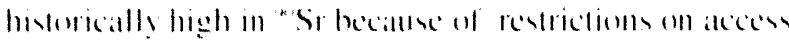
wadiallon protcollon ances

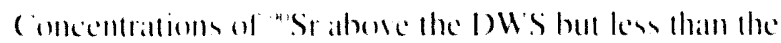

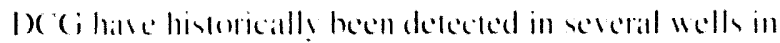

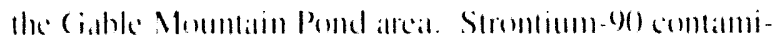
nation in that atrea resuled from the alecidental dischatrege

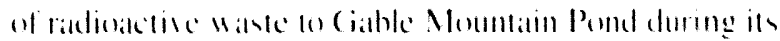

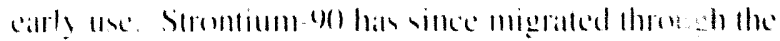

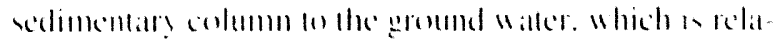

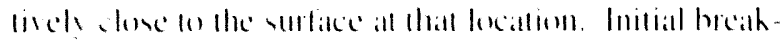
thregeh aceorred in lese in seme areas. The depth to hedroch is also small in the (iable Moumtam Pomd alleal.

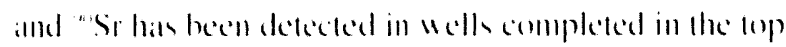

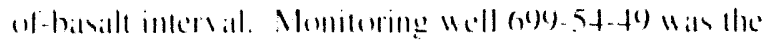

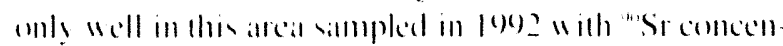

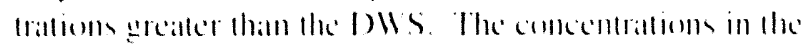

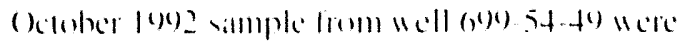
$+3.9 \mathrm{pr}(\mathrm{i} / 1$.

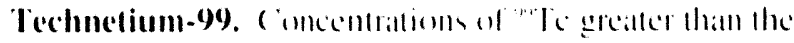
(2)(0)-p(

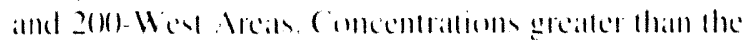

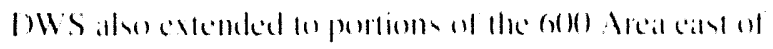

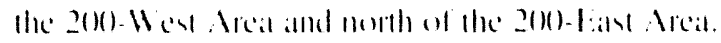

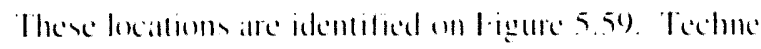

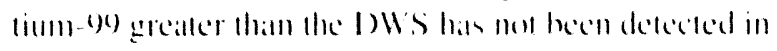

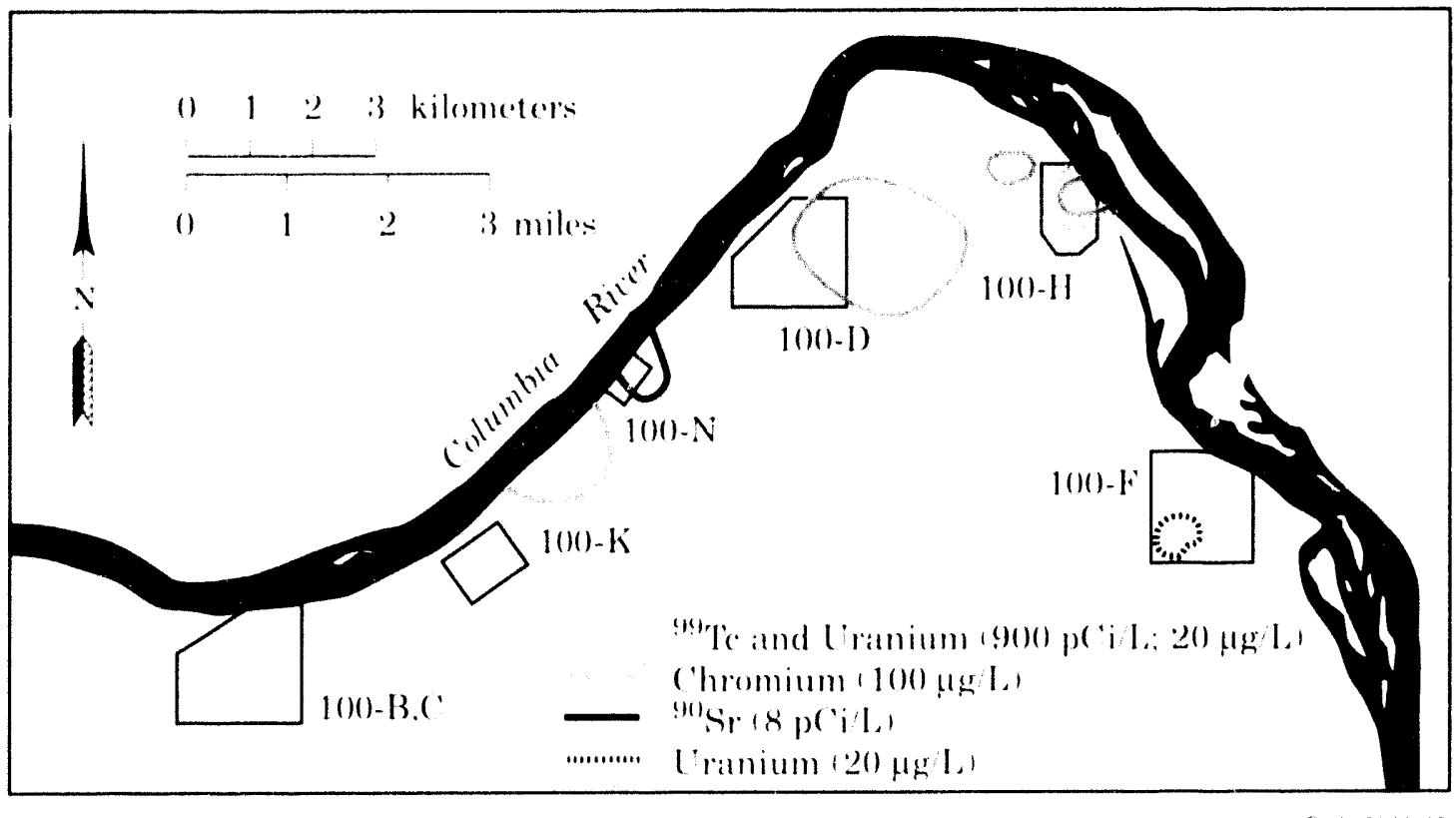

Figure 5.60. Distribution of Selected Contaminants Greater Than the Drinking Water Standard near the 100 Areas. 1992. Drinking water standard for each constituent is shown is parentheses. 


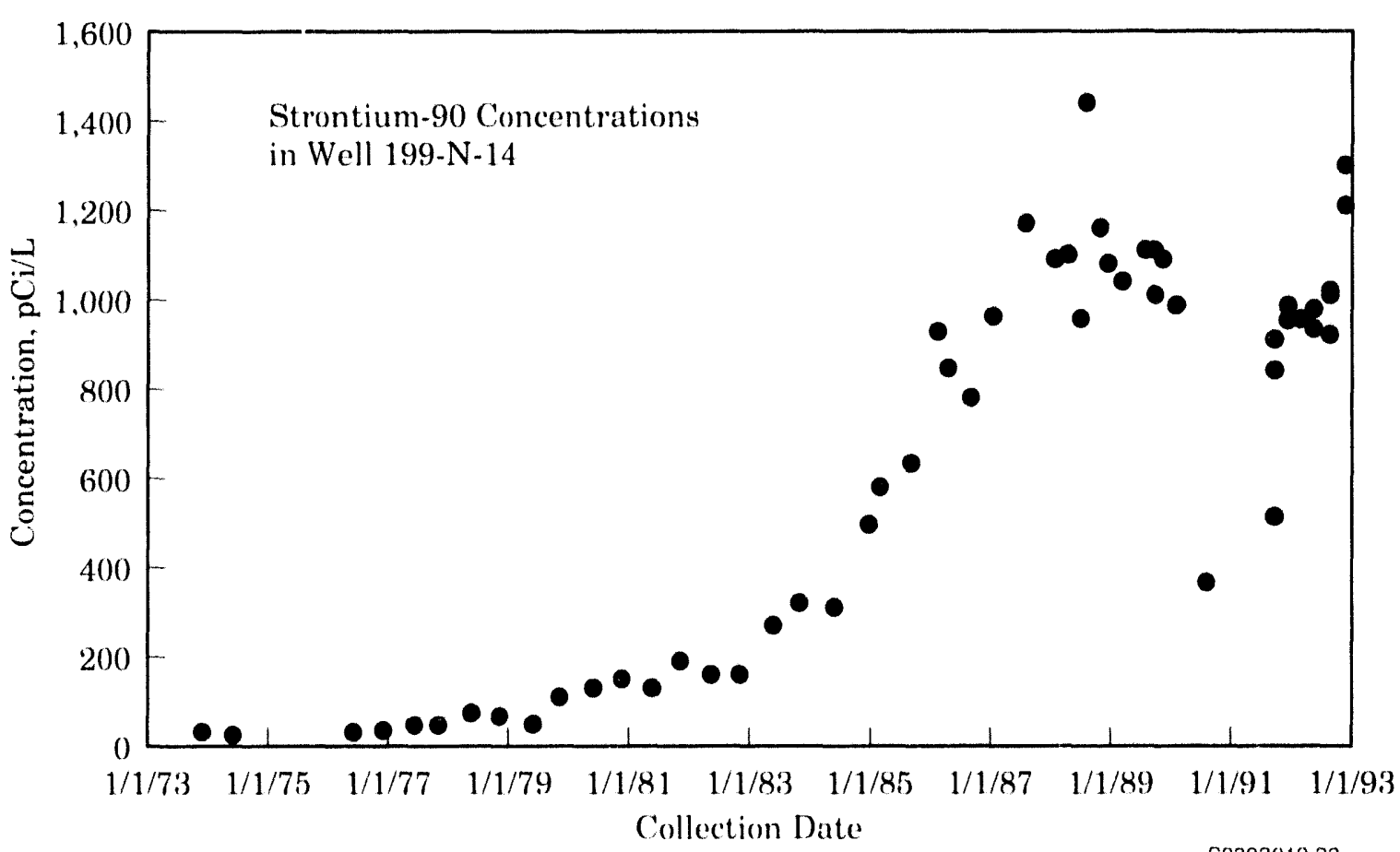

Figure 5.61. Strontium-90 ( $\left.{ }^{90} \mathrm{Sr}\right)$ Concentrations in Well 199-N-14, 1973 Through 1992

the $10(0-H$ Area since 1940 . Technetium is transponted in ground water ats a negattively charged (anionic) species that is highly mobile.

Antimony-125. Antimomy-125 has heen measured in the past in a lew wells in the $10(1)-N$ and $10(1)-K$ Areats. Concentrations measured in simples from these $1 w 0$ areas have heen as high as $30.5 \mathrm{p}$ ( $\mathrm{i} / \mathrm{h}$. near the $1325-\mathrm{N}$ 1.WDF in 1987. The DWS for "Sh for is 30) p(i/l.. and the $D C(i$ is $60.0(0)$ p(i/l. Antimony-125 wats nol detected at levels above the IJWS in 1992. Antimony125 hats a relatively short hall-lile $2.7 \mathrm{yr}$ ) and its lack of delection in recent years is attribulable in radionative decials:

Iodine-12\%. The presence of ${ }^{\text {nof }}$ in ground water is significant. hecause of its relatively lou D)WS (I pei/L.). ils perential for aceumulation in the enviromment as a result of fong-term releatses from nuclear fuel reprocessing facilities (Soldat 1976), and its relatively long hall-file (lo million sears). Al Hanford. We main contributor of "y lo ground water has heen licpuid discharges lo cribs in the 20) Areas. Assay of that isotope by high-semsitisily. direct-counting methods requires long connting limes with correspondingly low andylical throughput. The highest concentrations observed onsite ane downgradient from the PUREX and RED)OX plants, in the 2()()-East and 20()-West Areals. respectively. No ${ }^{\text {at }}$ samples were ahowe the DC(iol $5010 \mathrm{pC} \mathrm{i} / \mathrm{l}$.

The highest I concentrations in the 2(7)-Eats Area are in the merthwest near the 216-13Y Cribs and in the southeast neat the PURLXX Plant. The maximum concentra-

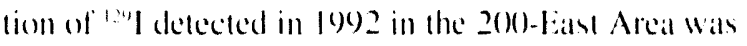
$16 \mathrm{pCi} / \mathrm{l}$. in well 209-1:17-20. This well is locilled south of the PUREX Plant. The "I plume from the PUREX area extends southeast into the o() Area and appears coincident with the 'H and nitrate plumes. The more limited exteme of the 1 plume shown in Figure 5.62 results from the lower intial concentrations of l" I than the initial concentrations of 'H and nitrate. The ent plume likely had the same sourees as the ' $\mathrm{H}$ and nitrate. lodine-129 has nearly the same high mobility in ground waller als 'H alld nitralle.

The highest ". I concentration observed in 1902 in Hanford ground waller were $52.9 \mathrm{pe}$ i/l. lound in well 2(y)-W14-12. The "'l plume liom the 2(x)-West Area extends into the $6(0)$ Area to the east. and is essentially concident with the 'H and nitrate plumes. 


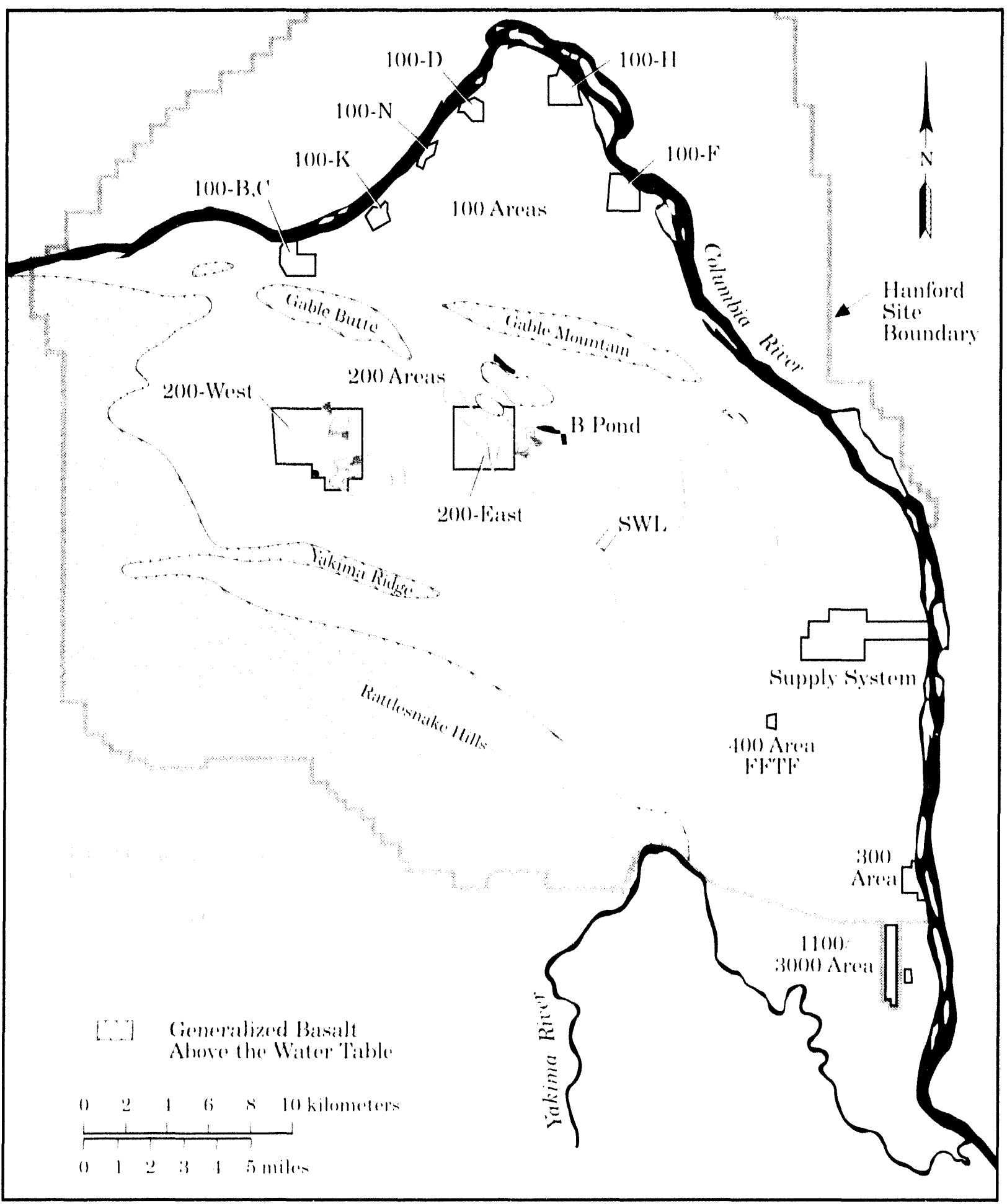

$\$ 9303012.41$

Figure 5.62. Distribution of lodine-129 (" II) in the Unconfined Aquifer, 1992 


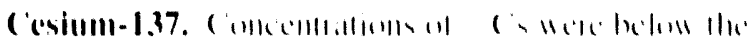

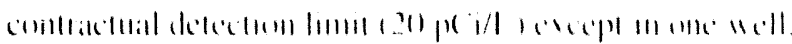

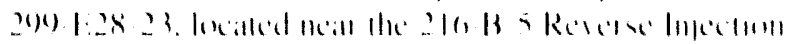

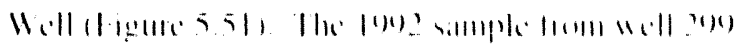

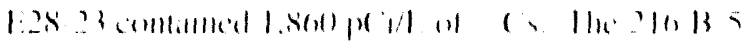

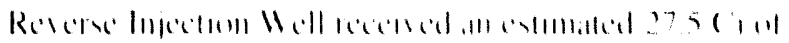

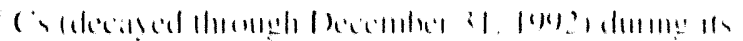

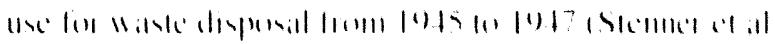
foss. The I)

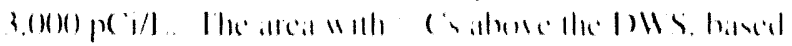

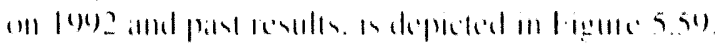

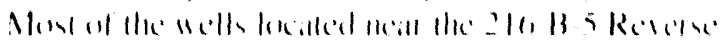

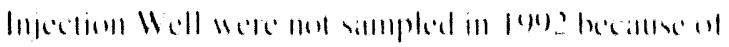

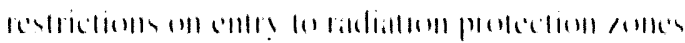

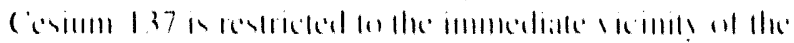

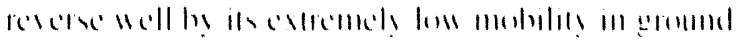
Hiller.

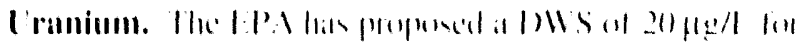

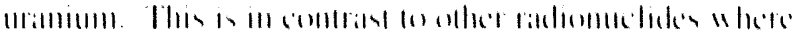

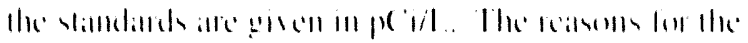

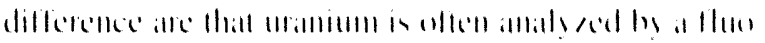

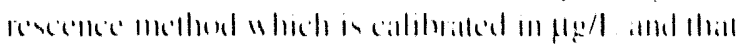

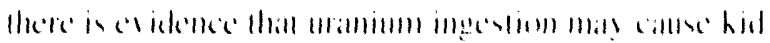

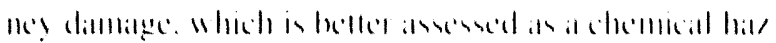

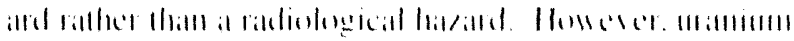

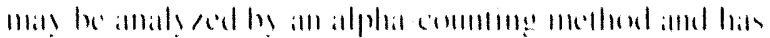

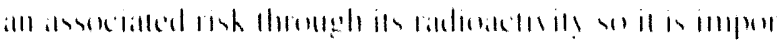

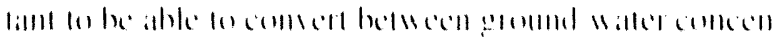

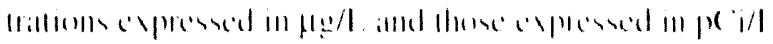

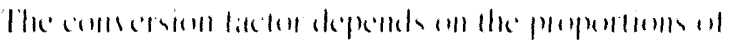

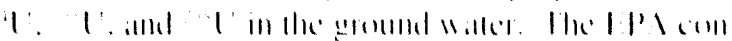

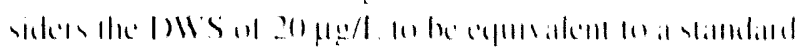

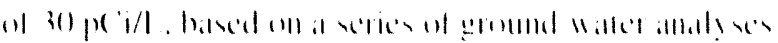

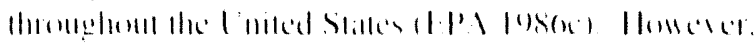

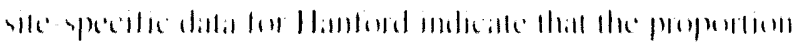

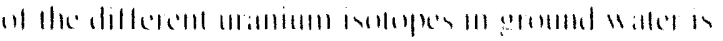

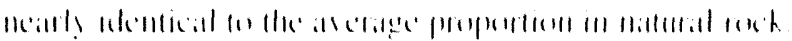

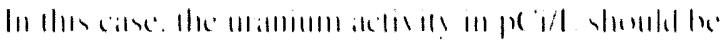

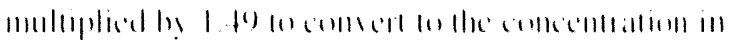

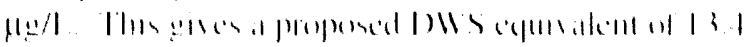

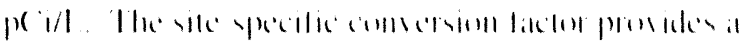

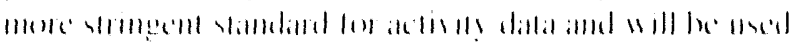
iII Ha' d/

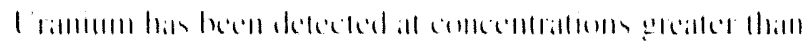

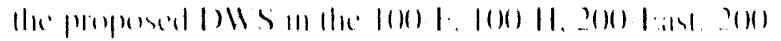

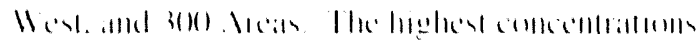

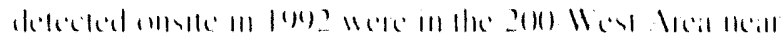

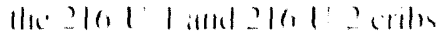

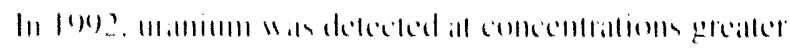

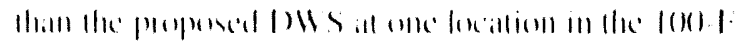

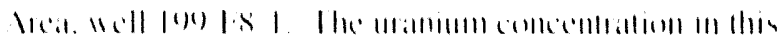

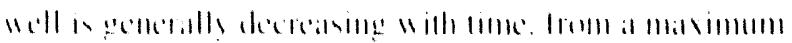

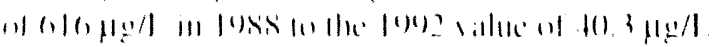

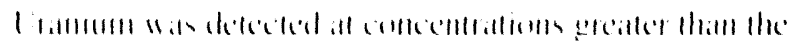

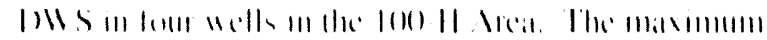

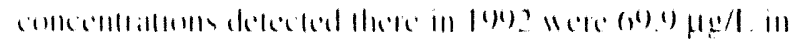
will $|(1)| 1$

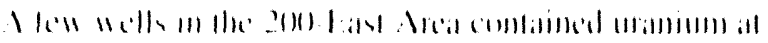

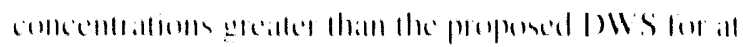

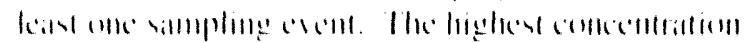

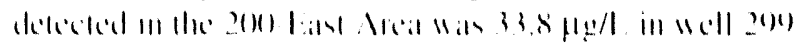

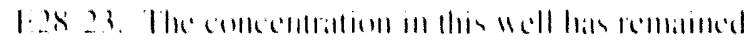

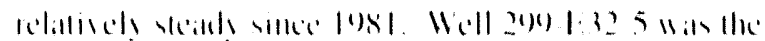

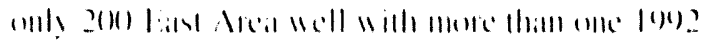

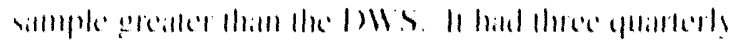

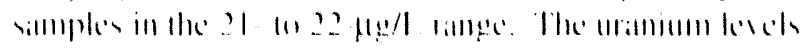

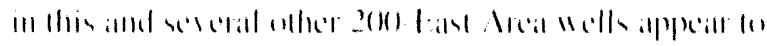

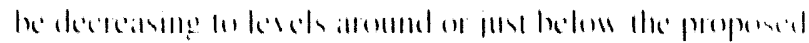
$111 \mathrm{~S}$

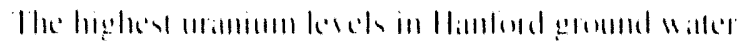

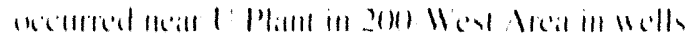

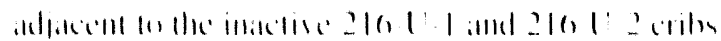

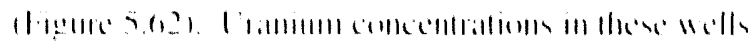

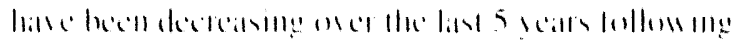

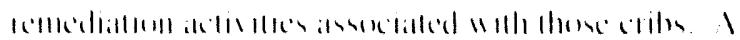

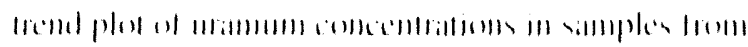

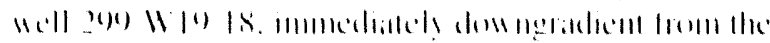

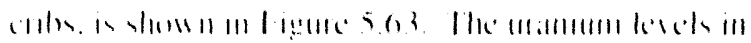

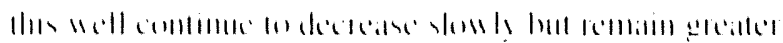

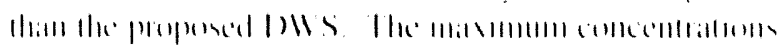

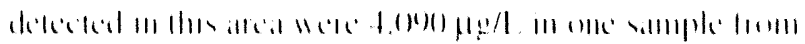

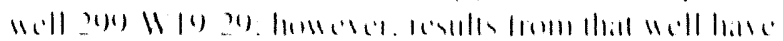

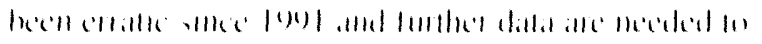

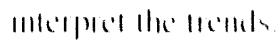

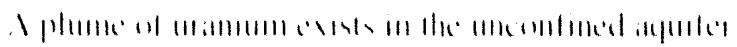

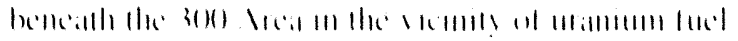

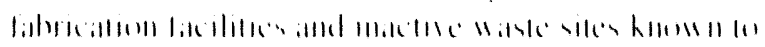

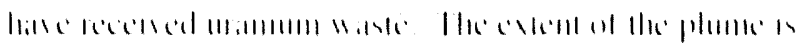

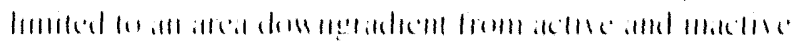

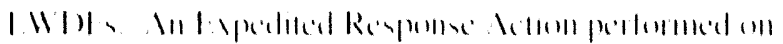
\|x

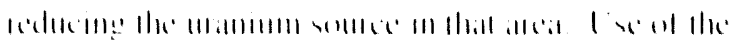

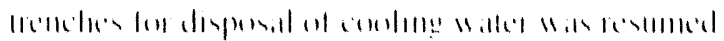

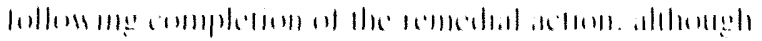




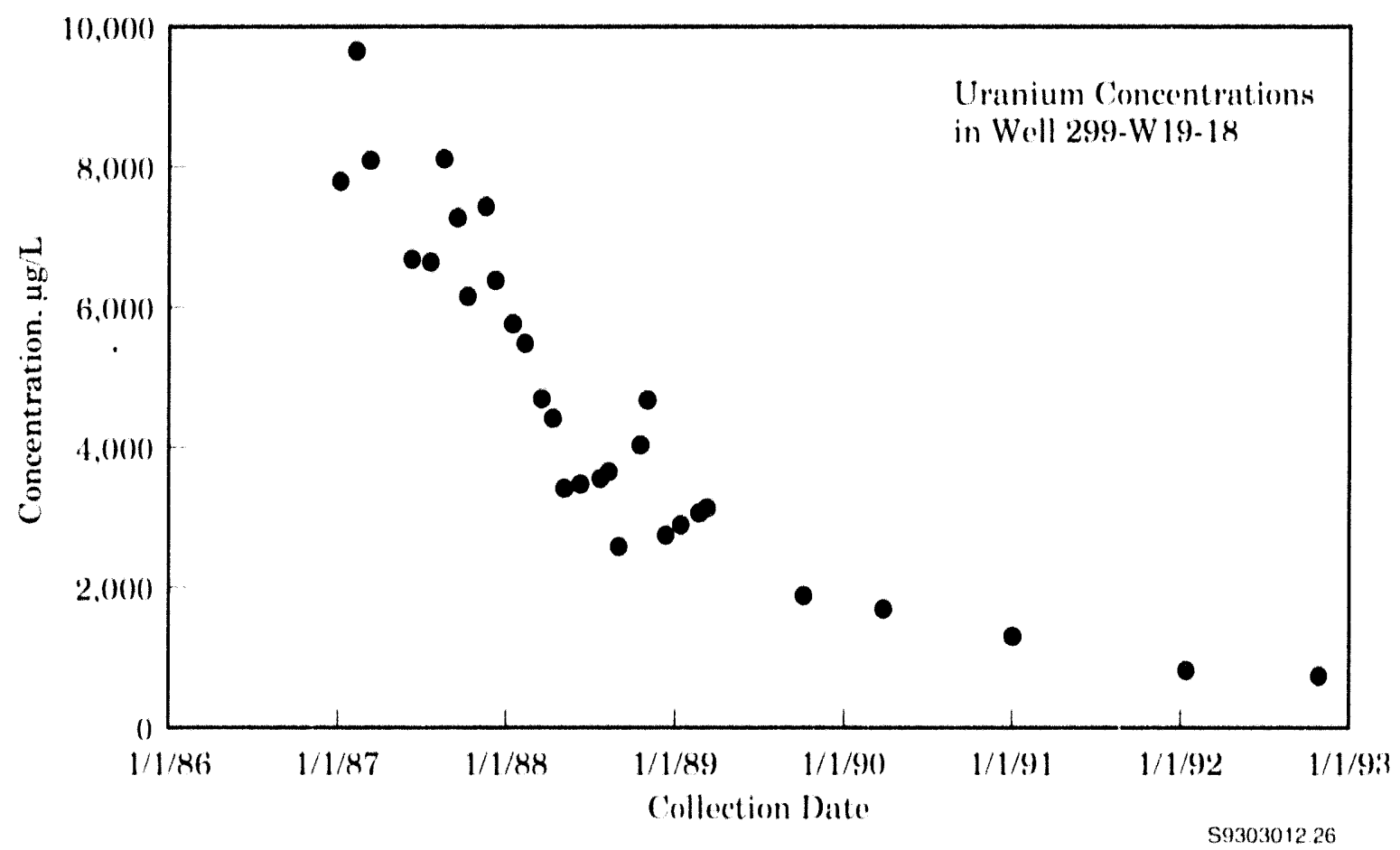

Figure 5.63. Uranium Concentrations in Well 299-W19-18, 1987 Through 1992

corrent dischatrge to the trenches is meth lower thatn in

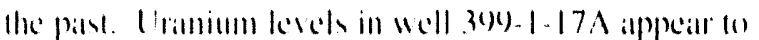

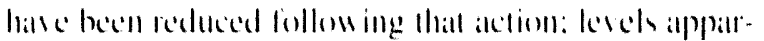
collty stabilised aboul a likctor of 10 below the maximum

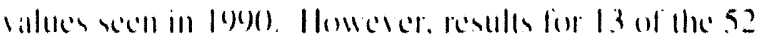

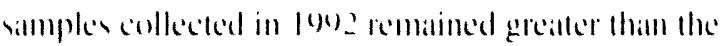
propured I) WS. A trend pled showing the uranium concentrillioms in thill well is shown in ligente 5.04 .

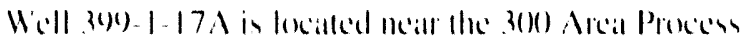

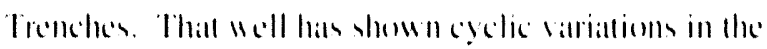

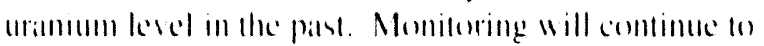
contirm the appartent impresement in mantum levels resulding term the remediation and llow reduction.

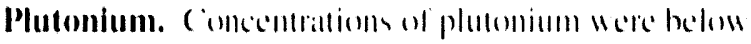

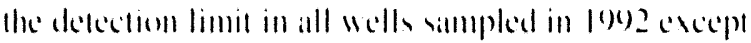

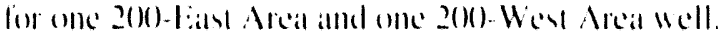

Ground willer simpled all 20(0)-tand Areal well 290-1:2823. which is lecilled neatr the 216-B-5 Reserse Injection

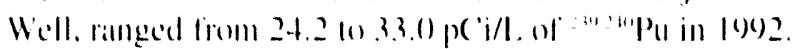

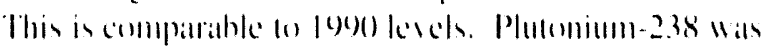

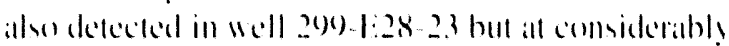

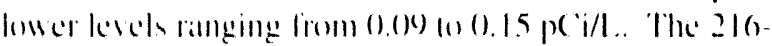

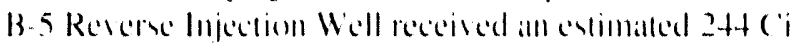

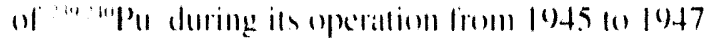

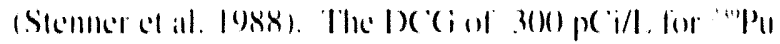

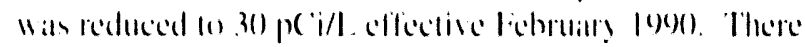

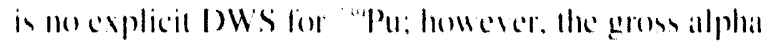
I)WS of $1.5 \mathrm{p}$ ('i/l. Mould he appliciahle all a minimum.

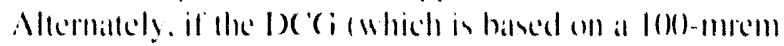

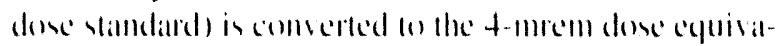

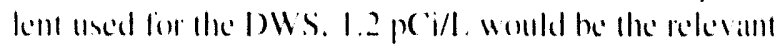
guideline. Plutenium is generally comsidered wo hind strongly lo sediments and thun has limited mohility in the inguilier.

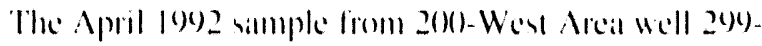
W23-13, an upgratlient wall for the S-SX single-shell

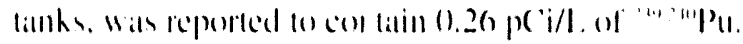

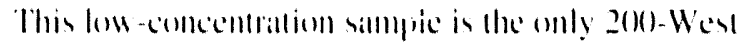
Areil sillmple in which plutonium was delected. and plu-

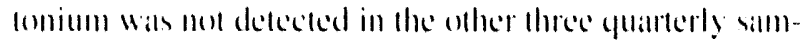
ples. Plutenium-239. 240 wats deteded in 199() and |90)

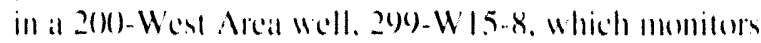

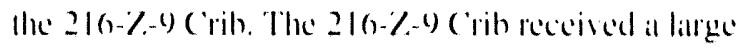
burden of plutenium and antericium from Z Plann licyuid

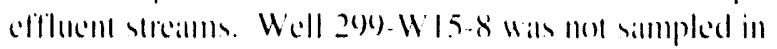

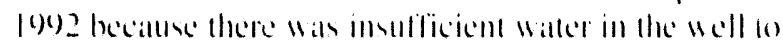
collect al silmple. 


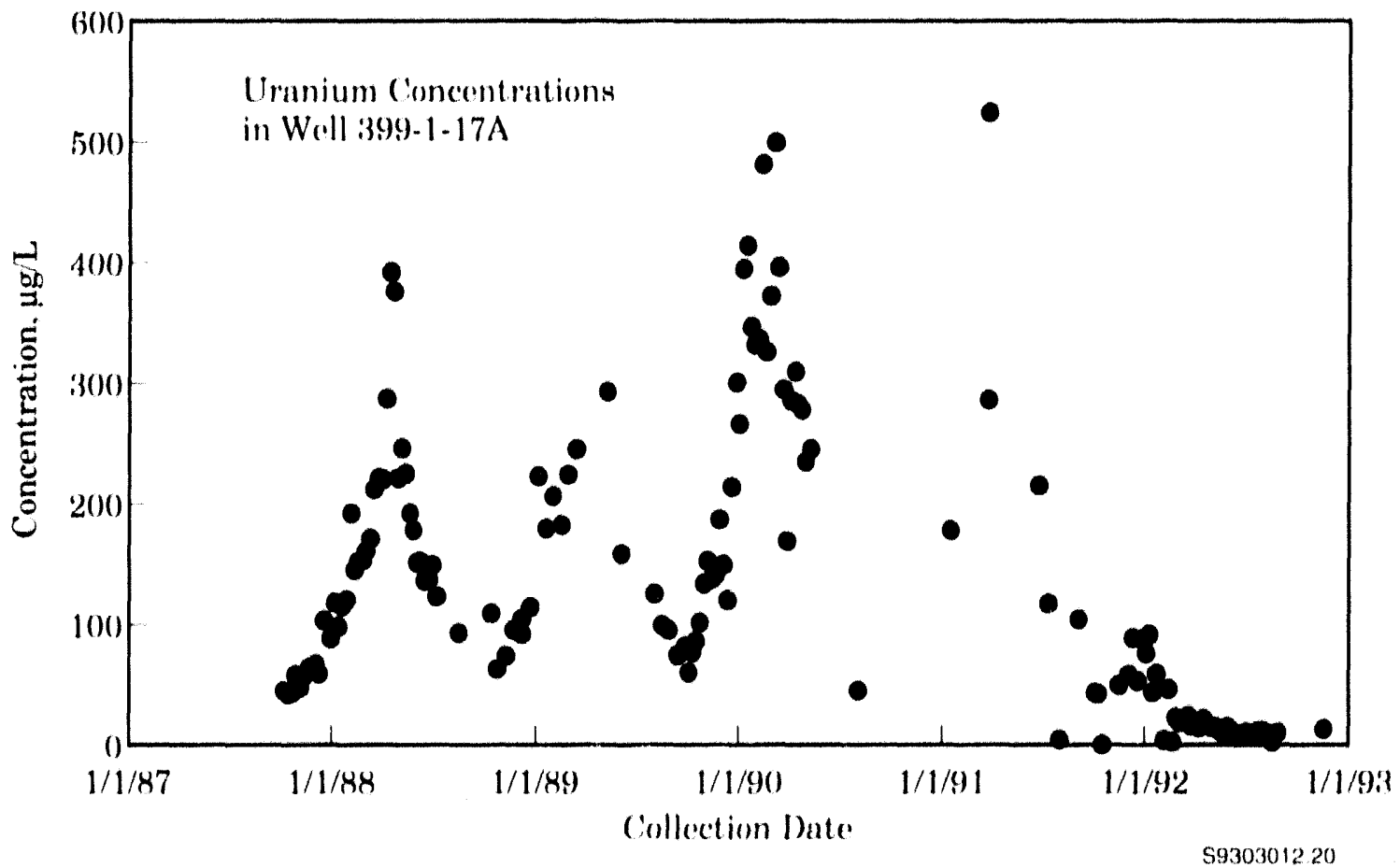

Figure 5.64. Uranium Concentrations in Well 399-1-17A, 1987 Through 1992

\section{Chemical Monitoring Results for the Unconfined Aquifer}

Chemical athaly aes performed on gromend-water simples hy varions momitoring programs all Hanford have identilicd eight hatatrdem chemicals accurring in gromend watle all concentrations grealler than existing or proposed lederal drinking waller standands. These alle nitrate.

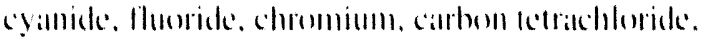

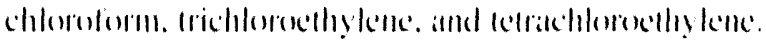

A mumber of the comstituems measured such as comder.

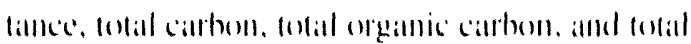

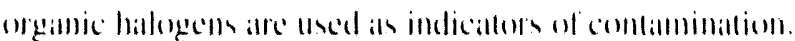
These will men be diectersed in detail in this repont

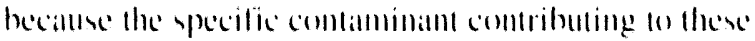

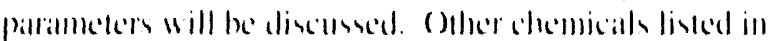
lable 5.25 alle indicallors of the natlual shemicall compo-

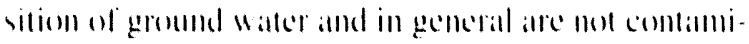
namb from coperalloms all Itantiond. These incluke

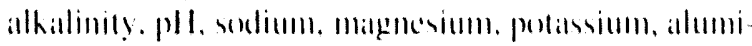
num. silicar. calcium, mangannese, and iron. ('hloride and

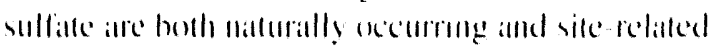

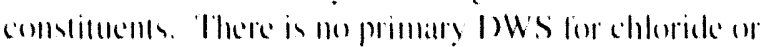

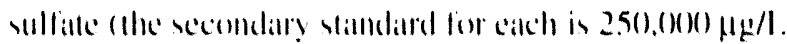
and is based onl asesthetic rather ham heallh comsideralioms so they will mos be discussed in detail. The

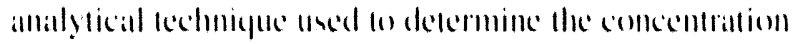

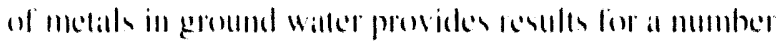
of comstillems that alle rallely observed above bilch-

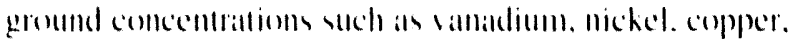

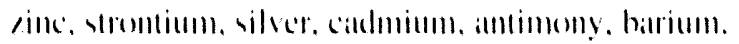
heryllium, and horon.

The bollowing subections present addelitional intormation

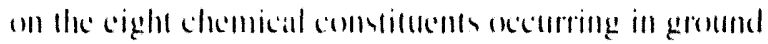

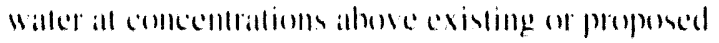
I)WS.

Nitrale, Most grommd-Waller simplen collected in love

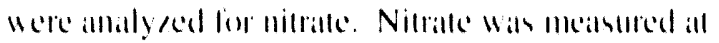
collecontratloms grealler llam lhe I)WS $(4.5 \mathrm{mg} / \mathrm{l}$. an No),

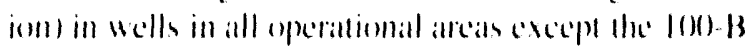
illid $f(0)$ Areats.

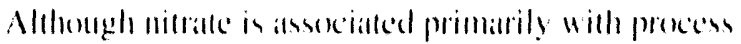

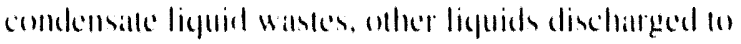

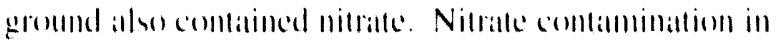

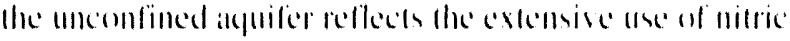




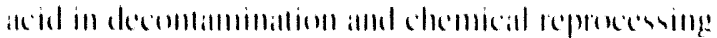

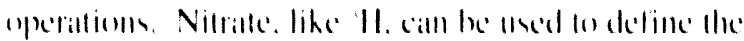

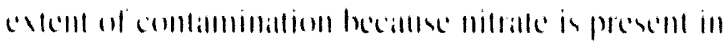

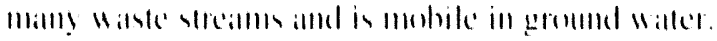

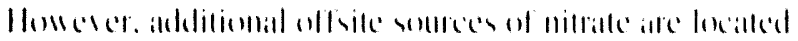

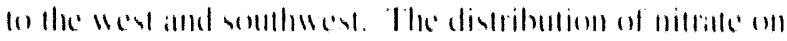

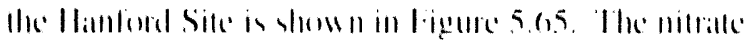

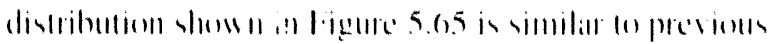
(iilluallioms.

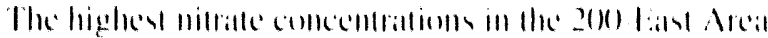

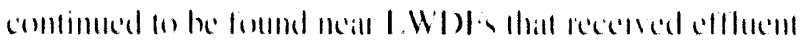

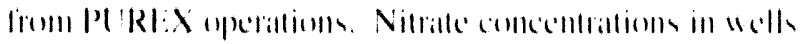

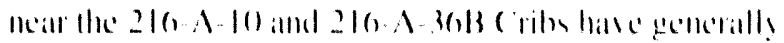

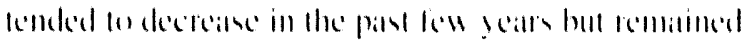

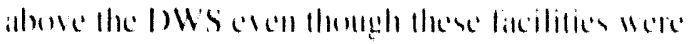
removed limm serice in 1987.

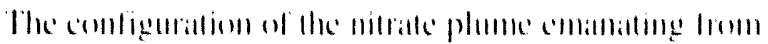

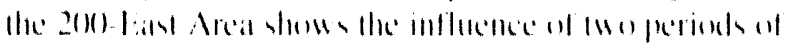
P RI:

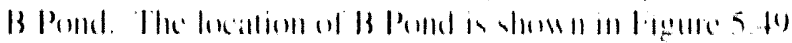

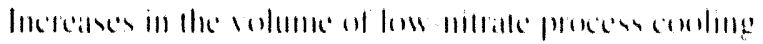

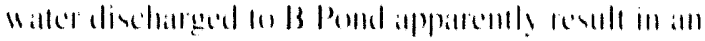

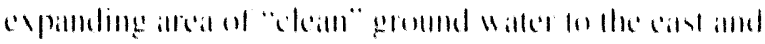

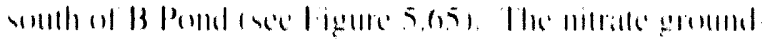

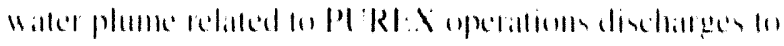

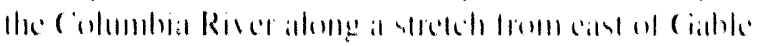

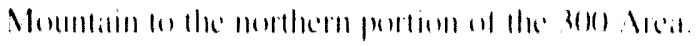

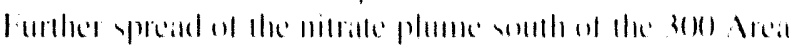

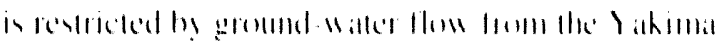

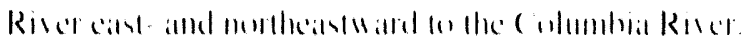

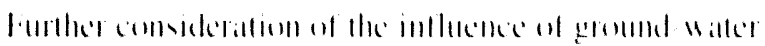

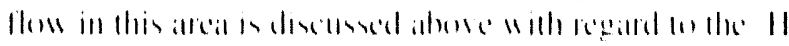
phumic

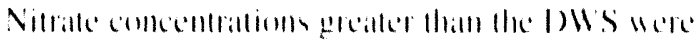

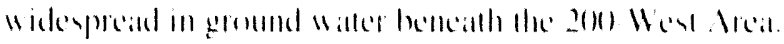

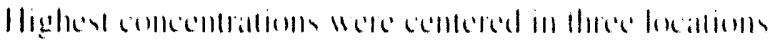

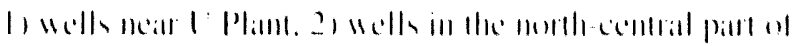

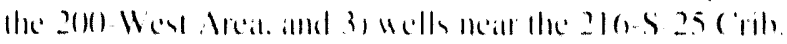

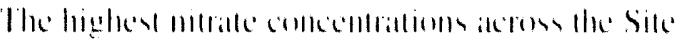

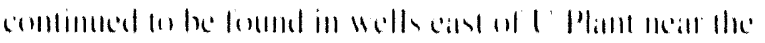

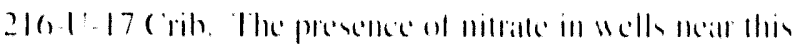

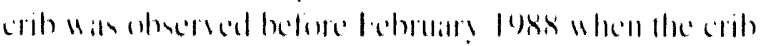

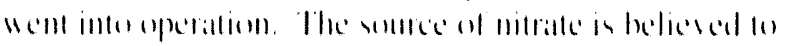

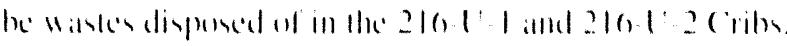

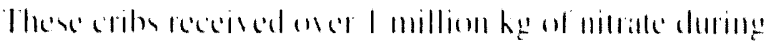

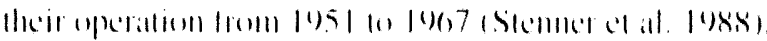

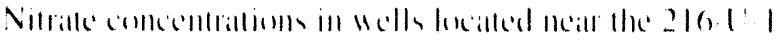

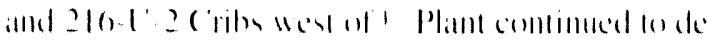

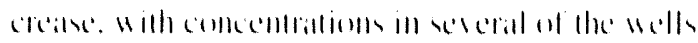

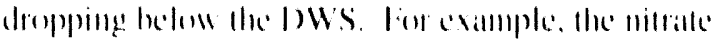

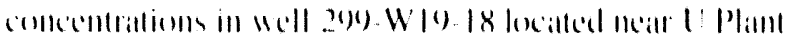

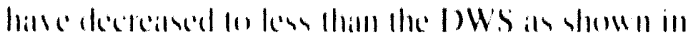
liguturesolo.

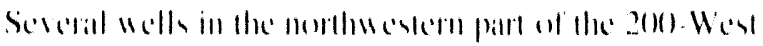

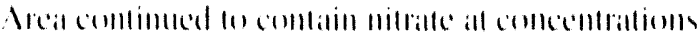

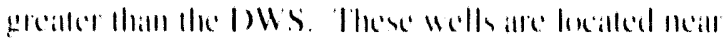

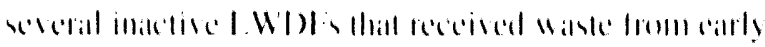

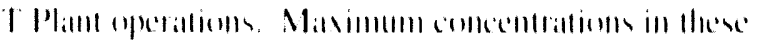

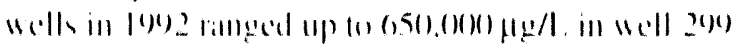

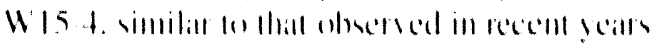

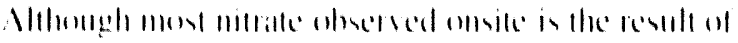

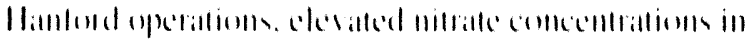

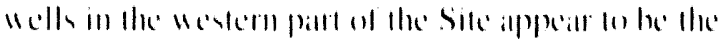

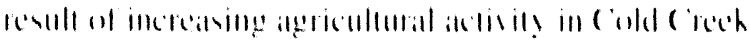

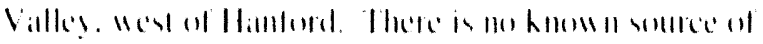

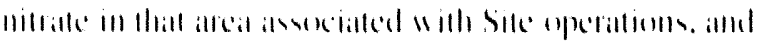

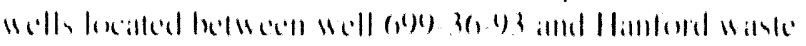

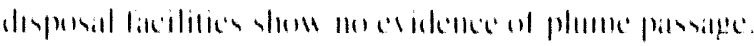

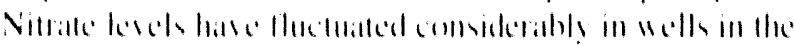

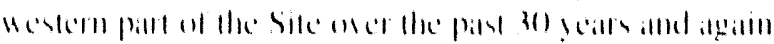

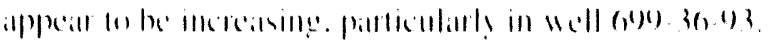

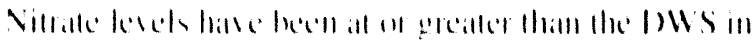
Hatl well sillce IDSi

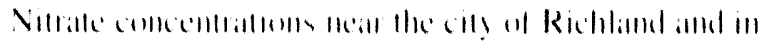

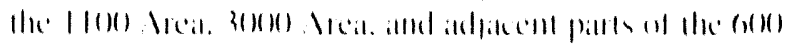

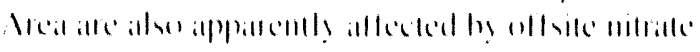

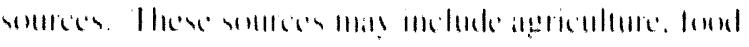

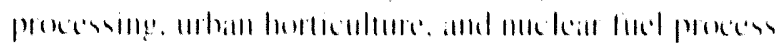

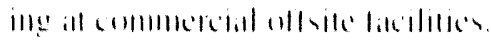

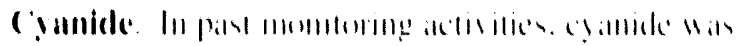

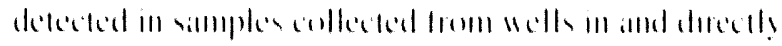

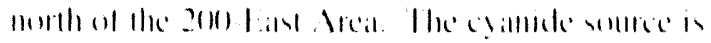

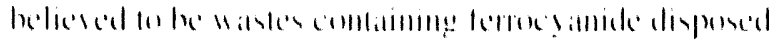

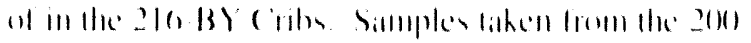

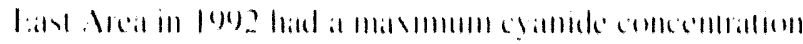

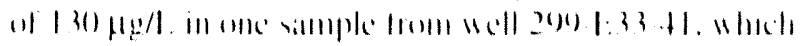

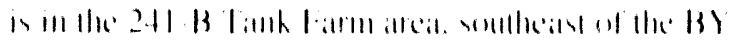

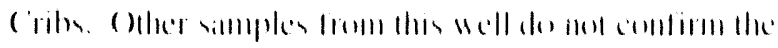

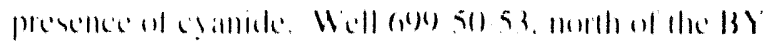

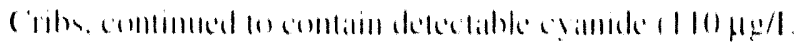

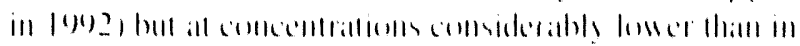

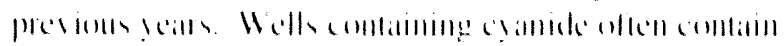

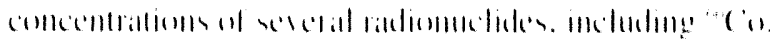

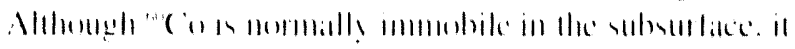

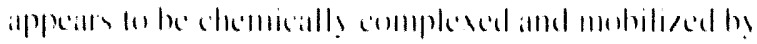

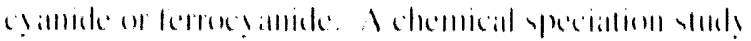




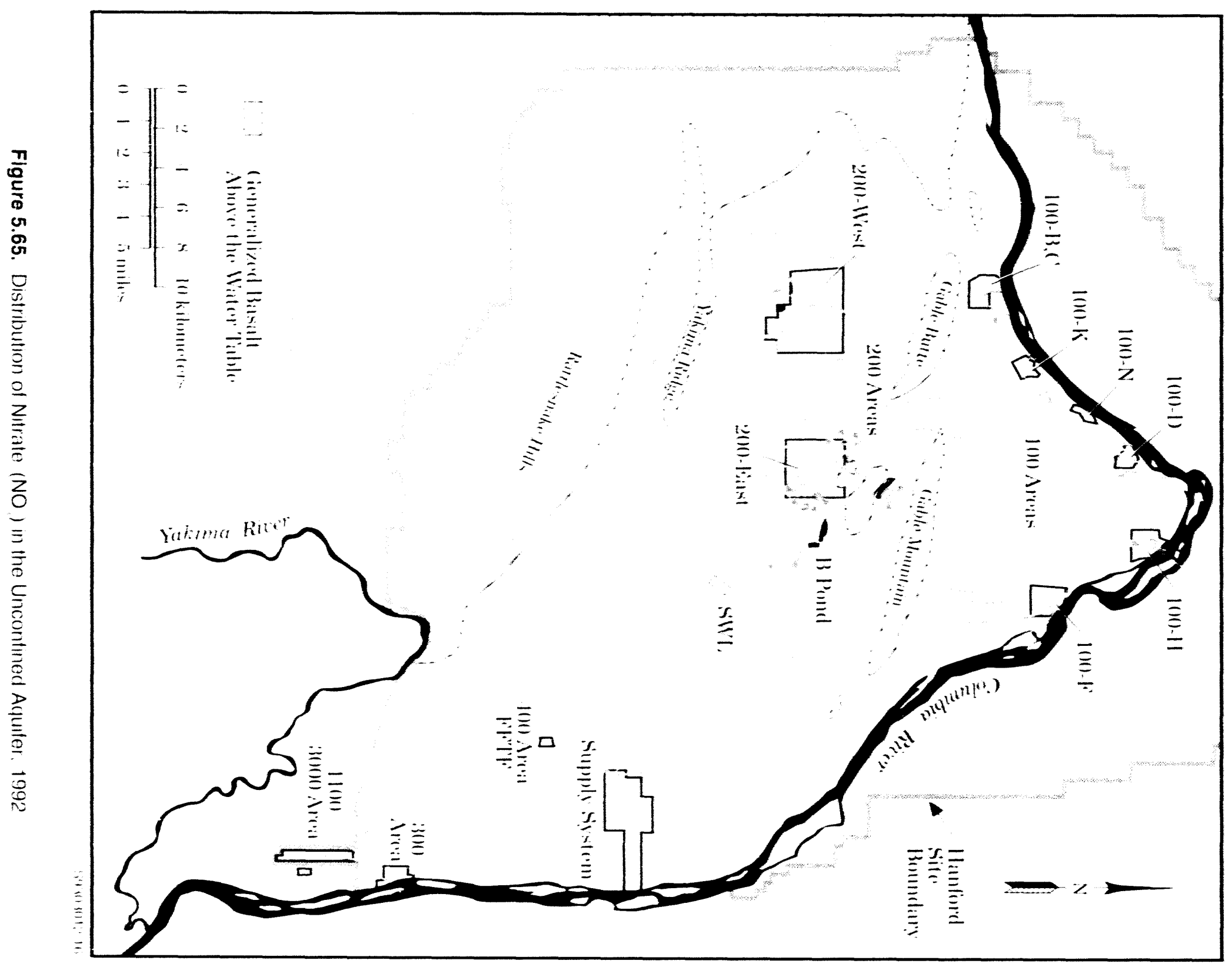




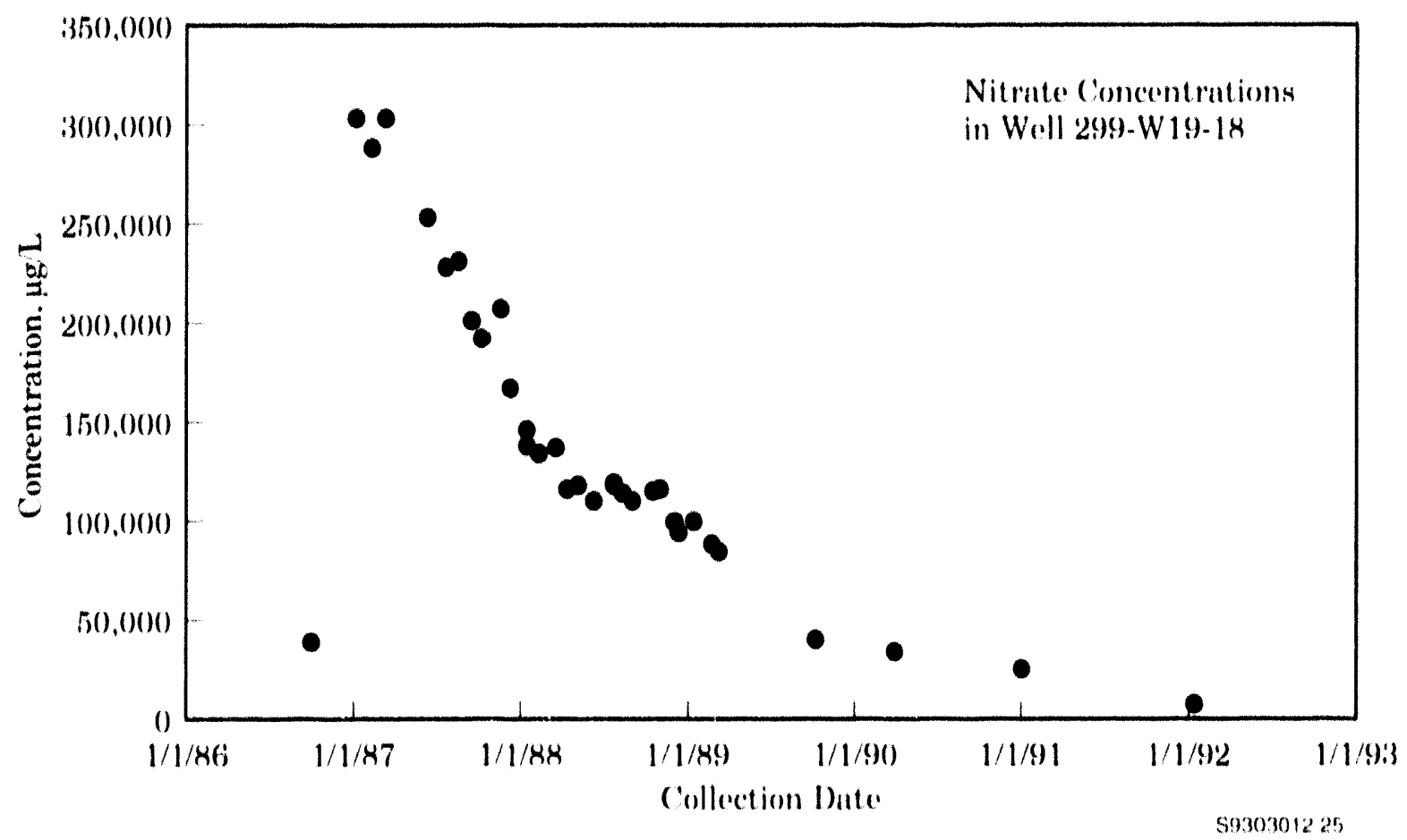

Figure 5.66. Nitrate (NO, ) Concentrations in Well 299-W19-18, 1986 Through 1992

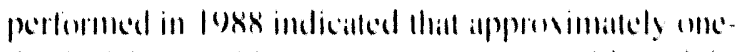

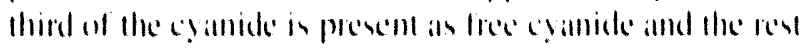

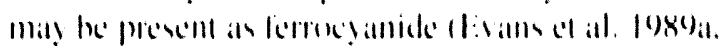
|บष()|,

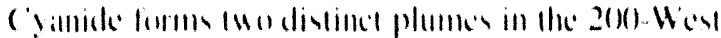

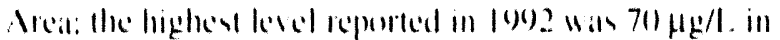

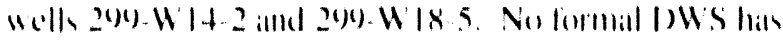

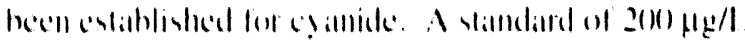

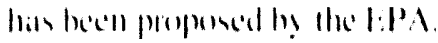

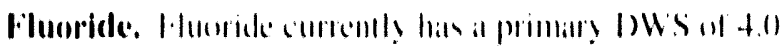

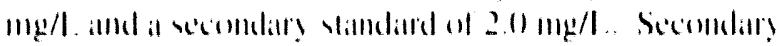

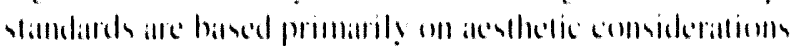

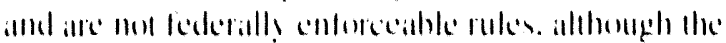

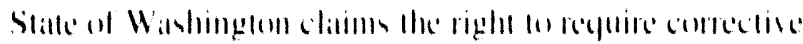

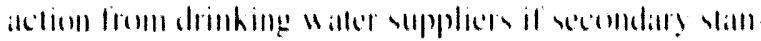

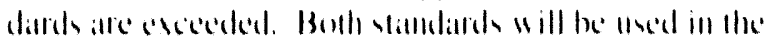

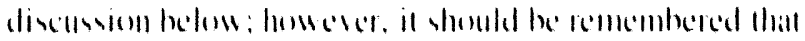

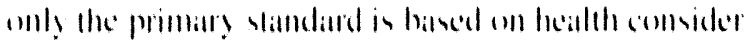

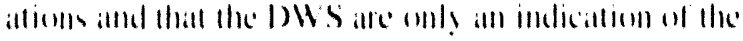

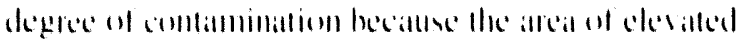

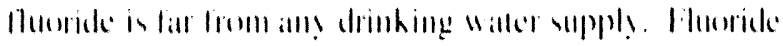

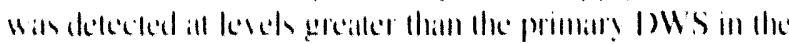

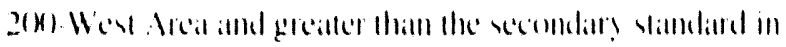

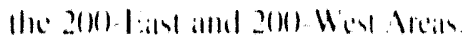

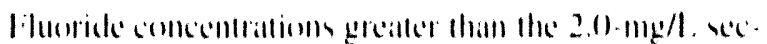

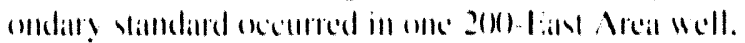

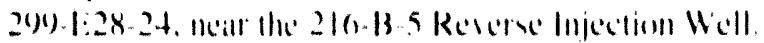
The masimum concentration deteded in fhe well in (1)() $2 แ a 2.7 \mathrm{~mL} / \mathrm{l}$.

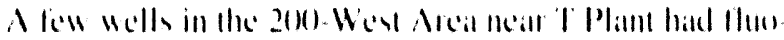

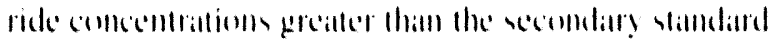

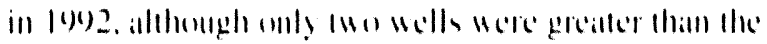

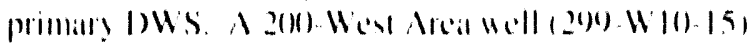

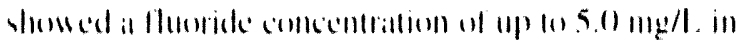
1902. Well 200 15 t bomed lle maximum flumide

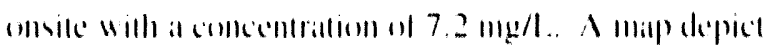

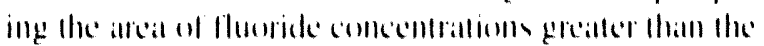

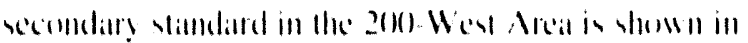

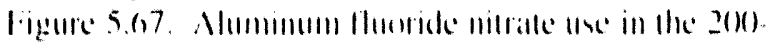

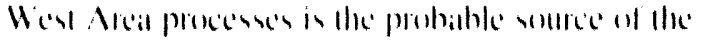
Honricke phume

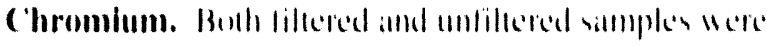

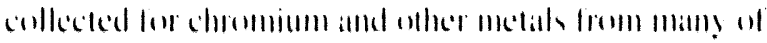

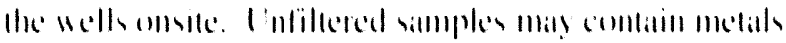

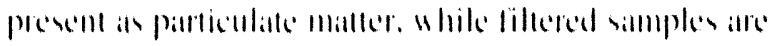

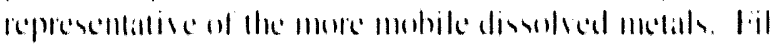

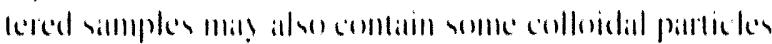

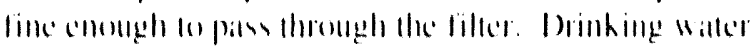




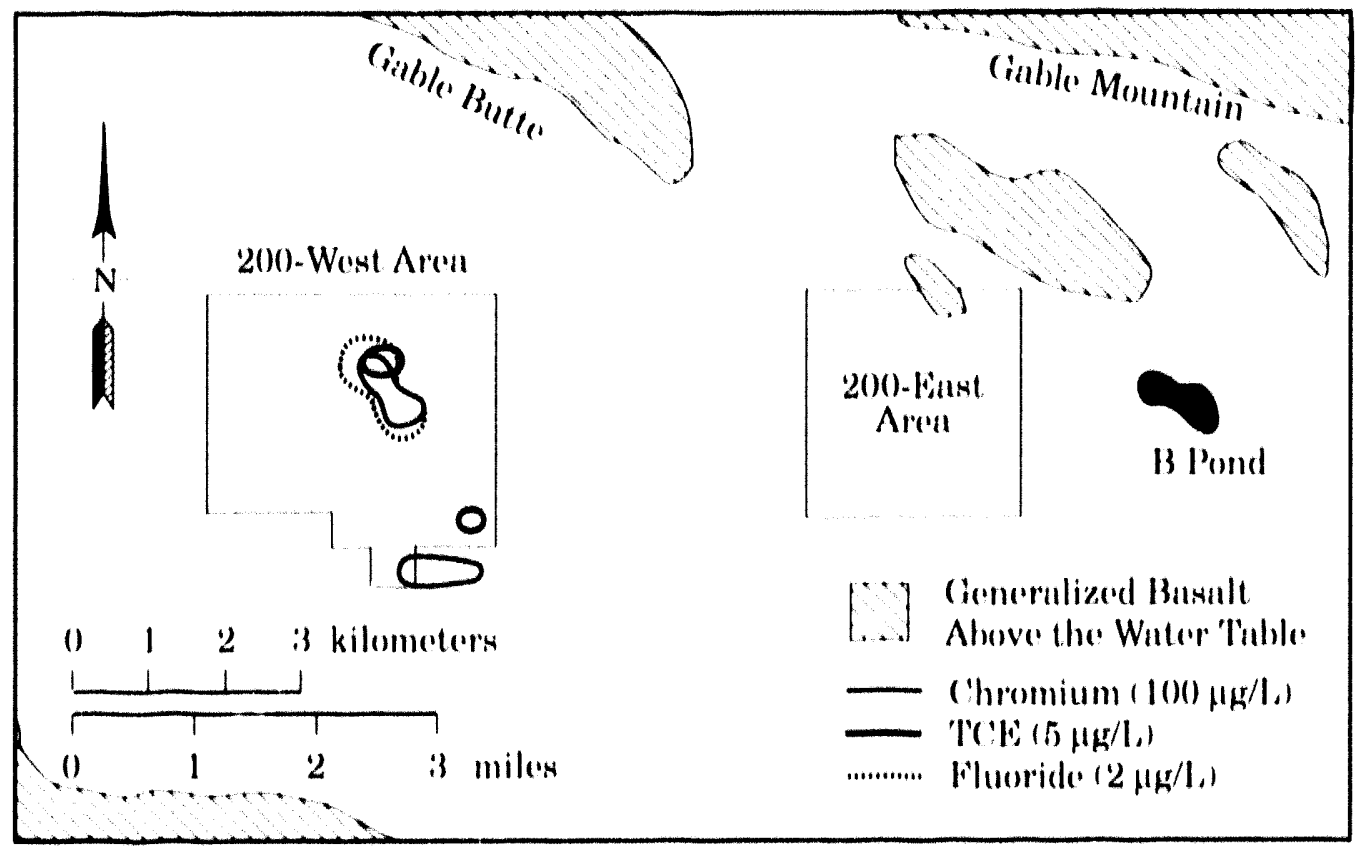

s930301:47

Figure 5.67. Distribution of Selected Hazardous Chemicals Greater Than the Drinking Water Standard near the 200 Areas, 1992. Drinking water standard for each constituent is shown in parentheses.

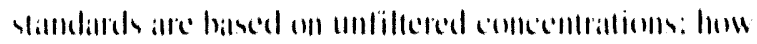

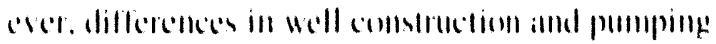
between momilonimg wells and waller supply wolls make

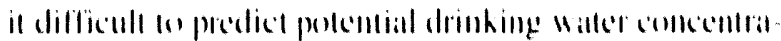

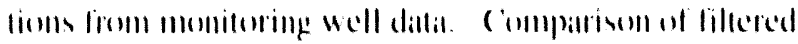

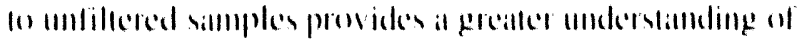

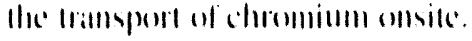

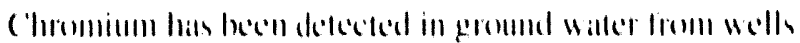

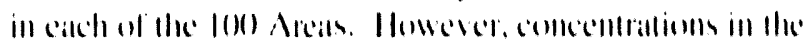

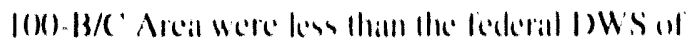

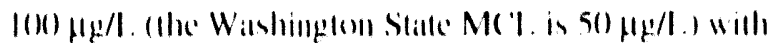

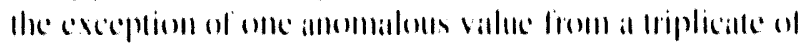
allalyses. The chromimm distribuliom in the lon) Areas is slown in ligume 5.00 .

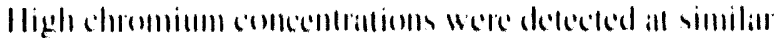
levels in beth liflered and undillered samples from the

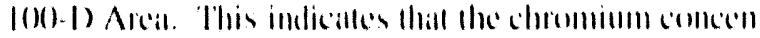

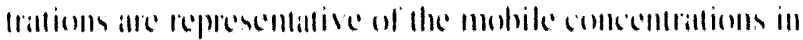
lhe gromend willer.

Relatively lew chromium antalyses anc anailable from the

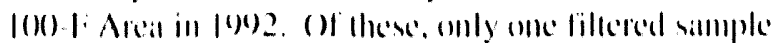

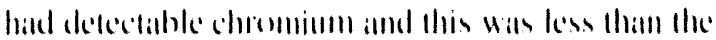
IIWS

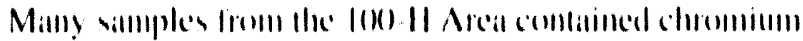

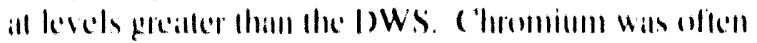
present all similial lenels in both fillered and unliltered

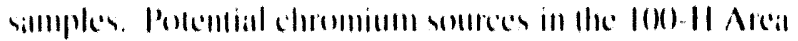

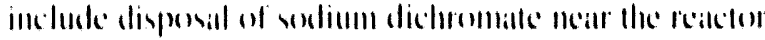

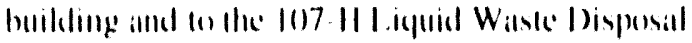

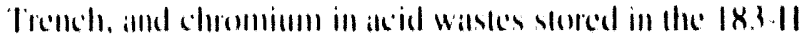

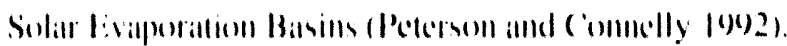

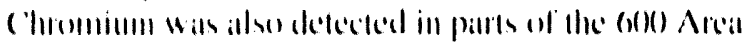

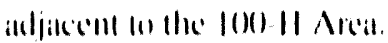

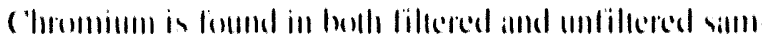

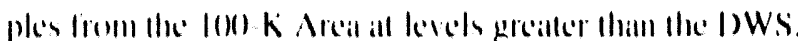

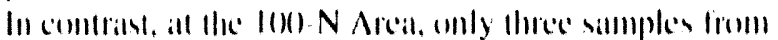

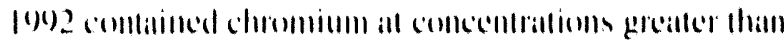

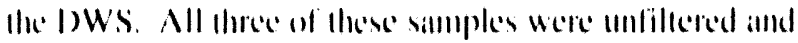

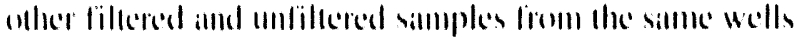
combanimed chromimm all levels less ham llo doWS. Thus,

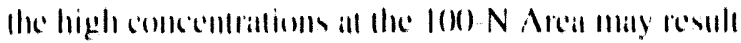

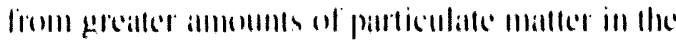
silimples.

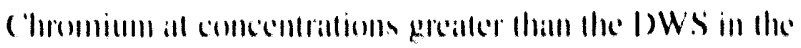
2(1) fand Area is lomml only in unfillered simples with

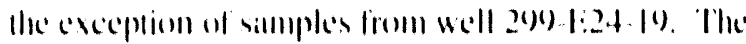




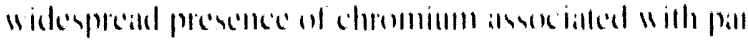

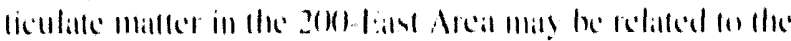

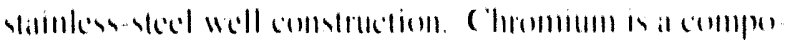

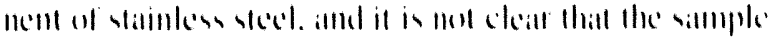

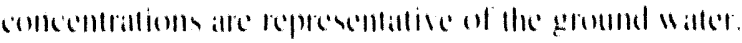

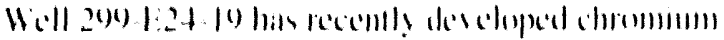

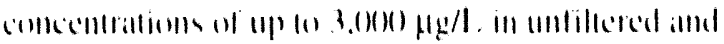

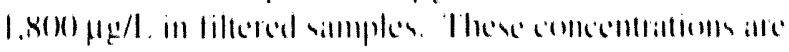

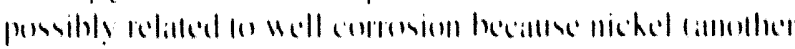

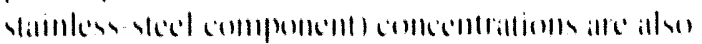

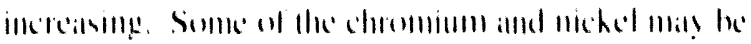

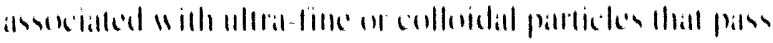

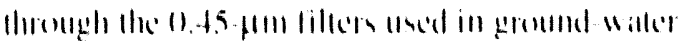
silmpling.

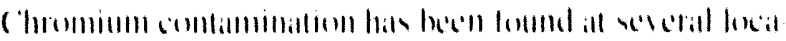

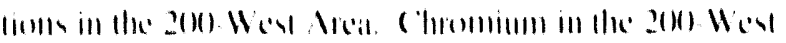

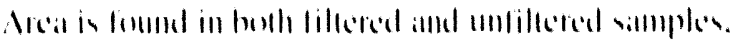

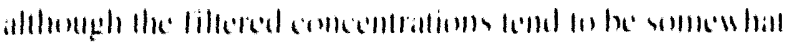

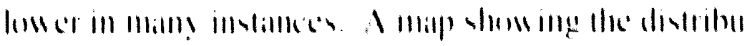

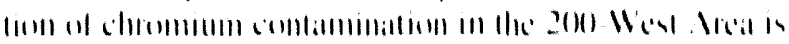
vlom in ligure 5,67

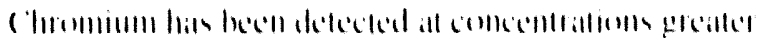

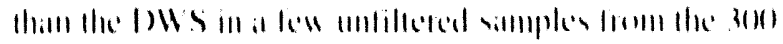

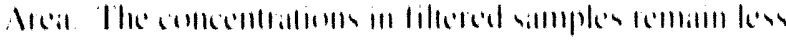

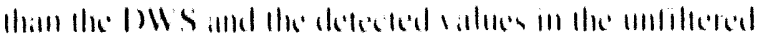

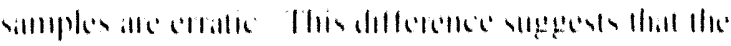
ligh s chrom

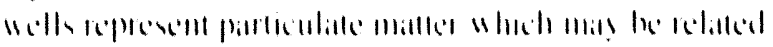

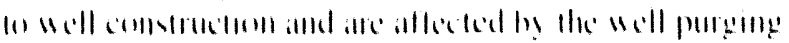

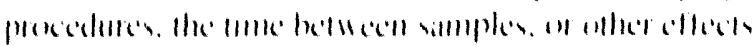

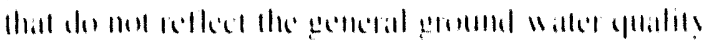

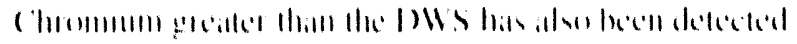

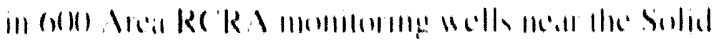

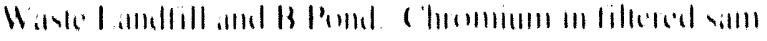

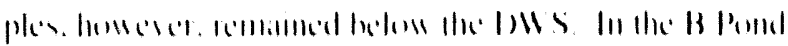

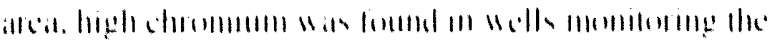

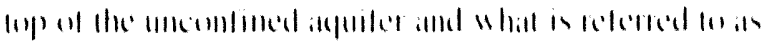

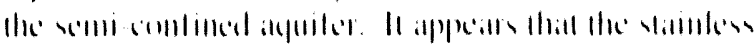

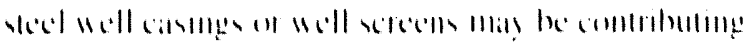

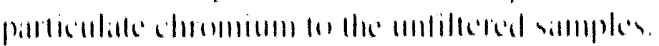

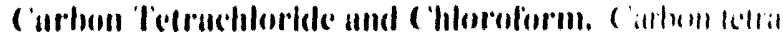

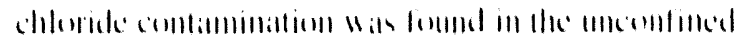

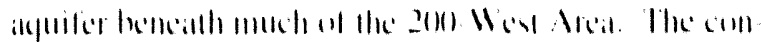

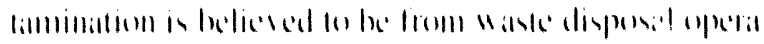

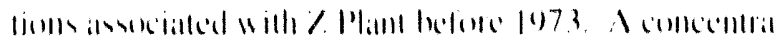

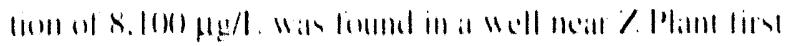

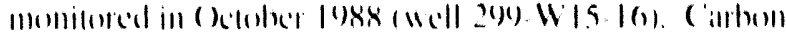

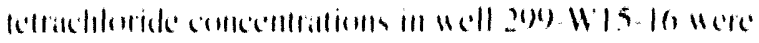

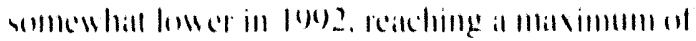

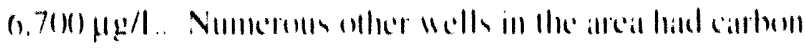

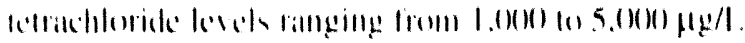

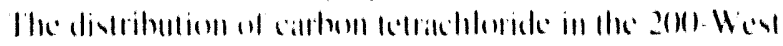

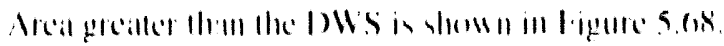

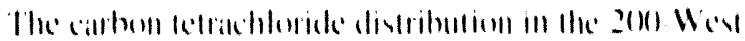

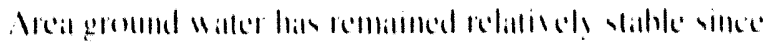

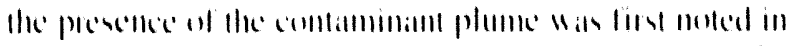

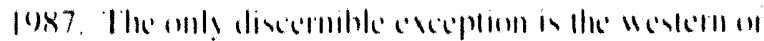

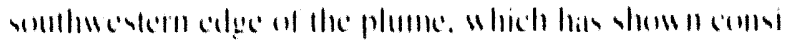

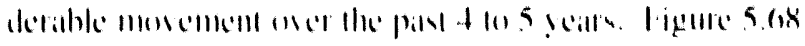

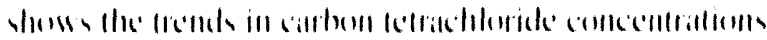

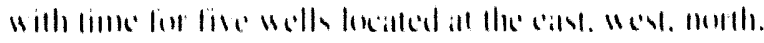

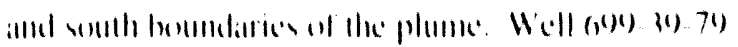

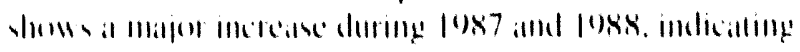

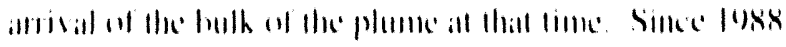

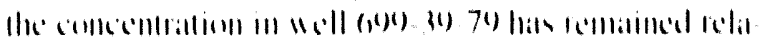

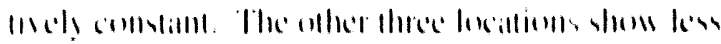

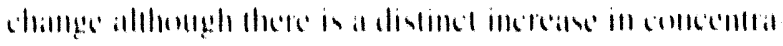

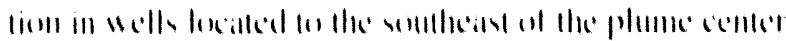

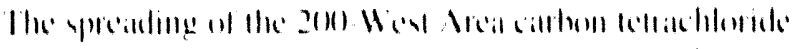

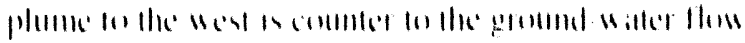

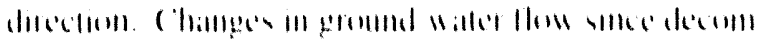

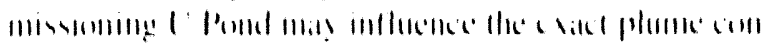

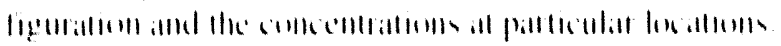

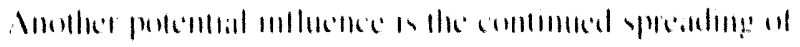

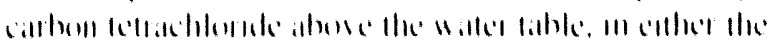

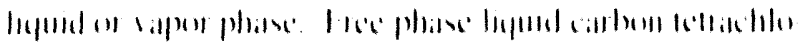

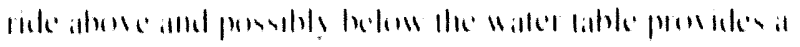

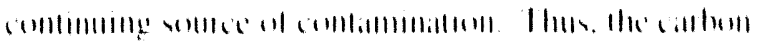

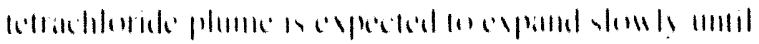

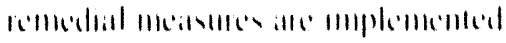

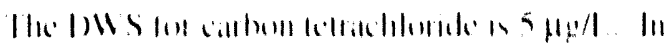

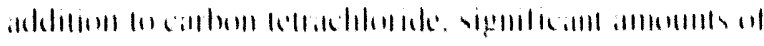

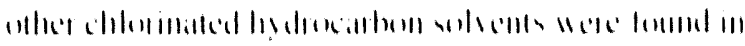

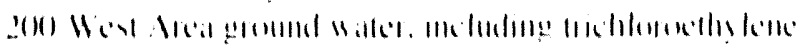

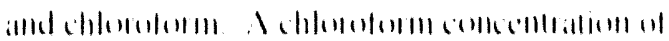

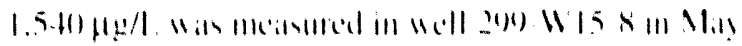

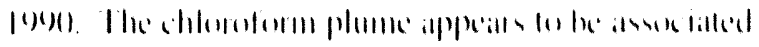

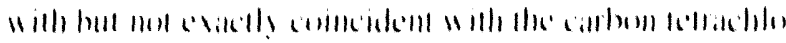

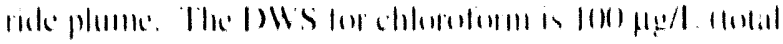

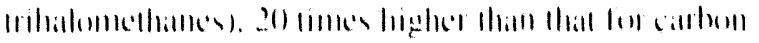

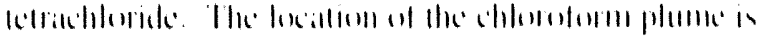

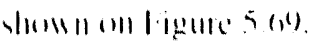




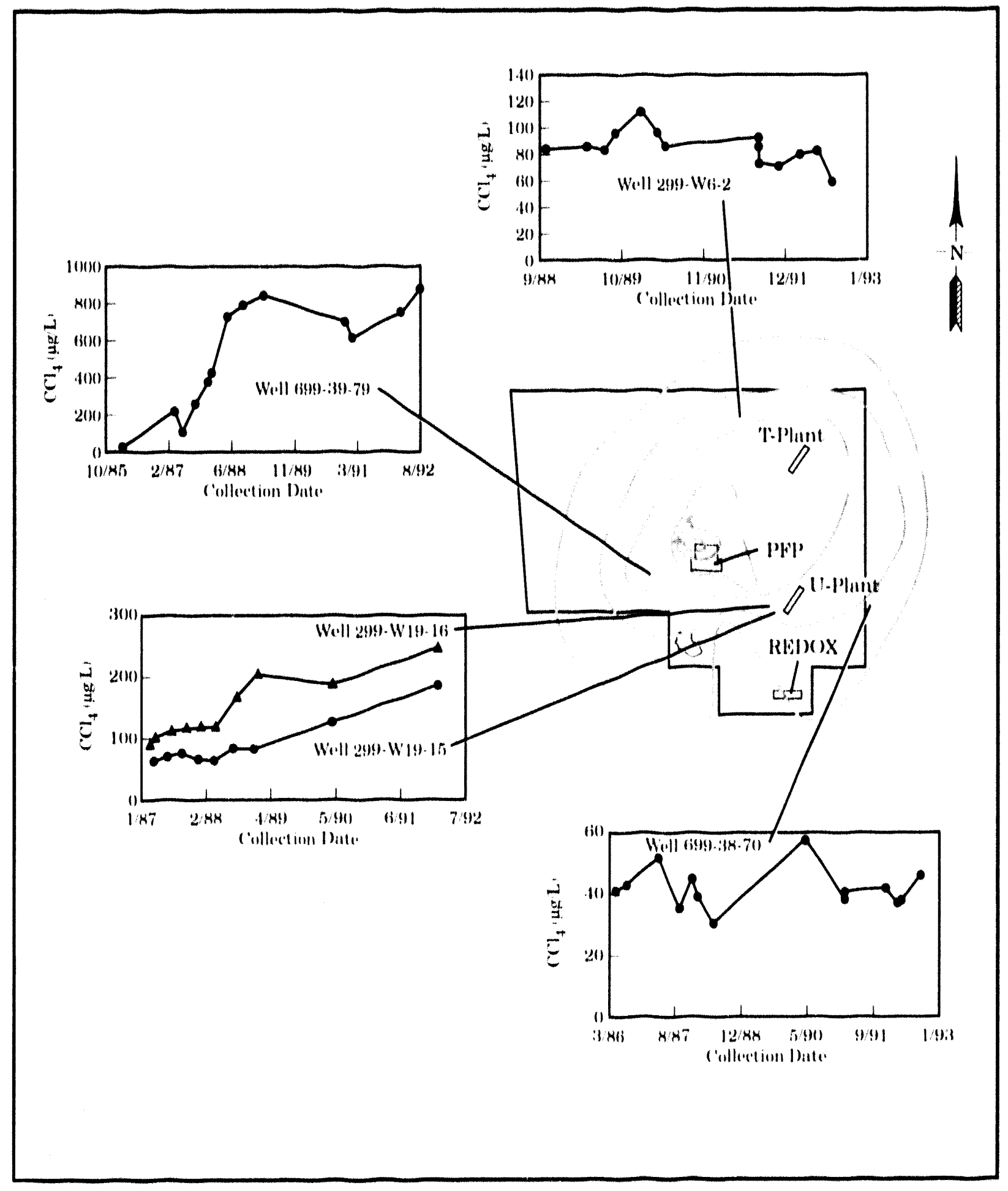

5930301255

Figure 5.68. Distribution of Carbon Tetrachloride $\left(\mathrm{CCl}_{4}\right)$ in the 200-West Area, 1992 
Trichlorocthylene. Trichlomethy lene hais al I)W

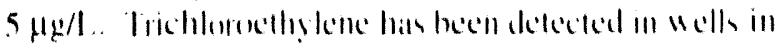

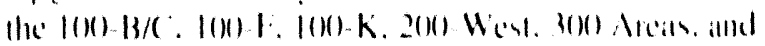

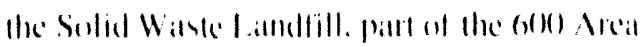

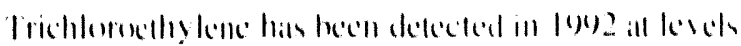

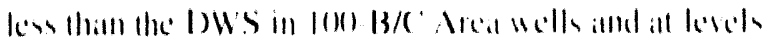

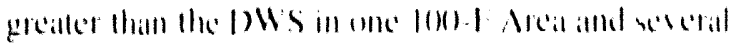

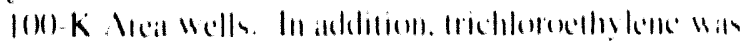

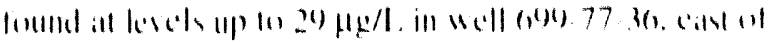

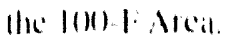

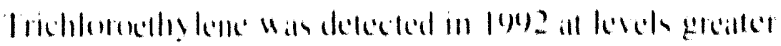
Whall the I)

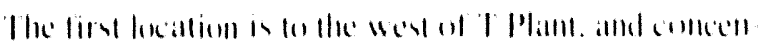

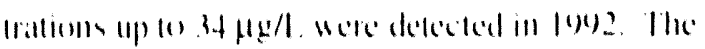

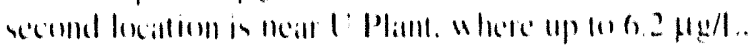

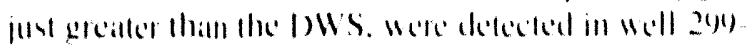

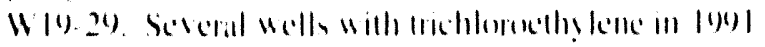

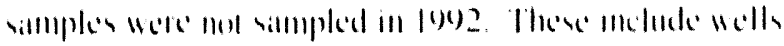

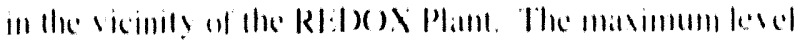

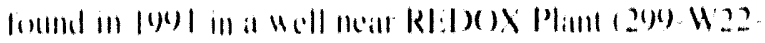
2011 wis $3+\mu \mathrm{g} / \mathrm{L}$.

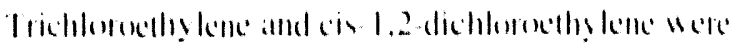

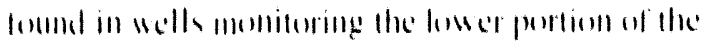

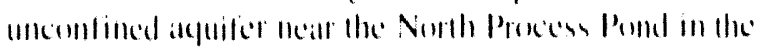

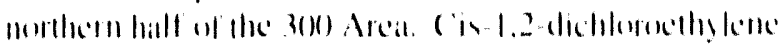

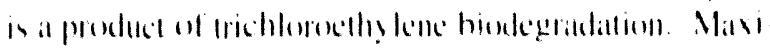

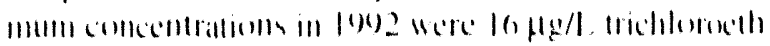

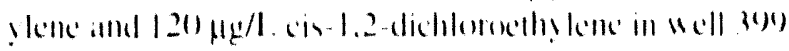

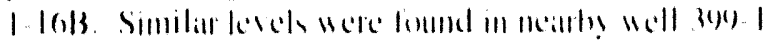

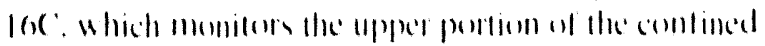

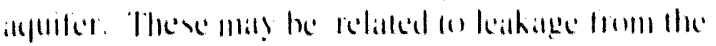

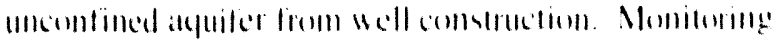

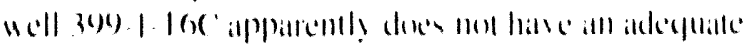

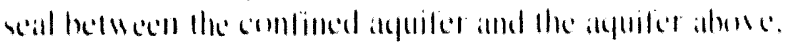

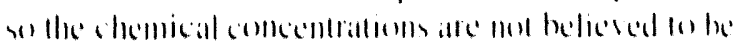

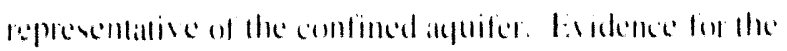

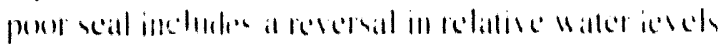

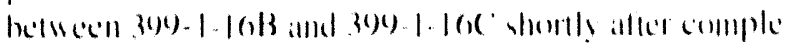

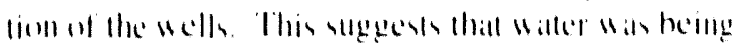

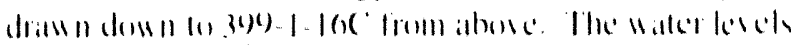

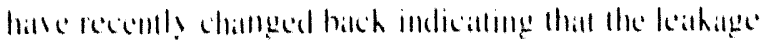

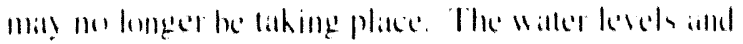

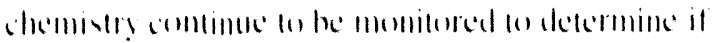

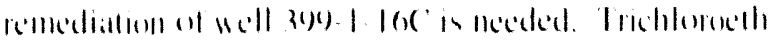

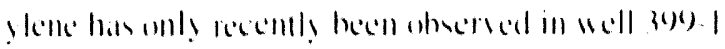

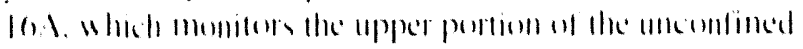

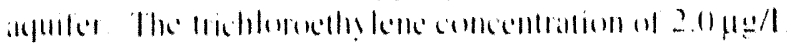

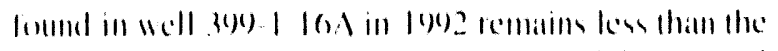

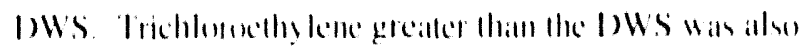

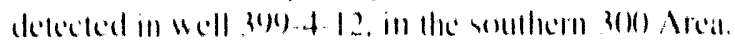

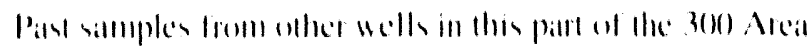

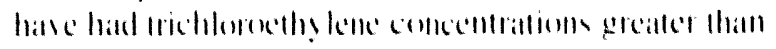
the llws

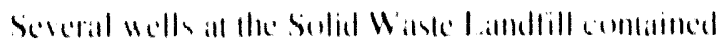

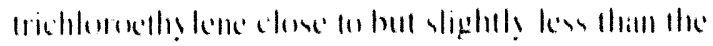

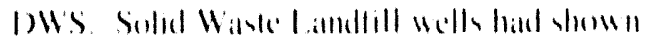

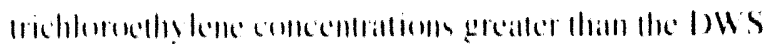

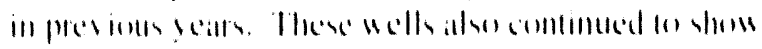

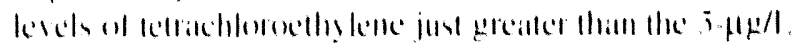

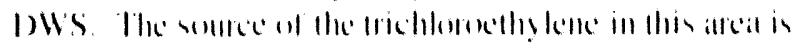

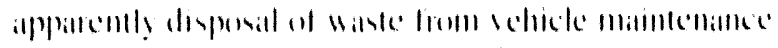

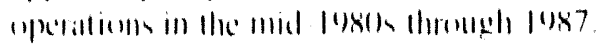

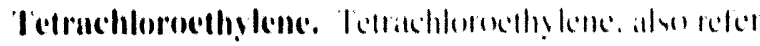

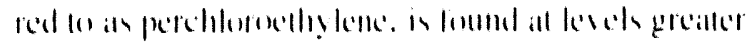
Hhan the DWS in a mumber of atreins al the Sile includeng

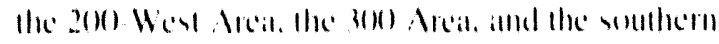

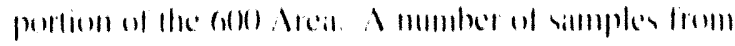
"10

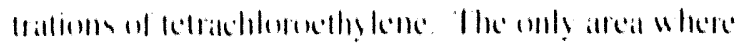

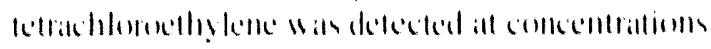

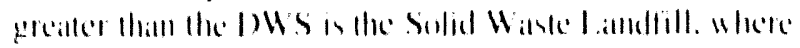
the collcentrations reathed a max

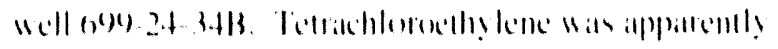

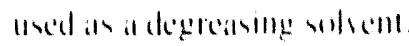

\section{Radiological and Chemical Monitoring Results for the Confined Aquifer}

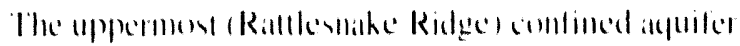

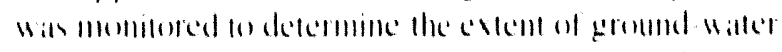
interaction betwesen the continted and ansomfined

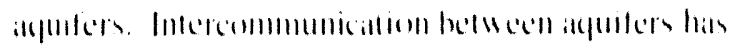

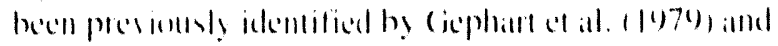

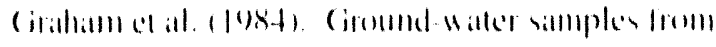

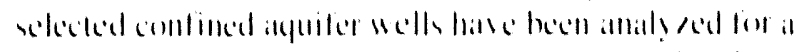

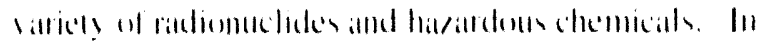

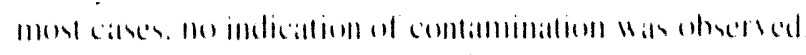

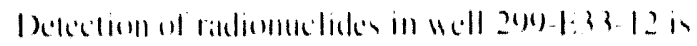

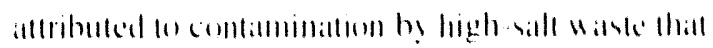

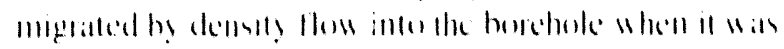

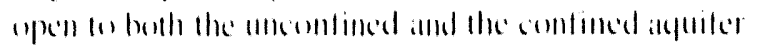

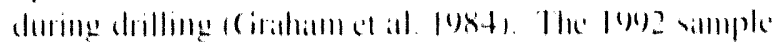

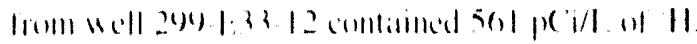




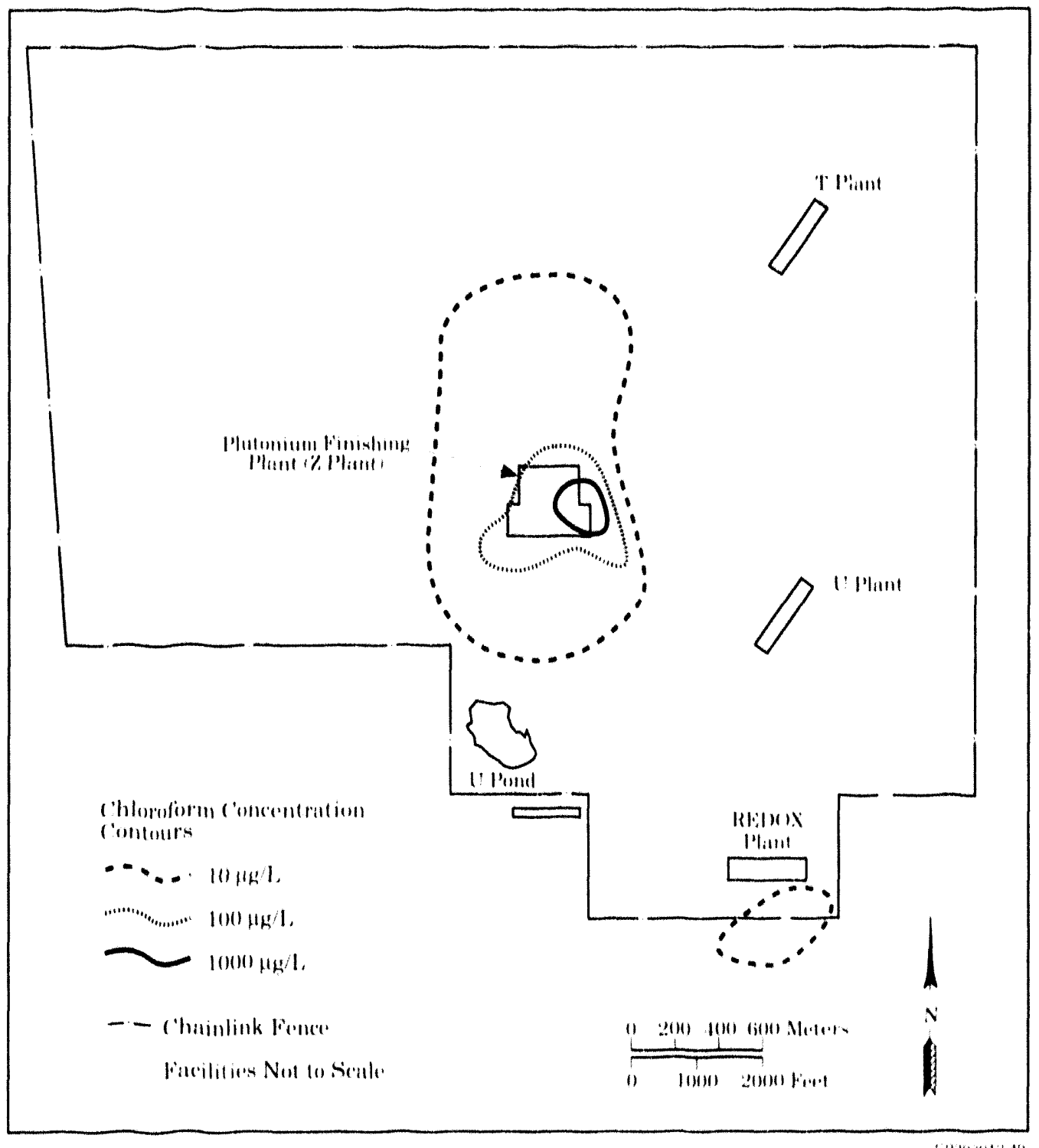

'94010119'49

Figure 5.69. Distribution of Chloroform in the Unconfined Aquiler near the 200-West Area, 1992

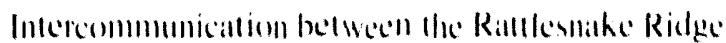

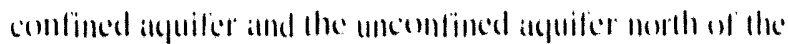
200) lats Area was indicalcel by nillatce concentralloms in well (6) $-47-50$, which were 8.80()$\mu g / 1$. in 10)2. This well is localled neatr all crosiomil window tan areal where

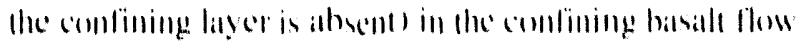

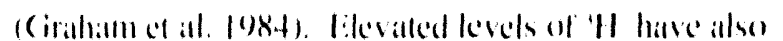

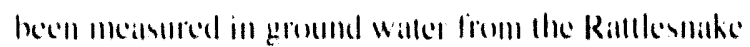
Ridge inlerhed in well (10).+2.+4)( This well comlaimed

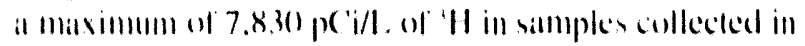

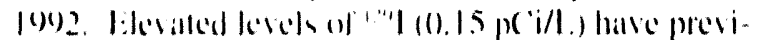

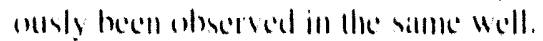




\section{Potential Radiation Doses from 1992 Hanford Operations}




\subsection{Potential Radiation Doses from 1992 Hanford Operations}

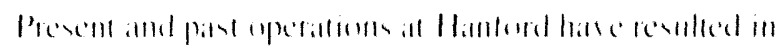

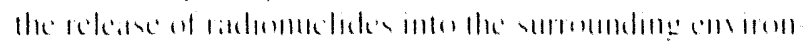

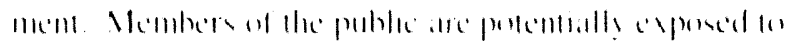

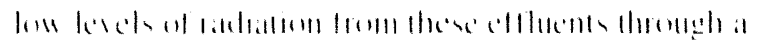

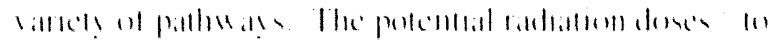

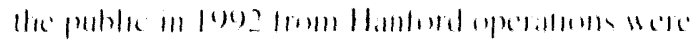

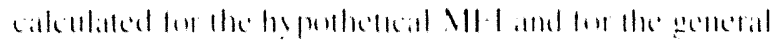

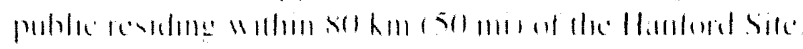

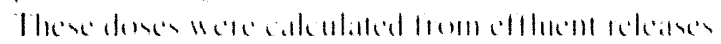

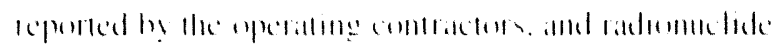

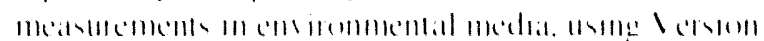

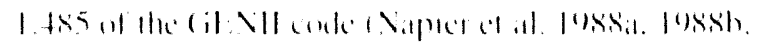

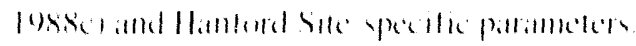

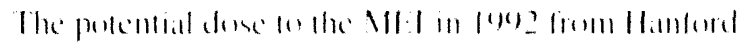

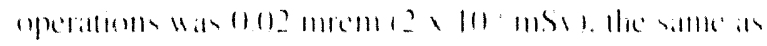

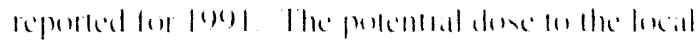

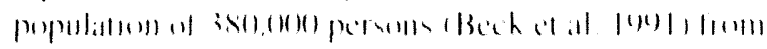

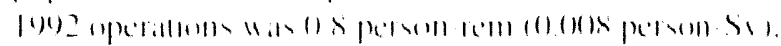

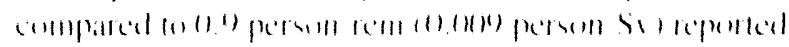

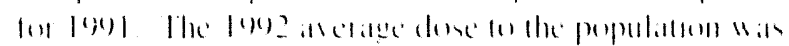

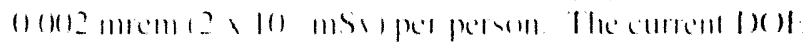

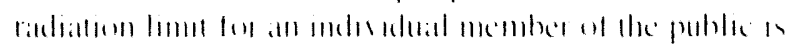

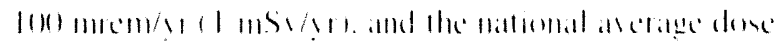

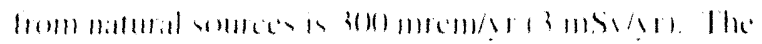

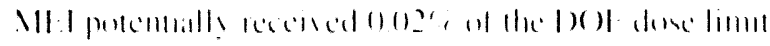

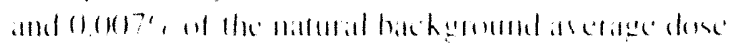

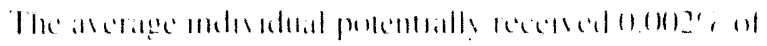

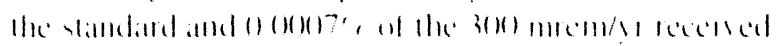

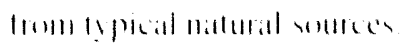

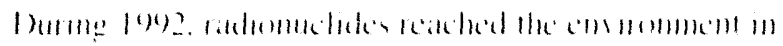

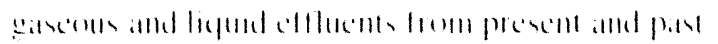

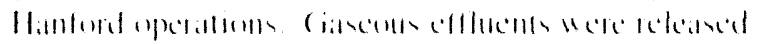

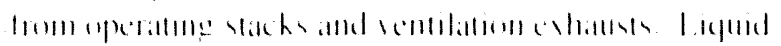

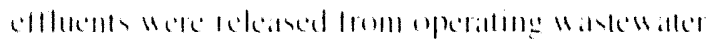

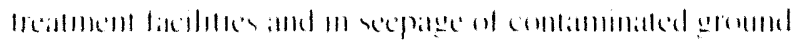

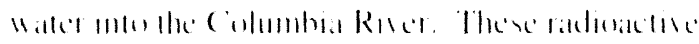

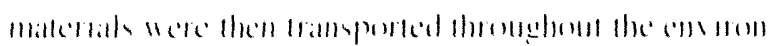

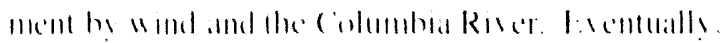

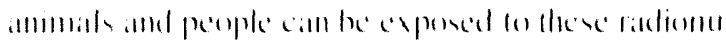

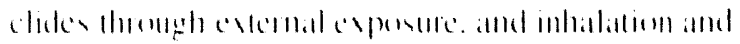

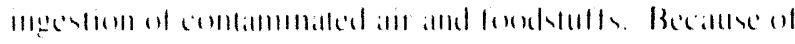

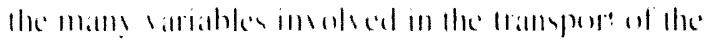

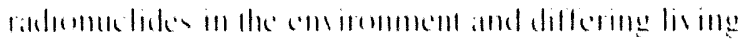

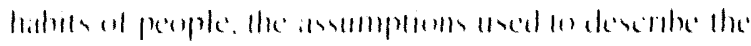

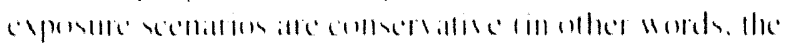

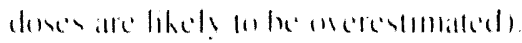

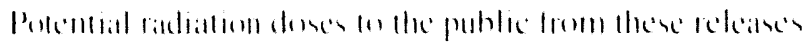

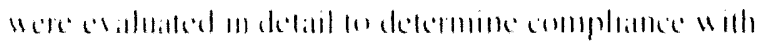

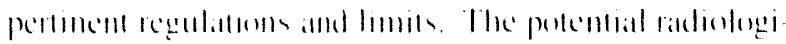

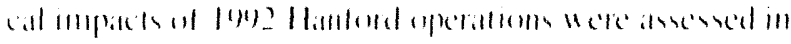

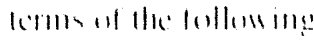

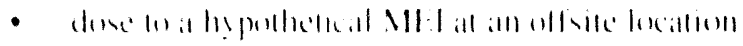

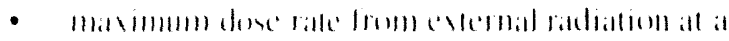

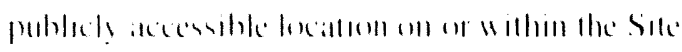
mominlars

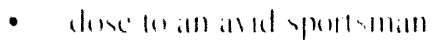

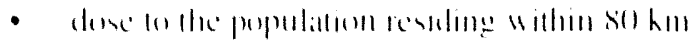
(8) milot the (1)

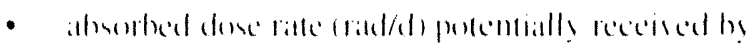

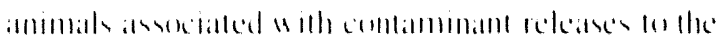
(i)hmhbia River

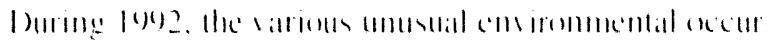

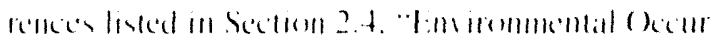

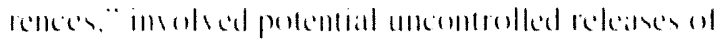

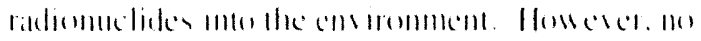

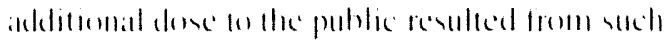
acturrences

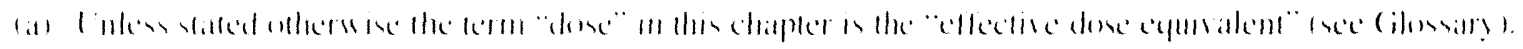




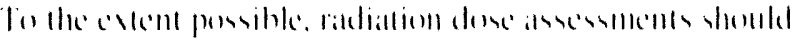

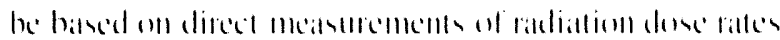

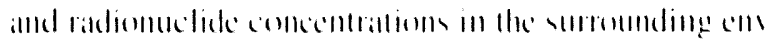

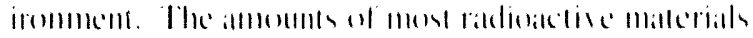

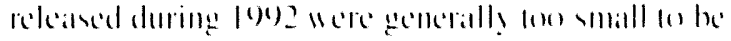

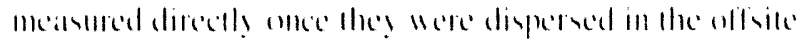

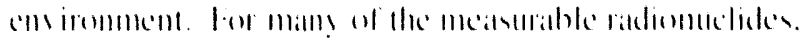

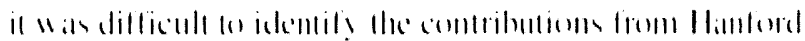

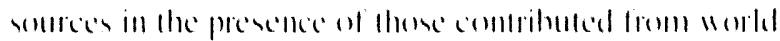

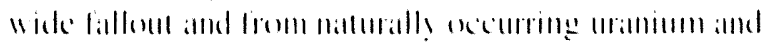

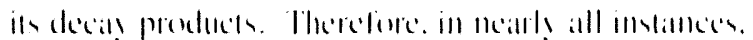

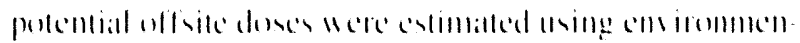

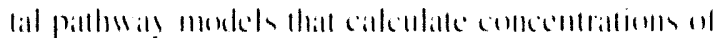

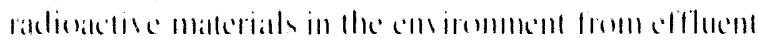

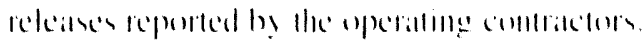

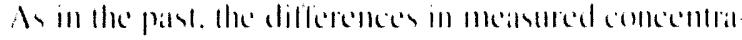

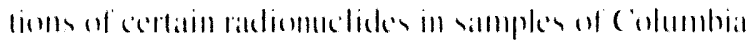

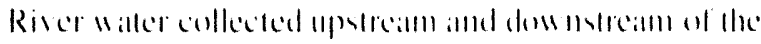

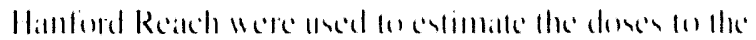

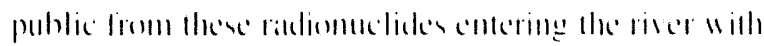

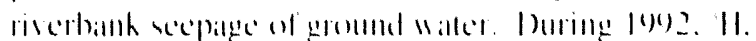

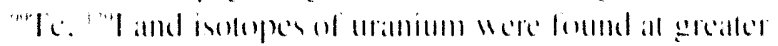

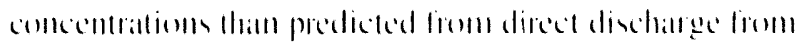
lhe $10(1)$ and $3(0)$ : Areits

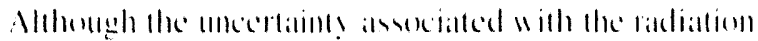

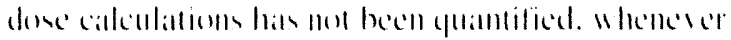

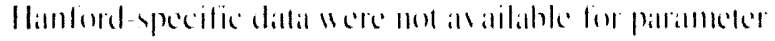

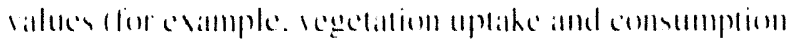

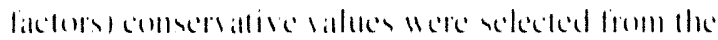

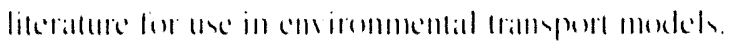

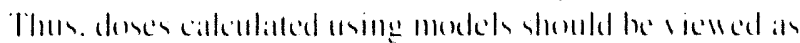

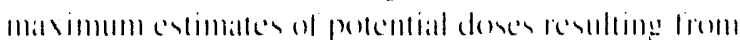
Ilantiond nperations

\section{Maximally Exposed Individual Dose}

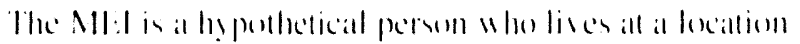

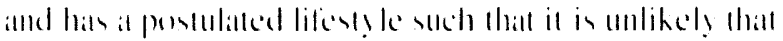

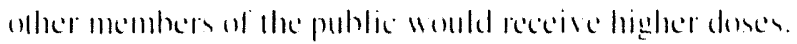

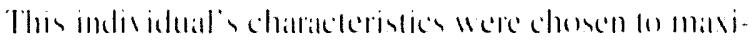

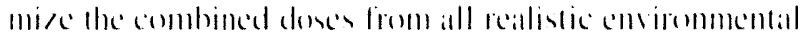

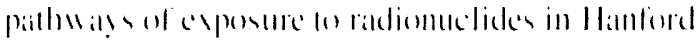

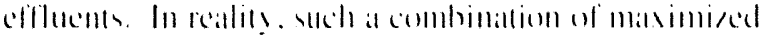

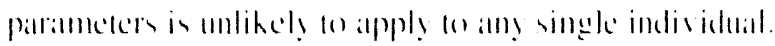

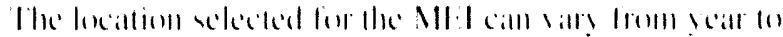

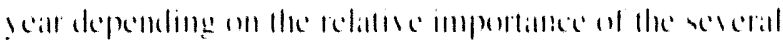

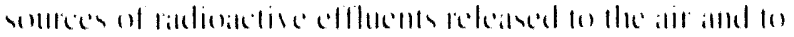

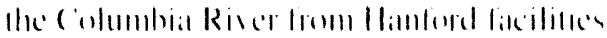

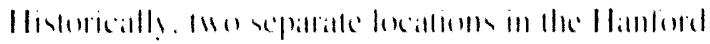

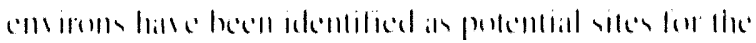

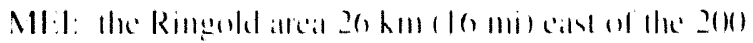

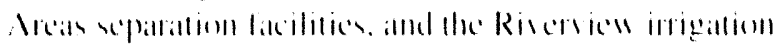

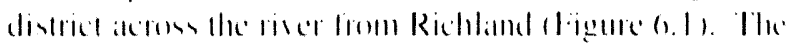

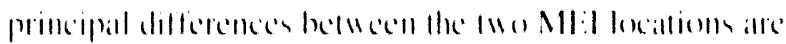

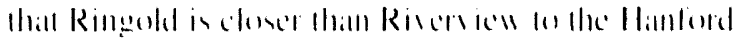

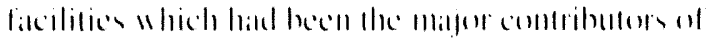

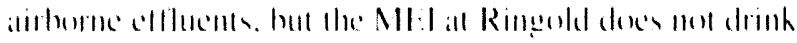

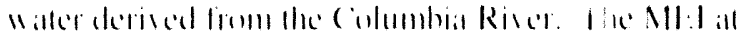

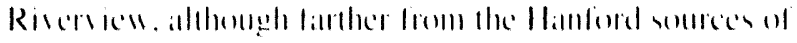

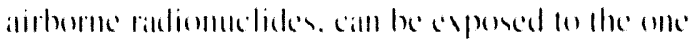

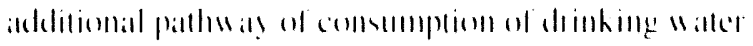
deried from the collumbia Rirer.

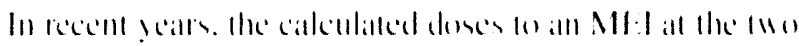

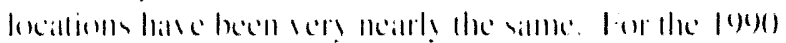

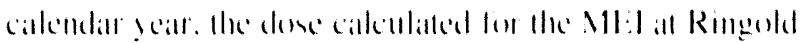

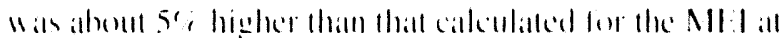

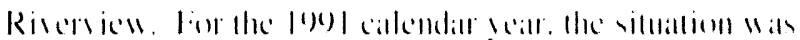

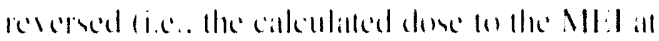
Rireniew was 5'; higher than that cillewalled lon the

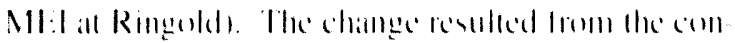

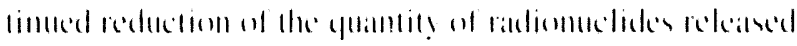

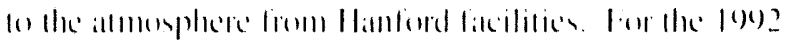

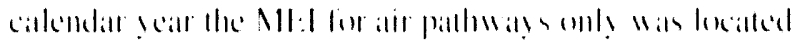
all Ringerdel while lhe Mll: l for all pathwall sombimed Waill Riscrick

The follow ing exporate pallowas were included in the

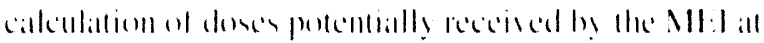

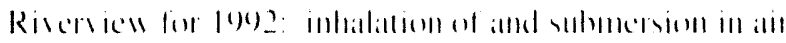

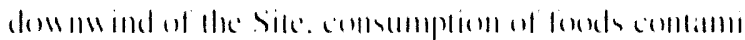

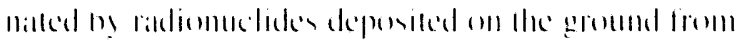

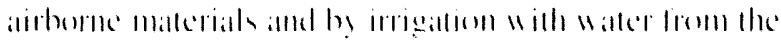

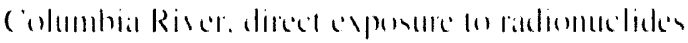

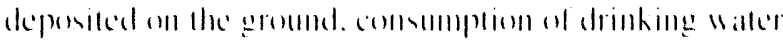

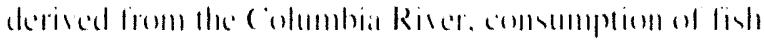

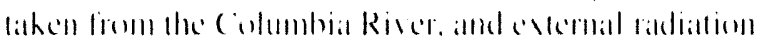

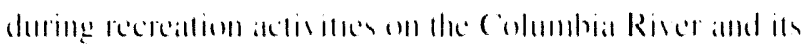

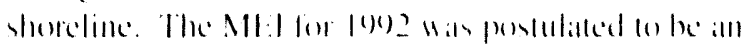
indis idual 11 lu:

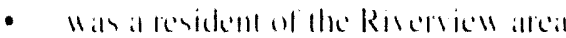




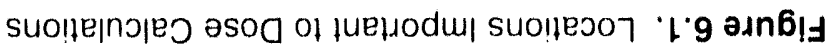

li: 89080265

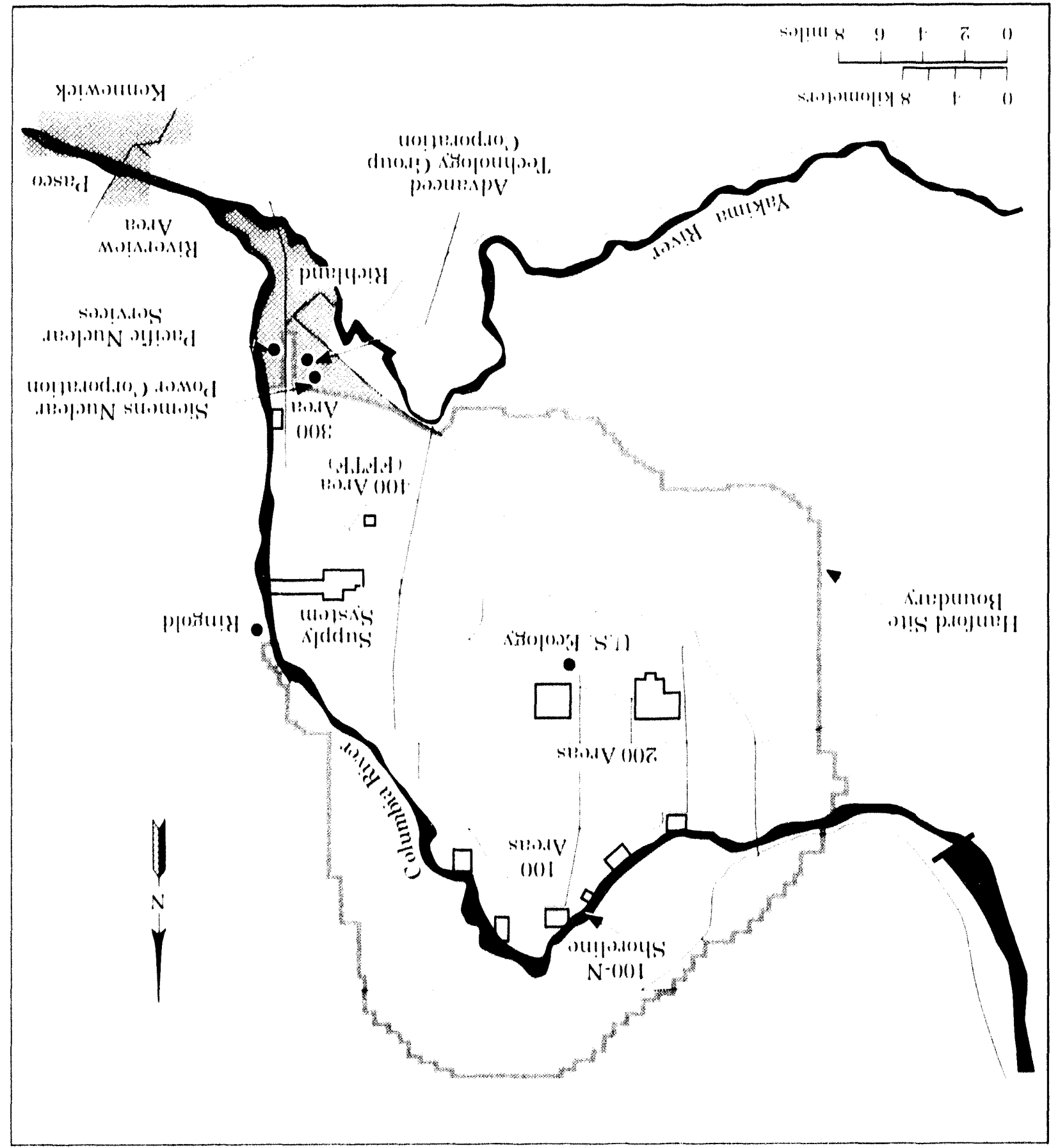




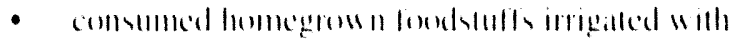
(i)lumbiar Rirer Walle

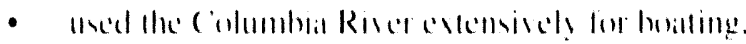
stimminge. and lishomg, and combumled the lish cillyghil

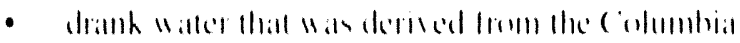

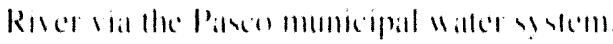

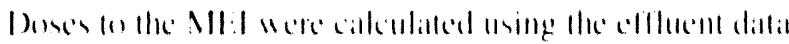

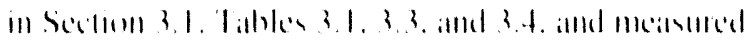

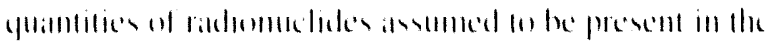

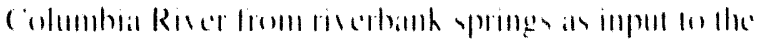

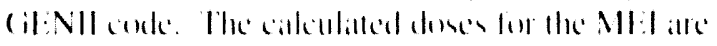

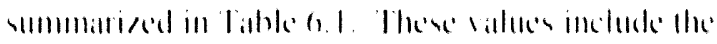

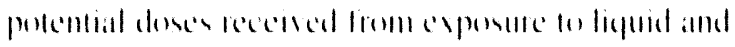

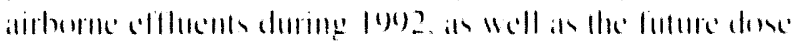

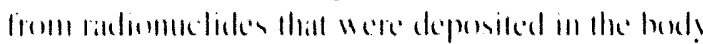

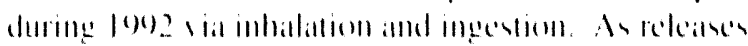

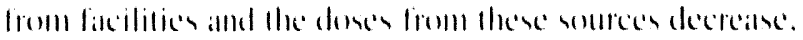

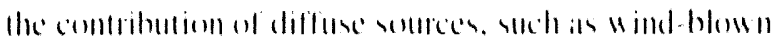

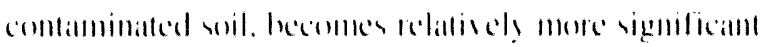

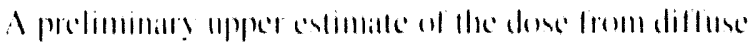

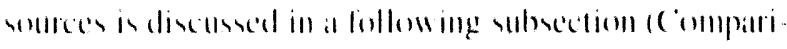

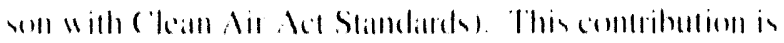

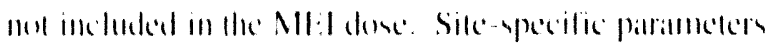

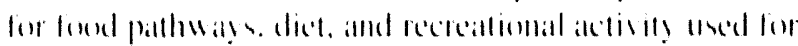

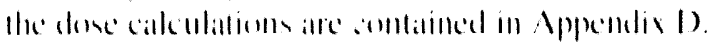

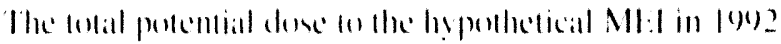

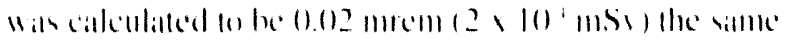
as calculated for in foul. The primally pallowas

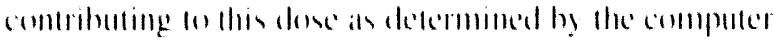
cilculintioms werk

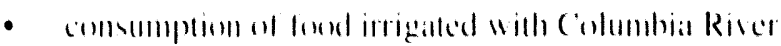

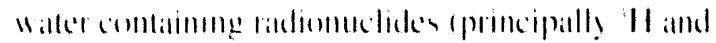
(n) $\left.(4)+()^{\prime}\right)$

Table 6.1. Doses to the Hypothetical Maximally Exposed Individual from Hanford Operations, 1992

\begin{tabular}{|c|c|c|c|c|c|c|}
\hline & & & & 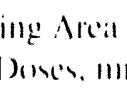 & mition & \\
\hline & & I(1) & $2(10)$ & $3(11)$ & $4(0)$ & Pithwill \\
\hline 1:1llucm & P'ullu:!l! & Arein & Arein & Arria & Areia & |intil \\
\hline Air & Evamal & 4100 & 5,101 & $3 \times 10 \%$ & $2 \backslash 10$ & 310 \\
\hline & Inhialittion & $6 \times 10^{\prime}$ & $7 \times 101$ & 1110 & 7,1110 & $4 \times 101$ \\
\hline & Inods & $6 \backslash 111$ & 0.01013 & $7 、 10^{\prime}$ & 2111 & 0.10114 \\
\hline Willer & Rorrantion & 2110 & 1,101 & $3 \times 111$ & $\cdots+1$ & $1 \times 10^{\circ}$ \\
\hline & Ionk & $5 \times 10^{4}$ & 0.011 & 1,101 & & $(1.11)$ \\
\hline & $1 \cdot 34 h^{2}$ & +1101 & 0.10115 & $2 \times 10^{\circ}$ & & 0.111160 \\
\hline & Drinhing waller & 310 & 0.10114 & 1,10 & $\cdots$ & 01.1114 \\
\hline & Tolal & 0.001 & 0.12 & (1).(1)1 & $(1 .(1)(0)(1), 3$ & 0.112 \\
\hline
\end{tabular}

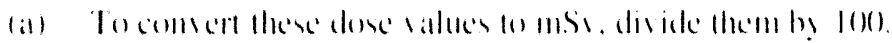

(h) Valuer munded aller iddling.

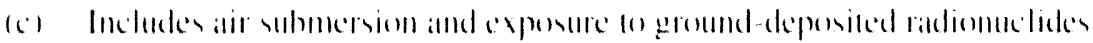

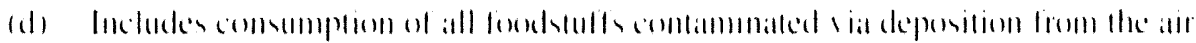

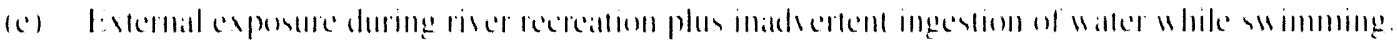

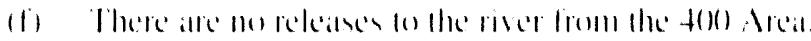

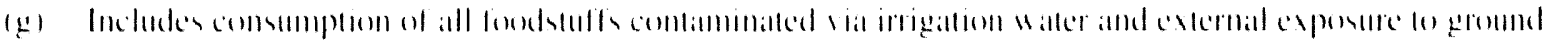

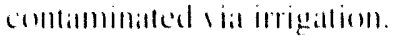

(h) Comsmmplimm af lish lishen from the colmmbial Riser. 
- comsumption of tish containing radionnclicles

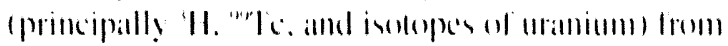
lhe (olumbia River (234).

- comsumplinen of foud containing ladionuclides

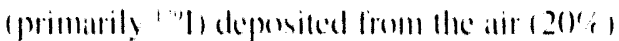

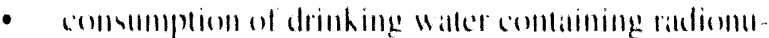

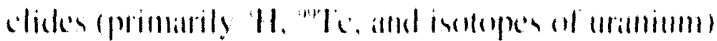
from the (inlumbia Risell ld'i).

The duse limit lon any member on the public bom all

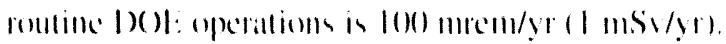

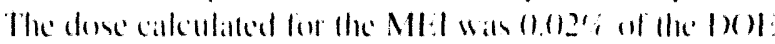
limiil.

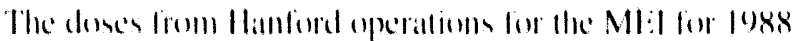

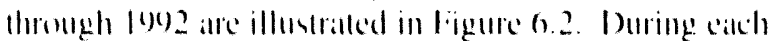

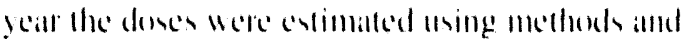

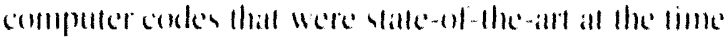

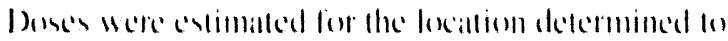

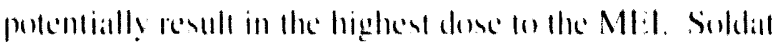

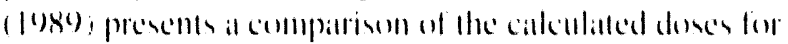

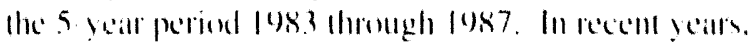

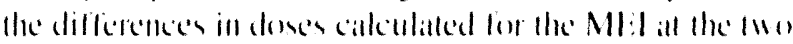

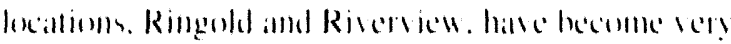
stimill.

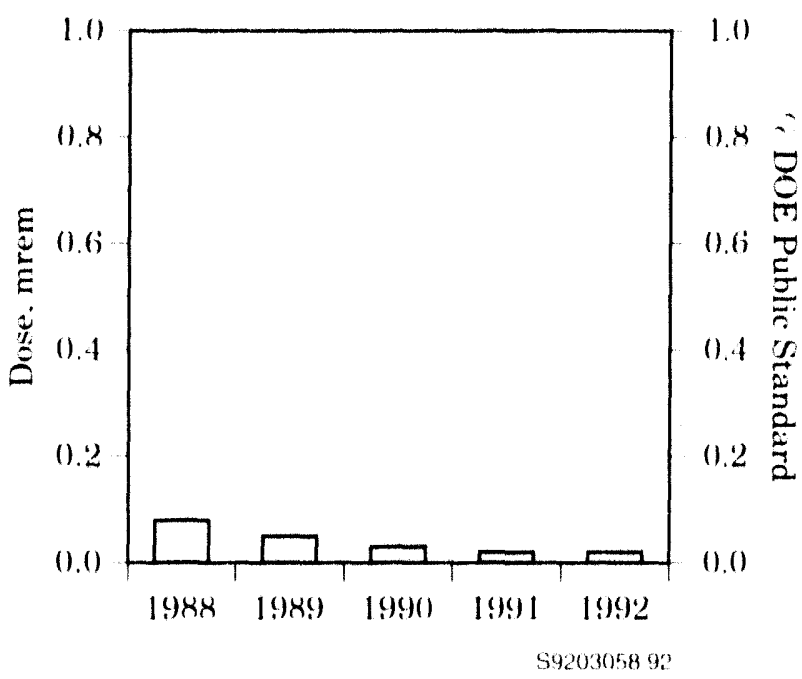

Figure 6.2. Calculated Effective Dose Equivalent to the Hypothetical Maximally Exposed Individual, 1988 Through 1992

\section{Special Case Exposure Scenarios}

While chantereristion that define the standind and historicall MI:I are selected lo definc a high expostare

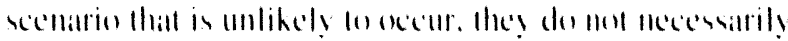

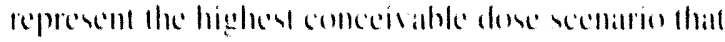

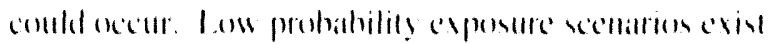
thall could conceis ahly resull in somewhall heler doses.

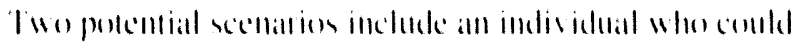
yend time all the Site homblatry lesiltom wilh the maximum evemal radiation dose ralle, and a yontumant

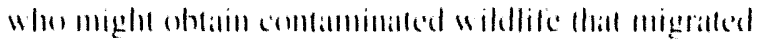

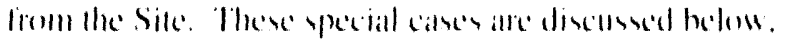

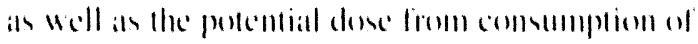

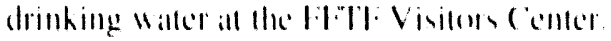

\section{Maximum "Boundary" Dose Rate}

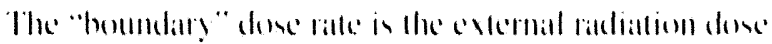

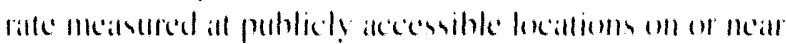

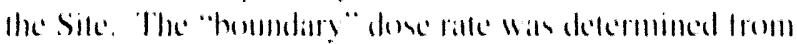

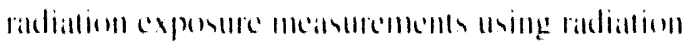

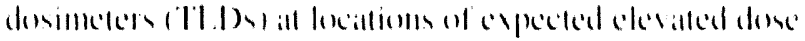

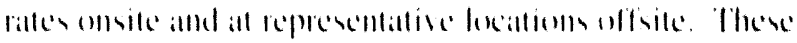

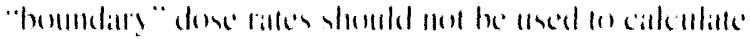

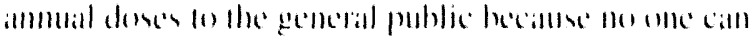

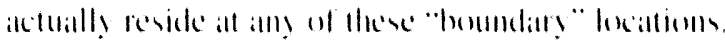

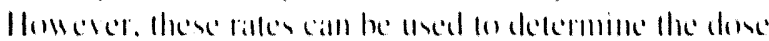
(1) a sereific indis idual who might spend some lime all lhall locillioll

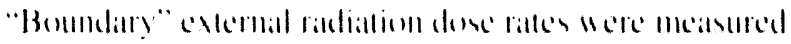

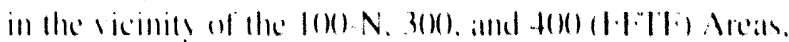

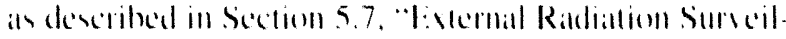

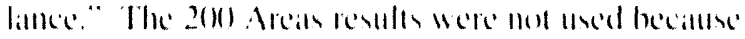

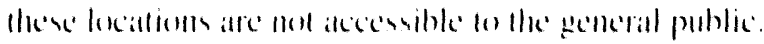

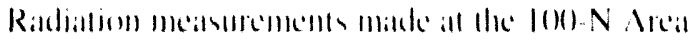

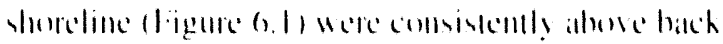

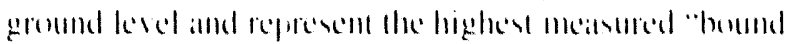
ary" dese ralle. The columbia Rirer prowides public

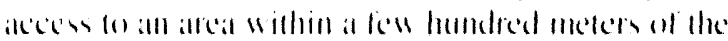

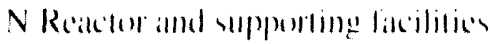

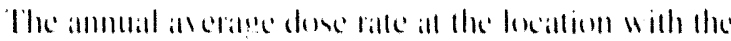

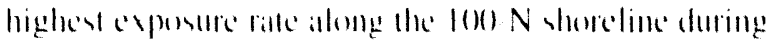

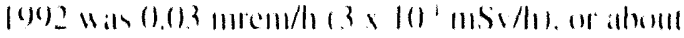




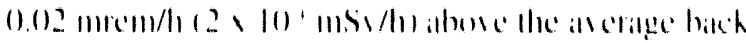

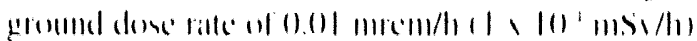

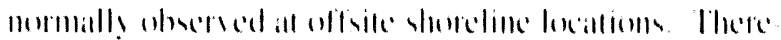

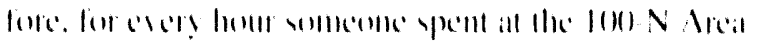

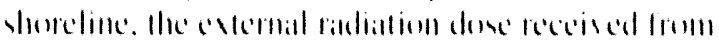

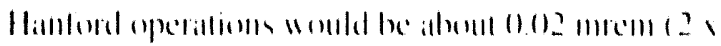

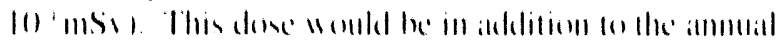

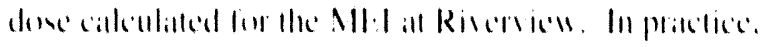

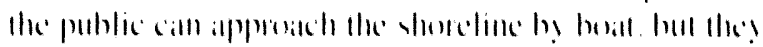

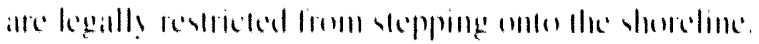

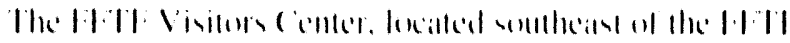

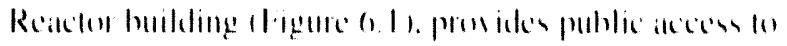

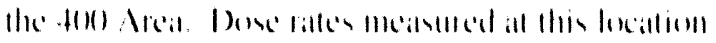

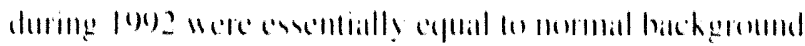

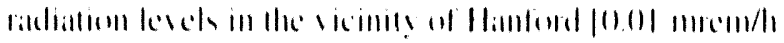

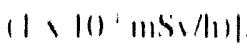

\section{Sportsman Dose}

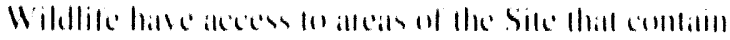

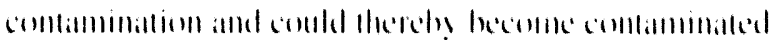

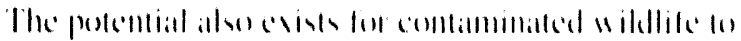

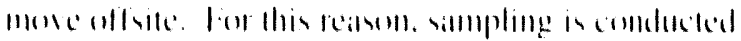

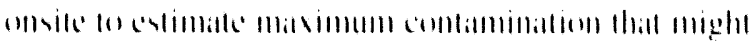

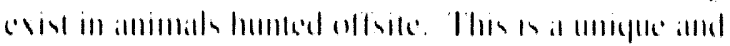

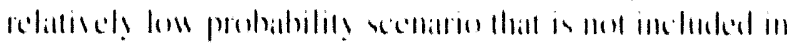

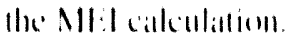

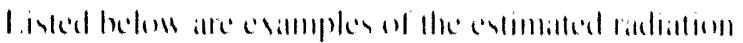

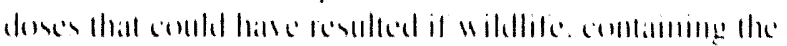

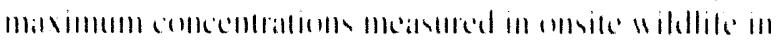

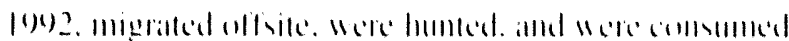

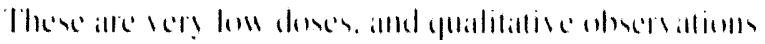

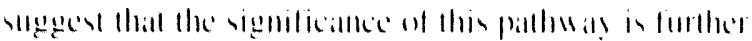

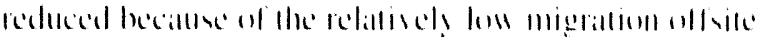

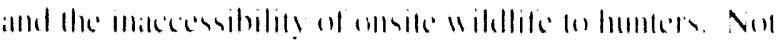

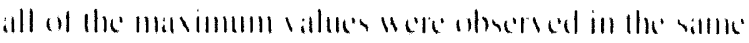

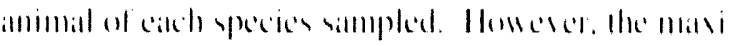

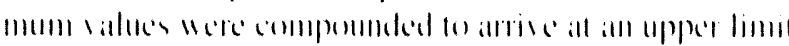

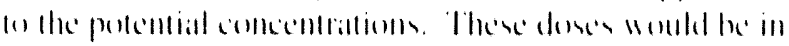

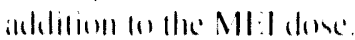

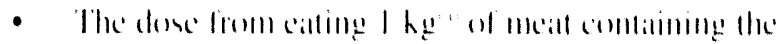

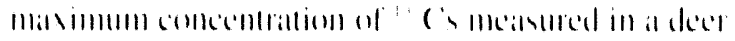

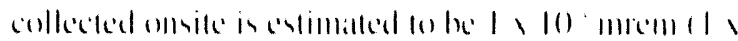
(1) IISi)

(a) $1 k !=2.211$

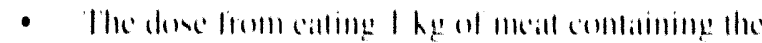

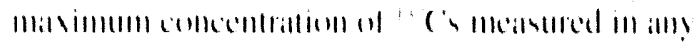

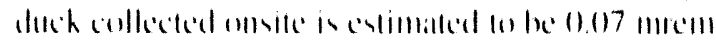

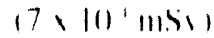

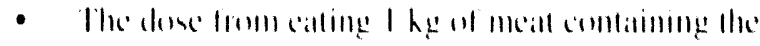

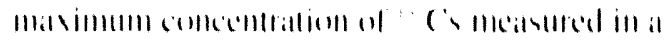

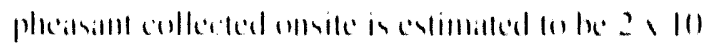
mirmI I 111 mSin)

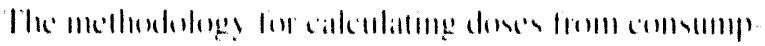

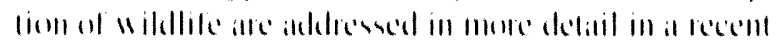
(1)

\section{FFTF Visitors Center Drinking Water}

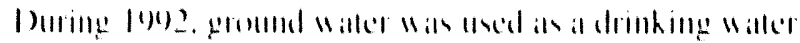

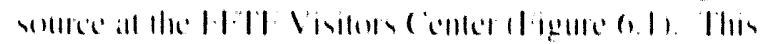

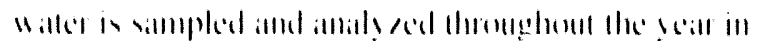

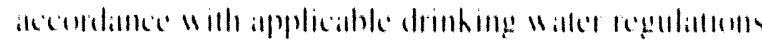

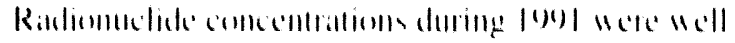

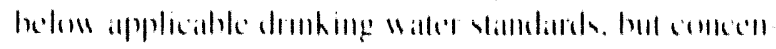

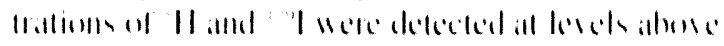

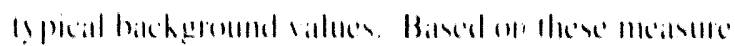

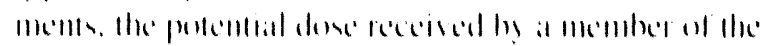

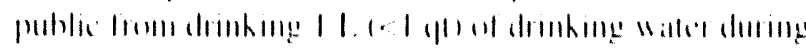

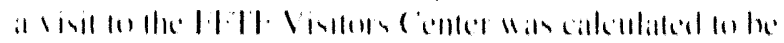

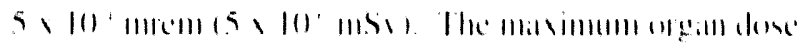

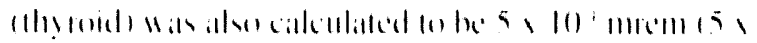

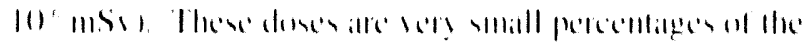

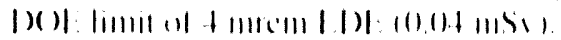

\section{Comparison with Clean Air Act Standards}

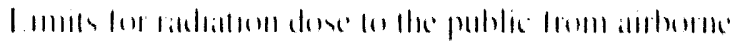

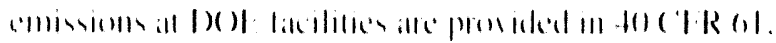

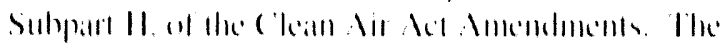

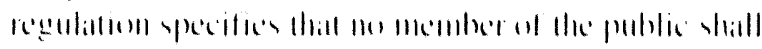

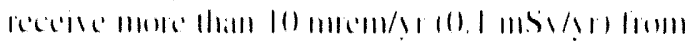

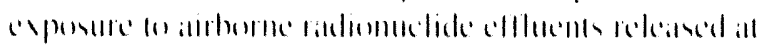

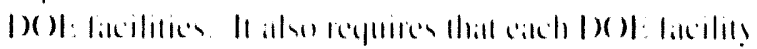

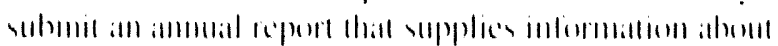

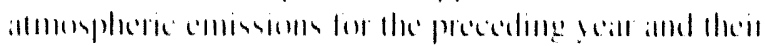

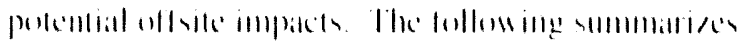

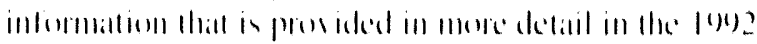

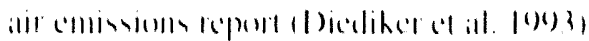




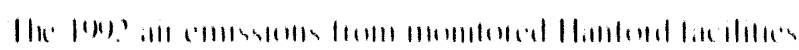

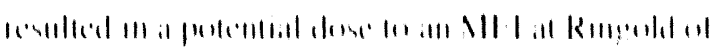

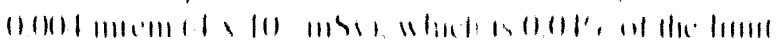

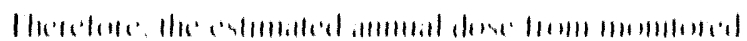

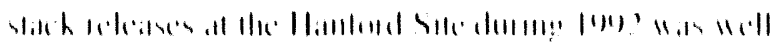

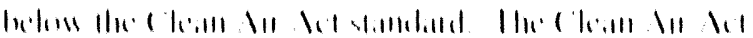

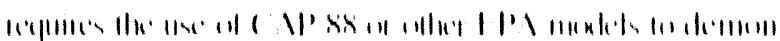

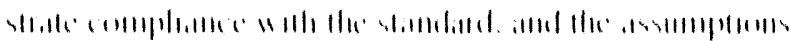

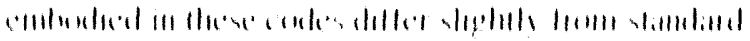
abs

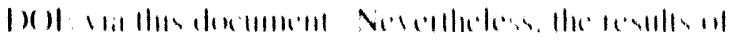

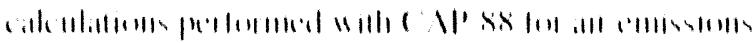

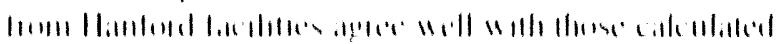

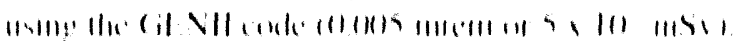

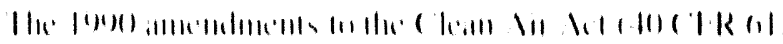

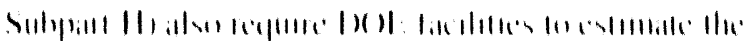

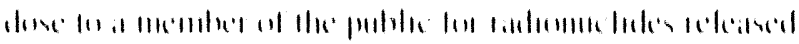

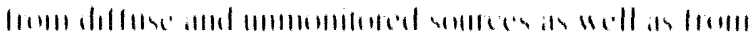

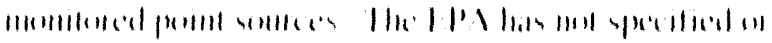

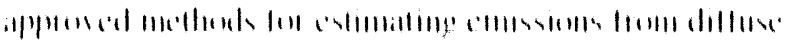

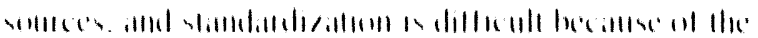

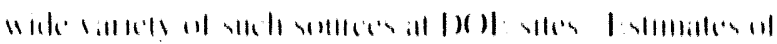

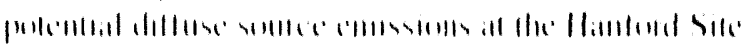

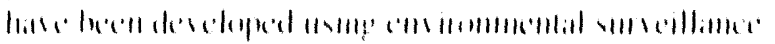

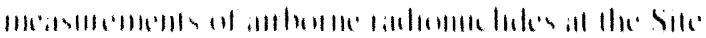

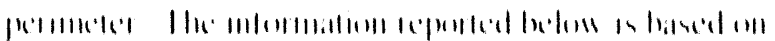

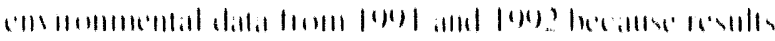

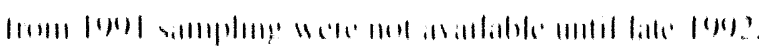

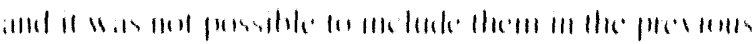
"1p)

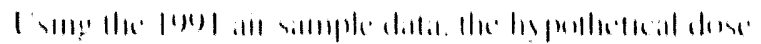

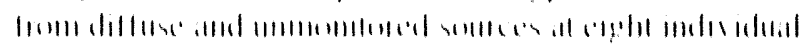

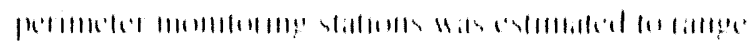

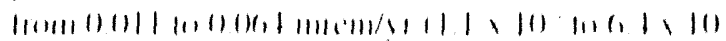

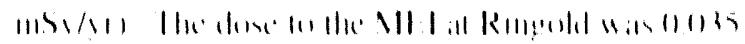

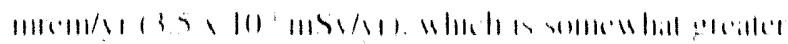

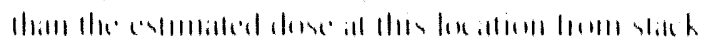

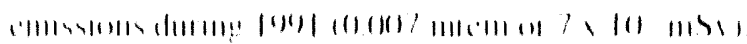

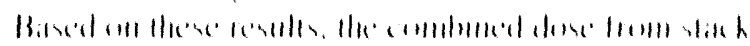
' 'III)

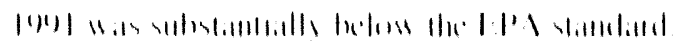

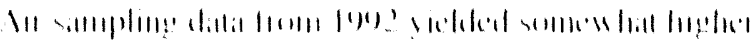

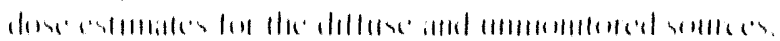

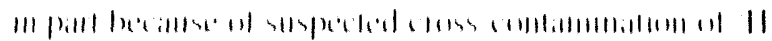

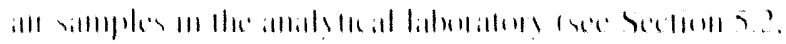

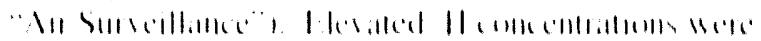

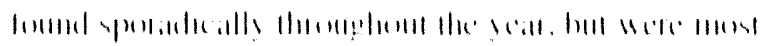

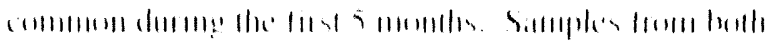

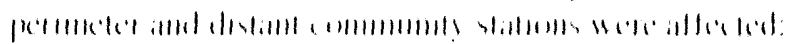

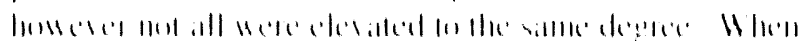

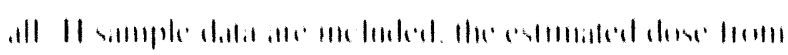

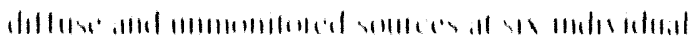

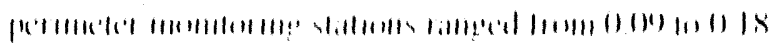

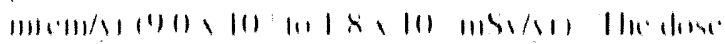

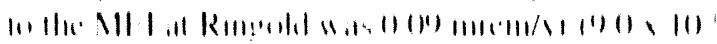

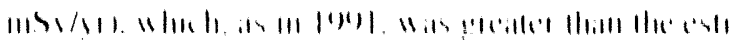

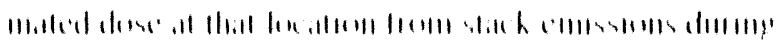

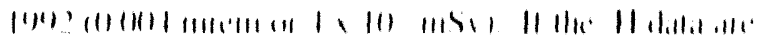

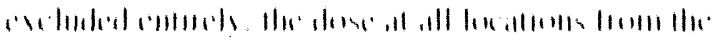

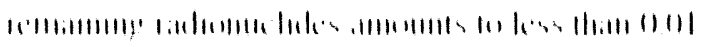

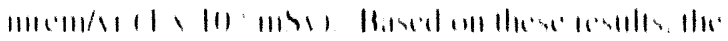

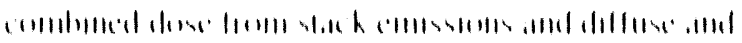

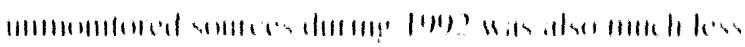

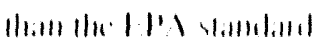

\section{Population Dose}

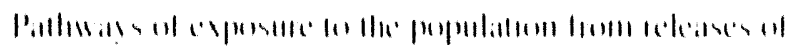

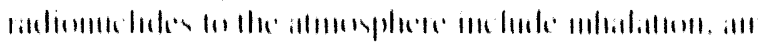

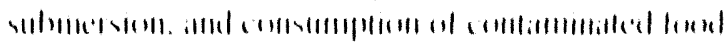

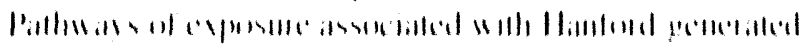

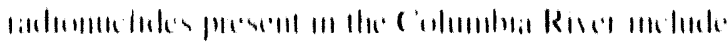
(1)16)

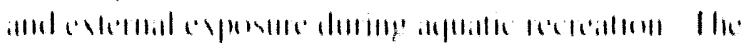
He:

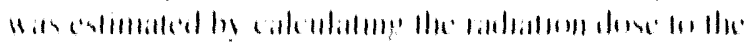

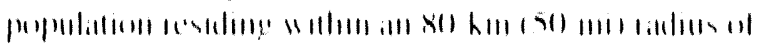

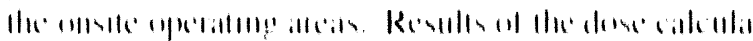

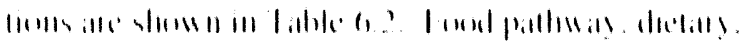

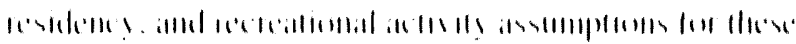

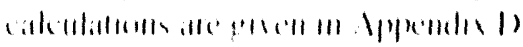

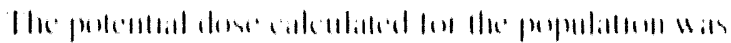

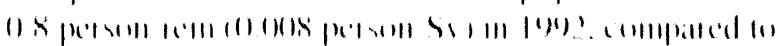

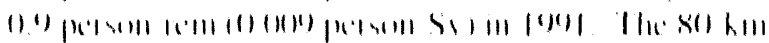

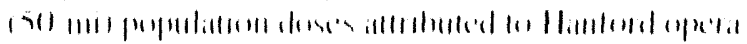

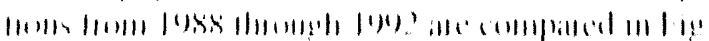
IIIC. 11

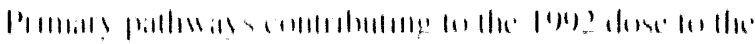

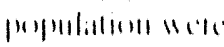

- $\quad$ (1)1

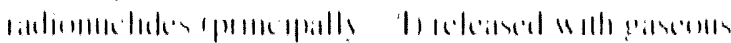

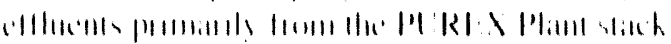

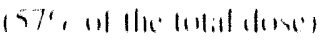

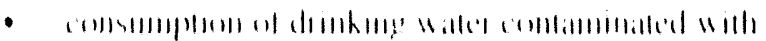

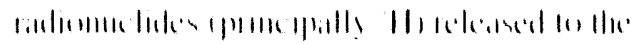

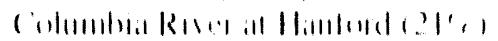


Table 6.2. Population Doses Irom Hanford Operations, 1992

\begin{tabular}{|c|c|c|c|c|c|c|}
\hline \multirow[b]{3}{*}{ I:lllue'n! } & \multirow[b]{3}{*}{ Palliway } & \multicolumn{5}{|c|}{ 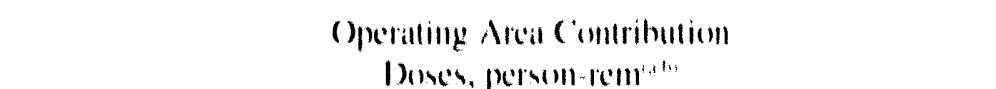 } \\
\hline & & $100)$ & $2(1)$ & $3(1)$ & $f(x)$ & Pallowaly \\
\hline & & Areas & Arean & Areia & Areil & Toliil \\
\hline \multirow[t]{3}{*}{ Air } & lixlemal & $11 .(1)(1)(1) 1$ & $0.10(0) 10$ & $0.610(1)(0) 2$ & 0.11113 & 0.01013 \\
\hline & Inhialialion & 0.0013 & 0.1 & 0.111 & 0.0011 & 0.1 \\
\hline & londsit: & $0.0101(12$ & 11.4 & 0.114 & $0.01(1) 2$ & 0.4 \\
\hline \multirow[t]{5}{*}{ Willer } & Recreations" & $(1 .(1)(1)(1))$ & 0.0101015 & $0 .(1)(1)(1) 2$ & $\ldots \cdot n$ & $0.0(0)(1) .5$ \\
\hline & londs"." & 0.00006 & 0.011 & $(0.0(1) 101$ & $\ldots$ & 0.01 \\
\hline & lish & 0.101012 & $0 .(1012$ & $0.00(1)(1) 6$ & $\ldots$ & 0.0112 \\
\hline & Drinking Waller & $(1)(1)(1) 1$ & 11.2 & $(0.010104$ & $\cdots$ & 0.2 \\
\hline & Tutul & 0.010 .5 & 0.7 & 0.115 & 0.6114 .4 & 0.8 \\
\hline
\end{tabular}

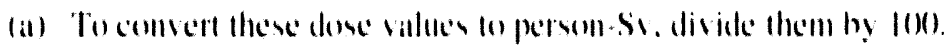

(h) Vallues roumded allerer inddinte.

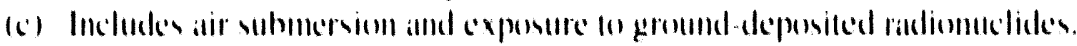

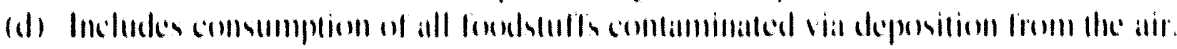

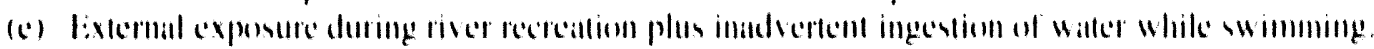

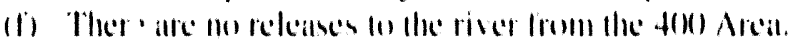

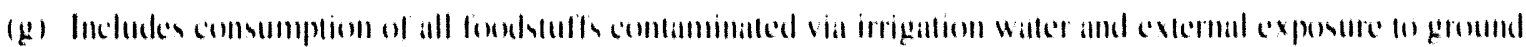
comllamininted via irrifillions.

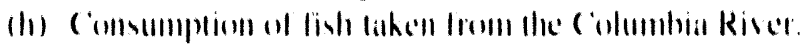

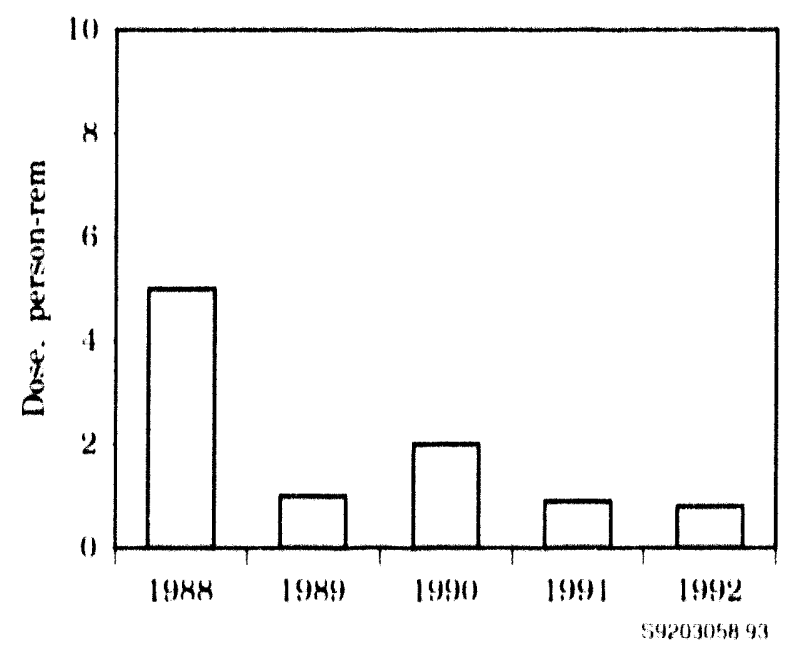

Figure 6.3. Calculated Effective Dose Equivalent to the Population Within $80 \mathrm{~km}(50 \mathrm{mi})$ of the Hanford Site, 1988 Through 1992

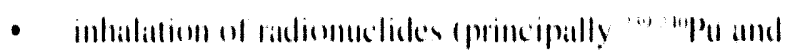

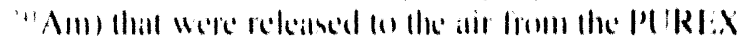
Plinl slack (1)? ).

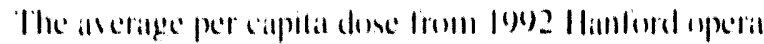
lioms, hascol on a population of $38(1),(1)(1)$ within $80 \mathrm{~km}$

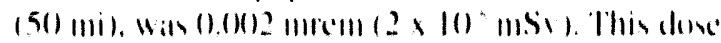

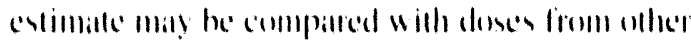

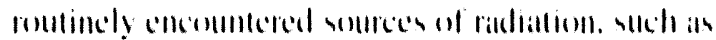

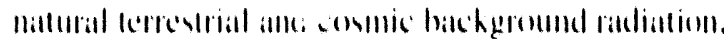

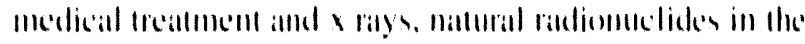

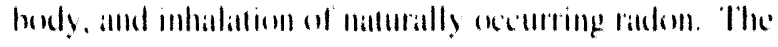

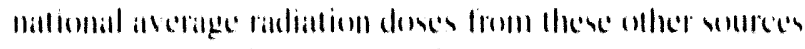

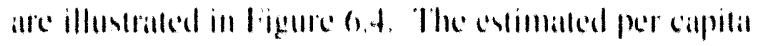

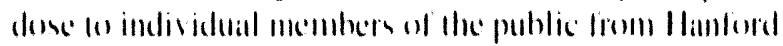

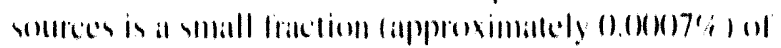

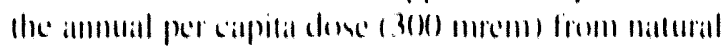
bicherentumb sources.

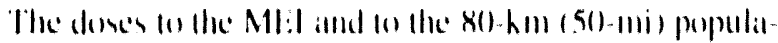

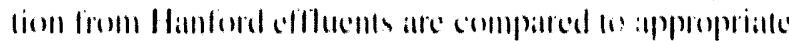
stamdards and nallual back kgromend radiantion in Table 6.3. 


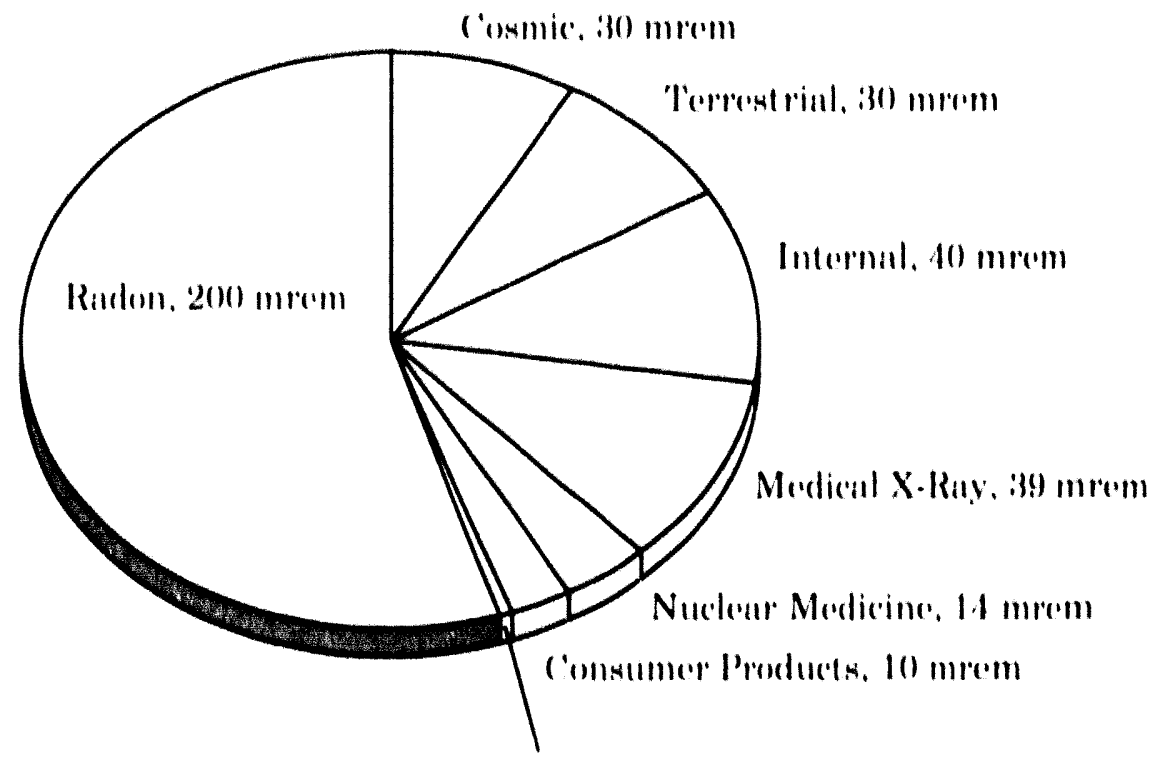

\begin{tabular}{|c|c|c|}
\hline \multirow{3}{*}{ 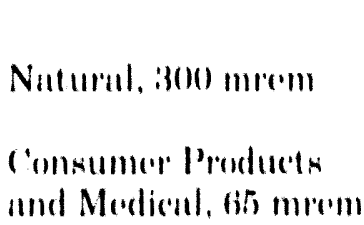 } & \multicolumn{2}{|l|}{ (other, 2 mrem } \\
\hline & ()ocupuntionul & 1 mrem \\
\hline & 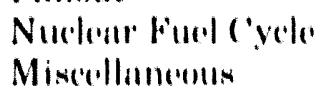 & $\begin{array}{l}0.04 \mathrm{mrcmm} \\
0.014 \mathrm{mrem}\end{array}$ \\
\hline
\end{tabular}

5920305494

Figure 6.4. National Annual Average Radiation Doses from Various Sources (mrem) (NCRP 1987)

Table 6.3. Summary of Doses to the Public in the Vicinity of Hanford from Various Sources, 1992

\section{Sintive}

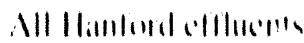

()( ) limili

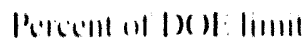

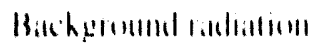

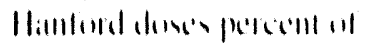

bichermulnd

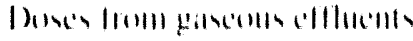

I:I'I ait stamblamd

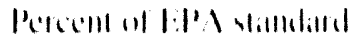

Marumumu Lunlividual.

$$
\text { IIII)II }
$$

$$
11112
$$

(111)

$$
\text { (1)11)" }
$$

(1II)

$$
\begin{aligned}
& 0111179 \\
& 111111.4 \\
& 111 \\
& 11.114 \%
\end{aligned}
$$

x(1) hIII l'(1)ulallinil. persill reme

11.8

1111,11111

$11.1111117 \%$

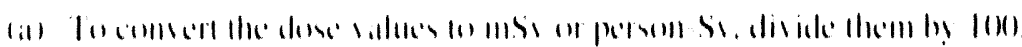

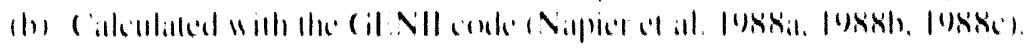

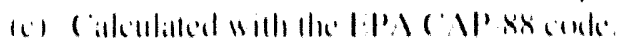




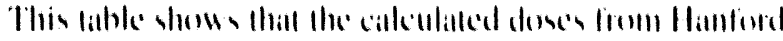

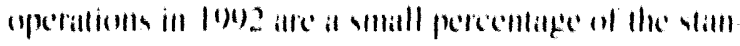
dards and ol mallural bicteheround.

\section{Doses from Other Than DOE Sources}

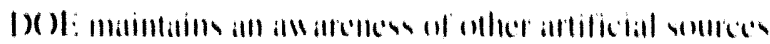

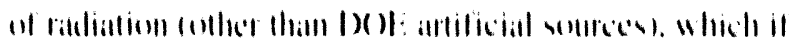

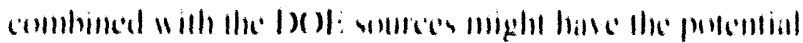

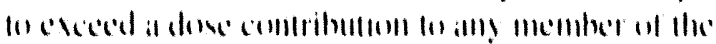

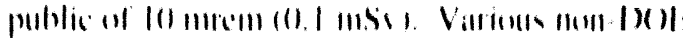

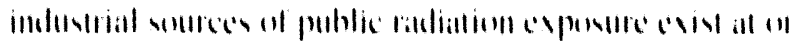

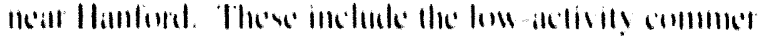

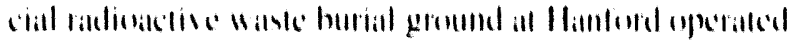

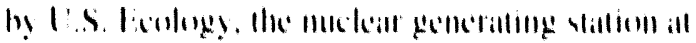

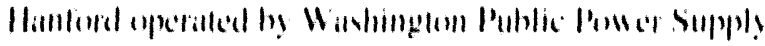

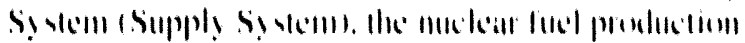

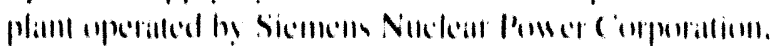

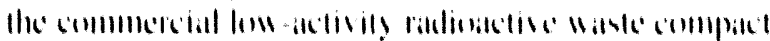

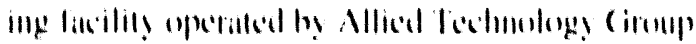

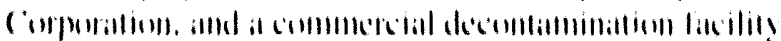

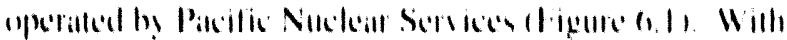

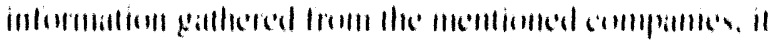

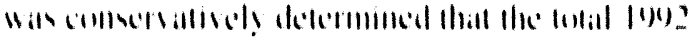

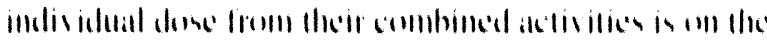

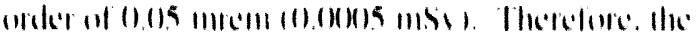

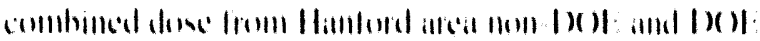

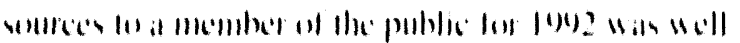

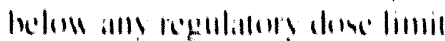

\section{Hanford Public Radiation Dose in Perspective}

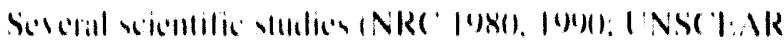

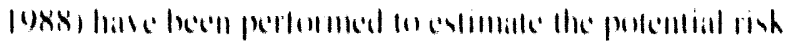

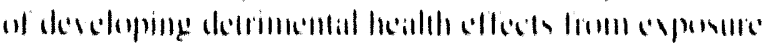

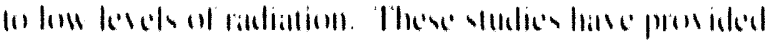

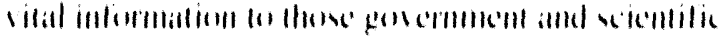

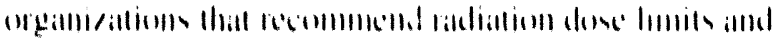

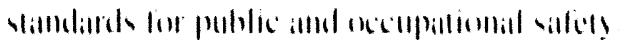

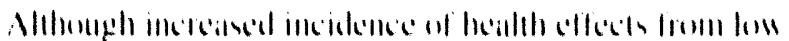

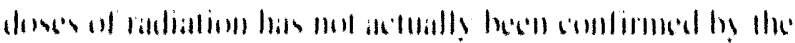

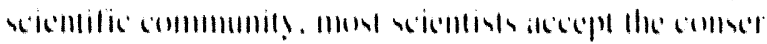

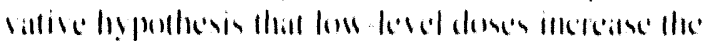

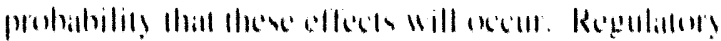

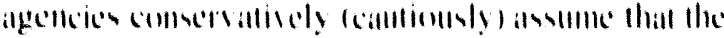

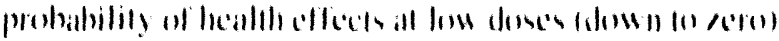

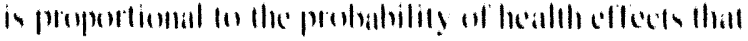

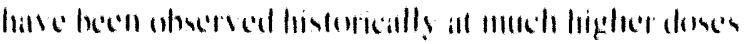

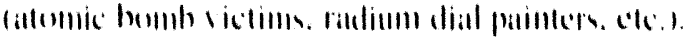

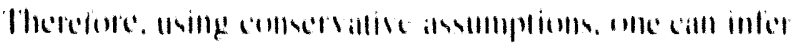

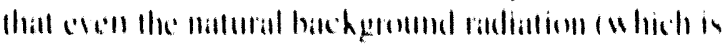

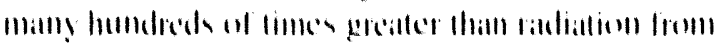

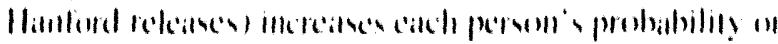

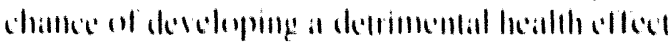

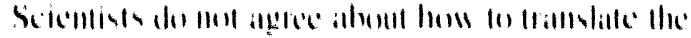

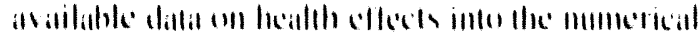

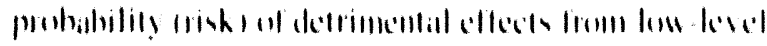

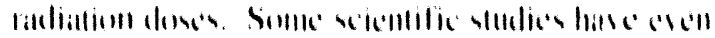

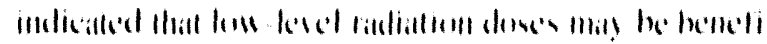

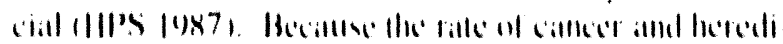

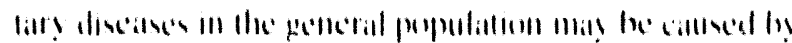

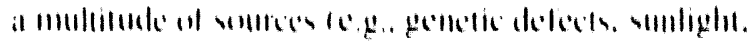

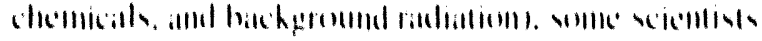

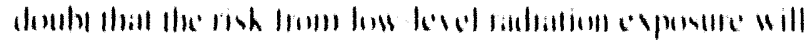

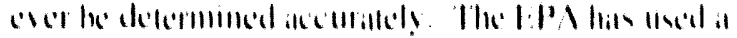

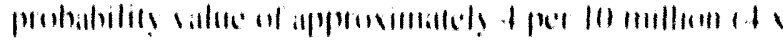

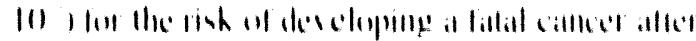

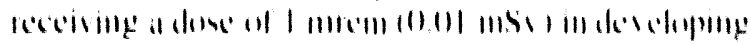

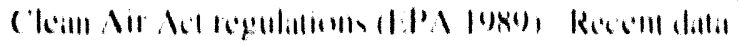

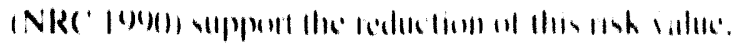

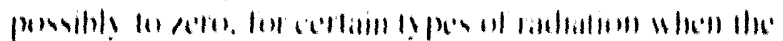

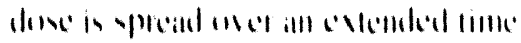

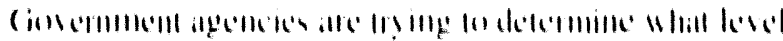

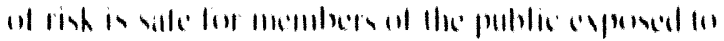

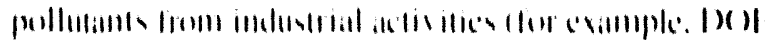

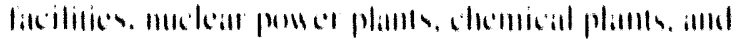

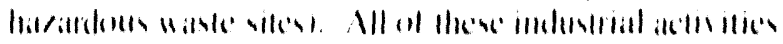

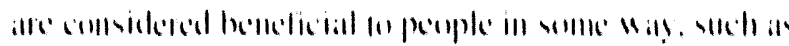

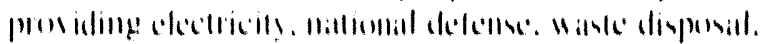

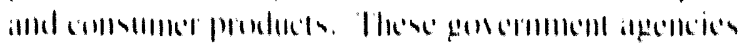

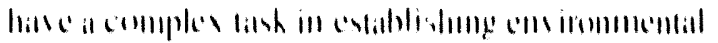

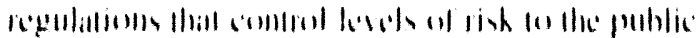

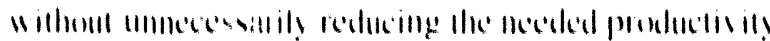
of Ha indlustry

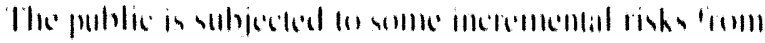

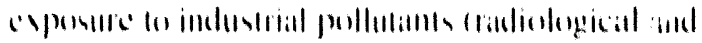

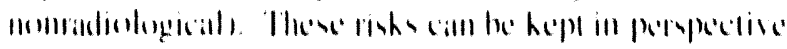

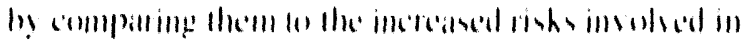

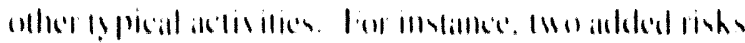

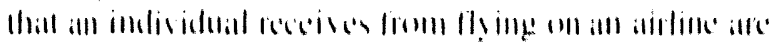

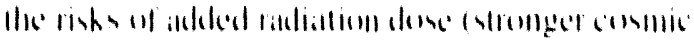

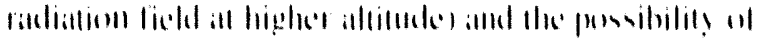

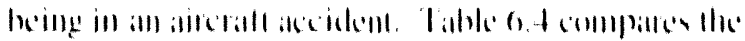




\section{Table 6.4. Estimated Risk from Various Activities and Exposures ${ }^{|a|}$}

\section{Activity or Lxponute Per Your}

Riding or dris ing in a parsenger solicke (300) miles)

Home ancidems

Drinking I cinn of beer or tomences of wille per dis!

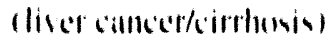

Ploisure bouting (atcidellts)

Pirciarms, ypurting tadcidents)

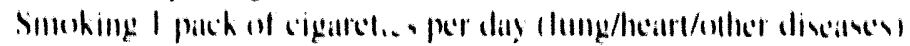

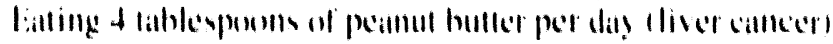

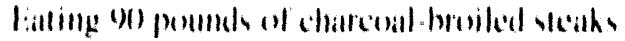

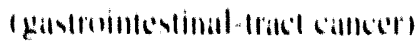

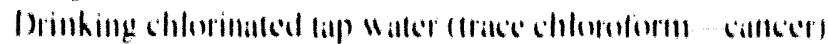

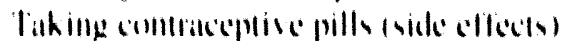

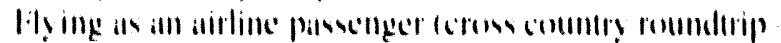

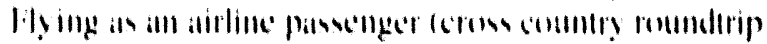

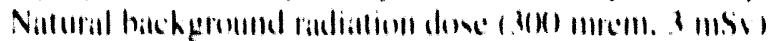

Donce of I merem (1). (1) misin

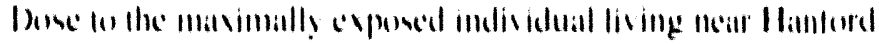
in 114). (1).11. III)
Rish ofl lintiality

$$
\begin{aligned}
& 2 \times 10 \\
& 1001 \times 10^{n+1+1} \\
& 10 \times 11)^{\circ} \\
& \text { (1) } \times 10^{\text {not }} \\
& \text { 11) } 11010^{\text {tat }} \\
& 3(1)(1) \times 10 \text { ) } \\
& x, 10 \\
& \text { 1) } 111 \\
& \text { (1) } 111 \\
& 201111 \\
& x, 111^{\ldots+\ldots .} \\
& 1111.5,1110 \\
& \text { (1) } 11120,111 \text {. } \\
& \text { (1) } 110.4 \text { (1) } \\
& \text { (1) } 1111.1618 \times 111 \text {. }
\end{aligned}
$$

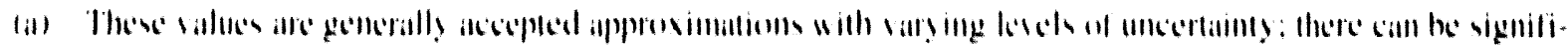

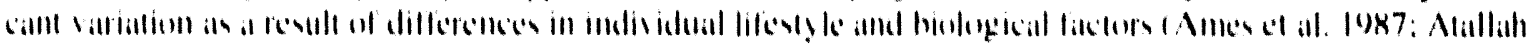

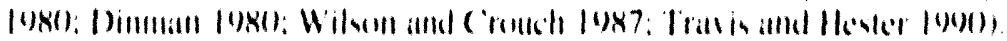

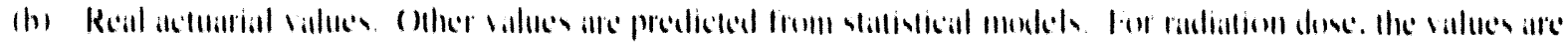

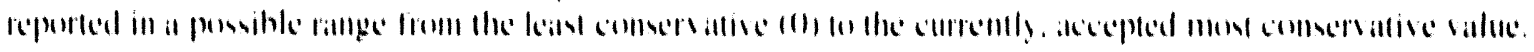

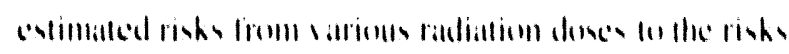

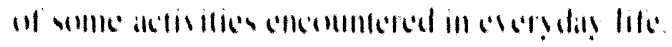

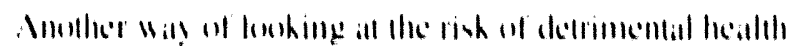

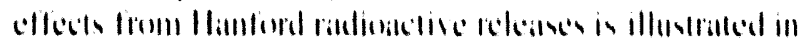

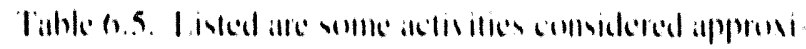

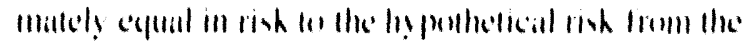

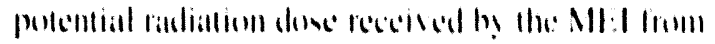
Hanford rekeisen in low?

\section{Dose Rates to Animals}

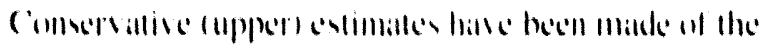

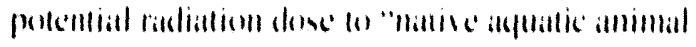

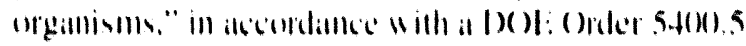

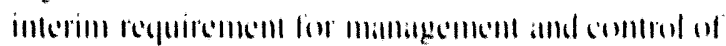

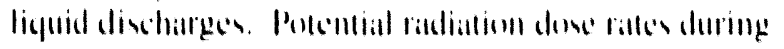

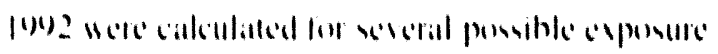

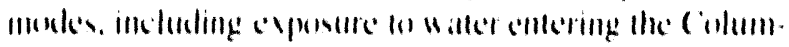

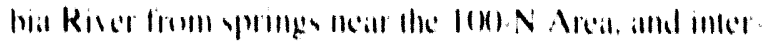

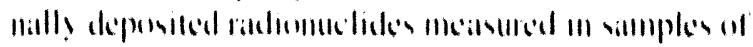

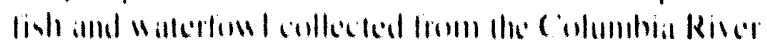

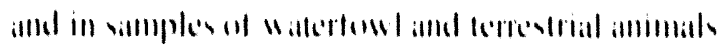
collectid omsite.

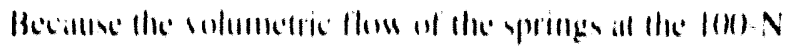

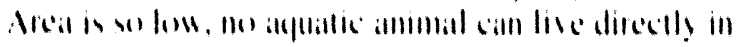

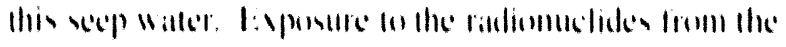

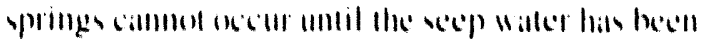

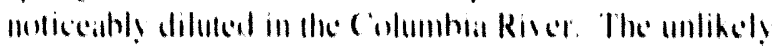

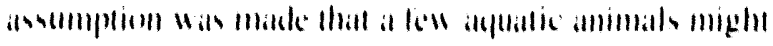

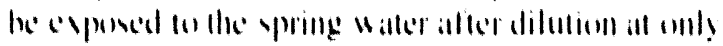

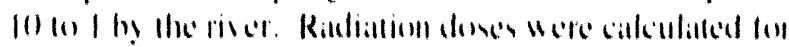

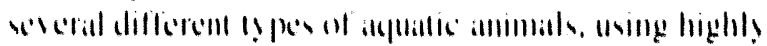

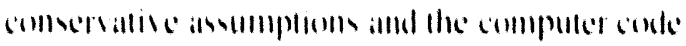


Table 6.5. Activities Compareble in Risk to That from the 0.02-mrem Dose Calculated for the 1992 Maximally Exposed Individual

Driving of ridling in a call $2 \mathrm{~km}(1.2 \mathrm{mi})$

Smoking $1 / 60)$ of a cigitrelle

lyimg $5 \mathrm{~km}$ (1.1 mil on a commmercial airlime

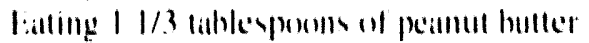

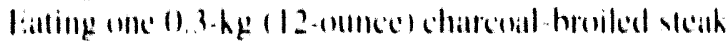

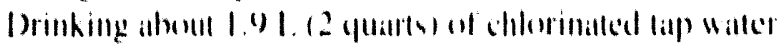

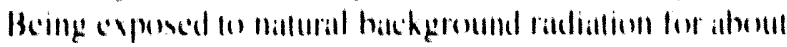

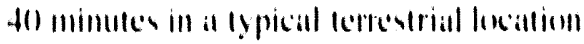

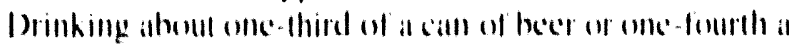

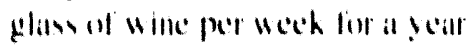

( RITR2 (Bather and Suldall l(4)2). The animal receiving

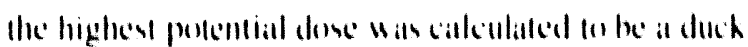

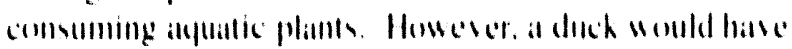

fo spend sots of is time in the anc tenth spring watter comsuming anly plants growing there foreceive a radiattion dose of I rad/d. Thus, it is highly unlikely that ally mative atyuatic animal orgamism andually received a

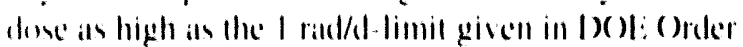
91011.5

Boses were also estimaled for clams. fish. and wallerfowl exposed for colmmbia River watler combaiming a mixture of all the radiontedides reatehing the (olumbia River

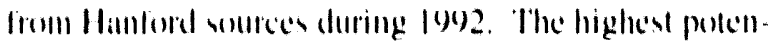
lial dose was lore a plant eationg doch, $2 \times 10 \mathrm{rad} / \mathrm{d}$.

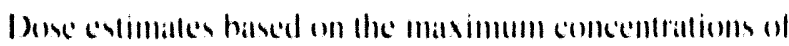

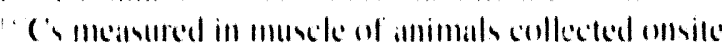

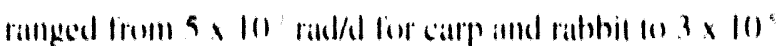

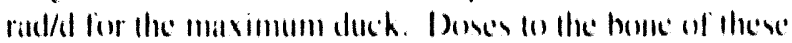

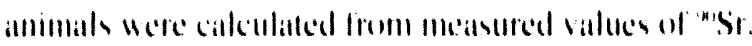

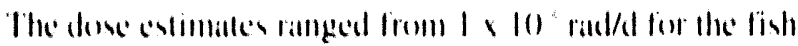
In6, 10 rad/d for rithbil and deer. 
Quality
Assurance

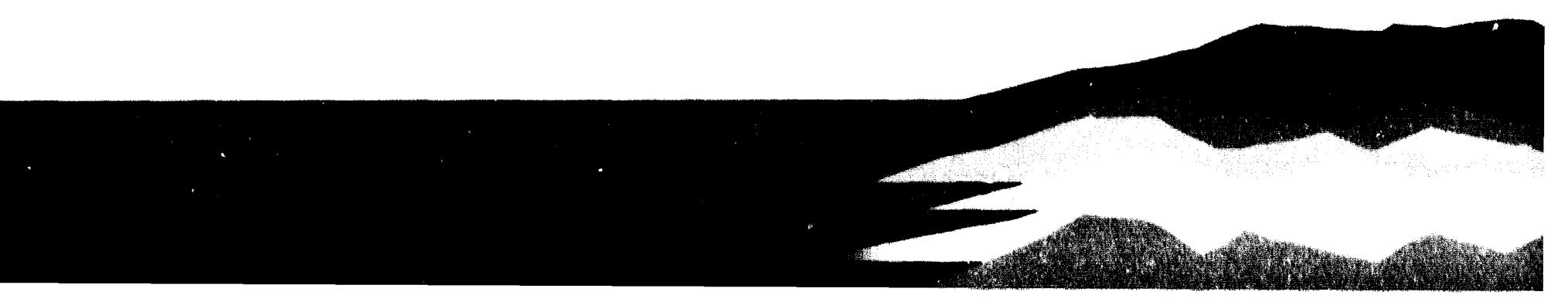




\subsection{Quality Assurance}

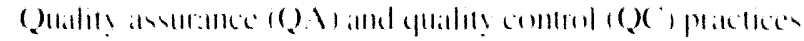

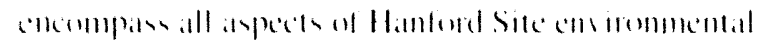

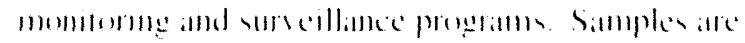

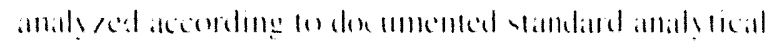

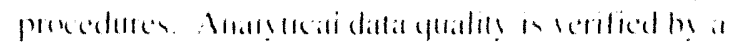

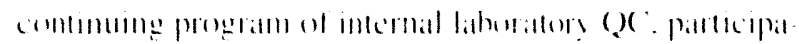

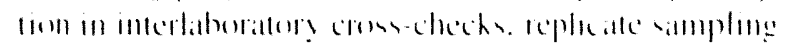

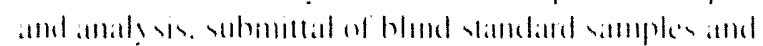

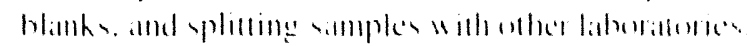

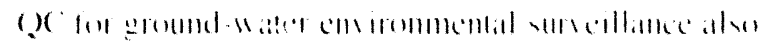

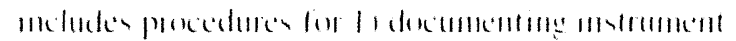

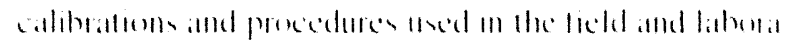

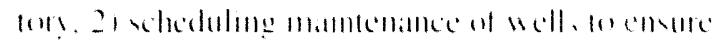

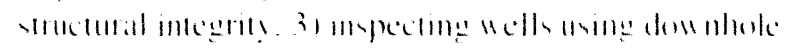

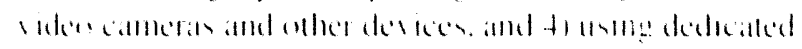

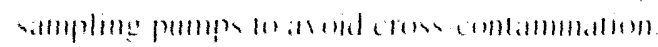

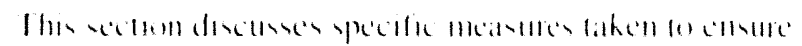

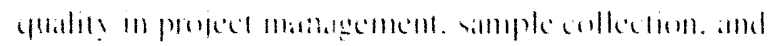
inalunal revelk,

\section{Environmental Surveillance}

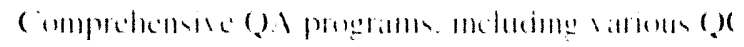

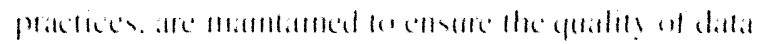

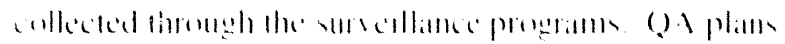

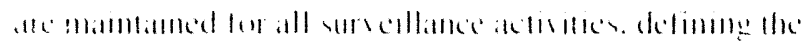

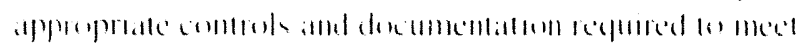

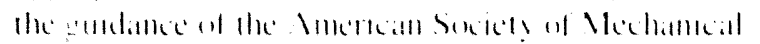

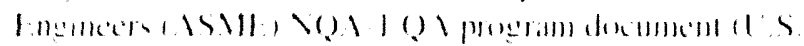

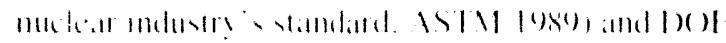
(biks

\section{Project Management Quality Assurance}

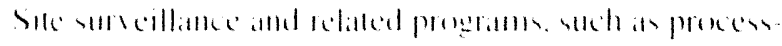

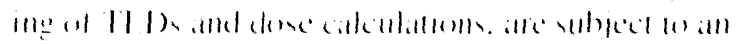

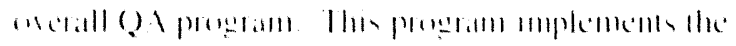

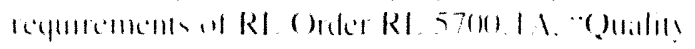

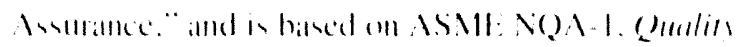

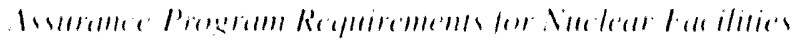
1.SMI 1989). The program is defimed in a (2) mamual

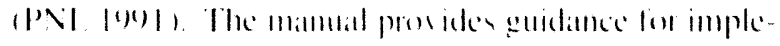

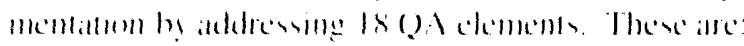

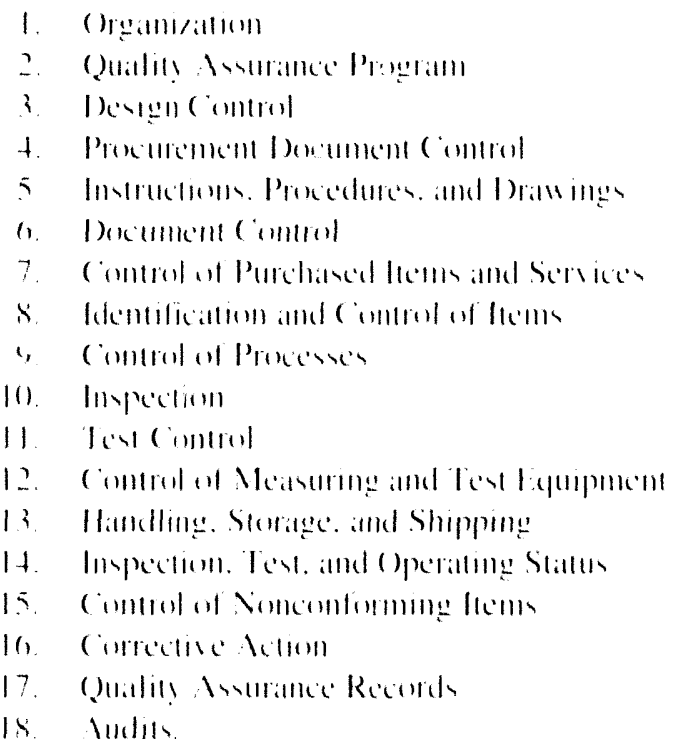

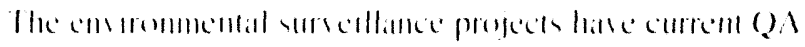

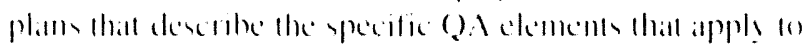

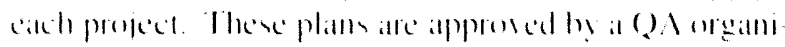

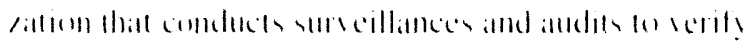

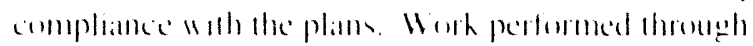

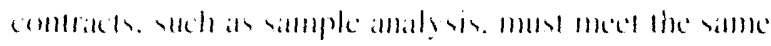

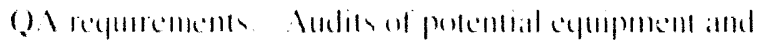
xerses -

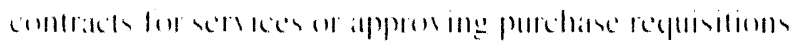

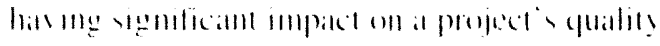

\section{Sample Collection Quality Control}

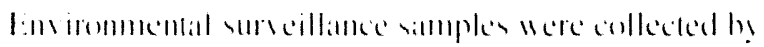

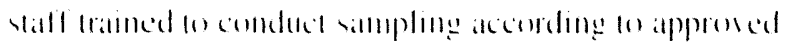

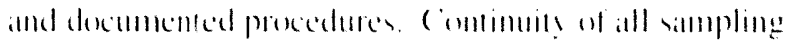

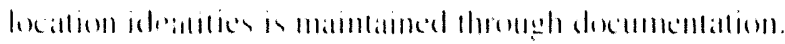


Silmples for ground-water hat atrdous chemical momiloring are sealed with evidence tape to prevem tampering and transpented to he laboratory in aceordance with the

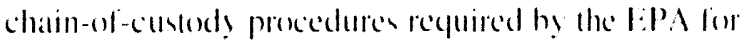
RCRA monitoring programs.

\section{Analytical Results Quality Control}

Routine radionementeal analyses for envirommental surveillance samples are performed hy International

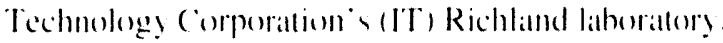
Analytical yualieg at the laboralory is craluatcel in a number of ways. It"s Richland laboratory participalles in

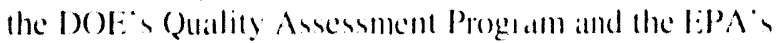
Lahoratory Intercomparison Sudies. PNI. conducts an additional (Q) program. IT"s Richland laboratory also matintains an internal ()$^{\circ}$ program. which PNI. aludits and reviews. ()ther atudits and compartisoms atte condected on specilic lypes of simples. A limal or chech of data is performed by a computeried screening of results agatimst project-specilic criteriat. Anomalous results are reported, and discrepancion resoleded and documented. Additional information on these efforts is provided in the following subsections.

\section{U.S. Department of Energy and U.S. Environmental Protection Agency Comparison Studies}

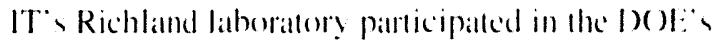

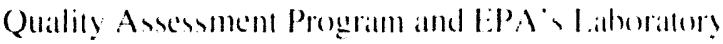
Intercomparisen Studies Program. The pe programm provide standard samples of hatome com mommental medial

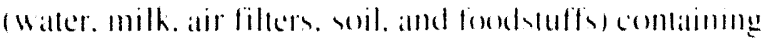

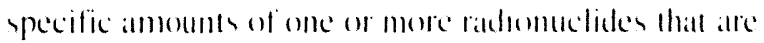
unkmown by the participatting laborallory. Sfler sample

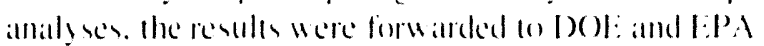

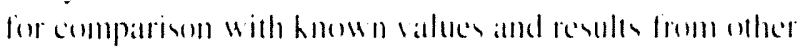
lahoratorices. Bohl I:PA and D)( )I hate (stahlished eri-

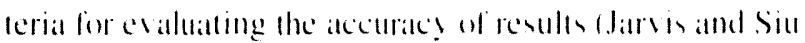
1981: Sanderson 1985). Summaries of the 1992 2 resuld for the programs atre pros ided in Tables 7.1 and 7.2 Approximately 93\% at the resulte during the seatr were within the typically ured "3-sigma comtrol limits" $1+3$

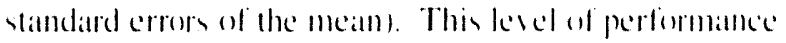
was determined to he acceptahle and was ammeng the hes of participating radioghemistry labonatories.

\section{Pacific Northwest Laboratory Evaluations}

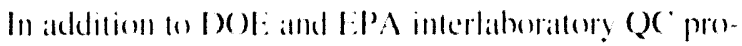

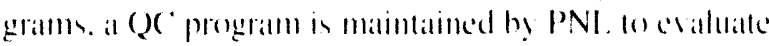
analetical contratefor precision and accurates and an

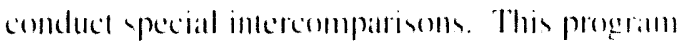
includer the use of hlind samples and replicate simples. Blind standatrd og simples and hlanhs are prepaned and submilted fo chech the alectrrac! and precision of lt" amalyses. The methods med to delermine accutacy and precision acceptahility Here tathen from the $E: P$ and 1)( )I: (danvis and Siu 1981: Simderson 1985). In 1902. blind and hlank simples were submitled in air liltem. soil. Watcr. and wime. () serall. It reported that 75't of reyuested (2) antalyes mere within comerol limits (Table 7.31. This progeram identified al posential problem in the galloma spectrosenpl soliw alle, which has heen corrected. The deficiency insoled short-lived radionuclides thall are mot momally forme in eme irommental media corrently suhmitted lon analysis.

PNI. also participalles in a Qualily Asumance Tark Force (Q) ATP). a program condected hy the Washingen Statce Department of Social and Healde Services. Tiwo environmental media samples and an I:PA reference waller

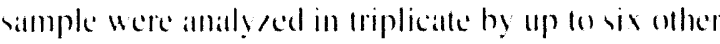
participanting laboratories in the Pacilic Northeses Table 7.t). The l'T resuls submilled by PNI. companed

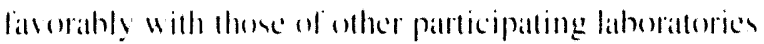

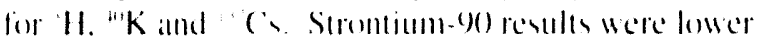

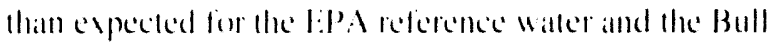
Run I athe sediment.

\section{Laboratory Internal Quality Control Programs}

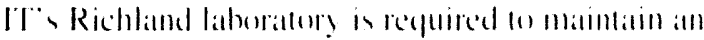

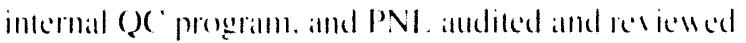

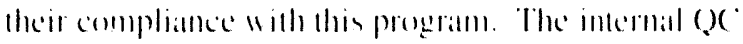

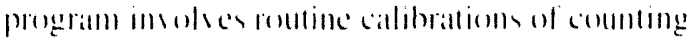

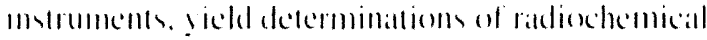

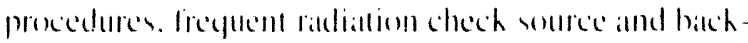
ground conums. replicalle and ypihed simplen antals ses, maintenance of control chants to indicatte analytleal

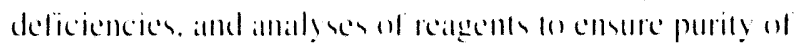

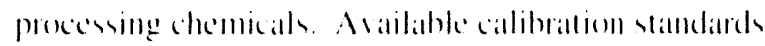

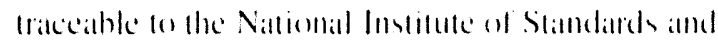

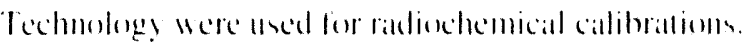


Table 7.1. International Technology Corporation Performances on DOE Quality Assessment Program Samples, 1992

\begin{tabular}{|c|c|c|c|}
\hline & & $\begin{array}{l}\text { Number } \\
\text { of Results }\end{array}$ & $\begin{array}{l}\text { Number } \\
\text { Within Control }\end{array}$ \\
\hline Simple Mediat & Radtionuclider & Reported & I.imits'st \\
\hline Air fillets & 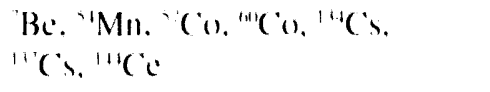 & 7 & 6 \\
\hline Suil & 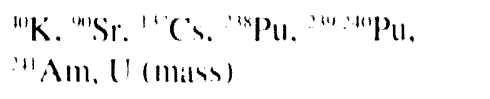 & 7 & 5 \\
\hline Vegetitlion & "K. "Cs & 2 & 2 \\
\hline Witler & 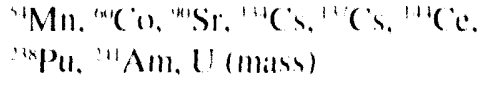 & 111 & () \\
\hline
\end{tabular}

Table 7.2. International Technology Corporation Performances on EPA Intercomparison Program Samples, 1992

\begin{tabular}{|c|c|c|c|}
\hline Simmple Media & Rindionuclides & $\begin{array}{l}\text { Number } \\
\text { of Results } \\
\text { Reported }\end{array}$ & $\begin{array}{c}\text { Number } \\
\text { Within Combrol } \\
\text { L.imits'..1 }\end{array}$ \\
\hline Willer & 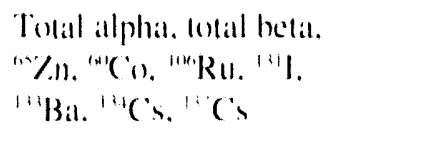 & 3.3 & 3.3 \\
\hline Witer & $\because$ Rat, "Rat. U (mass), "*a" Pu & 11 & 11 \\
\hline Willer & "Sr. "'Sr & 0 & 6 \\
\hline Willer & $\mathrm{H}$ & 2 & 2 \\
\hline Milk & "Sir. "Sir. "II. "S & $x$ & 5 \\
\hline Air lilters & $\begin{array}{l}\text { Totial alphat, total betat } \\
\text { "Sis." "S }\end{array}$ & 12 & 12 \\
\hline
\end{tabular}

(a) Control limits are from Jarvis and Siu (1981). 
(4)

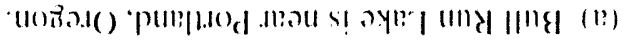

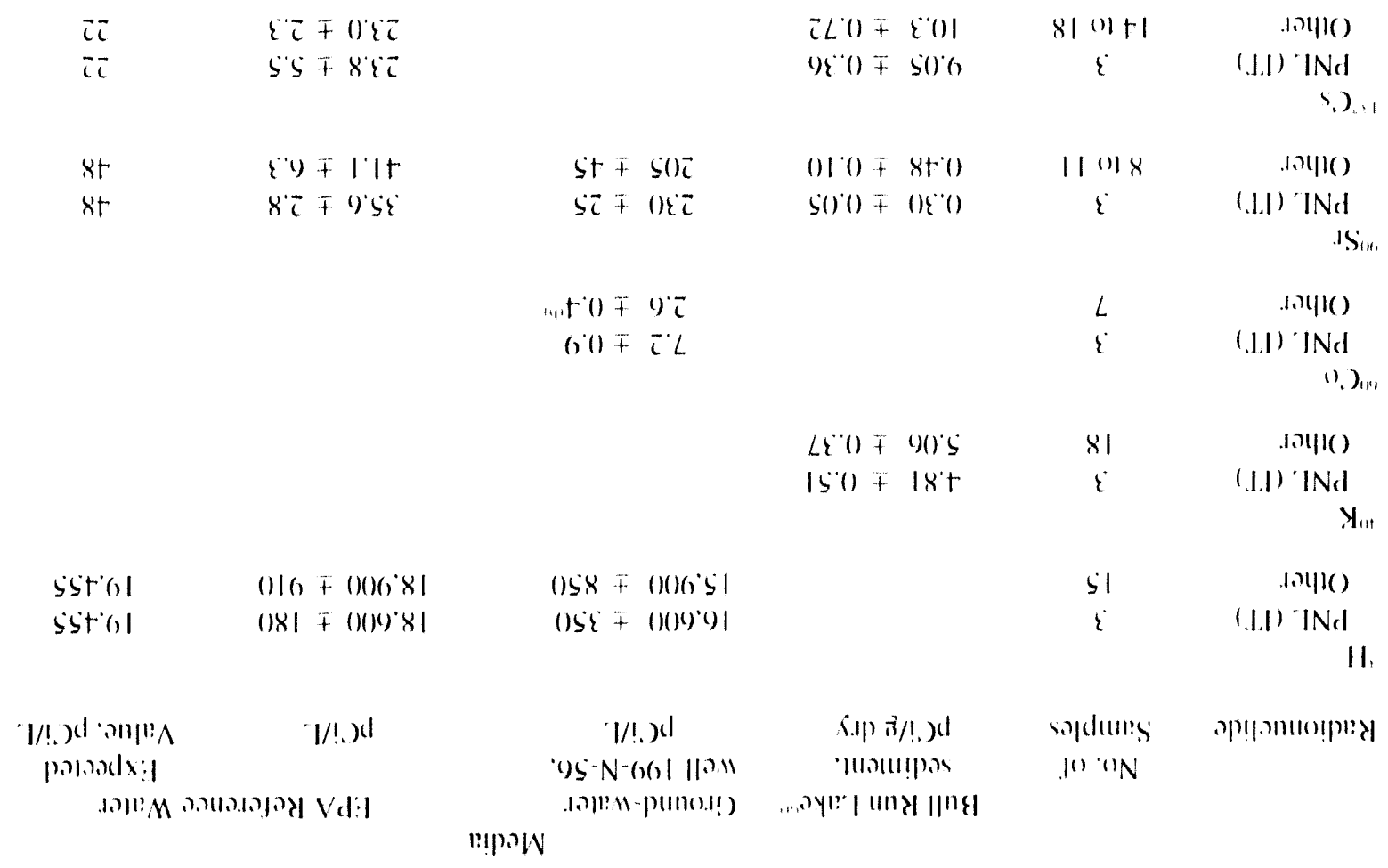

solnoje

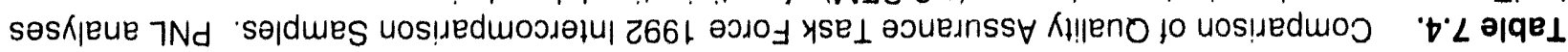

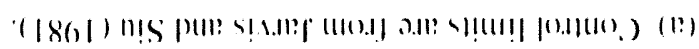

\begin{tabular}{|c|c|c|c|}
\hline 11 & 81 & $s_{1,1}, 11 W_{1}, H$ & $\because ! M$ \\
\hline & & 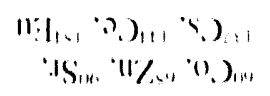 & \\
\hline$\angle 1$ & $1 t$ & 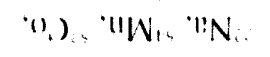 & . I. III:M \\
\hline ¿l & 伭 & 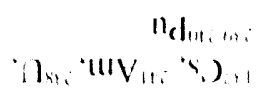 & l!ns \\
\hline & & 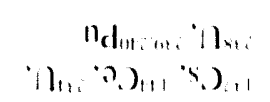 & \\
\hline$t i$ & $+\bar{\imath}$ & $(1))_{11}(1), \cdot 11 W_{n}=$ & $4.1 .11 ! 1.1 ! \mathrm{V}$ \\
\hline „1.s!!แu!। & 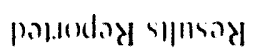 & 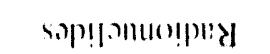 & 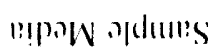 \\
\hline (1).1|110.) $41 ! \mid 1 ! M$ & $\|.1,4\| m \mathrm{~N}$ & & \\
\hline $\operatorname{my} \| 11 \ldots \mathrm{N}$ & & & \\
\hline
\end{tabular}




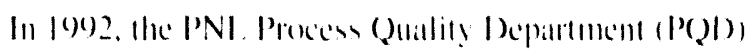
comdected onse formal alldil and participalled in lano

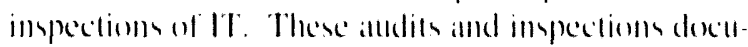
ment conformance with combatctual reguirements of the

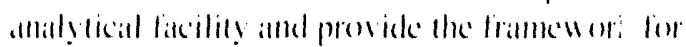
identilying and resolving polential performance prohlems. Reyponses lo aldedil and imspection lindinges atre documented he wrillen commonnication, or follow - up

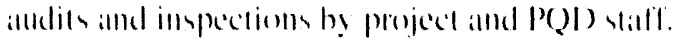

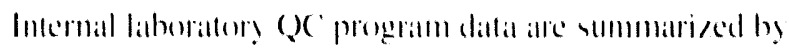

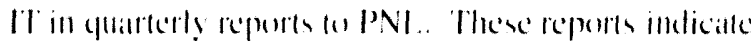

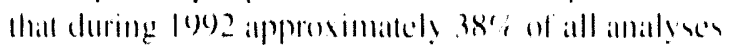

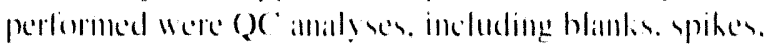

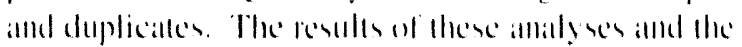

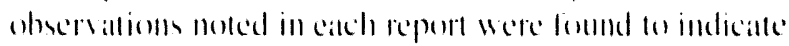

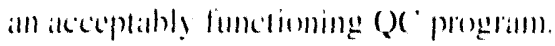

Verilicallom ar minimum dedectable concentration

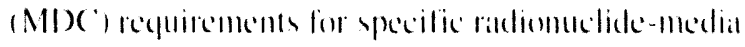

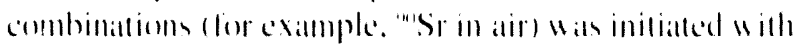

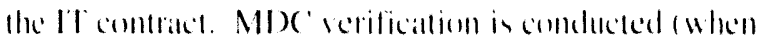

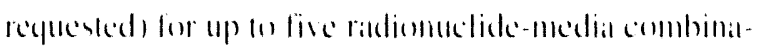

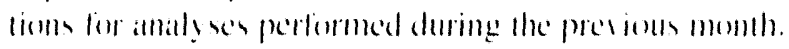

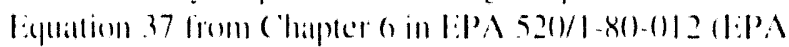

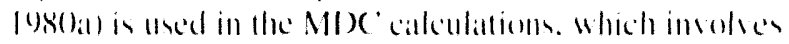

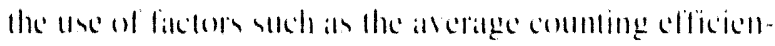

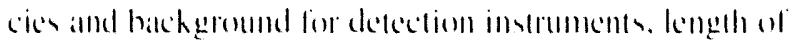

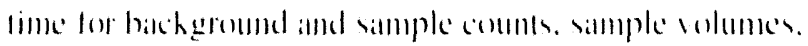

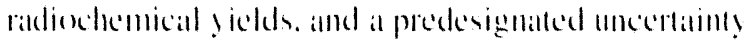

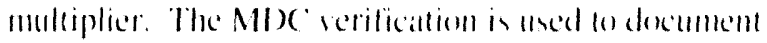

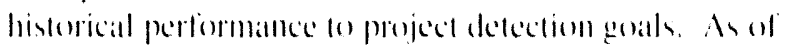

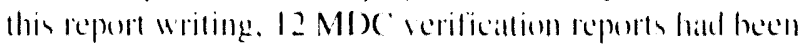

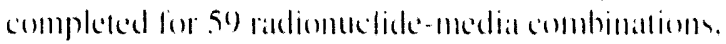

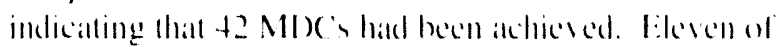
the seventeen radionuclide-medias combinatloms not

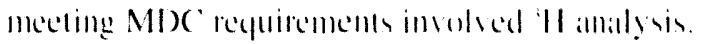

\section{Sample-Specific Audits and Comparisons}

Additional andiss and comparisoms are condected on sereral specifie typesol silmples. The State of Washinglon roulindy collected samples of various ensirommental media and measured external radiallion kevels all mulaple locillioms during ly92. The resules from the stalle monitoring program were reviewed and indicated good agreement hetween Tl. D meanuremems (approximaltely $10 \%$ vatriation). fotal betat meanturements

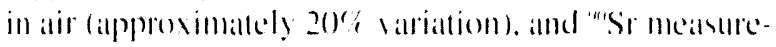
ments in allalla bapproximatcly $10 \%$ i variationt.

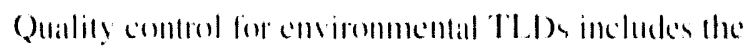

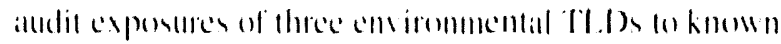
Valles of radiation (between 16 and 29) mR ). which are

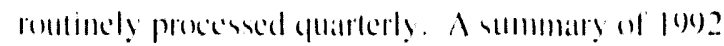

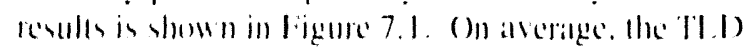
meisurements were hiasted 24: lower ham the known

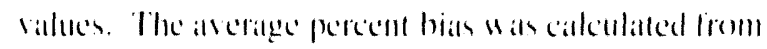

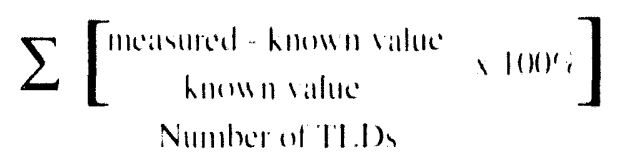

\section{Effluent Monitoring}

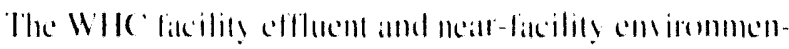

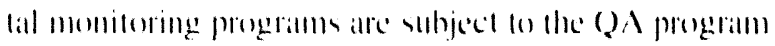

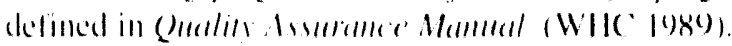

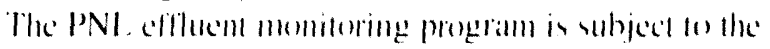

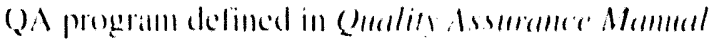

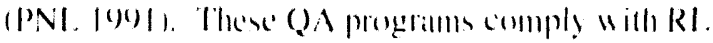

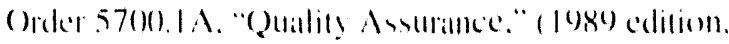

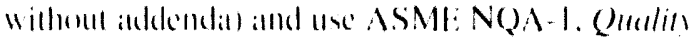

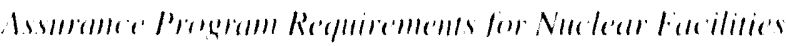

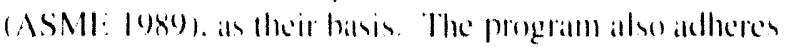

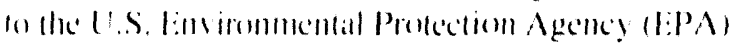

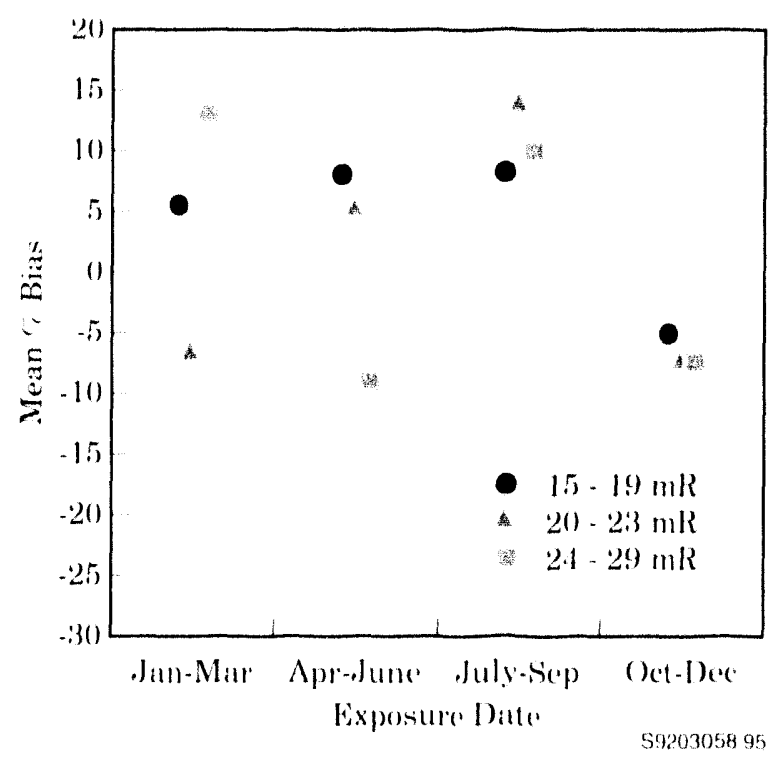

Figure 7.1. Comparison of Thermoluminescent Dosimeter Results with Known Exposures, 1992 


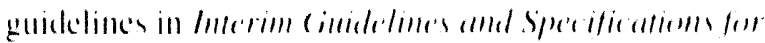

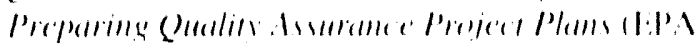

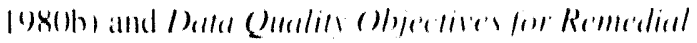

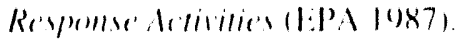

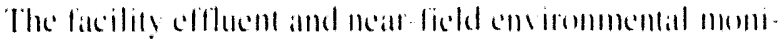

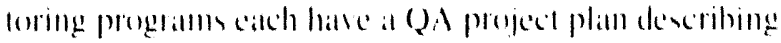

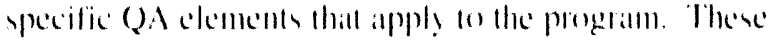

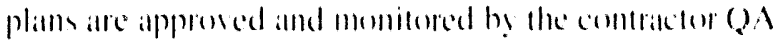

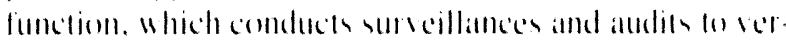
ily compliance with the plams. Wort performed through

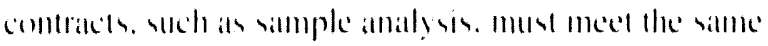

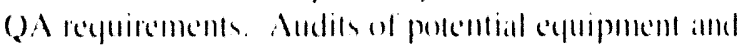

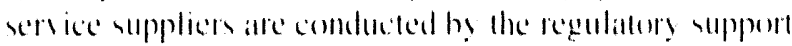

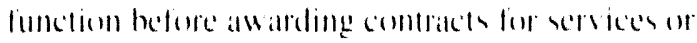

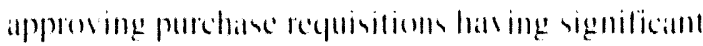
impact on a project s qualit!.

\section{Sample Collection Quality Assurance}

Eilluent and near-lateility operational monitoring silmples

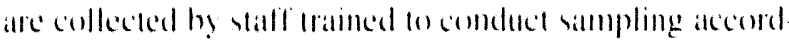
ing lo approsed and documented procedures. I ncallioms

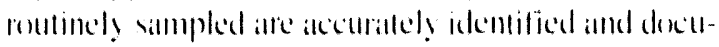

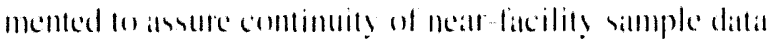

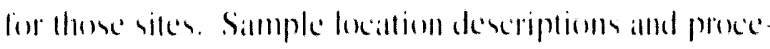

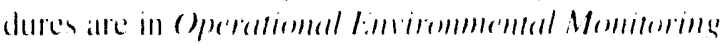

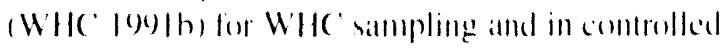
procedure lor PNI, sampling.

\section{Analytical Results Quality Assurance}

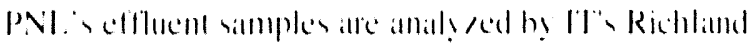

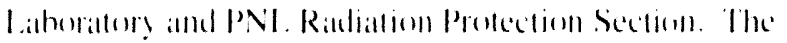

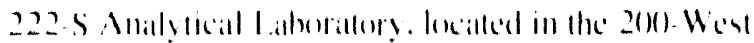

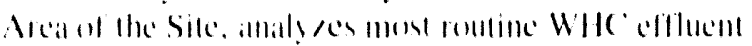

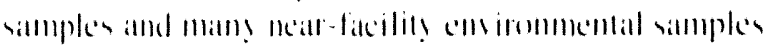

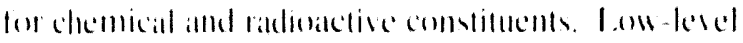

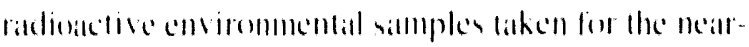
fatcilits ems irommental momitoring program atre sent to

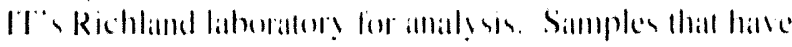

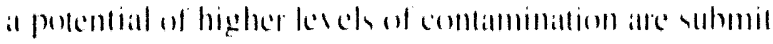

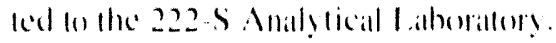

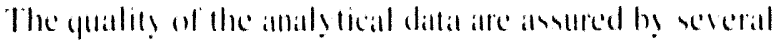
meims. Counting romm imstruments, for imstallece. alle

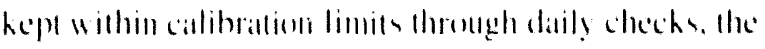
resuls of which ane sored in al compuler database to

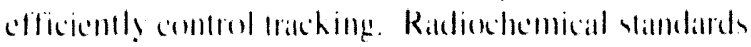
med in analy ses alle regulanly measured and the resuls,

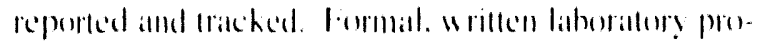
cedures are used in analy ging salmples. Analy lical pro.

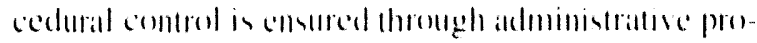

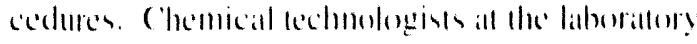

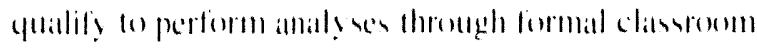
and in- lle-joh llatining.

for years the 222.5 Analytical Lathoratery has partici. palled in the I:I'A I:m irommental Monitoring and Sur-

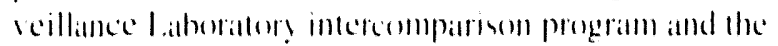

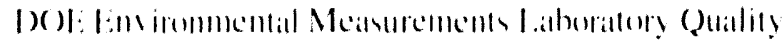

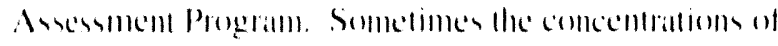
the stambards alle kes than the established delection

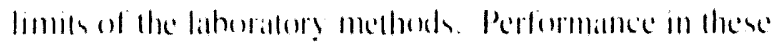

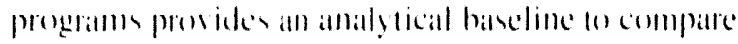

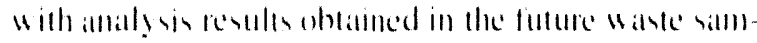
pling and shanacterisation lacilit!. Analytical summartes

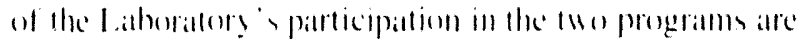
shown in Tables 7.5 and 7.6 . 
Table 7.5. 222-S Analytical Laboratory Performance on DOE Quality Assessment Program Samples, 1992

\begin{tabular}{|c|c|c|c|}
\hline Sample Media & Radionuclides & $\begin{array}{l}\text { Number } \\
\text { of Resulss } \\
\text { Reponted }\end{array}$ & $\begin{array}{c}\text { Number } \\
\text { Wilhin Control } \\
\text { l.imits:" }\end{array}$ \\
\hline Air liblers & 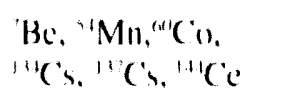 & 13 & 12 \\
\hline Soil & "K. "Cs & 4 & 3 \\
\hline Vegetation & "K. "'Cs & 5 & 5 \\
\hline Watcr & 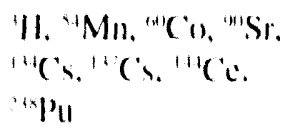 & 11) & 19) \\
\hline
\end{tabular}

(a) Control limits are from Sanderson (198.5).

Table 7.6. 222-S Analytical Laboratory Performance on EPA Intercomparison Program Samples, 1992

\begin{tabular}{|c|c|c|}
\hline & & $\begin{array}{l}\text { Number } \\
\text { of Results }\end{array}$ \\
\hline Sample Media & Radionaclides & Reported \\
\hline
\end{tabular}

Air fillers

Totil illphit.

6

$3^{w+1}$ total betit. "'c's

Alphia. betit. and gaimmia

Total allphita.

34

$26^{\text {thi }}$ emilters in watter lotial betil"." "Io, "'Zn. "'"Ru. " "Bit.

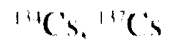

Willer

U (1titurill). " "Pu

6

4

Tritium in waller

'H

3

3

(a) Control limits ane from Jarvis and Sill (|) 191 ).

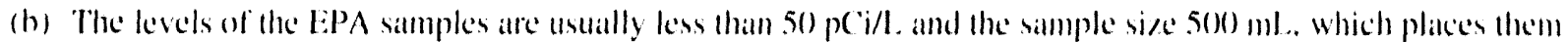
all or below backgromen levels an the 222-S Analytical laborittory.

(e) Performance differences exist in inalyzing betal samples beciluse the instruments at the 222-S Antalytical

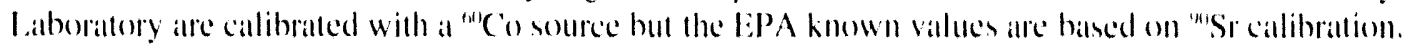


References

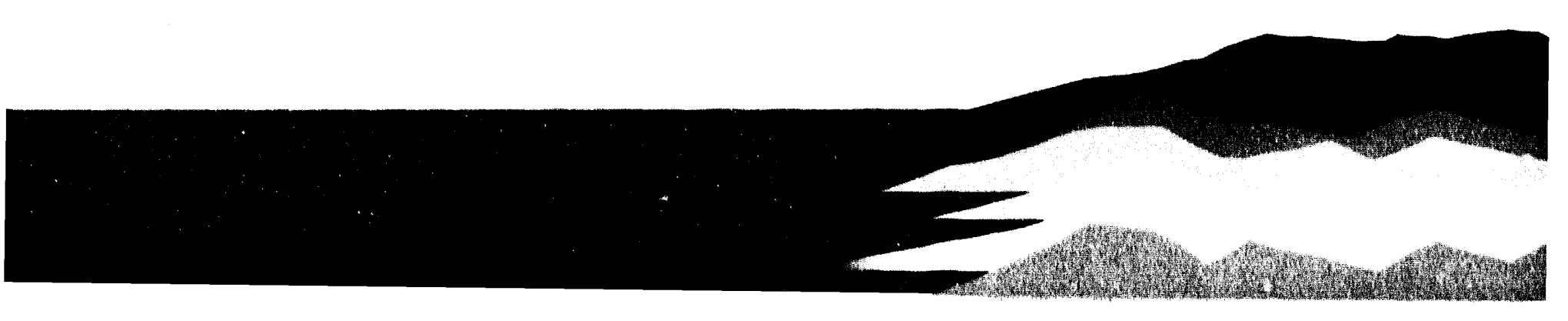




\subsection{References}

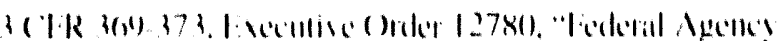

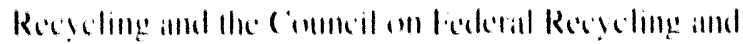

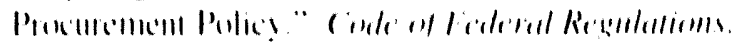

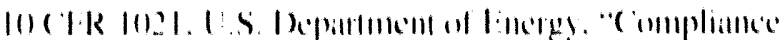

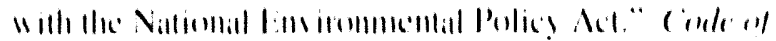
leatiral herulatroms

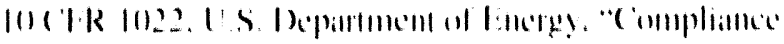

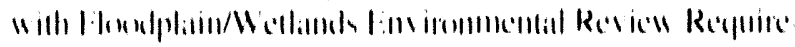

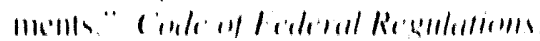

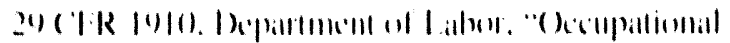

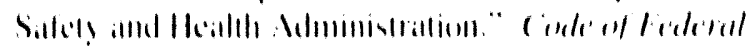
Re bullatim!l

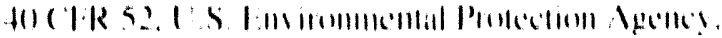

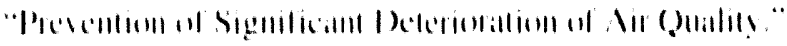

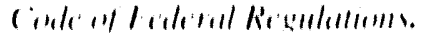

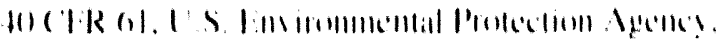

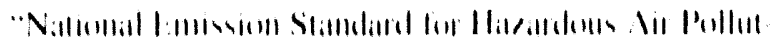

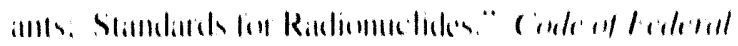

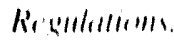

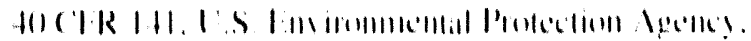

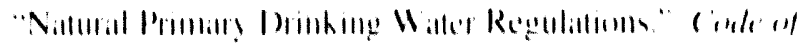

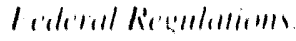

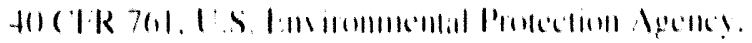

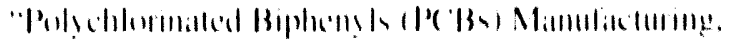

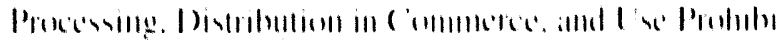

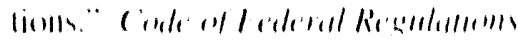

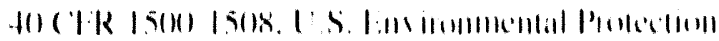

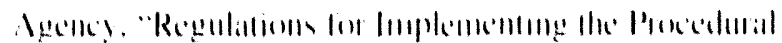

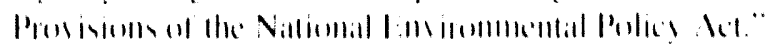

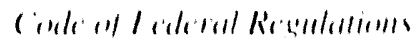

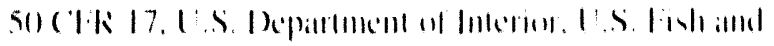

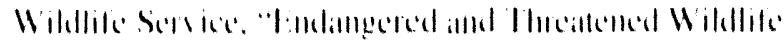

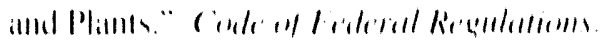

56 l R (1)

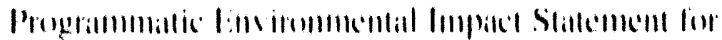

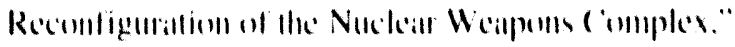

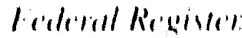

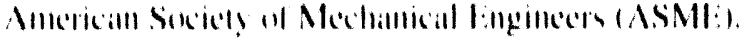

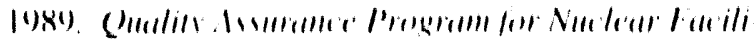

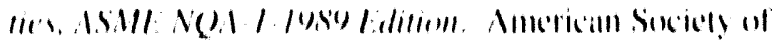

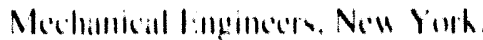

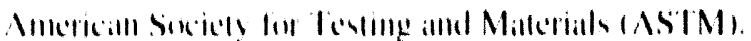

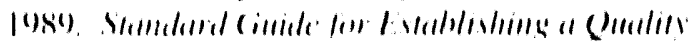

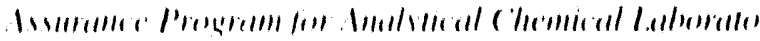

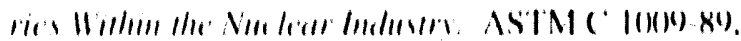

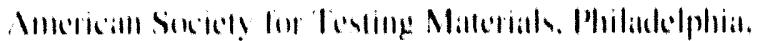
Penlm,liallia

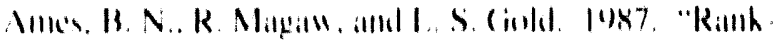

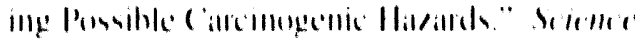

$231.271 .2 \times 11$.

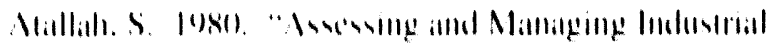

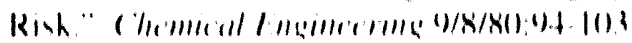

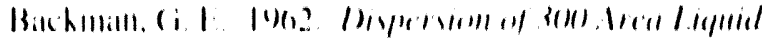

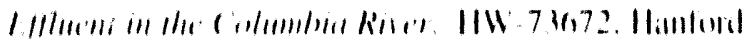

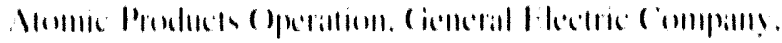
Richlimll. Warhim!ton.

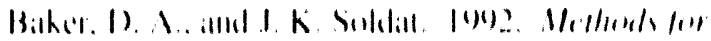

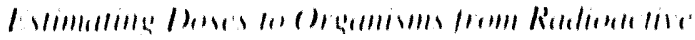

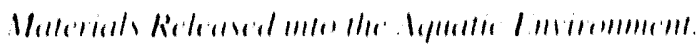

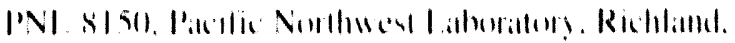
II

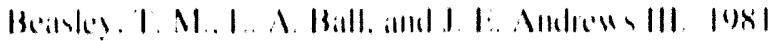

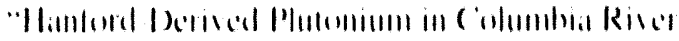

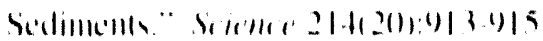

Bach. 1) M.. B. A. Nipled. M. I. Sionl. A. (i. Thumman.

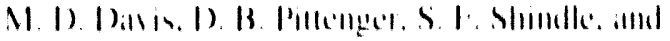

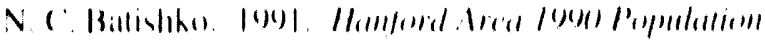

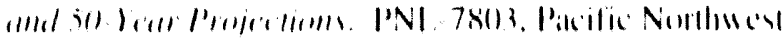

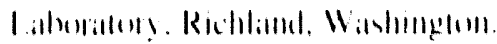




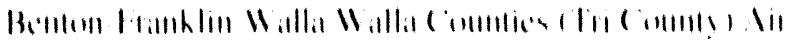

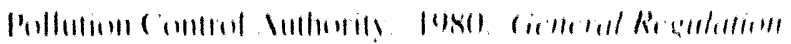

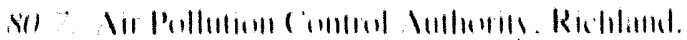

II ishrrethell.

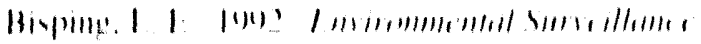

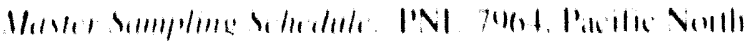

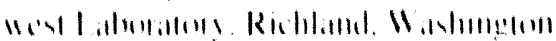

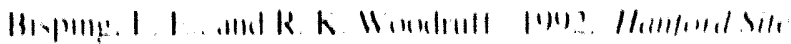

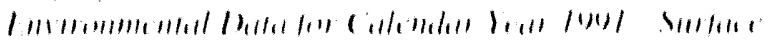

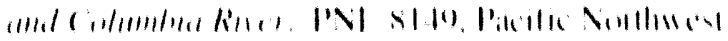

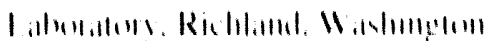

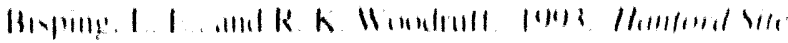

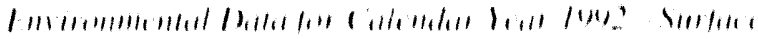

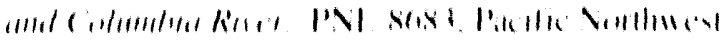

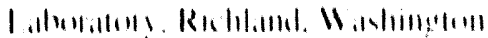

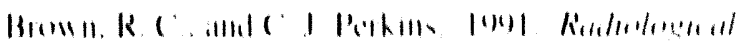

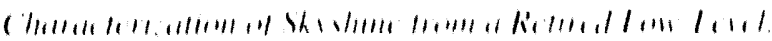

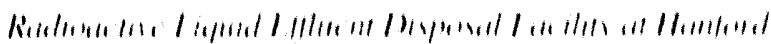

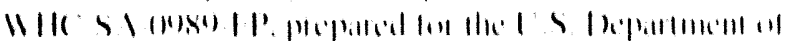

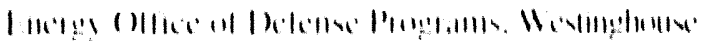

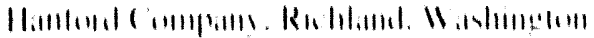

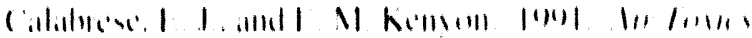

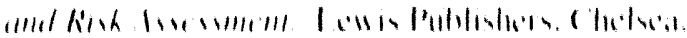
Nhelintill

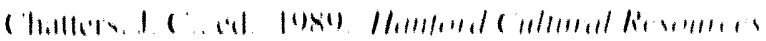

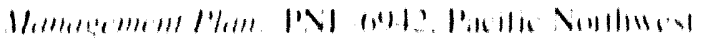

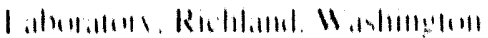

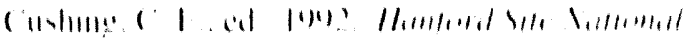

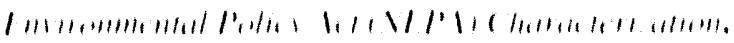

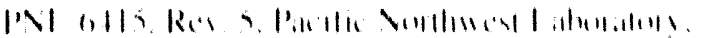

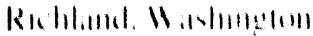

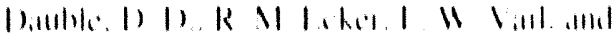

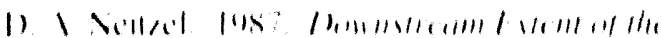

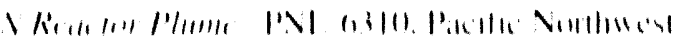

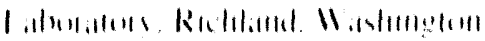

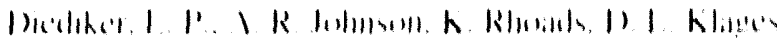

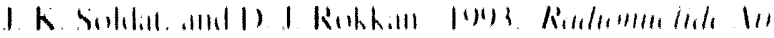

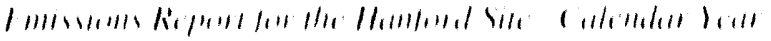

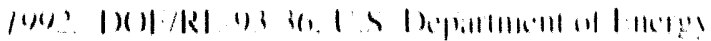

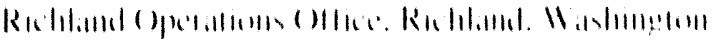

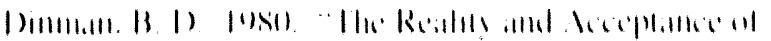

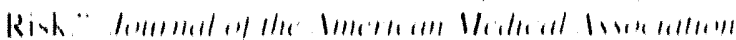
$1.111111111,1 \% 2,12 x$

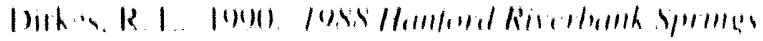

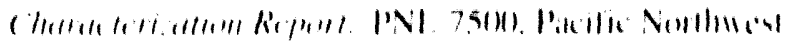

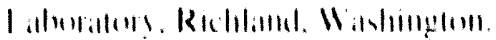

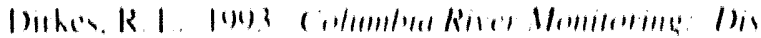

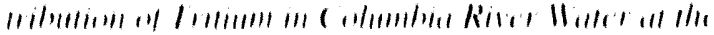

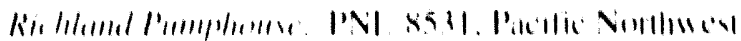

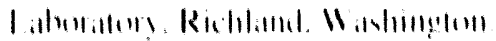

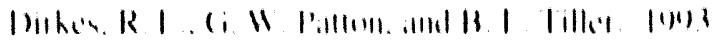

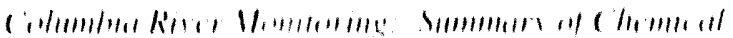

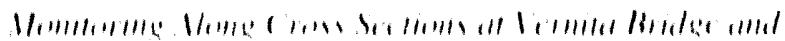

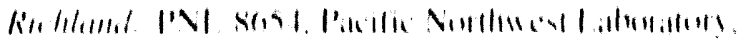

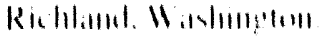

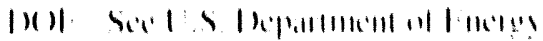

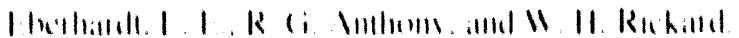
fuse "Som

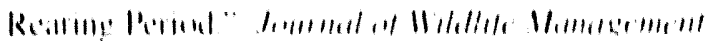
$\$ 1,17,17$

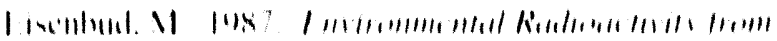

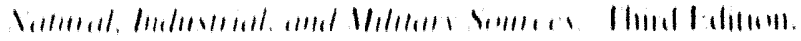

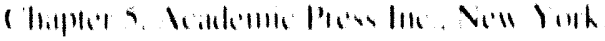

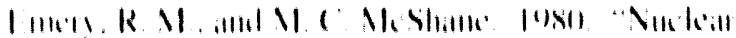

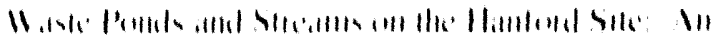

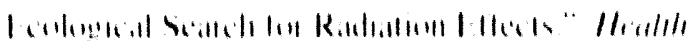
$1 \% 111$, is 757 sill

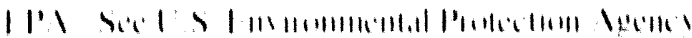

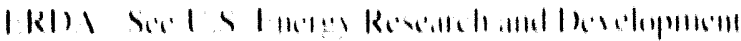

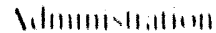

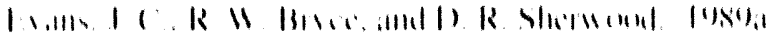

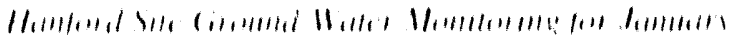

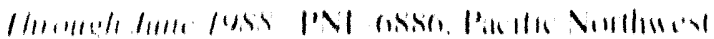

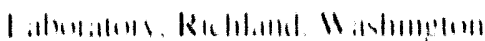

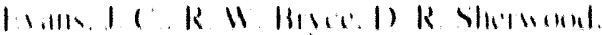

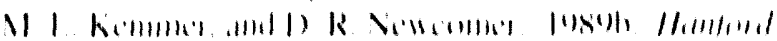

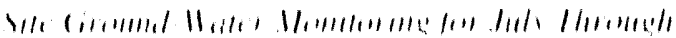

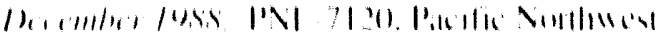

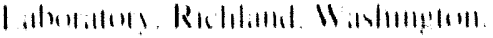

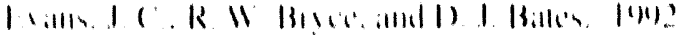

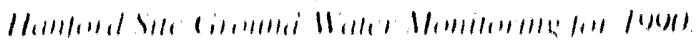

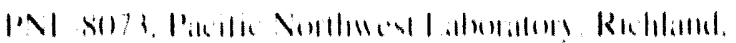
II 


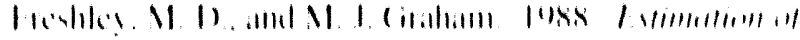

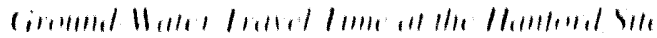

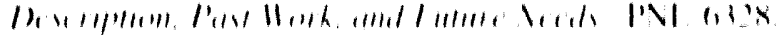

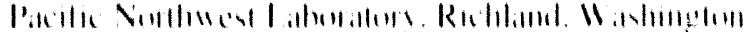

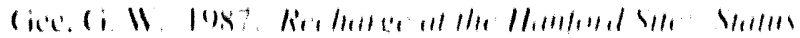

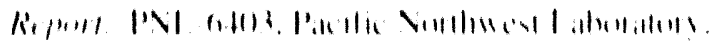

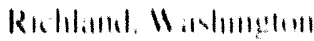

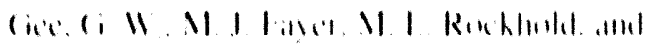
II 1 (

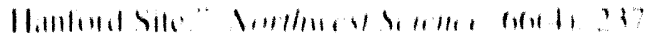

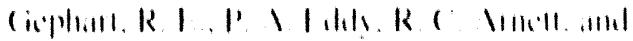

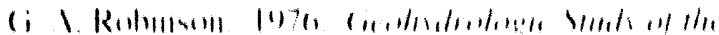

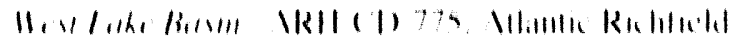

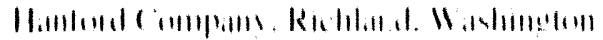

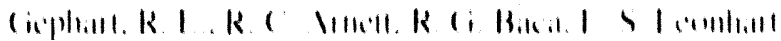

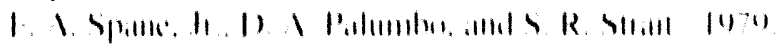

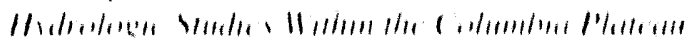
II,

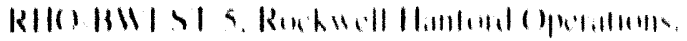

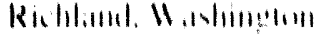

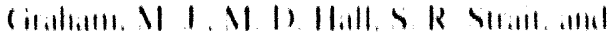

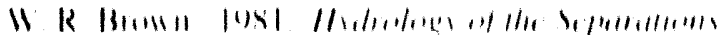

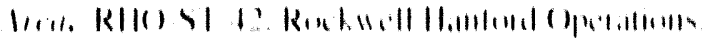
Rithlinl. II, Nhum:tu川

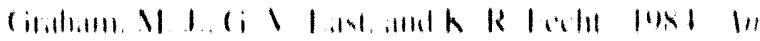

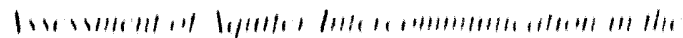

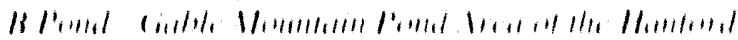

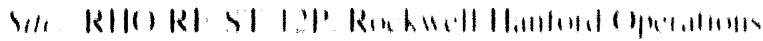

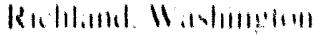

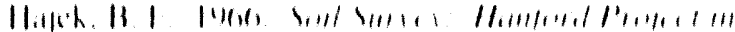

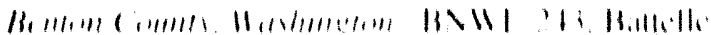

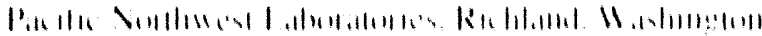

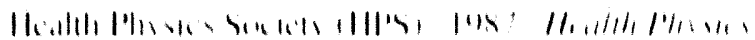

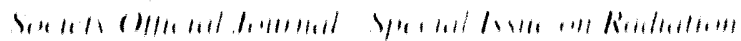
$1111111,11,1$,

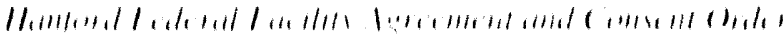

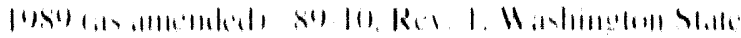

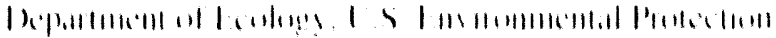

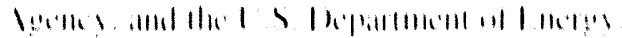

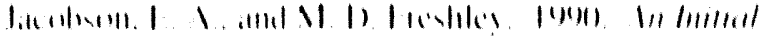

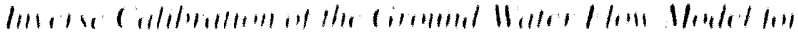

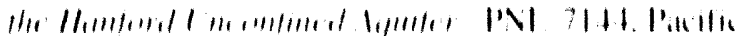

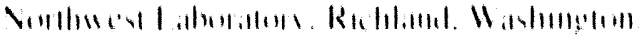

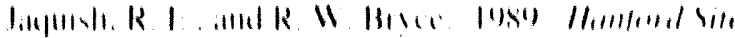

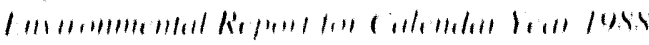

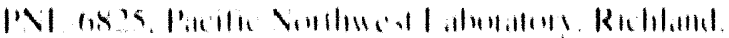
$11,1,111,1,1111$

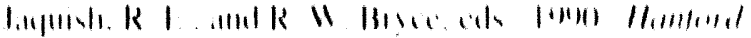

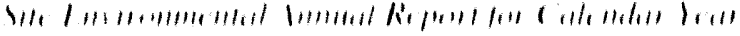

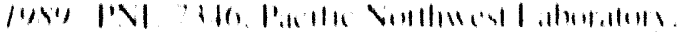

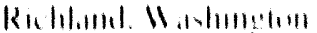

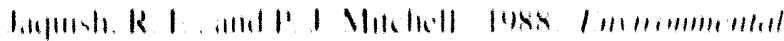

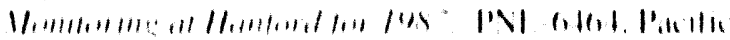

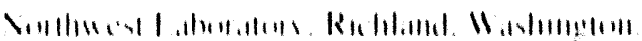

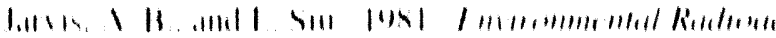

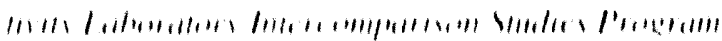

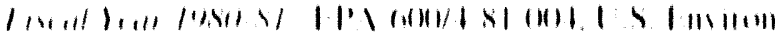

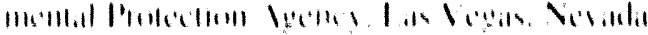

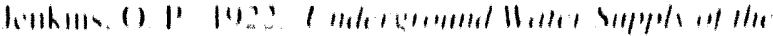

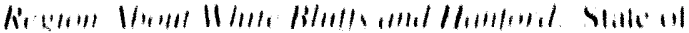

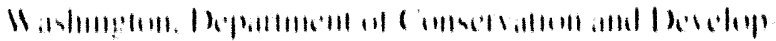

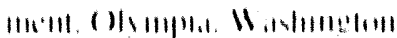

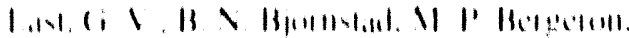

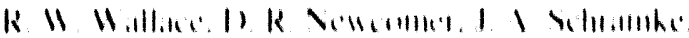

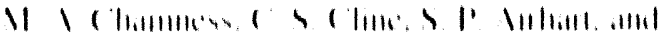

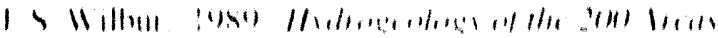

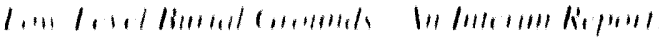

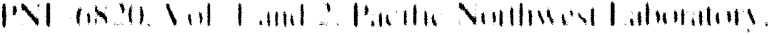

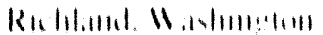

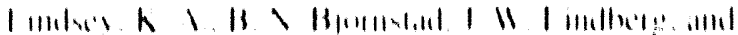

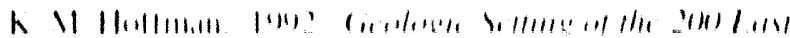

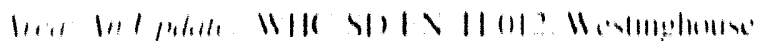

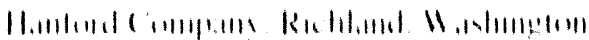

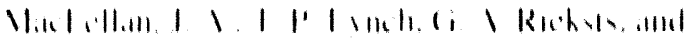

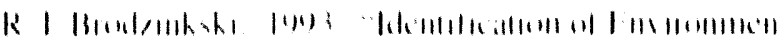

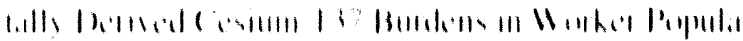

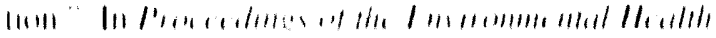

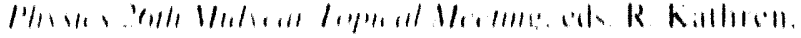

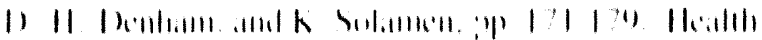
InI 


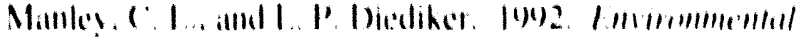

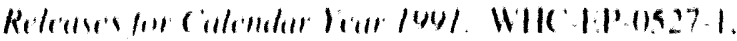

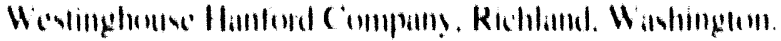

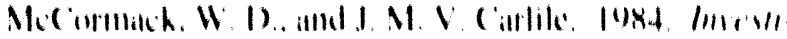

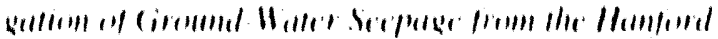

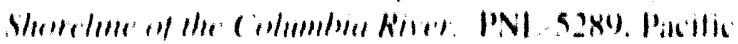
Northwenl ahorallons. Kichland. Wishimplom

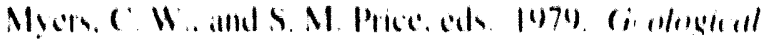

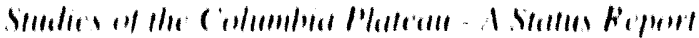

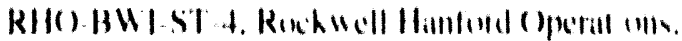
Rishlatud. Wishlinglon

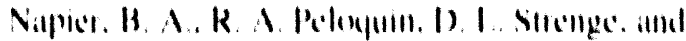

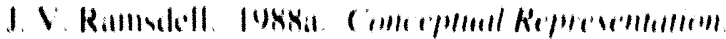

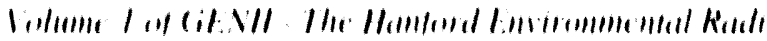

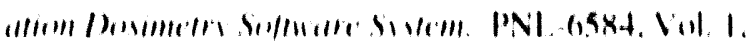

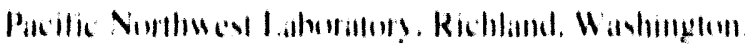

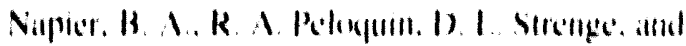

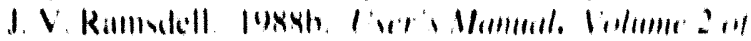

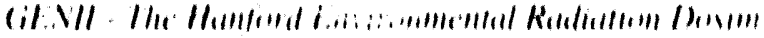

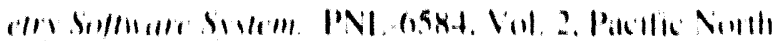

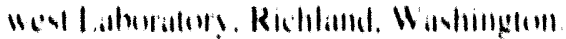

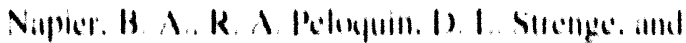

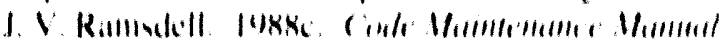

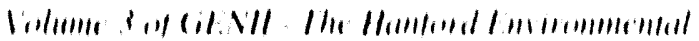

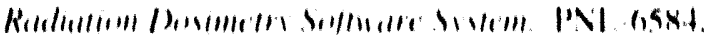

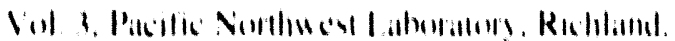
Hawlingluin.

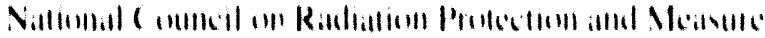

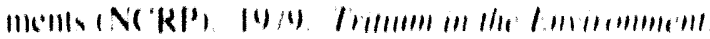
N('RP'Repunt Vu 6.. Wishimglum. I)

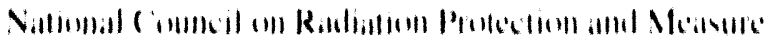

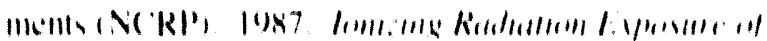

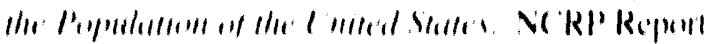
Nis, 43. Batherdir. Mansliand

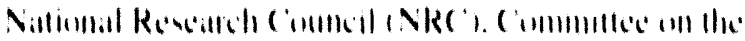

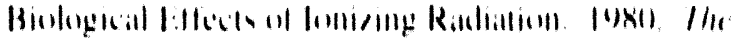

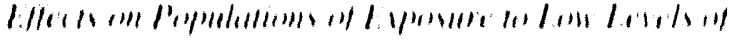

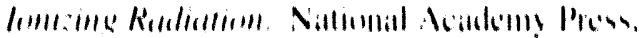
Washinglon, 1)

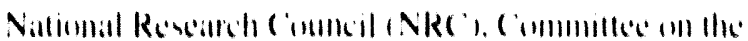

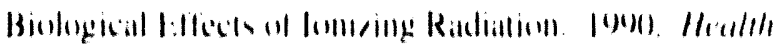

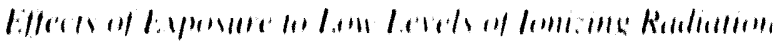

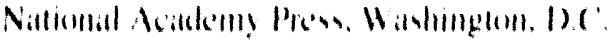

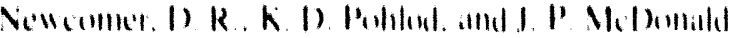

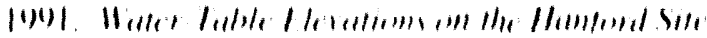

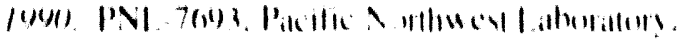
Richlind. Washunglun

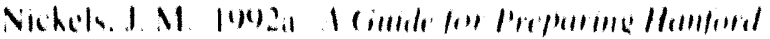

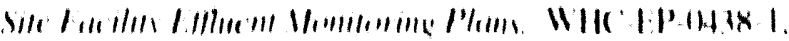

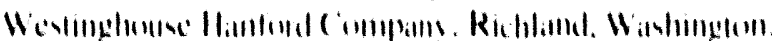

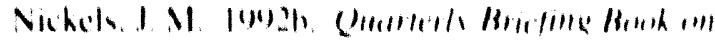

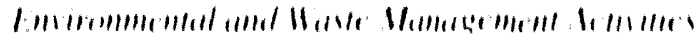

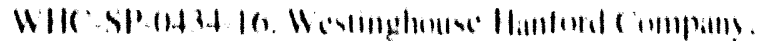
Richland. Wishomglum

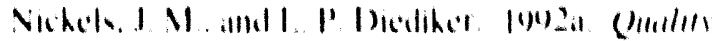

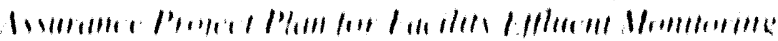

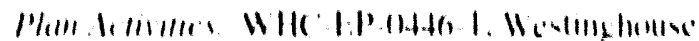

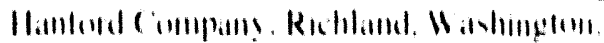

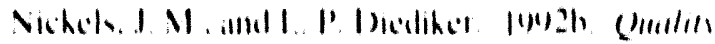

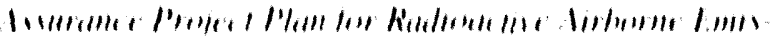

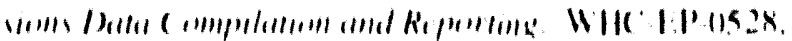

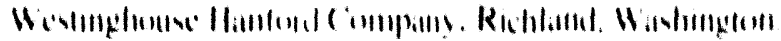

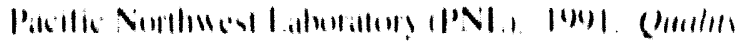

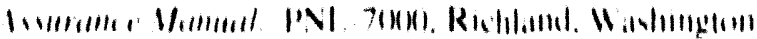

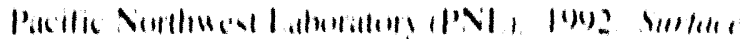

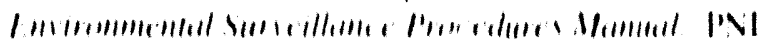

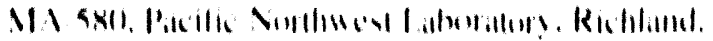
Wishollglull

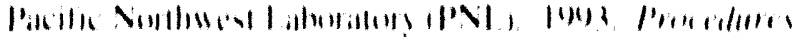

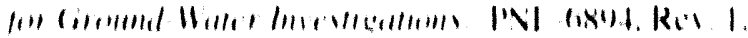

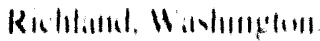

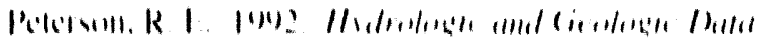

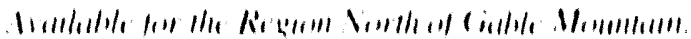

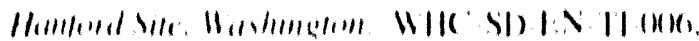

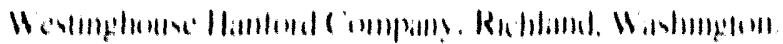

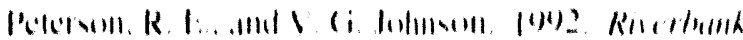

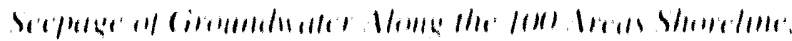

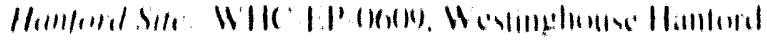

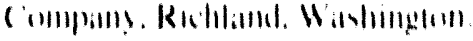

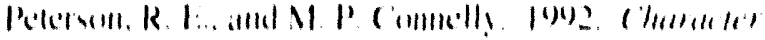

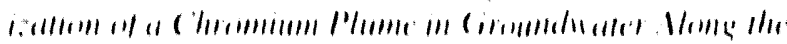

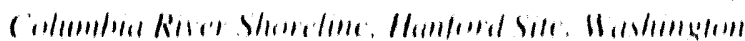

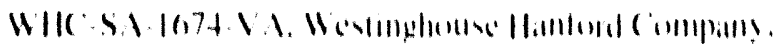
Richlamd. Winhimetom. 


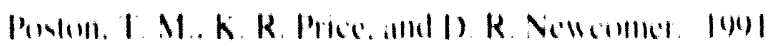

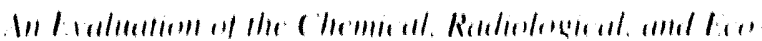

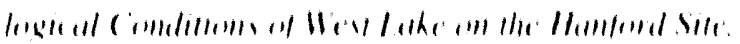

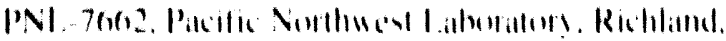
Hawhimglinn

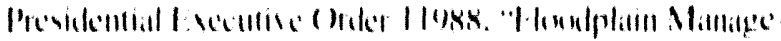

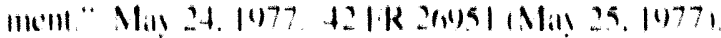

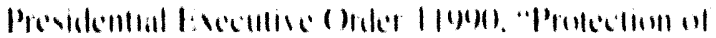

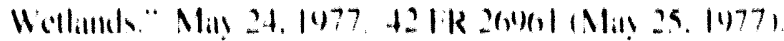

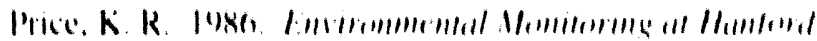

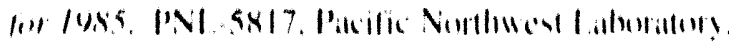
Rishland. Hashinglant.

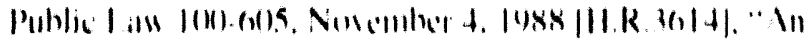

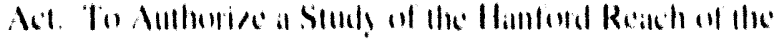

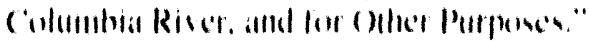

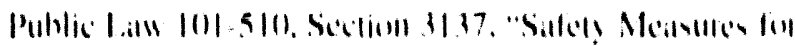

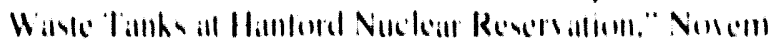

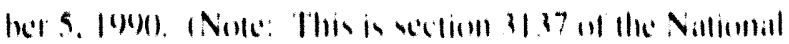

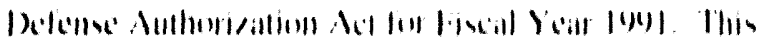

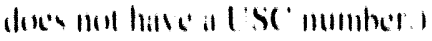

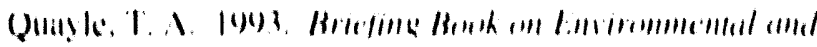

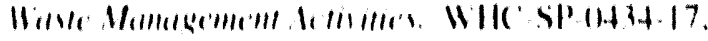

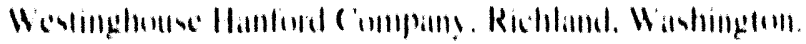

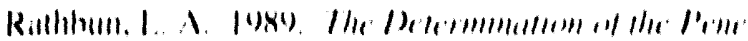

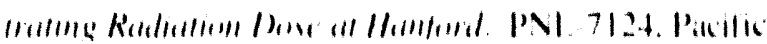

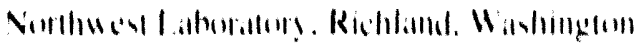

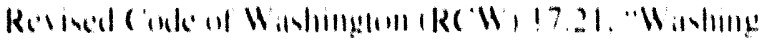

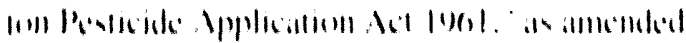

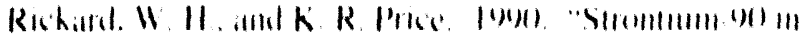

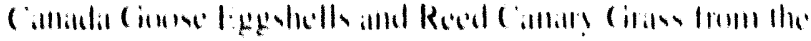

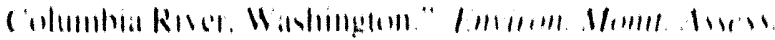
11)11:1.7:71.76

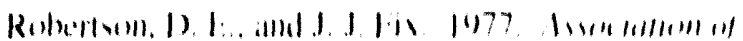

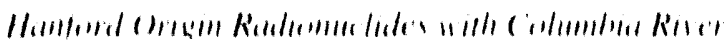

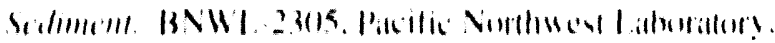
Ruhlambl. Wishinglun

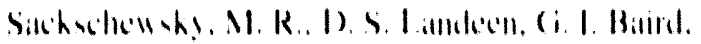

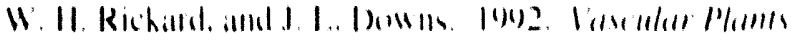

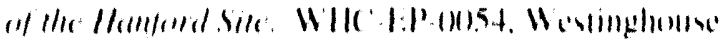
Himford ('mmpin!s. Richlimd, Washinglon.

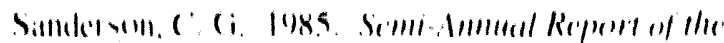

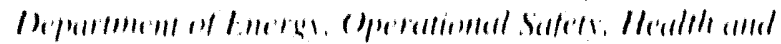

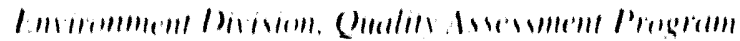

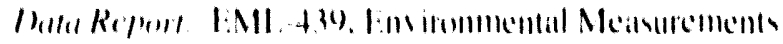

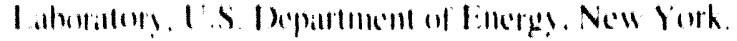

Simmidt, I W. A, K, Johmom, s M Mckimmer. and

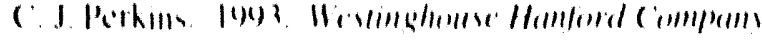

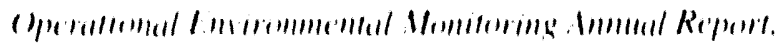

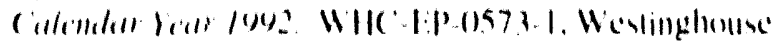

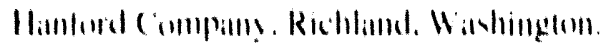

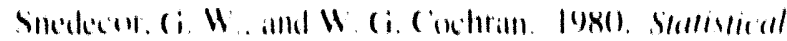

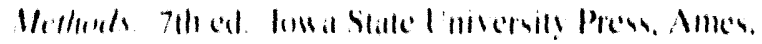
liwia.

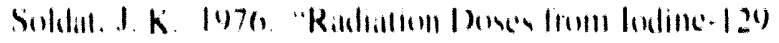

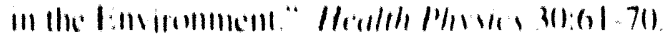

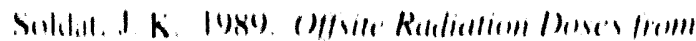

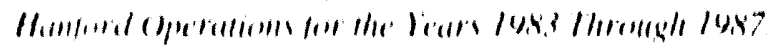

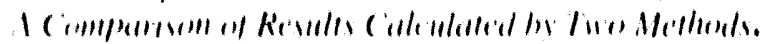

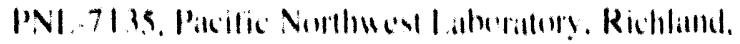
Wavling!luI.

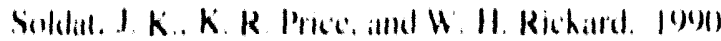

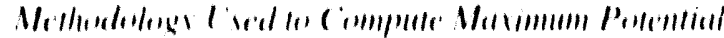

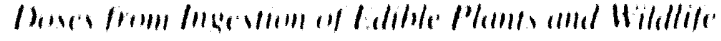

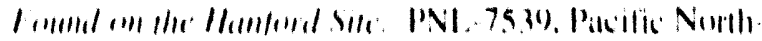

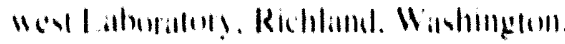

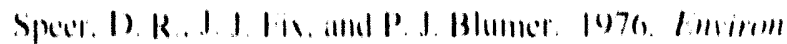

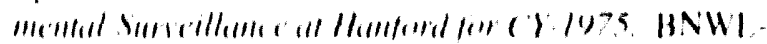

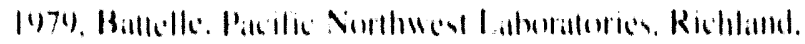
WiNhIIILL)

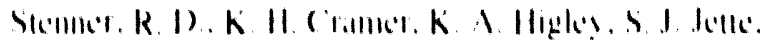

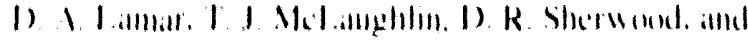

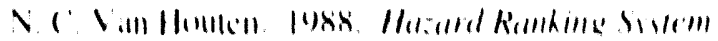

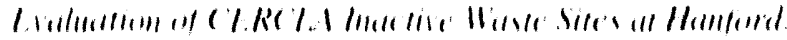

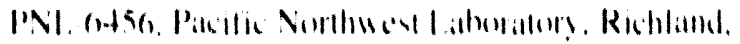
IIathilgh!n

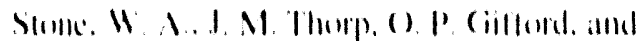

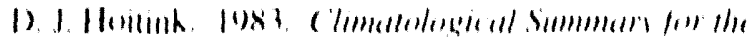

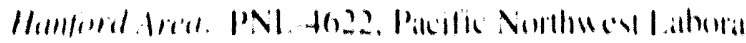
lar). Richlimsl. Wirhimglon.

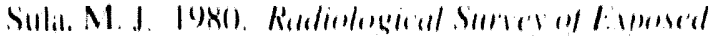

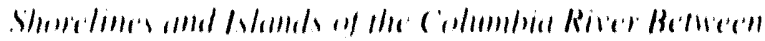

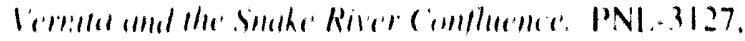

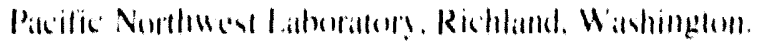




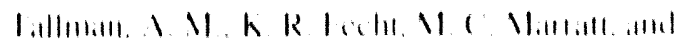

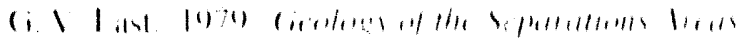

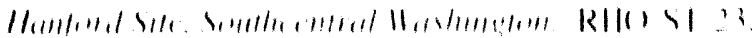

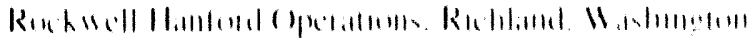

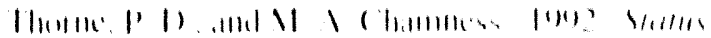

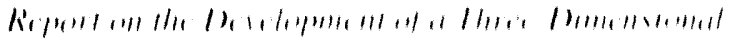

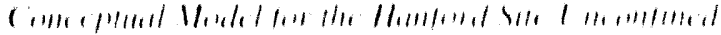

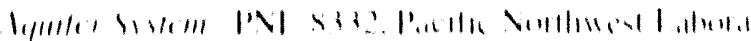

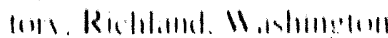

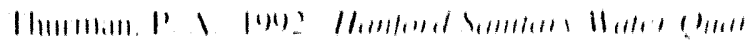

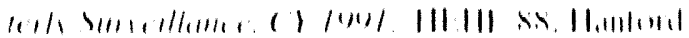

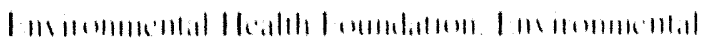

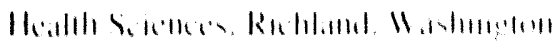

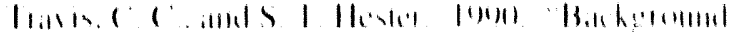

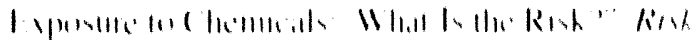
lnalw $1011+$

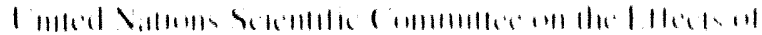

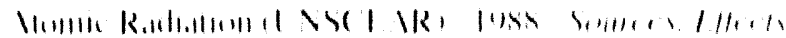

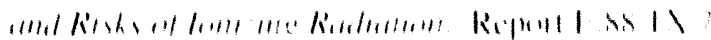
I nited Villulls. Vell link

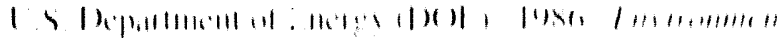

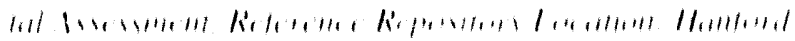

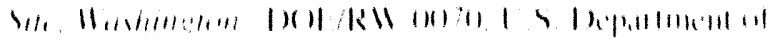

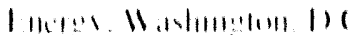

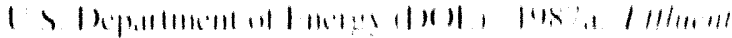

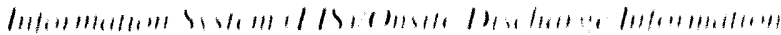

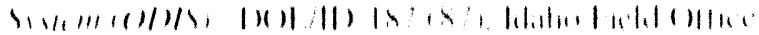

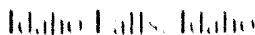

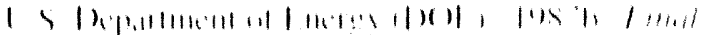

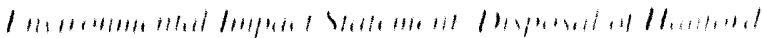

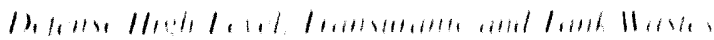

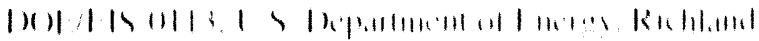
$11,1,111 m \cdot 1, \cdots$

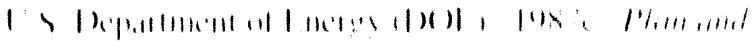

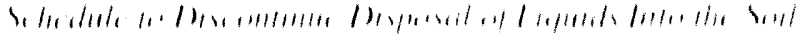

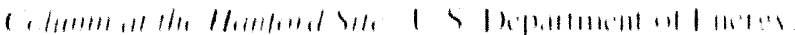

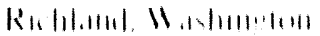

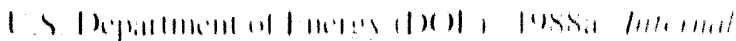

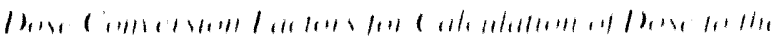

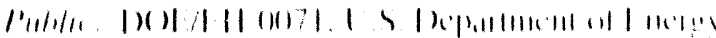

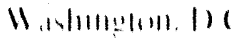

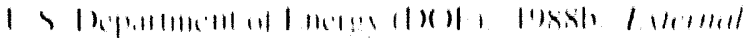

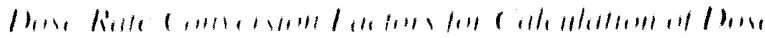

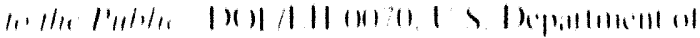

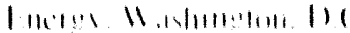

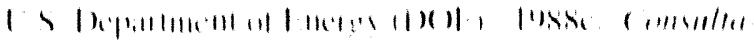

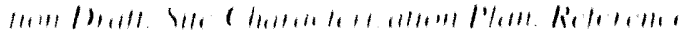

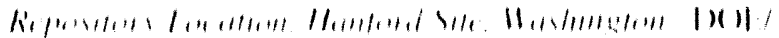

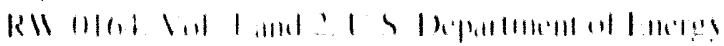

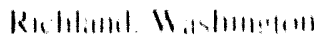

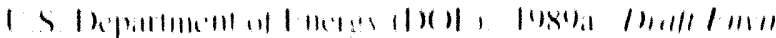

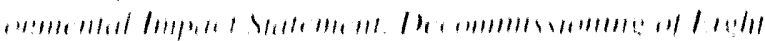

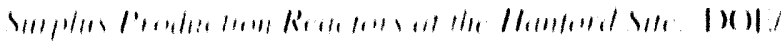

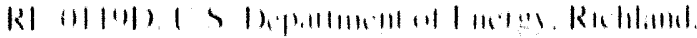
II, whIII!) 1111

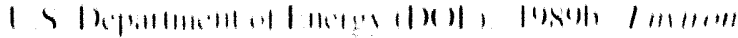

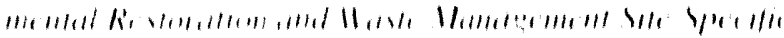

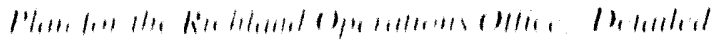

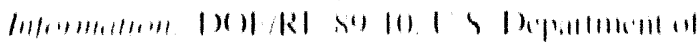

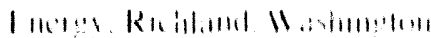

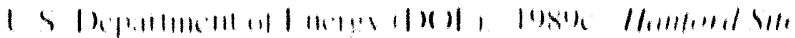

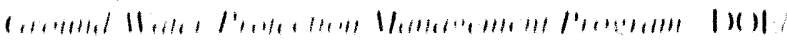

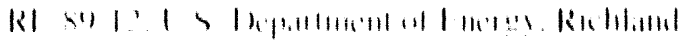

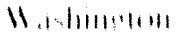

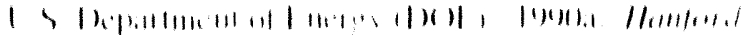

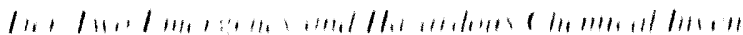

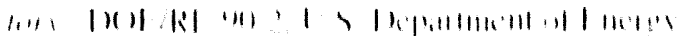

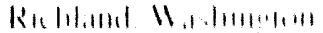

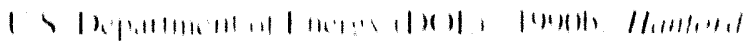

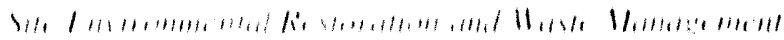

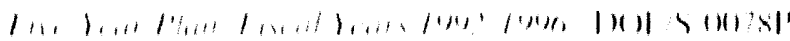

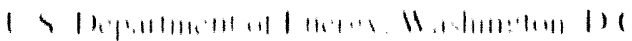

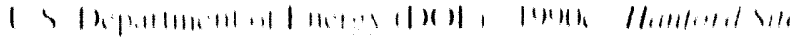

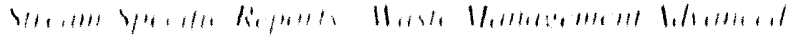

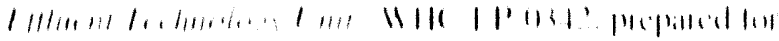

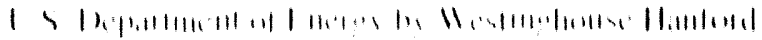

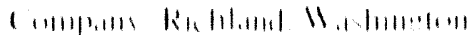

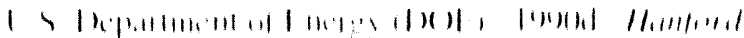

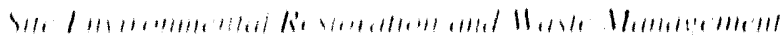

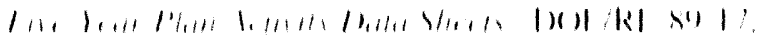

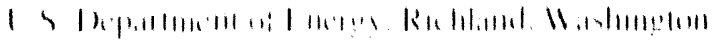

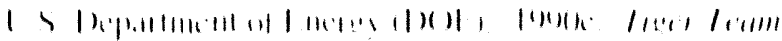

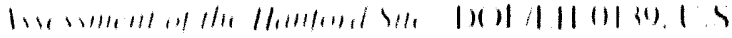

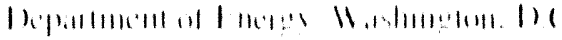




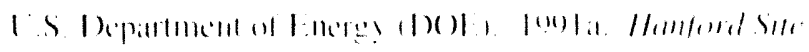

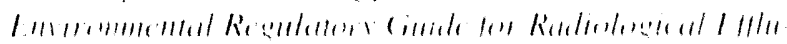

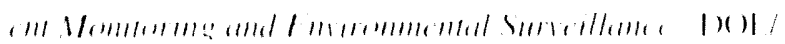

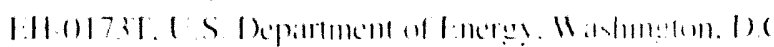

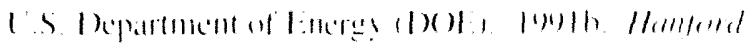

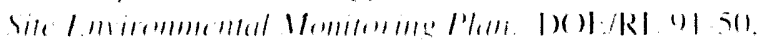

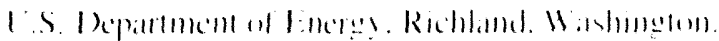

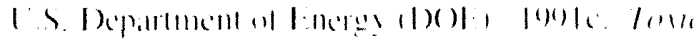

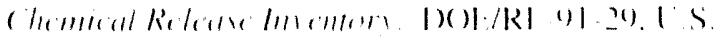

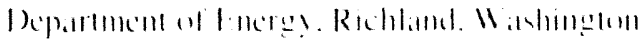

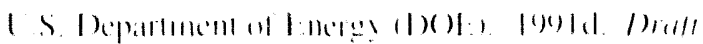

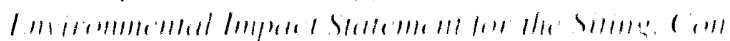

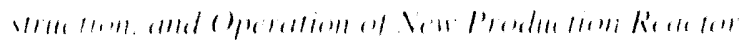

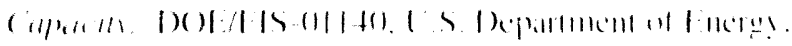
II (1)

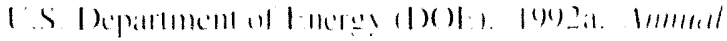

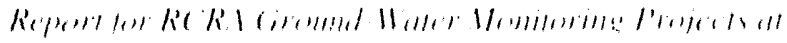

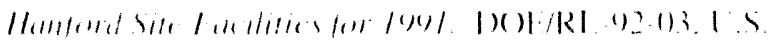
1)

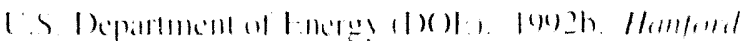

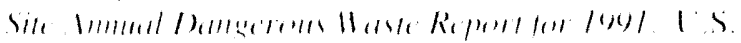

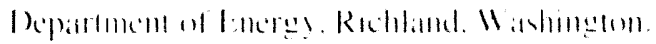

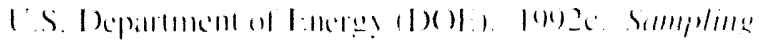

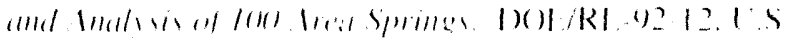

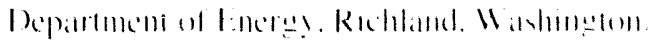

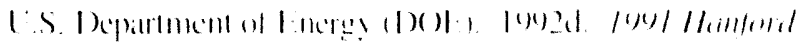

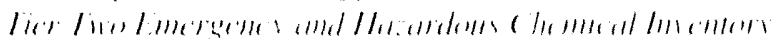

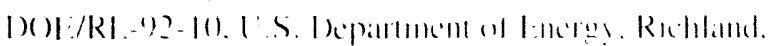
Hikhim!toll.

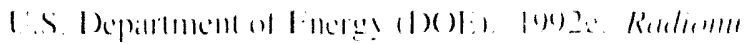

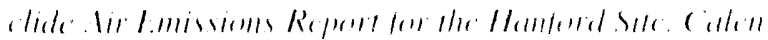

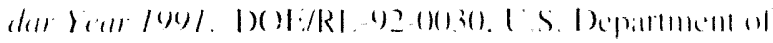
lacre!. Richlambl. Marhinglon.

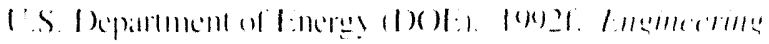

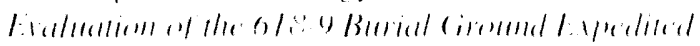

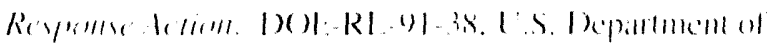

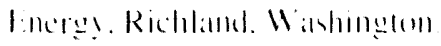

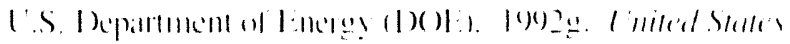

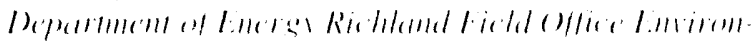

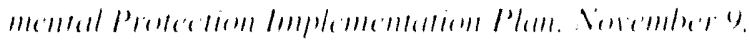

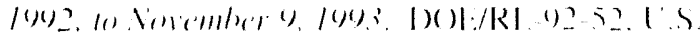

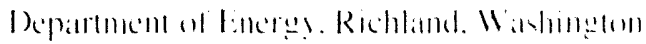

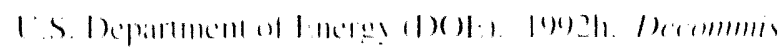

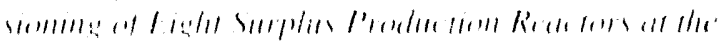

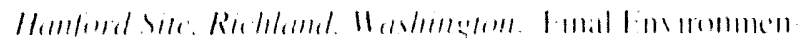

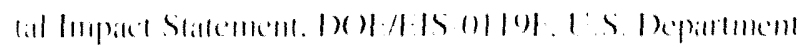

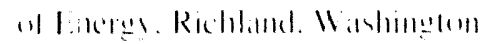

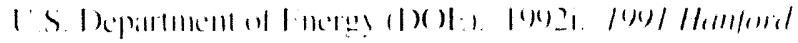

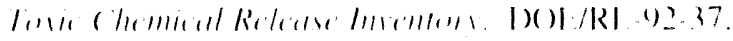

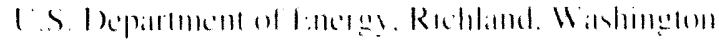

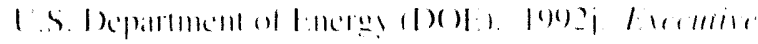

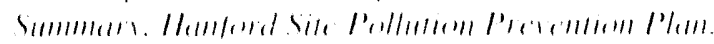
() 11 WIII:-11,1)

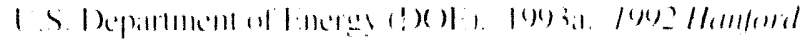

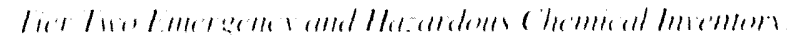

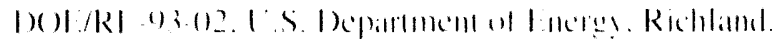
Hahmelon.

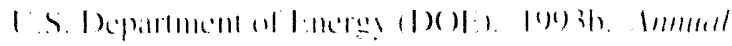

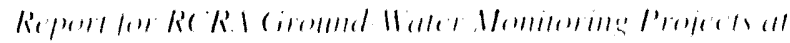

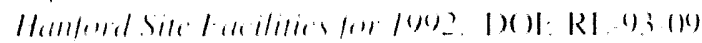

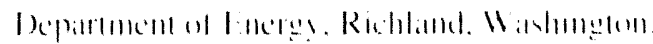

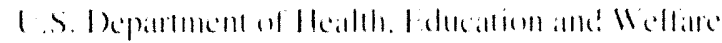

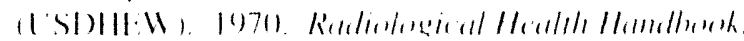

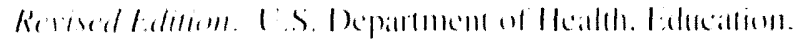

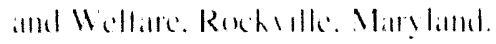

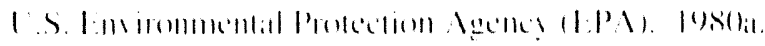

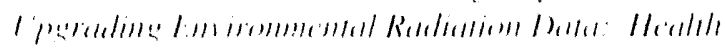

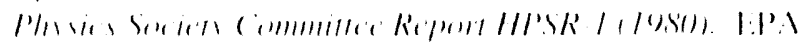

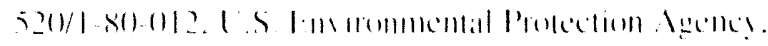
II: Mhim!n. I).

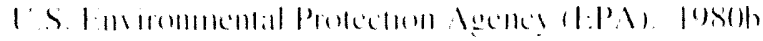

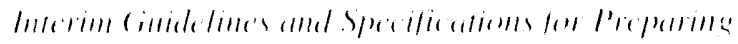

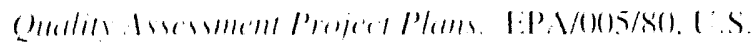

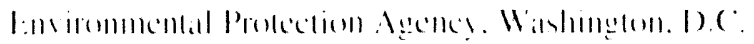

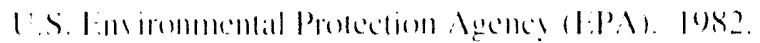

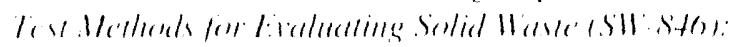

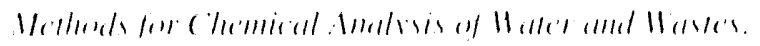

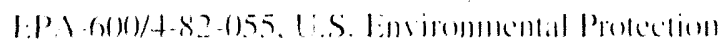

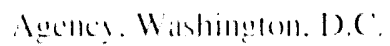

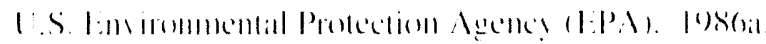

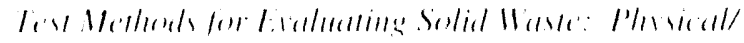

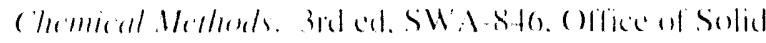

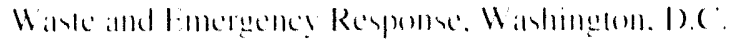


1.S. Emirommental Protection Agency (l:PA). I 980 h.

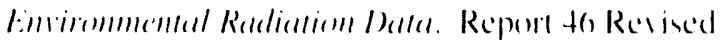

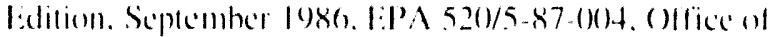
Radiation Programs. Fassern Lam irommental Raddiation

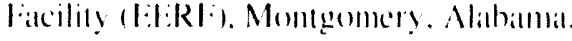

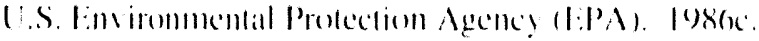

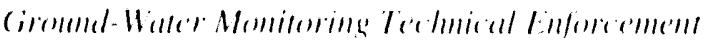

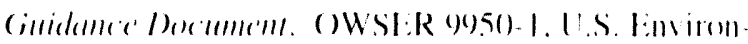
mental Prolection Agency. Wibhinglon. 1).

U.S. Insirommental Prolection Agency (l:PA). 1987.

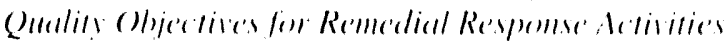

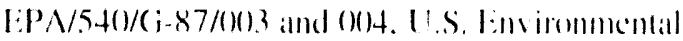
Protection Agency. Washington. 1).('.

U.S. Envirommental Profection Agency (LPA). IOS8.

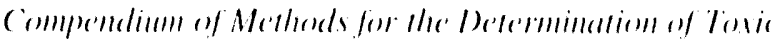

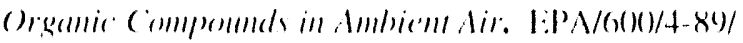
017. Atmompleric Rescitrch and Ixposure Assessment I ahoratory. U.S. Envirommental Protection Agency. Rescanch Triangle Pank. North Carolinat.

1).S. Panvirmmental Protedion Agency (I:PA). 1989).

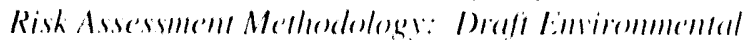

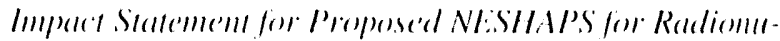

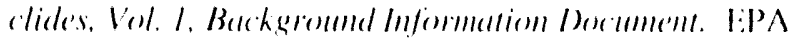
52()/1-89-()(15. U.S. Envirommental Protection Agency. Washingtom. 1).('

U.S. Cieological Survey (lISGS). 1988. Waller Resomeres

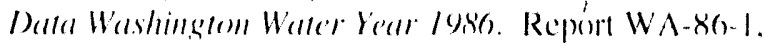
U.S. Cieological Survey. I) nerver. (oloraldo.

Washington Administrallice (ode (WAC) 16-228. (ieneral Pesticide Regulattions. Washimgton State I department of Agriculture.

Wathington Administrattive Code (WAC) 17.3-160. Minimum Standards for Comstruction amd Matintenance of Wells, Washinglon State Department of licology.

Washington Administrative Code (WAC) 17.3-30). Dangerous Waste Regulations. Washinglon Siate Department of lioblogy.

Washington Administrative Code (WAC) 17.3-.30). Pollution Prevention Planning. Washinglon State Department of licology.

Washington Administrative Code (WAC') $246-247$. Radiation Protection--Air Emissions, Washington Stalle Department ol Health.

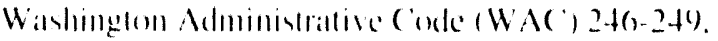

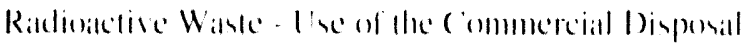

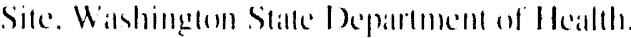

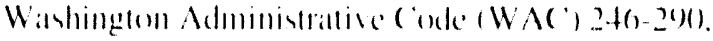

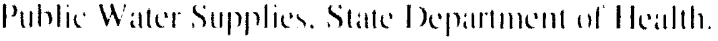

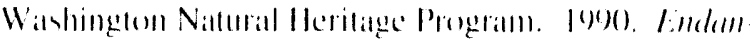

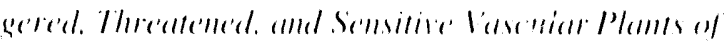

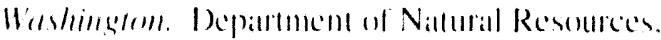
()lympiat. Washingtont.

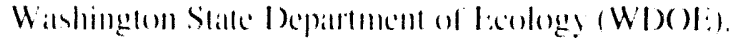

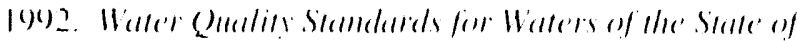

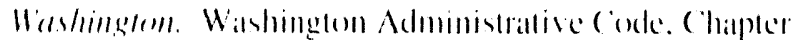
17.3.201a. ()! milpia. Watshington.

Warthimgen Stalle Iepartment of Social and Heath

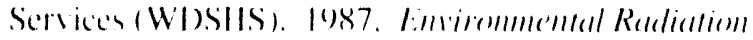

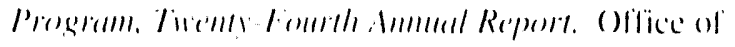
Radiantom Prolection. ()lympial. Washingenton.

Westinghouse Hamford (omprans (WHC). 1989. Qualim Ansmance Mammal. Whic ( M-t-?. Westinghouse Hamtind (ompany. Richland. Washinglonn.

Westimghouse Hanford (ompany (WH('). I90).

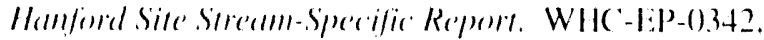
Westinghouse Hanford (ompany. Richland. Washinglon.

Westinghouse Hanlond (immpany (WHC). 199) la. Emirommemal compliance. WIIC'-('M-7-5.

Westinghouse Hanford (ompany. Richland, Walshington.

Westinghouse Hanford ('ompany (WHe'). 199)

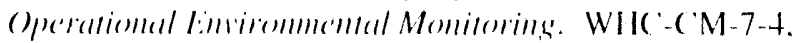
Westinghouse llanlond (ompany Richland. Walshingten.

Wilson. R. and E.S.C. Cromch. 1987. "Risk Assessmem and comparisoms: An Introduclions." Sicience 236 (.7790):2(17-270.

Wondrull. R. K.. R. W. Hanl, M. (i. Helly, and

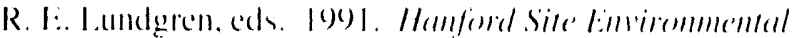

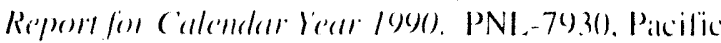
Northwest I aboratory. Richand. Washinglon.

Woudrull, R. K.. R. W. Hanl. and R. I: I andgren, eds.

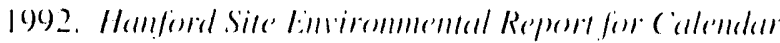
Yoal |y9l. PNI, Rl +8. Pacilic Northwest l aboratory. Richland. Washington. 


\section{U.S. Department of Energy Orders}

1)()H: (Mrder. $50(0) .3 \mathrm{~A}$. "()ceurrence Reporting and Processing of (Operations Intormation."

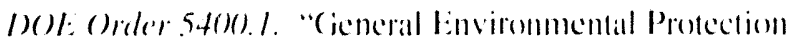
Programm."

D) OE: (order 540().5, "Ratdiation Protection of the Public and the Enviromment."

1)()E (Order .5ft(). II). "Nattional Envirommental Policy Act Compliance Program."

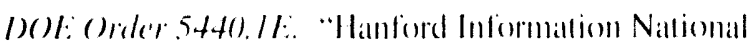
Imvirommental Policy Act Compliance Program."

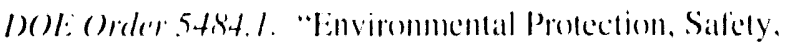
and Heallh Profection Information Reporting Reyuirements."

RL (Mider. 57(N). LA. "Quality Assurance."

Dot: ()rder .5820.2A. "Rardionetive Waste Management." 


\section{Acts}

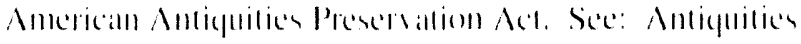

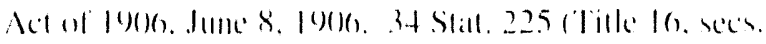
$+31,+32.4331$.

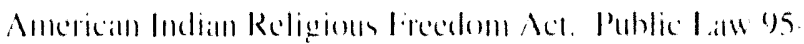

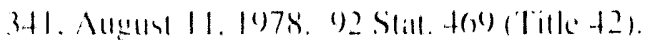

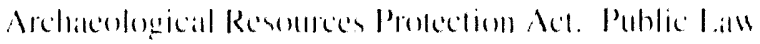

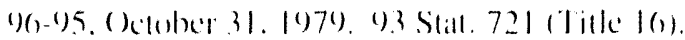

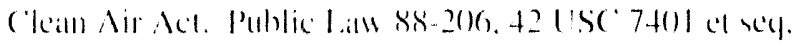
ar ankmeled.

('leall Waller Acl. Public l all 95-217. 1)ecember 27. 1977. 91 Stall. 1560 and Public I and 90 148.

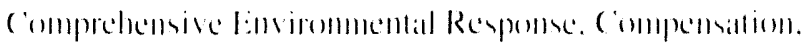

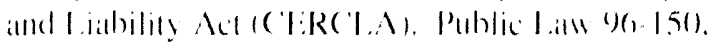

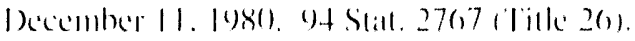

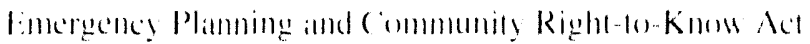

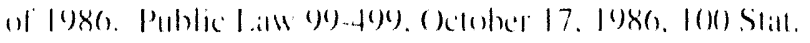

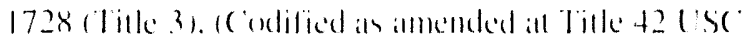
$110(1)$ el sey.).

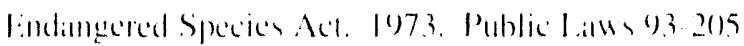
lhrough 10). 7017.
Pederal Inscedicide. Irumgicide, and Rodenticide Ace.

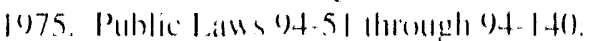

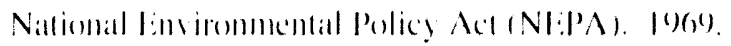

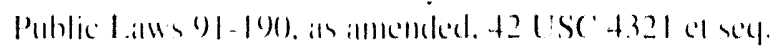

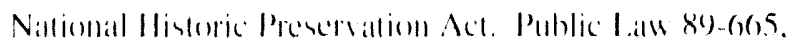

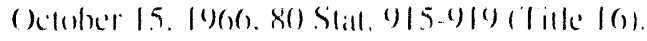

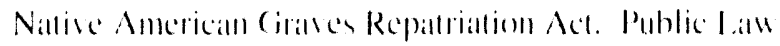

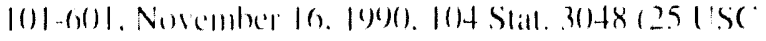
3()()$)(14 \times(y)$

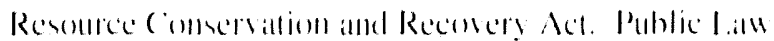

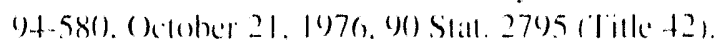

Salle Drinking Waller Acl. Public l alu 9.3-52.3.

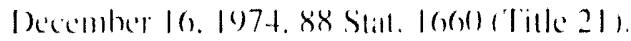

Supertund Ancondments and Reatuhnrisation Act. Public

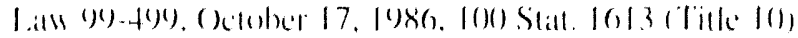

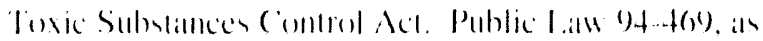

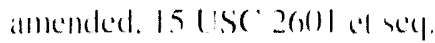


Appendixes 


\section{Appendix A}

Monitoring Results for 1992 


\section{Appendix A}

\section{Additional Monitoring Results for 1992}

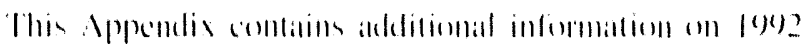

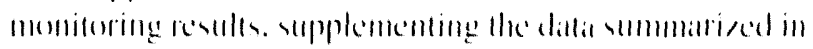

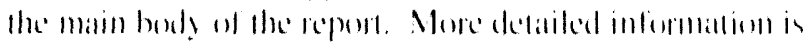

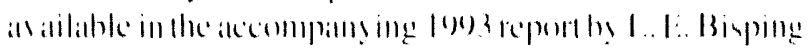

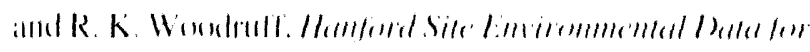

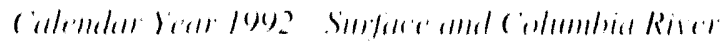

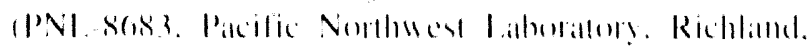
Wabhimg(o)1 
Table A.1. Radionuclide Concentrations Measured in Columbia River Water at Priest Rapids Dam. 1992 Compared to Values from the Previous 5 Years

\begin{tabular}{|c|c|c|c|c|c|c|c|}
\hline \multirow[b]{2}{*}{ Radionuedide" } & \multirow{2}{*}{$\begin{array}{l}\text { Nin at } \\
\text { Samples }\end{array}$} & \multicolumn{2}{|l|}{1092} & \multirow{2}{*}{$\begin{array}{l}\text { Nit. of } \\
\text { Samples }\end{array}$} & $\begin{array}{l}1487-1941 \\
\text { Concentrati }\end{array}$ & pCill & \multirow{2}{*}{$\begin{array}{c}\text { Dranking } \\
\text { Water } \\
\text { Standard }\end{array}$} \\
\hline & & Maximum & Average & & Maximum & Average & \\
\hline \multicolumn{8}{|l|}{ Composite System } \\
\hline Alphat & 12 & $0.8 \pm 0.7$ & $0.3 \pm 67 \%$ & 59 & $1.7 \pm 1.2$ & $0.56 \pm 18 \%$ & 15 \\
\hline Betat & 12 & $34 \pm 2.2$ & $0.7 \pm 114 r$ & 59 & $5.2 \pm 2.5$ & $1.5 \pm 27 \%$ & 50 \\
\hline$H$ & 12 & $114 \pm 4$ & $50 \pm 24 \%$ & 59 & $107 \pm 6$ & $01 \pm 7 \%$ & $20 .(x \times)$ \\
\hline "Sr & 12 & $0.1: \pm 0.14$ & $0.09 \pm 11 \%$ & 58 & $0.18 \pm 0.08$ & $0.10)=10 \%$ & 8 \\
\hline${ }^{n} T i$ & 12 & $11.32 \pm 0.11$ & $0.10 \pm 120 \%$ & 34 & $4.1 \pm 1.2$ & $-10.34 \pm 141 \%$ & (x) \\
\hline $\mathrm{I}$ & 12 & $0.27 \pm 0.14$ & $0.23 \pm 4 \%$ & 58 & $0.34=0.0 \mathrm{x}$ & $0.23 \pm 4 \%$ & $\ldots$ \\
\hline 1 & 12 & $0.02 \pm 0.01$ & $0(x)=50 \%$ & 58 & $0.14 \pm 0.02$ & $0 .(x) \pm 22 \%$ & $\ldots$ \\
\hline$\therefore \mathrm{C}$ & 12 & $0.24 \pm 0.05$ & $0.18 \pm 11 \%$ & 58 & $0.37 \pm 0.96$ & $0.19 \pm 5 \%$ & ... \\
\hline I-Total & 12 & $0.49 \pm 0.07$ & $0.42 \pm 78$ & 58 & $0.57 \pm 0.07$ & $043 \pm 5 \%$ & $\ldots$ \\
\hline \multicolumn{8}{|c|}{ Continuous System } \\
\hline 10 & 4 & 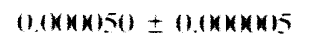 & $0.600023 \pm 91 \%$ & 16 & 0.omknus \pm 0 ormanoms & 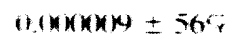 & 1 \\
\hline PuP & 4 & $\ln (x) x+4 \pm 0.0 \times x) 2$ & $0 .(0 \times 0) 3 \pm 67 \%$ & 19 & $0.06010 \pm 0.00000 \mathrm{x}$ & $\arg (x)=3 \pm 334$ & $\cdots$ \\
\hline D & 4 & $0.6 k$ ok $3 \pm 0.0$ kn)ly & $0.00020 \pm 145 \%$ & 19 & $0.0 \times 0) 14 \pm 0.0 \times m \times 07$ & $0.000005 \pm+4 r_{r}$ & $\cdots$ \\
\hline
\end{tabular}

(a) Vaximum values are \pm 2 vigma counting errors. Alerages are \pm 2 times the standard error of the calculated mean. expresed as a percentage.

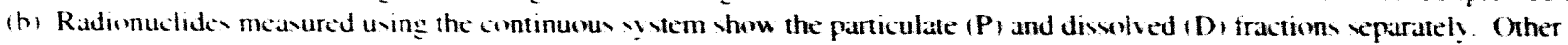

radionuclides are haved on samples collected by the compowite ststem isee text).

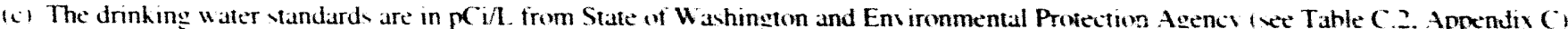

(d) Dashes indicate no concentration guides provided in drinking water standard. 
Table A.2. Radionuclide Concentrations Measured in Columbia River Water at the 300 Area. 1992 Compared to Values from the Previous 5 Years

\begin{tabular}{|c|c|c|c|c|c|c|c|}
\hline \multirow[b]{3}{*}{ Radionutide" } & \multirow{3}{*}{$\begin{array}{l}\text { No of } \\
\text { Samples }\end{array}$} & \multicolumn{2}{|l|}{$109=$} & \multicolumn{3}{|c|}{$|407-100|$} & \multirow{3}{*}{$\begin{array}{c}\text { Drinking } \\
\text { Water } \\
\text { Stundard }\end{array}$} \\
\hline & & Concentration. $\mathrm{pCiL}$ & $\left(10^{\circ} \mathrm{uCl} / \mathrm{mL}\right.$ & $\therefore 0.01$ & Cincentrat & $\mathrm{K} / \mathrm{L}$ & \\
\hline & & Mavimum & Average & Samples & Mavimum & Aieritge & \\
\hline \multicolumn{8}{|l|}{ Composite System } \\
\hline Total alpha & 4 & $1.1 \pm 0.6$ & $0 . x=50 \%$ & 211 & $1.41=0.45$ & $0.6=17 \%$ & 15 \\
\hline Total heta & + & $1.5 \pm 104$ & $1.1=27 \%$ & 20 & $2 x=15$ & $1.3=31 \%$ & 50 \\
\hline$H$ & 4 & $14^{2} \pm+4$ & $157=140$ & 14 & $20.6=5$ & $1+x=11^{2}$ & $20,000 \mathrm{~m})$ \\
\hline$* \mathrm{Sr}$ & + & $0.11=0.113$ & $0.10=23 \%$ & 20 & $0.4 i=0 . .4$ & $0.11=30 \%$ & $s$ \\
\hline${ }^{*} \mathrm{~T}$ & 4 & $0.48=0.16$ & $0.30=50 \%$ & 12 & $5 \pm \pm 1$ & $4^{-}=155^{2}$ & whr \\
\hline$\therefore \mathrm{l}$ & 4 & $0.3 x \pm 0.137$ & $0.34 \pm \omega$ & 20 & $0.4=0.07$ & $0.31=7 \%$ & $\ldots$ \\
\hline 1 & 4 & $0.02 \pm 0.02$ & $0.02 \pm 50 \mathrm{r}$ & 20 & $001=0102$ & $0.010=$ aro & $\cdots$ \\
\hline $\mathrm{l}$ & 4 & $0.40=0.07$ & $0.2 s \pm 24$ & 20 & $0.30=0.05$ & $11.24=x$ & $\ldots$ \\
\hline ( - Toual & 4 & $0 .-4=0.10$ & $0.6 \%=17 \%$ & 20 & $0.77 \pm n(n)$ & $0.54=7 \%$ & $\cdots$ \\
\hline \multicolumn{8}{|c|}{ Continuous System } \\
\hline 10 & 4 & 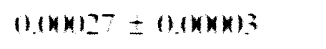 & $0 t x(x) 17 \pm+78$ & in & alkm) & $\theta(x)(x):=23^{+}$ & 1 \\
\hline$P u P$ & 4 & 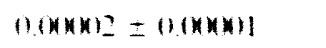 & brmmont \pm 359 & 19 & $0.6)_{2} 01=0(m \times)$ & $0.10013=154 \%$ & $\cdots$ \\
\hline $\mathrm{D}$ & + & $0 .(6 m) 5 x=0(x) 26$ & oranuth $=175$ & 19 & 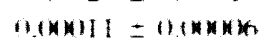 & o(x)mos $=33$ & $\ldots$ \\
\hline
\end{tabular}

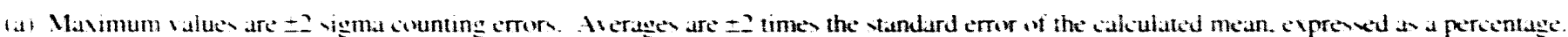

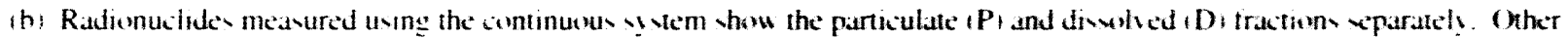

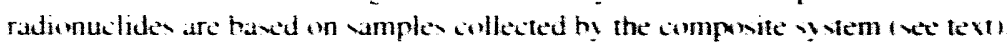

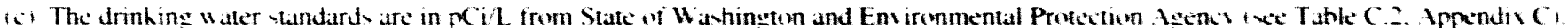

(d) Suspected outler. Alerage concentration influerced th this alue.

(e) Darhe indicate no concentration gaide prow ided in drink ing a ater tandard. 
Table A.3. Radionuclide Concentrations Measured in Columbia River Water at the Richland Pumphouse. 1992 Compared to Values from the Previous 5 Years

$1(x)=$

\begin{tabular}{|c|}
\hline Radionuclide \\
\hline Compoxite System \\
\hline Tolal alpha \\
\hline Tinall betat \\
\hline$H$ \\
\hline${ }^{\mathrm{S}_{\mathrm{r}}}$ \\
\hline${ }^{\prime \prime T}$ \\
\hline $\mathrm{I}$ \\
\hline 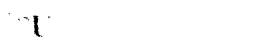 \\
\hline$\cdot \mathrm{c}$ \\
\hline l Total \\
\hline
\end{tabular}

\section{Continuous System}

In

PuP

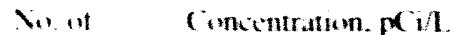
sample
$1114+1102$

$10.7 x=11.10$

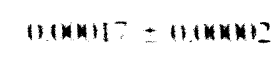

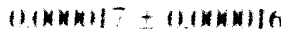

$110 \mu \mathrm{ComL}$, Arerage

$0.4 \pm 56$,
$1.2 \pm 56$,

$12 \pm 51 \%$

1) $(x)=11 \%$

$11.21=114 *$

$0.2 x \pm 14 \%$

10.010 \pm tor?

$1122=4 \%$

$1151=12 \%$

No. af
Sumple

Sumples

50
54
50
58
34
$5 x$
58
58
$5 x$

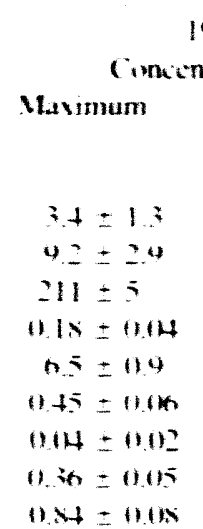

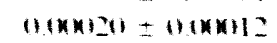

aimaliz $=31 x$

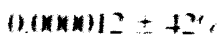

binmox $=11$
$|9 x-|(\infty) \mid$

Ril

Acoraste

Drinkins

Itat

stanutiru

$n^{2}=26$

$1+ \pm 2 \%$,

$121=7 \%$

6.111 = $11 \%$

$11.3 n=1-x$

$0.27=5 \%$

(1) $10 \pm 21 \%$

$10.20=5 \%$

$11+5=-1$.

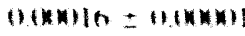

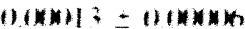

nimels \pm binxtin

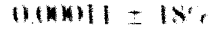

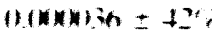

aromit $\pm 1: 5$

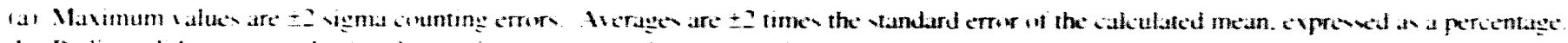

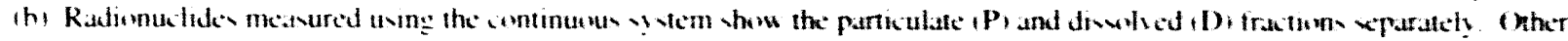

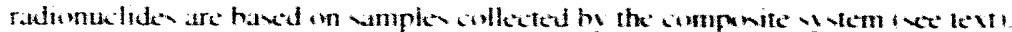

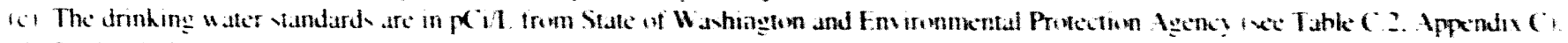

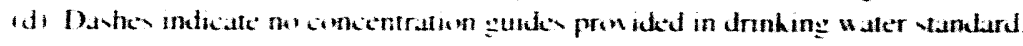

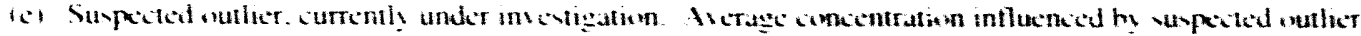


Table A.4. Radionuclide Concentrations Measured in Columbla River Water Along Cross Sections Established at the Vernita Bridge and Richland Pumphouse. 1992

\section{Ni1. (1) \\ Rislininumlick \\ Sillinplu}

Vorrillin IIridge

11

SI

i.

1

1

$1|11,1|$

Klohlund P'umphouss

II

" $\mathrm{Si}$

il

1

' 1

1. Thial

\begin{tabular}{|c|c|}
\hline 16 & 1310 \\
\hline 16 & $11.11+11.113$ \\
\hline 16 & $11.311+111$ \\
\hline 16 & $110101+01110$ \\
\hline 10 & $11.21+11.14$ \\
\hline 10 & $0.51 \pm 11.177$ \\
\hline
\end{tabular}

$126+4$

$11.13+11.04$

$10.37+11015$

$0111+1002$

$11.34+11015$

$1171+11018$
Concintritlun. pei/l Mliinim!n!

Aretige

$$
\begin{aligned}
& 17+2 \\
& \text { (1)(10) }+110(1) \\
& 11.24+0101 \\
& 11.1112+11.0107 \\
& 11.1 \times, 1102 \\
& 0.410112
\end{aligned}
$$

51) $\div 7$ $11.1152: 11117$ $1125+11111$ 11.1610 + 11161) (1.2) 1110111

11) $46+11112$

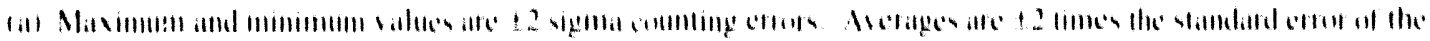
calculated metan I? $\mathrm{Sl}$ Mll. 


\begin{tabular}{|c|c|c|c|c|c|}
\hline \multirow[b]{2}{*}{ Analwa } & \multirow[b]{2}{*}{ Inits } & \multicolumn{4}{|c|}{ Vernits Bridge t up iream, } \\
\hline & & $\begin{array}{l}\text { Wo in } \\
\text { Sumples }\end{array}$ & Naxumum & Minimum & $\begin{array}{l}\text { Annual } \\
\text { Alcrage }\end{array}$ \\
\hline Temperature: & $\mathrm{C}^{\circ}$ & $\therefore x=$ & $20+4$ & $3 \mathrm{~s}$ & $11 x$ \\
\hline Diwwhed wygen & mo/ 1 & 5 & 140 & 40 & 11.5 \\
\hline Turthidit: & $\operatorname{si}$ & 5 & 15 & 116 & 1.0 \\
\hline $\mathrm{pH}$ & H unis & 5 & $\times 4$ & 7.8 & $x 2$ \\
\hline Fecal coliform & $=I(x) \mathrm{nL}$. & 5 & $:$ & $<1$ & $<2$ \\
\hline Surp inded whids. $105 \mathrm{C}$ & 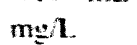 & 5 & 4 & 1 & 26 \\
\hline Diwolied whis. Ixic & $m=1$ & 5 & sh & 81 & $8:$ \\
\hline Specitix conductance & $\mathrm{uS} / \mathrm{cm}$ & 5 & 147 & $1: 3$ & 141) \\
\hline Hardnes, a $\mathrm{CaCO}$ & $\mathrm{mo} / \mathrm{L}$ & 5 & 70 & 54 & $\omega$ \\
\hline Pho-phorus | (xul & $m=1$ & 5 & 001 & anol & $\langle 0.11$ \\
\hline Chromum. divalied & $u=1$ & 5 & $\mathbf{N R}$ & NR & $\mathrm{NR}$ \\
\hline Nitrugen. Kkeldathl & $m y l$ & 5 & 0.2 & a.2 & (1).2 \\
\hline Total oreanic cartum & $\mathrm{mgh}$ & 5 & 22 & $1:$ & 16 \\
\hline Iron. divulued & $4=1$ & 5 & $:$ & $<3$ & $<3$ \\
\hline Ammonia divolied & $m=1$ & 5 & an: & 0,11 & \\
\hline
\end{tabular}

\begin{tabular}{|c|c|c|c|c|}
\hline \multicolumn{5}{|c|}{ 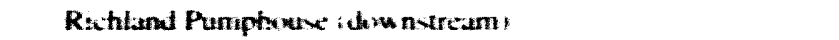 } \\
\hline $\begin{array}{l}\text { No of } \\
\text { Sampler }\end{array}$ & Mavimum & Minumum & $\begin{array}{l}\text { Annual } \\
\text { tveragete }\end{array}$ & $\begin{array}{c}\text { State } \\
\text { Standart }\end{array}$ \\
\hline 365 & 21.1 & 34 & 12.1 & 20 + muximum \\
\hline 4 & $1: x$ & 4.5 & 11.3 & * $\{$ minimum: \\
\hline 4 & 20 & 0.2 & 1.1 & $5+$ bahiground \\
\hline 4 & $x+4$ & $\$ .1$ & s.2 & $6.5 \cdot 8.5$ \\
\hline 4 & 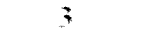 & 1 & 2.5 & [(n) \\
\hline 4 & 4 & 2 & ; & $\ldots$ \\
\hline 4 & 4 & 74 & (4) & $\ldots$ \\
\hline 4 & $14 \mathrm{~s}$ & $1: 3$ & $1+10$ & ... \\
\hline 4 & 69 & 54 & 6.3 & -.. \\
\hline+ & $0.1 \mathrm{~s}$ & $\langle 0,01$ & $\cos { }^{\circ}$ & -.. \\
\hline+ & 2 & $<1$ & $<1$ & $\cdots$ \\
\hline 4 & 4.10 & $<1.2$ & $<13$ & ... \\
\hline 4 & 22 & 12 & 1.5 & ... \\
\hline 4 & 5 & $<;$ & $<35$ & -.- \\
\hline 4 & 00,8 & siol & $<0,02$ & -.. \\
\hline
\end{tabular}

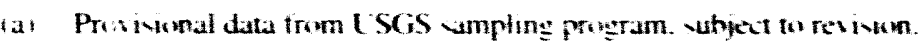

(h) Sec Appendic $C$

(c) Mavnum and minmum reprevent dall derage

id Vephelometric turhidit unit

(e) Annual inedian.

(f) Dasher indicate no vandard as ailable.

191 H Siemenvim.

thi $\mathrm{NR}=$ not repurted 


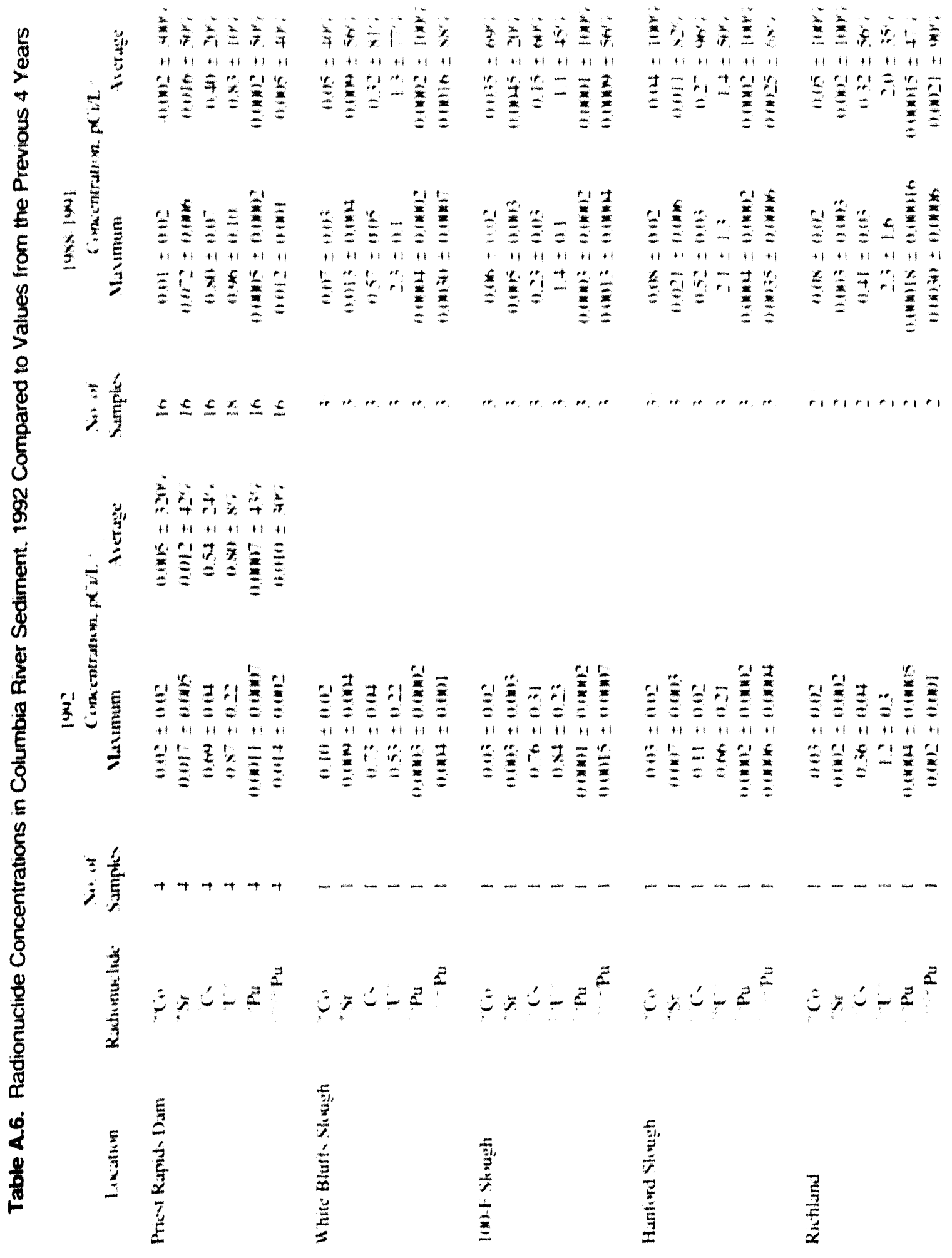




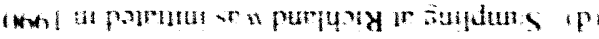

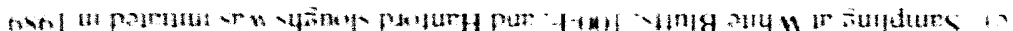

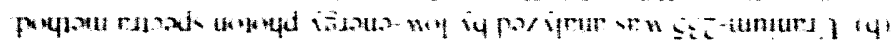

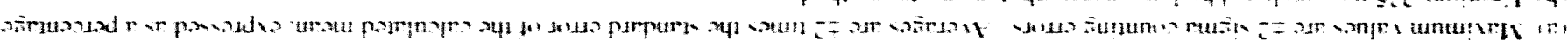

\begin{tabular}{|c|c|c|}
\hline$A A^{-}=6100 \mathrm{~A}$ & Einno= Enon & 41 \\
\hline$\therefore t=-$ thento & 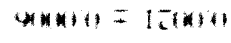 & 41 \\
\hline$A 1=11 !$ & $111=: 1$ & 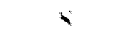 \\
\hline$=240$ & $s+n=n+1$ & 41 \\
\hline$=4200$ & sonot = Twin & 41 \\
\hline$a=t a$ & $\tan =\mathrm{H}$ & 41 \\
\hline and 1 & unumpl & -pulury \\
\hline
\end{tabular}
$|+\infty|+\infty) \mid$

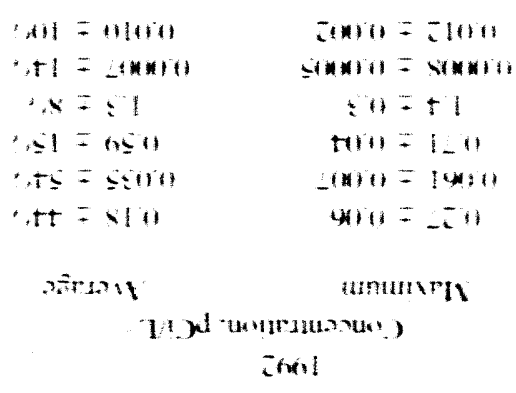

$n_{d}$

$n_{d}$

1

is

$\because 3$

umg ind

mpoundpy

ant?

(pluoo) Sנea人

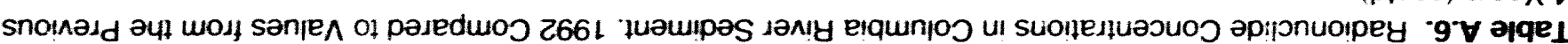


Table A.7. Summary of Cesium-137 $\left({ }^{137} \mathrm{Cs}\right)$ in Milk, 1992 Compared to Values from the Previous 5 Years $(\mathrm{pCi} / \mathrm{L})$

1902

\begin{tabular}{|c|c|c|c|c|}
\hline Radionuclide & Maximumm & Mcian".." & $\begin{array}{l}\text { No. Iess Than } \\
\text { Detection" }\end{array}$ & Maximum't. \\
\hline \multicolumn{5}{|l|}{ Downwind } \\
\hline $\begin{array}{l}\text { Walhluke Area } \\
\text { Sagemonor }\end{array}$ & $\begin{array}{l}2.24 \pm 190 \% / \\
6.91 \pm 6(1 \% / \%\end{array}$ & $\begin{array}{l}0.32 \pm(040 \% \\
1.07 \pm 8(1) \%\end{array}$ & $\begin{array}{r}4 \text { of } 4 \\
23 \text { of } 27\end{array}$ & $\begin{array}{l}7.69 \pm 10(0) \\
8.55 \pm 4.35\end{array}$ \\
\hline \multicolumn{5}{|l|}{ Upwind } \\
\hline Sunnyside & $103 \pm .3(0) \%$ & $-(0.93 \pm 170 \%$ & 4014 & $10.8 \pm 4(6)$ \\
\hline
\end{tabular}

|9087-1991

No. I.ess Than
Meintin

Table A.8. Strontium-90 $\left({ }^{90} \mathrm{Sr}\right)$ in Leafy Vegetables, 1992 Compared to Values from the Previous 5 Years (pCi/dry weight)

1992

Raldionuclide Maximum':" Meann"
No. Less Than Detection"."
$1987-1991$

Maximum Mean' $^{(1)}$
No. Less Than Detection's

\section{Downwind}

Wahluke Area

Salgemoor $\mathrm{NS} "$ (0.011 $\pm .50 \%$

Riverview (). (0) $12 \pm 220 \%$

NS
$0.0096 \pm 40 \%$
$\ldots$

NS
() of 3
1 of 31

$0.021 \pm 30 \%$

(). $(0.5 .3 \pm 20 \%$ (). () () $27 \pm .30 \%$

$0.010 \pm 60 \%$

$0.0(0) 71 \pm 120 \%$

(). ()(5) $\pm(50 \%$

3 of 8

1 of 1

Upwind

Sunnyside

$0.027 \pm 30 \%$

$(1.024 \pm 10 \%$

0013

$0.026 \pm .30 \%$

$0.0 .59 \pm 60 \%$

80115

(a) Maximum is \pm 2 sigma analytical propagated error, expressed at a percentage.

(b) Mean is \pm 2 SEM. expressed als a percentage.

(c) Number of samples less than detection out of number of samples analyzed.

(d) $N S=$ no sample. 
1992 Environmental Report

Table A.9. Cobalt-60 ( $\left.{ }^{60} \mathrm{Co}\right)$, Strontium-90 $\left({ }^{90} \mathrm{Sr}\right)$, and Cesium-137 $\left({ }^{137} \mathrm{Cs}\right)$ in Riverview Carrots, 1992 Compared to Values from the Previous 5 Years (pCi/dry weight)

(1) 2

\begin{tabular}{|c|c|c|}
\hline Resdionuclide & Maximum'".' & Meiall'": \\
\hline 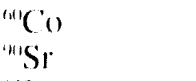 & $\begin{array}{l}0.0(0) 11 \pm 70 \% \\
0.012 \pm+(0 \%)\end{array}$ & $0.0(0)+60 \pm 0 \%$ \\
\hline${ }^{\mathrm{C} S \mathrm{~S}}$ & $0.01025 \pm 260 \%$ & \\
\hline
\end{tabular}

No. Less Thiml

Delection".

0 of 1
1 of 3
101

$|987-199|$

\begin{tabular}{|c|c|c|}
\hline Maximum'".' & Meanth' & $\begin{array}{l}\text { No. I.ess Than } \\
\text { Detection". }\end{array}$ \\
\hline$(0.00(0,9) \pm 1.40 \% /$ & $0.00(0) .34 \pm 6.30 \%$ & 15 of 18 \\
\hline$(0.013 \pm 40 \%$ & $0.0006 \pm .30 \% \%$ & 3 of 15 \\
\hline (). $.0070 \pm 70 \%$ & $0.000071 \pm 230 \%$ & 14 of 1.5 \\
\hline
\end{tabular}

(a) Maximum is \pm 2 sigmia analyticial propagalled error. expressed ars a perentage.

(b) Mean is \pm 2 SEM. expressed als a percentige.

(c) Number of simmples less than detection out of number of simples analyned. 
Table A.10. Annual Average ( \pm 2 SEM) Concentration of Strontium-90 $\left({ }^{90} \mathrm{Sr}\right)$ in Alfalfa, 1982 to 1992 (pCi/g dry weight)

\begin{tabular}{|c|c|c|c|c|c|c|c|c|c|c|c|}
\hline & $14: 2$ & 145: & 1484 & 1485 & 19sit & 148 & $14 x+5$ & $14: 4$ & 1440 & 1991 & $1402=$ \\
\hline Benton Cit & $197=10 \%$ & $10.1152= \pm+66^{\prime}$ & $0.153 \pm 3 \times$ & $1000=11 \%$ & $11.2114=18^{\prime}$ & $11.043 \pm 53$ & $11.151=7$ & $0.1501=+4$ & $(1) \cdot 1+1=11^{2}$ & is & $0.119 \pm 578$ \\
\hline Horn Rapid, Richland & is & ws & is & is & is & NS & is & is & $10.11 t=29$ & is & $01139 \pm 19 r^{\prime}$ \\
\hline Mone- Late & $11032 \pm 31^{t}$ & $0(14+1) \pm 35 \%$ & $0.223 \pm 134$ & $0.191 \pm 2 \%$ & 1.1.143 $\pm 29 \%$ & $0.161 \pm t \%$ & $0.202=1 \times 6$ & $0.67=4+i$ & $11.667=+5 \%$ & is & $0.0151 \pm 48$ \\
\hline Rivenion & $0.040=11 \%$ & $0(x)=16$ & $0.125 \pm 5 \%$ & $0.111 \pm+1^{\circ}$ & $0.15+ \pm 45$ & $0063+=69$ & $19.245 \pm 11$ & $11.240=230$ & $0155 \pm 126$ & $11.1^{2}= \pm 14$ & $0.107 \pm 15 \%$ \\
\hline Sagemen & $0.117 \pm 3.9$ & $(9020)=30^{2}$, & $11.1 .5=19 \%$ & $0.015=12 \%$ & $01.192=35 \%$ & $0.112 \pm 5 \%$ & $0.17+ \pm 25 \%$ & $0.11 \times 1 \pm 56$ & 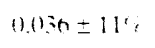 & $0.18010=7 \%$ & $0.1157 \pm 59 \%$ \\
\hline Summide & $01 x_{2}=1+\%$ & $10.02 \pm n^{-} r$ & $0.091=33$ & 0.1995259 & $19.118=360$ & $0.071 \pm 1+\%$ & $000-6=8$ & $0.114 \pm 3$. & is & is & $0.06 x=91 x^{2}$ \\
\hline Wahluke & $n \cdot k(k)=0^{-r}$, & $0.000 \pm 73$ & $0062 \pm 39 \sigma^{\prime}$ & $0.110 \pm 13 \%$ & $0.214 \pm 19 c^{\circ}$ & $0.23=4 r$ & $0.153 \pm 8 \%$ & $0.045 \pm 21 \%$ & $0.0136=11 \%$ & is & $0.1150 \pm 80 \%$ \\
\hline
\end{tabular}

(a) $\mathrm{XS}=$ ne ample 
Table A.11. Summary of Strontium-90 ( $\left.{ }^{(91)} \mathrm{Sr}\right)$ in Carp Carcass and Cesium-137 $\left({ }^{137} \mathrm{Cs}\right)$ in Carp Muscle, 1992 Compared to Values from 1990 and 1991 (pCi/g wet)

1() 2

No.

Miximumu!n

"Sr in Carcass

1()()-N Area

$0.011+70 \%$

(). (1) $46 \pm 20 \%$

$0.025 \pm .5(1 \%)$

() ol 1

$(0.420)+2(1 \%$

() of 5

$0.0 .36 \pm .30 \%$

$0.110 \pm 2(1 \%$

() of 1

3015

${ }^{137}$ Cs in Muscle

1()() N Areal

$$
\begin{aligned}
& 0 .(1) \pm 0(0 \% / \\
& 0.02 \pm 10(0 \% / 0.01 \pm .5(0 \%
\end{aligned}
$$$$
0.04 \pm .30 \%
$$

$3(0)$ Arcil

$<(1)(1)+19() \%$
$|9()-|(9) \mid$

No.

less Than

betectiont"$$
0.02 \pm .5(0 \%
$$$$
50110
$$

Vantigedu

$<0.0(1) 4 \pm 70 \%$

5 of 5

(1). () $1 \pm(0() \%$

$0.0(1) 7 \pm 40 \%$

6011.3

(a) Maximum is the concentration in pe $\mathrm{i} / \mathrm{g} \pm 2$ sigma analylical propagalled error, expressed als a percentage.

(b) Mean is $p(\mathrm{i} / \mathrm{g} \pm 2$ standard error. expressed as a pereentage.

(c) Number of samples less than detection out of number of samples antly aed.

(d) Collecled in 199() and 1991 . 
Table A.12. Concentrations of Strontium-90 ( $\left.{ }^{90} \mathrm{Sr}\right)$ in Whitefish Carcass and Cesium-137 $\left({ }^{137} \mathrm{Cs}\right)$ in Whitefish Muscle, 1992 Compared to Values from the Previous 5 Years (pCi/g wet weight)

1以2 2

No. Simnles

Lers Than

I. aciation

Maximum!'."'

Mc:ill't:
Delection"
$|987-199|$

No. Simlunples

Less Thion

M:เximum!n!'
Detection".

\section{"sir in Carcuss}

()(1)- $\mathrm{N}(1) \mid()(1)-1)$

Areas

$$
0.0 .32 \pm 20 \%
$$

$0.013 \pm+(1) \%$

() (1) 19
() 115

$0 .(1)+1+20 \%$

$0.019) \pm 2(1 \%$

(1) of 36

$3(0)$ Areia

(1). (1)+6. $\pm 2(1) \%$

(1). (1) $25 \pm 50 \%$

(1). $10.36 \pm .30 \%$

(1).019) $\pm(0) \%$

0 of 5

Kenle Riverts

$0.1148+4(1 \%$

(1). $(1.35 \pm 20 \%$

() of' 9

\subsection{Cs in Mluscle}

1)(0)-N (1) ] (1)(1)-1)

$$
\text { Arcis }
$$

$$
\begin{aligned}
& 0.17 \pm 20 \% \\
& 0.013 \pm 0.30 \%
\end{aligned}
$$

(1.). $4 \pm 76 \%$

3019

(1.) $11 \pm 70 \%$

7 of 10

Kertle River'tit

(a) Maximum is the concentration in pe $\mathrm{i} / \mathrm{g} \pm 2$ sigma total error. expressed an a percentage.

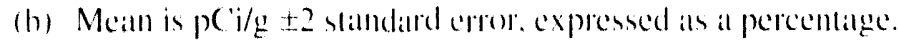

(c) Number of simples less thatn detection out of mumber of simples analyod.

(d) Collected in |c)l. 
Table A.13. Summary of Plutonium-238 ( $\left.{ }^{238} \mathrm{Pu}\right)$ and Plutoniurn-239,240 (239.240 Pu) in Rabbit Liver, 1992 Compared to Values from the Previous 5 Years ( $\mathrm{pCi} / \mathrm{g}$ wet weight)

$\mid(x) 2$

No. l,cess Than

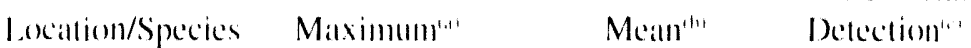

$1987-1001$

No. less Than Meill't" Detectionth

:"N'II

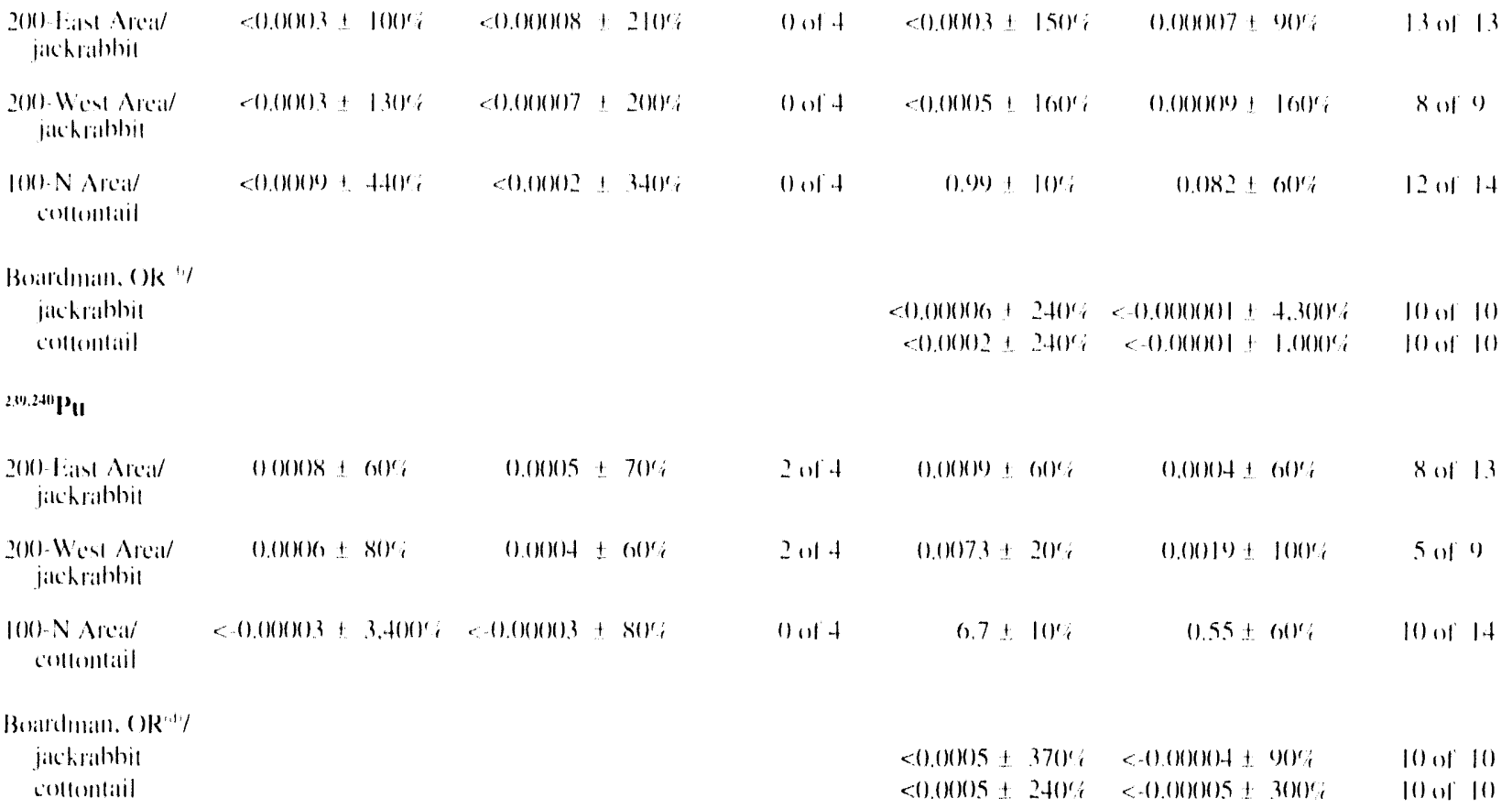

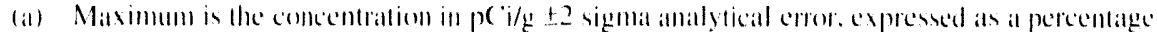

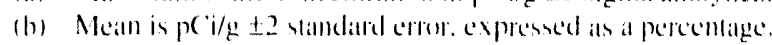

(c) Number of simples less tham delection out al mumber of samples antaly aed.

(d) (illected in ly)(). 
Table A.14. Summary of Strontium-90 ( $\left.{ }^{911} \mathrm{Sr}\right)$ in Deer Bone and Cesium-137 ( $\left.{ }^{137} \mathrm{Cs}\right)$ in Deer Muscle, 1992 Compared to Values from the Previous 5 Years ( $\mathrm{pCi} / \mathrm{g}$ wet weight)

1942

No.

Less Thill

L.ocillioll

Maximumul'

Mciall'"' $\mid 987-1901$

No.

less Than

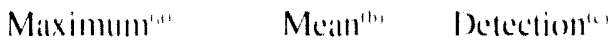

"Sr in Bone

200 A seats

$$
\text { NSi't }
$$

NS

I()(1)-N Areil

$20.8 \pm 3(1 \%$

$8.1 \pm 20 \%$

NS

$0.71 \pm 30 \%$

(0. $.59 \pm 4(1) \%$

2012

Stevens county

(1). $8 \pm 2(1) i$

$(0.59 \pm .30 \%$

(1) of ?

$58.3 \pm 2()^{\prime} \%$

$15.3 \pm 190 \%+01+$

${ }^{1.77} \mathrm{C}$ s in Mluscle

$2(1)$ Areils

$0.0006 \pm .50 \%$

NS

() of 2

NS

NS

NS

I()(1)-N Area

$0 .(1) \pm 40 \%$

$0.0104 \pm 0.01 \%$

1 of 1

0..0106 $\pm 80 \%$

(). $0015 \pm 40 \%$

1 of 2

Stevens Comnty

$0.52 \pm 10 \%$

$(1 .+3 \pm 40 \%$

1013

$0.0 .3 \pm .30 \%$

$0.01+140 \%$

2 of 4

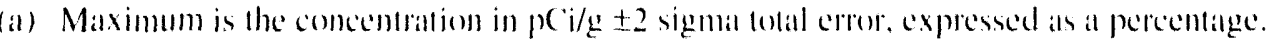

(b) Mean is $p(\mathrm{r} / \mathrm{g} \pm 2$ standand error. expressed als a percentige.

(c) Number of samples less than cielection out of number of simples allalyged.

(d) $\mathrm{NS}=$ no simple. 


\section{Onsite}

thoue 100-D Pumphoure

I Mile NE of lo()-N Area

1 Mike E of loot-N Area

I(k) Area Fire Station

200 -East NCentral

Eof $2(0)-$ East

2(0)-Fun SE

$\mathrm{SW}$ of $\mathrm{BC}$ Crih

$S$ of $2(x)-E$ ari

E of $200-1100$

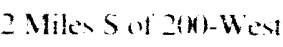

VE of Fas Flux Ten Facilits

IFFTF,

SE of FFTF

$x$ of $3(1)$ Area

Hantord Tounsite

Wie Barticade

lo()-N Spring Shoreline

1(x)-N Shore Hanford

Generating Plant i HGP)

\section{Onsite Average}

\section{Offsite}

Riverview

Biers Landing

Sayemoror

Tas lor Flats No. 2

IV End Fir Road

Ringold

Bers Ranch

Wahluhe Slope No. 2

Vernita Bridge"

Yasima Barricade

Ranlesnatke Spring-

Arid Land Ecology (ALE,

Prover Barricade

$S$ of $3(x)$ Area

$\begin{array}{ll}0.31 \pm 0.01 & 0.11 \pm 0.01 \\ 0.33 \pm 0.01 & 0.2 \pm \pm 0.01 \\ 1.1 \pm 0.1 & 0.25 \pm 0.02 \\ 0.34 \pm 0.02 & 0.57 \pm 0.012 \\ 0.24 \pm 0.02 & 0.59 \pm 0.012 \\ 0.02 \pm 0.01 & 0.04 \pm 0.01 \\ 0.11 \pm 0.01 & 0.23 \pm 0.01 \\ 0.35 \pm 0.02 & 0.71 \pm 0.27 \\ & 0.14 \pm 0.01 \\ 0.109 \pm 0.01 & 0.09=0.01 \\ & 0.07 \pm 0.01 \\ 0.24 \pm 0.01 & 0.13 \pm 0.01 \\ 0.29 \pm 0.01 & 0.13 \pm 0.01 \\ 0.18 \pm 0.01 & 0.14 \pm 0.01\end{array}$

$0.18 \pm 0.01$

$0.17 \pm 0.01$

$0.31 \pm 0.01$

$0.58 \pm 0.01$

$0 .+1=0.02$

$0.13 \pm 0.01$

$0.12 \pm 0.01$

$0.2 x=0.01$

$0.50 \pm 0.02$

$0.1+1 \pm 10.01$

$0.05 \pm 0.01$

$0.16 \pm 0.01$

$0.15 \pm 0.01$

$0.2 x \pm 0.01$

$0.11 \pm 0.01$

Benton Cit!
$0.31 \pm 0.16$

$0.05=0.01$

$0.04 \pm 0.01$

$0.10 \pm 0.01$

$0.05 \pm 0.01$

$0.21 \pm 0.01$

$0.20 \pm 0.01$

$0.07 \pm 0.011$

$0.06 \pm 0.01$

$0.12 \pm 0.011$

(1). $40 \pm 0.183$

$0.24 \pm 0.01$
$0.31 \pm 0.13$

$0.23=0.111$

$0.10 \pm 0.01$

$0.20 \pm 0.010$

$0.06 \pm 0.01$

$0.01 \pm 0.01$

$0.26=0.01$

$0.10 \pm 0.011$

$0 .(09) \pm 0.111$

$0.07 \pm 0.01$

$0.07 \pm 0.01$

$0.14 \pm 0.01$

$0.34 \pm 0.01$

$0.11 \pm 0.01$

$0.27 \pm 0.01$

$0.43 \pm 0.12$
$11.23 \pm 0.08$

$0107+0101$

$0.13 \pm 0.101$

$0.03 \pm 0.01$

0. $16 \pm 0(01$

$0.20 \pm 0.01$

$0.15 \pm 0.01$

$0.04 \pm 0.01$

$0.07 \pm 0.01$

$0.12 \pm 0.01$

$0.13 \pm 0.01$

$0,1(k) \pm 0 .(H)$

$0.26 \pm 0.01$

$0.2+ \pm 0.01$

$0.30 \pm 0.01$

$0.17 \pm 0.01$
$2.70 \pm 0.0714$

$$
\begin{aligned}
& 0.152 \pm 0.012 \\
& 0.16 \pm 0.012 \\
& 0.131 \pm 0.011 \\
& 0.119 \pm \pm 0.0129 \\
& 0.3+5 \pm 0.013 \\
& 0.173 \pm 0.0142 \\
& 0.102 \pm 0.10996 \\
& 0.214 \pm 0.013 \\
& 0.374 \pm 0.018 \\
& 0.157 \pm 0.0118 \\
& 0.0959 \pm 0.00718 \\
& 0.1516 \pm 0.160765 \\
& 0.167 \pm 0.00936 \\
& 0.0574 \pm 0.6062 \\
& 0.23 \pm 0.016 \\
& 1.47 \pm 0.063
\end{aligned}
$$

$0.235 \pm 0.0125$

$0.6311 \pm 0.10445$

2.70

$0.294+0.229$

$0.11 x \pm 0.122$

$0.122 \pm 0.1010956$ $0.0375 \pm 0.10172$ $0.0224=0.10458$

$0.121=0.01012$

$0.138 \pm 0.012$

$0.0628 \pm 0.100766$

$10.145 \pm 0.0111^{7}$

$0.121 \pm 0.04181$

$0.155 \pm 0.007 \mathrm{sh}$

$01623 \pm 0.00781$

$0.146=0.00655$

$0.0 \times 51=0.00 \times 16$

$0.2113 \pm 0.011+$

0.1.32 20.00015 
Table A.15. Strontium-90 ( $\left.{ }^{\circ} \mathrm{S} r\right)$ Concentrations in Soil. 1987 Through 1992 (contd)

\begin{tabular}{|c|c|c|c|c|c|c|}
\hline \multirow[b]{2}{*}{ Location } & \multicolumn{6}{|c|}{$p C l=t$ dr weight } \\
\hline & 1957 & 1988 & 1459 & $1 \overline{c x} \mid$ & 1441 & 1002 \\
\hline Sunny-ide & $0.25 \pm 0.12$ & $0.26 \pm 0.06$ & $0.13 \pm 0.01$ & $0.3+5 \pm 0.0134$ & $0.0243 \pm 0.1 \times 1314$ & $0.135= \pm 0.04400$ \\
\hline Walla Walla & $0.02 \pm 0.01$ & $0.04 \pm 0.01$ & $0.01 \pm 0.01$ & $011455 \pm 010101554$ & & \\
\hline Mañ Dam & $0.107 \pm 0.1 \mathrm{k}$ & $0.13 \pm 0.01$ & $0.04 \pm 0.01$ & $0.1784=0.101844$ & & \\
\hline Mone lake & $0.16 \% \pm 0.02$ & $0.01 \pm 0.111$ & $0.05 \pm 0.01$ & $1016612 \pm 0.06186$ & $0.0157=011 \times 429$ & \\
\hline Wahturnat & $0.12 \pm 0.12$ & $0.25 \pm 0.111$ & $0.12 \pm 0.01$ & $0.0496=01.105094$ & & \\
\hline Connell & $0.12 \pm 0.11$ & $0.09 \pm 0.01$ & $0.14=0.011$ & $0.214 \pm 0.0125$ & $10.164+=0.11116$ & \\
\hline Othello & $0.15 \pm 0.018$ & $0.04 \pm 061$ & $0.118 \pm 0.01$ & $0.0759=0.007 \mathrm{~s}$ & & \\
\hline Yahima & $0.06 \pm 0.04$ & $0.00 \pm \pm 0.01$ & $0.10 \pm 0.01$ & $0.126 \pm 0.141852$ & $0.119 \pm 0.01111$ & $0.1452=0.10515$ \\
\hline Offsite A verage & $0.12 \pm 0.0 .8$ & $0.16=0.04$ & $0.13 \pm 0.0=$ & $0.107 \pm 0.104 .54$ & $01.33=010555$ & $010+5=000369$ \\
\hline
\end{tabular}

(a) Blank tield indicater no data.

(h) Indiv idual result are \pm 2 igma counting errors. Alerage are \pm 2 time the tandard error of the calculated mean.

(c) Perimeter lacation onvite near Site heundary 


\section{Onsite}

Those (10)-D Pumphruxe

1 Mile NE of I(K)-N Areat

I Mike for lo(k)-N Area

(a) Area Fire Station

2(k)- East N Central

Eof Z(x)-Eat

2(x)-East SE

SU of BC Crith

Sof z(x)-Eave

E of $2(0)-10 \times 1$

z Miles $S$ of zorther

NE of FFTF

SE of FHT

Vif $3(x)$ Ares

Hanford Townsite

Hic Barricak

1(1)-N Spring Shoreline

(In)-X Shore IHCiP)

\section{Onsite A verage}

\section{Offsite}

Riverifer

Buer landing

Satermax

Taslor Flat No. 2

W End Fir Rorad

Ringold

Berg Rinch

Wathluhe Slope No. ?

lerniza Bridece

Yahima Barricade

Rattlenahe Spring

il.

Promer Barricade

$S$ of $3(x)$ Are:

Benton Cit?

\begin{tabular}{|c|c|}
\hline & $0.801 \pm 0.05$ \\
\hline $1.1 \pm 0.1$ & $0.74 \pm 0.05$ \\
\hline $1.3 \pm 0.1$ & $1.2 \pm 0.1$ \\
\hline $10 \pm 0.1$ & $26 \pm 0.1$ \\
\hline $10.69 \pm 0.04$ & $1.8 \pm 0.1$ \\
\hline $0.61 \pm 0.04$ & $1.6 \pm 0.1$ \\
\hline $0.01 \pm 0.02$ & $0.04 \pm 0.02$ \\
\hline $0.13 \pm 0.193$ & $0.5 x \pm 0.14$ \\
\hline \multirow[t]{2}{*}{$1.2 \pm 0.1$} & $5.4 \pm 1.7$ \\
\hline & $0.49 \pm 0.14$ \\
\hline \multirow[t]{2}{*}{$0.3: \pm 0.03$} & $0.24 \pm 0.03$ \\
\hline & $0.12 \pm 0.12$ \\
\hline $1.2 \pm 0.1$ & $0.51 \pm 0.04$ \\
\hline $1.1 \pm 0.1$ & $0.5 S \pm 0.16$ \\
\hline $159+00$ & \\
\hline
\end{tabular}

$0.46 \pm 0.05$

$0.6 . \pm 0.05$

$1.1 \pm 0.1$

$18 \pm 0.2$

$2.1 \pm 0.1$

$059+0104$

$0.52 \pm 0.14$

$0.80 \pm 0.14$

$3.0 \pm 0.1$

$0.63 \pm 0.14$

$0.19 \pm 0.02$

$0.22 \pm 0.03$

$1.2 \pm 0.1$

$1.2 \pm 0.1$

(1.39 \pm 0.44

$20 \pm 26$

$24 \pm 3.2$

$2.1 \pm 2.3$

$0.45 \pm 0.14$

$0.86+1105$

$0.23 \pm 11.03$

$0.12 \pm 0.02$

(1.) \pm 10.16

$0.23 \pm 0.04$

$1.0 \pm 0.1$

$0: 1+014$

$0.16 \pm 0.03$

$0.0 \mathrm{x} \pm 0.112$

$0.32 \pm 0.14$

$1.1 \pm 0.1$

$0.65 \pm 0.04$
$1.3 \pm 0.1$

$0.52 \pm 0.0 .13$

$1.0 \pm 0.1$

$0.34 \pm 011.14$

$0.2 x \pm 0.03$

$1 . x \pm 0.16$

$0.35+0.03$

$0.22 \pm 0.03$

$0.14 \pm 0.04$

$0.08 \pm 0.02$

$0.0 \times \pm 0.02$

$10 \pm 0.1$

$0.33 \pm 0.03$

$0.77 \pm 0.14$

$0.91 \pm 0 .(6)$
$11.6 x \pm 0.14$

$0.12 \pm 0.12$

$0.79 \pm 0.166$

$1.3 \pm 0.1$

$17 \pm 0.1$

$053+0.04$

$0.25 \pm 0.103$

$0.60 \pm 0.05$

$0.54+014$

$0.46 \pm 0.113$

$0.96 \pm 0.16$

$0.56 \pm 0.155$

$1.1 \pm 0.1$

$0.76 \pm 0.15$
: sto

$076+1002+6$

$0.652 \pm 0.044$

$0.76, \pm 0.06)$

$10.312 \pm 0.1042$

$0.205=0.1029$

$1.1:=0.053$

$0 .+10 x \pm 11035$

$0.346 \pm 110.32$

$0.357=0.1441$

$3.86=01.1015$

$16+01005$

$0.496 \pm 0.043$

$0.3 \times 7=0.038$

$0.1+2=0.02+$

$0.7(\mu) \pm 0.143$

$0.271 \pm 10.02 x$

$0.66=0.04$

$0.11=01022$

$1.14 \pm 0.1405$

$0.37 \pm 0.0307$

$0.2401=0.192$

$6.725 \pm 0.0337$

$1.78 \pm 0.115$

$0.597=0.038$

$11+73+00$ in

$0.147 \pm 100.34$

$0.552 \pm 0057$

$0+21+0(k) 1$

$0.116 \pm 0.02+4$

$0.102 \pm 0.11216$

$0.5 \times 3 \pm 0.4422$

$0.6 .7 \pm 0.14 .21$

$0.22+ \pm 0.129$

$0.726=0.15$

$61.447 \pm 0.1777$ 
Table A.16. Cesium-137 ( ${ }^{137} \mathrm{Cs}$ ) Concentrations in Soil, ${ }^{\text {(a) }} 1987$ Through 1992 (contd)

\begin{tabular}{|c|c|c|c|c|c|c|}
\hline \multirow[b]{2}{*}{ Location } & \multirow[b]{2}{*}{1987} & \multicolumn{4}{|c|}{$\mathrm{pCi} / \mathrm{g}$ (dry weight $)^{\mathrm{h}}$} & \multirow[b]{2}{*}{1992} \\
\hline & & 1988 & 1989 & 1990 & 1991 & \\
\hline nyside & $0.29 \pm 0.04$ & $1.0 \pm 0.3$ & $0.48 \pm 0.04$ & $1.22 \pm 0.06+11$ & $0.1668=0.025 x$ & $0.720 \pm 0.0315$ \\
\hline Ila Walla & $0.07 \pm 0.02$ & $0.23 \pm 0.03$ & $0.07 \pm 0.03$ & $0.333 \pm 0.0619$ & & \\
\hline Nary Dam & $0.30 \pm 0.47$ & $0.48 \pm 0.04$ & $0.54 \pm 0.04$ & $0.276 \pm 0.0348$ & & \\
\hline ses Lake & $0.16 \pm 0.09$ & $0.38 \pm 0.04$ & $0.18 \pm 0.03$ & $0.243 \pm 0.0346$ & $-0.10017 \pm 0.0201$ & \\
\hline shtucna & $0.38 \pm 0.17$ & $0.97 \pm 0.06$ & $0.91 \pm 0.05$ & $0.288 \pm 0.0497$ & & \\
\hline nnell & $0.35 \pm 0.61$ & $0.46 \pm 0.04$ & $0.67 \pm 0.05$ & $1.14 \pm 0.0005$ & $0.334 \pm 0.0388$ & \\
\hline ello & $0.76 \pm 0.42$ & $0.22 \pm 0.03$ & $0.54 \pm 0.04$ & $0.34 \pm 0.1413$ & & \\
\hline ima & $0.16 \pm 0.16$ & $0.11 \pm 0.02$ & $0.56 \pm 0.04$ & $0.599 \pm 0.0459$ & $0.324 \pm 0.0353$ & $0.45 \pm 0.0271$ \\
\hline [isite Average & $0.3 x \pm 0.12$ & $0.59 \pm 0.18$ & $0.74 \pm 0.27$ & $0.483 \pm 0.1902$ & $0.542 \pm 0.317$ & $0.547 \pm 0.2891$ \\
\hline
\end{tabular}

(a) Blank field indicater no data.

(b) Individual result are \pm 2 sigma counting errors. Averages are \pm 2 times the standard error of the calculated mean

(c) Perimeter lication onsite near Site bxundary. 
Onsite

thone 1(x)-D Pumphoure

1 Mile NE of l(W)-N Are:

I Mile E of I(k)-N Area

110) Area Fire Station

zmi-East N Central

$E$ of $2(x)$-East

201-Eas $\mathrm{SE}$

SW of BC Crith

$S$ of zm(t)-East

Eof $2(x)-H_{\text {en }}$

Miles $S$ of $2(x)-W e x$

NE of FFTF

SE of FFTF

$N$ of $3(x)$ Ared

Hantord Tom anite

W ve Barricate

I(w)-X Spring Shoreline

I(x)-N Shere (HGP)

\section{Onsite Average}

\section{Offsite}

Rivervieu

Biers Landing

Sistemon

Tavhor Flat No ?

W End Fir Road

Ringold

Bere Rinch

Wahluke Slope Xis. 2

Vernita Bridge

Yahima Barricatco

Rattlesnathe Springs

HLE

Pronver Barricade

Sof $31 x+$ trea

\begin{tabular}{|c|c|c|}
\hline & $0.11 \pm 0.01$ & $0.1177=0.002$ \\
\hline $0.023 \pm 0(x) 2$ & $0.019 \pm 0.002$ & $0.013 \pm 0(1 x) 1$ \\
\hline $0.017 \pm 0(x)$ & $0.027 \pm 11 .(x) 2$ & $0.026 \pm 0.0003$ \\
\hline $0.0131 \pm 0.002$ & $0.1 k 0 \pm 0.03$ & $0.081 \pm 0.0 \times 13$ \\
\hline $0 .(n)=0,(x) 1$ & $0.017 \pm 0 .(x) 1$ & $0.618=0.002$ \\
\hline $0.012 \pm 0.0(1) 1$ & $0.036 \pm 0.0012$ & $0.011 \pm 0.001$ \\
\hline$(r .(x))=0 .(x))$ & $0 .(001 \pm 0.001$ & $0.014 \pm 0.0001$ \\
\hline $0(x) s \pm \theta(x)]$ & $0013=0.001$ & $0.015=0.1002$ \\
\hline \multirow[t]{2}{*}{$0.17 \pm 0.111$} & $0.67 \pm 0.12$ & $0.53 \pm 00.01$ \\
\hline & $0.015 \pm 0.001$ & $0.022 \pm 0.1 \times 12$ \\
\hline \multirow[t]{2}{*}{$0.0 k 6= \pm 0 .(n) 1$} & $0.005 \pm 0.0001$ & $0.0 \times 4 \pm 0.001$ \\
\hline & $10(x)= \pm 11(x) 1$ & $0.6 \times k \pm 0 .(0) 1$ \\
\hline $0.014=0.0011$ & $0.011=0 .(x) 1$ & $0.024 \pm 0.002$ \\
\hline $0.1114=$ & $0.021 \pm 0.002$ & $0.027 \pm 0.1003$ \\
\hline & $0(x) x-00 u$ & \\
\hline
\end{tabular}

$0.126 \pm 0.027$

$0.111 \pm 0.11$

$0.051 \pm 1.0 k 4$

$0.015 \pm 0.0601$

$0.608 \pm 0 .(n)$

$0.0(x)=0 .(x)$

$0 .(1) 9=0 .(x) 2$

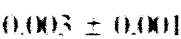

$0.017 \pm 0.002$

$0 .(x)=0.1 \times n)$

$0 .(x)= \pm 0.001)$

$0.002 \pm 0 .(x) 1$

$0 .(x) \pm 0.00)$

6.112t $=0.1012$

o.tons $=0 .(x))$

$0.190+0.602$

$0.064=0,(x))$

$0 .(x) 4 \pm 0(0) !$

$0.033 \pm 0.602$

$0 .(\mathrm{ng}) \pm 0 .(\mathrm{x}) \mathrm{x}$

$0.007 \pm 0.000$

$0 .(6) 3 \pm 0.000)$

$0.003 \pm 0.001$

$0(0) 3 \pm 0(0)$

$0.026 \pm 0.602$

$0.016=0 .(x)$

$0.017=0.0(x) 1$ ofos $\pm 0(0 \times) 1$

$0.0 \mathrm{it}=0.00 \mathrm{2}$

$0.019=0 .(20) 1$

$0.015 \pm 0.001$

0.0. \pm 0.0 .012$

$0.024 \pm 0 .(x) 3$

$0 .(x)=0 .(x) 2$

$0.0 \times 5=0.001$

$0.013 \pm 0.002$

$0.1111 \pm 0 .(011$

$0.011 \pm 0.001$

$0.021=0.0 \times 12$

$0.01 \mathrm{~s} \pm 0.0 \times 2$

$0.025 \pm 0 .(0) 2$
Uting

$0013:=000012 x$

$0.1129 \pm 060129$
$100179+0.6219^{7}$

$0104 \times 5+0100645$

$0.017 \pm 0$ onelt

$0.108455 \pm 0.40121$

$0 .($ B) $794 \pm 0.60114$

$0.00 \times 4+ \pm 0.00151$

6.1104 =0.0011

$0.656 \pm 0.0125$

$0.2 \times h \pm 0.005+h$

a00214 $\pm 0.002 \times 9$

0.615 = 0.6011s

nomisos = now.

$001^{7}=$ - nominl

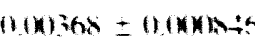

$1101168=0.101255$

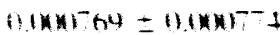

$13024=010117$ $0.10434=0.1001004$

$\left.000^{274}=0.16 \times 4\right)$

1)012?+0IMANE

$1111145=0140212$

$111138=0.1 \times 1415$

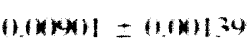

$0(x) 1+1=0.6 \times 07 \mathrm{hl}$

hompio \pm ofollos

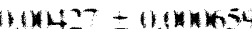

$00204=0101155$

onknal = menkawn

$116112=11.5011$

$00124 \pm 0.601+4$

6015: $=0,00214$

$100214=0100175$

$0.01502=0.04 m 3 x$

$00201=0.0475$ 
Table A.17. Plutonium-239.240 (239200 Pu) Concentrations in Soil. 1987 Through 1992 (contd)

\begin{tabular}{|c|c|c|c|c|c|c|}
\hline \multirow[b]{2}{*}{ Location } & \multirow[b]{2}{*}{$14 \times 7$} & \multicolumn{5}{|c|}{ Concentration. $\mathrm{pCl} / \mathrm{g}$ deg wetght } \\
\hline & & 145 & $14 \times 9$ & $\log (x)$ & $|94|$ & 1492 \\
\hline Benton City & $n(114 \pm 0(n) 1$ & $0014 \pm 0(6) 2$ & $0(1015 \pm 0(x) 1$ & & & \\
\hline Sunnı̣ side & 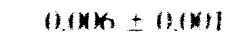 & $0.023 \pm 0.006$ & $0.111 \pm 0.002$ & $0.0291 \pm 0.60327$ & boMms & $0(x) 7 t a=0000119$ \\
\hline Walla Walla & $0 .(60174 \pm 0.16)^{2}$ & $0(n) z=0 .(x) 1$ & $0 .(x) 1 \pm 0 .(x) 1$ & $0.0136 k \pm 0.00 m 5+h$ & & \\
\hline MeNan Dam & $0,0 k=0,0 k \pi$ & $\theta(x) \pm \theta(0))$ & $0 .(n \times) \pm 0 .(x) 2$ & fingor $7+160 x+7 \times 3$ & & \\
\hline Mone lake & $0(x) 2 \pm 0(x) 1$ & $0.00 \mathrm{ks} \pm 0.001$ & $0(0) 2 \pm 0,0) 1$ & $0.00412 \pm 0.000546$ & 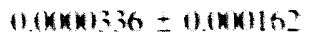 & \\
\hline Washitucna & $0.0)_{2}=0(m):$ & $0016=0,0) 1$ & $0017 \pm 0.012$ & $0(0) 26 \pm 0.005573$ & & \\
\hline Connell & $0,607 \pm 00011$ & $0(x)=0(0) 1$ & $0.010) \pm 0 .(x) 2$ & $0.0104=0.0012 ?$ & $0.001349 \pm 0.600778$ & \\
\hline Othelle: & $0013 \pm 11011$ & $0 .(604=0 .(0) 1$ & $0.00 x \pm 0.002$ & $0.100765=0.16 \times 1878$ & & \\
\hline Yahima & $0.100:=0.001:$ & $0(n):-n(n) 1$ & $0.010=0.1011$ & $0.010 \mathrm{k} \pm 0 .(x) 111$ & $0.0 \times 1 \times n)=0.00153$ & $010070=0.001107$ \\
\hline Offsite Average & $0011=0104$ & $0013 \pm 0(x) ;$ & $00013 \pm 04(01155$ & $01 \times 0865 \pm 0.104$ & $010 \times 45: \pm 0004 \times 1$ & $0.0114 \pm 0.10075$ \\
\hline
\end{tabular}

(a) Blarih tield indicate ne datiat.

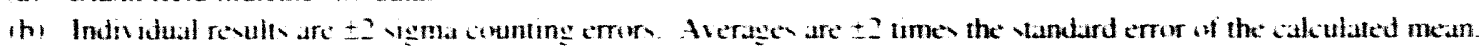

ic) Perimeter lixation unvite near Site moundary 
Table A.18. Uranium Concentrations in Soil. 1987 Through 1992

Lination

\section{Tesite}

Atore (10)-D) Pumphenuse

I Mile NE of IInI-X

I Mike Eufluo-N

Ifxi Area Fire Station

zon-Fan $\times$ Central

Fof $z(x)-$ Fan

2(1)-Las SE

SW of BC Crith

$S$ of $2(x)$ - East

Eof zix)-We

a Miks of zent-Wey

NE थ FTH

SE of PFTF

Sol Bal tred

Hantord Tounde

Wie Barncade

IIkI-. Spring Shureline

Iot-N shore tHCP)

\section{Onsite Average}

\section{Ofisite}

Rivervieu

Bier Landm:

Sidgeminer

Tanler Hat Ne?

W End Fir Rind

Ringovd

Berg Ranch

Wahluhe Slope No. 2

Vernita Bridec

Yahmia Barricade

Rattlensike Sprong

ALE

Pronuer Barricade

So of 3at Area

Benton Cin!

Tetal I ranium. ix $U / \mathrm{s}$ idn weight)

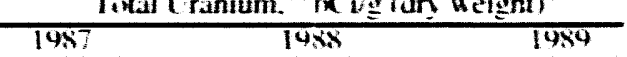

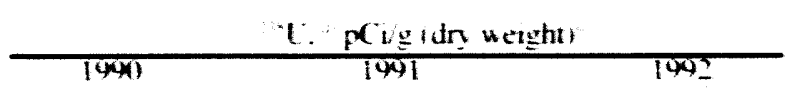

$1.31=0.03 x$

$14 \pm 0.159$

$1.31 \pm 0.137$

$1.2 \pm 0.10 \mathrm{x}$

$1.17 \pm 0.158$

$1.77 \pm 0.29$

$1.3 \pm 01132$

$1.2 \pm 0.14 x$

$1 . x 9 \pm 0.17$

$1.04=0.150$

$1.5=0.124$

$1.31=0.124$

$1.31 \pm 11.165$

$1.48 \pm 11.165$

$2.17 \pm 0.145$

$1.6 x=0.121$

$1.25 \pm 0.0767$

$1.104=0.114$

$107 \pm 0.4$

$100 \pm 0.453$

$14 \pm 0.147$

$1.15+0.1+2$

l.t.t

$1-2+0127$

$1.16=0.11 \mathrm{x}$

$1.22 \pm 01131$

$1.50=0.12 x$
1.127

$1.56 \pm 01.15=$

$0.761=0.176$

$0911=0224$

$0.72=0.214$

$0.57=0.24 \quad 1.85 \pm 0.113$

$04: \pm 0.55$

$6.97 \pm 6.27$

$0.55 \pm 01.15$

$0.07 \pm 11.15$

$0.35 \pm 0.11$

$0.86 \pm 0.34$

$0.83 \pm 0.35$

$0.17=0.05$

$0.55 \pm 0.46$

$0.5 n=0.21$

$(1.5)=0 . i^{4}$

0.501011 .24

$1.1 \pm 0.3$

$0.5 x=0.32$

$1.0 \pm 0.5$

$10.27=00 \mathrm{x}$

$0.2 x \pm 0.65$

$0.15 \pm 0.13$

$0.78 \pm 0.45$

$0.58 \pm 0.20$

$0 .+10 \pm 0.19$

$0.44 \pm 0.25$

$0.68 \pm 0.36 \quad 15 \pm 0.3$

$0.81 \pm 0.43 \quad 0.61 \pm 0.22$

$0.94 \pm 0.33 \quad 1.1 \pm 0.3$

$0.39 \pm 0.11$

$1.1 \pm 0.4$

$0.15 \pm 0.19$
$1.4 \pm 0.16 \mathrm{~s} \quad 1.75=6.13 \quad 0.52 \pm 0.36$

$1,2=0.10 \%$

$1.5 \pm 0.0811$

$1.56 \pm 01122$ 


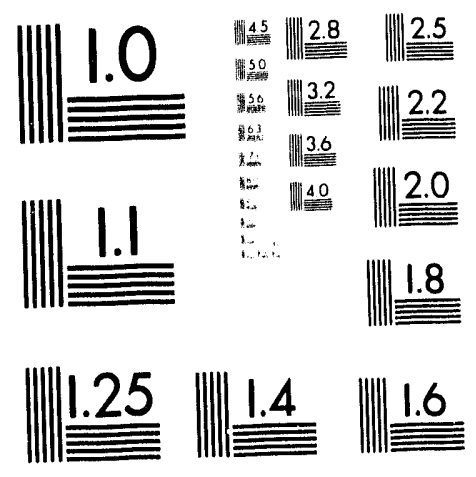



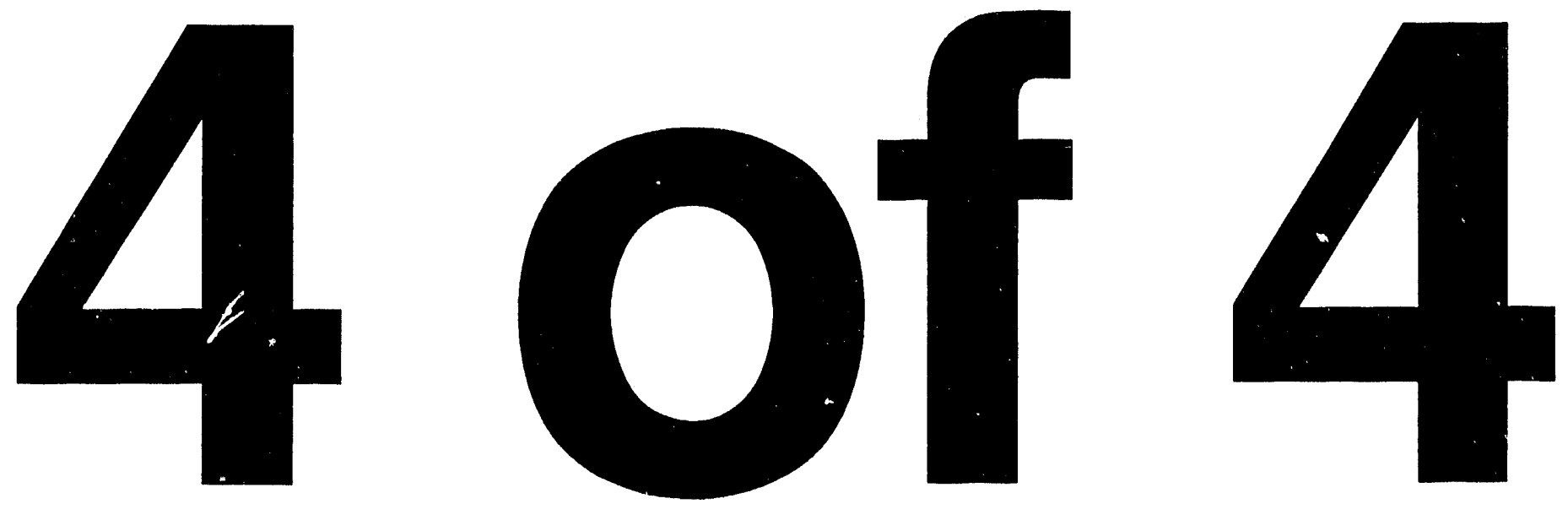
Table A.18. Uranium Concentrations in Soil, (a) 1987 Through 1992 (contd)

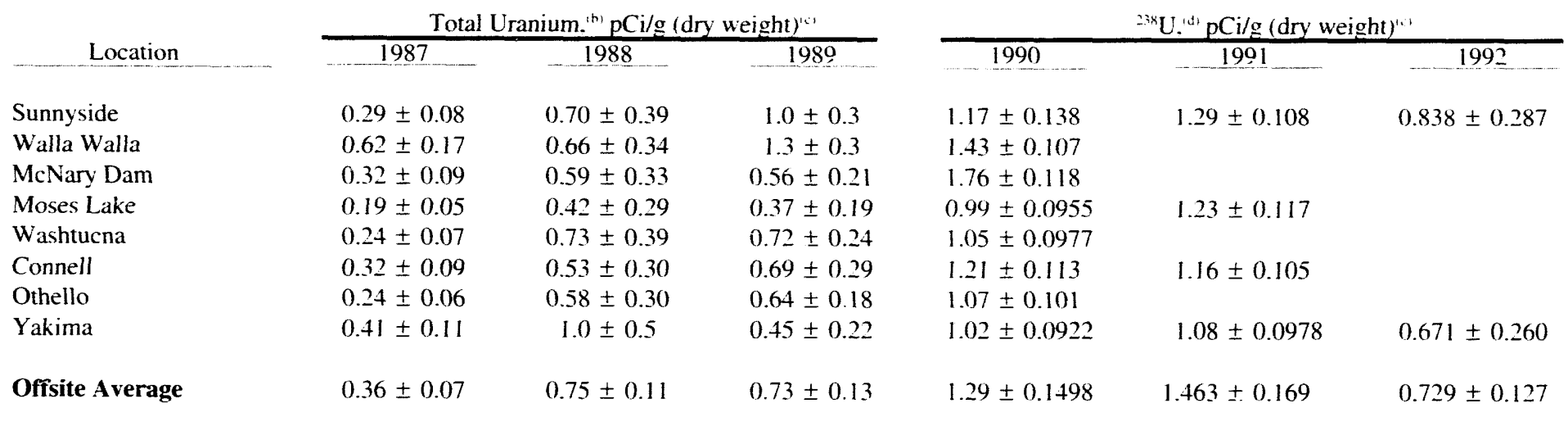
(a) Blank field indicates no data.
(b) Total uranium leached from sample; not directly comparable to ${ }^{2: 3} U_{\text {IEPS }}$.
(c) Individual results are \pm 2 sigma counting errors. Averages are \pm 2 times the standard error of the calculated mean.
(d) Uranium-238 analyzed by low-energy photon spectra (LEPS) method.
(e) Perimeter location onsite near Site boundary. 
Appendix B

Glossary 


\section{Appendix B}

\section{Glossary}

activation product - Material made radioactive by exposure lo radiation from a souree such as a nuclear reactor's neutrons.

air submersion dose - Radialion dose received from external exposure to radionctive materials present in the surrounding allmosphere.

aquifer - Permeable geologic unit that can transmit significant quantitics of water.

background radiation - Radiatton in the natural enviromment, including cosmic rays from space and radiation from naturally occurring radioactive elements in the air, in the earth, and in our bodies. In the United States. the average person receives about 300 ) millirems (merem) of background radiation per year.

bank storage - Hydrologic term that dexcribes river watter that flows into and is relained in permeable stream banks during periods of high river stage. Flow is reversed during periods of low river stage.

becquerel (Bq) - Unil of activity equal to one nuclear transformation per second ( $1 \mathrm{By}=1 / \mathrm{s})$. The conventional unit of activity, the curice is related to the becepuerel according to $1 \mathrm{Ci}=3.7 \times 10)^{11 \prime} \mathrm{Bc}$.

boundary dose rate - Dose rate measured or calculated al publicly aceessible locations on or near the Hanford Site.

composite sample - Sample formed by mixing discrele samples taken at different points in time or from different locallions.

confined aquifer - An aquiler hounded above and below by less permeable layers. Cround water in the conlined acuitier is under a pressure greater than almospheric pressule. continuous sample - Sample formed by the continuous collection of the medium or contaminants within the medium during the entire sample period.

controlled area - An area to which aceess is controlled to protect individuals from exposure to radiation or radioactive and/or hazardous materials.

cosmic radiation - High-energy suhatomic particles and electromagnetic radiation from outer space that hombard the earth. Cosmic radiation is part of natural hatckground radiation.

curie (Ci) - A unit of radioaktivity equal 10.37 hillion $\left(3.7 \times 10^{111}\right)$ nuclear transtormations per second.

decay - The decrease in the amount of any radioactive material with the passalge of time. due to the spontaneous emission from the atomic nuclej of nucleons or either alpha or beta particles, often aceompanied by gamma andiation. When a radioactive material decalys, the material may be converted to another radioactive species (decay product) or to a nonradioactive material.

Derived Concentration Guides (DC(i) - Concentrations of radionaclides in air and water that an individual could continuously consume, inhale or be immersed in at average annual rates, without receiving an effective dose equivalent of greater than 10() $\mathrm{mrem} / \mathrm{yr}$.

detection level - Minimum amount of a substance that can be measured with a $99 \%$ confidence that the antalyticall result is greater that $/ e r o$.

dispersion - Process whereby effluents are spread or mixed an they are mansported by ground water or atr.

dosimeter - Portable device for measuring the total accumulated exposture or absorbed dose from ionizing radiattion lields. 
Effective Dose - Sec "Erlective Dose Equivilent" under "Riadiation Done."

effluent - Liguid or giseous waste streams relealsed from al lacility.

effluent monitoring - Sampling or measuring specilic licpuid or gareous effluent streans for the presence of pollutints.

exposure - Subjecting al targed (usulally living tissue) to radiation or chemicals. Also used als a term describing external radtiation air ionization (see "Roentgen").

fallout - Riddioactive materials that atre released into the earth's almosphere following a nucleatr explosion or atmospheric release and that eventually fill to earth.

fission - A nuclear reatction involving the splitting or breaking apall of a nucleus into all least two sother nuckei, accompanied with a rekease of various lypers of energy. for example. when a heally allom, stech an uranium, is split, large amounts of energy including radiattion and neutrons are released allong with the new nuclei (which are fission products).

fission products - Flements formed from fissioning. Many fission preducts are radionative.

glaciofluvial sediments - Sedimentary deposits consisting of matlerial transported by. suspended in. or latid down by the mellwater streams flowing from melting glicier ice.

grab sample - A silmple thill is randemly collected or "grabbed" from the collection sile.

ground water - Subsurfice wather that is in the pore spateds of soil and geologic units.

gray (Cy) - Unio of absorbed dese in the International System of l'nits (St) ecyutil oo I joule per hilogram. I (iy $=1(0)$ riad.

half-life - Length of time in which a raddoactive substance will lose one half of its radionetivity by decily. Half-lives range from a friaterem of a second to billioms of years, and each radionuclide has a unicyue hall-life.

ion exchange - The reversible exchange of one specien of ion for a different species of ion within a medium. isotopes - Different forms of the same chemical element that are distinguished by different numbers of neutrons in the nucleus. A single element maly have many irotopes: some may he radioatetive and sone may be nonradioatetive (stable). For example. the three inotoper of hydrogen alre protium, deuterium. and tritium.

kurtosis - meatsure of the degree of peakedness of a data distrihution.

maximally exposed individual - A hypothetical member of the public residing rear the Hanlord Site who. by virtec of location and living hathits. could receive the highes possible radiation dose from radioade tive elflenents released from Hanlord.

mean - Average value of a series of meisurements.

The meilln. X. War computed an:

$$
\mathrm{x}=\frac{1}{n} \sum_{\mathrm{i}=1}^{11} \mathrm{x}_{\mathrm{i}}
$$

where $X$ is the ith measurement and $n$ is the number of meatsurements.

median - Middle value in a set of resulls when the datal are ranked in increasing or decreasing order.

millirem (mrem) - A unit of radiation dose cepuis allem

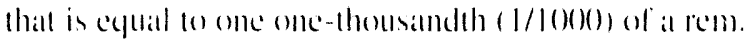

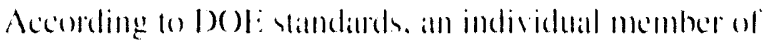
the public maly receive no more than l(x) mrem per yeate from al sikes soperation. This limil deen mot include radiation received for medical treatment or the approximatcly 300 memem that people receive annulilly from natural backerenund radiatlion.

minimum detectable concentration - Simallest anmem

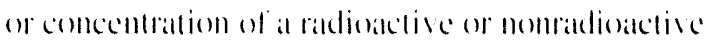
element that call be reliahly delected in al simple.

mode - the vallee of the piece of data that eccuss with the gereallest fresulency.

noble gas - Any of a group of ehemically and bio-

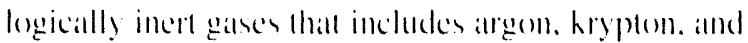
semon. These gatses are nol retained in the hedy follow. ing inhalation. The principal exposure pallhways from

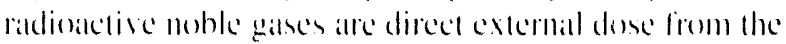
surrounding air (sec "Air Submersion Dose").

irradiation - Exposure an radiation. 
offsite locations - Sampling and measurement locations outside the Hanford Site boundary.

onsite locations - Sampling and measurement locattions within the Hantend Site boundary.

outfall - End of a drain or pipe that carries waste watter or other elfluents into a ditch. pond. or river.

plume - The cloud of a pollutant in air, surface water. or ground water formed aller the pollutant is released from at source.

plutonium - A heavy, radioatctive. anthropogenic metallic etement consisting of sereral isotopes. One important isolope is " $\mathrm{Pu}$. Which is produced by the irradiation of " $\mathrm{U}$. Routine analysis cammot distinguish

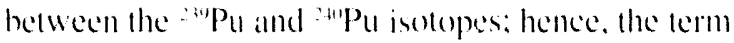
$\therefore$ :-2" Pu as used in this report is symbolic of the presence of ene or hollo of these isolopes in the analytical results.

radiation - The entergy emitted in the form of ratys of patticks, stech as those thrown oft hy transtorming (disintegrating) altoms. For this report. radiation refers to ionizing types of radiation: not radiowates. microwaves. radiant light, of other types of nonionizing radiation. The ionizing rays or particles typically comsist of alphat. betat or gamma radiattion.

- alpha radiation - Least penetrating lype of radialion. Alphat radiation can be stopped by a sheet of paper or the ouler deatl layer of stin. and call callse biological damage only if sufficient yatantities ance emilled inside the body.

- beta radiation - One form of radiation emilted from at nucleus during radiondetive decaly. Betat radiation cail be sopped hy an inch of word or at thin sheet of alluminum. and may calluc biological damatge if a sulticient amount is internal, or exciasionally extermal. (o) the body.

- external radiation - Radialion originatling from a source outside the hody:

- gamma radiation - Form of electromagnetic. highenceg! ratialton emilled from a nucleus. Ciamma ralys are ensemtially the same as s rays. They require heary shickling. stch as concrete or sted. fob be sopped. and maly callse biological damage when rriginatting intemally ore cxtermally to the bods in sufficient amounts
- internal radiation - Radiation originating from a source within the body as a result of the inhalationt. ingestion, skin absorption. or implantation of nattural or anthropogenic radionuclides in body tissues (e.g.. uranium dust in the lungs, radiofodine in the thyroid).

radiation dose - For the purpose of this report, radiation doses are defined als follows:

- absorbed dose - Amount of energy deposited by radiation in al given amount of material. Absorthed dose is measured in units of "rads" or "gratys."

- collective effective dose equivalent - Sum of the effective dose equivalents for individuals composing a delined population. The units lor this are "personrem" or "persom-sicuert."

- committed dose equivalent - Total dose equivalent accumblated in all organ or tissue in the 50 years following a single intake of radionetive materials into the body.

- dose equivalent - Producl ol the absorbed dose. the quality lactor, and any other modifying factors. The dose eyuisalent is a yuantity for comparing the biological effectiveness of different kinds of raddiation on a commonn scale. The unit of dose eyuivalemt is the rem. A millirem is one onethousandih of at rem.

- effective dose equivalent - A value used for estimatting the total risk of potential health effects from radiation exposure. This estimatle is the stum of the committed effective dose equivalent see above) from internal deposition of radionuclides in the hody and the effective dose equisalent from external radiation received during a year.

radioactivity - Property possessed by some isotopes of elements of emitting radiation (such as alphat. betil. or gammat ralys spontancously in their decaly process an stable element isotopes.

radioisotope - Radionctive isotope of a specilied element. Carhom-1t is al radoisotope of carbon. Tritium is a radioisotope of hydrogen.

- long-lived radioisotope - A radionuclide that decitys at stoh a slow rate that a guatntity will exist lore all extended period (lypically many years). 
- short-lived radioisotope - A radionuclide that decays so rapidly that a given quantity is transformed almost completely into decay products within a short period typically less than a lew moniths).

radionuclide - Radioactive atomic species or isolope of an element. There are several hundred known radionuclides, both anthropogenic and naturally occurring. Radionuclide and radioisotope are terms that are sometimes used interchangeably. allhough they are theoretically dilferent terms.

rem - Acronym for roentgen eyuivalent mant: a unit of dose equivalent that indicates the potential for impact on human cells.

risk - The probability that a detrimental health cflect will ocilli.

roentgen - Unit of $x$ ray or gamma radiation exposure in air. typicilly used for describing external radiattion levels. An exposure of I roentgen (R) is approximately equal to a 1 -rem dose to human tisstac.

sievert (Sv) - Unit of dose equivalent in the International System ol Inits (SI) eyual to $10($ ) rem.

skewness - meisure of the lack of symetry in a frequency distribution.

spent fuel - Nuclear fucl that has been exposed in a nuclear reactor; this fuel comtains uranium, activation products. fission products, and plutonium.

standard deviation - An indication of the dispersion or vartability of a set of results around their average.

standard error of the mean - An indiciltion of the dispersion or variability of an estimated mean from the average of other estimates of the same mean.

The standard error of $X$ was computed an

$$
S E=\sqrt{\frac{s^{2}}{n}}
$$

where $S^{-}$. the variance of the 11 measurements, was computed als

$$
S_{N}^{2}=\frac{1}{n-1} \sum_{i=1}^{n}\left(X_{i}-X\right)^{2}
$$

This estimater. S: includes the vartiance among the samples and the counting variance. The estimated $S$ : may oceassionally be less than the average counting variance.

taxon - A group of organism constituting one of the categorics or formal units in taxomomic classification (i.c.. kingdom. phylum, class. onder. lamily. genus, on species) and characteriad by common characteristics in varying degrees of distinction.

thermoluminescent dosimeter (TLD) - A mallerial that. after heing exposed to beta and/or gamma radiation. emits light when processed and heated. The amount of light emitted is proportional to the amount of radiation (dose) to which the TL. has heen exposed.

unconfined aquifer - An aquilier containing ground water that is not confined ahose by relatively impermeable rocks. The pressure at the lop of the unconfined aquiler is equal to that of the atmosphere. At Hantord. the unconfined ayuifer is the uppermost acputier and is most susceptible to contamination from Sitc operations.

uncontrolled area - Area on or near a nuclear facility to which public access is not restricted.

vadose zone - underground area from the surfice to the top of the water table or alquiler.

water table - Theoretical surfice represented by the elevation of water surlices in wells penctratting only at short distance into the unconfined alyuifer.

whole-body dose - Radiation dose that involves exposure of the entire body. Whole borly dose ypicilly refers lo external radiation exposure.

wind rose - Statr-shaped diagram showing how often winds of various speeds blow from different directions. 


\section{Appendix C}

\section{Applicable Standards and Permits}




\section{Appendix C}

\section{Applicable Standards and Permits}

()perations all the Hanterd Site musl comform to a valliely of gersermmentall standards and permits designed to ensure he biolegeical and phesical guality of the ensiron-

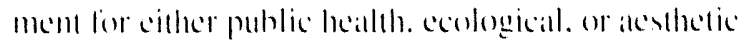
comsickerations. The primany ems ironmental youlity standards and permis applicable to Hanford operations in 1092 are listed in the following libles. The Stalle of

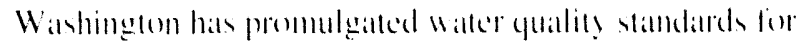
the (olumbia Rirer. Washingtem Administrative Code (W.AC) 17.3-20). The Hanlord Reich of the (olumbia

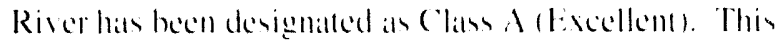
designallon reguires that the walle be thathle for substathtially all needs. including drinking Waller. recreation. and

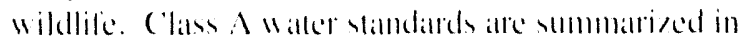
Tahk ('.1. Drinking water standards promulgaled hy the

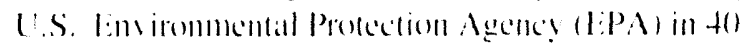

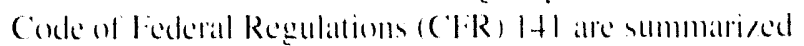

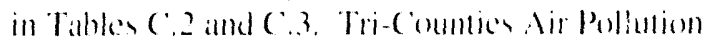

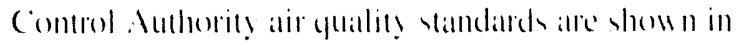

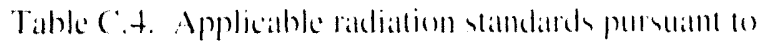

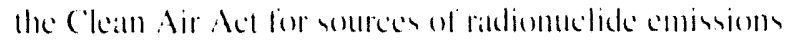

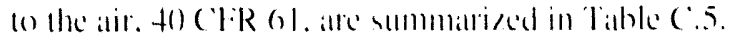

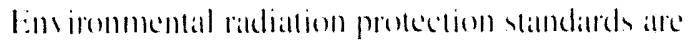

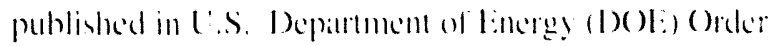

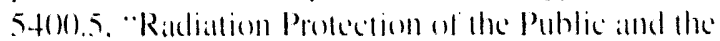

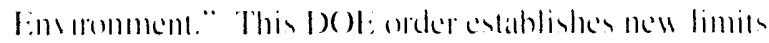

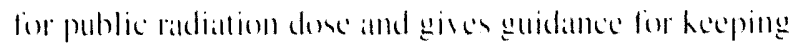

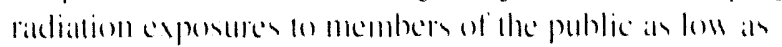

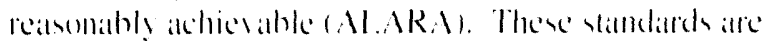
based on goudelimes recommended hy atulhoritatice

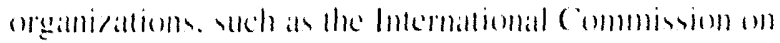
Raddologicial Protection and the Nattomal conncil an

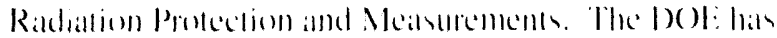

intialted a policy for erealling alld impkementing public radiation protection standard that alre genenally comsis-

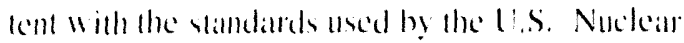
Regulatory ('ommisuson (NRC') in regulating and

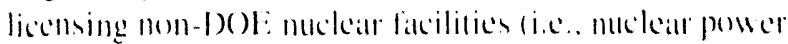

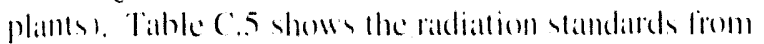
1)(3): ()rder $5 f(0) .5$. These standarde gotern allowable

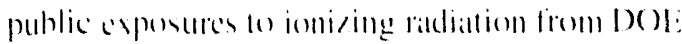
operitions.

In ()rder $5+100.5$, the Dole established Derived (anden-

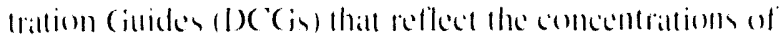
individual nuclides in walter or atr that would resull in an

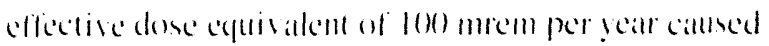
by ingestion of waller or inhalation of air al allerage

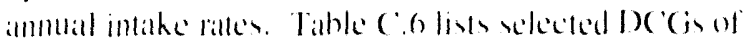

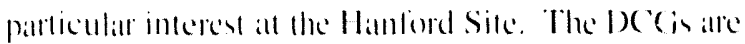
uscful reference values but do mot gemerally reperent comesntrattoms in the ens iromment that consure compli-

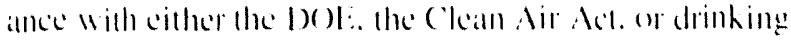
waller dose stamdands.

Permits required lor regulated releatses lo waller and ant hate been issuced by the ESA under the Natlomal Pollut-

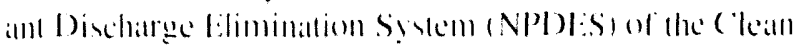
Hater det and the Presention of Signilicant Deterionaltion (PSD) requirements at the ('kan Air Act. N/wo. under atuthorily gramted hy the ( katm Air Act. the Washimglon Stall: Departmem of Heallh has instled al

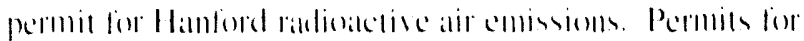

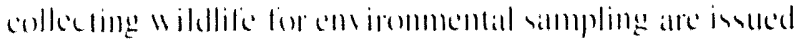

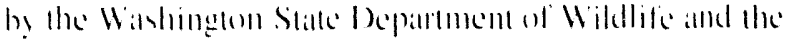

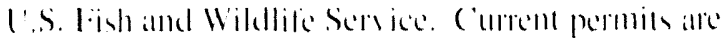
lived in lible $(9.7$. 


\section{Table C.1. Washington State Water Quality Standards for the Hanford Reach of the Columbia River}

Paramelet

Fecial coliform

Dissolved oxygen

Temperature

$\mathrm{pH}$

Turbidit!

Toxic, radionctive, or deleterious materials

Acshertic value
Permissible Levels

1) $\leq 10()$ organisms $/ 100 \mathrm{~mL}$.

2) $\leq 10 \%$ of samples maly exceed 200 organisms/lo() $\mathrm{ml}$.

$>8 \mathrm{mg} / \mathrm{L}$

1) $\leq 20 \mathrm{C}^{\circ}\left(68^{\circ} \mathrm{F}\right)$ due lo human ancivities

2) When natural conditions exceed $20 \mathrm{C}$. no temperature increalse in receiving water of greater than 0.3 C allowed: nor shall increases at any lime exceed $3+/(T+9)$. where $T=$ highest existing temperature in Coutside of dilution zonc.

1) 6.5108 .5 range

2) <().5 unit induced variation

$\leq 5$ NTU" over background turbidity

Concentrations shall be helow these of public health significance, or which catuse acule or chronic loxic conditions to lle alyuatic biota. or which may adversely affece ally waller use.

Shall not be impaired by the presence of mallerials or their effects. excluding those of natural origin. Which oflend the senses of sight. smell. fouch, or tasle

(a) NTU = nephelometric turbidity units. 
Table C.2. Selected Radiological Drinking Water Standards: U.S. Environmental Protection Agency, National Primary Drinking Water Regulations (40 CFR 141); and State of Washington, Rules and Regulations of the State Board of Health Regarding Public Water Systems (WAC 248-54)

$$
\text { Contaminint }
$$

Total alpna (excluding urimium)

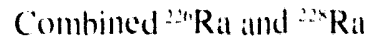

Radium-226 (State of Washington only)

Beta and gatmma radioactivity from anthropogenic radionuclides

$$
\begin{aligned}
& \text { L.imit } \\
& 15 \mu \mathrm{Ci} / \mathrm{L} \\
& 5 \mu \mathrm{Ci} / \mathrm{L} \\
& .3 \mu \mathrm{Ci} / \mathrm{L}
\end{aligned}
$$

Ammual average concentration shall not produce an annual dose from anthropogenic radionuclides equivalent to the total body or any internal organ dose greater thatn + mrem/yr. ll two or more radionuclides are present, the sum of their annual dose eciuivalents shall not exeeed + mrem/yr.

Compliance may be ansumed if annual average concentrations of total hetia. "H. and "'Sr atre less than 50, 20,000), and $8 \mathrm{pC}$ i/L.

\begin{tabular}{|c|c|c|}
\hline Riddionuclide & Critical Organ & Concentration. $\mathrm{pCi} / \mathrm{L}$. \\
\hline 'H & Whole hody & 20.0000 \\
\hline${ }^{14} \mathrm{C}$ & Fitly lissule & $2.0(x)$ \\
\hline " $\mathrm{Co}$ & Gl (LLL) $)^{\text {tal }}$ & 100 \\
\hline$" \mathrm{Sr}$ & Bone & 20 \\
\hline${ }^{* 14} \mathrm{Sr}$ & Bone martow & 80 \\
\hline${ }^{n} \mathrm{Sr}$ & Bone marrow & 8 \\
\hline$" \mathrm{Zr}$ & (il (LI.L) & 20() \\
\hline$" \mathrm{Nb}$ & (il (LLL) & .300 \\
\hline me & (il $(1,1.1)$ & $9(0)$ \\
\hline "'R"Ru & (i) (L.I.I) & 2010 \\
\hline$m \cdot R u$ & (il (1.l.1) & 30 \\
\hline 'Sh & (i) $(1.1 .1)$ & 3000 \\
\hline $1: " 1$ & Thyroid & 1 \\
\hline 1 & Thyroid & 3 \\
\hline$\because \mathrm{CS}$ & Gi (S) & 20.0000 \\
\hline " & Whole hodly & $2(00)$ \\
\hline
\end{tabular}
respectively.

The following list provides the annual average concentrations for anthropogenic radionuclides of interest. These radionuclides are atssumed to yield an annual dese of 4 mrem to the indicated organn. Dattat are taken from the Nationail Interim Primary Drinking Water Regulations, Table IV-2A (EPA 1976).

(a) Ciastrointestinal tract (lower large intestine).

(b) Stomich. 
Table C.3. Selected Chemical Drinking Water Standards: U.S. Environmental Protection Agency, National Primary Drinking Water Regulations (40 CFR 141); and State of Washington, Public Water Supplies (WAC 248-54)

\begin{tabular}{|c|c|}
\hline Chemical Comstituent & Concentratic \\
\hline As & $50 \mu \mathrm{g} / \mathrm{L}$. \\
\hline $\mathrm{Ba}$ & $1 \mathrm{mg} / \mathrm{l}$. \\
\hline $\mathrm{CCl}^{\prime}$ & $5 \mu \mathrm{g} / \mathrm{L}$. \\
\hline (id & 10 $\mu \mathrm{g} / \mathrm{l}$. \\
\hline $\mathrm{Cr}$ & $50 \mu \mathrm{g} / \mathrm{l}$. \\
\hline Cu & $1 \mathrm{mg} / \mathrm{l}$. \\
\hline $1:$ & $2 \mathrm{mg} / \mathrm{l}$. \\
\hline$H_{z}$ & $2 \mu g / l$. \\
\hline $\mathrm{NO}$ & $4.5 \mathrm{mg} / \mathrm{l}$. \\
\hline $\mathrm{Ph}$ & $.50 \mu \mathrm{g} / \mathrm{L}$. \\
\hline Si & $10 \mu z / l$. \\
\hline
\end{tabular}

Table C.4. Benton-Franklin-Walla Walla Counties (Tri-Counties) Air Pollution Control Authority Ambient Air Quality Standards ${ }^{(a)}$
Parameler
Type of Standard the
Simpling Period
Permissible Level

No

Secondiary and primany

Annual arerage

$100 \mu \mathrm{g} / \mathrm{m}^{3}$

(a) Benton-Franklin-Walla Walla ('ounties Air Pollution Control Authority (198()).

(h) Primary standiards for annbient air yuality detine levels of air yuality to protect the public health. Secondary standands deline levels of air cuallity to protect the public welfatre from any known or anticipated adverse effects of a pollutiont. 
Table C.5. Radiation Standards (Dose Limits ${ }^{(a)}$ ) for Protection of the Public from All Routine DOE Activities

All Pathways [limits from DOE Order 54(0).5]

The effective dose equivalent for any member of the public from all routine DOE activities'th shall not exceed the values given below.

Effective Dose Equivalent"

$\mathrm{mrem} / \mathrm{yr} \quad \mathrm{mS} \mathrm{S} / \mathrm{yr}$

Routine Public Dose

10()

5()()$+5$

Dose to Native Aquatic Animal Organisms from Liquid Discharges [interim limits from DOE Order 54(0).5]

Radioactive material in liquid wastes discharged to natural waterways shall not cause an absorbed dose 10 native aquatic animal organisms that exceeds I rad per day (10) mCy per day).

Drinking Water Pathway Only |limits from 40) CFR 141 and DOE Order 54(0).51

Radionuclide concentrations in DOE-(operated public drinking water supplies shall not cause persons consuming the water to receive an rffective dose equivalent greater than $4 \mathrm{mrem}(0 .() 4 \mathrm{mSv}$ ) in a year. DOE activities shall not cause private or puhlic drinking water systems downstream of the facility discharge to exced the radiological drinking water limils in 4 () CFR I 4 l (Table B.2).

Air Pathways Only llimits from t() (PFR oll

$$
\begin{aligned}
& \text { Erfective Dose Equivalent"' } \\
& \text { mrem/yr mSv/yr }
\end{aligned}
$$

Public Dose Limit all Location of Maximum Amnual Air Concentration als a Consequence of Routine Dot: Activitics:"
(). 1

(a) Radiation doses received from natural background, residual weapons lesting and nuclear accideme fallout. and medical consumer product exposures are excluded from the implementation of these dose limits.

(b) "Routine DOE activities" implies normal. planned activities and does not include actual or potential atecidental or unplanned releases.

(c) Effective dose eyuivalent is expressed in rem (or millirem) with the corresponding value in sievert (or millisievert) in parentheses.

(d) Authorized temporary annual dose limits maly be grealter than $10($ ) mrem/yr (hut cannot exceed 50() $\mathrm{mrem} / \mathrm{yr}$ ) if anusual circumstances exist that make avoidance of doses greater than 100 mrem to the public impracticable. The RL. is required on request and receive specific authorization from DOE:-HQ for an increase firom the routine public dose limil to a temporary anmual dose limit.

(c) Aborbed dose is expressed in rad (or milirad) with the corresponding value in graty (or millighay) in parentheses. 
Table C.6. Selected Derived Concentration Guides ${ }^{(a, b, c)}$

\begin{tabular}{|c|c|c|}
\hline Radionuclide & $\begin{array}{c}\text { Water, } \\
\mathrm{pCi} / \mathrm{L} \\
\left(10^{11} \mu \mathrm{Ci} / \mathrm{mL}\right)\end{array}$ & $\begin{array}{c}\text { Air, } \\
\mathrm{pCi} / \mathrm{m}^{3} \\
\left(10^{-12} \mu \mathrm{Ci} / \mathrm{mL}\right)\end{array}$ \\
\hline${ }^{3} \mathrm{H}$ & $2.00(0,0000$ & 100,000 \\
\hline${ }^{14} \mathrm{C}$ & 70.0000 & $50(0,0)(0)$ \\
\hline${ }^{4} \mathrm{Cr}$ & $1.000 .0(0) 00$ & $60,0(0)(0)$ \\
\hline${ }^{4} \mathrm{Mn}$ & $50,0(0)$ & $2.000)$ \\
\hline " $\mathrm{Co}$ & $5,(0)(0)$ & 80 \\
\hline$" \mathrm{Zn}$ & $9,000)$ & $6(0)$ \\
\hline${ }^{8 .} \mathrm{Kr}$ & $N S^{(d)}$ & $3 .(0)(0,000)$ \\
\hline${ }^{\prime \prime} \mathrm{Sr}$ & $1,000)$ & 50 \\
\hline "Te & 100,000 & $2,(0)()$ \\
\hline${ }^{1113} R u$ & 50,000 & 2.000 \\
\hline${ }^{11116} \mathrm{Ru}$ & 6.0000 & 30 \\
\hline${ }^{12.5} \mathrm{Sb}$ & 60,000 & 1.000 \\
\hline$\left.{ }^{1211}\right]$ & $5(0)$ & 70) \\
\hline 1.11 & $3,000)$ & $4(0)$ \\
\hline${ }^{177} \mathrm{Cs}$ & $3,000)$ & 40() \\
\hline${ }^{14} \mathrm{Ce}$ & $7,000)$ & $30)$ \\
\hline${ }^{n} \mathrm{U} U$ & $50(0)$ & 0.09 \\
\hline${ }^{21.5} \mathrm{U}$ & $6(0)$ & 0.1 \\
\hline${ }^{23} \mathrm{U} U$ & 600 & 0.1 \\
\hline${ }^{23 \times} \mathrm{Pu}$ & 4) & 0.03 \\
\hline${ }^{239} \mathrm{Pu}$ & 30) & 0.02 \\
\hline it(1) $\mathrm{Pu}$ & $30)$ & 0.). 2 \\
\hline
\end{tabular}

(a) Concentration of a specific radionuclide in water or air that could be continuously consumed or inhaled at average annual rates and not exceed an effective dose equivalent of $100 \mathrm{mrem} / \mathrm{yr}$. An exception is the limit for ${ }^{\times} \mathrm{Kr}$, which is based on the skin dose limit of 5 rem from immersion in a plume.

(b) Vilues in this table represent the lowest. most conservative derived concentration guides considered polentially applicable to Hanlord operattions, and maly be adjusted upward (larger) if aceurate solubility information is ivailiable.

(c) From DOE (Order 540().5.

(d) NS = No stindiard. 
Table C.7. Environmerital Permits

\section{Clean Water Act Permit}

NPDES Permit No. WA-()0)374-3, issued to the DOE, Richland Operations Office (RL) by Region 10 of the EPA, covers nomadioactive discharges to the Columbia River from eight outfills. The following are measurements required for NPDES-permitted discharges at Hanford:

\begin{tabular}{|c|c|c|c|}
\hline Measurement & $\begin{array}{l}\text { 1(0)-K Areal } \\
\text { (2 discharges) }\end{array}$ & $\begin{array}{l}\text { Lociation } \\
\text { (O)()-N Area } \\
\text { (5 discharges) }\end{array}$ & $\begin{array}{l}\text { 30) Area } \\
\text { (1 discharge) }\end{array}$ \\
\hline Flow rale & $x$ & $x$ & $\mathrm{x}$ \\
\hline Suspended solids & $x$ & $x$ & $x$ \\
\hline Temperature & $x$ & $x$ & $\ldots$ \\
\hline $\mathrm{pH}$ & $x$ & $x$ & $x$ \\
\hline Chlorine & $x$ & $x$ & ... \\
\hline Oil and grease & $\ldots$ & $x$ & $\ldots$ \\
\hline Heal discharged & -.. & $x$ & -.- \\
\hline Setuleable solids & $\ldots$ & $\ldots$ & $x$ \\
\hline Iron & $\cdots$ & $x$ & -.. \\
\hline Ammonial & $\cdots$ & $x$ & -.. \\
\hline Chromium & $\cdots$ & $x$ & $\ldots$ \\
\hline
\end{tabular}

(a) Dashed line indicates no measurement reguired.

\section{Clean Air Act Permits}

PSD Permit No. PSD-X8()-14, issted to RL by Region 10 of the EPA. covers emission of NO, to the atmosphere from the Piutonium Uranium Extraction (PUREX) Plant and the Uranium Trioxide (UO, ) Plant. No expiration date.

Radioactive Air Emission Permit No. FF-()1, issued to RL. by the DOH under authority granted by the Clean Air Act. covers operations on the Hanford Site having a potential to emit radioactive airborne effluents. Initially iseued August 15. 1991. the permit is for a 2 -year period.

\section{Wildlife Sampling Permits}

Scientific Study or Collection Permit No. (0)36, issued hy Washington State Department of Wildlife to Pateific Northwest Laborattory (PNL) for 1992. covers the collection of wildlife, including gamefish. for environmental monitoring purposes. Renewed annually.

Scientific Collection Permit No. 92-36, issued by Washington State Department of Fisheries 10 PNI. for 1992, covers the collection of food fish and shellfish for environmentil monitoring purposes. Renewed annually.

Federal Fish and Wildlife Permit No. 671877. issued by the U.S. Fish and Wildlife Serviee 10 PNL. covers the collection of migratory wildlife. Renewed annually (new rule).

Copies of the regulations concerning these permits may be obtained from the following organizaltions:

State of Washington

Department of Ecology

Olympia. WA $98.5(1) 4$

U.S. Envirommentil Protection Agency

Region 10

1200) Sixth Avenue

Sualle. WA 98I01
U.S. Department of Energy

Richland Operattions Office

Richland, WA 993.52 


\section{References}

40) CFR 61. US.S. Cowernment Printing (Office. "National Emissions Standard for Hazardous Air Pollutants: Standards for Radionuclides." Conde of Federal Re'unlations.

4) CTR 141. U.S. Ciovermment Printing Office. "National Primary Drinking Waller Regulations." code' of ficheral Regulations.

Benton-Franklin-Walla Walla Counties (Tri-Counties) Air Pollution Control Authority. I980). Cieneral Regulation S()-7. Air Pollution Comtrol Authority. Richland. Washington.

Clean Air Act. Public Law 88-206. as amended, 2 USC 74() 1 el sey.
Clean Water Act. Public Law 95-217. December 27 1977.91 Stat. 1506 and Public Law 96-148.

1)( )E: ()rder 5f(0).5. 1090. "Radiation Protection of the Public and the Environment."

U.S. Envirommental Protection Agency (EPA). 1976. National Interim I'rimary Drinking Waner Re'gulations. LPA-57()/9-76-()()3. Office of Watter Supply. Washing(on. D.C.

Washington Adminisllative Code (WAC') 17.3-2()1. Water Quality Standards for Wallers of the State of Washington. Washington State Department of Ecology.

Washington Administrative Code (WAC) 248-54, Public Watcr Supplies, Washington Statc Department of Social and Health Services. 1983. 
Appendix D

Dose Calculations 


\section{Appendix D}

\section{Dose Calculations}

The radiation dose that he public could hatse polentiall! received in loye from Hantord operatiom war calculated

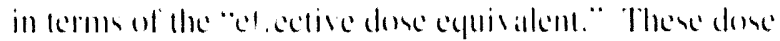

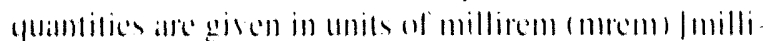

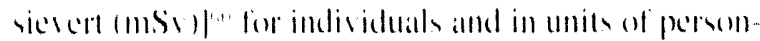

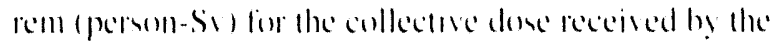
Intal populattom within an $8(1-\mathrm{km} 1.50$ mit radius of the

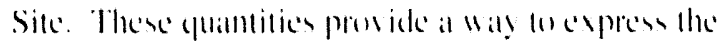

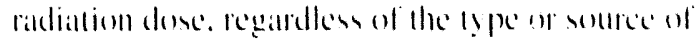
radiation of the meams hy which it is delisered. The

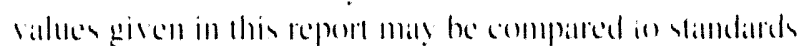

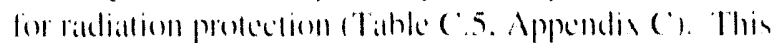

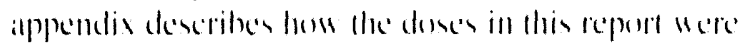
rilkulindel.

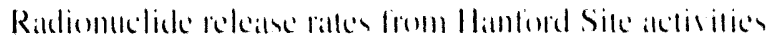

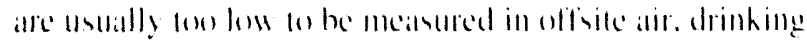

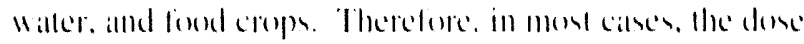
calculations mere based on measmemems make atl the point of release (stachs and cllluent streams). and ens $\mathrm{i}$ remmental concentrations were estimated from these

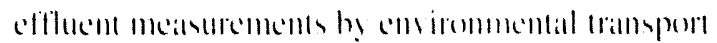
morkels.

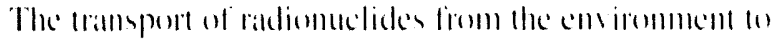

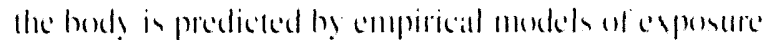

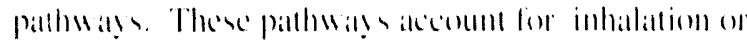
ingestion of radionmelides presem in air. Water. and

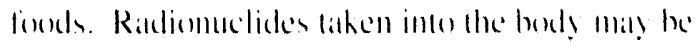
distributed among different organs and retained for

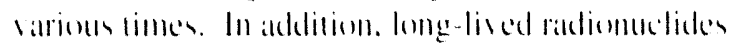
deperifed on the ground become possible souteres for long-term extermal expesume and aptatke hy agricullural

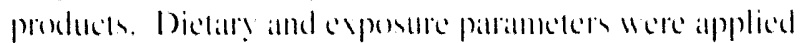

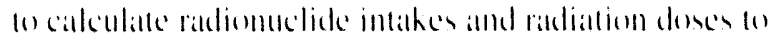
the public. Standardised computer prestam were used

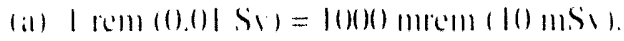

(1) perform the cinlubations. These progratms combain imlemally comsistent mathematlical molels that we vite-

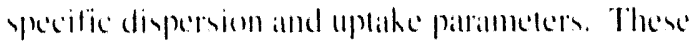
programs alre incorponalled in a master conde, (il: NII

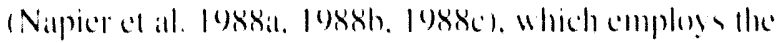
dowmetry medhodology dencribed in Internattomal (ommisson on Rathological Protection (IC RP) Repors

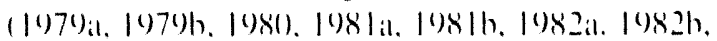

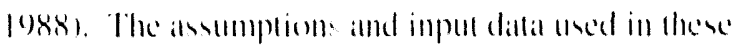
cialculations atre described below.

\section{Types of Dose Calculations Performed}

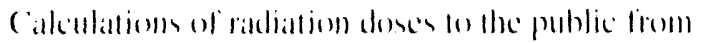

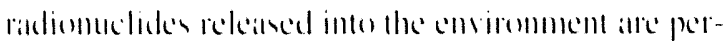
formed lo demomstrate compliance will applicable stamdind and regulations.

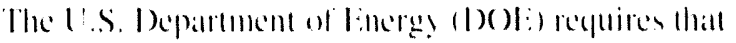

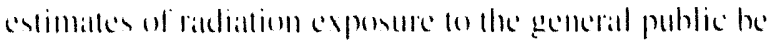

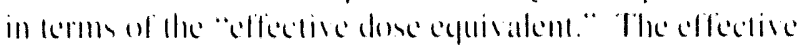

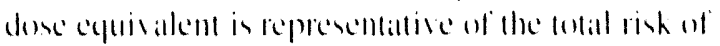

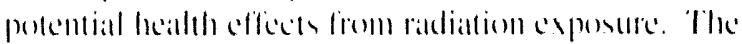

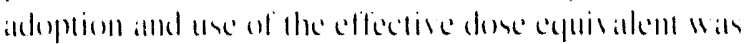

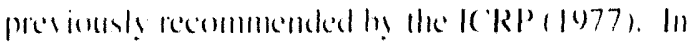

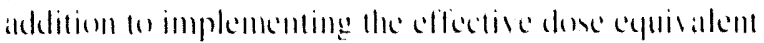

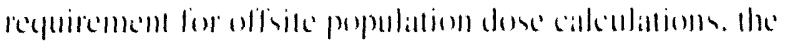

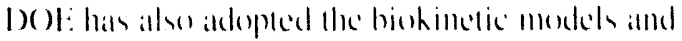

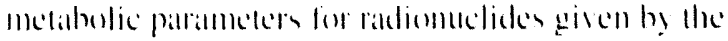

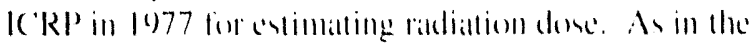

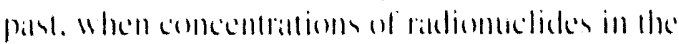

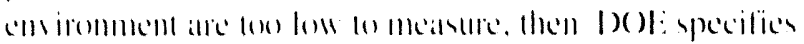

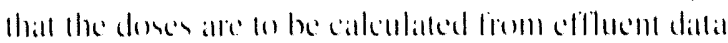

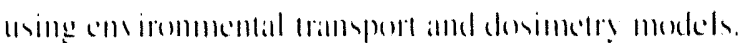




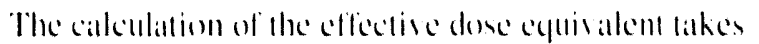

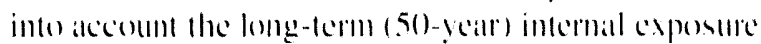

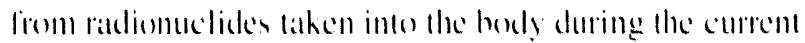

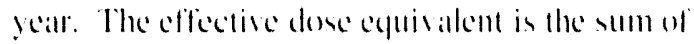
individual commitled (.50)-year' organ doses multiplised hy weighting factors that represc all the proportion of the

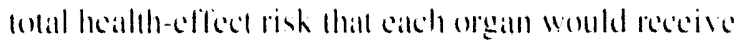
from unilom irratiation of the whole body. Internal organs maly also be irradialled from externall soluceson

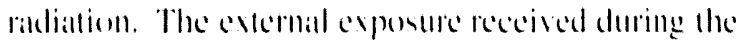
current year is added lo dhe commitled intermal dose 10 obtain the lotal eflective dose equisalent. In this report. the elfectise dose eyuivalent is expressed in rem for millirem). wilh the sorresponding value in sievert

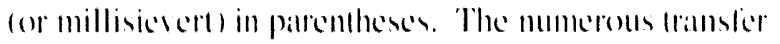

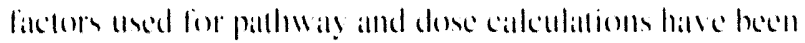
documented in (ilinII (Napier et all. Ieskat. lossh. I) (1)

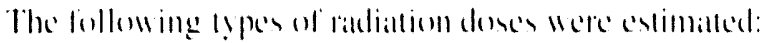

\section{1. "Boundary" Dose Rate (morem/h and merem/yr).}

The external radiation dose ralles during tha sat in

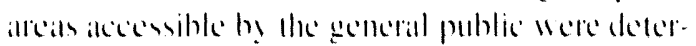

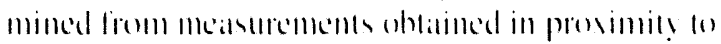
upcrating facilitics.

2. "Maximally Exposed Individual" Dose (mrem). The masimally expened indiridabl is a hy polledicall mémber of the public who lises all a locillion and har

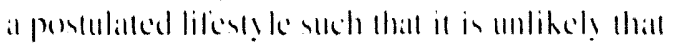

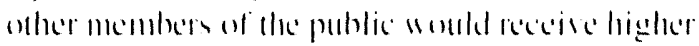

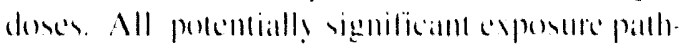

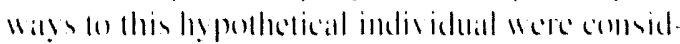
cred. including be following:

- inhaliation ol atrborne radiontelides

- submersion in airhorme radionnclicks

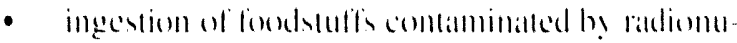

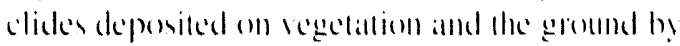

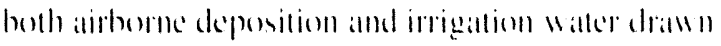

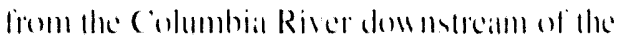
$N$ Reictur

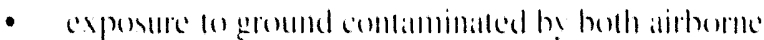
deponition and imigallom waller
- comsumption of santany Hater derived from the Columbial Riscr

- ingestion af fish laken fiom lle Colmmbia River

- recieation alonge lle columbia River. including bating. swimming. and shorelins activilies.

3. 8(1)-km Population Doses (person-rem). Regulattory limils hate not heen established for population

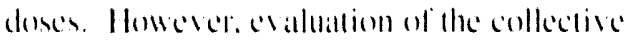

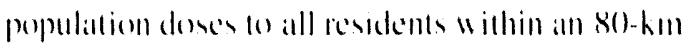
(.50-mi) radius of Hanford Site operatloms is reguired

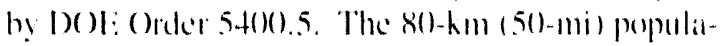
tion dose represents the summed prodects of the individual doses for the number of individuals

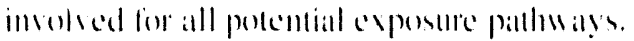

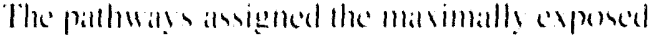
indiridual nere assumed a be applicialle to the

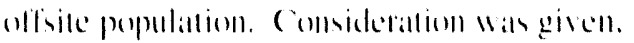

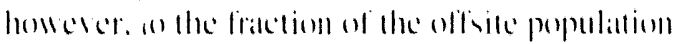

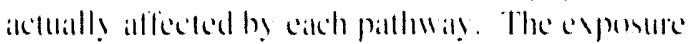

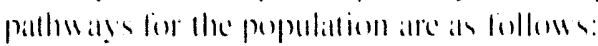

- Drinking Water. The citics ol Richland and larcon ohtain their municipall watter ditcetly. ind

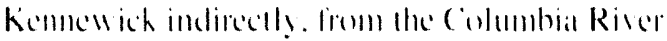
dommstram from the Hamford Sile. A total populat

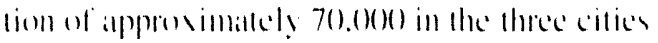
drinks waller derived from the (olumbia Riser.

- Irrigated foods. Columbia Rirer waller is will-

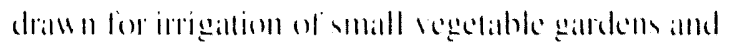

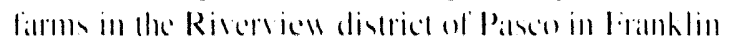

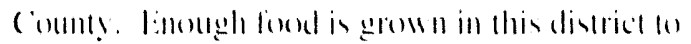

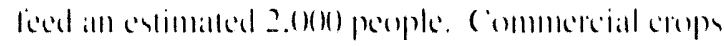
alle also irrigalled by Columbia Riser waller in lle

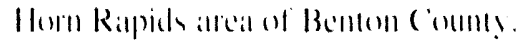

- River Recreation. These atetis itice include swimming. hoating. and shoreline recreation. An

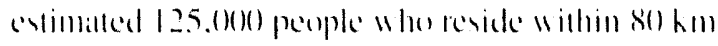
(.5) mis of the Hanfind Sitc anc assumed lo be allected hy these pallowas 
- Fish Consumption. Population doses from the comstmption of fish obtaincel locally from the Columbia River were calculated from an estimalled fotal ammual catch of $1.5 .00(0) \mathrm{kg} / \mathrm{yr}$ (without reference (o a specified human group of consumers).

\section{Data}

The dala that are needed to pertorm dose calculations based on measured effluent releases include intormation on initial transport through the almosphere or river.

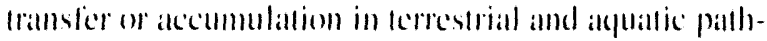
Ways, and public exposure. By comparisom, radiation dose calculations based on meatsured contedentations of radionuclides in lood recpuire datta describing only dietary and recreational alcivities and exposule limes. These dalla are discussed in the following sections.

\section{Population Distribution and Atmospheric Dispersion}

Geographic distributions of the penpulation residing within all 8()-km (50-mi) radius ol the four Hanford Site

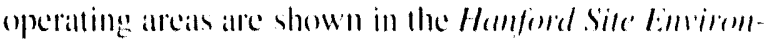

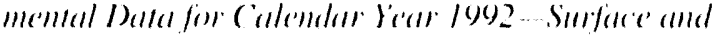
(ollumbia River (Bisping and Woodruft le9.3). These' distributions alle hased on 1990) Bureau of census dintat

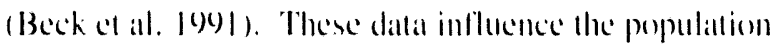
dose by providing estimales of the number of people exposed to radionetion material rekeses and their proximity to the poims of release.

Amospherie dispersion dallat are also shown in the

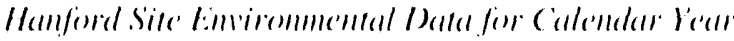

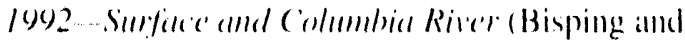
Woodrulf fo93). These dalla describe lle transport and dilution of airborne radiondetive matcrial, which influences the amounts of radiontedides being transponted through the air to specilic locations.

\section{Terrestrial and Aquatic Pathways}

Important parameters alfiecting the movement of radiomuclides within potential exposure pathways, such as irrigation rales. growing periods, and holdup periods, are listed in Table D.1. (ertain paramelers are specilic to eiller the lifestyles of "maximally exposed" or "atrerage" individuals.

\section{Public Exposure}

The potential offsite radiation dese is related to the extent af extermal exposture to or intake of radionuclides thatl alde released from Hantord Sile operations. Tables D.2 Ihrough 1).t give the parameters describing the diet. residency, and river recreation assumed for "maximally exposed" and "average" individuals.

\section{Dose Calculation Documentation}

The Hanliond Dose ( ) verview Pand has the responsibility for defining standard. dexumented compuler codes and

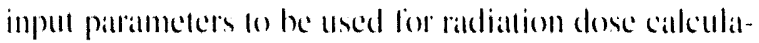
tions for the public in the vicinity of the Hanford Site. Only those procedures, models, and parameders defined by the Hanford Done ( ) verview Panel were used lo calculate the radiation doses. The calculations were then reviewed by the bose (O)erview Pancl. Summatries of dose calculation documentation for this report are shown in Tables D.5 (hrough D.9 and Hemford Site Limirom-

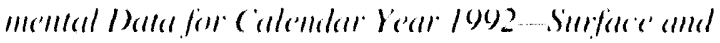

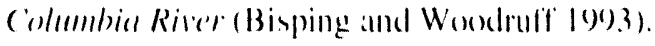


Table D.1. Food Pathway Parameters Used in Dose Calculations, 1992

\begin{tabular}{|c|c|c|c|c|c|}
\hline & \multicolumn{2}{|c|}{ Holdup, days (.." } & \multirow{2}{*}{$\begin{array}{l}\text { Cirowing } \\
\text { Period, } \\
\text { dalys }\end{array}$} & \multirow[b]{2}{*}{$\begin{array}{l}\text { Yicld, } \\
\mathrm{kg} / \mathrm{ml}^{\prime}\end{array}$} & \multirow{2}{*}{$\begin{array}{l}\text { Irrigattion } \\
\text { Rilte. } \\
\text { I./m1/month }\end{array}$} \\
\hline & $\begin{array}{l}\text { Maximally Exposed } \\
\text { Individual }\end{array}$ & $\begin{array}{l}\text { Averiage } \\
\text { Individual }\end{array}$ & & & \\
\hline Leally vegetables & 1 & 14 & $9)$ & 1.5 & 1.50 \\
\hline Other vegetiables & 5 & 14 & $9)$ & 4 & 170) \\
\hline Fruit & 5 & 14 & 9 & 2 & 150 \\
\hline Cercal & 180 & 180 & $9)$ & 0.8 & 0 \\
\hline Fgges & i & 18 & 9() & $(0.8$ & () \\
\hline Milk & 1 & 4 & & & \\
\hline Haly & $(1110)^{\prime \prime 11}$ & $(1)(1))$ & 4.5 & 2 & $2(0)$ \\
\hline Pasture & (0) & (1) & $.31)$ & 1.5 & $2(1)$ \\
\hline Red meill & 15 & 34 & & & \\
\hline Hay & $(1)(1))$ & $(1)(1))$ & 45 & 2 & 200 \\
\hline Cirain & $(18(1))$ & $(180)$ & 9() & 0.8 & () \\
\hline Pesultry & 1 & 34 & $9(1)$ & 0.8 & () \\
\hline Fish & 1 & 1 & $\cdots$ & $\cdots$ & $\cdots$ \\
\hline Drinking willer & 1 & 1 & -.. & $\cdots$ & $\cdots$ \\
\hline
\end{tabular}

(a) Holdup is the time between harvest and comsumption.

(b) Vallues in ( ) are the holdup in days between harvest and consumption by farm animals

Table D.2. Dietary Parameters Used in Dose Calculations, 1992

Maximailly

Exposed

Individual

Comsumprionn, $\mathrm{kg} / \mathrm{yr}$

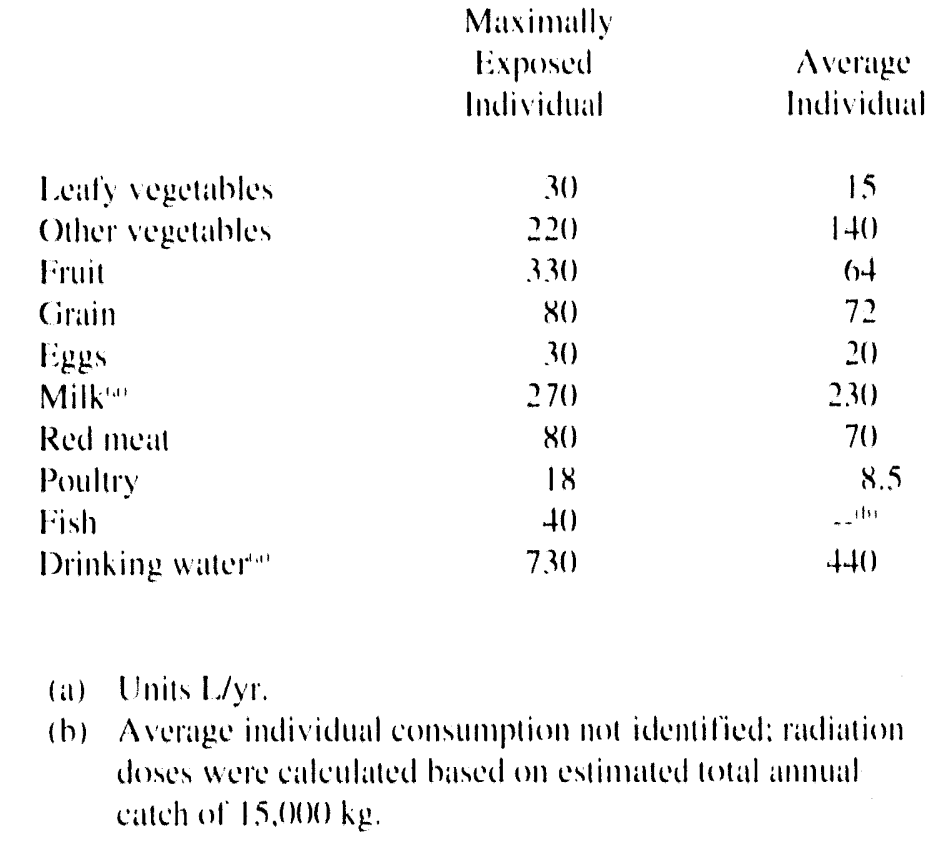


Table D.3. Residency Parameters Used in Dose Calculations, 1992

\begin{tabular}{|c|c|c|}
\hline & & \\
\hline & Maximally & \\
\hline Parameler & $\begin{array}{l}\text { Exposed } \\
\text { Individual }\end{array}$ & $\begin{array}{l}\text { Average } \\
\text { Individual }\end{array}$ \\
\hline Ground contamination & +.38 .3 & 2.920 \\
\hline Air sulbmersion & 8.760 & 8.760 \\
\hline nhallationn'."' & 8.7606 & 8.7606 \\
\hline
\end{tabular}

(a) Inhatittion rates: Adult $270 \mathrm{~cm} / \mathrm{s}$.

Table D.4. Recreational Parameters Used in Dose Calculations, 1992

\begin{tabular}{|c|c|c|}
\hline \multirow[b]{2}{*}{ Pantancler } & \multicolumn{2}{|c|}{ Вxposure, $b / y r^{x+1}$} \\
\hline & $\begin{array}{l}\text { Maximally } \\
\text { Exposed } \\
\text { Individual }\end{array}$ & $\begin{array}{l}\text { Average } \\
\text { Individual }\end{array}$ \\
\hline Shoreline & $5(0)$ & 17 \\
\hline Boatting & 1000 & 5 \\
\hline Swimming & $100)$ & 10 \\
\hline
\end{tabular}

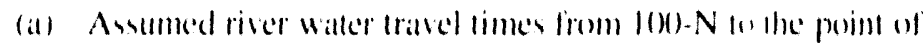
andittic recreatton were 8 h for the matsimally expmed individual and 1.3 h for the allerage individual. Correspondingly lesser timen were used for orther locations. 
Table D.5. Documentation of 100-N Area Airborne Release Dose Calculalions, 1992

Facility namc

Releilses

Meteorologicial conditions

$\mathrm{X} / \mathrm{Q}^{\prime}$

Roleisse height

Population distribution

Compuler code

Disc's cialculated

Pathwatys considered

filles addressed
1()(1)-N Areia

Sec Table 3.1

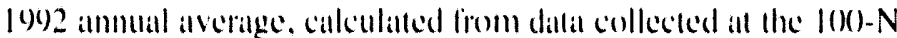
Area and the Hanford Meteorology Station from January 1902 through December 1992, using the compruter code HANCHI / see Table D-5. Bisping and Woudrufl (1003)1.

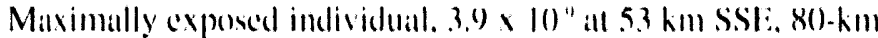
populattion. $1.8 \times 10)^{4} \mathrm{person-s/m1^{ \prime }}$

89)-m sflectirs stick height

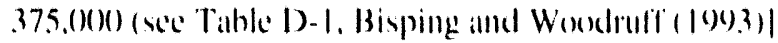

(iliNII, Varsion 1.48.5.12-.3-90)

Chronic, 1-year exposure, 50-year commitled intermal dose

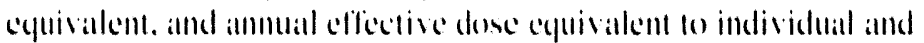
population

lixternal exposure fo plume and gromed deposits Inhulatlion

Ingestion of locially prodeced fords

Radionuclide l.ibrary, Rew, 7-1-()2

lined Tramster Library. Rev, 8-20-48

Fatermal Dose Fictor library. Res. 5-9-8x

Inlemal Dose Ficken Librals. Rev. 12-3-90) 
Table D.6. Documentation of 100-N Area Liquid Release Dose Calculations, 1992

Fiacility name

Releasces

Meall river llow

Shore-wideh fiactor

Population distribution

(impulet corde

Dose's calculated

Pilloways considered

Files addrensed
I()()-N Areia

Sece Tuble 3.5

101.0() $0)(\mathrm{dis}(2.860) \mathrm{m} / \mathrm{s})$

(1).2

70.0()() for drinking waller palloway 125.(6)() for alcuattic recreation

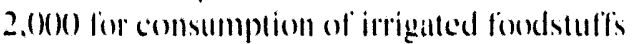
1.5.(1)(1) kg/yr lolal harvest ol Columbia River lish

(il:NII, Version 1,485, 12-3-4)

Chronic, 1-yeall expostlre, 5()-year commilled interthal dose equivalent. and anmual elfective dose eyuivalent to individual and p(1) ulation

Extermal expesture to irrigalled soil. lo river waller, and to shoreline sediments

Ingestion of drinking waller, alyuatic foods, and irrigalled farm products

Radionuclide Libraty, Rer. 7-1-92

Fond Transfer Library, Rev. 8-29-88

Extermal Dose factor Libraly. Res. 5.9-88

Incernal Dose Factor Library. Res. 12-3-90)

Bionccumulation Facdor Lihrary, Rev, 10-2(1-9) 
Table D.7. Documentation of 200 Area Airborne Release Dose Calculations, 1992

Facility name

Releisen

Meteorologicial conditions

$\mathrm{X} / \mathrm{Q}^{\prime}$

Release heigh:

Population distribution

Computer code

Dones cialculated

Pithwatys considercd

Files addresued
2(1) Areas

Sec Table 3.1

1992 annual average, cialculited from datta collected at the Hanford Meteorology Station from Jamuaty 1992 through December 1992. Using the compuler colle HANCHI see Tible D-6. Bisping and Woodruff (|(9),3)|

Maximally exposed individual. I x $10^{*}$ at $4.3 \mathrm{~km}$ SE: $8(1)-k m$ population. $1.7 \times 10)^{\prime}$ person! $\mathrm{s} / \mathrm{m}^{\prime}$

89-111 clfecive stack height

$.376 .(000)$ |see Tahle D-2. Bisping and Woodruff (199.3)|

(iENII, Version $1,485,12-3-9)$

Chronic, 1-year exposure, 5()-year committed intermal dose erruivalent, and annual effective (whole-body d dose equiv alent to individual and population

Extermal exposure to plune and ground deposits Inhalation

Ingestion of lecilly produced foods

Riddionuclide Library. Rer. 7-1-92

Fond Transfer Library, Rex. 8-29-88

External Dose fictor Library, Rev, 5-9-8x

Internall Dose Factor l.ibrary. Res. 12-3-9) 
Table D.8. Documentation of 300 Area Airborne Release Dose Calculations, 1992

Finility name

Releises

Meteorolngicial conditions

$\mathrm{X} / \mathrm{Q} \mathbf{Q}^{\prime}$

Releuse height

Population distrihution

Computer corde

Dones cialculatted

Pathwalys considered

Fillus addremed
$3(0)$ Areils

Sie Table 3.1

1992 annual ancrage. calculated from datta collected at the 300 Areal and the Meteorelogy Stattom from Jamtiary 1992 through Decernber l(9)2. using the compuler code HANCHII fee Fiable D-7, Bisping and Woodrufl (|(O) 3.)|

Maximally exposed individual, $8.9 \times 10^{*}$ in $1.3 \mathrm{~km}$ SSl:: $8(1)-\mathrm{km}$ population. 8.1$) \times 10^{\prime} \mathrm{pers}(\mathrm{m}-\mathrm{s} / \mathrm{m}$ '

10 III

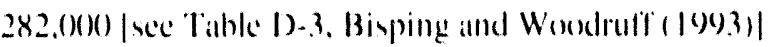

(iliNII, Version 1.485., 12-3-9)

Chronic, 1-year exposure, 50-year commilled internal dose equiv alent, and annual eflective (whoke-hody) dose equivalent lo individual and population

Extermal exposure bo plume and ground depenits Inhalation

lingestion of locially produced foods

Riddionuclide Library. Rev 7-1.92

Food Transfer Library. Rer, 8-29-8x

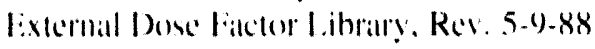

Internal Dose fictor l ibrary, Res. 12-3-9)() 
Table D.9. Documentation of 400 Area Airborne Release Dose Calculations, 1992

Facilits name

Relcanes

Meleorological comditions

$X / Q$

Relears height

Population distribution

Computer conde

Dones calculated

Patlowa! comsidered

Files addrened
3()() Arcias

Sec Talle 3.1

1992 anmual anerages caticulated from datat collected at the 401 Areal and the Mekorolog! Station from January 190? hrough December lye). using the computer code HANCHI l see Table D-8. Bisping and Wondrult (19093)|

Maximally exposed individual. $4.2 \times 10^{\times}$al $22 \mathrm{~km}$ SSE: 8()$-\mathrm{km}$ population. $5.6 \times 10^{\prime}$ persom-s/m

(1) 11

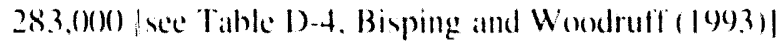

(iENII. Version 1.48.5. 12-3.90

Chronic, 1-vear exponsure. 5(1-sear commilted inlermal dose equiralent. and annual effective (whole-boly ) dose equivalent fo individuall and population

External exposure on plume and ground deposits Inhalation

Ingertion of locally produced londs

Radionuclide Lihran!. Re 7-1-9)

Food Trameler library. Rer. 8-29-88

Extemal Dome lackol l ihrary, Rex. 5-9-88

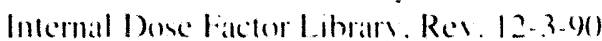




\section{References}

Beck. 1). M.. and B. A. Napier. M. J. Sicoll. A. (i. Thuman. M. D). Daris. D). B. Pillenger. S. I. Shindle. and N. C. Ballishho, |yo). Hanfond

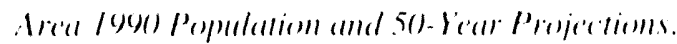
PNI. 780)3. Pacilic Northwest L ahoraltory. Richland. W:ahington.

Bisping. 1.1:.. and R. K. Woodrull. 1093. Ham!ond Sile

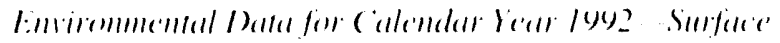

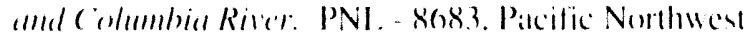
laborallory. Richland. Washinglon.

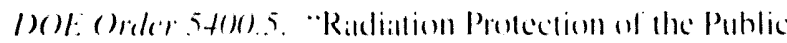
and the limiromment."

International Commossion on Radiolengical Protection

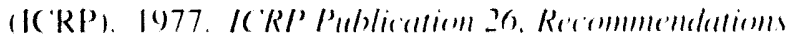

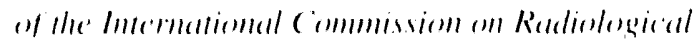
Promertiom. Amals of the IC RP. Vol. I. No. 3. Pergamon Press. Iimslord. Now York.

Intermattional Commimion on Raddological Protection

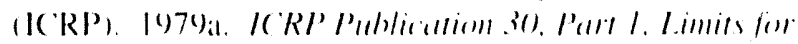

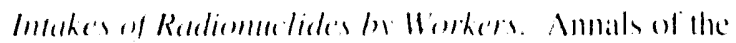

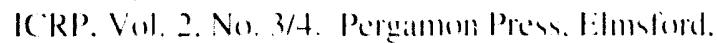
New rirk.

Internalional commission on Raddologeical Profection

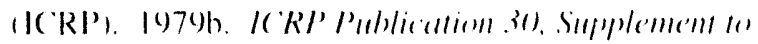

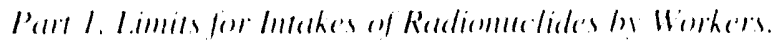
Anmals of the ICRP. Vol. 3. No. I-t. Pergammon Press. I:Imblurd. New York.

Internattional commission on Radiological Prolection

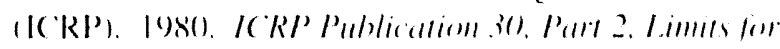

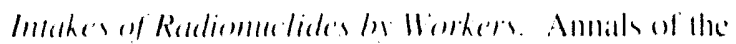
IC RP. Vul. 4. No. 3/4. Pergammon Press. I:lmstord. Nin link.

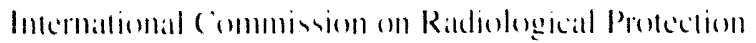

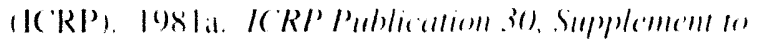

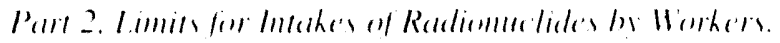

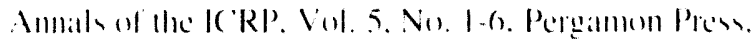
Elmofird. Nen Yonk.
International commission on Radiological Protection

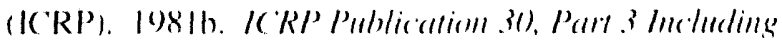

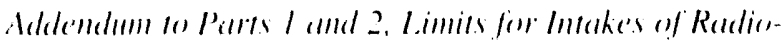
muclides by Workers. Ammals ol the IC'RI), Vol. 6, No. 2/ 3. Pergamon Press. Limsford. New York.

Intermational (ommission on Radiological Protection

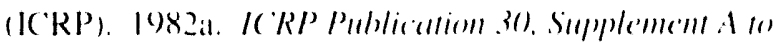

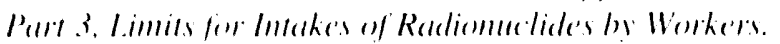
Anmals of the ICRP. Vol. 7. No, 1-3. Pergamom Press. likmord. New York.

Intcrmallomal commission on Radiologicall Protection

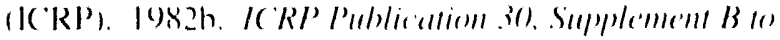

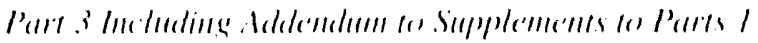

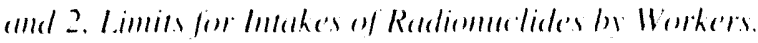
Anmals of the IC RP. Vol. \&. No. 1-3. Pergamon Press. limslord. Nen York.

Internattonal (ommission on Raddologeical Prolection

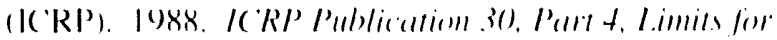

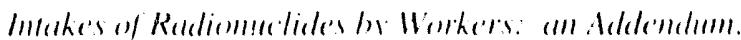
Ammuals of the IC RP. Vol. I9. No. 4. Pergamon Press. limstord. Now York.

Napicr. B. A.. R. A. Pelequin. 1). I. Strenges, and J. V.

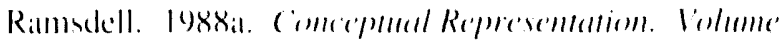

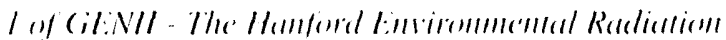

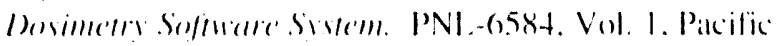
Northwest l abouratory. Richland. Washington.

Napice. B. A. R. A. Pelequmn. 1), I. Strenger, and I. V.

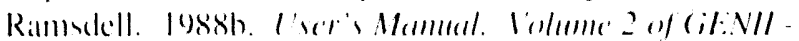

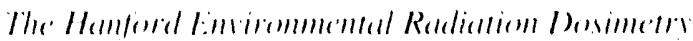

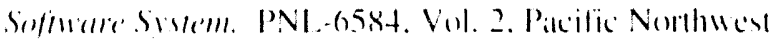
lathoratury. Richland. Washingum.

Napicr. B. A. R. A. Peloguin, D, I. Smenger, and J. V.

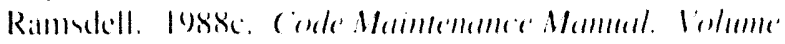

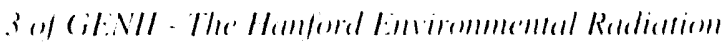

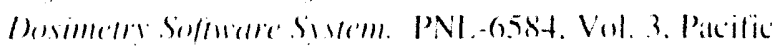

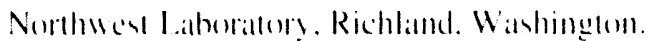




\section{Appendix E}

RCRA and CERCLA Monitoring Documents 


\section{Appendix E}

\section{RCRA and CERCLA Monitoring Documents}

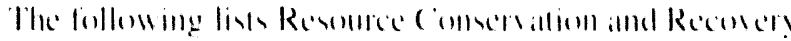

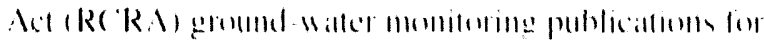
14)?:

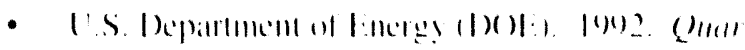

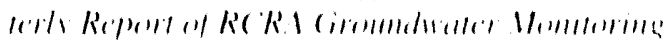

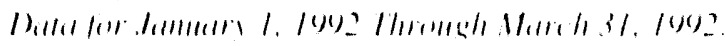

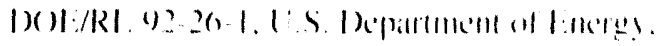
Richland. Washingeron.

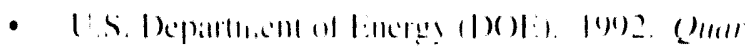

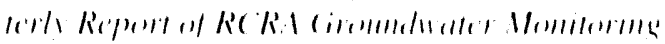

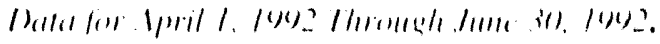

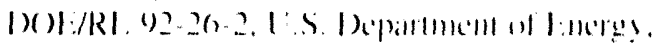
Richlamd. Winhlin!mon

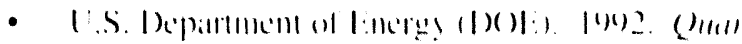

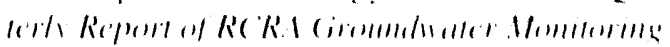

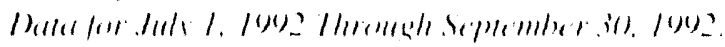

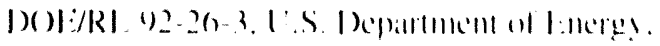
Richland. Hahhinglon.

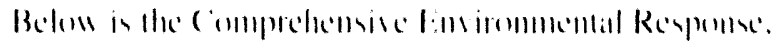

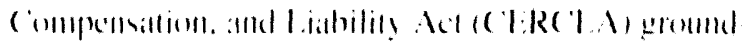

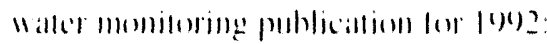

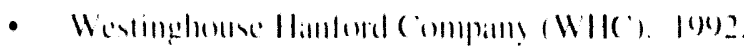

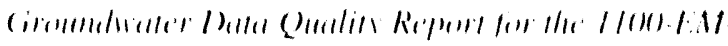

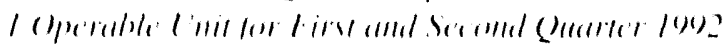

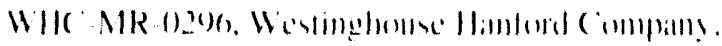
Richlamd. Wimbinglum.

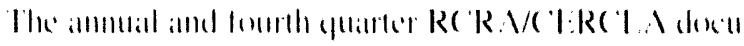

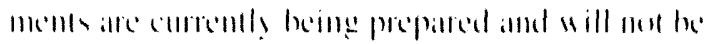

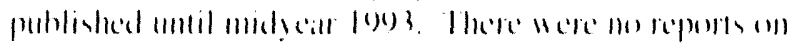

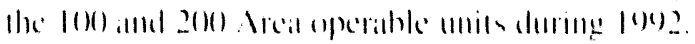




\section{Appendix F}

Radionuclides Detected by Gamma Spectroscopy 


\section{Appendix F}

\section{Radionuclides Detected by Gamma Spectroscopy (Gamma Scan)}

\begin{abstract}
(

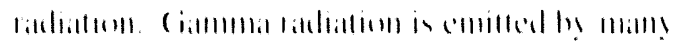

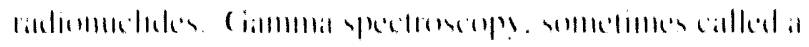

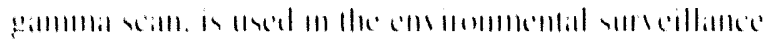

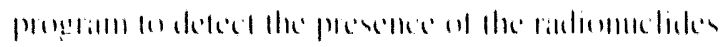

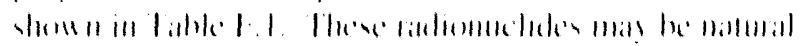

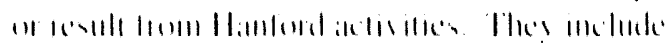

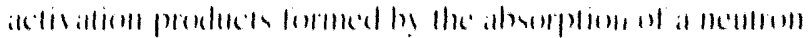

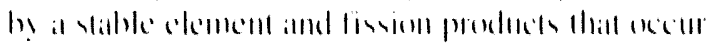

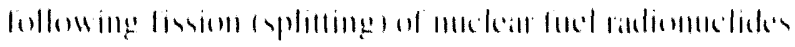

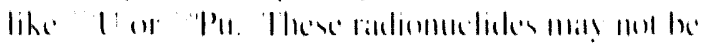

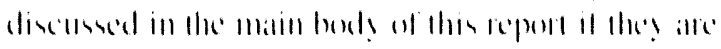

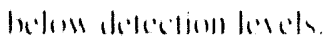

Tahle F.1. Radionuclides Analyzed by Gamma-Spectroscopy

\section{Rinlumumldk}

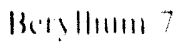

Sulium :.

Sollum 2.1

Putim, IIIII) fl)

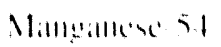

(i)ill 5 s

(inall (n)

|linl 51)

/1114: 15

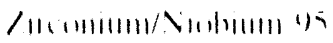

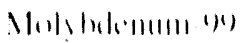

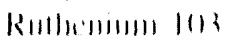

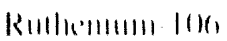

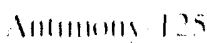

ladme | il

(i') 111111 ) 3.1

(ivilin) 13

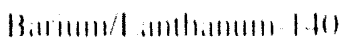

(imm!n $1+1)$

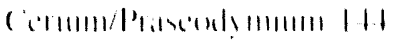

I

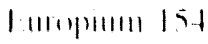

|atr|lum 159
Sim

Bi.

$\mathrm{Ni}$

Nil

h

III

(i)

(i)

10

111

$1 / \mathrm{Nh}$

111

R॥

"kı

Sh

I

(')

(

$13: 1 / 1,1$

( i

( i / /

I 11

111

1.11
Sumris 


\section{Appendix G}

Threatened and Endangered Species 


\section{Appendix G}

\section{Threatened and Endangered Species}

Threallemed and ondangered plants and animals idemtilied

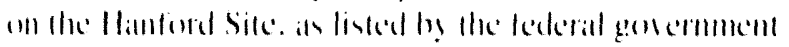

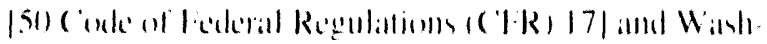

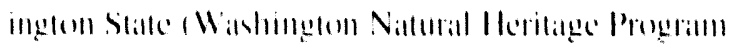

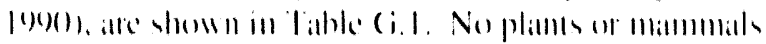

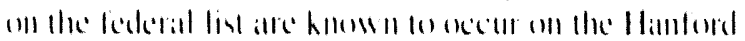

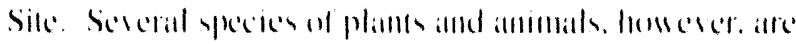

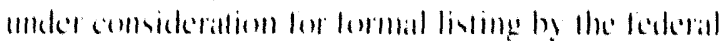

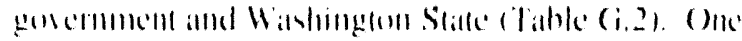

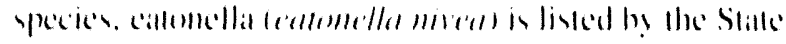

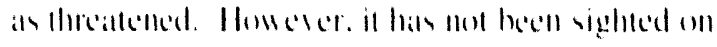

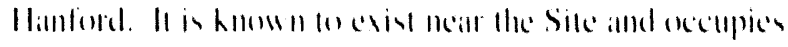

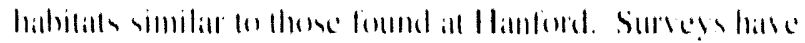

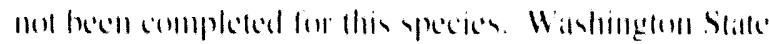

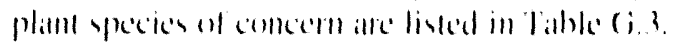

Table G.1. Threatened ( $T$ ) and Endangered (E) Species

(imm!nแ! Nimlle

Planls

(illumblat milk lodid

(i)lumbial sollumiter

Homed a descll patrole!

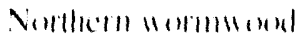

Bircls

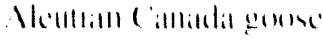

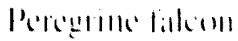

Ballil ciagle

While polliall

Samblhill stamk

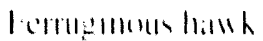

Mlunumuns

P!nII! rahhil
Sicintilic Nillle

lederial

Stilt:

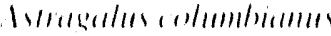

Rorilpled collumblicu

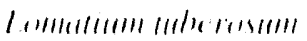

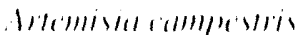

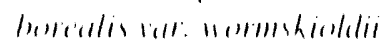

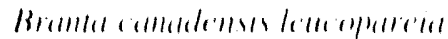

lalen peresermen

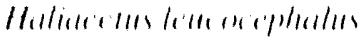

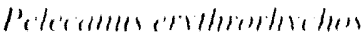

(i)m a rmendemsis

Bullererenellis

$\begin{array}{ll}1 & 1 \\ 1 & 1 \\ 1 & 1 \\ & 1 \\ & 1 \\ & 1\end{array}$


Table G.2. Candidate Species

(omIIIII N:III)

\section{Molluses}

Shortficied lians

(o)lumbial pehbleshail
Scientific Namle

Pederal

State

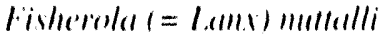

$x$

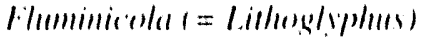

collumbiamu

$x$

$x$

Birds

(immonon loum

Swatllsoll, hallk

Forruginous haluk

Western sigge grouse

Silge sparrese

Burrowing ow

l.oggerdesill shrike

Northern gurhlaruk

Blach lern

\section{Mammuls}

Merriann ? shen

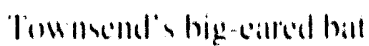

Pygm! ralhhil

\section{Plunts}

Columbial milh-1ctsh (columbia yellourcess

Hower - denert parreles!

Northern wormonenel
Cianion immere.

Batlen shominsomen

Buenere regalis

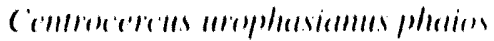

Amphispias helli

Alhene' comiculariou

Lamims lendenticiommes

drcipler gemilis

chlichomicas migen".

Sores merriomi

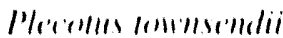

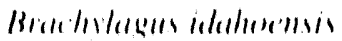

$x$

$x$

$x$

$x$

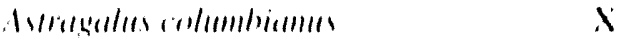

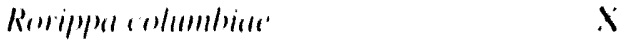

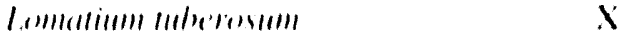

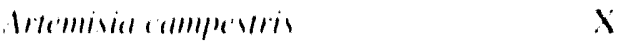

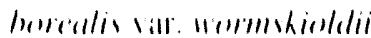




\section{Table G.3. Washington State Plant Species of Concern Occurring on the Hanford Site}

Commonon Name

Dense sedge

Ciray cryptantha

Bristly cyptantha

Shining flatsedge

Piper's daisy

Southern mudwort

Finse-pimpernel

Dwarl evening primrose

Toulh-sepal dodelet

Thompsorn's sandwort

Rohinson's onion

Syuill onion

Columbia River mugwort

Stalked-pod milkvetch

Medic milkichels

Crouching milksots

Rosy halsammour)

Palousc lhislle

Simoull clillhrake

fines-longute penslemon
Scientific Name

Stultus:":

cimesidensise

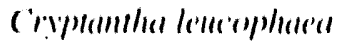

$S$

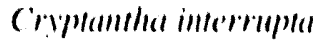

Cyperus rivelulas

Erigerom piperiames

Limose'lla acoulis

limdermia anagallide'a

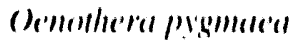

Cirserma de'miculater

Aremaria framklimii

1. thrmm!somii

Allium rohimsomii

Alliom suscillionicles

drememisia linalle'rema

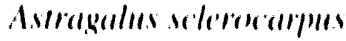

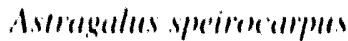

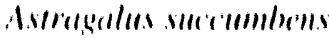

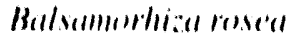

(iiverum herevifolium"

bellaras slalbella

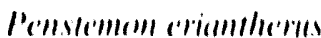

S

$S$

$S$

S

S

$\mathbf{S}$

$S$

MI

$\mathrm{M} 2$

M.3

M.3

$\mathrm{M} 3$

M.1

M.3

M.3

M.3

M.3

M.3

M3

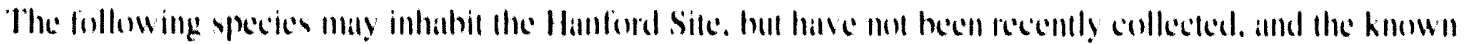

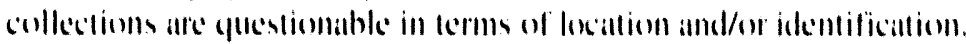

Palousc milknetch

fien-flowered blus-eyed Mary

('ugule tobicico
Astragalus arresellas

Collimsia sparsillora

Nicollialla cllte'llinalla
$S$

$S$

$S$

(a) Abbreviations: $S=$ Sensitive; laxa vulnerable an declining, and could hecome endangered or threalened withoul active management of remowal of theals: $M 1=$ Momilon (iromp l. Laxal for which there are

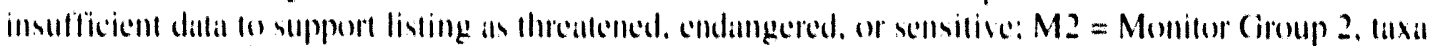

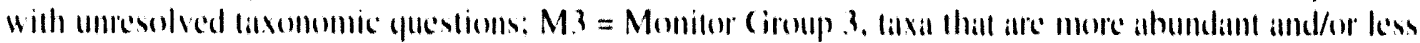
threallened than previously assumed. 


\section{References}

50) (TR 17. U.S. Fish and Wildlile Service, Departuneml of Interior. "I:ndangered and Thresilened Wildlite and

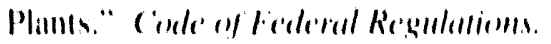

Washington Natlural Heritige Programe. 1940).

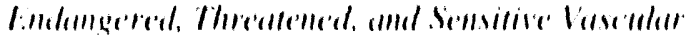

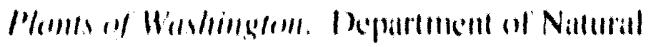

Resolureces, (Itympiar. Wirhinglon. 


\section{Distribution}

No, of'

('oplies

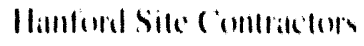

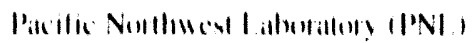

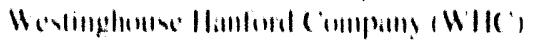

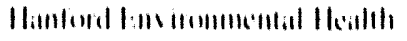

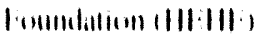

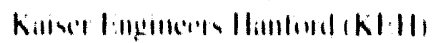

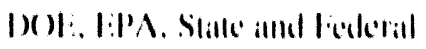

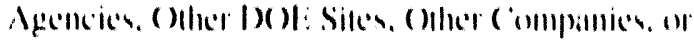

Nitclearl likilitic's

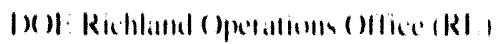

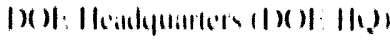

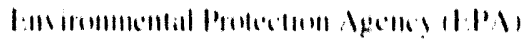

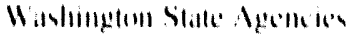

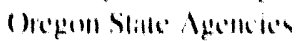

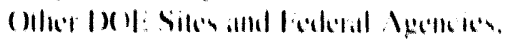

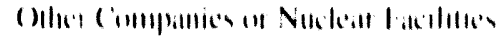

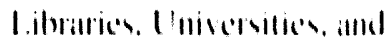

Shlows
No. of'

C'opleses

Public and likectedofflicials

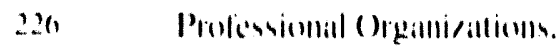

111 Public linterest cimoups. and

Nattive Americian (nflicilials

I'armers ('onlribuling Simllnkes

for Analysen

()whers ambl/mer Administratoms of Istands in the Hantond Reach of lle (inlumbia River

(1: roluding |)()):

Cimmonumity ()perithed

limirommonillial Surveillinter

Stallinll Mallingem

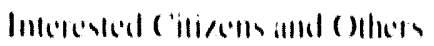

25

11

1)

7

5 

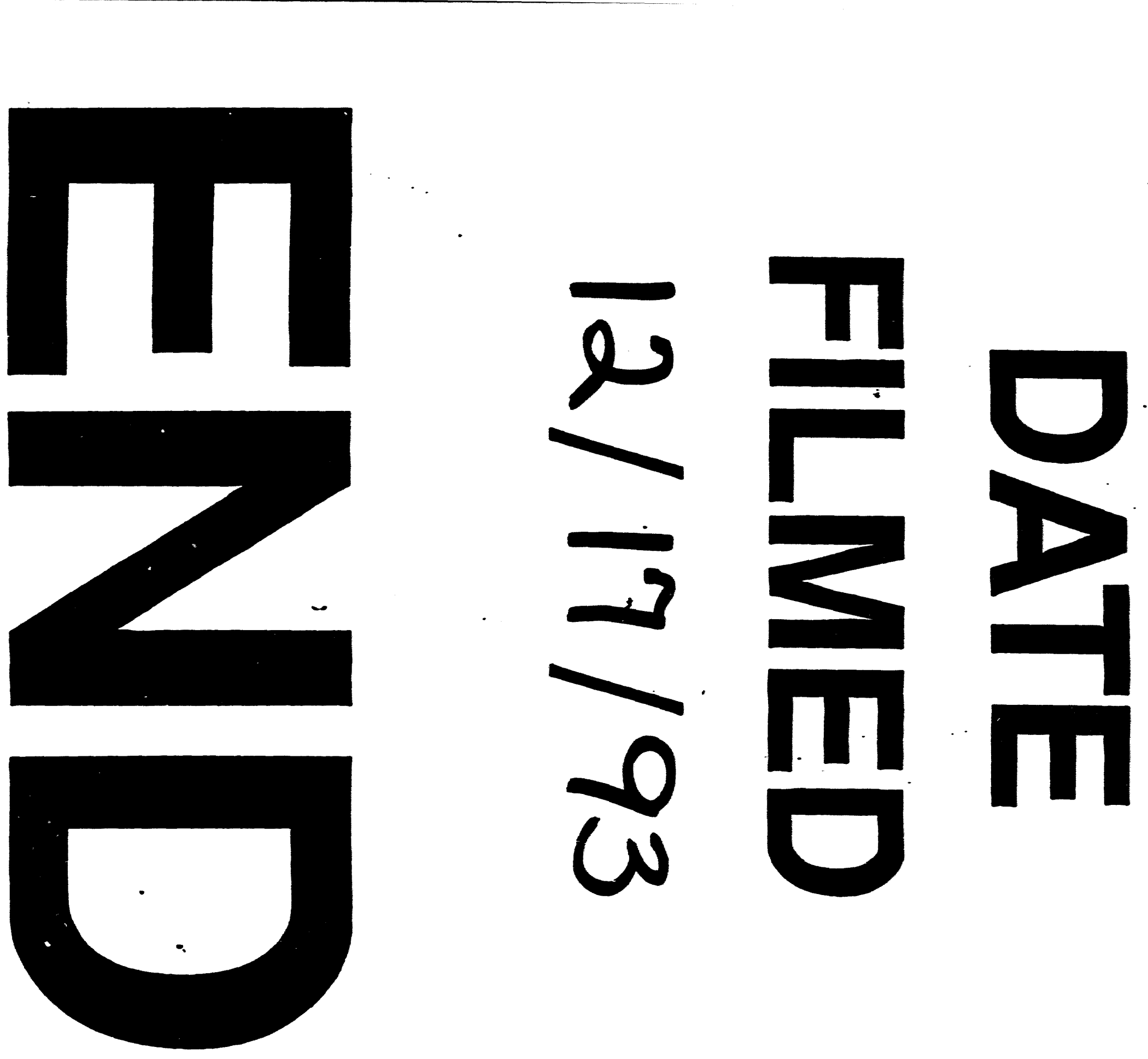
- 